TECEIVER

NOV 012020

OSTI

\title{
CsIX/TRU Grout Feasibility Study
}

\author{
S. J. Losinski \\ C. M. Barnes \\ B. K. Grover
}

Published November 1998

Idaho National Engineering and Environmental Laboratory Department High-Level Radioactive Waste Program

Lockheed Martin Idaho Technologies Company

Idaho Falls, Idaho 83415

\author{
Prepared for the \\ U.S. Department of Energy \\ Environmental Management \\ Under DOE Idaho Operations Office \\ Contract DE-AC07-941D13223
}


$\therefore \quad \div \quad \div \div$

, $\because$ 


\section{DISCLAIMER}

This report was prepared as an account of work sponsored by an agency of the United States Government. Neither the United States Government nor any agency thereof, nor any of their employees, make any warranty, express or implied, or assumes any legal liability or responsibility for the accuracy, completeness, or usefulness of any information, apparatus, product, or process disclosed, or represents that its use would not infringe privately owned rights. Reference herein to any specific commercial product, process, or service by trade name, trademark, manufacturer, or otherwise does not necessarily constitute or imply its endorsement, recommendation, or favoring by the United States Government or any agency thereof. The views and opinions of authors expressed herein do not necessarily state or reflect those of the United States Government or any agency thereof. 


\section{DISCLAIMER}

Portions of this document may be illegible in electronic image products. Images are produced from the best available original document. 


\begin{abstract}
A settlement agreement between the Department of Energy (DOE) and the State of Idaho mandates that liquid waste now stored at the Idaho Nuclear Technology Engineering Center (INTEC - formerly the Idaho Chemical Processing Plant, ICPP) will be calcined by the end of year 2012. This study investigates an alternative treatment of the liquid waste that removes undissolved solids (UDS) by filtration and removes cesium by ion exchange; followed by cement-based grouting of the remaining liquid into 55-gal drums. Operations are assumed to be from January 2008 through December 2012. The grouted waste will be contact-handled and will be shipped to the Waste Isolation Pilot Plant (WIPP) in New Mexico for disposal. The small volume of secondary wastes such as the filtered solids and cesium sorbent (resin) would remain in storage at the Idaho National Engineering and Environmental Laboratory for treatment and disposal under another project, with an option to dispose of the filtered solids as a remote-handled waste at WIPP.
\end{abstract}




\section{SUMMARY}

This report addresses regulatory requirements to process radioactive waste at the Idaho Nuclear Technology Engineering Center (INTEC) at the Idaho National Engineering and Environmental Laboratory (INEEL), proposes a process to prepare the waste for shipment to the Waste Isolation Pilot Plant (WIPP), and investigates the feasibility of two facility designs that will carry out the process.

There are two regulatory mandates for treatment of radioactive waste at INTEC: an October 16, 1995 Settlement Agreement between the DOE, the U.S. Navy, and the State of Idaho, and an April 3, 1992 Consent Order to a Notice of Noncompliance between the EPA and State of Idaho. Among other things, the Settlement Agreement requires that liquid sodium-bearing waste (SBW) be calcined by the end of year 2012. The Consent Order requires removal of liquid waste from the INTEC underground waste storage tanks. It also requires that DOE "cease use" of five of the eleven tanks, which are contained in pillar and panel vaults, by March 31,2009. The second modification to this Consent Order moved the date of "cease use" of these tanks up to June 30,2003. Closure of the remaining six tanks is scheduled to begin in October 2008. Because of the closure of these tanks, there will not be enough volume to hold all the liquid waste in the remaining tanks unless removal and processing of the waste has started on or before January 2008. This report investigates a waste treatment process that if used will meet this deadline.

There is, however, a need for an alternative to the calcining requirement because the New Waste Calcining Facility (NWCF) at INTEC is currently not operating, and this study assumes that operations will never resume. As an alternative to calcination, this study investigates treatment of the liquid waste by the Cesium Ion Exchange/Transuranic (CsIX/TRU) grout process. The CsIX/TRU grout process will remove undissolved solids (UDS) by filtration and remove cesium through ion exchange, followed by cement-based grouting of the remaining liquid into 55-gal drums. This operation will occur January 2008 through December 2012. The grouted waste will be shipped during this time to the WIPP in New Mexico for disposal.

This study investigated four possible absorbents for the primary wastes: grout, low-density silicabased absorbent (Ultrasorb), clay-based absorbent (Aquaset), and inorganic polymer absorbent (Acidbond). Each was evaluated on the following criteria: (1) process simplicity, (2) minimized solid waste volume, (3) mature development status, and (4) low cost. The evaluation selected grout for the waste form to use in this feasibility study because of the test data available for grouting of SBW, along with the results showing a higher volume for Ultrasorb, additional costs and a relatively small reduced volume for Aquaset, and the high absorbent cost and unknown long-term stability for Acidbond.

The WIPP Waste Acceptance Criteria (WAC) provide requirements for waste forms WIPP receives, all of which are met by the CsIX/TRU grout process waste form. The WIPP criteria most important to the process definition are:

- The waste must be contact-handled (200 mrem/hr or less at contact)

- The waste must contain more than $100 \mathrm{nCi} / \mathrm{g}$ TRU

- The waste must contain less than 2 liters of free liquid per 55-gal drum.

Environmental and regulatory aspects of the CsIX/TRU grout process were evaluated. The study concluded that the grouted waste form will meet WIPP waste acceptance criteria, although negotiations with WIPP will be necessary on issues of RCRA constituents, and characterization and inspection of waste. 
The study also considered disposal requirements for the small amount of secondary waste streams, such as the solids filtered from the liquid waste and the cesium-loaded ion exchange sorbent. These wastes will remain in storage at INTEC until they can be treated and disposed of under another project, such as a future Idaho vitrification plant to process the calcine or the Hanford vitrification plant. Another option is for the filtered UDS to be sent to WIPP for disposal.

This study investigated and prepared designs and facility layouts for two facility scenarios: one scenario constructs new buildings to house and operate all the process and waste storage equipment; the second scenario constructs new buildings for all operations except the most radiologically hazardous, which are housed instead in the NWCF building.

The study concluded that this process is feasible for the all-new-building scenario, with a total life cycle cost (escalated and discounted) of about $\$ 279$ million. However, the scenario of locating the process in the NWCF in time to meet the schedule requirements is not feasible, due primarily to the high personnel radiation exposure rate during rip-out and construction in the NWCF.

The process as presented in this report, although containing individual processes used separately in industry, has not been tested as described and hence has not been optimized. Because the primary goal is to design a feasible process, and because limited empirical data are available on the CsIX/TRU grout process concept, most assumptions and decisions have leaned toward the conservative or upper bounding case. Substantial reductions of both capital and operating costs and waste volumes can probably be achieved by simplifying and improving design concepts beyond those proposed here and acquiring supporting information. Research and testing are necessary to support this; the study recommends some specific future studies.

In order for the CsIX/TRU grout process concept and project to meet the 2012 date, it is imperative that actions begin soon on the following recommendations:

- Successful negotiation with DOE and/or the State of Idaho to reduce the number of Resource Conservation and Recovery Act (RCRA) listed hazardous waste numbers (HWNs) contained in the INTEC liquid waste from 105 to 4

- A ruling by DOE and/or NRC that SBW/Newly Generated Liquid Waste (NGLW) is not highlevel waste (HLW)

- Start and succeed in negotiations with WIPP to accept the waste

- Start the Line-Item Project funding cycle now to begin Conceptual Design no later than early FY-2001.

- Sample and analyze the Tank Farm solids (both suspended and settled), and have the Tank Farm Closure team determine what is to be done with the bulk settled solids in the tanks.

- Start the RCRA permitting process for the SBW CsIX/TRU grout treatment system.

- Start the National Environmental Policy Act (NEPA) process for SBW independently of the HLW \& FD (Facility Disposition) Environmental Impact Statement.

- Fund and aggressively pursue various studies investigating technical development issues. 


\section{ACKNOWLEDGEMENTS}

The authors wish to acknowledge the dedication, expertise, and input contributed by the following participants in this Feasibility Study:

Rick Adams - Capital Cost Estimates

Robert Carpenedo - Mechanical Engineer

Lynn Clapp - Architectural Engineer

Ron Dafoe - Mechanical Engineer

Howard Forsythe (NWCF evaluation)

Scott Jensen - Architectural/Structural Engineer

Cliff Olsen - Project Manager

Gary Stegen - HLW Technical Support

Robert Turk - Life Cycle Cost Estimates

Karen Williams - Project Lead 


\section{CONTENTS}

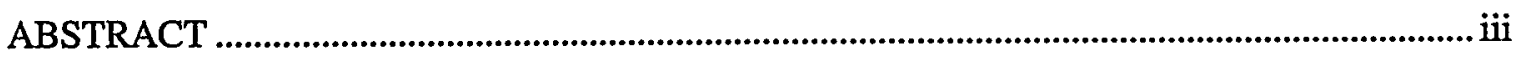

SUMMARY

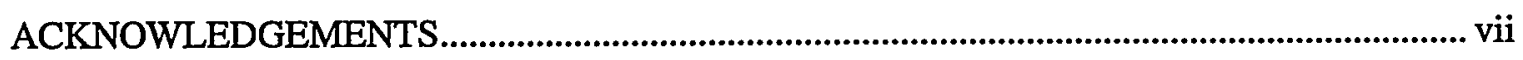

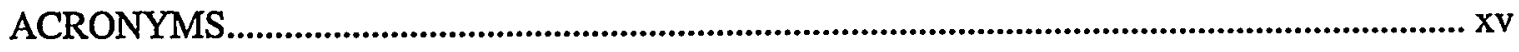

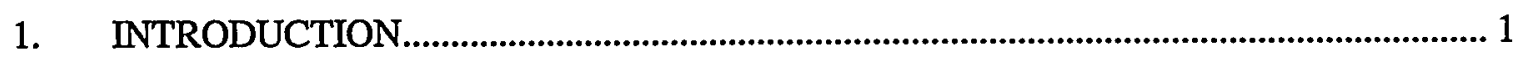

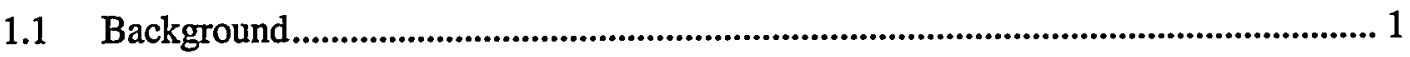

1.2 Objective and Scope of Work................................................................................... 2

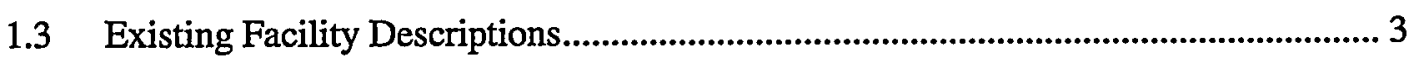

1.4 Concept Overview ...................................................................................................... 4

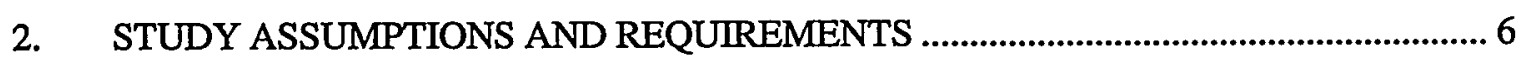

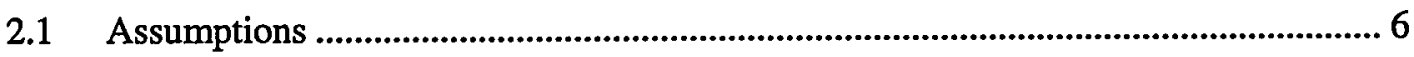

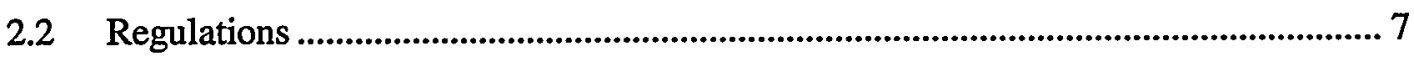

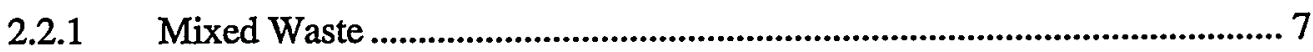

2.2.2 WIPP Waste Acceptance Criteria ........................................................... 8

2.3 Functional and Design Requirements ...................................................................... 11

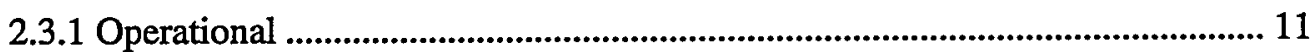

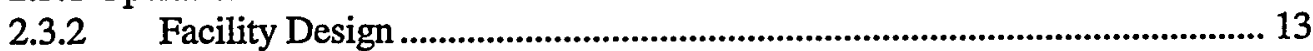

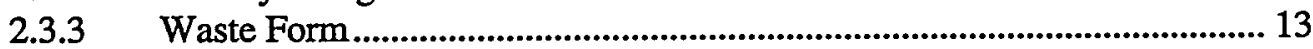

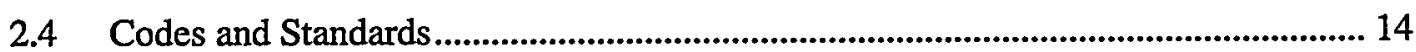

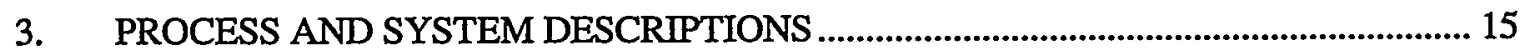

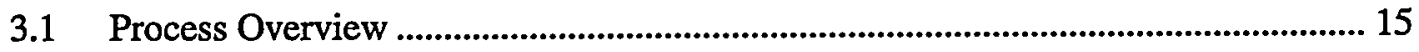

3.1.1 Process Description....................................................................................... 15

3.1.2 Waste Forms ........................................................................................ 16

3.1.3 Personnel Staffing ................................................................................... 19

3.1.4 Personnel Summary: ................................................................................. 20

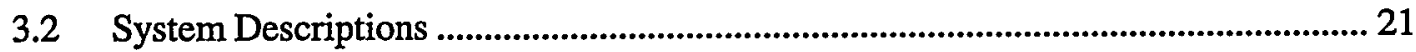

3.2.1 Tank Farm Liquid Waste Transfer.............................................................. 21

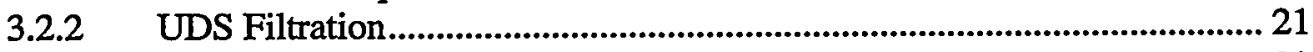

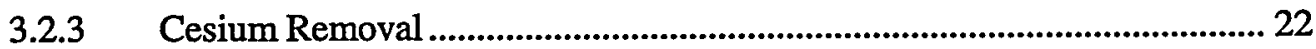




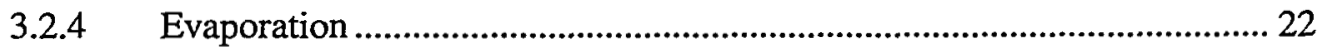

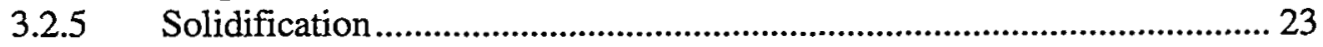

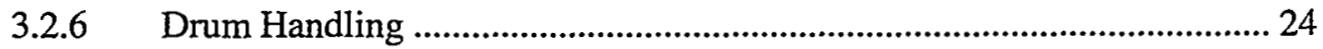

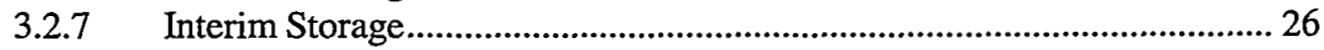

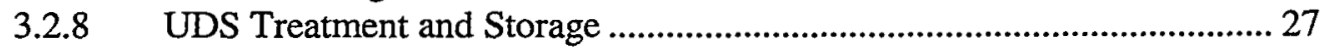

3.2.9 Spent Sorbent Treatment and Storage ........................................................ 28

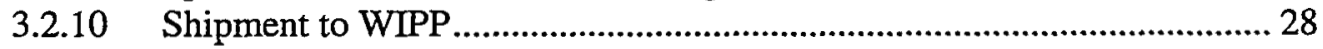

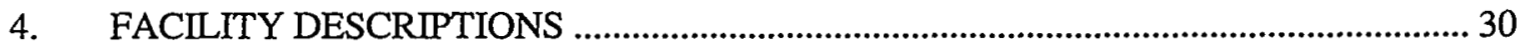

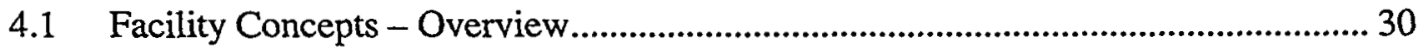

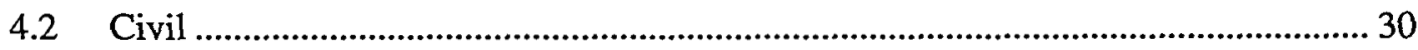

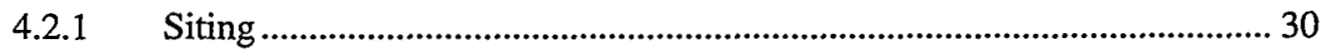

4.2.2 Surface Drainage, Erosion and Sediment Control ................................... 30

4.2.3 Soil Excavation and Shoring ............................................................... 31

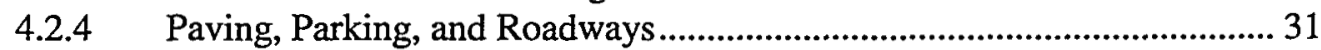

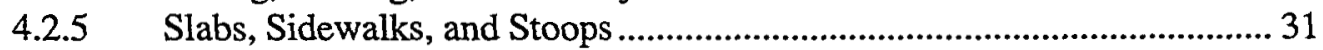

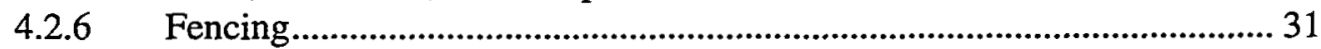

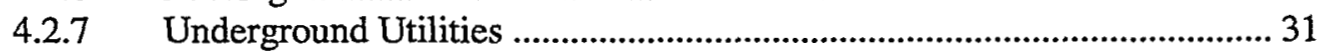

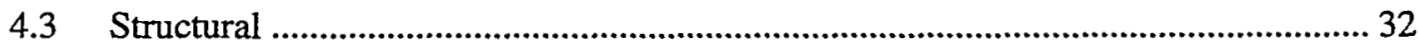

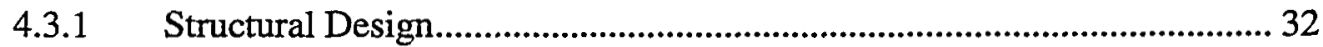

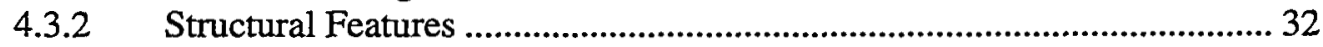

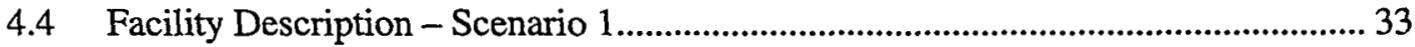

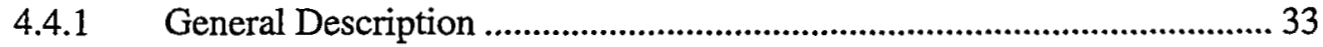

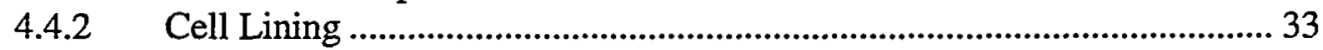

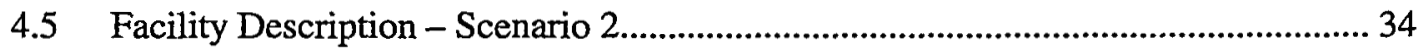

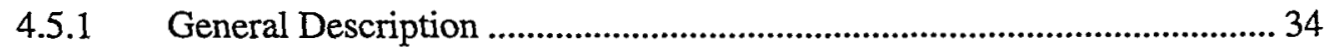

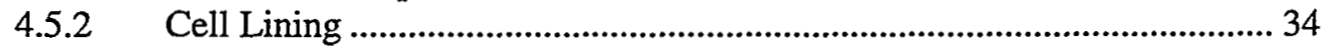

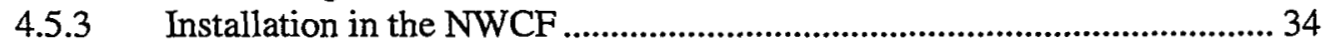

4.6 Interim Storage and TRUPACT Loading Facilities.................................................. 34

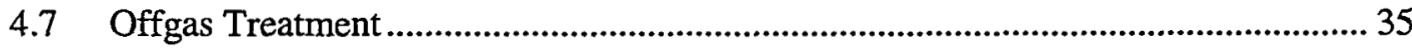

4.8 Heating, Ventilation, and Air Conditioning (HVAC)............................................ 35

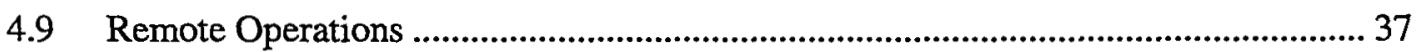

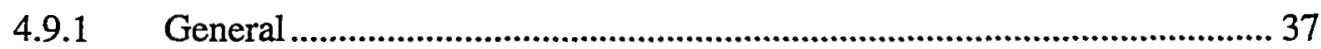

4.9.2 Equipment Used in Remote Operations .................................................. 38

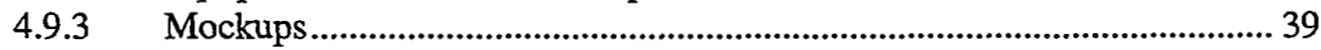

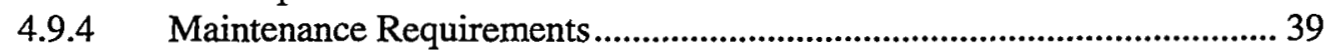

4.9.5 Remote Equipment Change/Cut-up/Disposal ......................................... 40

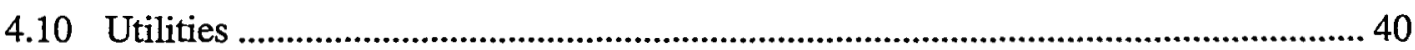

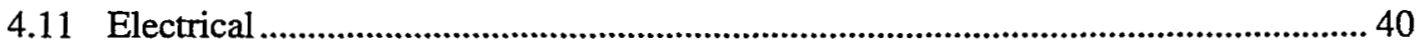

4.11.1 Available Electrical Utilities ...................................................................40 
4.11.2 Normal Power ............................................................................................ 41

4.11.3 Standby Power ............................................................................................ 41

4.11.4 Uninterruptable Power Supply (UPS) ........................................................... 42

5. METHOD OF PERFORMANCE ......................................................................................... 43

5.1 Design, Procurement, and Construction ..................................................................... 43

5.2 Work Breakdown Structure …………………................................................................ 43

$5.3 \quad$ Facility Decommissioning ................................................................................................ 43

6. ESTIMATED COST …......................................................................................................... 44

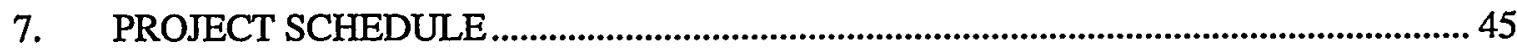

8. REQUIREMENTS AND ASSESSMENTS....................................................................... 48

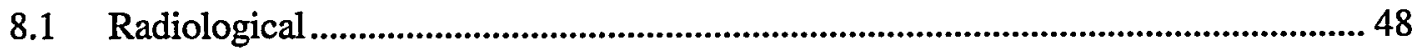

8.1.1 General ................................................................................................... 48

8.1.2 NWCF Radiation Exposure........................................................................ 48

8.2 Environmental......................................................................................................... 49

8.3 Quality .............................................................................................................. 50

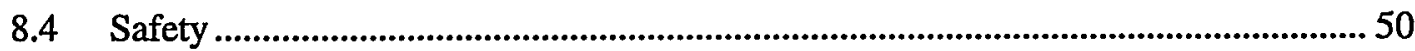

8.4.1 Facility Hazard Category and Performance Category...................................50

8.4.2 Industrial Safety ......................................................................................... 50

9. UNCERTAINTIES AND ISSUES ................................................................................ 53

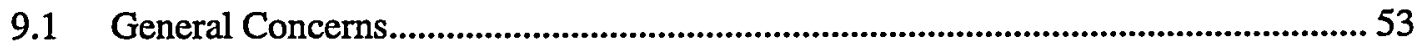

9.1.1 Behavior of UDS in the Process and Effects on Products............................ 53

9.1.2 TRU Waste Volume....................................................................................... 53

9.1.3 Ion Exchange Performance .......................................................................... 54

9.1.4 Liquid Waste Composition and Volume...................................................... 54

9.1.5 Processing and Disposition of UDS and Spent Sorbent............................... 54

9.1.6 Processing Grout Mixtures in Large-Scale Equipment.................................. 55

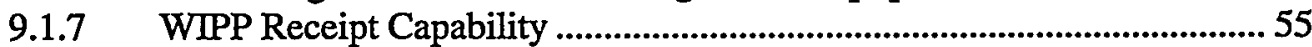

9.1.8 . Optimistic Schedule .................................................................................... 55

9.2 NWCF Facility Concerns .......................................................................................... 55

10. RECOMMENDATIONS ......................................................................................... 57

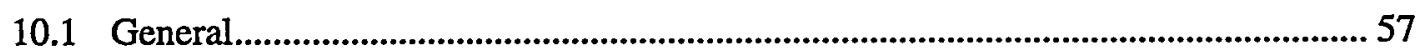

10.1.1 Negotiate Reduction in Number of HWNs in Waste ................................. 57

10.1.2 Obtain Ruling that Waste is not HLW .................................................... 57 
10.1.3 Begin Negotiations with WIPP .............................................................. 57

10.1.4 Start Line Item Project Funding Cycle..................................................... 57

10.1.5 Determine Fate of Solids in Tanks.................................................... 58

10.1.6 Start RCRA Permitting ................................................................... 58

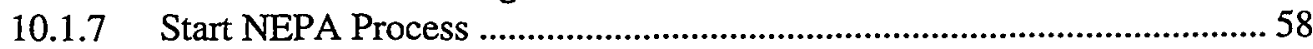

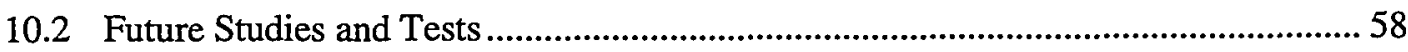

10.2.1 Tank Farm Liquid Waste Characterization ............................................. 58

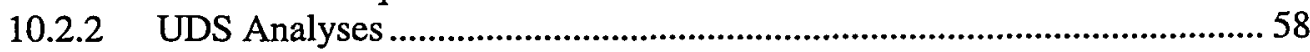

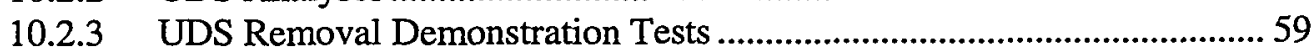

10.2.4 Ion Exchange Tests .............................................................................59

10.2.5 Final Waste Volume Optimization Studies..............................................5 59

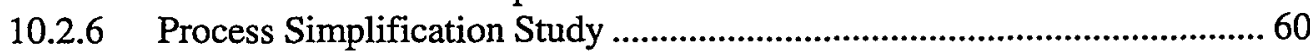

10.2.7 UDS and Spent Cesium Sorbent Processing ..........................................6 61

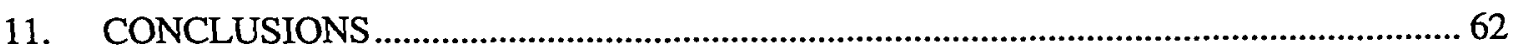

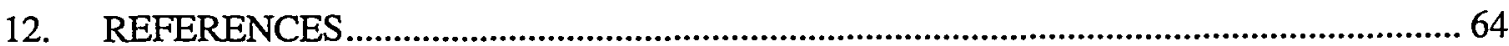

Appendix A - Assumption/Decision Table

Appendix B - Material Balance and Flow Diagrams

Appendix C - Cost Estimates

Appendix D - Drawings

Appendix E - Engineering Design Files

Appendix F - Equipment List

Appendix G - List of Applicable Regulations, Codes, and Standards

\section{FIGURES}

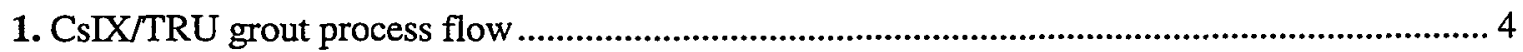

2. Work Breakdown Structure for the CsIX/TRU Grout Project ................................................ 43

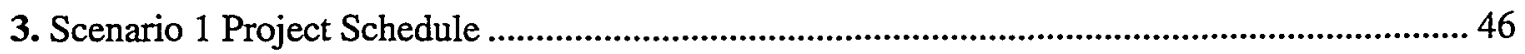

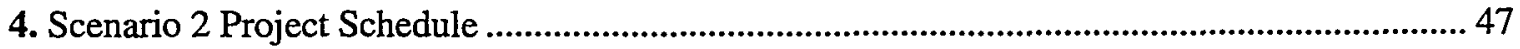




\section{TABLES}

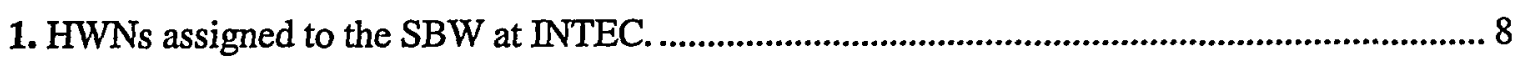

2. EPA Hazardous Waste Numbers Acceptable at WIPP........................................................

3. Overall Material Balance for Processing SBW and NGLW............................................. 16

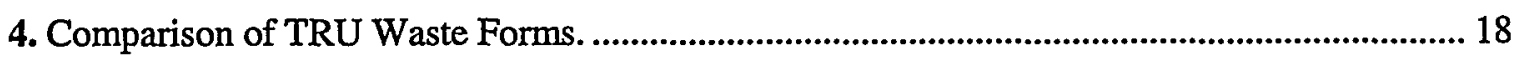

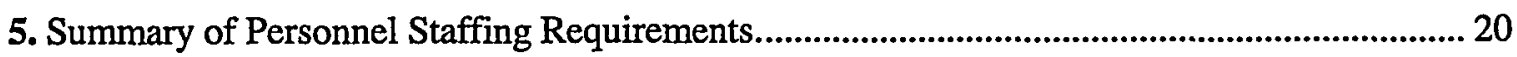

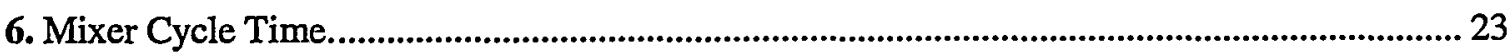

7. TRUPACT-II cask capacity for various types of containers .................................................... 29

8. Cost Estimates for the CsIX/TRU Grout Project............................................................................ 44 


\section{ACRONYMS}

$\mathrm{AE}$

CAO

CFR

$\mathrm{CH}$

CPP

CsIX

CST

D\&D

DOE

DOE-ID

EIS

EPA

EUSU

FD

FGE

GFE

HEPA

HF

HLLWE

HLW

HVAC

HWMA

HWN

ICPP

INTEC

INEEL

LDR

LET\&D

NGLW

NRC

NWCF

P\&V
(DOE-ID) Architectural Engineering (Standards)

Carlsbad Area Office

Code of Federal Regulations

Contact Handled

Chemical Processing Plant (recently renamed "INTEC")

cesium ion exchange

crystalline silicotitanate (a type of sorbent used to remove cesium)

. Decontamination and Decommissioning

U.S. Department of Energy

U.S. Department of Energy, Idaho Office

Environmental Impact Statement

U.S. Environmental Protection Agency

Electrical and Utility Systems Upgrade

Facility Disposition (EIS)

Fissile Gram Equivalent

Government-Furnished Equipment

High-Efficiency Particulate Air (filter)

uncomplexed hydrofluoric acid

High Level Liquid Waste Evaporator

High-Level Radioactive Waste

Heating, Ventilation, and Air Conditioning

Hazardous Waste Management Act (State of Idaho)

Hazardous Waste Number

Idaho Chemical Processing Plant (recently renamed "INTEC")

Idaho Nuclear Technology Engineering Center (previously named "ICPP")

Idaho National Engineering and Environmental Laboratory

land disposal restrictions

Liquid Effluent Treatment and Disposal

Newly Generated Liquid Waste

Nuclear Regulatory Commission

New Waste Calcining Facility

Piping and Valve (corridor) 


$\begin{array}{ll}\text { PC } & \text { Performance Category } \\ \text { PE } & \text { plutonium equivalent } \\ \text { PEWE } & \text { Process Effluent Waste Evaporator } \\ \text { QA } & \text { Quality Assurance } \\ \text { QAPjPs } & \text { Quality Assurance Project Plans } \\ \text { RCRA } & \text { Resource Conservation and Recovery Act } \\ \text { RH } & \text { Remote Handled } \\ \text { SBW } & \text { Sodium-bearing waste } \\ \text { SO } & \text { system operability (testing) } \\ \text { STD } & \text { Standard } \\ \text { SWB } & \text { (WIPP's) Standard Waste Box } \\ \text { TDOP } & \text { ten drum overpack } \\ \text { TRAMPACs } & \text { TRUPACT-II Authorization Methods for Payload Controls } \\ \text { TRU } & \text { transuranic } \\ \text { UCS } & \text { Utility Control System } \\ \text { UDS } & \text { undissolved solids } \\ \text { UPS } & \text { Uninterruptable Power Supply } \\ \text { WAC } & \text { waste acceptance criteria } \\ \text { WIPP } & \text { Waste Isolation Pilot Plant } \\ \text { WWIS } & \text { WIPP Waste Information System } \\ \end{array}$




\section{CsIX/TRU Grout Feasibility Study}

\section{INTRODUCTION}

This report addresses regulatory requirements to process radioactive waste at the Idaho Nuclear Technology Engineering Center (INTEC) at the Idaho National Engineering and Environmental Laboratory (INEEL), proposes a process to prepare the waste for shipment to the Waste Isolation Pilot Plant (WIPP), and investigates the feasibility of two facility designs that will carry out the process.

Treatment of radioactive waste at the Idaho Nuclear Technology Engineering Center (INTEC) at the Idaho National Engineering and Environmental Laboratory is mandated under a Settlement Agreement (October16, 1995) between the U.S. Department of Energy (DOE), the U.S. Navy, and the State of Idaho. Among other things, the Agreement requires that liquid sodium-bearing waste (SBW) be calcined by the end of year 2012. However, the New Waste Calcining Facility (NWCF) at INTEC is currently not operating, and this study assumes for conservatism (i.e., sizing of the CsIX TRU Grout plant) that NWCF operations will never resume.

An additional driver for scheduling of INTEC liquid waste management is the need to remove waste from the INTEC underground waste storage tanks. An April 3, 1992 Consent Order to a Notice of Noncompliance between the EPA and the State of Idaho requires that DOE "cease use" of five of the eleven tanks, which are contained in pillar and panel vaults, by March 31, 2009. The second modification to this Consent Order moved the date of "cease use" of these tanks up to June 30, 2003. Closure of the remaining tanks is scheduled to begin in 2008. Because of the early closure of these tanks, there will not be enough volume to hold all the liquid waste in the remaining tanks unless removal and processing of the waste has started early in 2008.

Because the NWCF is never expected to resume calcining operations, this study investigates an alternative treatment of the liquid waste by the Cesium Ion Exchange/Transuranic (CsIX/TRU) grout process. This process will remove undissolved solids (UDS) by filtration and remove cesium by ion exchange, followed by a cement-based grouting of the remaining liquid into 55-gal drums. Operation will occur January 2008 through December 2012. The grouted waste will be contact-handled and will be shipped to WIPP in New Mexico for disposal. A small volume of secondary wastes will be generated; the cesium sorbent will remain in storage at INTEC until it can be treated and disposed of under another project, such as the HLW Vitrification Facility. The filtered solids will be sent to WIPP or, if this is not possible, can also be stored at INTEC awaiting treatment and storage by another project such as the HLW Vitrification Facility.

\subsection{Background}

This study investigates treatment of the current INTEC tank farm liquid waste inventory, plus any newly generated liquid waste (NGLW) produced before January 2013 and stored in the INTEC tank farm. It assumes that the NWCF calciner will not be operational. The liquid waste is assumed to not be HLW.

The total volume of liquid waste that must be treated by the end of 2012 is about $1,200,000$ gal. After 2012, only about 5,500 gal of NGLW is expected to be generated per year. It would not be economical to operate the CsIX/TRU plant to treat this waste, as it amounts to only $2.4 \%$ of its normal operating capacity. Also, it is likely the waste produced after 2012 will not have sufficient TRU activity to meet the WIPP Waste Acceptance Criteria. The plant will not support other disposal options either; it does not treat Resource Conservation and Recovery Act (RCRA) wastes (e.g., remove mercury) nor does it prepare a "performance waste form" (i.e., with strong mechanical properties and capable of passing 
leach tests) or have the equipment to prove that it does (i.e., sampling or nondestructive examination stations). Therefore NGLW after 2012 would be kept in a storage tank for later treatment; other options are being considered for treatment of the waste generated after 2012.

To support tank closure schedule requirements, waste treatment operations must start on or before January 2008.

The WIPP Waste Acceptance Criteria (WAC) provides guidance for the waste form requirements, all of which are met by the CsIX/TRU grout process waste form. The WIPP criteria most important to the process definition are:

- The waste must be contact-handled ( $200 \mathrm{mrem} / \mathrm{hr}$ or less at the surface of the waste container)

- The waste must contain more than $100 \mathrm{nCi}$ TRU/g of waste matrix

- The waste must contain less than $0.5 \%$ (2 liters) of free liquid per 55-gal drum.

\subsection{Objective and Scope of Work}

The primary objectives of this study are to determine the feasibility of the CsIX/TRU grout process and estimate project cost and schedule necessary to implement the process.

This study targets the following two goals for treatment of the SBW and NGLW at INTEC:

- Remove the liquid waste from the tank farm tanks in time to meet the required 2009/2012 dates

- Bring the SBW and NGLW to final waste disposal forms which do not intermingle with the calcine high-level waste (HLW), and which meet the WIPP WAC.

The CsIX/TRU grout process and waste chemistry were evaluated in light of these goals. Various primary waste forms were investigated. The study also gave significant consideration to disposal requirements for secondary waste streams such as the solids filtered from the liquid waste, and the cesium-loaded ion exchange sorbent.

This study conservatively assumes that the NWCF will not start up again, so that the CsIX/TRU grout process must treat all liquid waste currently in the tank farm, plus all liquid waste that will be generated before December 31, 2012. Tank heel processing and tank closure will be accomplished by another project. For tanks closed in the operations timeframe of this study (i.e., through 2012), waste from those tank closure activities will be processed through this plant.

Environmental and regulatory aspects of the CsIX/TRU grout process were evaluated. A permitting strategy was developed to point out problem areas that might arise and make recommendations for their resolution. Permitting was included in the schedules and cost estimates.

This study investigated and prepared designs and facility layouts for two facility scenarios:

- Scenario 1 constructs new buildings to house and operate all the process and waste storage equipment;

- Scenario 2 constructs new buildings for all operations except the most radiologically hazardous, which are housed instead in the NWCF building. The use of the NWCF offers 
easier access to waste feed from the tank farm, use of the significant existing radiological shielding, and possible storage for the loaded sorbent and/or filtered UDS as well as their subsequent removal and transport to final processing. The study also evaluated whether installation of these systems inside the NWCF would trigger any problematic regulatory or environmental permitting requirements above that for a new facility.

\subsection{Existing Facility Descriptions}

INTEC - The Idaho Nuclear Technology and Engineering Center (INTEC), formerly the Idaho Chemical Processing Plant (ICPP), was constructed in 1951 and began operations in 1952. Within the confines of INTEC are all the facilities necessary to receive and store spent nuclear fuels, to process the fuels for recovery of uranium-235, and to handle waste generated by those functions. In 1992, however, DOE officially discontinued reprocessing fuel and changed the INTEC mission. The current mission includes receipt and storage of spent nuclear fuel and the isolation and solidification of the waste fission products from the spent reactor fuel recovery processes.

Tank Farm - The waste tank farm was designed and built to provide interim storage of highly radioactive liquid waste generated during fuel processing operations and process decontamination operations. The tank farm consists of eleven 300,000-gal underground storage tanks (VES-WM-180 through VES-WM-190). All of the tanks are connected by a system of lines, valves, and diversion boxes that allow transfers to and from other INTEC areas. Gravity, airlift plus gravity, or steam jet plus gravity supply the motive force for the transfers. Liquid wastes are stored in the tank farm until they can be converted to solid form in the NWCF.

NWCF - The NWCF began operations in 1982 and uses a fluidized-bed calcination process to convert highly radioactive liquid waste into a granular material called calcine. The calcine product is pneumatically transported to the Calcined Solids Storage Facilities (bin sets) for storage.

Evaporators - It is assumed that existing INTEC evaporator systems can be operated as necessary to support the SBW/TRU grout process:

HLLWE - The high-level liquid waste evaporator (HLLWE) is located in the NWCF. Many of the liquid waste solutions currently stored in the tank farm are not concentrated as much as feasible. As a result, the HLLWE was designed and built to concentrate liquid waste to maximize the storage capacity of the tank farm.

PEWE - Liquid wastes are collected and processed through the Process Equipment Waste Evaporator (PEWE). The PEWE concentrates this dilute waste within its operational capacity. This effectively reduces the volume of liquid waste, which must be stored and further treated. The evaporator concentrate is sent to the tank farm for interim storage. The condensate is sent to the Liquid Effluent Treatment and Disposal (LET\&D) facility for further processing.

$\underline{L E T \& D}$ - The LET\&D process concentrates the trace nitric acid in the PEW evaporator condensate by fractionating the waste into steam vapor and concentrated fractionator bottoms. The steam vapor is superheated and discharged to the INTEC stack for release to the environment. The concentrated bottoms are sent to the acid recycle system. 


\subsection{Concept Overview}

The basic CsLX/TRU grout process is described in EDF-CSIX-001 in Appendix B, and consists of the following major steps (see Figure 1):

1. Rough filtration of UDS from the liquid waste feed streams

2. Cesium removal by ion exchange (CsIX)

3. Neutralization as required before grouting

4. Grout mixing

5. Grout packaging in $\mathbf{5 5}$ gal drums

6. Sampling and inspection of grouted waste form to meet the WIPP WAC

7. Interim storage for grouted waste drums (since WIPP can not receive them as fast as they are produced)

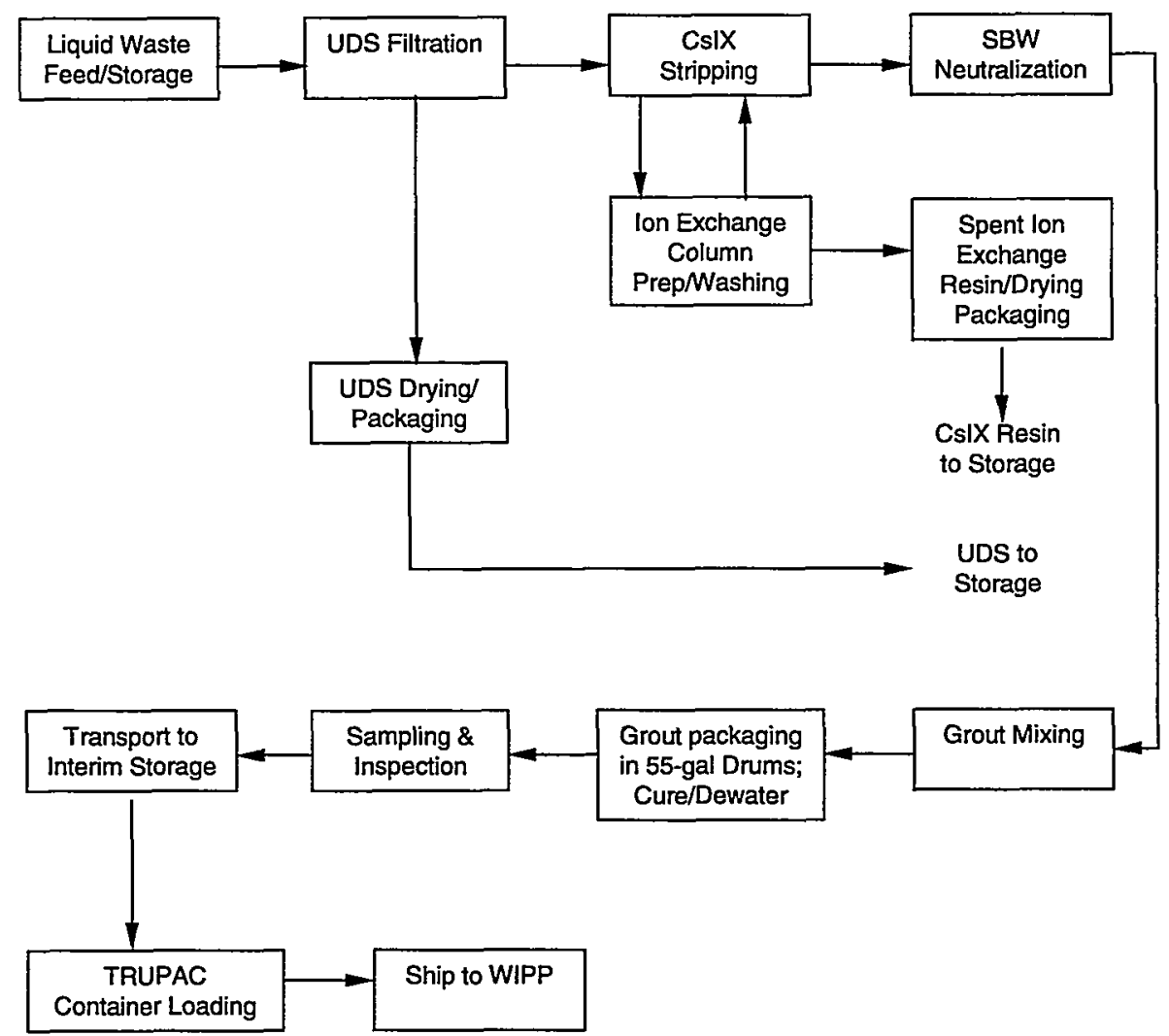

Figure 1. CsIX/TRU grout process flow.

For the first facility scenario, all of the above steps will be staged in new facilities. However, under the second facility scenario, the first two steps will be performed in the NWCF. 
Waste products from this process are expected to be:

- Dried waste UDS from liquid waste filtration - about $10 \mathrm{~m}^{3}$ (step 1)

- Dried waste sorbent from cesium removal from the liquid waste - about $10 \mathrm{~m}^{3}$ (step 2)

- Contact handled (CH) grouted TRU waste in 55-gal drums, from grouting and packaging the liquid waste-about $7,500 \mathrm{~m}^{3}$ in 38,000 drums (steps 4 and 5).

The first two waste types will be stored at INTEC until they can be treated and disposed of under another project such as the HIW Vitrification Facility. The third and primary waste type will be shipped to WIPP as quickly as INEEL shipping and WIPP receiving capabilities allow. An interim storage capacity of approximately 24,000 drums will be required at INTEC to accommodate the difference between the anticipated waste production rate of 146 drums/week and the anticipated WIPP receipt rate of 52 drums/week (see EDF-CsIX-002 in Appendix E for more details). 


\section{STUDY ASSUMPTIONS AND REQUIREMENTS}

\subsection{Assumptions}

See Appendix A for a list of the major assumptions and decisions made during this study. The following is a list of major process and design assumptions, garnered from Appendices $A$ and $B$, and from evaluation of environmental issues:

1. The liquid SBW and NGLW can be classified as TRU waste incidental to fuel reprocessing, and are not HLW (Assumption No. 1 in Appendix A).

2. The NWCF will not restart; waste inventories are as of April 1998 (No. 32 in Appendix A).

3. The waste will be assigned only four RCRA-listed hazardous waste numbers (HWNs), which will be acceptable at WIPP. (Three of these four RCRA-listed waste codes are already identified in Part A of the WIPP Permit; the fourth, U134 (uncomplexed hydrofluoric acid, HF), is not. This code must be delisted from the Idaho waste or it must be added to the WIPP Permit before the TRU waste can be shipped.) (No. 4 and 5 in Appendix A). In addition, the waste is assigned eight HWNs for the characteristically toxic metals (D004-D011).

4. A RCRA permitted interim storage facility will be required for the 55-gal drums filled with CH-TRU waste (No. 36 in Appendix A).

5. The WIPP WAC can be interpreted as-is (No. 14 in Appendix A). Any regulatory and volume issues involved with acceptance of the waste at WIPP will be addressed independently of this study.

6. UDS will only be removed from the tank liquid waste. No tank heel UDS will be removed. (No. 8 from Appendix A)

7. It is acceptable to continue to put liquid waste into the tank farm tanks, as it is generated, up to the end of 2012 (No. 21 from Appendix A).

8. The process will use an inorganic, commercially available sorbent, UOP's IONSIV IE-911 CST sorbent, to remove cesium (No. 2 from Appendix A).

9. The CsIX sorbent will not be regenerated (No. 3 from Appendix A).

10. Contamination of the CsIX sorbent (with UDS deposits, or by partial sorbtion of contaminants) need not be prevented except to keep the sorbent's cesium removal performance from degrading (No. 12 and 17 from Appendix A).

11. CsIX sorbent will be dried and stored in a pneumatically transferrable form by the CsIX/TRU Grout Project for future treatment and disposal under another Project. Filtered UDS will also be dried, but will be sent to WIPP for disposal or, if this is not possible, will also be stored for future treatment and disposal under another Project. (No. 16 and 18 in Appendix A). 
12. The treatment operating schedule is from January 1, 2008 through December 31, 2012. The process will operate 24 hours per day for 200 days per year. This assumes approximately $75 \%$ on-stream operating time at 75\% efficiency. (No. 27 and 28 in Appendix A).

13. The existing PEWE, HLLWE, and LET\&D fractionator will be available to concentrate NGLW through the end of 2012.

14. Following concentration in existing INTEC evaporators, no effort will be made to keep different types of NGLW waste streams separate from each other or from SBW in tank farm tanks.

15. The cost of either recycling or preventing off-spec waste product will be represented by selecting an off-spec scenario, such as the grout not setting up, and providing the system necessary to recycle the resulting off-spec waste product (No. 23 in Appendix A).

\subsection{Regulations}

This section outlines the major environmental regulations for the design of the CsIX/TRU grout process and associated activities. The primary drivers are the requirements for the final waste forms as identified in the WIPP WAC; this section provides the key waste form requirements. It draws from EDFWTS-003 and EDF-WTS-004 in Appendix E, and from Reference 1.

The permitting strategy consists of two parts that will have to be carried out in parallel, before construction can begin:

1. Permitting of the storage and treatment units necessary for the solids removal, Cs removal, TRU grouting, and storage as hazardous waste management units as required by the State of Idaho.

2. Negotiate with WIPP and the State of New Mexico by supplying documentation on a surrogate waste that the final TRU waste will meet the WIPP WAC. The demonstration would show that the waste will not be corrosive, ignitable, reactive, or contain free liquids $>0.5 \%$. In addition, the demonstration would show that the waste would generate head space gas $<500$ ppm TOC. This demonstration will be based on analytical data for the waste in the Tank Farm Facility (TFF) and surrogate testing of the organic waste loading that would be below the waste acceptance criteria.

\subsubsection{Mixed Waste}

The SBW stored in the tank farm is a radioactive and hazardous mixed waste. The hazardous constituents are subject to RCRA regulations as incorporated into the State of Idaho Hazardous Waste Management Act (HWMA) and the radioactive constituents are subject to DOE orders and regulations. The air emissions from the CsIX process will have to be below the emission limits for the INEEL.

The SBW is a mixture of decontamination and laboratory wastes that were corrosive at the point of generation and contain hazardous toxic metals and listed wastes. The listed waste constituents are designated by hazardous waste numbers (HWNs) F001, F002, F005, and U134. The characteristic constituents are designated by HWNs D002 and D004 through D011. Table 1 includes HWNs assigned to the tank farm waste, as well as 29 chemicals that are being evaluated to determine if the manner of their use would cause the application of additional listed HWNs to the waste. 
The evaluation being conducted for listed hazardous waste is assessing the past uses of chemicals to determine if any unused or off-specification chemicals were discharged to the PEW-TFF system that would result in assigning additional HWNs to the four listed HWNs. This determination consists of asking the laboratory personnel (1) was the chemical used at INTEC, (2) how was the chemical used, (3) in what form was the chemical discharged to the PEW-TFF system, and (4) was the chemical ever discharged as an unused or off-spec chemical product. The determination to date has identified chemicals used as solvents that require that the waste be assigned the F001, F002, and F005 HWNs. In addition, unused HF collected for purity analysis was complexed and discharged through lab drains into the system, which resulted in assigning the U124 HWN. To date, the usage and discharge of all the other chemicals was in a form that would not require the assigning of listed HWNs.

The major activities associated with the CsIX/TRU grout treatment process, which include UDS filtration, Cs removal, neutralization, grouting, and interim storage, will be subject to HWMA permitting and regulation as hazardous wastes.

The CxIX/TRU grout treatment process is expected to produce three wastes:

- Filtered UDS

- Cesium-bearing sorbent

- Grouted TRU waste.

These will all be considered listed waste, based on the EPA "derived-from" rule (i.e., residues derived from a listed waste must be managed as a listed waste). Treating a listed waste does not remove the listed designation or the requirement to manage the treated waste residue as a hazardous waste.

Table 1. HWNs assigned to the SBW at INTEC.

\begin{tabular}{|c|c|c|c|}
\hline D002 & *U002 & *U171 & *P098 \\
\hline D004 & *U003 & *U188 & *P104 \\
\hline D005 & *U012 & *U196 & *P105 \\
\hline D006 & *U044 & *U210 & *P106 \\
\hline D007 & *U080 & *U211 & *P119 \\
\hline D008 & *U108 & *U219 & *P120 \\
\hline D009 & *U122 & $* U 220$ & \\
\hline D010 & *U123 & *U225 & \\
\hline D011 & *U133 & *U226 & \\
\hline F001 & *U138 & *U227 & \\
\hline F005 & *U140 & $* \mathrm{U} 228$ & \\
\hline F002 & *U159 & & \\
\hline
\end{tabular}

* Hazardous waste constituents being evaluated for potential designation as listed waste in the SBW.

\subsubsection{WIPP Waste Acceptance Criteria}

This section discusses key parameters and outlines the steps necessary to qualify the TRU grout for disposal at WIPP. WIPP WAC are contained in Reference 2. The description of theWIPP WAC can be found in Reference 1.

TRU Waste Generator/Storage Sites participating in the National Transuranic Program must certify their TRU waste to the criteria and requirements defined in the WIPP WAC before transport and disposal at WIPP. Each participating Site shall submit copies of TRU Waste Certification Plans, TRUPACT-II 
Authorization Methods for Payload Control (TRAMPACs) and associated Quality Assurance (QA) plans, and TRU Waste Characterization Quality Assurance Project Plans (QAPjPs) to the Carlsbad Area Office (CAO) for review and approval.

Site-specific plans and procedures shall contain details of the processes, controls, techniques, tests, and other actions to be applied to each TRU waste payload container and/or waste stream. Method of compliance with each criterion and requirement shall be documented or specifically referenced. A waste stream is defined as waste material generated from a single process or activity that is similar in material, physical form, isotopic makeup, and hazardous constituents.

The primary reference document for establishing the RCRA waste characterization requirements for TRU mixed wastes is the WIPP Waste Analysis Plan. Sites must characterize their waste using the methods defined in the WIPP Waste Analysis Plan. The WIPP WAC identifies the RCRA HWNs acceptable at WIPP and the conditions for receipt of waste. Transuranic mixed waste shipped to WIPP does not have to be treated to meet the RCRA land disposal restrictions (LDR) for the constituents evaluated in the performance assessment. The performance assessment identifies the transuranic mixed wastes that are excluded from the RCRA LDR requirements when received at WIPP. The generator must report the type and quantity of hazardous constituents on the target analyte lists in the Site data package and the WIPP Waste Information System (WWIS) database. The target analyte list and HWNs acceptable at WIPP are identified in Table 2.

For compliance with 40 CFR 268, the State of New Mexico requires that the type and quantity of hazardous constituents on the target analyte lists be reported in the Site's data package and in the WWIS database. It is not clear at this time if hazardous constituents not identified in the performance assessment will require a new performance assessment, LDR treatment, be excluded by the Land Withdrawal Act, or require a separate petition to exclude. The current WIPP WAC and draft permit do not address the protocol necessary for the shipment and receipt of hazardous constituents not identified in Table 2. It is anticipated that a new performance assessment will be required as well as negotiations on concentration limits with the State of New Mexico, in order to allow the receipt of INTEC waste at WIPP.

Table 2. EPA Hazardous Waste Numbers Acceptable at WIPP.

\begin{tabular}{lll}
\hline D004 & D027 & F001 \\
D005 & D028 & F002 \\
D006 & D029 & F003 \\
D007 & D030 & F004 \\
D008 & D032 & F005 \\
D009 & D034 & P015 \\
D01 & D035 & \\
D01 & D036 & \\
D01 & D037 & \\
D01 & D038 & \\
D021 & D039 \\
D022 & D040 \\
D026 & D043 & \\
\hline
\end{tabular}




\subsubsection{WIPP WAC Required Chemical and Physical Properties for Mixed TRU Waste}

1. Free liquids $<0.5 \%$

2. Not corrosive, reactive, and ignitable

3. Not pyrophoric

4. Not have contained gases

5. Total VOCs in the headspace of a payload container is limited to $500 \mathrm{ppm}$.

\subsubsection{WIPP WAC Nuclear Property Limits for CH-TRU Waste}

1. $>100 \mathrm{nCi}$ TRU/g of waste matrix

2. $<200 \mathrm{mrem} / \mathrm{hr}$ contact dose rate per canister

3. $<200$ g Pu-239 fissile gram equivalent (FGE)/55-gal drum

4. <325 g Pu-239 FGE per Standard Waste Box (SWB)

5. $<325$ g Pu-239 FGE per ten-drum overpack (TDOP)

6. $<325$ g Pu-239 FGE/TRUPACT-II

7. Untreated CH-TRU waste shall not exceed 80 Plutonium Equivalent Curies (PE-Ci) of activity per 55-gal drum or $130 \mathrm{PE-Ci}$ of activity per SWB. 55-gal drums containing solidified/vitrified CH-TRU waste shall not exceed $1800 \mathrm{PE}-\mathrm{Ci}$ of activity per drum.

\subsubsection{WIPP WAC Nuclear Property Limits for RH-TRU Waste}

1. $>100 \mathrm{nCi}$ TRU/g waste matrix

2. $<1,000 \mathrm{rem} / \mathrm{hr}$ per canister at contact

3. $<1,000 \mathrm{Pu}-239$ equivalent activity $\mathrm{PE}-\mathrm{Ci} /$ canister

4. $<325 \mathrm{~g} /$ cask Pu-239 FGE

5. $<200 \mathrm{mrem} / \mathrm{hr}$ external dose rate at the surface of a loaded RH-TRU 72-B Cask

6. $<10 \mathrm{mrem} / \mathrm{hr}$ dose rate at the distance of 2 meters from a loaded RH-TRU 72-B Cask

7. No more than $5 \%$ of the RH canisters received at the WIPP are allowed to have dose rates $>100$ $\mathrm{rem} / \mathrm{hr}$. Neutron contributions are limited to $270 \mathrm{mrem} / \mathrm{hr}$. 


\subsection{Functional and Design Requirements}

\subsubsection{Operational}

\subsubsection{Schedule and Processing Rates}

The 10/16/98 Settlement Agreement between DOE, the U.S. Navy, and the State of Idaho requires that SBW be calcined by the end of 2012 . This project investigates treatment of the waste by the CsIX/TRU grout process in time to meet the same schedule. In addition, the process must remove waste from the INTEC tank farm in a timeframe and at a rate that meets the tank closure deadlines, as discussed below:

The proposed schedule for tank closure ${ }^{3}$ is as follows:

\begin{tabular}{|c|c|c|}
\hline Tank & Begin closure & Complete closu \\
\hline WM-182 & Oct 1, 1999 & Sept 30, 2005 \\
\hline WM-183 & Oct 1,2004 & Sept 30, 2006 \\
\hline WM-184 & Oct 1,2005 & Sept 30, 2007 \\
\hline WM-185 & Oct 1,2006 & Sept 30, 2008 \\
\hline WM-186 & Oct 1,2007 & Sept 30, 2009 \\
\hline WM-180 & Oct 1,2008 & Sept 30, 2010 \\
\hline WM-181 & Oct 1, 2009 & Sept 30, 2011 \\
\hline WM-187 & Oct 1,2011 & Sept 30, 2013 \\
\hline WM-188 & Oct 1,2012 & Sept 30, 2014 \\
\hline WM-189 & Oct 1,2013 & Sept 30, 2015 \\
\hline WM-190 & Oct 1,2014 & Sept 30, 2016 \\
\hline
\end{tabular}

The first five tanks listed above are contained in pillar and panel vaults. Use of these tanks must cease by June 30, 2003. According to the above schedule, closure for three tanks will have been completed by September 2007, closure for two other tanks will have been started, and the remaining six tanks will still be available to hold waste. Since each tank has an operational capacity of approximately 285,000 gal, the available volume by October 2007 will be about 1,710,000 gal. However, WM-190 is reserved for Type 2 NGLW that has a lower radionuclide content than the waste in the other tanks, which contain SBW and Type 1 NGLW. Thus only 5 tanks, or 1,425,000 gal of capacity, will be available for SBW and Type 1 NGLW by October 2007. By October 2008, the available capacity for SBW and Type 1 NGLW will be reduced to four tanks, or about $1,140,000$ gal.

The volume of existing SBW, after concentration by the HLLWE, will be about $1,000,000$ gal. The projected volume of NGLW generated from 1998 through 2008, after evaporation, is about 200,000 gal. Thus a tank capacity of at least $1,200,000$ gal is needed on January 1,2008 , and processing must begin 
early in 2008 to reduce the waste inventory to a maximum of $1,140,000$ gal by October 2008 . If the waste volume generation estimates and the HLLWE estimates are accurate, processing could begin at the latest by about May 2008, with enough tank capacity still available to meet demand. However, considering the potential inaccuracies of these estimates and to allow for operating tank capacities slightly less than 300,000 gal per tank, processing should begin by January 2008. Also, the processing rate of the CsIX/TRU grout process must be sufficient to support the closure schedule of one tank per year subsequent to 2009 , and the corresponding loss of 300,000 gal of capacity per year.

To meet this requirement, the CsLX/TRU plant must provide operating capability such that the projected amount of waste is less than the available storage volume:

\section{Tank Farm Volume, gal}

$\begin{array}{lcc}\text { Date } & \text { Available Storage } & \text { Projected Waste* } \\ \text { Oct 1, 2007 } & 1,425,000 & 1,200,000 \\ \text { Oct 1, 2008 } & 1,140,000 & 1,000,000 \\ \text { Oct 1, 2009 } & 855,000 & 750,000 \\ \text { Oct 1, 2010 } & 855,000 & 520,000 \\ \text { Oct 1, 2011 } & 570,000 & 290,000 \\ \text { Oct 1, 2012 } & 285,000 & 60,000 \\ \text { Oct 1, 2013 } & 0 & 0\end{array}$

*Assuming CsIX/TRU Grout Plant operations 24 hrs/day at 200 days per year

\subsubsection{NGLW Treatment and Storage}

From 1998 through 2012, approximately 470,000 gal of Type 1 NGLW and 4,100,000 gal of Type 2 NGLW will be generated. To avoid the costs of additional tankage and replacement evaporators, the existing INTEC evaporators are needed to concentrate this NGLW.

Following concentration in existing INTEC evaporators, no effort will be made to keep different types of NGLW waste streams separate from each other or from SBW in tank farm tanks.

\subsubsection{Process Systems and Equipment}

This study assumes that calcining operations in the NWCF will never restart and that degradation of calciner operability will be allowed in order to accommodate the installation of CsIX/TRU grout process equipment in Scenario 2. However, the following NWCF systems must remain operational:

- High Level Liquid Waste Evaporator (HLLWE)

- Filter Leach System 
- Decontamination Facility

- Building HVAC

- Vessel Off-gas

- Waste Water Collection

- Pneumatic Transfer System.

In addition, the CsIX/TRU process equipment must meet the following requirements:

- Valves, pumps and other mechanical equipment should have remote maintenance and operating capability.

- The UDS container storage location must have the capability to manage and store up to 50 radiologically shielded 55-gal drums

- The most radiologically hazardous equipment in the process, i.e., the CsIX columns, spent sorbent preparation tank, spent sorbent drier, spent sorbent storage tank, UDS slurry storage tank, and UDS drier must have adequate radiological shielding.

\subsubsection{Functional}

To produce a contact-handled waste form, radioactive cesium must be removed from the liquid waste before generating the final waste form.

TRU removal or concentration is not required to meet the WIPP WAC, which requires waste with a TRU concentration greater than $100 \mathrm{nCi} / \mathrm{g}$. The liquid waste contains approximately $1,200 \mathrm{nCi} / \mathrm{g}$ on average.

The CsIX/TRU grout process shall be capable of drying and storing the UDS and spent sorbent waste streams so that they are available for future pneumatic transfer by another project. In Scenario 2, the spent sorbent storage tank must be connected to the NWCF pneumatic transfer system for future removal of the dried spent sorbent, while in Scenario 1, a new pneumatic transfer system must be provided.

\subsubsection{Facility Design}

Facilities shall be provided to accommodate the CsIX/TRU process and related activities, located at INTEC, as close as possible to the INTEC tank farm.

Process facility feasibility designs shall be provided for two different approaches, one using all new facilities and the second using the existing NWCF to house at least the most radioactively hazardous parts of the CsIX/TRU processes.

\subsubsection{Waste Form}

The main waste product generated by the CsIX/TRU process must meet the WIPP WAC for contact handled waste (there is only limited space available at WIPP for remote handled waste). The process, including its waste-form characterization systems, must be configured such that it will be qualified to meet the WIPP WAC with minimal sampling of the waste-form product itself. 
The anticipated secondary waste streams of filtered UDS and cesium-loaded sorbent must be stored in a form that is pneumatically retrievable/transferable for future processing under another project.

\subsection{Codes and Standards}

The regulations, codes, and standards listed in Appendix $\mathrm{G}$ are those presently assumed to be applicable to the CsIX/TRU Process facilities. The facilities will be designed and constructed in accordance with the DOE-ID Architectural Engineering (AE) Standards, which invoke most of the required codes and standards. Some special systems will be designed to codes and standards not listed in the AE Standards.

Requirements for safety analysis, environmental evaluation and protection, project management, quality assurance and other activities necessary to support the design and construction of the facility are generally not included in the AE Standards, but the related references are included in Appendix G.

The following EDFs (Appendix E) contain additional information on laws, regulations, codes, and standards that may be applicable to this project.

- EDF-WTS-003, Regulatory Requirements for the Design, Construction, and Operations of the ICPP Proposed Waste Processing Facilities.

- EDF-WTS-004, Regulatory and Design Requirements for Waste Treatment Facilities. 


\section{PROCESS AND SYSTEM DESCRIPTIONS}

Section 3.1 contains a brief description of the CsIX/TRU grout process and summary material balance tables. The material balance details are provided in EDF-CsIX-001 in Appendix B. Section 3.2 describes the process equipment.

\subsection{Process Overview}

\subsubsection{Process Description}

SBW and NGLW will be transferred by steam or air jet from the INTEC tank farm to Liquid Waste Feed Tanks. From there the waste will be pumped through filters to remove UDS. Periodically these filters will be backflushed to transfer the UDS to a system that will dry them and place them in an interim storage location. The liquid waste will then pass through two in-series CsIX columns before entering the CsIX Effluent Hold Tanks.

The CsIX columns contain a crystalline silicotitanate (CST) sorbent that absorbs cesium ions in the liquid waste. The CST sorbent, which contains 10-25\% silicon dioxide, 25-40\% titanium dioxide, 10 $20 \%$ sodium oxide and $15-25 \%$ of a proprietary material, ${ }^{4}$ is inorganic, making it resistant to chemical and radiation degradation. It is also compatible with ultimate disposal by vitrification. The sorbent has been developed for application to DOE wastes for approximately the last 18 years. ${ }^{4}$ CST sorbent, which has a large affinity for cesium, even in the presence of high concentrations of sodium and high acidity, has recently been tested with SBW ${ }^{5,6}$ The absorption of cesium on the sorbent is irreversible, ${ }^{4}$ hence the cesium loaded sorbent becomes a secondary waste in the CsLX/TRU process.

The CsIX columns are designed to remove $99.9 \%$ of the cesium in the waste feed. The design includes three columns, two operating in series while the third is being loaded, unloaded, or is on standby. When breakthrough in the first of the two in-series columns is reached (and caught by the second column), the waste flow is stopped. The first column is valved out and the second column rotates to the lead location with the third column (recently recharged) rotated to the lag position. The spent sorbent in the first column is sluiced out and replaced with fresh sorbent. After removal, the slurry of spent sorbent is filtered, with liquid and fines being returned to an operating column, and the sorbent solid particles going to a drier. The sorbent then flows by gravity to its interim storage location in a spent sorbent storage tank.

The CsIX column effluent is pumped in batches from the CsIX Effluent Hold Tank to the Grout Feed Tank. In the Grout Feed Tank, solid calcium oxide is added to partially neutralize the waste. The neutralized waste is then fed to the grout mixer, where it is mixed with Portland cement, blast furnace slag, flyash, and cure-rate control chemicals. From the mixer, the uncured grout is transferred to 55-gal drums. The grout is allowed to cure in the drums and then sent through a dewatering process. The filled drums are subsequently placed in interim storage awaiting inspection and shipment to WIPP (see Figure 1 for an illustration of the process).

Table 3 summarizes the overall material balance for processing the SBW and all NGLW, including that generated through 2012 . 
Table 3. Overall Material Balance for Processing SBW and NGLW.

\begin{tabular}{lll}
\hline Feeds & Liters & $\mathrm{Kg}$ \\
\hline Composite SBW & $3.65 \mathrm{E}+06$ & $4.79 \mathrm{E}+06$ \\
Concentrated NGLW & $7.42 \mathrm{E}+05$ & $9.27 \mathrm{E}+05$ \\
Jet dilution water & $3.82 \mathrm{E}+05$ & $3.82 \mathrm{E}+05$ \\
Other water & $3.28 \mathrm{E}+05$ & $3.28 \mathrm{E}+05$ \\
CaOs & & $2.30 \mathrm{E}+05$ \\
Grout additives & & $7.97 \mathrm{E}+06$ \\
Cs Sorbent & & $8.97 \mathrm{E}+04$ \\
\hline Total feeds & & $1.46 \mathrm{E}+07$ \\
\hline
\end{tabular}

\section{Products}

\begin{tabular}{lcc}
\hline Grouted waste & $7.53 \mathrm{E}+06$ & $1.43 \mathrm{E}+07$ \\
UDS waste & $1.02 \mathrm{E}+04$ & $1.43 \mathrm{E}+04$ \\
Spent sorbent & $8.99 \mathrm{E}+03$ & $8.99 \mathrm{E}+03$ \\
Condensate to PEWE & $3.28 \mathrm{E}+05$ & $3.28 \mathrm{E}+05$ \\
\hline Total products & & $1.46 \mathrm{E}+07$
\end{tabular}

\subsubsection{Waste Forms}

A very brief evaluation was performed to determine the form of the TRU waste to be used in this study. The criteria used to select the waste form included:

- Minimized solid waste volume

- Low cost

- Process operational simplicity. Does the waste form require a mixer? Does the waste form require neutralization?

- Mature development status. Is there data to support waste loading, chemical interactions with the solidification media, final waste density, etc.? 
The four waste forms considered and their evaluation are as follows:

- Absorbed waste on a low density silica-based absorbent

The potential advantage of a low-density, silica- or polymer-based absorbent is process operational simplicity. A waste container would be filled with a given amount of absorbent and liquid waste would be added. The process would feature no mixing, no neutralization, quick curing, and little decontamination of equipment. Based on a brief review of commercial absorbents, Ultrasorb 244, a product of the Molton Corporation, was selected as representative of a high-capacity, inorganic absorbent for aqueous liquids. Ultrasorb 244, a granular diatomaceous earth, absorbs approximately $150 \%$ of its weight. Based on product literature for Ultrasorb, neutralization would not be required, as the product is stable with all acids except HF.

- Absorbed waste on an inorganic polymer absorbent

Inorganic polymer absorbents offer even higher absorption capacities, although at a higher cost. ACIDBOND A660, marketed by Nochar, was recently tested using INEEL wastes, including an SBW simulant. The tests showed a waste loading of 80-85 wt\% could be achieved, resulting in a volume increase of about $10 \%$. The resultant waste is a soft, rubber-like solid. Although the vendor had reported the product was an inorganic polymer, subsequent investigation revealed that it is a polyacrylate (organic)-based polymer. When nitrate salt solutions are solidified with AcidBond A660 and allowed to dry, the resultant material is readily ignitable. Heating tests of absorbed SBW suggest that the polymer undergoes a reaction with nitric acid present in SBW. Because of concerns about flammability and product stability, stabilization of SBW with ACIDBOND A660 was not recommended.?

- Absorbed waste on a clay-based absorbent

The potential advantage of a clay-based absorbent is a lower final waste volume. The Aquaset/Petroset products of Fluid Tech, Inc. were selected as representative of a clay-absorbent. Fluid Tech products have been tested and/or used in numerous nuclear waste applications, including some at the INEEL. Testing has not been done for solidification of SBW. For aqueous wastes containing a high level of dissolved salts, Fluid Tech literature recommends using 125$175 \mathrm{lb}$ of Aquaset II per $43-47$ gal of waste. For acidic wastes, neutralized to a pH of 5-10, the formulation is similar, 125 $175 \mathrm{lb}$ of a combination of Aquaset $I I$ and Petroset to $42-47 \mathrm{gal}$ of waste. Mixing of the waste with Aquaset/Petroset would be required.

- Grout

Grouting is a known technology with easily available and relatively inexpensive ingredients. An INEEL development program has been exploring the use of "performance" (high strength mechanical properties, leach resistant) grouting for solidification of INTEC liquid wastes. Before grouting in the CsIX/TRU grout process, the liquid waste would be neutralized to a specified set point of $\mathrm{pH} 1.3$ by mixing it with calcium oxide (lime). Solid ingredients-cement, blast-furnace slag, and fly ash - would then be added and blended with the liquid waste to form a grout product, which would be poured into 55-gal drums.

Table 4 compares the four waste forms. 
Table 4. Comparison of TRU Waste Forms.

\begin{tabular}{lcccc}
\hline & Grout & Ultrasorb & Aquaset & Acidbond \\
\hline Waste Volume, $\mathrm{m}^{3}$ & 7,500 & 12,500 & $6,100-6,800$ & 5,300 \\
Neutralization req'd & Yes(1) & No $(2)$ & Yes(3) & No \\
Mixing Required & Yes & No & Yes & No \\
Cost of absorbent & $\$ 430,000$ & $\$ 465,000$ & $\$ 1,800,000$ & $\$ 32,000,000$ \\
Test data available & Yes & No & No & No
\end{tabular}

Notes

1. Neutralization to $\mathrm{pH}$ of 1.3 with $\mathrm{CaO}$

2. Neutralization not needed except possibly to tie up HF

3. Neutralization to $\mathrm{pH} 7$ with $\mathrm{NaOH}$

The comparison shows that Ultrasorb results in a much higher volume of waste than the other alternatives. The range of waste volumes shown for Aquaset are based on (1) 45 gal of waste with $175 \mathrm{lb}$ of absorbent per drum and (2) $41 \mathrm{gal}$ of waste with $250 \mathrm{lb}$ of absorbent per drum. The higher waste loading is based on Fluid Tech literature. The lower loading is based on treatability study data for concentrated nitric acid waste performed at Sandia. ${ }^{4}$ As shown in Table 4, the volume of waste, when solidified with Aquaset/Petroset, is potentially 14-22\% less than when grouted; however, no equipment savings would be expected, and the cost of the solidification agents would be 3-5 times more than that of the grout ingredients.

Based on the limited information presently available for ACIDBOND, it appears that its use would result in about $30 \%$ less final waste volume than a grout product. Data is needed to evaluate the long-term stability of the product, its chemical interactions with SBW, the ability to vitrify the absorbed waste, and the potential for radiological decomposition or hydrogen generation.

The development program for grouting INTEC liquid wastes has focused on producing a highstrength grout, ${ }^{5.6}$ however, the WIPP WAC does not contain a strength requirement. It is expected that with further development, with the goal of minimizing waste volume and not being constrained by a grout strength requirement, the volume of final waste can be reduced to the range shown above for Aquaset or ACIDBOND. Grout was selected for the waste form to use in this feasibility study because of the test data available for SBW grouting, a higher expected volume for Ultrasorb, additional costs and a relatively small reduced volume for Aquaset, and the high absorbent cost and unknown long-term stability for ACIDBOND. Based on the SBW grouting data, ${ }^{5}$ there is high confidence that the grout volume shown in the material balance can be achieved. Should the CsIX/TRU grout process/project be carried into conceptual design, additional studies on waste forms are recommended. These studies should include testing evaporation of the ion exchange effluent, other grout formulations, waste loadings other than those tested to date, and absorbents that have the potential for minimizing the final waste volume. 
One final waste form, not considered in this study, is the crystalline solid produced when SBW is evaporated at $130-140^{\circ} \mathrm{C}$ and then cooled. Although this waste form is expected to be both hygroscopic and highly soluble, it may prove to be acceptable for disposal at WIPP. A recent small-scale test demonstrated the feasibility of the concept using a SBW simulant and achieved about $70 \%$ volume reduction. ${ }^{8}$ If this same volume reduction could be achieved for the entire SBW, the final waste volume would be only $1,400 \mathrm{~m}^{3}$.

\subsubsection{Personnel Staffing}

The following basis was used to estimate the number of personnel needed to operate the CsLX/TRU grout facilities:

- The CsIX/TRU grout processing facility will be in operation for approximately 5 years from January 2008 through December 2012.

- Shipment of CH TRU drums to WIPP is independent of the waste treatment process, in that this activity will be performed from the interim storage facilities location, and is anticipated to occur from January 2008 through December 2021 (EDF-CsIX-002, Interim Storage for CH TRU Waste Drums).

- Process operations are based on 24 hours per day, 7 days a week, with up-time of 200 days per year. The 200 days are rounded from 205 and based on previously used HLW program drivers stating that operations will be based on a $75 \%$ up-time at $75 \%$ efficiency $(0.75 \times 0.75 \times 365=205)$.

- Four rotating shifts of personnel will be required to support the schedule for main process and drum output handling/transfer to interim storage. Included in the four rotating shifts are personnel for process maintenance, QA, Safety, and Rad Con.

- New facility space will be required for final drum analysis to meet WIPP requirements. This space will be for videography (visual inspection of the head space area via camera) and head space gas analysis activities.

- Activities related to drum videography, drum head space gas analysis, and CH TRU drum shipments to WIPP occur only during the day shift.

- All personnel listed will be included for estimating life cycle costs.

- Office space is available in existing nearby facilities; therefore, new administrative facility space is not included in the Study. Personnel not housed in the process facility include the facility administration, engineering support, and training staffs. Operating costs for the facilities they use are a cost incurred by the project.

- A single day shift will include personnel for administration, administrative support, technical support, general building maintenance, and activities related to drum videography, drum head space gas analysis, and CH TRU drum shipments to WIPP.

- Management for support functions - QA, Safety, RadCon, Training, Engineering, Drafting, and Maintenance - is supplied from the existing INTEC infrastructure. Proportional costs for this management and their space are a cost incurred by the project. 
- Ancillary and support functions critical to the process will be housed within the same physical facility.

- INTEC will have infrastructure services and capabilities to accommodate some tasks not unique to this project, such as additional engineering and drafting support, common warehouse and storage, major support areas such as machine, weld, sheetmetal, fitter, electrician, and electronics/instrument shops, mock-up area(s), and laydown area(s).

- No special personnel requirements are required for the interim storage facilities that cannot be handled by day shift and rotating shift personnel.

\subsubsection{Personnel Summary:}

Total estimated number of personnel, summarized in Table 5, is 97, of whom 62 are considered radiation workers. This data was used in determining new facility space needs, performing life cycle cost analysis, and as input to the Project Data Sheet. See EDF-CsIX-003 in Appendix E for details.

Table 5. Summary of Personnel Staffing Requirements.

\begin{tabular}{|c|c|c|c|c|c|}
\hline . & Day Shift & $\begin{array}{l}\text { Rotating Shifts } \\
(4)\end{array}$ & $\begin{array}{l}\text { Radiation } \\
\text { Workers }\end{array}$ & $\begin{array}{l}\text { New Facility } \\
\text { Office Space }\end{array}$ & $\begin{array}{l}\text { New Facility } \\
\text { Ops/Maint }\end{array}$ \\
\hline $\begin{array}{l}\text { Facility } \\
\text { Admin }\end{array}$ & 6 & 0 & 0 & 0 & 0 \\
\hline $\begin{array}{l}\text { Engineering } \\
\text { Support }\end{array}$ & 3 & 0 & 0 & 0 & 0 \\
\hline Training & 2 & 0 & 0 & 0 & 0 \\
\hline $\begin{array}{l}\text { Operations } \\
\text { Admin }\end{array}$ & 3 & 8 & 0 & 3 & 0 \\
\hline $\begin{array}{l}\text { Process } \\
\text { Operators }\end{array}$ & 10 & 32 & 42 & 2 & 11 \\
\hline $\begin{array}{l}\text { QA, Safety, } \\
\text { RadCon }\end{array}$ & 2 & 12 & 5 & 3 & 0 \\
\hline $\begin{array}{l}\text { Process } \\
\text { Maintenance }\end{array}$ & 0 & 12 & 12 & 0 & 3 \\
\hline $\begin{array}{l}\text { Facility } \\
\text { Maintenance }\end{array}$ & $\underline{7}$ & $\underline{0}$ & $\underline{3}$ & $\underline{1}$ & $\underline{0}$ \\
\hline Total & 33 & 64 & 62 & 9 & 14 \\
\hline
\end{tabular}




\subsection{System Descriptions}

\subsubsection{Tank Farm Liquid Waste Transfer}

Major portions of the existing tank farm liquid waste transfer system, connecting the tank farm to NWCF, will be used to transfer liquid waste to the CsIX/TRU Grout Facility. Modifications to the transfer system are required for the stand-alone facility scenario, however, it is possible that the existing system will be adequate, without modification, for the NWCF scenario.

The transfer system uses steam jets and air lifts to transfer liquid waste from tanks WM-180,-181,$182,-183,-184,-185,-186,-187$, and -188 , and uses air jets to transfer liquid waste from tanks WM-189 and -190 . Receiving tanks in the CsIX/TRU Grout Facility will be capable of accepting liquid waste from all tanks.

Modifications to the existing steam and air jet transfer system, for the all-in-one facility scenario, include a tie-in to the existing transfer lines and the addition of a lift station which would develop adequate head pressure to deliver liquid waste to the new facility's liquid waste feed tanks.

Liquid waste will be transferred to the CsIX/TRU Grout Facility in batch mode. A batch mode was chosen because the existing transfer system is not capable of conveying liquid waste at the low (1gpm) feed rate used in the CsIX/TRU grout process, and sampling of waste materials for purposes of process control will be performed on a batch basis.

The CsIX/TRU grout process design includes two tanks capable of accepting liquid waste from the tank farm waste transfer system. Each tank is sized nominally at 3,500 gal capacity, which allows for a reasonable batch cycle time of approximately 2 hours, based on the tank farm transfer system feed rate.

\subsubsection{UDS Filtration}

The CsIX/TRU grout process design includes provisions for filtering out undissolved solids from the liquid waste before its introduction to the CsIX columns. The primary function of the filtration system is to protect the ion exchange resin bed from becoming blinded with solids particles. The design also includes provisions for drying and packaging the UDS collected during filtration.

Considerable uncertainty exists in the quantity and character of the suspended UDS in the waste stream. Limited data available on the particle size distribution shows that a large fraction of the UDS in the liquid waste is in the sub-micron size; this indicates a potentially significant filtration problem if these particles must be removed. An additional issue is that there is some evidence the sub-micron particles also carry a large fraction of the radionuclide inventory of the UDS. As recommended later in this report, empirical UDS data must be obtained on the liquid wastes to further refine the filtration process chosen for this study.

A combination of two vertical leaf pressure filters will provide continuous filtering capability at a capacity of $1.2 \mathrm{gpm}$. Filter media will be selected that removes particles larger than 10 microns. The filter will also be equipped with a scavenger leaf to allow draining of vessel water from the filter cake. At any given time, one filter will be on-line while the other is being serviced. The off-line filter will be drained and the filter cake back flushed with deionized water into a slurry tank. Filter transition from on-line to off-line will be initiated by setpoint pressure differential. 
Slurry from the slurry tank, assumed to be $10 \%$ solids by weight, will be metered at approximately $1.7 \mathrm{Kg} / \mathrm{min}$ to a rotary wiped film evaporator, where the slurry will be dried and sized to a flowable particulate solid. Solids from the evaporator will be stored in radioactively shielded 55 gal drums. Off-gas from the evaporator will be directed through a condenser before it is sent to the Vent Off Gas System. Condensed water will be returned to the HLLWE.

\subsubsection{Cesium Removal}

Cesium removal is proposed by ion exchange using a crystalline silicotitanate (CST) sorbent. The system includes three ion exchange columns nominally $16 \mathrm{in}$. diameter by $96 \mathrm{in}$. tall, containing $0.25 \mathrm{~m}^{3}$ of sorbent. At any given time, two columns will be on-line and configured for in-series flow at a nominal capacity of $1.2 \mathrm{gpm}$. The third column will be off-line ready for use. A column requiring sorbent recharge will be taken off-line after which the spent sorbent will be sluiced from the column and fresh sorbent added.

Fresh sorbent, received dry in 50-lb bags, will be preconditioned by mixing with water in the sorbent preparation tank. When a column is ready, fresh sorbent will be pumped to it, after which the sluice water will be gravity drained from the column.

Pressure drop through the ion exchange columns is expected to be less than 3 psi, based on theoretical calculations and experience operating similar ion exchange columns at ORNL. ${ }^{8}$

Lead-lag configuration and valve timing of the ion exchange columns will be automatically controlled, based on cesium breakthrough of the lead ion exchange column. When breakthrough of the lead column is detected by continuous in-line monitors located between the lead and lag columns, rotation of the columns will be sequenced so that the lag column will become the lead column and the recharged off-line column will become the lag column.

\subsubsection{Evaporation}

Evaporation of the CsIX effluent, before neutralization and grouting, was considered in this study, but was not included in the proposed CsIX/TRU process design.

Evaporation may reduce the final grout volume; however, no test data are available for grouting SBW more concentrated than bottoms from the HLLWE. Most SBW grouting tests have been done with simulants less concentrated than HLLWE bottoms $\left(0.77 \mathrm{M} \mathrm{H}^{+}, 2.67 \mathrm{M} \mathrm{NO}_{3}{ }^{-}\right)$. Very recent tests have used a waste composition based on HLLWE bottoms. The results from these tests should be reviewed do determine if modifications are needed to the design basis used for grouting in this study. If future tests show that waste more concentrated than HLLWE bottoms can be adequately grouted, evaporator tests are recommended as outlined in Section 10 of this report.

Added to the test data inadequacy is the lack of a good basis for design of an evaporator to concentrate the waste. As SBW is concentrated, precipitates will form at some point, which may cause deposition on evaporator walls or fouling on the evaporator heat transfer surfaces. The concentrated bottoms, which could thicken or solidify when cooled, may present additional handling problems. Maintenance of the evaporator may be problematic. Thus, to stay within the grouting parameters for which data is available, a pre-grouting evaporator system was not included in the proposed design. 


\subsubsection{Solidification}

SBW and NGLW, having passed through the CsIX columns, will then be grouted to form a solid TRU waste form. To do this, the liquid waste will be partially neutralized in grout feed tanks, mixed with cement ingredients, and then cast in 55-gal drums. The cement-based grout will be allowed to cure, be dewatered, and the drums finally sent to the interim storage facility for inspection and storage.

Neutralization of the liquid waste to a specified setpoint of $\mathrm{pH} 1.3$ will be accomplished by the addition of calcium oxide (lime) to a grout feed tank. Accurate $\mathrm{pH}$ measurement is difficult for a highly acidic mixture containing various ionic compounds. To more easily obtain accurate measurements, an automated system will titrate a known small volume of the liquid waste to a neutral $\mathrm{pH}$. Results of this titration will be utilized to calculate the quantity of $\mathrm{CaO}$ required to neutralize the grout feed tank to the setpoint.

Neutralized liquid waste will be metered to one of three grout mixers. Each ribbon blender type mixer is sized for a single 55-gal batch. Premeasured quantities of the cement, blast furnace slag, and fly ash solid ingredients will be added to the liquid waste in the mixer and thoroughly blended. The mixer will convey the resulting grout mixture into a casting head through which the 55-gal drums are filled.

Immediately at the end of a mix cycle, the next batch of liquid waste will be added to the mixer. This timing is required to prevent residual grout from hardening in the mixer. A wash/decontamination system, connected to the mixers through high-pressure nozzles, is provided for cleaning the mixers when they are turned off at the end of a production run or for maintenance purposes.

Table 6 provides a time estimate for the steps in a mixer cycle.

Table 6. Mixer Cycle Time.

\begin{tabular}{cll}
\hline Step & Description & Time (min) \\
\hline 1 & Operator Initiation of Mix & 10 \\
2 & Feed Liquid & 10 \\
3 & Operator Validation of Wet and Dry Ingredients & 5 \\
4 & Dry Ingredient Delivery & 15 \\
5 & Continuous Mix & 10 \\
6 & Cast Mix & 10 \\
7 & Operator Validation of Mix & 10 \\
\hline Total & & 70 \\
\hline
\end{tabular}




\subsubsection{Drum Handling}

\subsubsection{General}

The drum handling system, used for moving drums through the grouting, curing, and dewatering processes, is fully automated with operator oversight and override capability, and provides for minimal human contact with a drum. Although two facility scenarios are proposed for the CsIX/TRU grout process, both scenarios have similar drum handling features.

\subsubsection{New Drum Lag Storage}

The loading dock of the grout facility will temporarily store up to 630, or a two-week supply of, 55-gal drums used in the grout process. Each drum includes a drum body, a removable lid, two filters installed in the lid, and a cinch ring for securing the lid to the drum.

Each drum will have a bar-code label attached and will be scanned before being placed on the inlet conveyor to the air lock leading into the grout fill area. These initial drum preparation steps will be performed on a contact-handled basis. Once in position, the drum will be routed automatically by the conveyor system and can be tracked through the facility to the load-out dock. Although similar, there are variances in the layouts of the routing system for each of the proposed scenarios.

\subsubsection{Grout Filling}

Grout filling requires a drum to pass through two airlocks and three enclosures - an inlet airlock, an outlet airlock, a grout filling enclosure, a drum lid installation enclosure, and a decontamination booth. Each of these has a set of doors for drum entrance and a set for drum exiting. This arrangement provides contamination control as a drum passes through the stages of the grout filling system.

Drum introduction to the grout filling area is by the inlet conveyor that passes through the inlet airlock. Inside the grout fill area, the inlet conveyor becomes a staging line for the drums, which are positioned automatically at one of three grout filling station lines by the conveyor.

For each line, a drum will first enter the grout filling enclosure. A drum fill hood will be lowered and a valve will be used for on- and off-grout flow control at the same time the drum is being vibrated to help settle the grout. After filling is complete, the hood is raised and a time for residual grout dripping is allowed before the drum is moved to the next enclosure.

The next stop for the drum is the lid installation enclosure. Inside the enclosure, the drum is rotated 360 degrees on its axis while a mechanical arm is used to clean the drum lip. The lid is then installed and secured with the cinch ring. Filters installed in the lid are provided as an escape path for evaporated moisture as the grout cures.

Filled drums, from all three lines, are moved to a transferring section of the conveyor system. This section is used to send the drums to one of three decontamination booths. Each booth contains a video camera used to inspect a drum for visible contamination -spilled or dripped grout. A remote arm will make a RadCon swipe of any suspect areas with on-line counting of the swipe provided. For large areas of grout material, water or decontamination fluid spray will be available. Decontamination fluid will be collected at the bottom of the booth, sent to a collection tank, and then transported to the PEWE or LET\&D for concentration. It will eventually be routed back for treatment through the CsIX/TRU grout process. 
After decontamination is complete, the drums pass to the discharge conveyor, which positions the drums at the grout fill area exit airlock. Movement through this airlock places the drums in the curing cell.

See EDF-CsIX-005 in Appendix E for more information on the equipment and sequencing of the grout fill and drum handling system.

\subsubsection{Grout Cure Cell}

The grout cure cell encloses the cure line conveyor system, acting as the primary containment, and is fabricated from stainless steel for contamination control. Access panels and windows will be provided for maintenance activities and visual inspection of drum movement. Ventilation through the cell will remove the moisture given off by the curing process.

The cure line conveyor system includes two lines, one at floor level and the other directly over the first. The upper line is used as a back-up for the first or if more cure time is required. A drum is positioned at the appropriate cure line by a drum lift after which the conveyor moves the drum onto the line. Drum spacing on the line is set at 6 in.

The cure line conveyor will move a drum along the conveyor path for a period of 24 hours. When the drum reaches the end of the cure line, it will stop and be staged to enter into another drum lift. This lift will receive drums from both cure lines and will lower or pass the drum through to the second floor drum elevator, depending on the cure line. After being raised to the second floor, a drum is conveyed to the dewatering station area.

See EDF-CsLX-006 in Appendix $\mathrm{E}$ for more information on equipment and process sequencing for the grout curing line drum handling.

\subsubsection{Dewatering Station}

Nine dewatering stations are provided for the removal of residual water from the headspace of the drum after the grout has cured. These stations will dewater the drums for a 4-hour period with heated air.

Dewatering is performed through the filters in the drum lid, so, to locate the filters, the first stop for a drum is at an indexing/positioning table. The table is raised to an arm that indexes the drum when it is rotated and the arm comes in contact with a filter. The drum is lowered and conveyed to one of the dewatering stations. After being positioned in the station, a hooded manifold is lowered over the filters. The hoods will seal to the drum lid. Air heated to $150^{\circ} \mathrm{F}(65.5 \mathrm{C})$ will be drawn through one of the filters into the drum head space by the off-gas system which is exhausting the drum head space through the other filter. Dewatering will take place over a four-hour period.

After dewatering is complete, the manifold will raise and the dewatering station conveyor will move the drum back onto the staging conveyor line. The staging conveyor will then move the drum to the load-out conveyor, which is used to move the drum to a point where the drum is removed by a manual hydraulic lift and placed on a pallet for shipment to interim storage.

See EDF-CsIX-007 in Appendix E for more information on equipment and process sequencing for the dewatering process drum handling. 


\subsubsection{Drum Transport to Interim Storage Facilities}

The grout facility drum lag storage capacity will be 1 day's production or 38 drums. A covered flat bed truck will move these drums from the grout facility lag storage to the interim storage facility making three round trips a day, carrying four pallets of four drums each. This scenario provides a rate that exceeds the estimated grout-filled drum production rate. After receipt at the interim storage facilities, drums will be stacked and ready for future sampling and video inspection of the drum head space.

See EDF-CsIX-007 in Appendix E for more information on lag storage and transporting to interim storage. See EDF-CsIX-002 for more information on interim storage capacity requirements.

\subsubsection{Drum Head Space Inspection and Sampling}

Before shipment to WIPP, a gas sample must be taken from the drum head space to determine VOC and hydrogen levels. Because WIPP limits the amount of free liquid to less than $0.5 \%$ by volume, the head space must also be inspected for free liquid. A drum must be temperature conditioned for a 72 hour period before the sampling and inspection.

A drum will be placed in a temperature-controlled area of the interim storage for the 72-hour conditioning period after which it will be staged on a conveyor. The drum is initially conveyed to an indexing/positioning table for location and positioning of the drum lid filters. This process is identical to that used in the drum dewatering system. After indexing, the drum is passed through an airlock to a glovebox, where the inspection and sampling occur.

Once positioned in the glovebox, a clear hood will be lowered over the drum lid to provide additional containment. All subsequent inspection and sampling activities will occur within the hood environment. A filter removal/headspace sampler tool will lower to and engage one of the drum lid filters. The filter will be twisted off and allowed to drop into the headspace of the drum. A headspace gas sample will be taken as soon as the filter is removed. The filter removal/headspace sampler tool will be removed and a fiber optic video camera with lighting will be moved into position over the drum opening. The camera will be lowered through the opening and digitally record a 360 degree rotational view of the drum headspace. After video inspection, the camera will be removed and a new filter installed. The clear hood will raise from the drum and a mechanical arm will take a RadCon swipe of the drum lid surface. Online counting will be provided for swipe examination.

Once cleanliness is verified, the drum will pass through an exit airlock to the unloading conveyor, and be returned to interim storage or transported to the TRUPACT loading facility.

See EDF-CsIX-010 in Appendix E for more information on drum head space sampling and inspection.

\subsubsection{Interim Storage}

The grout-filled contact-handled TRU waste drums produced from the grout facility will be shipped to WIPP for final disposal. The drum production rate has been determined to be quite high relative to the rate at which WIPP could feasibly receive them (See EDF-CsLX-002, Appendix E). As a result, interim storage for up to approximately 24,000 drums will be required.

Data from EDF-CsIX-002, Interim Storage for CH TRU Waste Drums, in Appendix E, is summarized below: 
- Total number of drums produced $=38,000$.

- Average production rate over 5-year operating period $=633 /$ year, $146 /$ week, or $21 /$ day.

- $\quad$ Maximum actual daily rate considering efficiencies $=38$.

- $\quad$ Filled drum weight is $888 \mathrm{lb}(403 \mathrm{~kg})$.

- Maximum number of grout-filled drums per TRUPACT-II is 7.

- TRUPACT-II payload for CsIX/TRU drums is 6,864 lb (max allowed is 7,265 lb).

- Shipping rate is assumed to be 5 shipments (3 TRUPACT-IIs per shipment) every 2 weeks (105 drums).

- Interim storage capacity required is for approximately 24,000 (24,350 calculated) drums.

- Last date for drum shipment to WIPP is estimated to be December 2021.

The efficiencies referred to in the $3^{\text {rd }}$ bullet are $75 \%$ up-time with a $75 \%$ efficiency when up, based on a 24 hour per day operation, 7 days per week (Ref EDF-CsIX-003, Appendix E). The result is that drum production for an actual day must make up for the overall efficiency of $55 \%$ if the total drum production is to be completed in 5 years $-21 /$ day $/ 55 \%=38$.

The peak for interim storage will be the last month of drum production, which is assumed to be December 2012. The 24,000 drum interim storage requirement is more than the capacity $(17,000$ drum equivalent) of a single Type II RCRA qualified storage module currently used at RWMC for interim storage of TRU waste drums destined for WIPP. The interim storage facility proposed in this study is a single facility sized to the equivalent of two of the RWMC Type II modules. This extra capacity provides the space required for housing the drum head space inspection and sampling structure, equipment, and related activities.

\subsubsection{UDS Treatment and Storage}

The UDS drum handling and filling systems are similar for both facility scenarios. The systems for scenario 1 are located in the grout facility building, but for scenario 2 are in the NWCF.

When the UDS filter pressure drop reaches a predetermined level, the filter is taken off-line and back flushed with deionized water. The flush is collected in the UDS slurry tank. When the UDS evaporator is operating, the slurry leaves the tank and passes to the evaporator feed pump. From the feed pump, the slurry is fed to the UDS evaporator. The dried solids flow by gravity from the evaporator into a discharge tube. When the tube is full, a UDS drum is lifted into position and filled.

Because the UDS drum filling rate is low, at one drum per month, and space limitations in the NWCF are a concern, the drums will be shielded to a contact-handled level. Due to the shielded drum weight, a crane will handle these special containers before filling. First placed on a staging conveyor, a container will pass through the inlet and outlet doors of the decontamination booth and be positioned in the UDS loading station. The container will be raised to permit a dust-tight loading cone to mate with a dust tight cone on the drum lid, after which the loading cone will be opened to permit drum filling. Once the drum is filled, the drum movement process will be reversed and the drum moved back to the decontamination booth. 
The decontamination booth is similar to the one used in the drum grout-filling process. In addition to the RadCon swipe, counting, and decontamination capabilities, the booth will also be used to install a shield over the fill cone on the drum lid. The drum is then moved out of the decontamination booth to the staging conveyor. Drum handling after decontamination is manual. Generation of up to 50 UDS drums is anticipated over the entire process period.

From the staging conveyor, the drum is crane-loaded onto a pallet for movement to the UDS drum storage area. The storage space in scenario 1 will be in the southeast corner on the $1^{\text {st }}$ floor of the grout facility. For scenario 2 , storage will be near the new decontamination booth on the $3^{\text {rd }}$ floor of NWCF. When several drums have accumulated in the storage area, they will be moved to the loading area for shipment and disposal at WIPP in a SWB.

See EDF-CsIX-008 in Appendix E for more information on equipment and sequencing of the UDS filling and handling systems.

\subsubsection{Spent Sorbent Treatment and Storage}

When break-through is observed in the lead CsIX column, the column is taken off line and the sorbent removed. To do this, deionized water is added to the column at the ratio of 10 water volumes per sorbent volume. The sorbent/water mixture exits the column and enters the spent sorbent prep tank, where free liquid is decanted from the sorbent. The sluice water is pumped through the sorbent fines filter and is saved for reuse.

After the dewatering, the sorbent will be fed to a hollow flight drier for drying, using heat and hot air. The hollow flight drier is nominally sized to process moist sorbent at a rate of $2.1 \mathrm{l} / \mathrm{hr}$.

Dried sorbent will flow by gravity to a spent sorbent storage tank, sized at $15 \mathrm{~m}^{3}$ to hold all of the sorbent generated during the life cycle of the facility. The estimated total spent sorbent quantity is estimated to be $10.5 \mathrm{~m}^{3}$. The spent sorbent storage tank, when filled, will have a contact radiation reading of approximately $5,000 \mathrm{R} / \mathrm{hr}$. The spent sorbent storage tank is piped into the existing NWCF for the second facility scenario, or the all-in-one facility's pneumatic transport system for the first scenario, to provide a mechanism for future removal of the sorbent from the facilty under another project. The silica gel particle size is less than $60 \mathrm{~mm}$, and therefore, cannot be classified as debris and must be disposed of as mixed hazardous waste. Options for treatment, storage, and disposal of spent sorbent are discussed in more detail in EDF-CsIX-004 in Appendix E.

Test data is needed to better determine the quantity of water required for sluicing the spent sorbent, the behavior of the sorbent during sluicing (amount of fines generated), requirements for filtering the sorbent, behavior of the sorbent during drying, and properties of the dried sorbent.

\subsubsection{Shipment to WIPP}

The Carlsbad Area Office (CAO) at WIPP will provide a fleet of NRC-approved transportation packages for shipment of TRU waste from the INEEL to the WIPP. The CAO will grant or suspend the INEEL's authority to certify TRU waste and to use the TRUPACT-II cask based on an assessment of the INEEL TRU Waste program and its implementation. WIPP will be responsible for shipping the loaded packages from the INEEL to WIPP.

Table 7 lists capacity and weight limits for various types of containers that can be shipped in the WIPP TRUPACT-II cask. EDF-CsIX-002 in Appendix E discusses the packaging issue further. 
Table 7. TRUPACT-II cask capacity for various types of containers

\begin{tabular}{lll}
\hline Qty. & Container Type & Weight Limits \\
\hline 14 & 55-gal drums & $@<1,000 \mathrm{lb}$ each \\
2 & Standard waste boxes (SWB) & $@<4,000 \mathrm{lb}$ each \\
2 & SWB, containing one bin each & \\
2 & SWB, containing four 55-gal drums, each & $@<6,450 \mathrm{lb}$ \\
1 & Ten-drum over pack (TDOP), containing ten 55-gal drums & \\
1 & TDOP, containing one SWB & \\
1 & TDOP, containing one bin within a SWB & \\
1 & TDOP, containing four 55-gal drums within a SWB & $@<19,250 \mathrm{lb}$ \\
& TRUPACT-II & $@<8,000 \mathrm{lb}$ \\
\hline
\end{tabular}




\section{FACILITY DESCRIPTIONS}

\subsection{Facility Concepts - Overview}

This study evaluates two scenarios for the CsIX/TRU Grout Facilities:

- Scenario 1: This scenario requires construction of a single facility for housing the CxIX/TRU grout processes. Interim storage for the grout-filled waste drums and a facility for waste drum loading/shipping preparation are also required. The new process building will be similar to the NWCF in building type and construction materials. The interim storage and waste drum loading/shipping facilities will be similar to the RWMC Type II Storage Modules and TRUPACK-II Loading Station.

- Scenario 2: This scenario requires the use of the NWCF for the filtration and cesium removal portions of the process. This would require demolition and modification of portions of the NWCF. A new facility will be required for the grouting portion of the process. Interim storage and waste drum loading/shipping preparation facilities are identical to those in Scenario 1. Building types will be similar to those in Scenario 1.

\subsection{Civil}

\subsubsection{Siting}

Siting for the new processing facilities was evaluated against the following criteria:

- Proximity to the tank farm

- Utilities availability

- Economy of construction

- Land availability (future planned use)

- Vehicle accessability.

Limitations of the tank farm liquid transfer system make proximity to the tank farm a primary consideration. Four sites were selected for consideration - two directly east of NWCF across Hemlock Street, one east and beyond the perimeter fence, and one directly north of the tank farm. The importance of proximity to the tank farm reduced the selection possibilities to the site north of the tank farm. This preferred site is an environmentally controlled area. (See drawings in Appendix D.)

\subsubsection{Surface Drainage, Erosion and Sediment Control}

The area disturbed by this project will be within the INTEC boundaries. Most of the area is void of natural vegetation. The design will minimize the amount of soil that erodes from the site. Areas disturbed by construction activities will be stabilized with gravel.

The existing INTEC surface drainage system will be utilized with some slight modifications to drain surface water from the proposed project site. 


\subsubsection{Soil Excavation and Shoring}

Initial earthwork will consist of minimal vegetation clearing and grubbing and grading. Excavation is required for utilities, paving, footings, and foundations with no rock excavation anticipated. Sampling of the existing soil may be necessary before final design begins.

Shoring may be required if the actual selected site is close to the existing tank farm.

\subsubsection{Paving, Parking, and Roadways}

Paving will be provided in the truck access areas, a small parking area, and necessary access roadways. All roadways will be designed for the AASHTO HS-20 truck load with all access roads being at least $12 \mathrm{ft}$ wide.

\subsubsection{Slabs, Sidewalks, and Stoops}

Reinforced concrete stoops will be located at, and sloped away from, each exterior doorway.

Reinforced concrete slabs or pads will be provided for exterior utility equipment, exterior material storage bins and tanks, and exterior electrical equipment.

\subsubsection{Fencing}

Chain-link fencing will be provided around the Interim Storage and TRUPACT-II Loading Station Facilities if they will be located outside the existing INTEC perimeter fence.

\subsubsection{Underground Utilities}

Existing underground utility lines are shown on drawings $1-\mathrm{C} 1$ and $2-\mathrm{C} 2$. New underground utilities include:

- Potable water

- Fire water

- Sanitary sewer

- Steam

- Plant air. 


\title{
4.3 Structural
}

\subsubsection{Structural Design}

The CsIX/TRU Grout Facilities will be designed to resist all applicable loads as required by the DOE-ID Architectural Engineering (AE) Standards. Structural design for natural phenomena shall conform to the AE Standards and DOE STD 1020. The latter give requirements for three different performance categories of buildings. The assumed Performance Category (PC) for each building in the CsLX/TRU Grout Project is as follows:

\author{
Interim Storage Building $\quad \mathrm{PC}-2$ \\ TRU Pack Loading Station Building PC-2
}

$\underline{\text { Scenario } 1}$

New CsIX/TRU Grout Building $\quad$ PC-3

Scenario 2

NWCF Modifications $\quad$ PC-3

New Stand Alone Grout Building $\quad$ PC-2

Unless otherwise noted, structures, systems, or components important to safety or for protection of the environment will be designed in accordance with the requirements imposed by DOE STD 1020 for the performance category of the building where the structures, systems, or components are located. Office areas and systems or components that are not important to safety may be designed to PC-1 design requirements.

\subsubsection{Structural Features}

Process areas will be in cells with reinforced concrete superstructures. Cell walls will be fabricated from normal weight reinforced concrete. Thickness will vary as required by radiological shielding requirements. The minimum thickness for any load-bearing wall will be $12 \mathrm{in}$. See drawings 1-A1 through 1-A4 in Appendix D for assumed thickness of radiological shielding walls.

Reinforced concrete floors will be provided for all elevated floors unless otherwise noted.

\subsubsection{Footings and Foundations}

Reinforced concrete mats will be used as footings for the cell areas of all applicable buildings. Large equipment or tank footings will also be provided with mat footings. Depth will be as indicated on the drawings. Concrete load bearing and shear, not associated with cells, will have shallow reinforced concrete spread footings. Buildings with structural steel framing, as the main load-resisting members, will have reinforced concrete pier footings and a perimeter foundation wall. 


\subsubsection{Hatches and Hatch Covers}

The tops of all cells will have stepped concrete plug-type hatch covers that can be removed by the $3^{\text {rd }}$ floor overhead bridge crane.

\subsubsection{Building Framing Systems}

The cell areas will have reinforced concrete floors, walls, and ceilings for radiological shielding purposes. These components will also provide the main building framing in the cell areas. The walls will be designed to support all vertical loads, and resist horizontal loads as shear walls.

Building superstructures, containing support areas such as the office area and the main building HVAC equipment, will be an ordinary braced frame consisting of structural steel.

The drum storage buildings and the TRUPACT Loading Station Building will also be ordinary braced frames consisting of structural steel.

\subsection{Facility Description - Scenario 1}

\subsubsection{General Description}

For Scenario 1, the CsIX/TRU grout process, which includes the UDS filter system, the ion exchange system, and the grout system will be housed in a single new facility. This facility will be a multistory building with an approximate footprint of 14,000 square $\mathrm{ft}$. Drawings 1-A1 through 1-A4 in Appendix D illustrate the proposed floor plans for each level. Building sections are illustrated on drawing 1-A5.

Process equipment will be housed in reinforced concrete cells and corridors. Appendix F contains the CsIX/TRU Grout Project equipment list .

The periphery of the CsIX/TRU Grout Facility contains office space, change rooms, support areas, and truck delivery and loading areas. Most of these areas are at one end of the building. Stairways, corridors, and a freight elevator are provided for movement within the building.

A stack will be provided for exhausting ventilation air.

Two additional buildings are required for this scenario. The interim storage facility will provide space for drum storage and inspection, and the TRUPACT-II Loading station will provide the space and equipment needed for packaging the waste drums for shipment to WIPP.

\subsubsection{Cell Lining}

Process cells, the Piping and Valve (P\&V) corridor, and decontamination areas will be lined with 1/4-in.-thick stainless steel plates on the floors and 1/8-in.-thick stainless steel plates on the walls. Except for the UDS Dryer Cell and the Waste Sorbent Preparation Cell, which will be fully lined with stainless steel, only the lower $4 \mathrm{ft}$ of cell walls will be lined unless otherwise noted. 


\subsection{Facility Description - Scenario 2}

\subsubsection{General Description}

For scenario 2, the CsIX/TRU grout processes for UDS filtration and the cesium ion exchange system will be housed in the existing NWCF facility. This scenario is explored in detail in EDF-CsIX-009 in Appendix E.

The processes related to grouting will be housed in a new facility. This new facility will be a multistory building with a limited number of cell areas. Radiological shielding will generally be limited to 12-in.-thick reinforced concrete. Grouting equipment that will process radioactive materials will be housed in reinforced concrete cells and corridors. See Drawings 2-A1 through 2-A4 in Appendix D, and Appendix $\mathrm{F}$ for the equipment list.

The periphery of the new building contains office space, change rooms, support areas, and truck delivery and loading areas. Most of these areas are at one end of the building.

Stairways, corridors, and a freight elevator are provided for movement within the building.

A stack will be provided for exhausting ventilation air.

Two additional buildings, for interim storage and TRUPACT-II loading and identical to those in Scenario 1, are also required for this scenario.

\subsubsection{Cell Lining}

Cell lining in the existing NWCF cells will not be changed. The P\&V corridor and decontamination areas within the new grouting building will be lined with $1 / 4$-in.-thick stainless steel plates on the floors and 1/8-in.-thick stainless steel plates on the walls. Only the lower $4 \mathrm{ft}$ of cell walls will be lined unless otherwise noted.

\subsubsection{Installation in the NWCF}

Installation of CsLX equipment in the NWCF will take advantage of the existing NWCF infrastructure, which includes building utilities, HVAC, and off-gas systems. However, modifications are required to the NWCF to accommodate the CsIX equipment.

NWCF hot cell space was identified in the adsorber cell, calciner cell, valve cubicle, and hot sump tank removal cell sufficient to accommodate requirements of the CsLX/TRU grout process equipment installation. NWCF equipment will be used as much as possible to reduce the number of new items installed in the building (see Appendix $\mathrm{F}$ for the equipment list).

\subsection{Interim Storage and TRUPACT Loading Facilities}

The interim storage facility for this study is based on the capacity of the RWMC Type II Storage Modules. The proposed single building, as depicted in Drawings 1-C1, 2-C1, COM-A1, COM-A2, and COM-M1 (Appendix D), is twice the square footage of a Type II module. This will provide adequate interim storage space with room enough for the Drum Head Space Sampling and Inspection facility to be housed inside the structure. Not directly tied to the production of waste drums, but rather WIPP 
acceptance criteria, the facility for drum headspace sampling and inspection activities is located where the supply of drums to be surveyed are stored.

The RCRA-qualified interim storage facility is an environmentally unconditioned steel structure with a concrete floor measuring $240 \times 240 \times 24 \mathrm{ft}$ tall at eave elevation. Included in this facility is the Drum Head Space Sampling and Inspection building, which is an environmentally conditioned structure measuring $60 \times 60 \mathrm{ft}$ with an approximate ceiling height of $12 \mathrm{ft}$.

An additional facility, with two TRUPACT-II stations for the loading of drums for shipment to WIPP, is located next to the interim storage facility. This loading facility is based on the RWMC TRUPACT II loading station. The TRUPACT-II shipping container (Ref. EDF-CsIX-002) is specifically designed to accommodate 55-gal drums. Two TRUPACT-II loading stations are included in this facility because of the anticipated shipping rate. The TRUPACT-II loading station facility is an environmentally conditioned steel structure with a concrete floor measuring $80 \times 100 \times 24 \mathrm{ft}$ tall at eave elevation.

The interim storage and TRUPACT-II loading facilities are provided with overhead doors that allow the transport vehicles inside the facilities for loading and unloading activities. The Drum Head Space Sampling and Inspection building houses the drum conveyors, indexing table, glovebox, and airlocks required for the related sampling and inspection activities. Adequate space in the interim storage facility will be available for the movement of drums from their storage locations to and from the Sampling and Inspection building.

See EDF-CsIX-002 for a discussion of requirements for interim storage space, and EDF-CsIX-010 for information on drum head space sampling and inspection. Both are located in Appendix E.

\subsection{Offgas Treatment}

Offgas treatment is very similar for both scenarios. The offgas system will be connected to condensing vessels and the dewatering stations. The system will contain a condenser, demister, superheater, and double High-Efficiency Particulate Air (HEPA) filter housing just upstream of the fan. The removed moisture will be sent to the collection tank for reprocessing into grout. Exhaust offgas will be sent to the stack. Two independent systems will be installed. This will allow change-out of the double HEPA filters and failure of a system without facility shutdown. One of the two systems will be connected to the standby power source. See EDF-CsIX-013 in Appendix E for more information on the offgas system.

\subsection{Heating, Ventilation, and Air Conditioning (HVAC)}

In addition to the offgas system, the CsIX/TRU Grout building will be required to have two ventilation confinement zones per DOE Order 6430.1A. This section describes the two confinement zones, and the nonconfinement area. A ventilation confinement system will be provided, which, in conjunction with the physical barriers, maintains a continuous airflow pattern from areas of low potential contamination to areas of high potential contamination. The objectives of the confinement systems are to prevent the spread of radioactive and other hazardous materials to occupied areas and to minimize the release of radioactive and other hazardous materials in facility effluents.

- The primary confinement consists of the process hot cells and hot maintenance areas, which will be exhausted through two stages of HEPA filters. The primary confinement boundary will comprise hot cell walls, welded stainless steel ductwork, and HVAC equipment designed to maintain its structural integrity during and after operational and natural-phenomena design basis accidents. 
- The secondary confinement boundary consists of the operating corridors and other building structures that surround the primary confinement. The secondary confinement boundary contains all ventilation system equipment such as HEPA filter units and exhaust fans.

- The nonconfinement boundary consists of offices, control rooms and support areas outside the secondary confinement boundary. The nonconfinement zones do not require unique ventilation systems and will be maintained at a slight positive pressure with respect to ambient.

HEPA filters will remove contaminated particulates, but no carbon absorber beds will be provided in the primary filter trains, because semivolatile fission products and iodine will be removed by the offgas system.

EDF-CsIX-011 and EDF-CsIX-012 contain HVAC requirements as well as drawings depicting air flow diagrams of the HVAC systems for the CsIX/TRU Grout Facilities.

The facility will be provided with an independent chilled water system consisting of central chillers and pumps, delivering chilled water to various cooling coils. This system removes heat generated by process equipment because cooling will not be required for personnel comfort in the operating corridor. The facility will obtain steam for comfort heating from an INTEC central steam supply facility. The HVAC system will be controlled by a central electronic system located within the facility.

Redundancy will ensure proper ventilation confinement during HEPA filter changeout or fan maintenance. Exhaust ductwork will be located in areas that will not be normally occupied. HEPA filter housings will have the capability to be leak tested and tested for filter efficiency in place. Single stage HEPA filtration will be provided at the hot cell intakes to prevent possible contamination from momentary back flow to the occupied areas. Confinement boundaries will be sealed to maintain isolation when pressure differentials fall below normal conditions.

Confinement zones will be supplied by once-through ventilation. All the airflow from these zones will be HEPA filtered and discharged to the exhaust stack, with no air recirculation. The supply and exhaust fans for the hot cells can be powered from the INEEL site standby power grid (see Reference 9). This allows the ventilation confinement to be maintained in the hot cells during a loss of off-site power condition.

This facility will be designed with the following ventilation parameters:

- $\quad$ Eight air changes per hour for primary confinement

- Four air changes per hour for secondary confinement

- $20 \mathrm{cfm}$ per person for administrative areas

- One stage of testable HEPA filters for hot cell inlet

- Two stages of testable HEPA filters for hot cell exhaust

- $\quad$ One stage of testable HEPA filters for secondary confinement exhaust

- Once-through ventilation for primary and secondary confinement zones

- Zone pressures maintained per EDF-CsIX-011. 


\subsection{Remote Operations}

\subsubsection{General}

The CsIX/TRU grout facility design will allow remote replacement of moderately to highly contaminated process equipment that past experience has shown to be prone to failure. Equipment such as tanks, vessels, and columns, which have anticipated maintenance intervals of greater than 10 years, will be designed for hands-on maintenance.

The terms "remote maintenance" and "remote replacement" are generally used interchangeably. Radioactively contaminated items requiring maintenance at the CsIX/TRU Grout facilities will be remotely removed from service and decontaminated, after which hands-on maintenance will be performed.

Features of the CsIX/TRU Grout facilities, which will expedite the remote handling capabilities, include:

- Remote Handling and Replacement Cubicle or Piping and Valve (P\&V) Corridor - A radiologically shielded and enclosed area designed and equipped to provide remote handling and replacement for those items that must be isolated from the main process cells due to their high maintenance frequency and radiation hazard. Features required for direct maintenance will also be provided.

- Manipulator/Crane Parking and Maintenance Areas - Radiologically shielded areas designed for maintenance of major in-cell remote handling equipment. These areas will have the capability of being isolated and radiologically shielded from the $P \& V$ corridor during maintenance operations and will permit removal of the major remote handling equipment from the high radiation and contamination areas when the equipment is not being used.

- Remotely Operated Manipulators - Electro-mechanical manipulators with extended reach and intermediate handling capability, which can be controlled from a number of locations in the operating corridor.

- Remotely Operated Cranes - Conventional lifting and transport of heavy items inside the radiologically shielded areas of the facility controlled by remote means.

- Master-Slave Manipulators - Mechanical arms directly operated by personnel located at viewing stations when highly dexterous remote manipulation is required.

- Radiologically Shielded Viewing Windows - Stations that permit direct viewing of operational areas inside the radiologically shielded cells.

- Remotely Replaceable Lighting - Removable lighting units that provide adequate lighting in the radiologically shielded areas. Lighting units are replaced using remote handling equipment.

- Filtered Exhaust Ventilation - A remotely changeable system designed to achieve maximum radiological contamination control by filtering cubicle and corridor ventilation air at its exhaust point. Provisions for increased airflow during hatch removal will be provided. The preferred approach is the top-loading filter system for handling the changeout. 
- Smear Cell - A radiologically shielded area for limited decontamination, packaging, and transfer of failed equipment to other decontamination or waste disposal facilities/area. This area will be designed so that it can be intermittently isolated from the remote corridor to allow hands-on repair/smear of slightly radioactively contaminated equipment. This cell will be adjacent to or part of the decontamination cell.

- Decontamination Cell - A radiologically shielded area for decontamination, packaging, and transfer of failed equipment to other waste disposal facilities/areas.

- Hands-On-Maintenance (Repair) Area - A minimally radiologically shielded area for repair of decontaminated equipment.

\subsubsection{Equipment Used in Remote Operations}

High-maintenance equipment items include such items as valves, pumps, cell and vessel off-gas HEPA filters, and heat exchangers. These items will be grouped into common radiologically shielded areas and the areas provided with remote equipment, such as, master-slave manipulators, wall-mounted manipulators, bridge-mounted manipulators, bridge cranes, and jib cranes, and viewing windows. The remote areas must also be adequately illuminated with remotely replaceable lights, remote maintenance service stations, ${ }^{1}$ tool-drop ports, and work tables. Closed circuit video capability will also be provided if supplemental viewing is required. All in-cell remote handling equipment will be provided with redundant features and retrieval systems to minimize the effect of failures.

Equipment that is not prone to failure, such as tanks, vessels, columns, and airlifts, will also be located in radiologically shielded process cells, but the cells will not be equipped to permit remote replacement of these items. Rather, maintenance of these items will be hands-on after proper decontamination. These areas will be equipped for maintenance access and equipment removal without interference from adjacent equipment. Permanently installed working platforms with ladders for maintenance access will be considered on a case-by-case basis. Although certain equipment items are identified as hands-on maintenance items, to reduce operator radiological exposure, remote features such as quick-disconnects, remote flanges, captive bolts, and lifting bales may be incorporated into their design. The purpose of these features is twofold: (1) to permit potential hands-on maintenance activities to proceed more rapidly, and (2) to permit remote or partially remote operations, using overhead cranes and manipulators, to take place if the radiation fields are expected to be excessively high.

"Skyrights" will be provided to ensure equipment accessibility with overhead equipment. In addition, visibility, accessibility, and interferences must be considered during facility design. Center-ofgravity lifting bails will be provided on all remotely removable process equipment (e.g., valves). Remotely removable valves, pumps, and other equipment will be installed on self-aligning jumper assemblies to enhance remote removal and replacement.

Throughout the facility, remote concepts and equipment must be "proven" technology. Development items and unproven state-of-the-art items will not be included in the production facility unless the concepts/equipment can be proven by demonstration before incorporation. Remote tooling and equipment provisions must be maintained as practical, straightforward, and simple as possible. Standardization of sizes, shapes, and arrangement will also be maximized.

\footnotetext{
${ }^{1}$ A centralized grouping of selected utilities located in remote maintenance areas that can be accessed (connected/disconnected/used) by manipulators and used to support in-cell remote activities.
} 
A remote handling and replacement corridor, such as the $P \& V$ corridor, will be provided near those processing areas where radiation exposure and radiological hazards justify the additional facility cost. Failure-prone equipment will be located within this corridor. Basic equipment and process design will minimize the total amount of maintenance required. When maintenance or vessel inspection is required, the facility design will provide features that reduces radiation exposure to personnel. The operational features will include both remote and direct maintenance and will include less emphasis on remote capabilities as the process produces products with lower radiation fields and contamination levels.

Radiologically shielded viewing windows will provide unrestricted viewing of remote operations with additional viewing angles possible from adjacent windows. Remote operating and maintenance tasks will be located within the normal viewing angle of the windows. Remotely removable hot-side shields will be provided for the viewing windows where necessary.

\subsubsection{Mockups}

Mockups will be required for Scenario 2, for the rip-out and construction efforts of the NWCF. Costs for this mockup effort and associated training are included in this study's cost estimate.

All remote equipment, and major remote operations, will be mocked up in full scale (at the Remote Mockup and Test Facility located at the Test Reactor Area at the INEEL) and tested before design completion and procurement. Mockups will include mechanical equipment, remote handling equipment, viewing, and cell sizes and arrangements. Items being checked will include accessibility, interference, visibility, maintainability, operability, transfer routes, and lifting mechanisms and devices.

\subsubsection{Maintenance Requirements}

The facility will be designed to allow repair or replacement of any process component while minimizing personnel radiation or contamination exposures, maintenance cost, and downtime. All mechanical equipment, windows, video cameras, and other equipment in potentially radioactive or contamination areas will be sealed or otherwise designed to prevent penetration by contamination and/or decontamination solutions. Materials such as plastics and insulation that degrade in radiation environments will be excluded whenever possible. If used, they will be encased and shielded or otherwise protected from excessive radiation exposure. These materials, if installed, will be scheduled for periodic inspection and replacement.

The maintenance philosophy for radioactive process equipment will be to replace high maintenance items such as pumps and valves. These components will be isolated from the system, if required, uncoupled from the system, and removed to a decontamination area. The repaired or replacement component will then be reinstalled and returned to service. In selected cases, spare components will be available for service. Direct contact maintenance will be used for items that are not expected to fail more than once every 10 years and in areas where the component can be decontaminated and expected radiation levels will be minimal.

Equipment will be designed to permit decontamination, inspection, replacement, and/or repair with minimum radiation exposure to personnel. Cells will have provisions for visual inspection or radiation monitoring without exposing the inspector to radiation or contamination hazards. Access ports for transfer of small tools and equipment will be provided in areas requiring remote replacement or repair operations. Remote maintenance service stations will be located in selected remote maintenance cells and will be equipped with remote connectors for electrical, video, air and water utilities. 
Electrical systems will be designed to allow corrective and preventive maintenance on primary or emergency power systems without compromising safety or adequate environmental protection.

\subsubsection{Remote Equipment Change/Cut-up/Disposal}

Equipment in the $P \& V$ corridor that requires routine change-out will be capable of being remotely connected and disconnected, handled, and moved. The basic philosophy for a typical jumper change out would involve the use of an in-cell electro-mechanical manipulator and in-cell crane to remove the defective jumper to the decon cell area from the P\&V corridor. These areas must be next to each other to afford adequate crane coverage to perform initial decon activities. The crane maintenance and decon areas will have lower radiologically contaminated/radiation conditions, and provide the space for working on equipment that has come from the $\mathrm{P} \& \mathrm{~V}$ corridor, an area of higher radiologically contaminated/radiation conditions. Coverage by the $\mathrm{P} \& \mathrm{~V}$ corridor crane will overlap coverage by the bridge crane coverage over the crane maintenance, pre-decon, and decon areas. This coverage overlap allows remote transfer of equipment from the $\mathrm{P} \& \mathrm{~V}$ corridor up to the hands-on maintenance room area.

\subsection{Utilities}

The preferred site for the CsIX/TRU Grout facility is immediately north of the tank farm, and is near fire water, potable water, and sanitary sewer services. Steam, provided from the INTEC coal-fired plant, is available at the utility corridor near building CPP-606. Plant air will be provided from the new facility's own compressor.

Based on information taken from Reference 9, there is adequate capacity in the following systems to support CsIX/TRU Grout operations.

Raw Water - Raw water is pumped from two 3,000 gpm production wells. Current raw water usage at INTEC is $25 \%$ of production capability.

Treated Water - CPP-606 has the capability of producing 129 million gal/month, but present demand is $35-40$ million gal/month.

Demineralized Water - CPP-606 produces demineralized water. INTEC uses approximately 50,000 $\mathrm{gal} / \mathrm{month}$. With the addition of a planned reverse osmosis system in 1998, capacity will be increased to $200 \mathrm{gpm}$.

Potable Water - Present usage of potable water is $1.5 \mathrm{million}$ gal/month. This is $20 \%$ of the available production capacity.

\subsection{Electrical}

\subsubsection{Available Electrical Utilities}

The INTEC ties into the $138 \mathrm{kV}$ INEEL loop at Substation 2, which is located outside the area fence to the south. Substation 2 transforms the $138 \mathrm{kV}$ to $13.8 \mathrm{kV}$ and provides power to Substations 10 and 15 , located within the INTEC complex. Power at $13.8 \mathrm{kV}$ is then distributed from these substations through the complex. The Electrical and Utility System Upgrade (EUSU) project, currently in the construction phase, will install a new $13.8 \mathrm{kV}$ electrical distribution system throughout the INTEC complex. This new system will provide greater safety, additional capacity, and greater reliability. 
Standby power at INTEC is currently provided by each facility. Standby generators are located at the various facilities and operate as islands of power during a normal power outage. The EUSU project will construct a standby power plant, install new standby generators, and tie existing generators into the standby power system. After completion of the EUSU project, standby power will be distributed through the INTEC complex by the new $13.8 \mathrm{kV}$ distribution system and controlled through the Utility Control System (UCS), which also controls the normal power distribution system.

See EDF-CsIX-014 in Appendix E for details on the existing and required electrical utilities for the CsIX/TRU Grout project.

\subsubsection{Normal Power}

Normal power will be supplied to the CsIX/TRU facilities by $480 \mathrm{kV}$ feeders from Loadcenter 14 . A combination of new and existing duct banks will route the feeders.

The major electrical loads for the CsIX/TRU Grout project are from various lighting, heating, and motor loads. The motor loads are from both process and ventilation motors. See EDF-CsIX-014 for details.

Electrical power requirementsfor scenario 1, the all-new facility, are estimated to be $698 \mathrm{kVA}$ connected and $471 \mathrm{kVA}$ demand.

Electrical power requirements for scenario 2, the use of NWCF plus a new grout facility, are estimated to be $569 \mathrm{kVA}$ connected and $381 \mathrm{kVA}$ demand for all but the NWCF loads. There is probably a net decrease in the load to NWCF, although this has not been quantified. Other, unrelated activities will be occurring in the NWCF at the same time as the CsIX/TRU Grout Project. For cost estimating purposes, the CsIX/TRU Grout project's portion of the NWCF electrical consumption is estimated to be about onehalf of the current NWCF electrical usage. This assumption is the basis for estimating the CsIX/TRU Grout Project electrical costs within NWCF, plus the project's costs for such common building systems as HVAC, off-gas, and maintenance.

\subsubsection{Standby Power}

Standby power to the CsIX/TRU facilities will be provided over the normal power distribution system from Substation 60 . Shedding of the normal power loads will be performed by the UCS.

Standby power requirements for scenarios 1 and 2 are estimated to be $88 \mathrm{kVA}$ and $53 \mathrm{kVA}$ standby power, respectively. Standby loads consist of the following:

- UPS Normal and Bypass feeders

- Selected lighting and miscellaneous loads

- Exhaust and supply fans

- Other process loads

See EDF-CsIX-014 in Appendix E for details. 


\subsubsection{Uninterruptable Power Supply (UPS)}

A solid-state uninterruptable power supply (UPS), with a static transfer switch, will provide a 15minute battery backup and a feed to a 208Y/120 Volt panel. The UPS and the panel will be located in the electrical room and support the following loads:

- Voice paging/evacuation systems

- Environmental monitoring system

- Control system

- Other critical loads. 


\section{METHOD OF PERFORMANCE}

\subsection{Design, Procurement, and Construction}

Conceptual Design will most likely be accomplished by LMITCO Engineering personnel, whereas Title Design might be subcontracted to an Architectural Engineering firm. Any long-lead components will be provided as Government-Furnished Equipment (GFE). Procurement contracts for these items will be placed during the Title Design phase, if necessary, to ensure the equipment is available during the construction window identified in Section 7. Fabrication and installation of equipment will be accomplished by competitively bid fixed-priced construction contracts. Standard components will be procured during the construction work period by the Subcontractor. Force account workers might perform demonstration and installation/modification work inside the NWCF.

\subsection{Work Breakdown Structure}

Figure 2 shows the preliminary Work Breakdown Structure for the CsIX/TRU Grout Project.

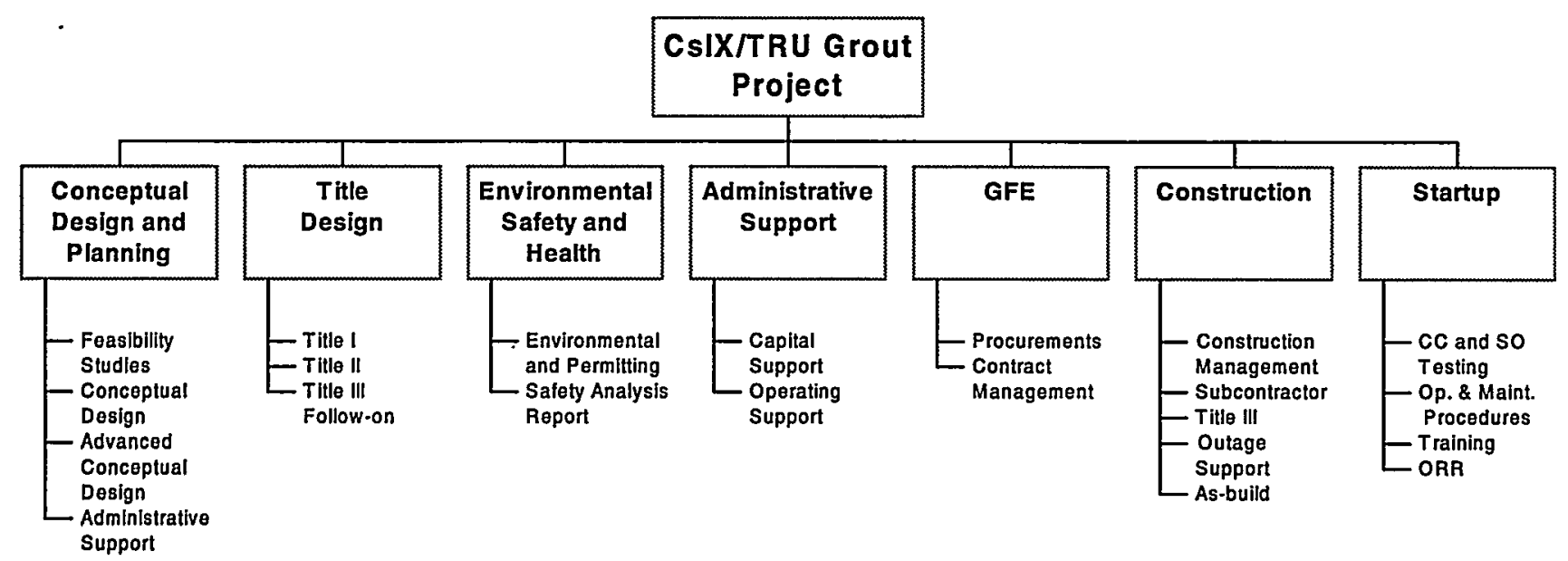

Figure 2. Work Breakdown Structure for the CsIX/TRU Grout Project.

\subsection{Facility Decommissioning}

A Decontamination and Decommissioning (D\&D) Plan will be written and in place before start of operations. The estimated cost to perform the D\&D work is summarized in Section 6 . Detailed and lifecycle cost estimates are contained in Appendix C.

If scenario 2 is selected, the existing NWCF D\&D Plan will be revised to reflect the modifications and additions generated by this project. 


\section{ESTIMATED COST}

The capital cost, also called total project cost (TPC), was estimated for this project. A TPC estimate was prepared for each of the two CsIX/TRU Grout Project scenarios. These estimates are divided into total estimated costs (TECs) and other project costs (OPCs). TECs include the title designs, project management (during title design and construction periods), construction and construction management, inspection, general and administrative costs, procurement, GFE, and all applicable escalation and contingency for the title design and construction periods. OPCs include conceptual designs, project management (during the conceptual and startup periods), environmental documentation, operations, support, final testing, and startup. To reflect the expected impact of inflation, the estimates are escalated to the future at a rate prescribed by "The Departmental Price Change Index Fiscal Year 2000 Guidance Anticipated Economic Escalation Rates, DOE Construction Projects and Operating Expenses," January 1998 update. The detailed cost breakdowns are included in Appendix $C$ and are summarized in Table 8.

A life-cycle cost (LCC) analysis was performed for each of the two scenarios. The LCC analyses estimated costs for preoperation, operation, and postoperation activities. LCC postoperation activities comprise decommissioning, decontamination, and demolition (DD\&D). The LCC analyses assume a 38year period (2000-2037) since this is the estimated time required to complete all anticipated CsIX/TRU Grout Project and remediation activities. A discounted LCC analysis assumes a constant-dollar basis, discounted at $6.10 \%$ per the Office of Management and Budget (OMB Circular A-94).

The LCC analysis for scenario 2 does not include DD\&D efforts for NWCF since it will be performed under another project (the NWCF closure project). For the preoperations portion of the cost analyses, the TPC estimates were used. Details on the LCC analyses are included in Appendix C and summarized in Table 8.

Table 8. Cost Estimates for the CsIX/TRU Grout Project.

\begin{tabular}{lcc}
\hline Cost Estimate & Scenario 1 & Scenario 2 \\
\hline Pre-Operations & & \\
$\quad$ Total Estimated Cost (TEC) & $\$ 102,000,000$ & $\$ 130,300,000$ \\
$\quad$ Other Project Cost (OPC) & $\$ 32,200,000$ & $\$ 39,800,000$ \\
Total Project Cost (TPC=TEC+OPC) & $\$ 134,200,000$ & $\$ 170,100,000$ \\
Discounted TPC & $\$ 92,400,000$ & $\$ 117,000,000$ \\
& & \\
Operations (with escalation \& contingency) & $\$ 367,400,000$ & $\$ 411,800,000$ \\
Discounted Operations & $\$ 181,600,000$ & $\$ 203,900,000$ \\
& & \\
Post Operations (DD\&D) & $\$ 48,122,000$ & $\$ 59,300,000$ \\
Discounted Post Operations & $\$ 5,000,000$ & $\$ 6,200,000$ \\
& & \\
Total Cost (unescalated, not discounted) & $\$ 301,100,000$ & $\$ 348,300,000$ \\
Total Cost (escalated, not discounted) & $\$ 549,700,000$ & $\$ 641,200,000$ \\
\hline Discounted Total Cost & $\$ 279,000,000$ & $\$ 327,000,000$ \\
\hline
\end{tabular}




\section{PROJECT SCHEDULE}

Project schedules were created for each of the scenarios and are given in Figures 3 and 4. For Scenario 1, the schedule shows the design and construction phases being completed in time to support the closure of a sixth INTEC tank farm underground waste storage tank starting in October 2008. The operations phase is scheduled to begin by January 2008 to permit the processing of all liquid wastes by the end of 2012. There is no contingency in the schedule before 2008; the funding, design, permitting, procurement, construction, system operability (SO) testing, and operations activities are all assumed to proceed relatively smoothly. If more time is needed for the funding cycle, design, or construction phases and the process ending date of December 2012 is to be met, then the operating window must be shortened accordingly by increasing the plant throughput capacity.

Note that it is not feasible for Scenario 2 to support the 2008 tank closure date nor the 2012 date with a 5-year operating window. This is primarily because the high radiation exposure that personnel would experience during rip-out and construction in the NWCF must be spread out over a longer time period and therefore will require a longer construction period. following:

This no-contingency schedule requires timely performance of many activities, including the

- The Line Item Project Funding Cycle must be started immediately to begin Conceptual Design by no later than early FY-2001.

- The RCRA permitting process for the SBW CsIX/TRU grout treatment system must be started in FY-2001 to support a construction start in FY-2005 at the latest. Obtaining this permit will take 3 to 4 years.

- The NEPA process for SBW must be started independently of the HLW \& Facility Disposition (FD) Environmental Impact Statement (EIS) if either

1. The SBW is not considered HLW and therefore not covered under this document, or

2. The HLW \& FD EIS record of decision (ROD) is delayed for various reasons and cannot support the schedule required for the SBW CsIX/TRU Grout Project. 


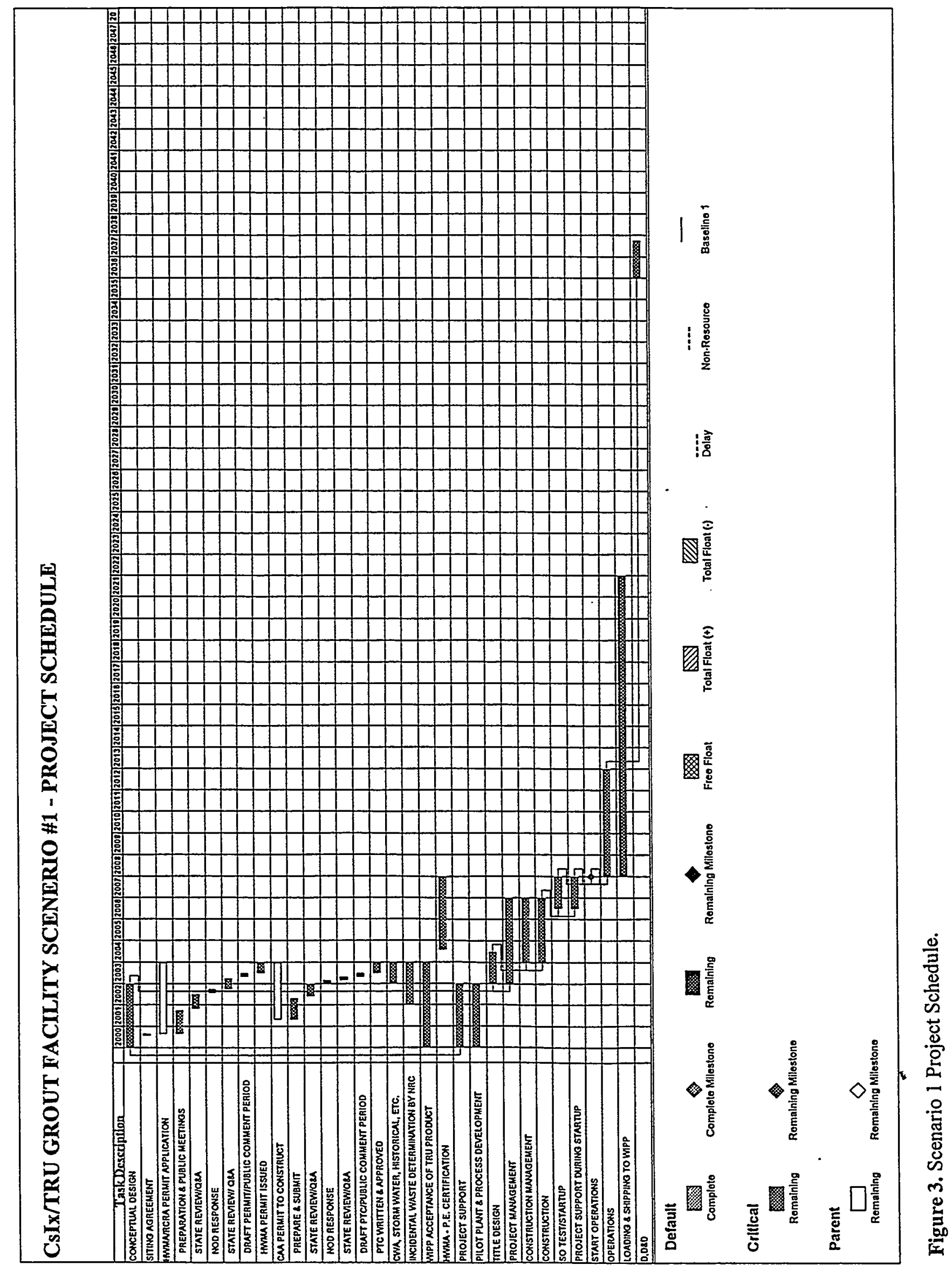




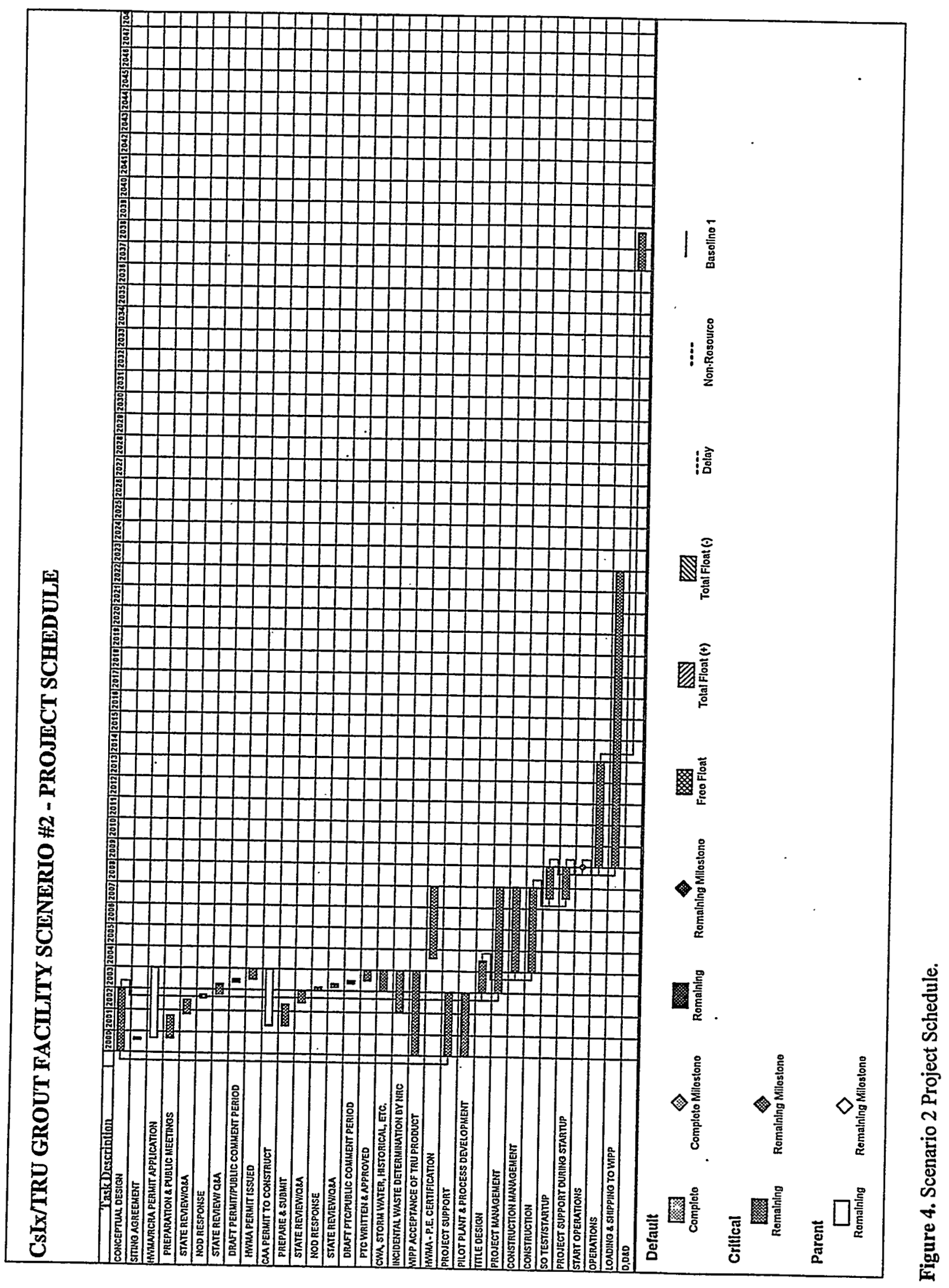




\section{REQUIREMENTS AND ASSESSMENTS}

\subsection{Radiological}

\subsubsection{General}

TRU radionuclides in the waste pose radiological hazards. While the external radiation exposure associated with TRU radionuclides is minimal, these materials do present a significant internal radiation exposure. Therefore, the engineered controls for this facility must include standard features for reducing and confining the loose contamination and shielding from radiation exposure.

The majority of the process systems are enclosed in a double confinement structure. A double confinement structure is defined as a series of physical barriers that, together with the ventilation system, minimizes the potential for release of radioactive material into work areas and the environment under normal and abnormal conditions. Radioactive materials are separated from the ambient environment by at least two barriers and from an operator by at least one barrier.

The ventilation system is designed to ensure air flow is from less contaminated areas to more contaminated areas. The primary containment is maintained at a negative pressure in relationship to the secondary containment and the secondary containment is maintained at a negative pressure in relationship to the environment. The ventilation system of the building is HEPA filtered.

The radiological contamination will be contained in the process system and the secondary confinement structures. This will be accomplished by use of radiological control work practices. These radiological work practices include the use of transfer sleeves for removal of radioactively contaminated material, maintaining the radiological contamination in the secondary confinement within established limits, and the use of airlocks for the removal of equipment and personnel from the secondary confinement.

See EDF-CsIX-015 and EDF-CsIX-016 in Appendix E for calculated radiation fields and shielding requirements for the CsIX/TRU grout waste form and facilities.

\subsubsection{NWCF Radiation Exposure}

The use of the NWCF in Scenario 2 provides the opportunity to use an existing facility and a significant portion of its equipment. However, rip-out of existing equipment and installation of new equipment for the CsIX/TRU process will require an estimated 40,000 hours of radiologically "hot" incell work, resulting in about 1,500 man-rem of exposure. This estimate assumes the calciner will be shut down permanently, so that the decontamination effort prior to any work would be very aggressive and effective. Decontamination methods would include a "sweep-out" with a final cold campaign, aggressive decontamination with strong acids or combinations that wouldn't normally be used due to potential corrosion damage to the vessels, and installation of local shielding. See EDF-CsLX-009 in Appenidix E for discussion. To limit the yearly personnel dose rate, this exposure level will require work to proceed over 6 years, resulting in at least a 1-year delay to the schedule so that the 2012 date is not met. Due to this, it is evident that it is not feasible for Scenario 2 to meet the 2012 schedule. 


\subsection{Environmental}

EDF-WTS-003, Regulatory Requirements for the Design, Construction and Operation of the ICPP Proposed Waste Processing Processes, located in Appendix E, provides an excellent description of the regulations and permitting activities that are required for this study; they are not repeated in this section.

A ruling is required by DOE and/or NRC that SBW/NGLW is not high-level waste (HLW). If the SBW is considered to be HLW, then a ruling should be sought that after removal of the cesium with the ion exchange system the cesium-free bulk liquid will no longer be $\mathrm{HLW}$, but instead low-level transuranic waste that can be disposed of at WIPP.

Another requirement will be successful negotiation with DOE and/or the State of Idaho of a reduction of the number of RCRA-listed codes contained in the INTEC liquid waste from 105 to 4, as argued in Reference 10. (This must also include negotiation that only these four codes are contained in the existing INTEC liquid waste management equipment system that will continue to be used for SBW/NGLW processing. The liquid waste management equipment system was assumed to be contaminated with all 105 codes due to applying the RCRA Part A HWNs to the PEW-TFF waste and application of RCRA "derived-from" and "mixture" rule concepts. This is important if, for any reason, previously processed wastes such as HLW calcine may perhaps not be recognized as having only these same four codes.) Reference 10 is a hazardous waste determination to assign the appropriate HWNs to the INTEC wastes by proving that they were not discharged to the PEW-TFF system as listed wastes versus "delisting" the wastes as provided for in RCRA law. Failure at this effort would most likely require treatment or separations processes to be added for the RCRA constituents in the waste, as well as development of a leach resistant "performance" waste form. This would require major changes to the process concept, adding to its complexity and cost.

It is also required that the regulatory process be addressed relative to listed HWNs not addressed by the WIPP performance assessment and not listed on the Part A portion of the WIPP RCRA Permit. At a minimum, a petition will be required to exclude the U134 (uncomplexed hydrofluoric acid, HF) HWN or modify the WIPP Permit's Part A to allow the receipt of waste with this U134 HWN. It appears that the TRU grout waste form would meet all of the technical requirements identified in the WIPP WAC. The Cs-bearing sorbent and filtered UDS waste streams generated from the CsIX process will have to be treated to the appropriate LDR 'standards and be excluded from futher hazardous waste management with a Petition to Exclude a Waste. Technically, the issue is not with achieving these LDR standards, since the concentration of listed constituents are minimal to start with and should not present an obstacle to delisting.

Negotiations with WIPP would also be desirable, as they have suggested, on issues such as bulk sampling of the waste feed to the CsIX/TRU grout plant to characterize the RCRA constituents, and characterization and inspection requirements for the final waste product (i.e., no-free-liquid determination, VOC analyses) with the goal of further reducing both capital and operational costs. WIPP personnel have suggested that these negotiations to reduce requirements are possible not only because of the homogenous nature of our feed (relative to "legacy wastes" which the WIPP WAC is primarily designed to protect against) but because of the control over the final waste form that would be achievable with proper system design. ${ }^{11}$

The only treatment WIPP requires for the hazardous constituents expected to be in the grouted TRU waste is to treat the corrosive characteristic of the waste by deactivation and to eliminate the free liquids. The free liquids will be hydrated in the matrix of the cement and will produce a dry solid waste form. 
Disposal of the cesium-loaded sorbent and filtered solids (UDS) should not present a problem. The sorbent and filtered UDS will contain sufficient TRU and Cs components to cause the waste to be greater than the Class $\mathrm{C}$ limits and would exhibit many of the characteristics of HLW.

The cesium-loaded spent sorbent, contaminated with transuranics from trace UDS, will be dried and stored for ultimate treatment and disposal by another project, probably in $\mathrm{HLW}$ glass in either the Idaho or Hanford vitrification plants. The solids will be disposed of at WIPP or, if this is not possible, treated in either the Idaho or Hanford vitrification plants and disposed of with the HLW.

The UDS filtrate contains transuranic constituents as well as other beta/gamma emitting radionuclides (e.g., cesium) in sufficient quantities that this waste product would probably qualify for disposal at WIPP. Otherwise, the filtrate could be treated by the vitrified waste process either at the INEEL or at Hanford.

The acceptance criteria for the disposal sites in the DOE complex where the final waste from the CsiX/TRU grout process might go are described in Reference 1.

The permitting of the facility, conceptual design, and negotiations on the waste acceptance agreement with WIPP must be conducted at the same time. The three issues are interlated and must be finalized before construction of the facility. If the approval for all three items cannot be achieved early in the conceptual design process, then an alternative process within the bounds of the HLW and FD EIS would have to be selected.

\subsection{Quality}

Appropriate quality program documentation will be generated and followed as necessary to confirm proper design, construction, and operation of the CsIX/TRU grout facility. Waste inspection requirements to assure compliance with WIPP WAC will be negotiated with WIPP; this study assumes that each waste drum must be visually inspected and a drum headspace gas sample taken and analyzed.

It is assumed that DOE-ID will continue to implement the quality assurance program described in DOE-RW-0333P, Quality Assurance Requirements and Description, and will apply it to this effort as well. The impact of implementing DOE-RW-0333P is reflected in the capital and life-cycle cost estimates generated by this Feasibility Study.

\subsection{Safety}

\subsubsection{Facility Hazard Category and Performance Category}

These categories are discussed under Section 4.3.1, "Structural Design."

\subsubsection{Industrial Safety}

The basic approach to worker safety for the CsIX/TRU Grout Project is the incorporation of standard engineering and administrative controls to separate the worker from physical, chemical, and radiation hazards. The LMITCO Health and Safety Program includes aggressive goals with respect to ALARA for radiation hazards and lost time worker injury rates for industrial workers. These goals are most effectively attained by physical separation of workers from the hazards associated with the processes contained within the project buildings. 


\subsubsection{Construction Hazards}

Construction hazards for this project are typical for most construction sites. For example, heavy equipment produces physical hazards of striking objects, crushing of hands or feet, and head injuries. Construction Subcontractors shall be required to comply with 10 CFR 1926, "Occupational Safety and Health for Construction," and monitored to ensure compliance.

\subsubsection{Systems Safety}

Manual and remote modes of system operation will allow standard physical lockout of either mode to ensure that operators do not initiate actions that could injure workers performing maintenance, repair, or troubleshooting tasks inside hazardous areas. Equipment and procedure to ensure safe shutdown of the different process systems will be provided as required by the hazards associated with the system.

Systems that may have high radiation fields will have remote repair or maintenance capabilities.

\subsubsection{Equipment Safety}

Equipment will comply with 10 CFR 1910, "Occupational Safety and Health Standards for General Industry." For example, equipment will be provided with guards, lockout/tagout features, operation and maintenance documentation, and any other items necessary to ensure safe operation of the equipment.

\subsubsection{Personnel Safety}

In general, workers will be physically separated from most hazardous systems by shielding walls, glove ports, viewing windows, booths, equipment guards, or other physical features. Administrative procedures will allow workers access to some systems for maintenance or repair of equipment. Entries to cells and shielded corridors are viewed as hazardous and nonroutine and will require the use of safety permits. A Radiation Work Permit will address radiological hazards. Personnel safety will be maintained by:

- Following administrative and procedural controls for entry into confinement areas.

- Incorporating engineering controls into the design for systems and equipment.

- Using personnel monitoring equipment.

- Using video monitoring inside confinement areas.

- Using lockout/tagout of remote controls and manual controls when working on equipment or systems.

- Using emergency procedures to initiate worker response to chemical spills/reactions, fires, and other emergencies.

- Using the buddy system when working inside confinement structures or other hazardous areas. 


\subsubsection{Emergency Preparedness}

Operations for this project will coordinate emergency preparedness planning with the INTEC Emergency Response Organization (ERO). The INTEC ERO will handle site emergencies.

Access for emergency vehicles and personnel will be provided as part of the project design. 


\section{UNCERTAINTIES AND ISSUES}

\subsection{General Concerns}

The CsIX/TRU grout process was conceived as an alternative method for processing INTEC liquid wastes. Data in support of the process design was taken from development work performed for other projects. As such, there are numerous areas where data is missing, resulting in various issues and uncertainties associated with the process. The process as presented in this report, although containing individual processes used separately in industry, has not been tested as described and hence not optimized. Because the primary goal was to design a feasible process, most assumptions and decisions have leaned toward the conservative or upper-bounding case. Substantial reductions in waste volumes and capital costs could be achieved by obtaining, for example, sample data on the liquid and solid waste properties, and by improving the process and acquiring supporting information. This section discusses these areas and other issues. Section 10 contains recommendations for obtaining additional information to provide high confidence in the process and reduce costs by optimizing it.

\subsubsection{Behavior of UDS in the Process and Effects on Products}

The very limited analytical data available on UDS results in considerable uncertainty in the assumed quantity and composition of the UDS. Limited analytical data, combined with minimal physical characterization of the UDS upon which to base its performance in the process, results in several uncertainties in this study. These uncertainties include:

- The effect of UDS that pass through the filtration system and ion exchange columns on the radiation level of the TRU solid product (i.e., the ability to formulate contact-handled waste with reasonable waste loadings)

- The effect of UDS on both the chemical and physical performance of the cesium sorbent

- The effect of radionuclide (transuranic) and hazardous chemical species (RCRA) on the classification of the spent cesium sorbent, and resulting requirements for its further treatment and ultimate disposal

- The amount of U-235 in the UDS composition, and the criticality level resulting from concentrating the solids through evaporation. Equipment selection and layout did not consider criticality issues.

\subsubsection{TRU Waste Volume}

One of the greatest uncertainties of the process is the final TRU waste volume. The CsIX/TRU grout process, as described in this report, defaulted to two conservative assumptions because of the lack of data beyond "performance" grout waste forms (high strength mechanical properties, leach resistant). The two assumptions are: (1) further evaporation of the CsIX effluent will not be performed before solidification, and (2) solidification will be by grouting. The grout formulation used in this study was not volumeoptimized to take advantage of the minimal requirements of the WIPP WAC. A brief evaluation, given in EDF-CsIX-001 in Appendix B, compares solidification with grout to absorption of the liquid waste on several absorbents, which shows a potential $30 \%$ reduction in waste volume. Other solidification methods that may significantly reduce waste volume include:

- Development of a grout loading/formulation specifically for the WIPP WAC 
- Selection of an absorbent capable of a high waste loading

- Evaporation of the waste further before grouting

- Evaporation of the waste to a solid form

- A combination of these ideas.

\subsubsection{Ion Exchange Performance}

The main requirement of the process is to produce a contact-handled waste product acceptable at and ready for shipment to WIPP. The major process step that fulfills this requirement is the removal of cesium by ion exchange. To date, the selected ion exchange process has only been demonstrated at a very small scale $\left(1 \mathrm{~cm}^{3}\right)$, with very limited amounts of surrogate wastes, and to only a small fraction of cesium-breakthrough. Thus, there are uncertainties in the performance of the cesium sorbent in a fullscale column, using actual wastes, and operated to breakthrough. An expectation, although not proven, is that minor components in the waste will not affect the ability of the sorbent to remove cesium. Another expectation is that major waste components, with their variable quantities, will not affect the sorbent's performance.

All of these will determine the need to remove the solids and at what level of filtration efficiency. If very high efficiencies are required, it could affect the viability the process concept.

\subsubsection{Liquid Waste Composition and Volume}

Incomplete analyses are available of liquid waste for all tank farm tanks. Most analyses do not report on trace species, including organics, which may affect classification of the final wastes, or even the ability to solidify the TRU waste. Only limited data is available on the radionuclide components. There is also uncertainty in the total liquid waste feed volume because of (1) the undetermined, as yet, sequence of blending tank farm liquids before evaporation in the HLLWE, (2) the amount of waste that will be calcined, and (3) the amount of NGLW that will be generated, including tank farm heels and flushes from tank closure activities, and the proportion of that waste that would be processed by the CsIX/TRU grout process.

Related to the last point, the Tank Farm Closure team has still not determined what is to be done with the bulk settled solids in the tanks. If these solids are going to be removed from the tanks, the bleedthrough of even trace levels of solids to the grout could cause significant problems in maintaining the WIPP CH limit for the waste. This is an important factor for the CsIX/TRU grout concept, and should be determined as soon as possible.

\subsubsection{Processing and Disposition of UDS and Spent Sorbent}

The requirements for final disposition of the filtered UDS and spent cesium sorbent have not been completely defined. As a result, there is uncertainty in defining the systems required to handle and store these wastes until their final disposition. Drying and handling characteristics, including pnuematic transport, of these products are largely unknown. The amount of backwash that will be required for processing UDS is also uncertain. A very conservative solids loading of $60 \mathrm{~g} / \mathrm{l}$ in the backwash was assumed. This single assumption, to a large degree, determines the size and hence the cost of the entire UDS processing system. 


\subsubsection{Processing Grout Mixtures in Large-Scale Equipment}

The requirements for mixing equipment, solids feed systems, cycle timing, grout rheology, and grout uniformity are not adequately defined. Also, little information is available on heat generation, water bleed, and other factors that require consideration in the cure cycle. Grout mixer cycle times directly influence the number and size of mixers that must be used to achieve the desired throughput. Cure cycle uncertainties directly affect the size of curing stations, ventilation and heat/ cooling loads, and the size/ need for dewatering stations.

\subsubsection{WIPP Receipt Capability}

Verbal discussion with WIPP personnel indicates that WIPP can receive the grouted waste on the schedule and at a shipment rate that is compatible with CsIX/TRU Grout Project objectives, and that prior negotiations and revision of Part A of the WIPP Permit will be successful. However, it is worth noting that WIPP is not yet receiving any waste, and the estimated average shipping rate of 5 shipments every 2 weeks through 2021 is perhaps an optimistic assumption.

\subsubsection{Optimistic Schedule}

The Project Schedule indicates that the 2012 date can be met for Scenario 1 if the funding, design, permitting, procurement, construction, SO testing, and operations activities all proceed relatively smoothly. However, no project of this complexity at the INEEL has proceeded in this short time in the recent past. This optimistic, no-contingency schedule is a concern. The schedule will become more realistic if future studies yield simpler design and operation. However, if funding, design, or construction take longer than is scheduled, so that less than 5 years will be available to process the waste, then it is possible that a larger scale plant with more capacity and higher throughput rate could be designed and built.

\subsection{NWCF Facility Concerns}

Scenario 2 was found to not be feasible to meet the 2012 date for processing the liquid waste, based primarily on the high radiation exposure that personnel would experience during rip-out and construction in the NWCF. If that factor could be overcome, however, the following additional issues should be considered when contemplating the installation of CsIX/TRU grout process equipment in the NWCF.

- The fundamental advantage of housing new processes in the NWCF is the possibility of utilizing the existing NWCF structure, radiological shielding, access to tank farm feed, utilities, chemical makeup, etc. With the NWCF and its equipment nearly 20 years old, finding spare parts and maintaining the aging equipment is becoming difficult. Spare parts are difficult to obtain, since many manufacturers no longer produce the equipment models installed in the NWCF.

- Previous INTEC experience with installing new processes in existing facilities has shown that cell penetrations are seldom in the correct locations nor are there sufficient penetrations for the new process. The scope of this problem is usually not fully understood until late in the title design phase causing escalation in the design and construction cost estimates.

- The building was designed for NWCF remote operations and not for CsIX/TRU grout operations. The cell windows, PARs, and master-slave manipulators may not be in the proper locations to provide for efficient remote activities. 
- Since new equipment will be installed in existing cells and some existing equipment will be utilized, SO testing will be conducted in a contaminated facility with contaminated equipment. As a result, the testing process will be more complicated, last longer, and be more expensive.

- Design philosophy at the INTEC has been to minimize the number of in-cell mechanical items such as valves and pumps. The CsIX/TRU grout process requires several mechanical items; however, the NWCF building scenario leaves few options for equipment placement. As a result, the proposed location for several pieces of mechanical equipment is in-cell, in direct violation of this design philosophy.

- The CsIX/TRU grout layout calls for spent sorbent to be sluiced approximately $100 \mathrm{ft}$ horizontally and $30 \mathrm{ft}$ vertically from the adsorber cell to the top of the calciner cell. Such a transfer is possible, however questions surround the practicality of doing so.

- Radiation fields in excess of $100 \mathrm{R} / \mathrm{hr}$ raise questions concerning the reliability and life span of electrical and mechanical components.

- NWCF filters are being stored in the hot sump tank removal cell while waiting to be processed through the filter leach process. The filters must be removed and leached before construction, otherwise an alternate storage location must be found. 


\section{RECOMMENDATIONS}

\subsection{General}

The following steps, all with the same relative schedule urgency (critical path), must be taken soon to make this process concept and project meet the 2012 "cease use" date for the Tank Farm tanks:

\subsubsection{Negotiate Reduction in Number of HWNs in Waste}

Reference 10 argues for successful negotiation with DOE and/or the State of Idaho to reduce the number of RCRA listed codes (hazardous waste numbers-HWNs) contained in the INTEC SBW/NGLW from 105 to 4. (This must also include negotiation that only these four codes are contained in the existing INTEC liquid waste management equipment system that will continue to be used for SBW/NGLW processing. The liquid waste management equipment system was assumed to be contaminated with all 105 codes due to applying the RCRA Part A HWNs to the PEW-TFF waste and application of RCRA "derived-from" and "mixture" rule concepts. This is important if, for any reason, previously processed wastes such as HLW calcine may perhaps not be recognized as having only these same four codes.) This document is a hazardous waste determination to assign the appropriate HWNs to the INTEC wastes by proving that they were not discharged to the PEW-TFF system as listed wastes versus "delisting" the wastes as provided for in RCRA law. Three of these four codes (F001, F002, and F005) are already accepted at WIPP; the fourth code, U134, (uncomplexed hydrofluoric acid, HF) will have to be proven to be complexed in the INTEC waste or added to the WIPP permit. Failure at this effort would most likely require treatment and/or separations processes to be added for the RCRA constituents in the waste as well as development of a leach resistant "performance" waste form requiring major changes to the process scheme, adding to its complexity and cost. As such, this issue should be settled as soon as possible.

\subsubsection{Obtain Ruling that Waste is not HLW}

A ruling by DOE and/or NRC should be pursued to the effect that SBW/NGLW is not high-level waste (HLW) or, if the SBW is considered to be HLW at present, that after removal of the cesium with the ion exchange system, the cesium-free bulk liquid will no longer be HLW but instead low-level transuranic waste that can be disposed of at WIPP.

\subsubsection{Begin Negotiations with WIPP}

Negotiations should be started with WIPP to accept the waste. Negotiations should cover at least these major issues: (a) overall volume of the waste, (b) receipt rate and schedule for the waste, (c) determination that SBW/NGLW satisfies the WIPP Land Withdrawal Act "defense waste" requirement, and (d) the need, as may be determined necessary, to revise various high-level WIPP documents such as the Performance Assessment or the Operating Permit (for example to add RCRA-listed code U134, HF). This should be started in FY-2001 at the latest.

\subsubsection{Start Line Item Project Funding Cycle}

The Line Item Project funding cycle must be started now to begin Conceptual Design by no later than early FY-2001. 


\subsubsection{Determine Fate of Solids in Tanks}

A decision is needed from the Tank Farm Closure team on what is to be done with the bulk settled solids in the tanks. If the Tank Farm Closure Risk Assessment were to determine that they must be removed, this would be quite a challenge. Not only would their removal be difficult but so would their separation and disposal (a minimal estimate of $1 / 4$ in. in all eleven tanks is $450 \mathrm{ft}^{3}$ of solids; a more realistic 2-in. estimate is $3,600 \mathrm{ft}^{3}$ ). The bleed-through of even trace levels of solids to the grout cause significant problems in obtaining the contact-handled dose rate for the waste. This is vitally important to the CsIX/TRU grout process concept and an answer is needed by mid FY-2000 at the latest.

\subsubsection{Start RCRA Permitting}

The RCRA permitting process for the SBW CsIX/TRU grout treatment system should be started soon. Obtaining this permit will take 3 to 4 years; it must be started in FY-2001 to support a construction start in FY-2005 at the latest.

\subsubsection{Start NEPA Process}

The NEPA process for SBW should be started independently of the HLW \& FD EIS if (1) the SBW is not considered HLW, as assumed by this study, and therefore not covered under the EIS document or (2) the HLW \& FD EIS record of decision (ROD) is delayed for various reasons and cannot support the schedule required for the SBW CsIX/TRU Grout Project.

\subsection{Future Studies and Tests}

It is recommended that the future studies listed in this section be funded and aggressiviely pursued, to reduce the uncertainties discussed in section 9.1 and to reduce project capital and operating costs. Work must proceed quickly to support starting Conceptual Design in FY-2001.

\subsubsection{Tank Farm Liquid Waste Characterization}

The results of sampling and analyses performed as part of the Tank Farm Closure and other programs should be used to prepare simulants for ion exchange tests and TRU waste minimization tests. Any continuation of design studies for the CsIX/TRU grout process should use the volumes of tank farm waste as currently provided and periodically updated on the High-Level Waste Systems Engineering web pages.

\subsubsection{UDS Analyses}

Characterization of UDS in the tank farm tanks, both suspended in the liquid and settled at the bottom, is needed to provide a basis for the design of UDS removal, handling, and packaging equipment, and to establish the required solids removal efficiency. Characterization of conditions, both before and after washing the tank, should include the total concentration and particle size distribution for each tank farm tank and the concentration of both hazardous and radionuclide species as a function of particle size. The radionuclide concentrations are of particular importance for determining the effect of UDS on the solidified TRU waste form, and as an important factor in the review for potential criticality or special nuclear material concerns. The concentration of fission products, notably ${ }^{137} \mathrm{Cs}$, in the UDS must be known to ensure that the final TRU waste can be contact-handled with reasonable waste loadings. There is a large variation between contents of different tanks, so UDS from more than just a few tanks must be characterized. 


\subsubsection{UDS Removal Demonstration Tests}

Testing is recommended to determine the degree of filtration required to avoid contaminating the cesium sorbent with TRU-containing solids, plugging the ion exchange column, and contaminating the TRU waste with high-dose contributing radionuclides. Additional to filter system testing, the tests should determine what fraction and UDS particle sizes pass through the CsIX column and what fraction and particle sizes are retained by the column. Follow-up testing of filtration equipment is recommended to demonstrate that the required removal efficiency of UDS can be achieved.

\subsubsection{Ion Exchange Tests}

The present data for the CST sorbent cesium loading is from 1- $\mathrm{cm}^{3}$ tests. Data from larger-scale tests are needed to confirm sorbent performance at increased volumes and to provide a basis for full-scale operations. Sorbent performance data are needed over the range of liquid waste compositions present in the tank farm tanks, particularly the range of cesium concentration, acid concentration, and other species that have a potential for chemical interaction with the sorbent. Long-term performance data are needed to discover kinetically slow chemical interactions of sorbent with the liquid waste, and to confirm models of sorbent performance from start of run to cesium breakthrough.

\subsubsection{Final Waste Volume Optimization Studies}

The goal of this recommended new study would be to define a process that minimizes the volume of waste shipped to WIPP. Three volume-minimization methods are recommended for study.

- Development of a high-waste-loading grout, meeting only the requirements of the WIPP WAC. Tests are recommended to better determine the limiting parameters for the grout including, but not limited to, minimum water, maximum solids, maximum nitrate, maximum chloride, and stability over time and temperature. Tests in which unneutralized SBW is grouted with Portland cement only are recommended.

- Use of an absorbent, such as an inorganic polymer absorbent or Aquaset/Petroset. This would include a thorough review of absorbents capable of absorbing high-solids-content acid solutions, followed by testing of the most promising.

- Evaporation of the ion-exchange effluent to a dry solid. Recent testing by R. J. Kirkham ${ }^{8}$ has shown that that a crystal-like solid product, less than $30 \%$ of the original volume, can be produced by evaporating a simulated SBW solution and cooling the concentrate. Additional testing of solidification by evaporation and cooling is recommended to verify Kirkham's preliminary test results. Tests for this scenario include determining offgas species during evaporation, determining the stability of the crystallized waste product, determining the type of evaporation and packaging equipment to be used in the process, and analysis of the materials of construction for this equipment. Possible problems with this process will need further evaluation. Preliminary reports indicate this waste form can be readily reliquefied upon application of heat. This could be a concern in certain transportation accident scenarios.

Development of the grout and testing of absorbents would be done in conjunction with evaporation tests that would concentrate the liquid to the limit set by the solidification agent. The possibility exists that the waste could be concentrated too much and thereby exceed the contact-handled dose rate. This could be mitigated in various ways by, for example, adding salts to the waste to make it solidify at a lesser concentration or providing some minor shielding in the waste package to reduce the field. Also, the possibility of sending remote-handled waste to WIPP could be further investigated. 
Should partial evaporation in conjunction with grouting or absorption prove optimal, the following is recommended:

- Determine the nature of precipitates as waste is concentrated

- Determine if the optimum evaporator location is upstream or downstream of cesium removal or if separate systems are required on both ends to provide certain process capabilities such as, for example, fine tuning of waste loadings or concentration of various secondary process waste waters such as resin sluice water, flitration system flush water, etc.

- Evaluate evaporation methods and related equipment types, followed by demonstration tests of the selected equipment.

- Determine the extent of evaporation possible before encountering operational problems with either the evaporator or downstream equipment.

\subsubsection{Process Simplification Study}

Some of the systems presented in the CsIX/TRU grout process design were chosen based on assumptions that simpler process concepts may not work. These assumptions were the direct result of insufficient information being available to confidently demonstrate that more simple concepts will be effective. Thus, the design presented may include process concepts that are more complex than actually required and could result in higher than necessary equipment, facility, and operational costs. After the project and process uncertainties and assumptions have been reduced or eliminated by, among other things, INTEC Technical Development empirical work, further engineering study is recommended to rework the design with a view toward both operational simplicity and cost savings. Future brain-storming sessions to develop creative ideas, studies, and decision making should continue to include personnel from the various INTEC organizations, such as Technical Development, NWCF Operations, and Engineering. Experts in particular areas, such as filtration and remote handling, should also be included. The following three ideas are presented as issues to be considered:

- Eliminate active mechanical dryers in-cell. This can be accomplished for spent sorbent if testing shows a method of rinsing and drying (heat only or heat plus drying/fluidizing air) that allows the media to be dried in-place in the ion exchange column itself or in place-in the storage tank(s) after being sluiced there. For the UDS, various options could be considered to avoid a mechanical dryer in-cell. One option would be to produce a high solids content sludge, filter cake, or filter media that is placed in a drum and dried inside the drum.

- Identify filtration options that are relatively simple and amenable to remote operation and retrofit. A separate comprehensive concept evaluation and selection study should be considered that identifies and evaluates options for filtration and solids dewatering. Subtasks include collection of information from vendors and people involved in operation of filtration and dewatering systems, site visits to locations using various technologies and system concepts, some scoping tests, and discussions with consultants or other experts with broad experience in filtration and solids dewatering systems.

- Use of out-of-cell valve and instrumentation boxes for most of the extensive valving and monitoring needed for the multi-column ion exchange system concept. The valve actuators and position switches and most of the waste stream radiation monitoring hardware would be located outside the box where they could be directly maintained. Valves and any other items inside the box would be contact maintained after draining and flushing the lines and opening 
the box. Or, use a modular, space efficient unit with the IX feed tank, columns, waste/flush tank, and pumps on a single skid (estimated size $4 \times 12 \mathrm{ft}$ ). Implementing these two concepts for Scenario 2 may allow the ion exchange system to be located in the NWCF calciner cell without removing the calciner, eliminating the need to clean out the NWCF absorber cell.

\subsubsection{UDS and Spent Cesium Sorbent Processing}

Tests are needed to determine the washing requirements, if any, and the drying requirements of both the spent cesium sorbent and the separated UDS (independently) and to demonstrate the use of equipment to dry both to free-flowing, pneumatically transportable solids. An important consideration in these washing. and drying tests is operational simplicity, to minimize maintenance on equipment that would process these high-radiation-field solids. As such, in-place dryers should be considered. 


\section{CONCLUSIONS}

- For the all-in-one grout building scenario (\#1), the CsIX/TRU grout process can feasibly be built and operated successfully in time to process all the liquid waste in the INTEC tank farm before the end of 2012, within a total life cycle cost (escalated and discounted) of $\$ 279$ million.

- Meeting the 2012 schedule requirement is not feasible for the scenario (\#2) of locating the most radiologically hazardous part of the CsIX/TRU grout process in the NWCF. This is in large part due to the high worker radiation dose (about 1,500 man-rem total) during the rip-out and construction phases in the NWCF. In addition, this scenario costs more (total life cycle cost, escalated and discounted $=\$ 327$ million), and there are many other concerns surrounding the practicality of this scenario. Therefore, this scenario should not be pursued.

- The grouted waste form will meet WIPP waste acceptance criteria. The solid waste shipped to WIPP will contain approximately $500 \mathrm{nCi} / g$ TRU on average. Negotiations with WIPP would be desirable, as they have suggested, on issues such as bulk sampling of the waste feed to the CsIX/TRU grout plant to characterize the RCRA constituents, and characterization and inspection requirements for the final waste product with the goal of further reducing both capital and operational costs. WIPP personnel have suggested that these negotiations to reduce requirements are possible not only because of the homogenous nature of our feed, but because of the control over the final waste form that would be achievable with proper system design.

- The four expected RCRA hazardous waste constituents in the SBW are identified in Part A of the WIPP Permit, with the exception of U134 (uncomplexed hydrofluoric acid, HF). This particular HWN must be delisted from the INTEC waste before the TRU waste can be shipped. If it cannot be delisted, then a performance assessment must be conducted for the addition of the U134 code to Part A of the WIPP Permit. (This performance assessment may need to address up to 30 chemicals, depending on the final assessment for designating listed HWNs for the SBW and NGLW.)

- The process as presented in this report, although containing individual processes used separately in industry, has not been tested as described and hence has not been optimized. Because of the primary goal of conceiving a feasible process, most assumptions and decisions have leaned toward the conservative or upper bounding case. Substantial reductions of both capital and operating costs and waste volumes can probably be achieved by improving the process and acquiring supporting information. Research and testing are necessary to substantiate this.

- It is imperatiave that action begins soon on the following recommendations (detailed in Section 10 ) in order for the CsIX/TRU grout process concept and project to meet the 2012 "cease use" date for the Tank Farm tanks:

- Successful negotiation with DOE and/or the State of Idaho to reduce the number of RCRAlisted codes (HWNs) contained in the INTEC liquid waste from 105 to 4

- A ruling by DOE and/or NRC that SBW/NGLW is not high-level waste (HLW)

- Start and succeed in negotiations with WIPP to accept the waste

- Start the Line Item Project funding cycle now to begin Conceptual Design by no later than early FY-2001. 
- Have the Tank Farm Closure team determine what is to be done with the bulk settled solids in the tanks.

- Start the RCRA permitting process for the SBW CsIX/TRU Grout treatment system.

- Start the NEPA process for SBW independently of the HLW \& FD EIS

- Fund and aggressively pursue various future studies, primarily investigating INTEC Technical Development issues. 


\section{REFERENCES}

${ }^{1} \mathrm{~J}$. Banaee, Standard Criteria of Candidate Repositories and Environmental Regulation for the Treatment and Disposal of ICPP Radioactive Mixed Wastes, INEEL/EXT-97-01147, Revision 2, February 1998.

${ }^{2}$ Waste Acceptance Criteria for the Waste Isolation Pilot Plant, DOE/WIPP-069, April 1996.

${ }^{3}$ W. B. Palmer, et al., ICPP Tank Farm Planning Through 2012, INEEL/EXT-98-00339, April 1998.

${ }^{4}$ J. E. Miller, N. E. Brown, Development and Properties of Crystalline Silicotitanate (CST) Ion Exchangers for Radioactive Waste Applications, SAND97-0771, April 1997.

${ }^{5}$ T. A. Todd, K. N. Brewer, D. J. Wood, P. A. Tullock, L. G. Olson, A. L. Olson, "Evaluation and Testing of Inorganic Ion Exchange Sorbents for the Removal of Cesium-137 from Idaho Chemical Processing Plant Acidic Tank Waste," Submitted to Separation Science \& Technology, December, 1997.

${ }^{6}$ K. N. Brewer, "Neutralization of SBW Prior to Cs Removal by CST, and a Review of DSI989-GES-028, 'SBW Treatment Ion Exchange Media Disposition,"' Lockheed Martin Idaho Technologies Company Interdepartmental Communication, KNB-08-98, July 20, 1998.

${ }^{7}$ M. D. Argyle, R. L. Demmer, "Tests of Acid-Bond A660 for Solidifying Sodium-Bearing Waste," LMITCO Interdepartmental Correspondence, MDA-02-98/RLD-05-98, October 12, 1998.

${ }^{8}$ R. J. Kirkham, "Sodium Bearing Waste Solidification by Evaporation," LMITCO Interdepartmental Communication, RJK-6-98, September 29, 1998.

${ }^{9}$ R. S. Garcia, Utilities and Infrastructure Report, INEEL/EXT-97-01398, February 1998.

${ }^{10}$ Kenneth L. Gilbert, Regulatory Analysis of INTEC Liquid Waste Stream U.S. Environmental Protection Agency Hazardous Waste Numbers, INEEL/EXT-97-01453, July 1998.

${ }^{11}$ Bob Kehrman and Jim Bosley, "WIPP Telecon for CsIx on August 19, 1998," Functional File No. BCCsIX-25. 


\section{Appendix A}

Assumption/Decision Table 


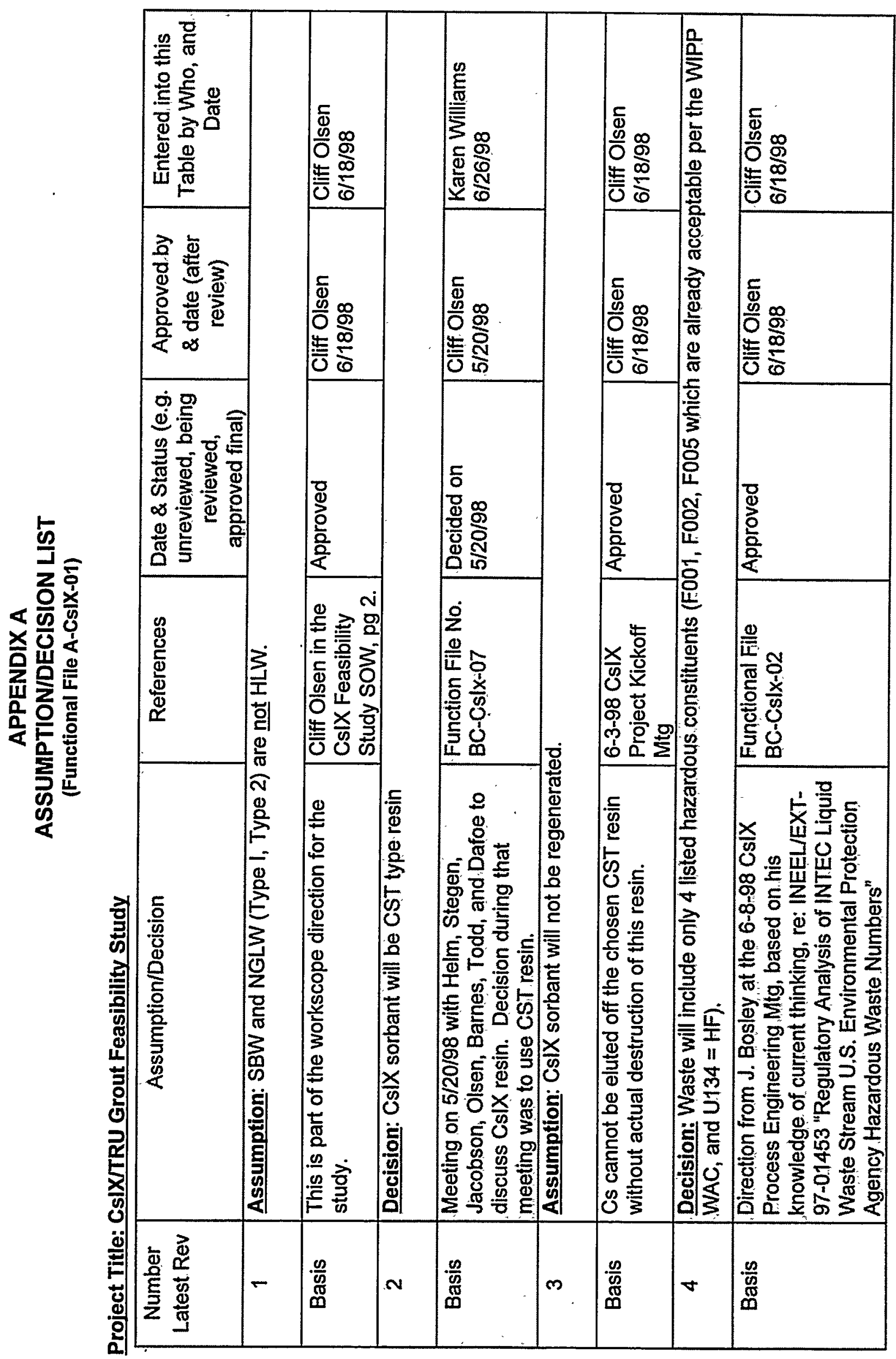




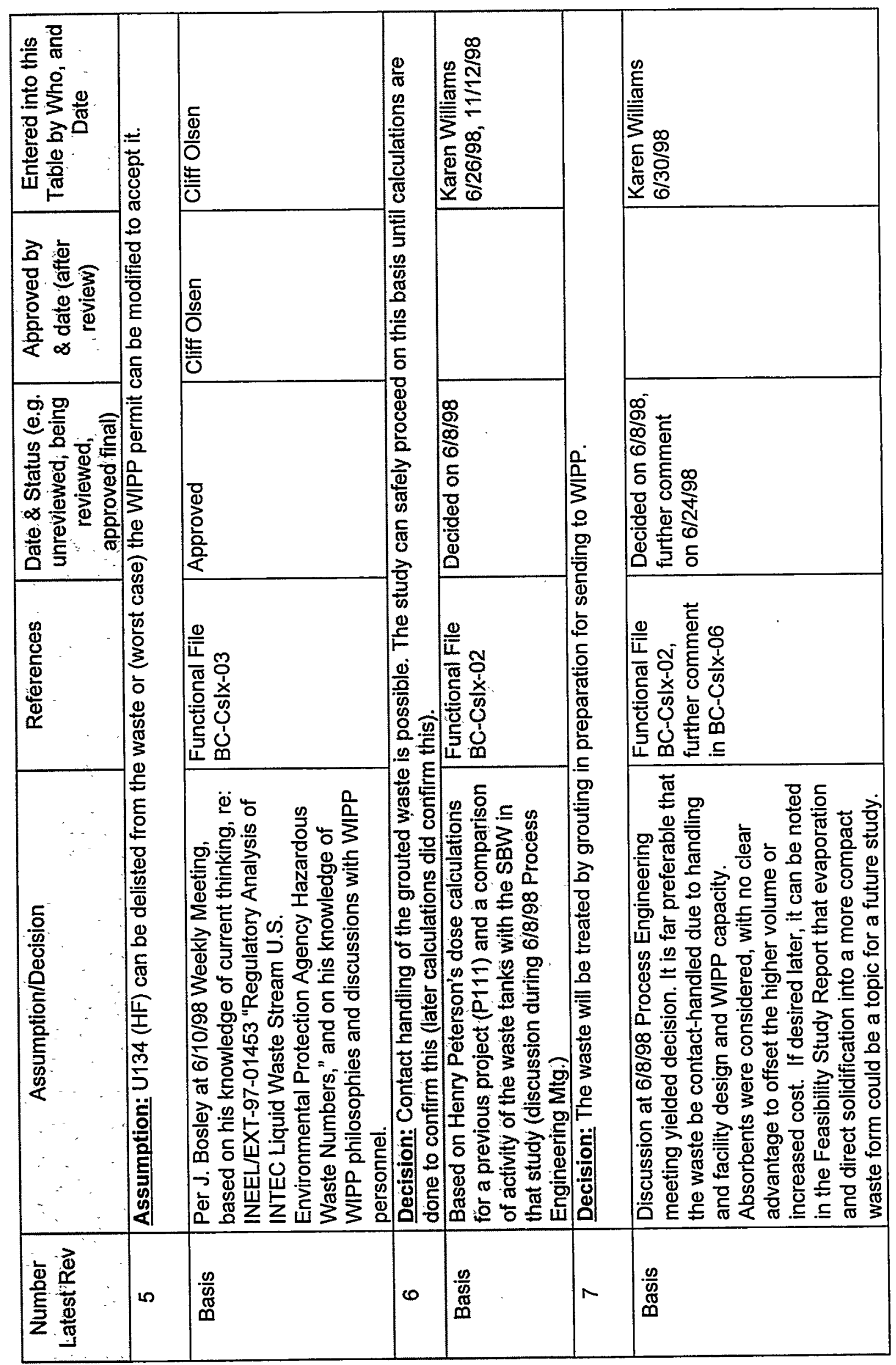




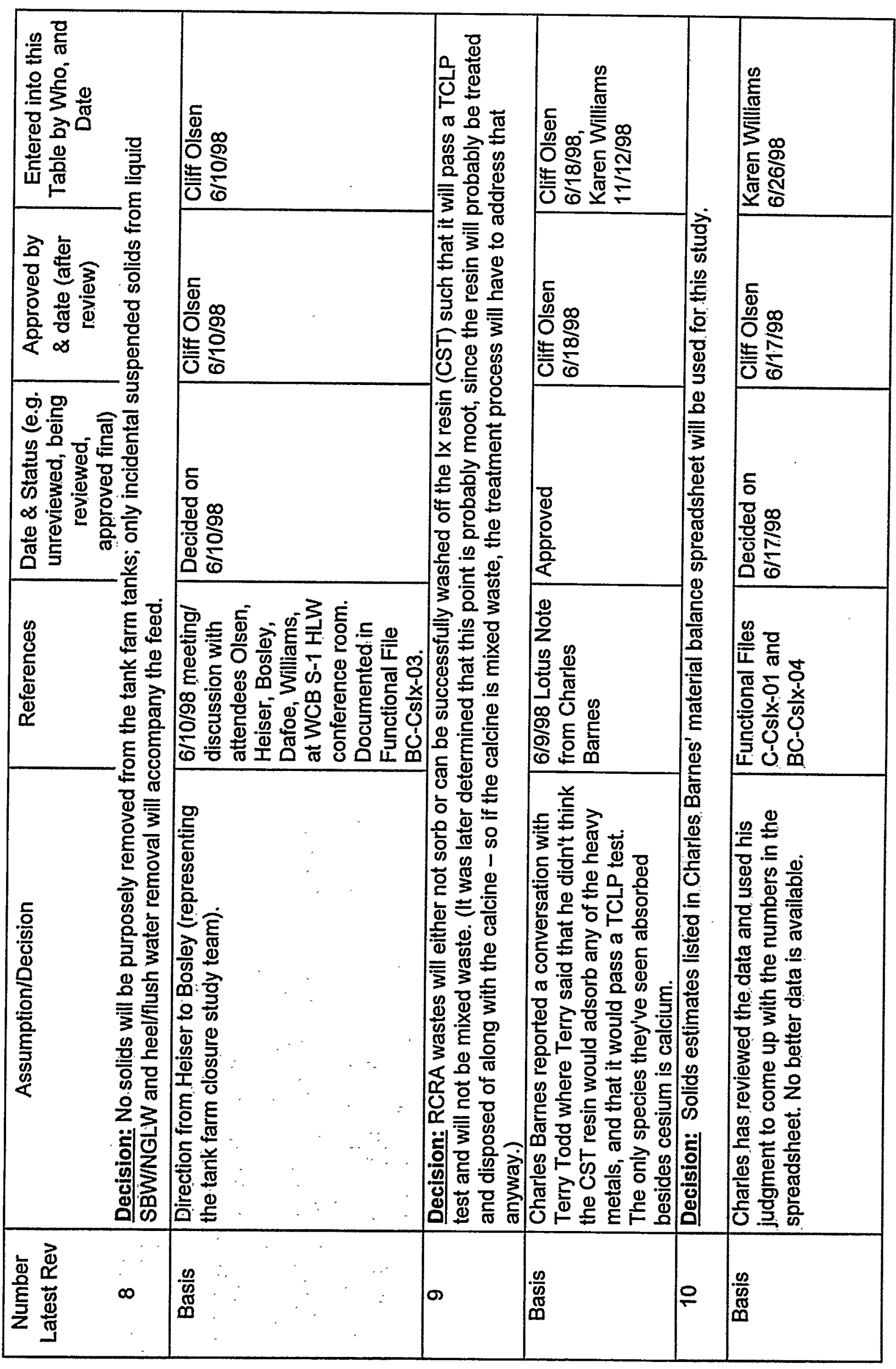




\begin{tabular}{|c|c|c|c|c|c|c|c|c|}
\hline 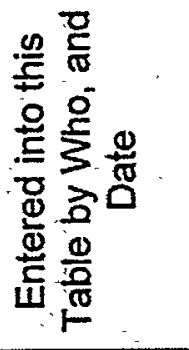 & 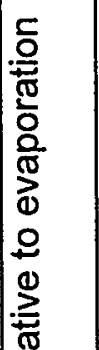 & 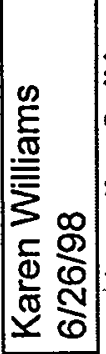 & 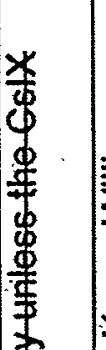 & 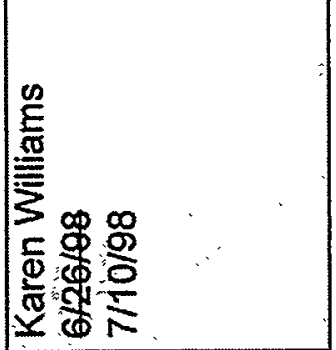 & 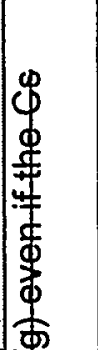 & 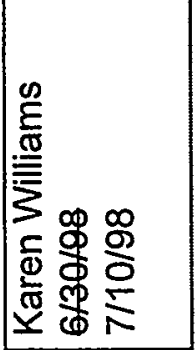 & 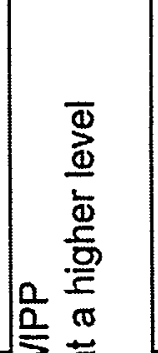 & 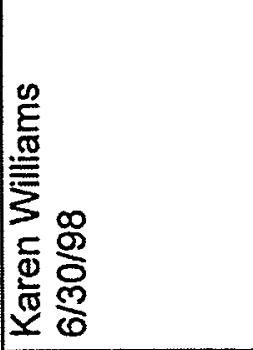 \\
\hline 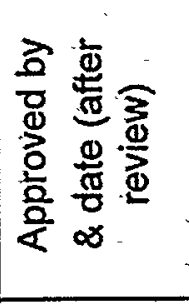 & 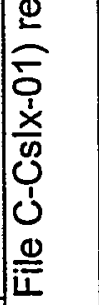 & & 串 & 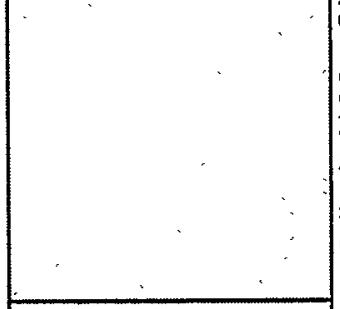 & 䒷 & & 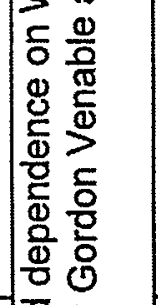 & 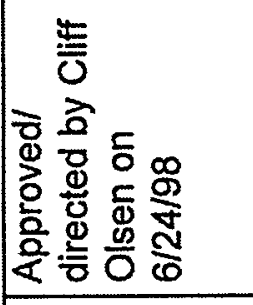 \\
\hline 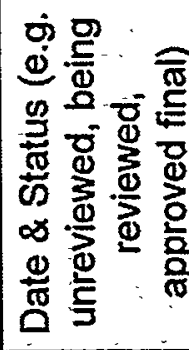 & 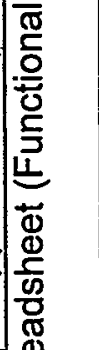 & 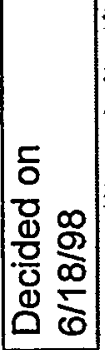 & 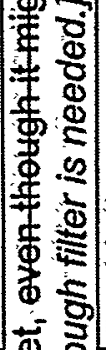 & 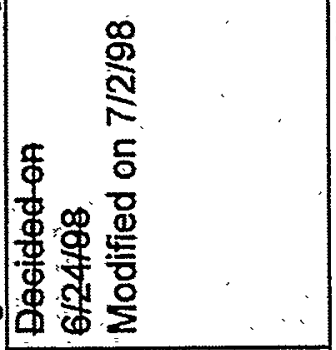 & 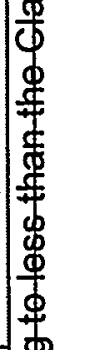 & 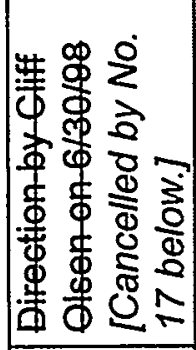 & 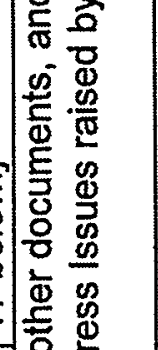 & 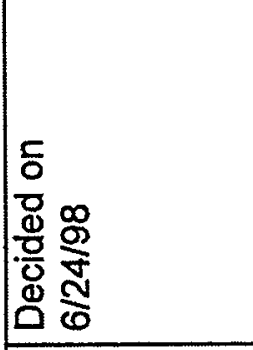 \\
\hline 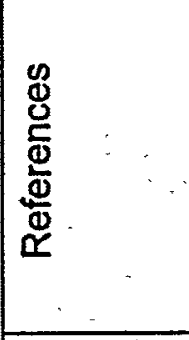 & 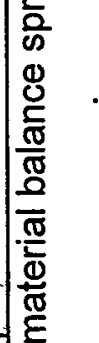 & 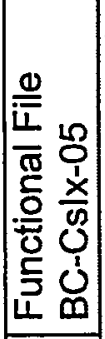 & 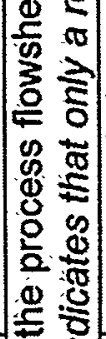 & 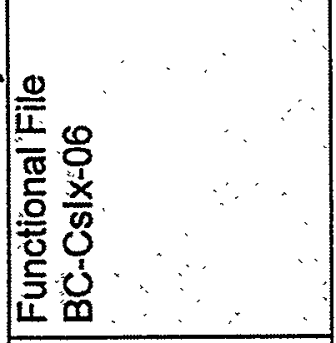 & 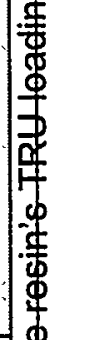 & 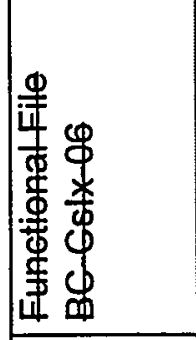 & 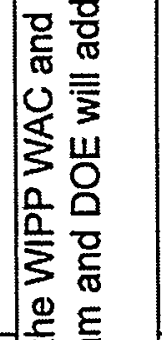 & 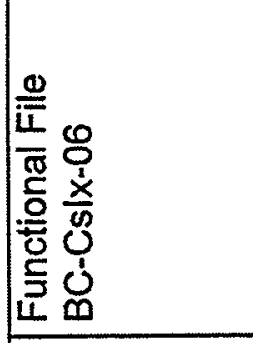 \\
\hline 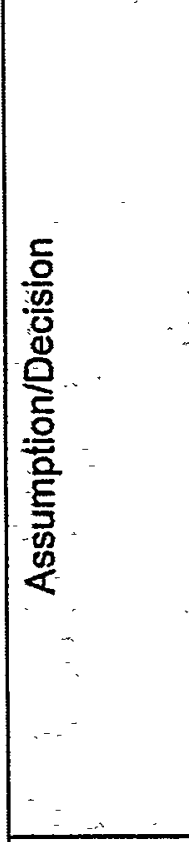 & 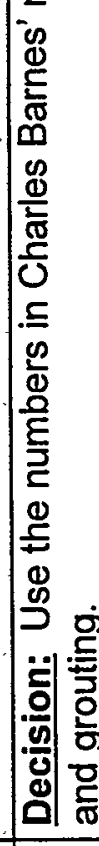 & 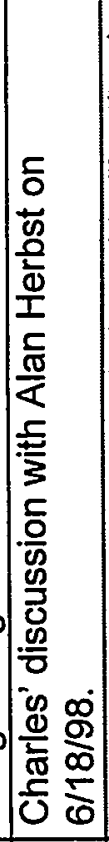 & 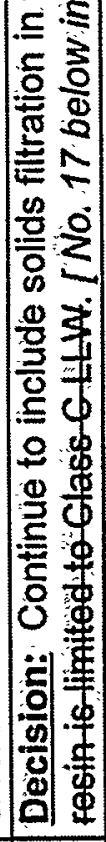 & 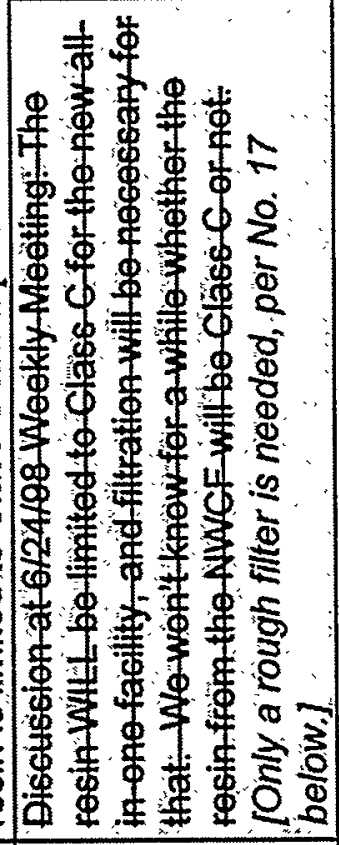 & 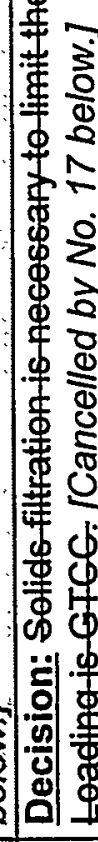 & 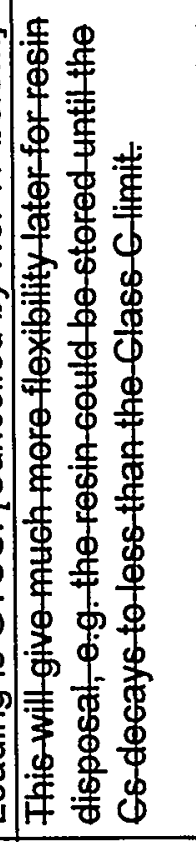 & 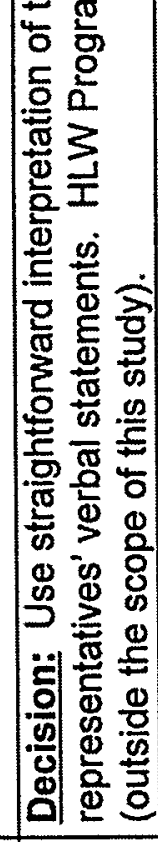 & 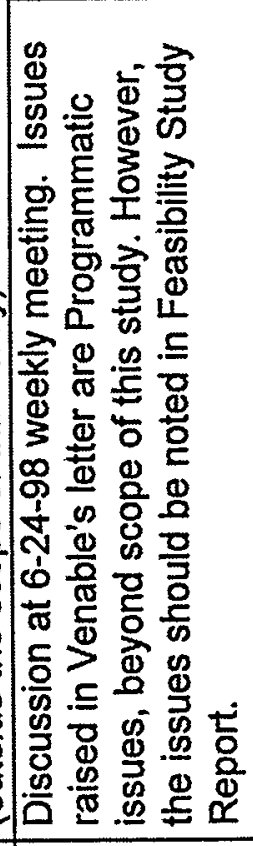 \\
\hline 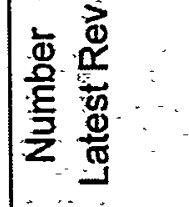 & $\mp$ & 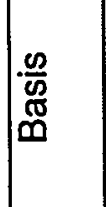 & $\stackrel{N}{*}$ & $\frac{\infty}{\frac{\infty}{\infty}}$ & $\stackrel{m}{\circ}$ & $\mid \frac{\infty}{2}$ & \pm & 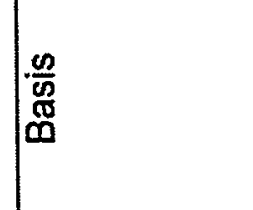 \\
\hline
\end{tabular}




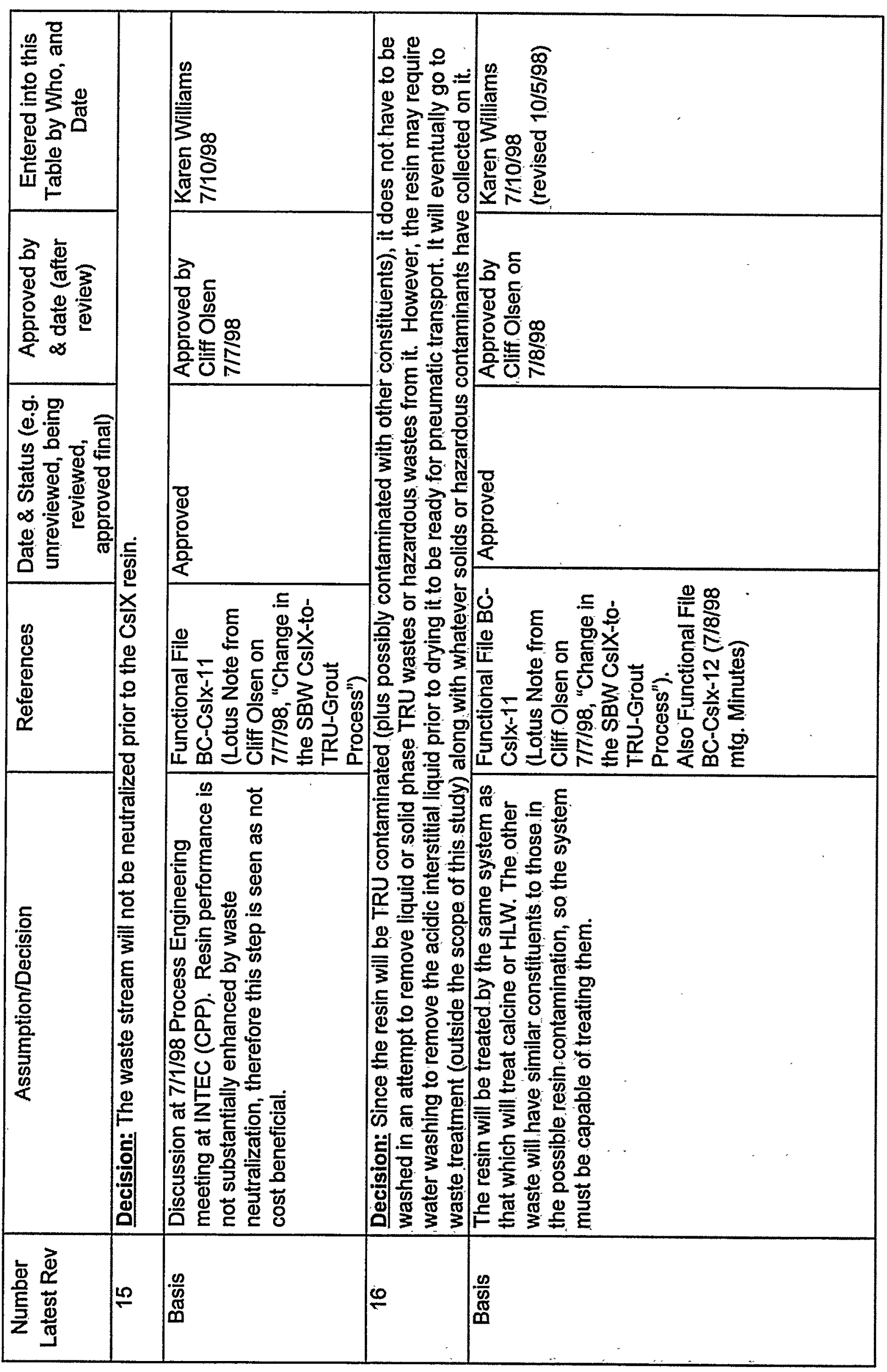




\begin{tabular}{|c|c|c|c|c|}
\hline 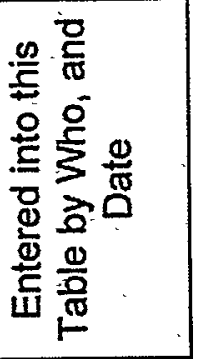 & & 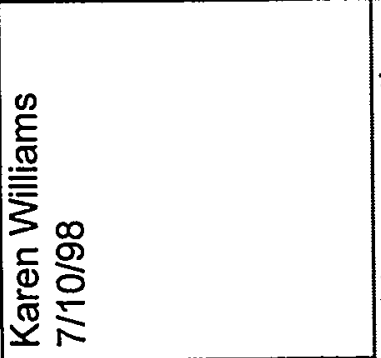 & 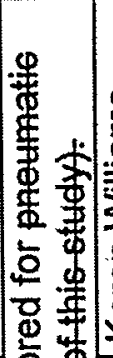 & 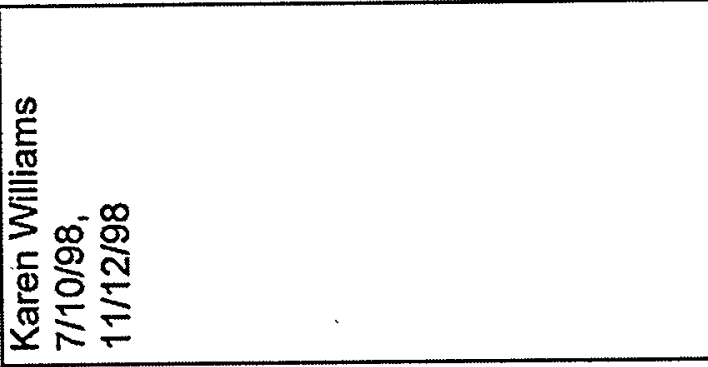 \\
\hline 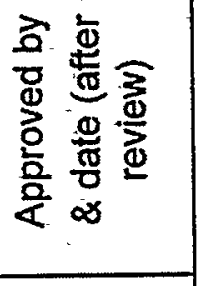 & & 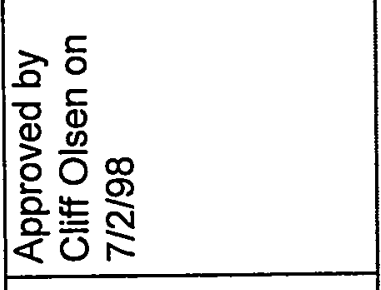 & 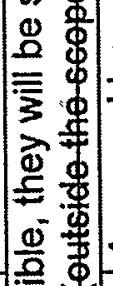 & 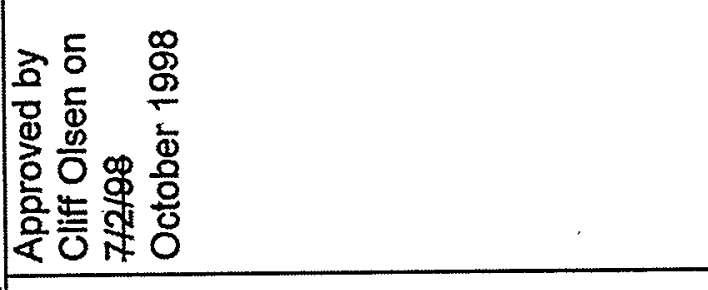 \\
\hline 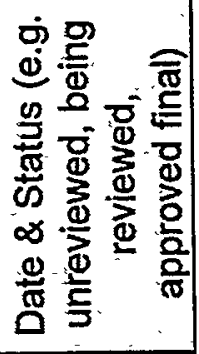 & 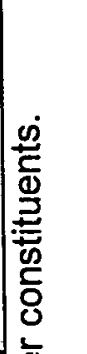 & 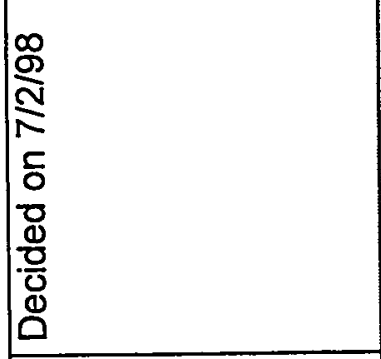 & 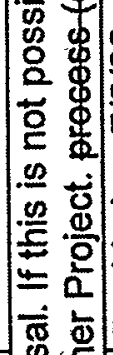 & 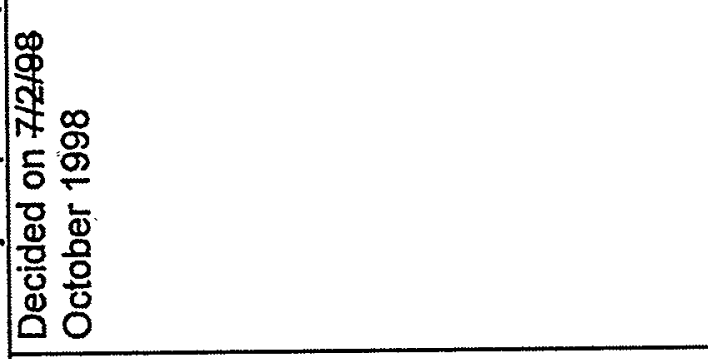 \\
\hline 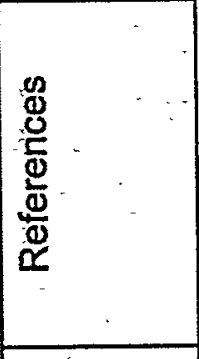 & 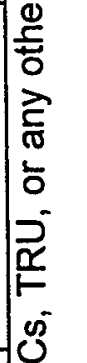 & 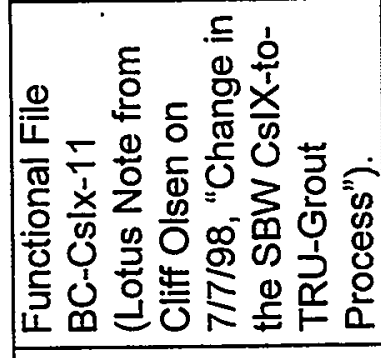 & 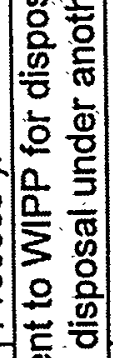 & 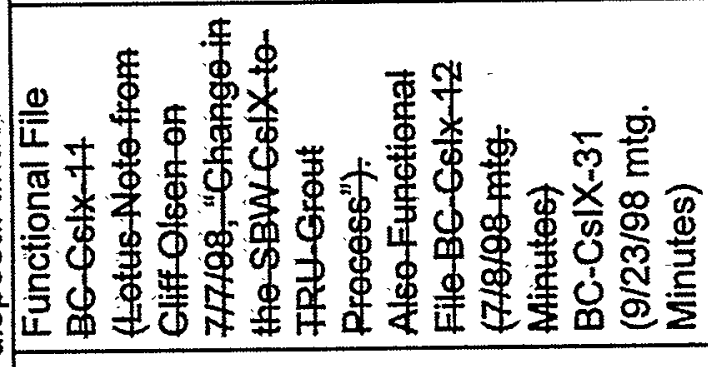 \\
\hline 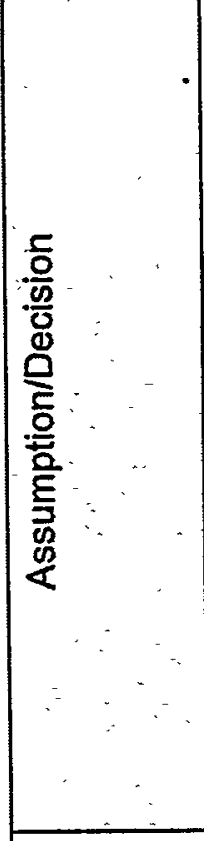 & 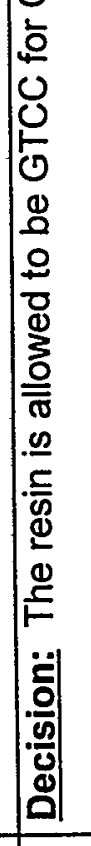 & 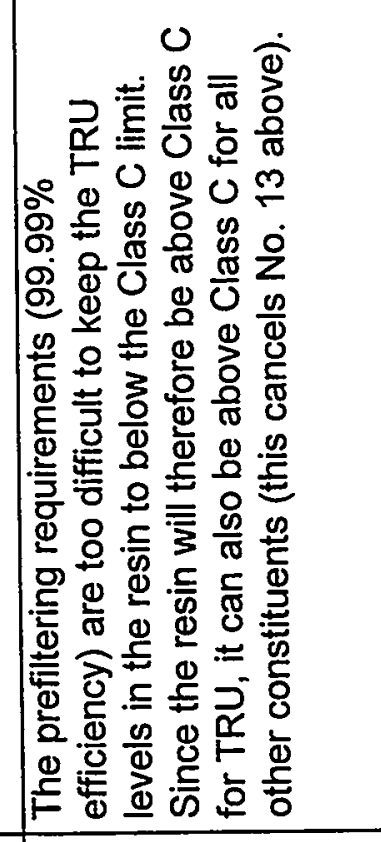 & 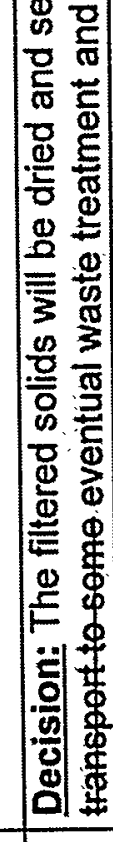 & 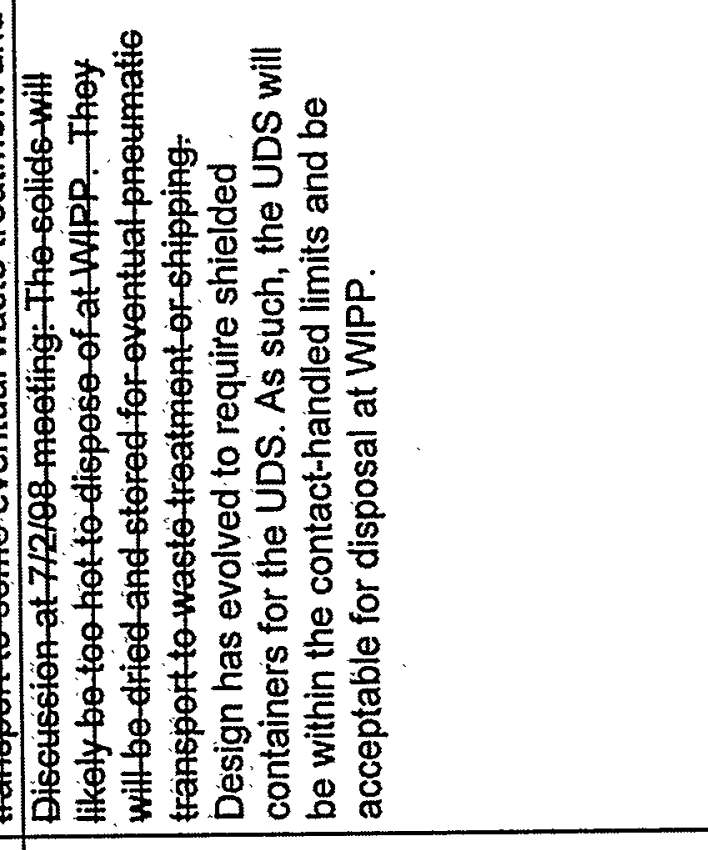 \\
\hline 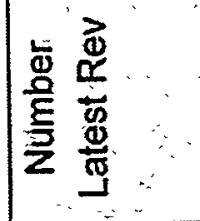 & $F$ & 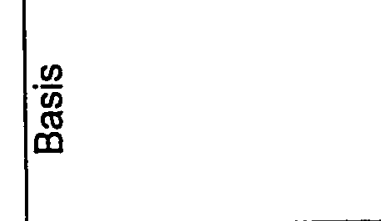 & $\stackrel{\infty}{\stackrel{0}{2}}$ & 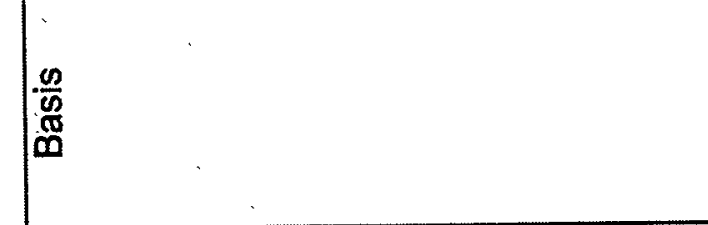 \\
\hline
\end{tabular}




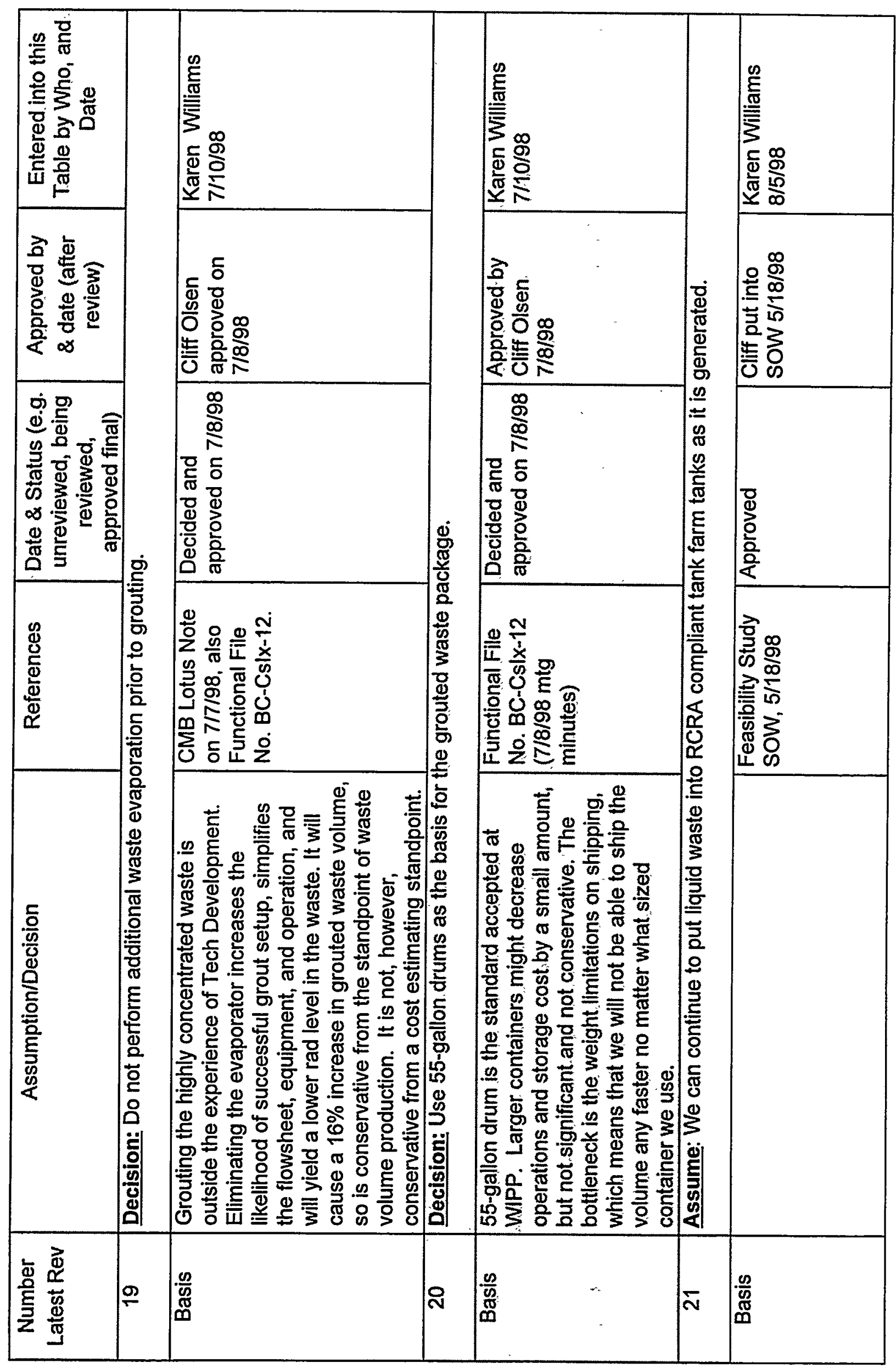




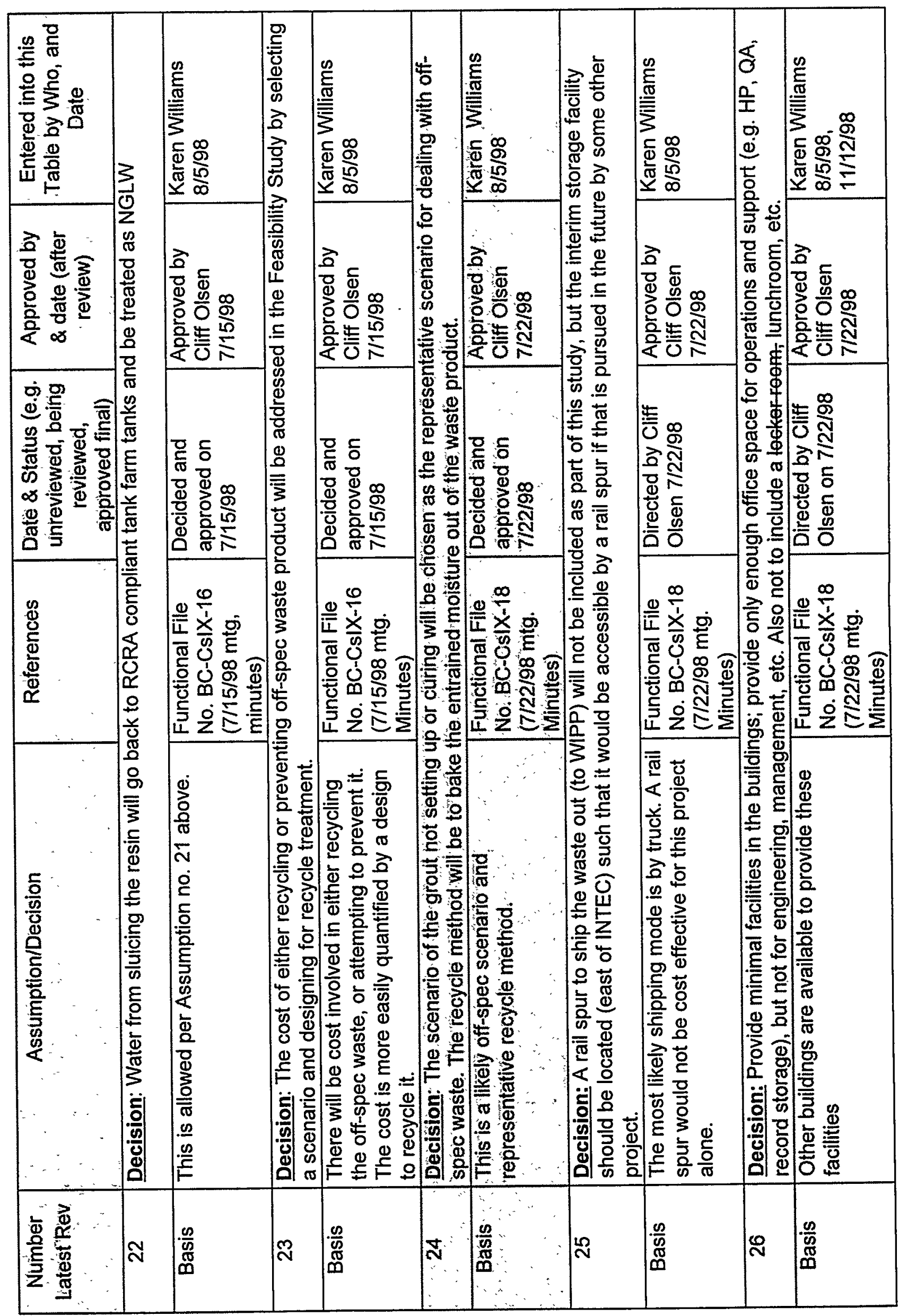




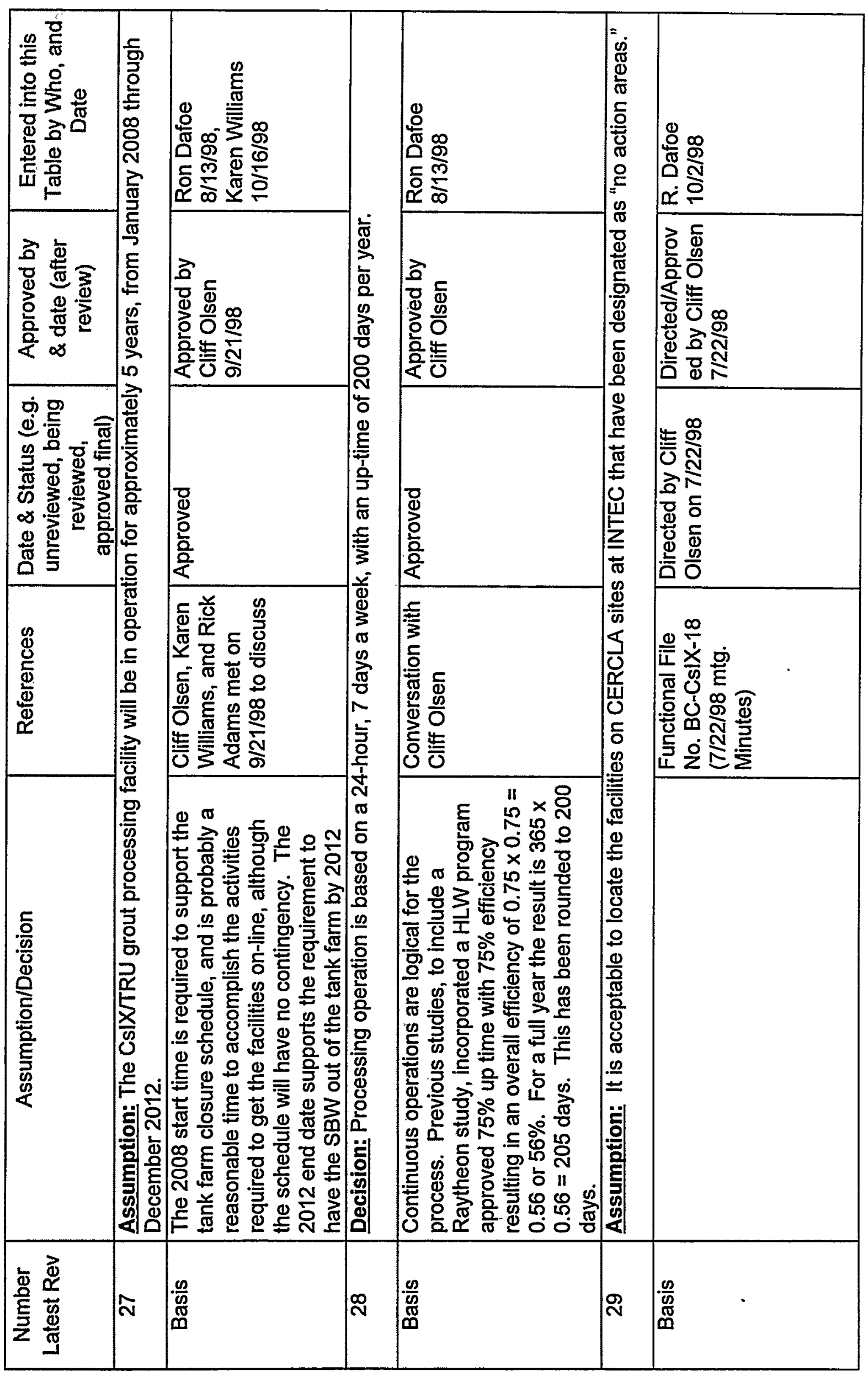




\begin{tabular}{|c|c|c|c|c|c|c|}
\hline 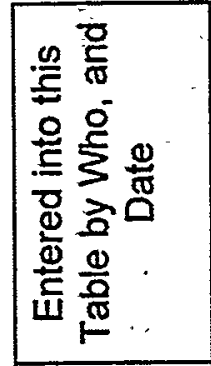 & 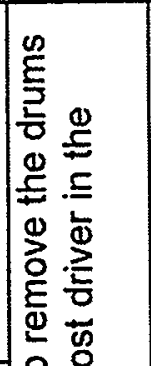 & 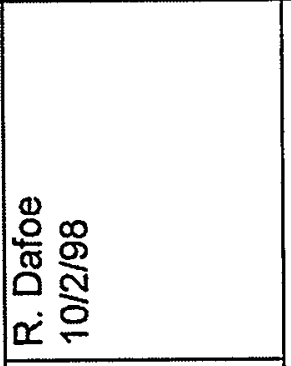 & & 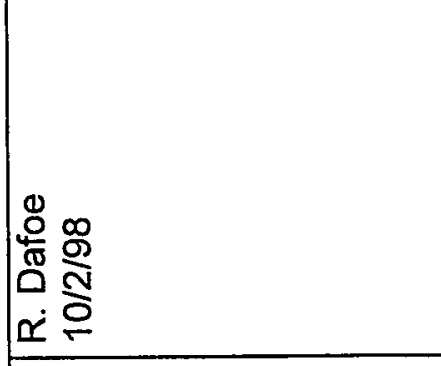 & & 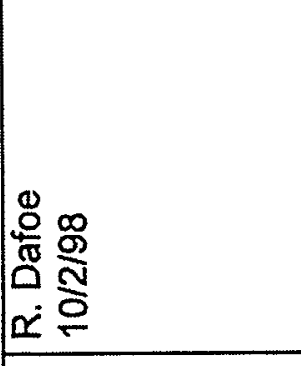 \\
\hline 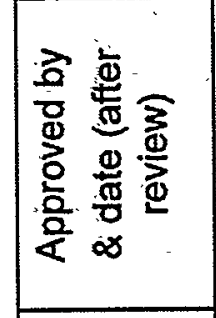 & 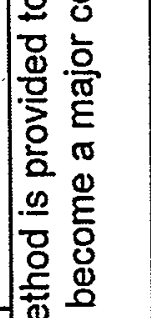 & 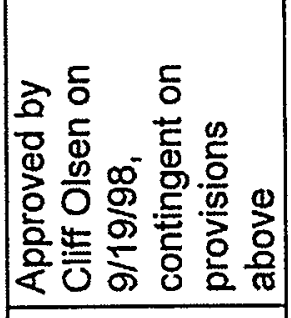 & & 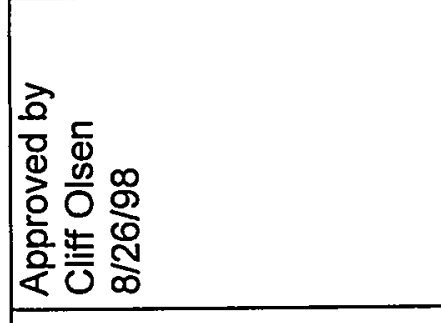 & 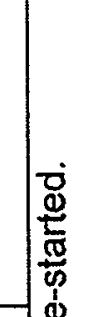 & 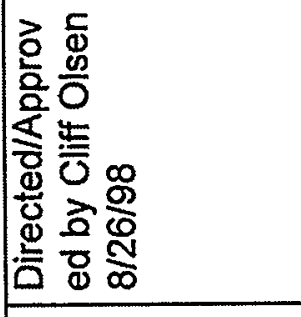 \\
\hline 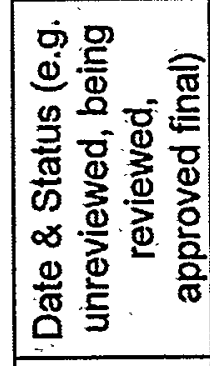 & 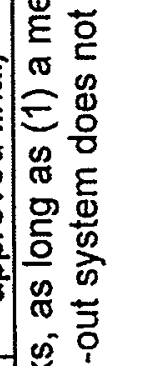 & 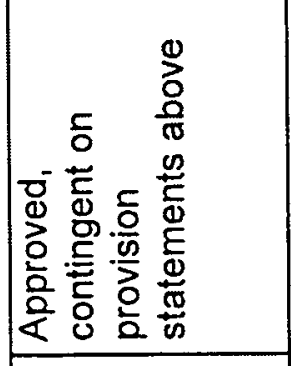 & & 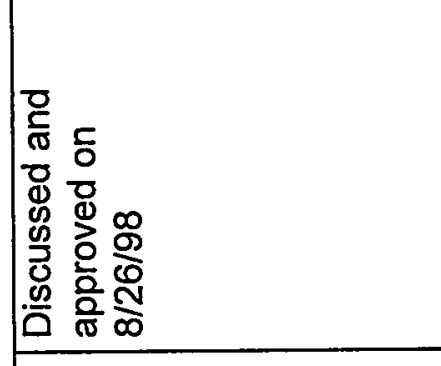 & 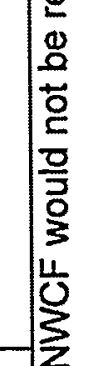 & 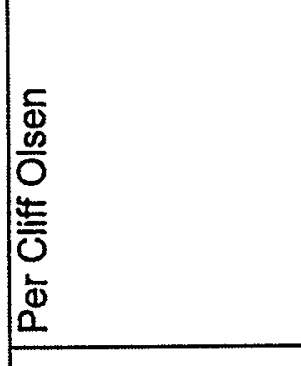 \\
\hline 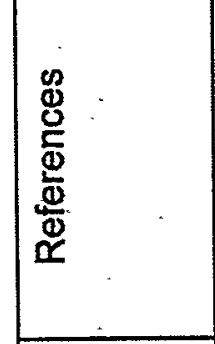 & 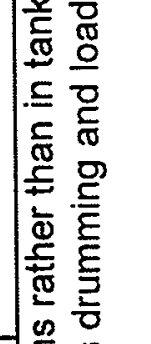 & 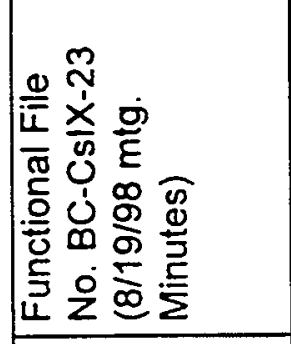 & 递 & 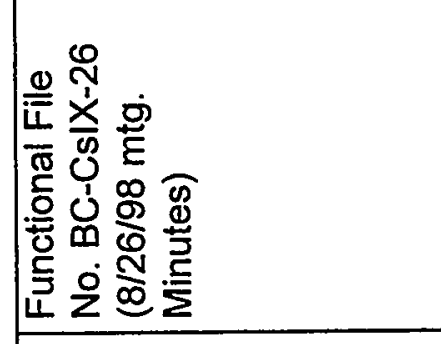 & 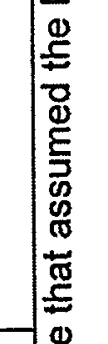 & 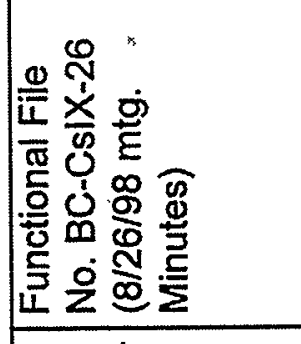 \\
\hline 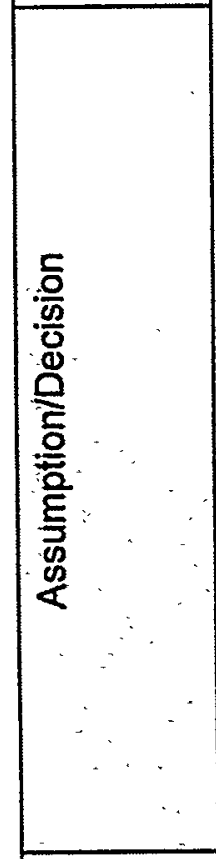 & 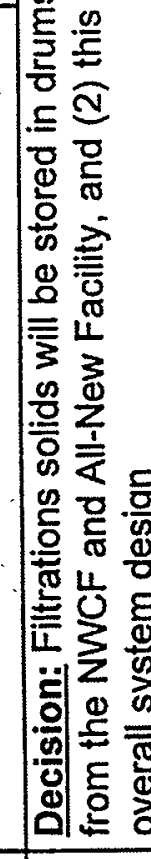 & 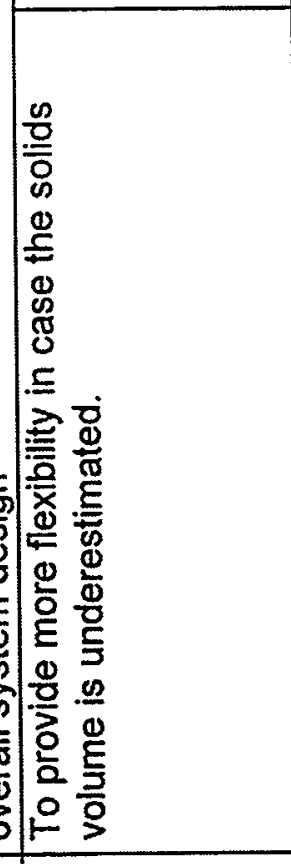 & 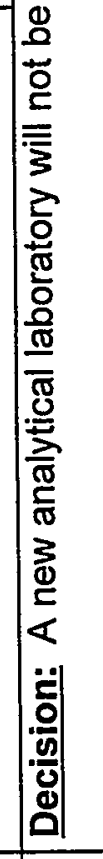 & 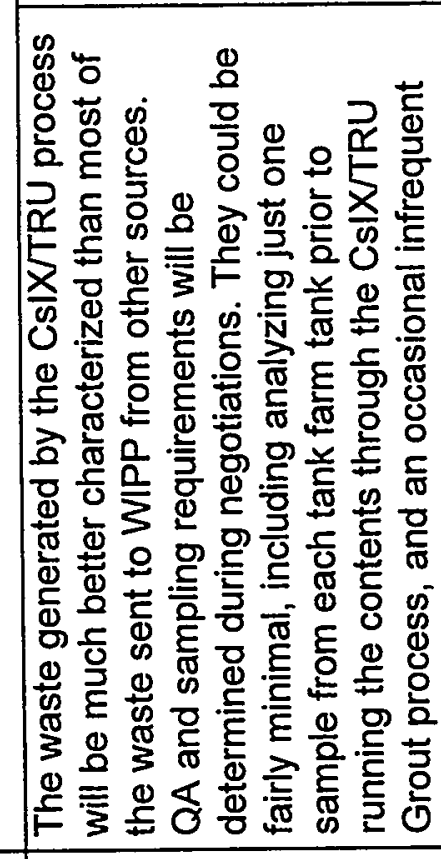 & 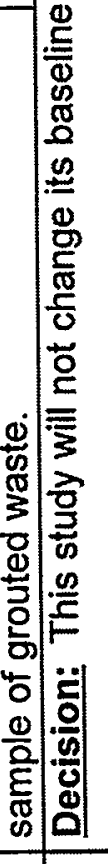 & 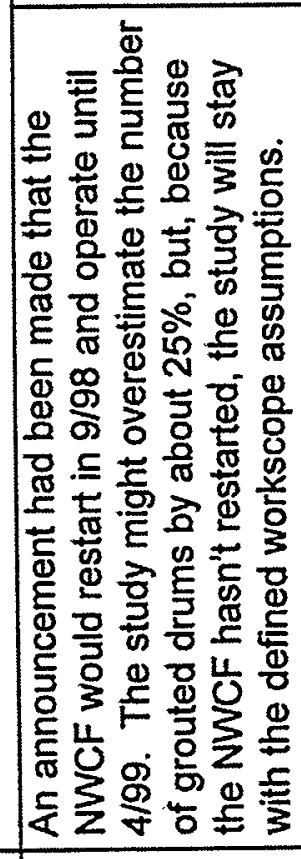 \\
\hline 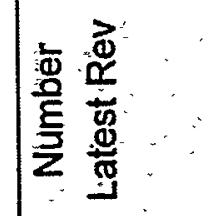 & p & $\mid \begin{array}{l}\frac{\mathscr{m}}{\tilde{D}} \\
\tilde{m}\end{array}$ & $\bar{m}$ & $\mid$ & ల్ల & 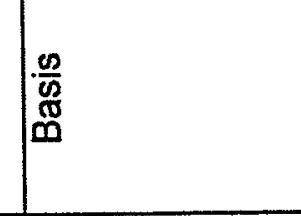 \\
\hline
\end{tabular}




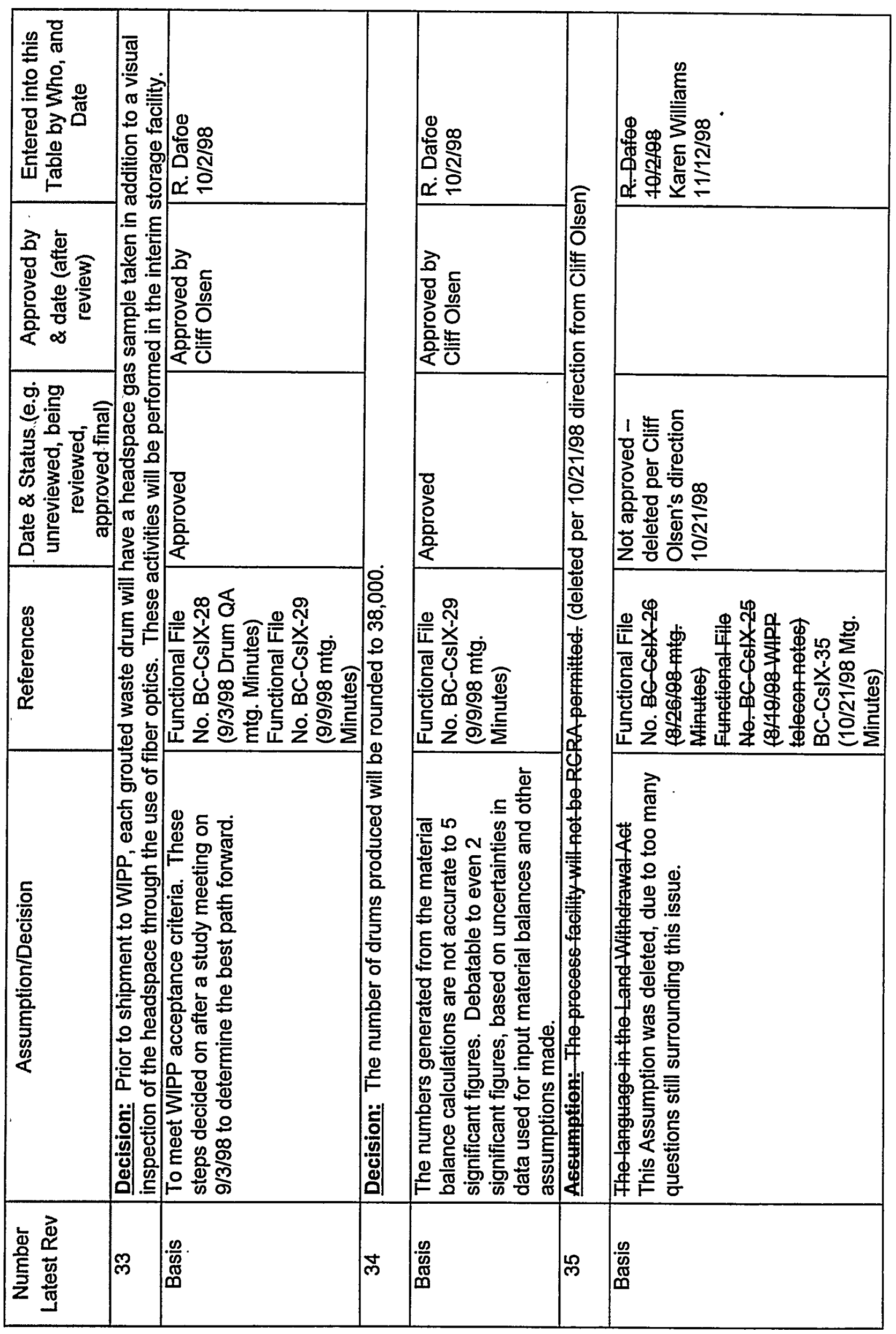




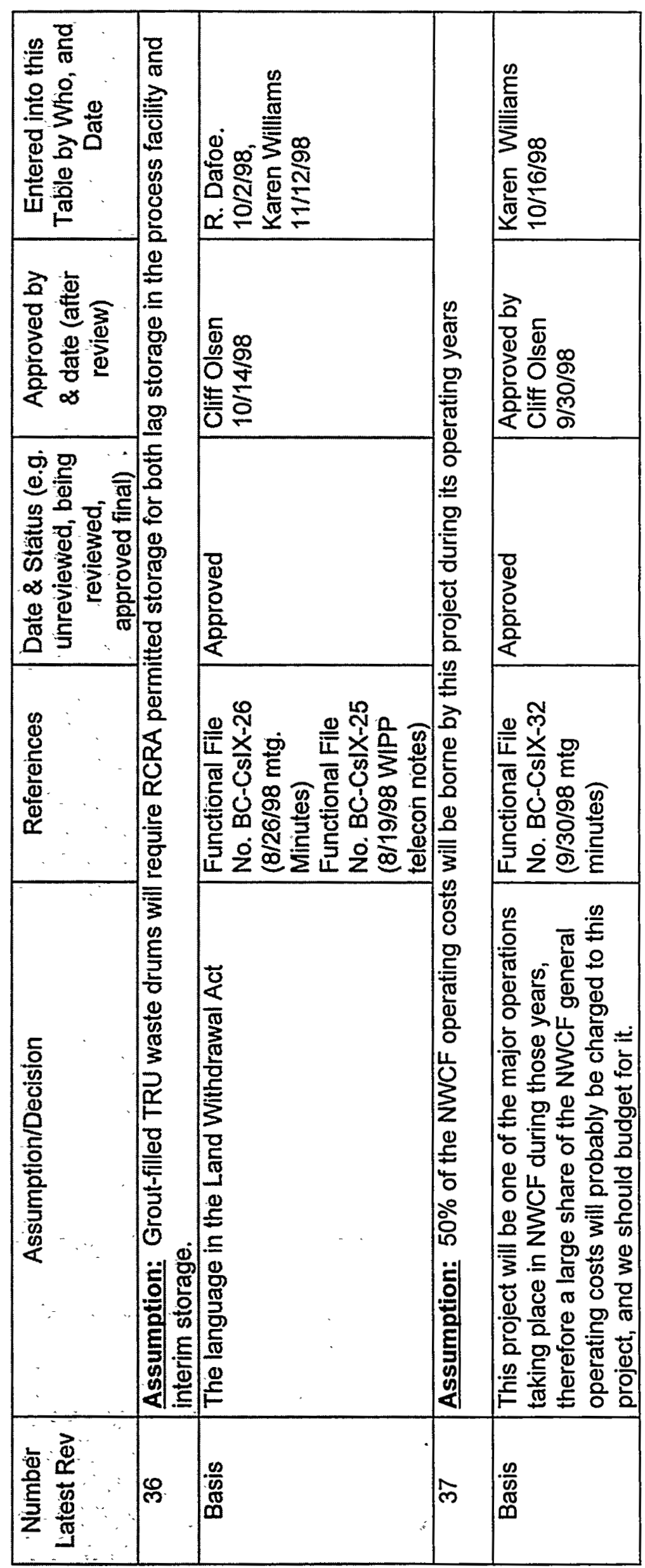


Appendix B

Material Balance and Flow Diagrams (Contained in EDF-CsIX-001) 
Project File Number $\quad$ 02BM1

Project/Task CsIX/TRU Grout Feasibility Study

Subtask Material Balance and Process Flow Diagrams

Title: CsIX/TRU Grout Process Basis, Description, Material Balance and Process Flow Diagrams

Summary: This EDF contains:

1. Assumptions used in calculating the material balance for the CsIX Grout Process

2. A brief comparison of alternative solidified waste forms for the TRU waste product

3. A brief discussion of the rationale for selecting the cesium sorbant

4. A.process description

5. Process flow diagrams

6. Material balance tables for the total composite waste

7. Material balance tables for WM-181, the expected highest dose rate case

8. Recommendations for development of the process.

Revision 1 contains a correction in ${ }^{137} \mathrm{Ba}$ activities for some streams in the material balance and minor other corrections.

Distribution (complete package): C. M. Barnes, S. J. Losinski, K. L. Williams, C. W. Olsen

Distribution (summary package only): J. J. McCarthy

\begin{tabular}{|c|c|c|c|c|c|}
\hline $\begin{array}{l}\text { Author } \\
\text { C. M. Barnes } \\
\text { emBanne }\end{array}$ & $\begin{array}{l}\text { Dept. } \\
4170 \\
10 / 21 / 98\end{array}$ & $\begin{array}{l}\text { Reviewed } \\
\text { S. J. Losinski } \\
\text { Af l. - l. }\end{array}$ & $\begin{array}{l}\text { Date } \\
10 / 21 / 98\end{array}$ & $\begin{array}{l}\text { Approved } \\
\text { K. L. Williams }\end{array}$ & $\begin{array}{l}\text { Date } \\
10 / 27 / 98\end{array}$ \\
\hline
\end{tabular}




\title{
1. Material Balance Assumptions
}

\author{
Feed Basis
}

1. The feed to the CsIX/TRU Grout process is assumed to include all tank farm liquid in tanks WM-180, $-181,-182,-183,-184,-185,-186,-187$, -188 , and -189 as of Jan, 1998 plus liquid waste (NGLW, types 1 and 2) generated 1998-2012.

\section{Tank Farm Volumes and Composition}

2. The volumes of tank farm waste are taken from the tank farm summary web page prepared by Dan Staiger, http://wcb08/nichtt/jm/tankdata/Tankmap.htm, with volumes as of April 30, 1998.

3. The chemical and radiological composition of tank farm waste is taken from tank farm web pages prepared by Dan Staiger, http://wcb08/nichtt/jm/tankdata/WM18i.htm, $\mathrm{i}=0,1,2 \ldots 9$, as updated 12/22/97 (WM-181, -183, -185, -186, 188), 12/23/97 (WM-180, -184, $187,189)$, or $3 / 17 / 98$ (WM-182).

4. Where chemical species concentrations were not available in the above source, data are taken from J. A. Nenni to W. B. Palmer, Alternative SodiumBearing Waste Calcination Flow Sheet Estimations, JAN-02-95, Sept. 21, 1995 or K. J. Rebish and J. A. Nenni to B. H. O'Brien and D. V. Croson, Tank Farm Inventory - June, 1994, KJR-02-94/JAN-03-94, June 23, 1994. Where a species concentration is not available in any source for a particular tank but is documented for other tanks, the unknown concentration is assumed to the same as the weighted average of the known tanks.

5. The nitrate concentration for each tank is adjusted to achieve a charge balance for all species.

6. The nonradioactive cesium concentration for each tank is assumed to be two times the total radioactive cesium concentration as of Jan, 1998. (Basis: discussions with Terry Todd, Ken Brewer and Gary Stegen.)

7. Concentrations of radionuclides are estimated when missing from the web page data based on concentrations in D. R. Wenzel, Evaluation of Radionuclide Inventory for Sodium Bearing Waste, EDF-FDO-006, CPP97080, Nov. 26, 1997. Estimates are as follows: 
7a. $A m-243=A m-241$ times ratio of Am-243/Am-241 from Wenzel

7b. $\mathrm{Cm}-242=\mathrm{Am}-241$ times ratio of $\mathrm{Cm}-242 / A m-241$ from Wenzel

7c. $\mathrm{Cm}-244=\mathrm{Cm}-242$ times ratio of $\mathrm{Cm}-244 / \mathrm{Cm}-242$ from Wenzel

7d. Th-230 $=$ U-233 times ratio of Th-230/U-233 from Wenzel

7e. Th-231 $=$ Th-230 times ratio of Th-231/Th-230 from Wenzel

7f. U-232 $=$ U-235 times ratio of U-232/U235 from Wenzel

7g. U-233 $=\mathrm{Np}-237$ times ratio of U-233/Np-237 from Wenzel

7h. $\mathrm{Bm}-137=\mathrm{Cs}-137$ times ratio of $\mathrm{Bm}-137 / \mathrm{Cs}-137$ from Wenzel

7i. $\mathrm{Ce}-144=$ composite $\mathrm{Ce}-144$ (ave of tanks for which have data)

7j. Co-60 = composite Co-60 (ave of tanks for which have data)

7k. Cs-135 = Cs-137 times ratio of Cs-135/Cs-137 from Wenzel

71. Eu-152 $=$ Eu-152 times ratio of Eu-152/Eu-154 from Wenzel

$7 \mathrm{~m}$. Eu-155 = Eu-152 times ratio of Eu-155/Eu-154 from Wenzel

7n. Pm-147 from Wenzel

7o. Pr-144 from Wenzel

7p. Ni-63 = composite Ni-63 (ave of tanks for which have data)

7q. Ru-106 = composite Ru-106 (ave of tanks for which have data)

7r. Sb-125 = composite Sb-125 (ave of tanks for which have data)

7s. Sm-154 from Wenzel

7t. Tc-99 from Wenzel

7u. $\mathrm{Y}-90=\mathrm{Sr}-90$

7v. $\mathrm{H}-3=$ composite $\mathrm{H}-3$ (ave of tanks for which have data)

$7 w$. $1-129=$ composite $1-129$ (ave of tanks for which have data)

8. Radionuclide activities are decayed to Jan. 2008. Decay products were not added except for ${ }^{137} \mathrm{Ba}$ from ${ }^{137} \mathrm{Cs}$ and ${ }^{90} \mathrm{Y}$ from ${ }^{90} \mathrm{Sr}$.

9. UDS chemical composition is based on tank farm solids analyses documented in M. C. Swenson to M. R. Christiansen, Historical Tank Farm Sample Results, MCS-27-92, December 17, 1992, Attachments 3-10. Calculation of chemical composition is as followed:

9a. Because of incomplete analyses for other tanks, only analyses for WM-180, WM-181 and WM-186 solids were used

$9 \mathrm{~b}$. $\mathrm{Zr}$ was assumed precipitated as a hydrated sulfate based on JDC-06-96 and Schi-28-96

9c. PO4 was assumed precipitated as AlPO4

9d. All other species assumed precipitated as oxides

9e. Analysis data was converted to compounds, and then the balance (1-total wt fraction) was assumed to be $\mathrm{SiO} 2$

9f. Analytical data for the three tanks was averaged assuming equal UDS content in liquid from each tank 
10. UDS fission product activity is also based on analytical data in MCS-2792

10a. Only analyses for WM-180, WM-181, WM-184 and WM-186 solids are used

10b. Analytical data for the tanks was averaged assuming equal UDS content in the liquid from each tank

10c. Activities were decayed from date of analyses to Jan, 2008.

Decay products were not included.

11. UDS actinide activity taken from an e-mail note from Arlin Olson to Vic Jacobson, 8/12/97 (and decayed from 1983 to Jan, 2008)

\section{HLLW Evaporation}

12. Tank farm liquid was assumed to be evaporated as follows:

12a. Liquid in WM-180, WM-183, WM-185, WM-188 \& WM-189 was not evaporated, having specific gravities of $1.28,1.24,1.28,1.32$ and 1.31 respectively.

12b. Based on a phone conversation with Frank Ward, liquid in WM-181 was combined with WM-184 and evaporated in a 1:1 blend. The evaporated volume was as given in R. E. Schindler, HLLWE Feed Blends for Wastes from WM-186, WM-184 and WM-181, July 28, 1998, Schi-1798.

12c. Liquid in WM-186 was evaporated to a volume given in $R$. $E$. Schindler, HLLWE Feed Blends for Wastes from WM-186, WM-184 and WM-181, July 28, 1998, Schi-17-98.

12d. Liquid in WM-183 and WM-187 was evaporated to a total dissolved solids concentration of about $530 \mathrm{~g} / \mathrm{l}$. This was equivalent to a concentration factor of 1.67 for WM-183 and 2.0 for WM-187.

\section{Newly Generated Liquid Waste Volumes and Composition}

13. NGLW volumes and compositions taken from the May, 1998 INEEL Liquid Waste Management Plan, draft to DOE-ID with exceptions as noted below. Volumes taken from Appendix $C$; compositions from Appendix $B$

14. Because tank farm heel volumes were included in the volume of the tank farm waste, they were deleted from the NGLW

15. Because the NWCF was assumed to be shut down as of 1998 , all NGLW generated from NWCF operations after 1999 was deleted

16. NWCF wastes generated in 1999 was adjusted to be nearly equal (to 
within $1 \%$ ) to that shown in Appendix $B$ for calciner closure flushes.

17. The composition of NGLW is assumed to be as follows:

Type 1 The average for the tank farm was used for concentrated Type 1 waste

Type 2 Group 1 The composition for waste 7o was used (for dilute waste)

Type 2 Group 2 The composition for waste 70 was used (for dilute waste)

Type 2 Group 3 The average for the tank farm was used for concentrated Type 1 waste

Type 2 Group 4 The composition of stream 7c was used for it's volume, the composition of $7 \mathrm{e}$ for the rest of group 4 wastes.

Type 2 Group 5 The composition for waste 5 was used (see PEW Descale worksheet)

Type 2 Group 6 The average for the tank farm was used for concentrated Type 1 waste

Type 2 Group 7 Since no compositions are available for this waste, all other streams were prorated up in volume to account for the Group 7 volume

\section{Tank Farm Liquid Transfer}

18. Transfer of liquid waste from the tank farm to the process feed tank by steam jet is assumed to add $8 \%$ to the volume.

\section{UDS Filtration \& UDS Product}

19. $80 \%$ removal of UDS by filtration is assumed.

20. The UDS filter backwash is assumed to have an average solids content of $60 \mathrm{~g} / \mathrm{l}$.

21. A density of $1.4 \mathrm{~g} / \mathrm{cm} 3$ is assumed for the dried UDS waste.

22. To bound composition and activities ranges of waste products, the material balance includes streams in which all of the $20 \%$ of UDS not removed by filtration is assumed to either remain with the ion exchange sorbant or pass through the ion exchange column and be mixed into the grout waste.

\section{Cesium Removal}


23. The assumed removal efficiency for cesium is $99.9 \%$. (Basis: Gary Stegen, memo to W. B. Palmer, Additional SBW Separations and Backup Options, Nov. 11, 1997. Later dose rate calculations have confirmed that this efficiency results in grout with a dose rate under $200 \mathrm{mR} / \mathrm{hr}$. Discussions with Terry Todd and Ken Brewer have confirmed that the assumed removal efficiency is achievable with the selected sorbant.)

24. It is assumed that no species other than cesium are removed in the ion exchange column.

25. Cesium is assumed to be removed with UOP's IONSIV IE-911 sorbant, a crystalline silicotitanate (CST). Cesium cannot be efficiently removed from this sorbant, hence it is not regenerated.

24a. The density of IONSIV IE-911 assumed to be $1.0 \mathrm{~g} / \mathrm{cm} 3$, as per phone conversation with Terry Todd.

$24 \mathrm{~b}$. The loading at change-out of the resin is obtained by prorating the value of $4.78 \mathrm{mg} \mathrm{Cs} / \mathrm{g}$ resin (at $9 \mathrm{mg} / \mathrm{l} \mathrm{Cs}$ ), obtained in e-mail note from Terry Todd, by the ratio of $\mathrm{Cs}$ in the feed

26. No pH adjustment will be made prior to ion exchange.

27. The amount of ions ( $\mathrm{Ca}+2$, others) released by the resin in exchange for $\mathrm{Cs}+$ is assumed negligible relative to the total solids in the IX effluent.

28. The spent Cs sorbant will not be washed before unloading to remove actinides or other species. The spent $\mathrm{Cs}$ sorbant will be sluiced out of the column with a volume of water 10 times that of the sorbant. (If future testing shows that this volume is inadequate to remove residual acidity and that acidity negatively affects the performance of the subsequent drying step or the sorbant storage, an in-column water wash step can be added to the process.)

\section{Neutralization and Grouting}

29. The ion exchange effluent will be neutralized with $\mathrm{CaO}$ to a pH of 1.3.

30. The volume of neutralized waste is assumed the sum of the ion exchange effluent and volume of water produced in the neutralization.

31. A waste loading of $40 \%$ (as liquid waste) is assumed for the grout. The grouted waste quantity is based on the liquid waste feed (tank farm liquid and NGLW) before dilution by jet transfer, water from sorbant sluicing, and neutralization. The $40 \%$ loading is equivalent, for the average material balance case, to a loading of $20 \%$ expressed as weight of waste solids per 
unit weight of grout. (Basis for loading: Phone conversation with Alan Herbst, July 7, 1998)

32. Grout additives are Portland cement, blast furnace slag and fly ash in a weight ratio of 1:3:1. (Basis: Phone conversation with Alan Herbst, July 7, 1998)

33. The density of the grouted waste is assumed to be $1900 \mathrm{~kg} / \mathrm{m} 3$. (Basis: Phone conversation between Syl Losinski and Alan Herbst, August xx, 1998)

\section{TRU Waste Form Evaluation}

A very brief evaluation was performed to determine the form of the TRU waste. Four waste forms were considered: grout, absorbed waste on a low density silicabased absorbant, absorbed waste on a clay-based absorbant, and absorbed waste on an inorganic polymer absorbant. The criteria used to select the waste form for the TRU waste included:

1. Process operational simplicity:

Does the waste form require a mixer?

Does the waste form require neutralization?

2. Solidified waste volume

3. Development status:

Is there data to support waste loading, chemical interactions with the solidification media, final waste density, etc.?

4. Cost.

The potential advantage of a low-density, silica- or polymer-based absorbant is process operational simplicity. A waste container would be filled with a given amount of absorbant and liquid waste would be added. No mixing, no neutralization, no curing, and no decontamination of equipment would be required. Based on a brief review of commercial absorbants, Ultrasorb 244, a product of the Molton Corporation, was selected as representative of a high-capacity, inorganic absorbant for aqueous liquids. Ultrasorb 244, a granular diatomaceous earth, absorbs approximately $150 \%$ of its weight. Based on product literature for Ultrasorb, neutralization would not be required, as the product is stable with all acids except HF.

Inorganic polymer absorbants offer even higher absorption capacities, although at a higher cost. ACIDBOND A660, marketed by Nochar, is presently being tested using INEEL wastes, including a SBW simulant. Although final test data is not 
available, based on tests of other wastes it is reasonable to expect that ACIDBOND A660 would absorb 85 lbs of waste per 15 lbs of absorbant, and result in a 10$20 \%$ volume increase over the original liquid volume. The resultant waste is a soft, rubber-like solid.

The potential advantage of a clay-based absorbant is a lower final waste volume. The Aquaset/Petroset products of Fluid Tech, Inc. were selected as representative of a clay-absorbant. Fluid Tech products have been tested and/or used in numerous nuclear waste applications, including some at the INEEL. Testing has not been done for solidification of SBW. For aqueous wastes containing a high level of dissolved salts, Fluid Tech literature recommends using 125-175 lbs of Aquaset II per 43-47 gallons of waste. For acidic wastes, neutralized to a $\mathrm{pH}$ of 5-10, the formulation is similar, $125-175 \mathrm{lbs}$ of a combination of Aquaset II and Petroset to 42-47 gallons of waste. Mixing of the waste with Aquaset/Petroset would be required.

Table 1 gives a comparison of the three waste forms.

Table 1. Comparison of TRU Waste Forms.

$\begin{array}{lcccc} & \text { Grout } & \text { Ultrasorb } & \text { Aquaset } & \text { Acidbond } \\ \text { Waste Volume, m3 } & 7,500 & 12,500 & 6,100-6,800 & 5,300-5,700 \\ \text { Neutralization req'd } & \text { Yes }(1) & \text { No }(2) & \text { Yes }(3) & \text { No } \\ \text { Mixing Required } & \text { Yes } & \text { No } & \text { Yes } & \text { No } \\ \text { Cost of absorbant } & \$ 430,000 & \$ 465,000 & \$ 1,800,000 & \$ 32,000,000 \\ \text { Test data available } & \text { Yes } & \text { No } & \text { No } & \text { No }\end{array}$

Notes

1. Neutralization to $\mathrm{pH}$ of 1.3 with $\mathrm{CaO}$

2. Neutralization not needed except possibly to tie up HF

3. Neutralization to $\mathrm{pH} 7$ with $\mathrm{NaOH}$

The comparison shows that Ultrasorb results in a much higher volume of waste than the other alternatives. The range of waste volumes shown for Aquaset are based on (1) $45 \mathrm{gal}$ of waste with $175 \mathrm{lbs}$ of absorbant per drum and (2) $41 \mathrm{gal}$ of waste with $250 \mathrm{lbs}$ of absorbant per drum. The higher waste loading is based on Fluid Tech literature. The lower loading is based on treatability study data for concentrated nitric acid waste performed at Sandia. ${ }^{1}$ As shown in the above table, the volume of waste when solidified with Aquaset/Petroset is potentially $14-22 \%$ less than when grouted; however, no saving would be expected on equipment, and the cost of the solidification agents would be 3-5 times more than grout.

Based on the limited information presently available for ACIDBOND, it appears to result in $20-30 \%$ less final waste volume than a grout product. Data from tests of ACIDBOND with SBW simulants is needed to confirm the above waste volume. 
Also data is needed to evaluate the long-term stability of the product. The disadvantage of the ACIDBOND product are the cost of the absorbant.

The development program for grouting INTEC liquid wastes has focused on producing a high-strength grout. ${ }^{2,3}$ The WIPP WAC does not contain a strength requirement. It is expected that with further development, with the goal of minimizing waste volume and not being constrained by a grout strength requirement, the volume of final waste can be reduced to the range shown above for Aquaset or ACIDBOND. Because of the test data available for grouting of SBW, along with the comparison above that show a higher volume for Ultrasorb, additional costs and a relatively small reduced volume for Aquaset, and the high absorbant cost and unknown long-term stability for ACIDBOND, grout was selected for the waste form to use in this feasibility study. Because of the SBW grouting data $^{2}$ there is high confidence that the grout volume shown in the material balance can be achieved. Should the CsIX/TRU Grout be carried into a conceptual design, additional studies on waste forms are recommended. These studies should include testing of evaporation of the ion exchange effluent, grout formulations and loadings other than those tested to date and absorbants that have the potential for minimizing the final waste volume.

\section{Rationale for Selecting the Cesium Sorbant}

lon exchange is a common separations process that in general is both highly efficient and highly selective. Removal of cesium by ion exchange has been tested or is being used at several DOE sites. No process other than ion exchange was considered for cesium removal.

The selection of the ion exchange resin or sorbant to remove cesium was based on the following factors:

1. The achievable efficiency of the process to remove cesium. A high efficiency is required in order to produce an effluent that, when solidified, will be contact handled.

2. The achievable selectivity of the process to remove cesium. The more selective for cesium, the lower the quantity of cesium-loaded waste is likely to be.

3. The available data for the sorbant/resin. The more data that is available for a process, the higher the confidence that expected efficiencies, selectivities, waste volumes, and costs can be achieved.

4. The composition of the cesium-loaded waste. The waste should be compatible with both anticipated future treatment requirements and anticipated storage requirements.

5. The availability and cost of the sorbant/resin needed for the process. 
Three ion exchange sorbants have been evaluated for cesium removal from INTEC liquid waste. ${ }^{5}$ All three have been shown to be effective for removing cesium from INTEC liquid waste, although one, a potassium copper hexacyanoferrate sorbant (FS-2), has recently been shown to absorb mercury, which reduces the sorbant's capacity for cesium. The availability of FS-2 is also questionable, being manufactured only in Russia. A second sorbant, an ammonium molybdophoshate on a polyacrilonitrile binder (AMP-PAN), contains $15 \%$ organic material. Storage of the spent AMP-PAN sorbant would be problematic because of the potential for radiolytic degradation of the organic material. Also, the spent sorbant with its high cesium content may be eventually disposed by processing in a high level waste melter. The organic material present in the AMP-PAN sorbant may pose difficulties in a glass melter. ${ }^{3}$ The third sorbant tested, a commercially-available crystalline silicotitanate (CST) is inorganic, and has been shown to be compatible with high level waste vitrification. ${ }^{4}$ Thus, based on the above factors, the CST sorbant, UOP's IONSIV IE-911, was selected for cesium removal from INTEC waste. Additional data is needed on removal of cesium from tank farm wastes with IONSIV IE-911, specifically data absorption of other species and data from largerscale tests.

\section{Process Description}

\section{Waste Transport from Tank Farm}

Transport of waste from the Tank Farm to the CsIX/TRU Grout Facility will use major portions of the existing Tank Farm Transport System which are capable of conveying waste from the Tank Farm to the NWCF. Modifications to the transport system are required for the stand-alone facility option, while it is possible that the existing system can function without modification for the NWCF option.

The existing system uses steam jets to transport waste from tanks WM-180,-181,$182,-183,-184,-185,-196,-187$, and uses air jets to transport waste from tanks WM-189, and -190 . Receiving tanks in the CsIX/TRU Grout Facility will be capable of accepting waste from all tanks.

Modifications to the existing steam and air jet transport system for the CsIX/TRU Grout Facility option include a tie-in to the existing transport lines and possible lift station which will develop adequate head pressure to deliver waste to the liquid waste feed tanks.

It is also assumed that waste will be transferred to the CsIX/TRU Grout Facility in batch mode. Two major reasons for this are that the existing transport system is not capable of conveying waste at the low $(1 \mathrm{gpm})$ steady state feed rate used in 
the CsIX/TRU Grout process, and, it is expected that sampling of waste materials for purposes of process control will be performed on a batch basis.

This design includes two tanks capable of accepting waste from the Waste Transport System. Each tank is sized nominally at 3500 gal capacity. This tank size allows for a reasonable transport batch cycle time, of approximately 2 hours, and is based on the feed rate of the existing Tank Farm Transport Systems.

\section{Solids Filtration}

This design includes provisions for filtration of the liquid waste prior to introduction to the ion exchange columns. The primary function of the filtration system is to protect the ion exchange resin bed from becoming blinded with solids particles. The design also includes provisions for drying and packaging the UDS collected during filtration.

A combination of two vertical leaf pressure filters will provide continuous filtering capability at a capacity of $1.2 \mathrm{gpm}$. Filter media will be selected which provides for removal of particles larger than 10 microns. The filter will also be equipped with a scavenger leaf which will allow draining of vessel water off of the filter cake. At any given time one filter will be on-line while the other is being drained and back flushed. The filter that is off-line will be drained and the filter cake back flushed with deionized water into a slurry tank. Filter transition (from on-line to offline) will be initiated by setpoint pressure differential.

Slurry from the slurry tank will be metered to a rotary wiped film evaporator where the slurry will be dried and sized to a flowable particulate solid. Slurry is assumed to be $10 \%$ solids by weight and will be fed to the evaporator at approximately 1.7 $\mathrm{Kg} / \mathrm{min}$. The evaporator will fill $\mathbf{5 5}$ gal drums with the particulate solids. Off-gas from the evaporator will directed through a condenser and then sent to the Vent Off Gas System. Condensed water will be returned to the HLLWE.

Considerable uncertainty exists in the quantity and character of the suspended UDS in the waste stream, which makes specification of the filtration system quite difficult. Limited particle size distribution data available shows that a large fraction of the UDS in the liquid waste is in the sub-micron size, which is an indication of a potentially significant filtration problem should these particles need to be removed. As discussed later in this report emperical filtration data must be obtained on the liquid wastes to further refine this filtration process. 


\section{Cesium Removal}

Cesium removal is accomplished in this design by the use of ion exchange columns packed with a crystalline silicotitanate (CST) sorbant. Three ion exchange columns are included in the design. The columns are nominally sized at $16^{\prime \prime}$ diameter by $96 "$ tall and will contain $0.25 \mathrm{~m}^{3}$ sorbant. At any given time, two columns will be on-line and configured for in-series flow at a nominal capacity of $1.2 \mathrm{gpm}$. The third column will be off-line while spent sorbant is sluiced from the column and fresh sorbant is added.

Pressure drop through the ion exchange columns is expected to be less than 3psi based on theoretical calculations and experience with operating similar ion exchange columns at $\mathrm{ORNL}^{7}$.

Lead-lag configuration and valve timing of the ion exchange columns will be automatically controlled, and is based on cesium breakthrough of the lead ion exchange column. When breakthrough of the lead column is measured, the columns will sequence so that the lag column will become the lead column, the newly charged fresh column will become the lag column

Spent sorbant sluiced from the ion exchange column will be dried and stored in a bulk storage tank. The spent sorbant will be decanted of free liquid in a waste resin preparation column. The waste resin preparation column feeds a hollow flight drier which, using heat and hot air, will dry the sorbant. The hollow flight drier is nominally sized to process $2.1 \mathrm{l} / \mathrm{hr}$ of moist resin

Dried sorbant will flow by gravity to a spent resin storage tank. The spent resin storage tank is sized at $15 \mathrm{~m}^{3}$ to hold all of the sorbant generated during the life cycle of the facility. The estimated total spent resin quantity is estimated to be $10.5 \mathrm{~m}^{3}$.

Fresh sorbant will be preconditioned in a resin preparation tank and then sluiced to the off-line ion exchange column.

\section{Solidification}

SBW and NGLW, having passed through the cesium ion exchange columns, will then be grouted to form a solid TRU wasteform. The liquid waste will be neutralized in grout feed tanks, mixed with cement ingredients, and then cast in a $55 \mathrm{gal}$ drum. The cement based grout will be allowed to cure, and the drum prepared for shipment to an interim storage facility. 
Neutralization of the liquid waste to a specified setpoint of $\mathrm{pH} 1.3$, will be accomplished by the addition of calcium oxide (lime) to a grout feed tank. Because it is difficult to accurately measure the $\mathrm{pH}$ of a highly acidic mixture containing various ionic compounds, an automated system will titrate a known small volume of the liquid waste to a neutral $\mathrm{pH}$. (It is easier to obtain accurate measurements at a neutral pH.) Results of this titration will be utilized to calculate the quantity of $\mathrm{CaO}$ required to neutralize the grout feed tank to the setpoint.

Neutralized liquid waste will then be metered to one of three grout mixers. Each ribbon blender type mixer is capable of mixing one 55 gal drum of grout.

Premeasured quantities of solid ingredients (cement, blast furnace slag, and fly ash) will then be added to the liquid waste and thoroughly blended. The mixer will convey the grout into a casting head which will fill the waiting 55 gal drum.

Upon completion of a mix cycle the next batch of liquid waste to be solidified will be added to the mixer. This will be performed to prevent residual grout from hardening in the mixer. High pressure wash/ decontamination nozzles are also provided in the mixer so that the grout mixers can be turned off at the end of a production run, or for maintenance purposes.

Table 2 gives an approximate mixer cycle time.

Table 2. Mixer cycle Time

\begin{tabular}{|c|l|l|}
\hline Step & Description & Time $(\mathrm{min})$ \\
\hline 1 & Operator Initiation of Mix & 10 \\
\hline 2 & Feed Liquid & 10 \\
\hline 3 & Operator Validation of Wet and Dry Ingredients & 5 \\
\hline 4 & Dry Ingredient Delivery & 15 \\
\hline 5 & Continuous Mix & 10 \\
\hline 6 & Cast Mix & 10 \\
\hline 7 & Operator Validation of Mix & 10 \\
\hline Total & & 70 \\
\hline
\end{tabular}

Cast drums of grout will be conveyed via a mechanical conveyor to a contamination check station to ensure that no external contamination of the 55 gal drum has occurred. After verification that the external surface of the container is clean, the drum will be moved to a cure station. The cure station will provide a 24 hour residence time so that the grout may harden.

After the grout has cured the $55 \mathrm{gal}$ waste drum will be moved, via a mechanical conveyor, to a dewatering station where hot air will be passed through the head 
space of the drum. Dewatering is performed to ensure that there is no free water in the headspace of the drum.

The cured waste drum will then be checked using video surveillance to ensure that the grout is properly set, and then properly labeled in accordance with applicable storage and shipping requirements. The labeled drum will be moved to the interim storage facility.

In conformance with WIPP waste acceptance requirements, grout drums will undergo a visual inspection for free liquids and sampling of the gas headspace for hydrogen, VOCs, and tritium. Equipment to perform the inspection and gas headspace sampling will be located in the interim storage facility

\section{Process Flow Diagrams}

Process flow diagrams for this process are attached at the end of this EDF.

\section{Process Flow Diagrams}

01 -Block Flow Diagram

02 -Materials Delivery/Storage

03 -Liquid Waste Feed/Storage

04 -UDS Filtration Drying/Packaging

05 - CsIX Stripping

06 - IX Column Preparation/Washing, Spent Resin Drying/Packaging

07 -SBW Neutralization

08 -Grout Mixing/Casting, Mixer Decontamination

09 -Waste Drum Decontamination

10 -Cure/Dewater Station

11 -Grout Rework

12 -Off-Gas Control System

13 -interim Storage Shipment

Also see the associated equipment list for the process equipment immediately following the process flow diagrams. 


\section{Material Balance for the Total Composite Waste}

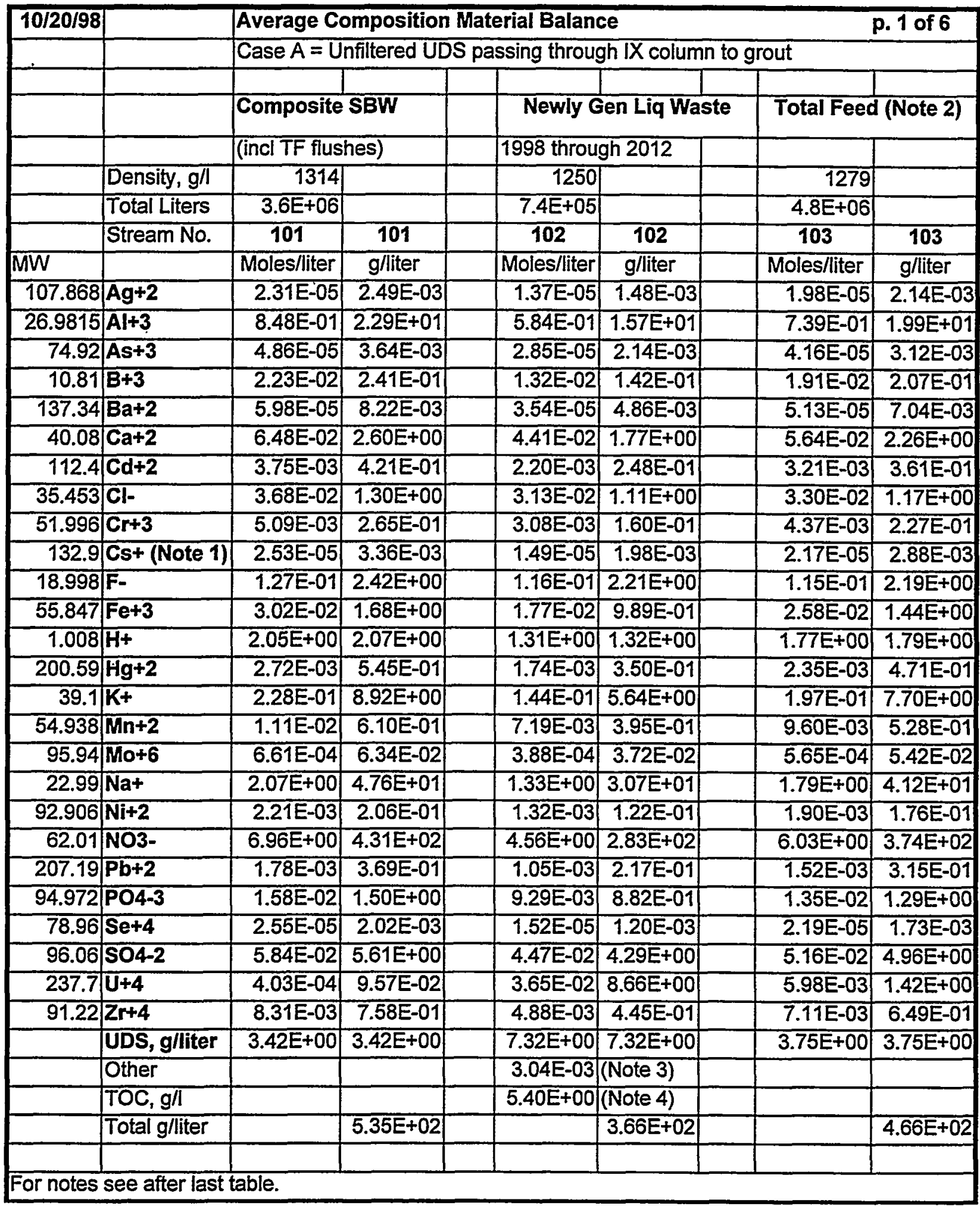




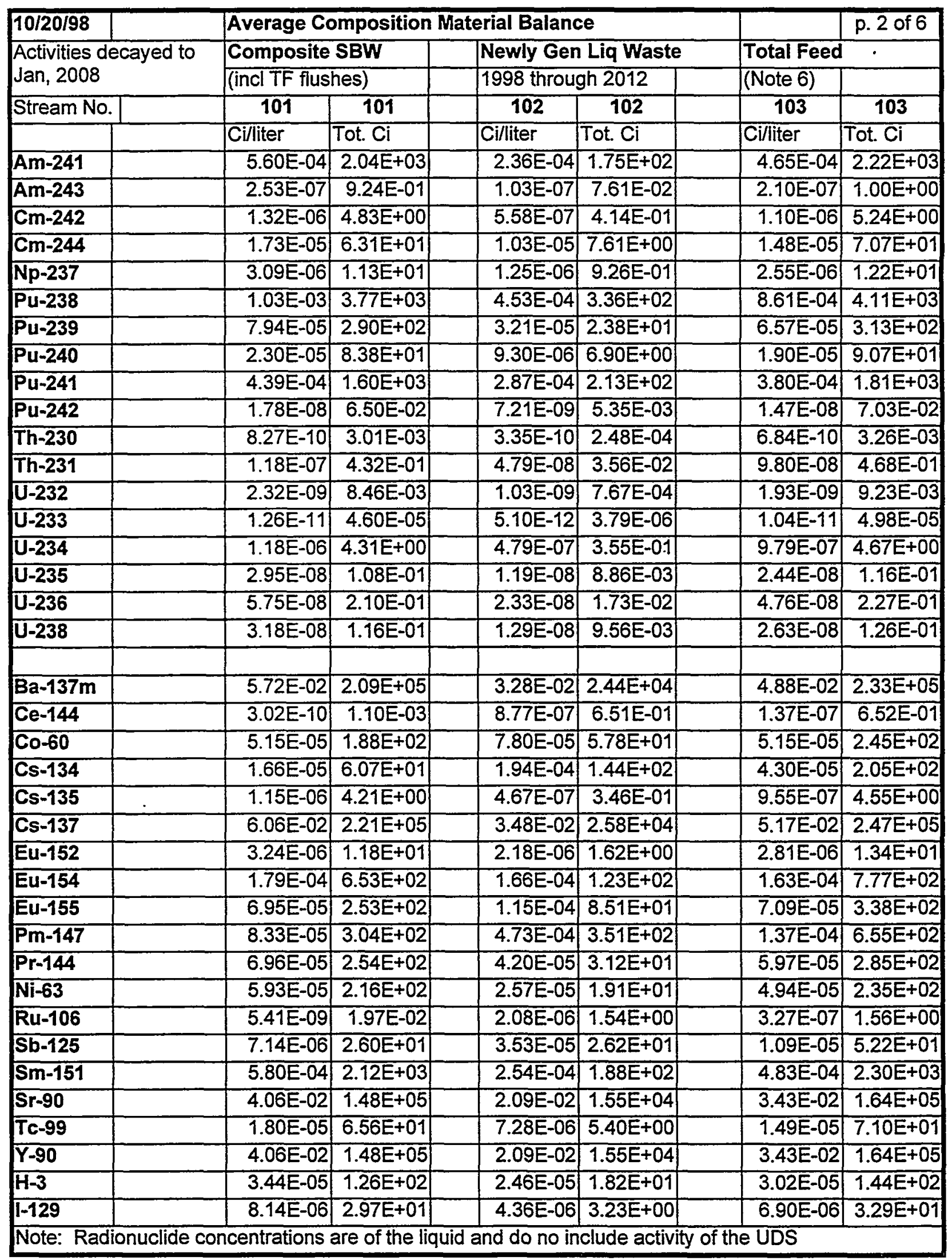




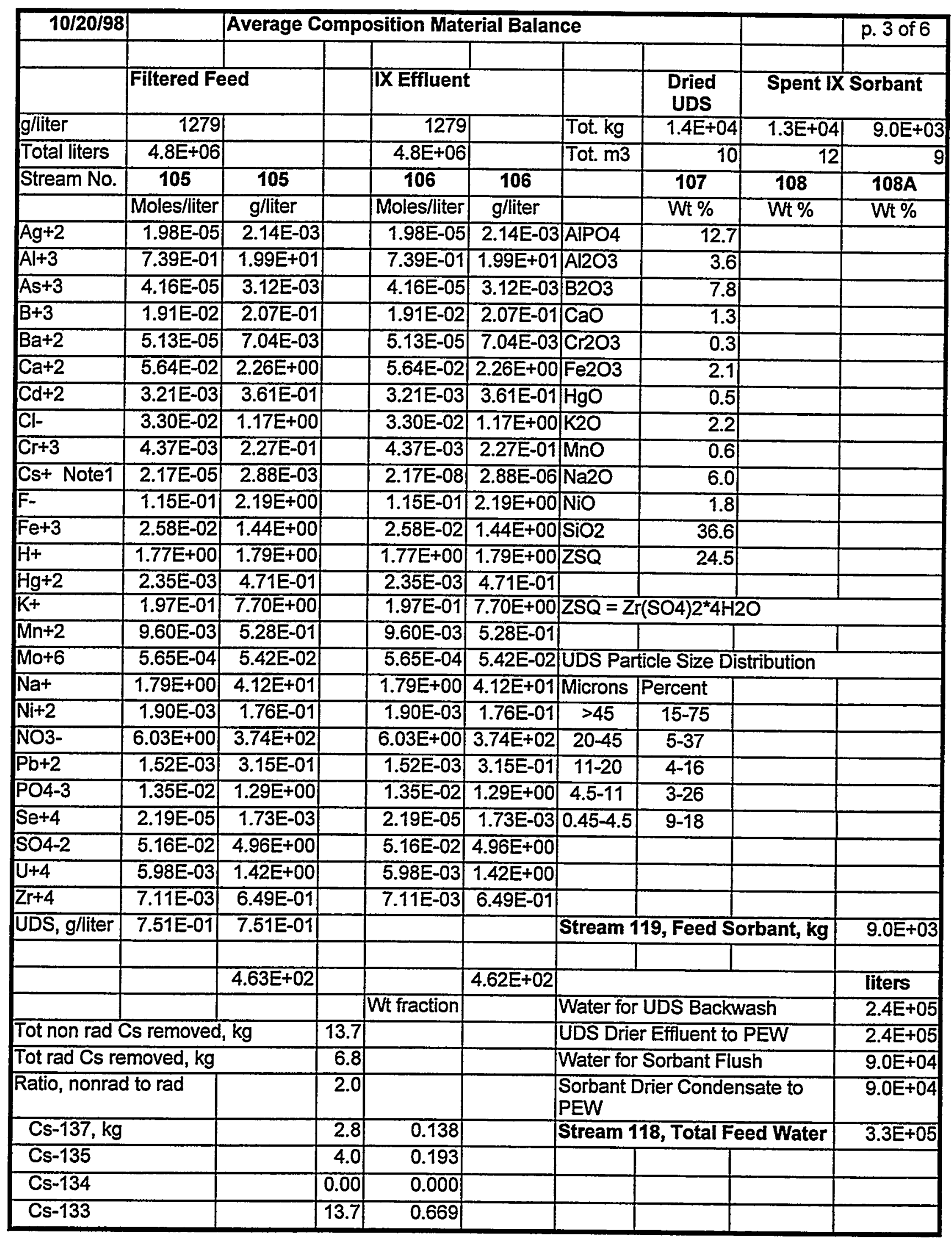




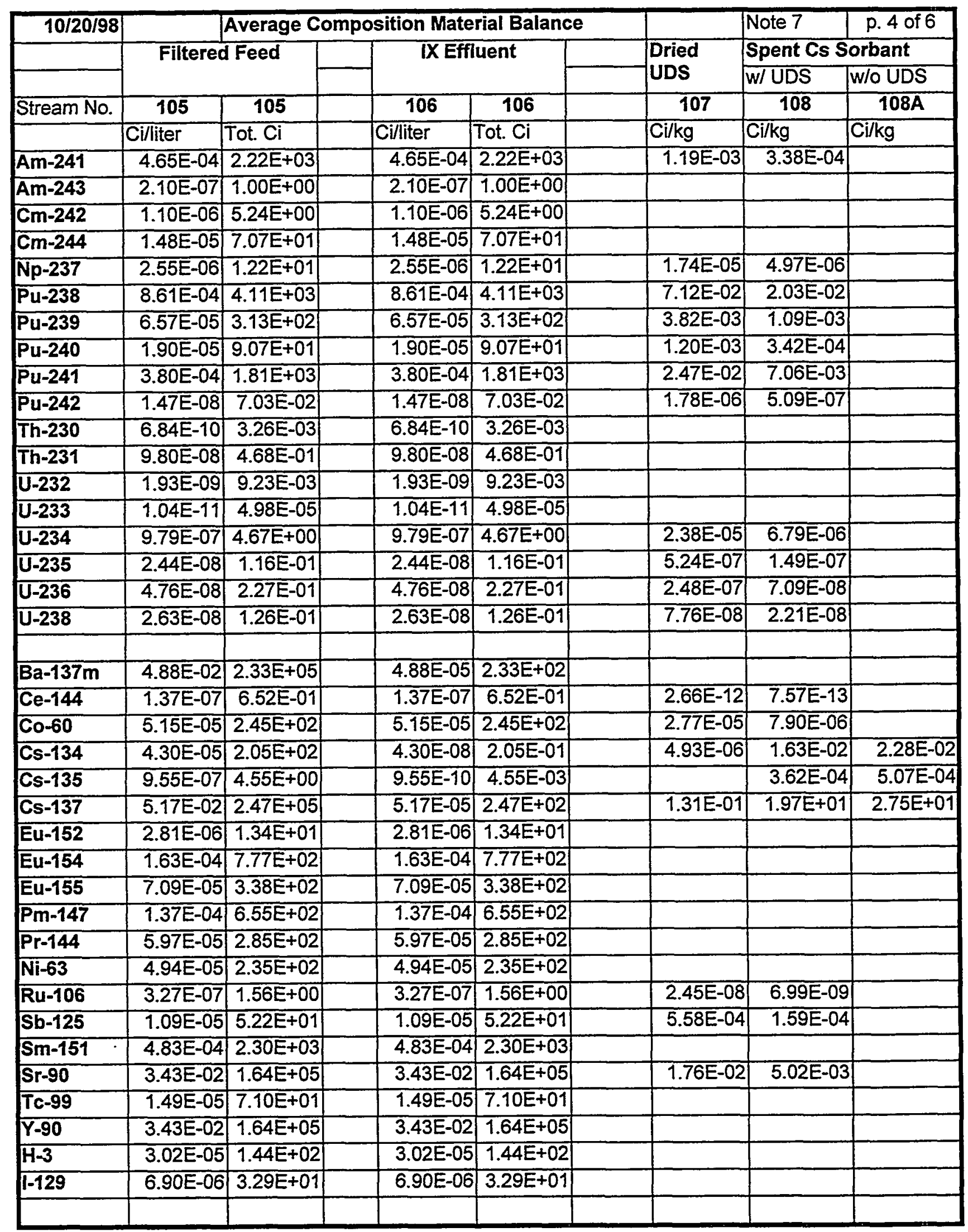




\begin{tabular}{|c|c|c|c|c|c|c|}
\hline \multirow[t]{2}{*}{$10 / 20 / 98$} & \multicolumn{5}{|c|}{ Average Composition Material Balance } & \multirow{2}{*}{ p. 5 of 6} \\
\hline & & & & Grouted Wa & & \\
\hline & \multicolumn{2}{|c|}{ Neutralized Waste } & & w/o UDS & w/UDS & \\
\hline & & & Density, $\mathrm{kg} / \mathrm{m} 3$ & 1900 & 1900 & \\
\hline Density, g/liter & 1310 & & Total $\mathrm{kg}$ & $1.4 \mathrm{E}+07$ & $1.4 \mathrm{E}+07$ & \\
\hline \multirow[t]{3}{*}{ Total Liters } & $4,844,104$ & & Total m3 & $7.5 E+03$ & $7.5 \mathrm{E}+03$ & \\
\hline & 113 & 113 & & 117 & $117 \mathrm{~A}$ & \\
\hline & Moles/liter & g/liter & & $\mathrm{g} / \mathrm{kg}$ & $\mathrm{g} / \mathrm{kg}$ & \\
\hline$\overline{A g+2}$ & 1.95E-05 & $2.10 \mathrm{E}-03$ & & $7.13 \mathrm{E}-04$ & $7.13 \mathrm{E}-04$ & \\
\hline$A \mid+3$ & $7.28 \mathrm{E}-01$ & $1.96 E+01$ & & $6.65 \mathrm{E}+00$ & $6.65 \mathrm{E}+00$ & \\
\hline$\overline{A s+3}$ & 4.10E-05 & 3.07E-03 & & $1.04 \mathrm{E}-03$ & $1.04 \mathrm{E}-03$ & \\
\hline $\mathrm{B}+3$ & 1.88E-02 & 2.03E-01 & & 6.89E-02 & $6.89 E-02$ & \\
\hline $\mathrm{Ba}+2$ & 5.05E-05 & $6.93 \mathrm{E}-03$ & & $2.35 \mathrm{E}-03$ & 2.35E-03 & \\
\hline$\overline{\mathrm{Ca}+2}$ & $9.03 \mathrm{E}-01$ & $3.62 E+01$ & & $1.23 \mathrm{E}+01$ & $1.23 E+01$ & \\
\hline $\mathrm{Cd+2}$ & $3.16 \mathrm{E}-03$ & 3.55E-01 & & $1.20 \mathrm{E}-01$ & $1.20 \mathrm{E}-01$ & \\
\hline Cl- & $3.25 E-02$ & $1.15 \mathrm{E}+00$ & & $3.90 \mathrm{E}-01$ & $3.90 \mathrm{E}-01$ & \\
\hline $\mathrm{Cr}+3$ & $4.30 \mathrm{E}-03$ & 2.24E-01 & & $7.58 \mathrm{E}-02$ & $7.58 \mathrm{E}-02$ & \\
\hline Cs+ (Note 1) & $2.13 E-08$ & 2.84E-06 & & 9.61E-07 & $9.60 \mathrm{E}-07$ & \\
\hline F- & 1.14E-01 & $2.16 \mathrm{E}+00$ & & 7.32E-01 & 7.31E-01 & \\
\hline $\mathrm{Fe}+3$ & 2.54E-02 & $1.42 E+00$ & & 4.81E-01 & 4.81E-01 & \\
\hline $\mathrm{H}+$ & $5.00 \mathrm{E}-02$ & $5.04 \mathrm{E}-02$ & & $0.00 E+00$ & $0.00 \mathrm{E}+00$ & \\
\hline $\mathrm{Hg}+2$ & 2.31E-03 & 4.64E-01 & & 1.57E-01 & 1.57E-01 & \\
\hline $\mathrm{K}+$ & $1.94 E-01$ & $7.58 \mathrm{E}+00$ & & $2.57 E+00$ & $2.57 \mathrm{E}+00$ & \\
\hline$M n+2$ & $9.46 \mathrm{E}-03$ & 5.19E-01 & & 1.76E-01 & $1.76 \mathrm{E}-01$ & \\
\hline Mo+6 & 5.57E-04 & 5.34E-02 & & 1.81E-02 & 1.81E-02 & \\
\hline Nat & $1.76 E+00$ & $4.06 \mathrm{E}+01$ & & $1.37 E+01$ & $1.37 \mathrm{E}+01$ & \\
\hline $\mathrm{Ni}+2$ & 1.87E-03 & 1.74E-01 & & $5.88 \mathrm{E}-02$ & $5.88 E-02$ & \\
\hline NO3- & $5.94 E+00$ & $3.68 \mathrm{E}+02$ & & $1.25 E+02$ & $1.25 E+02$ & \\
\hline $\mathrm{Pb}+2$ & $1.50 \mathrm{E}-03$ & 3.11E-01 & & $1.05 E-01$ & 1.05E-01 & \\
\hline PO4-3 & 1.33E-02 & $1.27 E+00$ & & 4.29E-01 & 4.29E-01 & \\
\hline $\mathrm{Se}+4$ & 2.16E-05 & $1.70 \mathrm{E}-03$ & & 5.77E-04 & 5.76E-04 & \\
\hline SO4-2 & $5.08 \mathrm{E}-02$ & $4.88 \mathrm{E}+00$ & & $1.65 \mathrm{E}+00$ & $1.65 \mathrm{E}+00$ & \\
\hline $\mathrm{U}+4$ & $5.88 \mathrm{E}-03$ & $1.40 \mathrm{E}+00$ & & 4.74E-01 & $4.74 \mathrm{E}-01$ & \\
\hline $\mathrm{Zr}+4$ & $7.00 \mathrm{E}-03$ & $6.39 \mathrm{E}-01$ & & $2.16 \mathrm{E}-01$ & 2.16E-01 & \\
\hline$S i+4$ & & & & & $4.28 \mathrm{E}-02$ & \\
\hline Tot g/liter & & $4.87 E+02$ & & $1.65 \mathrm{E}+02$ & $1.65 \mathrm{E}+02$ & \\
\hline \multicolumn{2}{|c|}{ Grout Additive Feeds } & & P. cement & $1.11 \mathrm{E}+02$ & $1.11 E+02$ & \\
\hline & Stream & $\mathrm{kg}$ & B.F. slag & $3.34 \mathrm{E}+02$ & $3.34 E+02$ & \\
\hline P. Cement & 114 & $1,593,482$ & Flyash & $1.11 \mathrm{E}+02$ & $1.11 \mathrm{E}+02$ & \\
\hline B. F. Slag & 116 & $4,780,446$ & Water & $2.78 E+02$ & $2.78 \mathrm{E}+02$ & \\
\hline Flyash & 115 & $1,593,482$ & & & & \\
\hline & & & & & & \\
\hline $\mathrm{CaO}$ & 109 & 230,320 & & & & \\
\hline
\end{tabular}


Functional File Number - SPR-CsIX-01 EDF Serial Number - EDF-CsIX-001, Rev 1

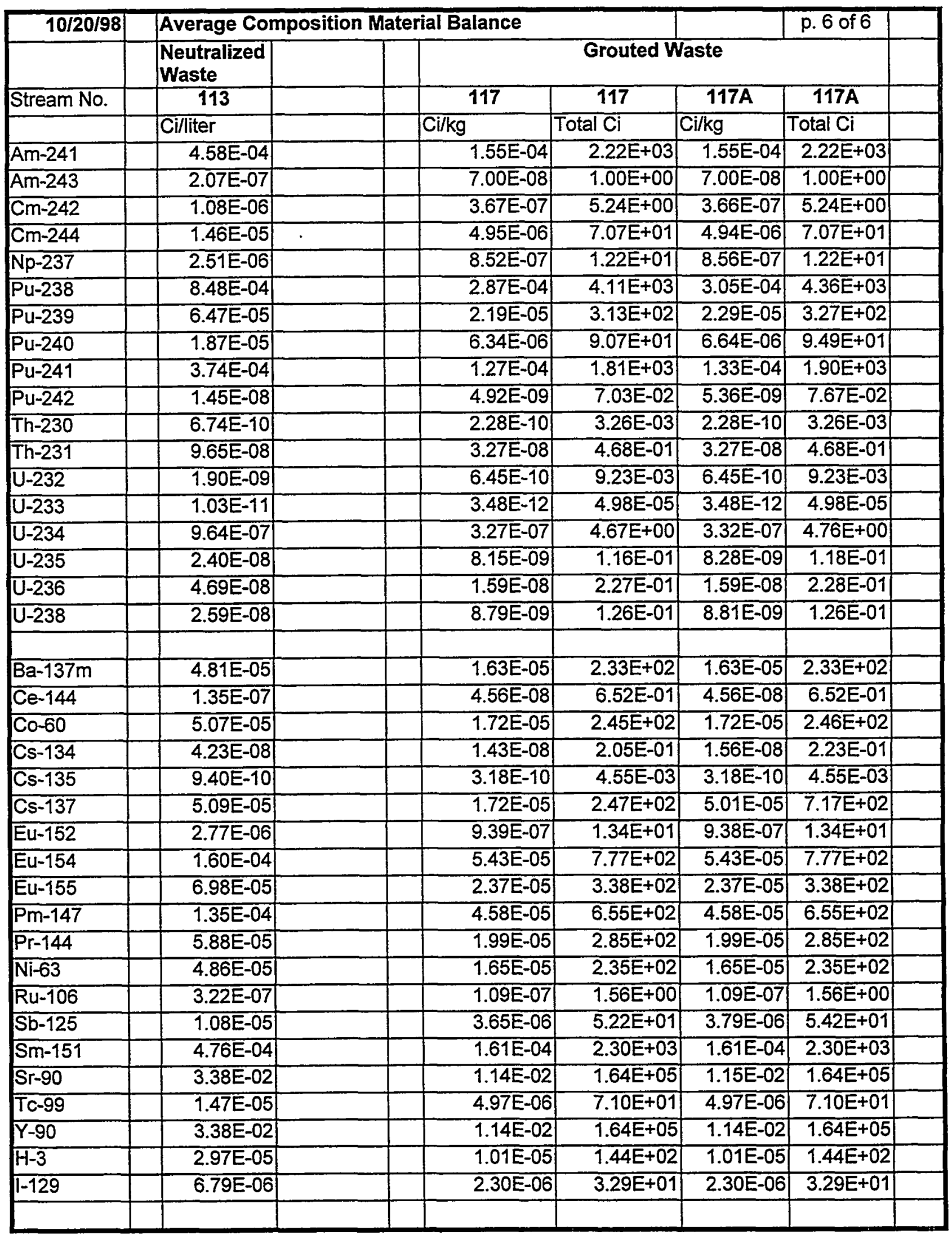


Notes for Average Composition Material Balance:

1. Cesium shown above is Cs-133 only

2. Total feed includes $8 \%$ dilution due to steam jet transfer

3. "Other" includes $\mathrm{Mg}$, Si and 13 other trace ions, which are not carried forward in the material balance.

4. Approx. $50 \%$ oxalic acid, $10 \%$ kerosene, $30 \%$ TEA, $10 \%$ DEA

5. Includes condensate from drying of UDS and spent Cs sorbant

6. Activities for liquid only, activity for UDS shown under stream 107

7. Data is not available to determine sorption of TRU or other radionuclides on sorbant. Thus actual activity of spent sorbant may be very different from what is shown here.

\section{Material Balance for Liquid Waste from WM-181}

Material balance tables are shown below for liquid from tank WM-181. Based on initial calculations, waste in WM-181, after evaporation, had the highest level of the radionuclides, ${ }^{137} \mathrm{Cs}$ and others, that contributed to the dose rate. The material balance for WM-181 was thus made for a "worst case". However, it was learned later in the study WM-181 will likely be blended with WM-184 or WM-186, and evaporated to a greater degree than was initially assumed. With this higher degree of concentration, along with tank heels that show higher radionuclide concentrations than the full tanks, the ${ }^{137} \mathrm{Cs}$ level in the composite waste became greater than the WM-181 case. This material balance is included for reference, but was not used for equipment sizing or selection in the study. 


\begin{tabular}{|c|c|c|c|c|c|c|c|}
\hline \multicolumn{5}{|c|}{ Material Balance - Worst Case (WM-181) } & \multirow{3}{*}{$\begin{array}{l}\text { After jet } \\
\text { Dilution }\end{array}$} & p. 1 of 4 & $10 / 20 / 98$ \\
\hline & & \multirow{2}{*}{\begin{tabular}{|l|} 
Before \\
Evap
\end{tabular}} & \multirow{2}{*}{\multicolumn{2}{|c|}{ After Evaporation }} & & \multicolumn{2}{|c|}{ Cs lon Exchange } \\
\hline & & & & & & Feed & Effluent \\
\hline \multicolumn{2}{|c|}{ Volume, liters } & $1.0 \mathrm{E}+06$ & $5.2 E+05$ & $5.2 E+05$ & $5.7 \mathrm{E}+05$ & $5.8 \mathrm{E}+05$ & $5.8 E+05$ \\
\hline \multicolumn{2}{|c|}{ Density, kg/l } & 1.16 & 1.30 & 1.30 & 1.28 & 1.27 & 1.27 \\
\hline \multicolumn{2}{|c|}{ Stream Number } & 100 & 101 & 101 & 103 & 105 & 106 \\
\hline & $M W$ & Moles/liter & Moles/liter & g/liter & Moles/liter & Moles/liter & Moles/liter \\
\hline$\overline{A g}+2$ & 107.868 & 3.04E-05 & \begin{tabular}{|l|}
$6.12 \mathrm{E}-05$ \\
\end{tabular} & $6.60 \mathrm{E}-03$ & 5.63E-05 & \begin{tabular}{|l|}
$5.50 \mathrm{E}-05$ \\
\end{tabular} & 5.50E-05 \\
\hline$A \mathrm{Al}+3$ & 26.9815 & $2.30 E-01$ & 4.63E-01 & $1.25 \mathrm{E}+01$ & 4.26E-01 & $4.16 \mathrm{E}-01$ & $4.16 \mathrm{E}-01$ \\
\hline$A s+3$ & 74.92 & 3.82E-05 & $7.68 \mathrm{E}-05$ & $5.75 \mathrm{E}-03$ & 7.07E-05 & 6.91E-05 & $6.91 \mathrm{E}-05$ \\
\hline $\mathrm{B}+3$ & 10.81 & $1.60 \mathrm{E}-02$ & $3.22 E-02$ & $3.48 \mathrm{E}-01$ & $2.96 \mathrm{E}-02$ & $2.90 \mathrm{E}-02$ & $2.90 \mathrm{E}-02$ \\
\hline $\mathrm{Ba}+2$ & 137.34 & $3.78 \mathrm{E}-05$ & $7.62 \mathrm{E}-05$ & 1.05E-02 & $7.01 \mathrm{E}-05$ & 6.85E-05 & $6.85 \mathrm{E}-05$ \\
\hline $\mathrm{Ca}+2$ & 40.08 & 4.60E-02 & $9.26 \mathrm{E}-02$ & $3.71 E+00$ & $8.52 \mathrm{E}-02$ & $8.33 \mathrm{E}-02$ & $8.33 \mathrm{E}-02$ \\
\hline$C d+2$ & 112.4 & $5.50 \mathrm{E}-03$ & $1.11 \mathrm{E}-02$ & $1.24 \mathrm{E}+00$ & $1.02 \mathrm{E}-02$ & 9.95E-03 & $9.95 \mathrm{E}-03$ \\
\hline $\mathrm{Cl}-$ & 35.453 & $1.26 \mathrm{E}-02$ & 2.54E-02 & 8.99E-01 & 2.33E-02 & $2.28 \mathrm{E}-02$ & $2.28 \mathrm{E}-02$ \\
\hline $\mathrm{Cr}+3$ & 51.996 & $3.00 E-03$ & $6.04 \mathrm{E}-03$ & $3.14 \mathrm{E}-01$ & 5.56E-03 & $5.43 \mathrm{E}-03$ & $5.43 \mathrm{E}-03$ \\
\hline Cst & 132.9 & $1.09 \mathrm{E}-05$ & $2.19 \mathrm{E}-05$ & 2.92E-03 & $2.02 \mathrm{E}-05$ & 1.97E-05 & 1.97E-08 \\
\hline F- & 18.998 & $9.40 E-02$ & $1.89 \mathrm{E}-01$ & $3.60 \mathrm{E}+00$ & $1.74 \mathrm{E}-01$ & $1.70 \mathrm{E}-01$ & $1.70 \mathrm{E}-01$ \\
\hline $\mathrm{Fe}+3$ & 55.847 & $1.30 \mathrm{E}-02$ & $2.62 \mathrm{E}-02$ & $1.46 \mathrm{E}+00$ & $2.41 E-02$ & $2.35 \mathrm{E}-02$ & $2.35 \mathrm{E}-02$ \\
\hline $\mathrm{H}+$ & 1.008 & $1.89 \mathrm{E}+00$ & $3.81 \mathrm{E}+00$ & $3.84 \mathrm{E}+00$ & $3.50 \mathrm{E}+00$ & $3.42 \mathrm{E}+00$ & $3.42 E+00$ \\
\hline $\mathrm{Hg}+2$ & 200.59 & $4.70 \mathrm{E}-04$ & $9.46 \mathrm{E}-04$ & $1.90 \mathrm{E}-01$ & $8.71 \mathrm{E}-04$ & $8.51 E-04$ & $8.51 \mathrm{E}-04$ \\
\hline $\mathrm{K}+$ & 39.1 & $1.50 \mathrm{E}-01$ & $3.02 \mathrm{E}-01$ & $1.18 \mathrm{E}+01$ & $2.78 E-01$ & $2.71 E-01$ & 2.71E-01 \\
\hline$M n+2$ & 54.938 & $1.29 \mathrm{E}-02$ & $2.60 \mathrm{E}-02$ & $1.43 \mathrm{E}+00$ & $2.39 E-02$ & $2.33 E-02$ & $2.33 \mathrm{E}-02$ \\
\hline Mo+6 & 95.94 & $5.47 E-04$ & $1.10 \mathrm{E}-03$ & $1.06 \mathrm{E}-01$ & $1.01 E-03$ & $9.90 \mathrm{E}-04$ & $9.90 \mathrm{E}-04$ \\
\hline $\mathrm{Na}+$ & 22.99 & $9.50 \mathrm{E}-01$ & $1.91 \mathrm{E}+00$ & $4.40 \mathrm{E}+01$ & $1.76 \mathrm{E}+00$ & $1.72 \mathrm{E}+00$ & $1.72 \mathrm{E}+00$ \\
\hline $\mathrm{Ni}+2$ & 92.906 & $1.30 \mathrm{E}-03$ & $2.62 \mathrm{E}-03$ & $2.43 \mathrm{E}-01$ & $2.41 E-03$ & $2.35 E-03$ & $2.35 E-03$ \\
\hline NO3- & 62.01 & $3.87 E+00$ & $7.79 \mathrm{E}+00$ & $4.83 E+02$ & $7.17 \mathrm{E}+00$ & $7.00 \mathrm{E}+00$ & $7.00 E+00$ \\
\hline$\overline{\mathrm{Pb}+2}$ & 207.19 & $1.10 \mathrm{E}-03$ & $2.21 \mathrm{E}-03$ & $4.59 \mathrm{E}-01$ & $2.04 \mathrm{E}-03$ & $1.99 \mathrm{E}-03$ & 1.99E-03 \\
\hline PO4-3 & 94.972 & $6.42 E-03$ & $1.29 \mathrm{E}-02$ & $1.23 \bar{E}+00$ & $1.19 \mathrm{E}-02$ & $1.16 \mathrm{E}-02$ & $1.16 \mathrm{E}-02$ \\
\hline $\mathrm{Se}+4$ & 78.96 & $3.01 E-05$ & 6.07E-05 & $4.79 \mathrm{E}-03$ & $5.58 \mathrm{E}-05$ & 5.45E-05 & $5.45 E-05$ \\
\hline SO4-2 & 96.06 & $2.50 \mathrm{E}-02$ & $5.03 \mathrm{E}-02$ & $4.83 E+00$ & $4.63 \mathrm{E}-02$ & $4.52 \mathrm{E}-02$ & $4.52 \mathrm{E}-02$ \\
\hline $\mathrm{U}+4$ & 237.7 & $3.19 E-04$ & $6.43 \mathrm{E}-04$ & $1.53 \mathrm{E}-01$ & $5.92 \mathrm{E}-04$ & $5.78 \mathrm{E}-04$ & $5.78 \mathrm{E}-04$ \\
\hline$Z r+4$ & 91.22 & $4.84 \mathrm{E}-03$ & $9.74 \mathrm{E}-03$ & $8.89 E-01$ & $8.96 \mathrm{E}-03$ & $8.76 E-03$ & $8.76 \mathrm{E}-03$ \\
\hline $\begin{array}{l}\text { UDS, } \\
\text { g/liter }\end{array}$ & & $1.70 \mathrm{E}-01$ & $3.42 E-01$ & 3.42E-01 & $3.15 \mathrm{E}-01$ & 3.07E-01 & \\
\hline & & & & & & & \\
\hline $\begin{array}{l}\text { Total } \\
\text { solids }\end{array}$ & & & & $5.77 E+02$ & & & \\
\hline & & & & & & & \\
\hline Notes: & & & & & & & \\
\hline 1. Ces & Wh & $s-135$ onily & & & & & \\
\hline & & & & & & & \\
\hline
\end{tabular}




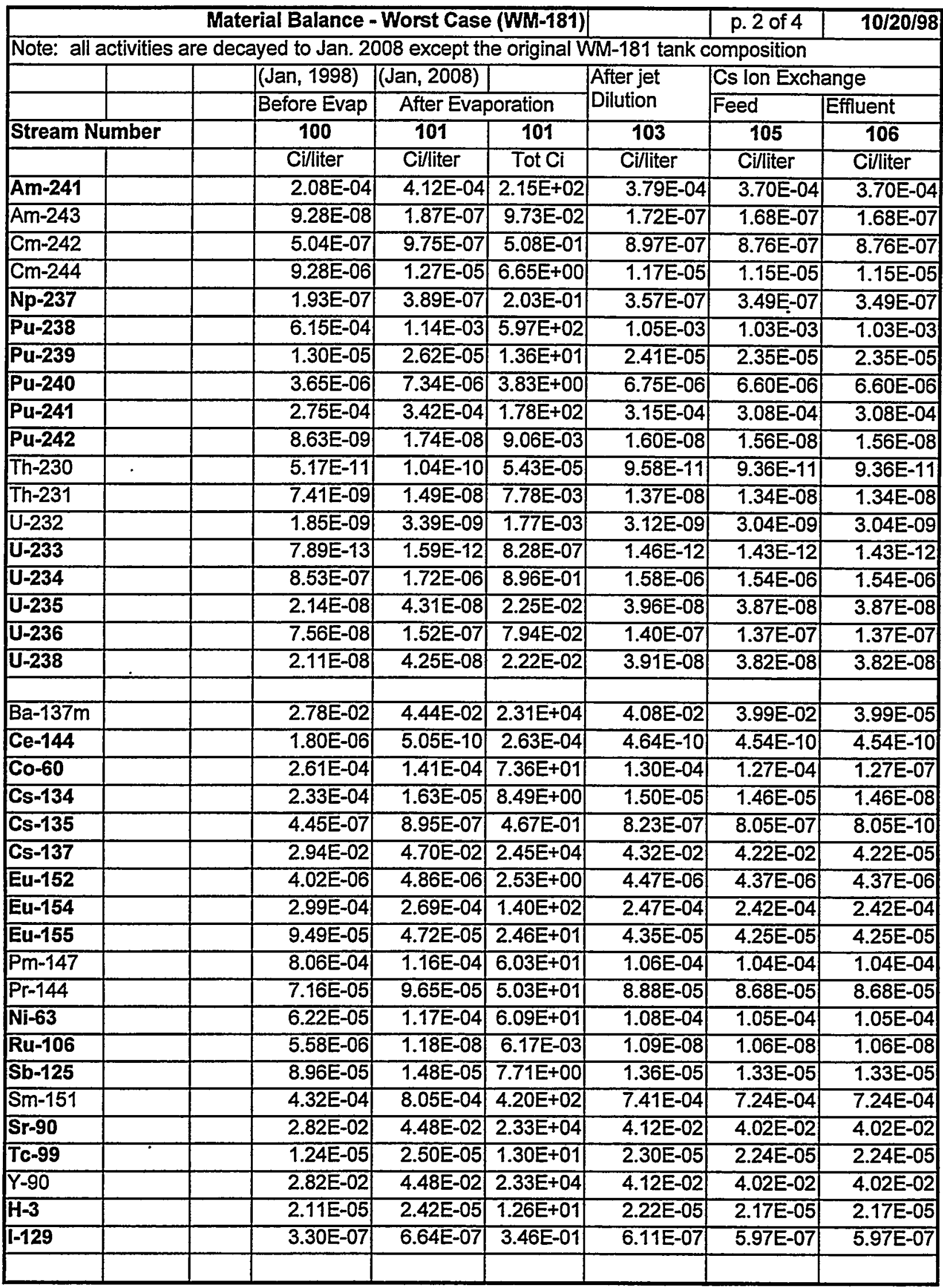




\begin{tabular}{|c|c|c|c|c|c|c|c|c|c|}
\hline \multicolumn{6}{|c|}{ Material Balance - Worst Case (WM-181) } & & \multirow{2}{*}{\multicolumn{2}{|c|}{\begin{tabular}{c|c|} 
p. 3 of 4 & $10 / 20 / 98$ \\
Grouted Waste
\end{tabular}}} & \\
\hline & \multicolumn{2}{|c|}{ Spent Cs Sorbant } & \multirow{2}{*}{$\begin{array}{l}\text { Dried } \\
\text { UDS }\end{array}$} & & \multicolumn{2}{|c|}{ Neutralized Waste } & & & \\
\hline & w/o UDS & w/UDS & & & & & w/o UDS & w/UDS & \\
\hline Vol, m3 & 1.1 & 1.12 & 0.10 & Vol, liters & $6.0 \mathrm{E}+05$ & $6.0 E+05$ & $8.9 E+05$ & $8.9 E+05$ & \\
\hline Density, kg/l & 1.0 & 1.0 & 1.4 & Dens., g/cm3 & 1.32 & 1.32 & 1.70 & 1.70 & \\
\hline \multirow[t]{3}{*}{ Stream No. } & $108 \mathrm{~A}$ & 108 & 107 & & 113 & 113 & 117 & $117 \mathrm{~A}$ & \\
\hline & & & Wt \% & & Moles/liter & g/liter & $\mathrm{g} / \mathrm{kg}$ & $\mathrm{g} / \mathrm{kg}$ & \\
\hline & & & & $\mathrm{Ag}+2$ & 5.34E-05 & 5.76E-03 & $2.03 E-03$ & 2.03E-03 & \\
\hline AlPO4 & & & 13.6 & $A \mid+3$ & $4.04 E-01$ & $1.09 \mathrm{E}+01$ & $3.84 \mathrm{E}+00$ & $3.84 \mathrm{E}+00$ & \\
\hline $\mathrm{B2O3}$ & & & 3.9 & $\overline{A s+3}$ & $6.70 \mathrm{E}-05$ & 5.02E-03 & 1.77E-03 & 1.77E-03 & \\
\hline $\mathrm{Al} 2 \mathrm{O3}$ & & & 1.4 & $B+3$ & $2.81 \mathrm{E}-02$ & $3.04 \mathrm{E}-01$ & 1.07E-01 & 1.07E-01 & \\
\hline $\mathrm{CaO}$ & & & 1.5 & $\mathrm{Ba}+2$ & $6.65 \mathrm{E}-05$ & $9.13 E-03$ & $3.22 \mathrm{E}-03$ & $3.22 \mathrm{E}-03$ & \\
\hline$\overline{\mathrm{C}} 203$ & & & 0.7 & $\mathrm{Ca}+2$ & $1.72 \mathrm{E}+00$ & $6.88 \mathrm{E}+01$ & $2.43 E+01$ & $2.43 E+01$ & \\
\hline $\mathrm{MnO}$ & & & 1.5 & $\overline{C d+2}$ & $9.66 \mathrm{E}-03$ & $1.09 E+00$ & $3.83 \mathrm{E}-01$ & $3.83 \mathrm{E}-01$ & \\
\hline $\mathrm{Na2O}$ & & & 3.2 & $\mathrm{Cl}$ & $2.21 E-02$ & 7.85E-01 & 2.77E-01 & 2.77E-01 & \\
\hline $\mathrm{NiO}$ & & & 3.9 & $\overline{C r+3}$ & $5.27 \mathrm{E}-03$ & 2.74E-01 & $9.66 \mathrm{E}-02$ & $9.66 \mathrm{E}-02$ & \\
\hline \multicolumn{2}{|c|}{$\mathrm{Zr}(\mathrm{SO} 4) 2^{*} 4 \mathrm{H} 2 \mathrm{O}$} & & 20.9 & $\overline{C s+}$ & 1.91E-08 & $2.54 E-06$ & $8.98 E-07$ & $8.98 \mathrm{E}-07$ & \\
\hline K2O & & & 5.8 & $F-$ & 1.65E-01 & $3.14 \mathrm{E}+00$ & $1.11 E+00$ & $1.11 \mathrm{E}+00$ & \\
\hline $\mathrm{HgO}$ & & & 0.013 & $\mathrm{Fe}+3$ & $2.28 \mathrm{E}-02$ & $1.28 \mathrm{E}+00$ & $4.50 \mathrm{E}-01$ & $4.50 \mathrm{E}-01$ & \\
\hline $\mathrm{Fe} 2 \mathrm{O3}$ & & & 2.7 & $\mathrm{H}+$ & $5.00 \mathrm{E}-02$ & $5.04 \mathrm{E}-02$ & $0.00 \mathrm{E}+00$ & $0.00 \mathrm{E}+00$ & \\
\hline \multirow[t]{2}{*}{$\mathrm{SiO} 2$} & & & 40.8 & $\mathrm{Hg}+2$ & $8.26 E-04$ & $1.66 \mathrm{E}-01$ & 5.84E-02 & $5.84 \mathrm{E}-02$ & \\
\hline & & & & $\mathrm{K}+$ & 2.63E-01 & $1.03 E+01$ & $3.63 E+00$ & $3.63 E+00$ & \\
\hline \multirow[t]{6}{*}{ Total kg } & 1,092 & 1,128 & 143 & $\mathrm{Mn}+2$ & $2.27 E-02$ & $1.24 \mathrm{E}+00$ & $4.39 \mathrm{E}-01$ & 4.39E-01 & \\
\hline & & & & Mo+6 & $9.61 E-04$ & 9.22E-02 & $3.25 E-02$ & $3.25 \mathrm{E}-02$ & \\
\hline & & & & $\mathrm{Nat}$ & $1.67 \mathrm{E}+00$ & $3.84 E+01$ & $1.35 \mathrm{E}+01$ & $1.35 \mathrm{E}+01$ & \\
\hline & & & & $\mathrm{Ni}+2$ & $2.28 \mathrm{E}-03$ & $2.12 \mathrm{E}-01$ & $7.48 \mathrm{E}-02$ & 7.48E-02 & \\
\hline & & & & NO3- & $6.80 \mathrm{E}+00$ & $4.22 E+02$ & $1.49 E+02$ & $1.49 \mathrm{E}+02$ & \\
\hline & Stream & & & $\overline{\mathrm{Pb}+2}$ & $1.93 \mathrm{E}-03$ & $4.00 \mathrm{E}-01$ & $1.41 \mathrm{E}-01$ & 1.41E-01 & \\
\hline Sorbant In & 119 & 1,128 & $\mathrm{~kg}$ & $\mathrm{PO4-3}$ & 1.13E-02 & $1.07 \mathrm{E}+00$ & $3.78 \mathrm{E}-01$ & $3.78 \mathrm{E}-01$ & \\
\hline \multirow[t]{2}{*}{ Water In } & 118 & 13,278 & liters & $\mathrm{Se}+4$ & $5.29 \mathrm{E}-05$ & 4.18E-03 & $1.47 \mathrm{E}-03$ & 1.47E-03 & \\
\hline & & & & SO4-2 & 4.39E-02 & $4.22 E+00$ & $1.49 E+00$ & $1.49 \mathrm{E}+00$ & \\
\hline \multirow[t]{14}{*}{$\mathrm{CaO}$} & 109 & 54,805 & $\mathrm{~kg}$ & $\mathrm{U}+4$ & $5.61 \mathrm{E}-04$ & 1.33E-01 & $4.70 \mathrm{E}-02$ & $4.70 \mathrm{E}-02$ & \\
\hline & & & & $\overline{Z r+4}$ & $8.50 \mathrm{E}-03$ & $7.76 \mathrm{E}-01$ & $2.74 \mathrm{E}-01$ & $2.73 E-01$ & \\
\hline & & & & & & & & & \\
\hline & & & & \multicolumn{2}{|c|}{ Total solids, g/liter } & $5.65 E+02$ & & & \\
\hline & & & & \multicolumn{2}{|c|}{ Waste solids, g/kg } & & 199 & 199 & \\
\hline & & & & Water, g/kg & & & 268 & 268 & \\
\hline & & & & \multicolumn{2}{|c|}{ Grout additives, g/kg } & & 533 & 533 & \\
\hline & & & & & & & & & \\
\hline & & & & Total kg & & & $1.7 E+06$ & 1.7E+06 & \\
\hline & & & & & & Stream No. & & & \\
\hline & & & & P. Cement, kg & & 114 & $1.8 \mathrm{E}+05$ & $1.8 \mathrm{E}+05$ & \\
\hline & & & & B. F. Slag, kg & & 116 & $5.4 E+05$ & $5.4 E+05$ & \\
\hline & & & & Flyash, kg & & 115 & $1.8 \mathrm{E}+05$ & $1.8 E+05$ & \\
\hline & & & & & & & & & \\
\hline
\end{tabular}




\section{Material Balance - Worst Case (WM-181)}

Note: all activities are decayed to Jan. 2008 except the original WM-181 tank composition

\begin{tabular}{|l|l|}
\hline p. 4 of 4 & $10 / 20 / 98$ \\
\hline
\end{tabular}

\begin{tabular}{|c|c|c|c|c|c|c|c|c|c|}
\hline \multirow{3}{*}{\begin{tabular}{|l|} 
\\
Stream No. \\
\end{tabular}} & \multicolumn{2}{|c|}{ Spent Cs Sorbant } & \multirow{2}{*}{$\begin{array}{l}\text { Dried } \\
\text { UDS }\end{array}$} & & \multirow{2}{*}{\multicolumn{2}{|c|}{ Neutralized Waste }} & \multicolumn{2}{|c|}{ Grouted Waste } & \\
\hline & w/o UDS & w/ UDS & & & & & \multirow{2}{*}{ w/o UDS } & \multirow{2}{*}{\begin{tabular}{|r|} 
W/UDS \\
$117 A$
\end{tabular}} & \\
\hline & $108 \mathrm{~A}$ & 108 & 107 & & 113 & 113 & & & \\
\hline & Ci $/ \mathrm{kg}$ & $\mathrm{Ci} / \mathrm{kg}$ & Ci/kg & & $\mathrm{Ci} / \mathrm{l}$ & Tot. $\mathrm{Ci}$ & $\mathrm{Ci} / \mathrm{kg}$ & $\mathrm{Ci} / \mathrm{kg}$ & \\
\hline$A m-241$ & & $3.75 \mathrm{E}-05$ & $1.19 \mathrm{E}-03$ & & $3.60 \mathrm{E}-04$ & $2.15 E+02$ & 1.27E-04 & $1.27 \mathrm{E}-04$ & \\
\hline Am-243 & & & & & 1.63E-07 & 9.73E-02 & $5.74 \mathrm{E}-08$ & 5.74E-08 & \\
\hline $\mathrm{Cm}-242$ & & & & & $8.50 \mathrm{E}-07$ & $5.08 \mathrm{E}-01$ & $3.00 E-07$ & $3.00 \mathrm{E}-07$ & \\
\hline $\mathrm{Cm}-244$ & & & & & 1.11E-05 & $6.65 \mathrm{E}+00$ & $3.92 E-06$ & 3.92E-06 & \\
\hline Np-237 & & 5.51E-07 & $1.74 \mathrm{E}-05$ & & 3.39E-07 & $2.03 E-01$ & 1.20E-07 & $1.20 \mathrm{E}-07$ & \\
\hline Pu-238 & & 2.25E-03 & $7.12 \mathrm{E}-02$ & & $9.98 \mathrm{E}-04$ & $5.97 \mathrm{E}+02$ & $3.52 E-04$ & $3.54 E-04$ & \\
\hline Pu-239 & & $1.21 \mathrm{E}-04$ & $3.82 \mathrm{E}-03$ & & $2.28 \mathrm{E}-05$ & $1.36 E+01$ & 8.05E-06 & $8.13 E-06$ & \\
\hline Pu-240 & & $3.79 \mathrm{E}-05$ & $1.20 \mathrm{E}-03$ & & $6.40 \mathrm{E}-06$ & $3.83 E+00$ & $2.26 \mathrm{E}-06$ & $2.28 \mathrm{E}-06$ & \\
\hline Pu-241 & & $7.82 E-04$ & $2.47 \mathrm{E}-02$ & & $2.99 \mathrm{E}-04$ & $1.78 E+02$ & 1.05E-04 & $1.06 \mathrm{E}-04$ & \\
\hline $\mathrm{Pu}-242$ & & 5.64E-08 & 1.78E-06 & & 1.52E-08 & $9.06 \mathrm{E}-03$ & $5.35 E-09$ & $5.38 \mathrm{E}-09$ & \\
\hline Th-230 & & & & & $9.08 \mathrm{E}-11$ & $5.43 \mathrm{E}-05$ & $3.20 \mathrm{E}-11$ & $3.20 \mathrm{E}-11$ & \\
\hline Th-231 & & & & & 1.30E-08 & $7.78 \mathrm{E}-03$ & $4.59 \mathrm{E}-09$ & 4.59E-09 & \\
\hline$U-232$ & & & & & 2.95E-09 & 1.77E-03 & $1.04 \mathrm{E}-09$ & 1.04E-09 & \\
\hline $\mathrm{U}-233$ & & & & & $1.39 \mathrm{E}-12$ & $8.28 \mathrm{E}-07$ & $4.89 E-13$ & $4.89 E-13$ & \\
\hline U-234 & & $7.52 \mathrm{E}-07$ & $2.38 \mathrm{E}-05$ & & $1.50 \mathrm{E}-06$ & $8.96 \mathrm{E}-01$ & $5.28 E-07$ & 5.29E-07 & \\
\hline $\mathrm{U}-235$ & & $1.66 \mathrm{E}-08$ & 5.24E-07 & & $3.76 \mathrm{E}-08$ & $2.25 \mathrm{E}-02$ & $1.33 \mathrm{E}-08$ & 1.33E-08 & \\
\hline U-236 & & $7.86 \mathrm{E}-09$ & $2.48 E-07$ & & $1.33 \mathrm{E}-07$ & 7.94E-02 & $4.68 \mathrm{E}-08$ & $4.68 \mathrm{E}-08$ & \\
\hline U-238 & & $2.46 \mathrm{E}-09$ & $7.76 \mathrm{E}-08$ & & 3.71E-08 & $2.22 \mathrm{E}-02$ & 1.31E-08 & $1.31 \mathrm{E}-08$ & \\
\hline & & & & & & & & & \\
\hline Ba-137m & & & & & 3.87E-02 & $2.31 E+01$ & $1.37 \mathrm{E}-05$ & 1.37E-05 & \\
\hline $\mathrm{Ce}-144$ & & $8.40 \mathrm{E}-14$ & $2.66 \mathrm{E}-12$ & & $4.40 \mathrm{E}-10$ & $2.63 E-04$ & $1.55 \mathrm{E}-10$ & $1.55 \mathrm{E}-10$ & \\
\hline Co-60 & & $8.76 \mathrm{E}-07$ & 2.77E-05 & & 1.23E-07 & $7.36 \mathrm{E}-02$ & $4.34 \mathrm{E}-08$ & 4.34E-05 & \\
\hline Cs-134 & 7.77E-03 & $7.52 E-03$ & 4.93E-06 & & $1.42 \mathrm{E}-08$ & $8.49 E-03$ & 5.01E-09 & $5.11 \mathrm{E}-09$ & \\
\hline Cs-135 & 4.27E-04 & $4.13 E-04$ & & & 7.81E-10 & $4.67 \mathrm{E}-04$ & $2.75 E-10$ & $2.75 E-10$ & \\
\hline Cs-137 & $2.24 E+01$ & $2.17 E+01$ & 1.31E-01 & & $4.10 \mathrm{E}-05$ & $2.45 \mathrm{E}+01$ & 1.45E-05 & 1.72E-05 & \\
\hline Eu-152 & & & & & $4.24 \mathrm{E}-06$ & $2.53 E+00$ & $1.49 \mathrm{E}-06$ & $1.49 \mathrm{E}-06$ & \\
\hline Eu-154 & & & & & $2.35 E-04$ & $1.40 \mathrm{E}+02$ & $8.27 E-05$ & 8.27E-05 & \\
\hline Eu-155 & & & & & 4.12E-05 & $2.46 \mathrm{E}+01$ & 1.45E-05 & $1.45 \mathrm{E}-05$ & \\
\hline Pm-147 & & & & & 1.01E-04 & $6.03 E+01$ & $3.56 \mathrm{E}-05$ & $3.56 E-05$ & \\
\hline Pr-144 & & & & & 8.42E-05 & $5.03 E+01$ & 2.97E-05 & 2.97E-05 & \\
\hline $\mathrm{Ni}-63$ & & & & & $1.02 E-04$ & $6.09 E+01$ & $3.60 \mathrm{E}-05$ & $3.60 E-05$ & \\
\hline Ru-106 & & $7.75 \mathrm{E}-10$ & $2.45 \mathrm{E}-08$ & & 1.03E-08 & $6.17 E-03$ & $3.64 \mathrm{E}-09$ & $3.64 E-09$ & \\
\hline Sb-125 & & 1.76E-05 & $5.58 \mathrm{E}-04$ & & $1.29 \mathrm{E}-05$ & $7.71 E+00$ & 4.55E-06 & $4.56 \mathrm{E}-06$ & \\
\hline Sm-151 & & & & & $7.03 E-04$ & $4.20 E+02$ & $2.48 \mathrm{E}-04$ & $2.48 E-04$ & \\
\hline Sr-90 & & $5.56 \mathrm{E}-04$ & 1.76E-02 & & $3.90 \mathrm{E}-02$ & $2.33 E+04$ & $1.38 \mathrm{E}-02$ & $1.38 \mathrm{E}-02$ & \\
\hline Tc-99 & & & & & 2.18E-05 & $1.30 E+01$ & $7.68 \mathrm{E}-06$ & 7.68E-06 & \\
\hline$Y-90$ & & & & & $3.90 \mathrm{E}-02$ & $2.33 E+04$ & $1.38 \mathrm{E}-02$ & $1.38 \mathrm{E}-02$ & \\
\hline $\mathrm{H}-3$ & & & & & 2.11E-05 & $1.26 \mathrm{E}+01$ & $7.43 \mathrm{E}-06$ & 7.43E-06 & \\
\hline $1-129$ & & & & & $5.80 \mathrm{E}-07$ & $3.46 \mathrm{E}-01$ & $2.04 \mathrm{E}-07$ & $2.04 E-07$ & \\
\hline & & & & & & & & & \\
\hline
\end{tabular}




\section{Process Development Recommendations}

As detailed in the Section 1, the CsIX/TRU grout process design is based on many assumptions, including assumptions regarding feed waste composition, assumptions about equipment performance, and assumptions about process requirements. This sections identifies tests that are recommended to better define process requirements and waste feed compositions, and to confirm the performance of process equipment. Development activities are recommended in three areas - final waste volume minimization, process equipment scale-up and demonstration, and feed and product characterization. Following a discussion of recommended development activities, Table $8-1$ presents relative priorities for these activities.

\subsection{Final Waste Volume}

Minimization of the final waste volume is critical to establishing the feasibility of the process. The total project cost will be highly dependent on this volume, as it will determine interim waste storage requirements and waste transportation costs. Also, minimization of the waste volume is important because of the limited space available at WIPP. To minimize the final waste volume, studies should be performed in two areas - grout and absorbant tests, and evaporation tests

\subsubsection{Final Waste Form Testing}

Testing of commercial absorbants is recommended to determine waste loadings using small batches of simulated waste. Testing is also recommended of alternative grout formulations with the potential for high waste loadings. The tests should include determination of the limiting parameters for the grout, including minimum water, maximum solids, maximum nitrate, and the sensitivity of the grout/absorbant formulation to waste composition. Limiting concentrations of waste constituents, e.g. acid for an absorbant or nitrate for grout, also need to be determined. The constraints for these tests is the WIPP Waste Acceptance Criteria, which include a limit for free liquids but do not include a waste strength requirement.

\subsubsection{Evaporation Testing}

In conjunction with these tests, waste evaporation tests are recommended to determine the maximum degree of evaporation possible based on the constraints of evaporator equipment and solidification requirements, e.g., water needed in the grout formulation. Testing of specific evaporation equipment, such as thin-film or wiped-film evaporators is recommended. Because of the tendency of the waste to form sticky-solids and solidify at relatively low temperatures, data on fouling, 
precipitation, and corrosion in an evaporator is needed to demonstrate its feasibility and performance

\subsection{Feed and Product Characterization}

Recommended characterization activities include characterization of feed waste undissolved solids (UDS) and determination of radionuclide and hazardous chemical species absorbed onto the ion exchange column sorbant. Behavior of the spent sorbant during sluicing, filtration and drying is also needed.

\subsubsection{UDS Characterization}

Characterization of UDS in the tank farm liquids is needed to provide a basis for the design of UDS solids removal, handling and packaging equipment, and to establish the required solids removal efficiency. Characterization should include the total concentration and total particle size distribution for each tank, and the concentration of both hazardous and radionuclide species as a function of particle size, and both before and after washing. The radionuclide concentrations are of particular importance to determine the effect of UDS that are not removed on the dose rate for the solidified TRU waste. The concentration of fission products, notably ${ }^{137} \mathrm{Cs}$, in the UDS must be known to ensure that final TRU waste is contact-handled.

\subsubsection{Spent Cs Sorbant Characterization}

To determine disposal options for the spent cesium ion exchange sorbant, characterization data is needed. Initial small-scale column tests could determine the extent, if any, to which hazardous metal and TRU radionuclide species are absorbed on the column, and whether these species are desorbed during sorbant removal. Tests are also needed to determine physical and drying properties of the spent sorbant, in order to adequately design for removal of the sorbant from the column, filtration of sluiced sorbant, and drying of the sorbant. Data needed includes the amount of fines generated during sluicing, the particle size distribution upstream of filtration and after drying, and thermal properties of the sorbant.

\subsubsection{Dried UDS Characterization}

Tests are needed to determine washing requirements for the separated UDS, and also the handling and processing requirements to dry the solids to a flowable, pneumatically-transferable material. 


\subsection{Process Equipment Demonstration Tests}

Testing is recommended to better establish the performance and process requirements of filtration equipment, the ion exchange sorbant, and the grouting process.

\subsubsection{Filtration Requirements and Demonstration Tests}

Testing is needed to determine the degree of filtration required to avoid contaminating the TRU waste with high-dose contributing radionuclides, contaminating the cesium sorbant with TRU-containing solids, and plugging the ion exchange column. The tests should include a determination of what fraction and UDS particle size range pass through the ion exchange column and what fraction and particle size range are retained by the sorbant. Testing of filtration equipment is then recommended to demonstrate that the required removal efficiency of UDS can be achieved.

\subsubsection{Ion Exchange Tests}

The present data for the CST sorbant cesium loading is from $1 \mathrm{~cm}^{3}$ column tests. Data from larger-scale tests in needed to confirm the performance at larger scale and to provide a base for scale-up to full scale. Performance data for the sorbant is needed over the range of compositions present in the tank farm, particularly the range of cesium concentration, acid concentration, and other species which have a potential for chemical interaction with the sorbant. Long term performance data is needed to discover kinetically slow chemical interactions of resin with the feed waste.

\subsubsection{Grouting Process Tests}

Should grouting prove to be the optimum solidification technology, tests are needed to establish the grouting process kinetics and thermodynamics. These would include tests to determine the grout viscosity versus time, the heat of grout formation, and the effect and requirement for plasticizers and cure enhancer or retarder additives.

\subsection{Development Recommendation Priorities}

Table 8-1 presents relative priorities for the recommended development activities. 
Table 8-1. Relative Priorities for CsIX/TRU Grout Process Development.

\begin{tabular}{|l|c|}
\hline Activity & Priority \\
\hline Final Waste Form (8.1.1) & 1 \\
\hline Evaporation (8.1.2) & 1 \\
\hline UDS Characterization (8.2.1) & 1 \\
\hline Cs Sorbant Loading for Hazardous and Non-Cs Radionuclides (8.2.2) & 2 \\
\hline UDS Washing and Drying Characterization (8.2.3) & 3 \\
\hline Filtration Requirements (8.3.1a) & 1 \\
\hline Filtration Equipment Demonstration (8.3.1b) & 2 \\
\hline Larger-Scale lon Exchange Tests (8.3.2a) & 1 \\
\hline Sorbant Performance Versus Feed Composition (8.3.2b) & 2 \\
\hline Sorbant Long-Term Performance. (8.3.2c) & 3 \\
\hline Grout Kinetic and Thermodynamic Tests (8.3.3) & 3 \\
\hline
\end{tabular}

\section{References}

1. S. Carson, Y.-C. Cheng, L. Yellowhorse, P. Peterson, Stabilization of Liquid Low-Level and Mixed Waste: A Treatability Study, SAND-96-0297C, 1996.

2. A. K. Herbst, D. W. Marshall, J. A. McCray, Idaho Chemical Processing Plant Low-Activity Waste Grout Stabilization Development Program FY-97 Status Report, INEEL/EXT-98-00116, February, 1998.

3. C. W. Olsen, Minutes From 5/20/98 Meeting with Terry Todd, BC-CSIX-07

4. A. K. Herbst, Idaho Chemical Processing Plant Low-Activity Waste Grout Stabilization Development Program FY-96 Status Report, AKH-07-96, September $18,1996$.

5. T. A. Todd, K. N. Brewer, D. J. Wood, P. A. Tullock, L. G. Olson, A. L. Olson, "Evaluation and Testing of Inorganic lon Exchange Sorbents for the Removal of Cesium-137 from Idaho Chemical Processing Plant Acidic Tank Waste," Submitted to Separation Science \& Technology, December, 1997.

6. M. K. Andrews, Glass Formulation Development in Testing for the Vitrification of Cesium-Loaded Crystalline Silicotitanate (CST), WSRC-MS-97-0041, June, 1997.

7. J.F. Walker, et al., "Cesium Removal Demonstration Utilizing Crystalline Silicotitanate Sorbent for Processing Melton Valley Storage Tank Supernate: Final Report., ORNL/TM-13503, March, 1998. 

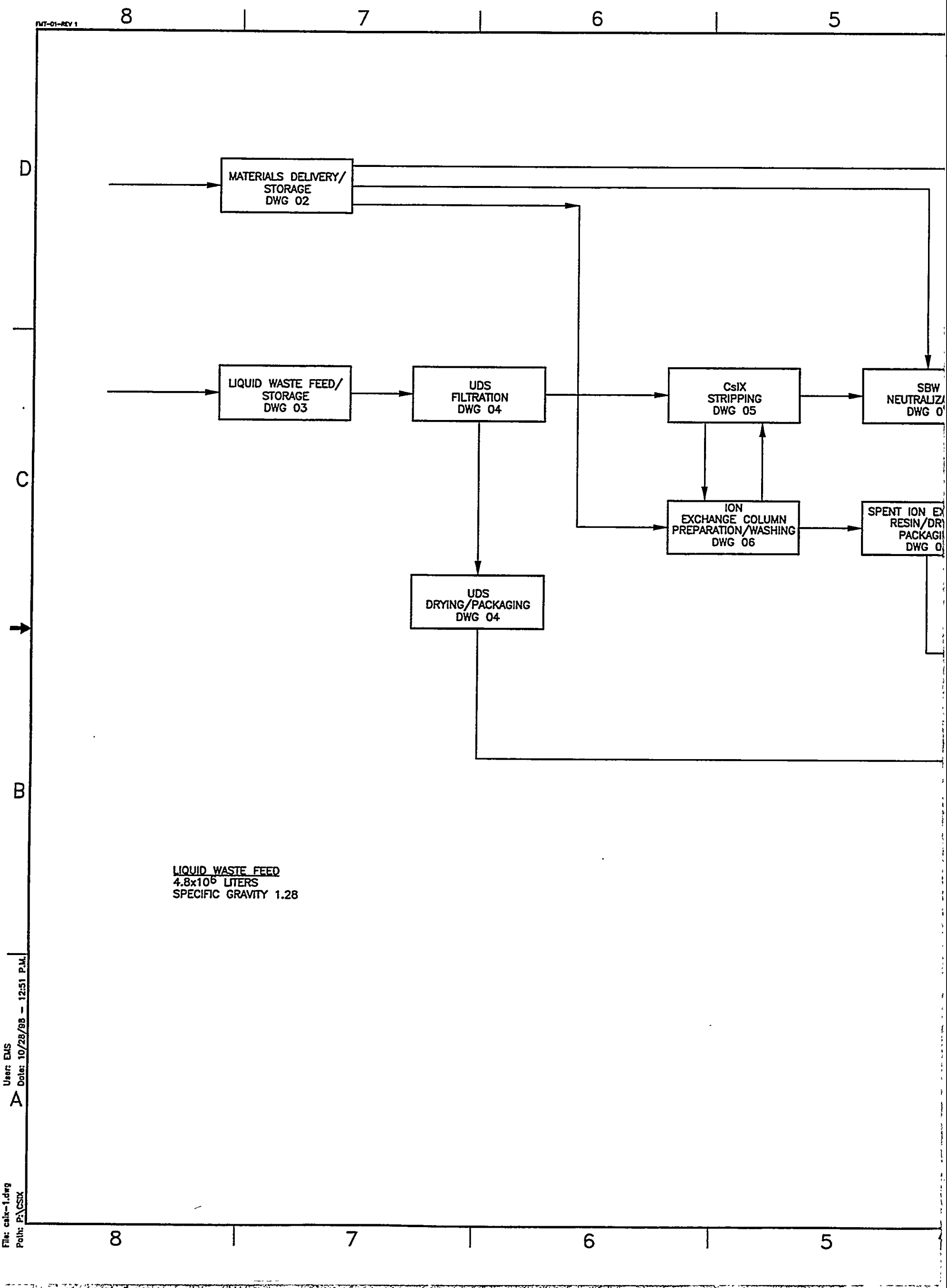


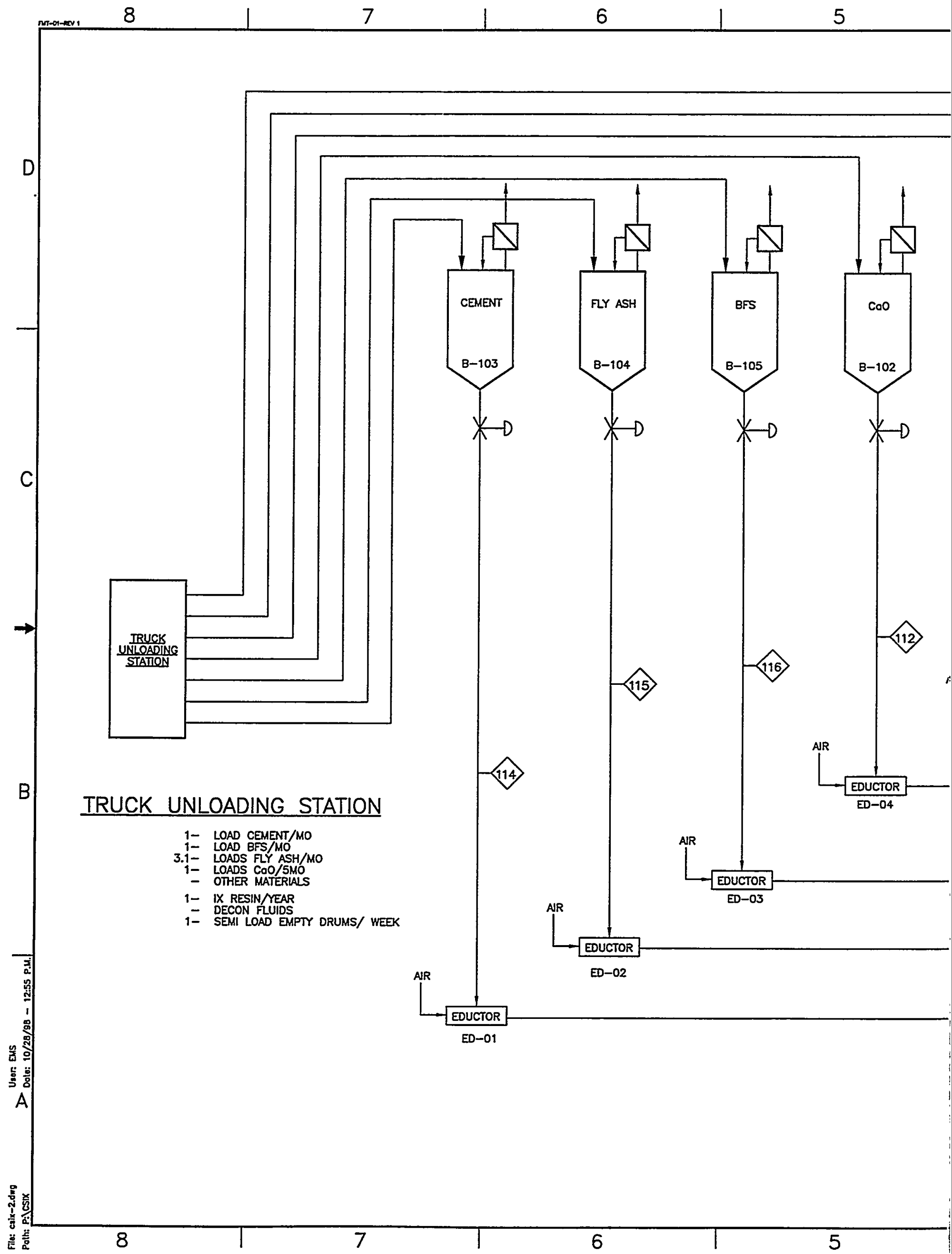




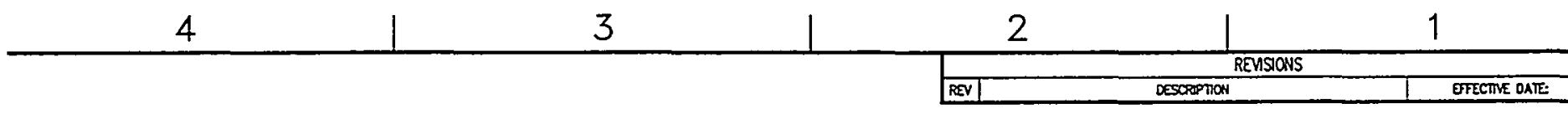
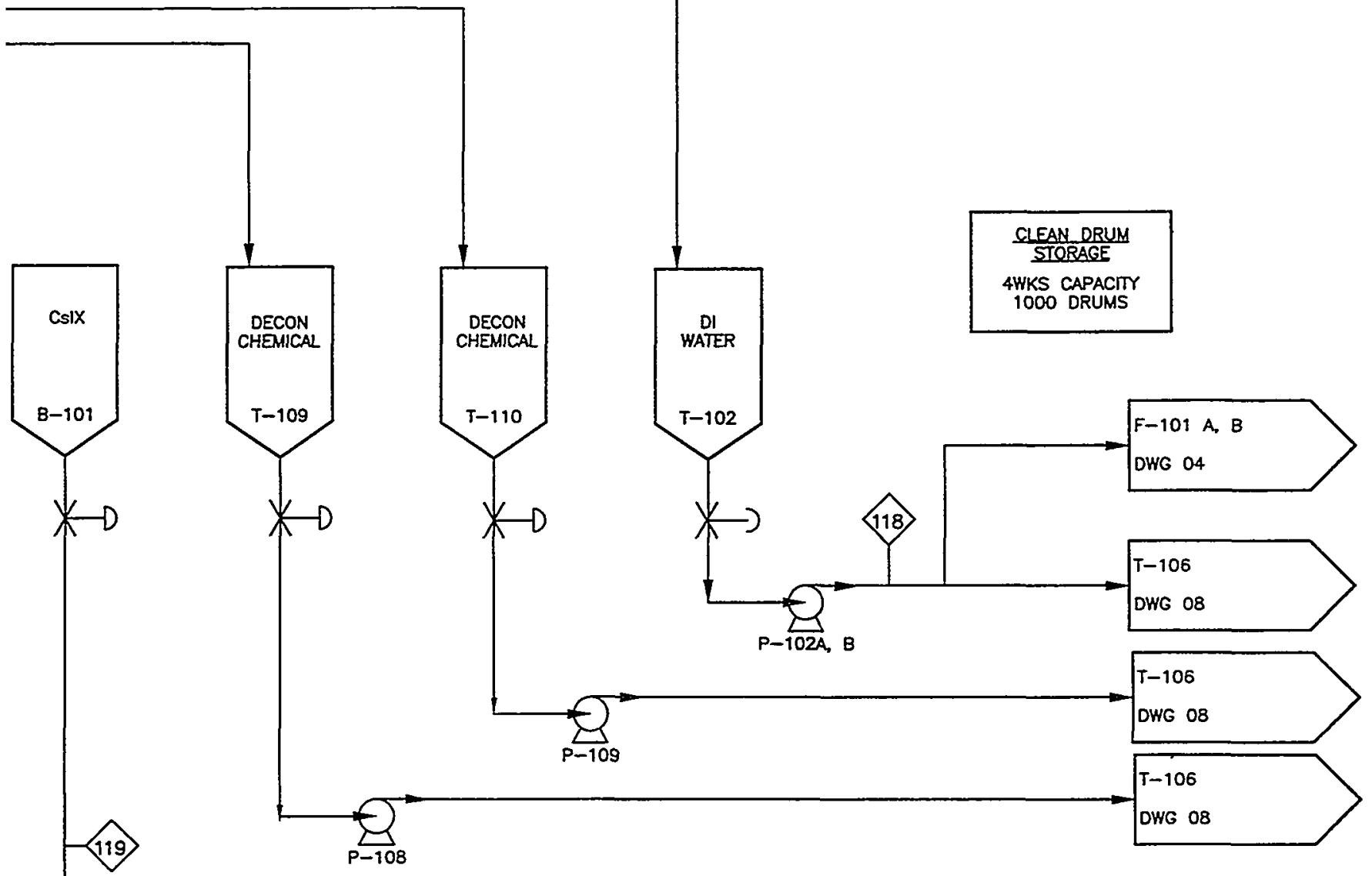


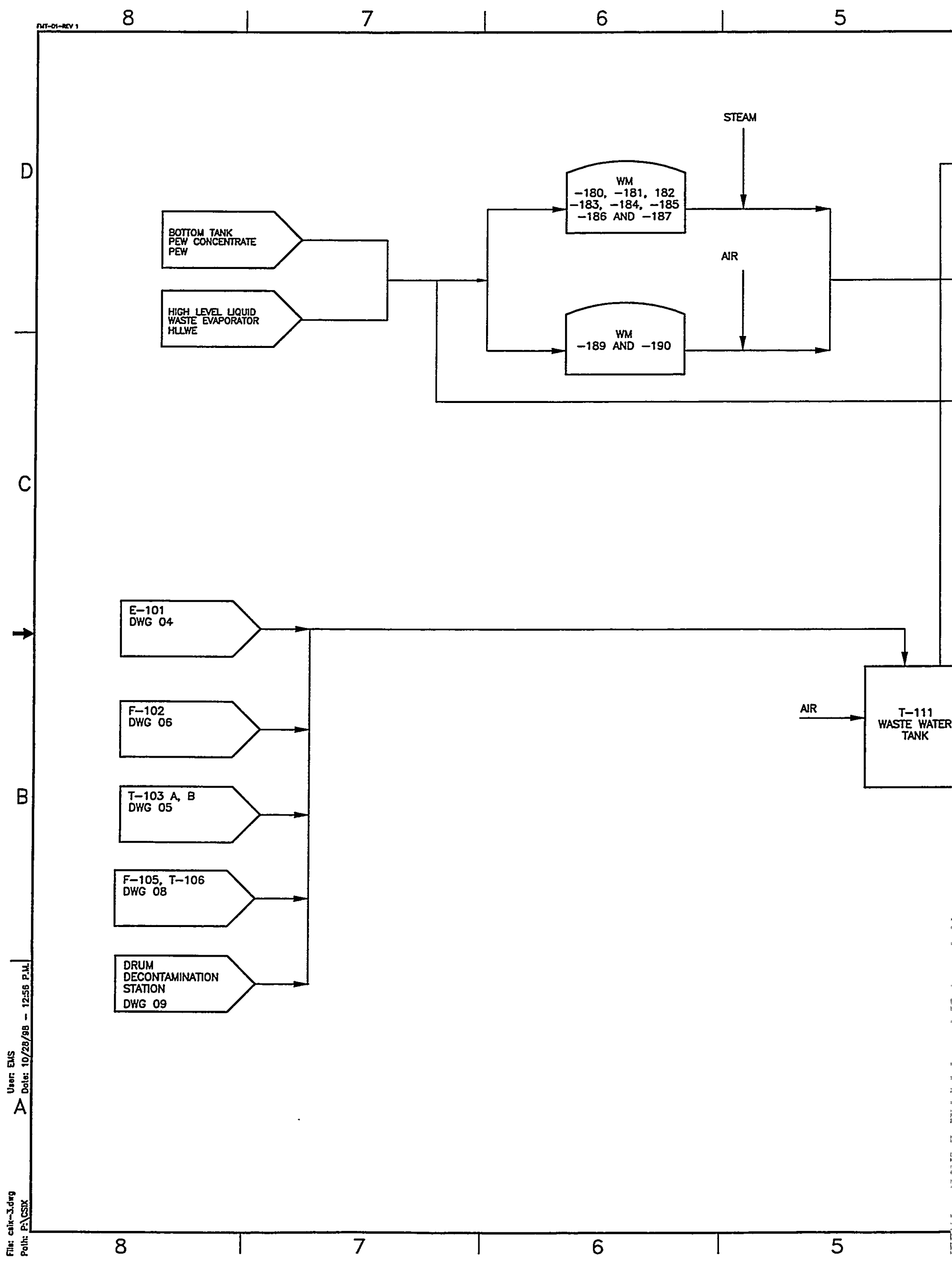




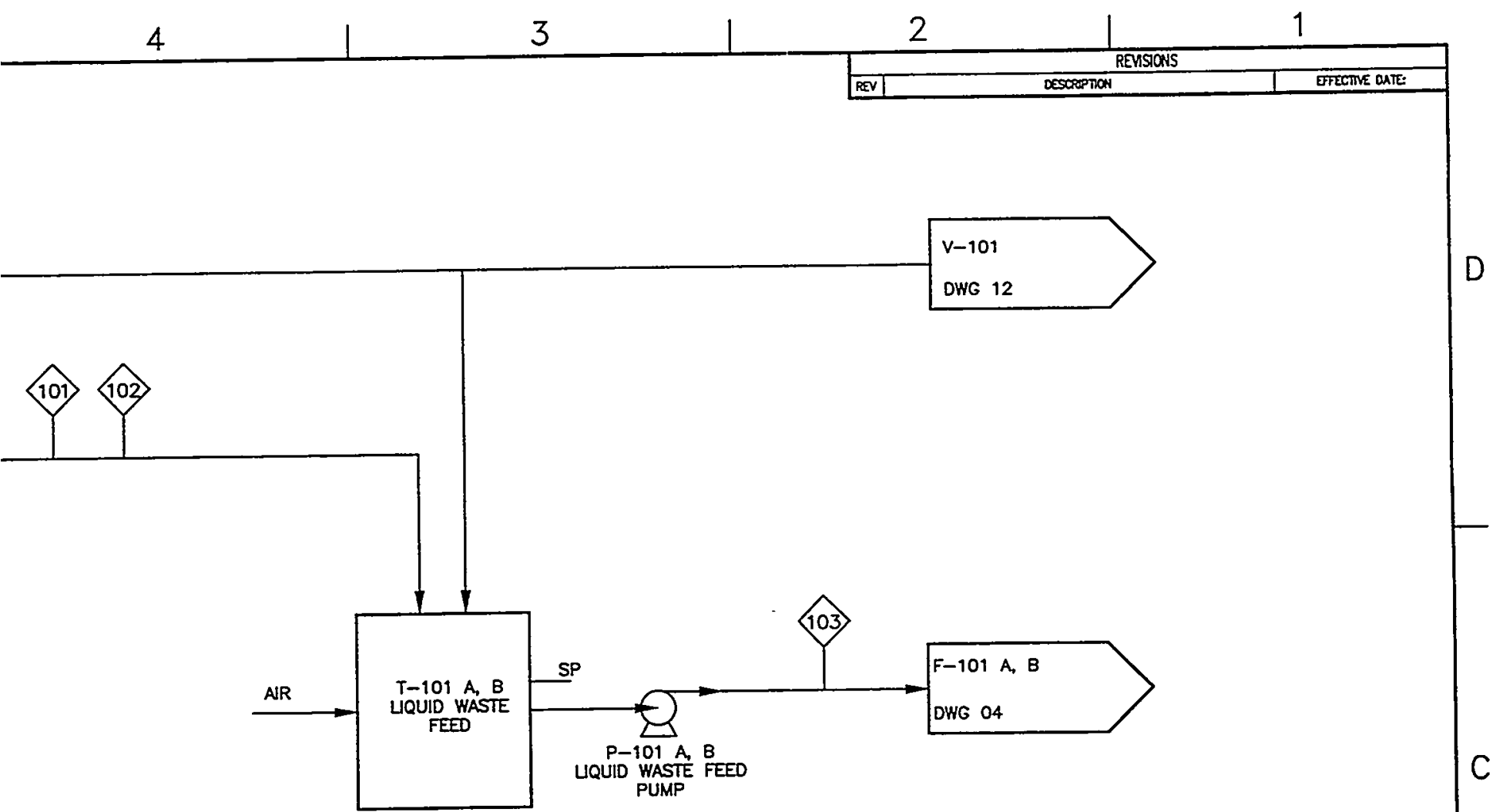

WASTE WATER PUMP

\begin{tabular}{|c|c|c|}
\hline SOOONTRLCT HO. & LOCKHEED NATTIN/ & \\
\hline REalsstre & \multirow{4}{*}{$\begin{array}{l}\text { CsIX/TRU GROUT FEASIBIUTY } \\
\text { STUDY } \\
\text { UQUID WASTE FEED/STORAGE }\end{array}$} & \\
\hline Desat & & \\
\hline Denut & & \\
\hline Prafet wa & & \\
\hline Prescocs & & \\
\hline 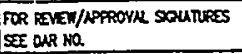 & 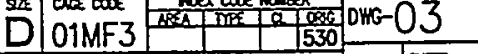 & 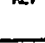 \\
\hline 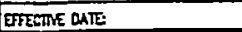 & SCNE NONE & \\
\hline
\end{tabular}




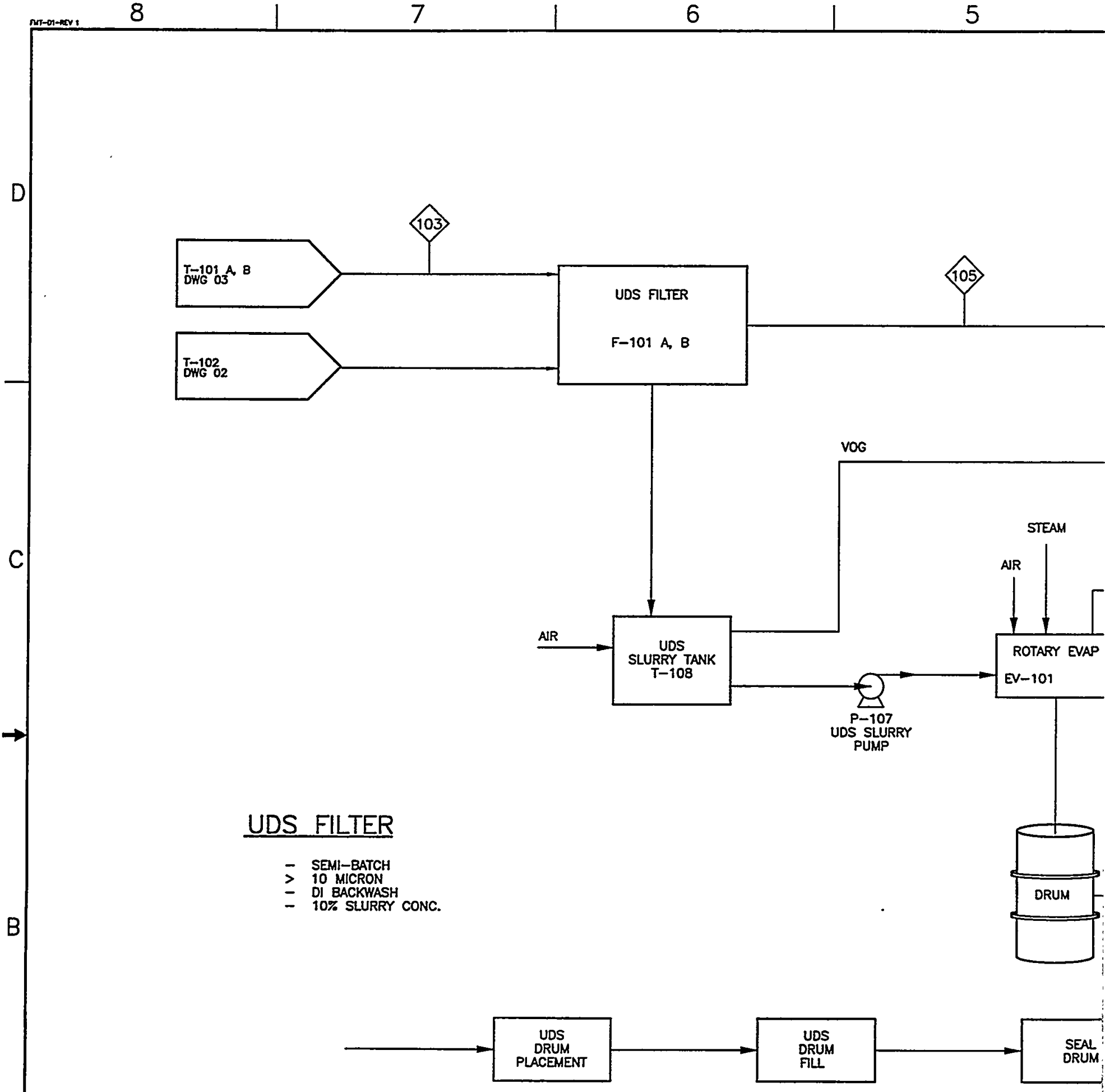

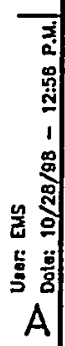

MECHANICAL DRUM TRANSFER STATION SEQUE 


\begin{tabular}{l|l|l|l|l|l|}
4 & 3 & 2 & \multicolumn{2}{|c|}{2} \\
\hline
\end{tabular}

$V-104 A, B, C$

DWG 05

E-103

DWG 12

SP

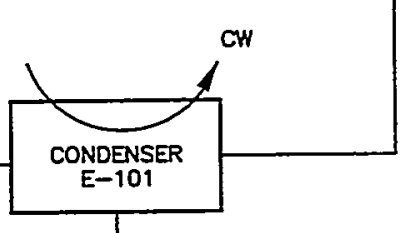

C

UDS STORAGE
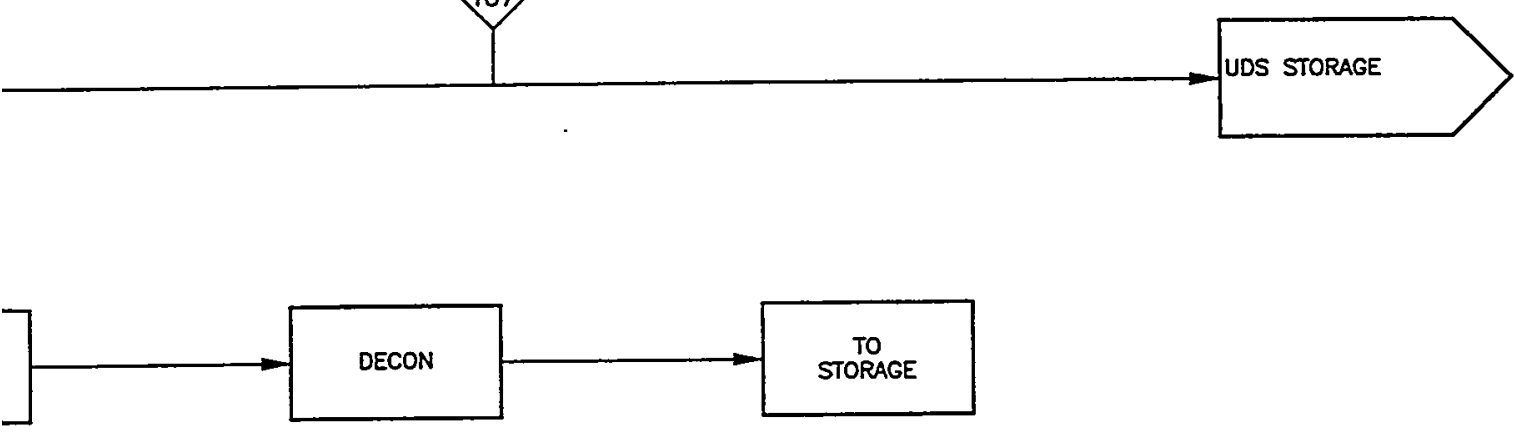

\begin{tabular}{|c|c|c|}
\hline SUOCONTRCT Ha & LOCKHEED MARTINA & \\
\hline Feacsters & \multirow{4}{*}{$\begin{array}{l}\text { CSIX/TRU GROUT FEASIBIUTY } \\
\text { STUDY } \\
\text { UDS FILTRATION DRMNG/PACKAGING }\end{array}$} & \\
\hline Desat & & \\
\hline Drant & & \\
\hline PRazer Ha & & \\
\hline \begin{tabular}{|l|l|} 
Pre cose & \\
\end{tabular} & & (BV \\
\hline 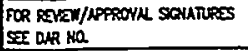 & 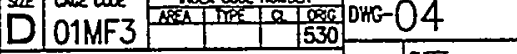 & 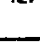 \\
\hline WHECTIS URT: & \begin{tabular}{|l|l|l|l|l|l|l|} 
SCNE NONE & SET \\
\end{tabular} & \\
\hline
\end{tabular}




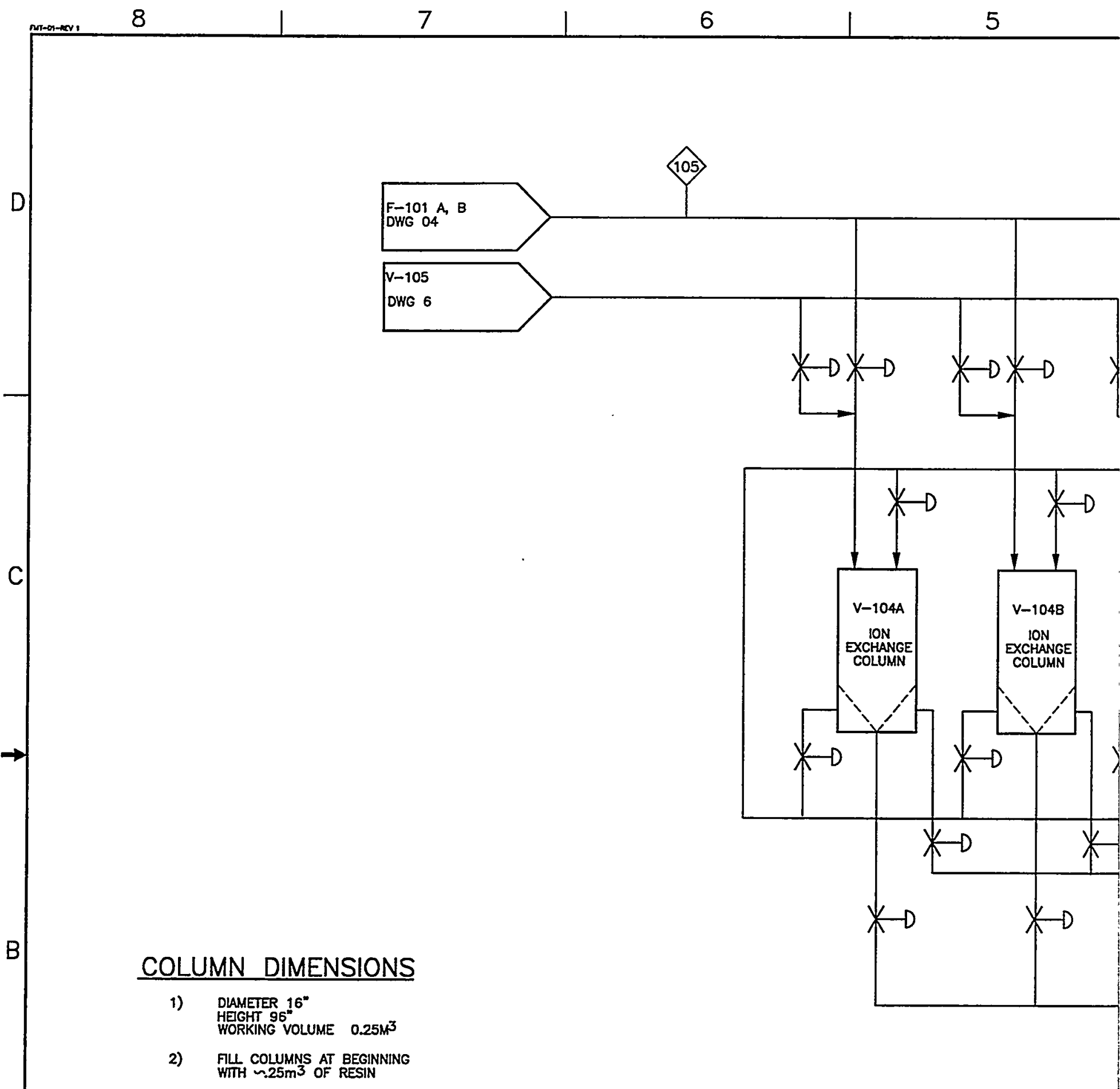




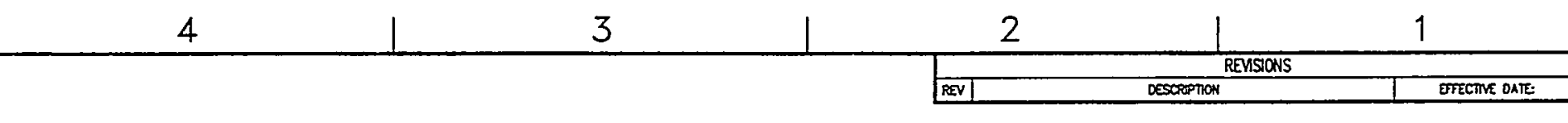

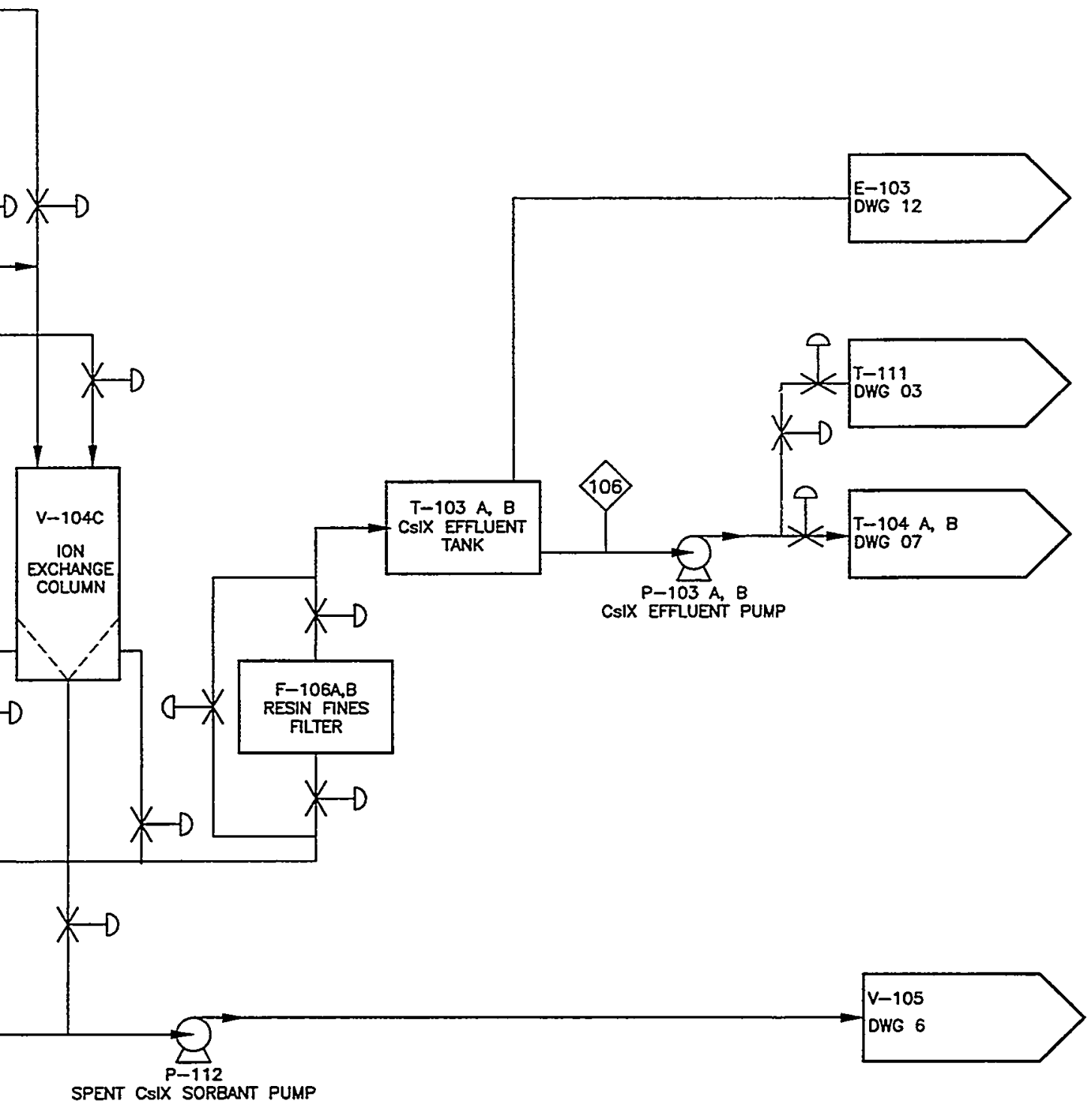

\begin{tabular}{|c|c|}
\hline SECONRUCT MO. & LOEKHEED WATTIN \\
\hline REOUSTIJE & \multirow{4}{*}{$\begin{array}{l}\text { CSIX/TRU GROUT FEASIBILTY STUDY } \\
\text { CSIX STRIPPING }\end{array}$} \\
\hline Dessat & \\
\hline prant EA SREL & \\
\hline \begin{tabular}{|l|l|} 
PRaser Ma & \\
\end{tabular} & \\
\hline \begin{tabular}{|l|l}
$\operatorname{spec} \cos$ & \\
\end{tabular} & \\
\hline 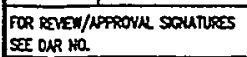 & 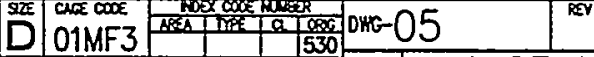 \\
\hline DFWTV OUTE & sare 1 OF \\
\hline
\end{tabular}




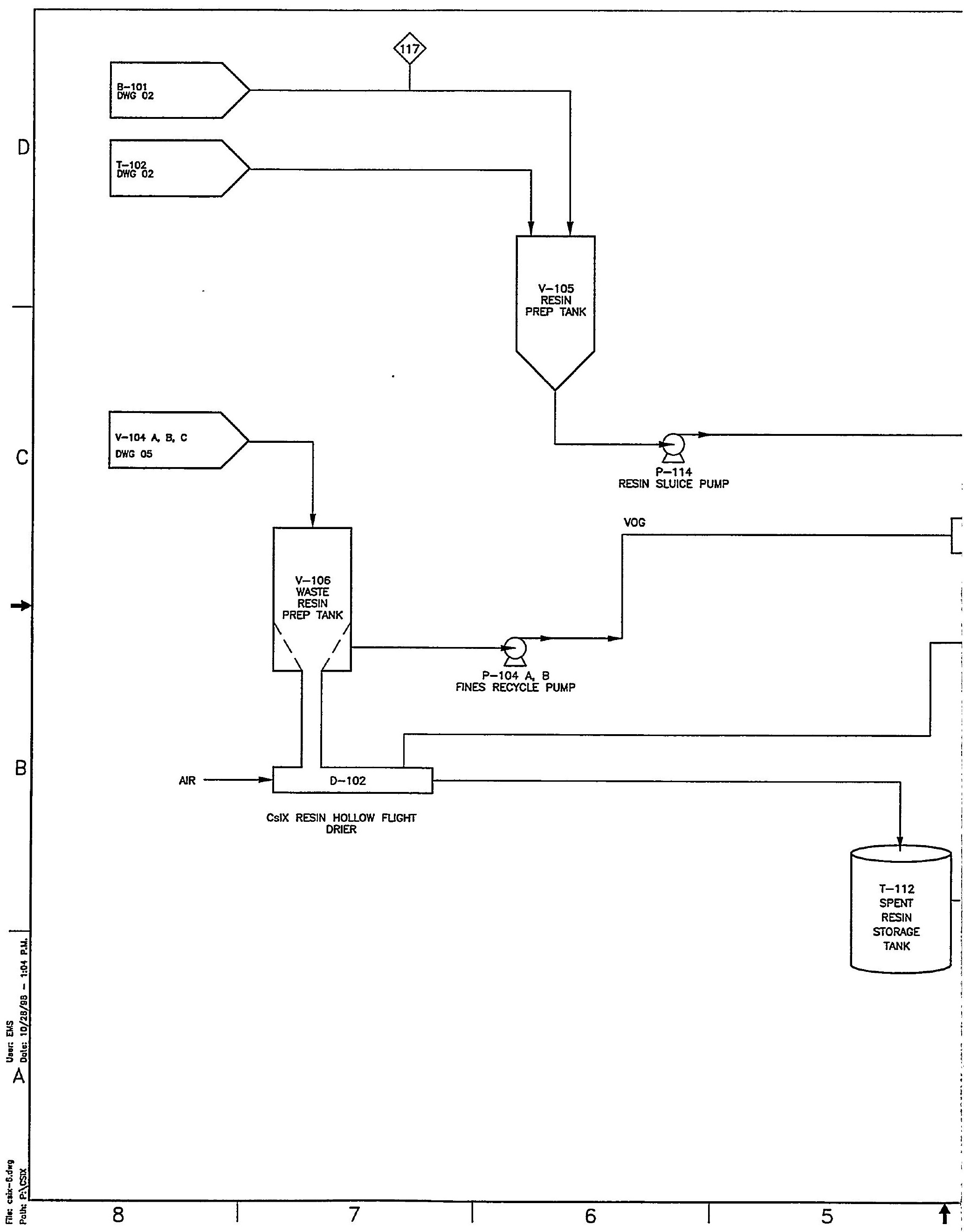


V-104 A, B, C DWG 05

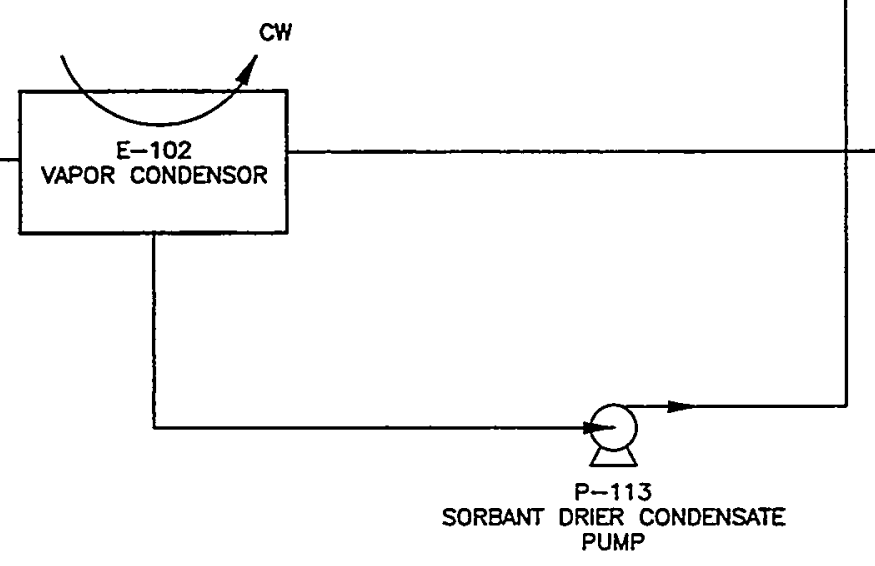

\section{$E-103$}

DWG 12

\begin{tabular}{|c|c|c|c|}
\hline SUPCOAIRUCT HO. & \multicolumn{3}{|c|}{ LOCKHEED MAATINA } \\
\hline FEOESTER & \multirow{5}{*}{\multicolumn{3}{|c|}{$\begin{array}{c}\text { CSIX/TRU GROUT FEASIBIUTY } \\
\text { STUDY } \\
\text { IX COLUMN PREPARATION/WASHING SPENT } \\
\text { RESIN DRYING/PACKAGING }\end{array}$}} \\
\hline cessat & & & \\
\hline DaAme & & & \\
\hline praect tho. & & & \\
\hline $\sec \infty \cos$ & & & \\
\hline 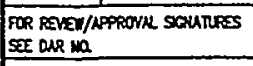 & 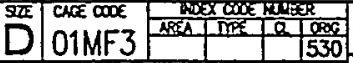 & OHG-06 & REV \\
\hline DFECMVE DATE: & ScNE NONE & Sext & \\
\hline
\end{tabular}




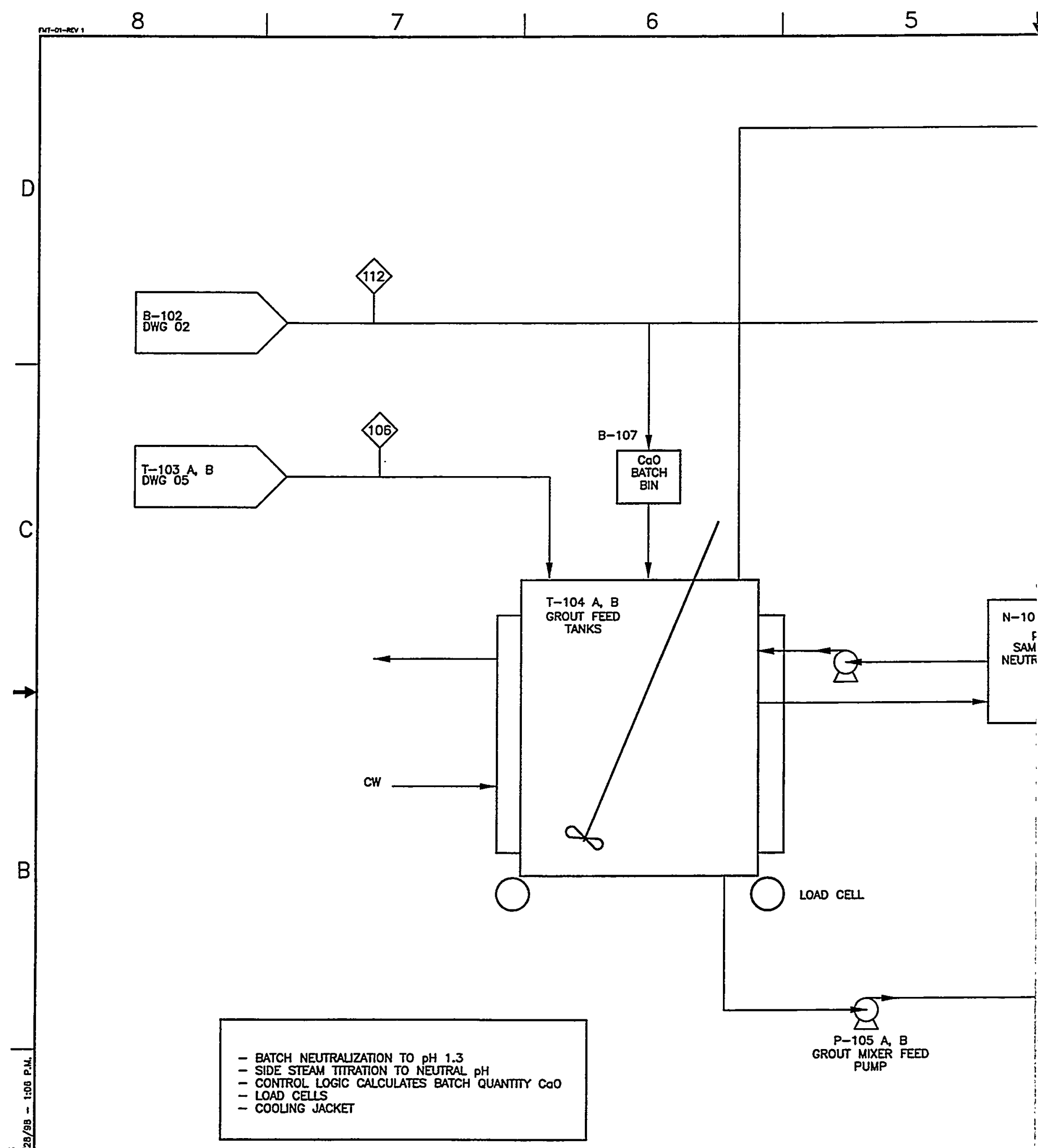

岩워

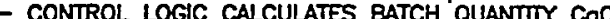

- LOAD cels

COOUNG JACKET 


$4 \quad 1$ 3

\begin{tabular}{|c|c|c|}
\hline SUECONRACT RO. & LOCKHEED WATTIN & \\
\hline KaUESTIP: & \multirow{4}{*}{$\begin{array}{l}\text { CSIX/TRU GROUT FEASIBILTY STUDY } \\
\text { SEW NEUTRALZATION }\end{array}$} & \\
\hline Dessat & & \\
\hline DRAM: AE QSOSOS & & \\
\hline \begin{tabular}{|l|} 
PROET KO \\
\end{tabular} & & \\
\hline Fosc 200 & & \\
\hline 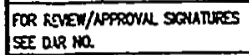 & 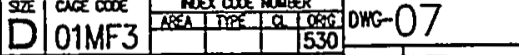 & kav \\
\hline ERTECTE QUTE & SCUE: NONE & \\
\hline
\end{tabular}




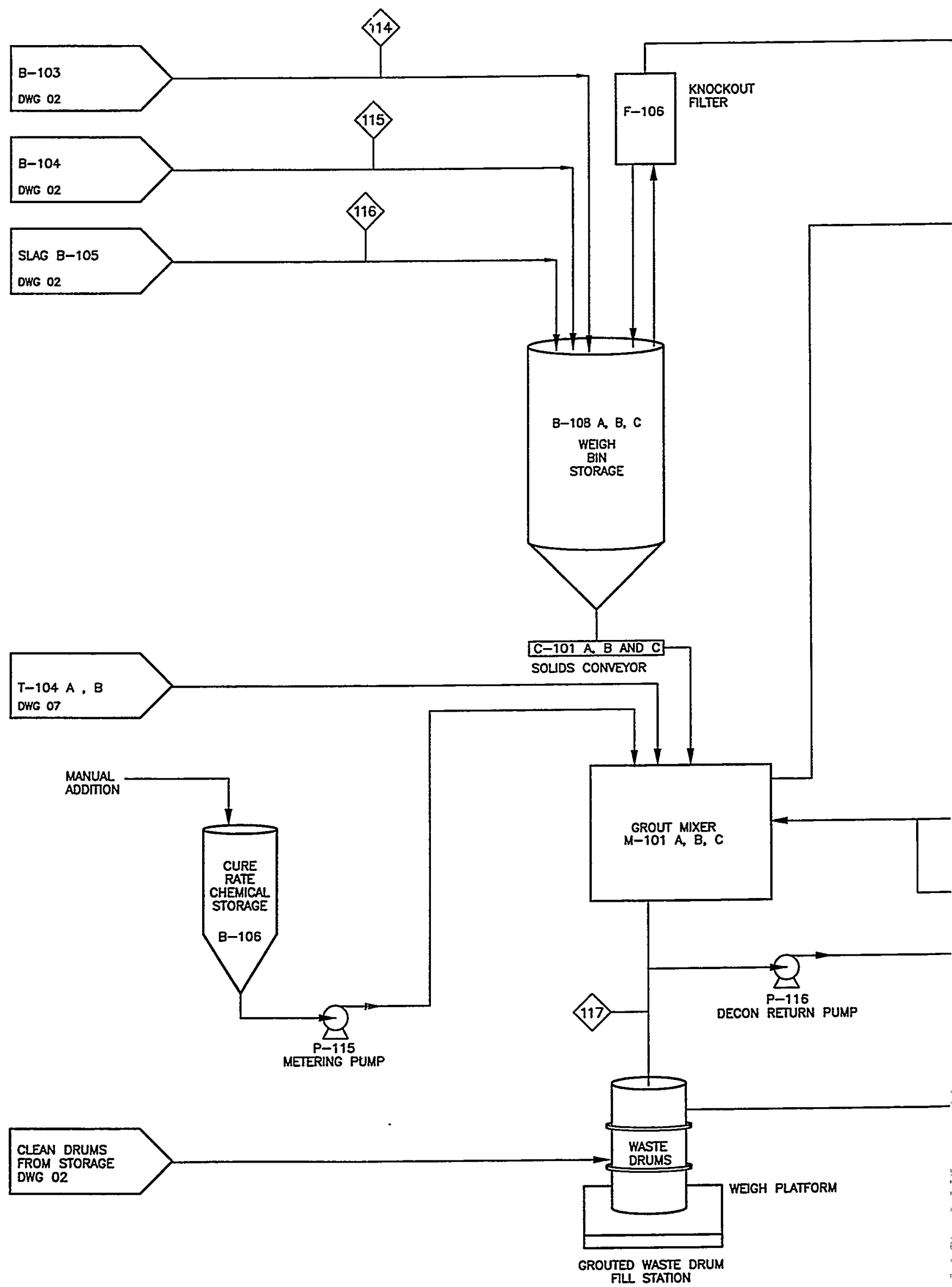

T-104 A, B

DHE 07 

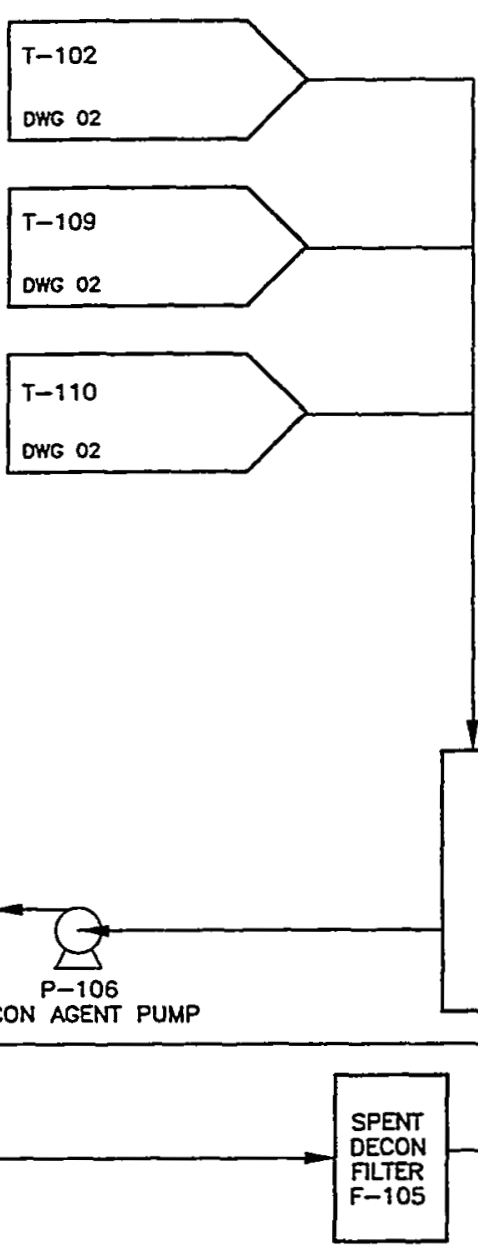

MIXER WASH

TANK

T-106 
r. D

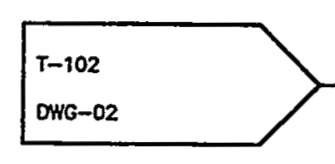

, C

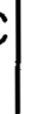

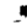

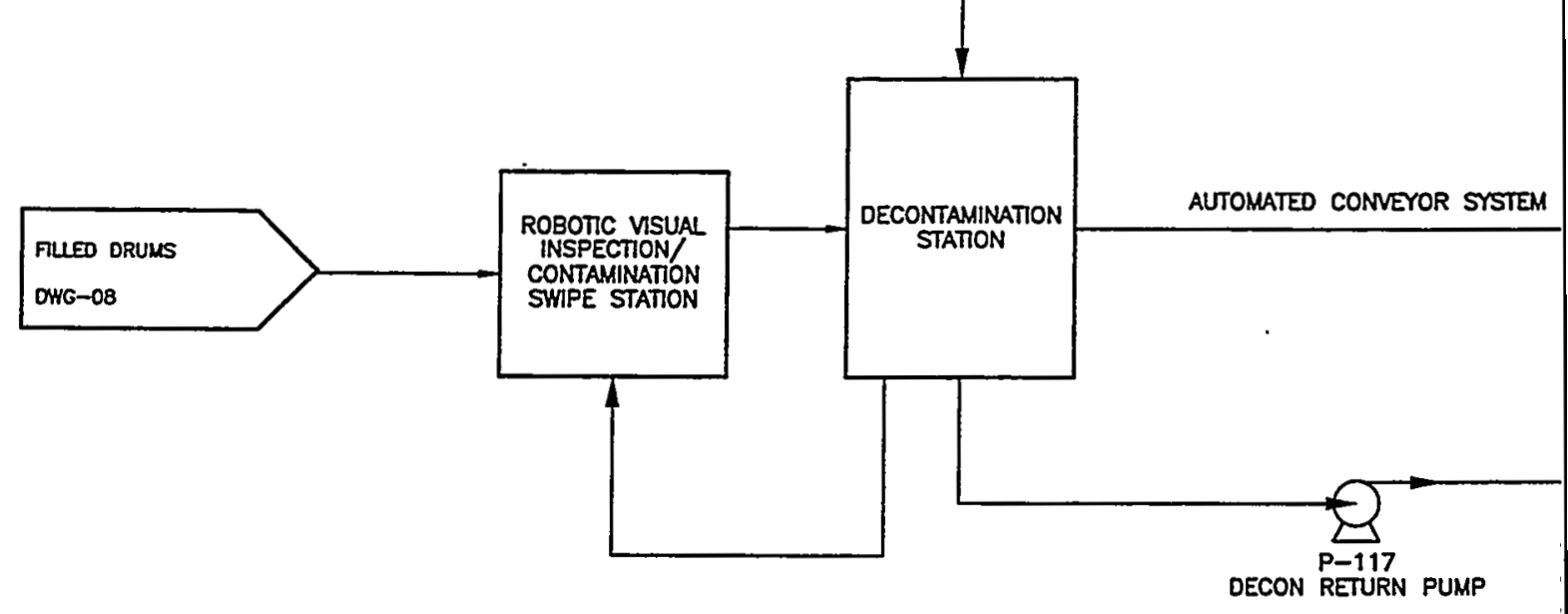

B

A

竞

A:

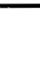


WASTE DRUMS

TO CURE

DWG 10

\begin{tabular}{|c|c|c|}
\hline sercanRet Ho. & LOCKHEED WATTIM & \\
\hline REQUESTER & \multirow{4}{*}{$\begin{array}{l}\text { CSIX/TRU GROUT FEASIBILTY STUDY } \\
\text { WASTE DRUM DECONTAMINATION }\end{array}$} & \\
\hline Dessot: & & \\
\hline \multirow{2}{*}{ DRAm: } & & \\
\hline & & \\
\hline $\sec 000$ & & \\
\hline 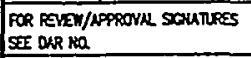 & 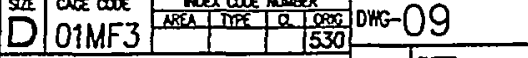 & Fey \\
\hline EFECWUE DATE: & SeNe NONE & \\
\hline
\end{tabular}




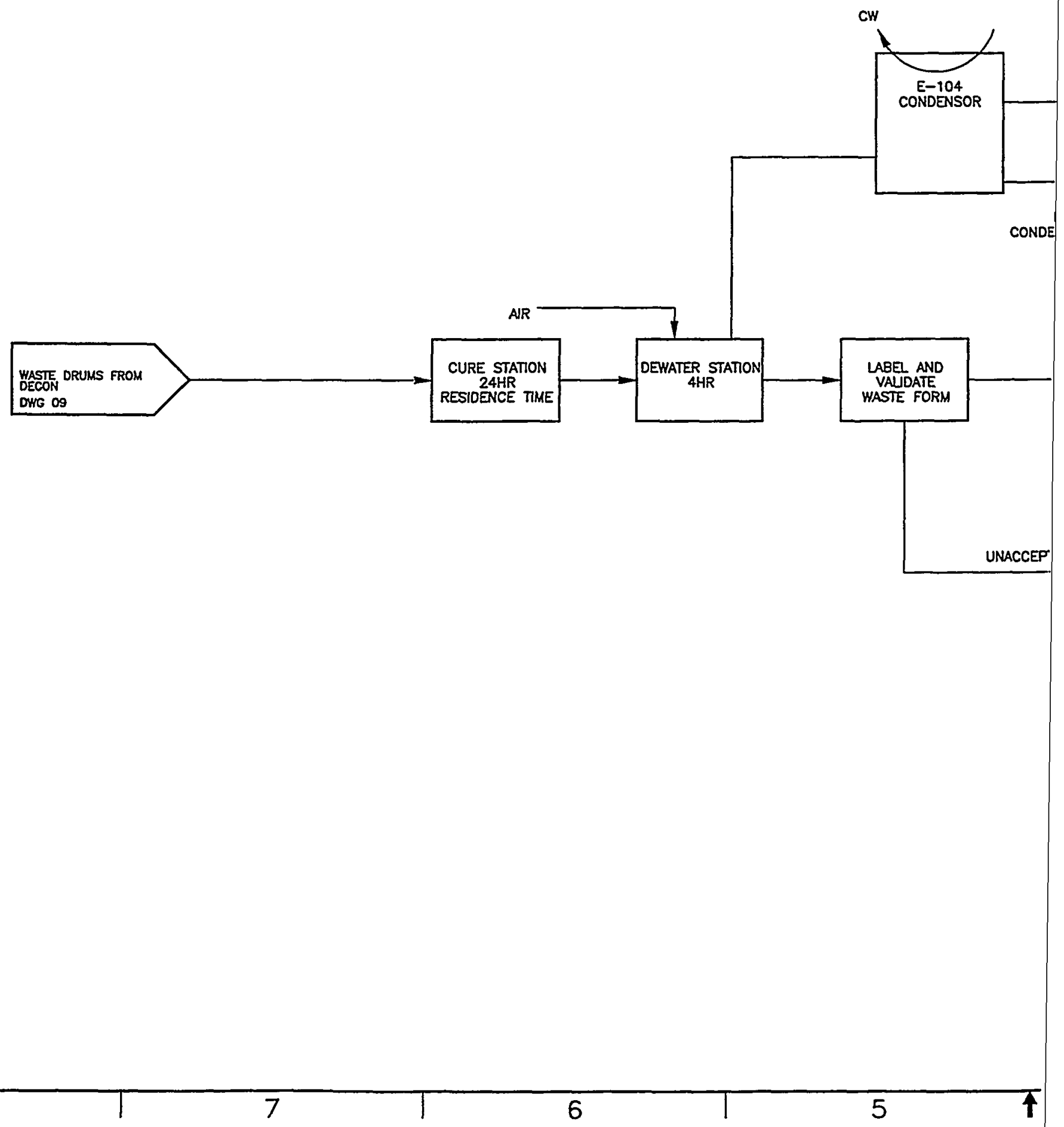




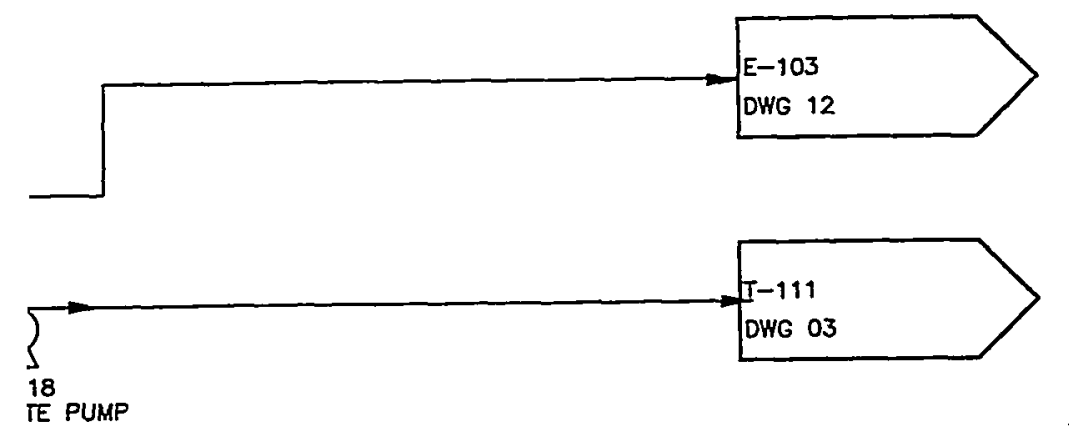

D

TRANSPORT GROUT

TO TEMPORARY

STORAGE

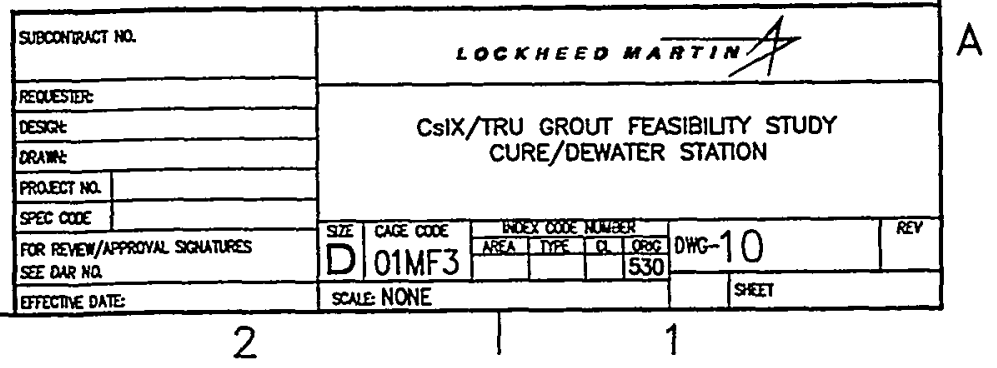


UNACCEPTABLE GROUT

DHG 10

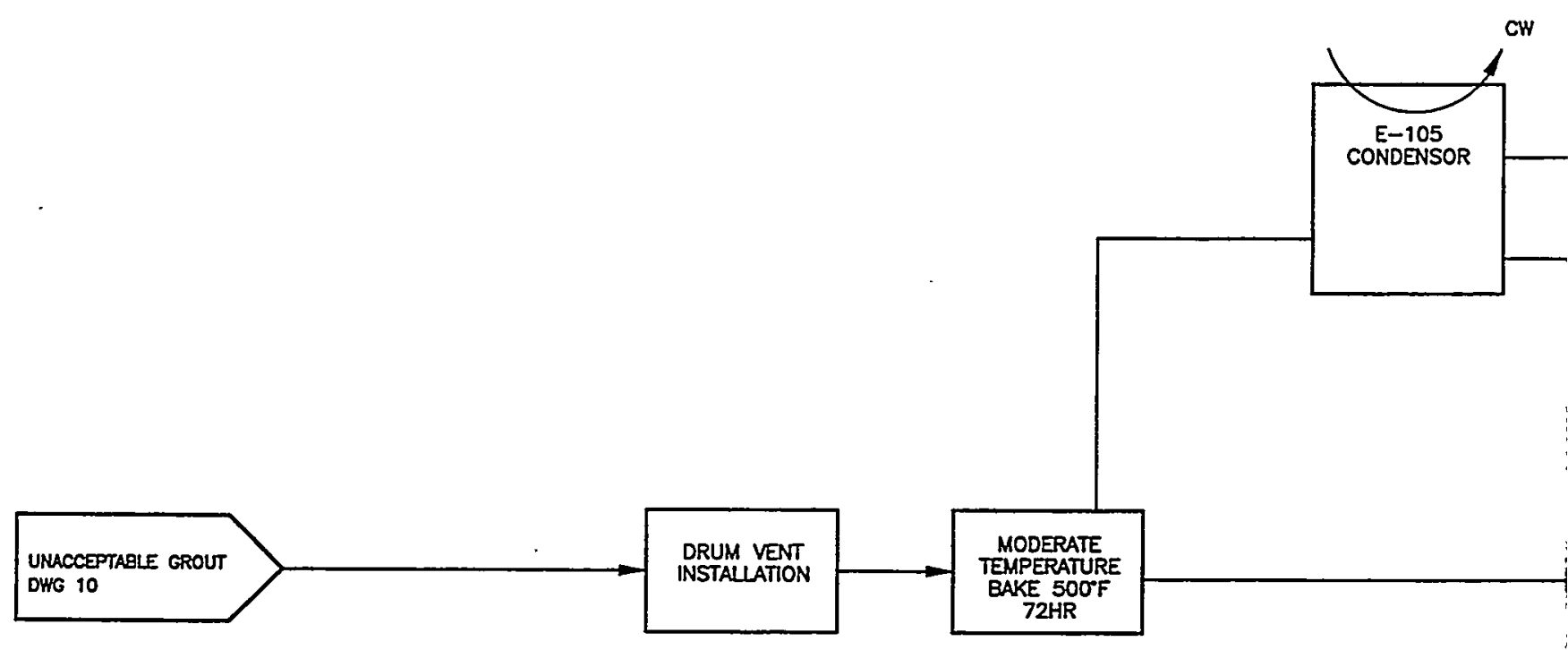




\begin{tabular}{|c|c|c|c|c|}
\hline SUBCOArRet Ho. & $\operatorname{LOCK}$ & HEED A A $\bar{A}$ & ATIN & \\
\hline FEOESTE: & \multirow{4}{*}{\multicolumn{3}{|c|}{$\begin{array}{l}\text { CSIX/TRU GROUT FEASIBILTY STUDY } \\
\text { GROUT REWORK }\end{array}$}} & \\
\hline Desset & & & & \\
\hline \multirow{2}{*}{\multicolumn{2}{|c|}{ GROUT REWORK }} & & & \\
\hline & & & & \\
\hline \multirow[b]{2}{*}{ 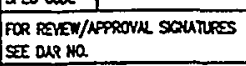 } & & & & \\
\hline & D & 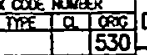 & DNG-11 & REV \\
\hline ETECTIE DARE: & SCNE: NONE & & SteEt & \\
\hline
\end{tabular}




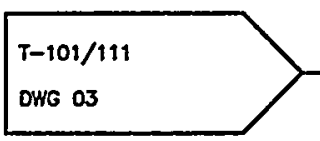

T-108, E-101

DWG 04

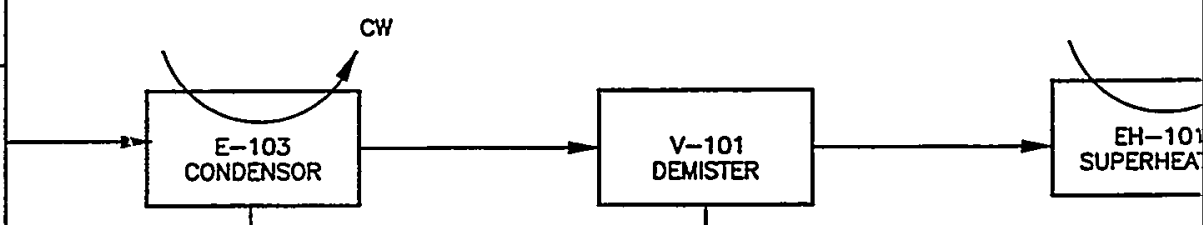

T-103 A B

DWG 05

M-101 $A$ B AND $C$

DWG 08

B

$$
\text { E-105 }
$$

DWG 11

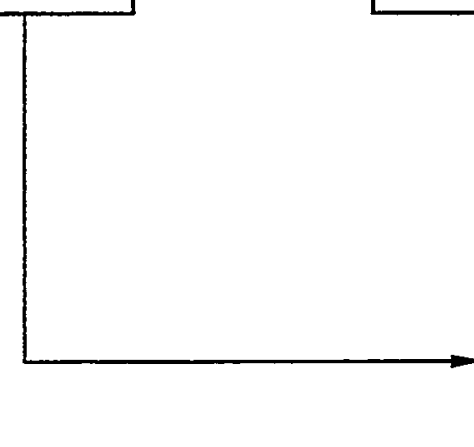




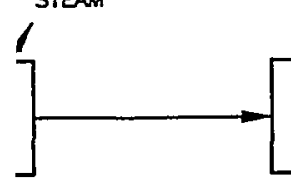

\begin{tabular}{|c|c|c|}
\hline SHecontinuet Ho. & LOCKHEED MATTINA & \\
\hline REOUSTER: & \multirow{4}{*}{$\begin{array}{l}\text { CSIX/TRU GROUT FEASIBILITY STUDY } \\
\text { OFF-GAS CONTROL SYSTEM }\end{array}$} & \\
\hline Desate & & \\
\hline Derme & & \\
\hline PRAECT Ma & & \\
\hline $\operatorname{seccccos}$ & & \\
\hline 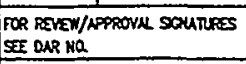 & 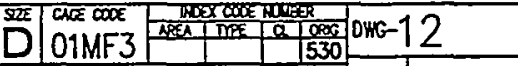 & $\overline{R E V}$ \\
\hline GFECMV DATE: & SCNE NONE & \\
\hline
\end{tabular}




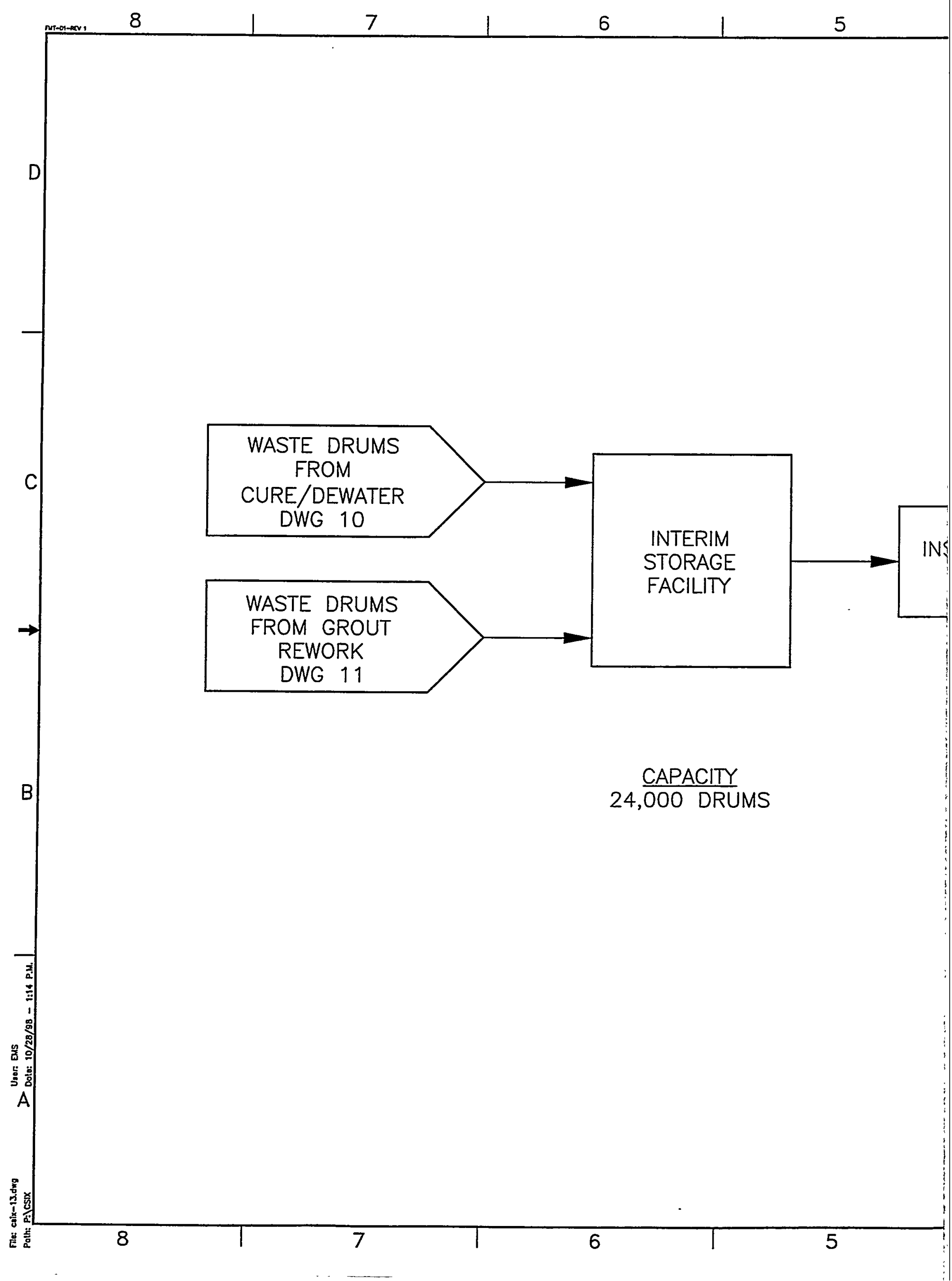




\begin{tabular}{l|ll|l|l|}
4 & 3 & 2 & 1 \\
\hline
\end{tabular}

TRUPAC

LOADING STATION
WASTE DRUMS

TO WIPP

\begin{tabular}{|c|c|}
\hline secomact ha & LOCKHEEDMATTIN/ \\
\hline REästre: & \multirow{4}{*}{$\begin{array}{l}\text { CSIX/TRU GROUT FEASIBILTT STUDY } \\
\text { INTERIM STORAGE/SHIPMENT }\end{array}$} \\
\hline Descite & \\
\hline DPArt: & \\
\hline Faret Ka & \\
\hline $\sec 00 x$ & \\
\hline 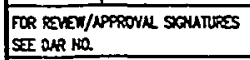 & 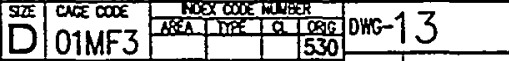 \\
\hline Brtime oure: & \begin{tabular}{|l|l|l|l|l|} 
SCUE NONE & SEXI \\
\end{tabular} \\
\hline
\end{tabular}




\section{Appendix C \\ Cost Estimates}

1. Capital Cost Estimate

2. Life Cycle Cost Estimate 
Lockheed Martin Idaho Technologies Company

INTERDEPARTMENTAL COMMUNICATION

Date: October 15,1998

$\begin{array}{llll}\text { To: } & \text { K. L. Williams } & \text { MS3765 }\end{array}$

From: $\quad$ R. D. Adams $\quad$ CDA MS3655 6-2963

Subject: $\quad$ CsIx/TRU GROUT FEASIBIITY STUDY -RDA-47-98

Cost Estimating has prepared Planning cost estimates for the subject project. These estimates were based on information received from August 20, 1998 to October 7,1998.

The Total Estimated Cost (TEC) for scenario \#1 of this project is $\$ 102,000,000$. The Total Estimated Cost (TEC) for scenario \#2 of this project is $\$ 130,300,000$. These costs include direct and indirect construction costs, G\&A, PIF, procurement fee, Engineering costs, Inspection costs, Project Management costs, Construction Management costs, escalation and contingency. The estimated costs have been escalated to the midpoint of the attached schedule. For more detailed information, please refer to the Cost Estimate Support Data Recapitulation form.

Attached for your review are the Summary Estimate sheet, the Cost Estimate Support Data Recapitulation form, detailed estimate sheets, Contingency Analysis sheet, and G\&A/PIF Calculation Sheet.

Also included is an Other Project Cost (OPC) estimate for scenario $\# 1$ of $\$ 32,200,000$ and for scenario $\# 2$ of $\$ 39,800,000$.

If you have any questions regarding these estimates, please contact me at 526-2963.

RDA

Attachments:

cc: Estimate File \#2484 \& \#2484A

R. J. Turk MS3875

R. D. Adams File

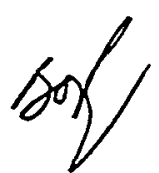


Lockheed Martin Idaho Technologies Company

\section{COST ESTIMATE SUPPORT DATA RECAPITULATION}

Project Title: CsIX TRU Grout Feasibility Study - Scenario \#1

Estimator: $\quad$ R. D. Adams

Date: $\quad$ October 15, 1998

Estimate Type: Planning

File:

Approved By:

I. SCOPE OF WORK: Brief description of the proposed project.

This project will construct a CsIx/TRU grout facility. This process includes undissolved solids (UDS) removal via filtration and cesium removal via ion exchange, followed by a cement-based grouting of the remaining liquid into 55-gallon drums. An interim storage facility and a TRUPAC loading facility will be built to temporarily house the waste drums and load them for shipment to the Waste Isolation Pilot Plant (WIPP) in New Mexico for disposal.

II. BASIS OF THE ESTIMATE: Drawings, Design Report, Engineers Notes and/or other documentation upon which the estimate is originated.

The estimate is based on the preliminary design drawings, the feasibility description, and an equipment list.

III. ASSUMPTIONS: Conditions statements accepted or supposed true without proof of demonstration. An assumption has a direct impact on total estimated cost.

1. Utilities and transfer lines are included in the estimate for the various facilities as needed.

2. All concrete surfaces in hot cells will be coated with stainless steel liner or epoxy coating as identified on the drawings.

3. Supervisor hours are figured at $5 \%$ of total labor hours.

4. Training hours are equal to $3 \%$ of total labor hours.

5. All building foundations start at $-6^{\prime}-0$ unless shown otherwise on the drawings.

6. Epoxy coating system is included on the Interim Storage Facility and TRUPAC Loading Station concrete floors.

7. All structural components of Grout facility are concrete except roof framing.

8. Grout Facility roof is standing seam-roofing panels over structural steel framing.

9. All overhead doors are motor-operated.

10. The exterior of the Grout Facility is left exposed concrete.

11. Included a concrete pad under the Grout Facility outside bin/tank area. 


\section{COST ESTIMATE SUPPORT DATA RECAPITULATION \\ - Continued -}

Project Title: CsIX TRU Grout Feasibility Study- Scenario \#1

File: $\quad 2484$

12. TRUPAC loading facility includes two 10ton-bridge cranes, work platforms, pallet scales, stretch wrap machine, and vacuum pump system.

13. TRUPAC station will require minimal H\&V (twelve $20 \mathrm{kw}$ electric unit heaters).

14. An exhaust stack with appropriate monitoring is required for the Grout Facility.

15. Interim Storage Facility includes a ventilation system not shown on the drawings.

16. Ten shielded containers are included for storage of UDS. The remaining 40 containers needed during production will be supplied by operations.

17. The initial charge of ion exchange resin is included in this estimate. The remaining resin required for production will be supplied by operations.

IV. CONTINGENCY GUIDELINE MMPLEMENTATION: The percentage used for contingency as determined by the contingency allowance guidelines can be altered to reflect the type of construction and conditions that may impact the total estimated cost.

Contingency for this scenario has been calculated to be $33.97 \%$. Most systems have not been detailed. Many changes to the assumed requirements will appear as these systems are developed further.

\section{OTHER COMMENTS/CONCERNS SPECIFIC TO THE ESTIMATE}

A Costs from the Detailed Cost Estimate sheets are direct costs for material, labor, equipment, and special subcontracts. Costs for corresponding divisions on the Cost Estimate Summary sheet include all applicable indirect costs (overhead, profit, commission on subcontracts, sales tax, and bond).

B. Costs for each activity represent present day costs escalated to the appropriate activity midpoint.

C. Subcontractor labor costs reflect INEEL Site Jurisdictional Agreement craft labor rates.

D. Costs for Lockheed Martin General and Administrative allowance (G \& A) and Performance Incentive Factor (PIF) have been included in this estimate. 
Lockheed Martir Idaho Technologies Co. ROV. 6/96
PROIECT NAME: CSIX/TRU GROUT FEASIBILITY STUDY SCENERIO \#1

LOCATION :: INEELMNTEC

REQUESTOR: K. L. WILLIIAMS
COST ESTIMATE SUMMARY

TYPE OF ESTIMATE: PLANNING PROJECT NO: 2484 PREPARED BY: R.D.ADAMS REPORT NAME: Cost Estimate Summary

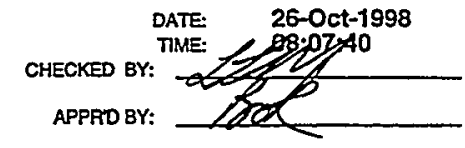

\begin{tabular}{|c|c|c|c|c|}
\hline $\begin{array}{l}\text { WBS } \\
\text { Element }\end{array}$ & Cost Estimate Element & $\begin{array}{c}\text { Total } \\
\text { Unescalated }\end{array}$ & Escalation & $\begin{array}{c}\text { Total } \\
\text { Incl Escalation }\end{array}$ \\
\hline 1.1 & ENGINEERING, DESIGN AND INSPECTION & & & $\$ 11,266,230$ \\
\hline 1.1.1 & DESIGN ENGINEERING TITLE I \& \| & $7,962,000$ & 875,820 & $8,837,820$ \\
\hline 1.1.2 & QUALITY ASSURANCE & $1,990,500$ & 437,910 & $2,428,410$ \\
\hline 1.2 & MANAGEMENT COSTS & & & $>\$ 11,544,900$ \\
\hline 1.2 .1 & PROJECT MANAGEMENT & $5,573,400$ & $1,114,680$ & $6,688,080$ \\
\hline 1.2 .2 & CONSTRUCTION MANAGEMENT & $3,981,000$ & 875,820 & $4,856,820$ \\
\hline 1.3 & CONSTRUCTION & & & $\$ 48,568,002$ \\
\hline 1.3.1 & GENERAL CONDITIONS & $2,496,329$ & 549,192 & $3,045,521$ \\
\hline 1.3.2 & SITEWORK & 863,098 & 189,882 & $1,052,980$ \\
\hline 1.3 .3 & CONCRETE & $4,209,942$ & 926,187 & $5,136,129$ \\
\hline 1.3 .5 & METALS & $1,406,944$ & 309,528 & $1,716,472$ \\
\hline 1.3 .7 & THERMAL \& MOISTURE PROTECTION & 205,553 & 45,222 & 250,775 \\
\hline 1.3 .8 & DOORS \& WINDOWS & 128,013 & 28,163 & 156,176 \\
\hline 1.3 .9 & FINISHES & $1,064,376$ & 234,163 & $1,298,539$ \\
\hline 1.3.10 & SPECIALTIES & 19,410 & 4,270 & 23,680 \\
\hline 1.3.11 & EQUIPMENT & $15,759,339$ & $3,467,055$ & $19,226,394$ \\
\hline 1.3.12 & FURNISHINGS & 221,438 & 48,716 & 270,154 \\
\hline 1.3.13 & SPECIAL CONSTRUCTION & $1,167,753$ & 256,906 & 1,424,659 \\
\hline 1.3.14 & CONVEYING SYSTEMS & $1,987,042$ & 437,149 & $2,424,191$ \\
\hline 1.3.15 & MECHANICAL & $6,457,307$ & $1,420,608$ & $7,877,915$ \\
\hline 1.3.16 & ELECTRICAL & 3,823,293 & 841,124 & 4,664,417 \\
\hline 1.5 & G\&APIF & & & $\gg \$ 3,178,566$ \\
\hline 1.5.1 & G\&A/PIF ADDER & $2,605,382$ & 573,184 & $3,178,566$ \\
\hline 1.5 .2 & PROCUREMENT FEES & $1,194,295$ & 262,745 & $>\$ 1,457,040$ \\
\hline & $\begin{array}{l}\text { SUBTOTAL INCLUDING ESCALATION } \\
\text { PROJECT CONTINGENCY }\end{array}$ & $63,116,414$ & $12,898,324$ & $\gg>\$ 76,014,738$ \\
\hline \multicolumn{4}{|c|}{ MANAGEMENT RESERVE- } & $\$ 5,320,361$ \\
\hline \multicolumn{4}{|c|}{ CONTINGENCY- } & $\$ 20,664,901$ \\
\hline & TOTAL ESTIMATED COST & & & $\$ 102,000,000$ \\
\hline
\end{tabular}

\section{PROJECT COST PARAMETERS}

EDI AS A \% OF CONST. + GFE $=23.00 \%$ 


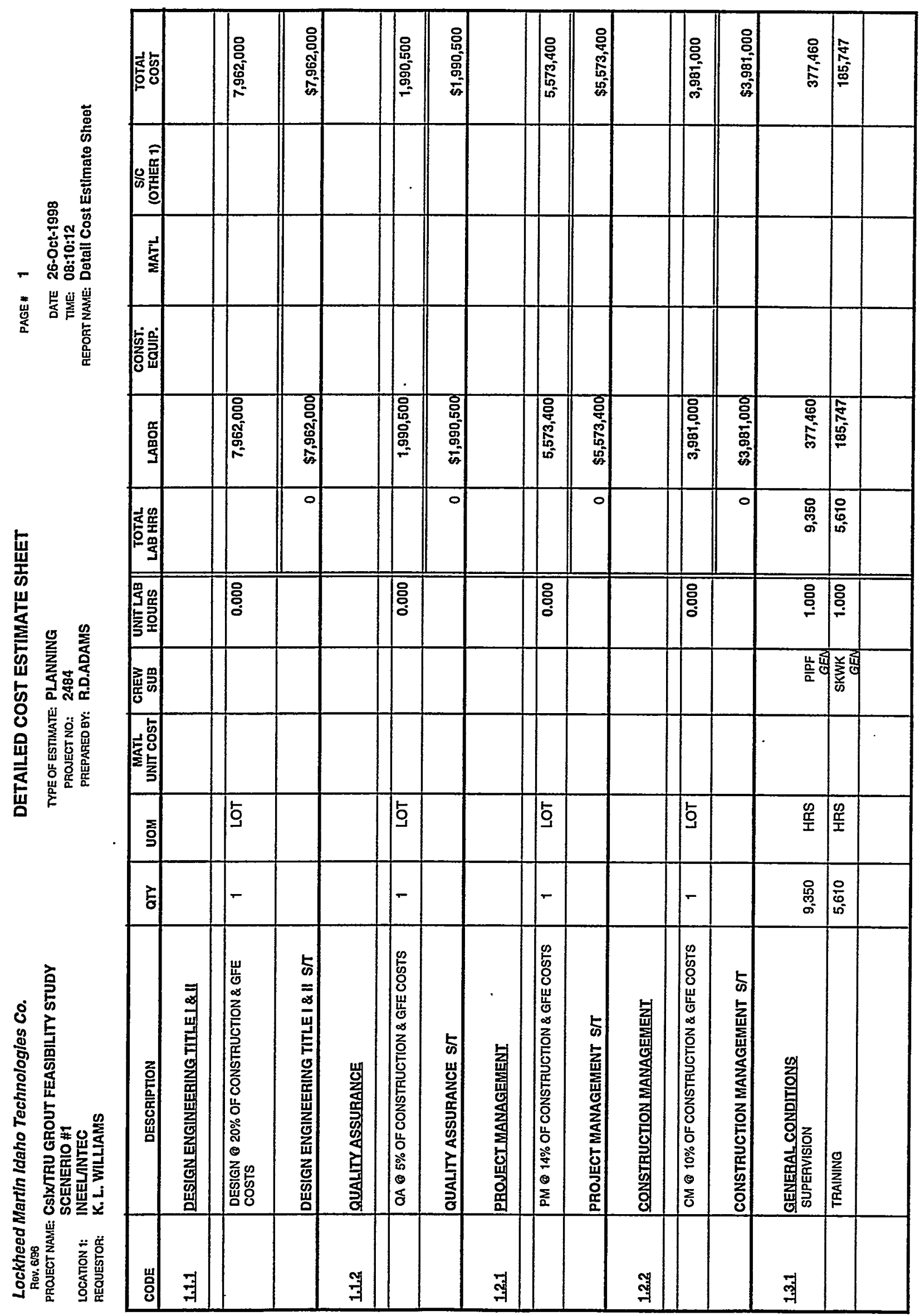




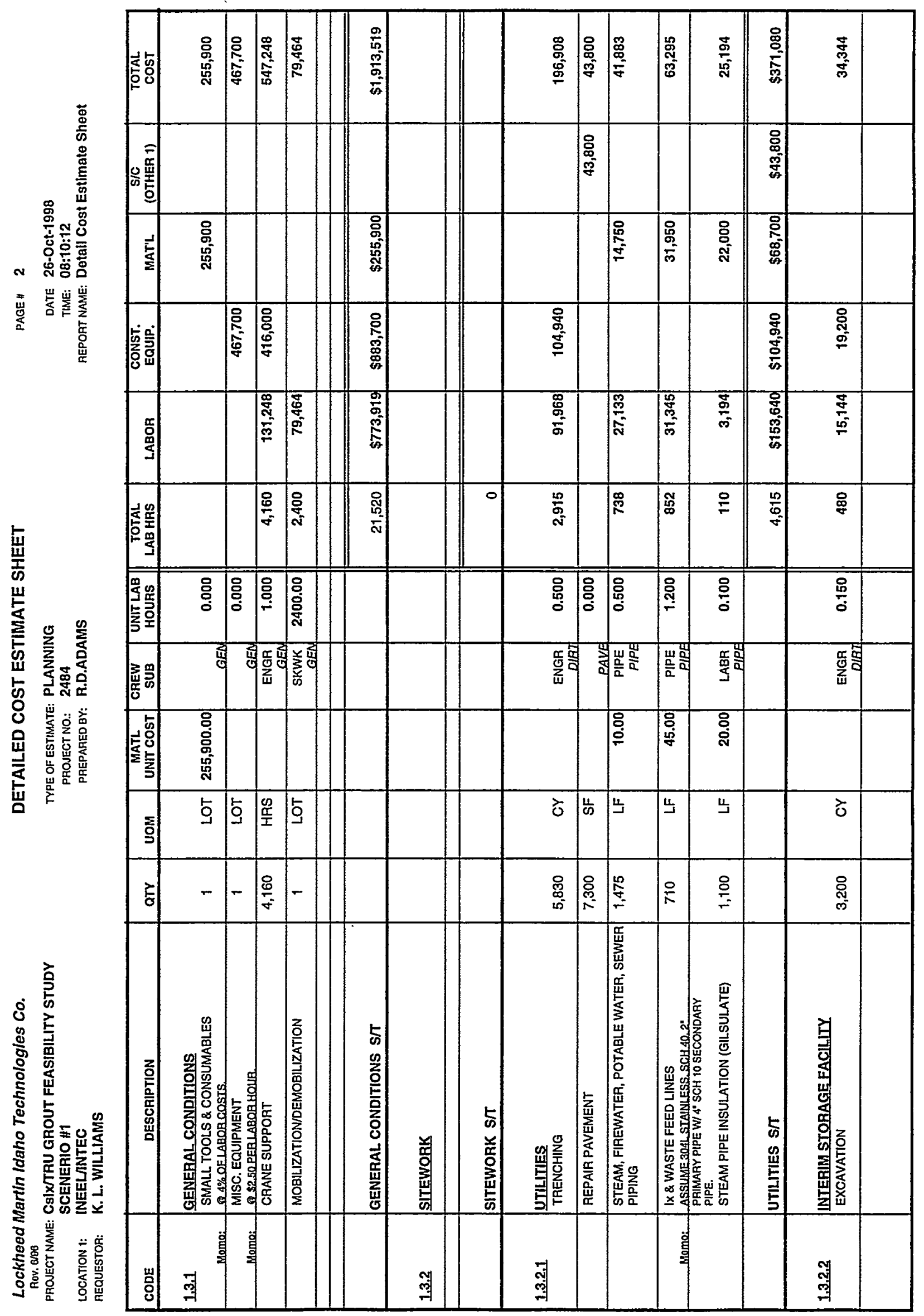




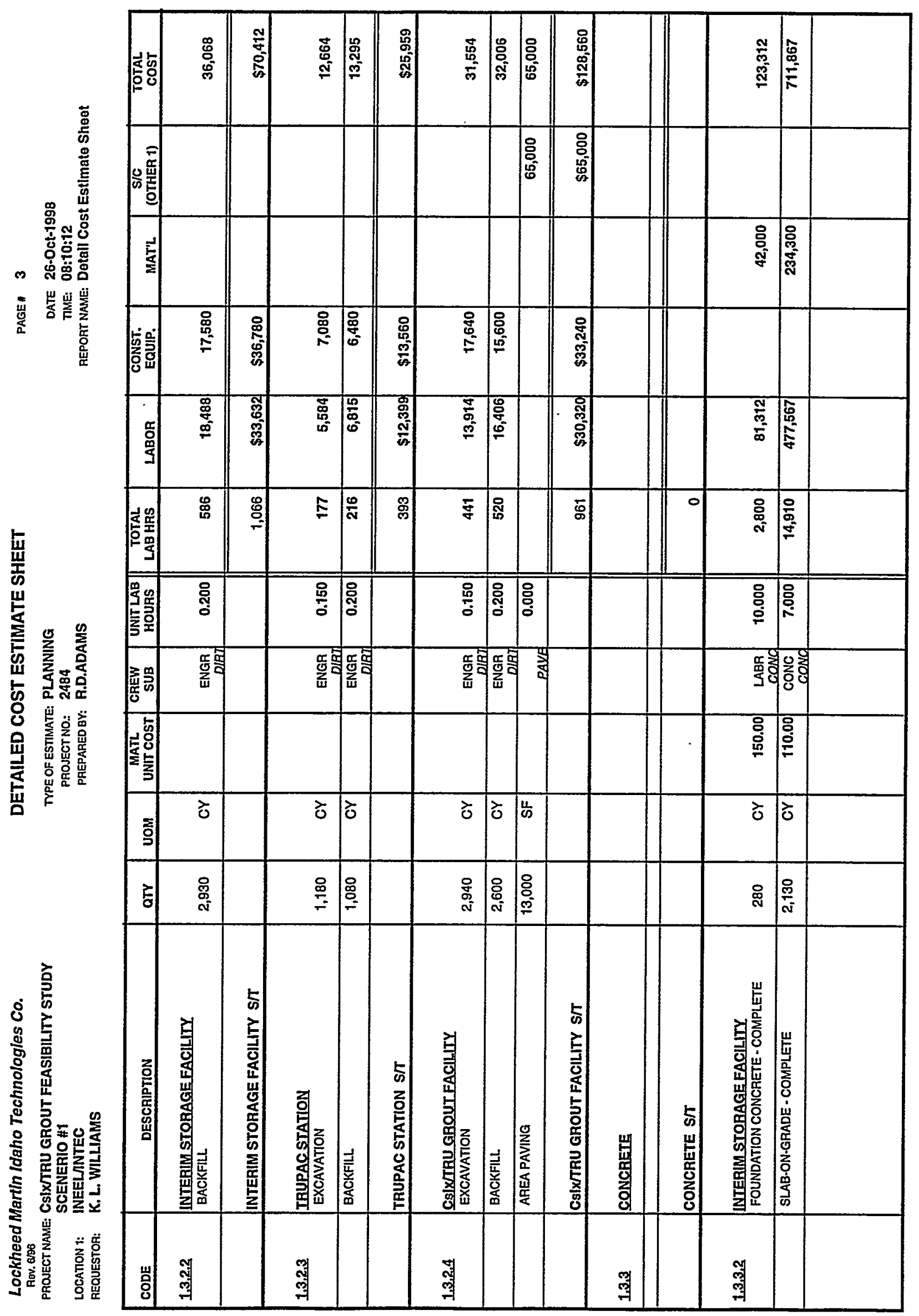




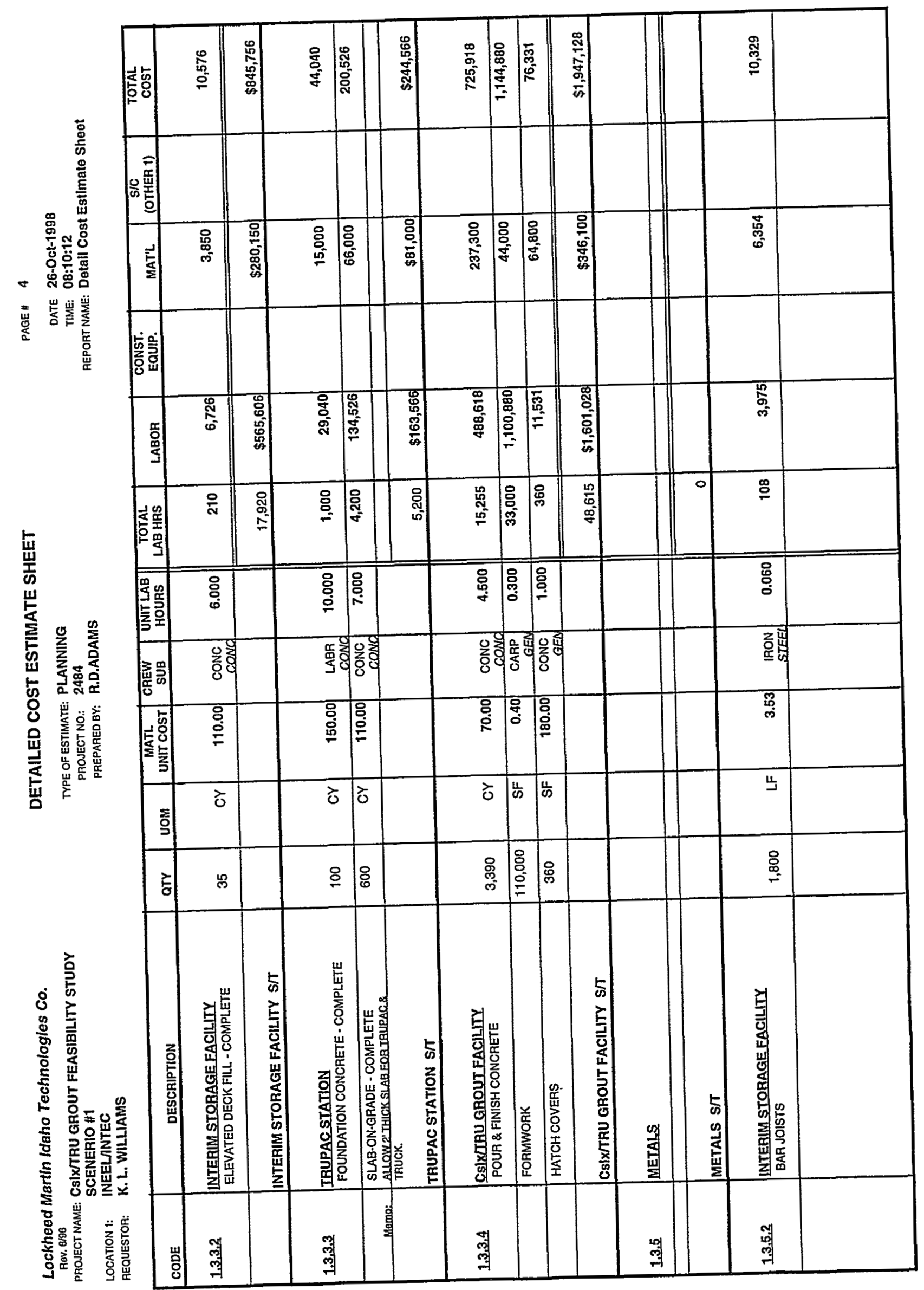




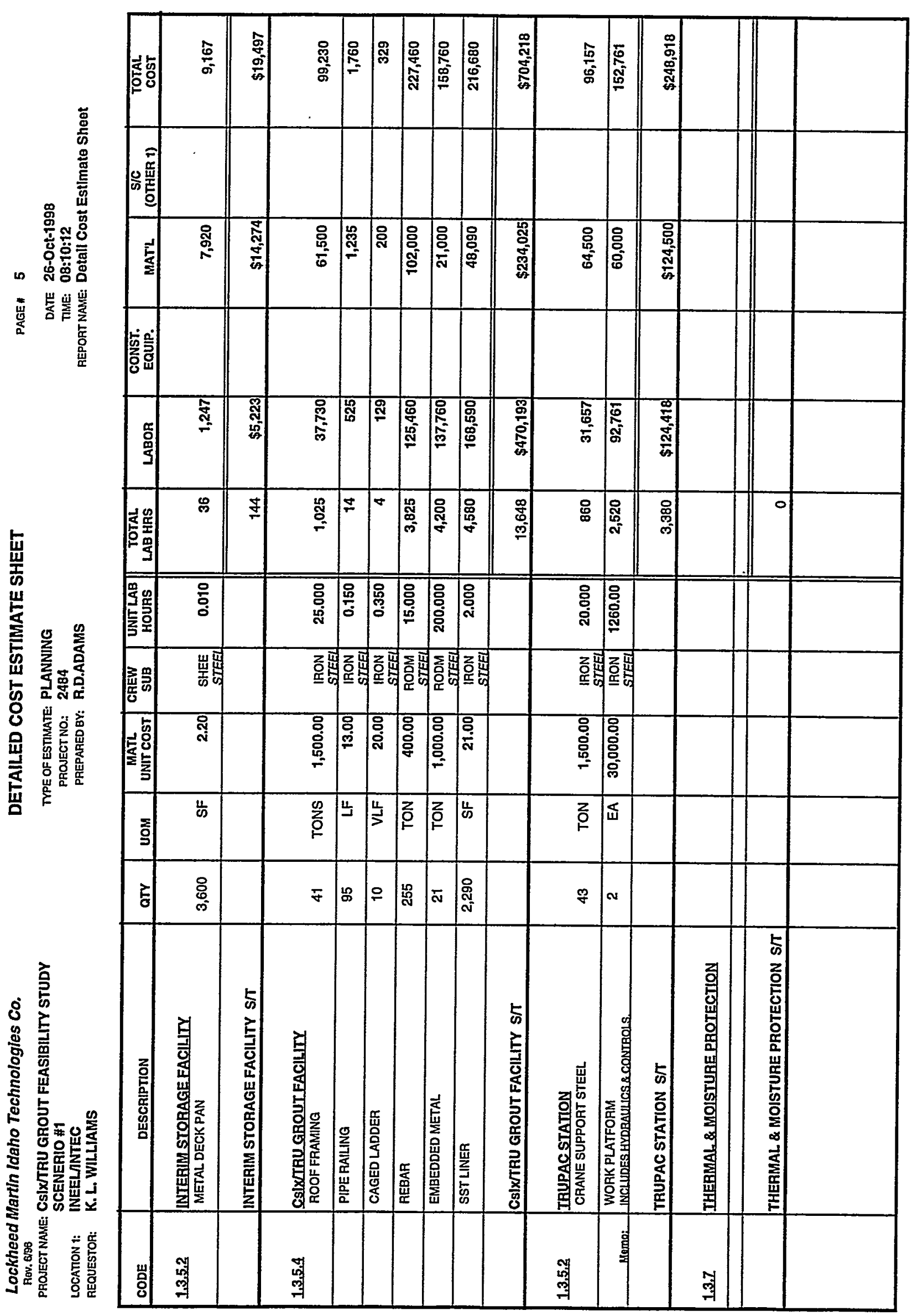




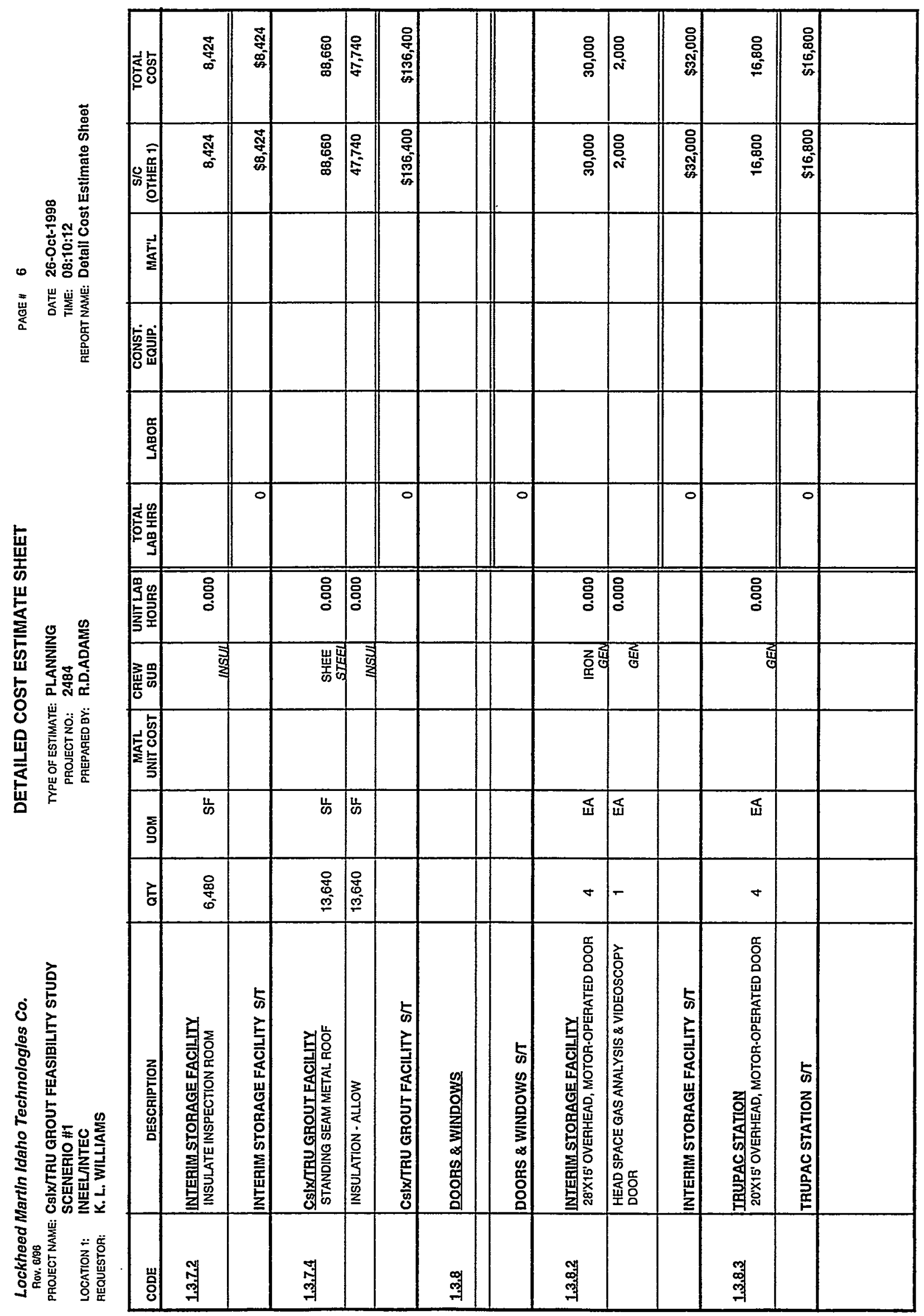




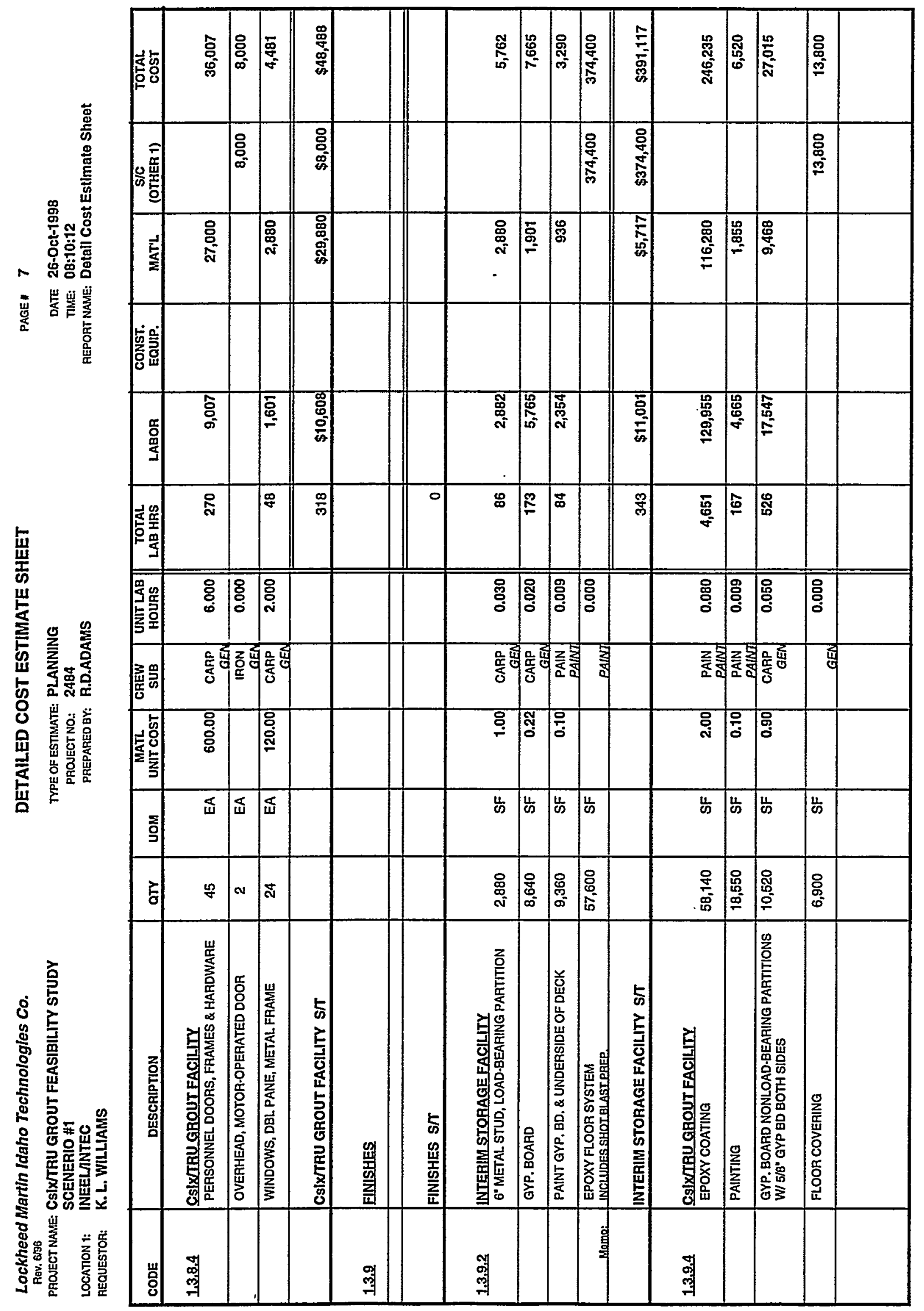




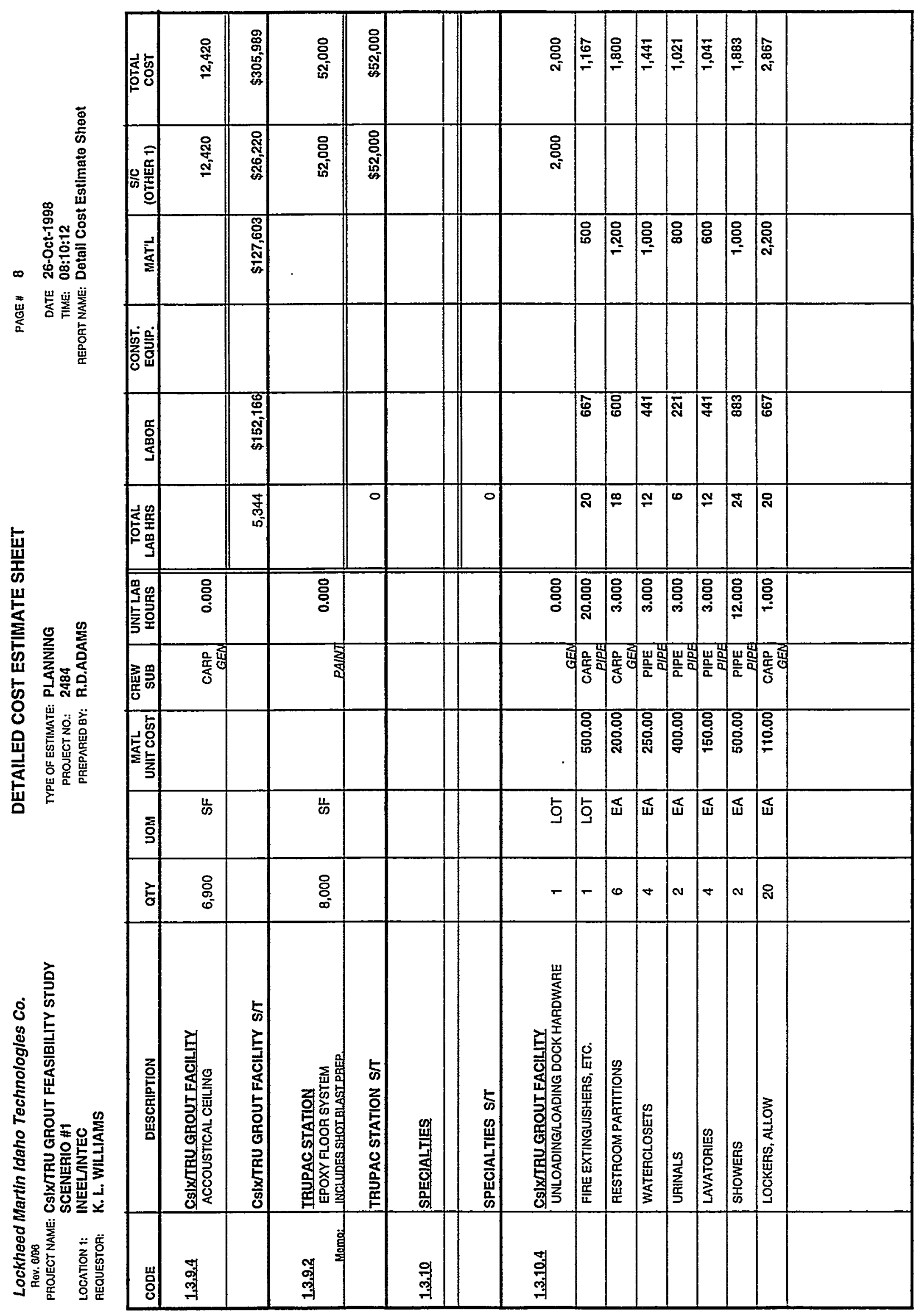




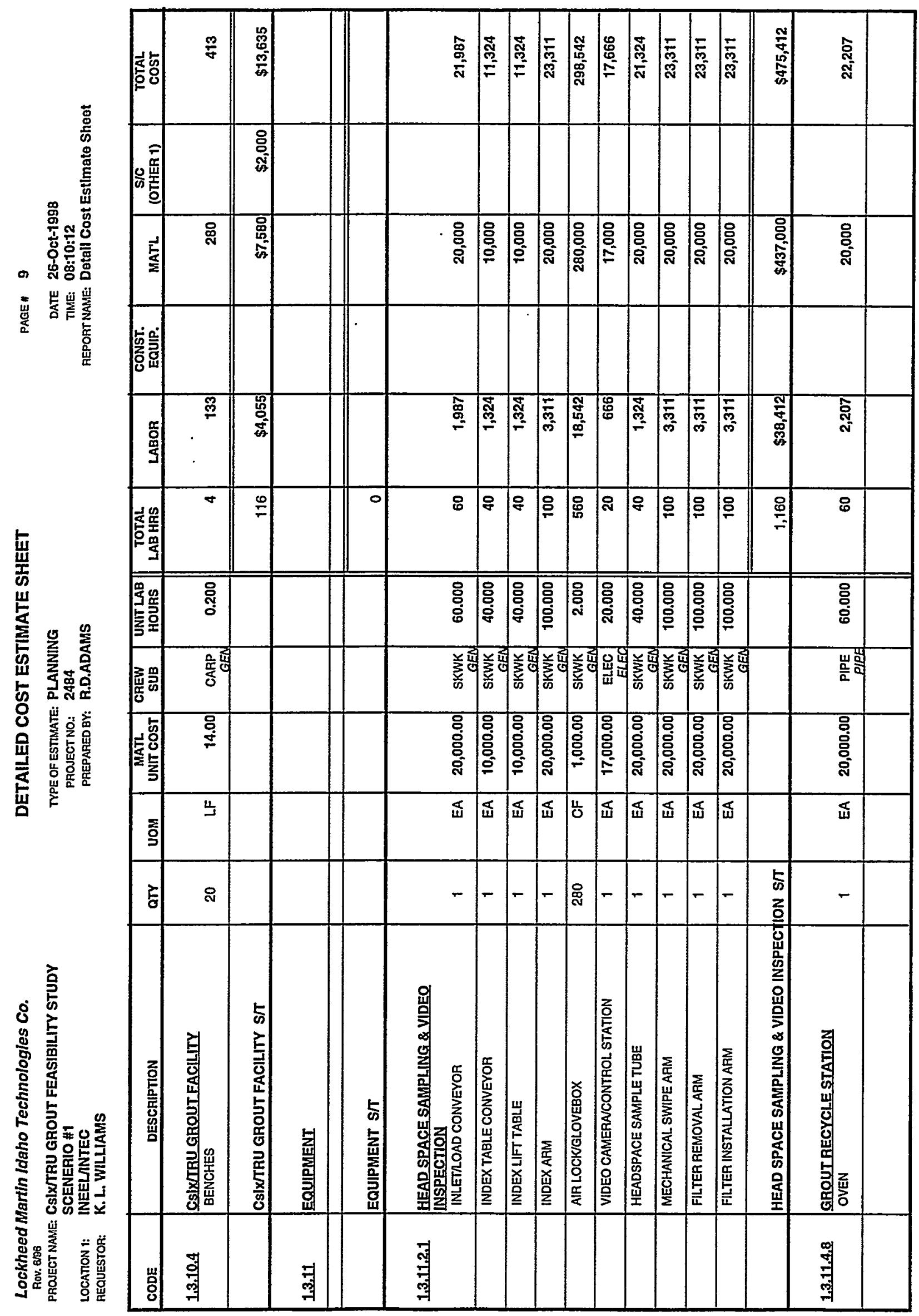




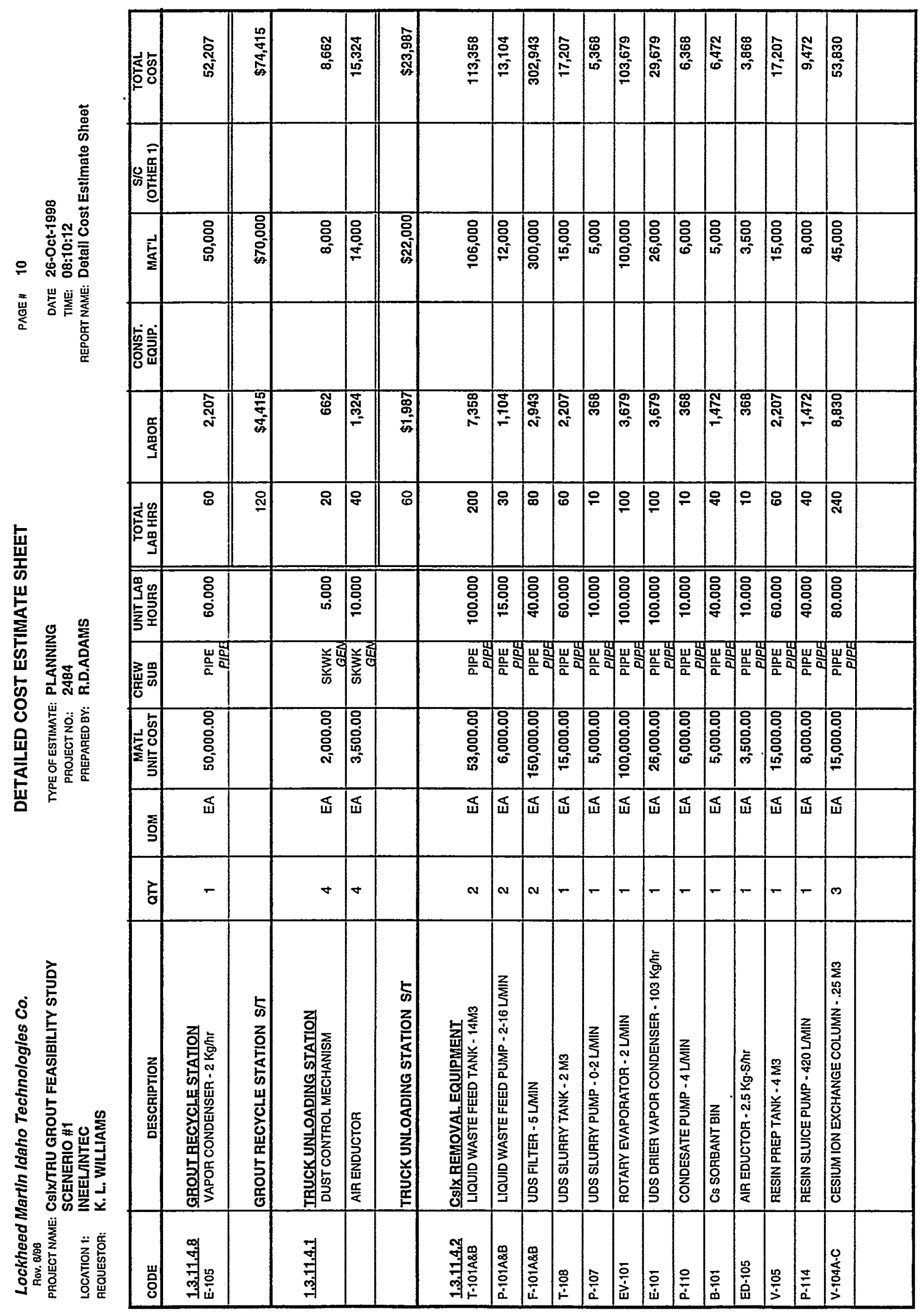




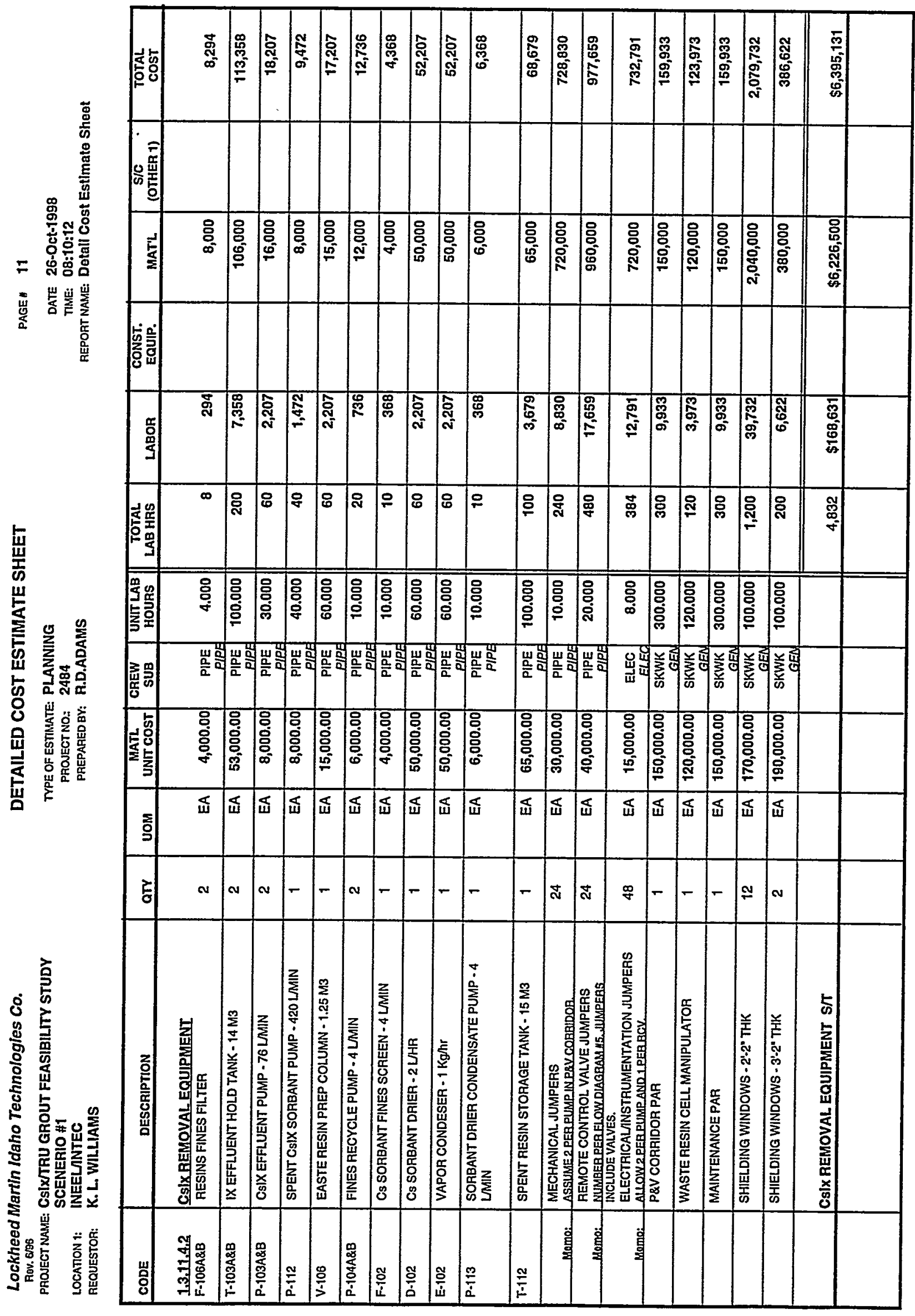




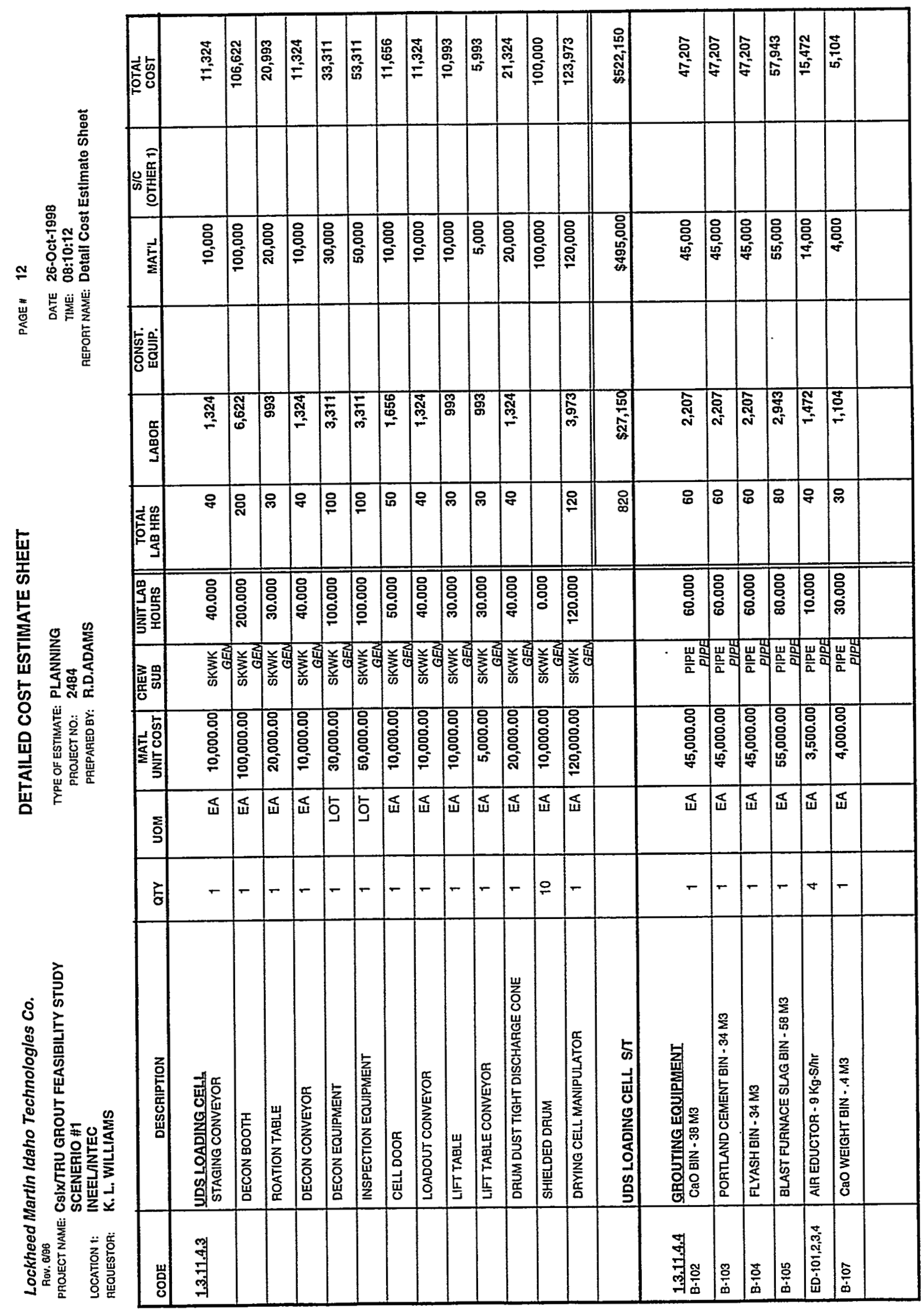




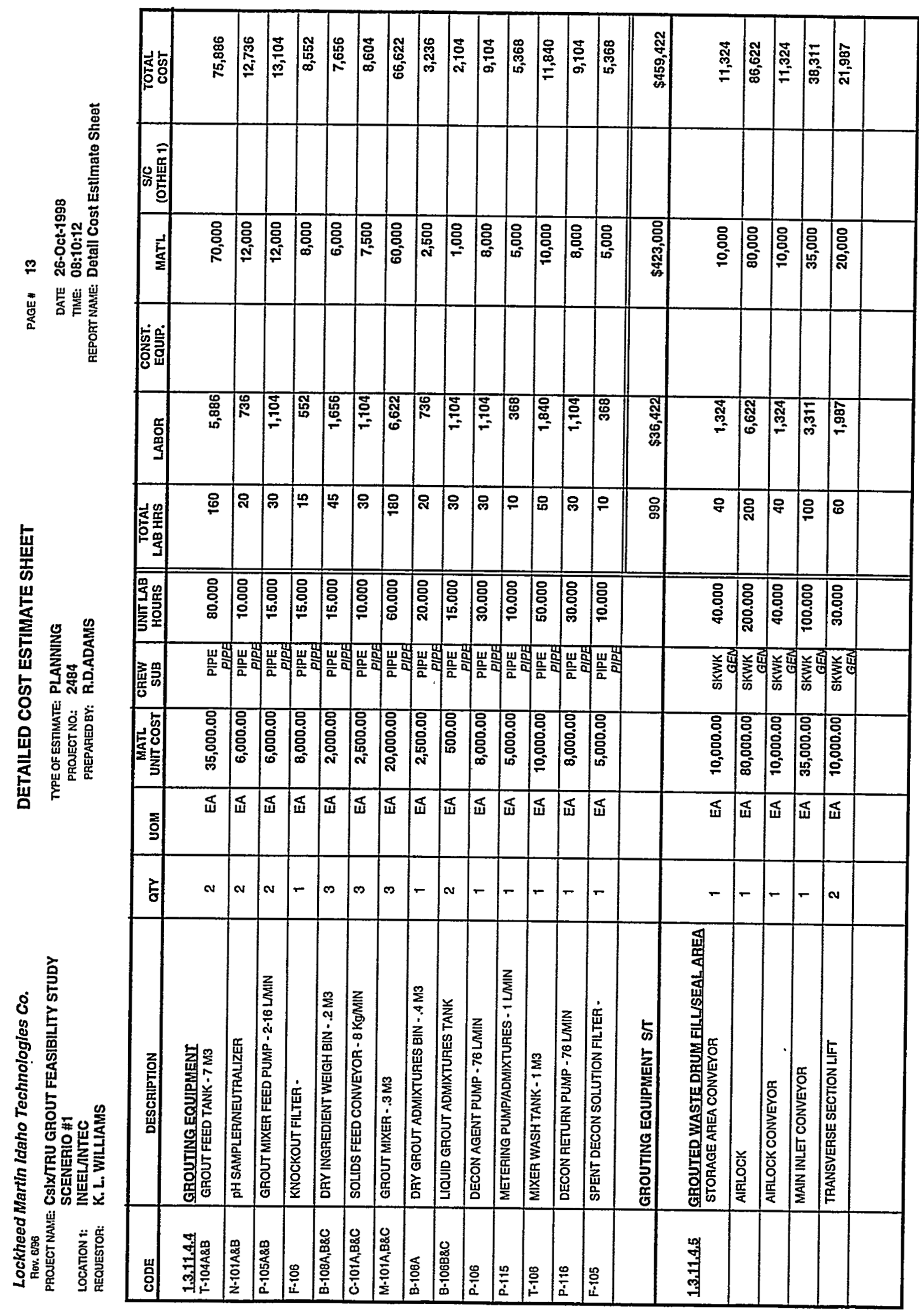




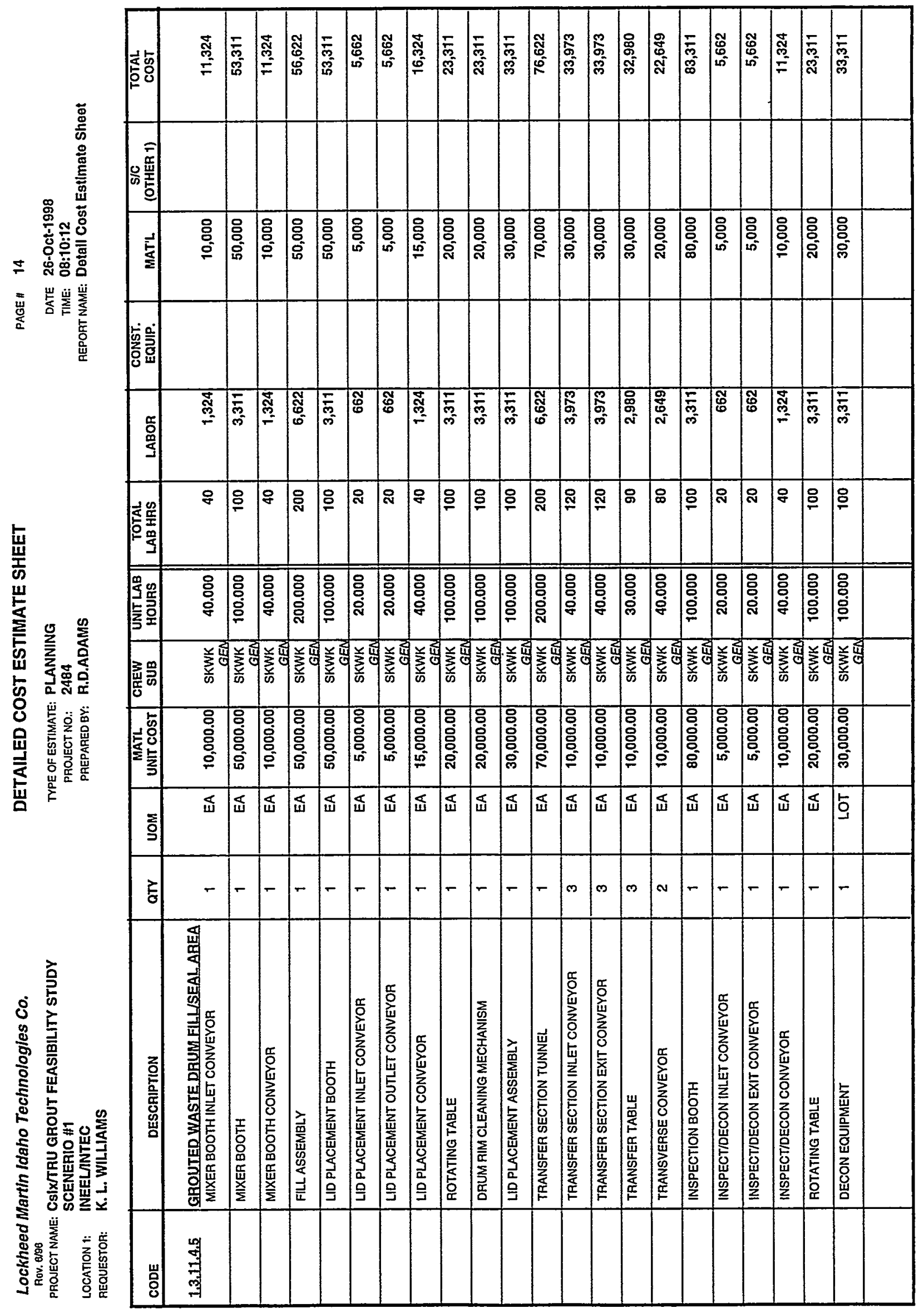




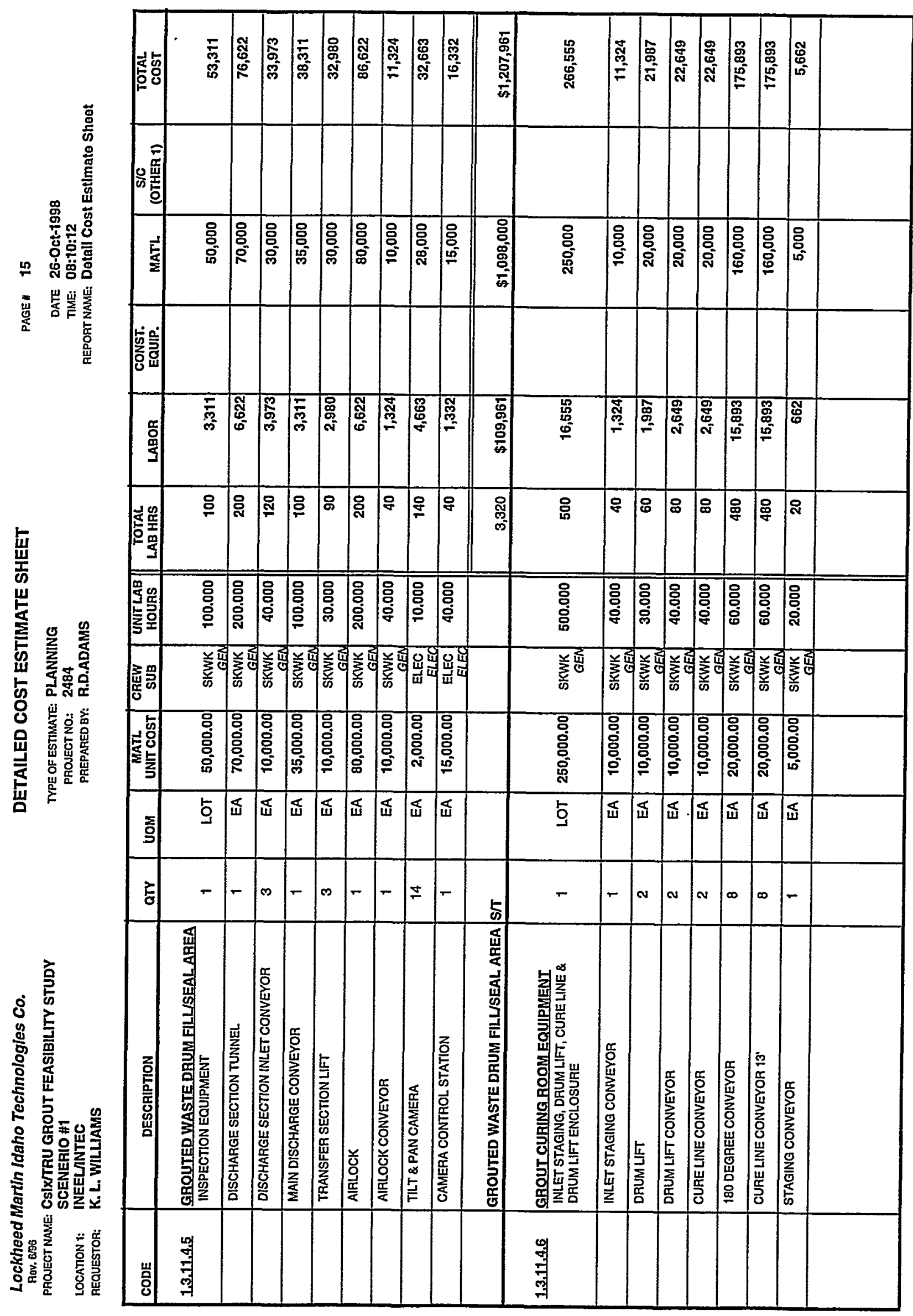




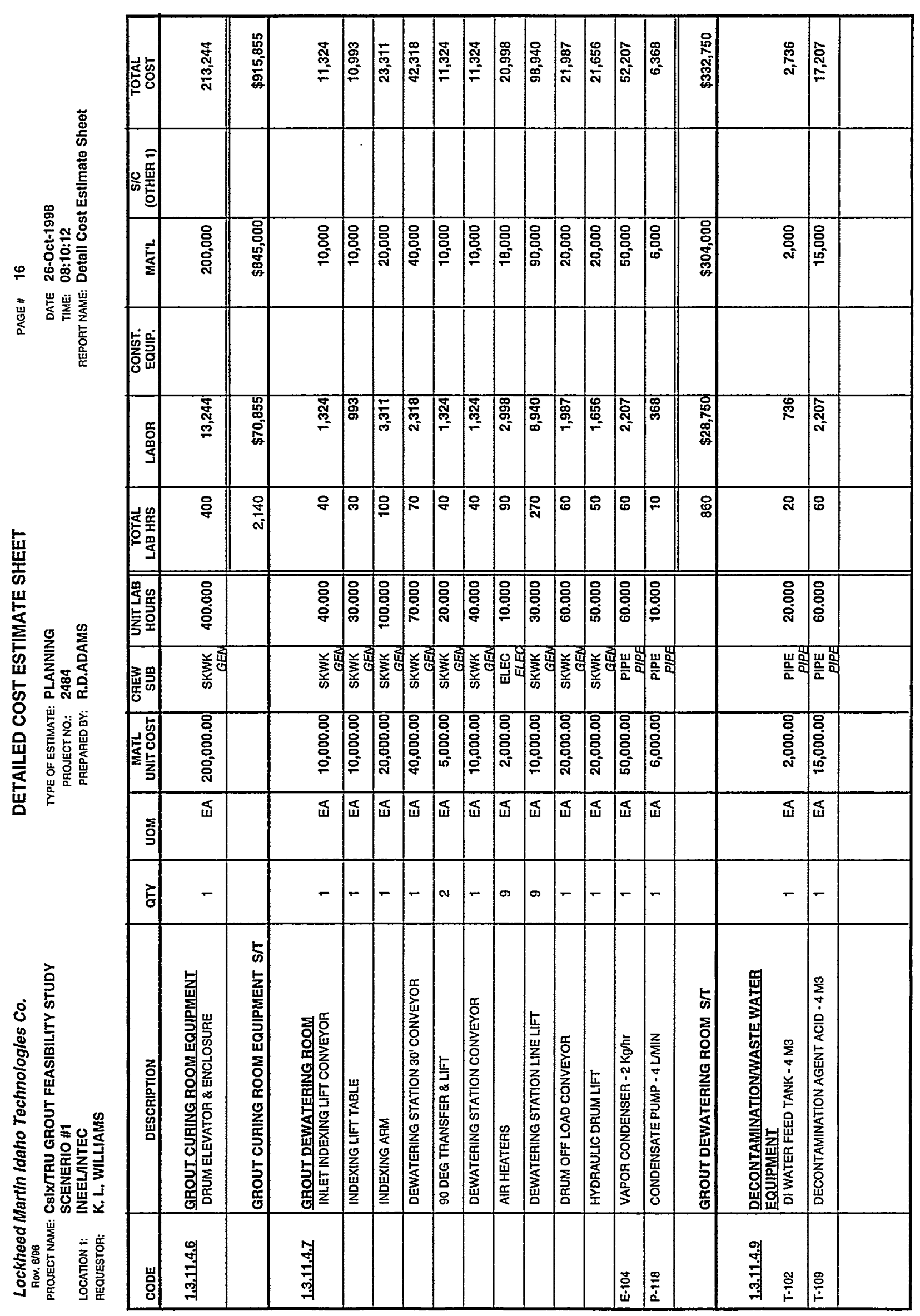




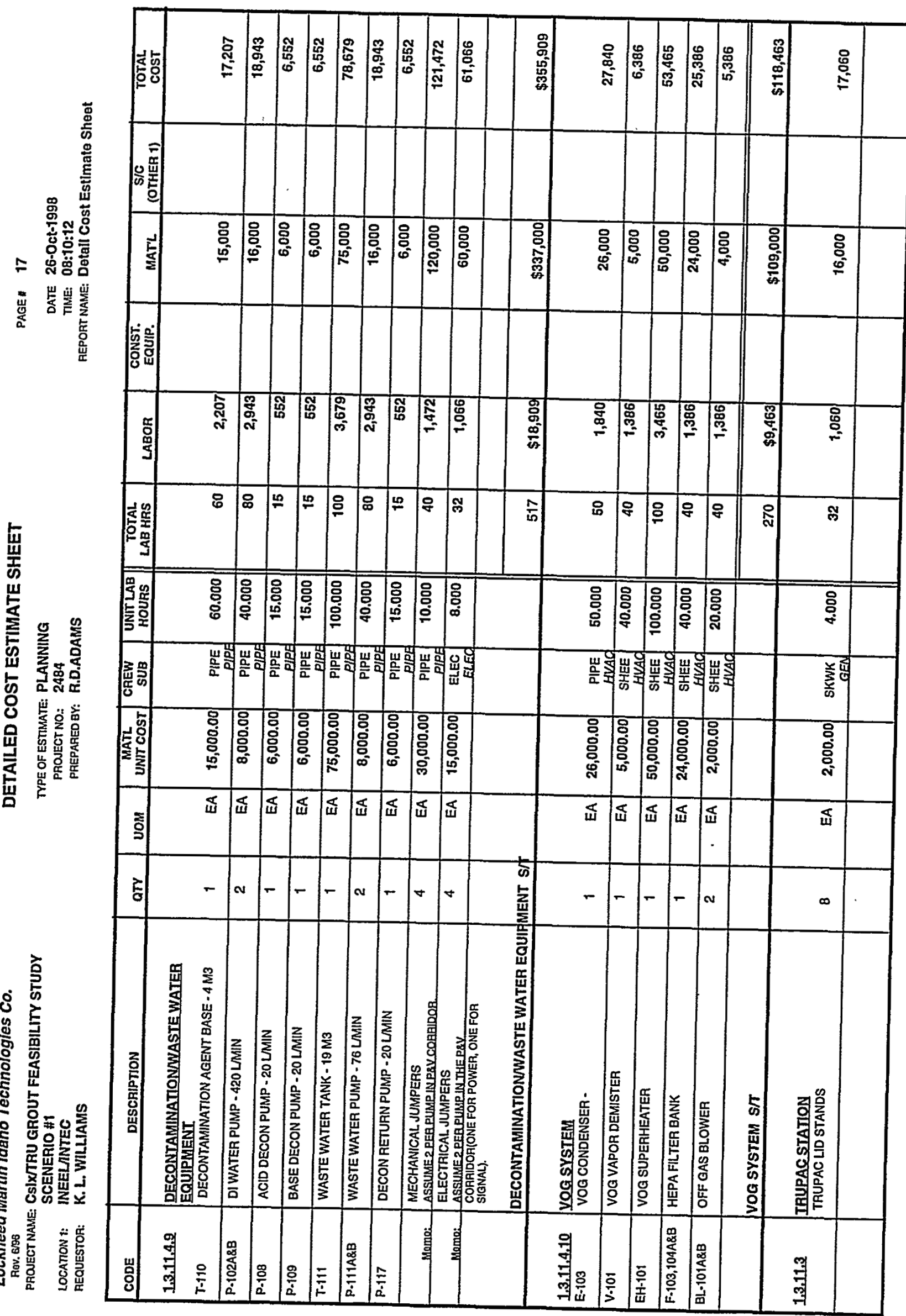




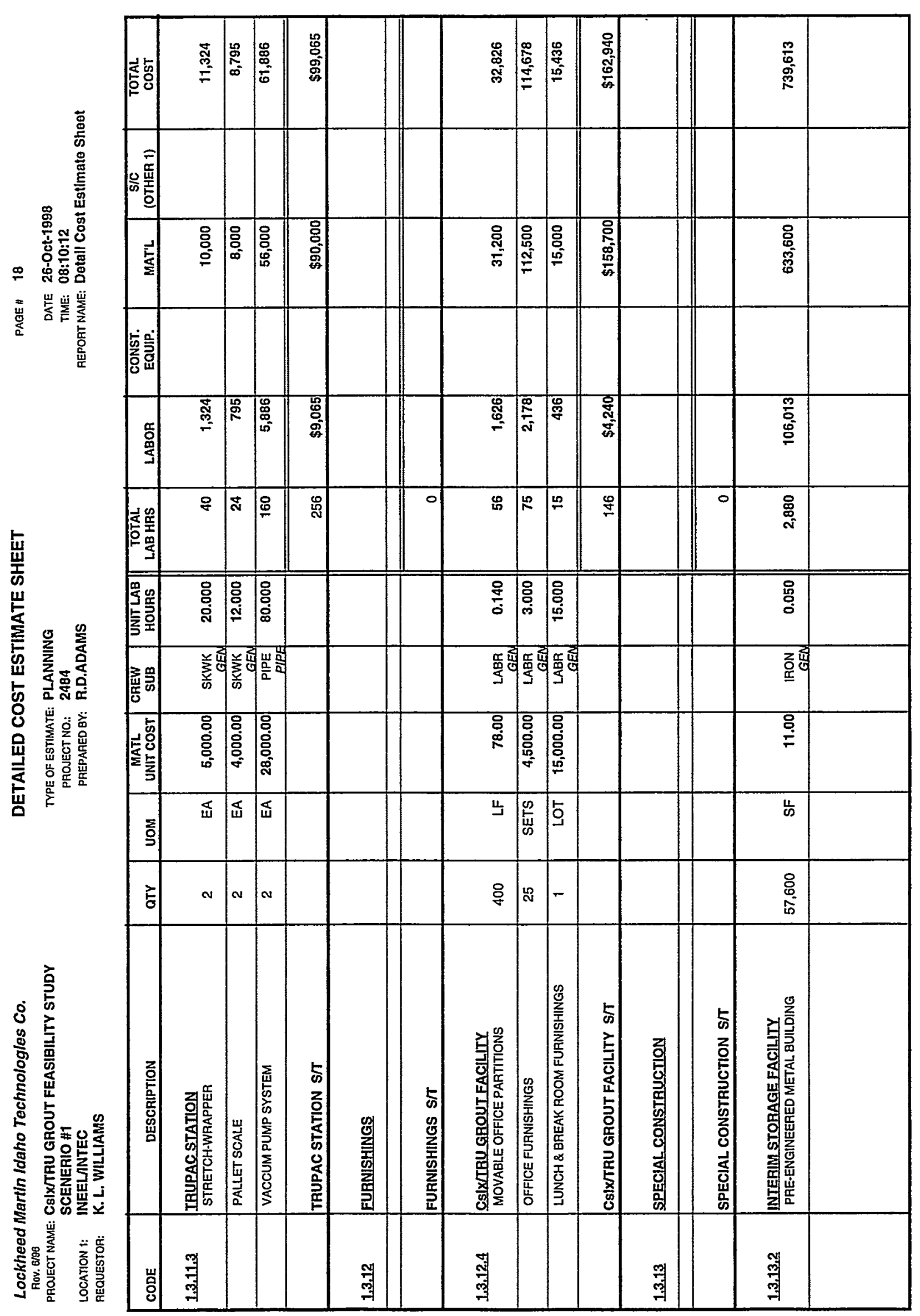




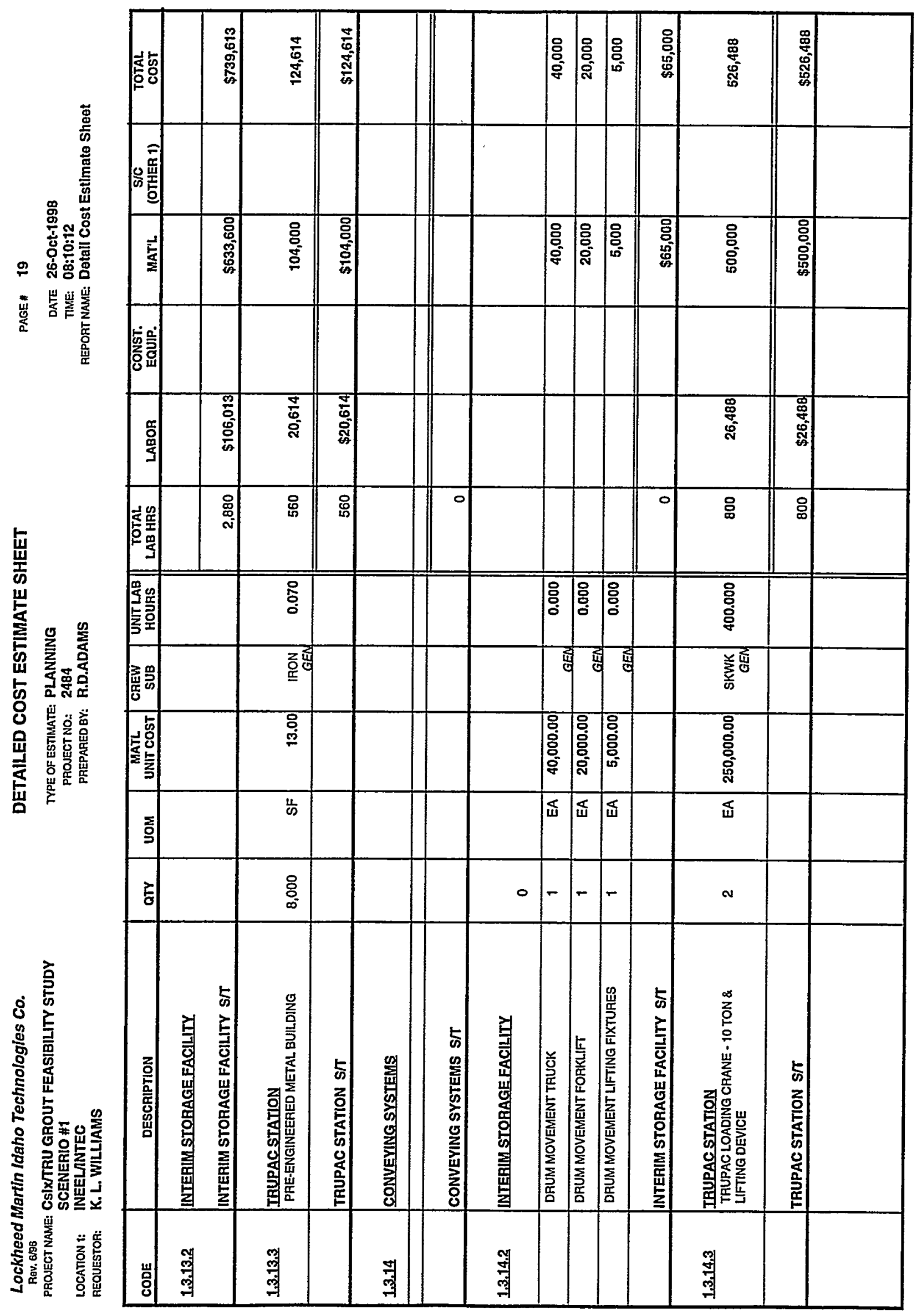




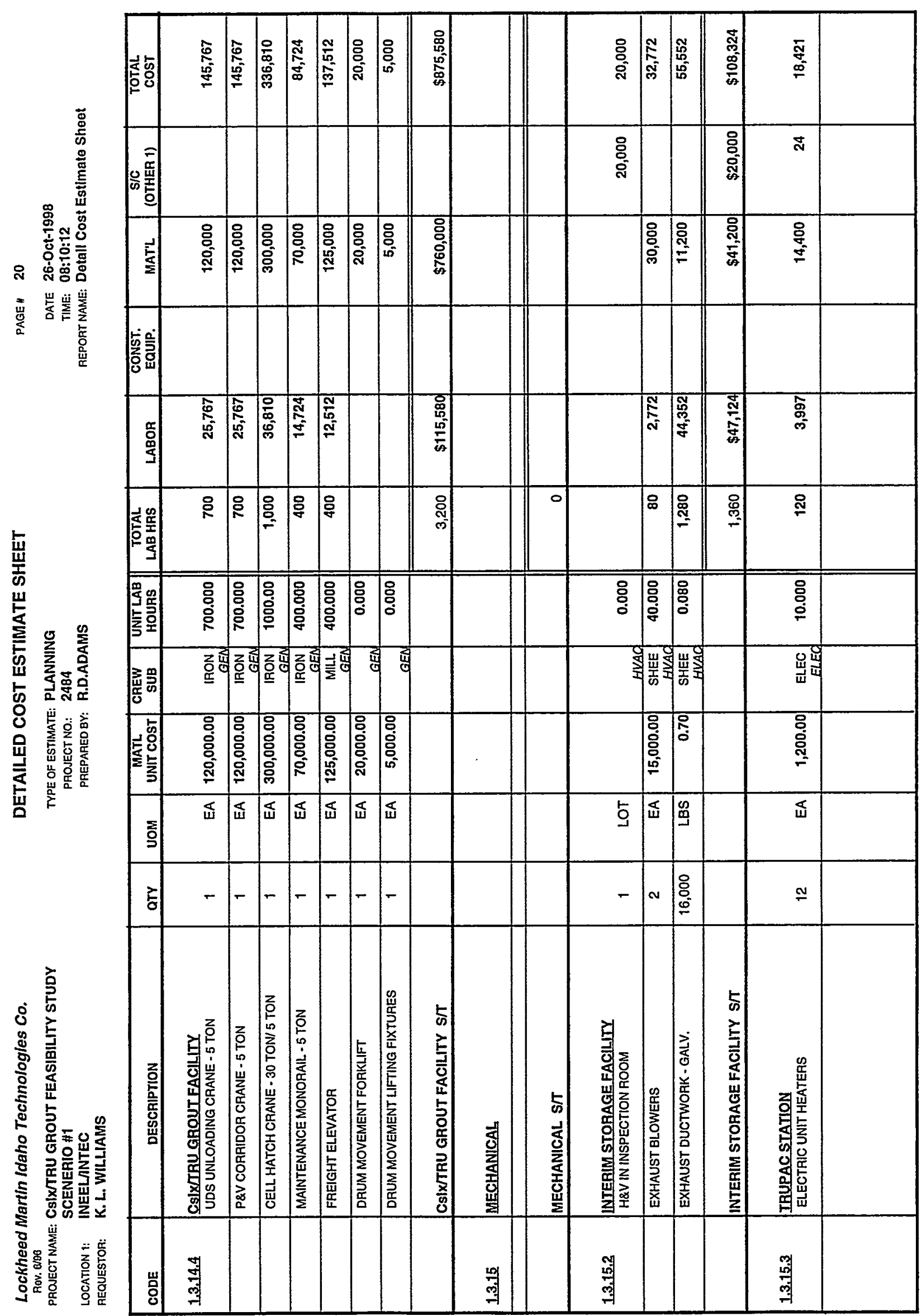




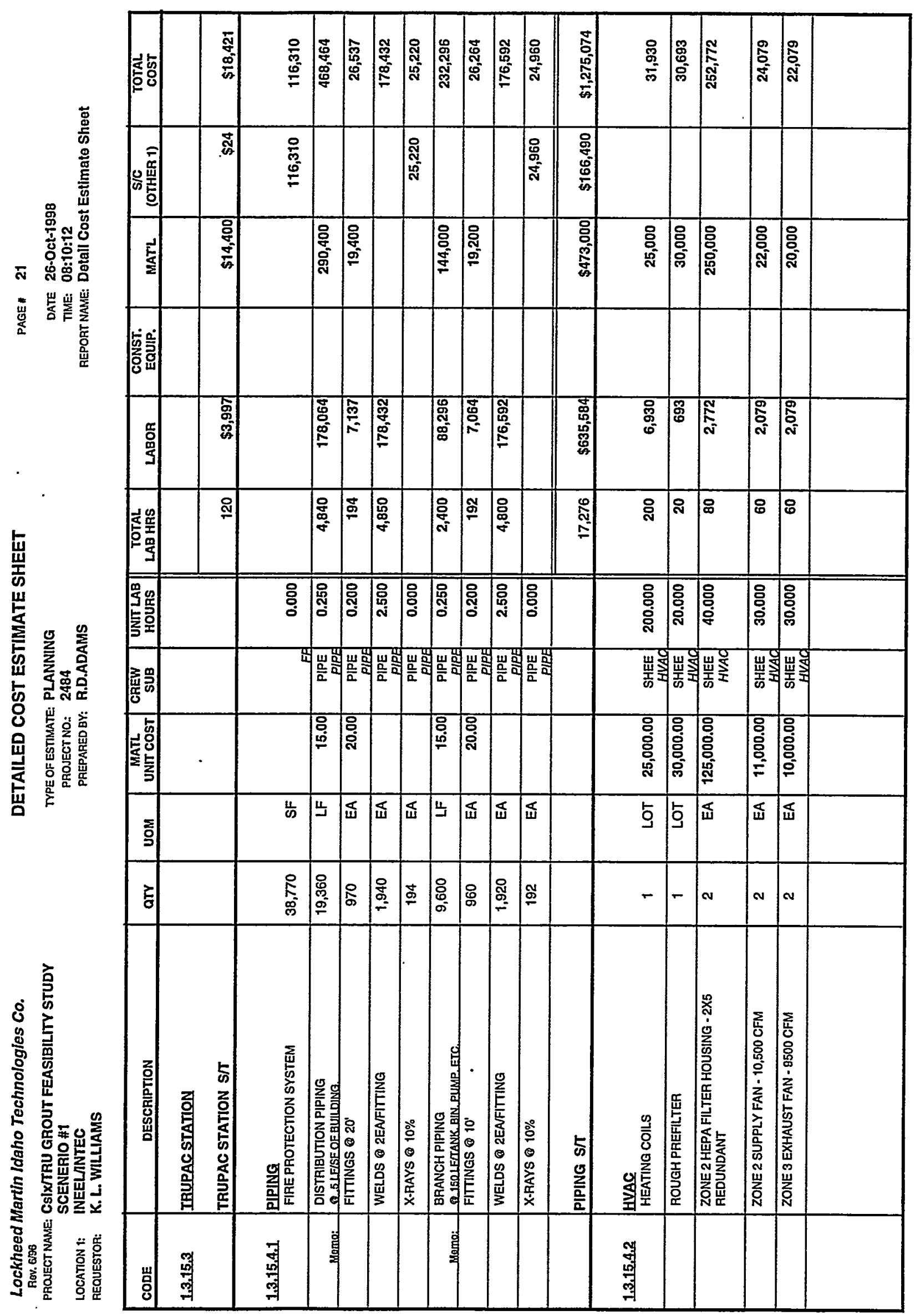




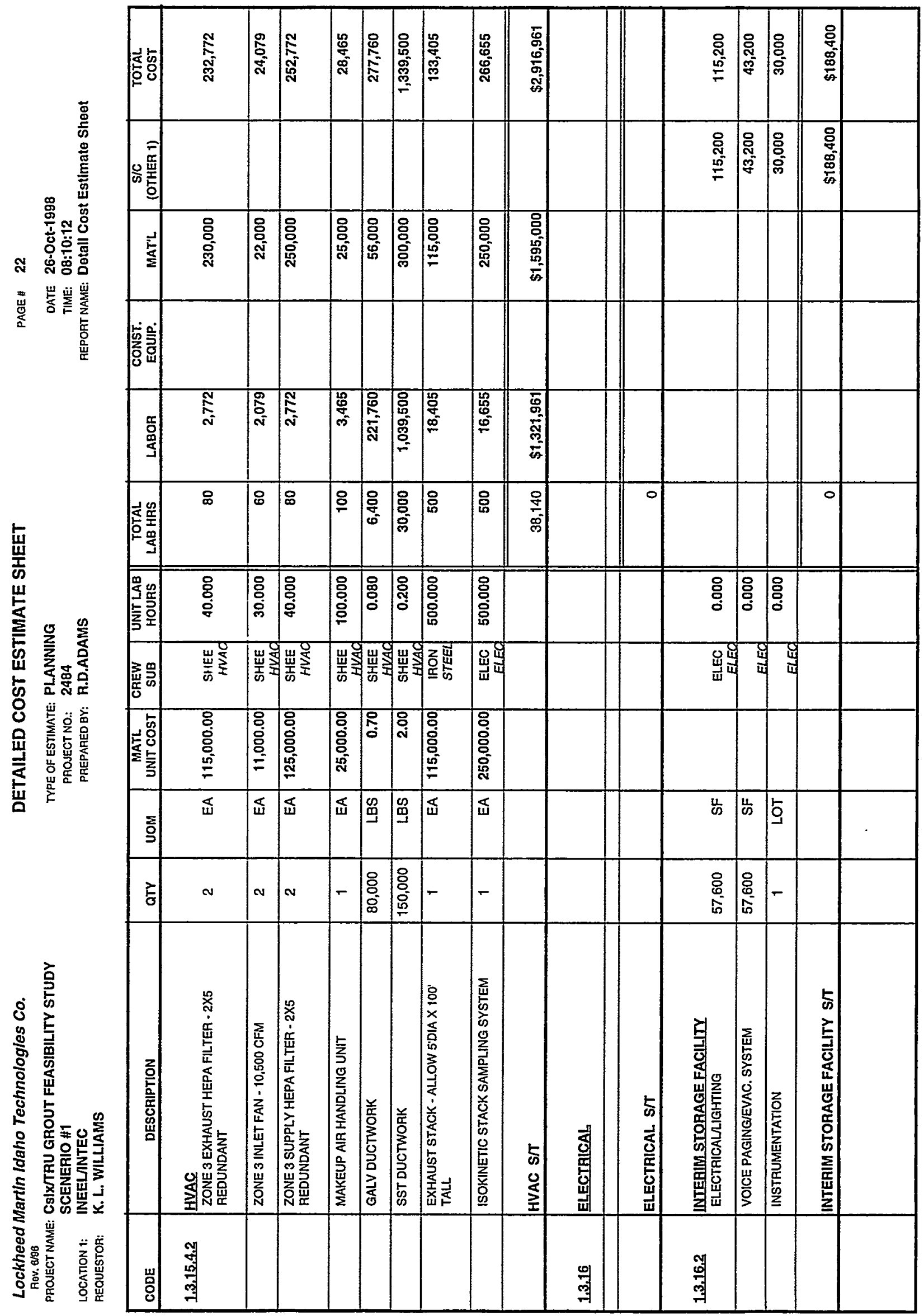




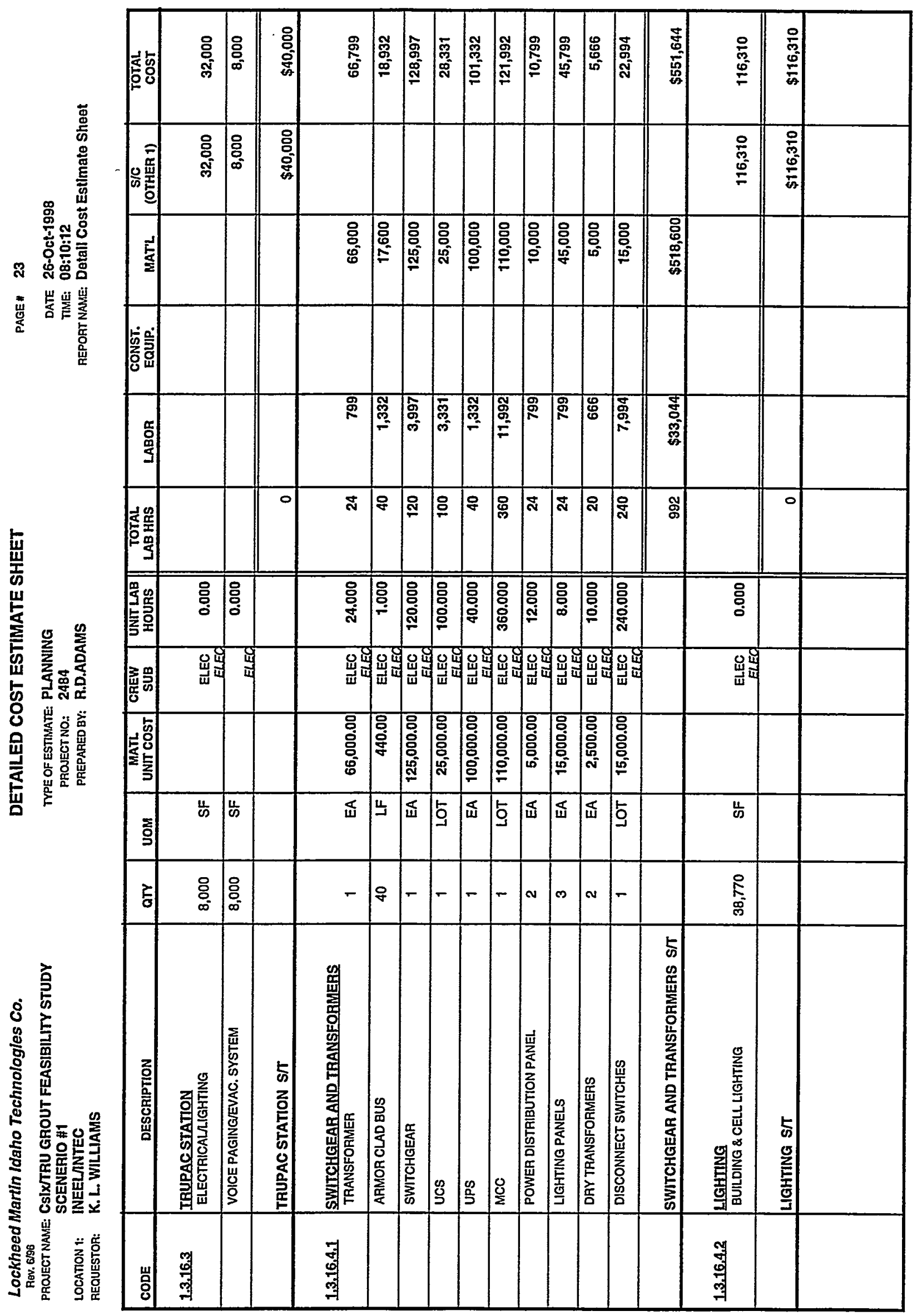




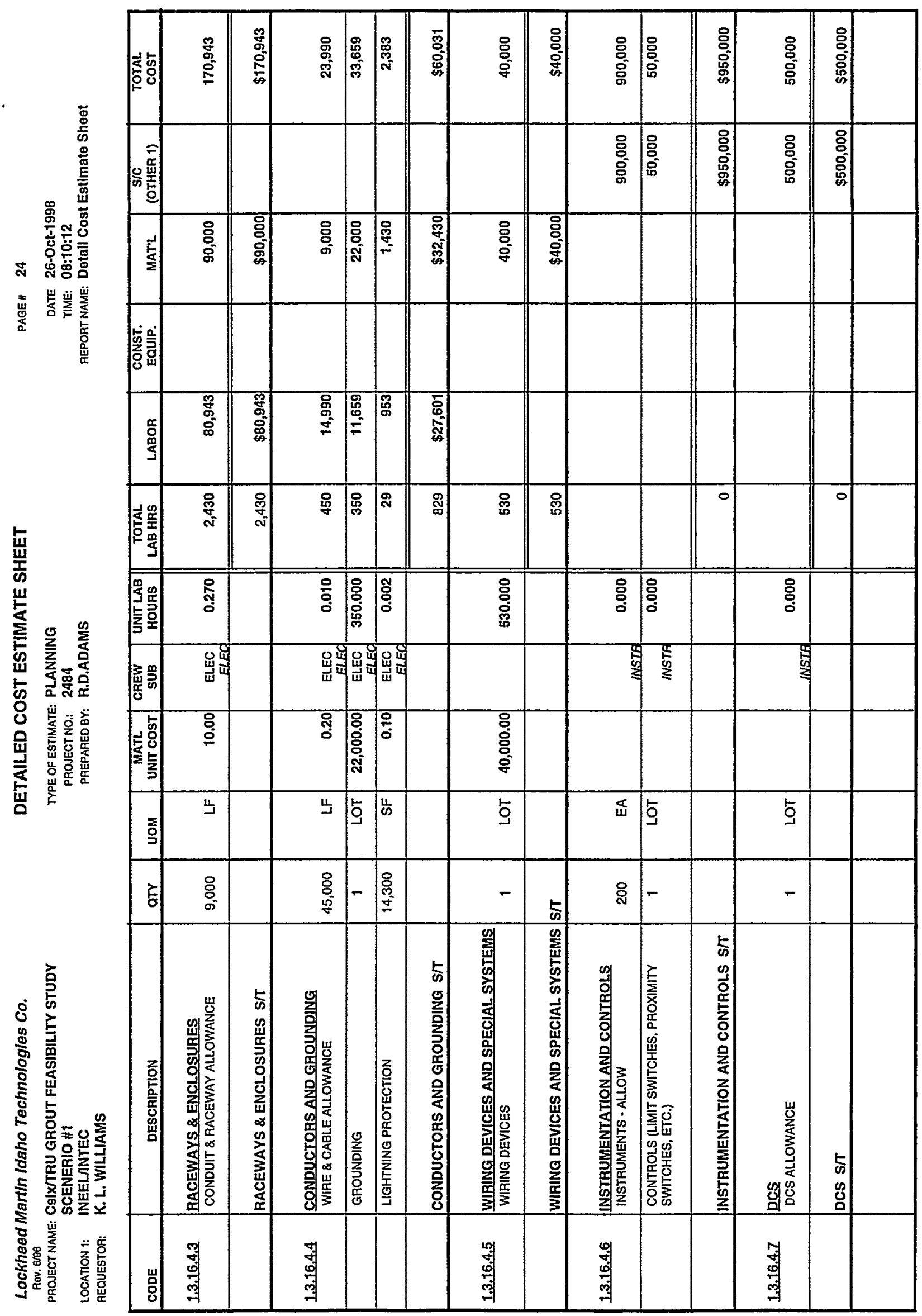




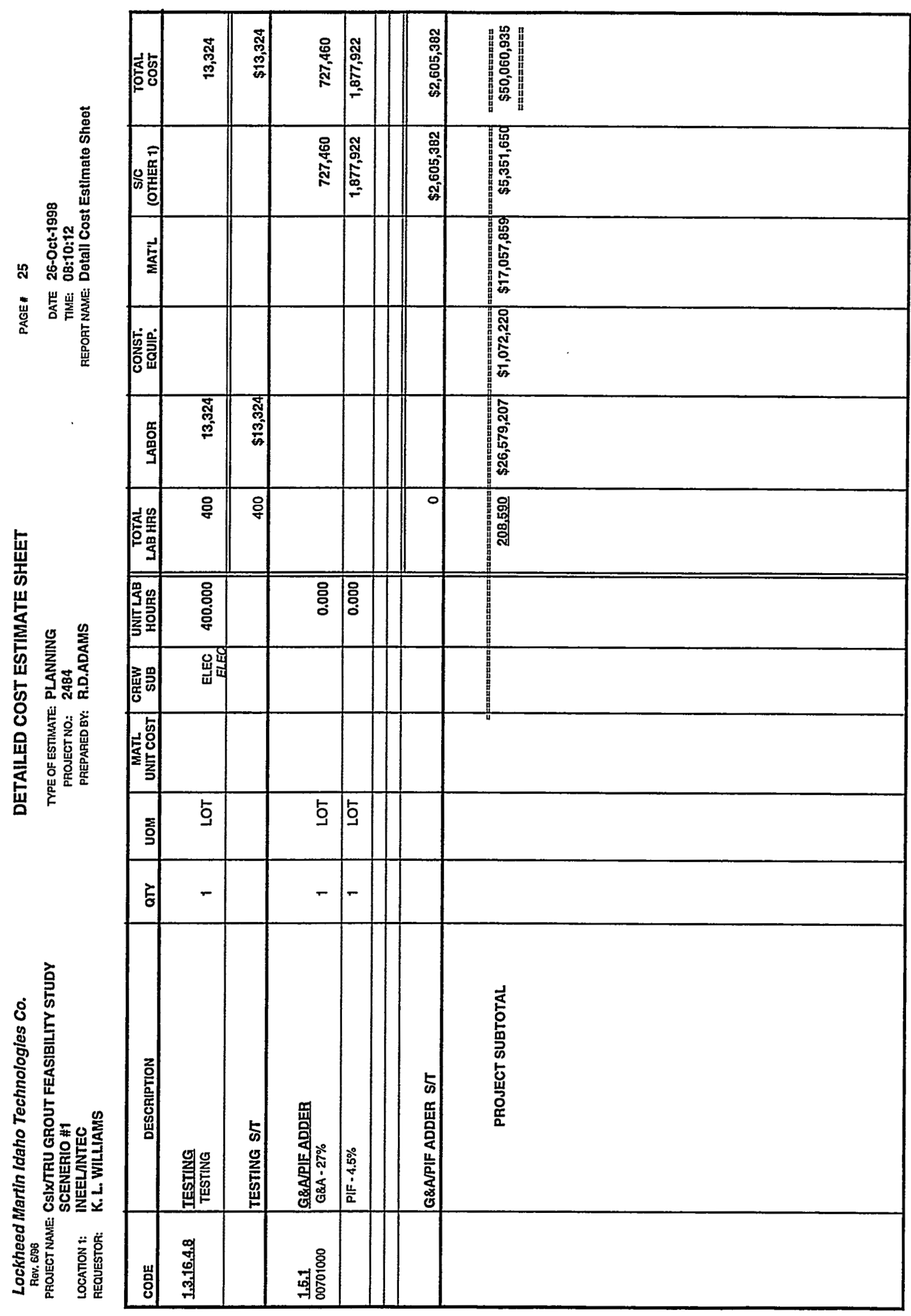


Lockheed Martin Idaho Technologies Co. PROJECT NAME: CSIX/TRU GROUT FEASIBILITY STUDY SCENERIO \#1 $\begin{array}{ll}\text { LOCATION 1: } & \text { INEELANTEC } \\ \text { REQUESTOR: } & \text { K. L. WILLLAMS }\end{array}$

\section{CONTINGENCY ANALYSIS}

TYPE OFESTIMATE: PLANNING PROIECTNO: 2484 PREPARED BY:

2484
R.D.ADAMS

DATE: 26-Oct-1998

TIME: 08:08:03

REPORT NAME: Contingency Analysis

\begin{tabular}{|c|c|c|c|c|c|c|c|c|c|c|c|}
\hline & PROB & IBLE \% VARIA & ION & & & & & & $\begin{array}{r}\text { PRO } \\
\text { CONTI }\end{array}$ & $\begin{array}{l}\text { CT } \\
\text { GENCY }\end{array}$ & SUMMARY \\
\hline $\begin{array}{l}\text { WBS } \\
\text { Element }\end{array}$ & Cost Estimate Element & Total Cost W/o & $\begin{array}{c}\text { \% Total } \\
\text { Cost }\end{array}$ & & $\begin{array}{l}\text { \% Var. } \\
\text { m Est. }\end{array}$ & Wt. \% & t Prob. & Contingency & $\%$ & Cost & Total Cost \\
\hline & & Contingency & & - & + & - & + & & & & by Element \\
\hline 1.1.1 & DESIGN ENGINEERING TILE $1 \& \|$ & $7,962,000$ & 10.47 & 10 & 35 & 1.05 & 3.67 & $3.195 \%$ & $9.36 \%$ & $2,432,288$ & $10,394,288$ \\
\hline 1.1 .2 & QUALITY ASSURANCE & $1,990,500$ & 2.62 & 10 & 35 & 0.26 & 0.92 & $0.799 \%$ & $2.34 \%$ & 608,072 & $2,598,572$ \\
\hline 1.2 .1 & PROJECT MANAGEMENT & $5,573,400$ & 7.33 & 10 & 35 & 0.73 & 2.57 & $2.236 \%$ & $6.55 \%$ & $1,702,602$ & $7,276,002$ \\
\hline 1.2 .2 & CONSTRUCTION MANAGEMENT & $3,981,000$ & 5.24 & 10 & 40 & 0.52 & 209 & $1.833 \%$ & $5.37 \%$ & $1,395,575$ & $5,376,575$ \\
\hline 1.3 .1 & GENERAL CONDITIONS & $2,496,329$ & 3.28 & 10 & 40 & 0.33 & 1.31 & $1.149 \%$ & $3.37 \%$ & 875,110 & $3,371,439$ \\
\hline 1.3 .2 & SITEWORK & 863,098 & 1.14 & 10 & 40 & 0.11 & 0.45 & $0.397 \%$ & $1.16 \%$ & 302,567 & $1,165,665$ \\
\hline 1.3 .3 & CONCRETE & $4,209,942$ & 5.54 & 10 & 40 & 0.55 & 222 & $1.938 \%$ & $5.68 \%$ & $1,475,833$ & $5,685,775$ \\
\hline 1.3 .5 & METALS & $1,406,944$ & 1.85 & 10 & 40 & 0.19 & 0.74 & $0.648 \%$ & $1.90 \%$ & 493,217 & $1,900,161$ \\
\hline 1.3 .7 & THERMAL \& MOISTURE PROTECTION & 205,553 & 0.27 & 10 & 40 & 0.03 & 0.11 & $0.095 \%$ & $0.28 \%$ & 72,058 & $2 \pi 7,611$ \\
\hline 1.3.8 & DOORS \& WINDOWS & 128,013 & 0.17 & 10 & 40 & 0.02 & 0.07 & $0.059 \%$ & $0.17 \%$ & 44,876 & 172,889 \\
\hline 1.3 .9 & FINISHES & $1,064,376$ & 1.40 & 10 & 40 & 0.14 & 0.56 & $0.490 \%$ & $1.44 \%$ & 373,127 & $1,437,503$ \\
\hline 1.3 .10 & SPECIALTIES & $\begin{array}{r}19,410 \\
\end{array}$ & 0.03 & 10 & 40 & 0.00 & 0.01 & $0.009 \%$ & $0.03 \%$ & 6,804 & 26,214 \\
\hline 1.3 .11 & EQUIPMENT & $15,759,339$ & 20.73 & 10 & 45 & 207 & 9.33 & $8.189 \%$ & $23.99 \%$ & $6,234,880$ & $21,994,219$ \\
\hline 1.3 .12 & FURNISHINGS & 221,438 & 0.29 & 10 & 35 & 0.03 & 0.10 & $0.089 \%$ & $0.26 \%$ & 67,646 & 289,084 \\
\hline 1.3.13 & SPECIAL CONSTRUCTION & $1,167,753$ & 1.54 & 10 & 40 & 0.15 & 0.61 & $0.538 \%$ & 1.58\% & 409,366 & $1,577,119$ \\
\hline 1.3.14 & CONVEYING SYSTEMS & $1,987,042$ & 2.51 & 10 & 45 & 0.26 & 1.18 & $1.033 \%$ & $3.03 \%$ & 786,135 & $2,773,177$ \\
\hline 1.3.15 & MECHANICAL & $6,457,307$ & 8.49 & 10 & 40 & 0.85 & 3.40 & $2.973 \%$ & $8.71 \%$ & $2,263,667$ & $8,720,974$ \\
\hline 1.3 .16 & ELECTPICAL & $3,823,293$ & 5.03 & 10 & 40 & 0.50 & 2.01 & $1.760 \%$ & $5.16 \%$ & $1,340,290$ & $5,163,583$ \\
\hline 1.5 .1 & G\&APIF ADDER & $2,605,382$ & 3.43 & 10 & 35 & 0.34 & 1.20 & $1.045 \%$ & $3.06 \%$ & 795,911 & $3,401,293$ \\
\hline 1.5 .2 & PROCUREMENT FEES & $1,194,295$ & 1.57 & 10 & 35 & 0.16 & 0.55 & $0.479 \%$ & $1.40 \%$ & 364,842 & $1,559,137$ \\
\hline & ESCALATION & $12,898,324$ & 16.97 & 10 & 35 & 1.70 & 5.94 & $5.175 \%$ & $15.16 \%$ & $3,940,396$ & $16,838,720$ \\
\hline & SUBTOTAL & $76,014,738$ & 100.00 & & & & & $34.130 \%$ & & & \\
\hline & CALCULATED CONTINGENCY & $25,943,705$ & & & & & & & & & \\
\hline & RESULTANT TEC & $101,958,443$ & & & & & & & & & \\
\hline & ROUNDED TEC & $102,000,000$ & & & & & & & & & \\
\hline & PROJECT CONTINGENCY & $25,985,262$ & & & & & & $34.18 \%$ & & & \\
\hline & MANAGEMENT RESERVE & $5,320,361$ & & & & & & & & & \\
\hline & CONTINGENCY & $20,664,901$ & & & & & & & & & \\
\hline & TOTAL ESTIMATED COST & $102,000,000$ & & & & & & & & $25,985,262$ & $102,000,000$ \\
\hline
\end{tabular}

CONFIDENCE LEVEL AND ASSUMED RISKS:

The Lockheed Idaho Technologies Co. Cost Estimate Contingency Analysis Model is based on the applied contingency and the assumptions upon which the estimate was predicated. The model is applied with a suggested risk level of $18 \%$ and a level of confidence of $90 \%$ the estimate will fall within the bid range. The Contingency Analysis is based on a weighted average to provide a

$90 \%$ probability of underrun and a $10 \%$ probability of overnun.
CONTINGENCY ANALYSIS GUIDE BY TYPE OF ESTIMATE

Guidelines established by DOE/FM 50, Cost Estimating Guide, Vol. 6 ,

Cost Guide, and as presented in the INEL Cost Estimating Guide.

PLANNING $20 \%-30 \%$ Experimental/Special Conditions............Up to 50\% Conceptual $15 \%-25 \%$ Experimental/Special Conditions.............Up to $40 \%$ TITLEI

TITLE ॥

TITLE II/AFC
$10 \%-20 \%$

Market Conditions 


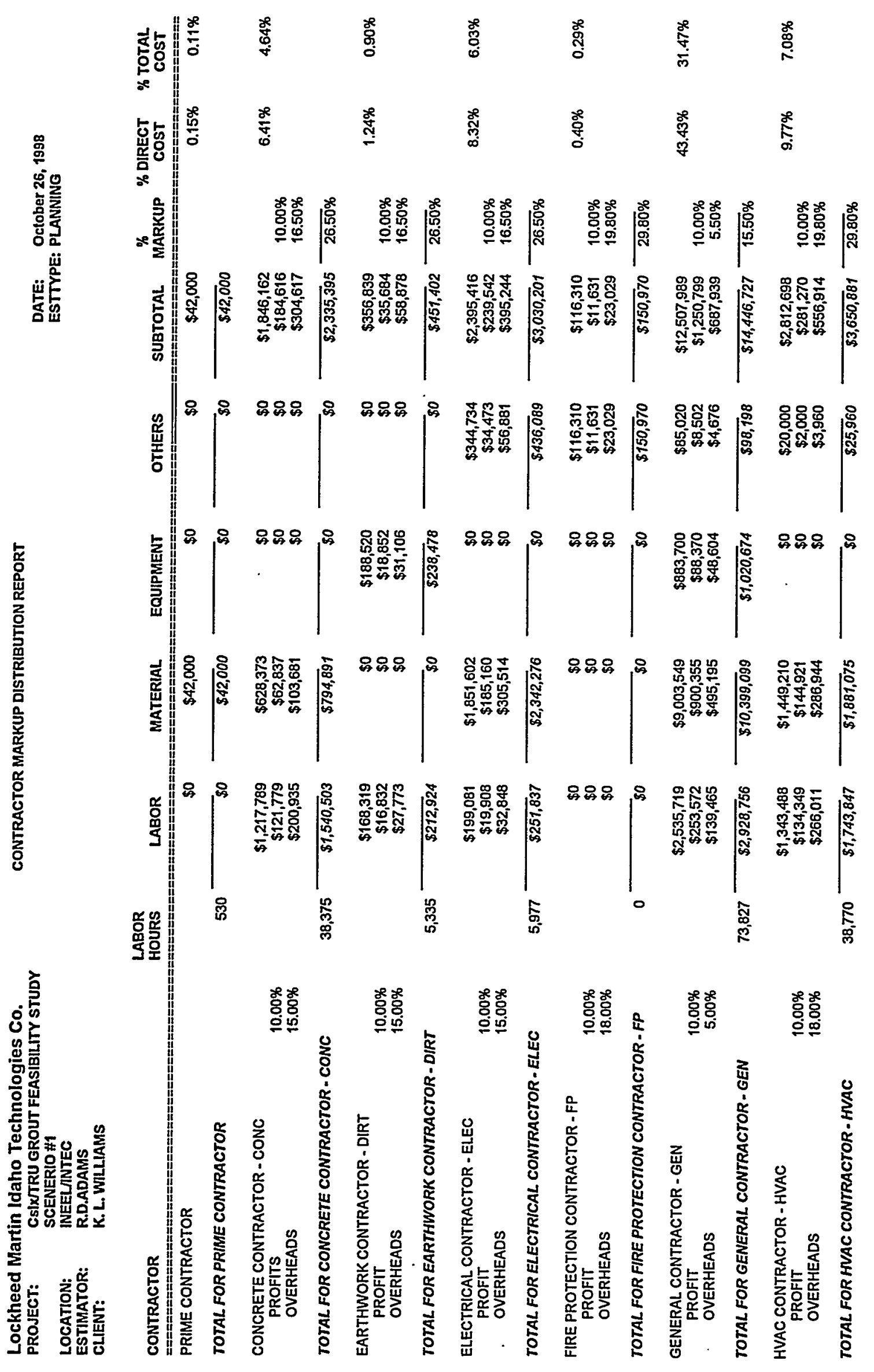




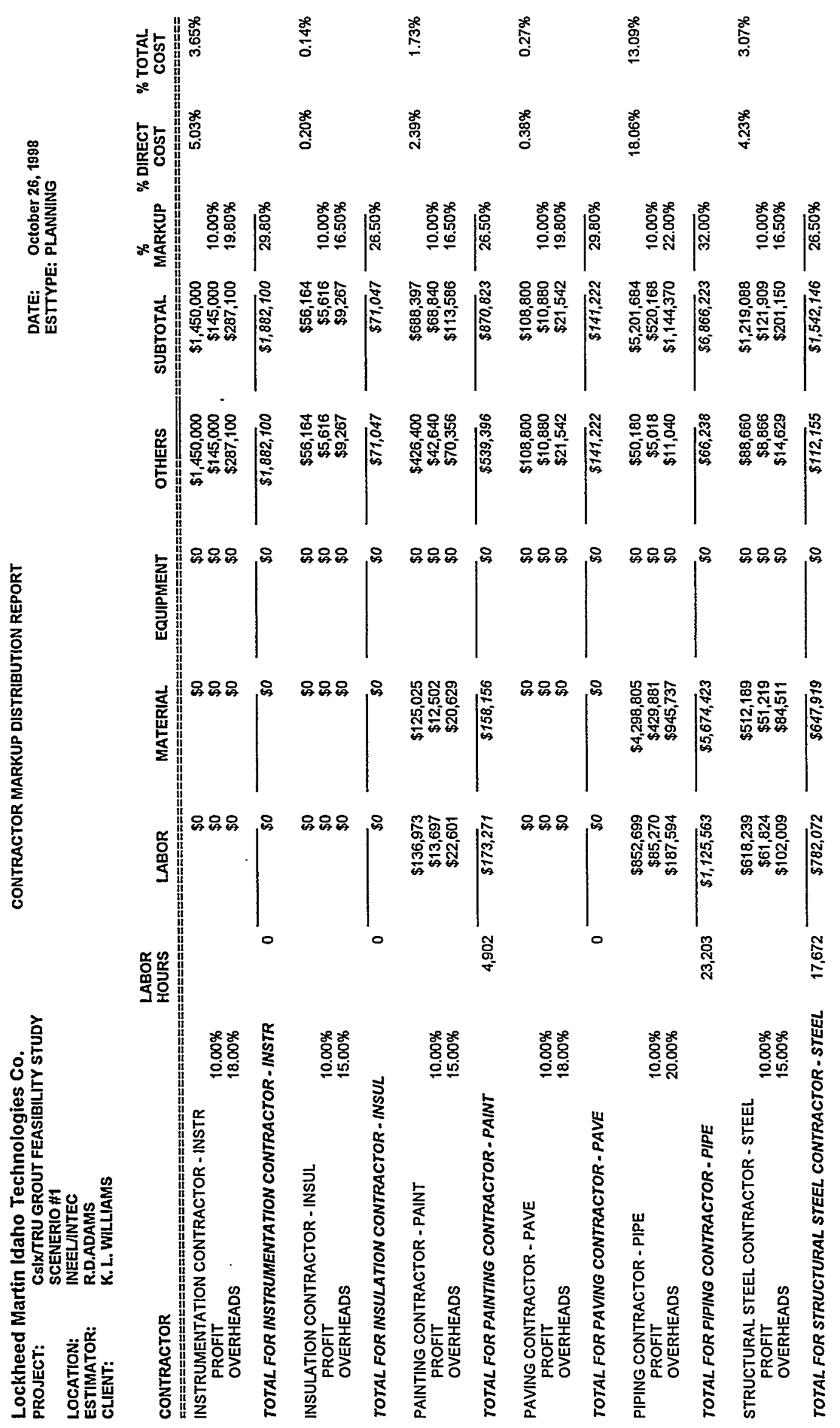




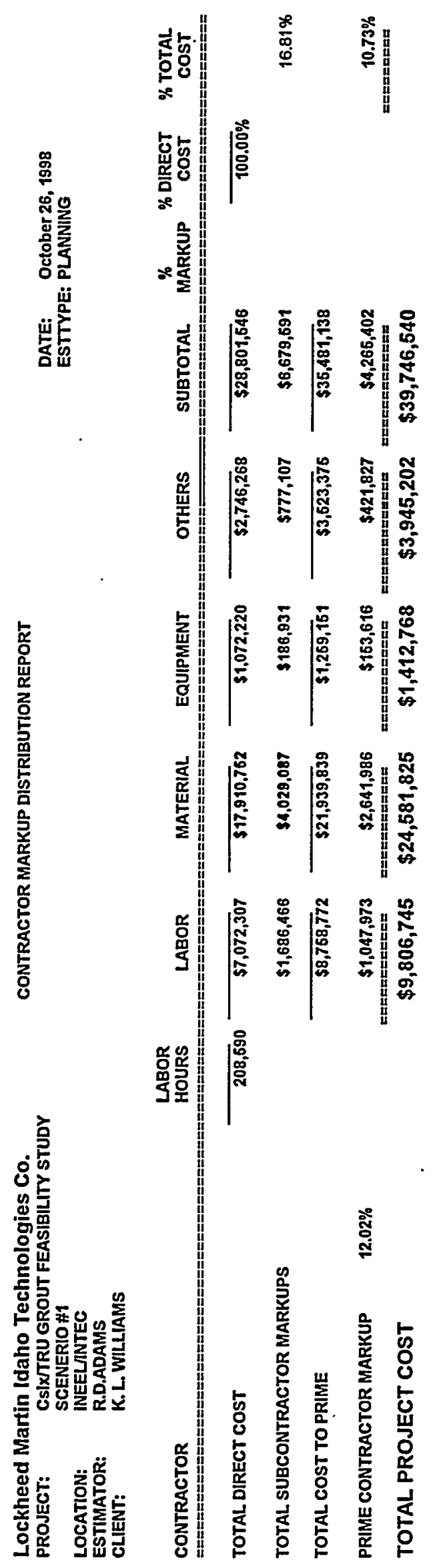




\section{FY-99 G\&A/PIF ADDER CALCULATION SHEET}

PROJECT:

DATE:

\section{CsIX/TRU GROUT FACILITY SCENERIO \#1}

PROCUREMENT FEE:

$10 / 14 / 98$

CONSTRUCTION $=$

$\mathrm{GFE}=$

Subtotal

FEE@3.0\%=
$\$ 39,809,836$

$\frac{\$ 0}{\$ 39,809,836}$

$\$ 39,809,836 \cdot 0.03=$

$\$ 1,194,295$

G\&A@ @27\% (with a ceiling of $\$ 500,000$ imposed per year)

CONSTRUCTION \$ OR CEILING * \# OF YEARS

YEARS OF CONST. $=3$

$\$ 1,500,000$

$\mathrm{GFE}=\quad \$ 0$

PROCUREMENT FEE =

$\$ 1,194,295$

Subtotal

$\$ 2,694,295$

FEE @ $27 \%=$

$\$ 2,694,295 \cdot 0.27=$

$\$ 727,460$

PIF@4.5\%

$\begin{array}{lr}\text { CONSTRUCTION }= & \$ 39,809,836 \\ \text { GFE }= & \$ 0 \\ \text { PROCUREMENT FEE }= & \$ 1,194,295 \\ \text { G\&A }= & \$ 727,460 \\ \text { Subtotal } & \$ 41,731,591\end{array}$

FEE @ $4.5 \%=$

$\$ 41,731,591 \cdot 0.045=\quad \$ 1,877,922$

TOTAL PROCUREMENT FEE:

$\$ 1,194,295$

TOTAL G\&A FEE:

$\$ 727,460$

TOTAL PIF:

$\$ 1,877,922$ 
Lockheed Martin Idaho Technologies Co: PROIECT NAME: CSIX/TRU GROUT FEASIBILITY STUDY

LOCATION 1: REQUESTOR: INEELINTEC

K. L. WILLIAMS SCENERIO \#1 - OPC

COST ESTIMATE SUMMARY

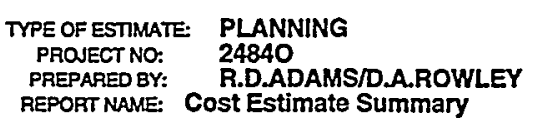

REPORT NAYE

Cost Estimate Summary

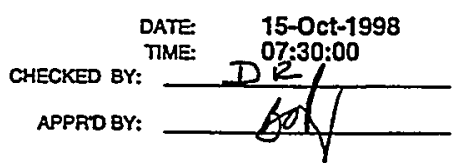

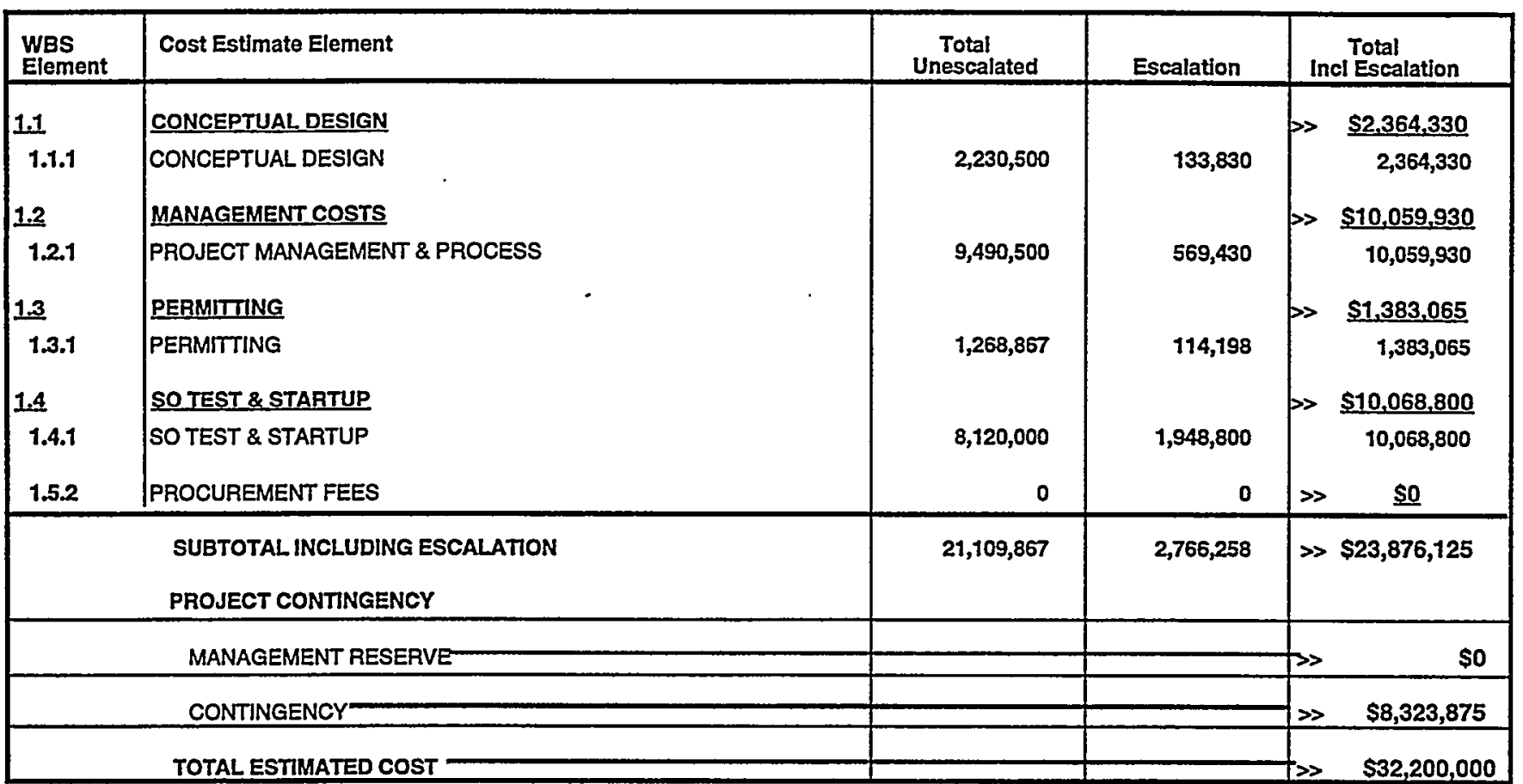

\section{PROJECT COST PARAMETERS}

EDI AS A $\%$ OF CONST. + GFE $=21.00 \%$

CONTINGENCY $=34.86 \%$ 


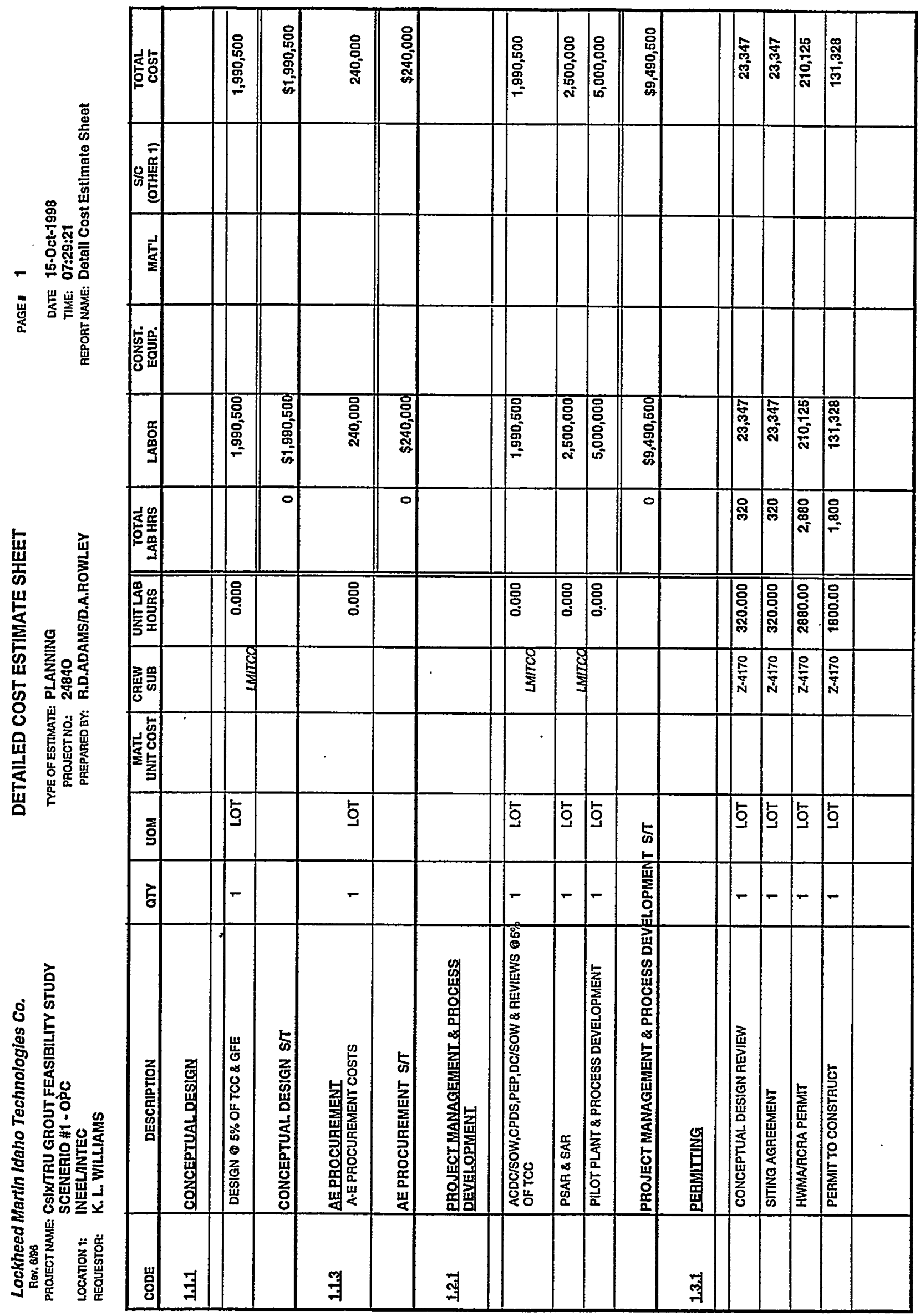




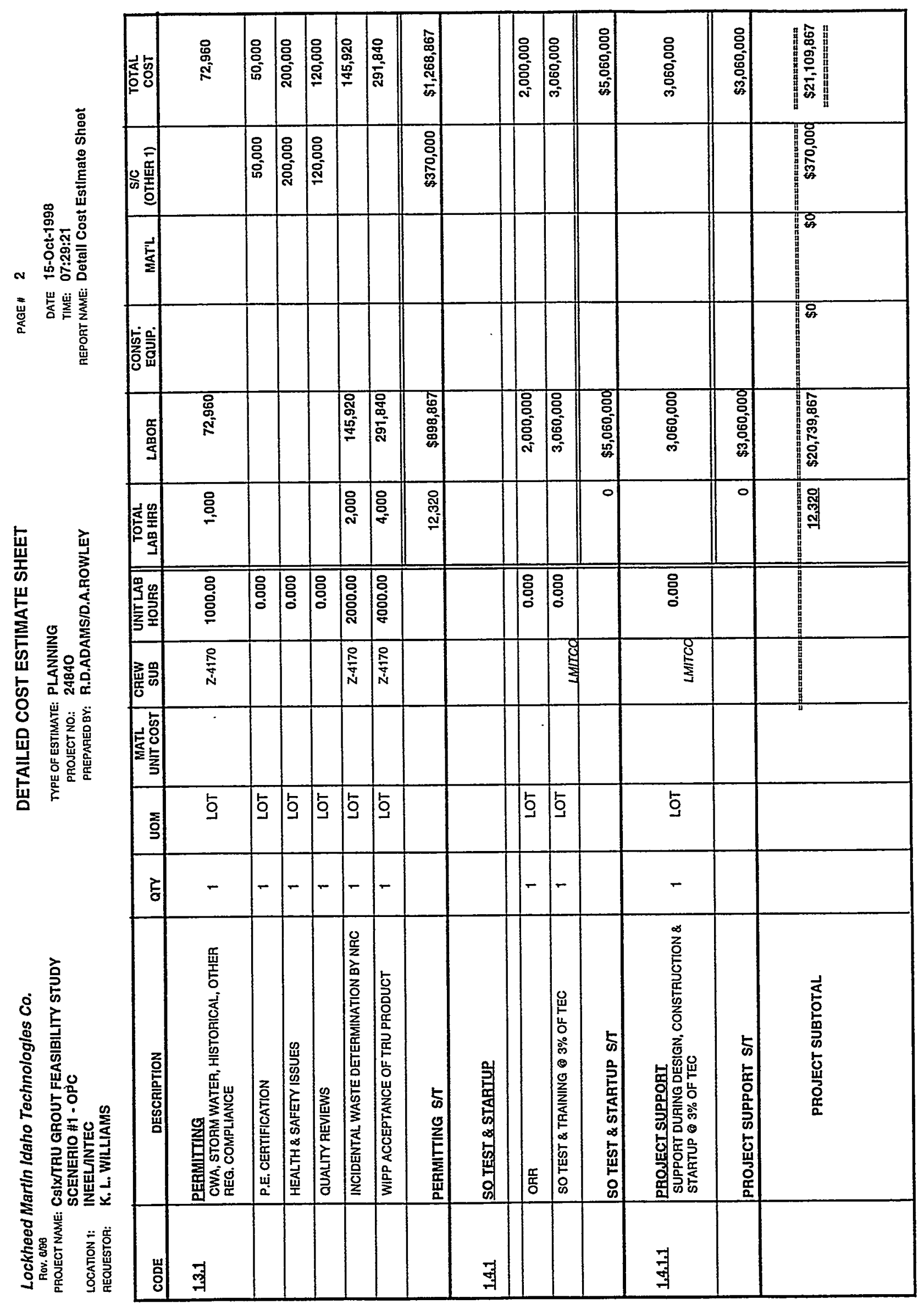


Lockheed Martin Idaho Technologies Co. PROJECT NAME: CSIX/TRU GROUT FEASIBILITY STUDY SCENERIO \#1 - OPC

LOCATION 1: INEELINTEC

REQUESTOR: K.LWILLIAMS

\section{CONTINGENCY ANALYSIS}

TYPE OFESTIMATE: PROSECT NO: PREPARED BY:

\author{
PLANNING \\ 24840 \\ R.D.ADAMSID.A.ROWLEY \\ DATE: $15-$ Oct-1998 \\ TIME: 07:29:54
}

REPORT NAME: Contingency Analysis

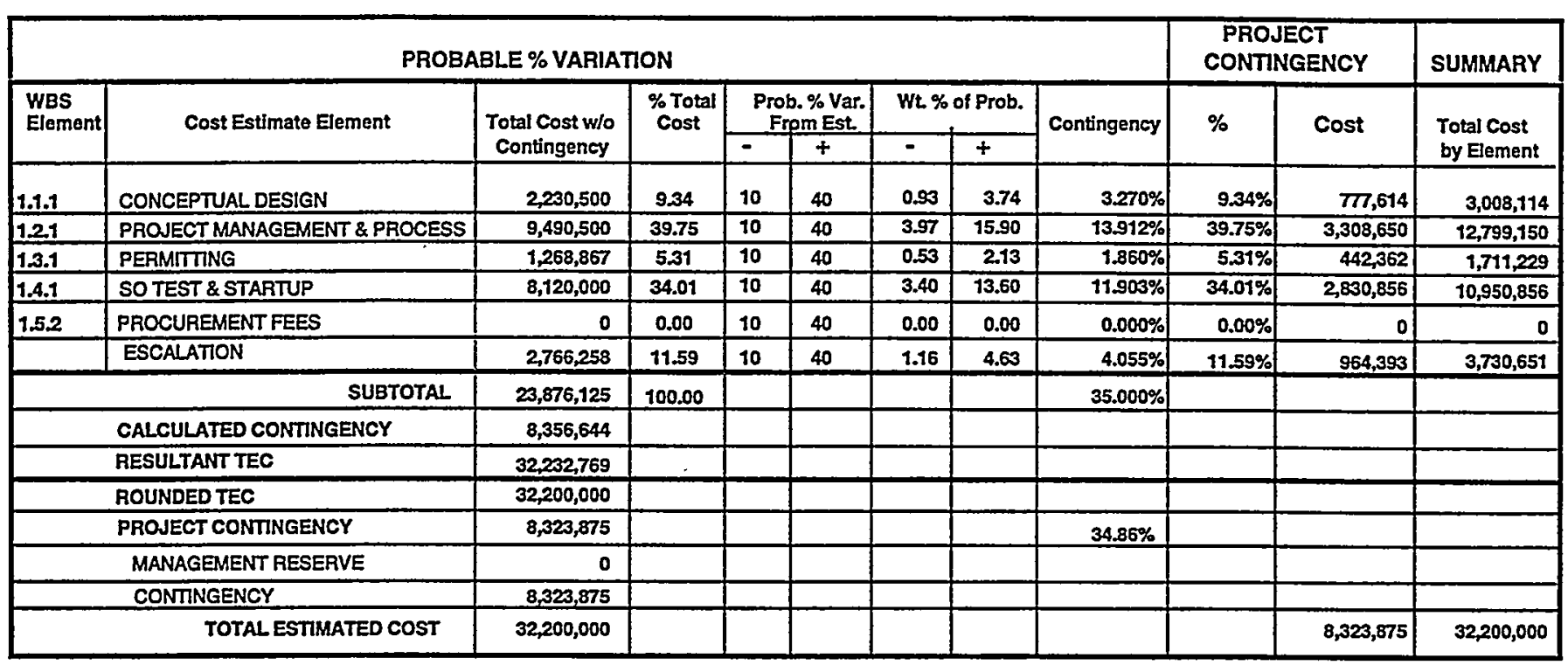

CONFIDENCE LEVEL AND ASSUMED RISKS:

The Lockheed Idaho Technologies Co. Cost Estimate Contingency Analysis Model is based on the applied contingency and the assumptions upon which the estimate was predicated. The model is applied with a suggested risk level of $18 \%$ and a level of confidence of $90 \%$ the estimate will fall within the bid range. The Contingency Analysis is based on a weighted average to provide a $90 \%$ probability of underrun and a $10 \%$ probability of overnun.
CONTINGENCY ANALYSIS GUIDE BY TYPE OF ESTIMATE

Guidelines established by DOE/FM 50, Cost Estimating Guide, Vol. 6 , Cost Guide, and as presented in the INEL Cost Estimating Guide. PLANNING $20 \%-30 \%$ Experimental/Special Conditions.............Up to 50\% Conceptual $15 \%-25 \%$ Experimental/Special Conditions.............Up to $40 \%$ TITLE I TITLE II/AFC
$5 \%-15 \%$

Market Conditions 
Lockheed Martin Idaho Technologies Company

\section{COST ESTIMATE SUPPORT DATA RECAPITULATION}

Project Title: CsIX TRU Grout Feasibility Study - Scenario \#2

Estimator: Adams / Rowley

Date: $\quad$ October 13, 1998

Estimate Type: Planning

File: Approved By:

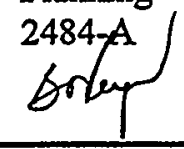

\section{SCOPE OF WORK: Brief description of the proposed project.}

This project consists of several parts. Cells in the existing New Waste Calcining Facility (NWCF) will be used to house the Cesium Ion Exchange equipment. A new facility will be built to grout the resulting liquid waste into drums. An interim storage facility and a TRUPAC loading facility will be built to temporarily house the waste drums and load them for shipment to WIPP.

The estimate includes all work associated with modifying the existing NWCF facility at INTEC to house a new Cesium Ion Exchange (CsIX) process. Modifications include:

A. Demolition of existing piping, structural steel and electrical in the calciner cell.

B. Removal of silica gel from the existing adsorber vessels.

C. Demolition of existing piping, structural steel, vessels, equipment, and electrical from the adsorber cell.

D. Demolition of other piping and electrical in NWCE as required.

E. Installation of a new partition wall inside the calciner cell.

F. Installation of new piping, equipment, and electrical in the calciner cell.

G. Installation of new piping, equipment, and electrical in the adsorber cell.

$\mathrm{H}$. Installation of new piping, equipment, and electrical in the valve cubicle.

I. Installation of new piping, equipment, and electrical in the hot sump tank removal cell.

II. BASIS OF THE ESTIMATE: Drawings, Design Report, Engineers Notes and/or other documentation upon which the estimate is originated.

The estimate is based on the preliminary design drawings, the feasibility description, equipment list, and discussions held with $\mathrm{H}$. S. Forsythe.

'III. ASSUMPTIONS: Conditions statements accepted or supposed true without proof of demonstration. An assumption has a direct impact on total estimated cost.

For NWCF: 


\begin{tabular}{|ll} 
& \multicolumn{1}{c}{ COST ESTIMATE SUPPORT DATA RECAPITULATION } \\
Project Title: & CsIX TRU Grout Feasibility Study- Scenario \#2 \\
File: & 2484-A
\end{tabular}

File: 2484-A

Page 2
A. Assume sufficient "cold" work will be available to enable workers to remain working after they have received their maximum annual radiation exposure.
B. Assume the radiation general body fields will not exceed the estimated fields provided by Maria Dumas and Griff Colson 10/1/98.
C. Assume the construction period will be four (4) years beginning in FY-04.
D. Assume the silica gel will be removed from the adsorber vessels and boxed as mixed waste. Final disposition and disposal costs are not included.
E. Personnel training estimates are based on workers being allowed to receive a total body exposure of $1.5 \mathrm{R}$ per year.
F. Assume LMITCO operations personnel will operate the overhead crane.

General:

1. Due to the amount of hot work in NWCF, this work will drive the schedule from three years to four years.

2. Utilities and transfer lines are included in the estimate for the various facilities as needed.

3. All concrete surfaces in hot cells will be coated with stainless steel liner or epoxy coating as identified on the drawings.

4. For non-NWCF work, supervisor hours are figured at $5 \%$ of total labor hours.

5. Training hours are equal to $3 \%$ of total labor hours.

6. All building foundations start at $-6^{\prime}-0$ unless shown otherwise on the drawings.

7. Epoxy coating system is included on the Interim Storage Facility and TRUPAC Loading Station concrete floors.

8. All structural components of Grout facility are concrete except roof framing.

9. Grout Facility roof is standing seam-roofing panels over structural steel framing.

10. All overhead doors are motor-operated.

11. The exterior of the Grout Facility is left exposed concrete.

12. Included a concrete pad under the Grout Facility outside bin/tank area.

13. TRUPAC loading facility includes two 10ton-bridge cranes, work platforms, pallet scales, stretch wrap machine, and vacuum pump system.

14. TRUPAC station will require minimal $\mathrm{H} \& \mathrm{~V}$.

15. An exhaust stack with appropriate monitoring is required for the Grout Facility.

16. Interim Storage Facility includes a ventilation system not shown on the drawings.

17. Ten shielded containers are included for storage of UDS. The remaining 40 containers needed during production will be supplied by operations.

18. The initial charge of ion exchange resin is included in this estimate. The remaining resin required for production will be supplied by operations. 


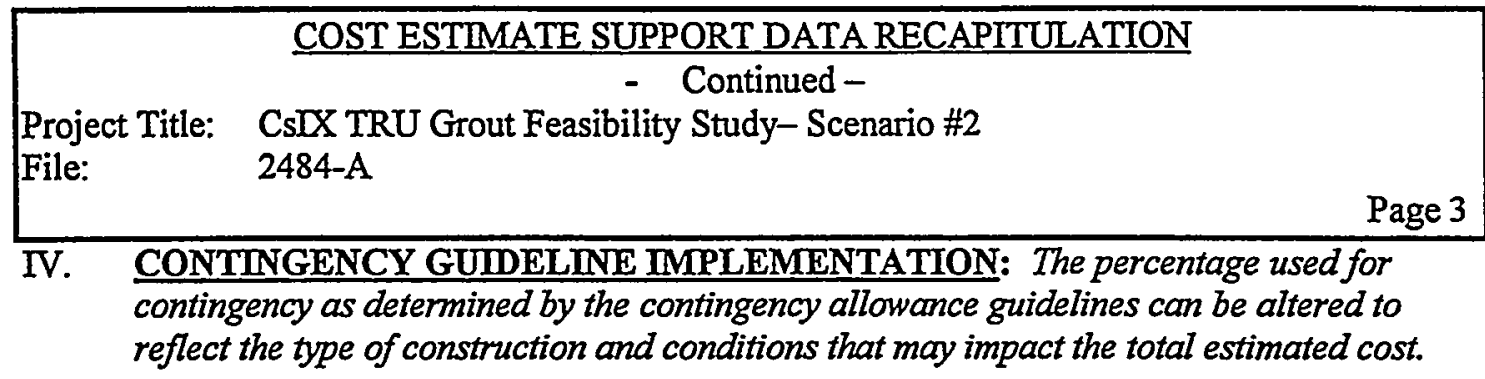

Contingency for this scenario has been calculated to be $35.2 \%$. Items of possible risk considered for this calculation are:
A. High to moderate levels of radioactive contamination. With regards to the ALARA program, work in the "hot" areas will be extremely difficult and radiation exposure to workers will be higher than desired.
B. The silica gel is anticipated to contain extremely high levels of radiation and is compounded by being a mixed waste for which a disposal process does not currently exist. It is possible that a new facility will have to be erected for the sole purpose of processing the contaminated silica gel for final disposal.
C. Due to the radiation fields, which are known to exist in NWCF, maintaining a constant work force will be difficult. Sufficient "clean" work will have to be worked concurrently with this scenario in order to sustain a constant work force.
D. It is anticipated that "travelers" (craftsmen from geographical areas other than the Idaho Falls / Pocatello area) will make up a majority of the work force. These workers tend to work for short periods of time and then return home. As a result the training allowance for this scenario appears to be high but could be substantially higher.
E. Most systems have not been detailed. Many changes to the assumed requirements will appear as these systems are developed further.

\section{OTHER COMMENTS/CONCERNS SPECIFIC TO THE ESTIMATE}

A. Costs from the Detailed Cost Estimate sheets are direct costs for material, labor, equipment, and special subcontracts. Costs for corresponding divisions on the Cost Estimate Summary sheet include all applicable indirect costs (overhead, profit, commission on subcontracts, sales tax, and bond).

B. Costs for each activity represent present day costs escalated to the appropriate activity midpoint.

C. Subcontractor labor costs reflect INEEL Site Jurisdictional Agreement craft labor rates.

D. Costs for Lockheed Martin General and Administrative allowance (G \& A) and Performance Incentive Factor (PIF) have been included in this estimate. 
Lockheed Martin Idaho Technologies Co. PROIECT NAME: CSIXJTRU GROUT FEASIBILITY STUDY LOCATION :: SCENERIO \#2 (NWCF)

REOUESTOR: K. L WILLIAMS
COST ESTIMATE SUMMARY

TYPE OF ESTIUATE PLANINING PROSECTNOTE PLANNING PROSECTNO: 2484A

PREPARED BY: R.D.ADAMS/D.A.ROWLEY CHECKED BY REPORT NAME: Cost Estimate Summary

\begin{tabular}{|c|c|c|c|c|}
\hline $\begin{array}{l}\text { WBS } \\
\text { Element }\end{array}$ & Cost Estimate Element & $\begin{array}{c}\text { Total } \\
\text { Unescalated }\end{array}$ & Escalation & $\begin{array}{c}\text { Total } \\
\text { Incl Escalation }\end{array}$ \\
\hline $\begin{array}{l}1.1 \\
1.1 .1 \\
1.1 .2 \\
1.2 \\
1.2 .1 \\
1.2 .2 \\
1.3 \\
1.3 .1 \\
1.3 .2 \\
1.3 .3 \\
1.3 .5 \\
1.3 .7 \\
1.3 .8 \\
1.3 .9 \\
1.3 .10 \\
1.3 .11 \\
1.3 .12 \\
1.3 .13 \\
1.3 .14 \\
1.3 .15 \\
1.3 .16 \\
1.4 \\
1.4 .1 \\
1.5 \\
1.5 .1 \\
1.5 .2 \\
\end{array}$ & 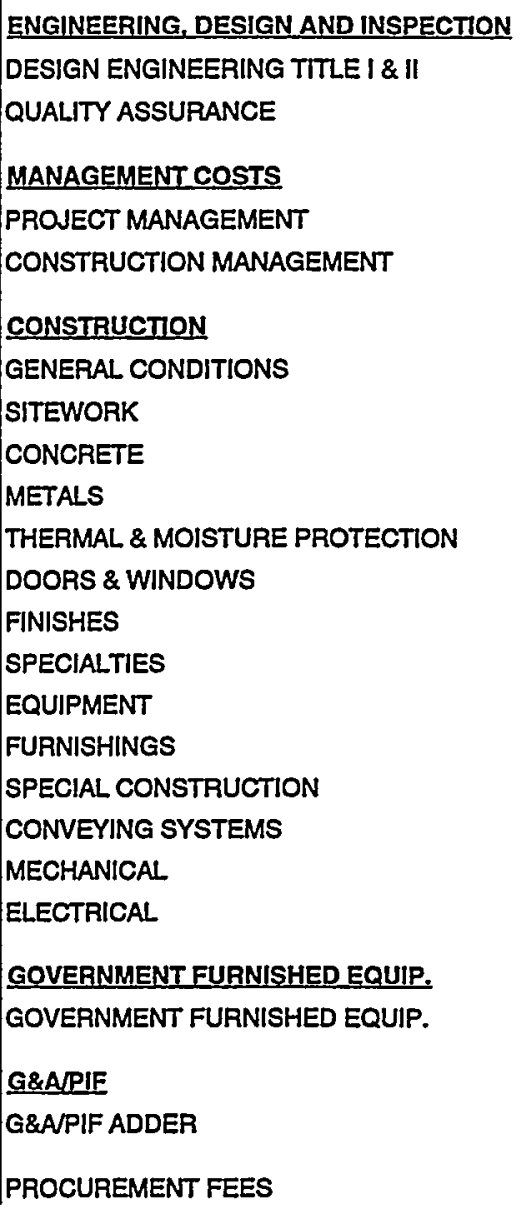 & $\begin{array}{r}9,877,200 \\
2,469,300 \\
\\
6,914,000 \\
4,161,000 \\
\\
6,676,627 \\
825,688 \\
2,631,536 \\
638,131 \\
105,632 \\
87,785 \\
824,547 \\
13,315 \\
12,321,818 \\
133,566 \\
1,503,468 \\
2,176,670 \\
8,925,228 \\
4,746,271 \\
\\
7,775,733 \\
3,745,643 \\
1,328,484\end{array}$ & $\begin{array}{r}1,669,157 \\
206,422 \\
657,884 \\
159,533 \\
26,408 \\
21,946 \\
206,137 \\
3,329 \\
3,080,454 \\
33,391 \\
375,867 \\
544,168 \\
2,231,307 \\
1,186,568 \\
\\
1,943,933 \\
\\
936,411 \\
332,121\end{array}$ & 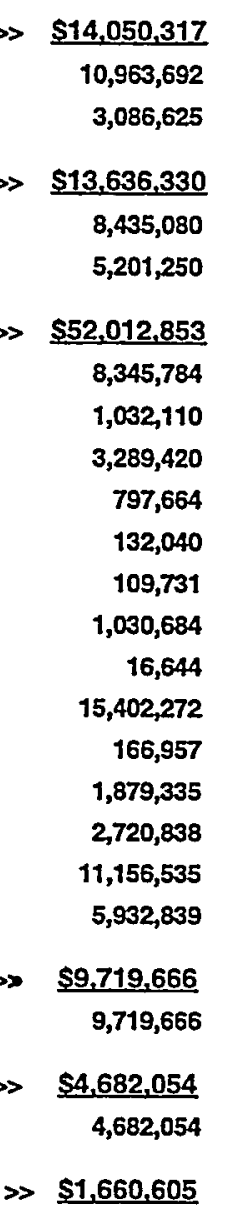 \\
\hline$\cdot$ & $\begin{array}{l}\text { SUBTOTAL INCLUDING ESCALATION } \\
\text { PROJECT CONTINGENCY }\end{array}$ & $77,881,642$ & $17,880,183$ & >> $\$ 95,761,825$ \\
\hline & MANAGEMENT RESERVE- & & & $\$ 6,807,518$ \\
\hline & CONTINGENCY & & & $\$ 27,730,657$ \\
\hline & TOTAL ESTMMATED COST & & & $\$ 130,300,000$ \\
\hline
\end{tabular}

PROJECT COST PARAMETERS

EDI AS A \% OF CONST. + GFE $=23.00 \%$

CONTINGENCY $=36.07 \%$ 


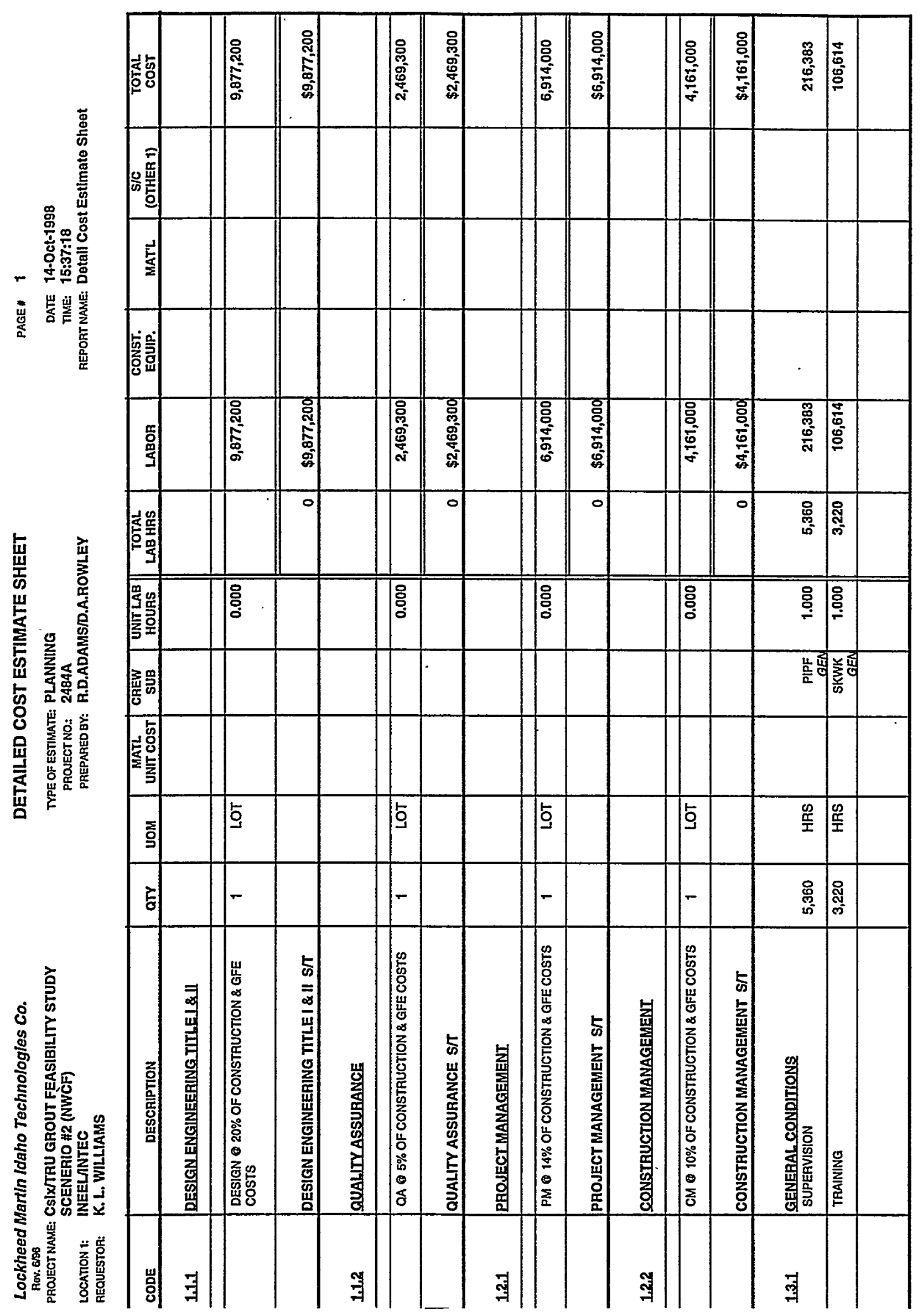




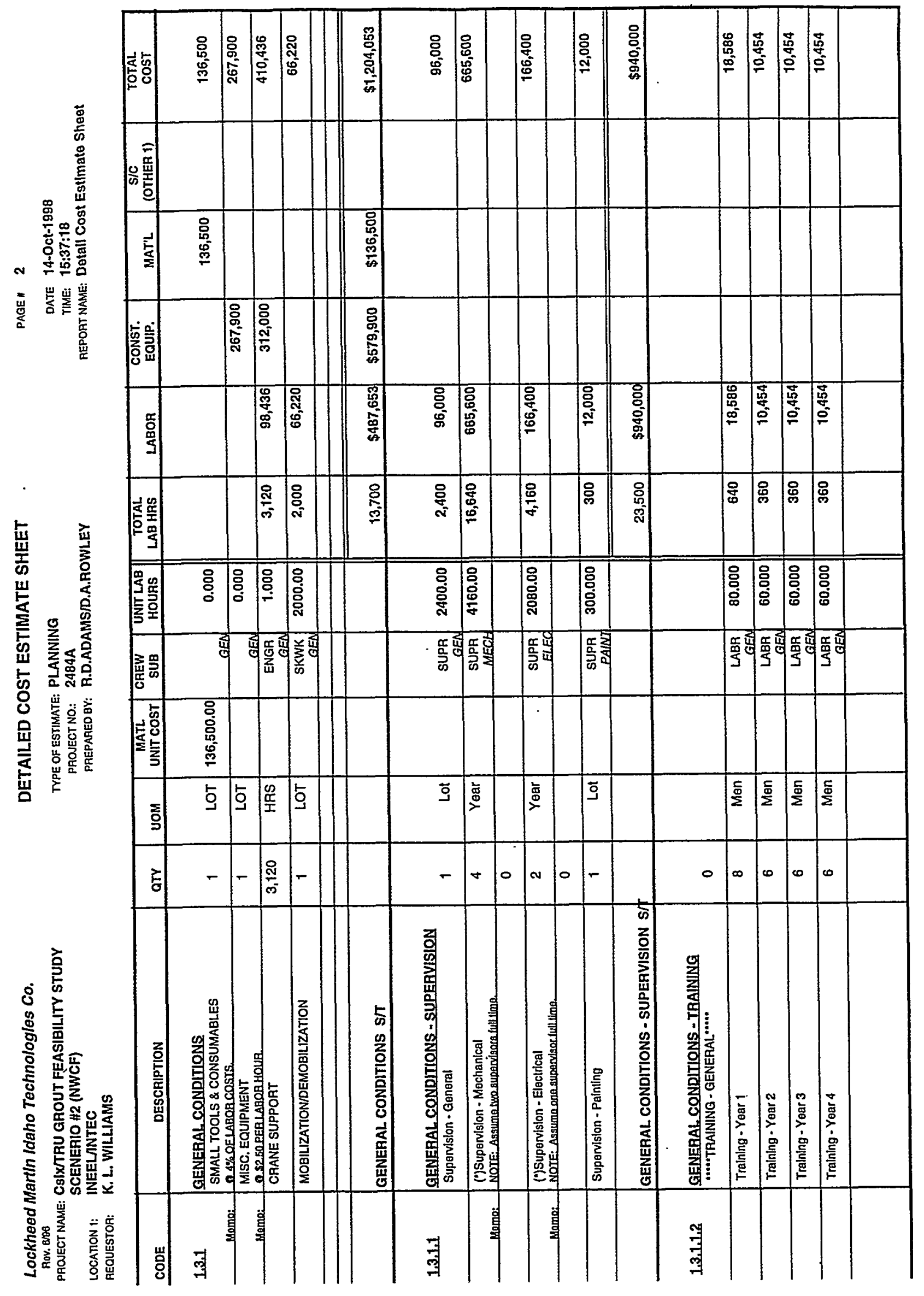




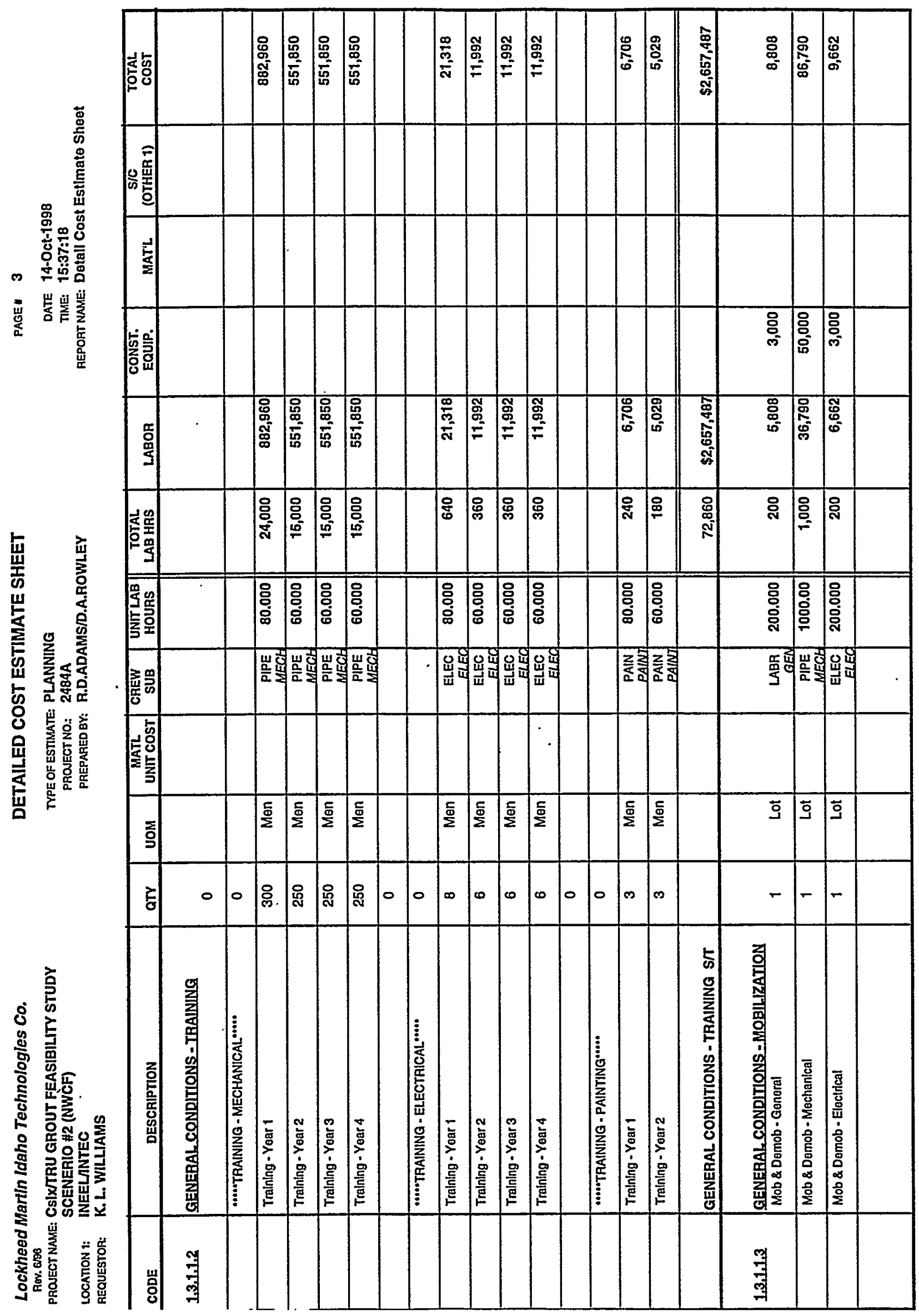




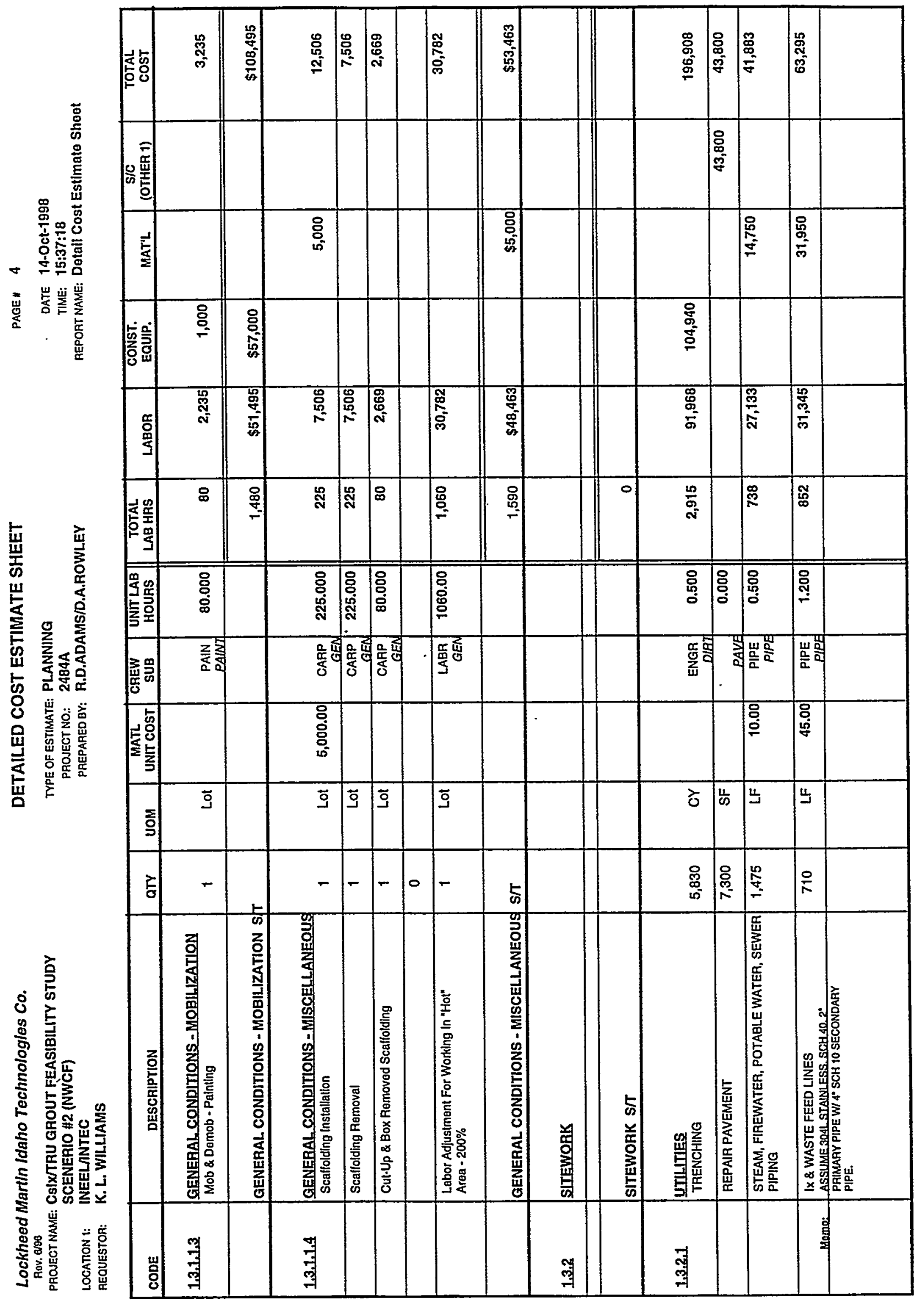




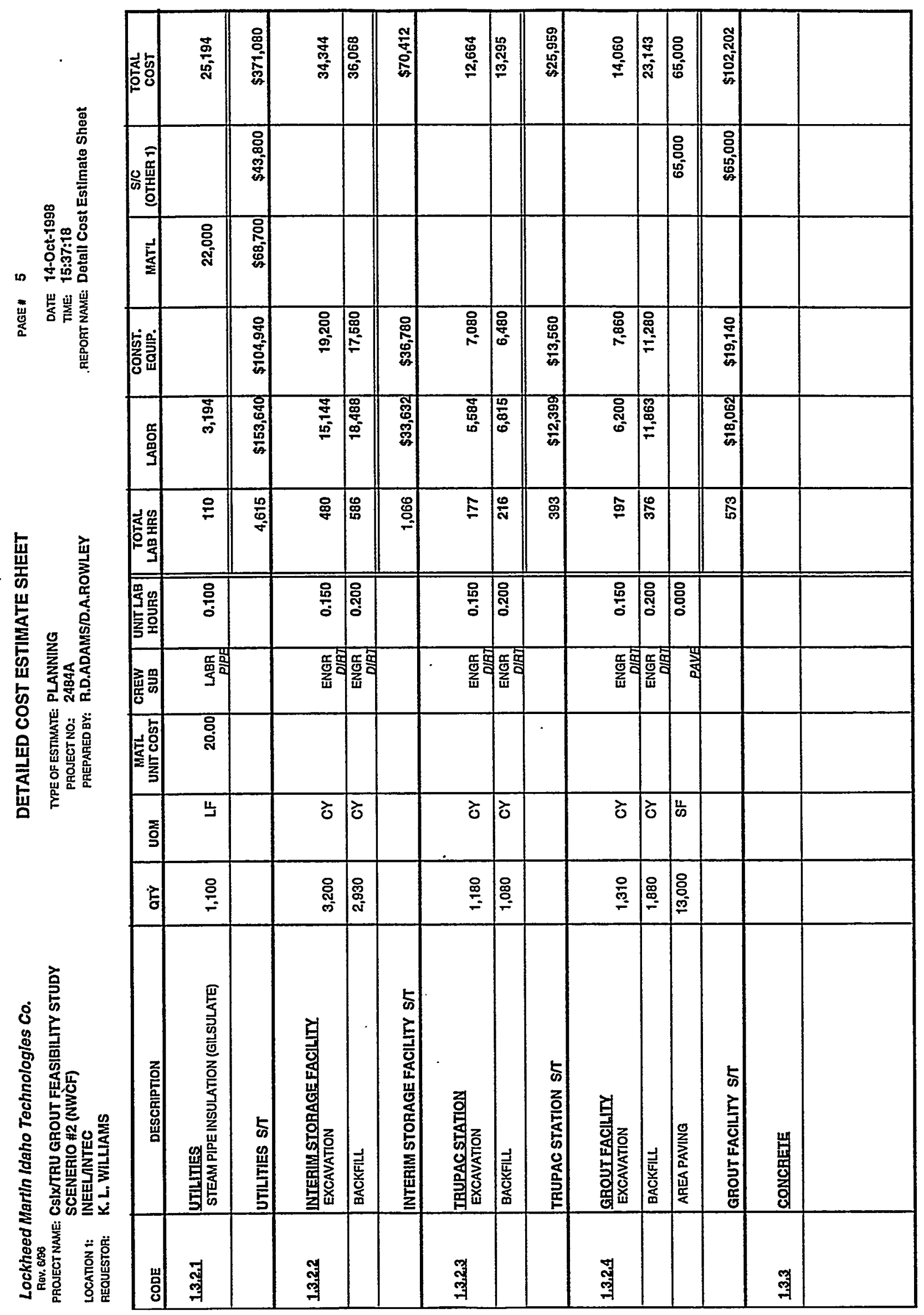




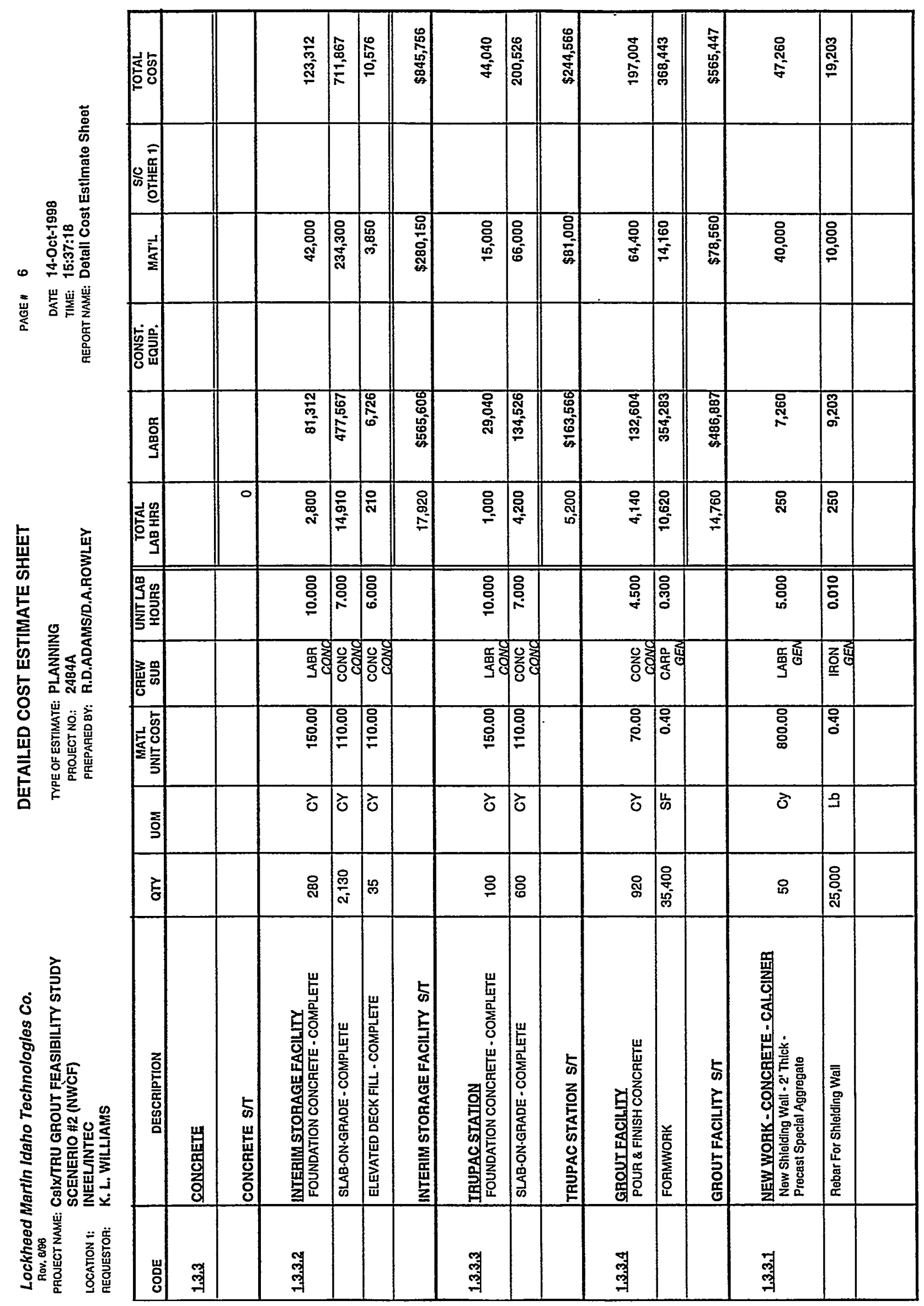




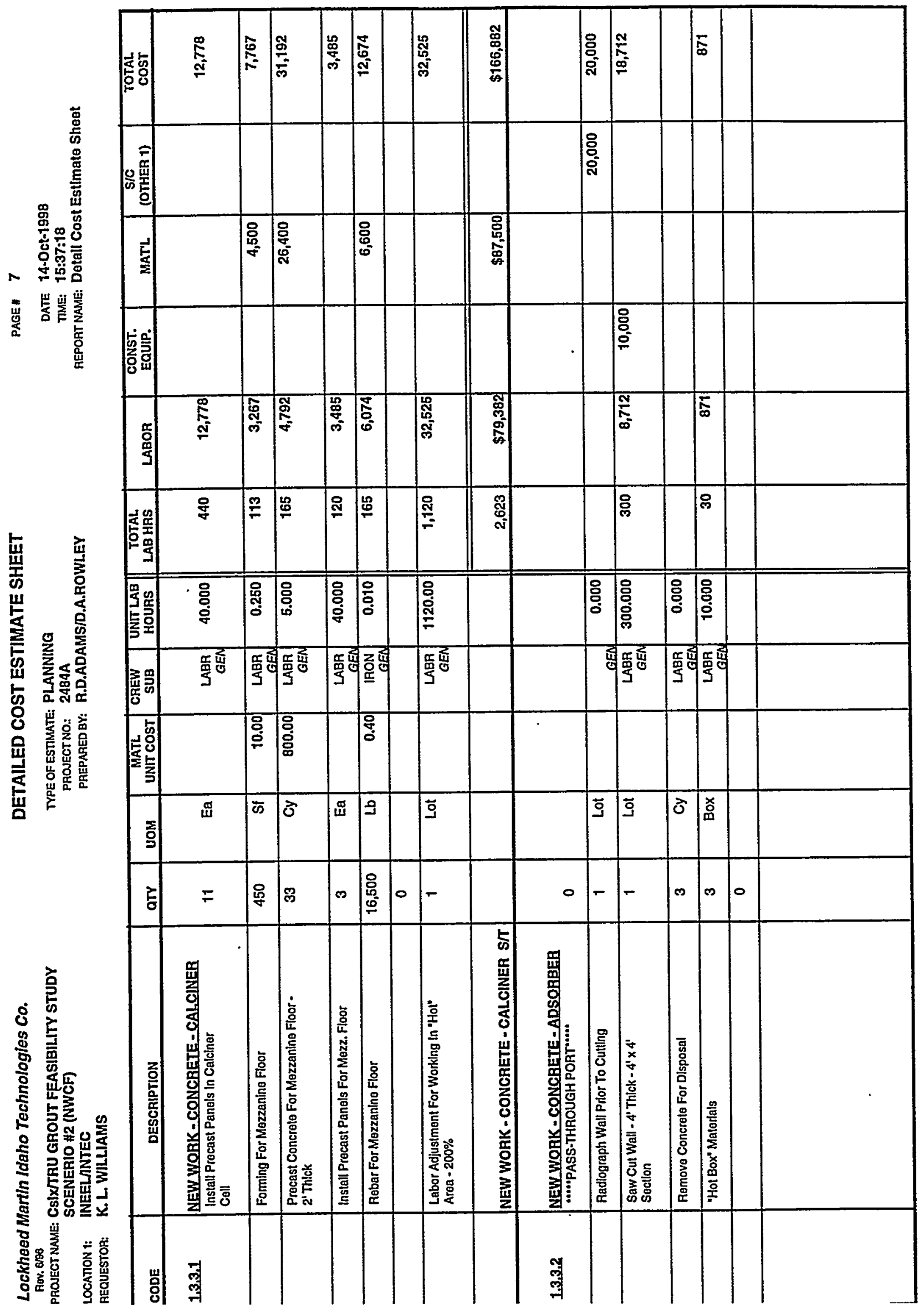




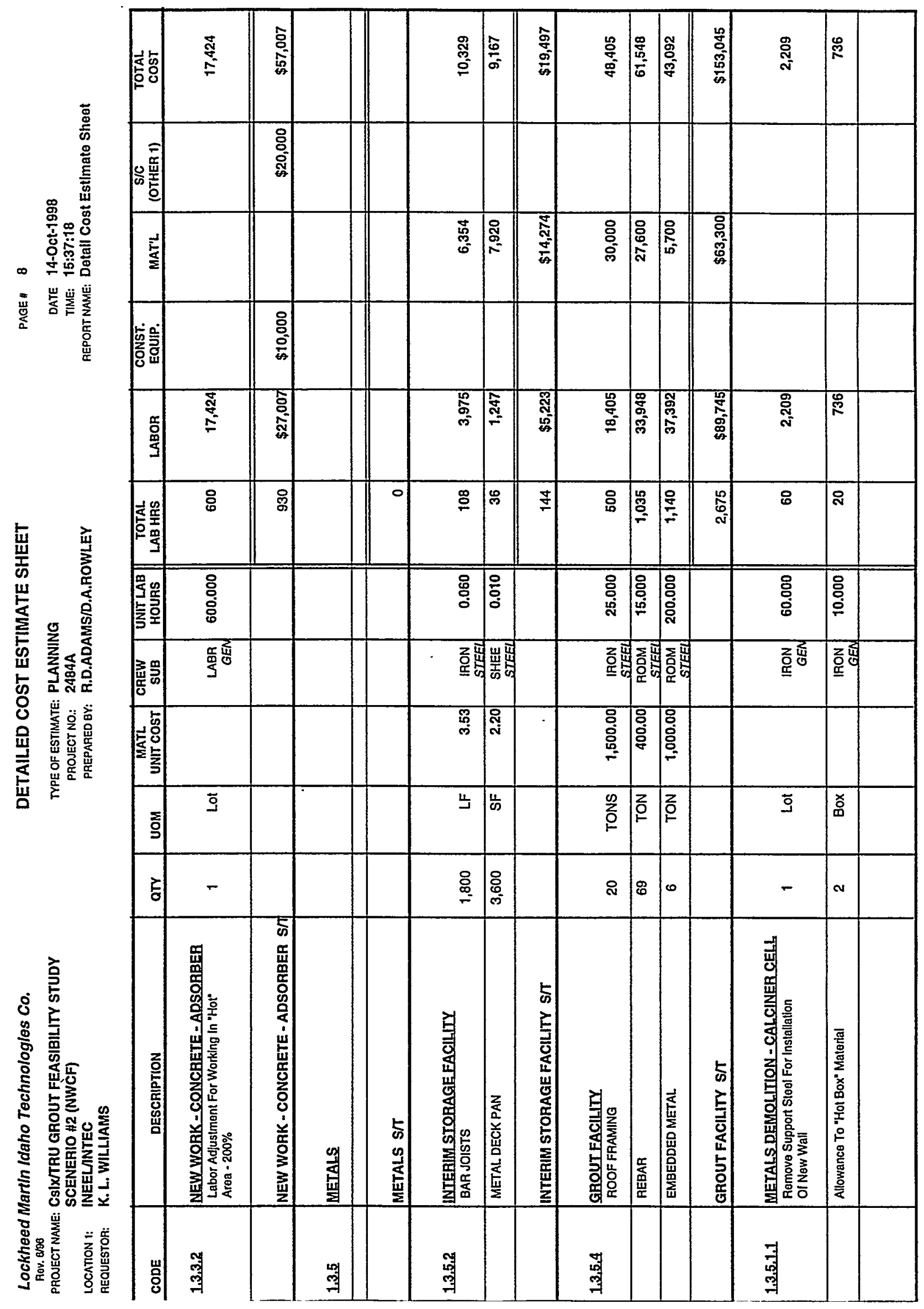




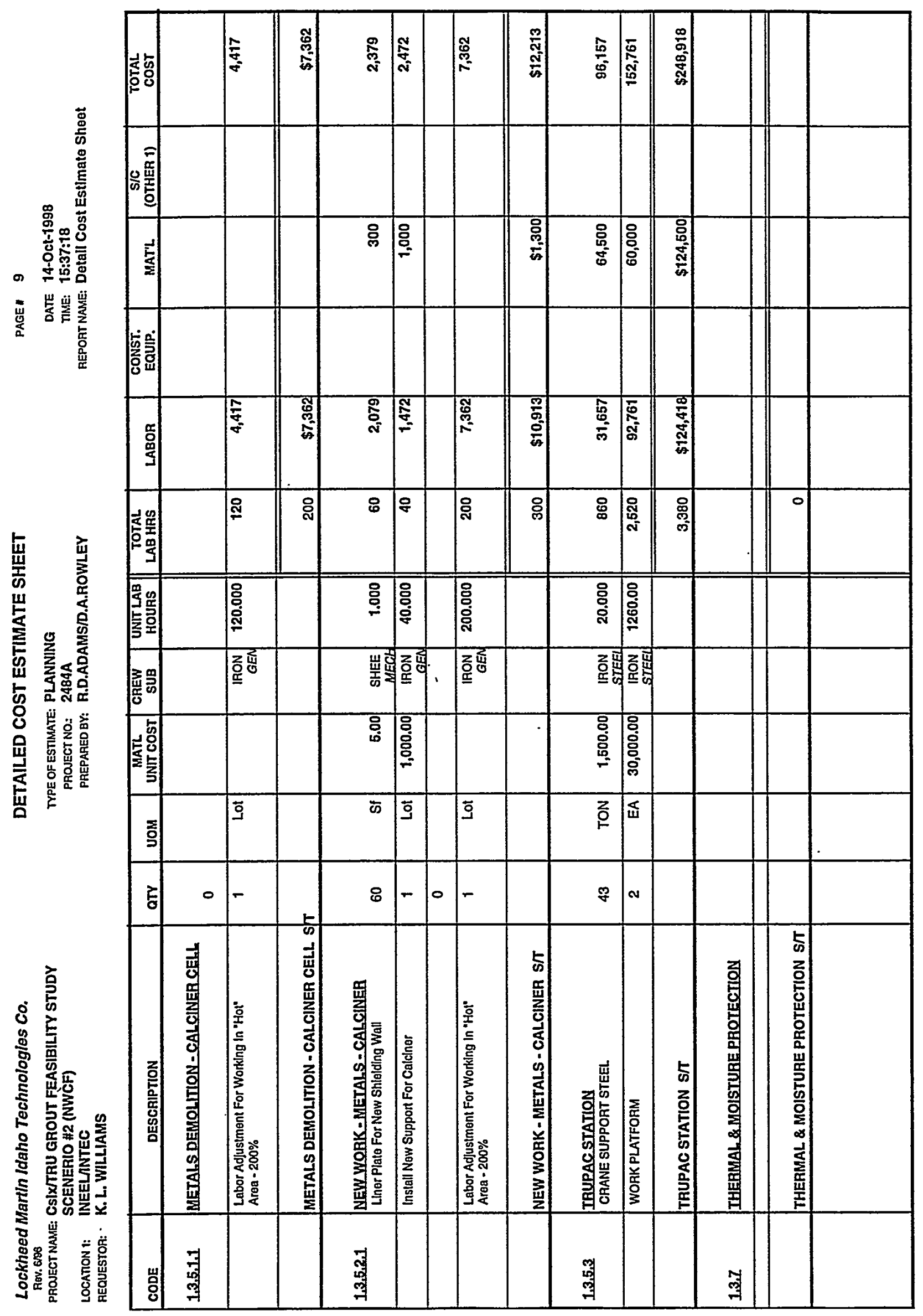




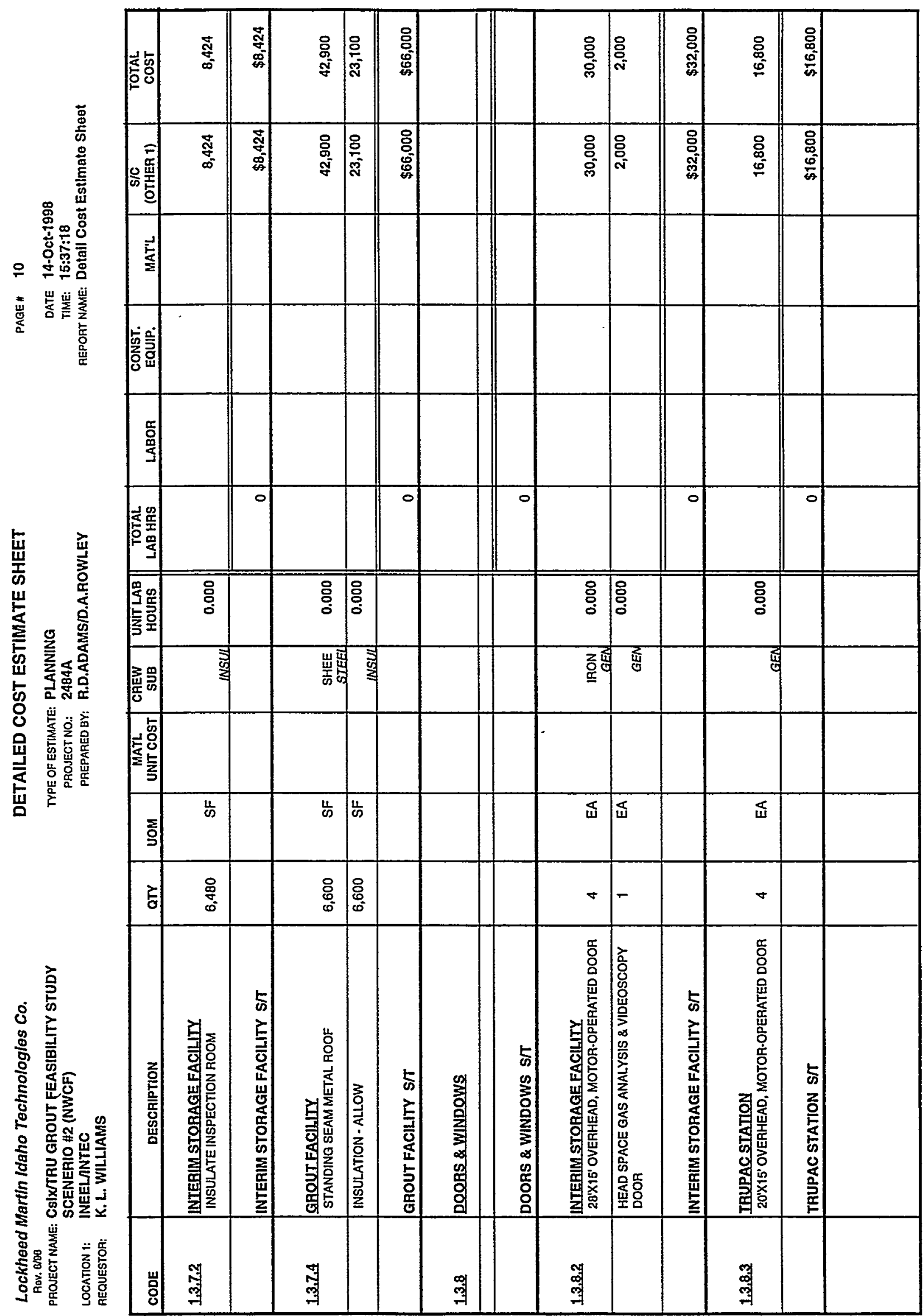




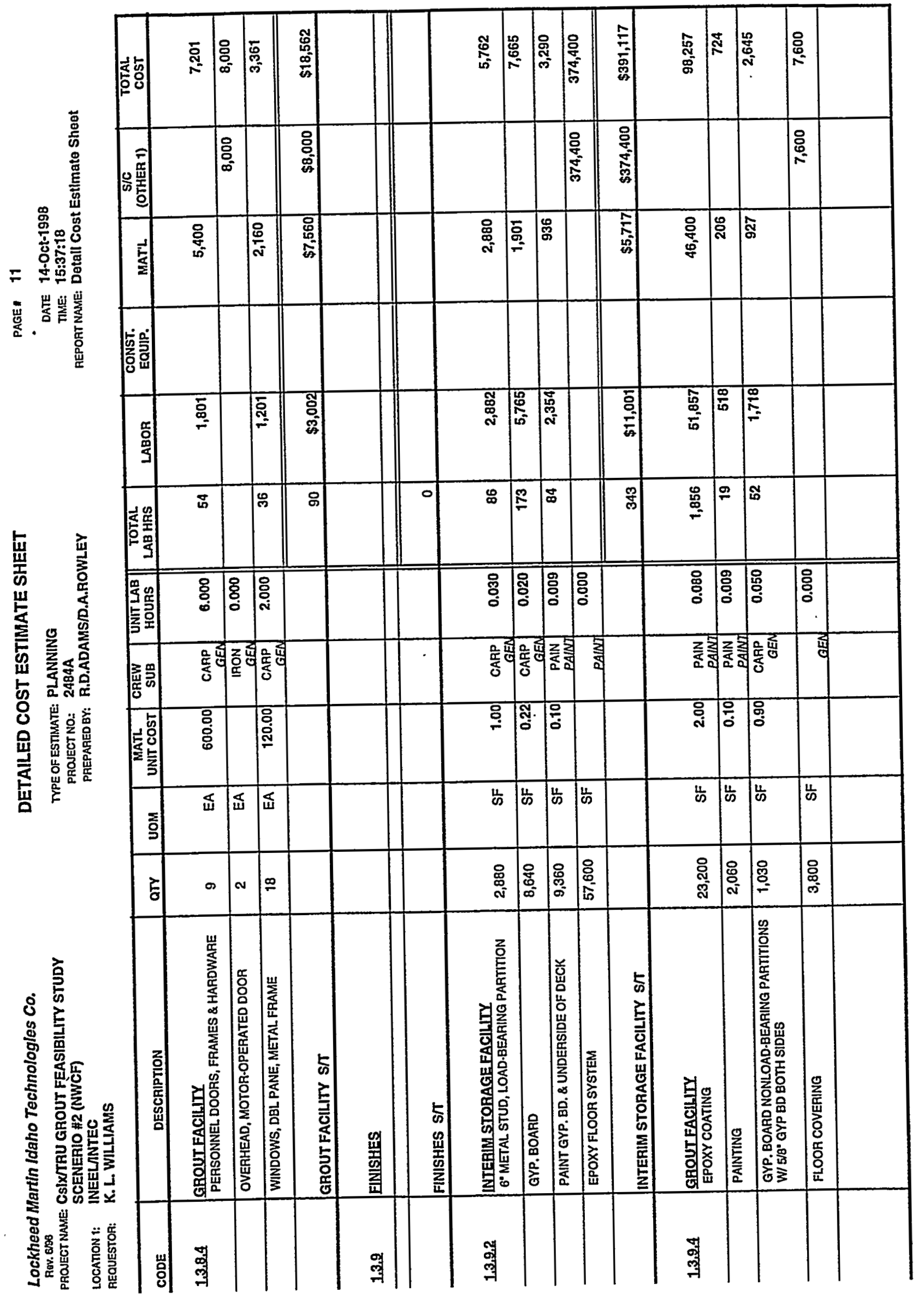




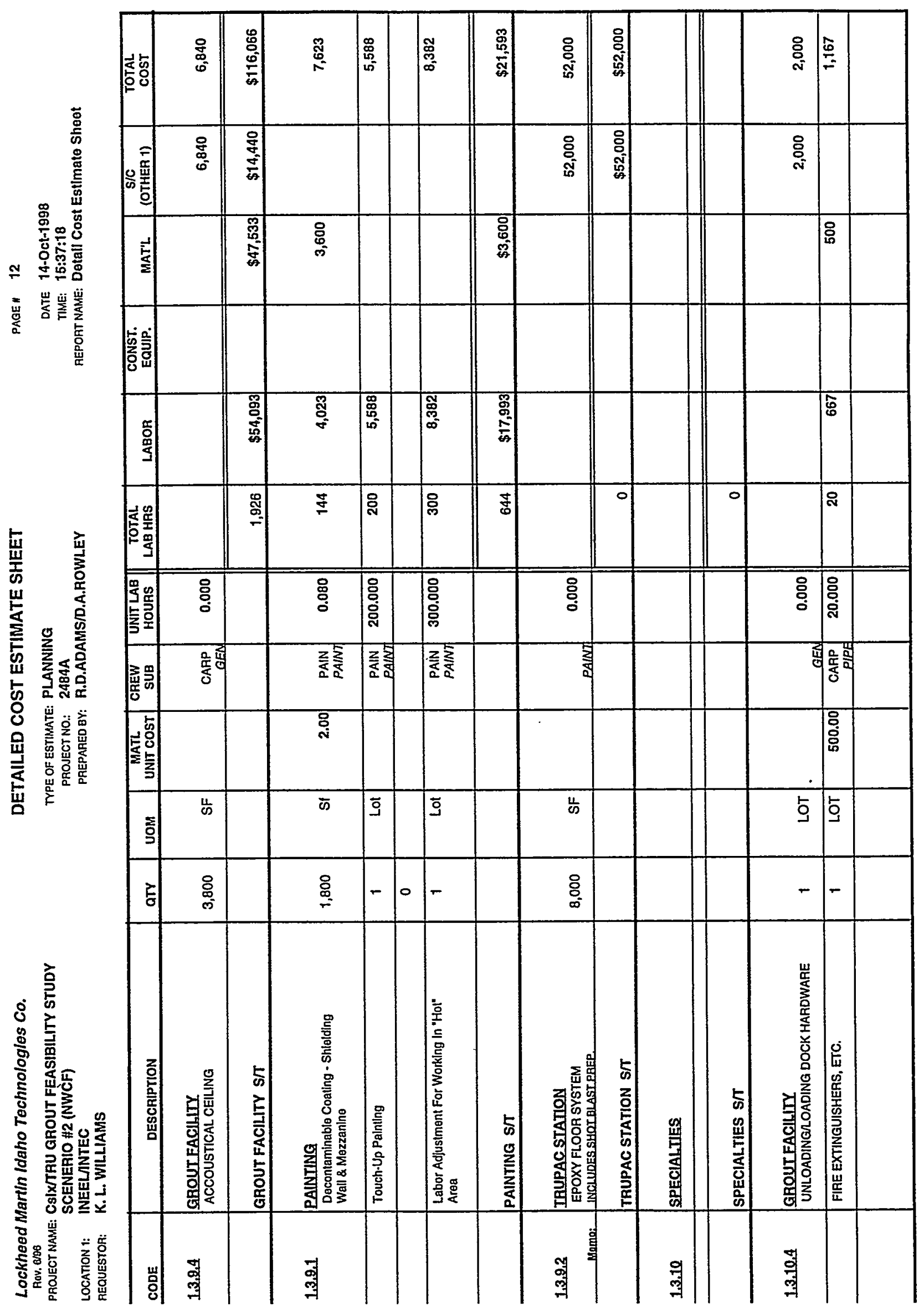




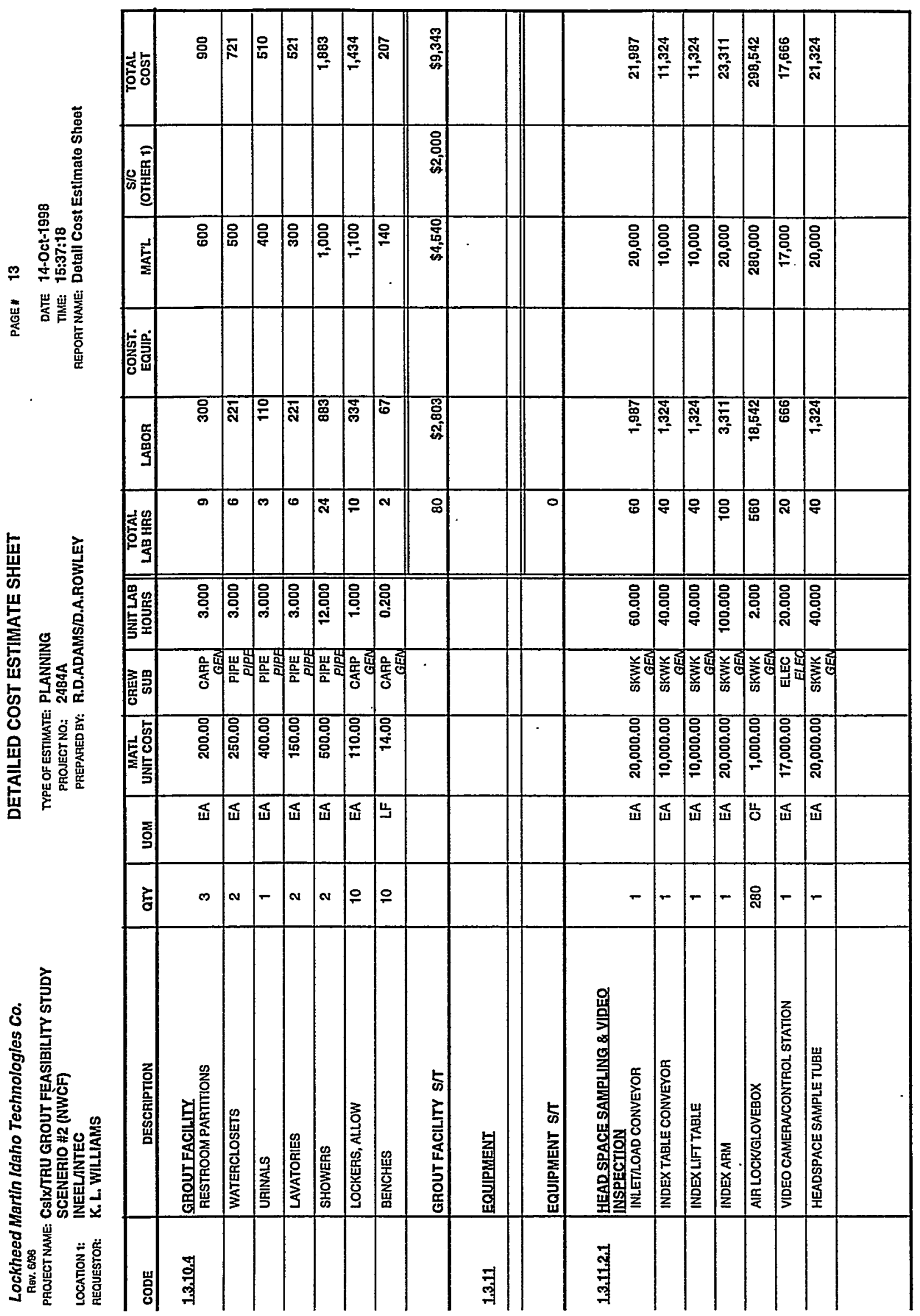




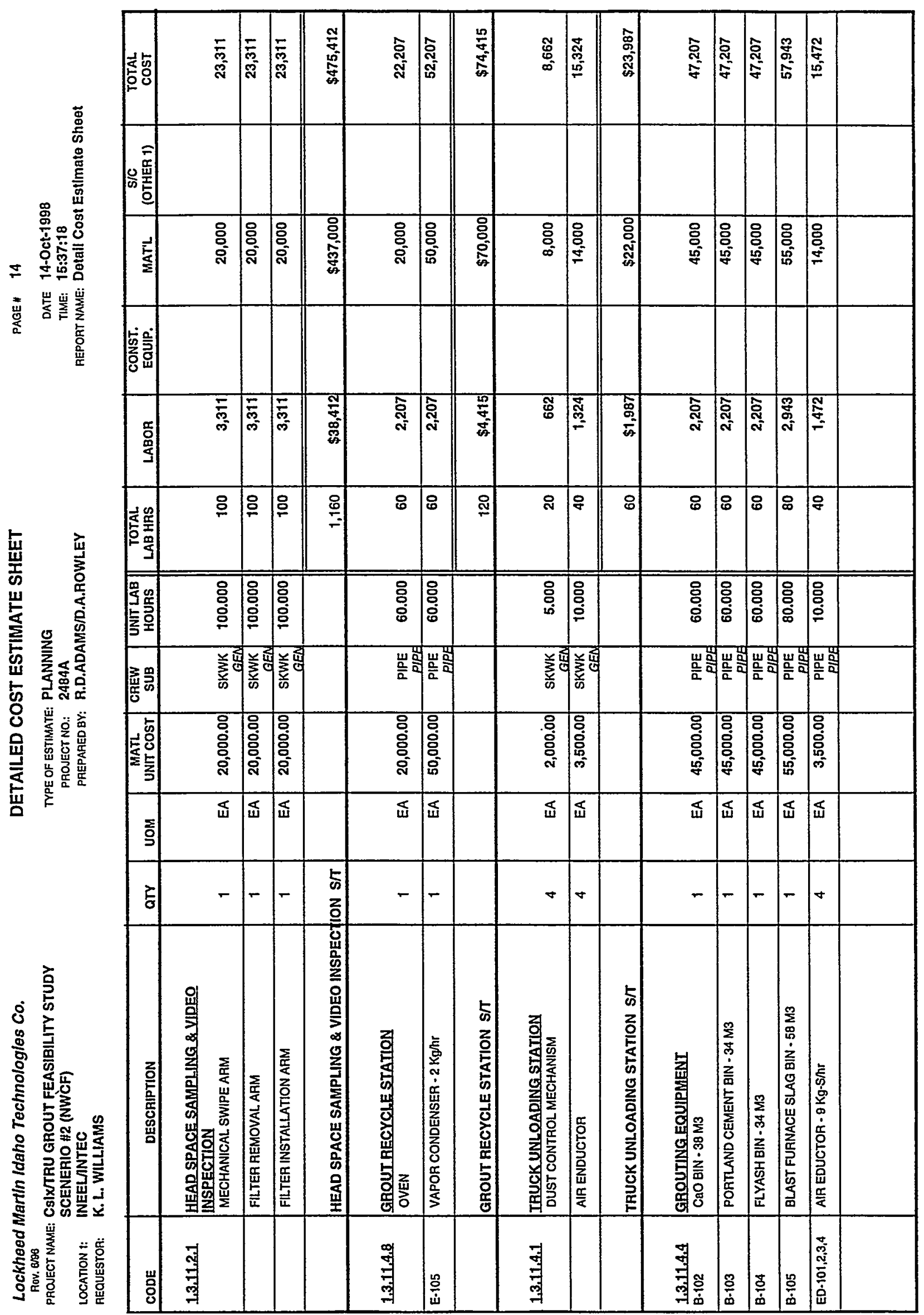




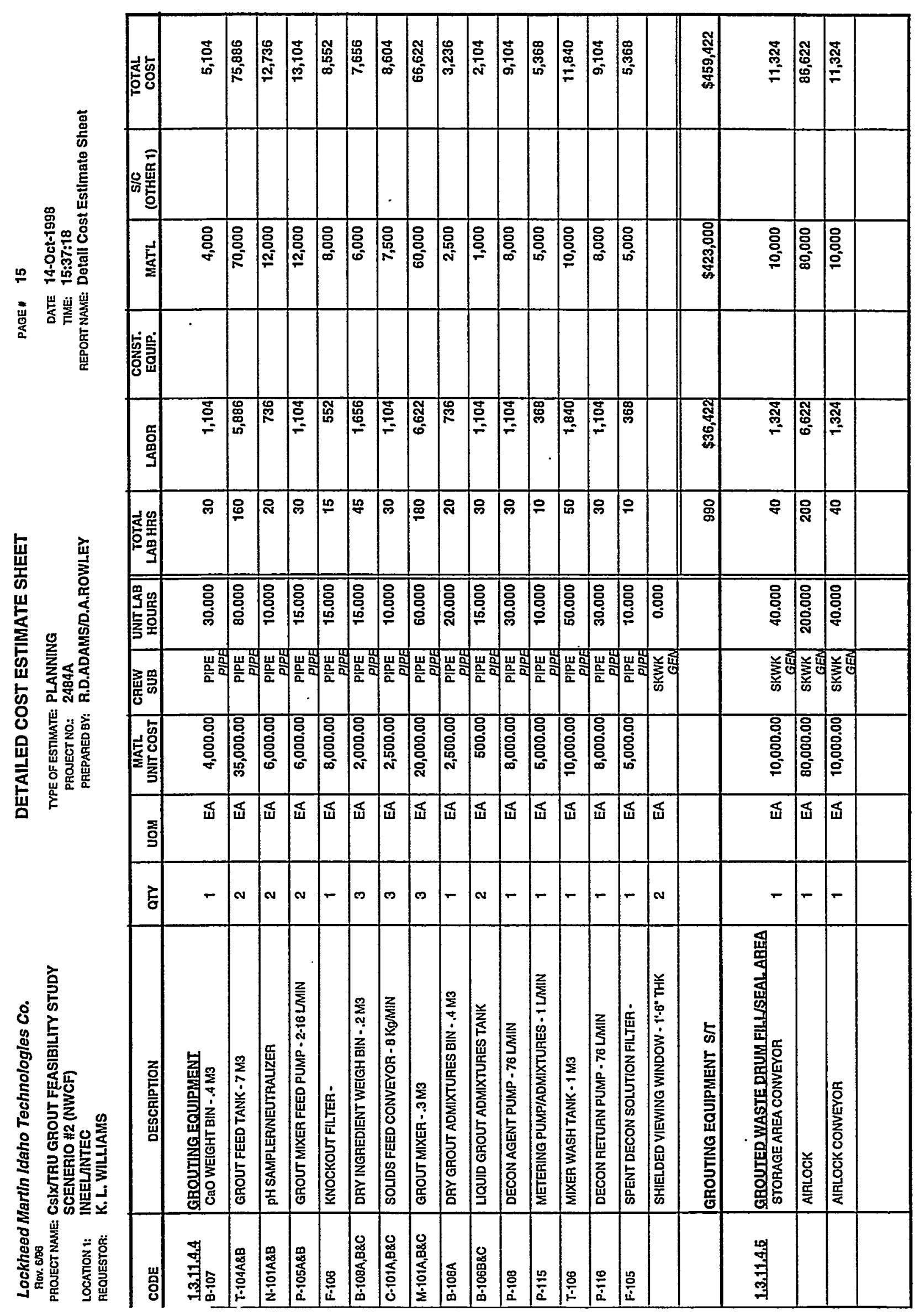




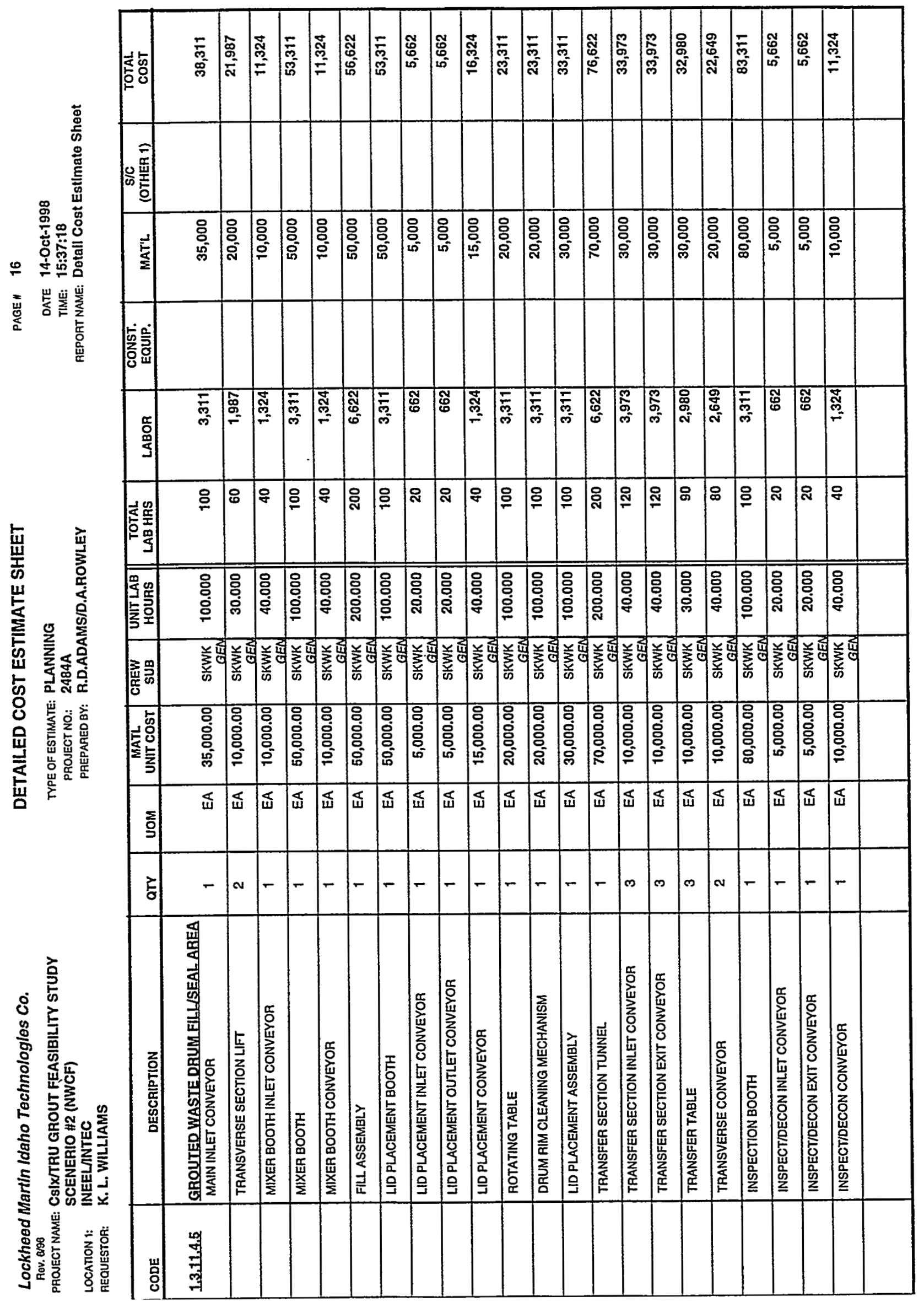




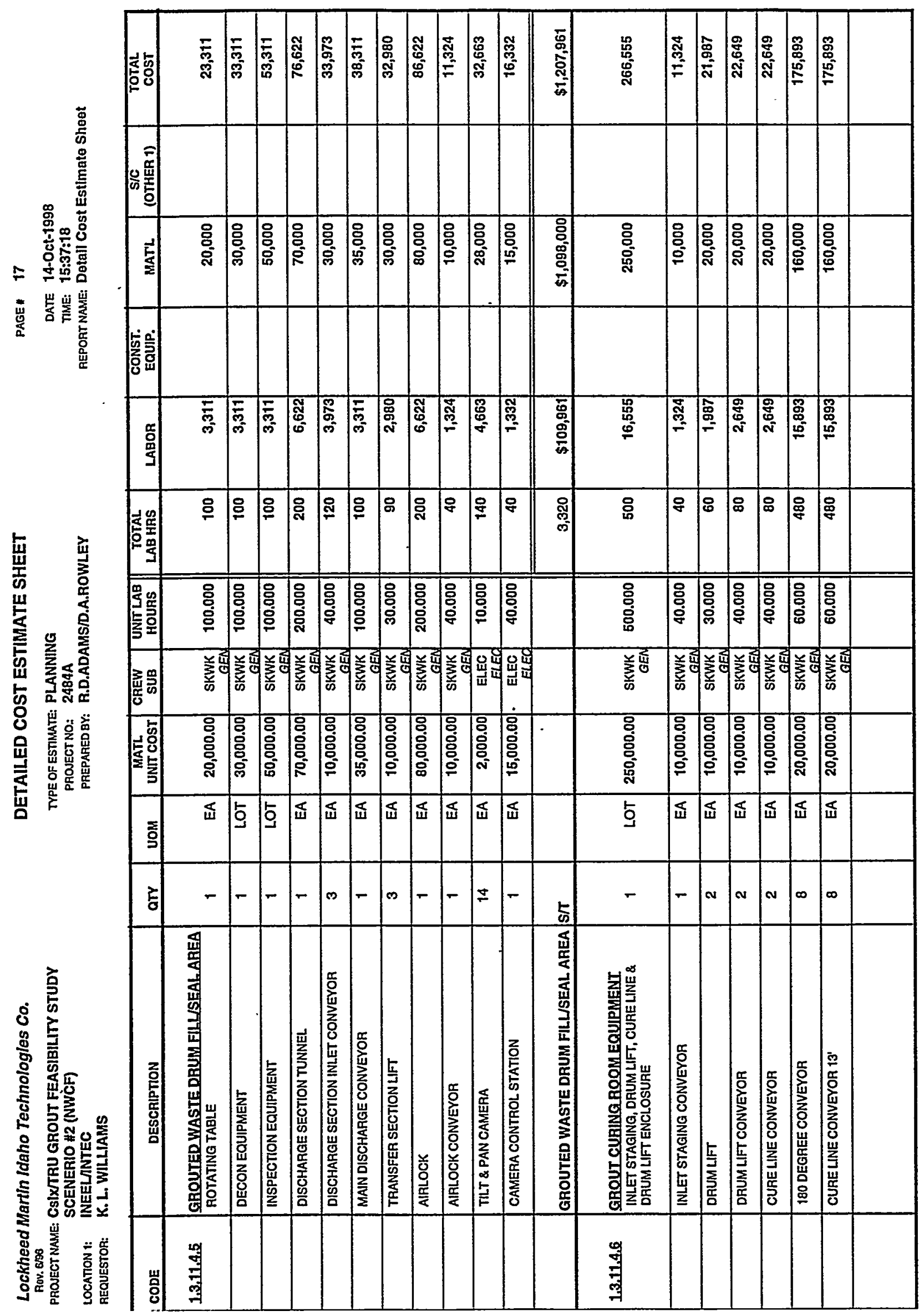




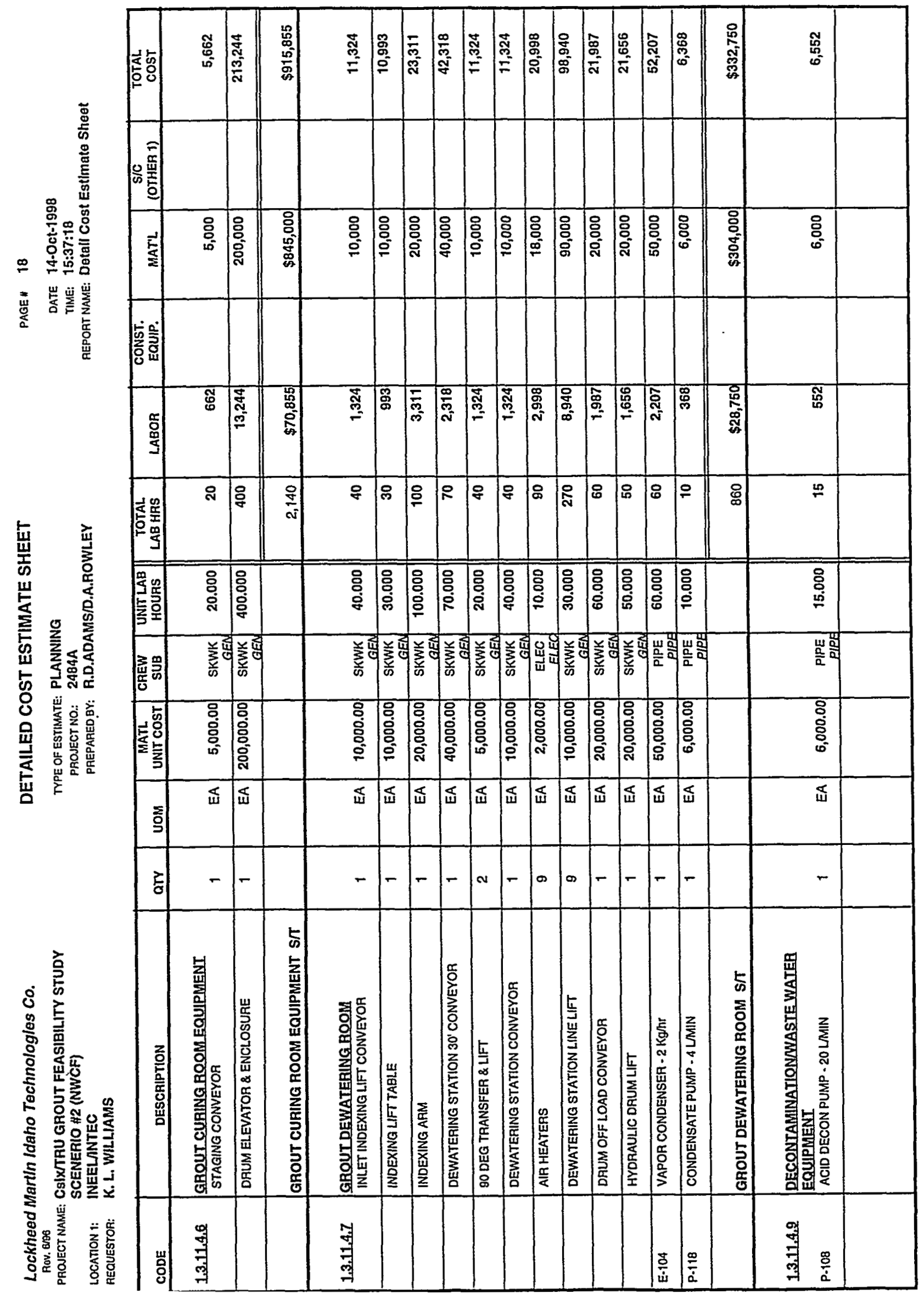




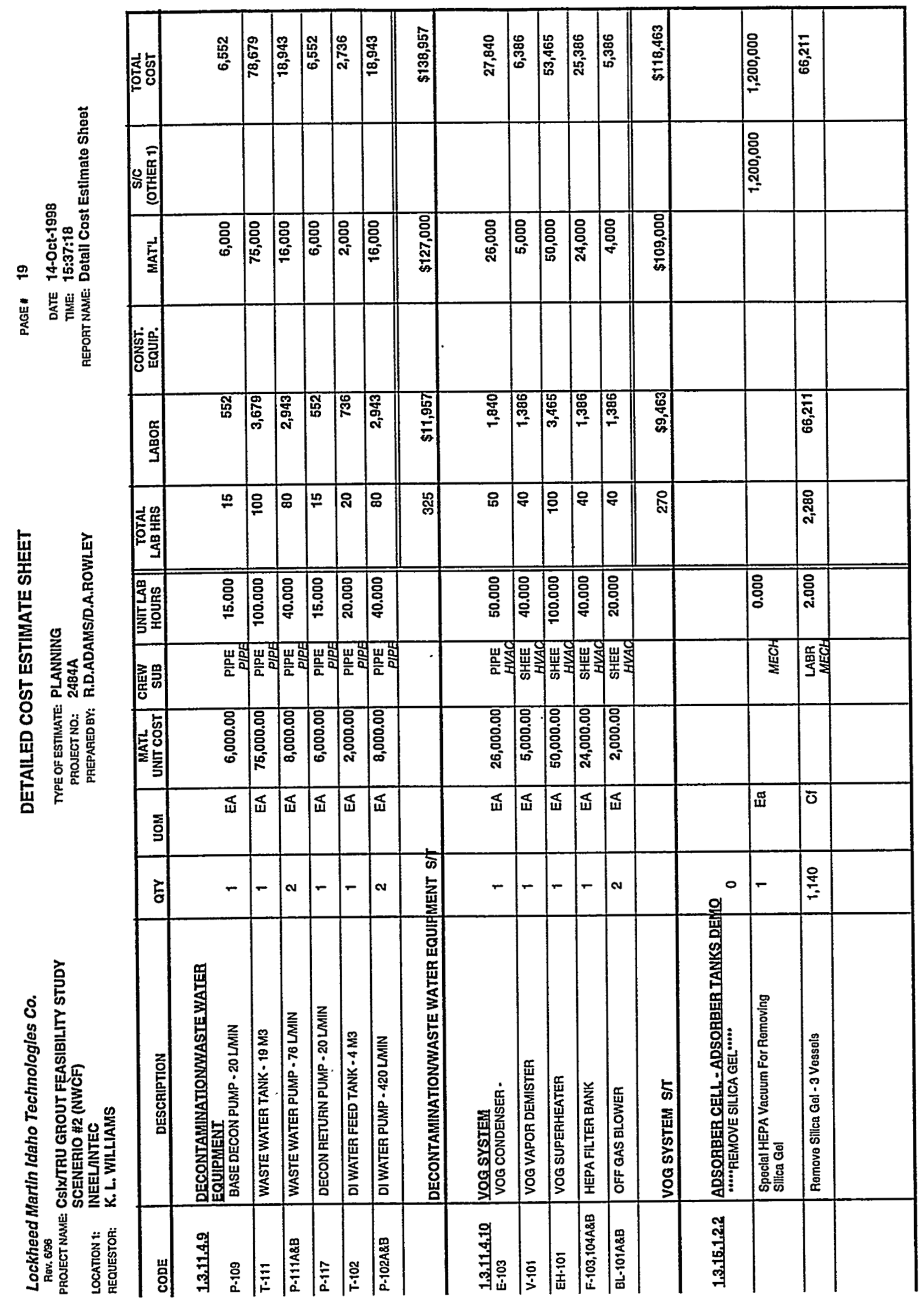




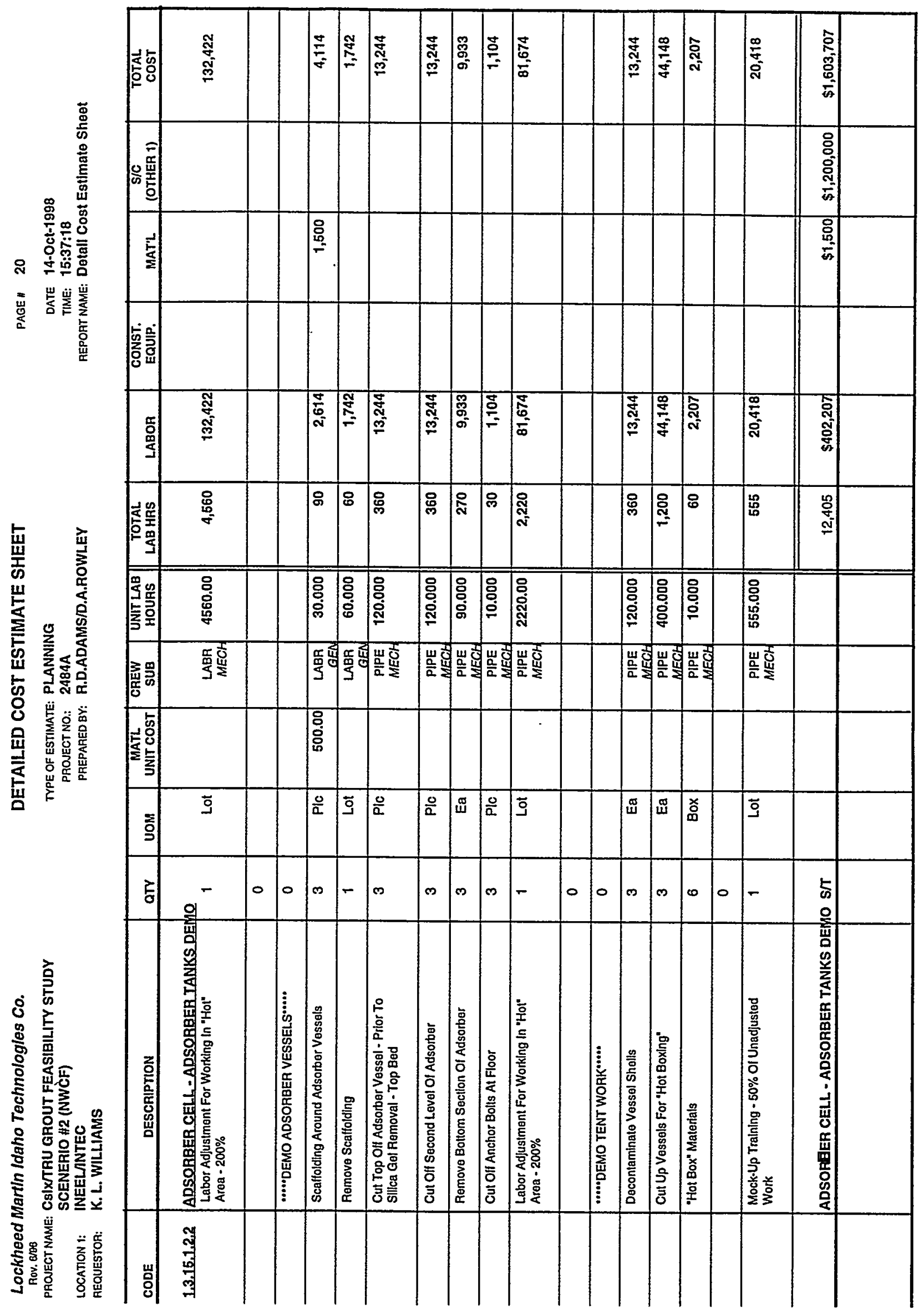




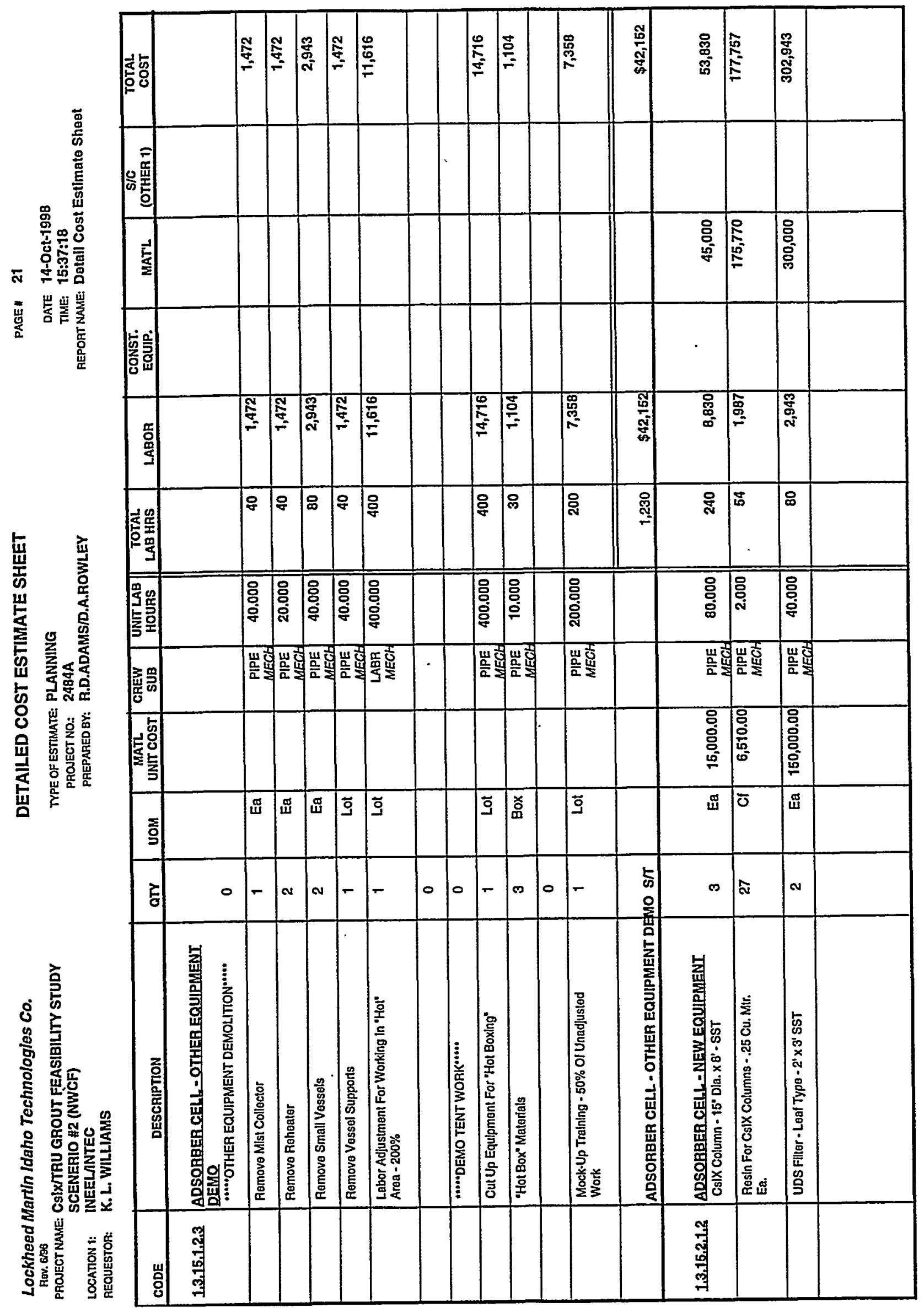




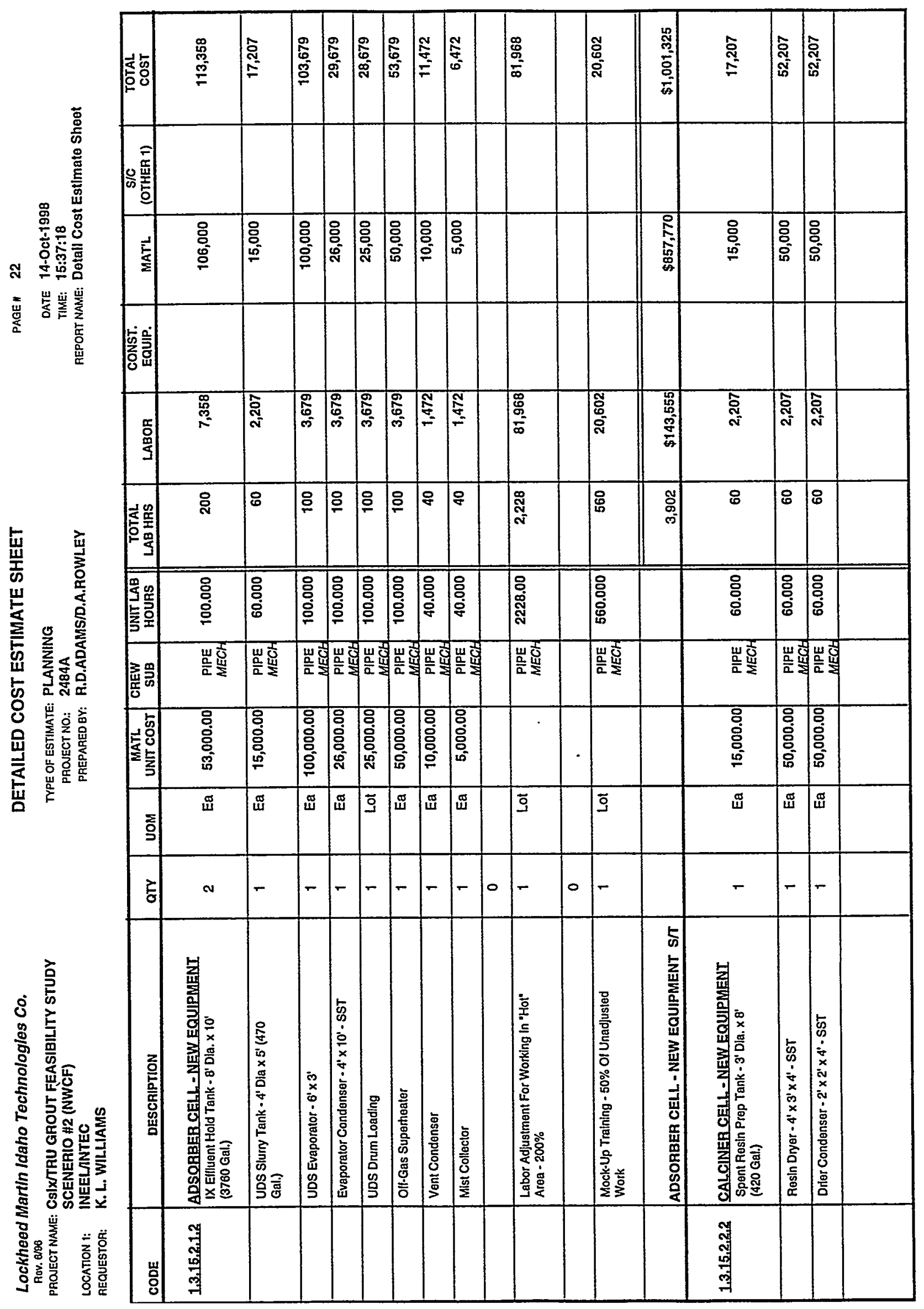




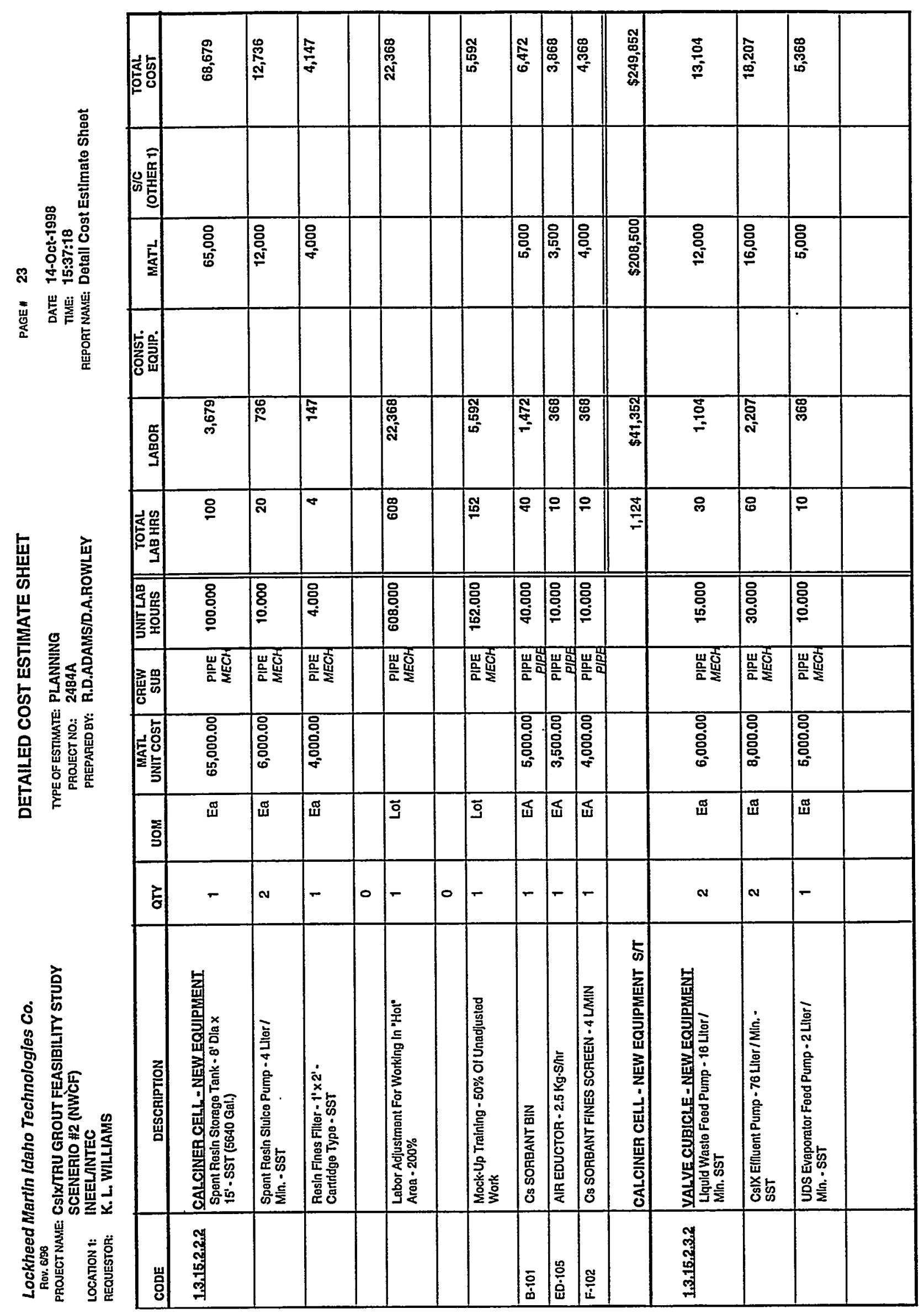




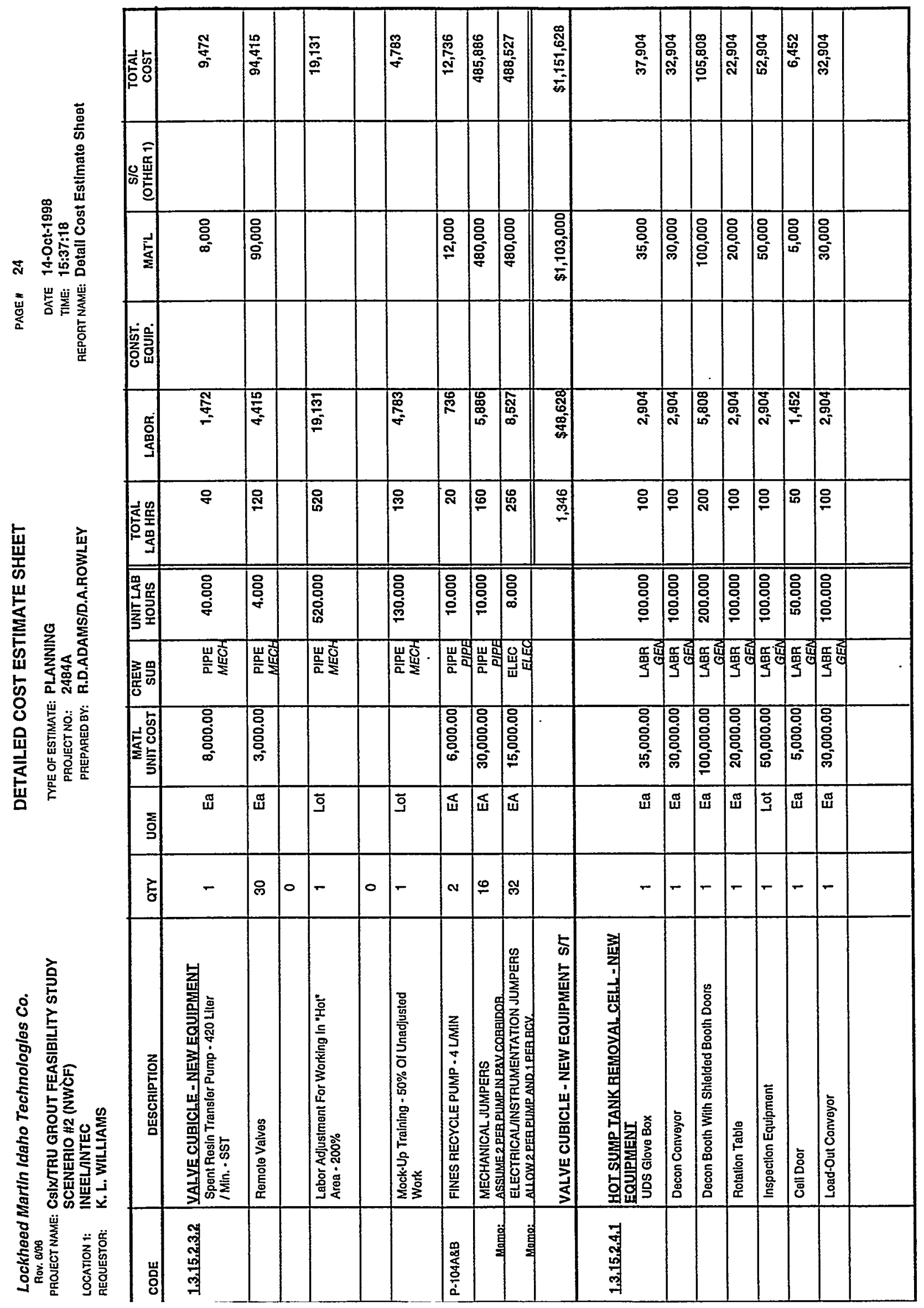




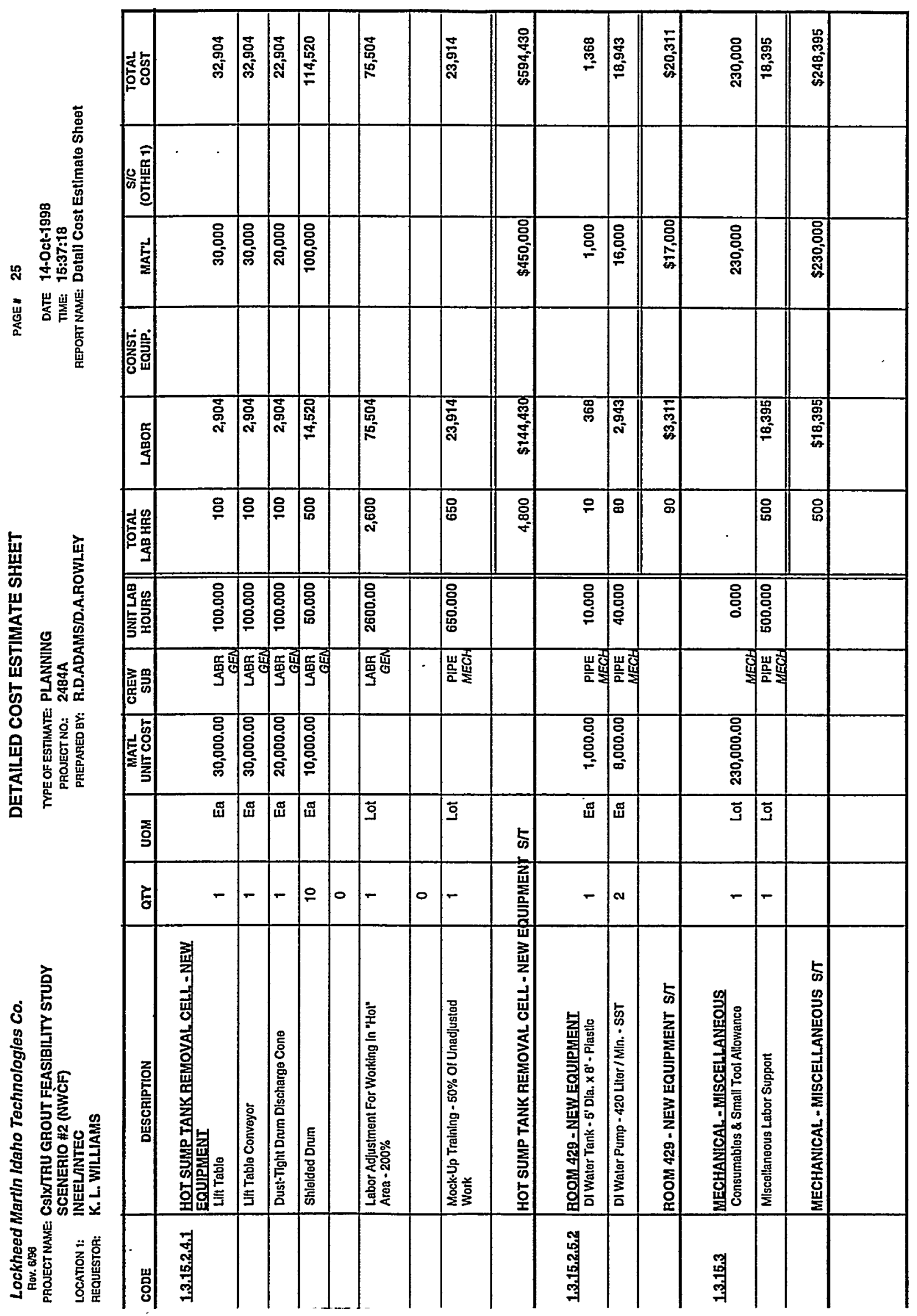




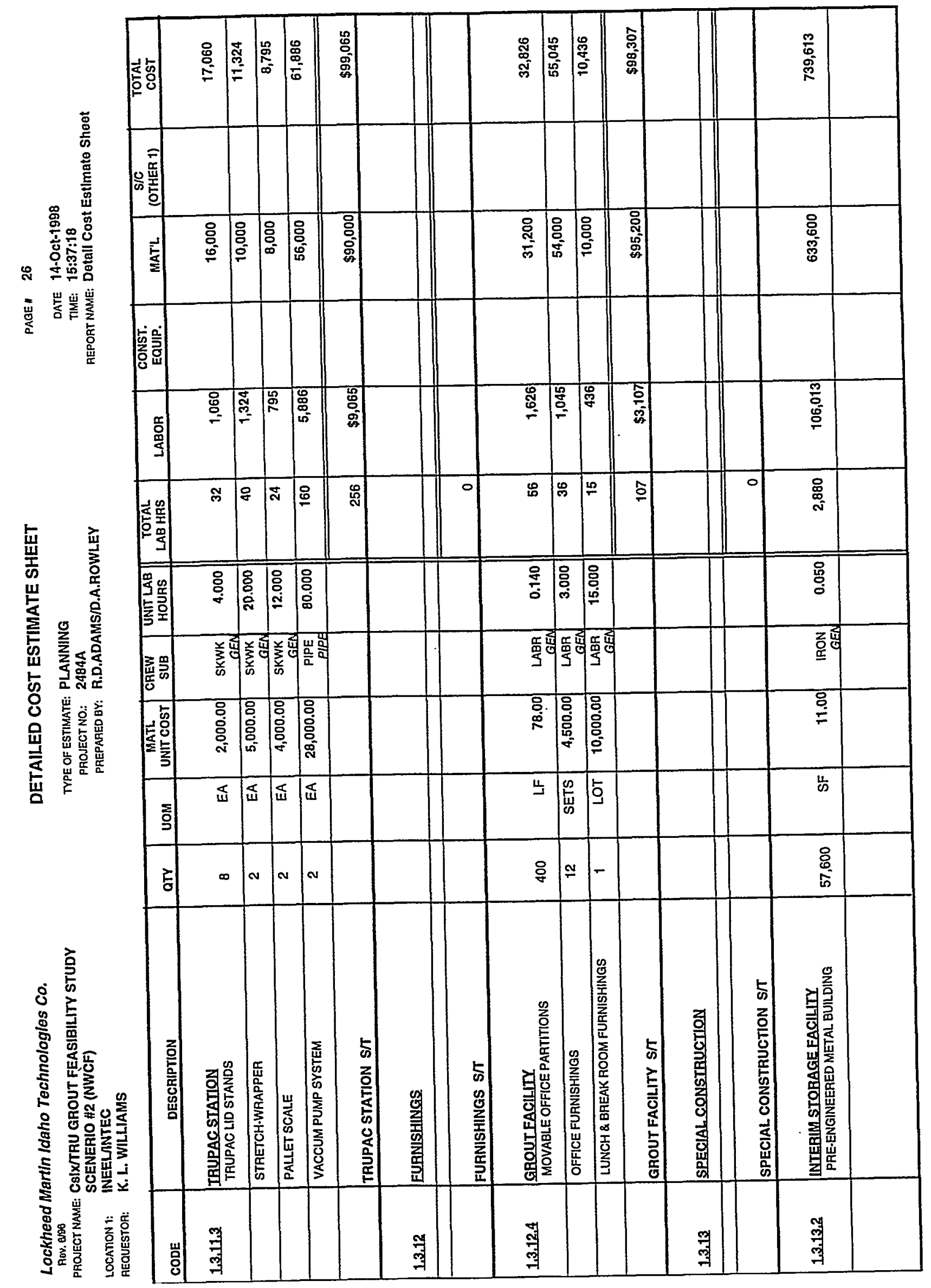




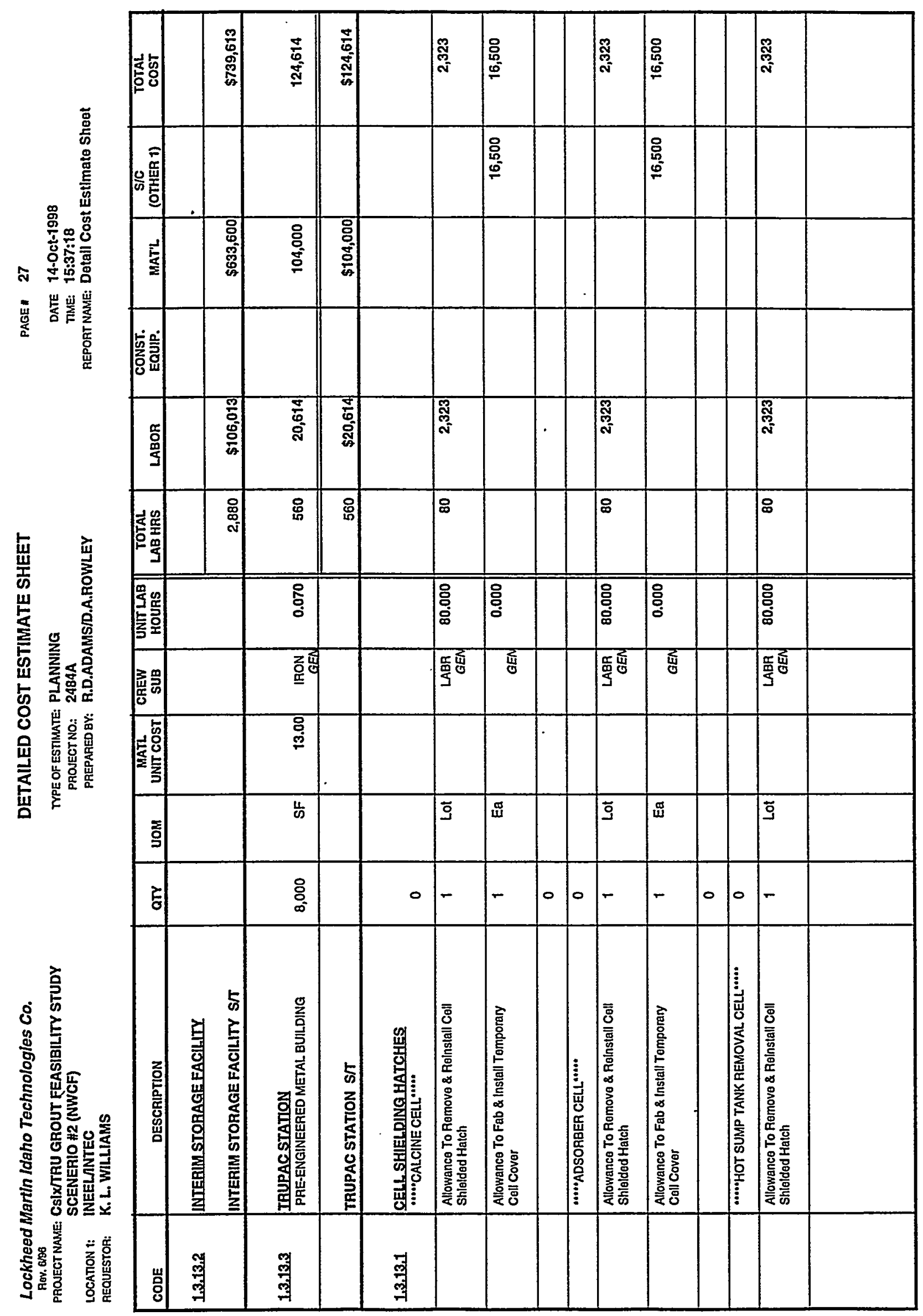




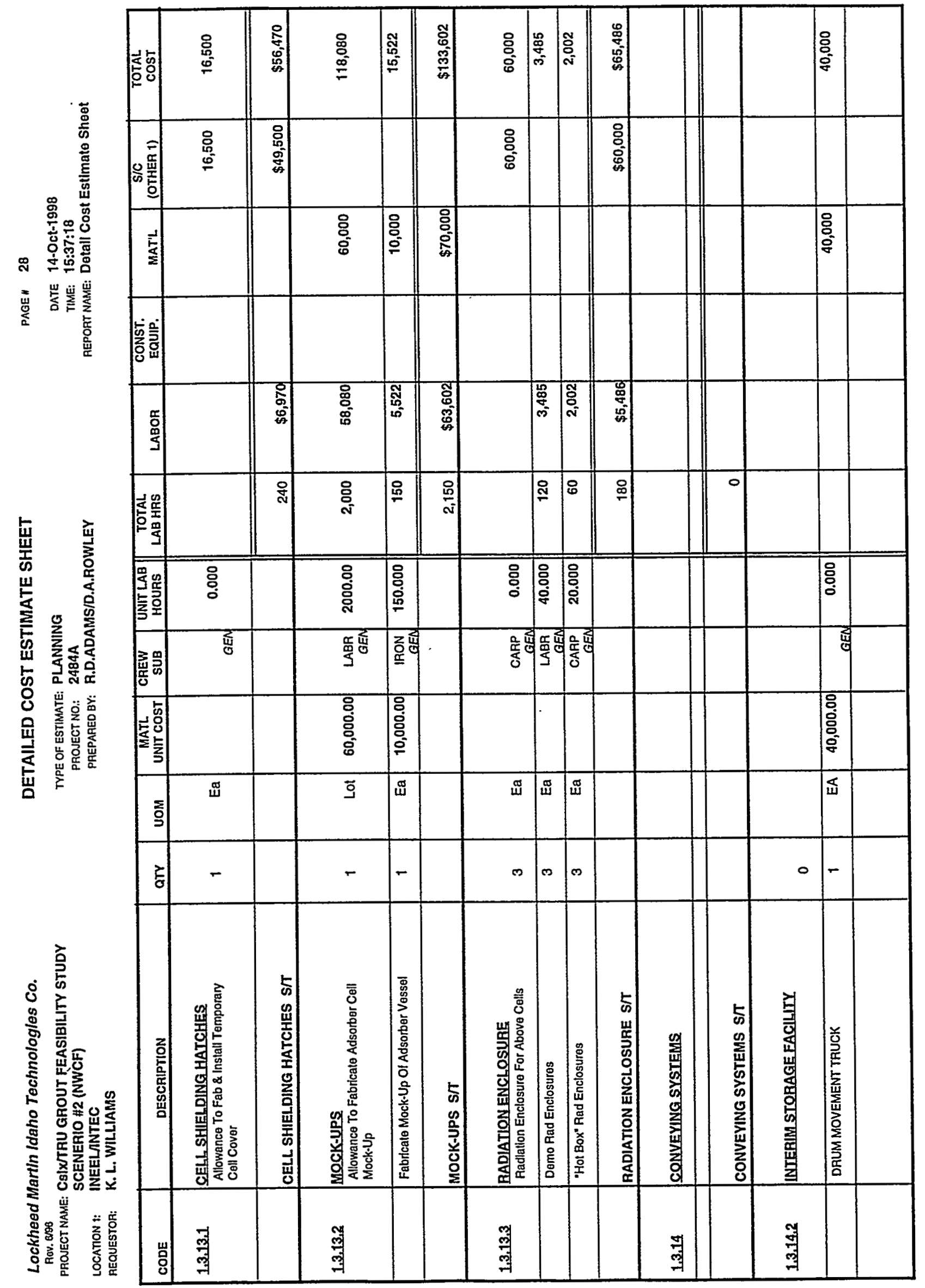




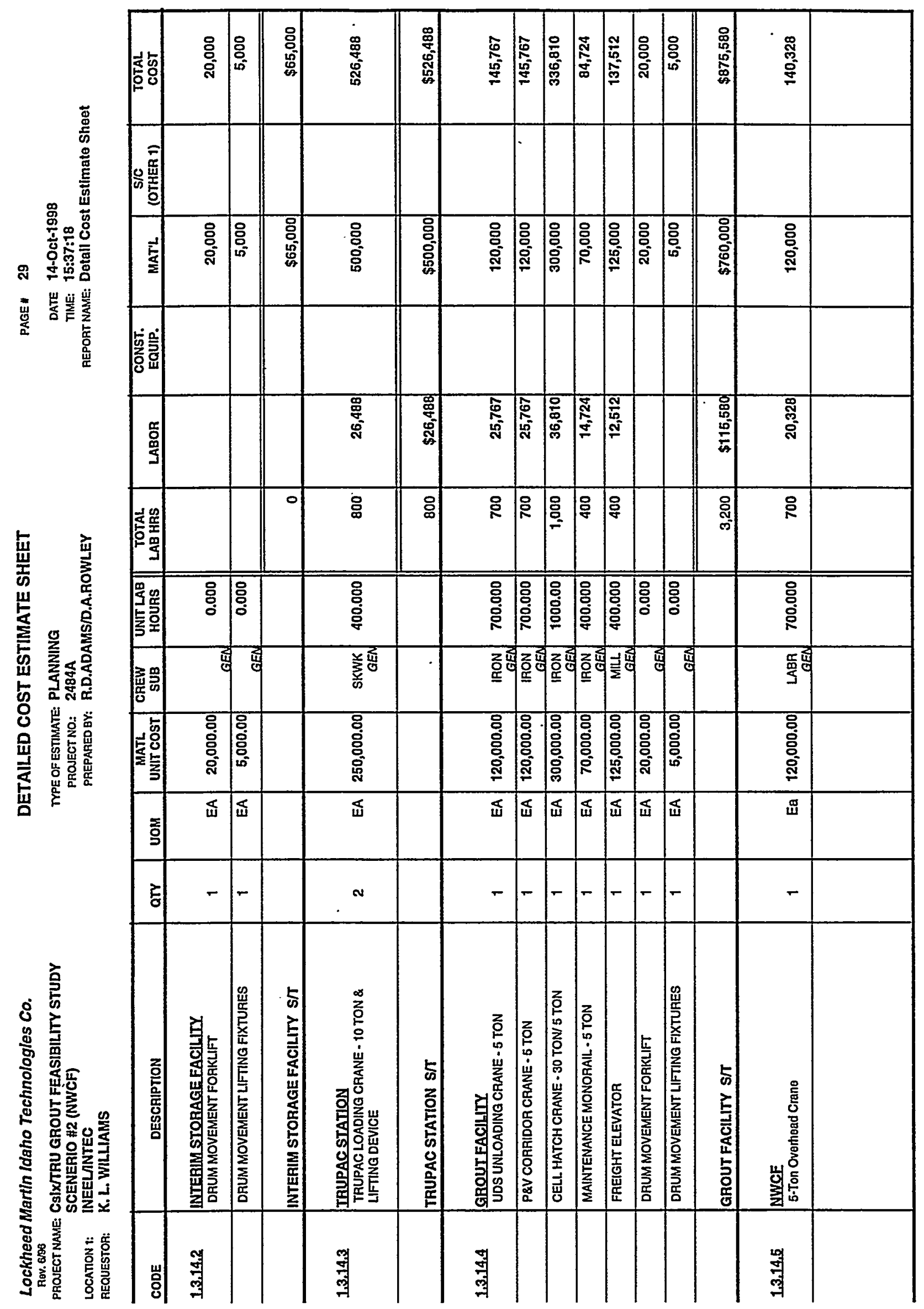




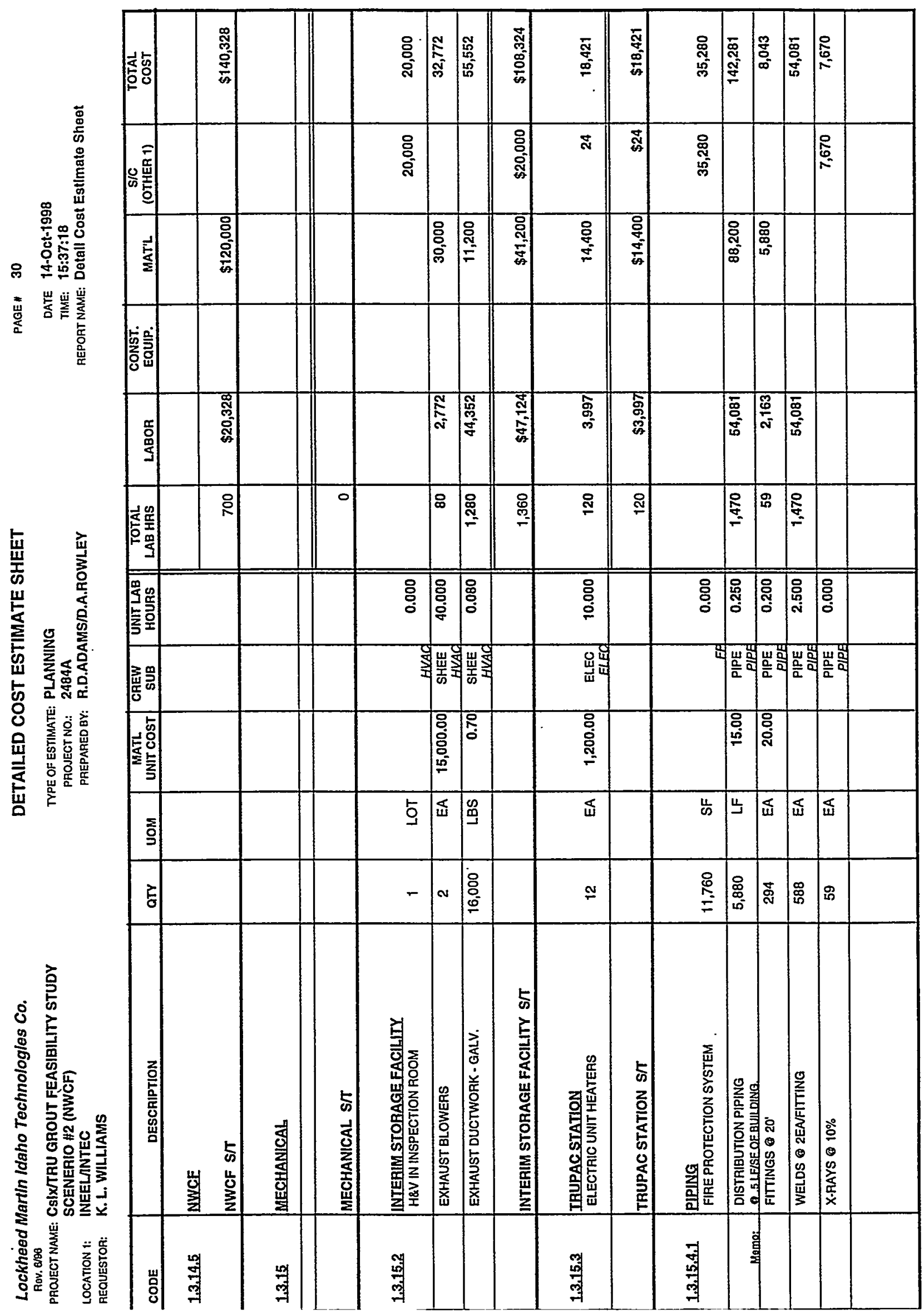




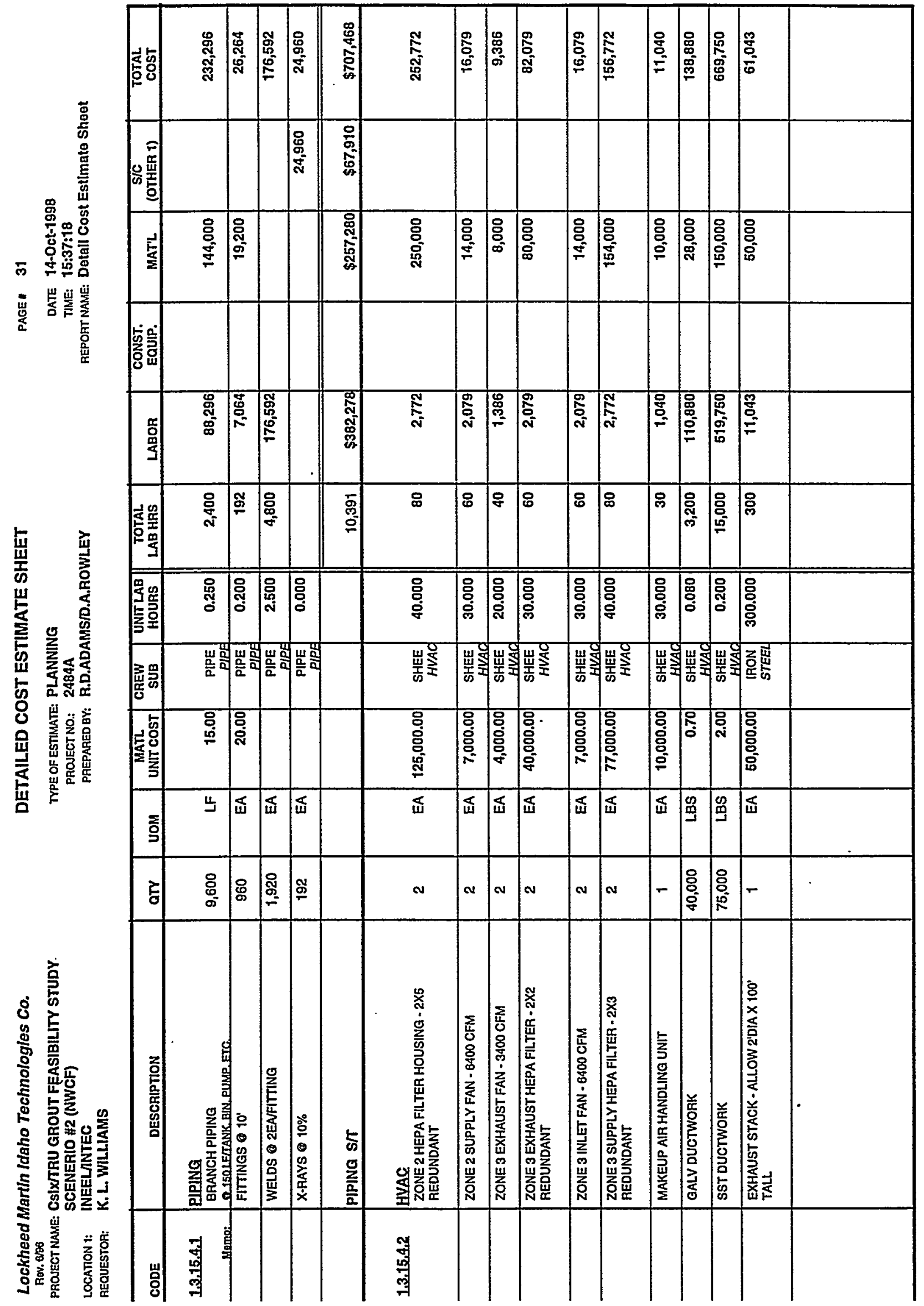




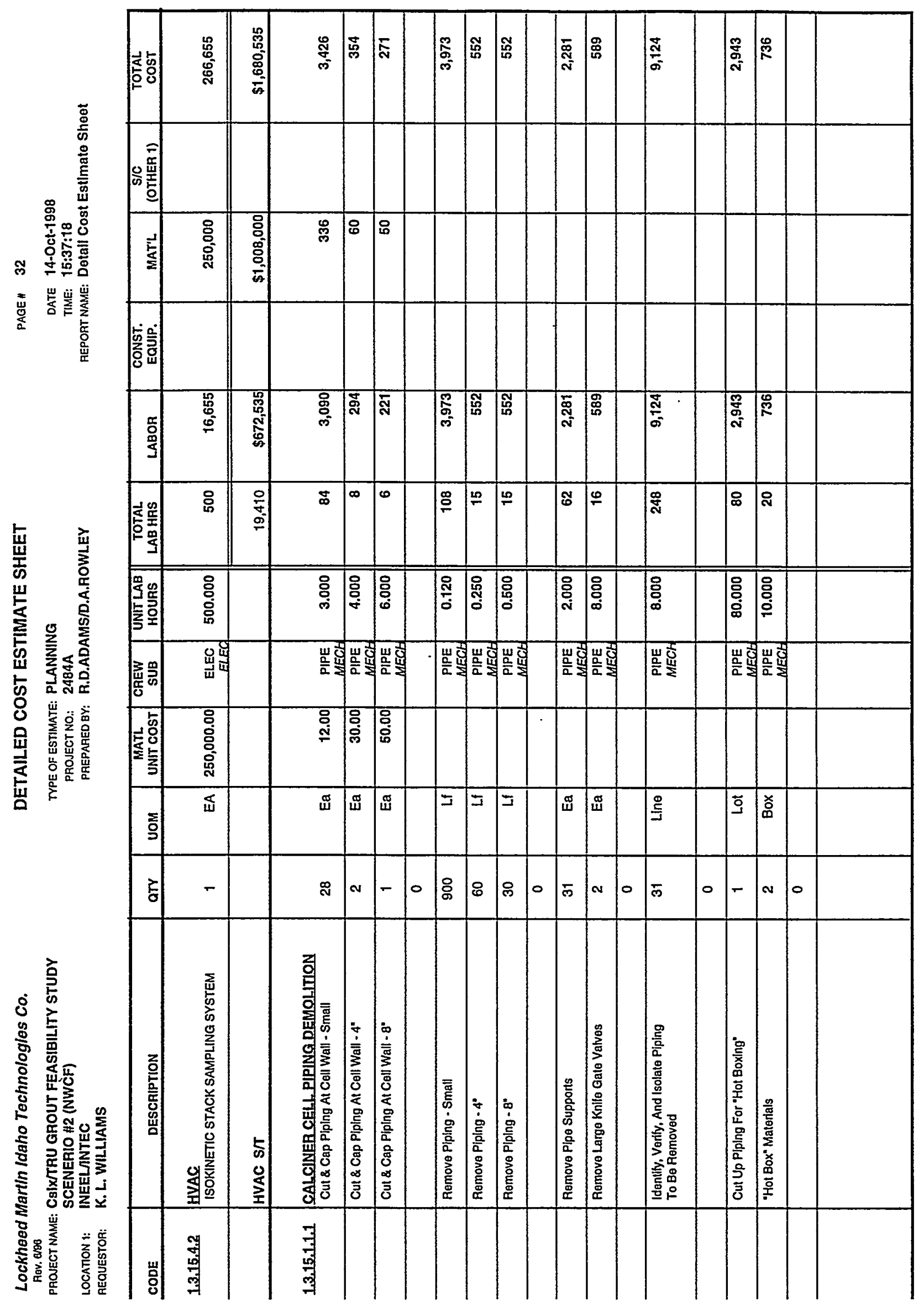




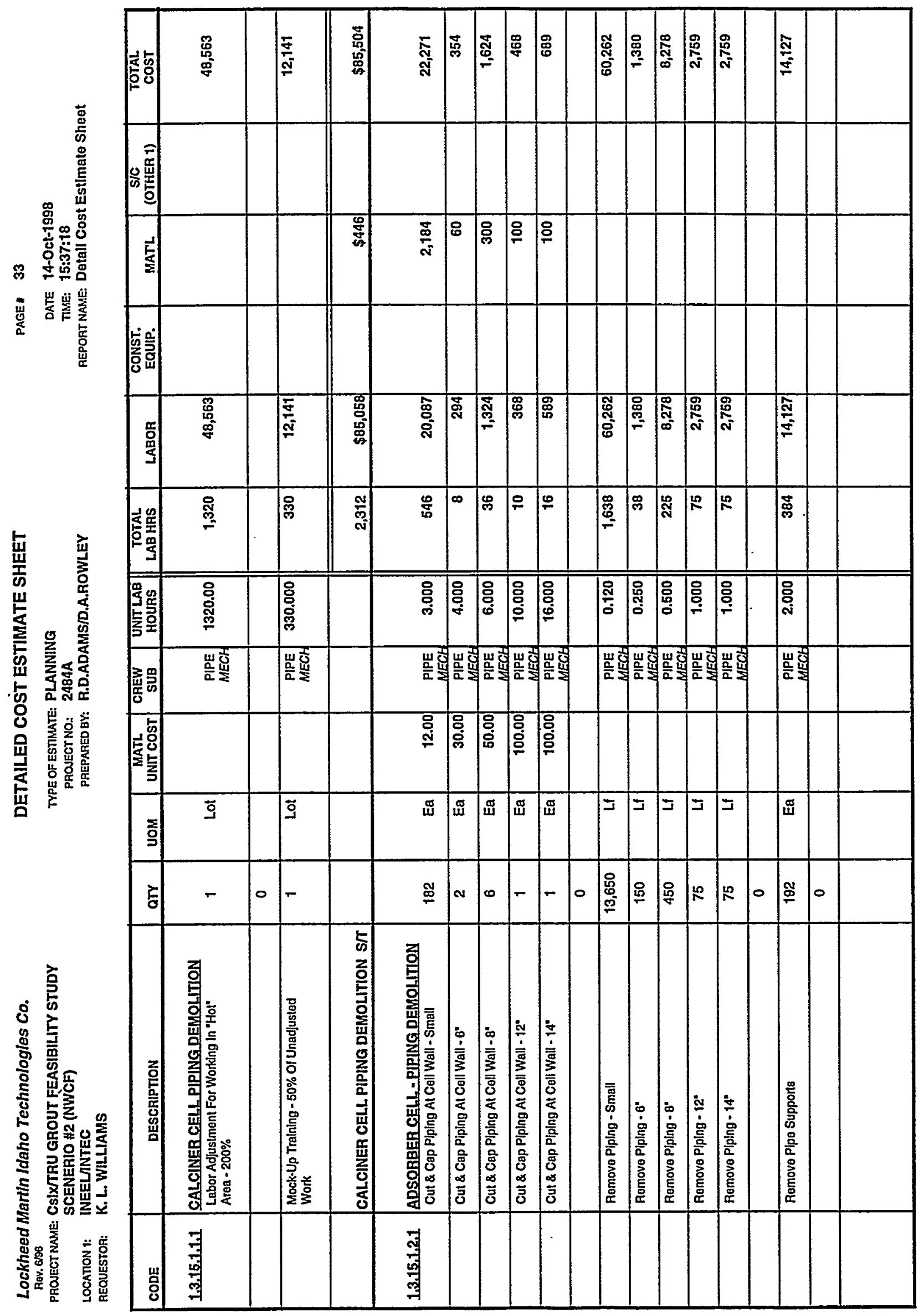




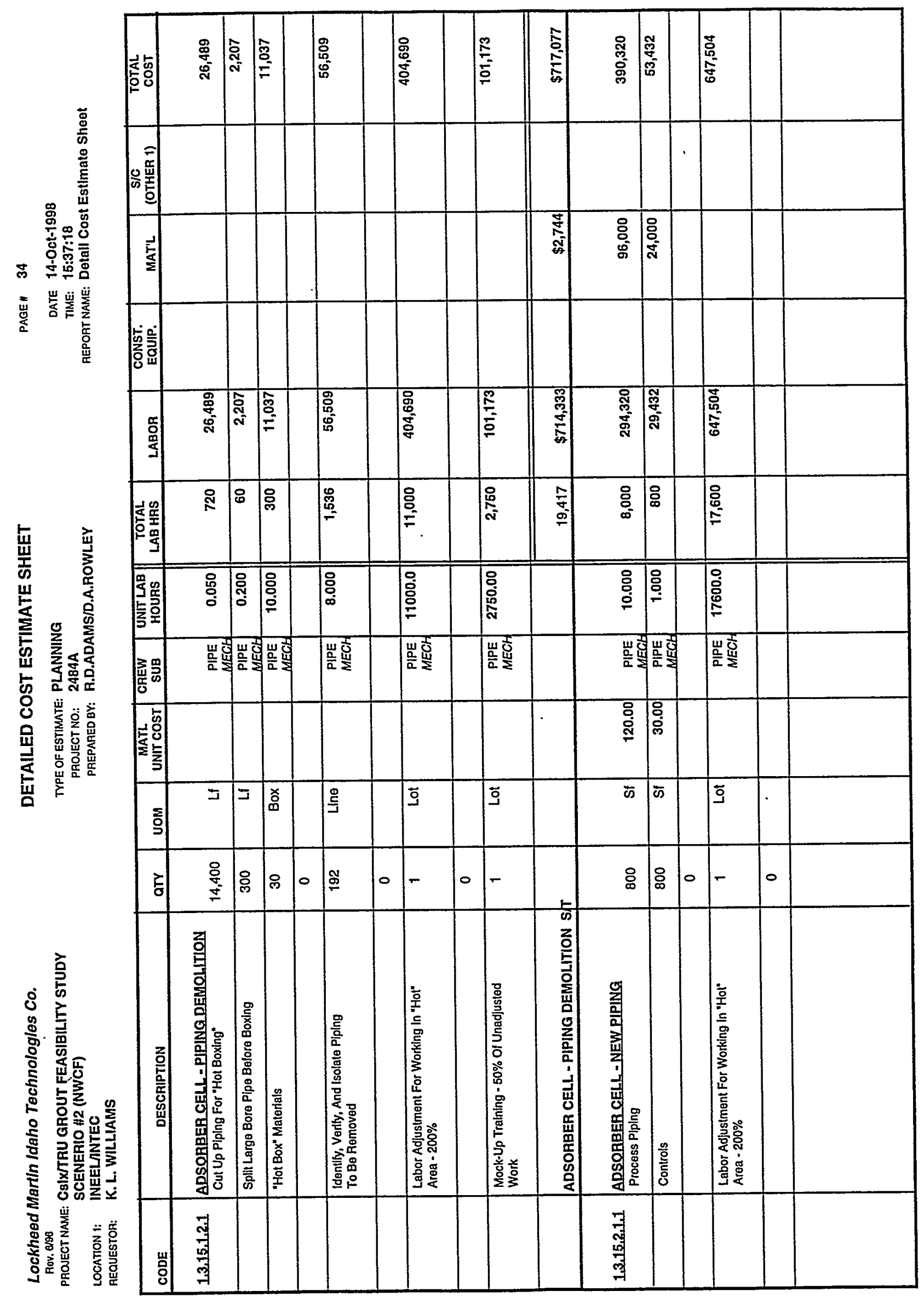




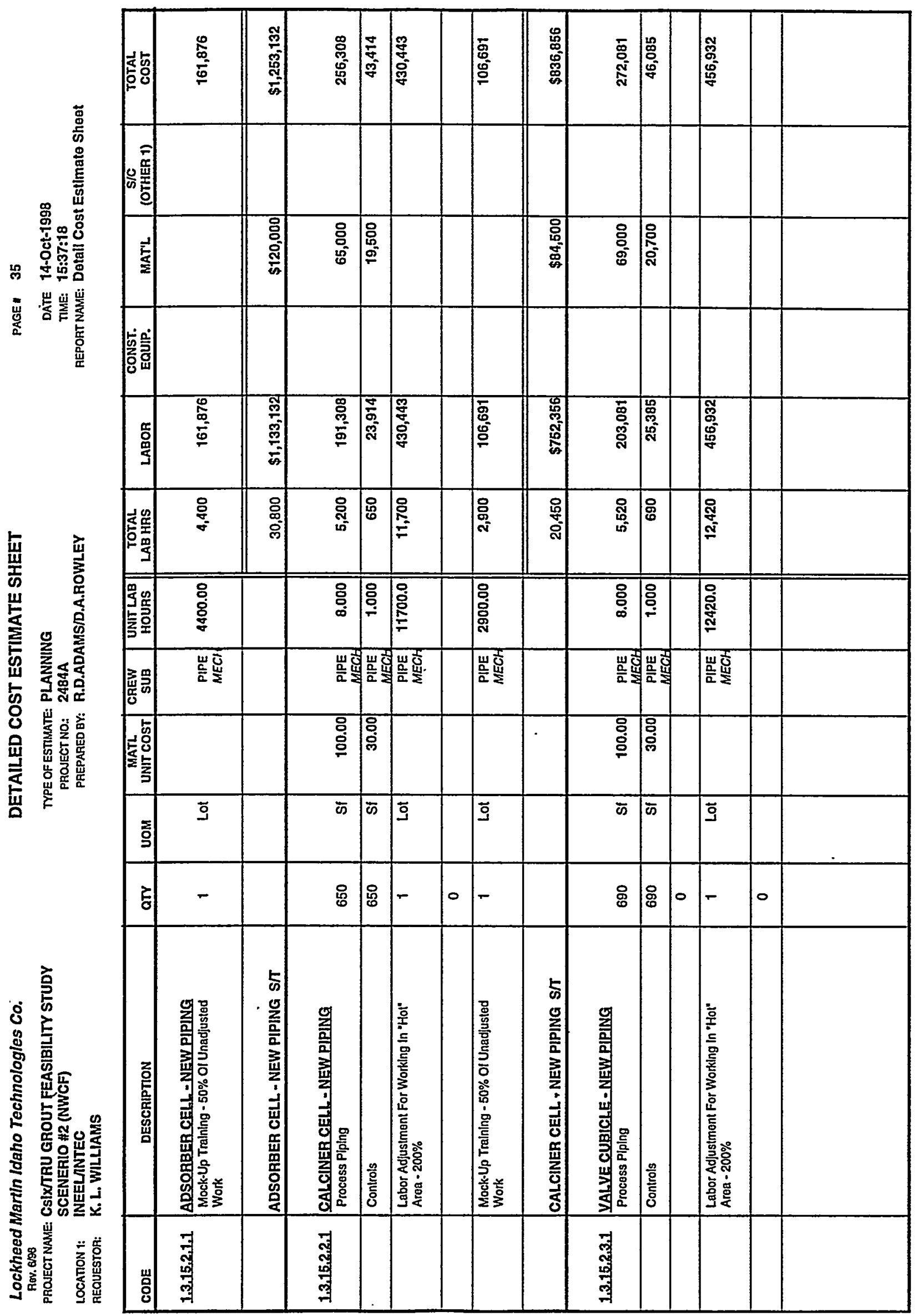




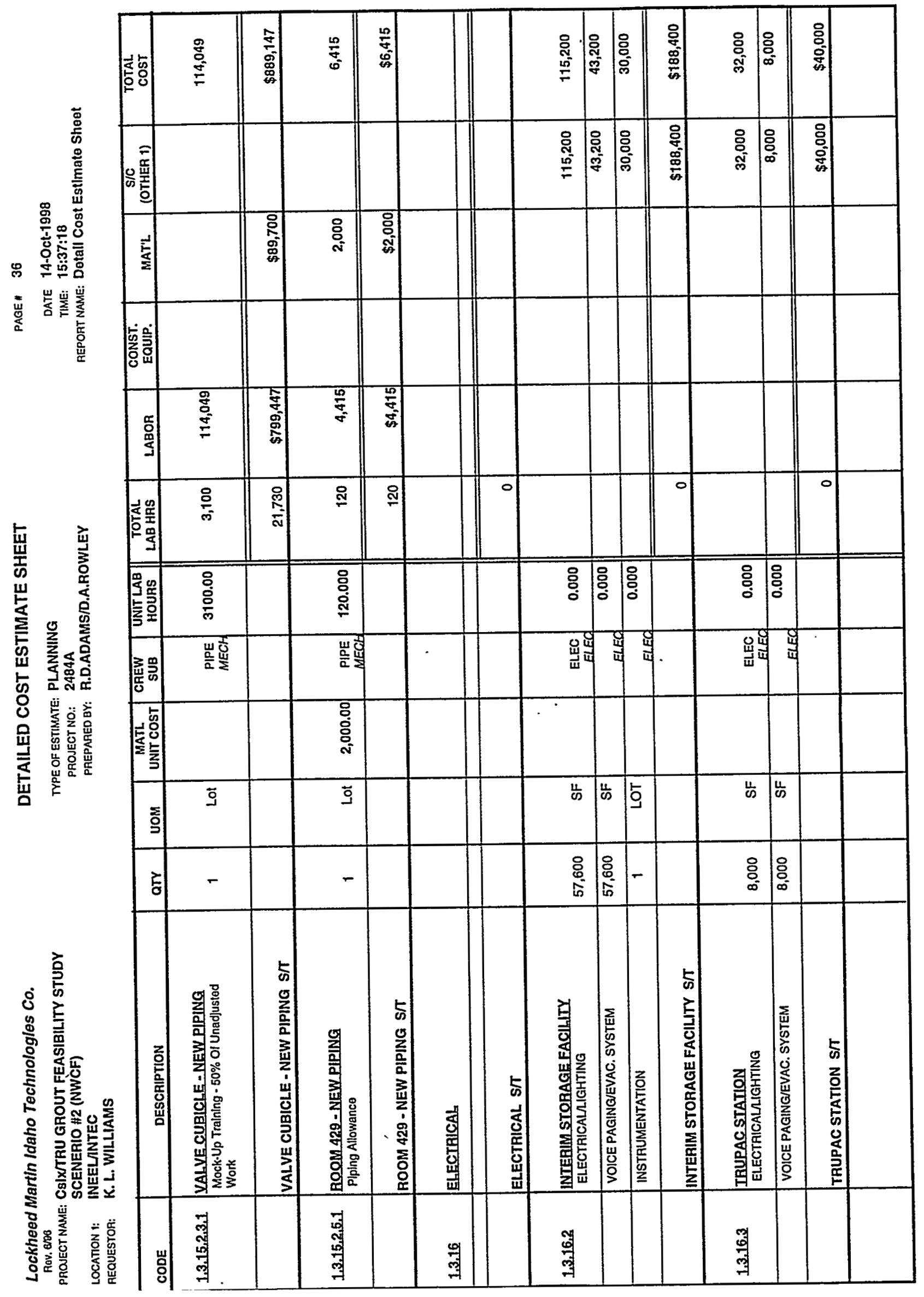




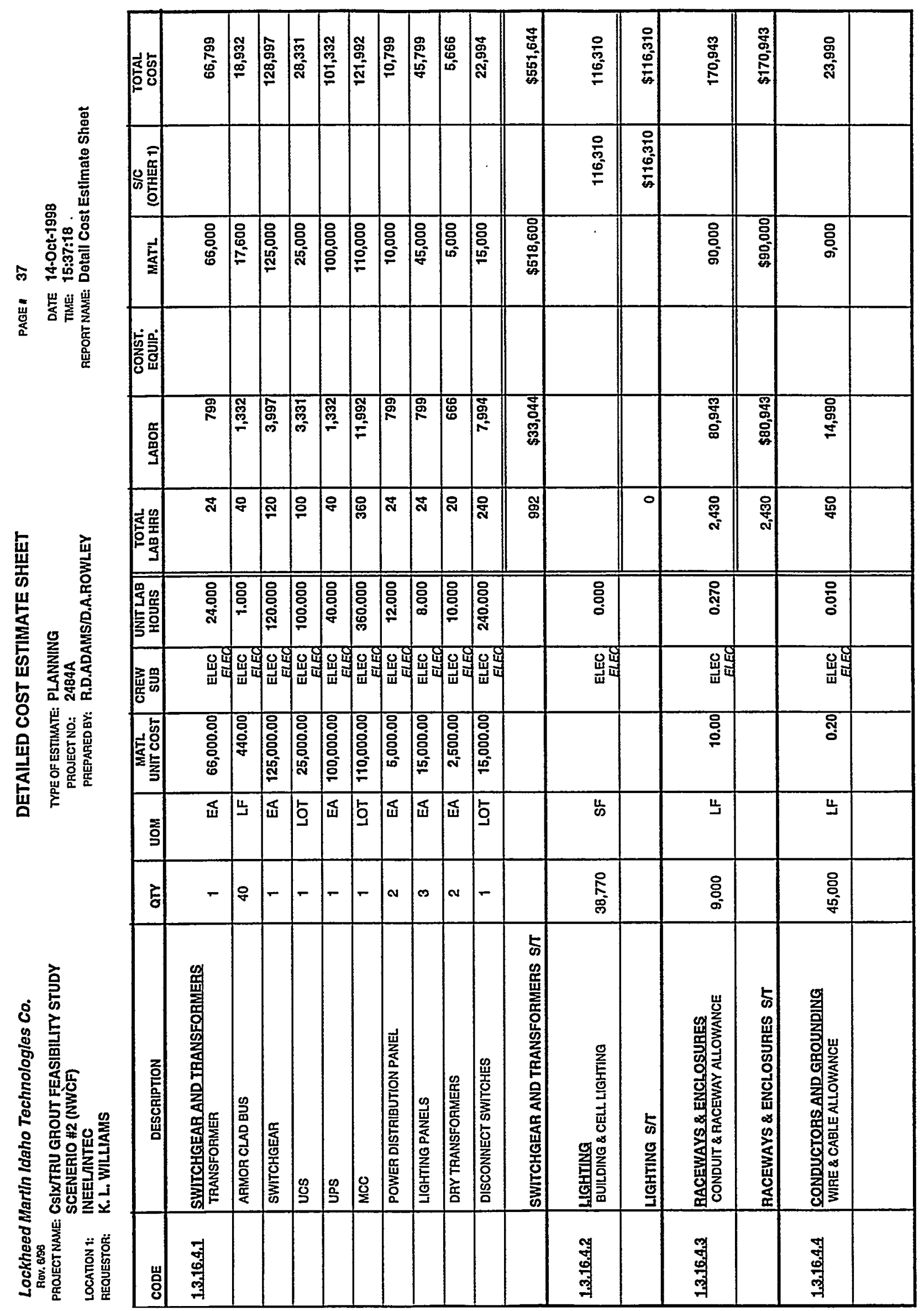




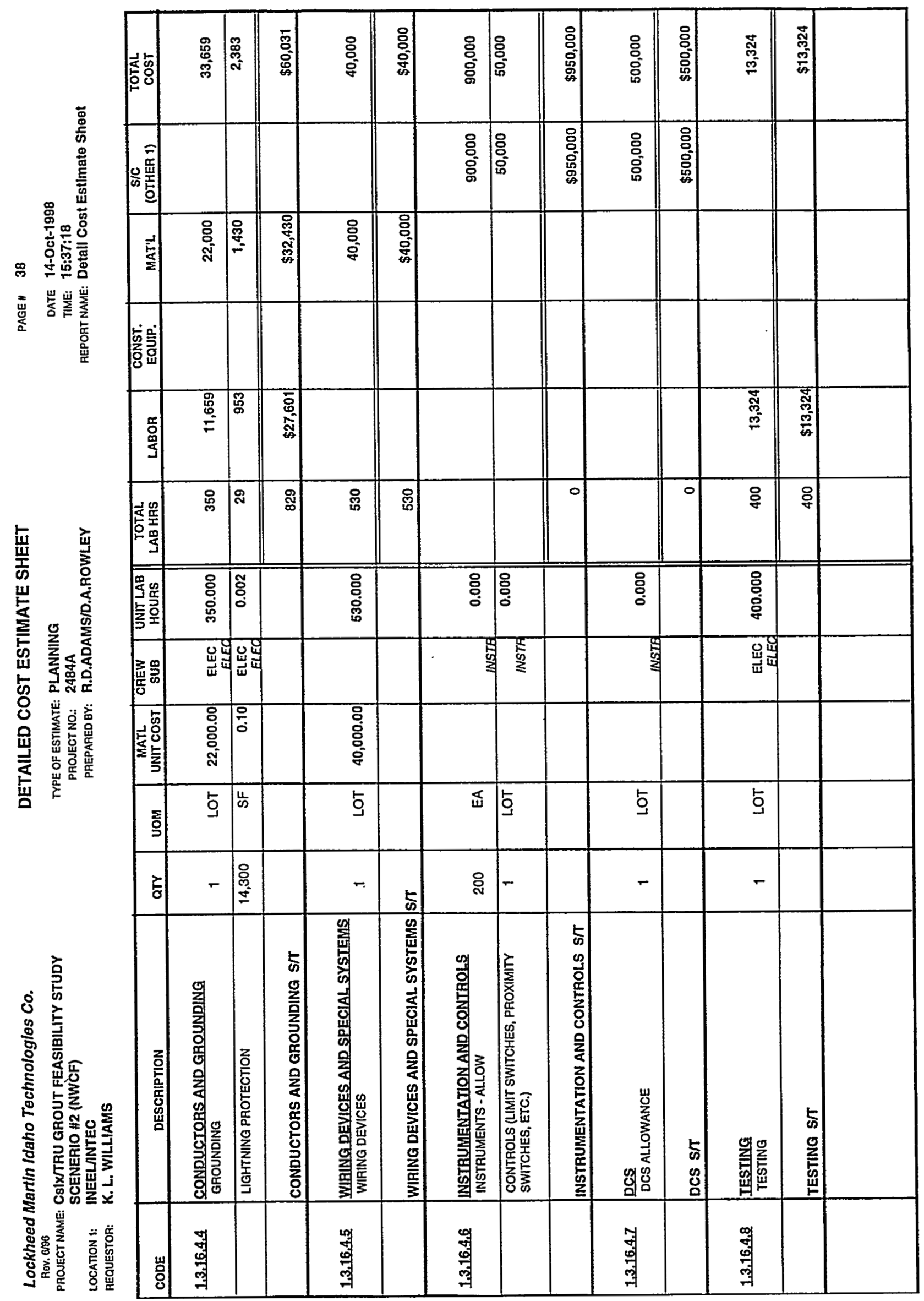




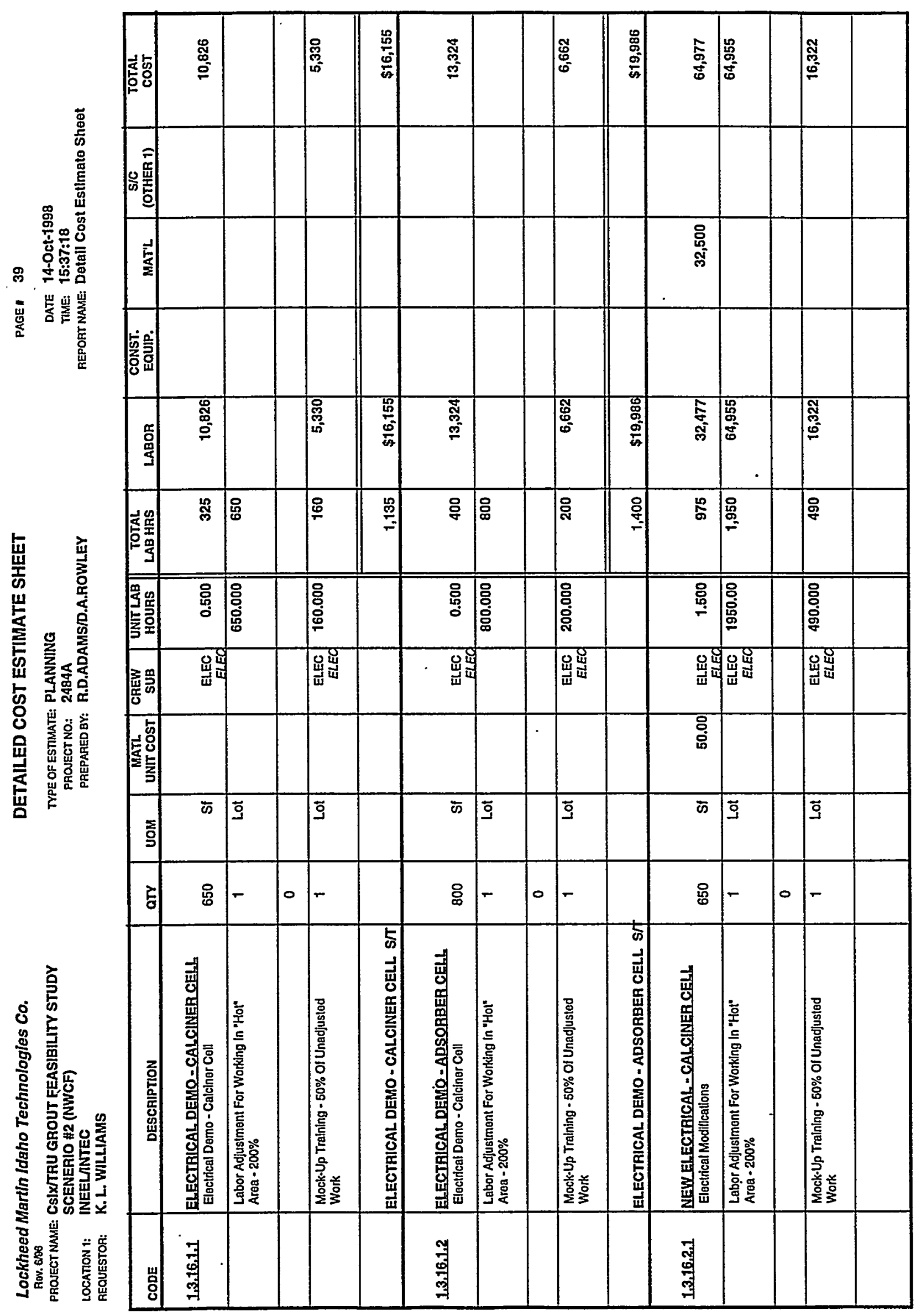




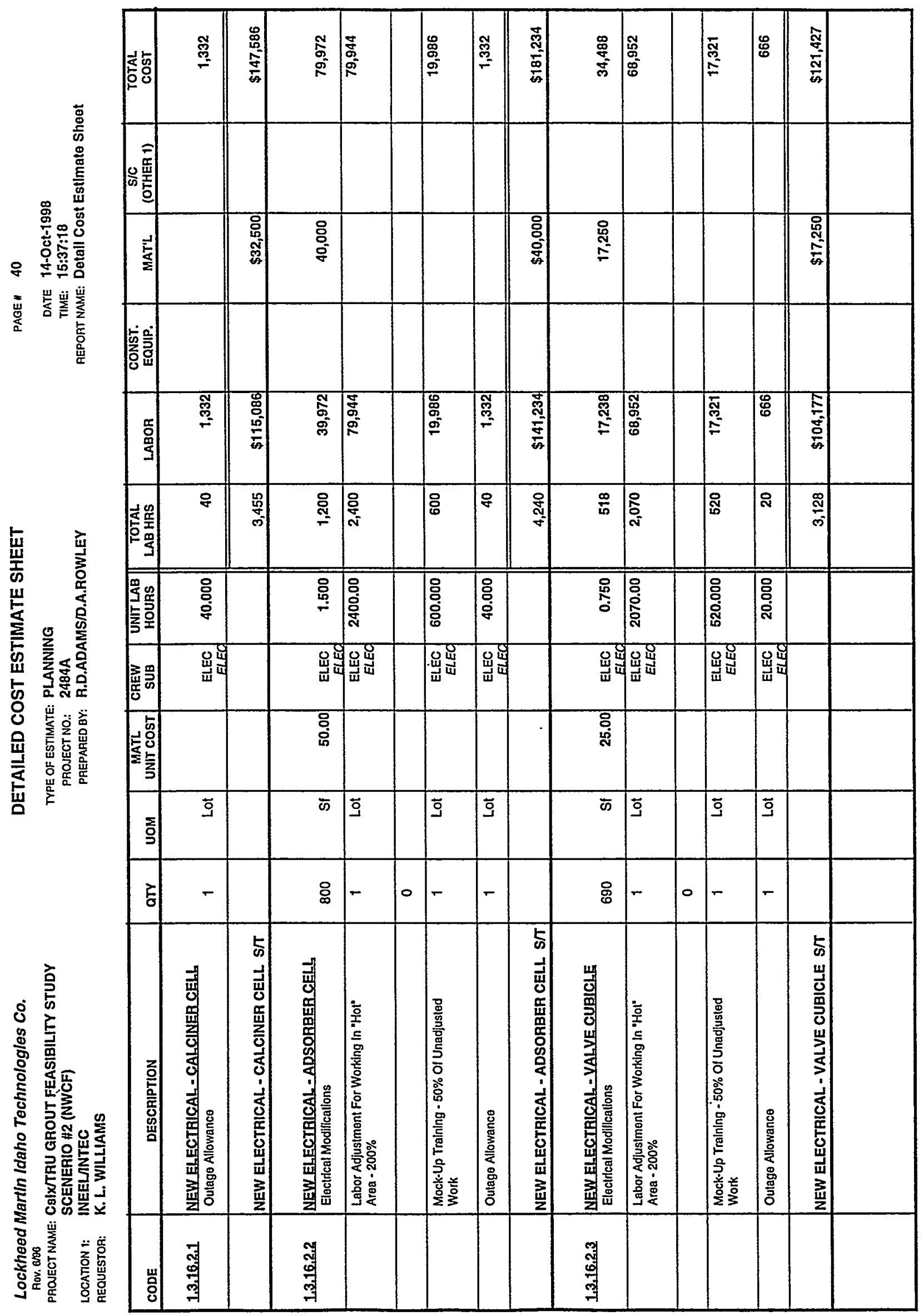




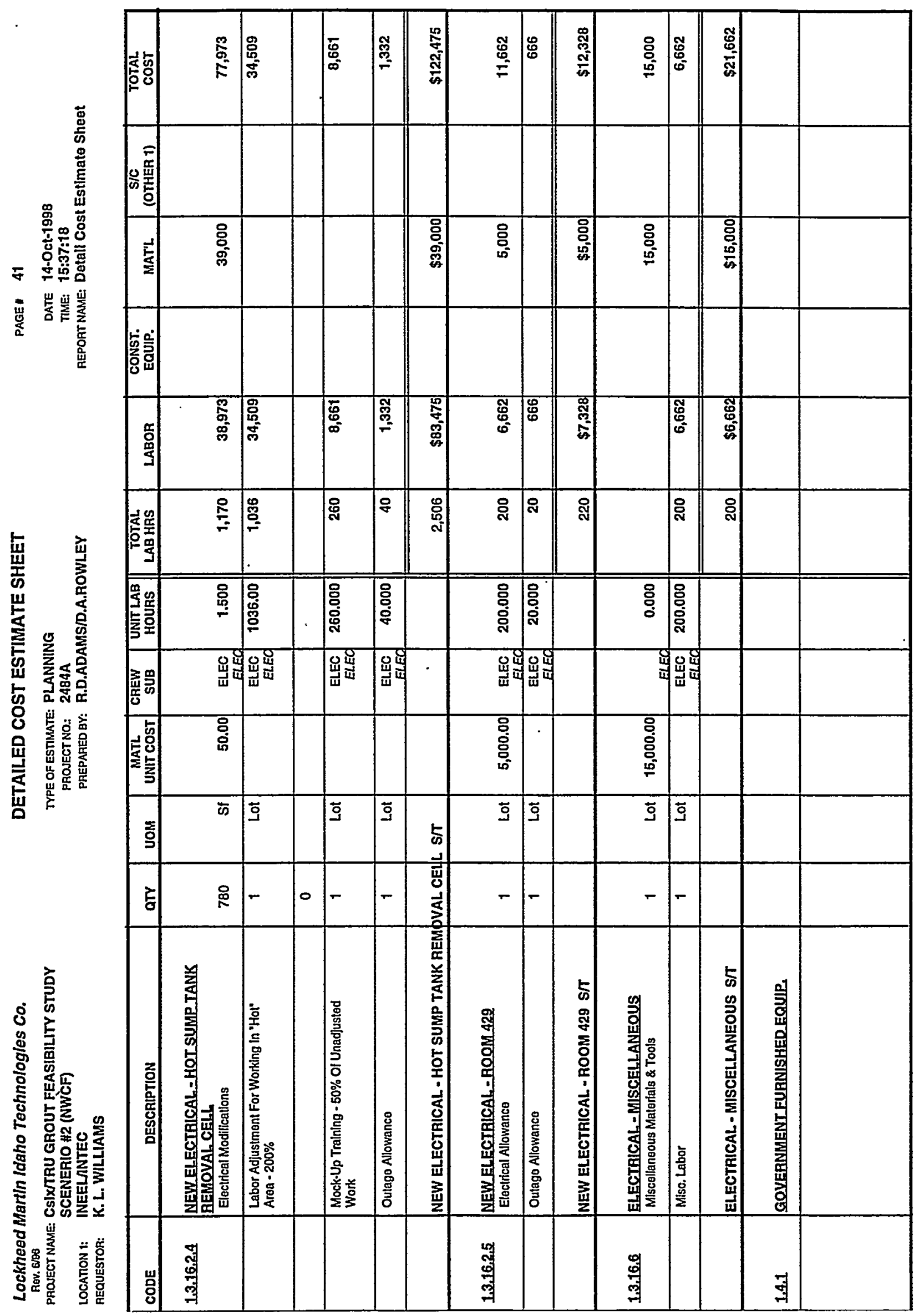




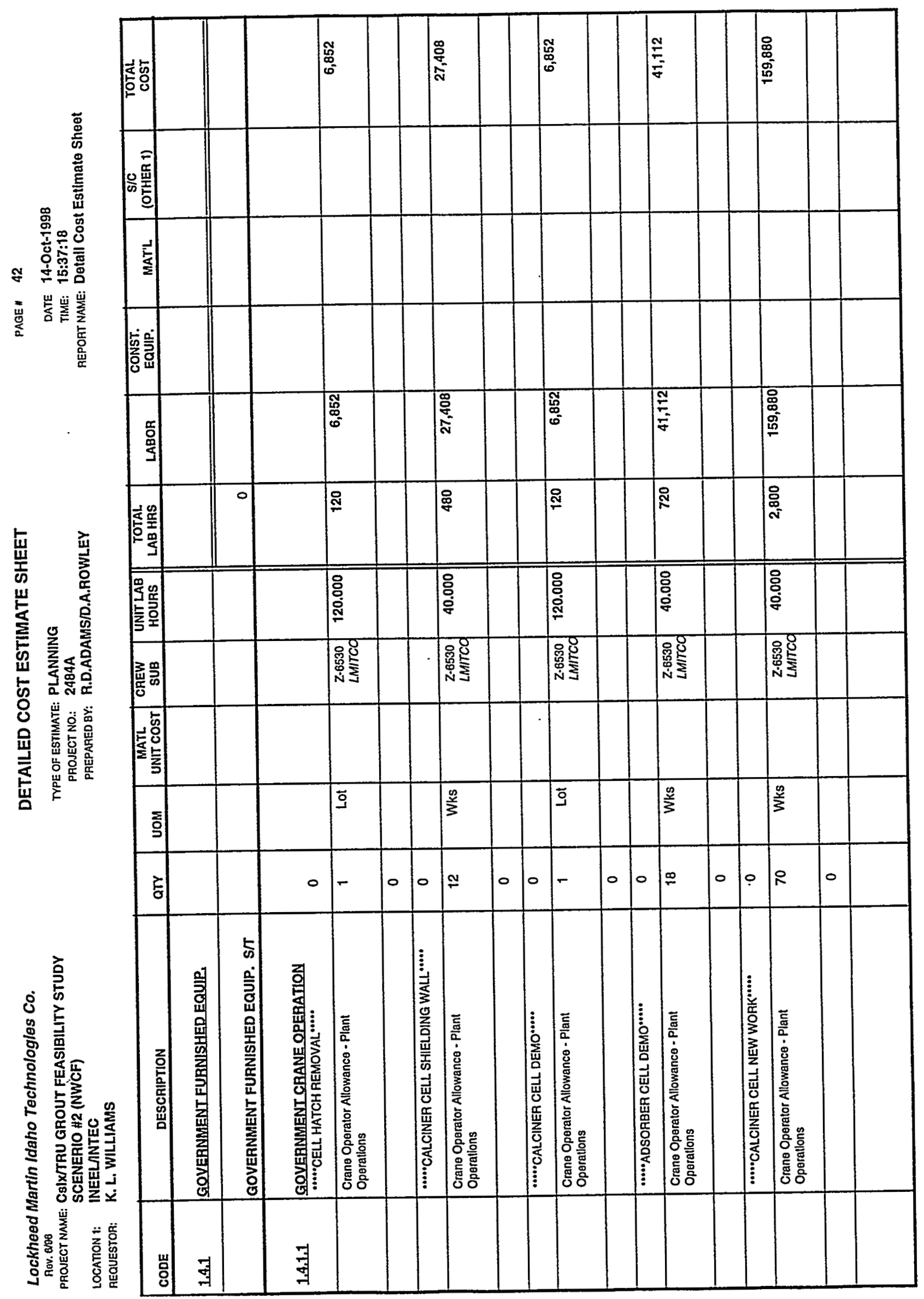




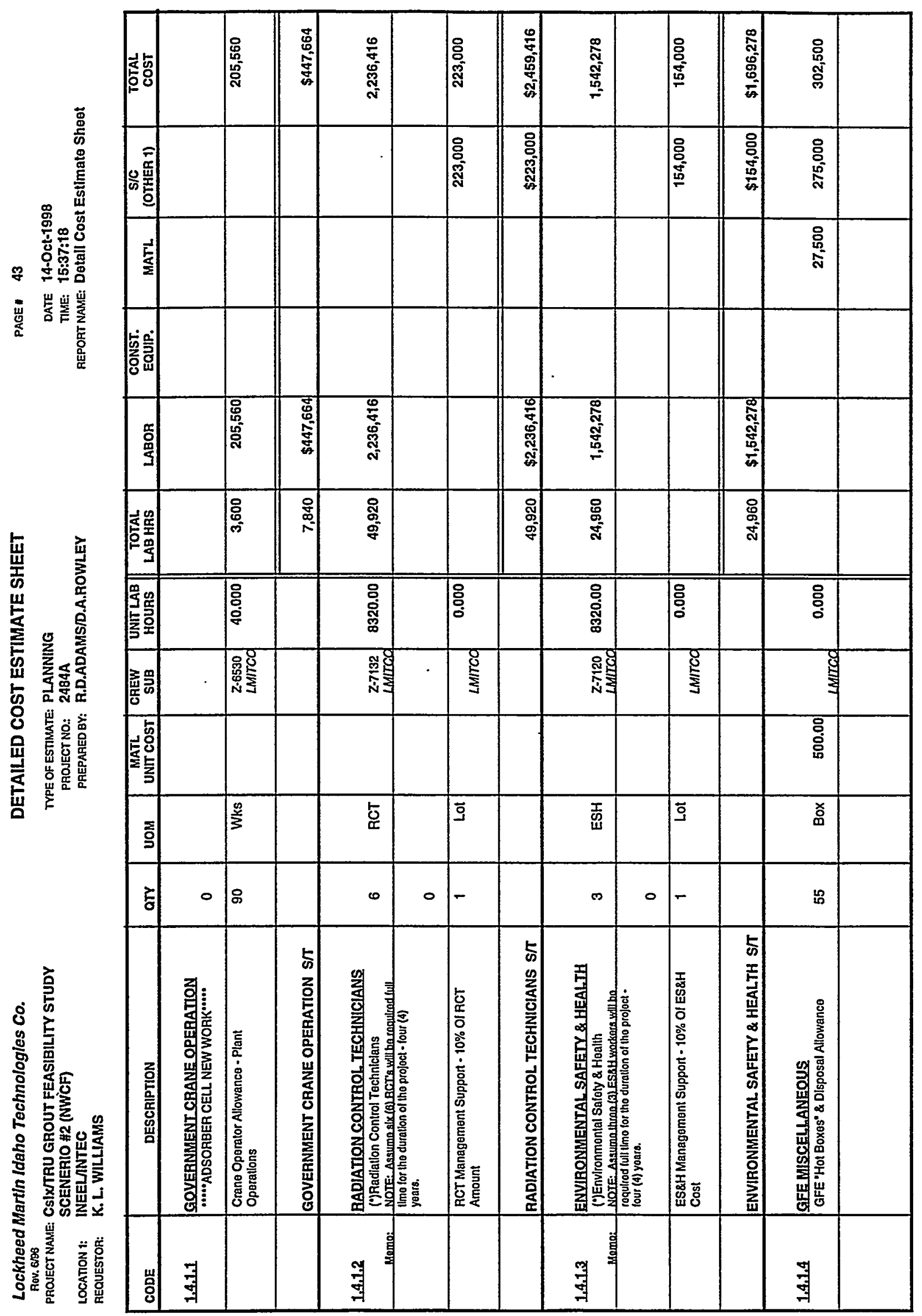




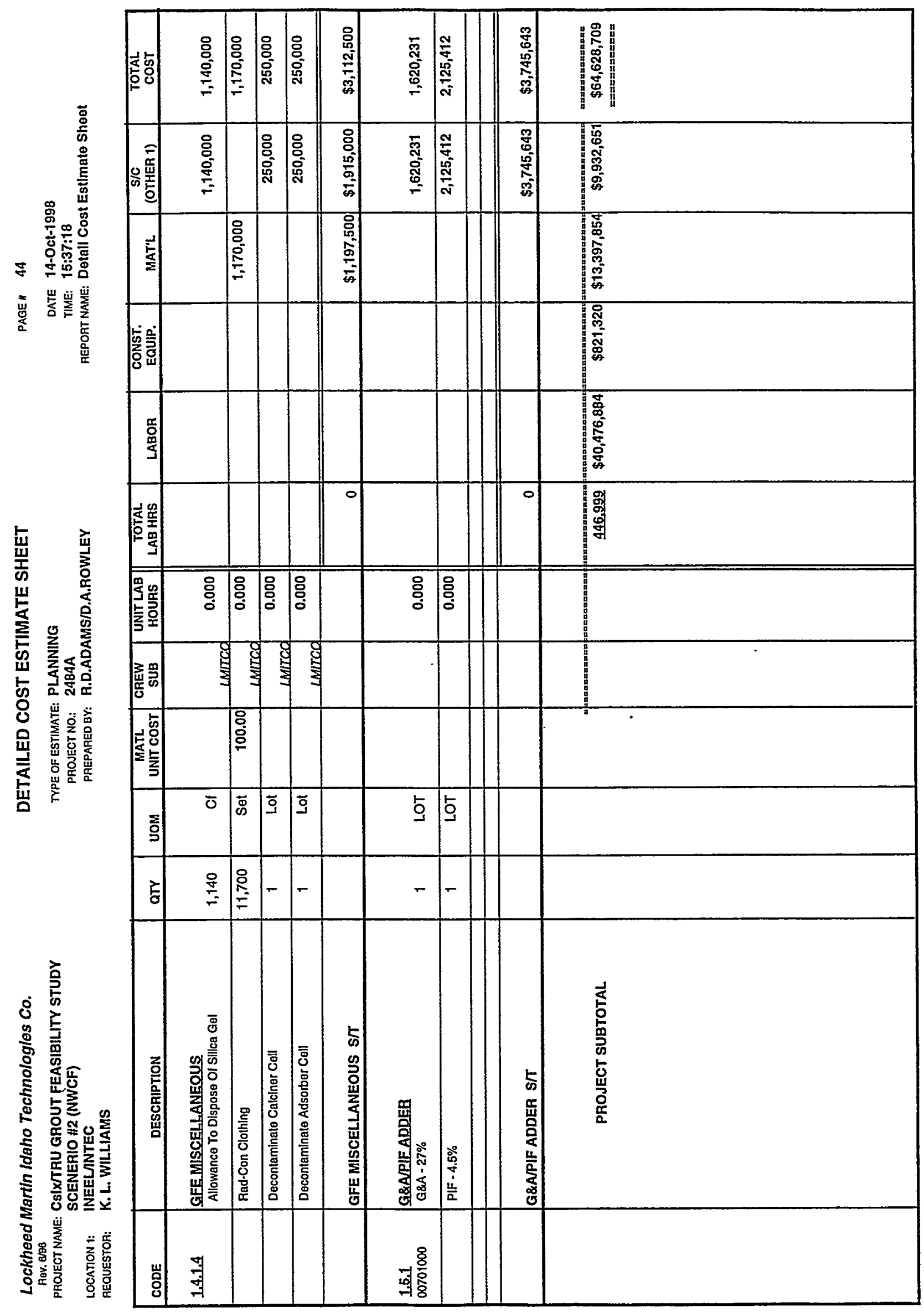


Lockheed Martin Idaho Technologies Co. PRONECT NAME: CSIXTRRU GROUT FEASIBILITY STUDY SCENERIO \#2 (NWCF) LOCATION 1: INEELANTEC REQUESTOR: K.L. WILLIAMS

\section{CONTINGENCY ANALYSIS}

TYPE OFESTIMATE: PLANNING PROSECT NO: PREPARED BY: 2484A
DATE: 14-Oct-1998

TIME: 15:41:30

REPORT NAME: Contingency Analysis

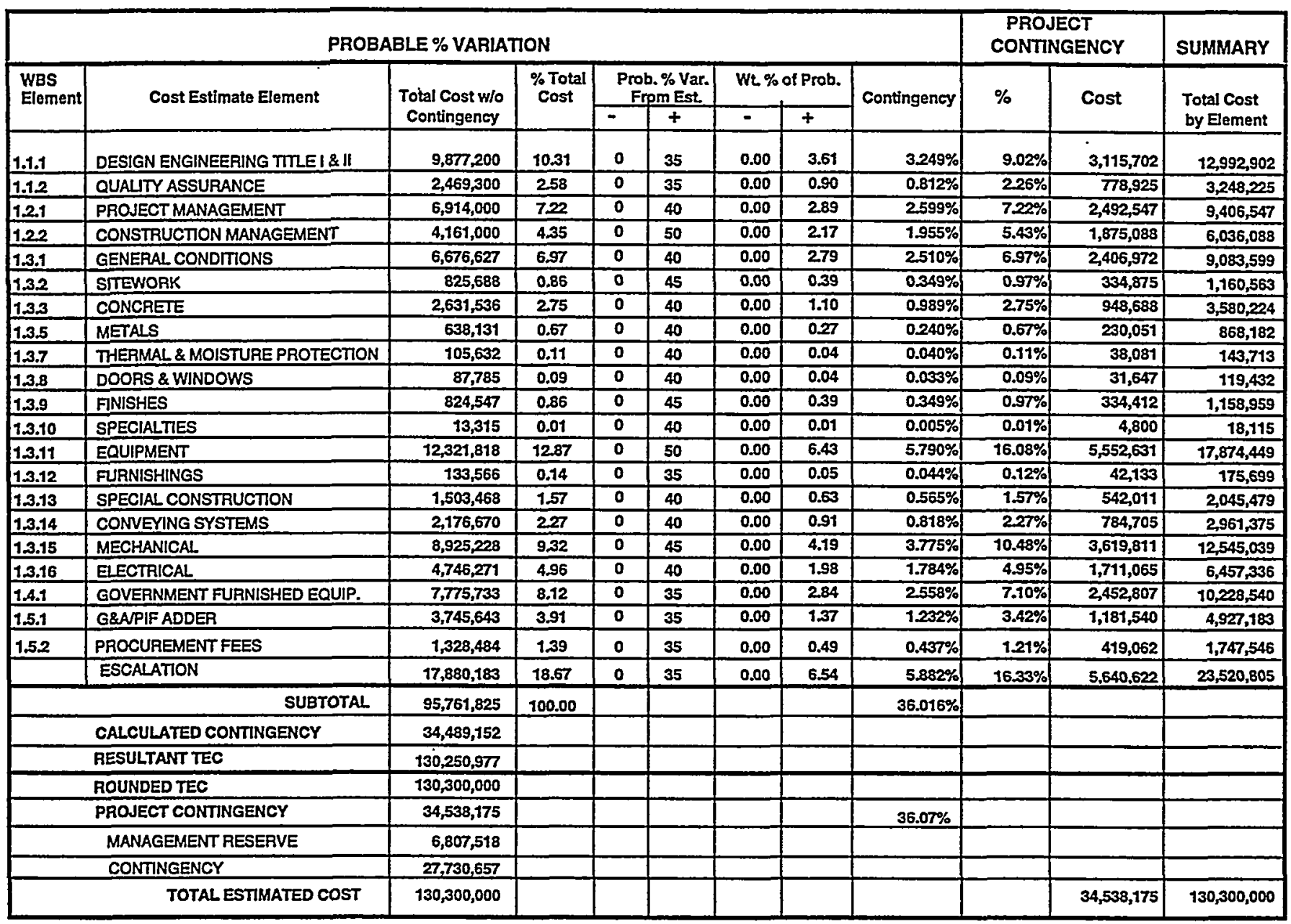

\section{CONFIDENCE LEVEL AND ASSUMED RISKS:}

The Lockheed Idaho Technologies Co. Cost Estimate Contingency Analysis Model is based on the applied contingency and the assumptions upon which the estimate was predicated. The model is applied with a suggested risk level of $18 \%$ and a level of confidence of $90 \%$ the estimate will fall within the bid range. The Contingency Analysis is based on a weighted average to provide a

$90 \%$ probability of underrun and a $10 \%$ probability of overrun.
CONTINGENCY ANALYSIS GUIDE BY TYPE OF ESTIMATE

Guidelines established by DOE/FM 50, Cost Estimating Guide, Vol. 6, Cost Guide, and as presented in the INEL Cost Estimating Guide. PLANNING $20 \%-30 \%$ Experimental/Special Conditions.............Up to $50 \%$ Conceptual $15 \%-25 \%$ Experimental/Special Conditions............Up to $40 \%$ TITLE TITLE II TITLE II/AFC
$10 \%-20 \%$ Market Conditions 


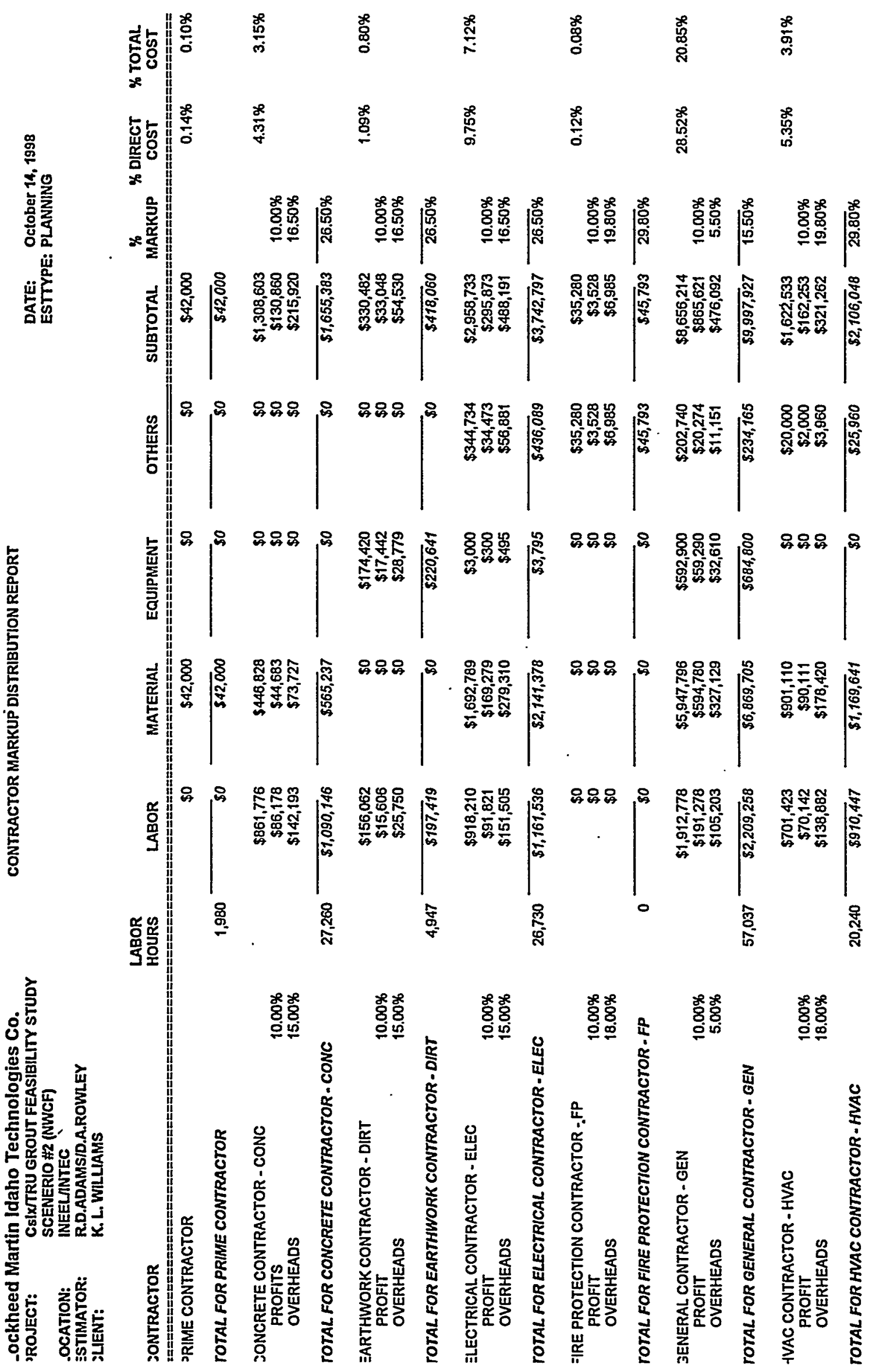




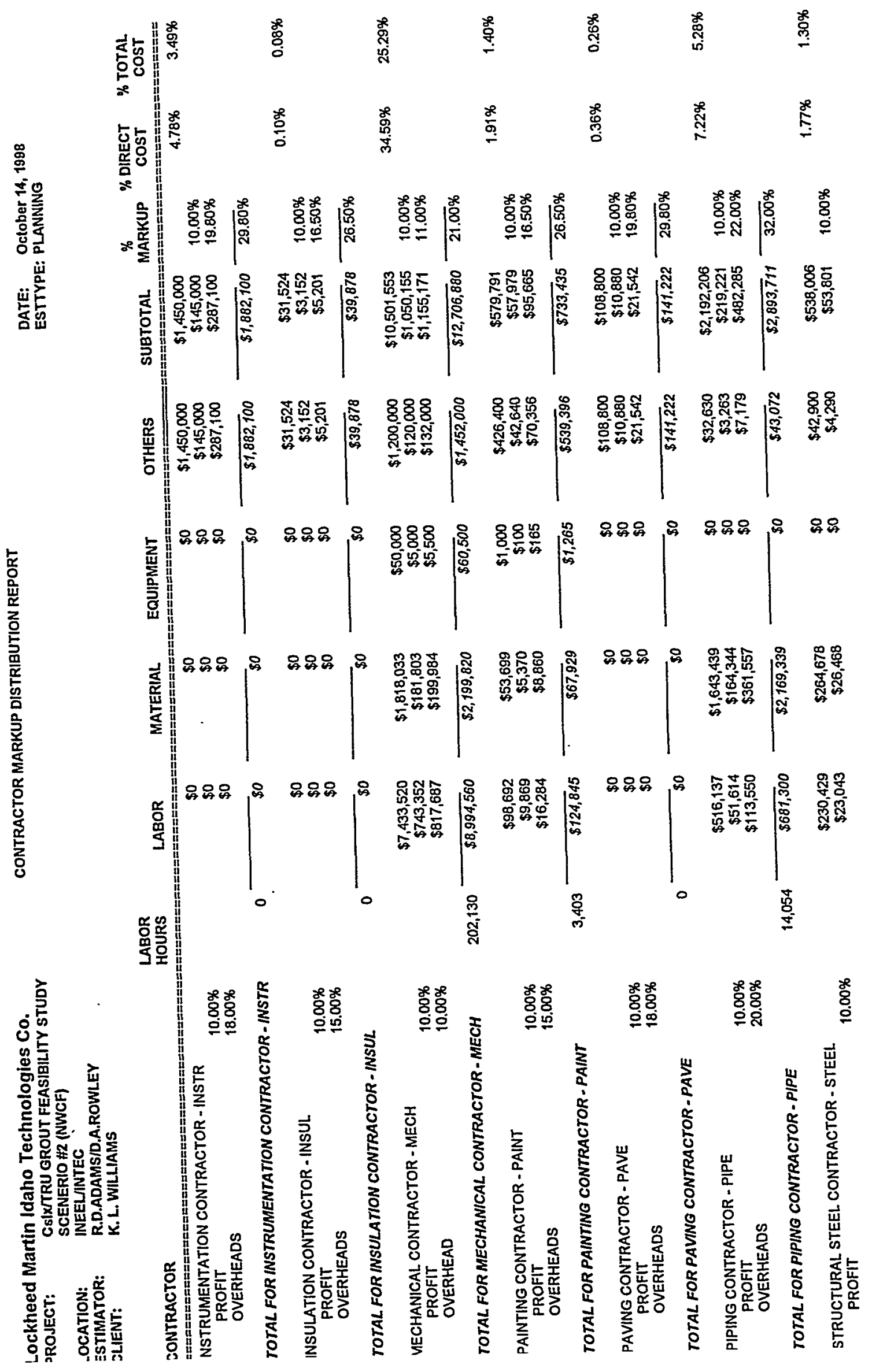




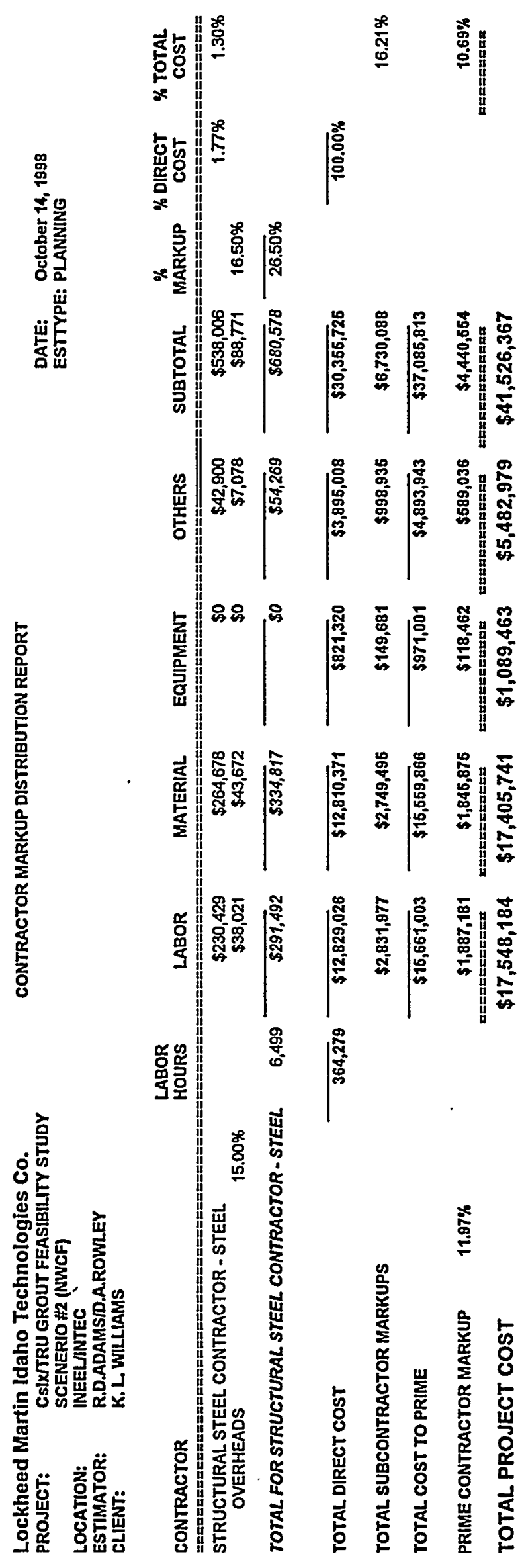




\section{FY-99 G\&AJPIF ADDER CALCULATION SHEET}

PROJECT: CSIX/TRU GROUT FACILITY SCENERIO \#2

DATE: 10/14/98

PROCUREMENT FEE:

\begin{tabular}{lr} 
CONSTRUCTION $=$ & $\begin{array}{r}\$ 41,610,282 \\
\text { GFE }= \\
\text { Subtotal }\end{array}$ \\
\cline { 2 - 2 } & $\$ 2,672,375$ \\
\hline FEE@3.0\%= & $\$ 44,282,657$ \\
& $\$ 44,282,657 \cdot 0.03=\quad \$ 1,328,480$
\end{tabular}

G\&A @ 27\% (with a ceiling of $\$ 500,000$ imposed per year)

CONSTRUCTION \$ OR CEILING * \# OF YEARS

YEARS OF CONST. $=4$

$\$ 2,000,000$

$\$ 2,672,375$

$\mathrm{GFE}=\quad \$ 2,672,375$

PROCUREMENT FEE $=$

$\$ 1,328,480$

Subtotal

$\$ 6,000,855$

FEE@ $27 \%=$

$\$ 6,000,855 \cdot 0.27=\quad \$ 1,620,231$

PIF @ 4.5\%

$\begin{array}{lr}\text { CONSTRUCTION }= & \$ 41,610,282 \\ \text { GFE }= & \$ 2,672,375 \\ \text { PROCUREMENT FEE }= & \$ 1,328,480 \\ \text { G\&A }= & \$ 1,620,231 \\ \text { Subtotal } & \$ 47,231,367\end{array}$

FEE@ $4.5 \%=$

$\$ 47,231,367 \cdot 0.045=\quad \$ 2,125,412$

TOTAL PROCUREMENT FEE:

$\$ 1,328,480$

TOTAL G\&A FEE:

$\$ 1,620,231$

TOTAL PIF:

$\$ 2,125,412$ 
Lockheed Martin Idaho Technologies Co. PROJECT NAME: CSIXITRU GROUT FEASIBILITY STUDY SCENERIO \#2 (NWCF) - OPC REOUESTOP: K L WILLIAMS
COST ESTIMATE SUMMARY

TYPE OF ESTIMATE: PLANNING PROSECT NO: 2484AO PREPARED BY: R.D.ADAMS/D.A.ROWLEY CHECKED BY: REPORT NAME: Cost Estimate Summary

\begin{tabular}{|c|c|c|c|c|}
\hline $\begin{array}{l}\text { WBS } \\
\text { Element }\end{array}$ & Cost Estimate Element & $\begin{array}{c}\text { Total } \\
\text { Unescalated }\end{array}$ & Escalation & $\begin{array}{l}\text { Total } \\
\text { Incl Escalation }\end{array}$ \\
\hline $\begin{array}{l}1.1 \\
1.1 .1 \\
1.2 \\
1.2 .1 \\
\frac{1.3}{1.3 .1} \\
\frac{1.4}{1.4 .1} \\
1.5 .2\end{array}$ & $\begin{array}{l}\text { CONCEPTUALDESIGN } \\
\text { CONCEPTUAL DESIGN } \\
\text { MANAGEMENT COSTS } \\
\text { PROJECT MANAGEMENT \& PROCESS } \\
\text { PERMITTING } \\
\text { PERMITTING } \\
\text { SO TEST \& STARTUP } \\
\text { SO TEST \& STARTUP } \\
\text { PROCUREMENT FEES }\end{array}$ & $\begin{array}{r}2,709,300 \\
11,469,300 \\
1,268,867 \\
10,318,000 \\
0\end{array}$ & $\begin{array}{r}162,558 \\
688,158 \\
114,198 \\
2,785,860 \\
0\end{array}$ & $\begin{array}{l}\gg \frac{\$ 2,871,858}{2,871,858} \\
\gg \quad \frac{\$ 12,157,458}{12,157,458} \\
\gg \quad \frac{\$ 1,383,065}{1,383,065} \\
\gg \frac{\$ 13,103,860}{13,103,860} \\
\gg \quad \text { S0 }\end{array}$ \\
\hline & $\begin{array}{l}\text { SUBTOTAL INCLUDING ESCALATION } \\
\text { PROJECT CONTINGENCY }\end{array}$ & $25,765,467$ & $3,750,774$ & $\gg \$ 29,516,241$ \\
\hline & MANAGEMENT RESERVE- & & & \$o \\
\hline & CONTINGENCY- & & & $\$ 10,283,759$ \\
\hline & TOTAL ESTIMATED COST - & & & $\$ 39,800,000$ \\
\hline
\end{tabular}

EDI AS A $\%$ OF CONST. + GFE $=20.00 \%$ 


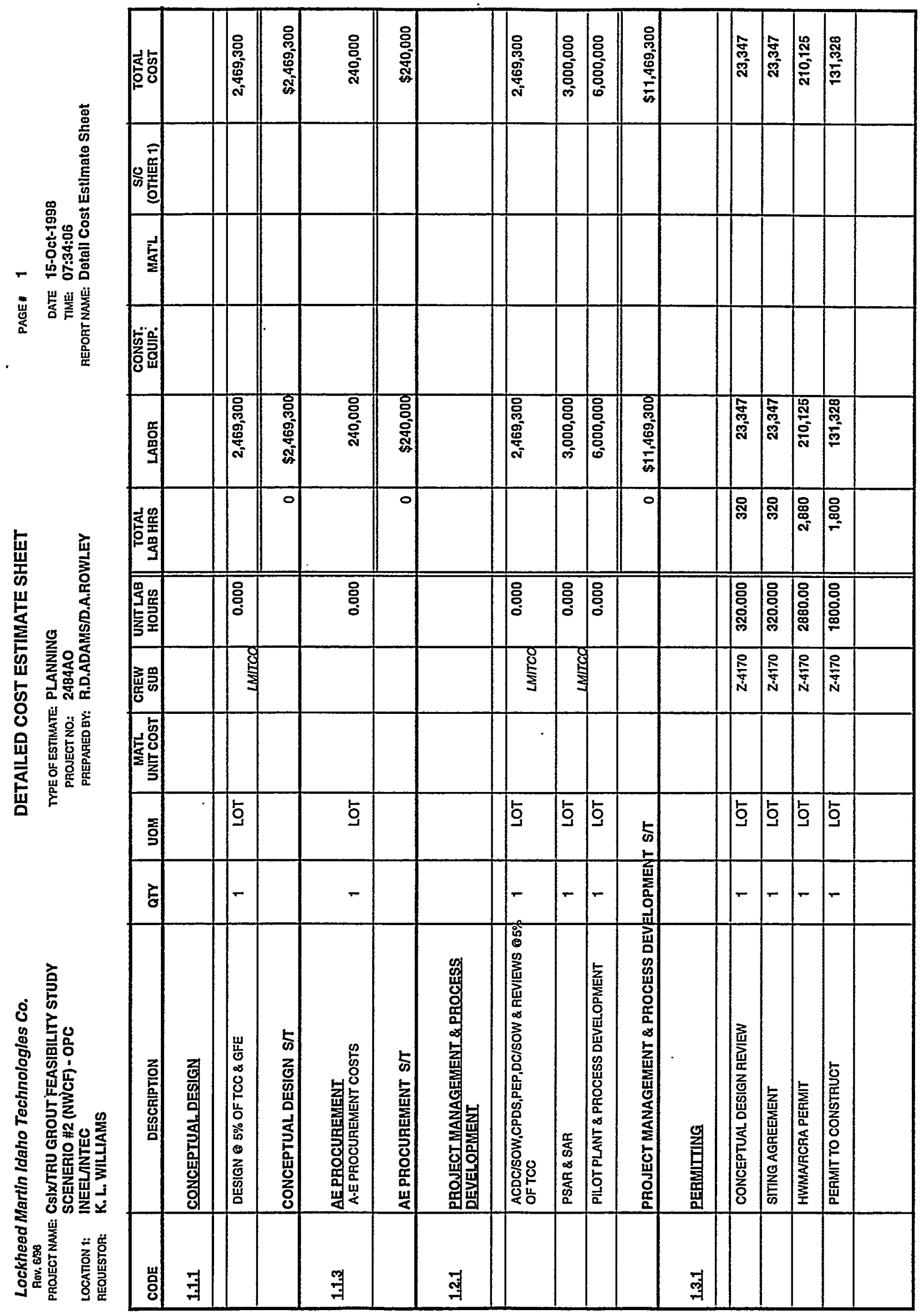




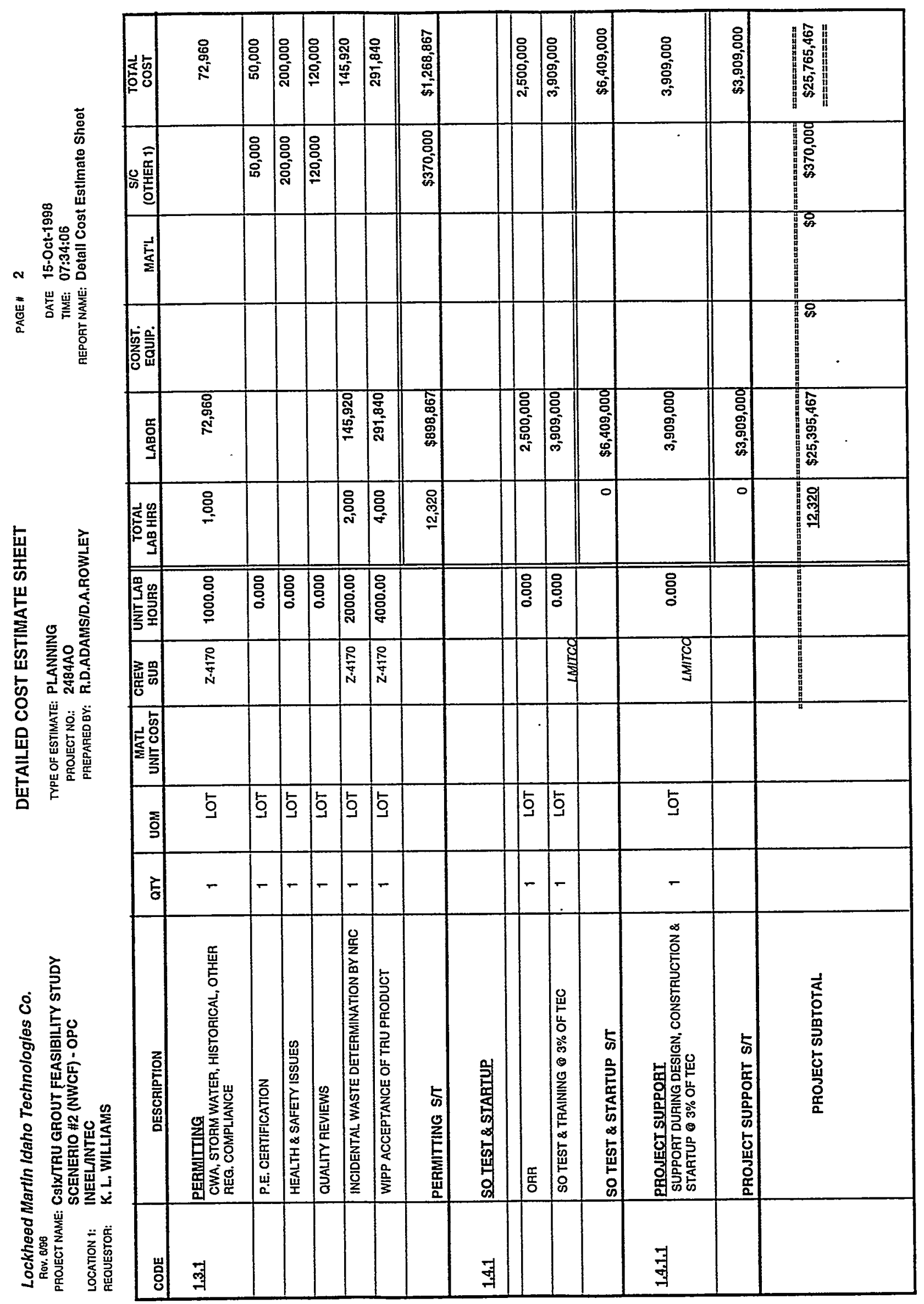


Lockheed Martin Idaho Technologies Co. PROUECT NAME: CSIXTRU GROUT FEASIBILITY STUDY SCENERIO \#2 (NWCF) - OPC

AEQUESTOR: K.L.WILLIAMS

\section{CONTINGENCY ANALYSIS}

$\begin{array}{clr}\text { TYPE OFESTIMATE: } & \text { PLANNING } & \text { DATE: } \\ \text { PRONECT NO: } & \text { 2484AO } & \text { TIME: } 07: 35: 08 \\ \text { PREPARED BY: } & \text { R.D.ADAMS/D.A.ROWLEY } & \end{array}$

REPORT NAME: Contingency Analysis

\begin{tabular}{|c|c|c|c|c|c|c|c|c|c|c|c|}
\hline \multicolumn{9}{|c|}{ PROBABLE \% VARIATION } & \multicolumn{2}{|c|}{$\begin{array}{c}\text { PROJECT } \\
\text { CONTINGENCY }\end{array}$} & \multirow{3}{*}{$\begin{array}{l}\text { SUMMARY } \\
\text { Total Cost } \\
\text { by Element }\end{array}$} \\
\hline \multirow[t]{2}{*}{$\begin{array}{l}\text { WBS } \\
\text { Element }\end{array}$} & \multirow[t]{2}{*}{ Cost Estimate Element } & \multirow{2}{*}{$\begin{array}{c}\text { Total Cost w/o } \\
\text { Contingency }\end{array}$} & \multirow[t]{2}{*}{$\begin{array}{l}\text { \% Total } \\
\text { Cost }\end{array}$} & \multicolumn{2}{|c|}{$\begin{array}{l}\text { Prob. \% Var. } \\
\text { From Est. }\end{array}$} & \multicolumn{2}{|c|}{ Wt $\%$ of Prob. } & \multirow[t]{2}{*}{ Contingency } & \multirow[t]{2}{*}{$\%$} & \multirow[t]{2}{*}{ Cost } & \\
\hline & & & & - & + & - & + & & & & \\
\hline 1.1 .1 & CONCEPTUAL DESIGN & $2,709,300$ & 9.18 & 10 & 40 & 0.92 & 3.67 & $3.213 \%$ & $9.18 \%$ & 943,948 & $3,653,248$ \\
\hline 1.2 .1 & PROJECT MANAGEMENT \& PROCESS & $11,469,300$ & 38.86 & 10 & 40 & 3.89 & 15.54 & $13.600 \%$ & $38.86 \%$ & $3,996,021$ & $15,465,321$ \\
\hline 1.3 .1 & PERMITING & $1,268,867$ & 4.30 & 10 & 40 & 0.43 & 1.72 & $1.505 \%$ & $4.30 \%$ & 442,086 & $1,710,953$ \\
\hline 1.4 .1 & SO TEST \& STARTUP & $10,318,000$ & 34.96 & 10 & 40 & 3.50 & 13.98 & $12.235 \%$ & $34.96 \%$ & $3,594,896$ & $13,912,896$ \\
\hline \multirow[t]{2}{*}{1.5 .2} & PROCUREMENT FEES & 0 & 0.00 & 10 & 40 & 0.00 & 0.00 & $0.000 \%$ & $0.00 \%$ & 0 & 0 \\
\hline & ESCALATTON & $3,750,774$ & 1271 & 10 & 40 & 1.27 & 5.08 & $4.448 \%$ & $12.71 \%$ & $1,306,808$ & $5,057,582$ \\
\hline & SUBTOTAL & $29,516,241$ & 100.00 & & & & & $35.000 \%$ & & & \\
\hline & CALCULATED CONTINGENCY & $10,330,684$ & & & & & & & & & \\
\hline & RESULTANT TEC & $39,846,925$ & & & & & & & & & \\
\hline & ROUNDED TEC & $39,800,000$ & & & & & & & & & \\
\hline & PROJECT CONTINGENCY & $10,283,759$ & & & & & & $34.84 \%$ & & & \\
\hline & MANAGEMENT RESERVE & $\mathbf{0}$ & & & & & & & & & \\
\hline & CONTINGENCY & $10,283,759$ & & & & & & & & & \\
\hline & TOTALESTIMATED COST & $39,800,000$ & & & & & & & & $10,283,759$ & $39,800,000$ \\
\hline
\end{tabular}

\section{CONFIDENCE LEVEL AND ASSUMED RISKS:}

The Lockheed Idaho Technologies Co. Cost Estimate Contingency Analysis

Model is based on the applied contingency and the assumptions upon which

the estimate was predicated. The model is applied with a suggested risk level

of $18 \%$ and a level of confidence of $90 \%$ the estimate will fall within the bid range.

The Contingency Analysis is based on a weighted average to provide a

$90 \%$ probability of underun and a $10 \%$ probability of overrun.
CONTINGENCY ANALYSIS GUIDE BY TYPE OF ESTIMATE Guidelines established by DOE/FM 50, Cost Estimating Guide, Vol. 6 , Cost Guide, and as presented in the INEL Cost Estimating Guide.

PLANNING

$20 \%-30 \%$

Experimental/Special Conditions.............Up to $50 \%$
Conceptual

$15 \%-25 \%$

TITLE ।

TITLE II

TITLE II/AFC

$10 \%-20 \%$

$5 \%-15 \%$

Market Conditions 


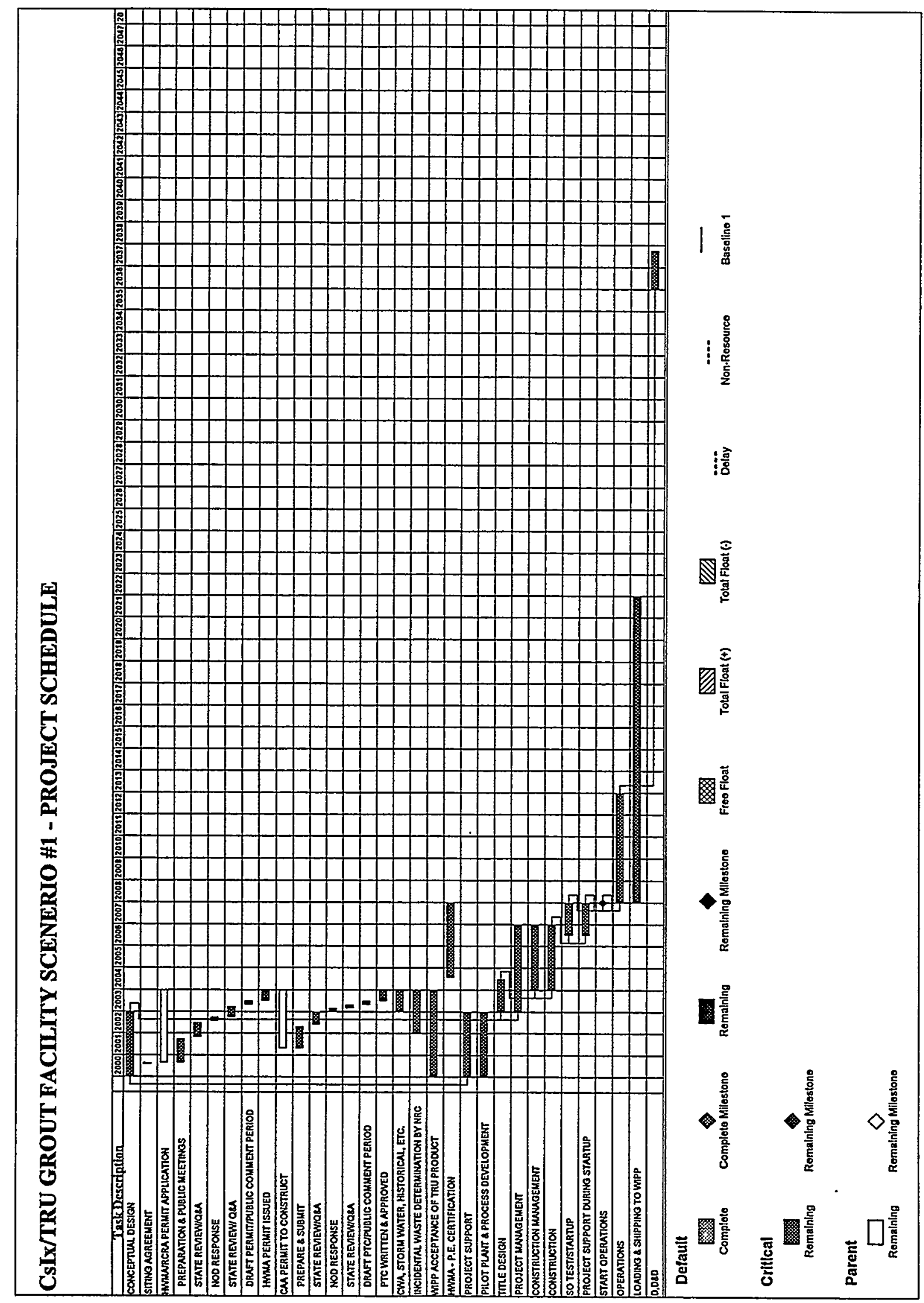




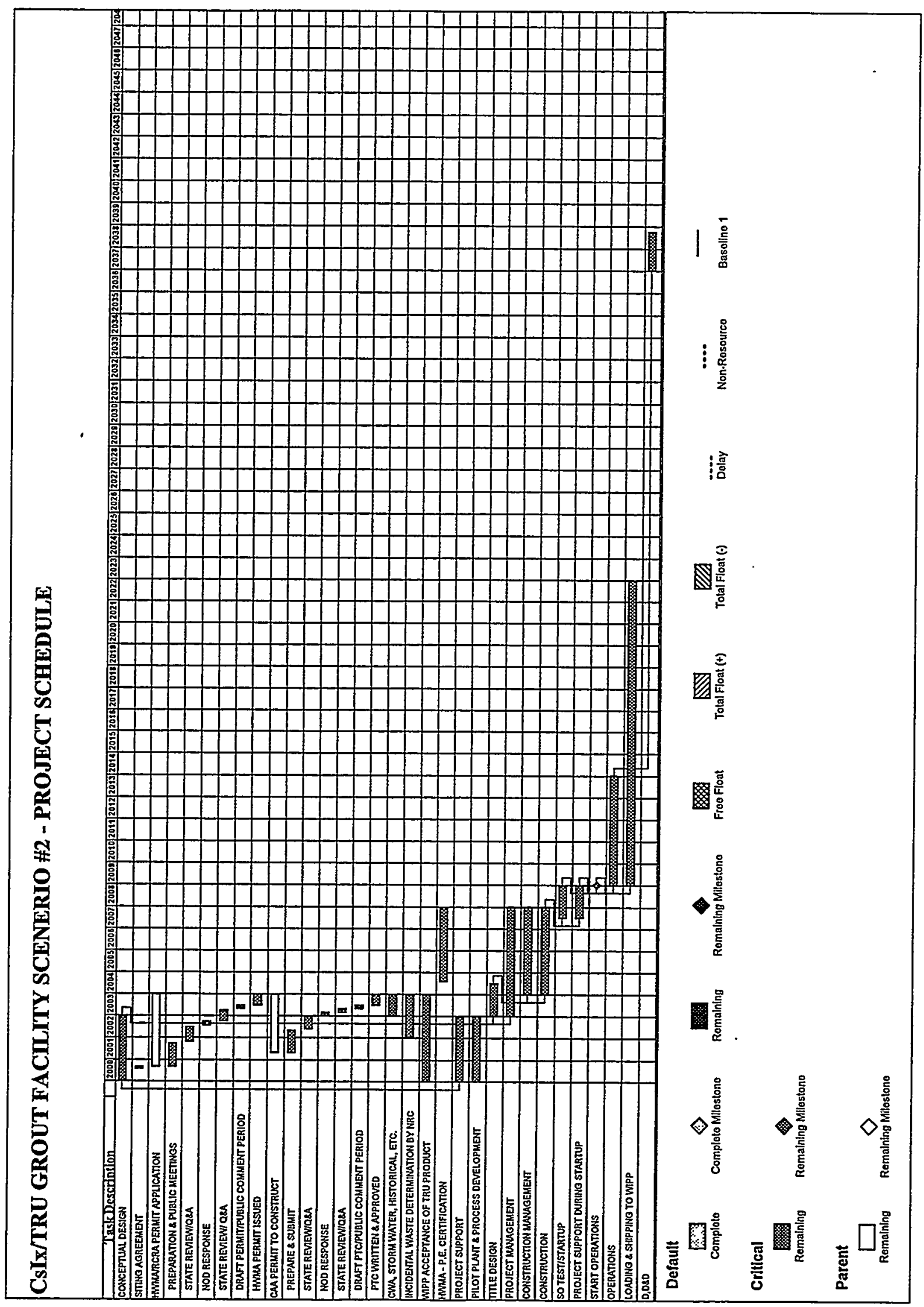


Date: $\quad$ November 9,1998

To: $\quad$ K. L.Williams . MS 3765 6-6760

From: R. J. Turk fYflech MS 3875

Subject: $\quad$ ECONOMIC AND LIFE CYCLE ANALYSIS CONDUCTED for the CsIX/TRU GROUT FACILITY-RJT-84-98 (REV1)

\section{Purpose:}

As requested, an Economic and Life-Cycle Cost (LCC) has been conducted to evaluate the CsIx/TRU GROUT FACILITY. This facility is proposed to process undissolved solids removal via filtration and Cesium removal via . ion exchange, followed by a cement-based grouting of the remaining liquid in 55 gallon drums. This economic analysis is based on information provided from Charles Barnes, Jim Bosley, Lynn Clapp, Ron Dafoe, Blair Grover, Tom Hipp, Scott Jensen and Syl Losinski. R. D. Adams provided cost estimates.

\section{Methodology:}

The Economic Evaluation assumed a 38-year period, (2000-2037) since this is the estimated time required to complete all of the anticipated remediation activities. The LCC evaluated the initial development, construction, operating and post operating costs over the life-cycle. A discounted LCC analysis assumes a constant-dollar basis, discounted at $6.10 \%$ per the Office of Management and Budget (OMB) Circular A-94. All costs are conservatively discounted assuming the end-of-year convention.

\section{Assumptions:}

The scope of work and requirements of all related activities are vague at this time. Facility and processing costs were developed from historical experience associated with DD\&D work at the INEEL. The LCC analysis was generated to match cost estimating cost structure. These costs include Permitting, Direct and Indirect Construction, G\&A, Procurement Fee, Engineering, Inspection, Project Management, Construction Management, Escalation and Contingency costs. The design period is assumed to be accomplished in three years with construction completed in 
KL. Williams

November 9, 1998

RJT 84-98 (rev 1)

Page 2

three years, followed by 1.25 years of start-up and testing. Labor rates were assumed as follows: Managers, \$125/hr; Engineers, $\$ 108 \$ / \mathrm{hr}$; Other Technicians $\$ 85 / \mathrm{hr}$; Administration/Support staff $\$ 65 / \mathrm{hr}$; Operators and Maintenance personnel $\$ 65 / \mathrm{hr}$. The operational period for this facility was assumed to be five years, followed by material placed in interim storage awaiting final disposition, followed by sixteen months of post-operations activities.

Utilities were assumed to cost $\$ 0.082 / \mathrm{kwh}$ for the facility. Due to this project's lack of complexity and relative cleanliness this analysis assumed a decommissioning cost equal to $20 \%$ of the unescalated engineering design cost, decontamination costs equal to $5 \%$ of the unescalated pre-operation cost, and demolition costs equal to $8 \%$ of the unescalated total pre-operation cost. DD\&D activities for scenario two do not include DD\&D efforts for NWCF due to being incorporated by anther study.

\section{Results:}

Scenario One has a Discounted LCC of $\$ 279$ million.

Scenario Two has a Discounted LCC of $\$ 327$ million.

Attachments:

cc: R. J. Turk File

$R$ D. Adams 


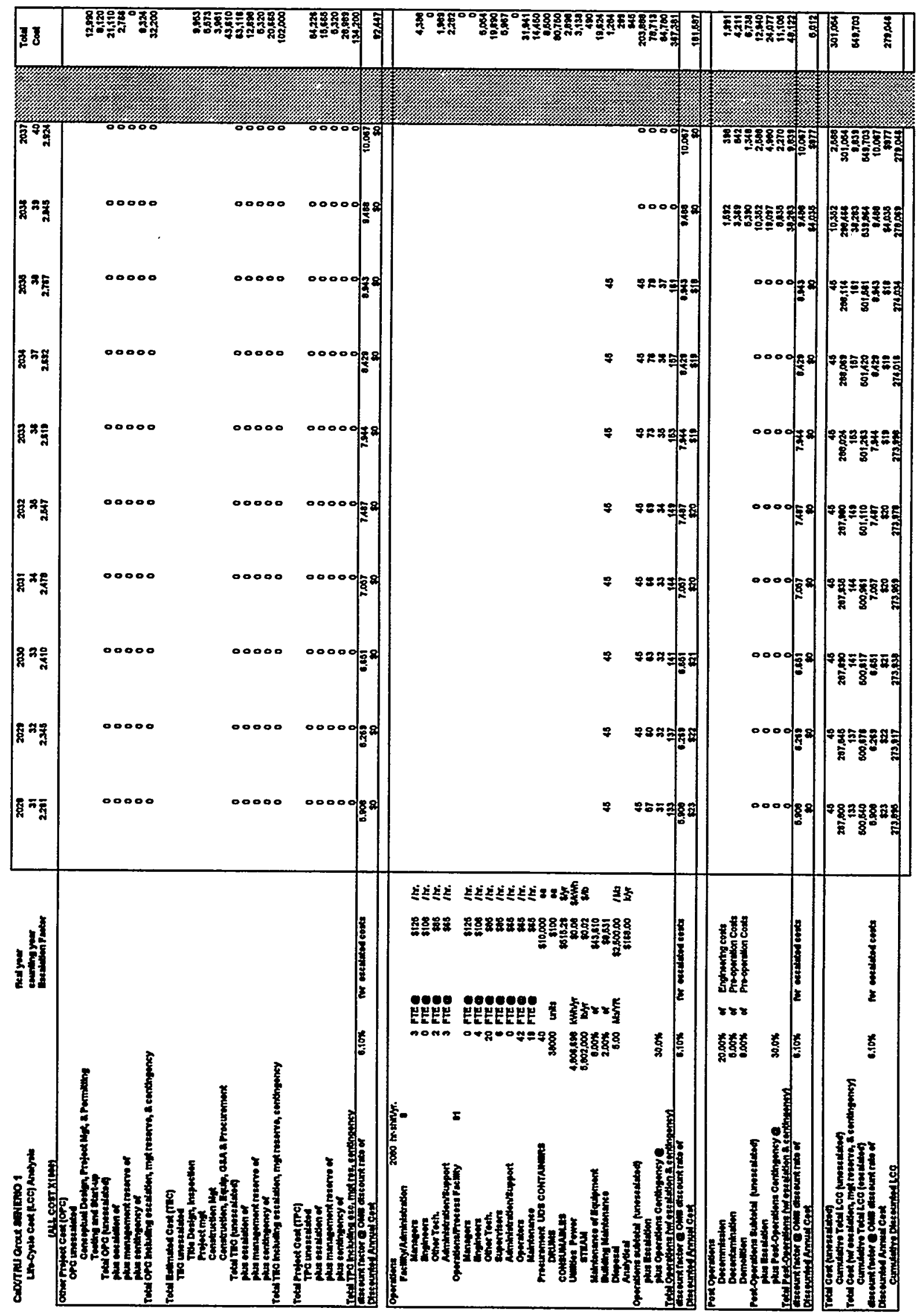




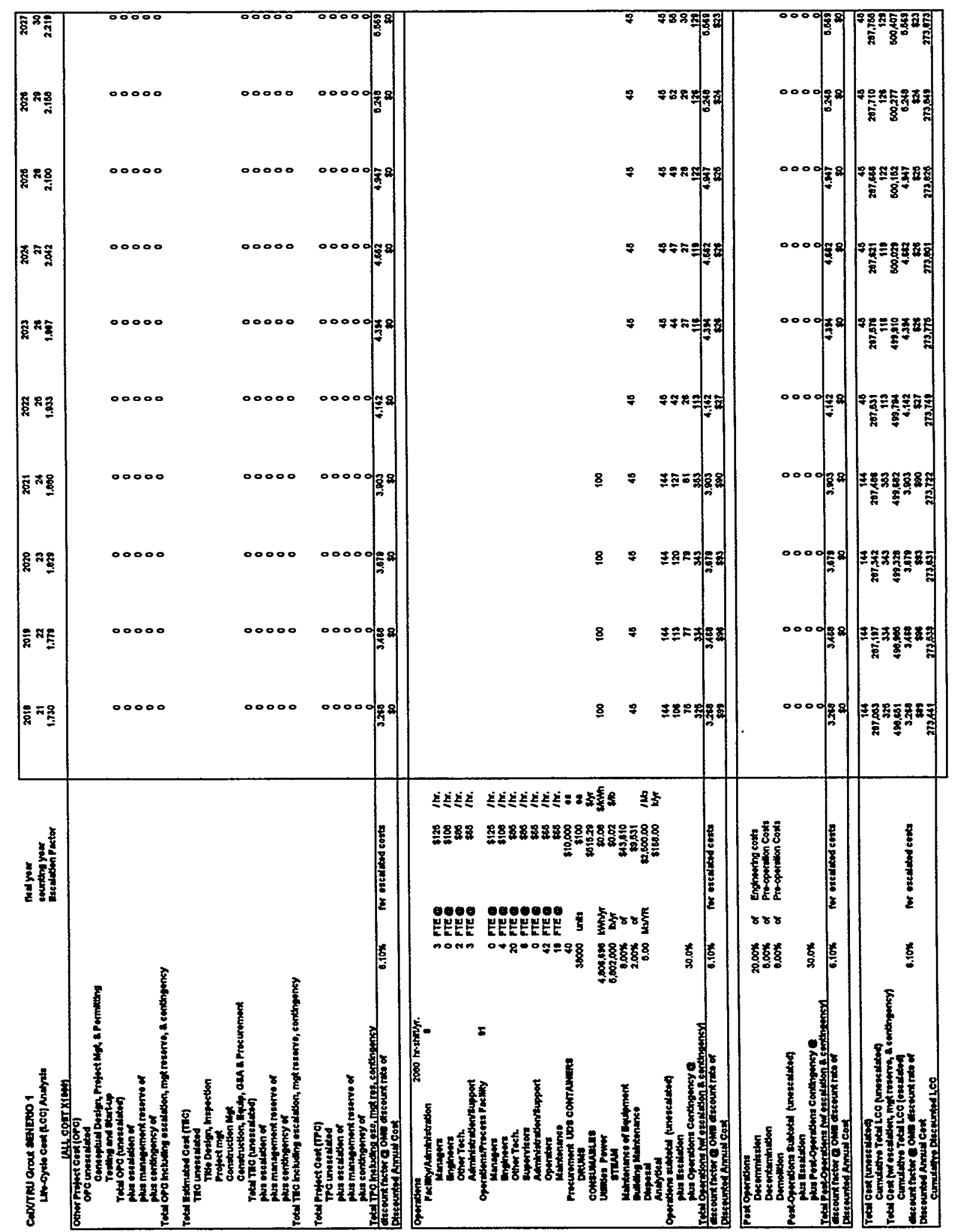




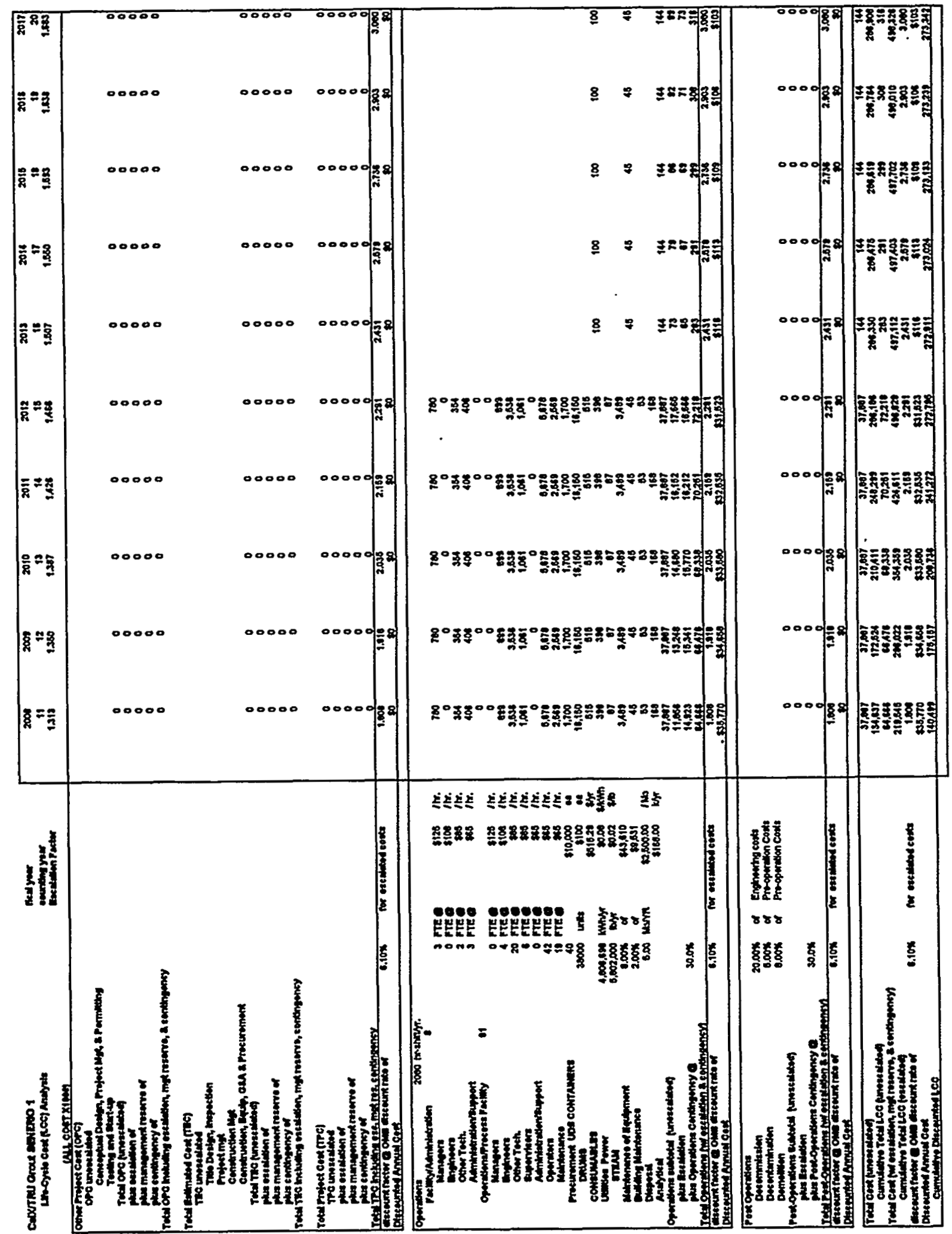




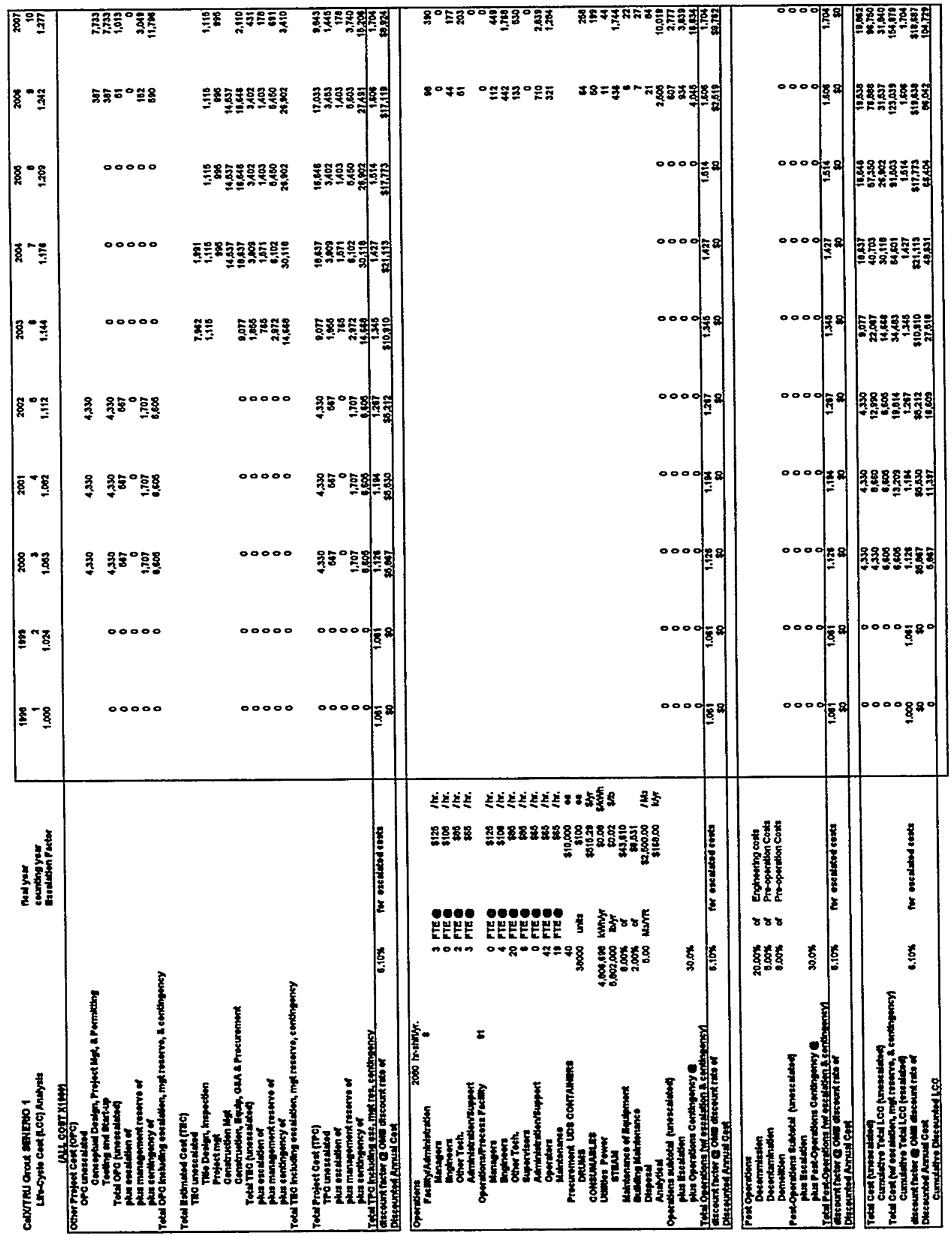




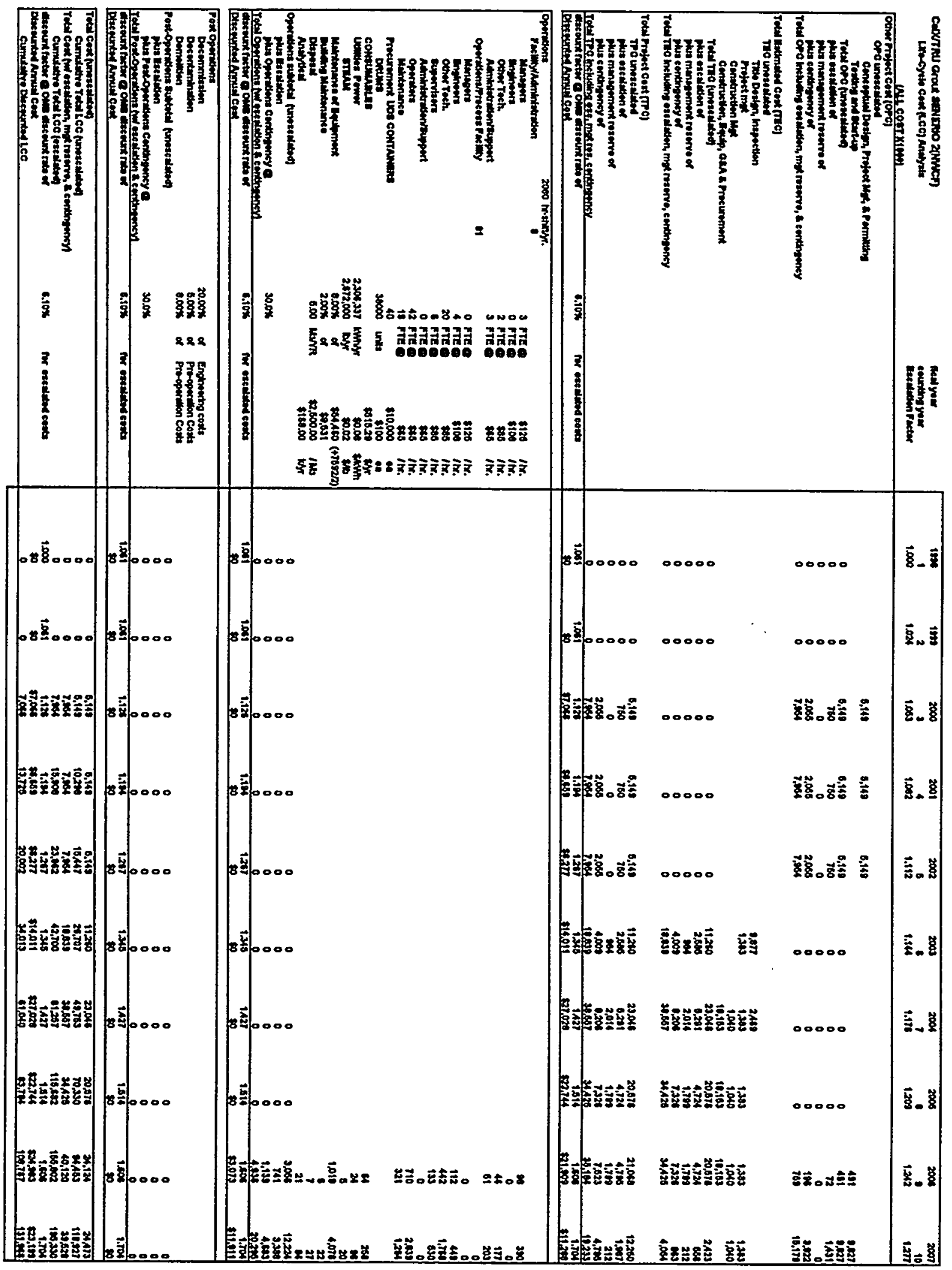




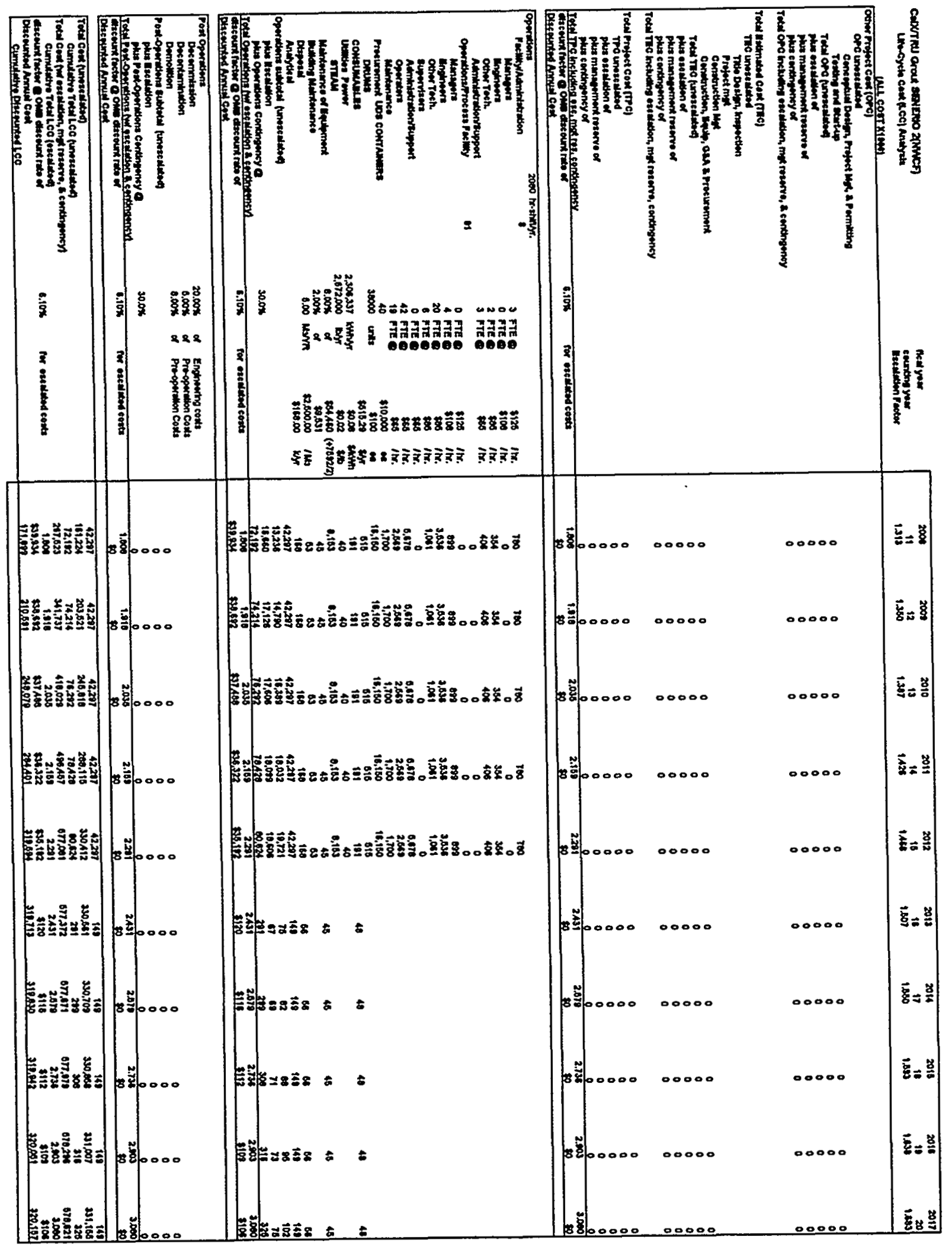




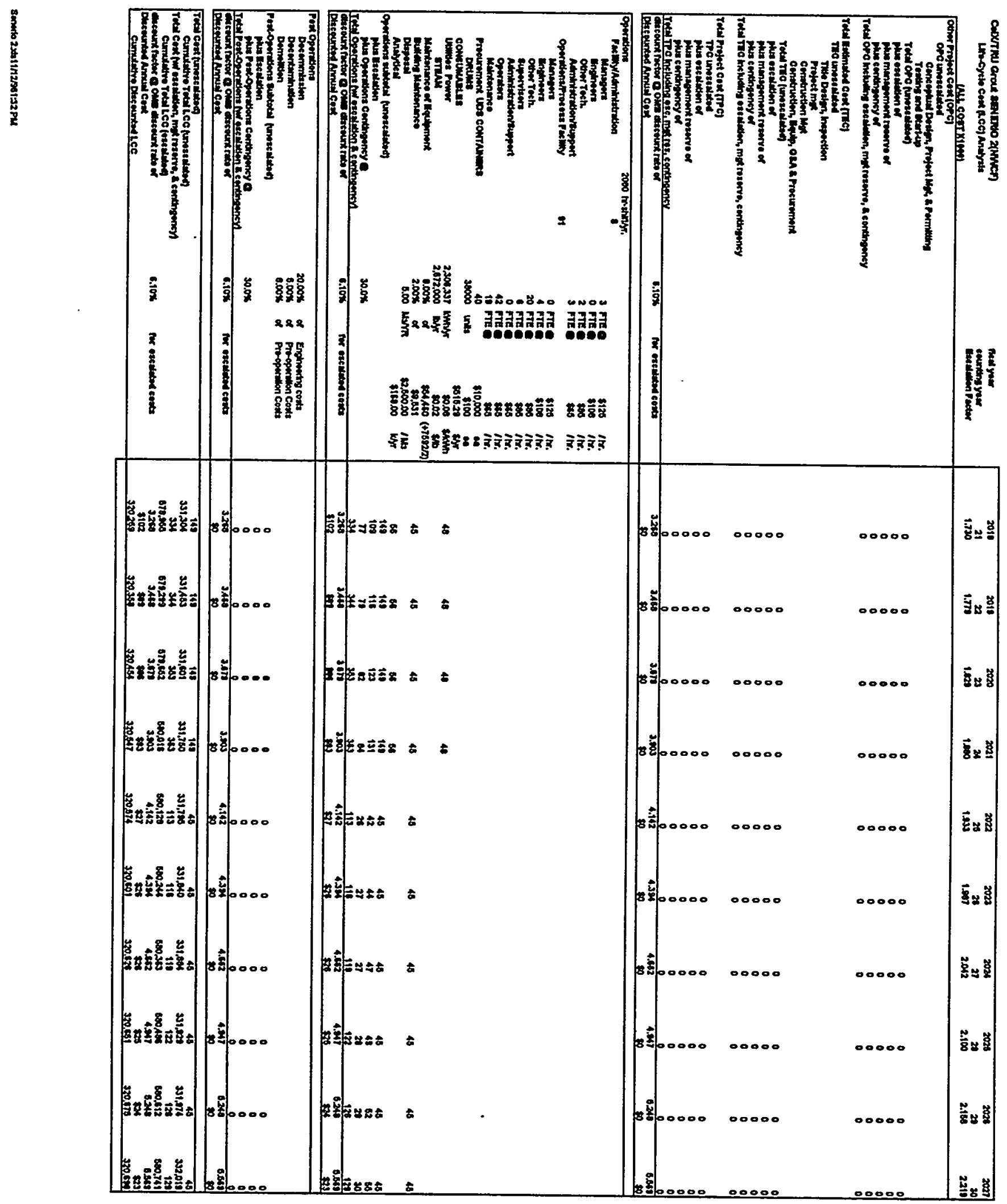




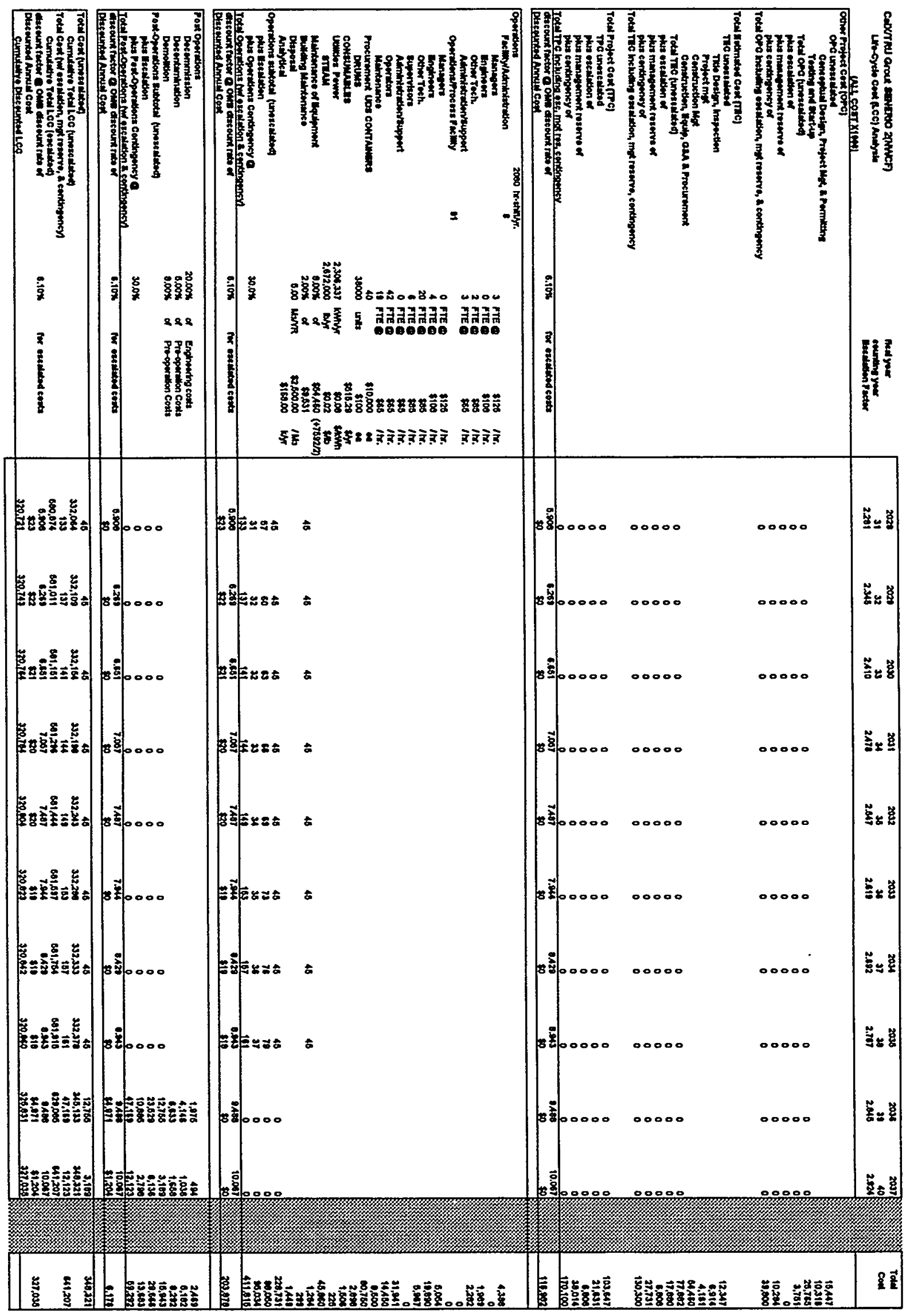




\section{Appendix D}

\section{Drawings}

Index: Sheet T-1

Scenario 1: Sheet 1-xx

Scenario 2: Sheets 2-xx

Common to both scenarios:

Sheets COM-xx 


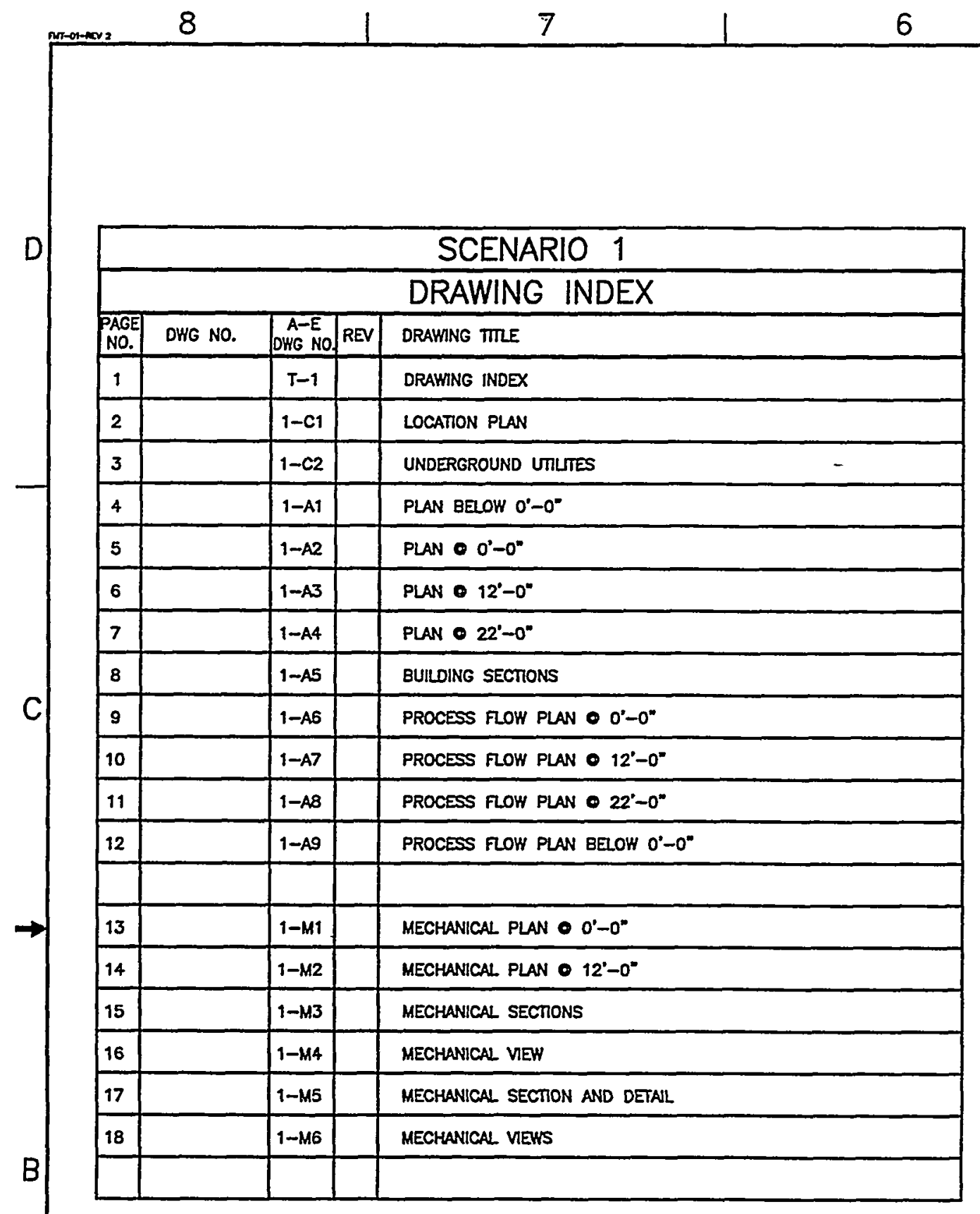

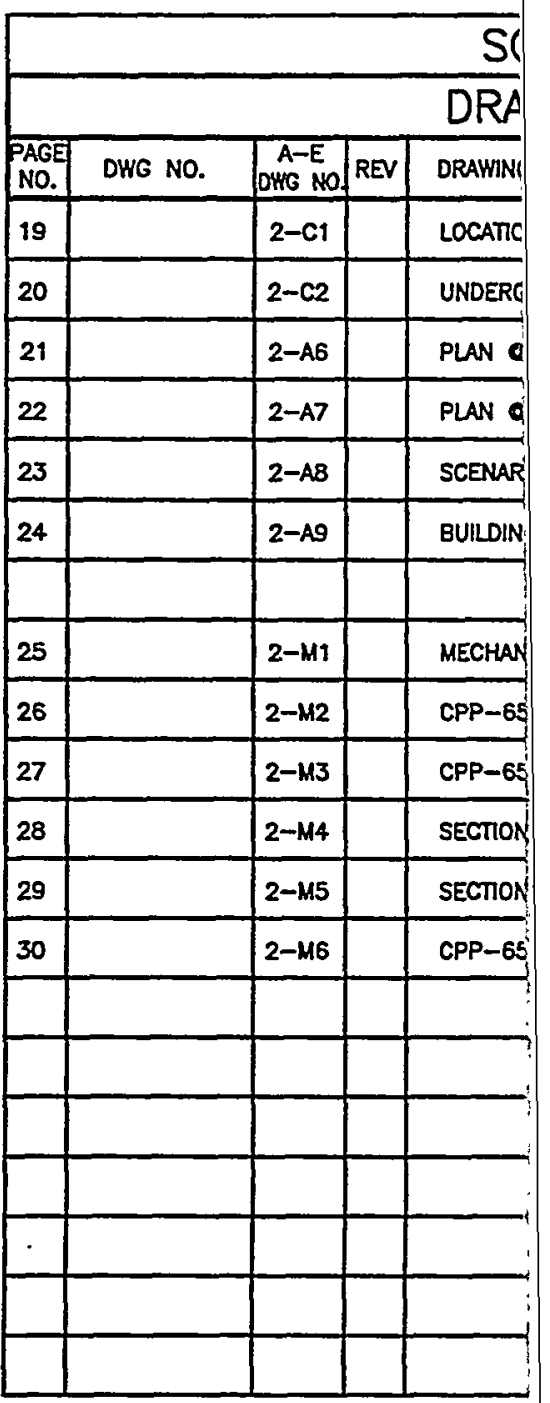




\section{NARIO 2}

ING INDEX

TLE

TLE

DLN

JND UTIUTES

$-0^{\circ}$

$0^{*}$

DLAN $020^{\circ}-0^{\circ}$

\section{EECTONS}

$\angle$ PLAN $\bullet 0^{\circ}-0^{\circ}$

3RD LEVE DENOUTON PLAN

3RD LEVE, PLAN

IST LEVEL PLAN

\begin{tabular}{|l|l|l|l|l|}
\hline \multicolumn{5}{|c|}{ SCENARIO 1 \& \& 2} \\
\hline \multicolumn{3}{|c|}{ DRAWING INDEX } \\
\hline $\begin{array}{l}\text { PAGE } \\
\text { NO. }\end{array}$ & DWG NO. & $\begin{array}{c}\text { A-E } \\
\text { DHG }\end{array}$ & REV & DRAWING MIIE \\
\hline 31 & & COM-AI & & INTERIM STORAGE FACIUTY PLANS \\
\hline 32 & & COM-A? & & INTERIM STORAGE FACIUTY ELEVATIONS \\
\hline & & & & \\
\hline 33 & & COM-M1 & & MECHANICAL INTERIM STORAGE FACIUTY PLAN \\
\hline & & & & \\
\hline
\end{tabular}

\begin{tabular}{|c|c|c|c|c|c|}
\hline FOR DRUNAG NDEX SEE DRULWG NO. & sracorrinuct ta & \multirow{2}{*}{\multicolumn{4}{|c|}{ LOCKHEED WATTIN }} \\
\hline \multirow{6}{*}{ NO SCALE } & FOLETIE: & \multirow{5}{*}{\multicolumn{4}{|c|}{$\begin{array}{l}\text { CSIX/TRU GROUT PROJECT } \\
\text { SCENARIO } 1 \& \& 2 \\
\text { DRAHING INDEX }\end{array}$}} \\
\hline & ocsest & & & & \\
\hline & DRANA BSEARE & & & & \\
\hline & \begin{tabular}{|l|l} 
Faret & \\
\end{tabular} & & & & \\
\hline & \begin{tabular}{|l|l|} 
SFE $\operatorname{cose}$ & \\
\end{tabular} & & & & \\
\hline & 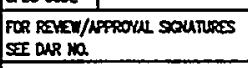 & $\left.\right|_{01153} ^{\sin }$ & DOMG- & & $\mathbf{k a t}$ \\
\hline QUNUTY LEVE: & ETECTRE OATE: & 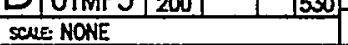 & sing & $T-1$ & \\
\hline
\end{tabular}




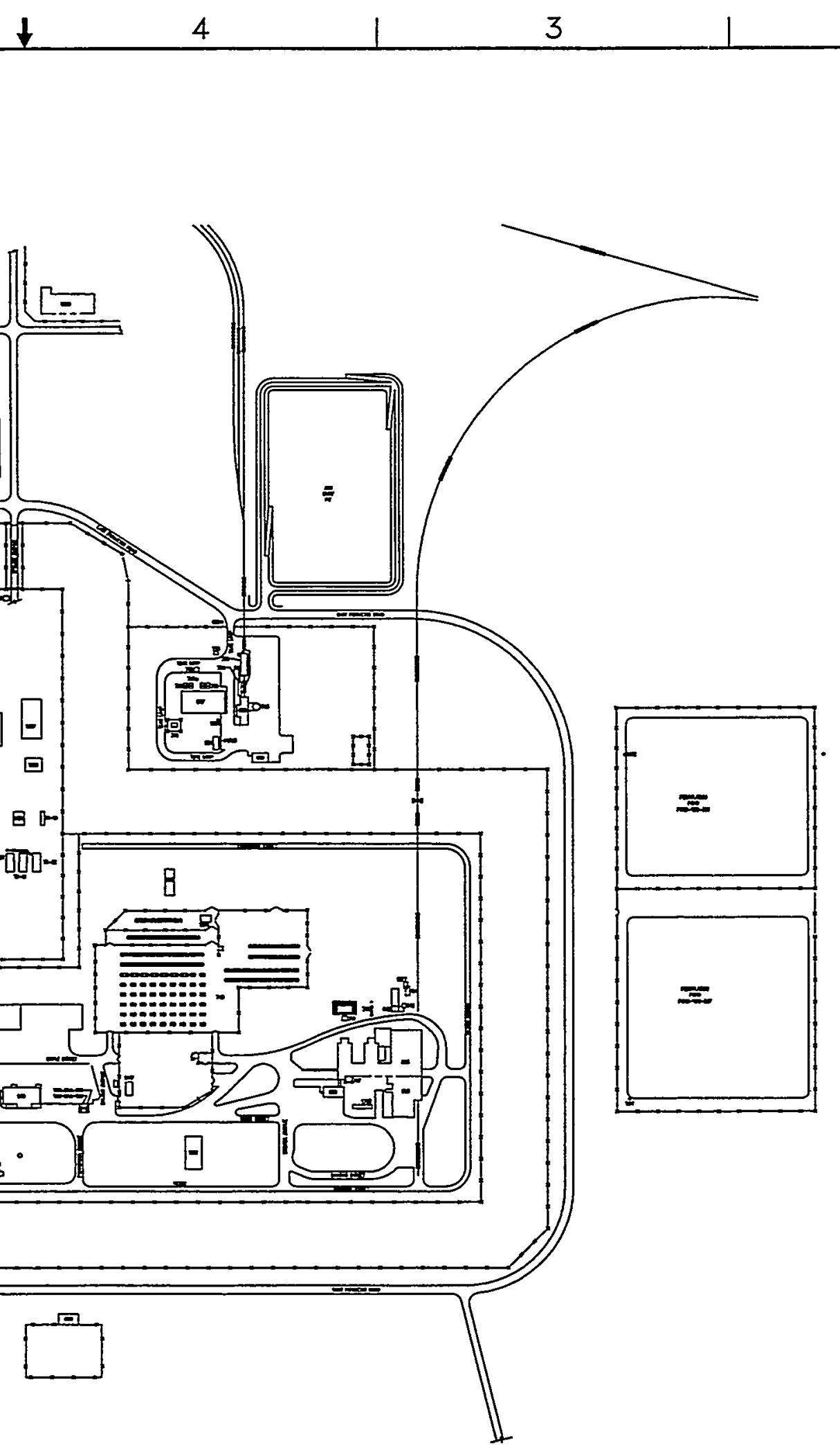

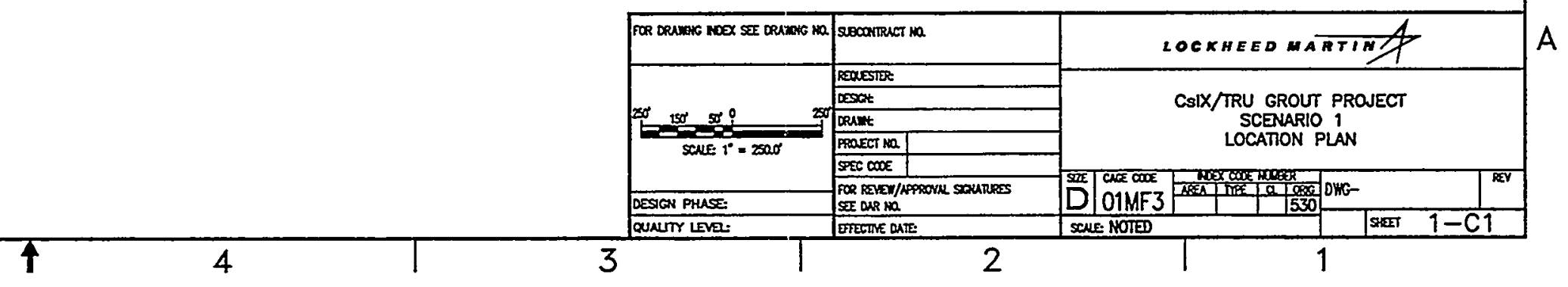




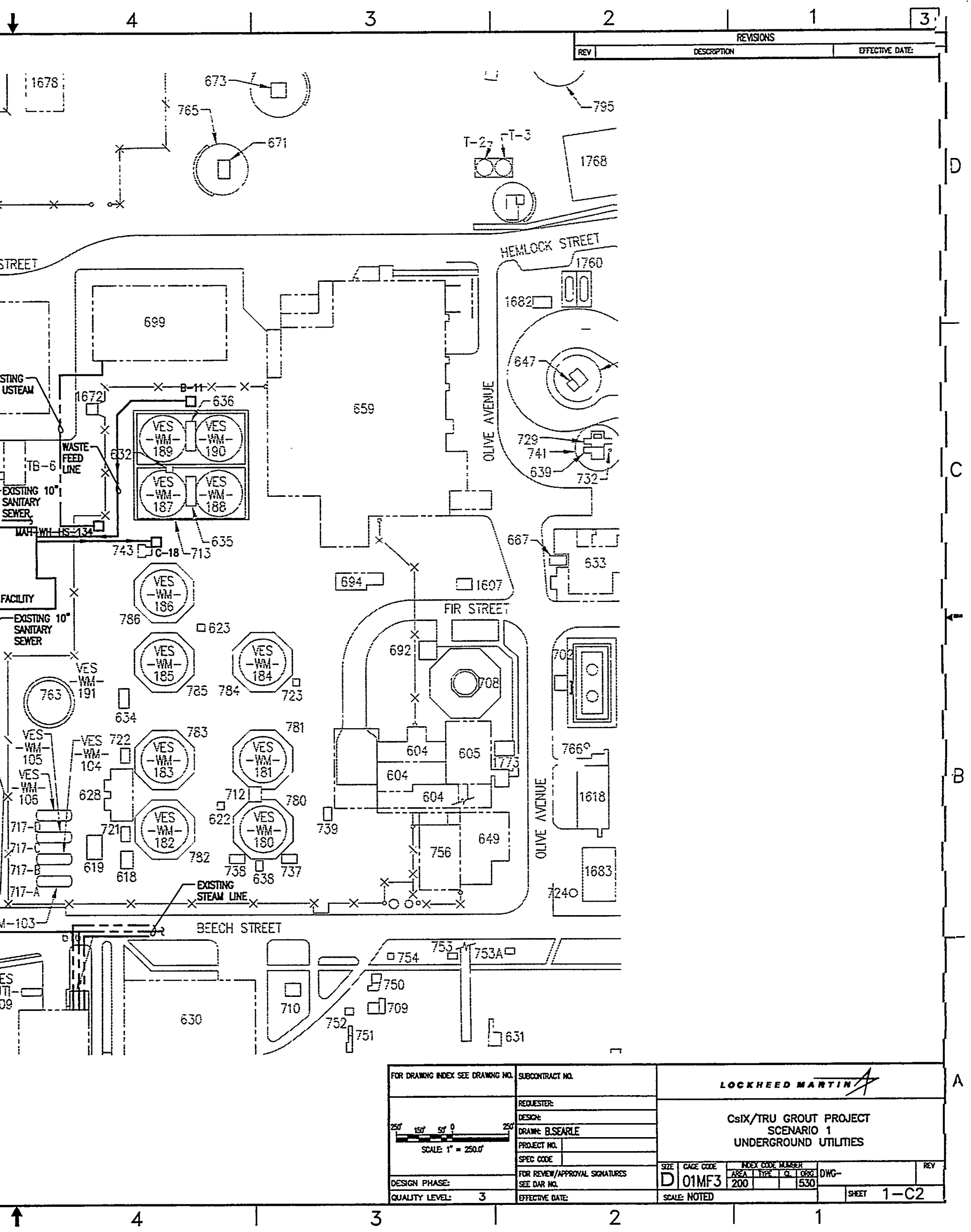




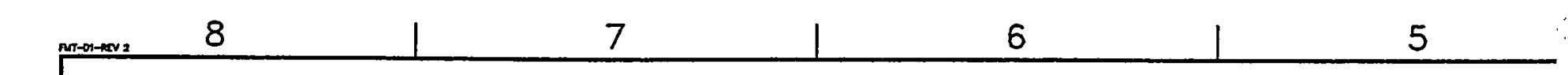

D
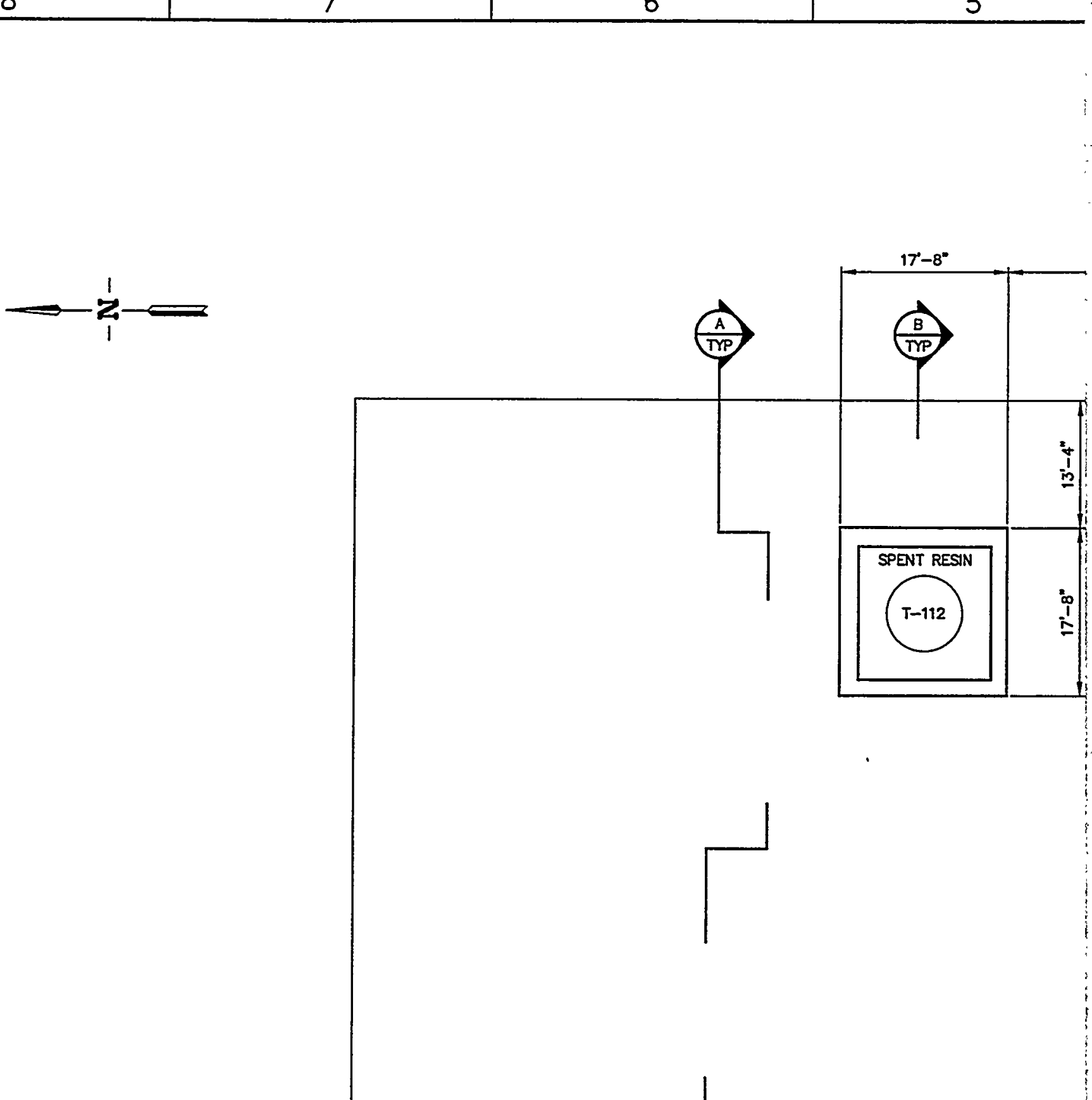

B

c
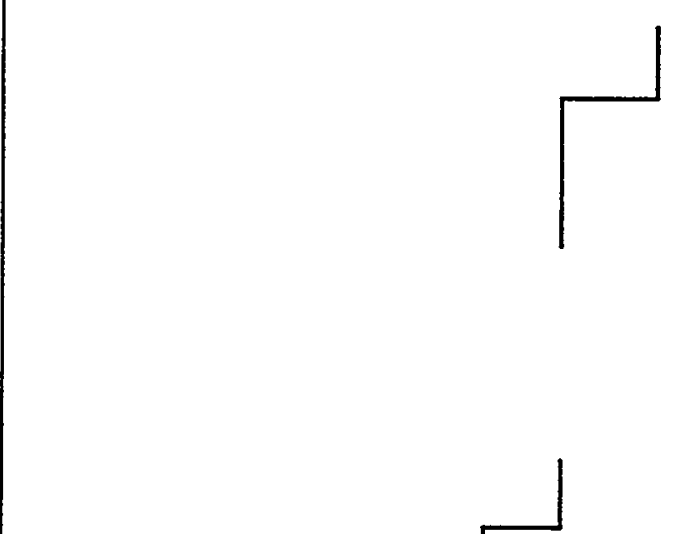

(1)

$$
\text { A }
$$




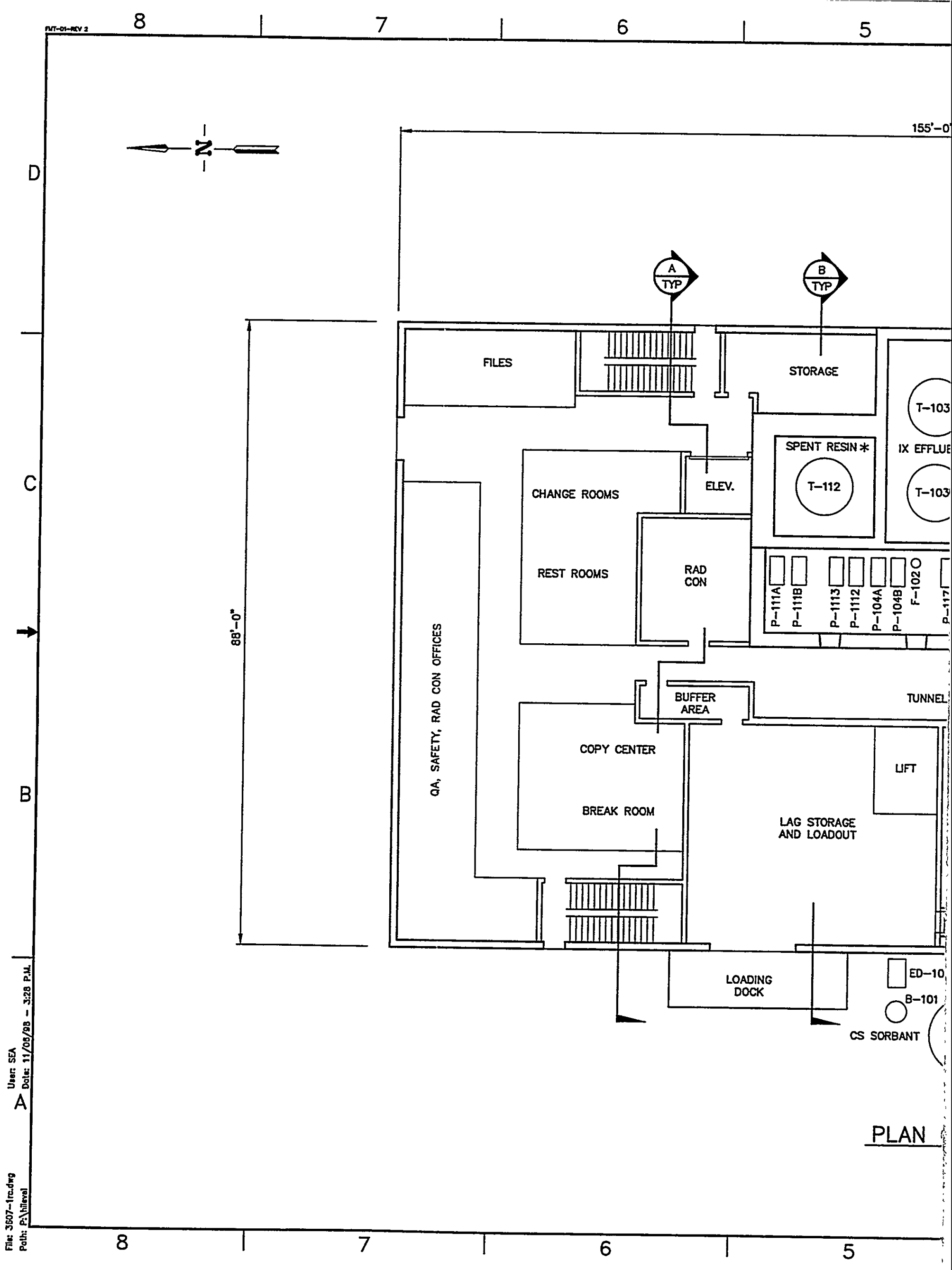




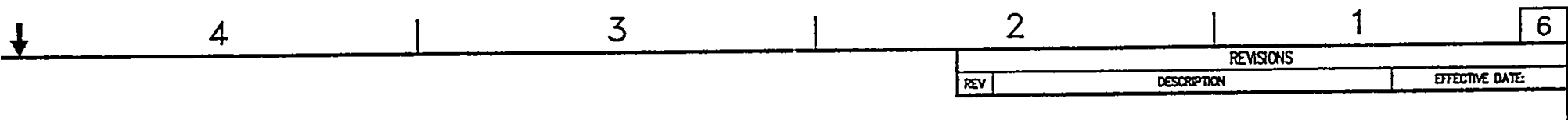

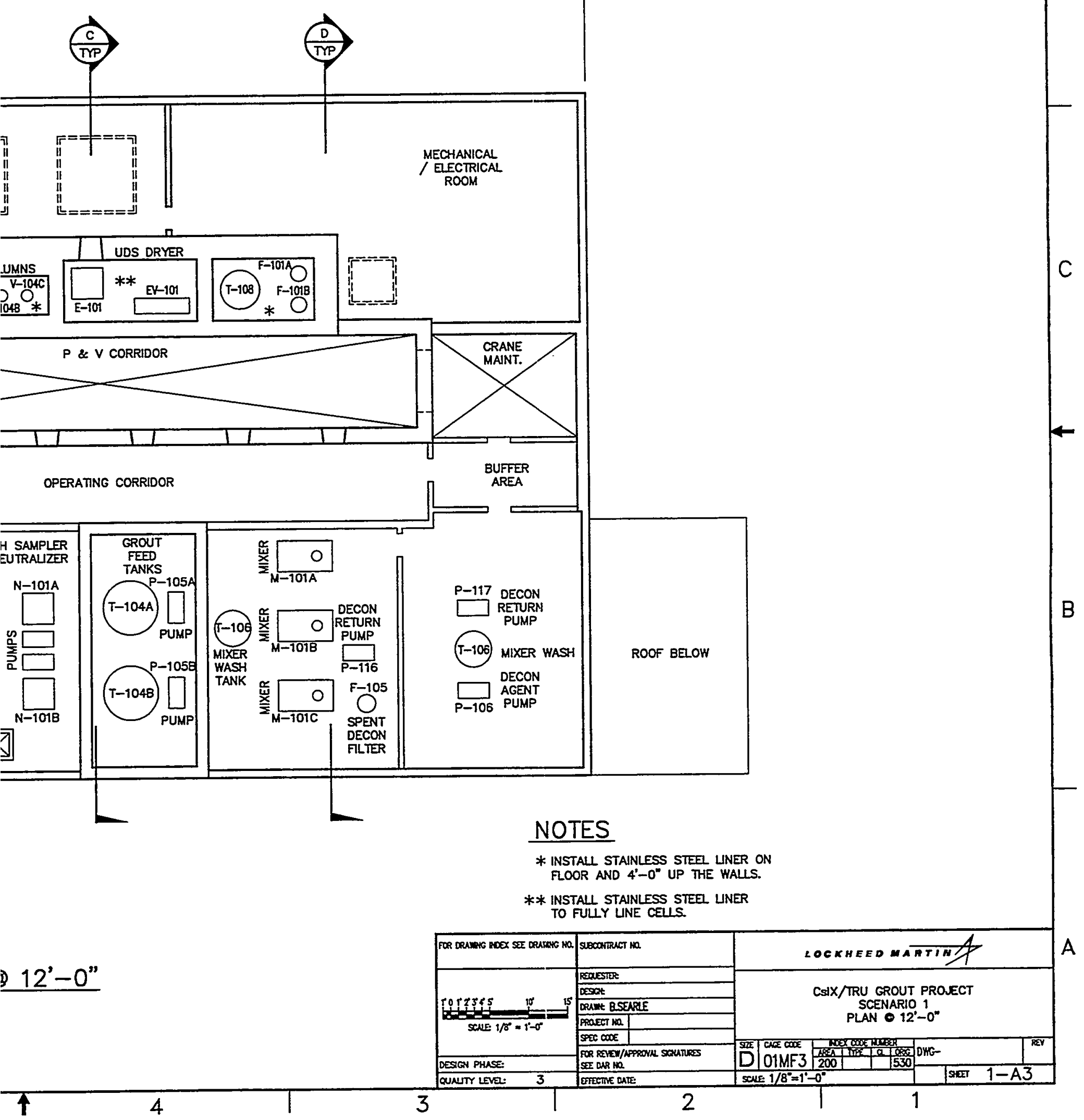




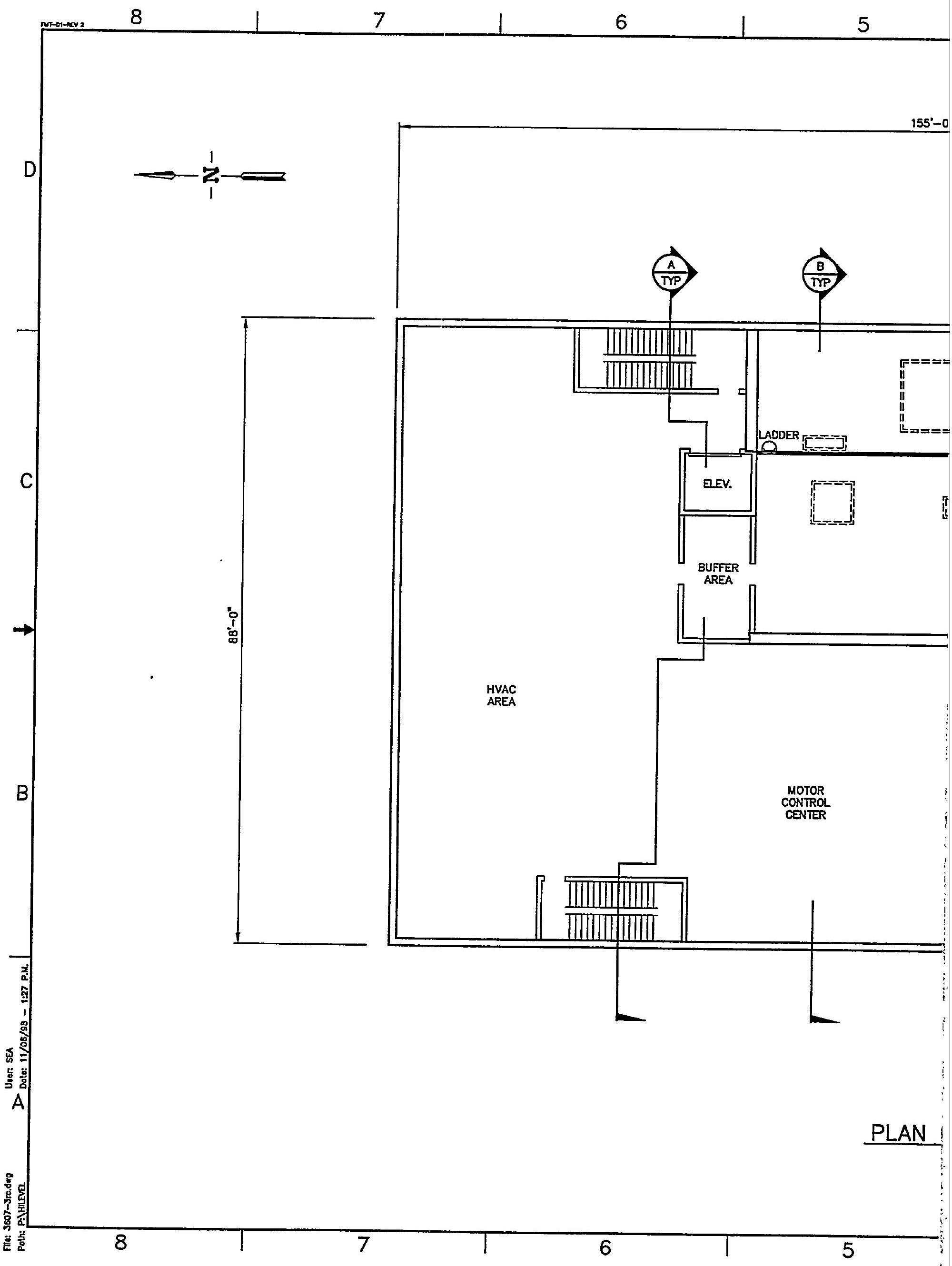




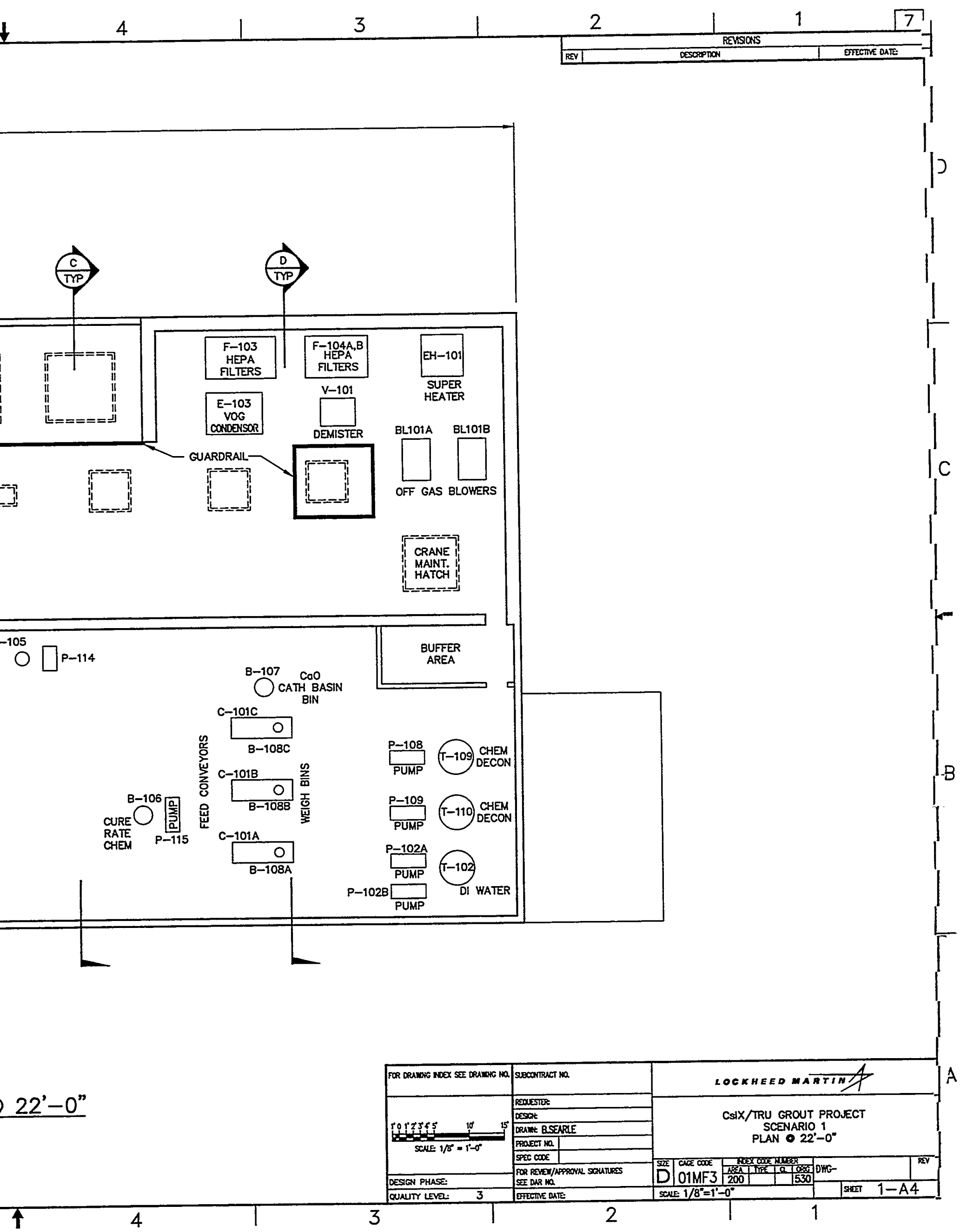




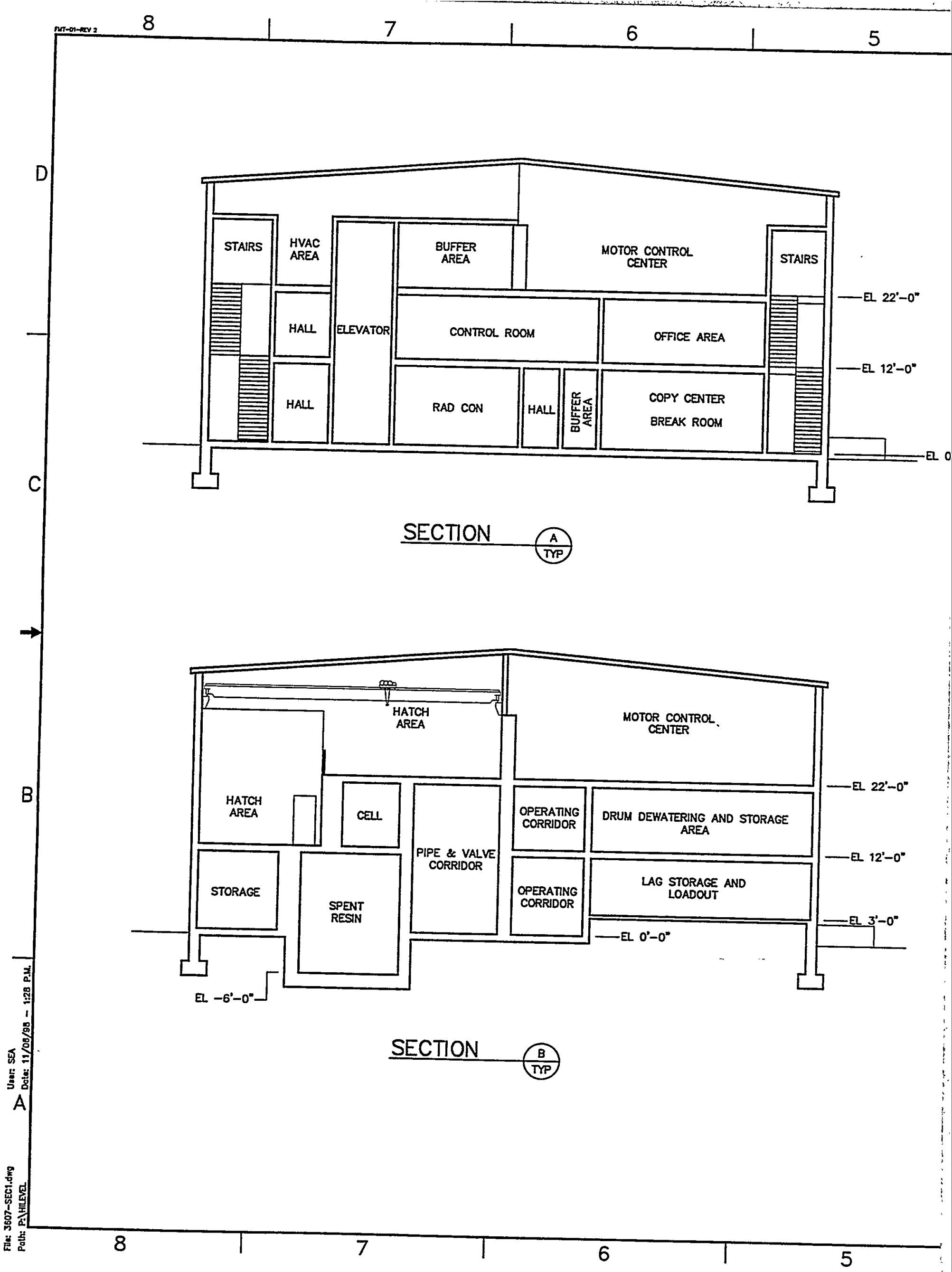




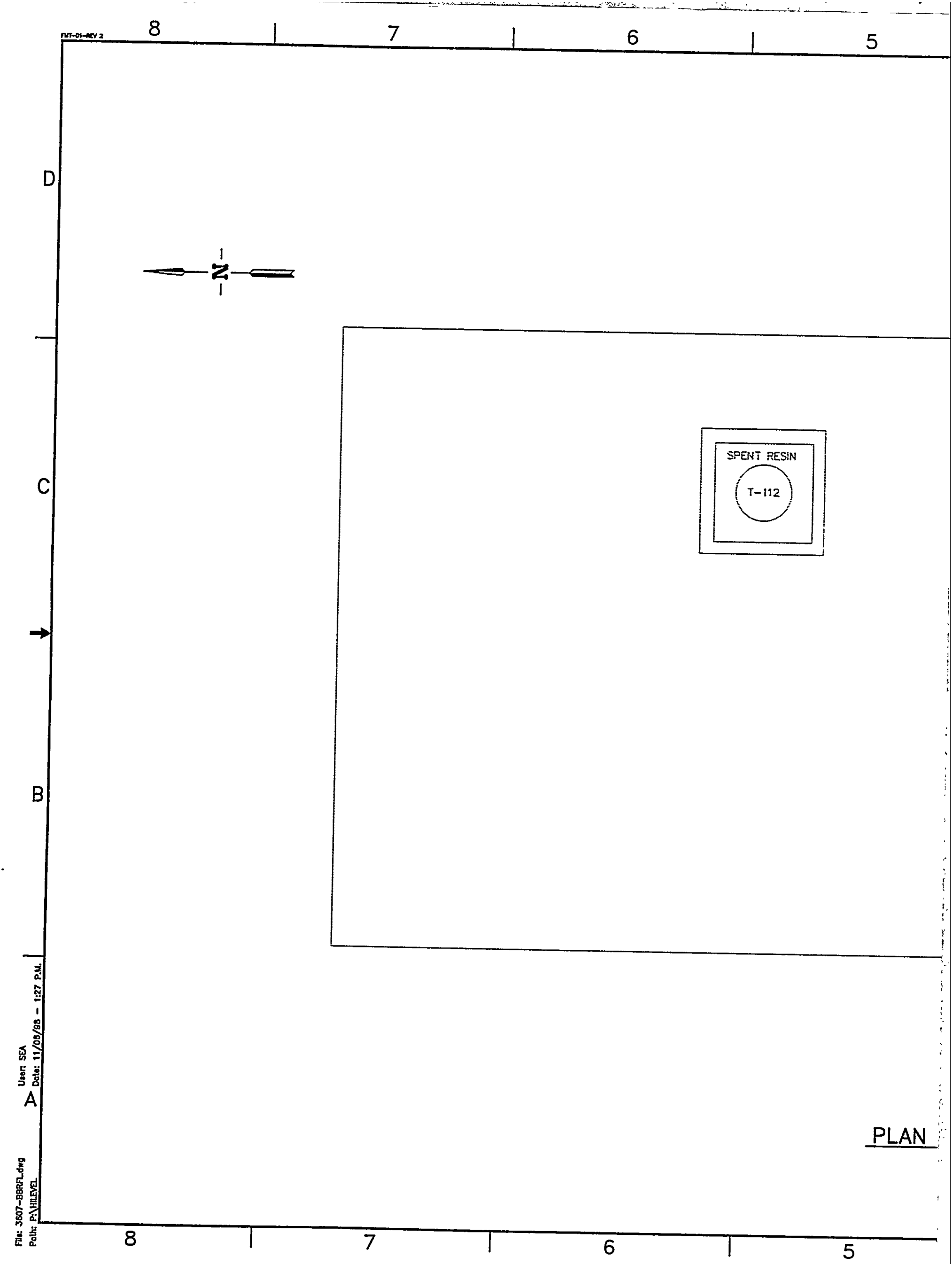





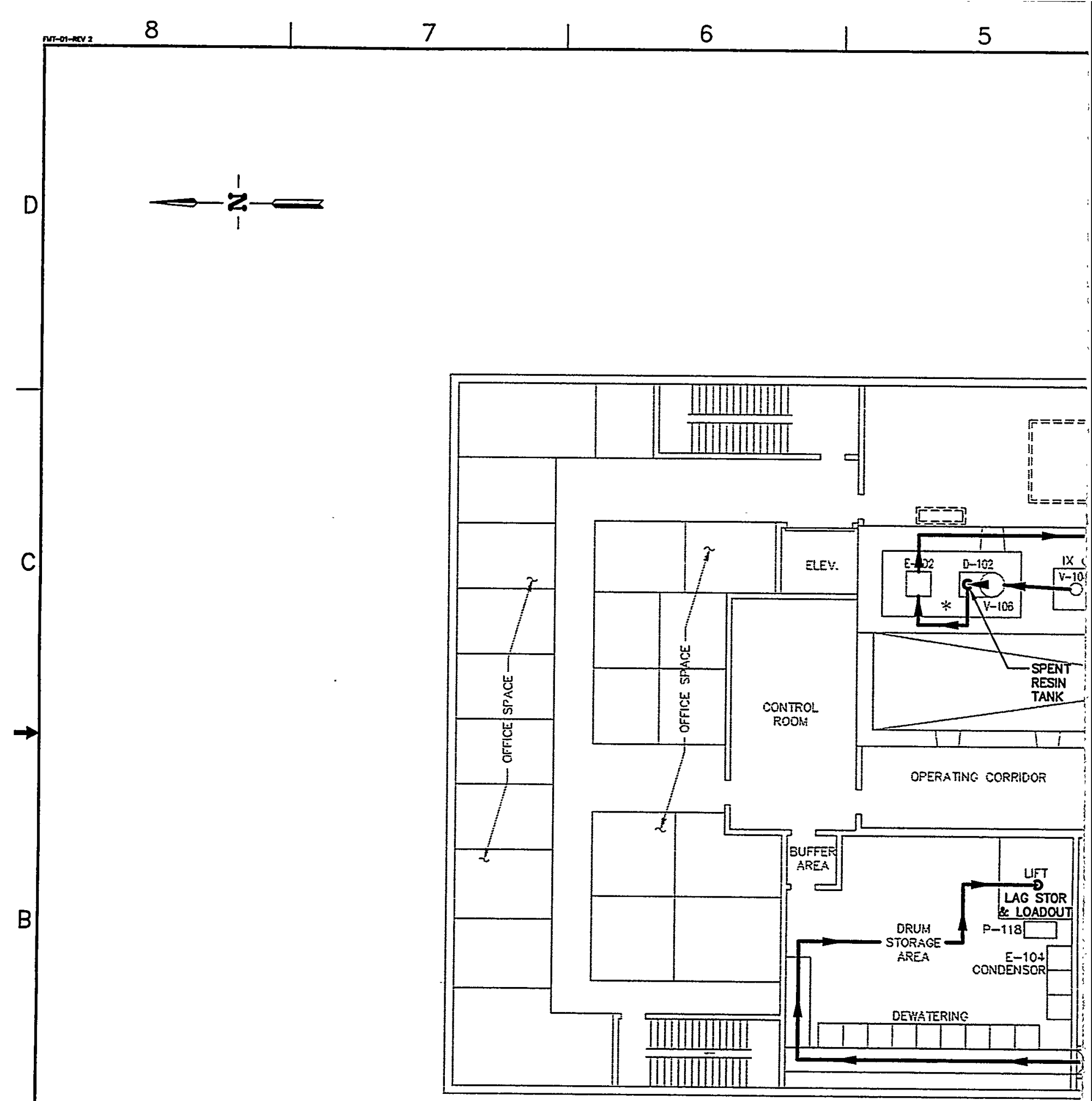




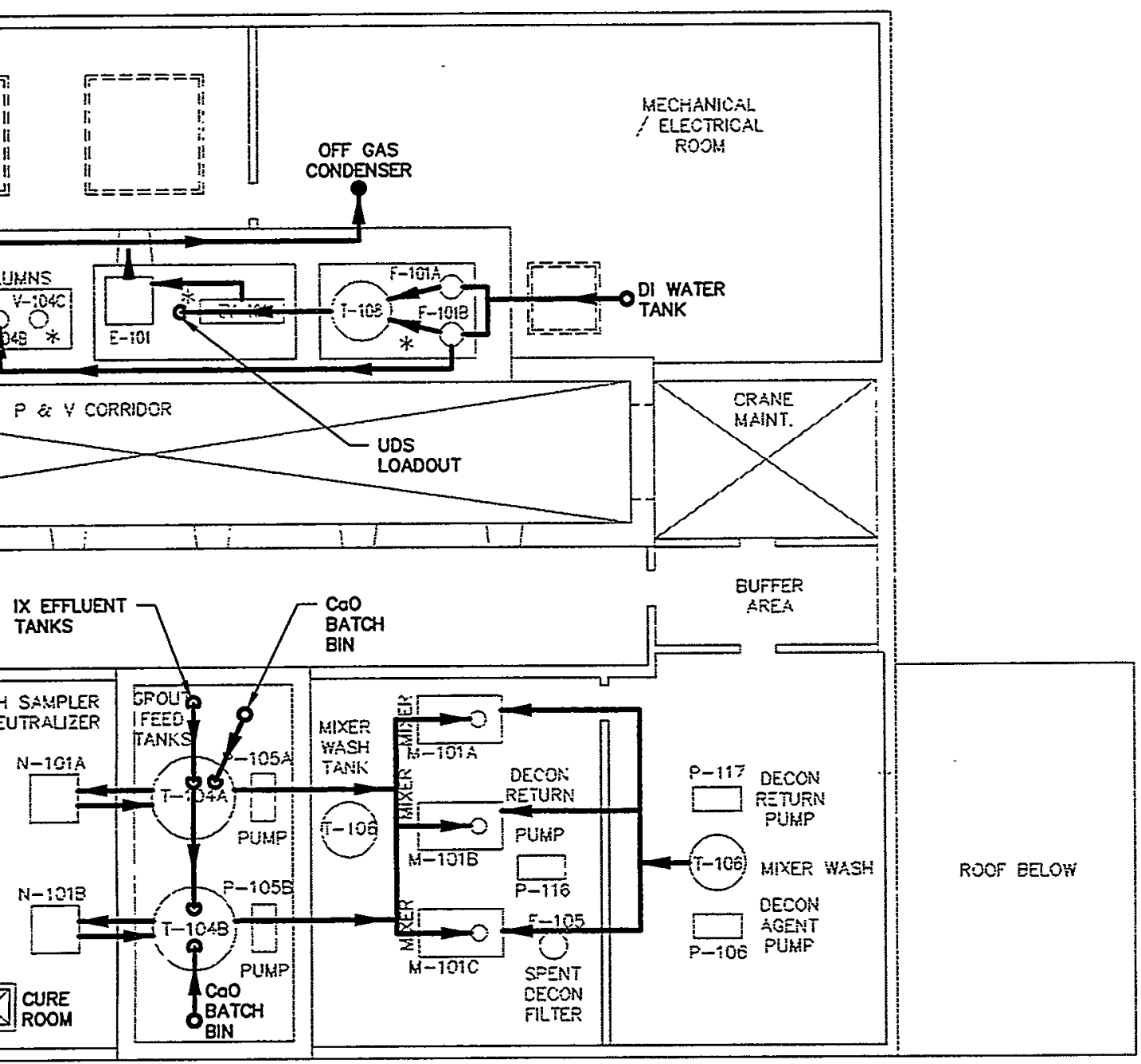

LEGEND

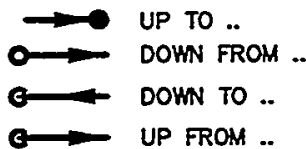

$12^{\prime}-0^{\prime \prime}$

\begin{tabular}{|c|c|c|c|c|}
\hline 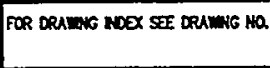 & secariruct ino. & \multicolumn{3}{|c|}{ LOCKHEEO MATTIN } \\
\hline \multirow{5}{*}{ 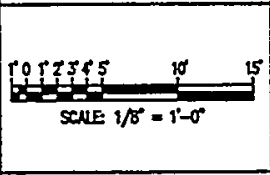 } & 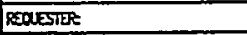 & \multirow{4}{*}{\multicolumn{3}{|c|}{$\begin{array}{l}\text { CSIX/TRU GROUT PROJECT } \\
\text { SCENARIO } 1 \\
\text { PROCESS FLOW } \bullet 12^{\prime}-0^{*}\end{array}$}} \\
\hline & pesat & & & \\
\hline & DRum: QSERARE & & & \\
\hline & \begin{tabular}{|l|l|} 
PFOEET Ma. & \\
\end{tabular} & & & \\
\hline & \begin{tabular}{|l|l|}
$\operatorname{sax} \cos \mid$ & \\
\end{tabular} & \multirow{2}{*}{ 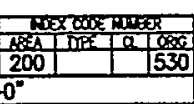 } & \multirow{2}{*}{ gूo } & \multirow[b]{3}{*}{$1-A 8$} \\
\hline DESGG PHASE: & 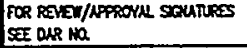 & 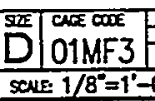 & & \\
\hline QUNATY LEVE: & EFFECIME WATE: & & 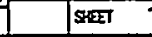 & \\
\hline
\end{tabular}




$+\quad 4$

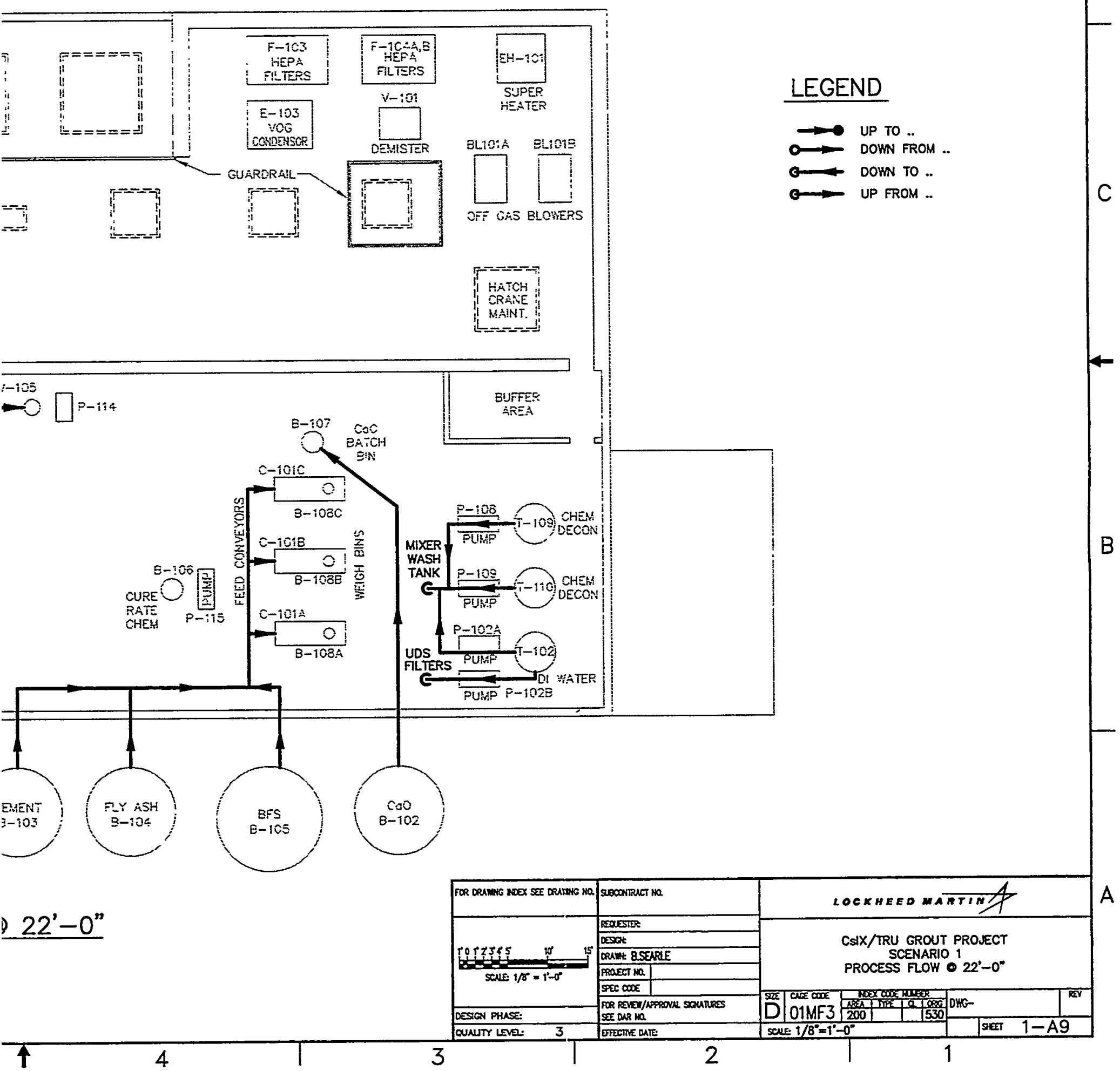




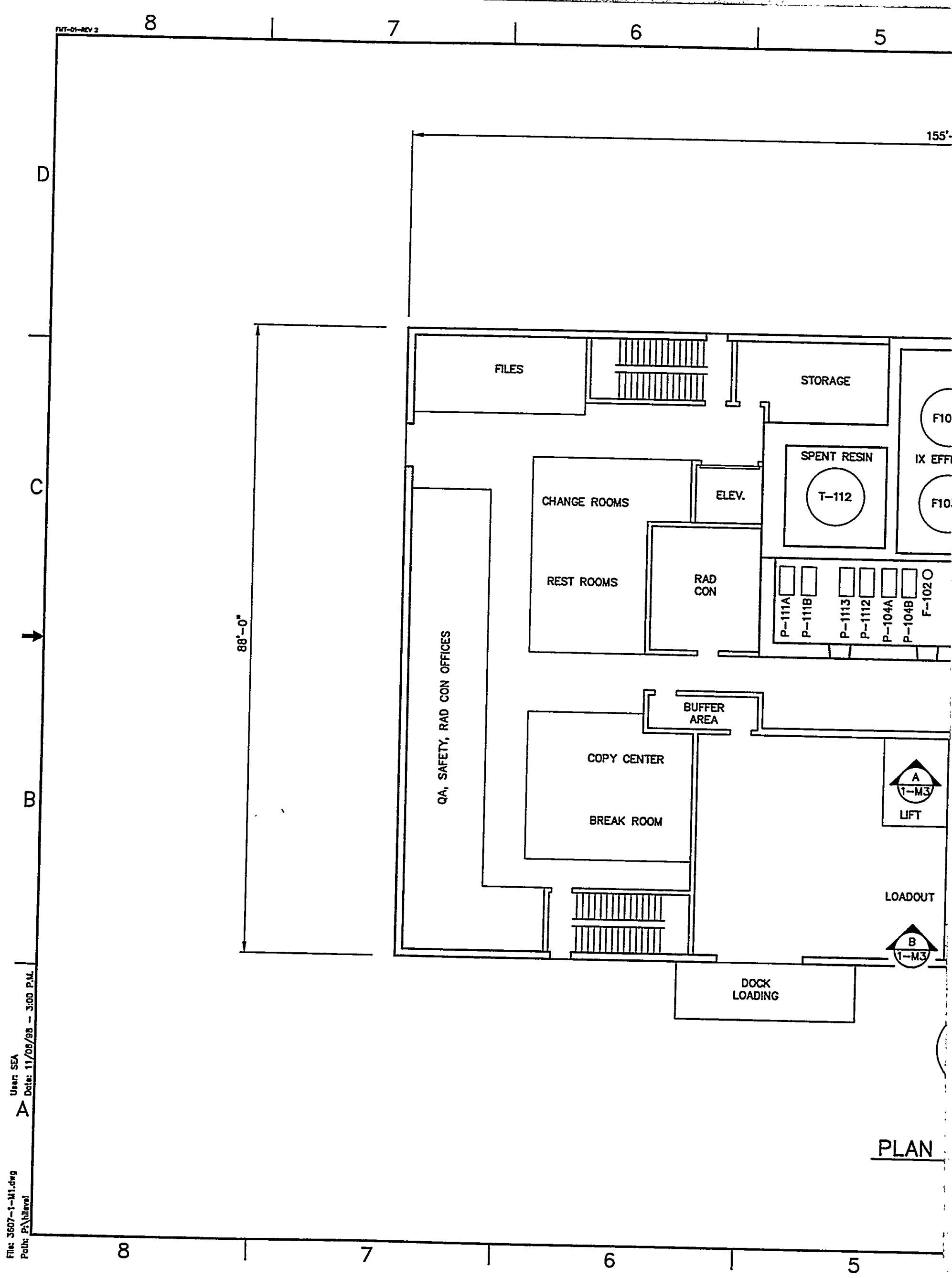




$+4$

2 1

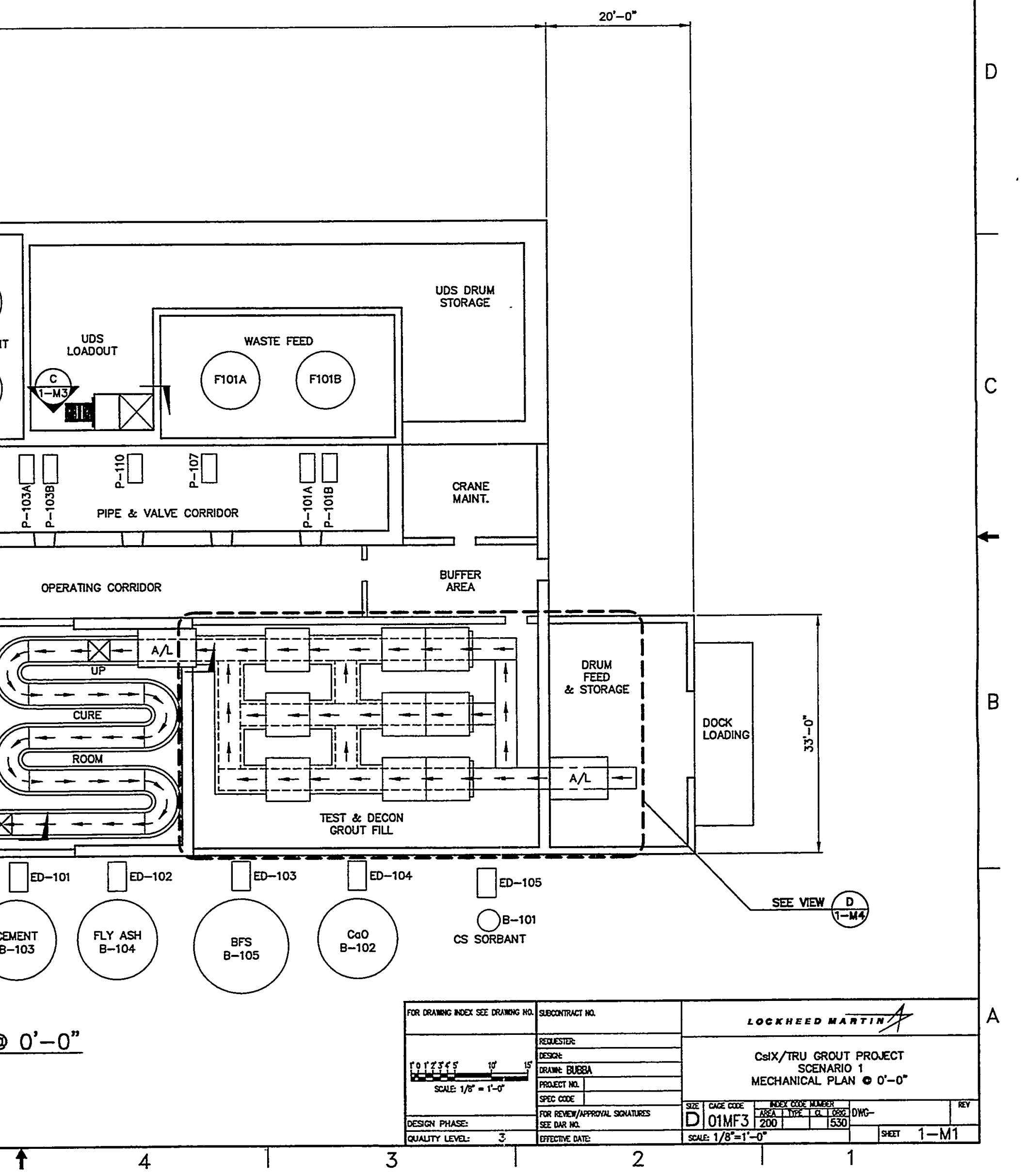




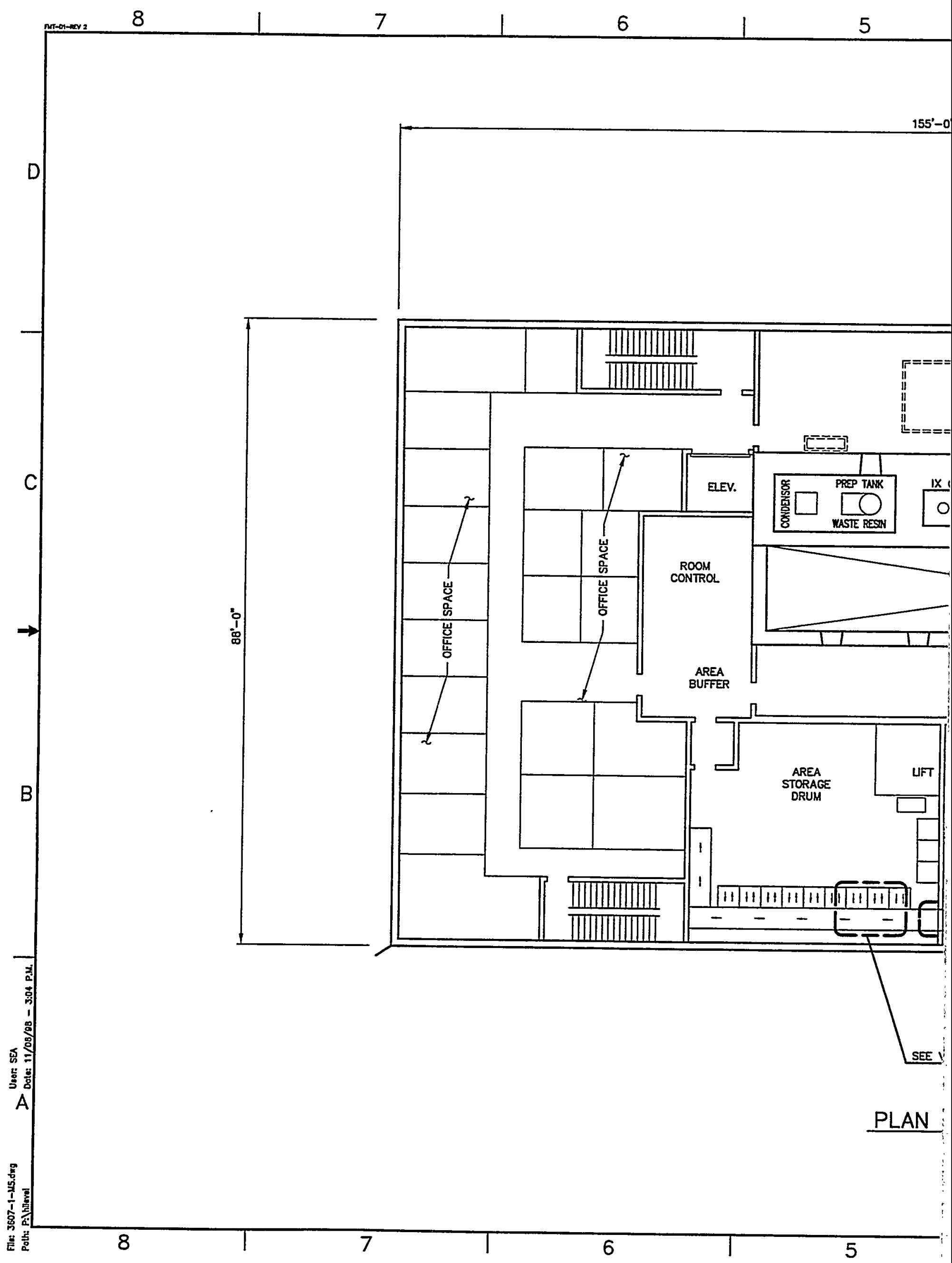




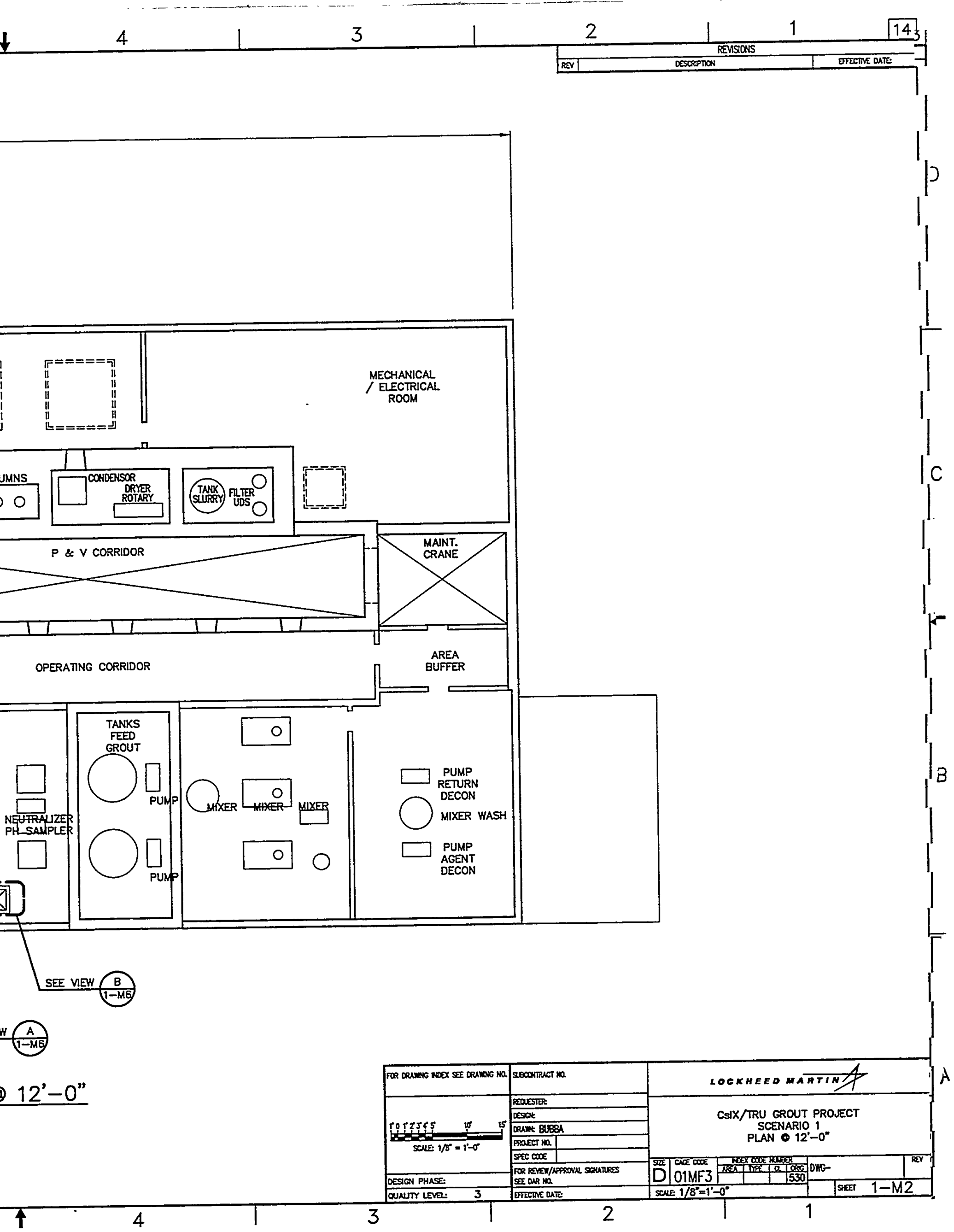




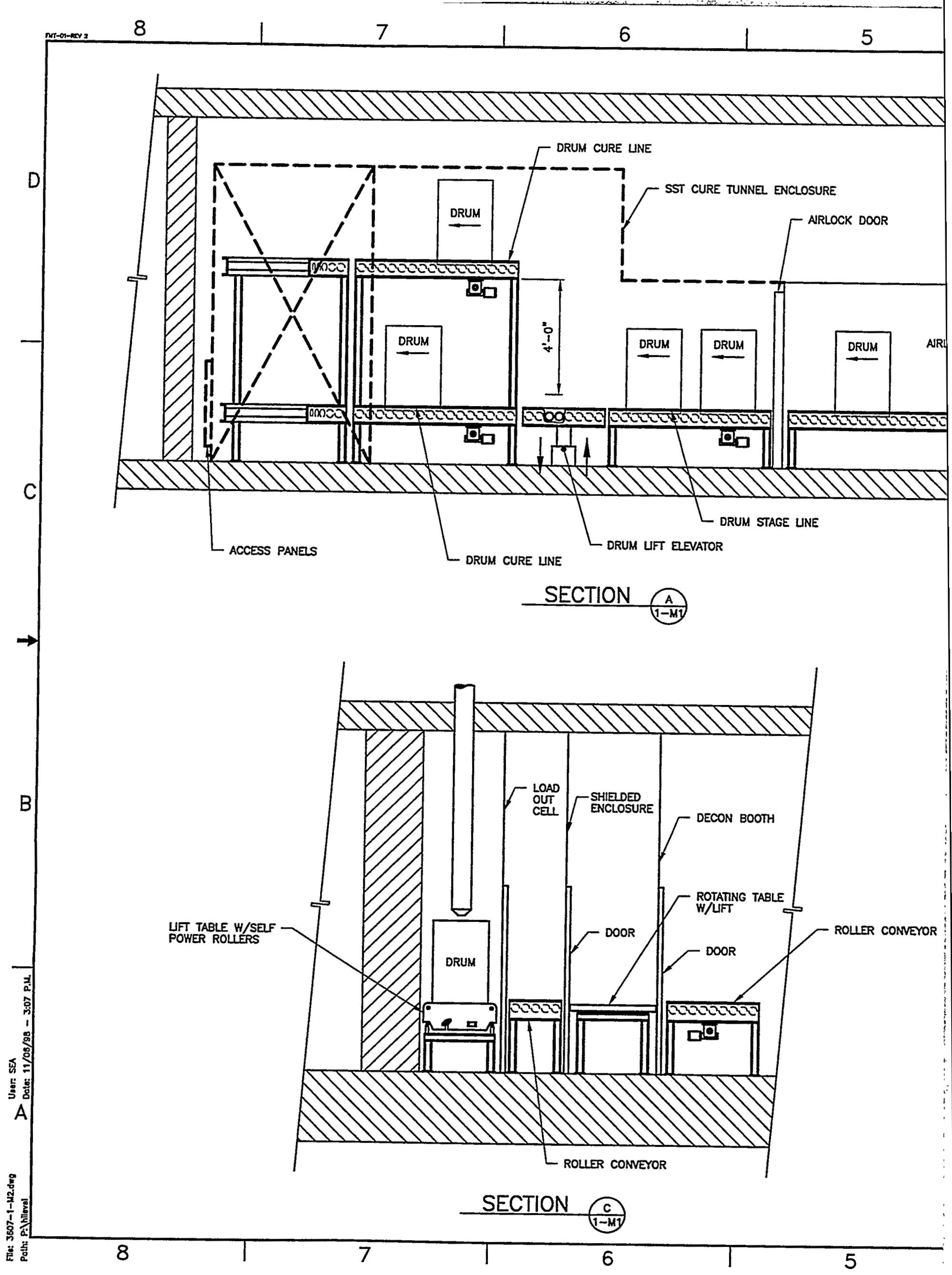



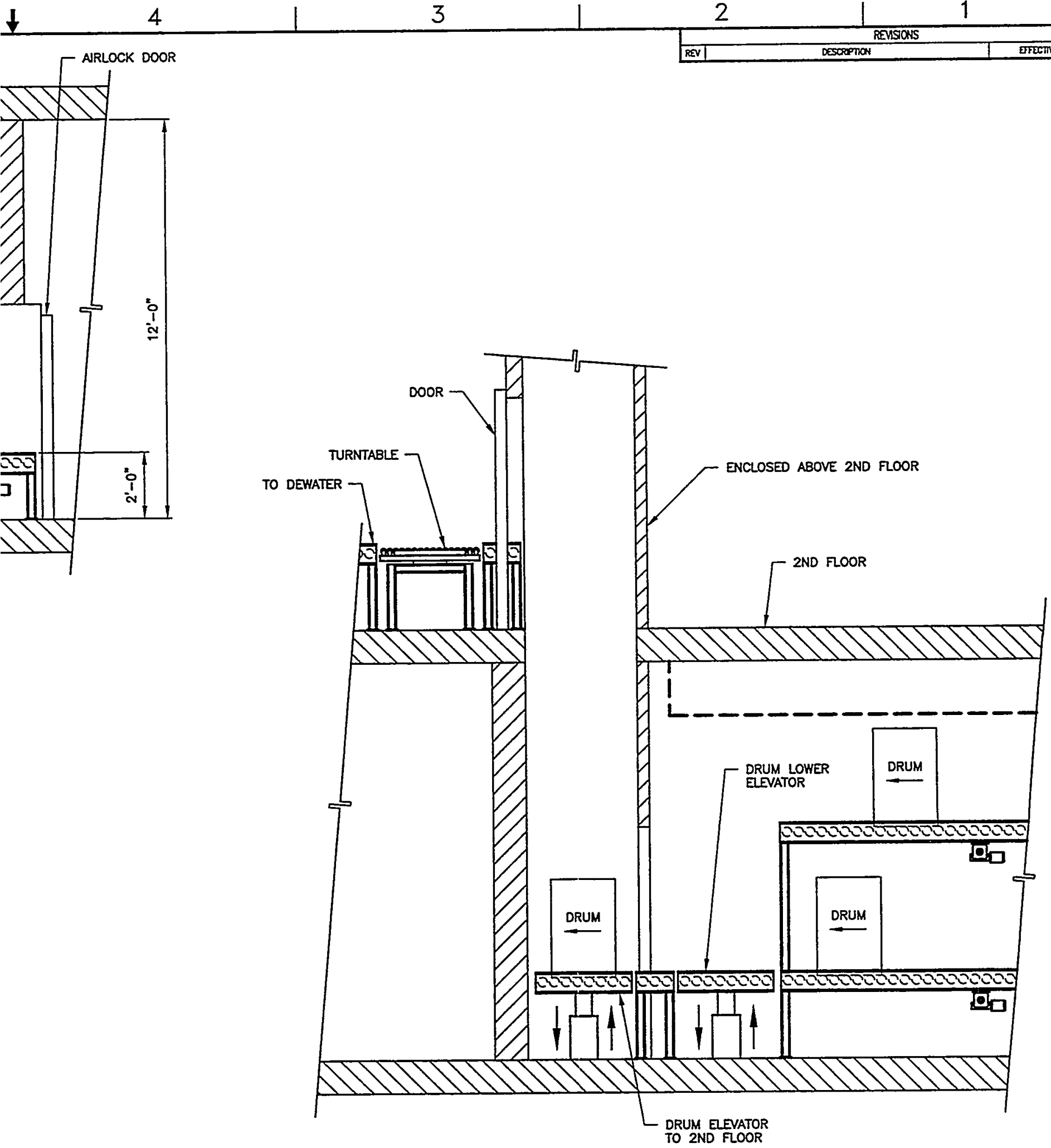

C

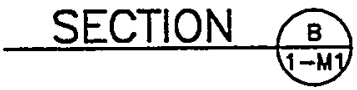

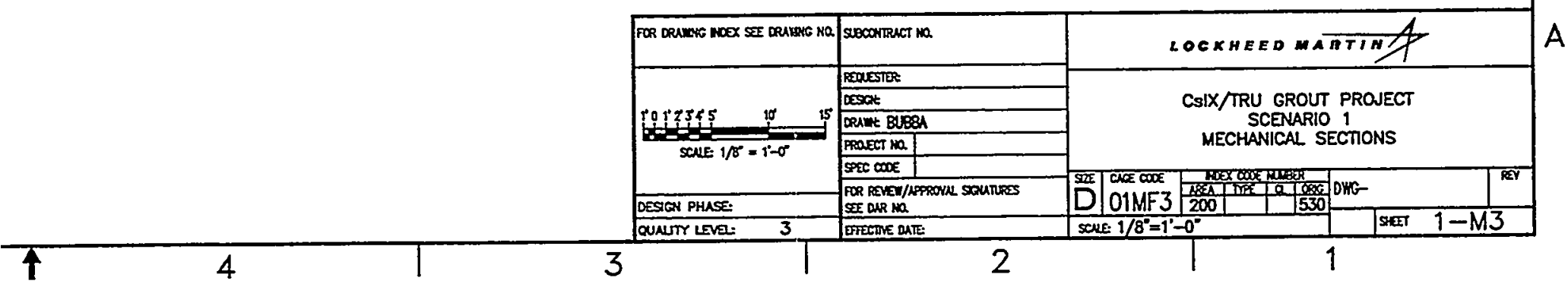




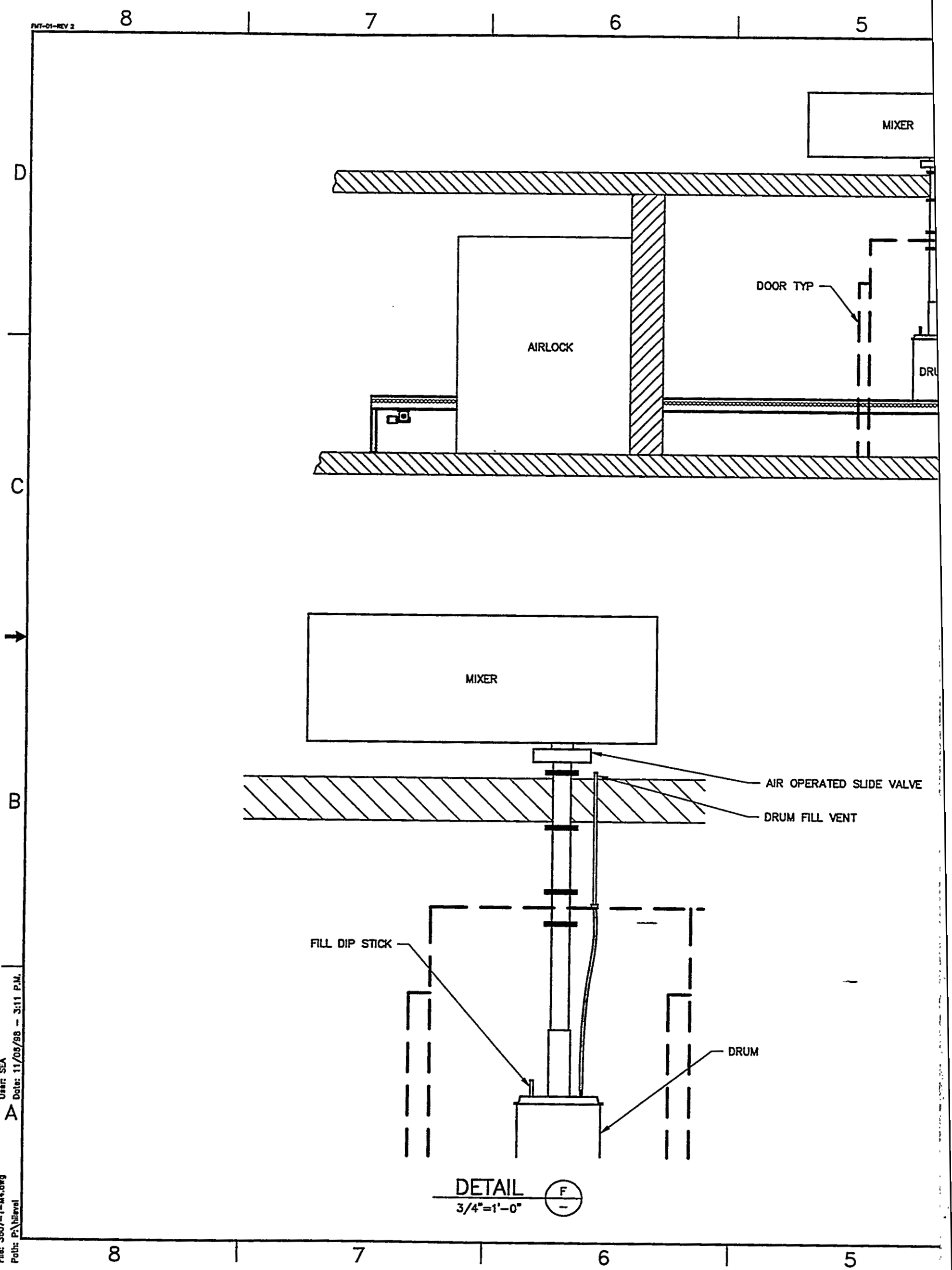




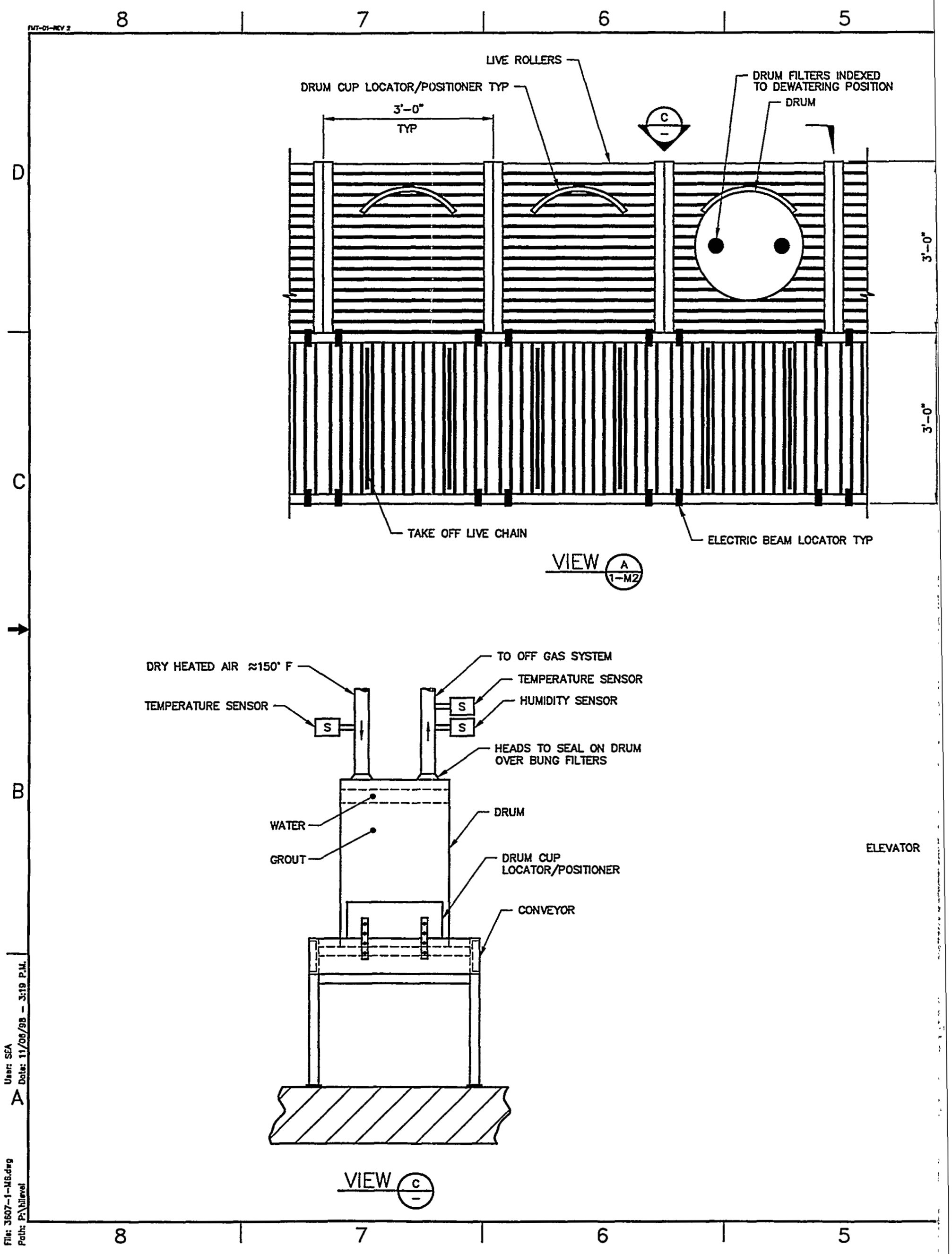




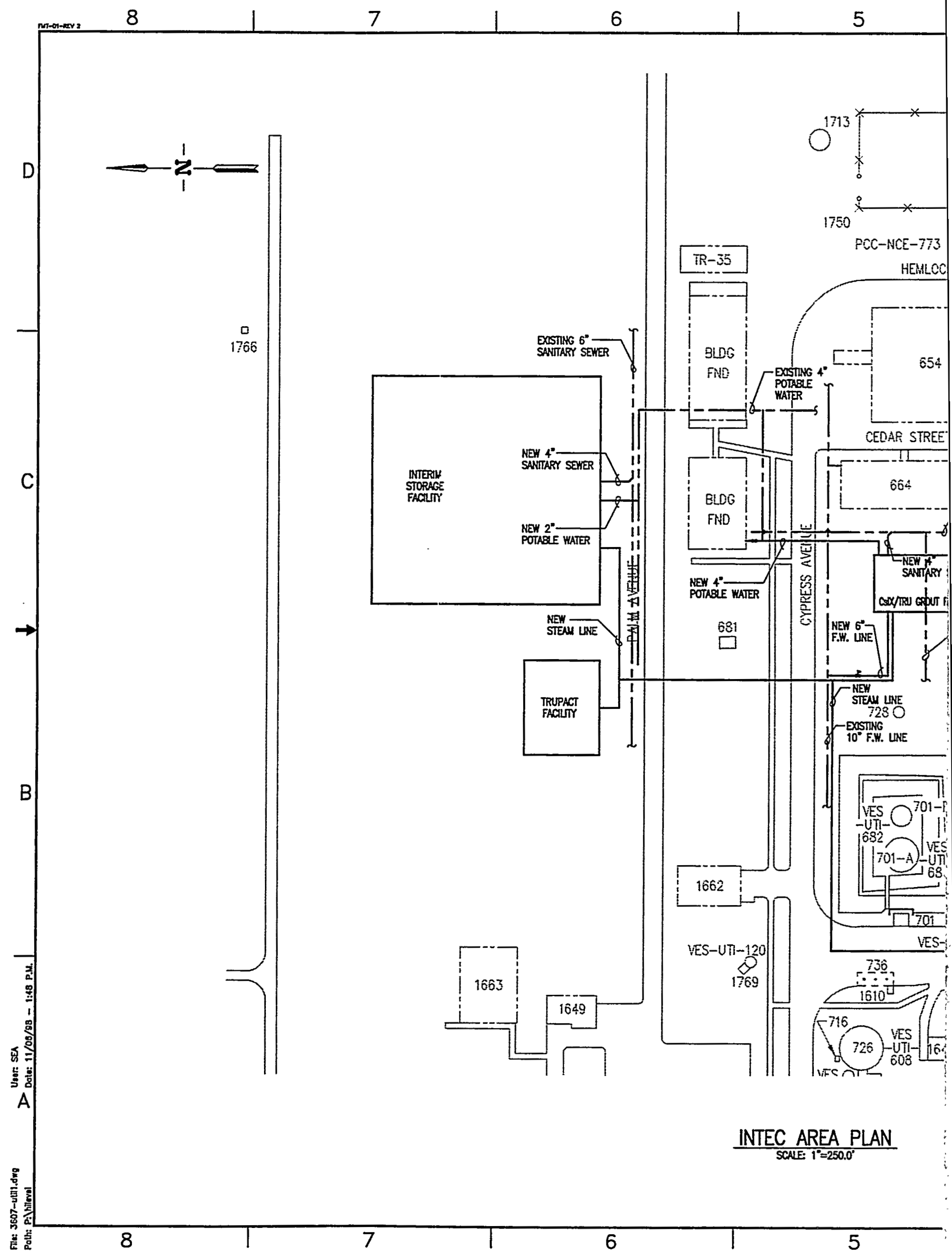




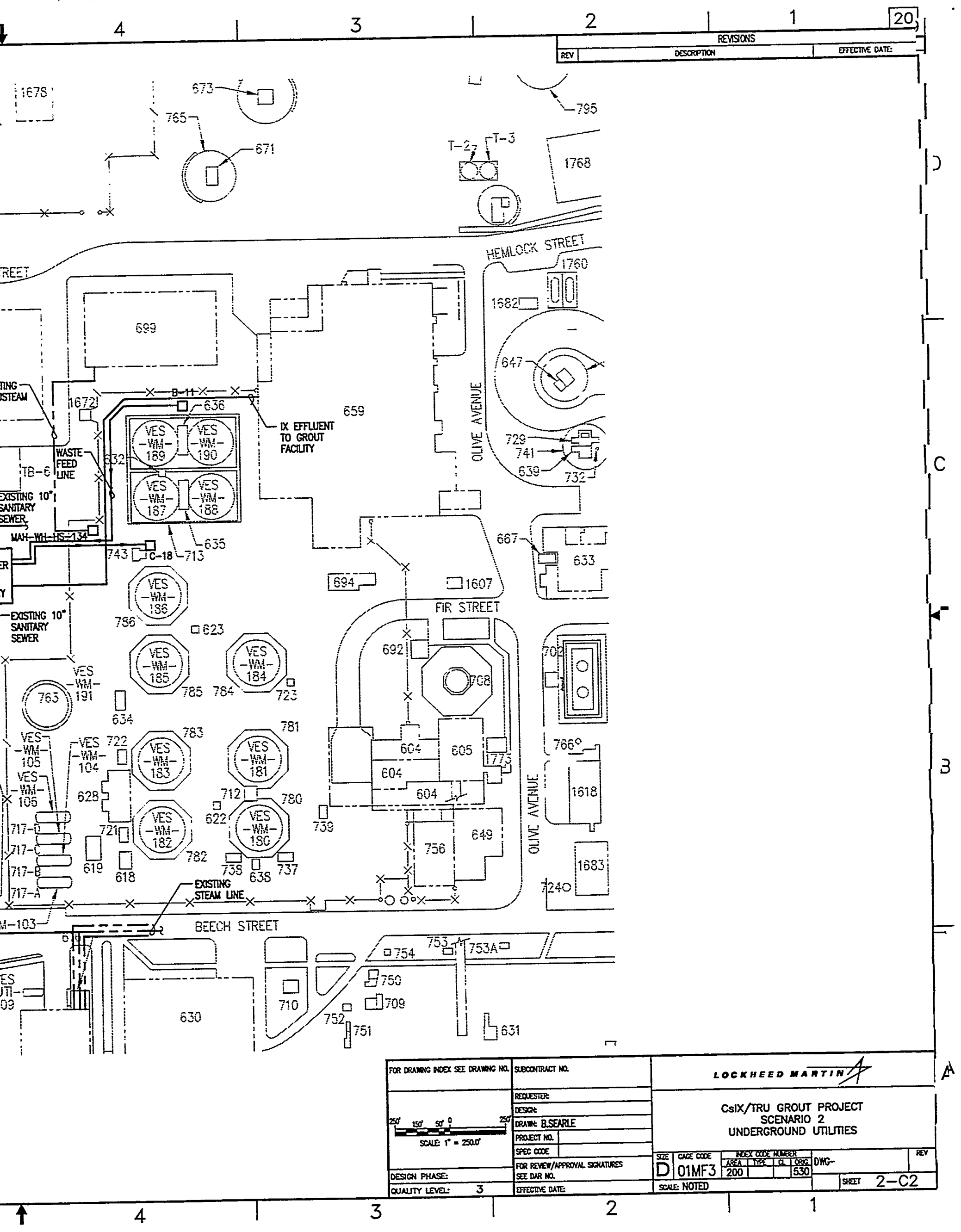




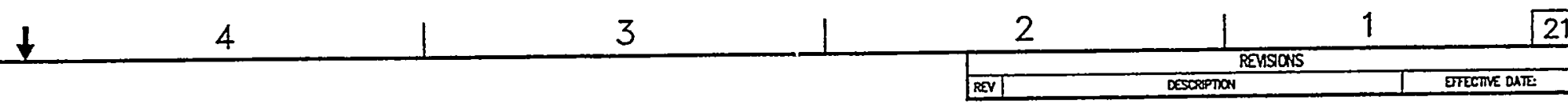

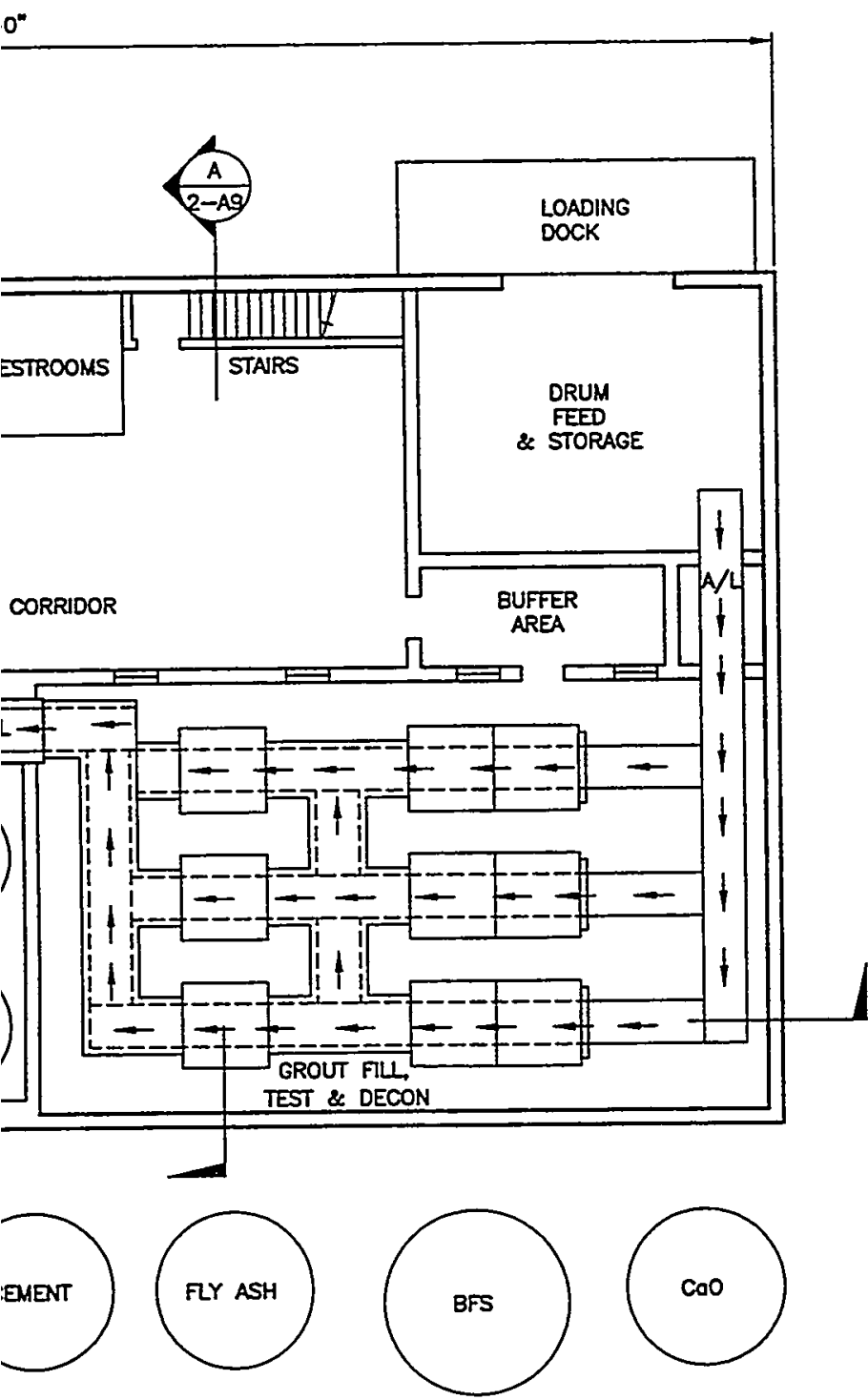

C

0'-0"

\begin{tabular}{|c|c|c|}
\hline 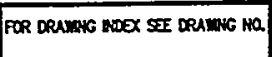 & secontruct ma & LOCKHEED MATTINA \\
\hline 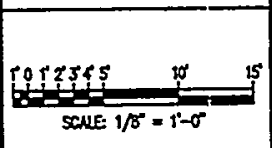 & 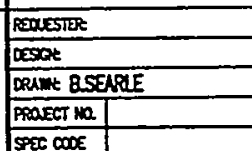 & $\begin{array}{l}\text { CSIX/TRU GROUT PRONECT } \\
\text { SCENARIO } 2 \\
\text { PLAN O } 0^{\prime \prime}-0^{*}\end{array}$ \\
\hline & 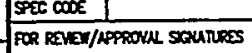 & SFE \\
\hline DESGN PHASE: & 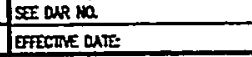 & 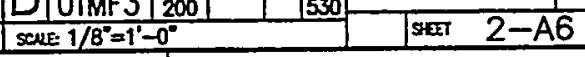 \\
\hline
\end{tabular}




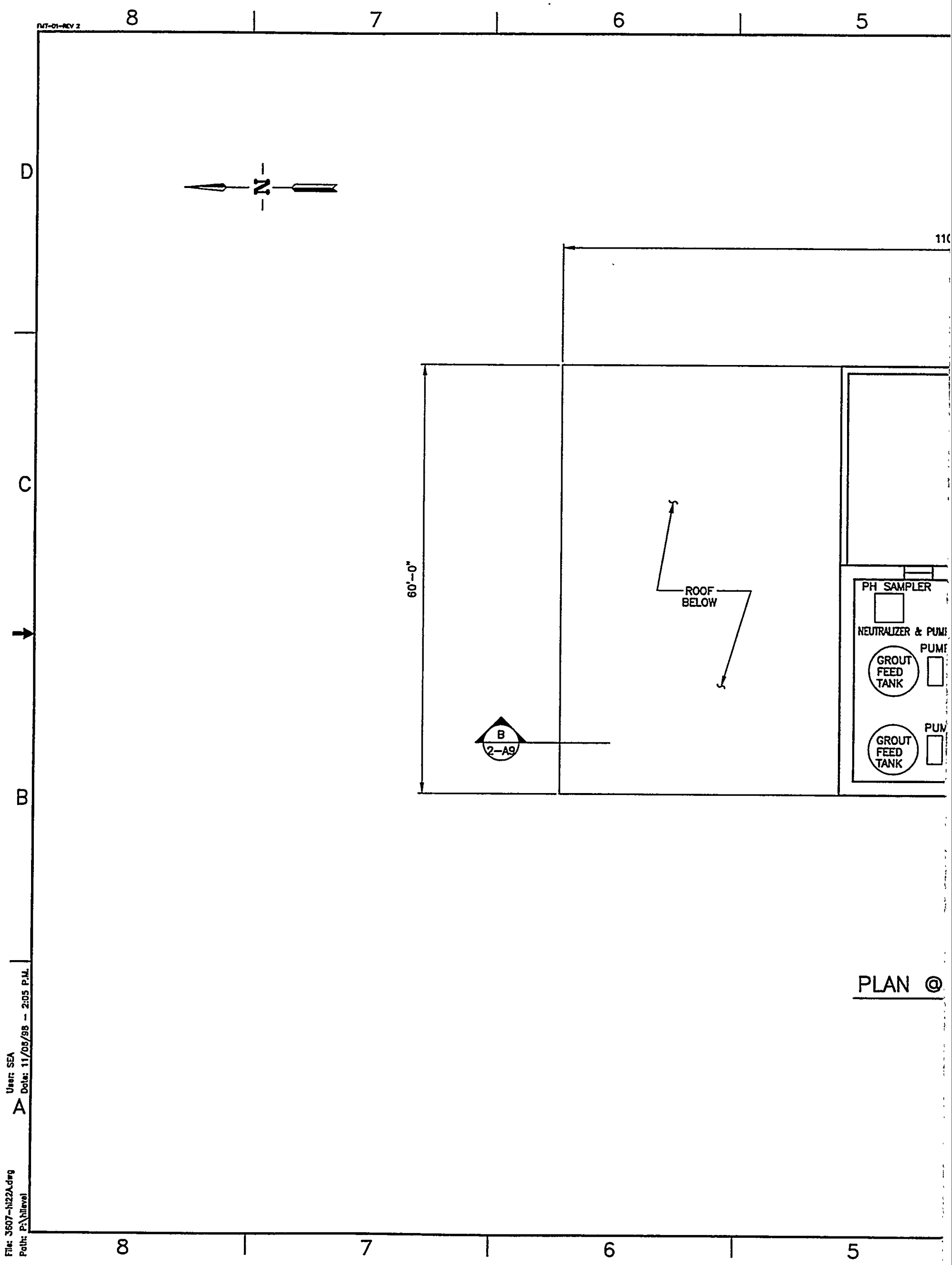




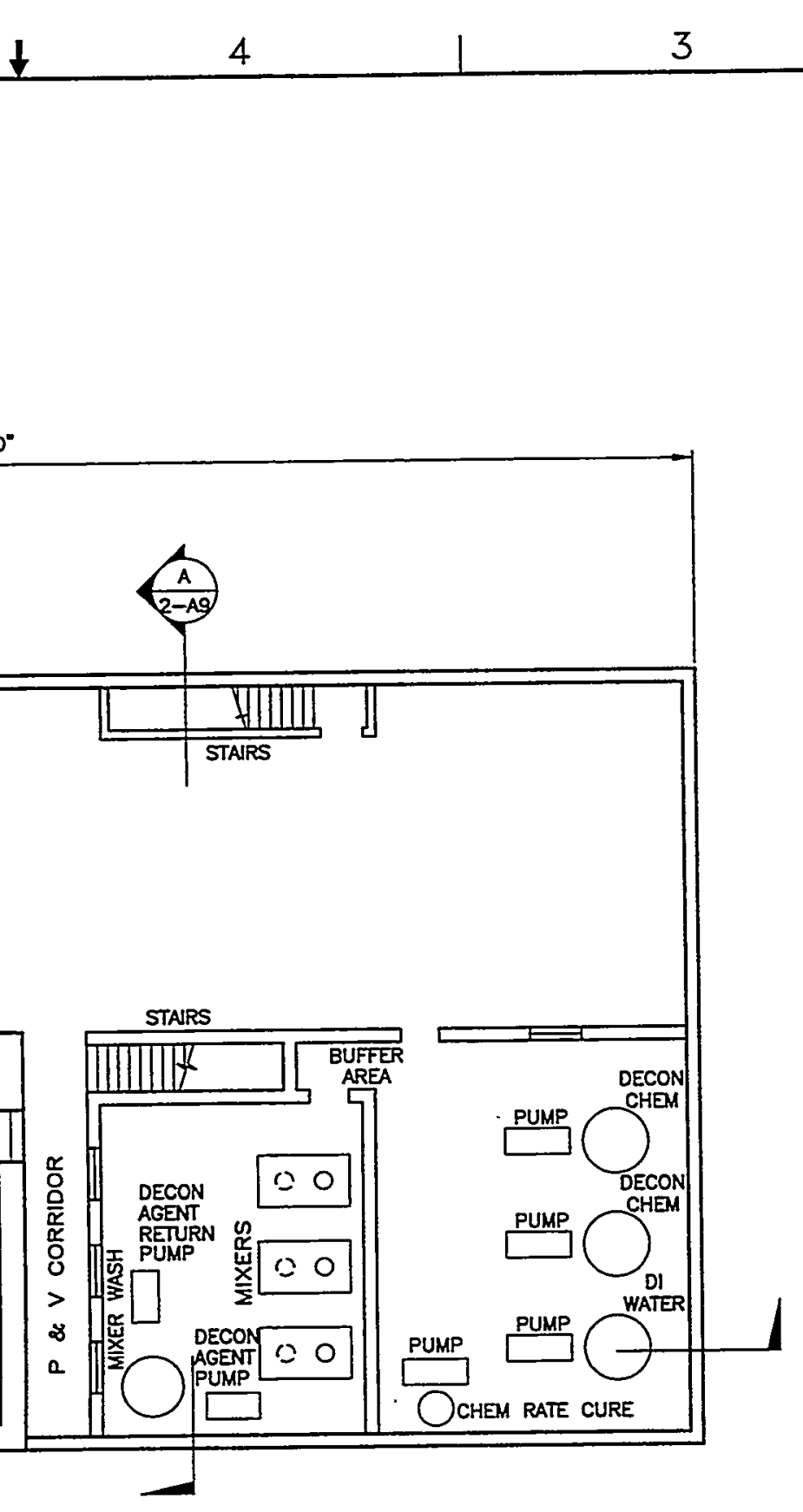

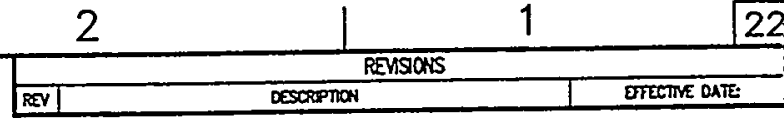

\begin{tabular}{|c|c|c|c|c|}
\hline 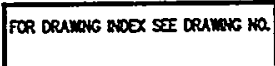 & seocorract ha. & \multicolumn{3}{|c|}{ LOCKHEED MARTIN/ } \\
\hline \multirow{5}{*}{ 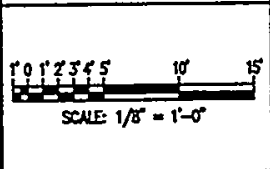 } & \multirow{3}{*}{ 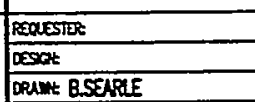 } & \multirow{4}{*}{\multicolumn{3}{|c|}{$\begin{array}{l}\text { CSIX/TRU GROUT PROJECT } \\
\text { SCENARIO } 2 \\
\text { PLAN } \odot 10^{\circ}-0^{*}\end{array}$}} \\
\hline & & & & \\
\hline & & & & \\
\hline & \begin{tabular}{|l|l|} 
Pangt Ma. & \\
\end{tabular} & & & \\
\hline & \begin{tabular}{|l|l|} 
sece cose & \\
\end{tabular} & 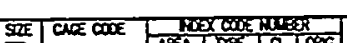 & & $\bar{k}$ \\
\hline DESGN PHUSE: & 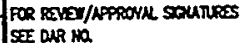 & 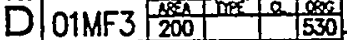 & DHo- & \\
\hline QUNUTY LEVE: & EFECWNE MUE: & scus: $1 / 8^{\circ}=1^{1}-0^{\circ}$ & TSET & $2-A 7$ \\
\hline
\end{tabular}




\begin{tabular}{lll|l|l|l|}
\hline 4 & 3 & 2 & 1 & 23 \\
\hline
\end{tabular}

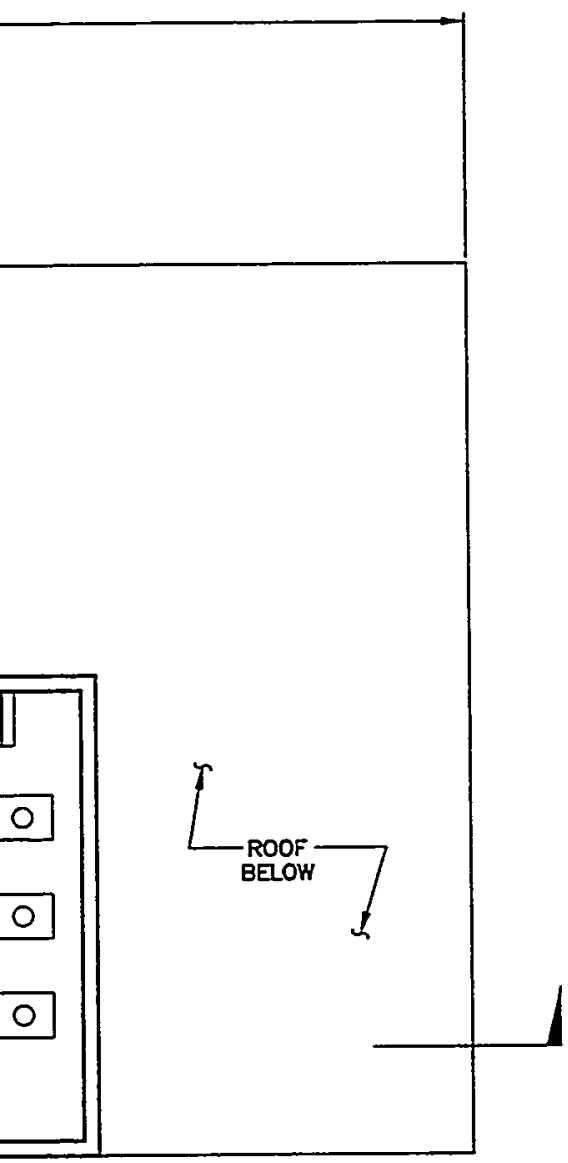

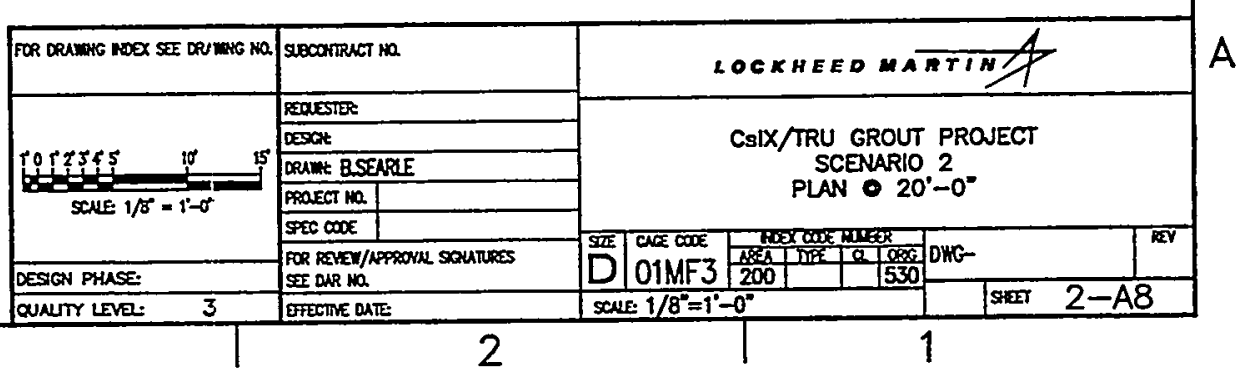




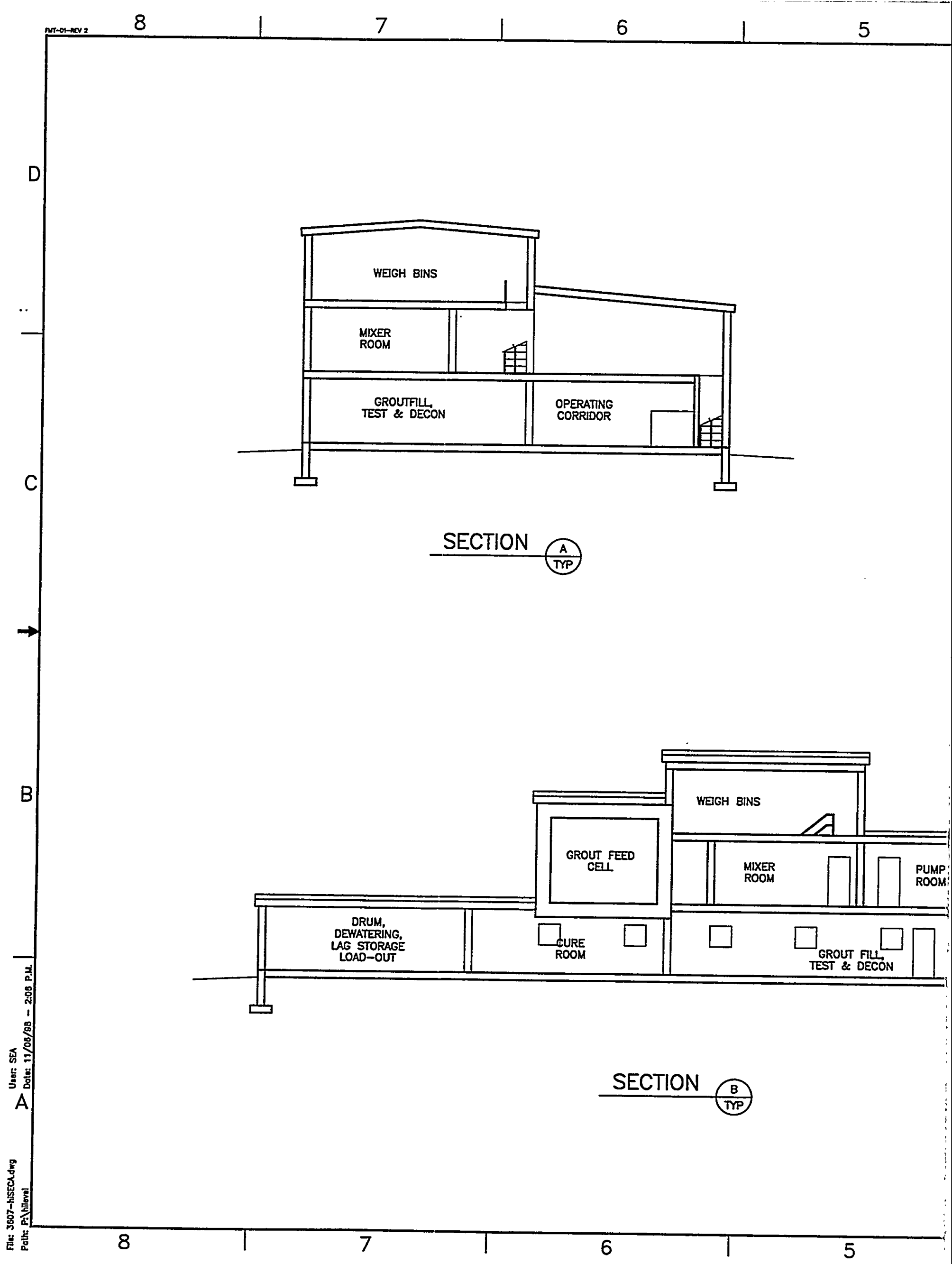




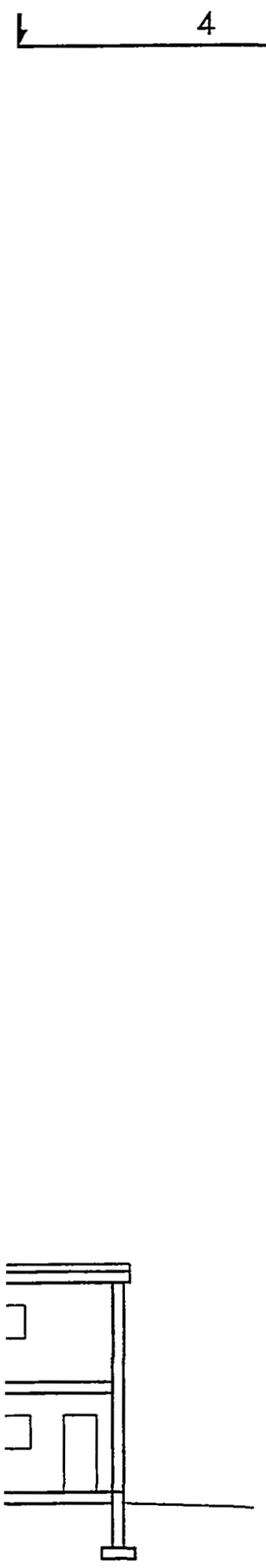

\begin{tabular}{|c|c|c|c|c|}
\hline 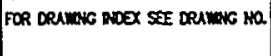 & seocontruct to. & LOCXHEED WA & & \\
\hline \multirow{6}{*}{ 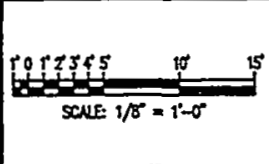 } & REQTESTER: & \multirow{4}{*}{\multicolumn{2}{|c|}{$\begin{array}{c}\text { CSIX/TRU GROUT PRONECT } \\
\text { SCENARIO } 2 \\
\text { BUILDING SECTIONS }\end{array}$}} & \\
\hline & Desset & & & \\
\hline & Daut B.SERRE & & & \\
\hline & \begin{tabular}{|l|l|} 
PREET MO & \\
\end{tabular} & & & \\
\hline & $57 x \cos$ & & & \\
\hline & \multirow{2}{*}{ 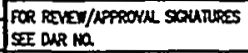 } & 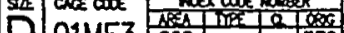 & \multirow{2}{*}{ DWw } & Ret \\
\hline DESTGN PHASE: & & $207 M+5|200| \frac{1530}{2}$ & \multirow{2}{*}{\multicolumn{2}{|c|}{ अस्थ $\frac{1}{2-A 9}$}} \\
\hline QUNUTY LEVE: & ETECTRE QRTE & SCNE: $1 / 8^{\circ}=1^{\prime}-0^{\circ}$ & & \\
\hline
\end{tabular}




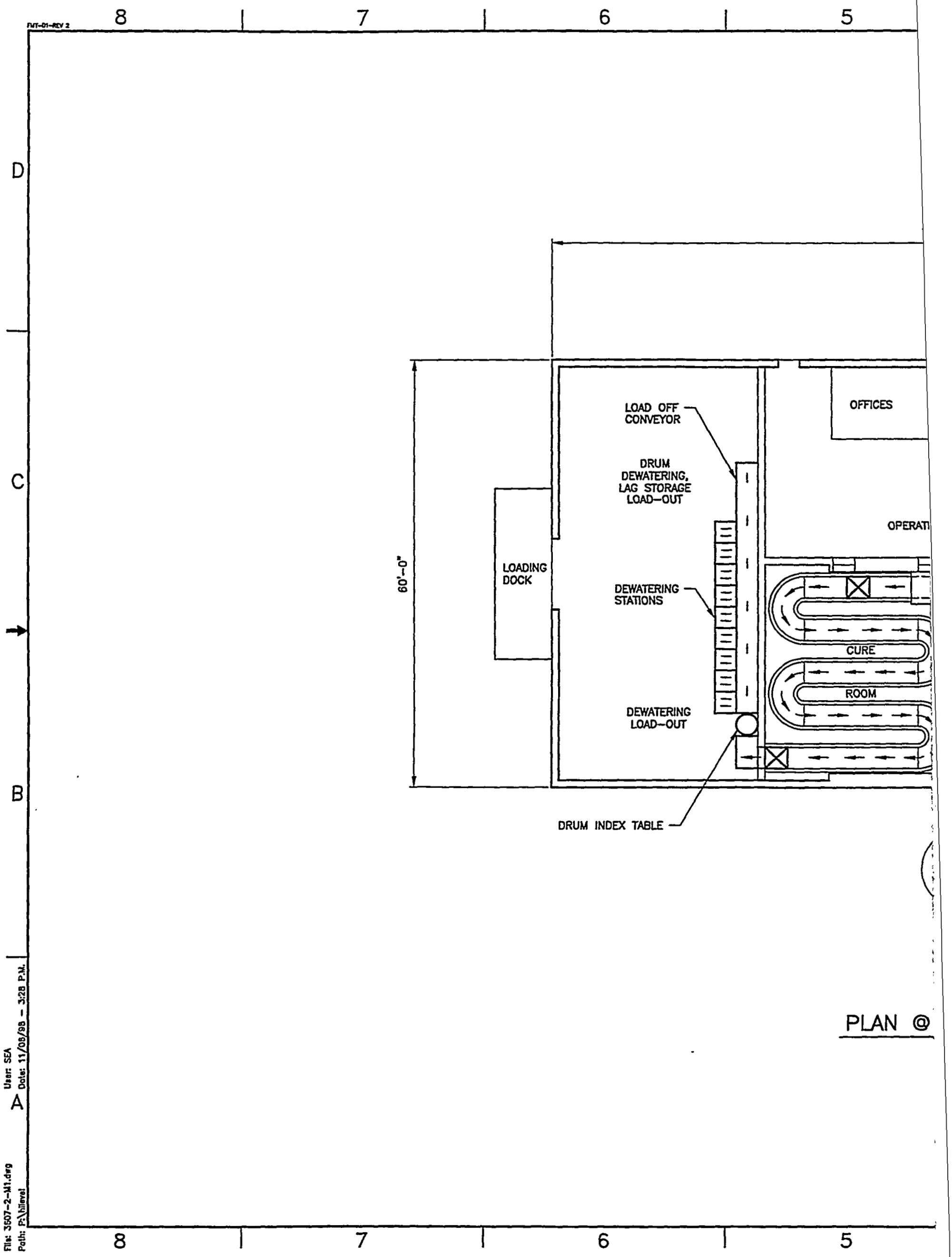




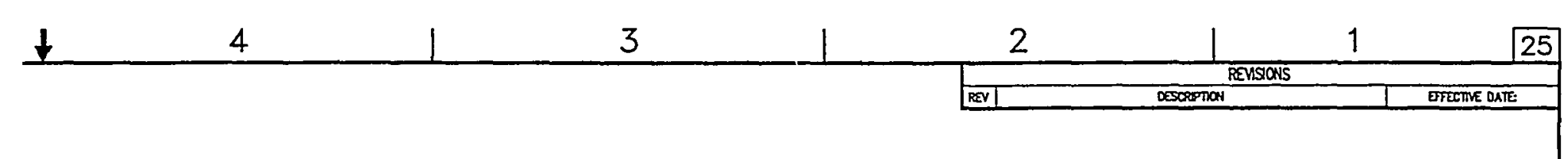

$$
\text { O" }
$$
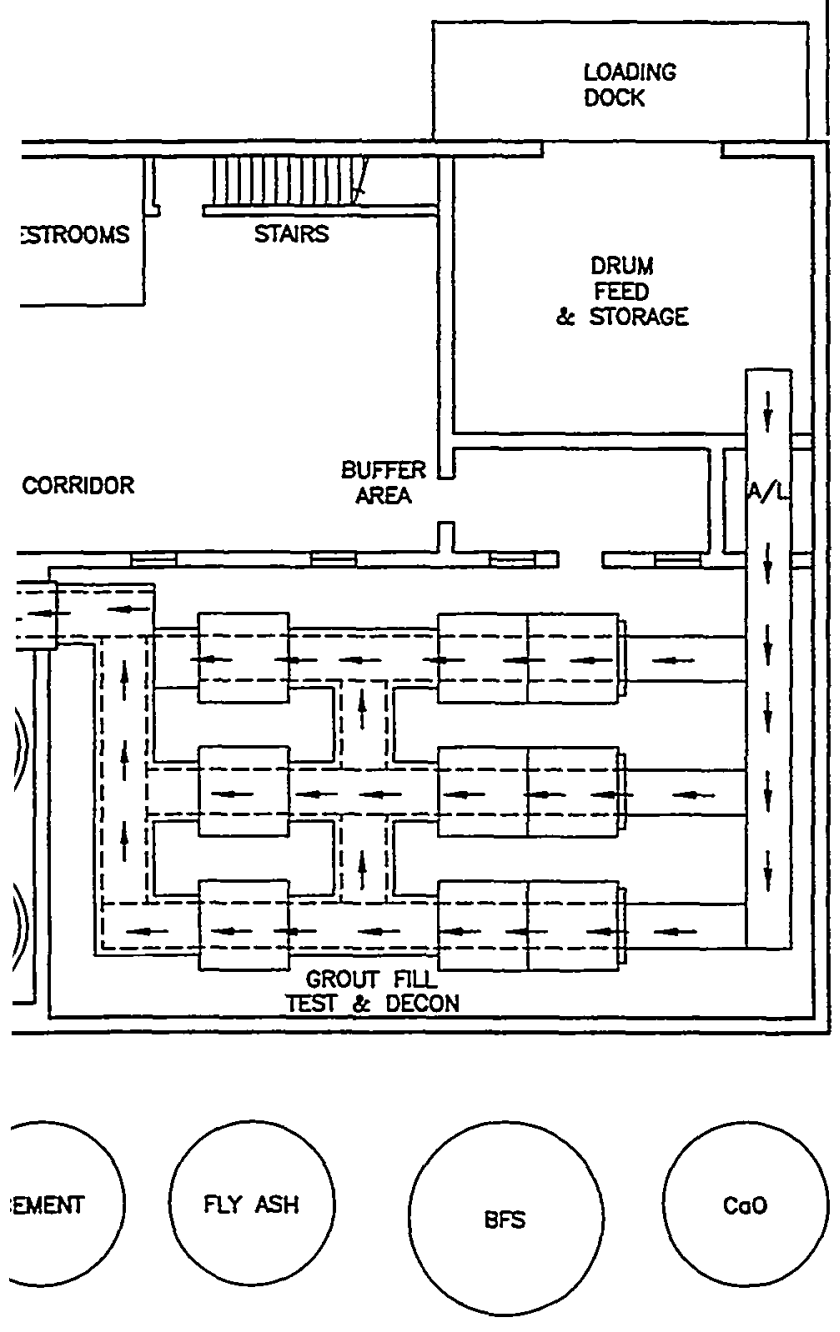

$0^{\prime}-0 "$

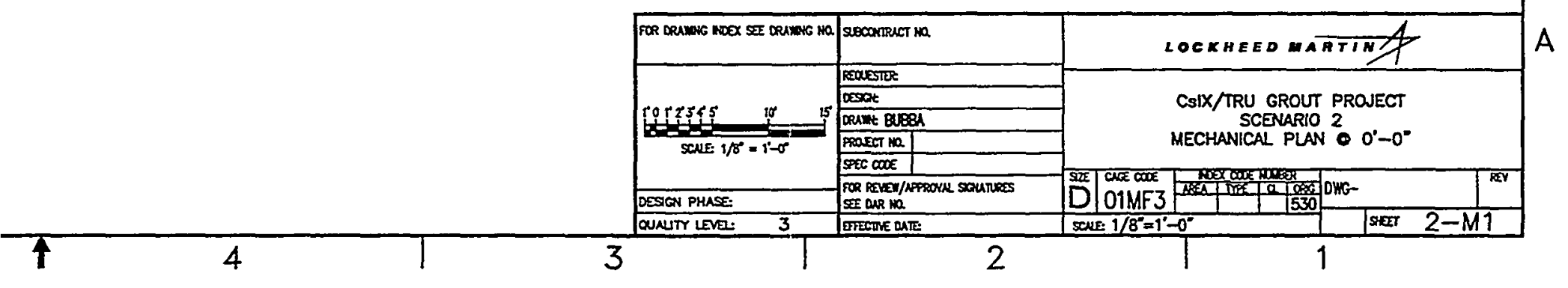




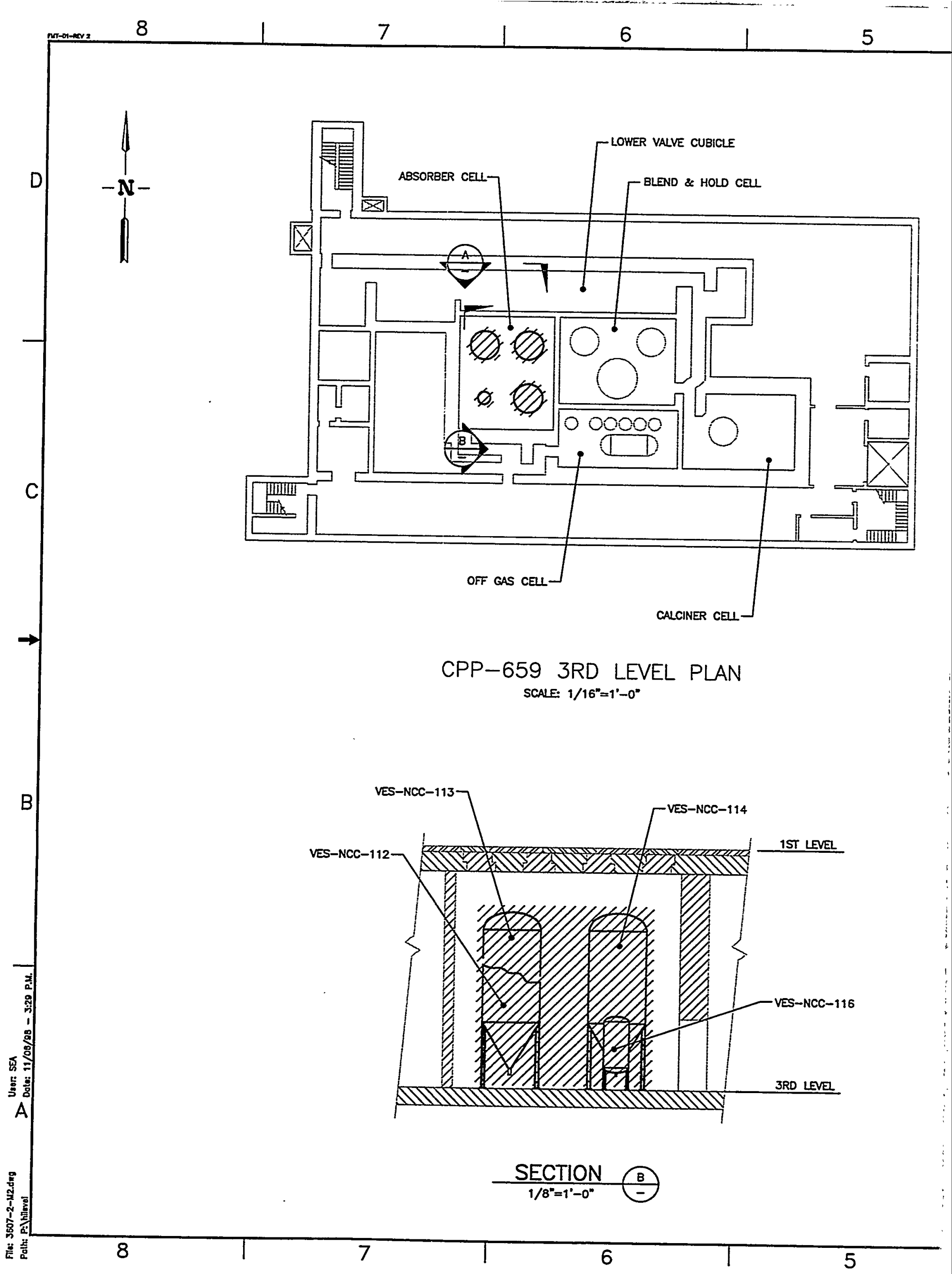




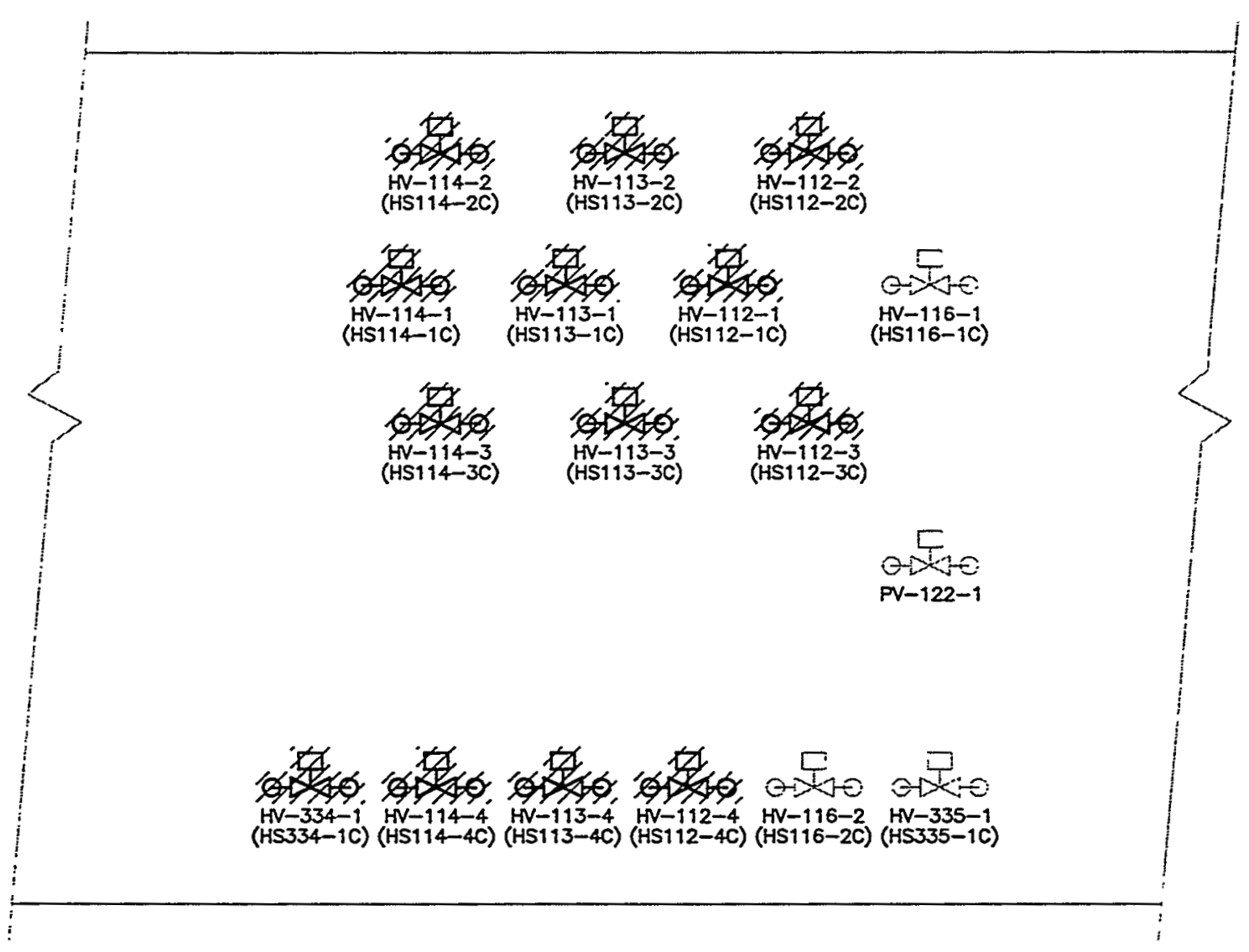

\section{$\frac{\text { SECTION }}{1 / 4^{n}=11^{\circ}-0^{n}}$}

\section{NOTES}

1. CROSS HATCHED EQUIPMENT INDICATES DEMOUTION.

2. NOT ALL OF THE EQUIPMENT TO BE DENOLTONED IS SHOWN ON DRAWING. FOR COMPLETE UST OF EQUIPMENT TO BE DEMOLTIONED SEE ABSORBER CELL DEMOLTION UST BELOW.

ABSORBER CELL DEMOLITION LIST

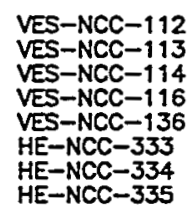

\begin{tabular}{|c|c|}
\hline 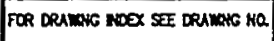 & suboontruet Ma \\
\hline \multirow{5}{*}{ NO SCALE } & REMEstrer \\
\hline & peogot \\
\hline & DRAIII: BUBSA \\
\hline & \begin{tabular}{|l|} 
PRaset Ma \\
\end{tabular} \\
\hline & $\sec 000$ \\
\hline DESGON PHASE: & \multirow[t]{2}{*}{ 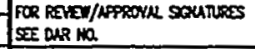 } \\
\hline & \\
\hline
\end{tabular}

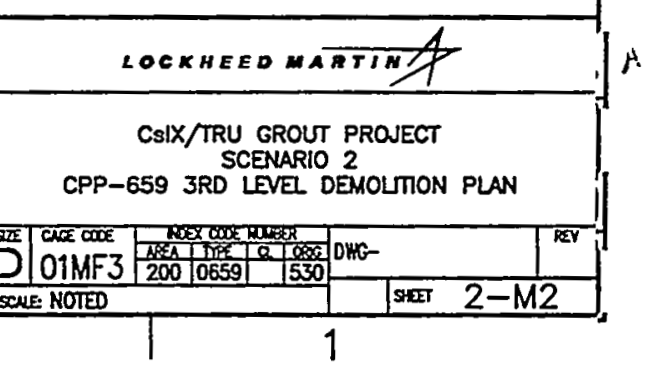




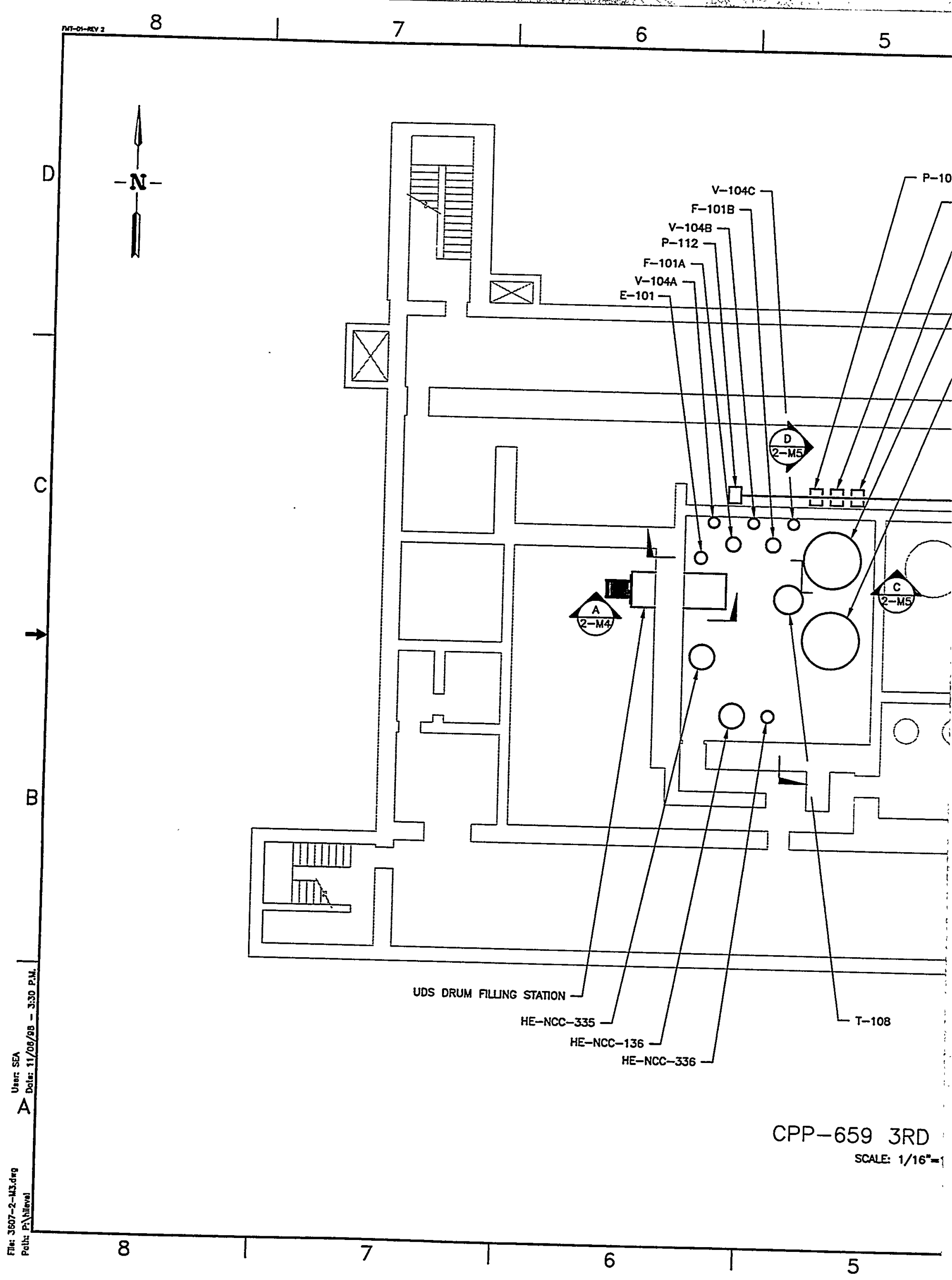




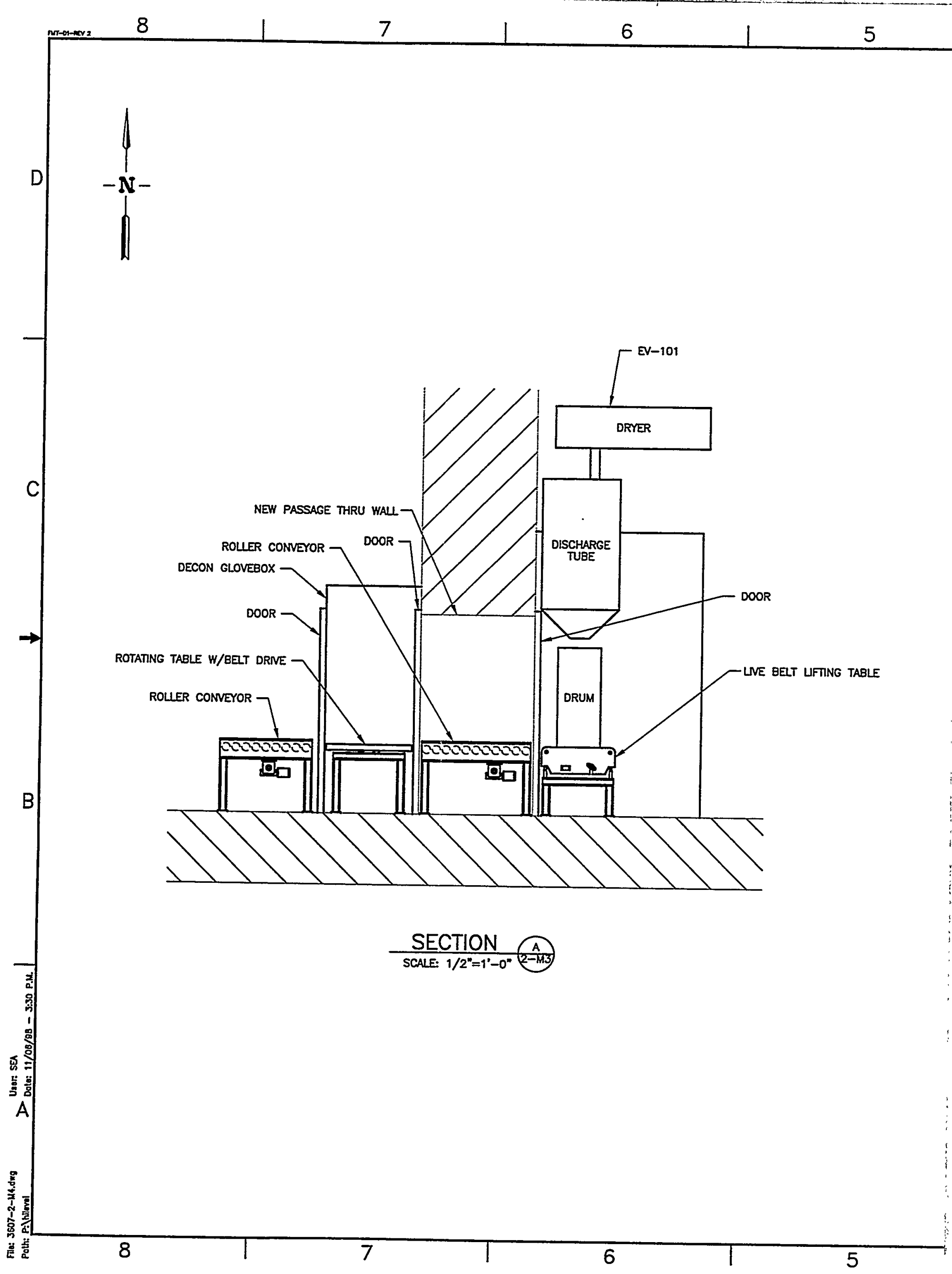




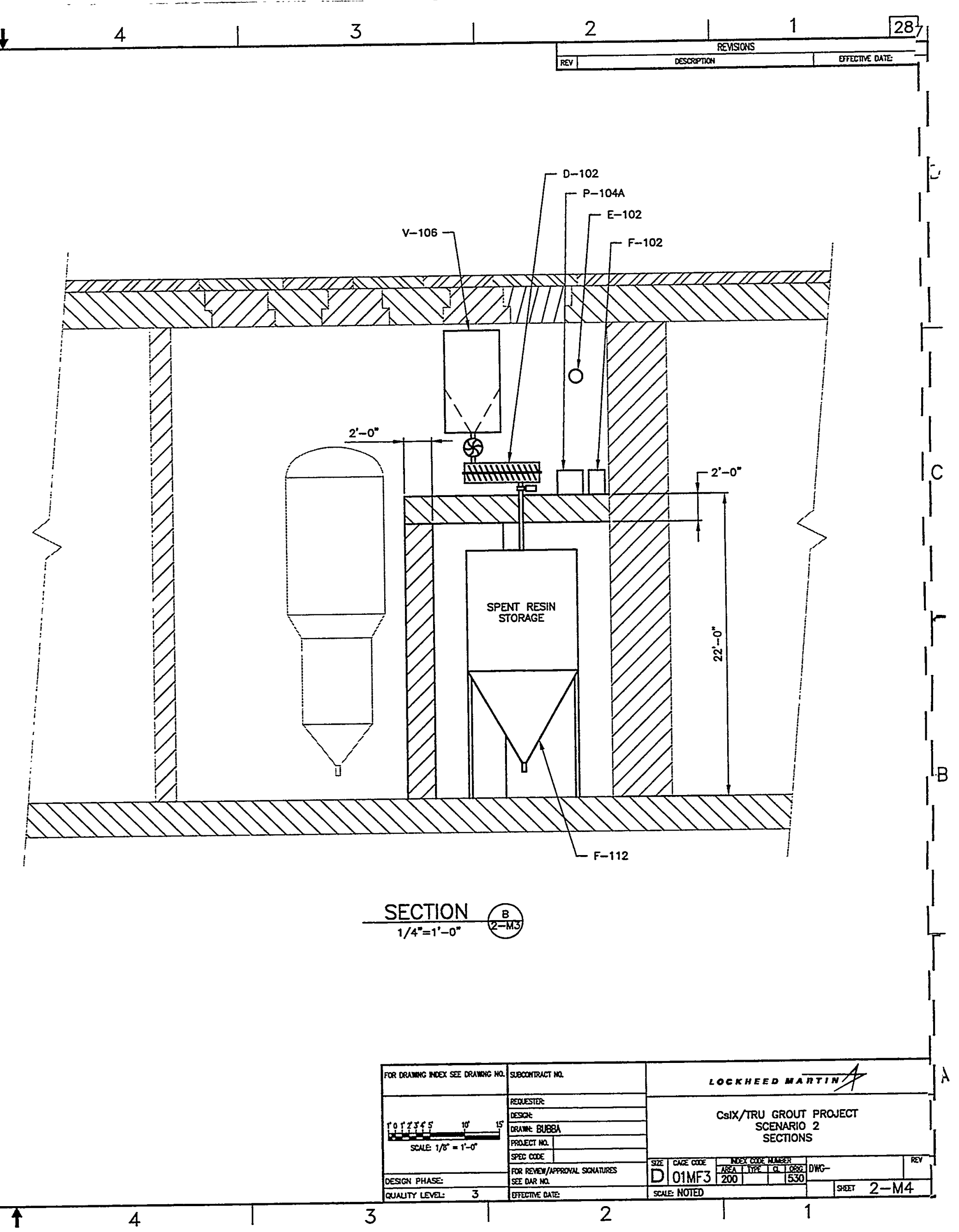




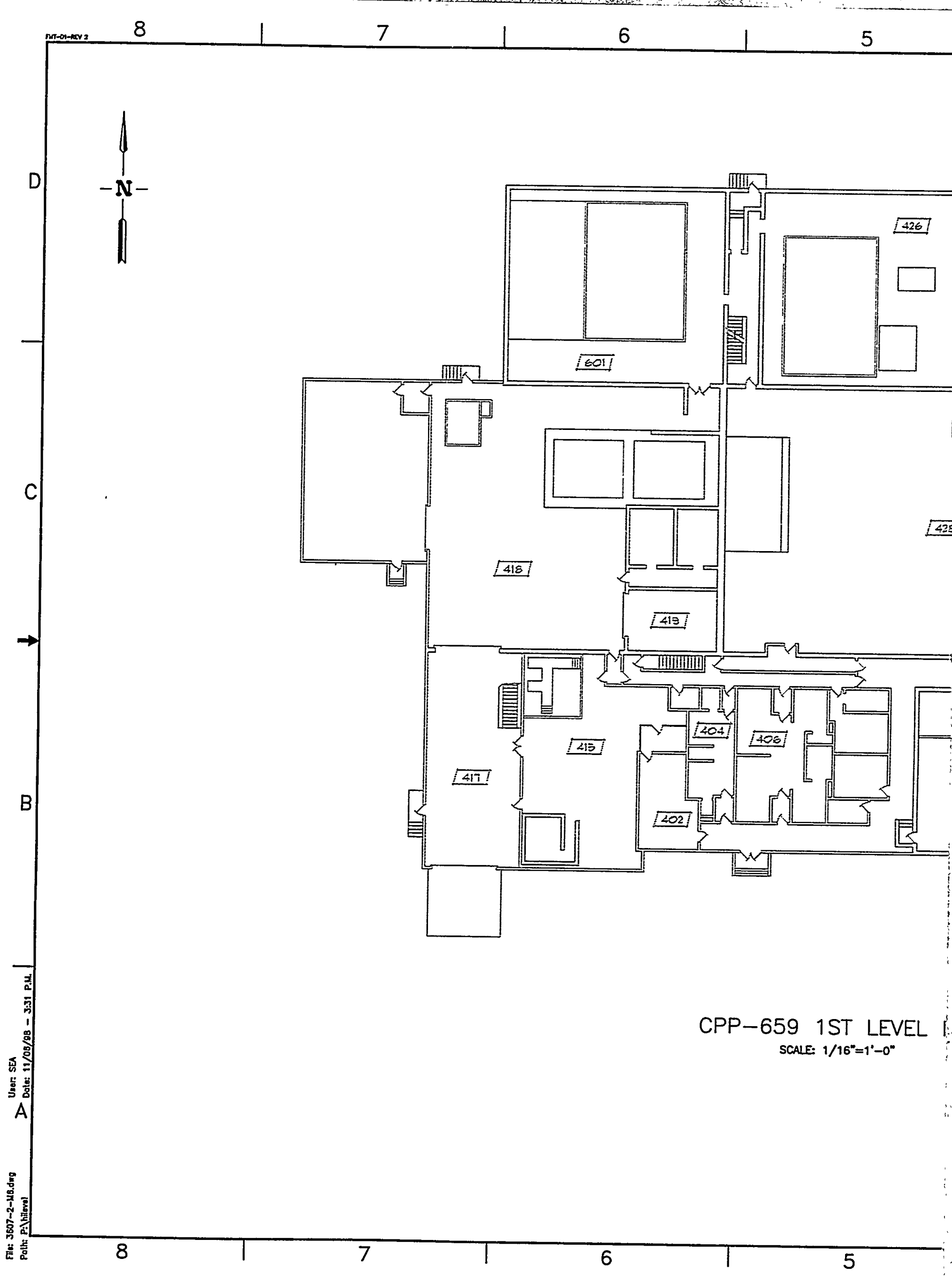




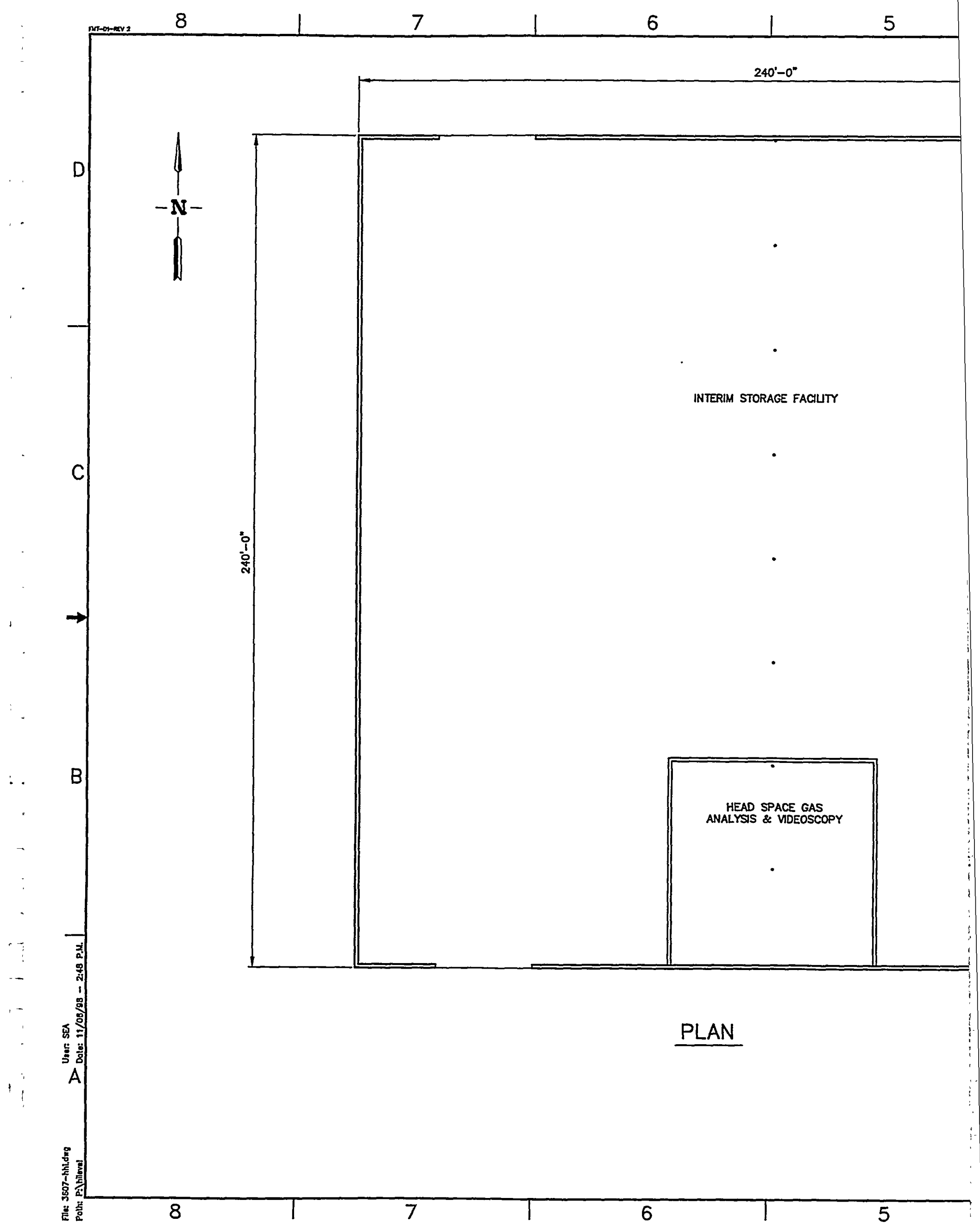




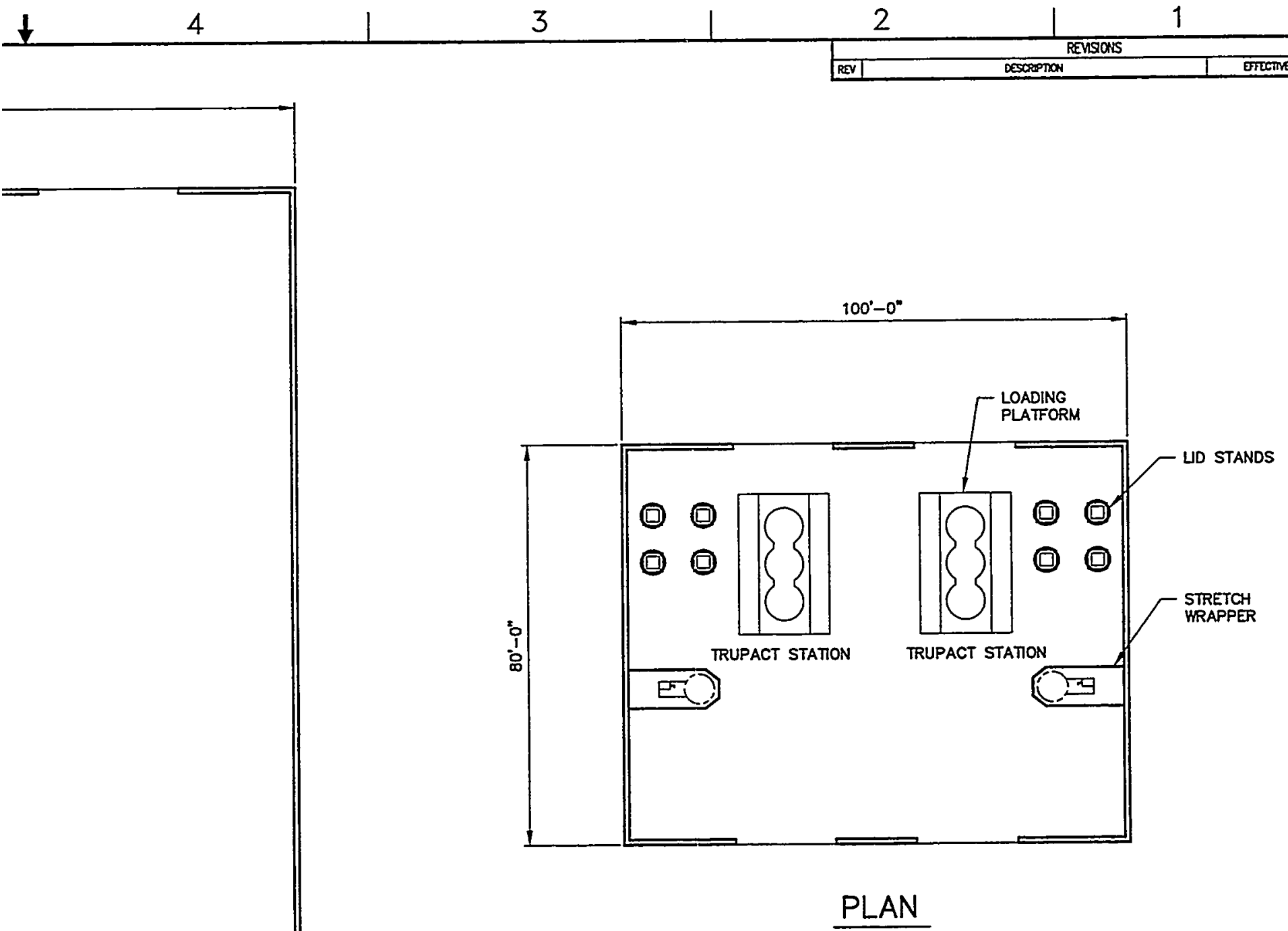

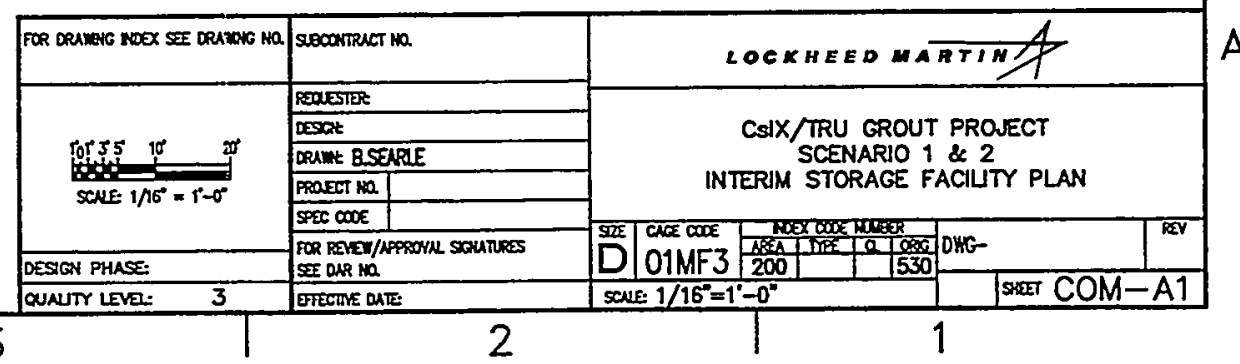




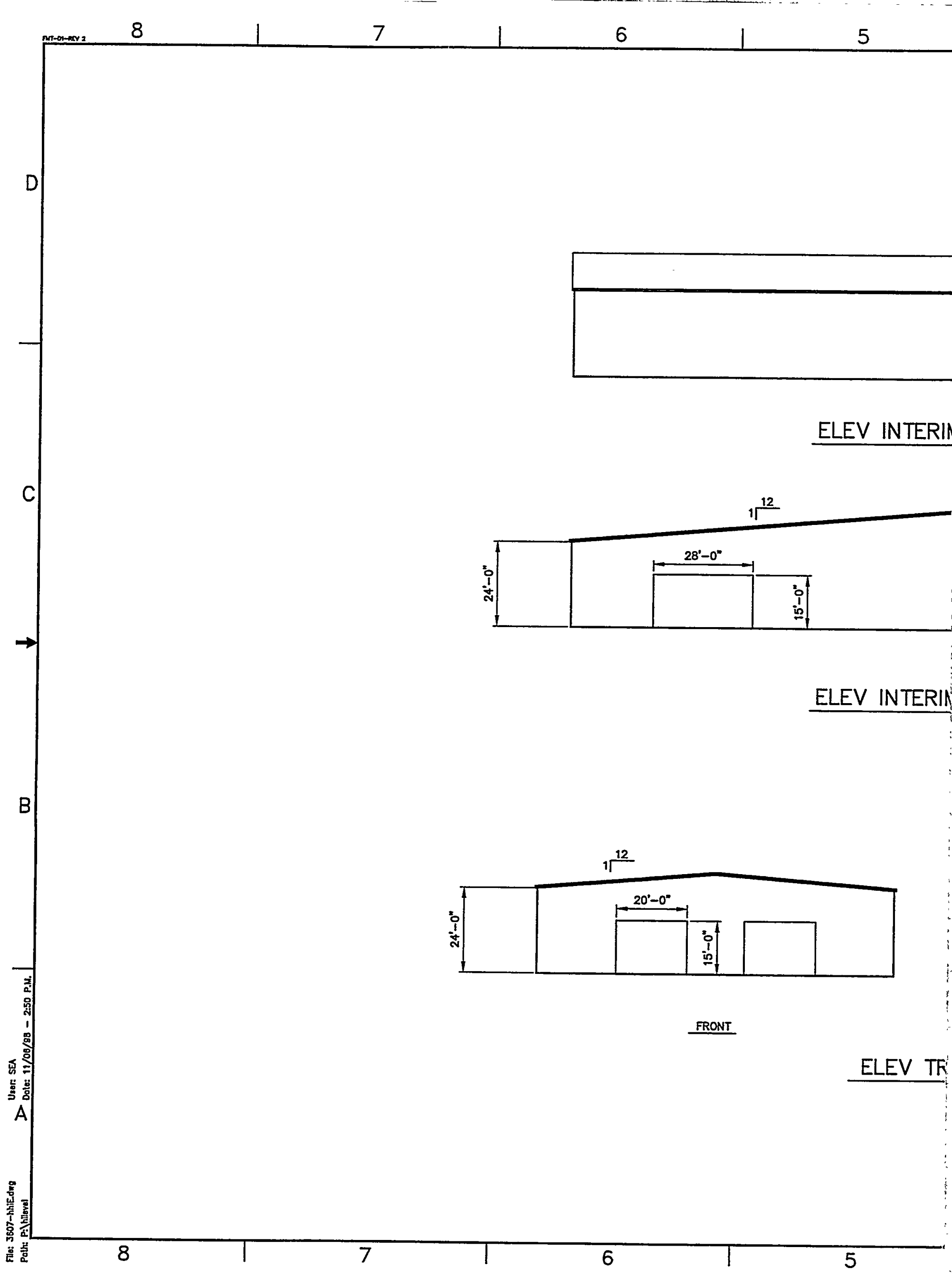




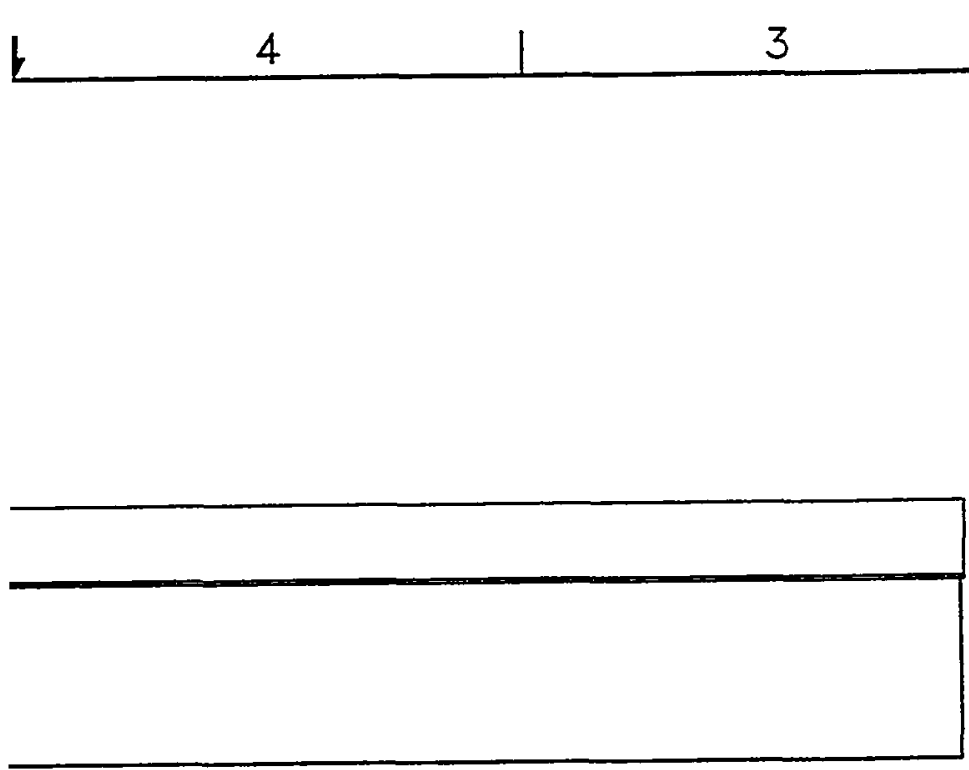

FOR DRAWHE RDOX SEE DRAWAC Ma
succonsuct Mo pequestix: ossat

DAANE B.SEARIE FraEeT in $\operatorname{sexc} c \mid$

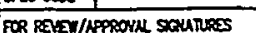




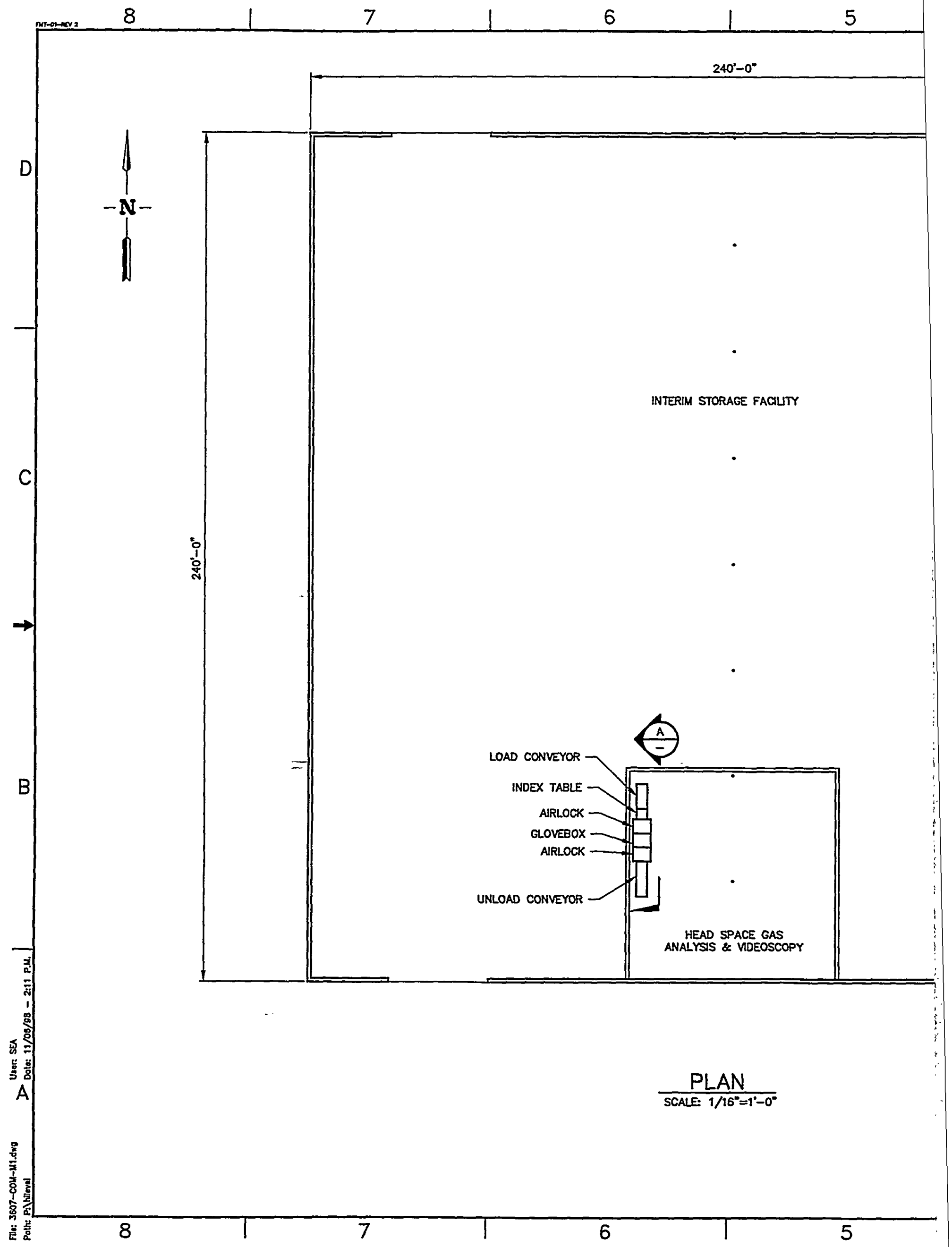




\section{Appendix E}

\section{Engineering Design Files}

EDF-CsIX-001 "CsIX/TRU Grout Process Basis, Description, Material Balance and Process Flow Diagrams" -in Appendix B

EDF-CsIX-002 "Interim Storage for CH TRU Waste Drums"

EDF-CsIX-003 "Personnel Staffing and Space Requirements for CsIX/TRU Grout Operations"

EDF-CsIX-004 "SBW Treatment - Ion Exchange Media Disposition Concepts"

EDF-CsIX-005 "Handling of Containers Through the Grouting Area"

EDF-CsIX-006 "Handling of Containers Through the Cure Cell"

EDF-CsIX-007 "Handling of Containers Through the Dewatering Process"

EDF-CsIX-008 "UDS Drum Filling and Handling"

EDF-CsIX-009 "Equipment Installation in NWCF"

EDF-CsIX-010 "Drum Head Space Sampling and Inspection"

EDF-CsIX-011 "HVAC - Scenario 1"

EDF-CsIX-012 "HVAC - Scenario 2"

EDF-CsIX-013 "Off-Gas"

EDF-CsIX-014 "Electrical Requirements"

EDF-CsIX-015 "CsIX/TRU Component Projected Radiation Fields"

EDF-CsIX-016 "Determine Facility Shielding Requirements"

Not generated by this Feasibility Study, but appended for reference (not for review):

EDF-WTS-003 "Regulatory Requirements for the Design, Construction, and Operations of the ICPP Proposed Waste Processing Facilities"

EDF-WTS-004 "Regulatory and Design Requirements for Waste Treatment Facilities" 
Project File Number $\quad$ 02BM1

Project/Task CsIX/TRU Grout Feasibility Study

Subtask D.4 Interim Storage Design

\section{Title: Interim Storage for $\mathrm{CH}$ TRU Waste Drums}

\section{Summary:}

This EDF contains the calculations used to determine how much interim storage capacity will be required for Contact Handled (CH) TRU waste drums produced during the CsLX/TRU Grout process. Also included is a brief guidance on current interim storage facilities, similar to those required for this study. The reference material - grout quantity and density - is documented in EDF-CsIX-001, CsIX/TRU Grout Process Basis, Description, Material Balance, and Process Flow Diagram.

\section{Conclusions:}

The numbers indicate an accuracy greater than really exists. Drum production rates and totals have been rounded and will vary from that listed when improved reference data becomes available.

- Total number of drums produced $=38,000$.

- Average production rate over 5 year operating period $=633 /$ year, $146 / \mathrm{week}$, or $21 /$ day.

- Maximun actual daily rate considering efficiencies $=38$.

- Filled drum weight is $888 \mathrm{lb}(403 \mathrm{~kg})$.

- Maximum number of grout filled drums per TRUPACT-II is 7 .

- TRUPACT-II payload for CsIX/TRU drums is $6864 \mathrm{lb}$ (max allowed is $7265 \mathrm{lb}$ ).

- Shipping rate is assumed to be 5 TRUPACT-II shipments (3 TRUPACT-IIs per shipment) every 2 weeks (105 drums).

- Interim storage capacity required is for approximately 24,000 ( 24,350 calculated) drums.

- Last date for drum shipment to WIPP is estimated to be December, 2021.

- Type II Storage Modules at RWMC should be the basis for estimating size and cost.

- The RWMC TRUPACT-II loading station should be the basis for estimating size and cost.

Distribution (complete package): R. E. Dafoe, MS 3765, C. W. Olsen, S. J. Losinski, MS 3625, MS 3211, K. L. Williams, MS 3765, Project File, a copy will be included in report INEEL/NT98-00869

Distribution (summary package only):

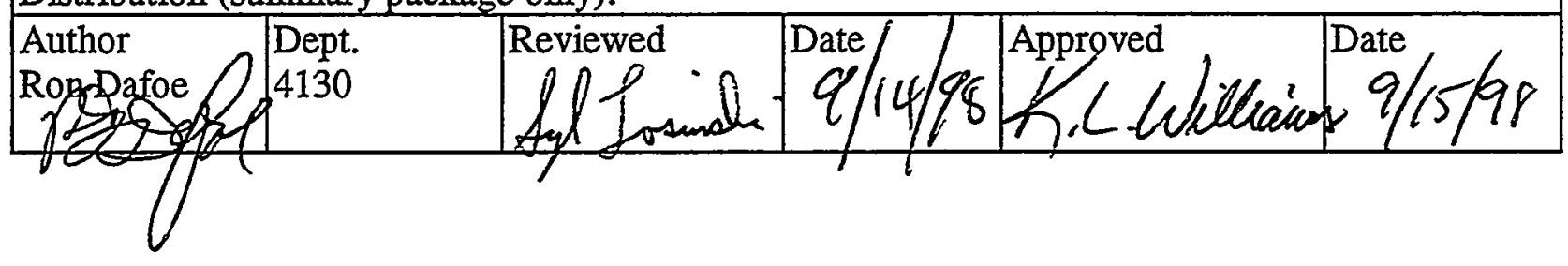




\section{Introduction:}

The number of drums to be generated is based on the material balance information provided in project EDF-CsLX-001, CsIX/TRU Grout Process Basis, Description, Material Balance, and Process Flow Diagrams. The volume of grout material produced, per the EDF, is $7500 \mathrm{~m}^{3}$ at a density of $1.9 \mathrm{~kg} / \mathrm{l}$. These quantities are used in the calculations shown in the latter pages of this EDF, with some of the following summary taken from the tables:

\section{Production Rate:}

Using a drum loading of $0.2 \mathrm{~m}^{3}$ of grout per drum, the total production of CH TRU waste drums is approximately 38,000 . For the operating period of 5 years, this converts to approximate averages of 633 drums per month, 146 per week, or 21 per day. Considering operating up-time and efficiency estimates of approximately 55\% (EDF-CsLX-003), any given day's output would be closer to $38(21 / 0.55)$ drums a day.

\section{TRUPACT-II Loading:}

The NuPac TRUPACT-II SAR (Rev.3, July 1989) states that the total payload for a TRUPACTII is $7265 \mathrm{lb}$, which, in addition to a maximum 14 55-gal drums, with contents, includes the inner components required to package the drums in the TRUPACT-II. The estimated drum weight from the CsIX Grout process is $888 \mathrm{lb}(403 \mathrm{~kg})$, which includes an estimated empty drum weight of $50 \mathrm{lb}$. Thus, the loading of a TRUPACT-II with these drums will be limited to 7. The remaining space in the TRUPACT- $\Pi$ is assumed to be filled with empty drums. This results in an estimated TRUPACT-II payload of approximately $6864 \mathrm{lb}$. TRUPACT-IIs are currently shipped in sets of 3 on a single trailer.

A major assumption is that the CsIX/TRU Grout process drum production rate will be greater than the rate at which the drums can be shipped to WIPP, their assumed ultimate destination. Functional File BC-CsIX-25, dated 8/25/98, which documents the 8/19/98 TELECON with WIPP about acceptance of waste product from SBW treatment by the CSIX/TRU Grout process, under item 8 , indicates that WIPP will size its TRUPACT-II fleet and handling capability to match the generator's needs. To avoid the need for interim storage, approximately one TRUPACT-II shipment, which includes 3 TRUPACT-II containers, or 21 drums, would have to made each day of the year. With up-time and efficiencies included, the actual rate would be at least 2 shipments a day. RWMC personnel indicate that it will be a struggle for them to make one shipment a day (Functional File No. BC-CsIX-21, Notes from 8-12-98 CsIX/TRU Grout Weekly Technical Status Meeting). For the purpose of this EDF, the assumption is that 2-1/2 shipments per week (5 every 2 weeks) (105 drums) can be made and that WIPP will be able to handle that rate. 


\section{Interim Storage:}

The following table shows the effect that varying shipping rates has on interim storage capacity required (see tables for details).

$\begin{array}{llll}\text { \# of drums } & \text { \# of TRUPACT } & \text { \# of drums in storage at } & \text { Date of last } \\ \text { per week } & \text { loads per week } & \text { INTEC by end of 2012 } & \text { Shipment } \\ \text { to WIPP } & \text { to WIPP } & & \text { to WIPP }\end{array}$

$\begin{array}{llrr}42 & 2 & 27,080 & 05 / 2025 \\ 52.5 & 2.5 & 24,350 & 12 / 2021 \\ 84 & 4 & 16,160 & 10 / 2016 \\ 147 & 7 & 0 & 12 / 2012\end{array}$

This summary indicates that significant savings in interim storage capital and operating costs can be had for even an incremental increase from 2 shipments a week to 2-1/2 a week. Four shipments per week could reduce the total shipping timeframe by 5 years from the 2-1/2 shipments-per-week schedule.

\section{Interim Storage Facilities:}

The Type II storage modules at RWMC are RCRA buildings designed to hold 17,500 55-gallon drum equivalents of $\mathrm{CH}$ TRU waste. Because the interim storage requirements for the CsIX/TRU Grout Feasibility Study are similar to the Type II Storage Modules, the design and cost estimating efforts for this study should be based on these facilities. The original design and cost estimate information is readily available. An initial ROM for 2 such buildings for interim RCRA storage of the CsIX Grout process CH TRU waste drums was approximately $\$ 6 \mathrm{M}$. Not included in this figure is the cost for a TRUPACT-II loading station which will be required for this study. An existing loading station exists at RWMC and should be the basis for one in this study. 


\section{Calculation Details and Summaries:}

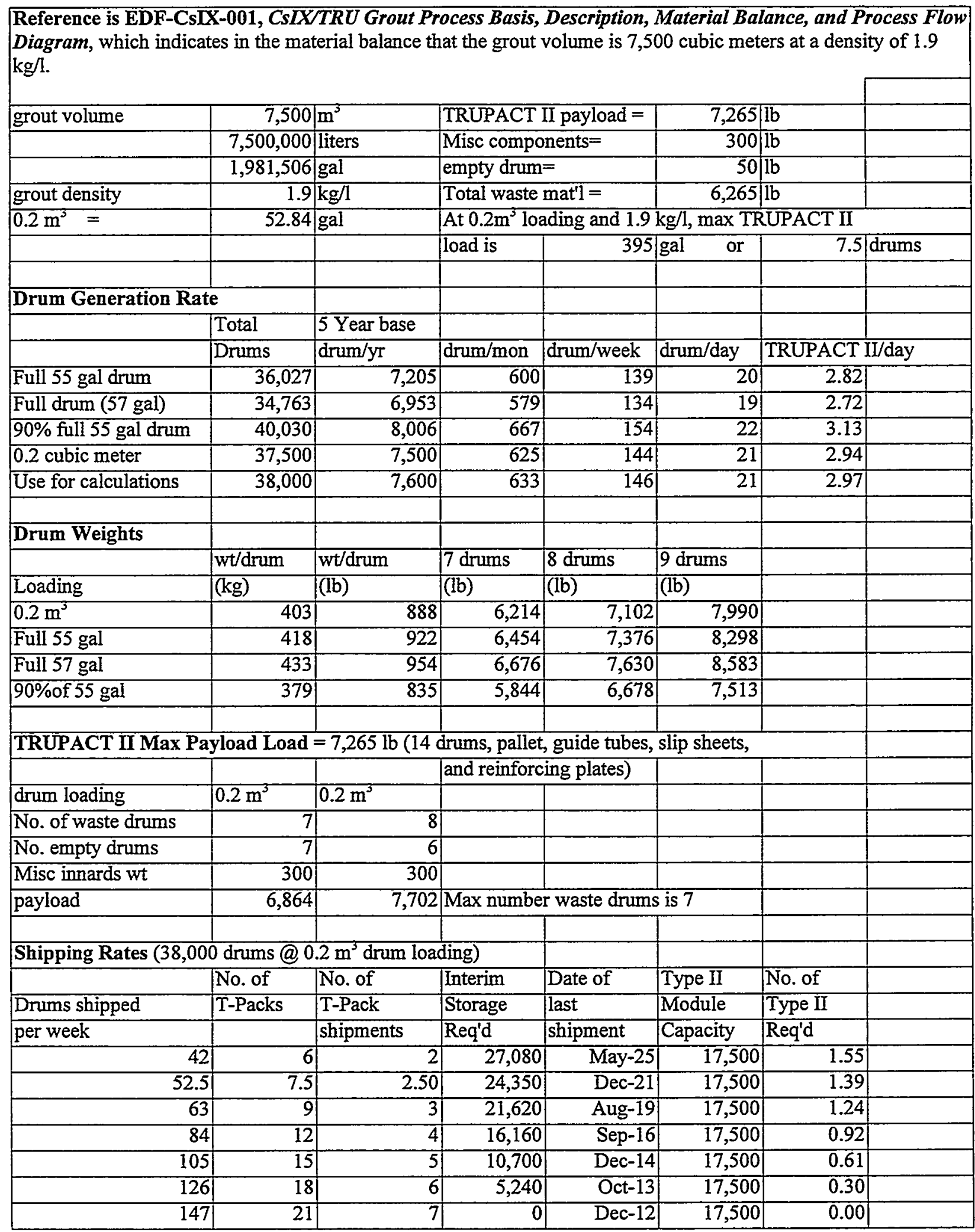


The following tables (Excel spreadsheet) were used to determine the amount of interim storage required for a given production rate and varying shipping rates.

There are 2 tables per page with each column and subsequent tables continuing on subsequent pages.

Total drums

38000

Int. Stor.

Req'd

$$
\begin{array}{ll}
0.2 \mathrm{~m}^{3} & \text { Shipped } \\
\text { drums } & \text { /week }
\end{array}
$$

Year Month generated $\begin{array}{ll}\text { Total drums } & 38000 \\ \text { Int. Stor. } & 24350\end{array}$

Req'd

$\begin{array}{rrrrrrrrrr}2008 & 1 & 633 & 182 & 451 & 2008 & 1 & 633 & 227.5 & 406 \\ 2008 & 2 & 633 & 182 & 903 & 2008 & 2 & 633 & 227.5 & 812 \\ 2008 & 3 & 633 & 182 & 1354 & 2008 & 3 & 633 & 227.5 & 1218 \\ 2008 & 4 & 633 & 182 & 1805 & 2008 & 4 & 633 & 227.5 & 1623 \\ 2008 & 5 & 633 & 182 & 2257 & 2008 & 5 & 633 & 227.5 & 2029 \\ 2008 & 6 & 633 & 182 & 2708 & 2008 & 6 & 633 & 227.5 & 2435 \\ 2008 & 7 & 633 & 182 & 3159 & 2008 & 7 & 633 & 227.5 & 2841 \\ 2008 & 8 & 633 & 182 & 3611 & 2008 & 8 & 633 & 227.5 & 3247 \\ 2008 & 9 & 633 & 182 & 4062 & 2008 & 9 & 633 & 227.5 & 3653 \\ 2008 & 10 & 633 & 182 & 4513 & 2008 & 10 & 633 & 227.5 & 4058 \\ 2008 & 11 & 633 & 182 & 4965 & 2008 & 11 & 633 & 227.5 & 4464 \\ 2008 & 12 & 633 & 182 & 5416 & 2008 & 12 & 633 & 227.5 & 4870 \\ 2009 & 1 & 633 & 182 & 5867 & 2009 & 1 & 633 & 227.5 & 5276 \\ 2009 & 2 & 633 & 182 & 6319 & 2009 & 2 & 633 & 227.5 & 5682 \\ 2009 & 3 & 633 & 182 & 6770 & 2009 & 3 & 633 & 227.5 & 6088 \\ 2009 & 4 & 633 & 182 & 7221 & 2009 & 4 & 633 & 227.5 & 6493 \\ 2009 & 5 & 633 & 182 & 7673 & 2009 & 5 & 633 & 227.5 & 6899 \\ 2009 & 6 & 633 & 182 & 8124 & 2009 & 6 & 633 & 227.5 & 7305 \\ 2009 & 7 & 633 & 182 & 8575 & 2009 & 7 & 633 & 227.5 & 7711 \\ 2009 & 8 & 633 & 182 & 9027 & 2009 & 8 & 633 & 227.5 & 8117 \\ 2009 & 9 & 633 & 182 & 9478 & 2009 & 9 & 633 & 227.5 & 8523 \\ 2009 & 10 & 633 & 182 & 9929 & 2009 & 10 & 633 & 227.5 & 8928 \\ 2009 & 11 & 633 & 182 & 10381 & 2009 & 11 & 633 & 227.5 & 9334 \\ 2009 & 12 & 633 & 182 & 10832 & 2009 & 12 & 633 & 227.5 & 9740 \\ 2010 & 1 & 633 & 182 & 11283 & 2010 & 1 & 633 & 227.5 & 10146 \\ 2010 & 2 & 633 & 182 & 11735 & 2010 & 2 & 633 & 227.5 & 10552 \\ 2010 & 3 & 633 & 182 & 12186 & 2010 & 3 & 633 & 227.5 & 10958 \\ 2010 & 4 & 633 & 182 & 12637 & 2010 & 4 & 633 & 227.5 & 11363 \\ 2010 & 5 & 633 & 182 & 13089 & 2010 & 5 & 633 & 227.5 & 11769 \\ 2010 & 6 & 633 & 182 & 13540 & 2010 & 6 & 633 & 227.5 & 12175 \\ 2010 & 7 & 633 & 182 & 13991 & 2010 & 7 & 633 & 227.5 & 12581 \\ 2010 & 8 & 633 & 182 & 14443 & 2010 & 8 & 633 & 227.5 & 12987 \\ 2010 & 9 & 633 & 182 & 14894 & 2010 & 9 & 633 & 227.5 & 13393 \\ 2010 & 10 & 633 & 182 & 15345 & 2010 & 10 & 633 & 227.5 & 13798\end{array}$


Total drums $\quad 38000$ Int. Stor.

27080

Req'd

$$
\begin{array}{ll}
0.2 \mathrm{~m}^{3} & \text { Shipped } \\
\text { drums } & \text { /week }
\end{array}
$$

Total drums Int. Stor.

Req'd
38000

24350 $\begin{array}{ll}0.2 \mathrm{~m}^{3} & \text { Shipped } \\ \text { drums } & \text { /week }\end{array}$

Year Month generated

42 remain

Year Month generated

\begin{tabular}{|c|c|c|c|c|c|c|c|c|}
\hline 2010 & 11 & 633 & 18215797 & 2010 & 11 & 633 & 227.5 & 14204 \\
\hline 2010 & 12 & 633 & 18216248 & 2010 & 12 & 633 & 227.5 & 14610 \\
\hline 2011 & 1 & 633 & 18216699 & 2011 & 1 & 633 & 227.5 & 15016 \\
\hline 2011 & 2 & 633 & 18217151 & 2011 & 2 & 633 & 227.5 & 15422 \\
\hline 2011 & 3 & 633 & 18217602 & 2011 & 3 & 633 & 227.5 & 15828 \\
\hline 2011 & 4 & 633 & 18218053 & 2011 & 4 & 633 & 227.5 & 16233 \\
\hline 2011 & 5 & 633 & 18218505 & 2011 & 5 & 633 & 227.5 & 16639 \\
\hline 2011 & 6 & 633 & 18218956 & 2011 & 6 & 633 & 227.5 & 17045 \\
\hline 2011 & 7 & 633 & 18219407 & 2011 & 7 & 633 & 227.5 & 17451 \\
\hline 2011 & 8 & 633 & 18219859 & 2011 & 8 & 633 & 227.5 & 17857 \\
\hline 2011 & 9 & 633 & 18220310 & 2011 & 9 & 633 & 227.5 & 18263 \\
\hline 2011 & 10 & 633 & 18220761 & 2011 & 10 & 633 & 227.5 & 18668 \\
\hline 2011 & 11 & 633 & 18221213 & 2011 & 11 & 633 & 227.5 & 19074 \\
\hline 2011 & 12 & 633 & 18221664 & 2011 & 12 & 633 & 227.5 & 19480 \\
\hline 2012 & 1 & 633 & 18222115 & 2012 & 1 & 633 & 227.5 & 19886 \\
\hline 2012 & 2 & 633 & 18222567 & 2012 & 2 & 633 & 227.5 & 20292 \\
\hline 2012 & 3 & 633 & 18223018 & 2012 & 3 & 633 & 227.5 & 20698 \\
\hline 2012 & 4 & 633 & 18223469 & 2012 & 4 & 633 & 227.5 & 21103 \\
\hline 2012 & 5 & 633 & 18223921 & 2012 & 5 & 633 & 227.5 & 21509 \\
\hline 2012 & 6 & 633 & 18224372 & 2012 & 6 & 633 & 227.5 & 21915 \\
\hline 2012 & 7 & 633 & 18224823 & 2012 & 7 & 633 & 227.5 & 22321 \\
\hline 2012 & 8 & 633 & 18225275 & 2012 & 8 & 633 & 227.5 & 22727 \\
\hline 2012 & 9 & 633 & $182 \quad 25726$ & 2012 & 9 & 633 & 227.5 & 23133 \\
\hline 2012 & 10 & 633 & 18226177 & 2012 & 10 & 633 & 227.5 & 23538 \\
\hline 2012 & 11 & 633 & 18226629 & 2012 & 11 & 633 & 227.5 & 23944 \\
\hline 2012 & 12 & 633 & 18227080 & 2012 & 12 & 633 & 227.5 & 24350 \\
\hline 2013 & 1 & 0 & 18226898 & 2013 & 1 & 0 & 227.5 & 24123 \\
\hline 2013 & 2 & 0 & 18226716 & 2013 & 2 & 0 & 227.5 & 23895 \\
\hline 2013 & 3 & 0 & 18226534 & 2013 & 3 & 0 & 227.5 & 23668 \\
\hline 2013 & 4 & 0 & 18226352 & 2013 & 4 & 0 & 227.5 & 23440 \\
\hline 2013 & 5 & 0 & 18226170 & 2013 & 5 & 0 & 227.5 & 23213 \\
\hline 2013 & 6 & 0 & 18225988 & 2013 & 6 & 0 & 227.5 & 22985 \\
\hline 2013 & 7 & 0 & 18225806 & 2013 & 7 & 0 & 227.5 & 22758 \\
\hline 2013 & 8 & 0 & 18225624 & 2013 & 8 & 0 & 227.5 & 22530 \\
\hline 2013 & 9 & 0 & 18225442 & 2013 & 9 & 0 & 227.5 & 22303 \\
\hline 2013 & 10 & 0 & 18225260 & 2013 & 10 & 0 & 227.5 & 22075 \\
\hline 2013 & 11 & 0 & $182 \quad 25078$ & 2013 & 11 & 0 & 227.5 & 21848 \\
\hline 2013 & 12 & 0 & 18224896 & 2013 & 12 & 0 & 227.5 & 21620 \\
\hline 2014 & 1 & 0 & 18224714 & 2014 & & & 227.5 & \\
\hline
\end{tabular}

52.5 remain 
Total drums $\quad 38000$

Int. Stor. $\quad 27080$

Req'd

$$
\begin{array}{ll}
0.2 \mathrm{~m}^{3} & \text { Shipped } \\
\text { drums } & \text { /week }
\end{array}
$$

Year Month generated

\begin{tabular}{|c|c|c|c|c|c|c|c|c|c|}
\hline 2014 & 2 & 0 & 182 & 24532 & 2014 & 2 & 0 & 227.5 & 2116 \\
\hline 2014 & 3 & 0 & 182 & 24350 & 2014 & 3 & 0 & 227.5 & 2093 \\
\hline 2014 & 4 & 0 & 182 & 24168 & 2014 & 4 & 0 & 227.5 & 20710 \\
\hline 2014 & 5 & 0 & 182 & 23986 & 2014 & 5 & 0 & 227.5 & 2048 \\
\hline 2014 & 6 & 0 & 182 & 23804 & 2014 & 6 & 0 & 227.5 & $202 t$ \\
\hline 2014 & 7 & 0 & 182 & 23622 & 2014 & 7 & 0 & 227.5 & $200^{\circ}$ \\
\hline 2014 & 8 & 0 & 182 & 23440 & 2014 & 8 & 0 & 227.5 & 1980 \\
\hline 2014 & 9 & 0 & 182 & 23258 & 2014 & 9 & 0 & 227.5 & 1957 \\
\hline 2014 & 10 & 0 & 182 & 23076 & 2014 & 10 & 0 & 227.5 & 1934 \\
\hline 2014 & 11 & 0 & 182 & 22894 & 2014 & 11 & 0 & 227.5 & 1911 \\
\hline 2014 & 12 & 0 & 182 & 22712 & 2014 & 12 & 0 & 227.5 & 1885 \\
\hline 2015 & 1 & 0 & 182 & 22530 & 2015 & 1 & 0 & 227.5 & 186 \\
\hline 2015 & 2 & 0 & 182 & 22348 & 2015 & 2 & 0 & 227.5 & 1843 \\
\hline 2015 & 3 & 0 & 182 & 22166 & 2015 & 3 & 0 & 227.5 & 1820 \\
\hline 2015 & 4 & 0 & 182 & 21984 & 2015 & 4 & 0 & 227.5 & 1798 \\
\hline 2015 & 5 & 0 & 182 & 21802 & 2015 & 5 & 0 & 227.5 & 1775 \\
\hline 2015 & 6 & 0 & 182 & 21620 & 2015 & 6 & 0 & 227.5 & 175 \\
\hline 2015 & 7 & 0 & 182 & 21438 & 2015 & 7 & 0 & 227.5 & $172 s$ \\
\hline 2015 & 8 & 0 & 182 & 21256 & 2015 & 8 & 0 & 227.5 & 170 \\
\hline 2015 & 9 & 0 & 182 & 21074 & 2015 & 9 & 0 & 227.5 & 168 \\
\hline 2015 & 10 & 0 & 182 & 20892 & 2015 & 10 & 0 & 227.5 & $166^{\prime}$ \\
\hline 2015 & 11 & 0 & 182 & 20710 & 2015 & 11 & 0 & 227.5 & 163 \\
\hline 2015 & 12 & 0 & 182 & 20528 & 2015 & 12 & 0 & 227.5 & 1616 \\
\hline 2016 & 1 & 0 & 182 & 20346 & 2016 & 1 & 0 & 227.5 & 1593 \\
\hline 2016 & 2 & 0 & 182 & 20164 & 2016 & 2 & 0 & 227.5 & 1570 \\
\hline 2016 & 3 & 0 & 182 & 19982 & 2016 & 3 & 0 & 227.5 & 1547 \\
\hline 2016 & 4 & 0 & 182 & 19800 & 2016 & 4 & 0 & 227.5 & 152 \\
\hline 2016 & 5 & 0 & 182 & 19618 & 2016 & 5 & 0 & 227.5 & 1502 \\
\hline 2016 & 6 & 0 & 182 & 19436 & 2016 & 6 & 0 & 227.5 & $147 s$ \\
\hline 2016 & 7 & 0 & 182 & 19254 & 2016 & 7 & 0 & 227.5 & 1456 \\
\hline 2016 & 8 & 0 & 182 & 19072 & 2016 & 8 & 0 & 227.5 & 143 \\
\hline 2016 & 9 & 0 & 182 & 18890 & 2016 & 9 & 0 & 227.5 & 1411 \\
\hline 2016 & 10 & 0 & 182 & 18708 & 2016 & 10 & 0 & 227.5 & 138 \\
\hline 2016 & 11 & 0 & 182 & 18526 & 2016 & 11 & 0 & 227.5 & $136 t$ \\
\hline 2016 & 12 & 0 & 182 & 18344 & 2016 & 12 & 0 & 227.5 & 1343 \\
\hline 2017 & 1 & 0 & 182 & 18162 & 2017 & 1 & 0 & 227.5 & 1320 \\
\hline 2017 & 2 & 0 & 182 & 17980 & 2017 & 2 & 0 & 227.5 & 1297 \\
\hline 2017 & 3 & 0 & 182 & 17798 & 2017 & 3 & 0 & 227.5 & 1274 \\
\hline 2017 & 4 & 0 & 182 & 17616 & 2017 & 4 & 0 & 227.5 & 12520 \\
\hline
\end{tabular}

\begin{abstract}
42 remain
\end{abstract}
Total drums

38000

Int. Stor.

Req'd
24350

$0.2 \mathrm{~m}^{3} \quad$ Shipped

Year Month generated

52.5 remain 
Total drums $\quad 38000$

Int. Stor.

27080

Req'd

$$
\begin{array}{ll}
0.2 \mathrm{~m}^{3} & \text { Shipped } \\
\text { drums } & \text { /week }
\end{array}
$$

Year Month generated

\begin{tabular}{rr}
2017 & 5 \\
2017 & 6 \\
2017 & 7 \\
2017 & 8 \\
2017 & 9 \\
2017 & 10 \\
2017 & 11 \\
2017 & 12 \\
2018 & 1 \\
2018 & 2 \\
2018 & 3 \\
2018 & 4 \\
2018 & 5 \\
2018 & 6 \\
2018 & 7 \\
2018 & 8 \\
2018 & 9 \\
2018 & 10 \\
2018 & 11 \\
2018 & 12 \\
2019 & 1 \\
2019 & 2 \\
2019 & 3 \\
2019 & 4 \\
2019 & 5 \\
2019 & 6 \\
2019 & 7 \\
2019 & 8 \\
2019 & 9 \\
2019 & 10 \\
2019 & 11 \\
2019 & 12 \\
2020 & 1 \\
2020 & 2 \\
2020 & 3 \\
2020 & 4 \\
2020 & 5 \\
2020 & 6 \\
2020 & 7 \\
& \\
\hline
\end{tabular}

42 remain Year Month generated

38000

24350

Int. Stor.

Req'd
$0.2 \mathrm{~m}^{3} \quad$ Shipped

52.5 remain
$18217434 \quad 2017 \quad 5$

18217252

18217070

18216888

18216706

18216524

$182 \quad 16342$

18216160

18215978

18215796

18215614

18215432

18215250

18215068

18214886

$182 \quad 14704$

$182 \quad 14522$

18214340

18214158

18213976

18213794

18213612

18213430

18213248

18213066

18212884

$182 \quad 12702$

18212520

18212338

18212156

18211974

18211792

18211610

18211428

18211246

18211064

18210882

18210700

18210518

$2017 \quad 6$

20178

20179

$2017 \quad 10$

$2017 \quad 11$

$2017 \quad 12$

$2018 \quad 1$

$2018 \quad 2$

20183

20184

20185

$2018 \quad 6$

20187

$2018 \quad 8$

20189

$2018 \quad 10$

$2018 \quad 11$

$2018 \quad 12$

$2019 \quad 1$

20192

2019

2019

2019

2019

2019

2019

2019

2019

2019

2019

2020

2020

2020

2020

2020

2020

2020
5

6

8

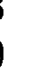

0

2

(n)

3

4

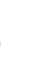

4
5

0 $227.5 \quad 12293$

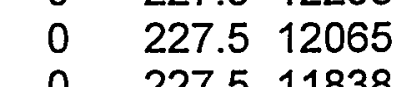

$\begin{array}{lll}0 & 227.5 & 11838\end{array}$

$0 \quad 227.511610$

$0 \quad 227.511383$

$0 \quad 227.511155$

$\begin{array}{lll}0 & 227.5 & 10928\end{array}$

$\begin{array}{lll}0 & 227.5 & 10700\end{array}$

$\begin{array}{lll}0 & 227.5 & 10473\end{array}$

$0 \quad 227.510245$

$0 \quad 227.510018$

$\begin{array}{lll}0 & 227.5 & 9790\end{array}$

$0 \quad 227.5 \quad 9562$

$0 \quad 227.5 \quad 9335$

$\begin{array}{lll}0 & 227.5 & 9107\end{array}$

$0 \quad 227.5 \quad 8880$

$0 \quad 227.5 \quad 8652$

$0 \quad 227.5 \quad 8425$

$\begin{array}{lll}0 & 227.5 & 8197\end{array}$

$\begin{array}{lll}0 & 227.5 & 7970\end{array}$

0 $227.5 \quad 7742$

0 $227.5 \quad 7515$

$0 \quad 227.5 \quad 7287$

$0 \quad 227.57060$

0 $227.5 \quad 6832$

$0 \quad 227.5 \quad 6605$

$0 \quad 227.5 \quad 6377$

$0 \quad 227.56150$

o $227.5 \quad 5922$

o $227.5 \quad 5695$

$\begin{array}{lll}0 & 227.5 & 5467\end{array}$

$0 \quad 227.5 \quad 5240$

$0 \quad 227.5 \quad 5012$

$0 \quad 227.5 \quad 4785$

$\begin{array}{lll}0 & 227.5 & 4557\end{array}$

$0 \quad 227.5 \quad 4330$

$\begin{array}{lll}0 & 227.5 & 4102\end{array}$

0 $227.5 \quad 3875$

$\begin{array}{lll}0 & 227.5 & 3647\end{array}$ 
Total drums $\quad 38000$ Int. Stor.

27080

Req'd

$$
\begin{array}{ll}
0.2 \mathrm{~m}^{3} & \text { Shipped } \\
\text { drums } & \text { /week }
\end{array}
$$

Year Month generated

$\begin{array}{rrrrr}2020 & 8 & 0 & 182 & 10336 \\ 2020 & 9 & 0 & 182 & 10154 \\ 2020 & 10 & 0 & 182 & 9972 \\ 2020 & 11 & 0 & 182 & 9790 \\ 2020 & 12 & 0 & 182 & 9608 \\ 2021 & 1 & 0 & 182 & 9426 \\ 2021 & 2 & 0 & 182 & 9244 \\ 2021 & 3 & 0 & 182 & 9062 \\ 2021 & 4 & 0 & 182 & 8880 \\ 2021 & 5 & 0 & 182 & 8698 \\ 2021 & 6 & 0 & 182 & 8516 \\ 2021 & 7 & 0 & 182 & 8334 \\ 2021 & 8 & 0 & 182 & 8152 \\ 2021 & 9 & 0 & 182 & 7970 \\ 2021 & 10 & 0 & 182 & 7788 \\ 2021 & 11 & 0 & 182 & 7606 \\ 2021 & 12 & 0 & 182 & 7424 \\ 2022 & 1 & 0 & 182 & 7242 \\ 2022 & 2 & 0 & 182 & 7060 \\ 2022 & 3 & 0 & 182 & 6878 \\ 2022 & 4 & 0 & 182 & 6696 \\ 2022 & 5 & 0 & 182 & 6514 \\ 2022 & 6 & 0 & 182 & 6332 \\ 2022 & 7 & 0 & 182 & 6150 \\ 2022 & 8 & 0 & 182 & 5968 \\ 2022 & 9 & 0 & 182 & 5786 \\ 2022 & 10 & 0 & 182 & 5604 \\ 2022 & 11 & 0 & 182 & 5422 \\ 2022 & 12 & 0 & 182 & 5240 \\ 2023 & 1 & 0 & 182 & 5058 \\ 2023 & 2 & 0 & 182 & 4876 \\ 2023 & 3 & 0 & 182 & 4694 \\ 2023 & 4 & 0 & 182 & 4512 \\ 2023 & 5 & 0 & 182 & 4330 \\ 2023 & 6 & 0 & 182 & 4148 \\ 2023 & 7 & 0 & 182 & 3966 \\ 2023 & 8 & 0 & 182 & 3784 \\ 2023 & 9 & 0 & 182 & 3602 \\ 2023 & 10 & 0 & 182 & 3420 \\ & & & & \end{array}$

Total drums $\quad 38000$ Int. Stor. 24350

Req'd

$$
\begin{array}{ll}
0.2 \mathrm{~m}^{3} & \text { Shipped } \\
\text { drums } & \text { /week }
\end{array}
$$

Year Month generated $\quad 52.5$ remain

$\begin{array}{rrrrr}2020 & 8 & 0 & 227.5 & 3420 \\ 2020 & 9 & 0 & 227.5 & 3192 \\ 2020 & 10 & 0 & 227.5 & 2965 \\ 2020 & 11 & 0 & 227.5 & 2737 \\ 2020 & 12 & 0 & 227.5 & 2510 \\ 2021 & 1 & 0 & 227.5 & 2282 \\ 2021 & 2 & 0 & 227.5 & 2055 \\ 2021 & 3 & 0 & 227.5 & 1827 \\ 2021 & 4 & 0 & 227.5 & 1600 \\ 2021 & 5 & 0 & 227.5 & 1372 \\ 2021 & 6 & 0 & 227.5 & 1145 \\ 2021 & 7 & 0 & 227.5 & 917 \\ 2021 & 8 & 0 & 227.5 & 690 \\ 2021 & 9 & 0 & 227.5 & 462 \\ 2021 & 10 & 0 & 227.5 & 235 \\ 2021 & 11 & 0 & 227.5 & 7 \\ 2021 & 12 & 0 & 227.5 & -220\end{array}$


Total drums $\quad 38000$

Int. Stor.

Req'd

$\begin{array}{ll}0.2 \mathrm{~m}^{3} & \text { Shipped } \\ \text { drums } & \text { /week }\end{array}$

Year Month generated
Total drums

Int. Stor.

Req'd
38000

24350

$\begin{array}{ll}0.2 \mathrm{~m}^{3} & \text { Shipped } \\ \text { drums } & \text { /week }\end{array}$

52.5 remain

$\begin{array}{rrrrr}2023 & 11 & 0 & 182 & 3238 \\ 2023 & 12 & 0 & 182 & 3056 \\ 2024 & 1 & 0 & 182 & 2874 \\ 2024 & 2 & 0 & 182 & 2692 \\ 2024 & 3 & 0 & 182 & 2510 \\ 2024 & 4 & 0 & 182 & 2328 \\ 2024 & 5 & 0 & 182 & 2146 \\ 2024 & 6 & 0 & 182 & 1964 \\ 2024 & 7 & 0 & 182 & 1782 \\ 2024 & 8 & 0 & 182 & 1600 \\ 2024 & 9 & 0 & 182 & 1418 \\ 2024 & 10 & 0 & 182 & 1236 \\ 2024 & 11 & 0 & 182 & 1054 \\ 2024 & 12 & 0 & 182 & 872 \\ 2025 & 1 & 0 & 182 & 690 \\ 2025 & 2 & 0 & 182 & 508 \\ 2025 & 3 & 0 & 182 & 326 \\ 2025 & 4 & 0 & 182 & 144 \\ 2025 & 5 & 0 & 182 & -38\end{array}$


Total drums $\quad 38000$

Int. Stor.

21620

Req'd

$$
\begin{array}{ll}
0.2 \mathrm{~m}^{3} & \text { Shipped } \\
\text { drums } & \text { /week }
\end{array}
$$

Year Month generated
63 remain
Total drums

Int. Stor.

Req'd
38000

16160

$\begin{array}{rrrrrrrrrr}2008 & 1 & 633 & 273 & 360 & 2008 & 1 & 633 & 364 & 269 \\ 2008 & 2 & 633 & 273 & 721 & 2008 & 2 & 633 & 364 & 539 \\ 2008 & 3 & 633 & 273 & 1081 & 2008 & 3 & 633 & 364 & 808 \\ 2008 & 4 & 633 & 273 & 1441 & 2008 & 4 & 633 & 364 & 1077 \\ 2008 & 5 & 633 & 273 & 1802 & 2008 & 5 & 633 & 364 & 1347 \\ 2008 & 6 & 633 & 273 & 2162 & 2008 & 6 & 633 & 364 & 1616 \\ 2008 & 7 & 633 & 273 & 2522 & 2008 & 7 & 633 & 364 & 1885 \\ 2008 & 8 & 633 & 273 & 2883 & 2008 & 8 & 633 & 364 & 2155 \\ 2008 & 9 & 633 & 273 & 3243 & 2008 & 9 & 633 & 364 & 2424 \\ 2008 & 10 & 633 & 273 & 3603 & 2008 & 10 & 633 & 364 & 2693 \\ 2008 & 11 & 633 & 273 & 3964 & 2008 & 11 & 633 & 364 & 2963 \\ 2008 & 12 & 633 & 273 & 4324 & 2008 & 12 & 633 & 364 & 3232 \\ 2009 & 1 & 633 & 273 & 4684 & 2009 & 1 & 633 & 364 & 3501 \\ 2009 & 2 & 633 & 273 & 5045 & 2009 & 2 & 633 & 364 & 3771 \\ 2009 & 3 & 633 & 273 & 5405 & 2009 & 3 & 633 & 364 & 4040 \\ 2009 & 4 & 633 & 273 & 5765 & 2009 & 4 & 633 & 364 & 4309 \\ 2009 & 5 & 633 & 273 & 6126 & 2009 & 5 & 633 & 364 & 4579 \\ 2009 & 6 & 633 & 273 & 6486 & 2009 & 6 & 633 & 364 & 4848 \\ 2009 & 7 & 633 & 273 & 6846 & 2009 & 7 & 633 & 364 & 5117 \\ 2009 & 8 & 633 & 273 & 7207 & 2009 & 8 & 633 & 364 & 5387 \\ 2009 & 9 & 633 & 273 & 7567 & 2009 & 9 & 633 & 364 & 5656 \\ 2009 & 10 & 633 & 273 & 7927 & 2009 & 10 & 633 & 364 & 5925 \\ 2009 & 11 & 633 & 273 & 8288 & 2009 & 11 & 633 & 364 & 6195 \\ 2009 & 12 & 633 & 273 & 8648 & 2009 & 12 & 633 & 364 & 6464 \\ 2010 & 1 & 633 & 273 & 9008 & 2010 & 1 & 633 & 364 & 6733 \\ 2010 & 2 & 633 & 273 & 9369 & 2010 & 2 & 633 & 364 & 7003 \\ 2010 & 3 & 633 & 273 & 9729 & 2010 & 3 & 633 & 364 & 7272 \\ 2010 & 4 & 633 & 273 & 10089 & 2010 & 4 & 633 & 364 & 7541 \\ 2010 & 5 & 633 & 273 & 10450 & 2010 & 5 & 633 & 364 & 7811 \\ 2010 & 6 & 633 & 273 & 10810 & 2010 & 6 & 633 & 364 & 8080 \\ 2010 & 7 & 633 & 273 & 11170 & 2010 & 7 & 633 & 364 & 8349 \\ 2010 & 8 & 633 & 273 & 11531 & 2010 & 8 & 633 & 364 & 8619 \\ 2010 & 9 & 633 & 273 & 11891 & 2010 & 9 & 633 & 364 & 8888 \\ 2010 & 10 & 633 & 273 & 12251 & 2010 & 10 & 633 & 364 & 9157 \\ 2010 & 11 & 633 & 273 & 12612 & 2010 & 11 & 633 & 364 & 9427 \\ 2010 & 12 & 633 & 273 & 12972 & 2010 & 12 & 633 & 364 & 9696 \\ 2011 & 1 & 633 & 273 & 13332 & 2011 & 1 & 633 & 364 & 9965 \\ 2011 & 2 & 633 & 273 & 13693 & 2011 & 2 & 633 & 364 & 10235 \\ & & & & & & & & & \end{array}$


Total drums $\quad 38000$ Int. Stor. $\quad 21620$

Req'd

$$
\begin{array}{ll}
0.2 \mathrm{~m}^{3} & \text { Shipped } \\
\text { drums } & \text { /week }
\end{array}
$$

Total drums Int. Stor.

Req'd
38000

16160

Year Month generated

63 remain

$\begin{array}{rrrrrrrrrr}2011 & 3 & 633 & 273 & 14053 & 2011 & 3 & 633 & 364 & 10504 \\ 2011 & 4 & 633 & 273 & 14413 & 2011 & 4 & 633 & 364 & 10773 \\ 2011 & 5 & 633 & 273 & 14774 & 2011 & 5 & 633 & 364 & 11043 \\ 2011 & 6 & 633 & 273 & 15134 & 2011 & 6 & 633 & 364 & 11312 \\ 2011 & 7 & 633 & 273 & 15494 & 2011 & 7 & 633 & 364 & 11581 \\ 2011 & 8 & 633 & 273 & 15855 & 2011 & 8 & 633 & 364 & 11851 \\ 2011 & 9 & 633 & 273 & 16215 & 2011 & 9 & 633 & 364 & 12120 \\ 2011 & 10 & 633 & 273 & 16575 & 2011 & 10 & 633 & 364 & 12389 \\ 2011 & 11 & 633 & 273 & 16936 & 2011 & 11 & 633 & 364 & 12659 \\ 2011 & 12 & 633 & 273 & 17296 & 2011 & 12 & 633 & 364 & 12928 \\ 2012 & 1 & 633 & 273 & 17656 & 2012 & 1 & 633 & 364 & 13197 \\ 2012 & 2 & 633 & 273 & 18017 & 2012 & 2 & 633 & 364 & 13467 \\ 2012 & 3 & 633 & 273 & 18377 & 2012 & 3 & 633 & 364 & 13736 \\ 2012 & 4 & 633 & 273 & 18737 & 2012 & 4 & 633 & 364 & 14005 \\ 2012 & 5 & 633 & 273 & 19098 & 2012 & 5 & 633 & 364 & 14275 \\ 2012 & 6 & 633 & 273 & 19458 & 2012 & 6 & 633 & 364 & 14544 \\ 2012 & 7 & 633 & 273 & 19818 & 2012 & 7 & 633 & 364 & 14813 \\ 2012 & 8 & 633 & 273 & 20179 & 2012 & 8 & 633 & 364 & 15083 \\ 2012 & 9 & 633 & 273 & 20539 & 2012 & 9 & 633 & 364 & 15352 \\ 2012 & 10 & 633 & 273 & 20899 & 2012 & 10 & 633 & 364 & 15621 \\ 2012 & 11 & 633 & 273 & 21260 & 2012 & 11 & 633 & 364 & 15891 \\ 2012 & 12 & 633 & 273 & 21620 & 2012 & 12 & 633 & 364 & 16160 \\ 2013 & 1 & 0 & 273 & 21347 & 2013 & 1 & 0 & 364 & 15796 \\ 2013 & 2 & 0 & 273 & 21074 & 2013 & 2 & 0 & 364 & 15432 \\ 2013 & 3 & 0 & 273 & 20801 & 2013 & 3 & 0 & 364 & 15068 \\ 2013 & 4 & 0 & 273 & 20528 & 2013 & 4 & 0 & 364 & 14704 \\ 2013 & 5 & 0 & 273 & 20255 & 2013 & 5 & 0 & 364 & 14340 \\ 2013 & 6 & 0 & 273 & 19982 & 2013 & 6 & 0 & 364 & 13976 \\ 2013 & 7 & 0 & 273 & 19709 & 2013 & 7 & 0 & 364 & 13612 \\ 2013 & 8 & 0 & 273 & 19436 & 2013 & 8 & 0 & 364 & 13248 \\ 2013 & 9 & 0 & 273 & 19163 & 2013 & 9 & 0 & 364 & 12884 \\ 2013 & 10 & 0 & 273 & 18890 & 2013 & 10 & 0 & 364 & 12520 \\ 2013 & 11 & 0 & 273 & 18617 & 2013 & 11 & 0 & 364 & 12156 \\ 2013 & 12 & 0 & 273 & 18344 & 2013 & 12 & 0 & 364 & 11792 \\ 2014 & 1 & 0 & 273 & 18071 & 2014 & 1 & 0 & 364 & 11428 \\ 2014 & 2 & 0 & 273 & 17798 & 2014 & 2 & 0 & 364 & 11064 \\ 2014 & 3 & 0 & 273 & 17525 & 2014 & 3 & 0 & 364 & 10700 \\ 2014 & 4 & 0 & 273 & 17252 & 2014 & 4 & 0 & 364 & 10336 \\ 2014 & 5 & 0 & 273 & 16979 & 2014 & 5 & 0 & 364 & 9972 \\ & & & & & & & & & \end{array}$


Total drums $\quad 38000$ Int. Stor.

21620

Req'd

$$
\begin{array}{ll}
0.2 \mathrm{~m}^{3} & \text { Shipped } \\
\text { drums } & \text { /week }
\end{array}
$$

Total drums Int. Stor.

Req'd
38000

16160

\section{$0.2 \mathrm{~m}^{3} \quad$ Shipped drums /week}

\begin{tabular}{|c|c|c|c|c|c|c|c|c|c|}
\hline 2014 & 6 & 0 & 273 & 16706 & 2014 & 6 & 0 & 364 & 9608 \\
\hline 2014 & 7 & 0 & 273 & 16433 & 2014 & 7 & 0 & 364 & 9244 \\
\hline 2014 & 8 & 0 & 273 & 16160 & 2014 & 8 & 0 & 364 & 8880 \\
\hline 2014 & 9 & 0 & 273 & 15887 & 2014 & 9 & 0 & 364 & 8516 \\
\hline 2014 & 10 & 0 & 273 & 15614 & 2014 & 10 & 0 & 364 & 8152 \\
\hline 2014 & 11 & 0 & 273 & 15341 & 2014 & 11 & 0 & 364 & 7788 \\
\hline 2014 & 12 & 0 & 273 & 15068 & 2014 & 12 & 0 & 364 & 7424 \\
\hline 2015 & 1 & 0 & 273 & 14795 & 2015 & 1 & 0 & 364 & 7060 \\
\hline 2015 & 2 & 0 & 273 & 14522 & 2015 & 2 & 0 & 364 & 6696 \\
\hline 2015 & 3 & 0 & 273 & 14249 & 2015 & 3 & 0 & 364 & 6332 \\
\hline 2015 & 4 & 0 & 273 & 13976 & 2015 & 4 & 0 & 364 & 5968 \\
\hline 2015 & 5 & 0 & 273 & 13703 & 2015 & 5 & 0 & 364 & 5604 \\
\hline 2015 & 6 & 0 & 273 & 13430 & 2015 & 6 & 0 & 364 & 5240 \\
\hline 2015 & 7 & 0 & 273 & 13157 & 2015 & 7 & 0 & 364 & 4876 \\
\hline 2015 & 8 & 0 & 273 & 12884 & 2015 & 8 & 0 & 364 & 4512 \\
\hline 2015 & 9 & 0 & 273 & 12611 & 2015 & 9 & 0 & 364 & 4148 \\
\hline 2015 & 10 & 0 & 273 & 12338 & 2015 & 10 & 0 & 364 & 3784 \\
\hline 2015 & 11 & 0 & 273 & 12065 & 2015 & 11 & 0 & 364 & 3420 \\
\hline 2015 & 12 & 0 & 273 & 11792 & 2015 & 12 & 0 & 364 & 3056 \\
\hline 2016 & 1 & 0 & 273 & 11519 & 2016 & 1 & 0 & 364 & 2692 \\
\hline 2016 & 2 & 0 & 273 & 11246 & 2016 & 2 & 0 & 364 & 2328 \\
\hline 2016 & 3 & 0 & 273 & 10973 & 2016 & 3 & 0 & 364 & 1964 \\
\hline 2016 & 4 & 0 & 273 & 10700 & 2016 & 4 & 0 & 364 & 1600 \\
\hline 2016 & 5 & 0 & 273 & 10427 & 2016 & 5 & 0 & 364 & 1236 \\
\hline 2016 & 6 & 0 & 273 & 10154 & 2016 & 6 & 0 & 364 & 872 \\
\hline 2016 & 7 & 0 & 273 & 9881 & 2016 & 7 & 0 & 364 & 508 \\
\hline 2016 & 8 & 0 & 273 & 9608 & 2016 & 8 & 0 & 364 & 144 \\
\hline 2016 & 9 & 0 & 273 & 9335 & 2016 & 9 & 0 & 364 & -220 \\
\hline 2016 & 10 & 0 & 273 & 9062 & & & & & \\
\hline 2016 & 11 & 0 & 273 & 8789 & & & & & \\
\hline 2016 & 12 & 0 & 273 & 8516 & & & & & \\
\hline 2017 & 1 & 0 & 273 & 8243 & & & & & \\
\hline 2017 & 2 & 0 & 273 & 7970 & & & & & \\
\hline 2017 & 3 & 0 & 273 & 7697 & & & & & \\
\hline 2017 & 4 & 0 & 273 & 7424 & & & & & \\
\hline 2017 & 5 & 0 & 273 & 7151 & & & & & \\
\hline 2017 & 6 & 0 & 273 & 6878 & & & & & \\
\hline 017 & 7 & 0 & 273 & 6605 & & & & & \\
\hline 017 & 8 & 0 & 273 & 6332 & & & & & \\
\hline
\end{tabular}

Year Month generated 63 remain Year Month generated 
Total drums $\quad 38000$

Int. Stor. $\quad 21620$

Req'd

$$
\begin{array}{ll}
0.2 \mathrm{~m}^{3} & \text { Shipped } \\
\text { drums } & \text { /week }
\end{array}
$$

Year Month generated

$\begin{array}{rrrrr}2017 & 9 & 0 & 273 & 6059 \\ 2017 & 10 & 0 & 273 & 5786 \\ 2017 & 11 & 0 & 273 & 5513 \\ 2017 & 12 & 0 & 273 & 5240 \\ 2018 & 1 & 0 & 273 & 4967 \\ 2018 & 2 & 0 & 273 & 4694 \\ 2018 & 3 & 0 & 273 & 4421 \\ 2018 & 4 & 0 & 273 & 4148 \\ 2018 & 5 & 0 & 273 & 3875 \\ 2018 & 6 & 0 & 273 & 3602 \\ 2018 & 7 & 0 & 273 & 3329 \\ 2018 & 8 & 0 & 273 & 3056 \\ 2018 & 9 & 0 & 273 & 2783 \\ 2018 & 10 & 0 & 273 & 2510 \\ 2018 & 11 & 0 & 273 & 2237 \\ 2018 & 12 & 0 & 273 & 1964 \\ 2019 & 1 & 0 & 273 & 1691 \\ 2019 & 2 & 0 & 273 & 1418 \\ 2019 & 3 & 0 & 273 & 1145 \\ 2019 & 4 & 0 & 273 & 872 \\ 2019 & 5 & 0 & 273 & 599 \\ 2019 & 6 & 0 & 273 & 326 \\ 2019 & 7 & 0 & 273 & 53 \\ 2019 & 8 & 0 & 273 & -220\end{array}$

Total drums $\quad 38000$

Int. Stor. $\quad 16160$

Req'd

$\begin{array}{ll}0.2 \mathrm{~m}^{3} & \text { Shipped } \\ \text { drums } & \text { /week }\end{array}$

84 remain 
Total drums $\quad 38000$

Int. Stor. $\quad 10700$

Req'd

$$
\begin{array}{ll}
0.2 \mathrm{~m}^{3} & \text { Shipped } \\
\text { drums } & \text { /week }
\end{array}
$$

Year Month generated
105 remain

$\begin{array}{rrrrrrrrrr}2008 & 1 & 633 & 455 & 178 & 2008 & 1 & 633 & 546 & 87 \\ 2008 & 2 & 633 & 455 & 357 & 2008 & 2 & 633 & 546 & 175 \\ 2008 & 3 & 633 & 455 & 535 & 2008 & 3 & 633 & 546 & 262 \\ 2008 & 4 & 633 & 455 & 713 & 2008 & 4 & 633 & 546 & 349 \\ 2008 & 5 & 633 & 455 & 892 & 2008 & 5 & 633 & 546 & 437 \\ 2008 & 6 & 633 & 455 & 1070 & 2008 & 6 & 633 & 546 & 524 \\ 2008 & 7 & 633 & 455 & 1248 & 2008 & 7 & 633 & 546 & 611 \\ 2008 & 8 & 633 & 455 & 1427 & 2008 & 8 & 633 & 546 & 699 \\ 2008 & 9 & 633 & 455 & 1605 & 2008 & 9 & 633 & 546 & 786 \\ 2008 & 10 & 633 & 455 & 1783 & 2008 & 10 & 633 & 546 & 873 \\ 2008 & 11 & 633 & 455 & 1962 & 2008 & 11 & 633 & 546 & 961 \\ 2008 & 12 & 633 & 455 & 2140 & 2008 & 12 & 633 & 546 & 1048 \\ 2009 & 1 & 633 & 455 & 2318 & 2009 & 1 & 633 & 546 & 1135 \\ 2009 & 2 & 633 & 455 & 2497 & 2009 & 2 & 633 & 546 & 1223 \\ 2009 & 3 & 633 & 455 & 2675 & 2009 & 3 & 633 & 546 & 1310 \\ 2009 & 4 & 633 & 455 & 2853 & 2009 & 4 & 633 & 546 & 1397 \\ 2009 & 5 & 633 & 455 & 3032 & 2009 & 5 & 633 & 546 & 1485 \\ 2009 & 6 & 633 & 455 & 3210 & 2009 & 6 & 633 & 546 & 1572 \\ 2009 & 7 & 633 & 455 & 3388 & 2009 & 7 & 633 & 546 & 1659 \\ 2009 & 8 & 633 & 455 & 3567 & 2009 & 8 & 633 & 546 & 1747 \\ 2009 & 9 & 633 & 455 & 3745 & 2009 & 9 & 633 & 546 & 1834 \\ 2009 & 10 & 633 & 455 & 3923 & 2009 & 10 & 633 & 546 & 1921 \\ 2009 & 11 & 633 & 455 & 4102 & 2009 & 11 & 633 & 546 & 2009 \\ 2009 & 12 & 633 & 455 & 4280 & 2009 & 12 & 633 & 546 & 2096 \\ 2010 & 1 & 633 & 455 & 4458 & 2010 & 1 & 633 & 546 & 2183 \\ 2010 & 2 & 633 & 455 & 4637 & 2010 & 2 & 633 & 546 & 2271 \\ 2010 & 3 & 633 & 455 & 4815 & 2010 & 3 & 633 & 546 & 2358 \\ 2010 & 4 & 633 & 455 & 4993 & 2010 & 4 & 633 & 546 & 2445 \\ 2010 & 5 & 633 & 455 & 5172 & 2010 & 5 & 633 & 546 & 2533 \\ 2010 & 6 & 633 & 455 & 5350 & 2010 & 6 & 633 & 546 & 2620 \\ 2010 & 7 & 633 & 455 & 5528 & 2010 & 7 & 633 & 546 & 2707 \\ 2010 & 8 & 633 & 455 & 5707 & 2010 & 8 & 633 & 546 & 2795 \\ 2010 & 9 & 633 & 455 & 5885 & 2010 & 9 & 633 & 546 & 2882 \\ 2010 & 10 & 633 & 455 & 6063 & 2010 & 10 & 633 & 546 & 2969 \\ 2010 & 11 & 633 & 455 & 6242 & 2010 & 11 & 633 & 546 & 3057 \\ 2010 & 12 & 633 & 455 & 6420 & 2010 & 12 & 633 & 546 & 3144 \\ 2011 & 1 & 633 & 455 & 6598 & 2011 & 1 & 633 & 546 & 3231 \\ 2011 & 2 & 633 & 455 & 6777 & 2011 & 2 & 633 & 546 & 3319\end{array}$

38000

5240

Req'd

$\begin{array}{ll}0.2 \mathrm{~m}^{3} & \text { Shipped } \\ \text { drums } & \text { /week }\end{array}$

126 remain 
Total drums $\quad 38000$ Int. Stor. Req'd

$$
\begin{array}{ll}
0.2 \mathrm{~m}^{3} & \text { Shipped } \\
\text { drums } & \text { /week }
\end{array}
$$

Total drums

Int. Stor.

Req'd
38000

5240 $\begin{array}{ll}0.2 \mathrm{~m}^{3} & \text { Shipped } \\ \text { drums } & \text { /week }\end{array}$

126 remain

$\begin{array}{rrrrrrrrrr}2011 & 3 & 633 & 455 & 6955 & 2011 & 3 & 633 & 546 & 3406 \\ 2011 & 4 & 633 & 455 & 7133 & 2011 & 4 & 633 & 546 & 3493 \\ 2011 & 5 & 633 & 455 & 7312 & 2011 & 5 & 633 & 546 & 3581 \\ 2011 & 6 & 633 & 455 & 7490 & 2011 & 6 & 633 & 546 & 3668 \\ 2011 & 7 & 633 & 455 & 7668 & 2011 & 7 & 633 & 546 & 3755 \\ 2011 & 8 & 633 & 455 & 7847 & 2011 & 8 & 633 & 546 & 3843 \\ 2011 & 9 & 633 & 455 & 8025 & 2011 & 9 & 633 & 546 & 3930 \\ 2011 & 10 & 633 & 455 & 8203 & 2011 & 10 & 633 & 546 & 4017 \\ 2011 & 11 & 633 & 455 & 8382 & 2011 & 11 & 633 & 546 & 4105 \\ 2011 & 12 & 633 & 455 & 8560 & 2011 & 12 & 633 & 546 & 4192 \\ 2012 & 1 & 633 & 455 & 8738 & 2012 & 1 & 633 & 546 & 4279 \\ 2012 & 2 & 633 & 455 & 8917 & 2012 & 2 & 633 & 546 & 4367 \\ 2012 & 3 & 633 & 455 & 9095 & 2012 & 3 & 633 & 546 & 4454 \\ 2012 & 4 & 633 & 455 & 9273 & 2012 & 4 & 633 & 546 & 4541 \\ 2012 & 5 & 633 & 455 & 9452 & 2012 & 5 & 633 & 546 & 4629 \\ 2012 & 6 & 633 & 455 & 9630 & 2012 & 6 & 633 & 546 & 4716 \\ 2012 & 7 & 633 & 455 & 9808 & 2012 & 7 & 633 & 546 & 4803 \\ 2012 & 8 & 633 & 455 & 9987 & 2012 & 8 & 633 & 546 & 4891 \\ 2012 & 9 & 633 & 455 & 10165 & 2012 & 9 & 633 & 546 & 4978 \\ 2012 & 10 & 633 & 455 & 10343 & 2012 & 10 & 633 & 546 & 5065 \\ 2012 & 11 & 633 & 455 & 10522 & 2012 & 11 & 633 & 546 & 5153 \\ 2012 & 12 & 633 & 455 & 10700 & 2012 & 12 & 633 & 546 & 5240 \\ 2013 & 1 & 0 & 455 & 10245 & 2013 & 1 & 0 & 546 & 4694 \\ 2013 & 2 & 0 & 455 & 9790 & 2013 & 2 & 0 & 546 & 4148 \\ 2013 & 3 & 0 & 455 & 9335 & 2013 & 3 & 0 & 546 & 3602 \\ 2013 & 4 & 0 & 455 & 8880 & 2013 & 4 & 0 & 546 & 3056 \\ 2013 & 5 & 0 & 455 & 8425 & 2013 & 5 & 0 & 546 & 2510 \\ 2013 & 6 & 0 & 455 & 7970 & 2013 & 6 & 0 & 546 & 1964 \\ 2013 & 7 & 0 & 455 & 7515 & 2013 & 7 & 0 & 546 & 1418 \\ 2013 & 8 & 0 & 455 & 7060 & 2013 & 8 & 0 & 546 & 872 \\ 2013 & 9 & 0 & 455 & 6605 & 2013 & 9 & 0 & 546 & 326 \\ 2013 & 10 & 0 & 455 & 6150 & 2013 & 10 & 0 & 546 & -220 \\ 2013 & 11 & 0 & 455 & 5695 & & & & & \\ 2013 & 12 & 0 & 455 & 5240 & & & & & \\ 2014 & 1 & 0 & 455 & 4785 & & & & & \\ 2014 & 2 & 0 & 455 & 4330 & & & & & \\ 2014 & 3 & 0 & 455 & 3875 & & & & & \\ 2014 & 4 & 0 & 455 & 3420 & & & & & \\ 2014 & 5 & 0 & 455 & 2965 & & & & & \end{array}$


Total drums $\quad 38000$ Int. Stor.

Req'd

$$
\begin{array}{ll}
0.2 \mathrm{~m}^{3} & \text { Shipped } \\
\text { drums } & \text { /week }
\end{array}
$$

Total drums

38000 Int. Stor.

Req'd 105 remain Year Month generated 126 remain

$\begin{array}{rrrrr}2014 & 6 & 0 & 455 & 2510 \\ 2014 & 7 & 0 & 455 & 2055 \\ 2014 & 8 & 0 & 455 & 1600 \\ 2014 & 9 & 0 & 455 & 1145 \\ 2014 & 10 & 0 & 455 & 690 \\ 2014 & 11 & 0 & 455 & 235 \\ 2014 & 12 & 0 & 455 & -220\end{array}$

Total drums

38000

Int. Stor.

0

Req'd

$$
\begin{array}{ll}
0.2 \mathrm{~m}^{3} & \text { Shipped } \\
\text { drums } & \text { /week }
\end{array}
$$

Year Month generated 147 remain

$\begin{array}{lllll}2008 & 1 & 633 & 637 & -4\end{array}$

Shipping faster than generating. 
Project File Number $\quad$ O2BM1

Project/Task CsIX/TRU Grout Feasibility Study

\section{Subtask D.5 Facility Design}

Title: Personnel Staffing and Space Requirements for CsIXTRU Grout Operations

\section{Summary:}

This EDF contains an estimate of the number of personnel, by discipline, required to staff the Cs/IX/TRU Grout operations, and space needs not part of the direct operations. Also included is a listing of the assumptions used as the basis for the estimate.

The CsIX/TRU Grout operations involves the processing of the SBW and NGLW contained in the INTEC tank farm into a CH TRU grouted waste form. The process includes the removal, via filtration, of undisolved solids for improved ion exchange efficiency, ion exchange removal of cesium, to reduce the final product to a radioactively contact handled level, and finally, mixing the resulting waste stream with a grout mixture to form a solid cement-like waste in 55-gallon drums. Waste drum production is assumed to be higher than the shipping rate, so interim storage capability will be required (EDF-CsIX-002 Interim Storage for $C H$ TRU Waste Drums). New facility space for drum videography (visual inspection of the head space area via camera) and head space gas analysis will be located within or near the interim storage facilities. These two activities and shipping activities at the interim storage location will be independent of the grouting operations.

Occupying space for personnel not directly related to the process operations is assumed to be available from the existing INTEC infrastructure. The project however, would still be required to fund the infrastructure required.

Conclusions: The total number of personnel is 97,71 of whom are considered radiation workers. Funding for personnel and the space they require, whether from the INTEC infrastrucure or new will be funded by the project. Occupying space for 23 individuals will be required in the new facilities.

Distribution (complete package): J. L. Clapp, MS 3650, R. E. Dafoe, MS 3765, S. A. Jensen, MS 3650, S. J. Losinski, MS 3625, C. W. Olsen, MS 3211, K. L. Williams, MS 3765, Project File, a copy will be included in report INEEL/INT-98-00869

Distribution (summary package only):

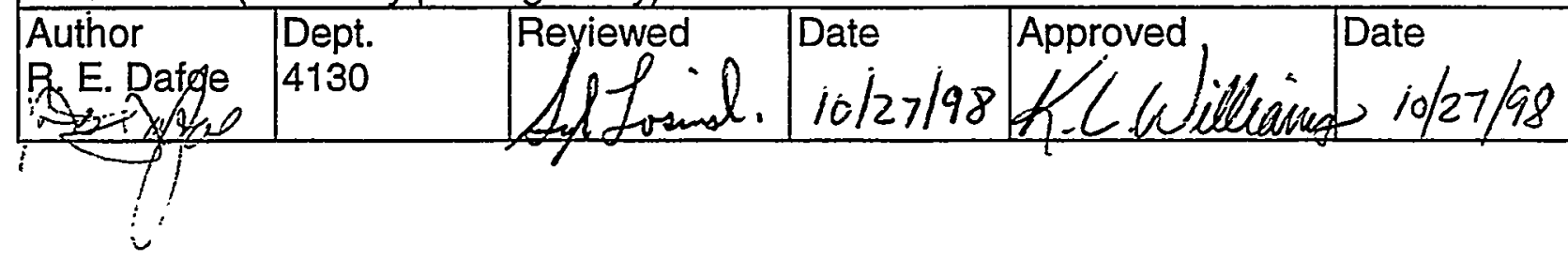




\section{PERSONNEL STAFFING ASSUMPTIONS/SUMMARY}

\section{Assumptions:}

- The CsIX/TRU grout processing facility will be in operation for approximately 5 years from January 2008 through December 2012.

- Shipment of CH TRU drums to WIPP is independent of the process, in that this activity will be performed from the interim storage facilities location, and is anticipated to occur from January 2008 through December 2021 (EDF-CSIX-002 Interim Storage for CH TRU Waste Drums).

- Process operations are based on twenty-four hours per day, 7 days a week, with up-time of 200 days per year. The 200 days is rounded from 205 and based on previously used HLW program drivers stating that operations will be based on a $75 \%$ up-time at $75 \%$ efficiency $(0.75 \times 0.75 \times 365=205)$.

- Four rotating shifts of personnel will be required to support the schedule for main process and drum output handling/transfer to storage. Included in the 4 rotating shifts are personnel for process maintenance, QA, Safety, and Rad Con.

- New facility space will be required for final drum analysis to meet WIPP requirements. This space will be for videography (visual inspection of the head space area via camera) and head space gas analysis activities.

- Activities related to drum videography, drum head space gas analysis, and CH TRU drum shipments to WIPP occur only during the day shift.

- All personnel listed will be included for estimating Life Cycle Costs.

- Office space is available in existing nearby facilities, therefore new administrative facility space is not included in the Study. Personnel not housed in the process facility include the facility administration, engineering support, and training staffs. Operating costs for the facilities they use are a cost incurred by the project.

- A single day shift will include personnel for administration, administrative support, technical support, general building maintenance, and activities related to drum videography, drum head space gas analysis, and CH TRU drum shipments to WIPP.

- Management for support functions - QA, Safety, RadCon, Training, Engineering, Drafting, and Maintenance - is supplied from the existing INTEC infrastructure. Proportional costs for this management and their space are a cost incurred by the project.

- Ancillary and support functions critical to the process will be housed within the same physical facility.

- INTEC will have infrastructure services and capabilities to accommodate some tasks not unique to this project such as additional engineering and drafting support, common warehouse and storage, and major support areas such as machine, weld, sheetmetal, fitter, electrician, and electronics/instrument shops, common warehouse and storage, mock-up area(s), and laydown area(s).

- No special personnel requirements are required for the interim storage facilities that can't be handled by day shift and rotating shift personnel.

- Space needs are based on an average $150 \mathrm{ft}^{2}$ allowance for office personnel and $500 \mathrm{ft}^{2}$ for a single shift of operations personnel. The office space allowance is slightly above the current $130 \mathrm{ft}^{2}$ used at INTEC to be conservative, considering the space is related to an operating facility and not typical office space. 


\section{Personnel Summary:}

Total estimated number of personnel is 97,71 of whom are considered radiation workers. New office space for 9 and operating space for 14 individuals is required. The operating space for 14 individuals includes 3 considered as field type office space for the shippers, gas analyzers, and radiography technicians. The estimates are based on the entire CsIX/TRU process being housed in all new facilities. For the process scenario which uses the NWCF, new facility personnel space can be reduced by what ever NWCF space could be obtained. Per the assumptions, new space for all personnel will not be required in the new facility. The New Facility Office Space and New Facility Ops/Maint Space personnel numbers, listed below, take into account shift personnel sharing common space. This data will be used in determining new facility space needs, life cycle cost analysis, and as input to the Project Data Sheet.

\begin{tabular}{|c|c|c|c|c|c|}
\hline & Day & Rotating & Radiation & New Facility & New Fa \\
\hline & Shift & Shifts (4) & Workers & Office Space & $\mathrm{Ops} / \mathrm{M}$ \\
\hline \multicolumn{6}{|l|}{ Space } \\
\hline Facility Admin & 6 & 0 & 0 & 0 & 0 \\
\hline Engineering Support & 3 & 0 & 0 & 0 & 0 \\
\hline Training & 2 & 0 & 0 & 0 & 0 \\
\hline Operations Admin & 3 & 8 & 0 & 3 & 0 \\
\hline Process Operators & 10 & 32 & 42 & 2 & 11 \\
\hline QA, Safety, RadCon & 2 & 12 & 14 & 3 & 0 \\
\hline Process Maintenance & 0 & 12 & 12 & 0 & 3 \\
\hline Facility Maintenance & 7 & 0 & 3 & 1 & 0 \\
\hline Total & 33 & 64 & 71 & 9 & 14 \\
\hline
\end{tabular}

\section{ADMINISTRATION AND SUPPORT REQUIREMENTS}

\section{Admin/Admin Support}

- Operations Managers

- Administrative Support

Secretarial/Clerical, Budget/Cost Control, Schedule

Physical requirements

File/Document Storage, Office Machines

- Waste Tracking/Data Acquisition Tech

(6)

Dav Shift

(Receipt, Entry, Examination, Sampling, Repack,

Storage/Shipping, etc., Manifest, Chain of Custody)

Physical requirements

File/Document Storage, Office Machines

- Document control personnel

Physical requirements

File/Document Storage Fire Vault, Library, Office Machines
Technical Support

(3)

Dav Shift

- Engineers

- Drafters 
Training

(2)

Dav Shift

- Trainers

Phvsical requirements

- Classroom(s)

- Storage for files/documents, training aids, audio/visual, etc.

\section{Admin Facility Maintenance}

(1)

Dav Shift

Janitor (admin areas)

Common Ancillary Space (Phvsical requirements onlv)

- Small Lunch Room

- Toilet Rooms

- Janitor Room

- Storage

- Circulation

- Electrical

- Communications

- Mechanical

DOE Facility Office(s) - (Physical requirements only)

- None

\section{PROCESS FACILITY/OPERATIONS}

Operations Administration

(11)

Dar Shift Rotating

Shift Supervisors

Operations Technicians

24

Process coordinator

$0 \quad 4$

Process Operators

(42)

Day Shift Rotating

- Material receipt

- SBW/NGLW retrieval from tank farm to feed tanks

- Feed sampling and related activities

- Raw material receipt - cement, slag, flyash, drums, etc.

- Solids and Cs removal processes

- Grouting processes

- Material handling

- Empty drum feed to process

- Full drum movement to lag and interim storage

- Shipping from interim storage (loading drums into 
TRUPACK II and prepping trucks)

- Filled drum videography

- Drum head space gas analysis

- QA

- Safety

14

- RadCon

04

Physical requirements

- Equipment storage (surveillance, analyzers, etc)

Process Maintenance

Electrical

Mechanical

I\&C
(12) Dav Shift Rotating

Process Facility Maintenance

(6) Day Shift Rotating

Electrical

Mechanical

I\&C Technicians

Maintenance Planner/PMs/Work Orders

Attendant

Janitor
$0 \quad 4$

$0 \quad 4$

$0 \quad 4$

\section{Process Facility Support (Phvsical Requirements onlv)}

- Anti-C Issue Rooms

- Change Rooms/Shower Rooms

- Personnel Decon Area

- Ready Room (Break Room)

- Toilet Rooms and Janitor Room

- Emergency Equipment Room

- Mechanical, Electrical and Communications

- Mechanical Shops - Minimal space for minor work. Most shop work done at INTEC common shops

- Instrument Shop - Minimal space for protected storage for calibrated instruments and minor work. Most shop work done at INTECV common shops

- Contaminated, Hot Maintenance Area

- Drum Handling Systems (i.e., carts) Survey, Decon and Maintenance areas

- Tool Room - Storage of Issue Tools

- Long Term Storage - Clean Containers

- Short Term Storage - Lag/Lead for drums 
Project File Number $\quad 02 \mathrm{BM} 1$

Project/Task CsIX/TRU Grout Feasibility Study

Subtask E. Studies

Title: SBW Treatment - Ion Exchange Media Disposition Concepts

Summary:

The attached report provides information on requirements and options for disposal of spent ion exchange media from treating Sodium Bearing Waste (SBW). Example concepts are also provided for intermediate handling, storage, and related process steps, and for fitting the process into NWCF. This is provided for information and is subject to review, iteration, and modification based on results of other parts of the study. Final process and equipment concepts in the CsIX/TRU Grout Feasibility Study are therefore likely to differ from examples described in the draft report.

Distribution (complete package):

Distribution (summary package only):

\begin{tabular}{|c|c|c|c|c|c|}
\hline $\begin{array}{l}\text { Author } \\
\text { G.E. Stegen }\end{array}$ & $\begin{array}{l}\text { Dept. } \\
\text { Cogema }\end{array}$ & Reyiewed 0 & $10 / 5 / 98$ & Approved & {$\left[\begin{array}{l}\text { Date } \\
\text { ic/5/ag }\end{array}\right.$} \\
\hline
\end{tabular}

See Management Control Procedure (MCP) 6 for instructions on use of this form. 


\title{
SBW TREATMENT \\ ION EXCHANGE MEDIA DISPOSITION
}

Gary E. Stegen

September 1998

Prepared for Lockheed Martin Idaho Technologies Company

Under Subcontract C95-175006

\author{
COGEMA Engineering Corporation
}

P.O. Box 840

Richland, Washington 99352

54-3000-101 (9/59) \{EF\} GEF014 


\section{TABLE OF CONTENTS}

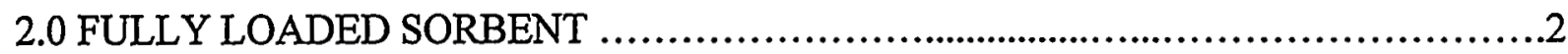

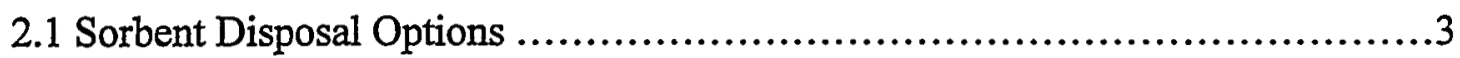

2.2 Spent Sorbent Intermediate Handling.........................................4

3.0 SORBENT STORAGE TANK OPTION.........................................

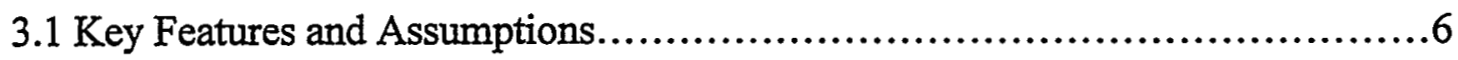

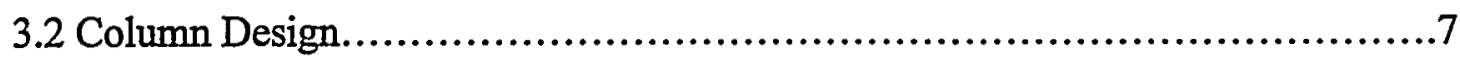

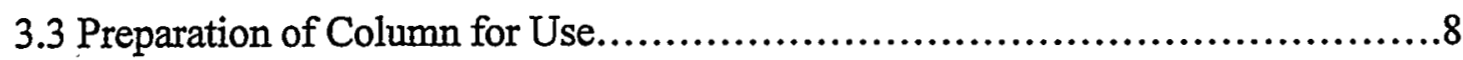

3.4 Removal and Storage of Spent Sorbent........................................ 8

3.5 NWCF Installation and Operating Concepts.................................9

4.0 FULLY LOADED SORBENT, MODULAR COLUMN...............................10

4.1 MCWP Features and Assumptions..............................................10

4.2 Modular Column/Waste Package................................................11

4.3 Preparation of Column for Use...................................................11

4.4 Preparation of Spent Sorbent Packages........................................12

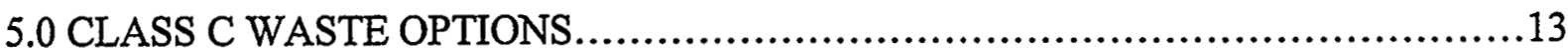

5.1 Disposal Requirements...................................................13

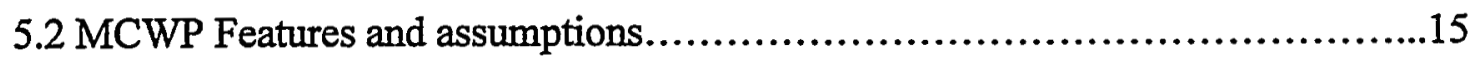

5.3 Modular Column/Waste Package...............................................16

5.4 MCWP Operations............................................................. 17

5.5 Sluice Out Ion Exchange Media to Transportation Package........................18

5.6 Filtration Requirements for Class C Options...................................18

5.7 Discussion - Class C Options....................................................19

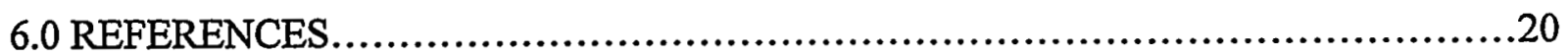

ATTACHMENT-SKETCHES AND BACKUP CALCULATIONS 
54-3000-101 (9/59) \{EF\} GEF014

DSI 


\title{
SBW TREATMENT, DISPOSITION OF SPENT ION EXCHANGE MEDIA
}

\author{
G. E. STEGEN \\ September, 1998
}

\subsection{INTRODUCTION}

Lockheed Martin Idaho Technologies Company (LMTCO) has initiated an engineering study to evaluate treatment options for sodium-bearing waste (SBW). Options currently under evaluation are based on partial decontamination of the SBW in order to produce contact handled TRU as the primary bulk waste form. Processes to be considered include cesium removal by ion exchange, filtration to remove suspended solids; and concentration, solidification, and packaging of the resulting partially decontaminated liquid waste. The process generates spent ion exchange media heavily loaded with radio-cesium (principally ${ }^{137} \mathrm{Cs}$ ). This paper provides a summary of requirements and concepts for disposal and intermediate handling of the resulting spent ion exchange media. Example design concepts for related systems components, comments on design considerations, and comments on ways the concepts might be implemented in existing NWCF cells are also provided. This is provided as input to the overall LMITCO study, which will include additional evaluation and comparison with other ideas before an actual implementation concept is recommended. Example concepts mentioned herein may therefore differ from the final study concept developed by LMITCO.

Four primary waste type and facility concepts are being considered in the study.

1. Production of a Class $\mathrm{C}$ waste form for disposal in a near surface low level waste disposal facility, with filtration and ion exchange performed in NWCF.

2. Ion exchange media waste form loaded to the maximum equilibrium cesium content (above Class C), and stored for future processing and disposal. Filtration and ion exchange is performed in NWCF.

3. Production of a Class $\mathrm{C}$ waste form for disposal in a near-surface, low-level waste disposal facility; with filtration and ion exchange performed in a new facility.

4. Ion exchange media waste form loaded to the maximum equilibrium cesium content (above Class $\mathrm{C}$ ), and stored for future processing and disposal. Filtration and ion exchange is performed in a new facility. 
As discussed in Section 4, Class C options have significant advantages in terms of disposal, however, ability to reliably produce a non-TRU sorbent that meets hazardous waste disposal requirements is uncertain. Significant additional work is required to develop and adequately evaluate this option. To limit the study effort and still produce a design concept with a high probability of working, a decision was made to limit process and facility concept development to fully loaded sorbent options ( 2 and 4 ). Additional evaluation of Class $C$ options may be considered in the future.

In all cases, it is assumed that a non-regenerable ion exchange media (or "sorbent") referred to as crystalline silicotitanate or "CST" is used for removal of cesium. Reference 1 provides an extensive summary of the development history and properties of this sorbent. Reference 2 provides results of initial tests of this sorbent with INEEL sodium bearing waste. Reference 3 provides results of a demonstration program in which about 30,000 gallons of radioactive tank waste were processed with this sorbent. While CST is considered a suitable and desirable sorbent, other sorbents may prove to be even better, and selection of CST should therefore be considered as only a study basis rather than a final selection.

The following sections discuss spent sorbent disposal and intermediate disposition methods. Examples discuss the NWCF retrofit cases; however, most of the information is also applicable to new facility cases.

\subsection{FULLY LOADED SORBENT}

For fully loaded sorbent options, cesium content is not limited to Class C. Cesium loading may conceivably increase to near chemical equilibrium with inlet feed, unless a reason is identified to stop loading earlier. Two options may be considered for TRU components: a) Allow the final spent sorbent to be contaminated above the TRU limit (100 nCi $/ \mathrm{g})$; or, b) Attempt to maintain spent sorbent as a non-TRU waste. As discussed in Section 5, the main differences for the nonTRU option are that suspended solids need to be efficiently removed, and absorbed TRU must be stripped from the spent sorbent. Implementation of the non-TRU option increases final disposal options but is likely to increase complexity and initial cost of processing the SBW.

Sorbent disposal and intermediate handling options are discussed in Sections 2.1 and 2.2, but a final disposition option recommendation and complete system concept for final disposition of the spent sorbent are not provided in the current study. Rather, two interim storage concepts for spent sorbent are provided. In the first "storage tank" option, sorbent is sluiced from fixed ion exchange columns into interim storage tanks. In the second "modular column" option, a modular single-use column concept is described. Both of these concepts are compatible with reasonably likely final disposal options. It is assumed that the final decision on disposition method will not be made for some time, conceivably after the sorbent is placed in interim storage. 


\subsection{Sorbent Disposal Options}

A significant number of intermediate and final sorbent disposition options may be considered. Primary ultimate disposition options include the following.

1. Co-processing in the Waste Treatment Facility (WTF) at INEEL. Spent sorbent is incorporated into the same waste form as other INEEL high level waste (HLW). The sorbent could be blended with other HLW feeds to the immobilization process or the sorbent could be immobilized separately using the same equipment to be used for $\mathrm{HLW}$. Immobilized sorbent would then be handled, stored, and disposed along with the other wastes.

2. Co-processing at another DOE site. Incorporate spent sorbent into immobilized high level waste produced at a different DOE site (Savannah River or Hanford). Sorbent is integral with, and disposed with other HLW at a Federal repository.

3. Package and directly dispose sorbent at a Federal repository without additional treatment.

4. Package and directly dispose at a Federal repository after heat treatment.

5. Package and directly dispose sorbent as greater than Class $\mathrm{C}$ low-level waste.

6. Store until decayed below Class $\mathrm{C}$. Then dispose as Class $\mathrm{C}$ waste. This is expected to require storage for roughly 60 years, possibly more.

7. Dispose (entomb) in the NWCF when it is closed.

\subsubsection{Discussion - Sorbent Disposal}

All of the listed options could be considered as potentially viable. If the waste treatment facility for immobilization and packaging of the HLW calcine is built at INEEL on a compatible schedule, the option of incorporating spent sorbent into the immobilized waste product appears reasonably feasible. Previous work has shown that spent CST sorbent is compatible with high level waste vitrification ${ }^{5}$. It can be blended with other HLW melter feeds, or it can be vitrified separately with a relatively high achievable waste loading ${ }^{5}$. Blending and vitrification along with high level waste eliminates the need to limit TRU level and also eliminates new disposal related hazardous waste issues. The previous WTF feasibility study already included blending of spent ion exchange media into $H L W$ melter feed, and this appears to be reasonably practical. Evaluation of CST vitrification is in progress at other DOE sites. Previous work has also shown 
that simply heating the CST will produce a very durable waste form ${ }^{6}$. While not directly tested, it also appears likely that blending of CST with waste feed would also be compatible with the direct cementitious and HIP waste form options. Shipment to an offsite plant is also likely to be feasible. However, increased cost, risk, inefficiencies, and institutional/political difficulties makes this less attractive than onsite co-processing unless the INEEL WTF is substantially delayed, there is a need to remove the sorbent from the NWCF, and transfer to the binsets is for some reason prohibited. The option of direct disposal in the repository could also be pursued; however, a significant effort would be required to attempt to get approval of this new waste type.

Options 5, 6, and 7 presume a non-TRU waste and therefore require efficient filtration and methods that assure TRU is not retained on the sorbent. There is a reasonable probability that Nevada Test Site and/or Hanford will be able to accept greater than Class C low-level waste (non-TRU) in the future. If so, this could prove to be an attractive disposal option. As discussed in Section 5 (Class C option), the ability to reliably produce a non-TRU product is uncertain, and at a minimum will require a more complex process system. Therefore, a decision was made to defer development of a design concept for the non-TRU option in the current study in order to focus available time and resources on the other options that appear to be lower risk.

\subsubsection{Conclusions}

Co-processing with HIW calcine at INEEL (Option 1d) and disposal as low level waste (options 5,6 , and 7), are most likely to be the preferred options. Ability to reliably produce a non-TRU product is uncertain, and additional work is needed to define a process and equipment concepts for the low-level waste options.

\subsection{Spent Sorbent Intermediate Handling}

There are several options for intermediate handling and storage of the spent sorbent. Options that have been identified are briefly described below. All assume that interim storage is as a dry retrievable solid.

1. Retrieve dry spent sorbent using a pneumatic/vacuum line and transfer to the existing bin-sets using existing pneumatic transfer systems. The sorbent is later retrieved from the binsets and processed along with the stored calcine when the waste treatment facility is implemented. The WTF calcine processing system will be designed to accommodate sorbent mixed in with calcine.

2. Retrieve dry spent sorbent using a pneumatic/vacuum line and transfer out of the NWCF using portions of the existing pneumatic transfer system. However, the sorbent is not delivered to the bin sets. Rather, the pneumatic transfer lines are modified by the WTF project to deliver the sorbent directly to the WTF where it is processed for disposal. 
3. Retrieve dry spent sorbent from the storage container using a pneumatic/vacuum line and transfer into a package used for shipment.

4. Entomb spent sorbent in the NWCF when it is closed. Either leave spent sorbent in interim storage containers, or retrieve and transfer to engineered, qualified containment/disposal packages placed in NWCF.

5. Use a modular column waste package concept in which spent sorbent would remain during intermediate storage, handling, and transportation (if used).

\subsubsection{Discussion - Intermediate Handling}

If the sorbent is water washed and dried at moderate temperature, it is expected to be inert and relatively easy to resuspend for pneumatic conveying. Sorbent bulk density $(1 \mathrm{~g} / \mathrm{cc})$ is a little lower than calcine $(1.2$ to $1.6 \mathrm{~g} / \mathrm{cc}$ ). Typical sorbent particle size is in the range of 60 to 30 mesh, ${ }^{1}$ which is slightly larger than typical calcine. The existing pneumatic transfer system is likely capable of transferring the $-30 /+60$ mesh sorbent, however, a slightly smaller sorbent size could be specified if needed to facilitate pneumatic transfer. Some limited testing could verify resuspension and transfer of spent sorbent.

If on-site co-processing in the WTF is the selected disposition method, a decision to either keep the sorbent segregated, or to mix it with calcine can be made during conceptual design of the WTF. Either should be practical from a process and equipment point of view, and the choice requires optimization work which cannot be performed until other portions of the WTF conceptual design are defined. With the 1997 WTF FS process design, sorbent mixed with calcine would not likely be dissolved and would therefore be mixed with undissolved solids from calcine dissolvers. In the FS design, spent sorbent sluiced from ion exchange was blended with undissolved calcine solids prior to interim storage, followed by transfer to vitrification. Thus, the end point for sorbent mixed with calcine is the same as in the FS, and impacts from blending with the calcine appear minimal. In the future WTF design, process flowsheets may change. However, if the designers know the calcine will contain some ion exchange sorbent, it should not be a substantial additional complication to accommodate it in the process design. If necessary, a digestion step with HF or concentrated caustic could be considered to partially dissolve the sorbent.

Because the WTF will already be using pneumatic transfer of calcine and the NWCF is equipped with pneumatic transfer capabilities, it seems certain that pneumatic transfer out of the NWCF will be preferred over package transfer for the on-site co-processing option. Slurry transfer might also be considered during the WTF conceptual design, depending on compatibility with 
the balance of the WTF process and facility design. The suggested spent sorbent storage concept should also be compatible with slurry transfer. However, the spent sorbent drying step could likely be avoided if it is known that slurry transfer is to be used.

The option of entombing spent sorbent in the NWCF when it is closed would presumably require that the sorbent be non-TRU. If the sorbent is non-TRU, it seems reasonable that placement of the sorbent in qualified 300+ year disposal containers which are entombed in concrete inside the NWCF, should provide an acceptable, low-risk, and possibly low-cost disposal option. However, increased complexity required to assure the sorbent is non-TRU, regulatory requirements, and licensing costs could offset facility and disposal cost savings. A more complete analysis of regulatory requirements, compliance strategy, compliance costs, and probability of success is needed to evaluate benefits of pursuing this option.

The option for offsite disposal as greater than class $\mathrm{C}$ waste is also viable provided feasibility of producing a non-TRU, non-hazardous sorbent product is verified. Efforts are ongoing to implement disposal of greater than Class $C$ low-level waste which will presumably eventually be successful. To keep these options open, it is necessary to assure the spent sorbent is non-TRU.

\subsection{Sorbent Storage Tank Option}

This section discusses an interim storage option for spent ion exchange media that would use storage tanks.

\subsection{Key Features and Assumptions}

The ion exchange system and spent media interim storage concept includes the following key features and assumptions.

1. Three, or possibly four ion exchange columns are provided to allow one to be off-line for bed replacement while a minimum of two columns in series continue to operate.

2. When cesium capacity of the first column in series is expended, the feed is replaced with flushes of dilute acid followed by water. Spent sorbent is then sluiced from the bed using a recirculating water stream. Sorbent slurry flows to a storage tank where sorbent particles settle out and water is recirculated until spent sorbent is completely flushed from the column.

3. In order to keep the system simple and reliable, it is assumed that no attempt is made to certify spent sorbent as non-TRU. If carryover of solids through the ion exchange beds 
and downstream processes proves acceptable, it could be feasible to get by with a simple system with little or no filtration except for that provided by the ion exchange bed. Possible plugging of the sorbent bed and impacts to downstream processing of the treated liquid (such as increased dose rate from SBW suspended solids and ion exchange media fines carryover) need to be evaluated prior to implementing a simple system without filtration. Certification that the sorbent is Non-TRU could be considered, but would face difficulties discussed for the Class $\mathrm{C}$ option in Section 5.

4. When a spent sorbent storage tank is filled, the sorbent is dried by draining free water followed by blowing or drawing warm air through the sorbent. Supplemental heating of the outside of the tanks may also be needed (such as a steam coil or jacket).

5. No further treatment is provided beyond dilute acid and water flushes of the spent sorbent bed followed by drying in the storage tank.

\subsection{Column Design}

The example column concept consists of a stainless steel canister with outside diameter of about 2 feet and a total height of about 6 feet [these dimensions are preliminary, and may be adjusted if needed]. All penetrations are made through a flange welded to the top of the canister. Upflow or downflow designs can be considered. For upflow, the inlet pipe passes down through the bed of ion exchange media to the distributor screens at the bottom. Feed material flows up through the bed, through media retainer screens, and out through a penetration in the top flange. Auxiliary penetrations are provided for filling the column with ion exchange media, sluicing out spent sorbent, draining the liquid, instrumentation, and spares. The column concept is similar to the modular column/waste package described in Section 4.2, except a closure plate and sealing ring is not required and one or two sluice out pipes are provided which penetrate through the screens into the bed area.

Assuming 2 foot outside diameter, $3 / 8$ inch wall thickness, and a net loaded bed height of $5 \mathrm{ft}$, sorbent volume is calculated at 14.7 cubic feet or 0.42 cubic meters per bed load. Based on a nominal 1 gallon per minute feed rate, the lead column bed will need to be changed out after every three to four weeks of continuous operation. The suggested column size provides a reasonable bed change-out frequency, and a relatively low ratio of flow rate to bed volume, which is expected to provide a high decontamination factor for cesium. (based on one gallon per minute feed and two columns in series with 14.7 cubic feet of bed in each, the ratio is 0.27 bed volumes per hour). 


\subsection{Preparation of Column for Use}

After emptying spent sorbent, the column is prepared for reuse. It is filled with sorbent, fines are flushed out with water, and the sorbent is preconditioned if needed. The sluice system valves are closed, and the column may be reconnected to the ion exchange system when desired.

\subsection{Removal and Storage of Spent Sorbent}

Sorbent is removed from the bed by increasing upflow of water until the bed begins to expand or fluidize, and then drawing the sorbent slurry out through one or more dip tubes located in the bed. Some of the finer sorbent particles are also elutriated in the bulk fluid. The streams from the column flow to the storage tank where solids settle, while decanted water is passed through a strainer and is recirculated for sluicing additional sorbent. Sorbent from multiple batches is transferred to the same tank, where solids continually build up. Sluice water is left in the tank until the tank is filled with sorbent. Liquid is then drained and the sorbent is dried. After drying, sorbent may be stored in the tank indefinitely. Any surplus sluicing water is filtered and transferred to the ion exchange feed tank.

If solids removed from the SBW are to be packaged and loaded out separately for disposal, care must be taken to avoid transfer of ion exchange media to the main undissolved solids filtration system. Ion exchange media transferred to the upstream solids filtration system would come to equilibrium with cesium content of the raw feed. Bulk ion exchange media loaded to equilibrium with SBW is estimated to have a contact dose rate on the order of $10,000 \mathrm{R} / \mathrm{hr}$. Inclusion of even a small fraction of ion exchange media with the solids could substantially increase their dose rate making removal and handling of the undissolved solids more difficult than expected. Ion exchange media carried downstream could also cause operational problems. This media will come to equilibrium with product cesium concentrations. If uniformly dispersed, this may not be a major problem, however if ion exchange media collects in low points, tank bottoms etc. it could develop a significant dose rate. Guard filters, and provisions for detecting and flushing radioactive solids that may accumulate should be included in the system design.

As discussed in sections 2.1 and 2.2, there are a large number of options for final disposal of the sorbent, and for intermediate handling of the sorbent. A decision on the preferred approach requires decisions concerning other issues that are not likely to be resolved for some time. Therefore, a system for retrieval, handling and processing of the sorbent is not fully designed or installed initially. Rather, features such as ports in the top of the storage tanks are provided to facilitate retrieval, and the sorbent flushing and drying approach is designed to produce a free flowing dry sorbent that can be readily retrieved using a pneumatic suction system. This storage concept is expected to be compatible with all reasonably expected disposition options without incurring significant avoidable future liabilities. 


\subsection{NWCF Installation and Operating Concepts.}

The calciner cell or the pump and valve corridor could be considered for the ion exchange process and spent sorbent storage tanks. Other areas such as the absorber cell or offgas cell could also be considered but would require more dismantling and removal of existing equipment. The calciner cell has piping connections for feeding solids into the existing pneumatic transfer system, and appears to have sufficient room for the ion exchange module and spent sorbent storage tanks. It also has more shielding than the pump and valve corridor. Based on a cursory evaluation, the calciner cell appears to be a logical location for the ion exchange columns and spent sorbent storage tanks.

Two tanks with 6 foot diameter and 7 foot working solids depth should be sufficient to hold all of the spent sorbent. Assuming approximately 12 inches of steel or lead shielding on the cell side of the tanks, it appears there is sufficient space in the calciner cell to accommodate these tanks plus a module with three ion exchange columns, a feed tank and a waste/flush tank. Some existing piping in the cell may need to be removed, but most of the piping and the calciner vessel should not need to be removed. However, this leaves little or no space for filtration within the calciner cell.

Features for future retrieval of the stored sorbent need to be considered in laying out the storage tanks. Spare ports on top of the tanks are needed that can be opened for insertion of retrieval vacuum lines. If feasible, height of the sorbent storage tanks could be limited so there is enough space above the tanks to install the remote sorbent retrieval system inside the cell. If this is not feasible, it could be necessary to construct a shielded enclosure in the operating area above the calciner cell for some of the retrieval equipment.

Extensive valving is required for switching the various feed, product, and flush flows for the column modules. It is probably best to put this valving in contact-maintained shielded valveboxes located in the operating corridor or in the operating area above the cells. Valve actuators and position switches could be located outside the valve boxes allowing direct hands-on maintenance. For maintenance of valves inside the box, the lines would be drained and flushed before opening the box, in order to facilitate contact maintenance. If the filtration system is located in the pump and valve corridor, and the ion exchange is located in the calciner cell, transfer lines between the two systems will be needed. If a convenient in-cell route is not available, a small lightly shielded pipeway could be put in the operating corridor or the operating area above the cells, tying in to the ion exchange valve box. Due to the much higher dose rates, increased difficulty in flushing, and desirability of keeping the piping short, valves for the spent sorbent sluicing lines probably need to be inside the cell. 


\subsection{FULLY LOADED SORBENT, MODULAR COLUMN}

For this option a "modular column waste package" (MCWP) concept is used in which the ion exchange column is a single use unit that also serves as the waste handling and storage package. The following sections discuss a preliminary concept based in the MCWP option.

\subsection{MCWP Features and Assumptions}

The proposed ion exchange media packaging and disposal concept for the MCWP option includes the following key features and assumptions.

1. A modular ion exchange column is used. After use, the column is mechanically closed and is used as the primary container for handling and storage. The combined unit is referred to herein as a modular-column waste-package or MCWP.

2. When the cesium capacity of the modular-column is expended, the column is flushed with dilute acid followed by water. It is then drained, and dried out by blowing or drawing heated air through the bed, and the penetrations are plugged. A bolted closure plate may be added if needed for handling or transportation.

3. If needed, external surfaces of the MCWP are decontaminated with a high pressure water spray, and the container is moved to a storage location in the NWCF where it remains until ready for the next disposal step which may be determined after the sorbent is placed in interim storage.

4. During operation, content of cesium and other radioisotopes are estimated for each MCWP by material balance.

5. It is assumed that acid and water flushes adequately strip weakly sorbed hazardous metals so that the resulting waste form passes TCLP leach criteria. It is further assumed that flushing decontaminates the sorbent well enough that delisting will be granted if needed for any listed wastes present in the SBW. These assumptions [if verified] allow the MCWP to be stored, transported and disposed as non-hazardous waste.

6. Options available for intermediate handling and disposal include all of those discussed in sections 2.1 and 2.2. In order to facilitate removal of the contents, one or more ports will be provided to allow vacuum lines to be inserted to suck the sorbent out of the MCWP.

7. The example MCWP is nominally 2 feet outside diameter by $6 \mathrm{ft}$ to $15 \mathrm{ft}$ tall. For 
MCWP transportation options, a nominal $6 \mathrm{ft}$ size could be used to allow 4 units to fit in a Chem-Nuclear Systems CNS 8-120 B type B shipping cask. Or $2 \mathrm{ft}$ outside diameter by $10 \mathrm{ft}$ or $15 \mathrm{ft}$ length could potentially be transported in the same casks to be used for transportation of HLW glass canisters by the Savannah River or Hanford sites respectively. Package details, including lifting features (lugs, bails etc.), must be worked out as needed to facilitate movement and storage in the NWCF and possibly transportation to another facility.

\subsection{Modular Column/Waste Package}

A preliminary concept for the MCWP is shown in the Attachment. The MCWP consists of a stainless steel canister with outside diameter of about 2 feet and a total height of about 6 to $15 \mathrm{ft}$. Note that these dimensions are preliminary, and may be adjusted to facilitate handling and storage within the NWCF, and removal of spent sorbent from the facility for shipment. All penetrations are made through a flange welded to the top of the canister. The inlet pipe passes down through the bed of ion exchange media to the distributor screens at the bottom. Feed material flows up through the bed through media retainer screens and out through a penetration in the top flange. A downflow arrangement could also be used if desired. Auxiliary penetrations (not all shown) are provided for filling the MCWP with ion exchange media, removing spent media, draining the liquid, instrumentation, and spares. A ring with a sealing surface is provided for installation of a bolted closure plate when the MCWP is readied for storage or shipping.

Assuming 2 foot outside diameter, $3 / 8$ in. wall thickness, and $6 \mathrm{ft}$. total height with a loaded bed height of $5 \mathrm{ft} 3$ inches, sorbent volume is calculated at 15.5 cubic feet or 0.44 cubic meters per package. If integral shielding of each MCWP is needed to facilitate handling, the useable volume may be significantly reduced. For example, a 2 inch thick wall, 2 foot outside diameter and a net bed depth of 5 feet results in a sorbent volume of 10.9 cubic feet ( 0.31 cubic meters), a $30 \%$ reduction from the thin walled example above.

For the fully loaded sorbent case, about 8 to 12 cubic meters of sorbent is required. At 0.44 cubic meters of sorbent per package, a total of about 18 to 27 MCWPs [2 ft X6ft] is estimated to be required to process the total waste inventory. Layouts and remote handling equipment concepts need to be developed to evaluate feasibility of fitting this quantity in the existing NWCF cells, however, the quantity does not appear intractable. Larger column size can also be considered to help facilitate storage.

\subsection{Preparation of Column for Use}

Prior to transferring each MCWP into the NWCF hot cells, it is filled with sorbent, fines are flushed out with water, the sorbent is preconditioned if needed, and the unit is tested. Excess liquid is then drained and the unit is ready for transfer to the cell. 


\subsection{Preparation of Spent Sorbent Packages}

It is assumed that at least 2, and possibly 3 MCWPs are operated in series. The first column in series is replaced when loaded to near full saturation, and a new column is placed in series after the remaining partially used column(s). Following is a preliminary list of steps in preparing a used MCWP prior to transfer to storage.

1. Line feed to the ion exchange system is shut off.

2. System feed is replaced with flush solution. The study flowsheet is based on flushing with 10 column volumes of dilute acid followed by 10 column volumes of water (10 times the gross volume of one MCWP, or about 5,000 liters). Flush solution from the spent column passes through the downstream columns and exits as other treated waste. It is assumed that this flush will remove any sorbed heavy metal sufficiently to allow the sorbent to pass the TCLP test. Similar sorbent used for treatment of tank waste at Oak Ridge was found to pass the TCLP test'. It may not be essential to flush the column with acid for the fully loaded case, however, it is recommended that basic hardware for both acid and water flush of the column be included in the preliminary design concept.

3. The spent MCWP is physically disconnected from the balance of the ion exchange system. The feed line is removed and connected to the following column and a new second column is connected. Ion exchange processing in the other columns can now proceed while the spent column is prepared for removal from the cell.

4. Water is drained from the spent column. An auxiliary drain line extends to the bottom of the vessel to facilitate complete draining.

5. After the column is drained, remaining water is evaporated by blowing or drawing heated air through the column. The nominal study basis is 30 SCFM of air heated to $190^{\circ} \mathrm{F}$ at the inlet to the column. For the 6-ft example column, this is estimated to completely dry out the bed in about 4 to 6 days.

6. Remaining lines are removed and the penetrations are plugged. The closure plate is then bolted in place.

7. The package is washed with a water spray to remove surface contamination.

8. The package is moved to a storage rack, where it remains until decisions are made and 
additional features are provided for the next step in processing. The most likely disposition is expected to be pneumatic transfer of the spent sorbent to the WTF either directly or via the binsets.

9. Detailed operating sequence and equipment concepts for removing contents from MCWPs, or removing the packages from the cell is to be developed after decisions on disposition methods have been made.

\subsection{CLASS C WASTE OPTIONS}

For Class C options it is assumed that spent sorbent is to be disposed of at an offsite low level waste (LLW) disposal facility. Disposal facilities currently in operation are limited to disposal of LLW within Class $C$ limits specified in 10CFR61. The radioisotopes of concern for meeting these limits are ${ }^{137} \mathrm{Cs}$ and transuranics (TRU). To satisfy these limits, cesium content of the loaded media will be controlled (limited) to keep the resulting package within the maximum Class $\mathrm{C}$ limits $\left(4,600 \mathrm{Ci}^{137} \mathrm{Cs}\right.$ per cubic meter). Design features and operating practices will also be provided to assure transuranic isotope content is less than the Class $\mathrm{Climit}$ of $100 \mathrm{nCi} / \mathrm{g}$. It is also assumed that it will be demonstrated that the resulting loaded media is not a hazardous waste. If it is found to be infeasible to operated the process to produce spent sorbent that is not hazardous waste, additional treatment will be needed to render spent sorbent suitable for disposal.

Two primary packaging suboptions may be considered for Class $C$ spent sorbent: a) The "modular column waste package" (MCWP) concept in which the ion exchange column is a single use unit that also serves as the waste package; and b) an approach in which spent sorbent is sluiced out of the column directly into a waste disposal package or liner. The first option is similar to the fully loaded MCWP option. For the second option, location for filling the waste package can be chosen to facilitate loading for shipment. The package could be located near the shipping cask during sluicing and drying in order to reduce required equipment and to reduce risk from moving and loading the high dose rate sorbent package into the shipping cask. The following sections discuss Class $\mathrm{C}$ disposal requirements and preliminary example implementation concepts.

\subsection{DISPOSAL REQUIREMENTS}

The Nevada Test Site (NTS) and the Hanford Site are the most likely locations for disposal of Class $\mathrm{C}$ ion exchange media produced by treating the SBW. Baseline criteria for acceptance of waste at the Hanford Low-Level Burial Grounds (LLBG) are described in Reference 4. The LLBG includes both lined and unlined portions. The lined portions are permitted to accept 
certain additional hazardous waste types that cannot be disposed in the unlined portions. Key requirements related to acceptance of INEEL ion exchange media are summarized below.

- The waste must be within Class $\mathrm{C}$ limits described in 10CFR61. Primary radioisotopes of concern relative to these limits are ${ }^{137} \mathrm{Cs}$ and alpha emitting transuranics (TRU).

- The generator must implement and maintain a waste certification program to ensure that any waste meets the acceptance requirements. Concentration of each "major radionuclide" must be established. A quality assurance program that complies with DOE $5700.6 \mathrm{C}$ is also required. Hanford would retain joint responsibility to assure that the waste meets the acceptance requirements.

- The generator must establish and maintain a waste minimization program to assure that the amount of radioactive waste generated and/or shipped is minimized.

- The generator must determine the physical and chemical characteristics of the waste with sufficient accuracy to properly designate the waste. This applies to both radioactive waste classifications and hazardous waste classifications. Guidelines on what constitutes acceptable knowledge of the waste characteristics are given in Reference 4.

- Free liquids must be absorbed or stabilized in accordance with Reference 4 Appendix E.

- Hazardous waste originally designated only with characteristic waste number D001 through D043 can be accepted in unlined portions of the LLBG if it is no longer hazardous and meets all applicable treatment standards of 40CFR268. In addition, waste with codes F001 through F005, waste with F039 containing F001 through F005 constituents, and Washington State only dangerous waste may be accepted at the lined portions of the LLBG. All wastes must meet applicable treatment standards of the land disposal restrictions.

- If radiolytic heat generation exceeds 3.5 watts per cubic meter, the package must be evaluated and approved. Class $C$ spent sorbent is expected to exceed this limit.

- Mitigation measures must be used if radiolysis will produce gas concentrations exceeding the lower explosive limits. (Note: Additional evaluation is needed to determine if radiolytic gas generation from the sorbent is significant relative to this requirement.)

- The spent sorbent must either be packaged in an approved high integrity container or stabilized in concrete or other stabilization agent. Stabilized waste must meet the leach index and compressive strength requirements of the NRC Branch Technical Position Paper on Waste form, Section C.2 and Appendix A.

- If the waste contains mobile radionuclides above a threshold value, additional stabilization requirements could be imposed. Mobile radionuclides identified in Reference 4 include: ${ }^{3} \mathrm{H}$, ${ }^{14} \mathrm{C},{ }^{36} \mathrm{Cl},{ }^{79} \mathrm{Se},{ }^{93} \mathrm{Mo},{ }^{99} \mathrm{Tc},{ }^{129} \mathrm{I},{ }^{187} \mathrm{Re}$, and all uranium isotopes. If the acid and water flushing process described herein is followed, it is not expected that mobile isotopes will be a significant concern.

- Limits are established for removable contamination on accessible surfaces of the waste package.

- For all important-to-safety components the QA Program must meet DOE/RE/0333P 


\section{Requirements.}

Certain waste types or waste characteristics are prohibited and may not be disposed at the Hanford LLBG. Prohibited waste types of interest for the INEEL spent sorbent case include the following.

- Waste containing free liquids

- Waste that poses substantial hazards due to formation of flammable or toxic gases, vapors or fumes.

- Wastes with RCRA U, $\mathrm{P}$, and $\mathrm{K}$ waste number and $\mathrm{F}$ codes other than listed above cannot currently be disposed at the LLBG.

- Packages and sacrificial rigging shall not contain regulated materials such as lead.

\subsection{MCWP Features and Assumptions}

The proposed ion exchange media packaging and disposal concept for the MCWP Class $\mathrm{C}$ option includes the following key features and assumptions.

- A modular ion exchange column is used. After use, the column is mechanically closed and is used as the primary container for handling and disposal. The combined unit is referred to herein as a modular-column waste-package or MCWP.

- When the cesium capacity of the modular column is expended, the column is flushed with dilute acid followed by water. It is then drained, and dried out by blowing heated air through the bed, and the penetrations are plugged.

- If needed, external surfaces of the MCWP are decontaminated with a high pressure water spray, and the container is ready to be moved from the NWCF hot cells to a shielded cask used for shipment to a Class $C$ low level waste disposal facility.

- During operation, cesium curie content for each MCWP is calculated by material balance to verify the overall package contains less than the Class $\mathrm{C}$ cesium limit.

- The waste is certified as non-TRU based on: a) Prior testing, to demonstrate that TRU absorbed from solution is adequately stripped by acid and water flushes; and, b) Primary filtration of TRU suspended solids followed by a backup guard filter to assure that the media is not contaminated with significant quantities of TRU solids. This approach is assumed to be acceptable to the disposal facility. However, the process control approach and associated operational requirements must be agreed to by the disposal facility operator. ${ }^{4}$.

- It is assumed that the acid and water flushes adequately strip weakly sorbed hazardous metals so that the resulting waste form will pass the TCLP leach criteria. It is further assumed that flushing decontaminates the sorbent well enough that delisting will be granted if needed for any listed wastes present in the SBW. These assumptions [if verified] allow 
the MCWP to be disposed as non-hazardous Class C low level waste.

- The example MCWP is nominally 2 feet outside diameter by $6 \mathrm{ft}$ tall with final finished sizing to allow 4 units to fit in a Chem-Nuclear Systems CNS 8-120 B type B shipping cask. If needed to facilitate loading and unloading, a moderate thickness of integral shielding could be included in MCWP to achieve a moderate reduction in the surface dose rate of the filled MCWP. Package details, including lifting features (lugs bails etc.) and acceptable surface dose rates, must be worked out with the disposal facility operator.

- Discussions with Hanford personnel indicate that Hanford could accept the spent sorbent if it is less than Class C, and is either not hazardous waste or else it is treated to meet the land disposal restrictions. Cesium and TRU are the only components that appear to be significant considerations in meeting the Class $\mathrm{C}$ limits. The waste package must not contain free liquids, and the waste must either be stabilized or else packaged in a qualified high integrity container. Oak Ridge is also working with NTS, which has indicated it can accept their similar spent sorbent if it is within Class C limits and is not hazardous waste ${ }^{3}$.

- It is probably not cost effective to stabilize the waste or qualify the MCWP as a high integrity container (300year physical integrity after burial). It is therefore assumed that the MCWP will be placed in a qualified high integrity container prior to disposal (either before shipment or at the disposal facility). These details need to be finalized before the detailed package configuration can be finalized.

\subsection{Modular Column/Waste Package}

An example concept for the MCWP is shown on Page 1 of the Attachment. The MCWP consists of a stainless steel canister with outside diameter of about 2 feet and a total height of about 6 feet. Note that these dimensions are preliminary, and may be adjusted to facilitate removal of spent sorbent from the facility for shipment, or to allow the system to be fit into existing cells. All penetrations are made through a flange welded to the top of the canister. The inlet pipe passes down through the bed of ion exchange media to the distributor screens at the bottom. Feed material flows through the bed, through media retainer screens, and out through a penetration in the top flange. A downflow arrangement could also be used if desired. Auxiliary penetrations (not all shown) are provided for filling the MCWP with ion exchange media, draining the liquid, instrumentation, and spares. A ring with a sealing surface is provided for installation of a bolted closure plate when the MCWP is readied for shipping.

Assuming 2 foot outside diameter, $3 / 8$ inch wall thickness, and a loaded bed height of $5 \mathrm{ft} 3$ inches, sorbent volume is calculated at 15.5 cubic feet or 0.44 cubic meters per package. If integral shielding of each MCWP is needed to facilitate handling, the useable volume may be significantly reduced. For example, a 2 inch thick wall, 2 foot outside diameter and a net bed depth of 5 feet results in a sorbent volume of 10.9 cubic feet $(0.31$ cubic meters), a $30 \%$ reduction from the thin walled example above. 
Based on a total ${ }^{137} \mathrm{Cs}$ inventory of about $250,000 \mathrm{Ci}$, Class $\mathrm{C}$ limit of $4,600 \mathrm{Ci}{ }^{137} \mathrm{Cs}$ per cubic meter, and assumed average loading efficiency of $90 \%$ of the Class $C$ limit; about 60 cubic meters of sorbent are required. At 0.44 cubic meters of sorbent per package, 137 MCWPs is estimated to be required to process the total waste inventory. Assuming 4 MCWPs per waste shipment if the Chem-Nuclear Systems CNS 8-120 B type B shipping cask is used, 35 shipments to the Class C LLW disposal facility are estimated.

\subsection{MCWP Operations}

Prior to transferring each MCWP into the NWCF hot cells, it is filled with sorbent, fines are flushed out with water, the sorbent is preconditioned if needed, and the unit is tested. Excess liquid is then drained out and the unit is ready for transfer to the cell.

It is assumed that at least 2, and possibly 3 MCWPs are operated in series. The first column in series is replaced when loaded to approximately the Class $\mathrm{C}$ limit, and a new column is placed in series after the remaining partially used column(s). The following discusses the steps in preparing a used MCWP prior to transfer out of the cell.

1. Feed to the ion exchange system is shut off.

2. System feed is replaced with flush solution. The study flowsheet is based on flushing with 10 column volumes of dilute acid followed by 10 column volumes of water $(10$ times the gross volume of one MCWP, or about 5,000 liters). Flush solution from the spent column passes through the downstream columns and exits as other treated waste.

The 10 column-volume acid flushing allowance is based on Table 10 of Reference 1 , which shows a Kd value for $\mathrm{Pu}$ of $3.8 \mathrm{ml} / \mathrm{g}$ at a pH of 0.58 . Based on an average bed density of about 1 $\mathrm{g} / \mathrm{ml}, 3.8$ bed volumes would be required to flush $\mathrm{Pu}$ from the column with an ideal vertical step f77unction breakthrough curve. The assumed 10 column-volumes provides 2.6 times this minimum value. Additional data is needed to determine distribution coefficients for all of the transuranics, and to verify that they can actually be reliably stripped with an acceptable quantity of acid. Data is also needed to determine the preferred acid concentration. It is assumed that this flush will also remove any sorbed heavy metal sufficiently to allow the sorbent to pass the TCLP test. Similar sorbent used for treatment of tank waste at Oak Ridge was found to pass the TCLP test ${ }^{3}$. Limited data in Reference 1 also suggest that many of the characteristic hazardous metals are not significantly sorbed. However, the data are not complete, and additional tests are needed to verify that the assumed flushes are adequate to assure spent sorbent is not a characteristic hazardous waste.

3. The spent MCWP is physically disconnected from the balance of the ion exchange system. The feed line is removed and connected to the following column and a new second column is 
connected. Ion exchange processing in the other columns can now proceed while the spent column is prepared for removal from the cell.

4. Water is drained from the spent column. An auxiliary drain line extends to the bottom of the vessel to facilitate complete draining.

5. After the column is well drained, remaining water is evaporated by blowing or drawing heated air through the column. $30 \mathrm{SCFM}$ of air heated to $190^{\circ} \mathrm{F}$ at the inlet to the column is estimated to completely dry out the bed in about 4 to 6 days.

6. Remaining lines are removed and the penetrations are plugged. The closure plate is then bolted in place.

7. The package is decontaminated to remove surface contamination and smear tested.

8. The package is moved to a storage rack where it remains until removal from the cell.

9. The operating sequence and equipment concepts for removing the package from the cell and loading into a transportation cask have not been developed at this time. For the NWCF retrofit case, these may present a significant challenge.

\subsection{Sluice-Out Ion Exchange Media to a Transportation Package}

For the Class C option, another approach is to sluice the spent sorbent directly from the column into a transportation package or liner. This approach has a number of advantages and is already used in the nuclear industry. After the sorbent is transferred to the liner, drainable water is pumped out, and residual water is either removed by evaporation or taken up by drying or solidification agents added to the liner.

\subsection{Filtration Requirements for Class C Options}

For the Class $\mathrm{C}$ options, it is essential to avoid production of spent ion exchange media that cannot be certified to be non-TRU. Calculations summarized in the Attachment indicate that less than $0.4 \%$ of the estimated SBW solids would be sufficient to contaminate the entire quantity of spent ion exchange media to the $100 \mathrm{nCi} / \mathrm{g}$ TRU limit. High reliability, and ability to defend the non-TRU certification to outside parties is essential for disposal as Class $\mathrm{C}$ waste. In specifying the required filtration efficiency an approximate safety factor of 10 is therefore recommended to allow for batch to batch variation in solids content of filter feed, uncertainties in measurements and analyses, and for the fact that some residual TRU will also be present from liquid phase 
adsorption. If it is assumed that most of the solids passing through the filtration could become attached to the media, and a safety factor of 10 is provided, the allowed penetration is $.04 \%$ and the required filtration efficiency is estimated at $99.96 \%$.

\subsection{Discussion - Class C Options}

The Class $\mathrm{C}$ options have the significant advantage that there are currently disposal facilities in operation that can accept the spent ion exchange media if the acceptance requirements can be met. These options also have a number of uncertainties, risks, and disadvantages. Additional test data is needed to verify that a non-TRU product that meets hazardous waste disposal requirements can be reliably produced. Production of spent sorbent that does not meet these requirements would result in an "orphan" waste with no current disposal mechanism. It could presumably be disposed by vitrification with $\mathrm{HLW}$ as in the fully loaded options, however, the volume to be stored, handled, and disposed would be much larger than for the fully loaded options if cesium levels are restricted to Class $\mathrm{C}$.

Additional engineering studies and test data is also needed to develop a design concept for filtration, concentration and solidification of filtered solids, and related support systems for the Class $\mathrm{C}$ option. Due to the limited time and resources and need for additional data, a decision was made to defer additional development of the Class $\mathrm{C}$ options for the current study. These may be considered further at a later date. 


\subsection{REFERENCES}

1. Miller, J. E., and N. E. Brown, "Development and Properties of Crystalline Silicotitanate (CST) ion exchangers for Radioactive Waste Applications, "SAND97-0771, Sandia National Laboratories, April 1997.

2. Todd, T. A., et. al., "Evaluation and Testing of Inorganic Ion Exchange Sorbents for the Removal of Cesium-137 from Idaho Chemical Processing Plant Acidic Tank Waste,." paper submitted for publication in Separations Science and Technology, December 1997.

3. Walker, J. F., et. al., "Cesium Removal Demonstration Utilizing Crystalline Silicotitanate Sorbent for Processing Melton Valley Storage Tank Supernate: Final Report," ORNL/TM13503, March 1998.

4. Hanford Site Solid Waste Acceptance Criteria, HNF-EP-0063, Rev. 5 (DRAFT), April 28, 1998.

5. Ferrara, D. M., et. al., "Fiscal Year 1997 Final Report for Task Plan SR-16WT-31 Task B, Vitrification of Ion Exchange Material," WSRC-TR-97-00320, Westinghouse Savannah River Company, September 30, 1997.

6. Balmer, M. L., and B. C. Bunker, "Inorganic Ion Exchange Evaluation and Design Silicotitanate Ion Exchange Waste Conversion," PNL-10460, Pacific Northwest Laboratory, Richland Washington, March 1995. 
ATTACHMENT-SKETCHES AND BACKUP CALCULATIONS 


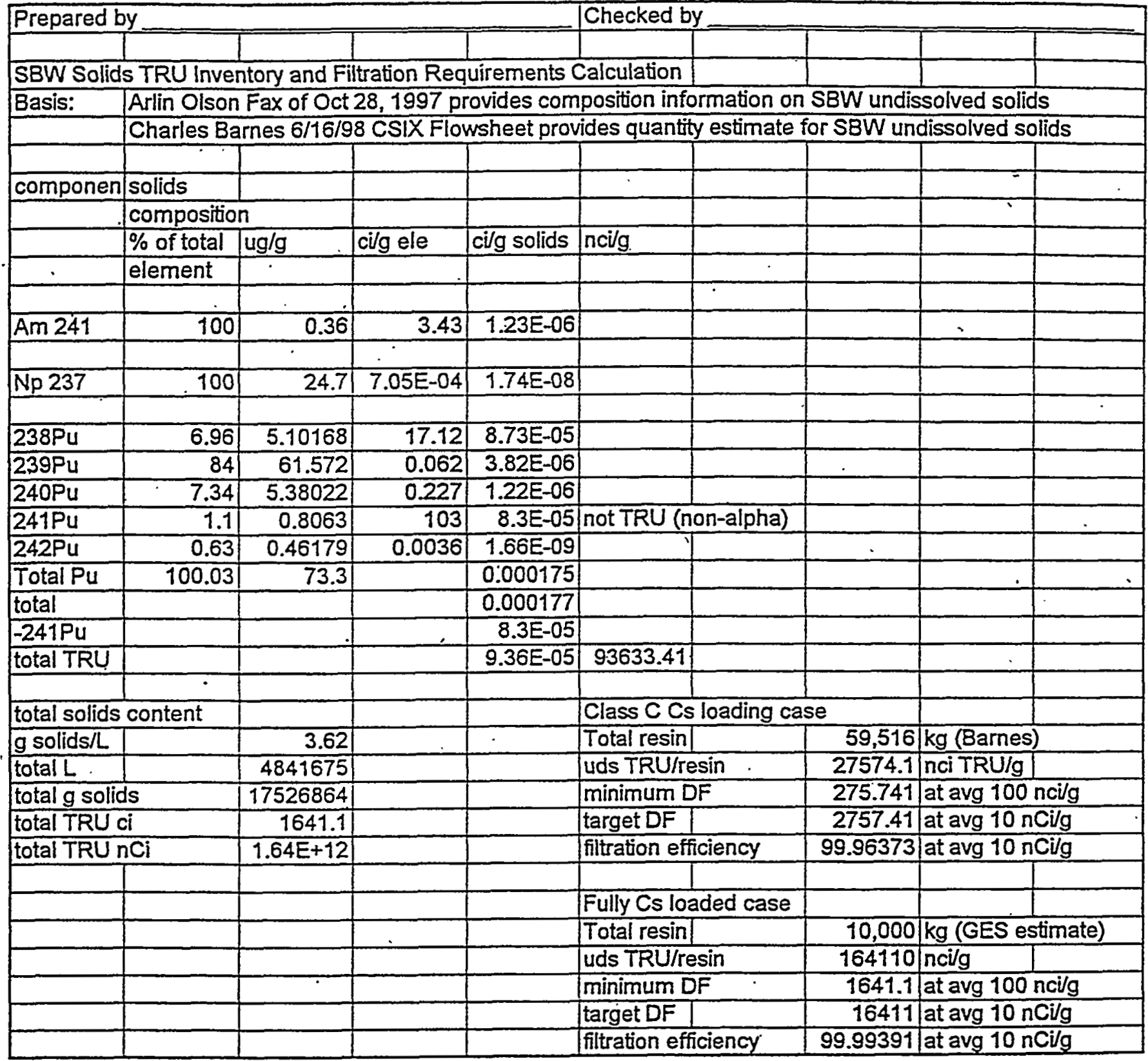


Attackment is Modslar Column Woste Packare

ENGINEERING WORKSHEET

Page 1 of

$\therefore$ r.

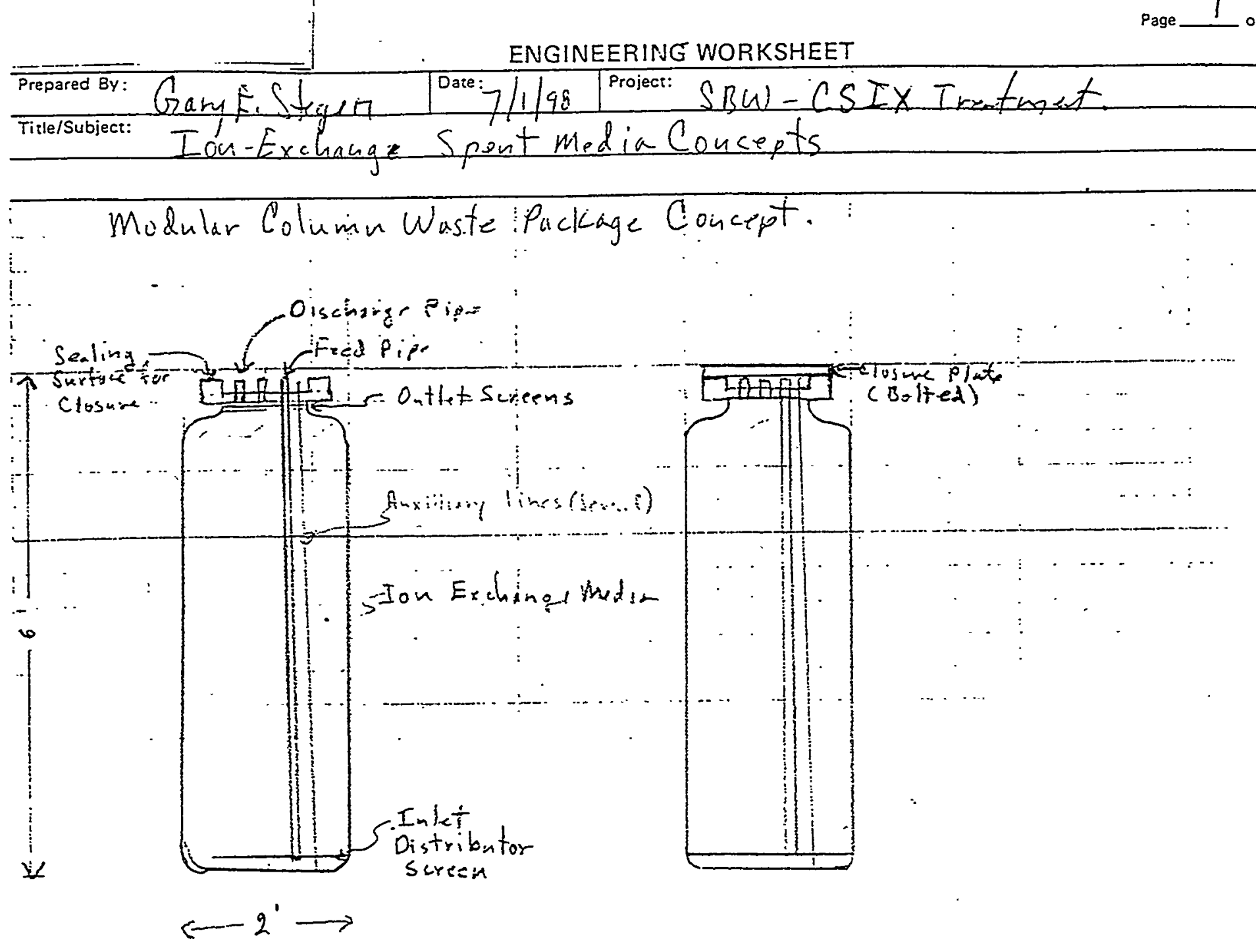


Project File Number

Project/Task CsIX/TRU Grout Feasibility Study

Subtask D.4 Handling \& Storage

Title: Handling of Containers Through the Grouting Area

\section{Summary:}

The final major step in the CsIX/TRU process is the encapsulation, in grout, of the dry product from the process. This EDF describes the method and equipment proposed for moving an empty 55-gal drum to the grout filling station and from the filling station through a number of intermediate stations to a curing cell.

EDF Contents:

- Attachment A - Detailed Process Description w/Assumptions \& Requirements

- Attachment B - Equipment List for the Grouting Area

- Attachment $\mathrm{C}$ - Sequence of Moving a Drum Through Grouting Area

- Attachment D - Sketches

Distribution (complete package): C. W. Olsen MS 3211; K. L. Williams MS 3765; Project File; A copy of this EDF will be included in External Report No. INEEL/EXT-98-00869.

Distribution (summary package only): None

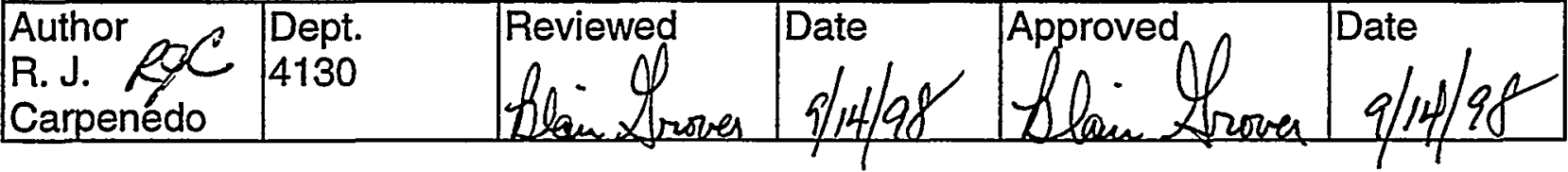




\section{Attachment A \\ CsIX/TRU Feasibility Study \\ Handling of Containers Through the Grouting Area}

\section{General}

This EDF provides information on a method for filling 55-gal drums with grout produced in the CsLX/TRU process and moving the drums through an alpha contaminated environment. The filled drum will be checked for exterior contamination and cleaned if necessary then labeled and cured. After a specified curing, the drums will be dewatered then sent to the drum storage facility. Final examination will be performed just prior to shipment to WIPP.

\section{Assumptions}

The following assumptions have been made in the development of this design.

1. There will be no regular sampling of drums. The process will be qualified and only random verification sampling and testing of the grout will be done.

2. The grouted waste package will be a 55-gal, DOT-17C drum with a full removable head.

3. The filled drums will be considered contact handled, as will be maintenance activities for the drum line activities.

4. The ventilation scheme for the drum movement will be based on alpha type considerations.

\section{$\underline{\text { Requirements }}$}

- The design of the drum handling portion of the facility will comply with the existing environmental regulations and standard criteria pertaining to the design, construction, operations and performance of the proposed CsLX facility at INTEC.

- The structures, systems, and components associated with the Separations Facility will conform to the applicable national, state, local, and DOE requirements. Specifically, the requirements mandated by the RCRA for hazardous waste treatment, storage and disposal facilities apply. The DOE requirements in DOE 420.1 and the applicable DOE Directives apply to this portion of the facility.

- The filling process will begin January 2008 and will be completed by December 2012.

- The facility will be operated 24 hours a day, 200 days a year and produce 45 drums per day.

- The waste will be treated by grouting in preparation for sending to WIPP.

- The specific gravity of the grout is 1.9 and the total grouted waste volume is $7500 \mathrm{~m}^{3}$. 
Attachment A

CsIX/TRU Feasibility Study

Handling of Containers Through the Grouting Area

\section{Process Description}

A drum, without a lid, is retrieved from storage and placed on the inlet conveyor where it is given a unique tracking number. An operator starts the conveyor sending the drum through the grouting cell airlock. Once in the grouting cell, the drum is directed to one of three filling stations as determined by process control system. Drum movement is by a self-powered roller conveyor system set to operate at $10 \mathrm{fpm}$. Direction changes are completed by a small belt conveyor system that lifts the drum slightly above the inlet conveyor level and drives it perpendicular to the inlet conveyor.

The drum is driven into the mixer booth where a grout fill assembly apparatus is lowered into the top portion of the drum. A valve on the bottom of the mixer is opened and grout is gravity fed into the drum. The weight of the drum is monitored during the fill and controls the level in the drum. The maximum weight of a drum will be set at $900 \mathrm{lbs}$. (this assumes that the specific gravity of the mixture is 1.9). After the mixer valve is closed, the fill assembly and drum are vibrated to dislodge any residual grout and level the grout in the drum. The grout fill assembly is raised and the drum moved out of the mixer booth and into the lid placement booth. The mixer booth and the lid placement booth are stainless steel gloveboxes connected together and isolated from each other by a sealed door.

The filled drum is driven into position in the lid placement booth by a small belt conveyor located at the same elevation as the inlet conveyor. The belt conveyor is then lowered placing the drum on a table capable of rotating $360^{\circ}$. A robotic arm with a wiper attachment is moved so that the wiper contacts the rim of the drum. The drum is rotated and the wiper cleans the rim of grout residue, if any, prior to placement of the lid. The wiper is deposited in a collection can and will be disposed of as low-level waste. The lid placement device is lowered placing a lid, with filters installed, on the drum. Next the belt conveyor is raised lifting the drum off the table. In sequence, the outlet conveyor is started, the lid placement booth outlet door is opened and the small belt conveyor is started moving the drum out of the booth.

The drum is driven into the transfer section where it is directed to any one of the three inspection/decon booths. The transfer sections and the transfer tables are enclosed in a stainless steel tunnel arrangement that is integrally connected to the lid placement booths and the inspection/decon booths. Sealed doors provide isolation between the units. The transfer tables allow a drum to be driven perpendicular to the inlet conveyor. A small belt conveyor in the transfer table is raised to match the elevation of the conveyor sections in the transfer section. The drum is driven onto the belt conveyor and stopped. The belt conveyor is lowered placing the drum onto the table which is then turned $90^{\circ}$ redirecting the drum to the open inspection/decon booth. This action has to take place twice for a drum to be redirected. 
Attachment A

CsIX/TRU Feasibility Study

Handling of Containers Through the Grouting Area

The drum is driven into position in the inspection/decon booth by a small belt conveyor located at the same elevation as the inlet conveyor. The belt conveyor is then lowered placing the drum on an indexing table capable of rotating $360^{\circ}$. The table is actuated and the drum exterior surface is visually inspected using a remote camera. This is the only operator involvement required during the filling operation. If the operator sees an area that looks like it may have grout on it, he can program the coordinates into a robotic arm that will take a swipe of the area. The arm can be moved up and down and across the top of the drum so that all surfaces can be checked. The swipe is placed in an on-line alpha counter. If contamination is found, the robotic arm will be used to wipe the area with a damp cloth. The indexing table can be programmed to rotate over a small angle just enough to cover the suspected area. The area will be swiped again to verify the contamination has been removed. The cloth and the swipes will be disposed of as low-level waste. If needed, the drum can be sprayed with water or decon solution and then vacuum dried. The solution will be collected, filtered and pumped back into the system for treatment. Next the belt conveyor is raised lifting the drum off the indexing table. The inspection/decon booth is a stainless steel glovebox connected to the transfer section and the discharge section and isolated from them by sealed doors. To move the drum out of the booth, the inspection/decon booth outlet conveyor is started, the outlet door opened and the belt conveyor started.

The drum is driven into the discharge conveyor section where it is positioned on the discharge conveyor. The required direction change is made by a small belt conveyor system that moves the drum into position above the discharge conveyor level. The belt conveyor is lowered placing the drum on the discharge conveyor that runs perpendicular to the belt conveyor. The drum is then moved through the curing cell airlock into the curing cell. The discharge conveyor section is enclosed in a stainless steel tunnel arrangement that is integrally connected to the inspection/decon booths. Sealed doors provide isolation between the units.

If a drum goes through the farthest mixer line and back through the closest inspection/decon booth and has to be deconned, it is estimated that from the time it is placed on the inlet conveyor in the storage area to time it enters the curing tunnel it will take approximately 65 minutes. With three identical processing lines and a required processing rate of one drum every half-hour, the speed through a line is adequate.

\section{Area Description}

The drum fill area is located on the west side of the LAW treatment facility between the drum receiving/storage area and the curing area. It is a single room on the ground floor level measuring approximately $37 \mathrm{ft}$. by $48 \mathrm{ft}$. by $10 \mathrm{ft}$. high with personnel access through an airlock off the operating corridor. Drums enter the drum fill area on the southwest corner from the receiving/storage area and exit on the northeast corner into the curing area through airlocks. The airlocks prevent the spread of contamination if there is a release in the room. Inside the drum fill area are the gloveboxes for the 
Attachment A

CsIX/TRU Feasibility Study

Handling of Containers Through the Grouting Area

different processing stations. The glovebox concept is employed to prevent the spread of contamination during the filling process and prior to placement of a lid. The room is a zone 2 ventilation area with the zone 1 area being the interior of the gloveboxes. Access to the equipment in the gloveboxes is through either gloveports or removable covers on the boxes. All operations in this area are automated and require no operator action. The walls separating the drum fill area from the storage area, the curing area and the operating corridor are 6 inch thick concrete walls providing shielding for the operators. Viewing windows will be provided in both the area walls and the glovebox walls. All maintenance tasks will be performed hands-on. No remote capabilities will be provided. 


\author{
Attachment B \\ CsIx/TRU Feasibility Study \\ Equipment List for Grouting Area
}

Storage area conveyor - self powered rollers, $6 \mathrm{ft}$. long, $1200 \mathrm{~W}$

Stainless steel airlock enclosure, 7 ga.

Airlock doors (2) - electric motor driven, $1 / 2 \mathrm{HP}$

Airlock conveyor - self powered rollers, $5 \mathrm{ft}$. long, $900 \mathrm{~W}$

Main inlet conveyor w/3 $90^{\circ}$ transfer sections - self powered rollers, $24 \mathrm{ft}$. long, transfer sections to be belt driven w/lifting mechanism, $3 \mathrm{ft}$. long, $4500 \mathrm{~W}$ for conveyors and $1.5 \mathrm{HP}$ for transfer section lifts

Mixer booth inlet conveyor - self powered rollers, $3 \mathrm{ft}$. long, $600 \mathrm{~W}$

Mixer booth - stainless steel glovebox, 7 ga., w/electric motor driven entry and exit doors, $1 / 2 \mathrm{HP}$ each

Mixer booth conveyor - self powered rollers, $6 \mathrm{ft}$. long, $2200 \mathrm{~W}$

Fill assembly - telescoping fill tube, electric motor driven, $1 / 2 \mathrm{HP}$

Lid placement booth - stainless steel glovebox, 7 ga., w/electric motor driven exit door, $1 / 2$ HP

Lid placement inlet and exit conveyor sections - self powered rollers, $1.5 \mathrm{ft}$. long, $600 \mathrm{~W}$ each

Belt driven conveyor section w/lifting mechanism $-3 \mathrm{ft}$. long, $2 \mathrm{HP}$

Rotating table - stainless steel, electric motor driven $1 \mathrm{HP}$

Drum rim cleaning mechanism - remote arm w/wiper assembly, $1 / 4 \mathrm{HP}$

Lid placement assembly - stainless steel, $2 \mathrm{HP}$ total

Transfer section tunnel - stainless steel glovebox, 7 ga., 5 sections

Transfer section inlet and outlet conveyors ( 3 places) - self powered rollers, $3 \mathrm{ft}$. long, $1200 \mathrm{~W}$ each (6 total)

Transfer tables (3) w/belt driven conveyor sections $-3 \mathrm{ft}$. dia., stainless steel, $90^{\circ}$ operation of table, reversible belt driven conveyors, belt driven conveyor sections have electric driven lifting mechanism, $1 \mathrm{HP}$ for table, $2.5 \mathrm{HP}$ for belt driven conveyor and lift

Transverse conveyor sections (2) - self powered rollers, $5.5 \mathrm{ft}$. long, $1000 \mathrm{~W}$

Inspection/decon booth - stainless steel, $7 \mathrm{ga}$., w/electric motor driven entry and exit doors, $1 / 2 \mathrm{HP}$ each

Inspection/decon booth inlet and exit conveyor sections - self powered rollers, 1.5

ft. long, $600 \mathrm{~W}$ each

Belt driven conveyor section w/lifting mechanism $-3 \mathrm{ft}$. long, $2.5 \mathrm{HP}$

Rotating table $-3 \mathrm{ft}$. dia., stainless steel, electric motor driven $1 \mathrm{HP}$

Decon equipment including solution collection system $-1200 \mathrm{~W}$

Inspection equipment - automatic swipe of area of concern, remote mechanism, on-line counting, $3000 \mathrm{~W}$

Discharge section tunnel - stainless steel glovebox, 7 ga., 3 inlet sections plus one long tunnel

Discharge section inlet conveyor (3) - self powered rollers, $3 \mathrm{ft}$. long, $1200 \mathrm{~W}$ each 
Main discharge conveyor w/ $390^{\circ}$ transfer sections - self powered rollers, $24 \mathrm{ft}$. long, transfer sections to be belt driven w/lifting mechanism, $3 \mathrm{ft}$. long, $9000 \mathrm{~W}$ for conveyors and $2.5 \mathrm{HP}$ for transfer section lifts

Stainless steel airlock enclosure, 7 ga.

Airlock conveyor - self powered rollers, $5 \mathrm{ft}$. long, $1800 \mathrm{~W}$

Airlock doors (2) - electric motor driven, $1 / 2 \mathrm{HP}$

Tilt \& pan cameras w/zoom capability (14 req'd), - $400 \mathrm{~W}$ per camera 
Attachment $\mathrm{C}$

CxIX/TRU Feasibility Study

Sequence of Moving a Drum Through The Grouting Area

Retrieve drum w/o lid from storage

Place drum on conveyor

Place unique label on drum

Sequence through grouting cell airlock

Stop conveyor when drum reaches airlock inlet door

Open grouting cell airlock inlet door

Start both conveyors (one inside airlock and then one the drum is on)

Move drum into airlock

Stop both conveyors

Close airlock inlet door

Pause to let airflow to adjust

Open grouting cell airlock outlet door

Start both conveyors (one in grouting area and then one inside airlock)

Move drum into grouting cell (drum will automatically stop at the correct fill line based

on computer decision)

Stop both conveyors

Close grouting cell airlock outlet door

Raise belt drive conveyor to match elevation of correct fill line

Start mixer booth inlet conveyor

Start belt drive conveyor moving drum to mixer inlet

Stop conveyors when drum reaches mixer inlet door

Sequence through mixer booth

Open mixer inlet door

Start conveyor inside mixer booth

Start mixer inlet conveyor moving drum into position in mixer booth

Stop both conveyors

Close mixer inlet door

Lower grout fill assembly to mate with drum

Open valve on bottom of mixer

Fill drum, vibrate to settle and level grout

- Raise fill assembly

Pause to let residual material drip off

Sequence through lid placement booth

Open mixer outlet/lid placement inlet door

Start lid placement inlet conveyor

Raise belt drive conveyor to match elevation of inlet conveyor

Start belt drive conveyor

Start mixer conveyor moving filled drum into position in lid placement booth

Stop conveyors

Close mixer outlet/lid placement inlet door

Lower belt drive conveyor placing drum on rotating table

Move wiper to contact drum rim

Start table rotating drum and cleaning rim of grout residue, if any

Stop table 
Attachment C

CXIX/TRU Feasibility Study

Sequence of Moving a Drum Through The Grouting Area

Move wiper and deposit wiper pad in disposal port

Actuate lid placement device placing lid on drum

Sequence into transfer section

Open lid placement booth outlet door

Start transfer section inlet conveyor

Start lid placement booth outlet conveyor

Raise belt drive conveyor to match lid placement outlet conveyor elevation

Start belt drive conveyor moving drum into transfer section

Stop conveyors

Close lid placement outlet door

Sequence through transfer section (computer selects inspection/decon booth that is available)

Raise belt drive conveyor in transfer table to match elevation of conveyor sections in

transfer section

Start belt drive conveyor and downstream conveyor section if drum is to be moved. straight ahead

Start inlet transfer conveyor moving drum to inlet of inspection/decon booth

If the inspection/decon booth that is open is not directly ahead of the drum then the steps are as follows:

Raise belt drive conveyor in transfer table to match elevation of conveyor sections in transfer section

Start belt drive conveyor

Start inlet transfer conveyor moving drum to transfer table

Stop conveyors

Lower belt drive conveyor onto transfer table

Rotate table 90 degrees to direct drum to correct inspection/decon booth

Raise belt drive conveyor in transfer table to match elevation of conveyor sections in transfer section

Repeat these steps until drum is positioned in front of inspection/decon booth

Sequence through inspection/decon booth

Open inspection/decon inlet door

Start inspection/decon inlet conveyor

Raise belt drive conveyor to match elevation of inlet conveyor

Start belt drive conveyor

Start inspection/decon inlet conveyor moving drum into position in inspection/decon booth

Stop conveyors

Close inspection/decon inlet door

Lower belt drive conveyor placing drum on rotating table

Start table rotating drum and inspecting for contamination

Decon as necessary and vacuum drum removing liquid

Stop table

Sequence onto discharge conveyor

Open inspection/decon outlet door

Start discharge inlet conveyor

Start inspection/decon booth outlet conveyor moving drum to the discharge conveyor 
Attachment C

CXIX/TRU Feasibility Study

Sequence of Moving a Drum Through The Grouting Area

Stop conveyors and close inspection/decon booth outlet door

Raise belt drive conveyor on discharge conveyor to match elevation of discharge inlet conveyor

Start belt drive conveyor

Start discharge inlet conveyor moving drum onto belt drive conveyor

Stop then lower belt drive conveyor placing drum on discharge conveyor

Start main discharge conveyor moving drum to curing cell airlock

Sequence through curing cell airlock

Stop discharge conveyor when drum reaches airlock inlet door

Open curing cell airlock inlet door

Start both conveyors (one inside airlock and then the discharge conveyor)

Move drum into airlock

Stop both conveyors

Close curing cell airlock inlet door

Pause to let airflow to adjust

Open curing cell airlock outlet door

Start both conveyors (one in curing cell and then one inside airlock)

Move drum into curing cell

Stop both conveyors

Close curing cell airlock outlet door 


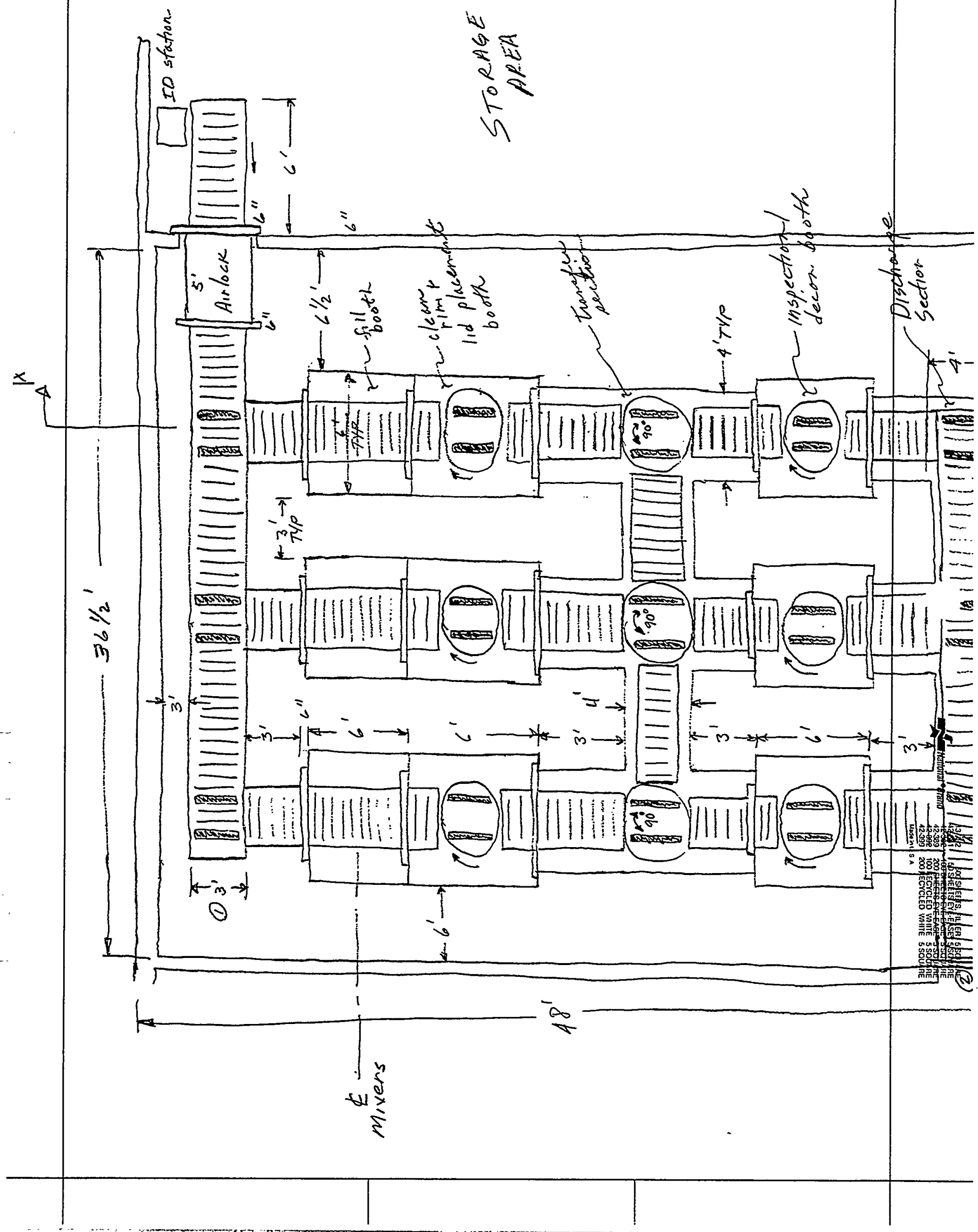




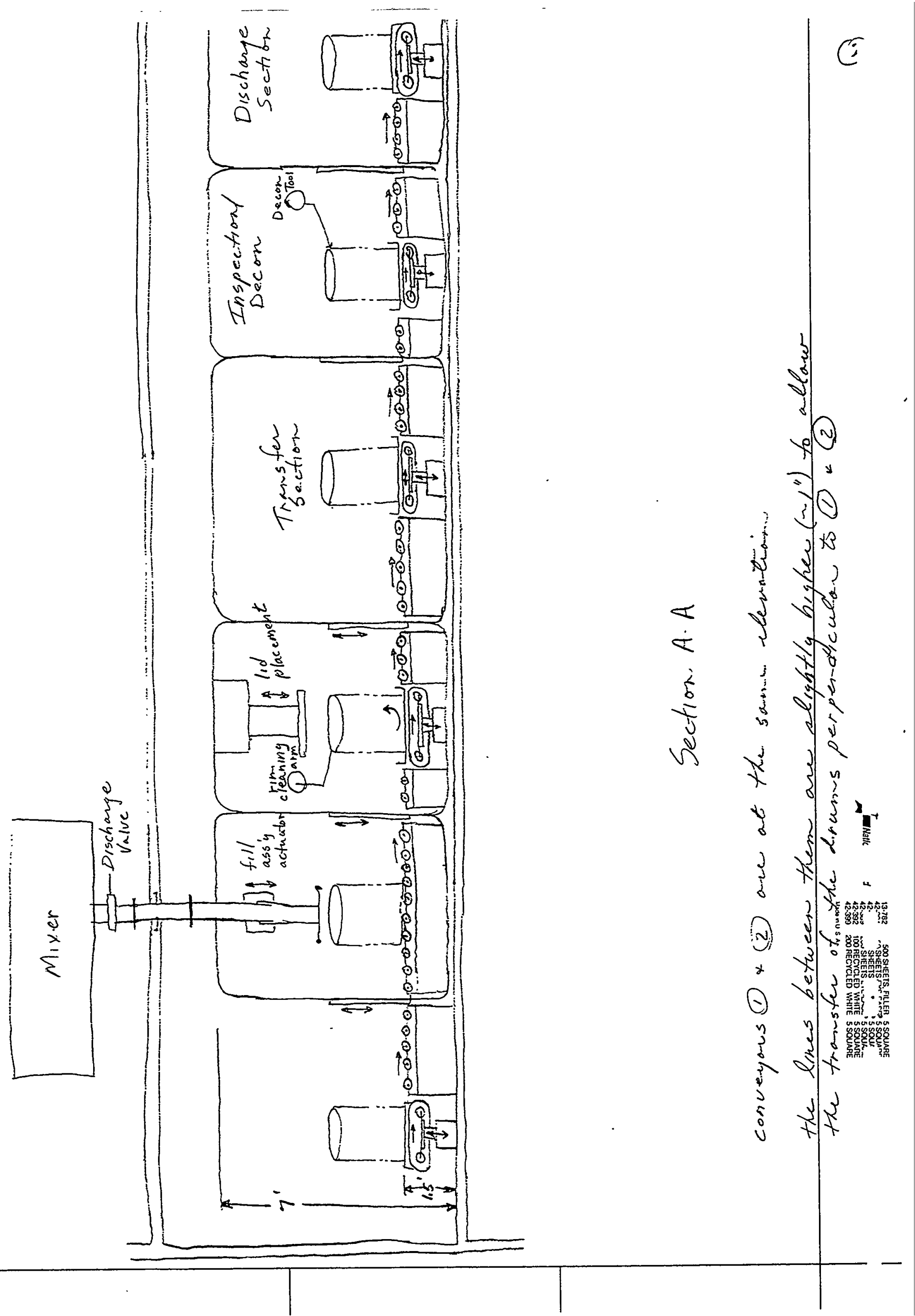



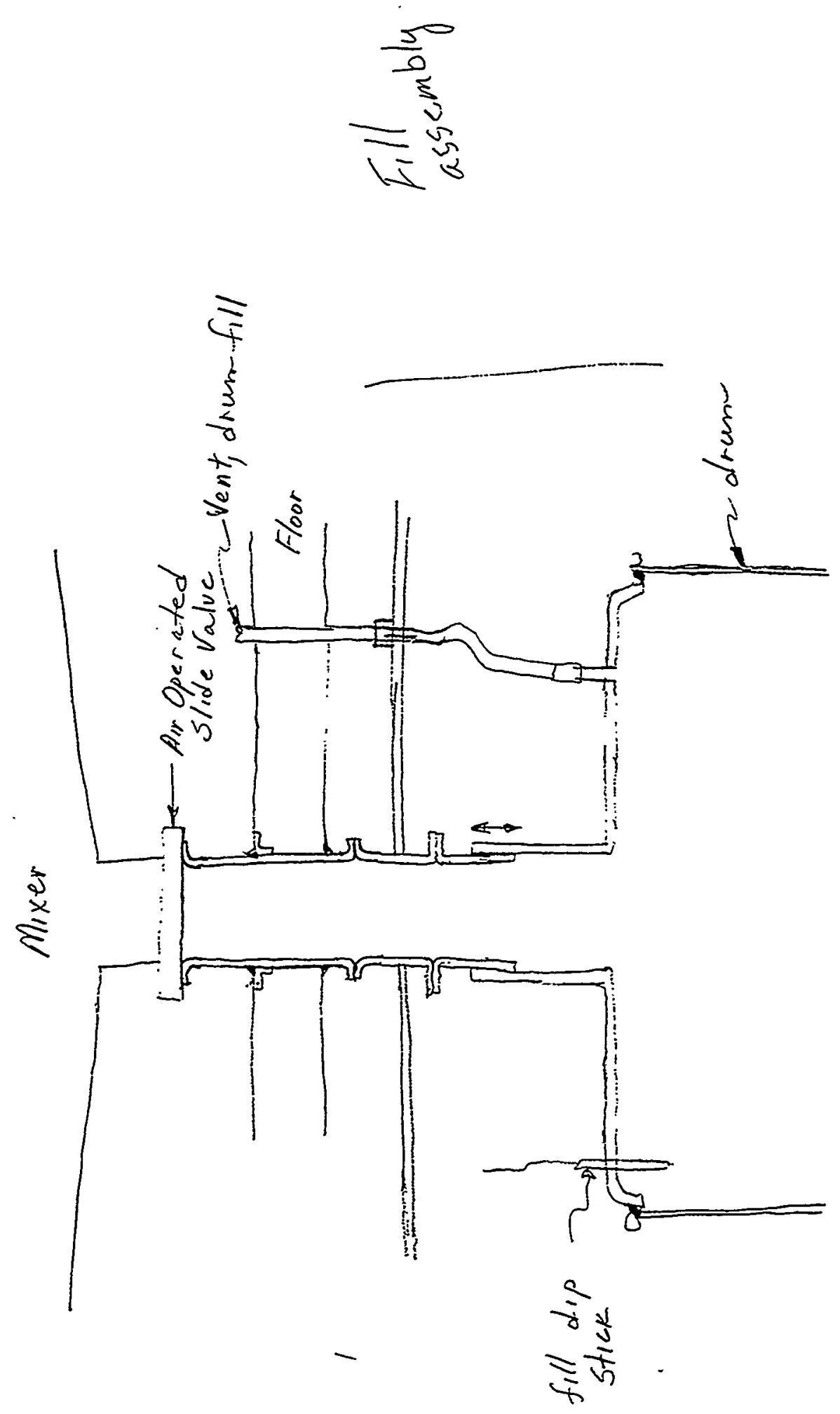
Project File Number $\quad 02 B M 1$

Project/Task CsIX/TRU Grout Feasibility Study

Subtask D.4 Handling \& Storage

Title: Handling of Containers Through the Curing Area

Summary:

This EDF describes movement of the grout filled 55 gallon drums through a curing cell after being filled and decontaminated. The curing process will be a 24-hour period and the drums will then be passed to a dewatering station. The equipment required for Option 1 and 2 are identical but the floor layouts are different.

EDF Contents:

- Attachment A - Detailed Process Description w/Assumptions \& Requirements

- Attachment $B$ - Equipment list for the cure cell

- Attachment $C$ - Sequence of moving a Drum through the cure cell.

- Attachment D - Sketches

Distribution (complete package): C. W. Olsen MS 3211; K. L. Williams MS 3765; Project File; A copy of this EDF will be included in External Report No. INEEL/EXT $=98-00869$.

Distribution (summary package only):

\begin{tabular}{|c|c|c|c|c|c|}
\hline $\begin{array}{l}\text { Author } \\
\text { B. K. } \\
\text { Grover }\end{array}$ & $\begin{array}{l}\text { Dept. } \\
4130\end{array}$ & Revieweg & $9 / 28 / 98$ & K.C.Culleaim & $9 / 28 / 98$ \\
\hline
\end{tabular}




\author{
Attachment A \\ CsIX/TRU Feasibility Study \\ Handling of Containers through the Curing Area
}

\title{
General
}

This EDF describes the method of transporting and providing curing time for the grout filled 55-gallon drum containers produced in the CsIX/TRU grouting process. After the containers have been filled with grout, sealed, and decontaminated, a 24hour curing period will be required. After the curing period the containers will then be sent to a dewatering station and then to an interim storage facility.

\section{Assumptions}

1. Containers will be clean/decontaminated prior to entrance into the cure cell.

\section{Requirements}

1. The cure cell drum handling equipment must provide a remote automated curing period as determined by the process.

2. A 24-hour period is required for the grout placed in the drums prior to dewatering and shipment to the interim storage facility.

3. Maintenance performed on the automated handling equipment will be contact handled. No remote maintenance will be required in the cure cell and all equipment will be stainless steel for decontamination.

4. A redundant cure line will be réquired to provide for an equipment failure or in case the cure period needs to be increased.

5. The drum handling equipment must accept 45 drums per day when in operation.

\section{Process Description}

The fully automated conveyor system will require sequencing by a logic control system with an attending operator with oversight and override capabilities. After the drum has been filled with grout, a lid installed, and decontaminated, it will be transported through an air lock on a roller conveyor to the cure cell. After entering the cell the drum will stage on a 6-foot conveyor for entrance to a drum elevator. Once the elevator is determined to be vacant the drum will enter. 
There will be two cure line conveyor systems. One system will be 2 feet above the floor and the second will be located directly over the first. Vertical distance between the cure lines will be 4 feet. If one of the conveying systems is inoperative the drum will be transferred to the operating cure line. Drums will be placed on the conveyor 6 inches apart. The cure line conveyors will operate at a speed of 4.75 feet per hour for a 24-hour cure period and traveling a distance of 114 feet. This rate may change or both conveyor systems will be used simultaneously if additional cure time is determined to be required.

The cure line conveyors will be encased in a stainless steel tunnel and ventilated to remove moisture produced from the curing process. The SST tunnels will have removable panels on the sides for access to maintenance items. Grease lines and other required lubrication lines will be placed on the exterior of the tunnel for ease of access. Windows will be incorporated into the tunnel for sight of the drum movement and visual inspection of the cure line conveyor equipment.

Once the drum has traveled to the end of the cure line it will enter into the lowering elevator. If the drum is on the top cure line conveyor it will enter and be lowered to the floor level conveyor system and staged to the second floor elevator. If the drum is on the floor level cure line it will stage to the second floor elevator.

Once the second floor elevator is determined to be vacant the drum will enter and be lifted to the second floor. Upon reaching the second level the elevator will stop and a set of doors on the second floor will open. The drum will be transferred past the doors into the dewatering area and the doors closed. 


\section{Attachment B \\ CsIX Feasibility Study \\ Equipment list for the Cure cell}

Inlet staging conveyor - SST, self powered rollers, $6 \mathrm{ft}$ long, 1200 Watt

Inlet staging conveyor enclosure with windows and access panels, $7 \mathrm{ga}$.

SST.

Drum lift - SST, $3 \mathrm{ft} \times 3 \mathrm{ft}, 4-\mathrm{ft}$ lift, $1.5 \mathrm{Hp}$.

Drum lift conveyor - SST, self powered rollers, $3 \mathrm{ft}$ long, $1200 \mathrm{Watt}$

Drum lift enclosure with windows and access panels, 7 ga. SST.

Cure line conveyor $-114 \mathrm{ft}$, self powered rollers and Chain driven

Conveyor - SST, self powered rollers, 2 ea. $-6 \mathrm{ft}$ long, 1200 Watt ea.

180-degree conveyor - SST, chain driven, 8 ea. $-4.5 \mathrm{ft}$ radius, $2 \mathrm{Hp}$ ea.

Conveyor - SST, chain driven, 8 ea. - $13 \mathrm{ft}$ long, $1 \mathrm{Hp}$ ea.

Cure line conveyor enclosure with windows and access panels, 7 ga. SST.

Drum lift - SST, $3 \mathrm{ft} \times 3 \mathrm{ft}, 4-\mathrm{ft}$ lift, $1.5 \mathrm{Hp}$.

Drum lift conveyor - SST, self-powered rollers, $3 \mathrm{ft}$ long, $1200 \mathrm{Watt}$

Drum lift enclosure with windows and access panels, $7 \mathrm{ga}$. SST.

Stage conveyor to elevator - SST, self powered rollers, $1.5 \mathrm{ft}$ long, 400 Watt

Drum elevator to $2^{\text {nd }}$ floor $-\mathrm{SST}, 3 \mathrm{ft} \times 3 \mathrm{ft}, 12-\mathrm{ft}$ lift, $2 \mathrm{Hp}$.

Drum elevator enclosure with windows and access panels, 7 ga. SST.

Drum elevator doors, $2^{\text {nd }}$ floor - motor driven, SST, $1 / 2 \mathrm{Hp}$. 
Receive drum from grout cell air lock

Stop conveyor when drum reaches lifting elevator

Verify elevator is vacant

Start conveyor and move drum into elevator and stop conveyor

Determine which cure line conveyor drum will go to

Lift drum to $2^{\text {nd }}$ cure line if required

Start conveyor and move drum onto cure line

Cure line conveyor transports drum for 24 hours

Stop conveyor when drum reaches lowering elevator

Verify elevator not in use

Start conveyor and move drum into elevator and stop conveyor

Lower drum to floor cure line conveyor if required

Start conveyor and move drum to $2^{\text {nd }}$ floor staging conveyor

Stop conveyor when drum reaches $2^{\text {nd }}$ floor elevator

Verify elevator is not in use

Start conveyor and move drum into $2^{\text {nd }}$ floor elevator and stop conveyor

Elevator lifts to $2^{\text {nd }}$ floor and stops

Open elevator doors on $2^{\text {nd }}$ floor

Start conveyor and move drum through doors and stop conveyor

Close elevator doors

Elevator returns to $1^{\text {st }}$ floor 


\section{Attachment D \\ CsIX Feasibility Study \\ Sketches and drawings}




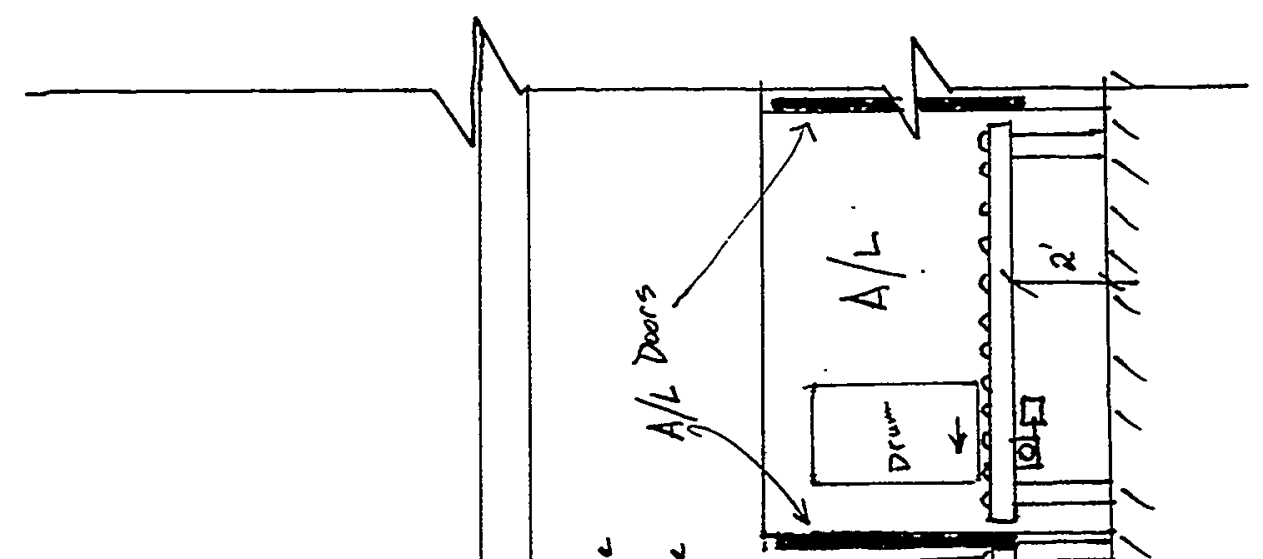

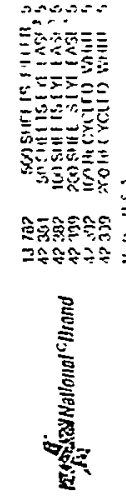

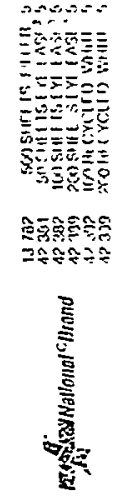
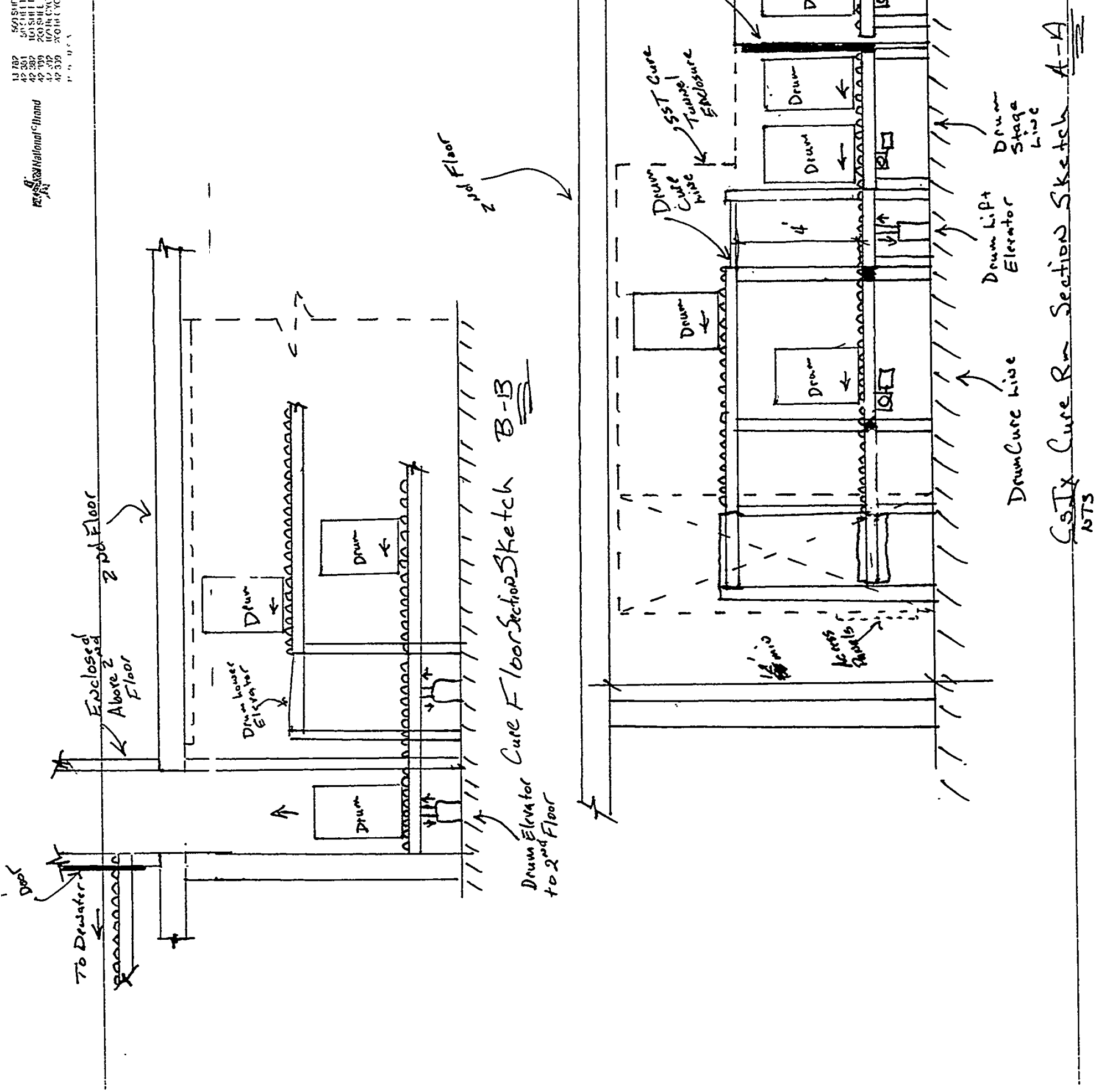
Project File Number $\quad$ 02BM1

Project/Task CsIX/TRU Grout Feasibility Study

Subtask D.4 Handling \& Storage

Title: Handling of Containers through the Dewatering Process

Summary:

This EDF describes the movement of the cured 55-gallon grout drums through the automated dewatering stations and the process by which it is accomplished. The dewatering process is an automated 4 hour period of water removal from the drum head space. The stations are described in addition to the sequencing the drums must go through. Dewatering must be completed before the drums are placed on a pallet and shipped in a covered flat bed truck to the interim storage facility. Option 1 and option 2 are very similar in operation and equipment requirements.

Differences are only in the layout due to the facility.

EDF Contents:

- Attachment A - Detailed Process Description w/Assumptions \& Requirements.

- Attachment $B$ - Equipment list for the Dewatering stations.

- Attachment $\mathrm{C}$ - Sequence of moving a Drum through the Dewatering area.

- Attachment D - Sketches

Distribution (complete package): C. W. Olsen MS 3211; K. L. Williams MS 3765; Project File; A copy of this EDF will be included in External Report No. INEEL/EXT $=98-00869$.

Distribution (summary package only): \begin{tabular}{l|l|l}
\hline Author & Dept. & Reviewed \\
\hline
\end{tabular} B. K. 4130 Grover

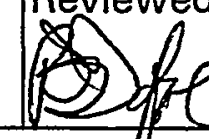




\author{
Attachment A \\ CsIX/TRU Feasibility Study
}

Transportation of 55 gallon drum containers through Dewatering

General

This EDF describes the method of dewatering the headspace of the 55-gallon drum containers after a 24-hour cure period in the CsIX/TRU grouting process. After passing through the Cure cell, any remaining water in the head space or void portion of the drum must be removed. WIPP acceptance requirements dictate that there should be no more than $1 \%$ liquid by volume in the drums. The cured drums may have residual moisture in the void space or head of the drum due to the lid being installed prior to the curing period.

\title{
Assumptions
}

1. Drum lid filters will not degrade passing water vapor.

2. Loading of drums to pallets after completion at the dewatering stations will be contact handled.

3. Lag storage of palletized drums will be on the second floor in the dewatering area and on the first floor load-out dock.

4. A 120 air change per hour (approximately 1 CFM) on the drum head space at 150 degrees $F(65.5 \mathrm{C})$ temperature is required for water removal. See attachment $D$ for supporting information.

5. Pallets of drums will be removed from the facility on a flat bed truck in numbers of 4 pallets or 16 drums. Loading, round trip to interim storage, and unloading will take 2 hours per trip. Three trips per day in covered flat bed truck to interim storage will be sufficient for drum production rates. See attachment $D$ for supporting information.

\section{Requirements}

1. A 4-hour dewatering period is called for from the process. See EDF-CsIX-001

2. The dewatering process must be an automated operation with operator oversight and override. 
3. 9 dewatering stations will be required. 8 stations for accepting a new drum every 30 minutes for 4 hours and 1 redundant or backup station.

4. After dewatering, the drums must be placed on a pallet in the facility for shipment to the interim storage facility.

5. There must be enough lag storage at the grouting facility to accommodate a one day production run of drums. This entails 38 drums or with 4 drums per pallet, 9-1/2 pallets of drums. Maximum pallet stacking height is two.

\section{Process Description}

The handling and dewatering system is fully automated with the attending operator having oversight and override capabilities. After the containers have been through the 24-hour cure period any residual moisture in the head of the drum will be removed as the drums pass through the dewatering stations.

As the drum enters the dewatering station area from the elevator doors it will stage on an indexing lift and rotation table. The drum lids contain two identical filters. The indexing table will locate the drum lid filters and position them for alignment in the dewatering stations. As the drum exits the elevator doors, the conveyor will stop it on the indexing table. The table will lift the drum up a predetermined height to an arm that has a roller located on the end of it. Once this roller has made contact with the drum lid, the drum will then be rotated. As the drum rotates, the roller will rotate on the top of the drum and when a filter is contacted the arm will pivot. This pivoting motion will be used to flag the filter location. Once the filter has been oriented, the drum will lower back to the conveyor line and move forward to one of the nine dewatering stations.

Once the drum has been oriented to enter the dewatering stations, a side lift conveyor will raise the drum to enter one of the vacant dewatering stations. After the drum has entered the dewatering station, it will travel until it reaches the back drum location support. After positioning in the dewatering station, a manifold will lower from above the drum. The manifold will consist of two 2 -inch lines with hoods that seal to the drum lid around the filters. One line will connect to the offgas system pulling air from the drum. The other line will contain electric resistance heaters and heat the air entering into the drum. Air will be taken from the space, heated, pulled through the drum head space, and into the off-gas system. See sketches in Attachment $E$ for more detail. After a 4-hour period in the station the drum will be shifted back onto the main conveyor line and moved to the end conveyor for loading onto a pallet. 
Once the drum has traveled to the end of the conveyor system it will be removed with a manually operated hydraulic drum lift and placed on a pallet. Drums will be stacked 4 to a pallet, see drawing $\# 449511$ for pallet details. The pallet will then stage to be lowered to the first floor on the loading dock elevator for shipping in a covered flat bed truck to the interim storage facility. The lag storage on the second floor will be 4 pallets. First floor lag storage will be 6 pallets. 


\section{Attachment B CsIX/TRU Feasibility Study \\ Equipment list for Dewatering stations}

Inlet indexing lift conveyor - SST, self powered rollers, $3 \mathrm{ft}$ long, 1200 watts Indexing lift table - SST, 18 inch diameter, $1.5 \mathrm{Hp}$ Indexing table rotation motor - $1 / 2 \mathrm{Hp}$ Indexing arm - Roller assembly arm, no power required

Dewatering station conveyor - SST, self powered rollers, $30 \mathrm{ft}$ long, 5600 watts 90-degree transfer and lift - SST, chain transfer, $3 \mathrm{ft}$ long, $1.5 \mathrm{Hp} \mathrm{ea.}$ Station conveyor - SST, self-powered rollers, $3 \mathrm{ft}$ long, 1200 watts Air heaters - electric heaters, 9 ea., 100 watts ea.

Dewater station line raise and lower -9 ea., $1 / 2 \mathrm{Hp}$ ea.

Drum load off conveyor - SST, self-powered rollers, $10 \mathrm{ft}$ long, 2200 watts 90 degree transfer and lift - SST, chain transfer, $3 \mathrm{ft}$ long, $1.5 \mathrm{Hp}$ Manual operated hydraulic drum lift 


\section{Attachment C \\ CsIX/TRU Feasibility Study \\ Sequencing of moving a Drum in/out of Dewatering station}

Receive drum from cure cell elevator doors

Stop drum over indexing table

Raise drum to indexing arm

Rotate drum to locate filters

Lower drum to conveyor

Determine dewatering station to receive drum and start conveyor

Stop conveyor at dewatering station

Raise drum on 90-degree chain transfer section

Start chain conveyor and self-powered roller conveyor

Stop conveyors when in position and lower chain transfer section

Lower dewatering manifold onto drum

Start dewatering process for 4 hours

Raise dewatering manifold

Raise chain transfer section and start both chain and roller conveyor systems

Move drum to main dewatering conveyor and stop conveyors

Lower chain transfer section

Start Main dewatering conveyor and move drum to end of conveyor

Stop conveyor on chain transfer section

Raise chain transfer section

Start chain transfer and load out conveyor

Move drum to end of load out conveyor and stop

Move drum to pallet

Fill pallet with 4 drums

Stage pallet to dock load out elevator

Load pallet into load dock elevator

Lower elevator to first floor dock

Stage pallet to be loaded into covered flat bed truck

Load pallet into covered flat bed truck

Ship to interim storage facility 


\section{Attachment D \\ CsIX/TRU Feasibility Study \\ Supporting calculations}

Drum head space dewatering:

Drums will be filled to $95 \%$ of their volume:

$$
\begin{aligned}
& \text { Total drum volume }=8.05 \mathrm{ft}^{3}\left(0.228 \mathrm{~m}^{3}\right) \\
& \text { Grout volume }=8.05 \mathrm{ft}^{3} \times 95 \%=7.65 \mathrm{ft}^{3}\left(0.216 \mathrm{~m}^{3}\right)
\end{aligned}
$$

Head space remaining is the difference of the two volumes divided by the area of the drum.

$$
\begin{aligned}
& \text { Volume }=8.05-7.65=0.4 \mathrm{ft}^{3} \\
& \text { Height }=(8.05-7.65) / 2.76=.14 \mathrm{ft}=1.73 \text { inches }(4.4 \mathrm{~cm})
\end{aligned}
$$

Assume 120 air changes per hour ventilation to remove the water.

$$
0.4 \mathrm{ft}^{3} \times 120 \mathrm{AC} / \mathrm{hr}=48 \mathrm{ft}^{3} / \mathrm{hr}=0.8 \mathrm{ft}^{3} / \mathrm{min}=0.37 \mathrm{l} / \mathrm{s}
$$

Heating required for $70 \mathrm{~F}$ to $150 \mathrm{~F}$ rise.

$$
\mathrm{Q}=0.92 \times 0.8 \times 80=58.8 \mathrm{Btu} / \mathrm{hr}=17.2 \text { watts }
$$

Assume that heating system will require back up and controls. Each system will be 50 watts, of which 2 will be required.

Drum transport from grout facility to interim storage facility:

Drum will already be placed on a pallet and be ready for loading into the cover flat bed truck. It is assumed the truck can hold 4 pallets of drums.

Loading of 4 pallets into truck at grout facility $-45 \mathrm{~min}$ Driving time between facilities - $30 \mathrm{~min}$ Unloading 4 pallets at interim storage facility $-45 \mathrm{~min}$

$$
\text { Total } 120 \mathrm{~min}(2 \mathrm{hr})
$$

Trips made per day assuming 8 hour working day.

Mobilization $\quad-2 \mathrm{hrs}$

Trips ( $3 @ 2 \mathrm{hr}$ ea.) - $6 \mathrm{hrs}$ - which is 12 pallets or 48 drums per day. 
Attachment $E$

CsIX/TRU Feasibility Study

Sketches and Drawings 


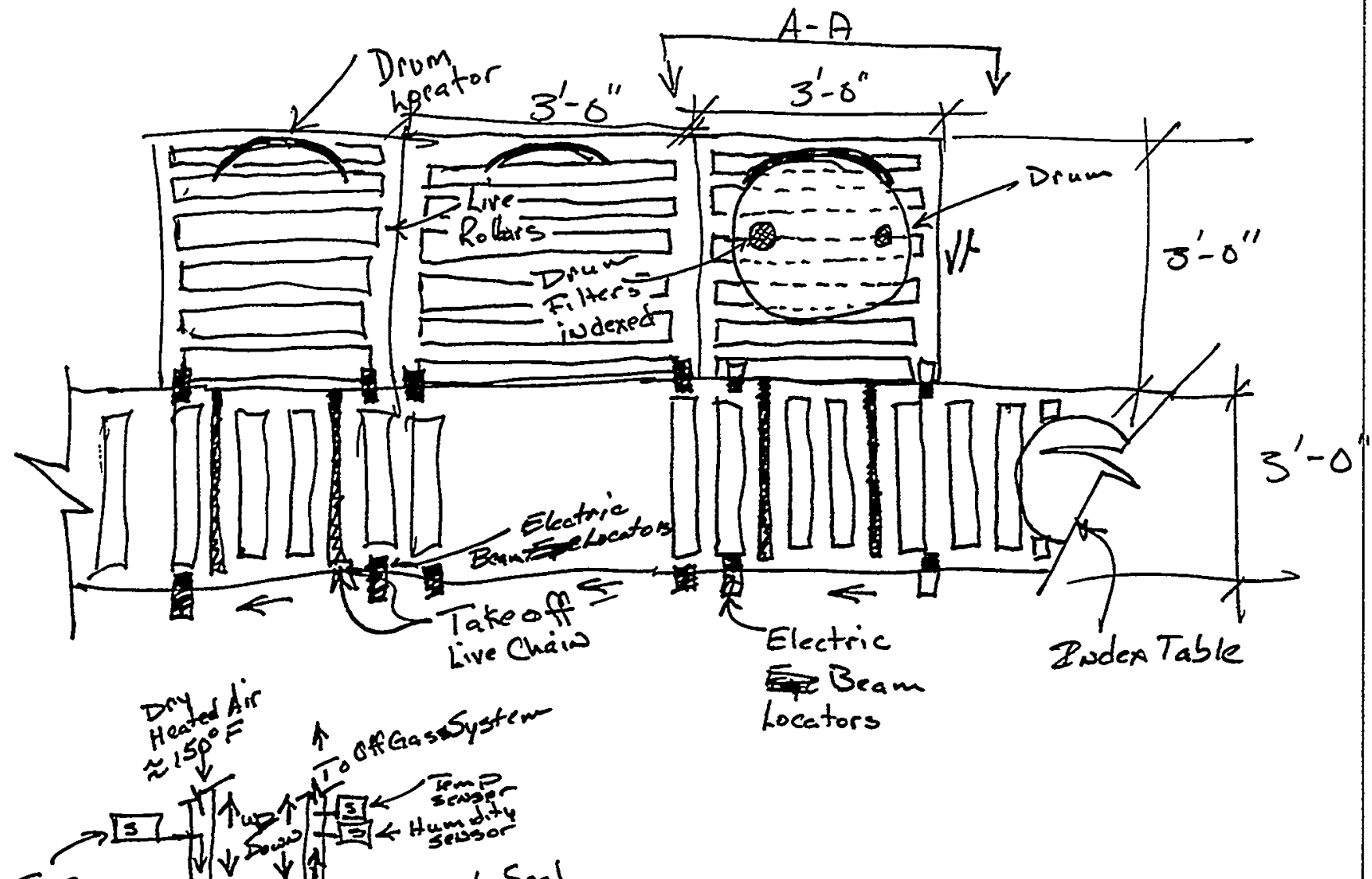

Tenpor

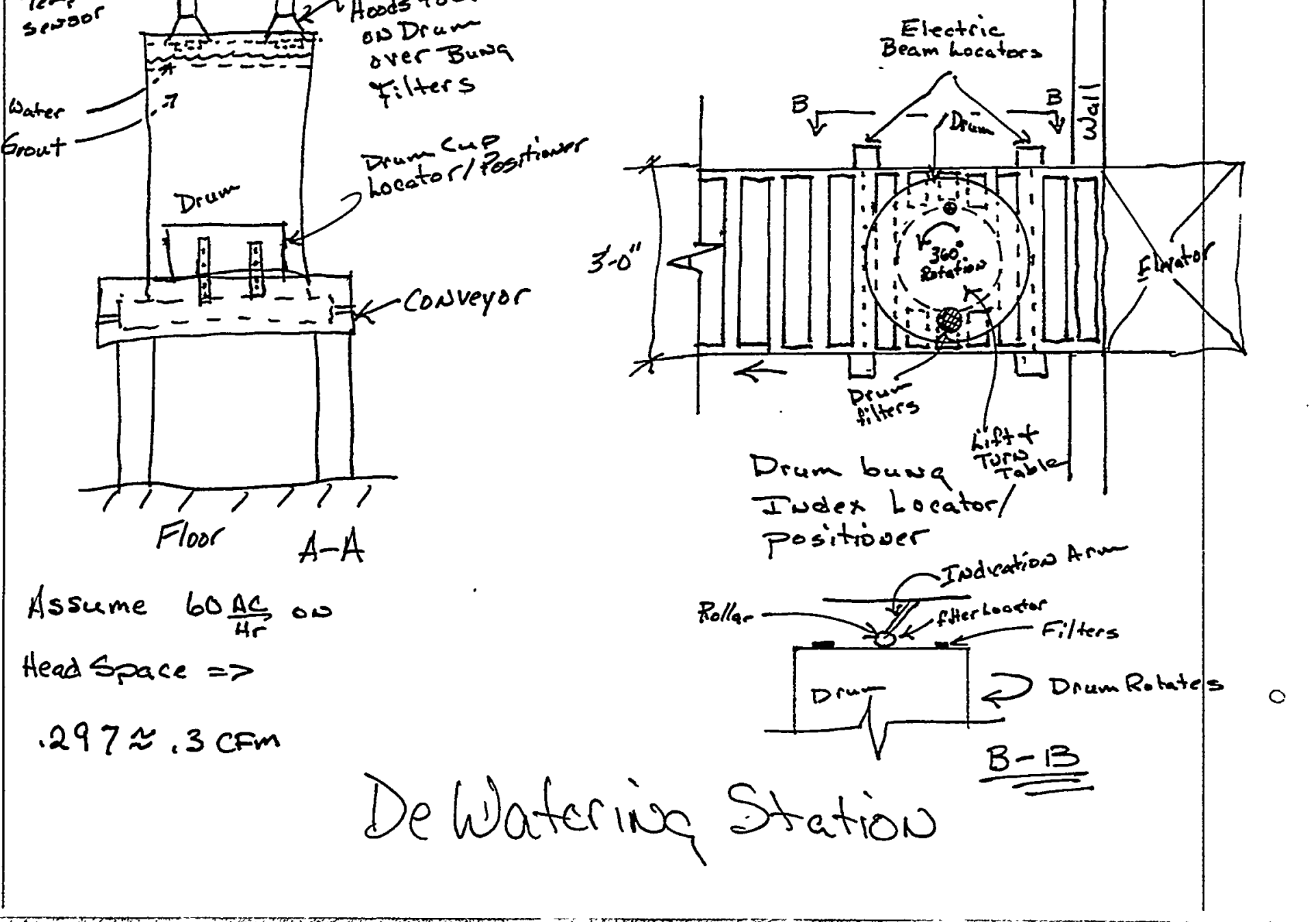




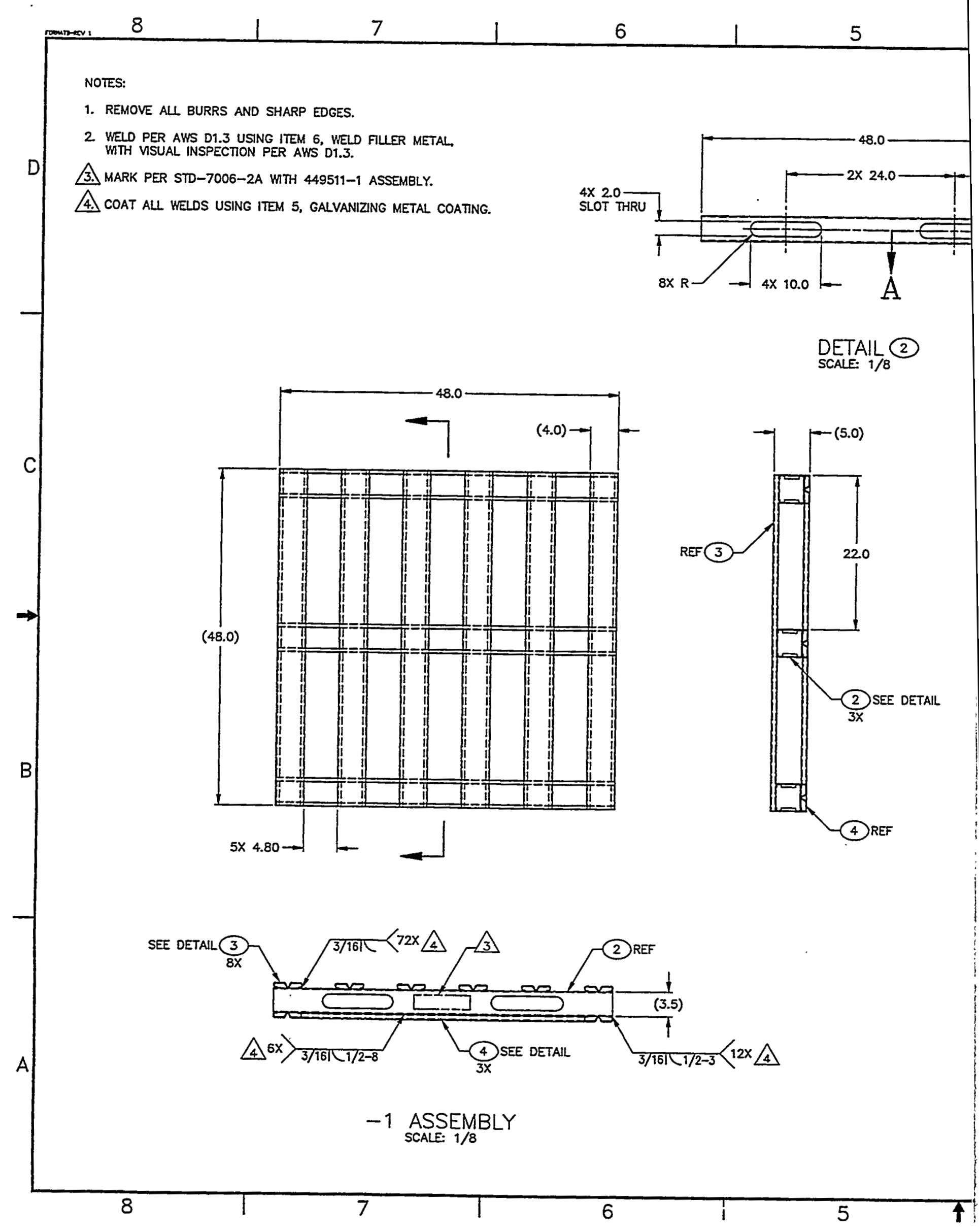




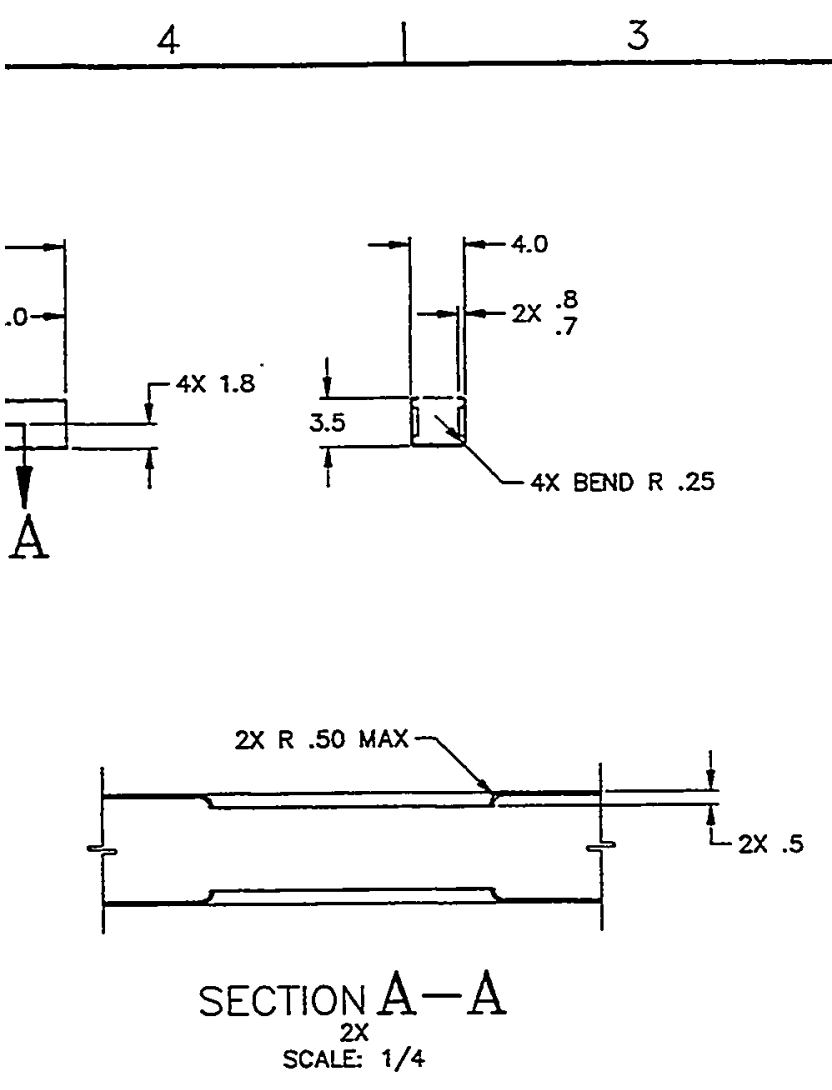

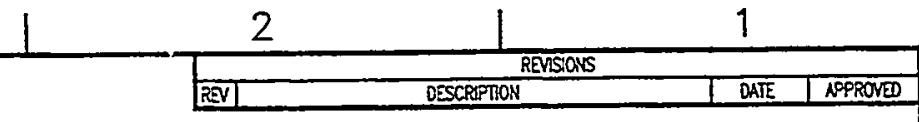

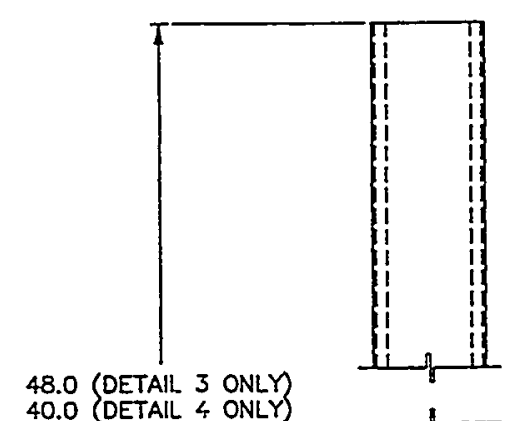

D

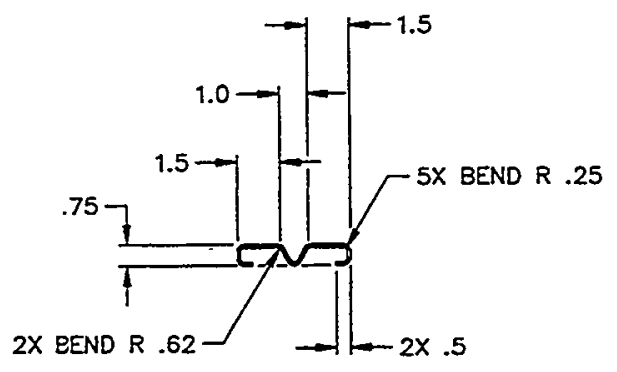

DETAIL $3(4)$

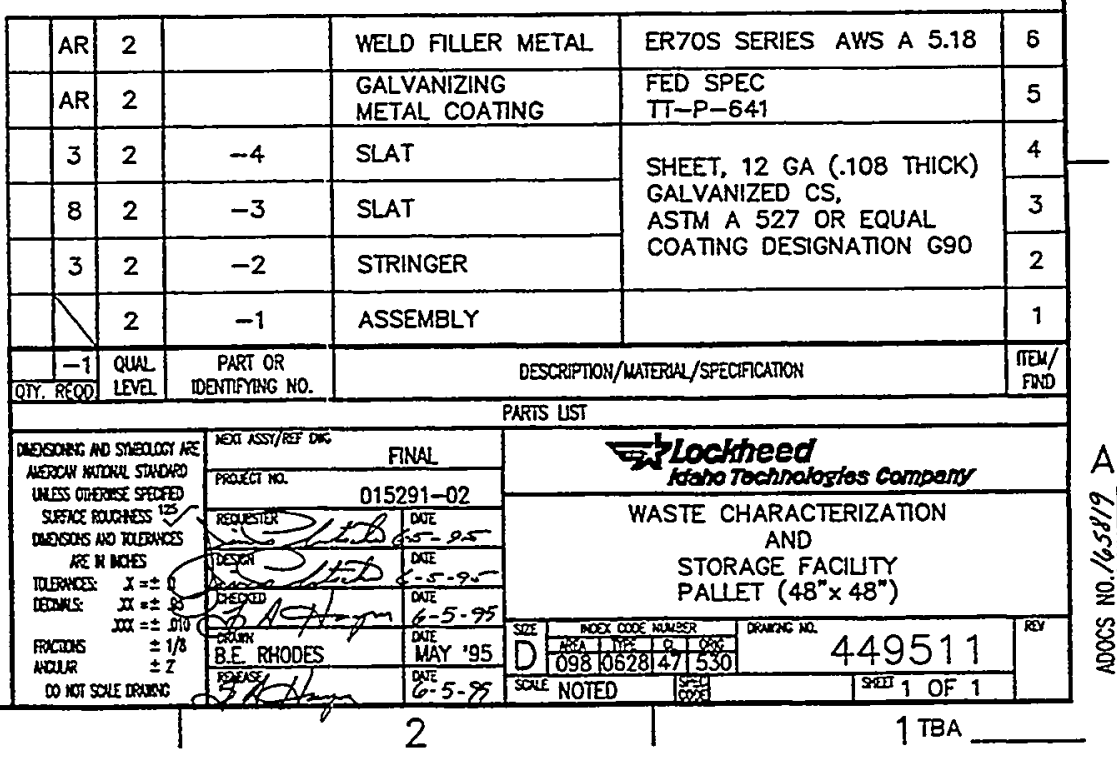


Project File Number $\quad$ 02BM1

Project/Task CsIX/TRU Grout Feasibility Study

Subtask D.4 Handling \& Storage

Title: UDS Drum Filling and Handling

Summary:

This EDF documents the requirements, assumptions, and description for the Undissolved Solids (UDS) drum filling and drum handling system. Both scenarios use the same configuration for UDS handling.

EDF Contents:

- Attachment A - Detailed Process Description w/Assumptions \& Requirements.

- Attachment B - Equipment list for the UDS Drum Filling and Handling System.

- Attachment $\mathrm{C}$ - Sequence of moving a Drum through the UDS Drum Filling and Handling system.

- Attachment D - Sketches and Drawings

Distribution (complete package): C. W. Oisen MS 3211; K. L. Williams MS 3765; Project File; A copy of this EDF will be included in External Report No. INEEL/EXT $=98-00869$.

Distribution (summary package only):

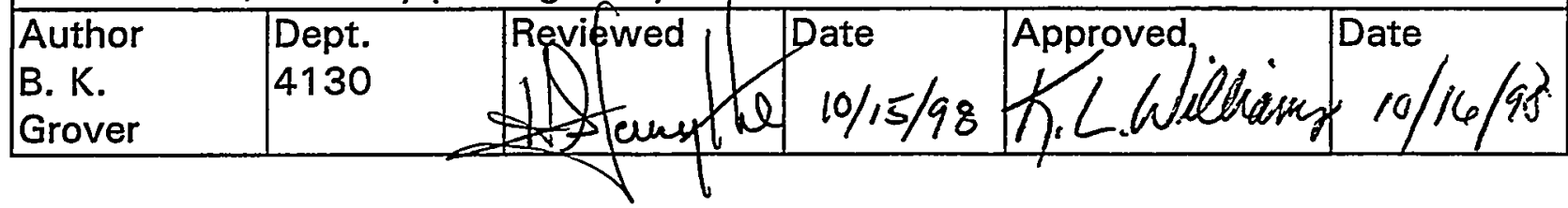




\author{
Attachment A \\ CsIX/TRU Feasibility Study
}

General

This EDF describes the process by which the UDS are conveyed into a 55-gallon $\left(0.2 \mathrm{~m}^{3}\right)$ drum and transported to a storage area within the grouting facility or NWCF. UDS produce a higher radiation field than the grout and require remote handling and special shielded drums. UDS will be stored in the new grouting facility or in the NWCF awaiting treatment from another project or shipment to WIPP.

\title{
Assumptions
}

1. Drums will be shielded to provide contact handling and filled weight will be less than $3000 \mathrm{lbs} .(1360 \mathrm{~kg})$.

2. After UDS drums have been filled, decontamination will allow contact handling in both NWCF and the grouting facility.

3. One UDS drum will be filled per month and exposure time periods to personnel will be low.

4. NWCF UDS storage area floor loading will not exceed capacity of the facility floor.

\section{$\underline{\text { Requirements }}$}

1. UDS drums must have no more than 200-mR/hr contact exposure. Shielding must be provided to maintain minimum exposure limits.

2. Decontamination accommodations must be provided for UDS drums after filling. Remote decontamination mechanisms and online counting will be required

3. UDS drum filling and handling must be a remotely operated automated system.

4. Transfer of the UDS into the drums must be a dust tight configuration for contamination control. 
The automated UDS drum handling system will be sequenced by a logical control system and be overseen by an attending operator. Drum movement into the decontamination booth and fill cell will be fully automated. Handling of the drum to the fill area from storage and back into the storage area will be by personnel. Lifting of a UDS drum onto the staging conveyor will be from an overhead crane. The drum will then be moved into the fill cell, filled, decontaminated in the booth, and then removed for personnel handling. The drum will then be stacked in the storage area of the grout facility or NWCF. The UDS will be disposed of by a future project or shipped to WIPP for permanent storage.

Approximately fifty 55 -gallon $\left(0.2 \mathrm{~m}^{3}\right)$ drums will be required through the life of the CsIX/TRU process, see EDF-CsIX-002. These drums are shielded to protect personnel from radiological exposure.

The lid/fill mechanism is a cone shaped lid that raises and provides an opening into the drum. This cone is air operated and opens the tube discharge cone holding the UDS. Engagement of the cones will be from a lift table.

The engagement of the drum cone to the discharge cone will occur when the fill cell lift table raises the drum a predetermined height. Once engaged, the UDS will be gravity fed into the drum. By design, the cone can be pulsed to dislodge or break up the UDS in the discharge tube. After the drum filled, the cone will close and the drum will lower from the fill cone. The drum will then be moved to the decontamination booth for clean up. After decontamination, the drum will be moved out to an area where personnel can move it to a storage area.

The process is very similar in both scenarios. The only variance is where the filled drums are stored. The filled drums will be stored and wait for processing by a future project or shipment to WIPP for permanent storage. Further development on the drums is recommended. 


\section{Attachment B \\ CsIX/TRU Feasibility Study \\ Equipment list for the UDS Drum Filling and Handling system}

Staging/Unloading conveyor - SST, chain driven, $4 \mathrm{ft}$ long, $1 \mathrm{Hp}$

Electric doors - Decontamination booth, SST, electric driven, $1 / 2 \mathrm{Hp}$

Decontamination Booth - shielded, SST

Rotation table - SST, $3 \mathrm{ft}$ diameter, $1 \mathrm{HP}$

Decontamination conveyor - SST, self powered rollers, $30 \mathrm{ft}$ long, 5600 watts

Decontamination Equipment - 1200 watts

Inspection Equipment - Area swipe remote mechanism, on-line counting, 300 watts

Electric doors - SST, electric driven, $1 / 2 \mathrm{Hp}$

Load out conveyor - SST, self powered rollers, $1.5 \mathrm{ft}$ long, 600 watts Lift table - SST, Air operated

Lift table conveyor - SST, self-powered rollers, $3 \mathrm{ft}$ long, 1200 watts

Drum dust tight discharge cone - SST, air operated

Shielded drum with dust tight inlet cone - SST inner liner with shielding material around exterior of drum - estimated 50 required over life of grout production run 


\section{Attachment C \\ CsIX/TRU Feasibility Study \\ Sequencing of moving a Drum through the UDS Drum Filling and Handling system}

Once a drum is moved into position from the storage area, a crane will be used to lift the drum onto the staging conveyor. Once on the staging conveyor, the stage conveyor will move the drum to the doors of the decontamination booth. Once the booth has been reached, the doors to the booth will open. As the doors open the staging conveyor will move the drum through the doors into the decontamination booth. Once inside the booth, the doors will close.

When the drum conveyor has stopped in the decontamination booth, a mechanical arm will drop from the ceiling of the booth and remove a shielded cap from the top of the drum fill cone. The cone will be placed to the side and reinstalled after the drum has been filled. Shielding of the fill cone is required due to the relatively high radiation field of the UDS. Once the cone shield has been removed, the doors to the filling cell will open and the decontamination booth conveyor will start and move the drum into the fill cell.

When the drum enters into the fill cell on the conveyor, it will be on top of a lifting table. Once the drum is in the predetermined position in the fill cell, the conveyor will stop and the doors to the decontamination booth will close. The lift table will raise the drum a predetermined height to a discharge cone. The discharge cone is on the end of a collection tube that the UDS evaporator is connected to. When the UDS evaporator discharges the UDS, particulates are collected in the tube with the filling cone. Once the cones on the drum and discharge tube had been engaged, the filling cone raises, pneumatically, and the UDS flow into the drum. The filling process is dust tight and no outside surfaces of the drum are exposed to the UDS.

When the UDS drum is full, measured by weight from the lift table, the fill cone will be lowered. The discharge tube and UDS drum are thus closed. The drum will be lowered back to the conveyor plane and is ready to be moved into the decontamination booth for clean up and reinstallation of the cone shielding.

After the lift table is lowered to the conveyor line, the doors to the decontamination booth will open. The conveyor will start and move the UDS drum into the decontamination booth. Once in the booth, the conveyor will stop and the doors to the booth from the fill cell will close. The decontamination process will now start.

Areas of concern and the fill cone will be swiped with the mechanical arm located in the decontamination booth. The swipe will then be placed into an on-line counter and determined if contamination is present. The area can be cleaned with 
the mechanical arm or if deemed necessary, the entire drum can be washed down with decontamination fluid. If decontamination fluid is used, it will be collected at the bottom of the booth and gravity fed to the waste collection system and routed back through the CsIX/TRU grout process. When the level of cleanliness has been reached, the mechanical arm will reinstall the cone shield enclosure.

After installation of the cone shield, the doors to the decontamination booth will open and the decontamination booth conveyor will start. The conveyor will move the drum through the doors onto the staging conveyor and stop. The doors to the decontamination booth will close and the drum is ready for removal from the staging conveyor. The drum is now contact handled to storage.

The overhead crane will lift the drum from the staging conveyor to a pallet. The pallet will be lifted by pallet jack and taken to the temporary storage in the grout facility or stacked with the overhead crane in the filling area of NWCF. 
431.02

$06 / 17 / 97$

Rev. \#04

\section{ENGINEERING DESIGN FILE}

\section{Attachment D \\ CsIX/TRU Feasibility Study \\ Sketches and Drawings}

Functional File Number - SPR-CsIX-06

EDF Serial Number - EDF-CsIX-008

Page 1 of 1 


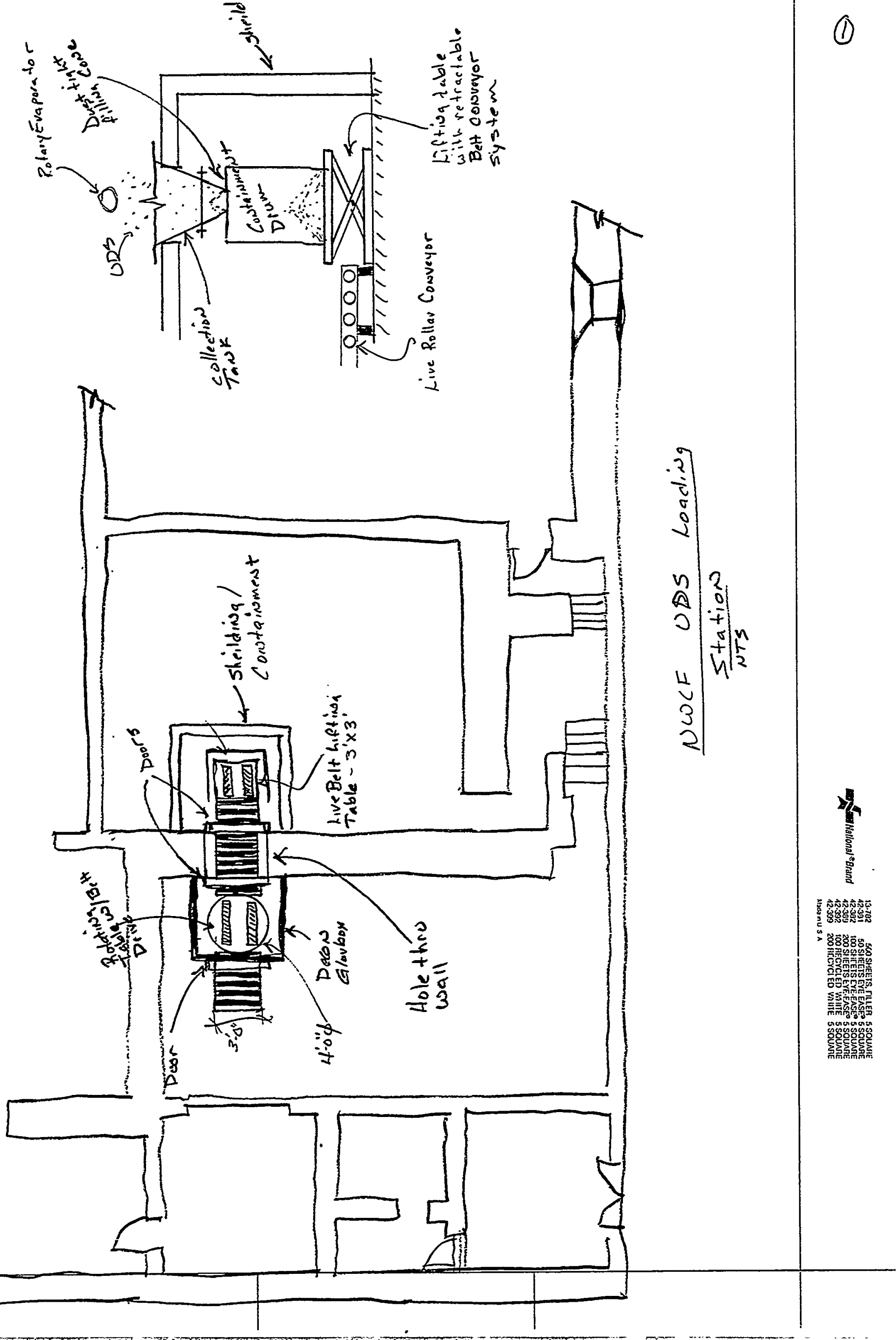


Project File Number $\quad$ O2BM1

Project/Task CsIX/TRU Grout Feasibility Study

Subtask Equipment Installation in NWCF

Titie: Installation of CsIX Equipment in NWCF

Summary:

This EDF evaluates the feasibility of installing the CsIX filtration and cesium removal processes within existing cells at the NWCF. The evaluation revealed the CsIX equipment can be installed in the calciner cell, adsorber cell, valve cubicle and hot sump tank removal cell.

\section{EDF Contents:}

- Attachment A - Evaluation of NWCF Hot Cell Space and Equipment Lay-out

- Attachment B - Equipment List

- Attachment C - Shielding Requirements

- Attachment D - NWCF Cell Radiological Filed Estimates

- Attachment E - NWCF Lay-out Drawings

Distribution (complete package): H.S. Forsythe, K. L. Williams, C. W. Olsen

Distribution (summary package only): J. J. McCarthy

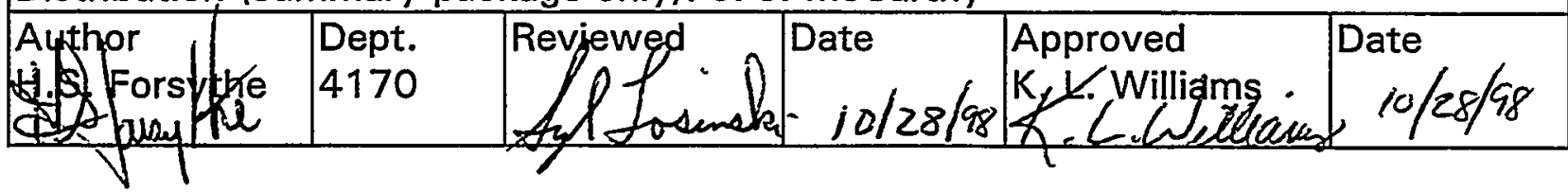




\author{
Attachment A \\ CsIX/TRU Feasibility Study \\ Evaluation of NWCF Hot Cell Space and Equipment Lay-out
}

\title{
Introduction
}

The CsIX/TRU Grout Feasibility Study investigates treatment of tank farm liquid waste via cesium removal followed by grouting into 55-gallon drums. The study examines two facility layout scenarios: one scenario constructs new buildings to house the processes; the second scenario constructs buildings for the grouting process leaving the most radiologically hot processes to be housed in the New Waste Calcining Facility (NWCF). This EDF evaluates the feasibility of placing the "hot" processes of filtration and cesium removal within existing cells at the NWCF.

\section{CsIX Equipment}

Based on the process defined in EDF-CsIX-001, five systems are required to accomplish the filtration and cesium removal steps. The five systems include solids processing, cesium ion exchange, waste water collection, process off-gas, and bulk chemical storage. NWCF equipment will be utilized as much as possible to reduce the number of new items installed in the building. The following tables identify the systems, the equipment required for each system, and NWCF equipment that can be substituted for required equipment.

Solids Processing - The solids filtration system includes provisions for accepting waste from the tank farm, filtering the liquid waste prior to introduction to the ion exchange columns, and drying and packaging the undissolved solids collected during filtration. The table below identifies the required equipment and the NWCF equipment that can be utilized.

\begin{tabular}{|c|c|c|}
\hline $\begin{array}{c}\text { Equipment } \\
\text { Number }\end{array}$ & Description & NWCF Equipment \\
\hline T-101-A,B & Liquid Waste Feed Tank & VES-NCC-102, -103 \\
\hline P-101-A,B & Liquid Waste Feed Pump & None \\
\hline F-101-A,B & UDS Filter & None \\
\hline T-108 & UDS Slurry Tank & None \\
\hline EV-101 & UDS Rotary Evaporator & None \\
\hline E-101 & UDS Evaporator Condenser & None \\
\hline P-110 & Condensate Pump & Gravity \\
\hline P-107 & Evaporator Feed Pump & None \\
\hline N/A & Drum Loading Station & None \\
\hline
\end{tabular}

The filtration system requires twelve components. VES-NCC-102 and -103 perform the same function as T-101-A, B; therefore they can be substituted for the liquid feed tanks. The evaporator condensate can flow by gravity to the 
wastewater collection tank, VES-NCC-119, thereby eliminating the need for a condensate pump.

Cesium Removal and Resin Drying- The cesium removal system includes provisions for removing cesium using three ion exchange columns, sluicing resin into and out of the ion exchange columns, and drying and storing the spent resin. The table below identifies the required equipment and the NWCF equipment that can be utilized.

\begin{tabular}{|l|c|c|}
\hline $\begin{array}{c}\text { Equipment } \\
\text { Number }\end{array}$ & Description & NWCF Equipment \\
\hline $\mathrm{V}-104-\mathrm{A}, \mathrm{B}, \mathrm{C}$ & CsIX Columns & None \\
\hline $\mathrm{T}-103-\mathrm{A}, \mathrm{B}$ & CsIX Effluent Tank & None \\
\hline $\mathrm{P}-103-\mathrm{A}, \mathrm{B}$ & CsIX Effluent Pump & None \\
\hline $\mathrm{P}-112$ & Spent Cs Resin Pump & None \\
\hline $\mathrm{V}-105$ & Resin Prep Tank & VES-NCM-120 \\
\hline $\mathrm{P}-114$ & Resin Transfer Pump & P-NCM-220 \\
\hline V-106 & Spent Resin Prep Tank & None \\
\hline $\mathrm{P}-104-\mathrm{A}, \mathrm{B}$ & Spent Resin Sluice Pump & None \\
\hline $\mathrm{F}-102$ & Resin Fines Filter & None \\
\hline $\mathrm{D}-102$ & Cs Resin Drier & None \\
\hline $\mathrm{E}-102$ & Resin Drier Condenser & None \\
\hline $\mathrm{P}-113$ & Condensate Pump & Gravity \\
\hline $\mathrm{T}-102$ & Spent Resin Storage Tank & None \\
\hline
\end{tabular}

The cesium removal system requires eighteen components. The new resin can be prepared in VES-NCM-120 and pumped to the ion exchange columns using P-NCM220. The drier condensate can flow by gravity to the wastewater collection tank, VES-NCC-119, thereby eliminating the need for a condensate pump.

Waste Water Collection - The waste water collection system includes provisions for collecting waste water and transferring it to a treatment process (PEW, HLLWE, etc.). The table below identifies the required equipment and the NWCF equipment that can be utilized.

\begin{tabular}{|c|c|c|}
\hline $\begin{array}{c}\text { Equipment } \\
\text { Number }\end{array}$ & Description & NWCF Equipment \\
\hline & & \\
\hline$T-111$ & Waste Water Tank & VES-NCC-119/122 \\
\hline P-111 & Waste Water Pump & JET-NCC-519 \\
\hline
\end{tabular}

The waste water collection system requires two components. The hot sump tanks, VES-NCC-119, -120 , can be used as the waste water collection tanks and JETNCC-519 and -520 can be used in place of the waste water pump. 
Process Off-gas - The process off-gas system provides off-gas for all tanks and vessels. The process off-gas system includes a condenser, demister, superheater, prefilter, HEPA filter and blower. The table below identifies the required equipment and the NWCF equipment that can be utilized.

\begin{tabular}{|l|c|c|}
\hline \multicolumn{1}{|c|}{$\begin{array}{c}\text { Equipment } \\
\text { Number }\end{array}$} & Description & NWCF Equipment \\
\hline E-103 & & \\
\hline V-101 & Off-gas Condenser & HE-NCC-336 \\
\hline EH-101 & Off-gas Demister & VES-NCC-136 \\
\hline F-103 & Off-gas Superheater & HE-NCC-335 \\
\hline F-104-A,B & Prefilter & F-NCC-130 \\
\hline BL-101-A,B & HEPA Filter & F-NCC-130 \\
\hline
\end{tabular}

The process off-gas system requires eight components. The NWCF Vessel Off-gas (VOG) and Process Off-gas (POG) systems can be used, thus eliminate the need for any additional equipment.

Bulk Chemical Storage - The bulk chemical storage system provides bulk storage for decontamination chemicals and demineralized water. The table below identifies the required equipment and the NWCF equipment that can be utilized.

\begin{tabular}{|c|c|c|}
\hline $\begin{array}{l}\text { Equipment } \\
\text { Number }\end{array}$ & Description & NWCF Equipment \\
\hline & & \\
\hline B-101 & Cs Resin Storage & None \\
\hline$T-109$ & Decon Chemical Storage & VES-NCM-117 \\
\hline$T-110$ & Decon Chemical Storage & VES-NCM-118 \\
\hline$T-102$ & DI Water Tank & None \\
\hline P-108 & Decon Chemical Pump & P-NCM-217 \\
\hline P-109 & Decon Chemical Pump & P-NCM-218 \\
\hline P-102-A,B & DI Water Pump & None \\
\hline
\end{tabular}

The bulk chemical storage system requires eight components. The NWCF decon tanks and pumps can be used for chemical storage. A new DI water tank and pump will be installed in room 429, as shown on drawing 2-M6, to supply DI water to the process. The cesium resin storage bin will be eliminated and instead of bulk shipments, resin will be received in 50-pound bags.

Based on the information presented in the tables above, eighteen pieces of NWCF equipment can be used, leaving twenty-seven major pieces of CsIX equipment to be installed. The NWCF scenario equipment list in included in Attachment B.

\section{NWCF Facility Requirements and Assumptions}

The installation of CsIX equipment will take advantage of existing NWCF infrastructure, which includes building utilities, HVAC, and off-gas systems. This 
EDF assumes these systems are adequate and that no changes or upgrades are needed to accommodate the new CsIX equipment.

However, modifications are required to the NWCF in order to accommodate the CsIX equipment. This EDF assumes the calcining process will no longer be needed and that calciner operability can be degraded as a result of new equipment installation. However, the following systems are needed, and their operational status cannot be degraded.

- High Level Liquid Waste Evaporator (HLLWE)

- Filter Leach System

- Decontamination Facility

- Building HVAC

- Vessel Off-gas

- Waste Water Collection

- Pneumatic Transfer System

In addition to the facility constraints listed above, the CsIX equipment has the following constraints that must be met.

- Valves, pumps and other mechanical equipment should have remote maintenance and operating capability.

- The UDS container storage location must have the capability to remotely manage and store up to fifty 55 gallon drums

- The ion exchange columns, spent resin prep tank, spent resin drier, spent resin storage tank, UDS slurry storage tank and UDS drier must have adequate shielding to protect operating personnel.

- The spent resin storage tank must have access to the NWCF pneumatic transfer system.

Sufficient hot cell space, meeting all of the constraints listed above, was identified in the adsorber cell, calciner cell, valve cubicle and hot sump tank removal cell.

\section{Cell Descriptions}

Adsorber Cell - The adsorber cell has approximately 800 square feet of floor space and is 34 feet high. The cell houses three ruthenium adsorbers, the adsorber superheater, the process off-gas reheater, the off-gas mist collector, the vent condenser, and the vent condenser knock out drum. If the adsorbers are removed, the cell will provide space to house the following CsIX equipment. 


\begin{tabular}{|c|c|}
\hline $\begin{array}{l}\text { Equipment } \\
\text { Number }\end{array}$ & Description \\
\hline & \\
\hline $\mathrm{V}-104-\mathrm{A}, \mathrm{B}, \mathrm{C}$ & CsIX Columns \\
\hline $\mathrm{T}-103-\mathrm{A}, \mathrm{B}$ & CsIX Effluent Tank \\
\hline $\mathrm{F}-101-\mathrm{A}, \mathrm{B}$ & UDS Filters \\
\hline $\mathrm{T}-108$ & UDS Slurry Tank \\
\hline $\mathrm{EV}-101$ & UDS Rotary Evaporator \\
\hline $\mathrm{E}-101$ & Evaporator Condenser \\
\hline $\mathrm{E}-103$ (HE-336) & Off-gas Condenser \\
\hline V-101 (VES-136) & Off-gas Demister \\
\hline EH-101 (HE-335) & Off-gas Superheater \\
\hline None & UDS Drum Loading \\
\hline
\end{tabular}

The demolition of the adsorber cell, shown on drawing 2-M2, will include removing all processing equipment in the cell, including the off-gas reheater (HE-335), vent condenser (HE-336) and vent knock out drum (VES-136). These off-gas components are vital components of the off-gas system and will be redesigned and re-installed in the adsorber cell during the construction phase.

As shown on drawings 2-M3 and 2-M5 section $\mathrm{C}$, the ion exchange columns will be mounted on the adsorber cell north wall near the ceiling, which will provide access to the valve cubicle for positioning of new valves. The contact radiation reading for a fully loaded resin column is estimated at $2,700 \mathrm{R} / \mathrm{hr}^{1}$. The existing shielding is adequate to reduce the body field in occupied areas to less than $0.1 \mathrm{mR} / \mathrm{hr}^{2}$.

The UDS drum filling station, shown on 2-M3, will be located adjacent to the adsorber cell west wall with an opening in the wall to allow UDS containers to pass between the hot sump tank removal cell and the adsorber cell. The filling station will be housed within a glove box enclosure (shown on 2-M4 section A) to keep the UDS containers free of external contamination. The containers will be shielded to provide contact handling, and the container filled weight will be less than 3000 lbs. Each container will hold $7.35 \mathrm{ft}^{3}$ of solids, which, based upon the anticipated solids listed in the material balance in EDF-CsIX-001, translates into approximately 50 containers. The UDS drum filling station is explained in further detail in EDFCsIX-008.

The UDS evaporator will be located above the drum loading station, and when filled with solids, will have a contact radiation reading of approximately $48 \mathrm{R} / \mathrm{hr}^{3}$.

The UDS evaporator can either be repaired remotely using the overhead $\mathrm{PaR}$ and crane or by hands-on maintenance. In either case, the radiation field in the cell must be reduced prior to maintenance activities. This is accomplished by removing solids from the evaporator and drum loading station, transferring ion exchange column resin out of the cell, and decontaminating process vessels.

1 EDF-CsIX-015,

2 Attachment B

${ }^{3}$ EDF-CsIX-015, 
Calciner Cell - The calciner cell has approximately 650 square feet of floor space and is about 34 feet high. The calciner vessel occupies the west half of the cell with the east half remaining relatively free of equipment. This open area to the east is an ideal location for the spent resin storage tank and provides clear access to the pneumatic transport line. In addition, with over 30 feet of cell height, the resin drier can be located above the storage tank with the spent resin prep tank located above the drier. This arrangement, as shown on 2-M4 section $B$, allows the resin to move by gravity from the prep tank to the storage tank. The proposed layout calls for the resin to be dewatered, dried and stored while the sluice water is filtered and returned to NCC-119. To accomplish these tasks the following equipment will be installed in the claciner cell.

\begin{tabular}{|c|c|}
\hline $\begin{array}{l}\text { Equipment } \\
\text { Number }\end{array}$ & Description \\
\hline & Spent Resin Prep Tank \\
\hline V-106 & Resin Drier \\
\hline E-102 & Resin Drier Condenser \\
\hline T-112 & Spent Resin Storage Tank \\
\hline P-104-A,B & Spent Resin Sluice Pump \\
\hline F-102 & Resin Fines Filter \\
\hline
\end{tabular}

The contact radiation reading at the spent resin storage tank, when full, is estimated at $5,000 \mathrm{R} / \mathrm{hr}^{4}$. The shielding required to achieve $0.1 \mathrm{mR} / \mathrm{hr}$ outside the cell is calculated to be 38 inches of concrete. Since the calciner cell wall thickness is 57 inches, the cell walls will provide adequate shielding.

Remote maintenance will be performed on the resin drier, sluice water pumps and fines filter using the overhead PaR and crane. The PaR and crane gain access to the cell through the cell hatches, and the radiation field at the top of the cell must be less than $200 \mathrm{mR} / \mathrm{hr}$ when the cell hatches are removed. This will be accomplished by enclosing the spent resin storage tank with concrete shielding walls measuring 24 inches as shown on 2-M3 and 2-M4 section $B$.

Valve Cubicle - The valve cubicle is about 60 feet long, 11.5 feet wide, and 34 feet high. High-maintenance valves for the incoming feed line, the blend and hold tanks, and the adsorber cell are located in this cubicle. Adsorber cell valves associated with the adsorber off-gas lines and drain lines will be removed. Drawing 2-M2, detail A, highlights the valves to be removed. The free wall space will be used for new valves and pumps affiliated with the filtration and Cs removal operations. The table below lists the pumps and the approximate number of valves to be located in the valve cubicle.

${ }^{4}$ EDF-CsIX-015,

${ }^{5}$ Attachment B 


\begin{tabular}{|c|c|}
\hline $\begin{array}{l}\text { Equipment } \\
\text { Number }\end{array}$ & Description \\
\hline & \\
\hline P-101-A,B & Liquid Waste Feed Pump \\
\hline P-107 & UDS Evaporator Feed Pump \\
\hline P-103-A,B & CsIX Effluent Pump \\
\hline P-112 & Spent Resin Transfer Pump \\
\hline Valves & $\sim 30$ \\
\hline
\end{tabular}

Following demolition, approximately 648 square feet of wall space is available for new valves. According to Rick Lane with Specialty Engineering (remotes), each valve requires 25 square feet. Therefore, approximately 25 valves can be placed in the valve cubicle. The other valves must be installed in-cell with remote maintenance capabilities.

Hot Sump Tank Removal Cell - This cell has 780 square feet of floor space, is 35 feet high and contains no equipment. Presently, spent/used NWCF HEPA filters are stored in this cell, which would be removed prior to construction activities. The cell is contaminated but can be cleaned to low levels.

UDS containers will pass through the wall between the hot sump tank removal cell and the adsorber cell into an enclosed decontamination glovebox. The UDS containers will be surveyed, decontaminated, and then stored in the cell. A 5-ton crane will be installed in the cell to move and stack the 2,000-pound containers. If the containers are stacked two high, approximately 200 square feet of floor space will be needed. The cell layout is shown on Drawing 2-M3 and 2-M4, detail A.

\section{Process Description}

Waste is transferred to the NWCF using the existing Tank Farm transfer system and is received in one of two blend tanks, VES-NCC-102 or -103, in the blend and hold cell. From the blend tanks, the waste passes through the north wall into the valve cubicle. The waste is then pumped to the UDS filters in the adsorber cell using one of two liquid waste feed pumps, P-101-A or $-B$, located in the northeast corner of the valve cubicle. From the filters, the waste enters one of three ion exchange columns mounted on the north wall near the ceiling of the adsorber cell. From the lead column, the waste then enters the top of the lag column exiting at the bottom to one of two CsIX effluent tanks, also located in the adsorber cell. When the effluent tank is full, the contents will be pumped to valve box B-11 and on to the grouting facility through a new transfer line.

When break-through is observed in the lead cesium ion exchange column, the column is taken off line and the resin removed and replaced with fresh resin. Deionized water is added to the column at the ratio of 10 water volumes per resin volume. The resin/water mixture exits the column and passes through the adsorber 
cell north wall into the valve cubicle to the spent resin sluice pump, P-112. The resin is then pumped from the valve cubicle to the calciner cell via the second level mezzanine and flow meter cubicle. In the calciner cell, the resin enters the spent resin prep tank, V-106, and is dewatered. The sluice water is pumped, P-104-A, B, through the resin fines filter, F-102, and back to VES-NCC-119. The dewatered resin is fed to the resin drier, D-102, and collected in the spent resin storage tank, T112. At a later date, the resin will be transported using the NWCF pneumatic transport line to the calcine bin sets or to a vitrification facility. The spent resin storage tank, when filled, will have a contact radiation reading of approximately $5,000 \mathrm{R} / \mathrm{hr}$.

New resin is received in 50 pound bags and stored in the calcium nitrate make up room, 427. Resin is mixed with water and prepared for use in VES-NCC-120. When a column is ready, fresh resin is pumped from VES-NCC-120 to the column using PNCC-220. The sluice water will drain by gravity from the column to VES-NCC-119. Once filled with fresh resin, the column is held in reserve until needed.

When the UDS filter pressure drop reaches a predetermined level, the filter is taken off-line and back flushed with deionized water. The flush is collected in the UDS slurry tank, T-108, located in the adsorber cell. When the UDS evaporator is operating, the slurry leaves $T-108$ and passes through the adsorber north wall into the valve cubicle to the evaporator feed pump, P-107. From the feed pump, the slurry is fed to the UDS evaporator, EV-101, located in the adsorber cell. The dried solids flow by gravity from the evaporator into a discharge tube. When full, a UDS container is lifted into position and filled. The container is then passed through the adsorber cell west wall into the hot sump tank removal cell. The container is surveyed, decontaminated and stored in the hot sump tank removal cell.

\section{Waste Management}

Waste generated during demolition of the adsorber cell and valve cubicle will be classified as mixed hazardous and will consist of silica gel, piping, adsorber vessels, and valves. According to Waste Generation Services ${ }^{6}$, the waste can be legally generated and would normally be subject to the one year LDR storage (40CFR268.50) prohibition. However, the one-year prohibition can be by-passed if the waste is included in the Site Treatment Plan maintained by Waste Generation Services.

Any waste generated that exceeds a $60 \mathrm{~mm}$ particle size can be classified as debris. Debris can be rendered nonhazardous provided it is rinsed for 15 minutes (40 CFR 268.45 Table One Item A-2) and passes a visual inspection for staining (clean debris surface inspection). In addition, the waste must be sized in a manner that will allow visual inspection of interior surfaces. Debris treatment must take place within 90 days of waste generation; otherwise the treatment process requires a RCRA permit.

${ }^{6}$ Conversation with Tom Shea on August 31, 1998. 
Unfortunately, the silica gel particle size is less than $60 \mathrm{~mm}$, and therefore, can not be classified as debris and must be disposed of as mixed hazardous waste.

All projected liquid waste from decontamination and debris treatment must be included in the Waste Minimization Plan ${ }^{7}$ before it can be processed through the PEW evaporators.

\section{NWCF Facility Concerns/lssues}

The following items should be considered when contemplating the installation of CsIX equipment in the NWCF.

a) The fundamental advantage of housing new processes in the NWCF is the possibility of utilizing the existing NWCF structure, shielding, access to tank farm feed, utilities, chemical makeup, etc. With the NWCF and its equipment nearly 20 years old, finding spare parts and maintaining the aging equipment is becoming difficult. Spare parts are difficult to obtain, since many manufacturers no longer produce the equipment models installed in the NWCF.

b) Previous INTEC experience with installing new processes in existing facilities has shown that cell penetrations are seldom in the correct locations nor are there sufficient penetrations for the new process. The scope of this problem is usually not fully understood until late in the title design phase causing escalation in the design and construction cost estimates.

c) The building was designed for NWCF remote operations and not for CsIX operations. The cell windows, PARs and masterslaves may not be in the proper locations to provide for truly remote activities.

d) The UDS may contain a high percentage of U-235. Concentrating the solids through evaporation could pose a criticality hazard and the equipment selection and layout did not consider criticality issues.

e) Since new equipment will be installed in existing cells and some existing equipment will be utilized, system operability (SO) testing will be conducted in a contaminated facility with contaminated equipment. As a result, the testing process will be more complicated, last longer, and be more expensive.

f) Design philosophy at the INTEC has been to minimize the number of mechanical items (i.e. valves, pumps, etc.) in cell. The CsIX process requires several mechanical items, and the NWCF building scenario leaves few options for equipment placement. As a result, several pieces of mechanical equipment were located in cell in direct violation of this design philosophy.

g) The CsIX layout calls for spent resin to be sluiced approximately 100 feet horizontally and 30 feet vertically from the adsorber cell to the top of the calciner cell. Such a transfer is possible, however questions surround its feasibility.

h) Radiation fields in excess of $100 \mathrm{R} / \mathrm{hr}$ raise questions concerning the reliability and life span of electrical and mechanical components.

${ }^{7}$ Conversation with Juile Tripp on September 1, 1998 
i) NWCF filters are being stored in the hot sump tank removal cell while waiting to be processed through the filter leach process at the rate of $0-100$ filters per year. The filters must be removed and leached prior to construction, otherwise an alternate storage location must be found.

\section{ALARA Estimate}

The table below lists the in-cell construction hours and general body field estimates ${ }^{8}$ for both demolition and construction activities. The total estimated exposure for installing equipment in the NWCF is 1,528 R.

\begin{tabular}{|c|c|c|c|c|c|c|}
\hline CELL & $\begin{array}{l}\text { DEMOLITION } \\
\text { (Hours) }\end{array}$ & $\begin{array}{c}\text { GENERAL } \\
\text { BODY FIELDS } \\
\text { (mR/hr) }\end{array}$ & $\begin{array}{l}\text { DEMOLITION } \\
\text { (R) }\end{array}$ & $\begin{array}{l}\text { NEW WORK } \\
\text { (Hours) }\end{array}$ & $\begin{array}{c}\text { GENERAL } \\
\text { BODY FIELDS } \\
\text { (mR/hr) }\end{array}$ & $\begin{array}{l}\text { NEW WORK } \\
\text { (R) }\end{array}$ \\
\hline CALCINER & 1.674 & 100 & 167 & 8,254 & 20 & 165 \\
\hline ABSORBER & 8,263 & 97 & 801 & 12,582 & 10 & 126 \\
\hline $\begin{array}{c}\text { FILTER CELL/NALVE } \\
\text { CUBICLE }\end{array}$ & 0 & & 0 & 7764 & 30 & 233 \\
\hline SUMP & 0 & & 0 & 1,911 & 5 & 10 \\
\hline MISC. & 225 & 85 & 19 & 305 & 20 & 6 \\
\hline TOTALS & 10,162 & & 988 & 30,816 & & 540 \\
\hline
\end{tabular}

${ }^{8}$ Attachment C 


\section{Attachment B \\ CsIX/TRU Feasibility Study Equipment List}

\begin{tabular}{|c|c|c|}
\hline Cell & $\begin{array}{c}\text { Equipment } \\
\text { No. }\end{array}$ & Description \\
\hline \multirow{14}{*}{ Adsorber Cell } & V-104-A & CsIX Column \\
\hline & V-104-B & CsIX Column \\
\hline & V-104-C & CsIX Column \\
\hline & F-101-A & UDS Filter \\
\hline & F-101-B & UDS Filter \\
\hline & T-103-A & IX Effluent Hold Tank \\
\hline & T-103-B & IX Effluent Hold Tank \\
\hline & $T-108$ & UDS Slurry Tank \\
\hline & EV-101 & UDS Evaporator \\
\hline & E-101 & Evaporator Condenser \\
\hline & None & UDS Drum Loading \\
\hline & $\mathrm{EH}-101$ & Off-gas Superheater (HE-NCC-335) \\
\hline & E-103 & Vent Condenser (HE-NCC-336) \\
\hline & $\mathrm{V}-101$ & Mist Collector (VES-NCC-136) \\
\hline \multirow{7}{*}{ Calciner Cell } & $\mathrm{V}-106$ & Snent Resin Pren Tank \\
\hline & $\mathrm{D}-102$ & Resin Drier \\
\hline & $\mathrm{E}-102$ & Drier Condenser \\
\hline & $T-112$ & Spent Resin Storage Tank \\
\hline & P-104-A & Spent Resin Sluice Pump \\
\hline & P-104-B & Spent Resin Sluice Pump \\
\hline & $\mathrm{F}-102$ & Resin Fines Filter \\
\hline \multirow{7}{*}{ Valve Cubicle } & $P-101-A$ & Liquid Waste Feed Pump \\
\hline & P-101-B & Liquid Waste Feed Pump \\
\hline & $P-103-A$ & CsIX Effluent Pump \\
\hline & P-103-B & CsIX Effluent Pump \\
\hline & $P-107$ & UDS Evaporator Feed Pump \\
\hline & $\mathrm{P}-112$ & Spent Resin Transfer Pump \\
\hline & Valves & 30 Remote Valves \\
\hline \multirow{2}{*}{$\begin{array}{l}\text { Hot Sump } \\
\text { Tank Removal } \\
\text { Cell }\end{array}$} & None & 5-Ton Crane \\
\hline & None & UDS Drum Glove Box \\
\hline \multirow{3}{*}{ Room 429} & $T-102$ & DI Water Tank \\
\hline & P-102-A & DI Water Pump \\
\hline & $\mathrm{P}-102-\mathrm{B}$ & DI Water Pump \\
\hline
\end{tabular}


Attachment $C$

CsIX/TRU Feasibility Study

Memo from H.K. Peterson 
Date: $\quad$ September 23, 1998

To:

H. S. Forsythe

MS 3625

$6-1603$

From:

H. K. Peterson

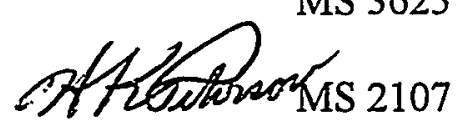

$6-8657$

Subject: $\quad$ SHIELDING REQUTREMENTS FOR HIGH LEVEL WASTE PROCESSING COMPONENTS LOCATED IN THE NWCF-- HKP-16-98

References: a) H. K. Peterson ltr to J. L. Clapp, SHIELDING REQUIREMENTS FOR HIGH LEVEL WASTE PROCESSING COMPONENTS, HKP-15-98, August 31, 1998

b) MicroShield, Version 5.01, Grove Engineering, Rockville MD

Shielding calculations have been made that define the shielding requirements for the high level waste processing components (Ref.a). These requirements were compared against the shielding available for the proposed component placement in the NWCF as illustrated in Sketches 1 through 5. As proposed, the component placement positions the components where they will be more than adequately shielded. The modifications to this NWCF arrangement involve shielding overpacks for the waste drums before they are moved out of the cell where they are loaded and involve a shielded platform above the spent resin storage tank (T-112). All calculations were performed with the MicroShield (Ref.b) computer code and are appended for reference.

As discussed and tentatively decided, the overpacks should reduce the waste drum surface radiation field to a contact-handleable field, i.e., $200 \mathrm{mR} / \mathrm{hr}$. The overpack thickness to accomplish this attenuation is 1.1 inches of lead $(\mathrm{Pb})$ or 3.5 inches of steel.

The shielded platform proposed for above the spent resin storage tank was also sized to attenuate the radiation field at the top of the cell to $200 \mathrm{mR} / \mathrm{hr}$. The thickness of this platform to accomplish this attenuation is $2.5 \mathrm{ft}$. of concrete or 6.25 inches of steel.

MicroShield input/output listings are on file with the Letter File copy of this analysis should they be required for reference or review. If there are any questions or comments concerning this analysis, please call me at the above-listed number.

\section{hkp}

cc:

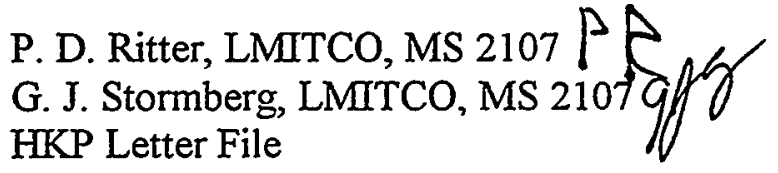




\author{
Attachment D \\ CsIX/TRU Feasibility Study \\ E-Mail from M.M. Dumas
}

Maria M. Dumas

10/1/98 10:28 AM

Subject: NWCF Cell Radiological Field Estimates

As requested, Griff Colson and I have come up with estimates for three NWCF process cells that are intended to be utilized in the CsIX process. We were asked to provide general body fields (GBF) after decontamination for the calciner cell (room 214), adsorber cell (room 206), and filter cell/valve cubicle (room 216). One year should be allowed to perform the necessary decontamination work prior to start of construction.

Calciner cell: Based on historical performance, this cell can be decontaminated to an average GBF of $300 \mathrm{mR} / \mathrm{hr}$ gamma. We believe that with use of considerable creative decontamination methods and with no regard to minimization of liquid waste generation that the cell can be decontaminated to $100 \mathrm{mR} / \mathrm{hr}$ gamma. Construction would then install permanent shielding walls in the cell and further in cell work would take place behind this shielding. The estimate for the GBF within this shielded area is $20 \mathrm{mR} / \mathrm{hr}$ gamma.

Adsorber cell: This cell has not been fully decontaminated in several years therefore estimates are based on the current undecontaminated radiological fields and a conservative assumption is made for what we reasonably believe we can decontaminate the cell to based on performance in other cells. After decontamination the cell's estimated GBF will be $300 \mathrm{mR} / \mathrm{hr}$ gamma. Construction would then remove the existing equipment from the cell in a manner to remove the equipment producing the higher fields first. After equipment removal the GBF is estimated to be $10 \mathrm{mR} / \mathrm{hr}$ gamma. Assume that the average GBF for the course of then entire ripout would be $97 \mathrm{mR} / \mathrm{hr}$ gamma.

Filter cell/valve cubicle: Based on historical performance, this cell can be decontaminated to an average GBF of $100 \mathrm{mRem} / \mathrm{hr}$. We believe that with use of considerable creative decontamination methods and with no regard to minimization of liquid waste generation that the cell can be decontaminated to $30 \mathrm{mR} / \mathrm{hr}$ gamma. This assumes that all filters that are currently stored in the area are removed and either leached or stored in other areas of NWCF. It is estimated that if any further reduction in GBF is made after equipment removal it will be negligible due to the small amount of equipment to be removed (adsorber cell valve loops) relative to what will remain in the cell (filter banks and blend and hold cell valve loops). 


\section{Attachment $E$ \\ CsIX/TRU Feasibility Study \\ Scenario 2 Drawings}

\begin{tabular}{|c|l|}
\hline Drawing Number & \multicolumn{1}{|c|}{ Description } \\
\hline $2 \mathrm{M}-2$ & Scenario 2, CPP-659 3 ${ }^{\text {rd }}$ Level Demolition Plan \\
\hline $2 \mathrm{M}-3$ & Scenario 2, CPP-659 3 ${ }^{\text {rd }}$ Level Plan \\
\hline $2 \mathrm{M}-4$ & Scenario 2 Sections \\
\hline $2 \mathrm{M}-5$ & Scenario 2 Sections \\
\hline $2 \mathrm{M}-6$ & Scenario 2, CPP-659 1st Level Plan \\
\hline
\end{tabular}




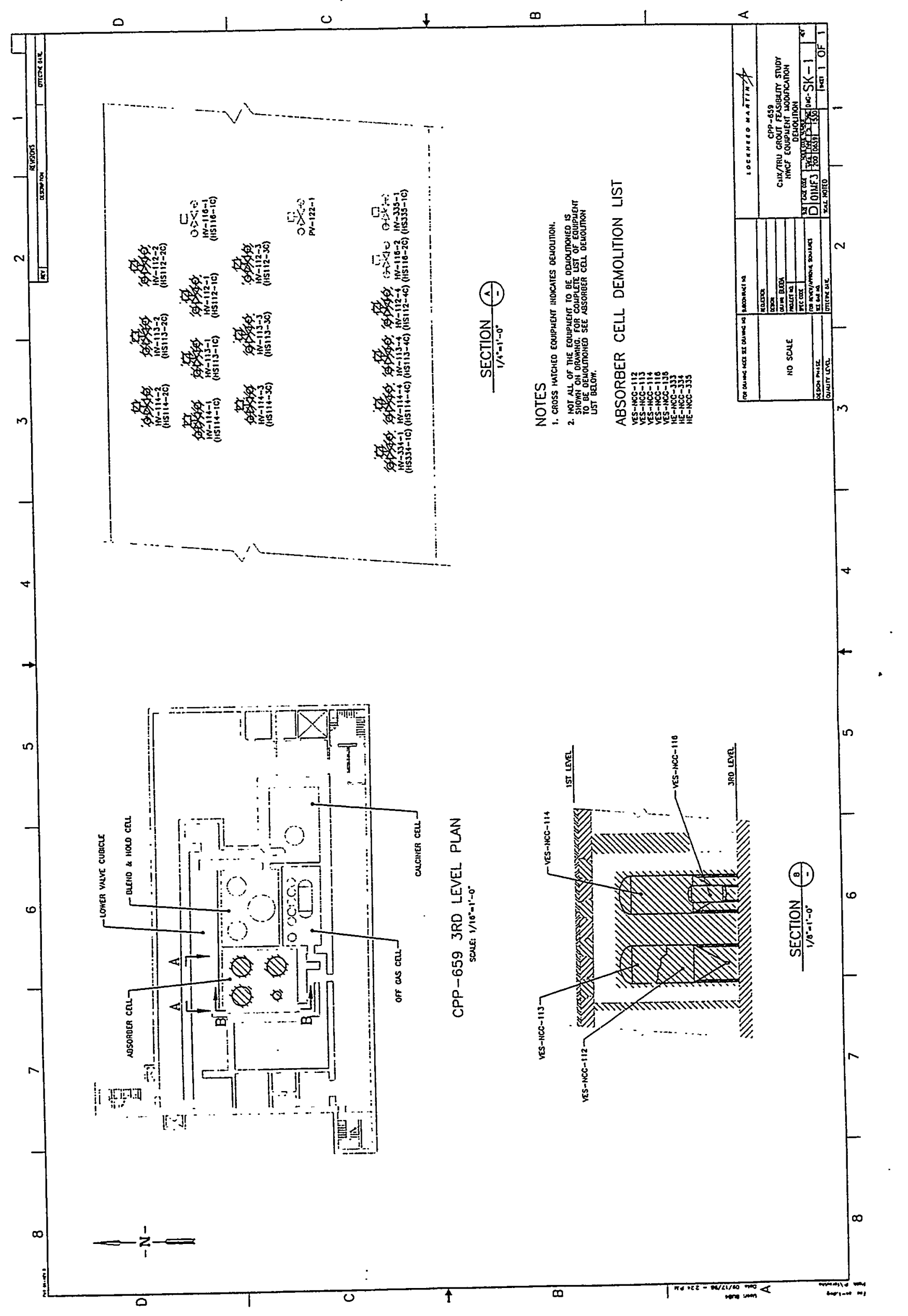




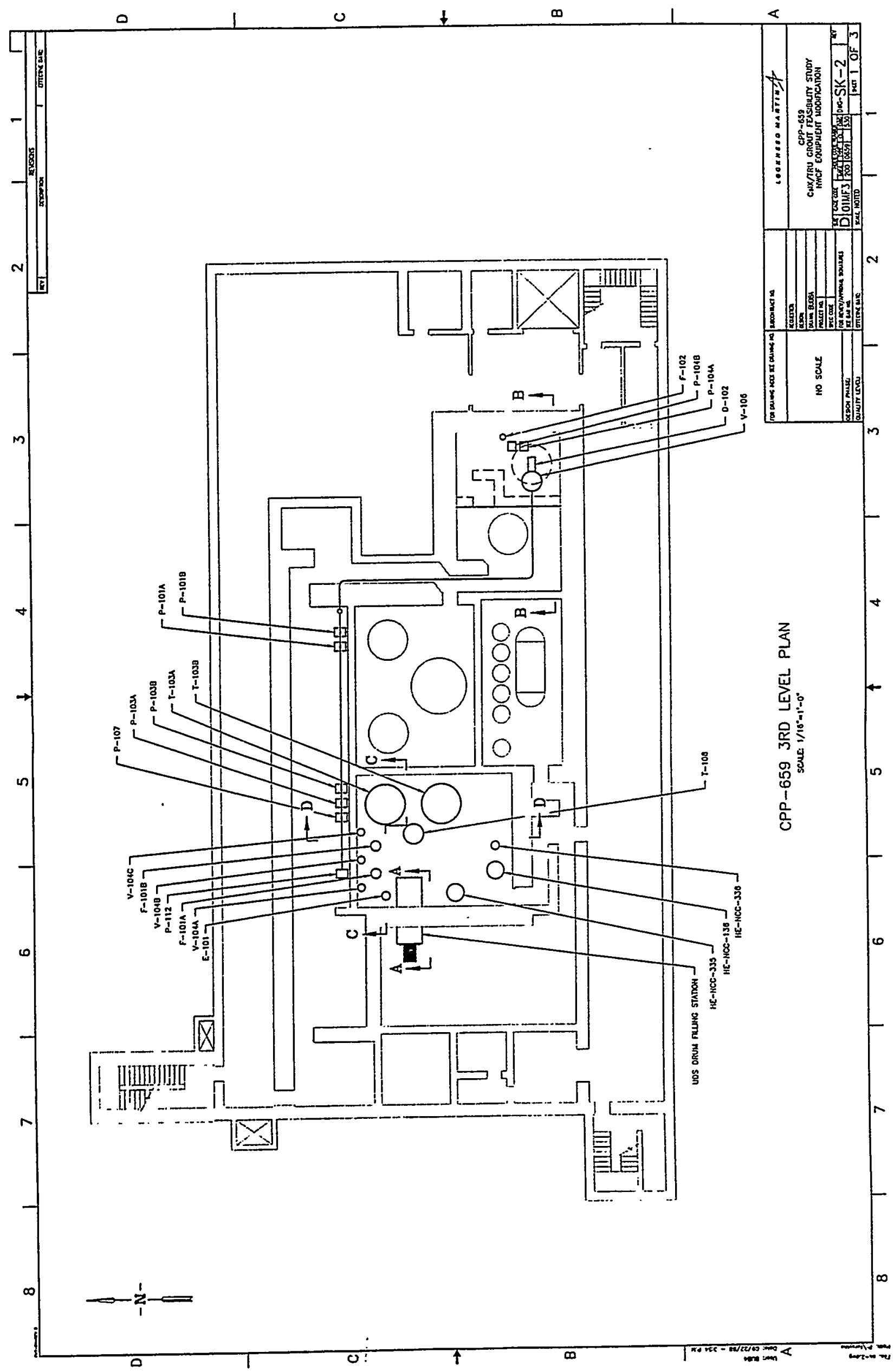




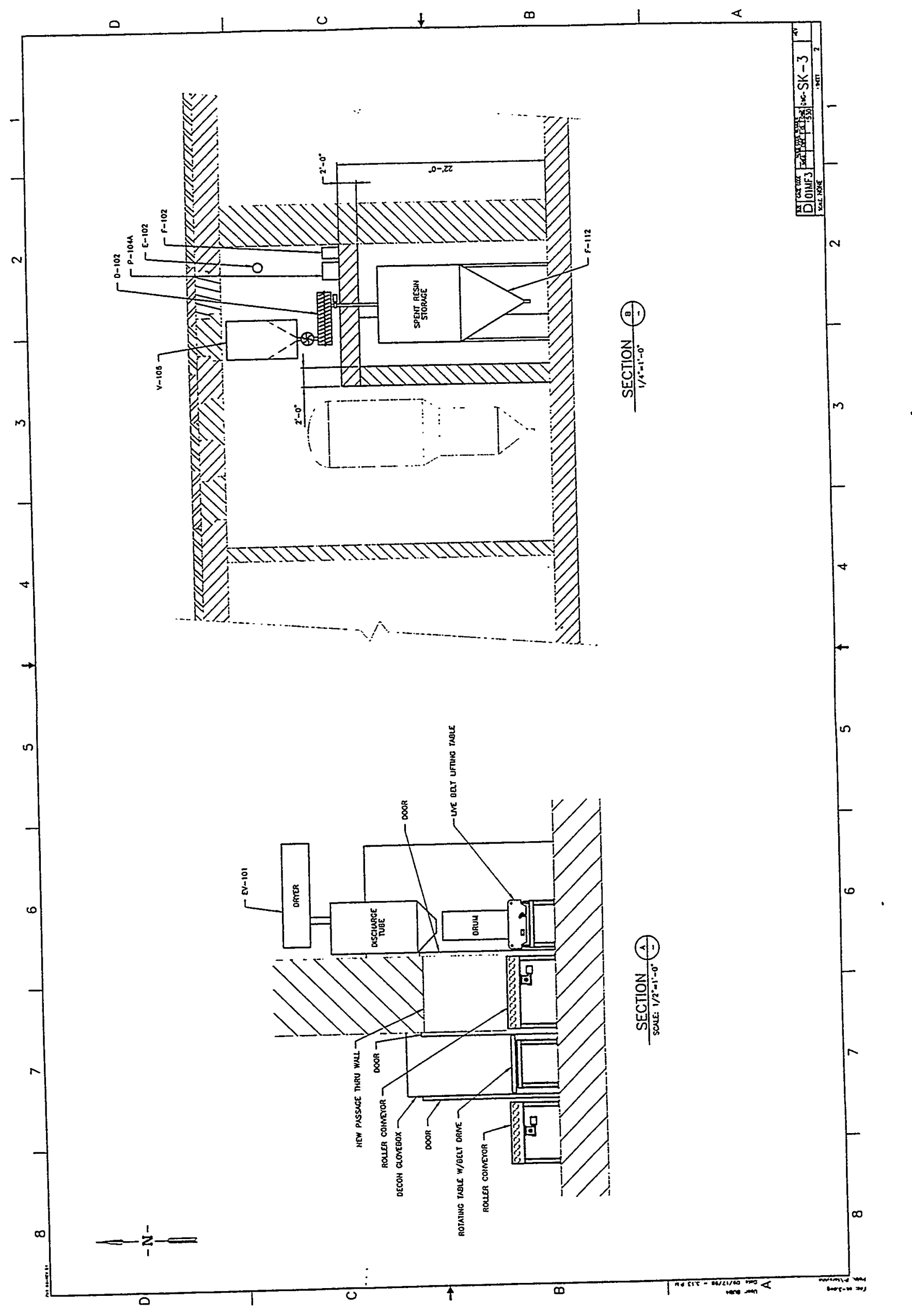




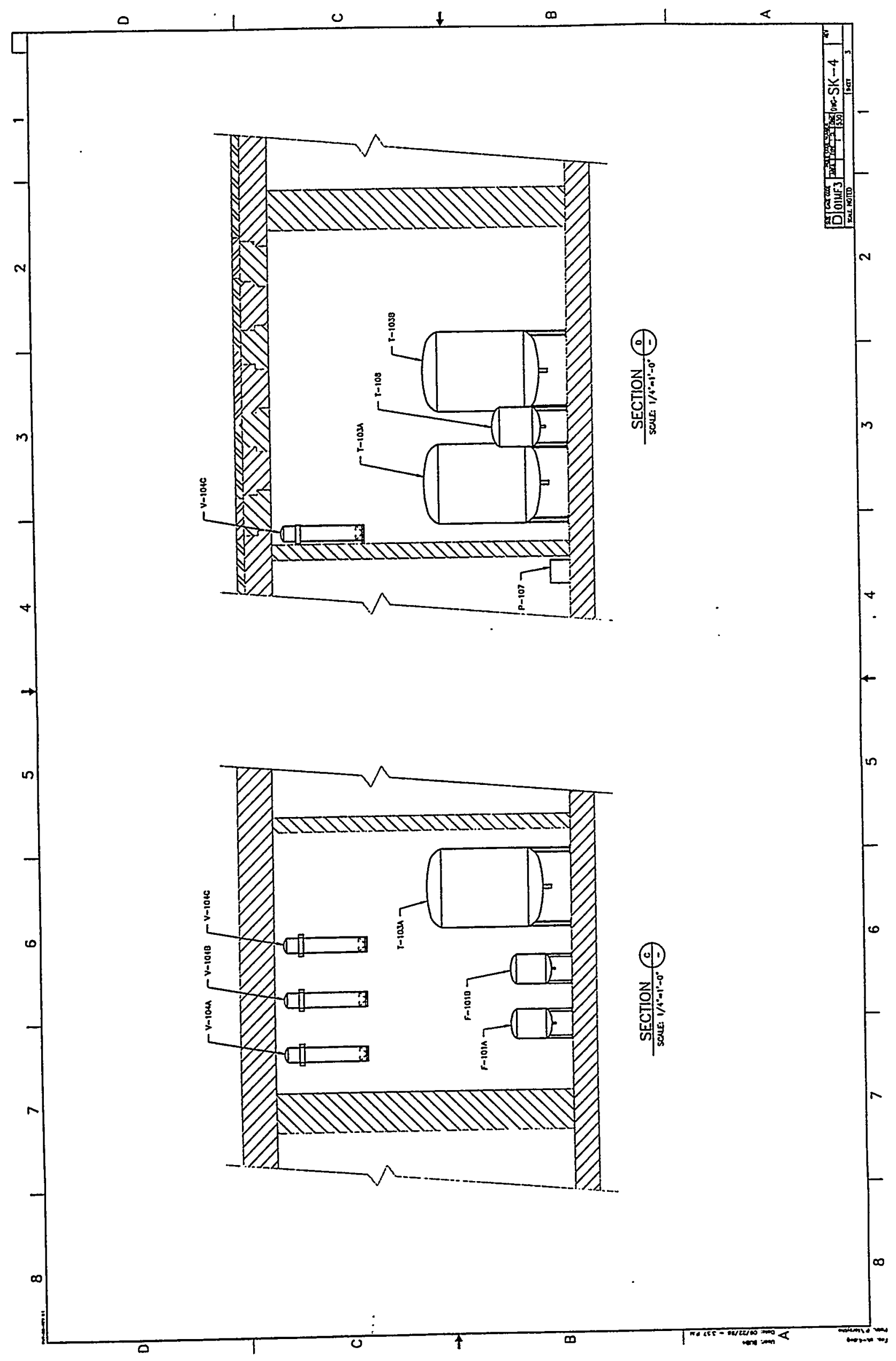




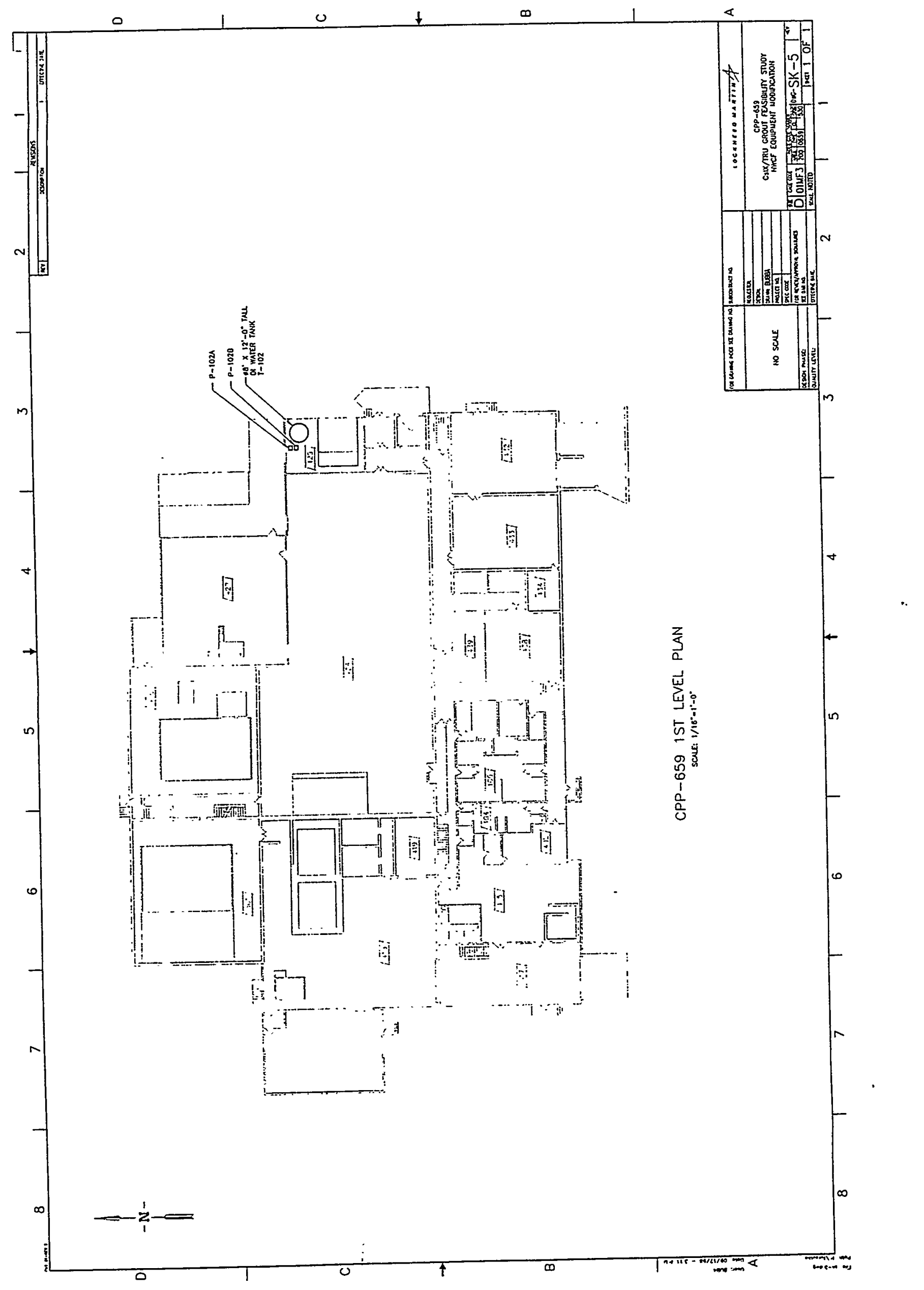


Project File Number $\quad 02 \mathrm{BM} 1$

Project/Task CsIX/TRU Grout Feasibility Study

Subtask D.4 Handling \& Storage

Title: Drum Head Space Sampling and Inspection

Summary:

This EDF documents the requirements, assumptions, description, and calculations for the Drum Headspace Sampling and Inspection system.

EDF Contents:

- Attachment A - Detailed Process Description w/Assumptions \& Requirements.

- Attachment B - Equipment list for the Sampling and Inspection stations.

- Attachment $\mathrm{C}$ - Sequence of moving a Drum through the Sampling and Inspection Station

- Attachment D - Sketches and Drawings

Distribution (complete package): C. W. Olsen MS 3211; K. L. Williams MS 3765; Project File; A copy of this EDF will be included in External Report No. INEEL/EXT $=98-00869$.

Distribution (summary package only):

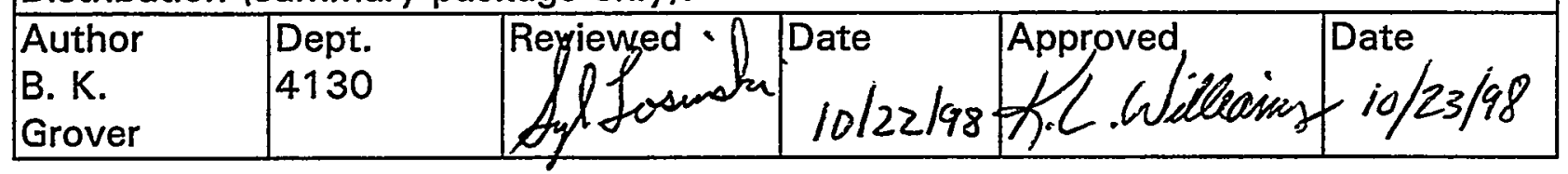


Attachment A

CsIX/TRU Feasibility. Study

\section{General}

This EDF describes the systems required to meet WIPP requirements for detection of free liquids in grout filled drums, and the need to take a gas sample of a drum's headspace.

The sampling and inspection will take place in a separate area within the interim storage facility. The drums will be stored in this temperature-controlled area for a 72-hour period prior to inspection and gas sampling. The system is remotely automated, but requires operator interface for inspection and drum feed. A drum oven will be used to evaporate off any liquid contents of the drums if inspection reveals the grout has not cured.

\section{Assumptions}

1. Baking of the drum contents will remove liquid if the grout did not cure properly.

2. Video inspection through the drum lid filter hole is attainable.

3. Hydrogen levels of the drum headspace will not require special combustion construction of the inspection booth.

4. Drum radiation levels are at contact limits.

\section{$\underline{\text { Requirements }}$}

1. A drum being sent to WIPP must not contain free liquids in excess of $0.5 \%$ of the volume of the drum.

2. Drum VOC and Hydrogen levels must be below WIPP prescribed levels.

3. A drum must be temperature conditioned for a 72-hour period prior to the sampling and inspection.

4. A drum-baking oven will be required to cure drum grout that did not set properly. 


\section{Process Description}

Pallets of drums will be moved from the Interim storage area to the conditioned space of the inspection and sampling area. Drum pallets will be stacked 2 high and require a 72-hour holding period. This time period allows the contents of the drum to reach a $70^{\circ} \mathrm{F}\left(21^{\circ} \mathrm{C}\right)$ temperature. Approximately 18 pallets (72 drums) must be maintained in the inspection area at all times. This allows the capacity of 1 TRUPACT shipment per day to WIPP if required.

After the 72-hour period has elapsed the drum will be placed on a staging conveyor. The drum bar code will be scanned to identify it for tracking and inspection results. The drum is initially conveyed to a locating/positioning table for positioning of the drum lid filters. This system is identical to that used in the drum dewatering system in the grouting facilities. After indexing, the drum is passed through an airlock to a glovebox where inspection and sampling occur.

Once positioned in the glovebox, a clear hood will be lowered over the drum lid to provide additional radiological containment. All subsequent inspection and sampling activities will occur within the hooded environment. A filter removal/headspace sampler tool will lower to and engage one of the predetermined drum lid filters. The filter will be twisted into the drum and allowed to drop into the headspace of the drum. During this same operation, a headspace gas sample will be taken as soon as the filter is removed. The filter removal/headspace sampler tool will be removed and a fiber optic video camera with lighting will be moved into position over the drum opening. The fiber optic camera will be lowered through the opening and digitally record a 360 degree rotational view of the drum headspace. Operator interface on the video inspection will be required. After video inspection, the camera will be removed and a new bung plug installed. The clear hood will rise from the drum and a mechanical arm will be used to take a RadCon swipe of the drum lid surface. On-line counting will be provided for swipe examination. Once cleanliness of the drum top is verified, the drum will pass through an exit airlock to an unloading conveyor.

If a drum is visually determined not to have set or cured, it will be removed from the unloading conveyor and moved to a drum oven. The oven will be steam heated and ventilated to the inspection booth exhaust system. Capacity of the drum oven will be 4 drums. The drums will be baked at $350^{\circ} \mathrm{F}\left(176.6^{\circ} \mathrm{C}\right)$ for a 72 -hour period to evaporate off any moisture that may be present in the mixture. Following this baking of the drum contents another headspace inspection will be performed.

When inspections are complete, the drums will be transferred to the TRUPACT II loading facility. Drums will be placed in TRUPACT shipping units and shipped to WIPP for storage. 


\section{Attachment B \\ CsIX/TRU Feasibility Study \\ Equipment list for Drum head Space Sampling and Inspection station}

Inlet/Load Conveyor - SST, chain drive, $10 \mathrm{ft}$ long, $2 \mathrm{Hp}$

Index Table - SST, self-powered rollers, $3 \mathrm{ft}$ long, 1200 watts

Index lift table - SST, 18 in. diameter, $1.5 \mathrm{Hp}$

Index table rotation - $1 / 2 \mathrm{Hp}$

Index arm - roller assembly, arm, no power required.

Airlock - 7 gauge, ST with window and HEPA filter

Airlock inlet doors - motor driven, SST, $1 / 2 \mathrm{HP}$

Airlock conveyor - SST, self powered rollers, $4 \mathrm{ft}$ long, 1600 watts

Glovebox - 7 gauge, SST with window and HEPA filter

Glovebox inlet doors - SST, motor driven, $1 / 2 \mathrm{Hp}$

Glovebox conveyor - SST, self powered rollers, $5 \mathrm{ft}$ long, 2000 watts

Video camera - hardened, $1 / 2$ in. optic lens, rotatable, 1500 watts

Headspace sample tube - SST, no power required

Mechanical swipe arm - Auto swipe of area, remote, on-line counting, 3000

watts

Hood enclosure - Clear, no power required

Filter removal arm - SST, automated, 400 watts

Filter installation arm - SST, automated, 400 watts

Glovebox outlet doors - SST, motor driven, $1 / 2 \mathrm{Hp}$

Airlock - 7 gauge, SST with window

Airlock conveyor - SST, self powered rollers, $4 \mathrm{ft}$ long, 1600 watts

Airlock outlet doors - SST, motor driven, $1 / 2 \mathrm{Hp}$

Unload conveyor - SST, Chain driven, $10 \mathrm{ft}$ long, $2 \mathrm{Hp}$

Govebox exhaust fan - 2 each, $2 \mathrm{Hp}$, one unit on back-up power

Airlock exhaust fan - 2 each, $1 \mathrm{Hp}$, one unit on back-up power

Drum Bake Oven - Steam heated, SST, Pallet type 


\section{Attachment C \\ CsIX/TRU Feasibility Study \\ Sequencing of moving a Drum through the Sampling and Inspection Station:}

A pallet of drums will be moved into headspace sampling and inspection area for 72-hour period. One drum will be removed from a pallet and placed on the staging conveyor to the inspection/sampling system. The bar code on the drum will be scanned for identification and inspection tracking. The staging conveyor starts and moves the drum to the locating/positioning table. Once the drum has reached the locating/positioning table, the staging conveyor stops.

The locating/positioning table raises the drum a predetermined height to the filter locating mechanical arm and stops. The table then rotates the drum for locating one of the lid filters. After the filter has been located, the drum has been positioned. The table then lowers the drum back to the staging conveyor.

When the drum has been lowered back to the conveyor, the doors to the inlet airlock will open and the conveyor moves the drum into the airlock. When the drum has moved into the airlock the conveyor stops and the inlet doors to the airlock close. Once the inlet doors to the airlock are closed the inlet doors to the inspection booth open. The airlock conveyor starts and moves the drum into the inspection booth. Once inside the inspection booth and positioned, the conveyor will stop. The inlet doors to the inspection booth close and inspection procedures begin.

A clear hood will lower from the ceiling of the booth and seal to the top of the drum. A filter/gas-sampler tool will move into position over the filter that was indexed. The tool will lower to the filter and engage into the filter. The filter will be twisted into the drum and allowed to drop into the drum headspace. A gas sample is drawn with the same tool as soon as the filter is removed. The filter/gas sampler tool is moved aside and a fiber optic camera with a lighted end will move into position over the filter hole. The camera will be lowered into the drum headspace and a 360-degree rotation will be made. Operator interface is required during this video inspection. Once the inspection is complete, the camera will raise and move aside. A plug installation tool will move into position over the drum filter hole. A new drum bung plug will lower on the installation tool end and be twisted into the drum lid. The plug tool will move aside and a RadCon mechanical swipe arm will move to the filter area. The RadCon arm will lower and swipe the area around the filter area. Once swiping is complete, the mechanical arm will raise and deposit the swipe in an on-line counter. Once cleanliness is verified, the mechanical arm and clear hood will rise and move aside. Inspection procedures are now complete. 
After the RadCon arm and clear enclosure hood are moved aside, the exit doors to the inspection booth will open. The inspection booth conveyor will start and move the drum into the inspection booth exit airlock. Once the drum is inside of the airlock, the conveyor will stop and the inspection booth exit doors will close. After the inspection booth exit doors are closed, the airlock exit doors will open and the airlock conveyor will start. The airlock conveyor will move the drum out of the airlock and onto the unloading conveyor. When the drum is out of the airlock, the airlock exit doors will close and the unloading conveyor will move the drum to the end of the conveyor line. When the drum has reached the end of the unloading conveyor line, the conveyor will stop.

Drums will be removed from the unloading conveyor and placed on pallets. The pallets will then be taken, via forklift, to the TRUPACT II loading station for shipment to WIPP. If a drum does not meet video inspection requirements, it will be baked in a steam heated drum oven. Drums placed in the oven will be baked for a 72-hour period at $350^{\circ} \mathrm{F}\left(176^{\circ} \mathrm{C}\right)$. After the drum has completed the baking period, it will be sent back to the beginning of the inspection system and inspected again. 
Attachment D

CsIX/TRU Feasibility Study

Sketches and Drawings 
Head Space Sampliwg + Video IInspection

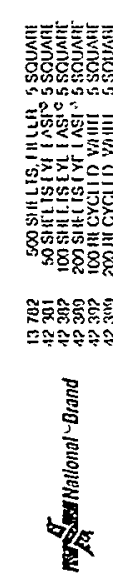

Interim Storage Facility
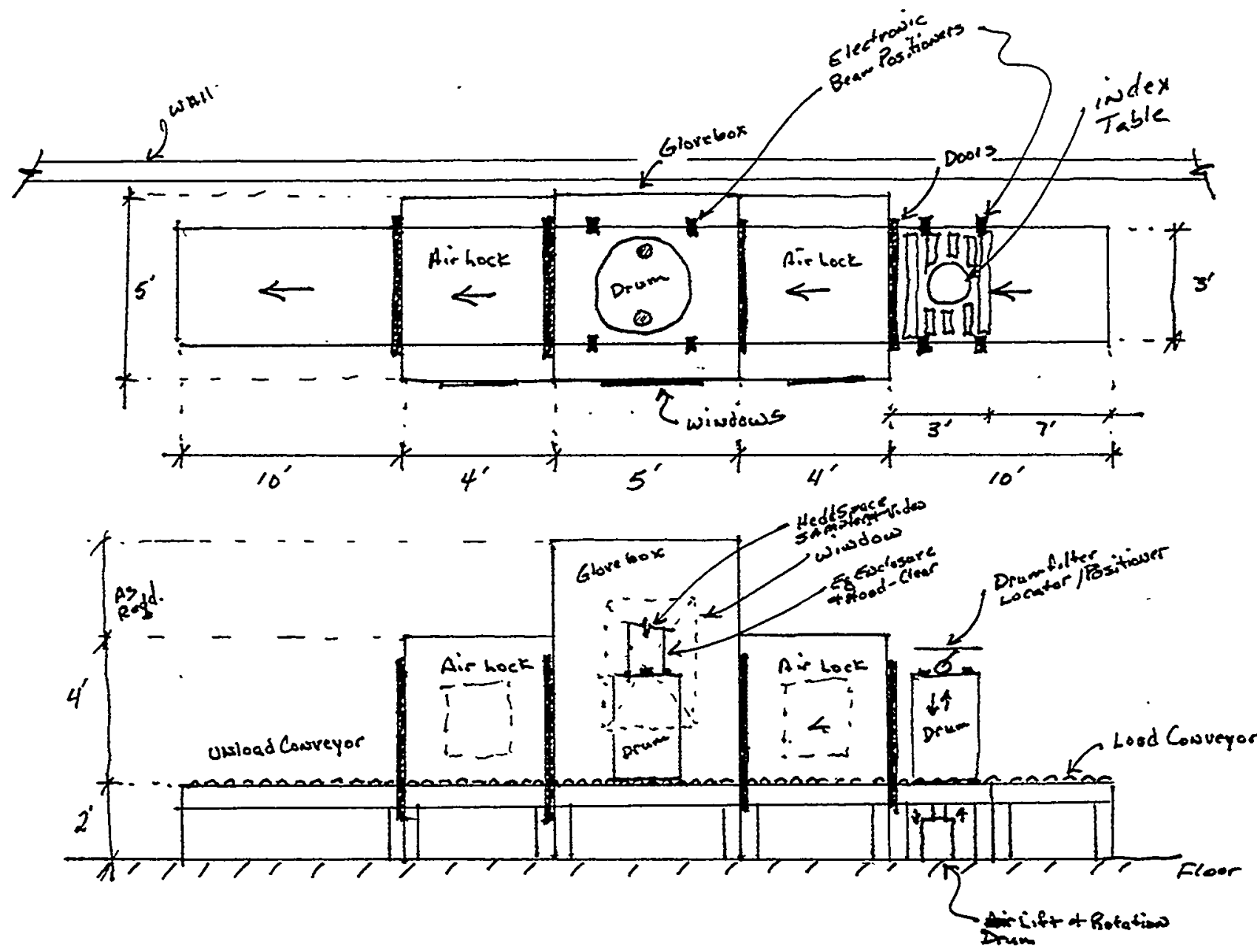
Project File Number $\quad$ O2BM1

Project/Task CsIX/TRU Grout Feasibility Study

Subtask D.5 Facility Design

\section{Title: HVAC - Scenario 1}

\section{Summary:}

This EDF describes documents requirements, assumptions, description, and calculations for the HVAC systems required for Scenario 1.

EDF Contents:

- Attachment A - Detailed Process Description w/Assumptions \& Requirements.

- Attachment B - Equipment list for the Scenario 1 HVAC Systems.

- Attachment $\mathrm{C}$ - Supporting Calculations

- Attachment D - Sketches and Drawings

Distribution (complete package): C. W. Olsen MS 3211; K. L. Williams MS 3765; Project File; A copy of this EDF will be included in External Report No. INEEL/EXT $=98-00869$.

Distribution (summary package only):

\begin{tabular}{|c|c|c|c|c|}
\hline $\begin{array}{l}\text { Author } \\
\text { B. K. } \\
\text { Grover }\end{array}$ & $\begin{array}{l}\text { Dept. } \\
4130\end{array}$ & Reviewed Pidf & Date $10 / 27 / 98$ & Approved $/$ K.L.W Jillaiaf $10 / 22 / 98$ \\
\hline
\end{tabular}




\author{
Attachment A \\ CsIX/TRU Feasibility Study
}

General

This EDF describes the HVAC system required for the Scenario 1 Facility. The Interim Storage Facility and TRUPACT II Loading Facility are included in this EDF and are common to both scenarios. The Off-Gas system is covered under EDFCsIX-013.

The HVAC system is comprised of three different zones for the Grouting facility. Each zone has its own air handling system. The primary and secondary zones have redundant systems with one redundant system connected to standby power for continuous operation. The office space has a split DX system with heating and the grouting process area has heating only. The Interim Storage Facility has both steam heating and DX cooling for the Headspace Sampling area within the storage unit. The TRUPACT II loading facility is heated with electric unit heaters. All three systems are as described below.

\title{
Assumptions
}

1. Eight air changes per hour for primary confinement.

2. Four air changes per hour for secondary confinement.

3. $20 \mathrm{cfm}$ per person of outside air for administrative areas.

4. Only roughing filters are required on secondary zone outside air intake.

5. Only primary zone return air will require redundant HEPA filtration prior to discharge to the stack.

\section{Requirements}

1. The CsIX/TRU Grout building will be required to have two ventilation confinement zones per DOE Order 6430.1A.

2. Ventilation for primary and secondary zones are once through systems.

3. Steam heating will be used where applicable.

4. HEPA filtration will be required on both the primary and secondary zones. 


\section{Process Description}

The HVAC system is comprised of three zones within the Grouting facility. These zones are primary confinement, secondary confinement, and office space. Pressure of the different zones are +0.1 inch W.C. for office space, -0.5 inch W.C. for secondary confinement, and -0.75 inch W.C. for primary confinement. Airflows and equipment will be as follows.

The office space of the facility will have a split direct expansion (DX) system with steam heating. The air handler will be located in the office space to eliminate the possibility of contamination and will provide 2800-CFM of air to the space. 285CFM of fresh air for occupants will be taken in through a louvered inlet. Heating will be from a steam coil and cooling will be from a DX unit. The total loads for the office space are 7 Tons of cooling and $120 \mathrm{MBH}$ of heating at the AE standards design conditions for the INEEL. Estimated annual energy consumption for heating the structure using a single measure check is $169.5 \times 10^{3} \mathrm{lbs}$. of steam per year. Annual cooling energy requirements using a single measure check are estimated at $11,168 \mathrm{kWh}$ per year. The condenser or cooling unit will be located on a slab outside of the office space on the north side of the facility. Outside air will be introduced through a louvered inlet on the side of the building. See calculations for details on heating and cooling loads.

The Heating and Ventilation system for secondary confinement will be located on the third floor on the north end of the facility. Outside air will be taken into the system from a louver on the north side of the facility. The air will be rough filtered, heated, and then distributed to various locations throughout the building. The secondary confinement air system will be the main source of heating the process side of the building. 10500-CFM of outside air is required for this system. CFM quantities are based on a volumetric basis. Secondary zone air requirements are 4 air changes per hour for the area served. Heating will be from steam coils and require $2917 \mathrm{MBH}$ of heating capacity. Estimated annual energy consumption for heating the building using a single measure check is $4.12 \times 10^{6} \mathrm{lbs}$. of steam per year. There will be two airhandlers for supply to the secondary confinement areas. The primary airhandling unit will be connected to backup power. There are two airhandlers for maintenance and failure purposes. Once air is passed through the secondary zone, it will be HEPA filtered prior to being supplied to the primary zone or discharge to the stack. Two $2 \times 5$ HEPA filter housings will be installed, one HEPA housing serve one airhandler. See airflow schematic in Appendix $D$ for more detail on airflow patterns and equipment layout.

Exhaust from the secondary confinement zone will be from a duplicate set of airhandlers. One of the airhandler units will be connected to backup power for maintenance and failure purposes. These exhaust airhandlers also supply air to the 
primary confinement zone and discharge to the stack. Air that is supplied to the primary confinement zones is exhausted by another set of airhandlers. Not all of the secondary confinement air is supplied to the primary confinement system and is sent to the stack.

The Ventilation system for the primary confinement zone will be located on the north side of third floor in the facility, see drawing 1-A3. The primary containment heating and ventilation system will receive air from the secondary containment system. The air discharged from the secondary zone is single HEPA filtered before entering into the primary zone. No heating or cooling capability is provided for the primary confinement zone system. Ductwork will be welded SST and be routed in areas not occupied by personnel. The primary zone system does not require the airflow that the secondary system does and for this reason, the secondary containment system bypasses 1000-CFM to the stack. See Scenario 1 airflow sketch in Appendix D for more detailed information on flow pattern. 9500-CFM of air is supplied to the primary confinement zone. CFM quantities are based on a volumetric basis. Primary confinement zone air requirements are 8 air changes per hour for the area served. The airhandler and HEPA filter system is redundant for maintenance and failure purposes. The primary airhandling unit will be connected to back up power. Once air has passed through the primary confinement zone, it will be double HEPA filtered and discharge to the stack.

The Interim Storage Facility has one area within the building that will be conditioned. This area is the headspace sampling area. The HVAC system in this area will be a split DX system. Heating will be from steam supplied to the facility. The air handler will be located above the headspace sampling area. The airhandler will deliver 3600-CFM of air to the space and requires a $107 \mathrm{MBH}$ heating coil. 200-CFM of outside air will be taken in through a louvered inlet on the wall of the facility. Estimated annual energy consumption for heating the inspection area using a single measure check is $1561.2 \times 10^{3} \mathrm{lbs}$. of steam per year. Estimated annual cooling energy using a single measure check is $19,553 \mathrm{kWh}$ per year. The 9-ton condensing unit will be located outside the facility on a concrete pad. There are two exhaust systems in this facility. The Inspection and Headspace sampling booth requires an exhaust system. This system will be connected to the inspection booth and the two air locks. The exhaust will be filtered with glovebox style HEPA filters. See EDF-CsIX-010 for more information on the inspection booth equipment. The main exhaust system is two $7.5 \mathrm{Hp}$ duel speed utility units that ventilates the interim storage area. 14000-CFM is exhausted by each of the two main exhaust systems for a total of $28000-C F M$ ventilation. These main exhaust systems provide $0.5 \mathrm{CFM} / \mathrm{ft}^{2}$ ventilation for the main interim storage area. Outside air is introduced into the facility by louvered inlets along the bottom of the outside walls. 
The TRUPACT II Loading Facility will be electrically heated with unit heaters. The basis for electric heating on this building was the existing unit that is located at RWMC. The heaters will be ceiling mounted and be $20 \mathrm{~kW}$ each. There will be 12 unit heaters required to heat the facility when being ventilated by the exhaust system at $A E$ design standard conditions. Estimated annual energy consumption for heating the building using a single measure check is $397,917 \mathrm{kWh}$ per year. The exhaust system is very similar to the Interim Storage Facility. The ventilation fans are a sidewall unit and louvered inlets on the bottom of the outside walls allow air to enter the facility. Each fan provides $2250-$ CFM of ventilation when in operation for a total of 4500-CFM. Information provided on the TRUPACT II loading facility is based on existing plans that are available. See Appendix $C$ for more information on calculated heating loads and Appendix $D$ for plans of the existing facility at RWMC. 


\section{Attachment B \\ CsIX/TRU Feasibility Study \\ Equipment list for the Scenario 1 HVAC System}

\section{Grouting Facility}

Office space airhandler - 2800-CFM, 2.75 inch W.C., steam heat coil, $3 \mathrm{Hp}$

Condenser -7 ton cooling capacity, DX, pad mounted, $7.8 \mathrm{kWatts}$

Secondary confinement - Backward inclined Utility fan, Class II, 10,500-CFM,

3.4 inch W.C., SST, $15 \mathrm{Hp}, 2$ each, one connected to backup power

HEPA filter housing $-2 \times 5,10,500-C F M$, SST, 2 each

Steam heat coils- $2917 \mathrm{MBH}$ total load

Primary confinement supply fan - Backward inclined utility fan, Class II,

10,500-CFM, 6.75 inch W.C., SST, $15 \mathrm{Hp}, 2$ each

Exhaust Fan - Backward inclined utility fan, Class II, 10,500-CFM,

6.75 inch W.C., SST, $15 \mathrm{Hp}, 2$ each

HEPA filter housing $-2 \times 5,10,500-C F M, S S T$, redundant filters, 2 each

\section{Interim Storage Facility}

Inspection/Storage Airhandler - 3600-CFM, 2.75 inch W.C., steam heat coil, $3 \mathrm{Hp}$ Condenser - 9-ton cooling capacity, DX, pad mounted, $10.2 \mathrm{kWatts}$ Exhaust fan - Backward inclined utility fan, $7.5 \mathrm{Hp}$, two speed motor, 14000-CFM, 2 each

\section{TRUPACT II Facility}

Electric Unit heater $-20 \mathrm{~kW}, 12$ each

Exhaust fan - Sidewall exhauster, 1/2 Hp, two speeds, 2250-CFM, 2 each 
431.02

$06 / 17 / 97$

Rev. \#04
ENGINEERING DESIGN FILE

\section{Attachment C \\ CsIX/TRU Feasibility Study \\ Supporting Calculations}

Functional File Number - SPR-CsIX-10

EDF Serial Number - EDF-CsIX-011

Page 1 of 1 


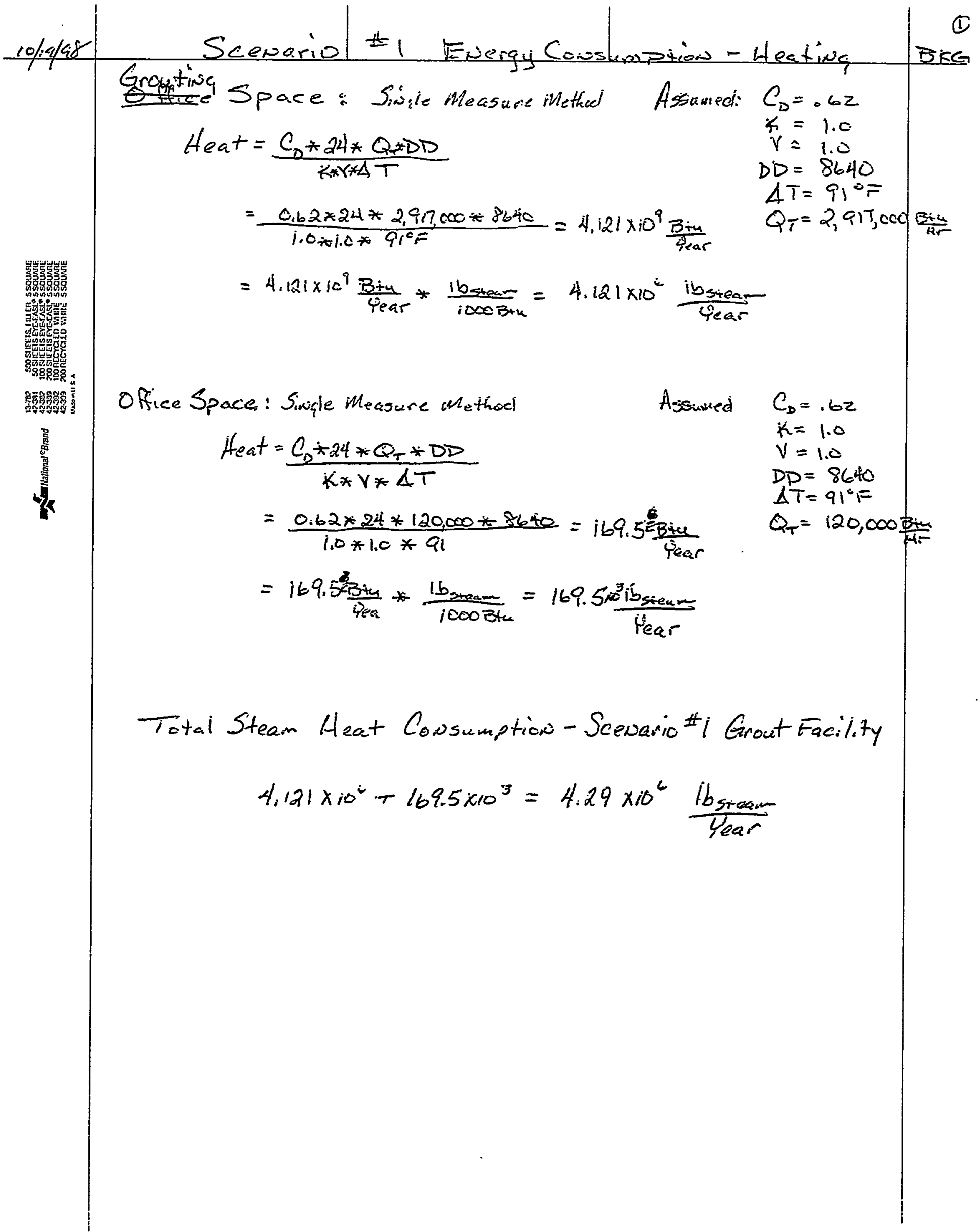




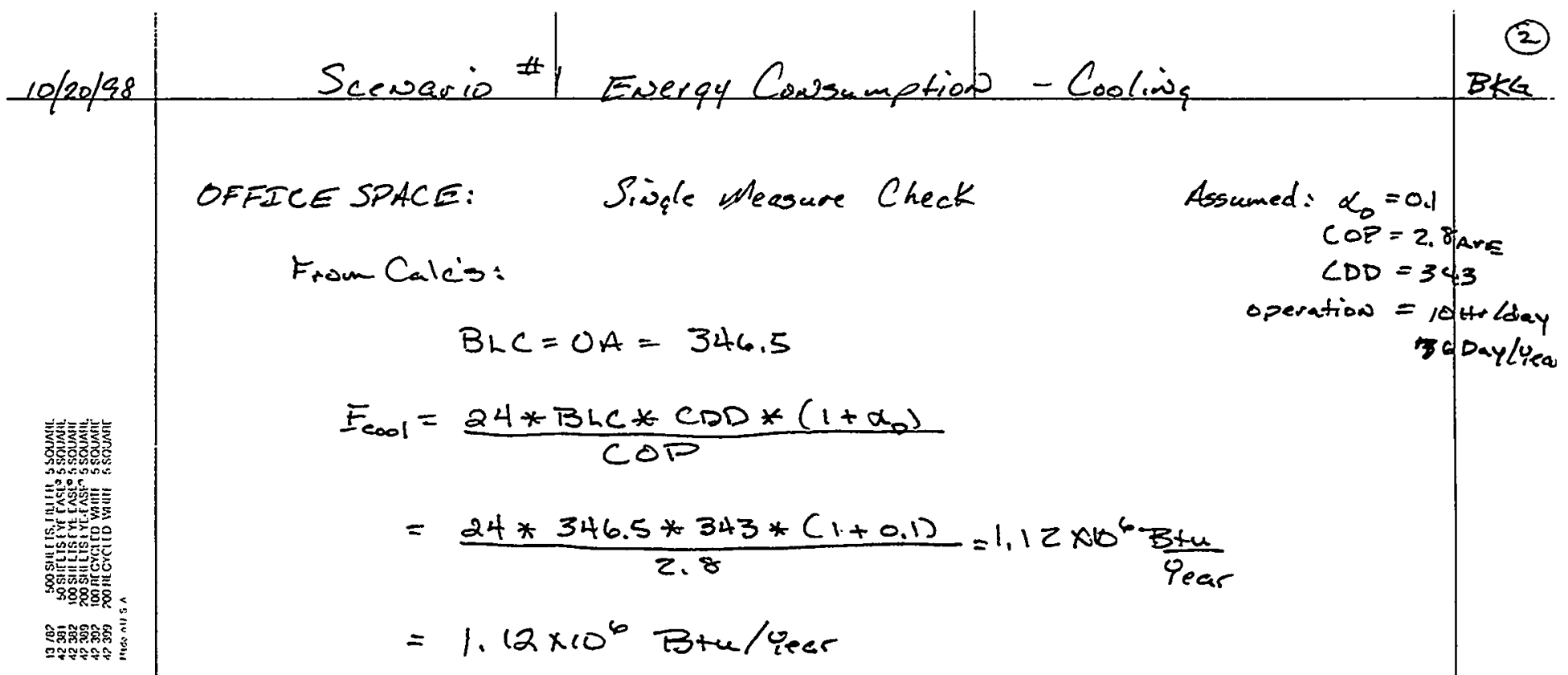

OTER GAINS from Cali's

$$
\text { OS }=5,244 \frac{B+4}{1+r}
$$

$E_{8}:=\frac{43,434}{48+n}$

Total $48,678 \frac{3+n}{+r}$

$$
E_{I 6}=48,678 * 10 * 76=36.99 \times 10^{6} \frac{\text { Btu }}{\text { Tear }}
$$

Total Energy Consumption:

$$
\begin{aligned}
E_{\text {tot. } 1} & =36.99 \times 10^{6}+1.12 \times 10^{\circ}=38.11 \times 10^{6} \frac{B_{\text {tu s }}}{\text { Year }^{\circ}} \\
& =11,168 \frac{\mathrm{k \omega H}}{\text { Year }_{\text {ear }}}
\end{aligned}
$$




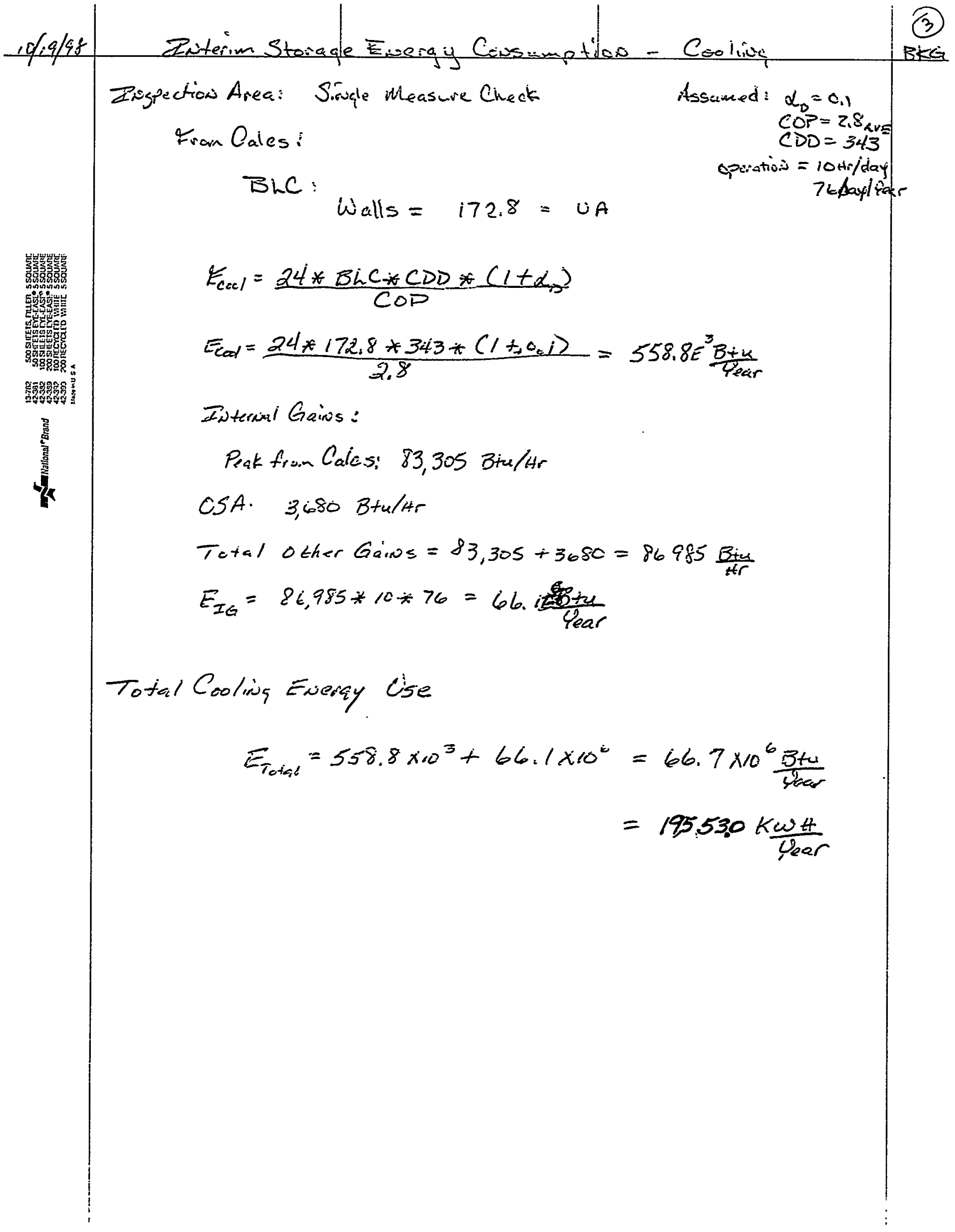




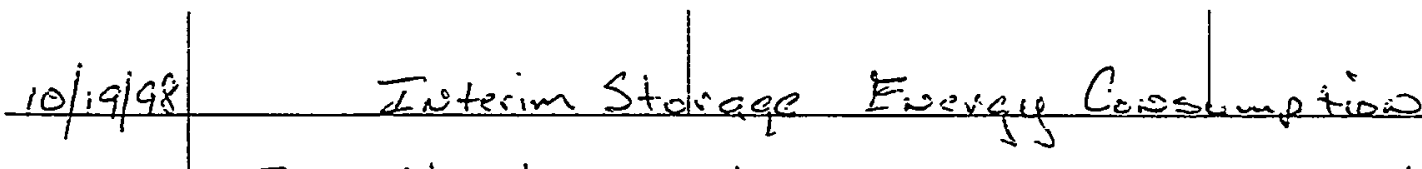

Inspection Area: Single Measure method

Assicumed:

$$
\begin{aligned}
& \text { Heat }=\frac{S_{0} * 24 * Q_{-} * D D}{K * V * \Delta T} \\
& =\frac{0.62 \times 24 * 107,000 * 8240}{1.0 * 1.0 * 91}=151.2 \times 10^{k} \frac{\mathrm{Bin}}{4 \mathrm{ear}}
\end{aligned}
$$

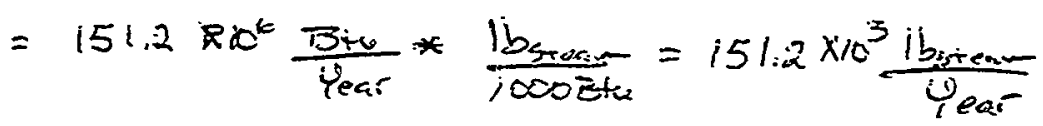

$$
\begin{aligned}
& \text { Total } 15 \% 2 \times 10^{3} \frac{i b_{\text {stan }}}{\text { year }}
\end{aligned}
$$

$(4)$

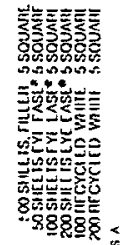

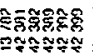

4 
$10,9 / 98$

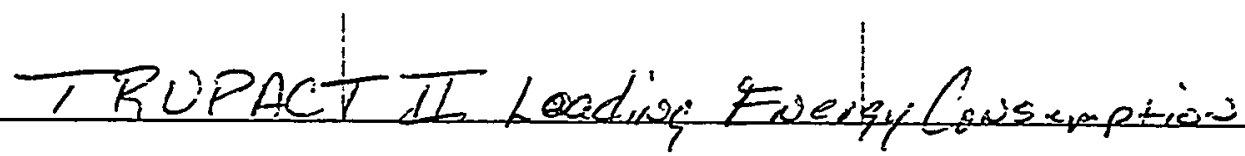

(5)

Loading Area: Single Measure Method

$$
\begin{aligned}
& \text { Heat }=\frac{C_{i} * 24 * Q_{T} * \Delta i D}{K * V * \Delta T} \\
& =\frac{0.80 * 24 * 745,000 * 8640}{10 * 1.0 \times 91}=1,35 \times 10^{9} \frac{3+u}{4 \text { ear }} \\
& =1,35 \times 10^{9} \frac{\text { Btu }}{\text { year }} \times \frac{\text { inst }}{3413 \text { Bice }}=397,917 \frac{\mathrm{KwH}}{Y_{\text {ear }}}
\end{aligned}
$$

Assumed: $C_{D}=0,80$

$K=1,0$

$v=1.0$

$D D=8640$

$\angle T=q_{i l}^{\circ}=$

$Q_{T}=7.450606 t$

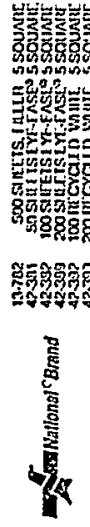




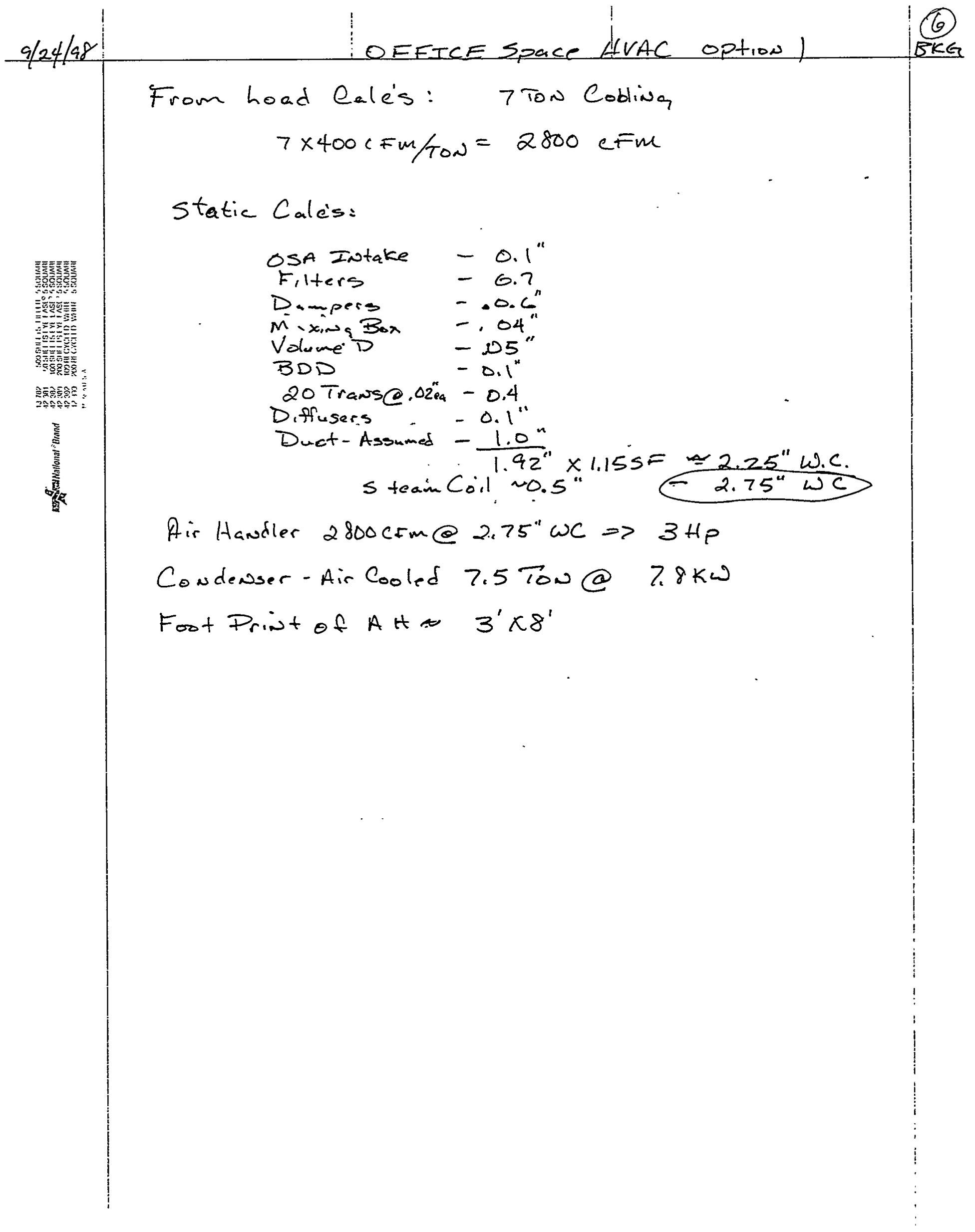


ZONEZ Air Reguirements

Assumed: Once thru Air

$1^{\text {st }}$ FloorAtea $\simeq 5000 \mathrm{ft}^{2} @ 12^{\prime}$ Herght $=60000 \mathrm{ft}^{3}$

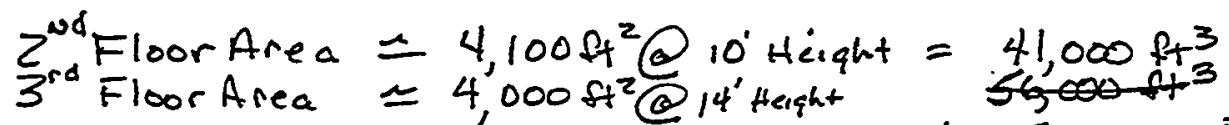

Total 157,000 $\mathrm{ft}^{3}$

$157,000 * 4$ A.C. $11 \mathrm{tr}=628,000 \mathrm{ft} / \mathrm{Ar}=10,500 \mathrm{cFm}$ 之 $18 H E P A S$

Supply Faw Cale's

S.P. Calés

OSA Intake - $0.1^{\prime \prime}$
Fiters
Heat Coils $0.1^{\prime \prime}$
Ductiog-Assumed - $0.75^{\circ}$
Total $2.75 * 1.155= \pm 3.4^{\prime \prime}$ W.C.

Eq: Reguire two Systems; owe for Backup t Change Oat of filters one hooked to Backup Pawer

Rea-Fass-10,500cFm@3.4"w.c., ClassII, H5ttp

Lea - HFPA Housings- $2 \times 5$ Redundawt, $55 T$ 


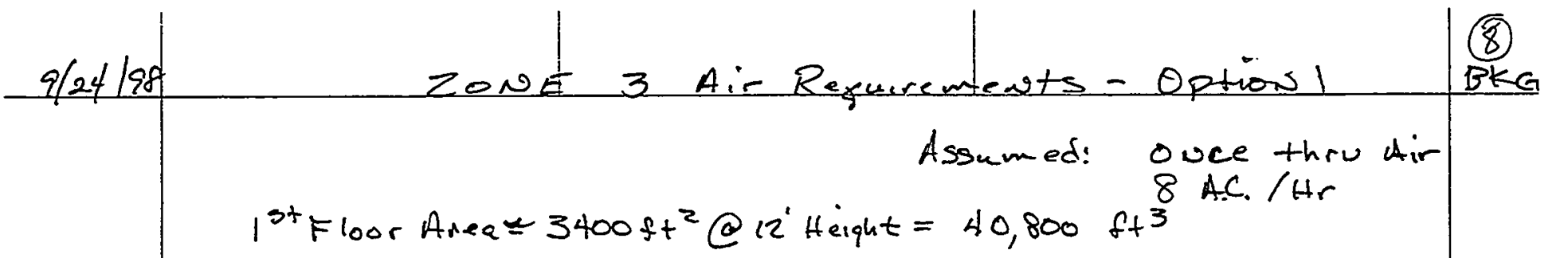

$z^{0 d}$ Floor Area $=3050 \mathrm{ft}^{2} @ 10^{\prime}$ Height $=30,500 \mathrm{ft}^{3}$

$$
\text { Total 7l, } 300 \mathrm{ft}^{3}
$$

$71,300 * 8$ A.C. $/ \mathrm{Hr}=570,400 \frac{\mathrm{ft}^{3}}{\mathrm{Hr}} \cong 9,500 \mathrm{CFm}-10 \mathrm{HEPA}=$ Exhausf:

$$
\text { SP Cale's }
$$

Space Static - $0.75^{\prime \prime}$

AEPA'S-Dirty - 4.0"

$$
\text { Ductiog-Assumed }-\frac{2.0^{\prime \prime}}{6.75^{\prime \prime} \omega . c^{*}}
$$

* Supply from zove 2 to Zave 3 Assume equal, HEPA's sized from Zove $Z$ supply Air

Eq:

Require two Systems, owe for Back up and Chawge out Exhaust frome owe 3

2ea - Fans - 9,500 cFm@6.75* W.C., Class II, ij5T, 15 HP

2ea - HEPA Housiags.- $2 \times 5$ Redundant, SST.

Supply to Zowe 3 :

200-Faws-10,500CFm@6.25"w.C., Class II, 5ST, 15 HP

2ea HEPA Housings - $2 \times 5$ Redundant, SST. *

* HEPA Housiags Sized from Air Supplyed to Zowe $Z$ urea. 


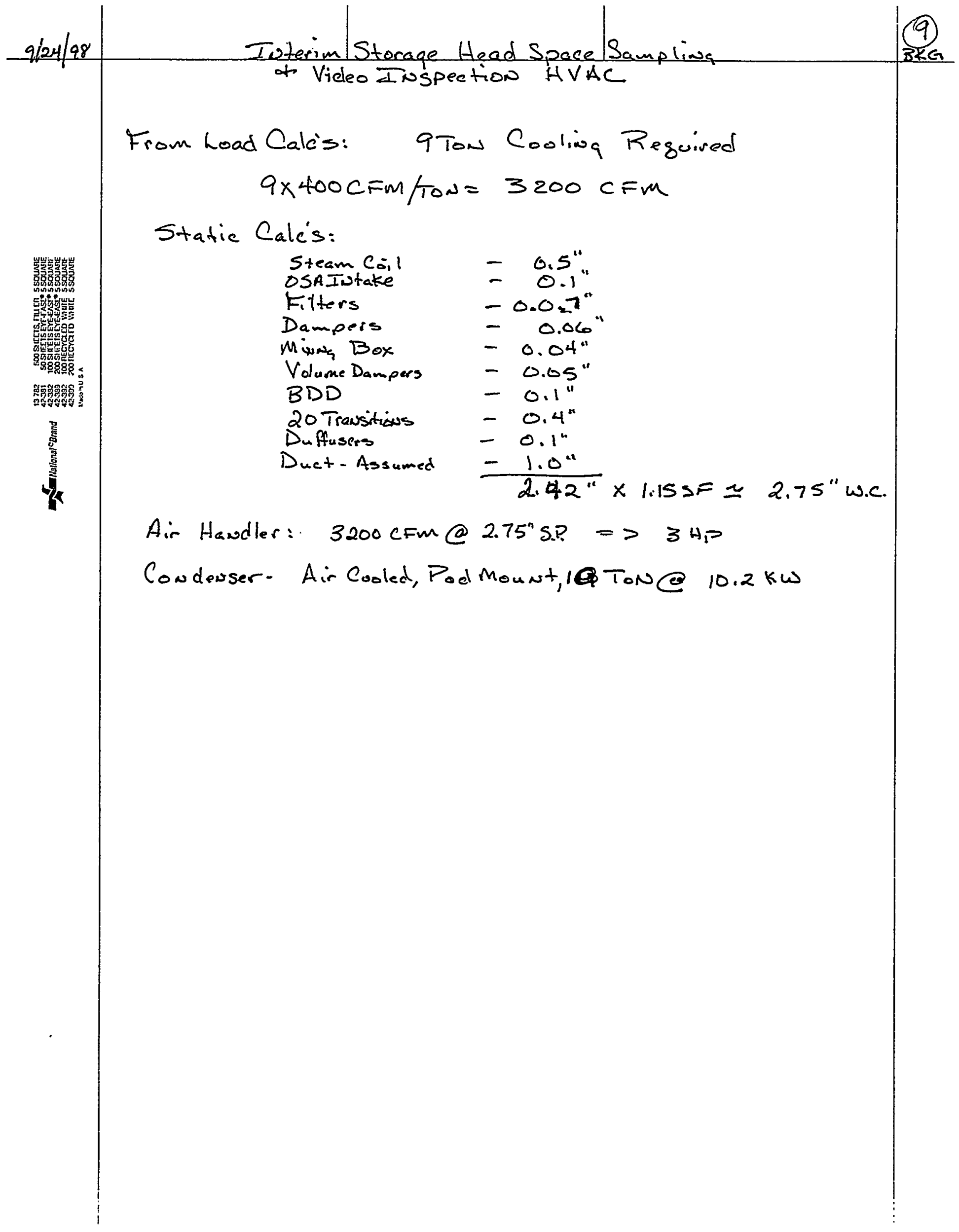




\begin{tabular}{|c|c|c|c|c|c|c|}
\hline Project: & CsIX/TRU Grout & Project & & & & \\
\hline Calculated by: & B. K. Grover & Date: & Sept 24,98 & Checked By: & $\mathrm{RH}$ & \\
\hline Zone: & Office space & & & Design TIme & 5 & $10 \mathrm{am}$ to $5 \mathrm{pm}$ \\
\hline$\overline{7}$ & & HEAT TRANSMIS & SION\& SOLARI & HEAT GAIN : & $\because \%$ & $\because$ \\
\hline COMPONENT & $\begin{array}{l}\text { Gross AREA } \\
\text { (FT2) }\end{array}$ & \begin{tabular}{|l|} 
Net AREA \\
(FT/2)
\end{tabular} & $\begin{array}{l}\text { TEMP DIFF } \\
\text { (DEG F) } \times\end{array}$ & "U'FACTOR= & $\begin{array}{l}\text { SENSIBLE } \\
\text { SEtu/h }\end{array}$ & IT LATENT \\
\hline ROOF $\because$ R & & & 62 & 0.04 & & \\
\hline ACTUAL AREA & 1,877 & 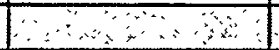 & 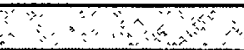 & $8+9$ & $\because \because$ & $\therefore$ \\
\hline NORTH WALL & 1,936 & 1,839 & 25 & 0.06 & 2,729 & \\
\hline N.E. WALL & & & 25 & 0.06 & & \\
\hline EAST WALL & 1,144 & 1,087 & 27 & 0.06 & 1,741 & \\
\hline S.E.WALL : & & & 27 & 0.06 & & \\
\hline SOUTH WALL & & & 31 & 0.06 & & \\
\hline S.W. WALL & & & 61 & 0.06 & & \\
\hline WEST WALL : & 880 & 836 & 72 & 0.06 & 3,572 & \\
\hline N.W. WALL & & & 55 & 0.06 & & \\
\hline GLASS TRANS & 198 & 198 & 20 & 0.55 & 2,178 & \\
\hline MISC. $\because$ & & & & & & \\
\hline MISC. & & & & & & \\
\hline COMPONENT & $A R E A(F T 2)$ & $x$ SHGF & CXCLF & $\mathrm{XSC}=$ & $2 \ldots$ & $\therefore+3$ \\
\hline N. GLASSSOLAR & 97 & 37 & 0.75 & 0.55 & 1,477 & \\
\hline N.E GLASS SOLAR & & 159 & 0.23 & 0.55 & & \\
\hline E. GLASSSOLAR & 57 & 215 & 0.23 & 0.55 & 1,556 & \\
\hline S.E SLASS SOLAR & & 179 & 0.29 & 0.55 & & \\
\hline S. GLASSSOLAR : & & 128 & 0.41 & 0.55 & & \\
\hline S.W. GLA'SS SOLAR & & 179 & 0.59 & 0.55 & & \\
\hline W. GLASS SOLLAR & 44 & 215 & 0.56 & 0.55 & 2,914 & \\
\hline N.W. GLASSSOLAR & & 159 & 0.51 & 0.55 & & \\
\hline SKYLIGHT SOLAR & & 254 & 0.56 & 0.50 & & \\
\hline 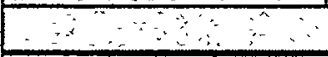 & $\therefore \therefore$ & 40 & 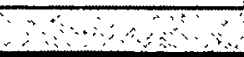 & 4 & $\because \therefore+\cdots$ & $\ldots+\cdots$ \\
\hline$\because=7$ & 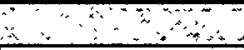 & INTERNAL HEAT & GAINS & Natis & nos & 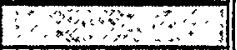 \\
\hline CFM per Person $=$ & 15 & People Coóling Lo & d'Factor (CLE) & 1.00 & 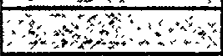 & 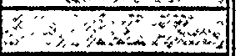 \\
\hline Number of Qccupants & 19 & Lighting Eooling $L$ & ad actor (OLF) & 0.89 & 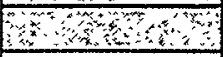 & $-a, b \quad n$ \\
\hline Btuper Óccupant & 255 & Sensibible $x \in L F$ & 255 & Sulatent & 4,845 & 4,845 \\
\hline Lighis & 2 & WHrfF^2 & 3,754 & $\times 3,413 \times \mathrm{CLF}=$ & 11,403 & \\
\hline Power & 5 & WWHREN & 9,385 & $\times 3413=3$ & 32,031 & \\
\hline 20 & $x_{n} x_{2}$ & sin & 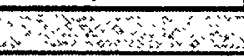 & $x_{2}$ & $x^{\prime} x^{\prime}$ & $3 \times, 94$ \\
\hline 4 & $A+4$ & OUTSIDE AIRHE & ATGAIN ${ }^{2}$ & ond & W & $x^{6} y^{n}+y^{2}+4$ \\
\hline 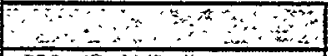 & CFM & ning & now & Constant & 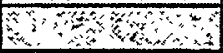 & and \\
\hline Sensible & 285 & Temp Diff. & 20 & 0.92 & 5,244 & - \\
\hline Lavent & 285 & Ratio Difference & -0.0022 & 4840 & - & Neg. Value \\
\hline 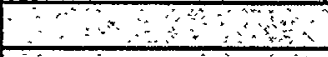 & 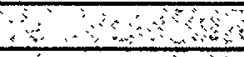 & 20 & 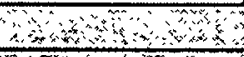 & SUBTOTAL & 69,690 & 4,845 \\
\hline$\therefore+$ & $\because \cdots$ & 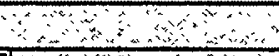 & Safety Factor = & 1.10 & 76,659 & 4,845 \\
\hline & & 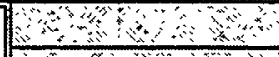 & $x^{3}+40+5$ & 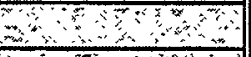 & 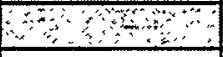 & $\cdots$ \\
\hline & & $5+\infty$ & SOn & tal Heât Gains & 76,659 & 4,845 \\
\hline & & Combined Total & Bas & $\mathrm{MBH}$ & 82 & \\
\hline & & 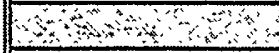 & end & TONS & 6.8 & \\
\hline & & Tofal Cooling Loa & 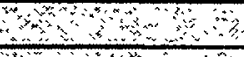 & BUTHAL & 43.4 & \\
\hline & & $2 \mathrm{mon}$ & 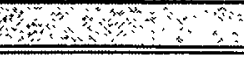 & $\mathrm{FtATON}$ & 276 & \\
\hline
\end{tabular}




\begin{tabular}{|c|c|c|c|c|c|}
\hline Project: & CsIX/TRU Grou & roject & & & \\
\hline Calculated by: & B. K. Grover & Dat & Sept 24,98 & Checked By: & $\mathrm{RH}$ \\
\hline Zone: & Office space & & & Design TIme & 4 \\
\hline & & Heałting Trănst & ssion Loss & & \\
\hline Exposuré & $\begin{array}{c}\text { Gross Area } \\
\mathrm{Ft}^{\prime \prime 2}\end{array}$ & $\begin{array}{c}\text { Nét Aréa } \mathrm{X} \\
\mathrm{F}^{\wedge}{ }^{2}\end{array}$ & $\begin{array}{l}\text { Temperature } x \\
\text { Difference }\end{array}$ & "Un Fàctồ = & Btu/Hr \\
\hline Roof & & & 91 & 0.04 & \\
\hline Walls & 3,960 & 3,762 & 91 & 0.06 & 20,317 \\
\hline Glazing & 198 & 198 & 91 & 0.55 & 9,910 \\
\hline Floor & & & 91 & 0.54 & \\
\hline & & & & . & \\
\hline & $\begin{array}{l}\text { Perimeter } \\
\text { Ftx }\end{array}$ & $\begin{array}{l}\text { Heat } \\
\text { Below } \mathrm{C}\end{array}$ & ade $x$ & $\begin{array}{l}\text { Temperature }= \\
\text { Difference }\end{array}$ & \\
\hline Basement Walls & & & & 91 & \\
\hline & & & & $\therefore$ & \\
\hline & $\begin{array}{l}\text { Heat Loss } x \\
\text { Coefficient }\end{array}$ & $\begin{array}{l}\text { Are } \\
\mathrm{Ft}\end{array}$ & & $\begin{array}{c}\text { Temperafure }= \\
\text { Difference }\end{array}$ & \\
\hline Basement Floors & & & & 91 & \\
\hline & & & & & \\
\hline & $\begin{array}{l}\text { Heấ Loss } x \\
\text { @ Grade }\end{array}$ & $\begin{array}{r}\text { Perim } \\
F \\
\end{array}$ & $2 x$ & $\begin{array}{l}\text { Temperature } \\
\text { Difference }\end{array}$ & $\therefore$ \\
\hline Floor@Grade & 0.65 & 134 & & 91 & 7,926 \\
\hline & & & & $\therefore$ & \\
\hline & & Infiltratiớn $\mathrm{Hea}$ & toss & & \\
\hline & 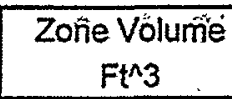 & $\begin{array}{l}\text { Air Chängès x } \\
\text { Per. Minute }\end{array}$ & $\begin{array}{c}\text { Air x } \\
\text { Constant }\end{array}$ & $\begin{array}{l}\text { Temperature }= \\
\text { Difference }\end{array}$ & \\
\hline Infilfration? & 41,294 & 0.0167 & 0.92 & 91 & 57,619 \\
\hline Room Height & 22.0 & $\therefore i$ & 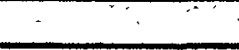 & $\because \cdots$ & \\
\hline & & Fresh Air Heati & Load & $\therefore$ & \\
\hline . & CFM $x$ & $\therefore$ Temp & $\begin{array}{l}\text { ature } x \\
\text { Degree }\end{array}$ & $\begin{array}{l}\text { Avr = : } \\
\text { Constant }\end{array}$ & 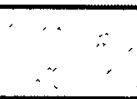 \\
\hline Fresh Air Intake, & 285 & 9 & & 0.92 & 23,860 \\
\hline & & $\because \quad \therefore$ & $\because$ & $\therefore \cdots$ & \\
\hline & & Total Heating, Lo & d... & $\mathrm{MBH}$ & 120 \\
\hline & & 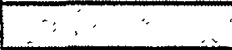 & $\because, \ldots, \cdots$ & $k \mathbf{k}$ & 35 \\
\hline & & $\therefore \quad \therefore, \ldots$ & $y^{\prime} \quad \ldots$, & $\therefore$ BtulFt² & 64 \\
\hline & & Outside Balance & oint Temperature & (dég $F)$ & 35 \\
\hline
\end{tabular}




\begin{tabular}{|c|c|c|c|c|}
\hline Project: & \multicolumn{2}{|l|}{ CsIXTRU Grout Project } & Latitude-degrees: & $\overline{43}$ \\
\hline Calculated by: & \multicolumn{2}{|l|}{ B. K. Grover } & Longitude - degrees: & 112 \\
\hline Site Location & \multicolumn{2}{|l|}{ INEEL } & Elevation - Ft: & 4938 \\
\hline 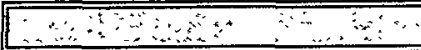 & \multicolumn{4}{|c|}{ Design Conditions } \\
\hline$\sqrt{2+360}$ & Outside Temperáture & Insidé Temperature & Deltat $T$ & Gooling Air Hümitity. \\
\hline Winter Dessign@ $97.5 \%$ & \begin{tabular}{|l|}
-19 \\
\end{tabular} & 72 & 91 & Ratio Difference \\
\hline Summer Design @ 2.5\% & 95 & 75 & 20 & -0.0022 \\
\hline
\end{tabular}

\begin{tabular}{|c|c|c|c|c|}
\hline$\therefore \quad, \cdots$ & $\because \cdots \cdots$ & Zone "U"Factors & 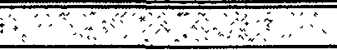 & $\therefore$ \\
\hline 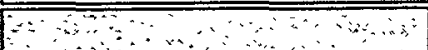 & $\therefore \quad \therefore$ Wall & 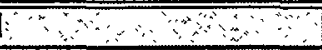 & Wall & 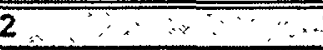 \\
\hline Walls & $\therefore$ Construction & "Value & Construction & "R" Vălựe \\
\hline Outside Air Tilm & 15 MPH Moving Air & 0.17 & 15 MPH Moving Air & 0.17 \\
\hline Qưtside Surfacê & & & & \\
\hline Outer Sheàthing & & & & \\
\hline Insulation & $\mathrm{R}-16$ & 16.00 & & \\
\hline insioe Surface & & & & \\
\hline Inside Air Film & Still Air & 0.68 & Still Air & 0.68 \\
\hline Other & & & & \\
\hline Other & & & & \\
\hline TótálR Factor & & 16.85 & & 0.85 \\
\hline$U^{\prime \prime}$ Factor $-T^{\prime} / R=$ & & 0.06 & & 1.18 \\
\hline 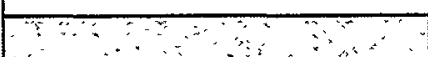 & : & $8 x^{2}$ & Floor & 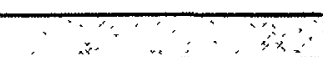 \\
\hline$\because$ Floors and Roofs & $\therefore$ Cónš́trứctiơn & R" Value & Construction & R" Value \\
\hline Qutside Air Film & $15 \mathrm{MPH}$ Moving Air & 0.17 & Still Air & 0.92 \\
\hline Outside Surface & & & & \\
\hline Oufer sheathing & & & & \\
\hline Insulation & R-22 & 22 & & \\
\hline Inside Suiface : & & & & \\
\hline Inside Air Film & Still Air & 0.61 & Still Air & 0.92 \\
\hline Other & & & & \\
\hline Other & & & & \\
\hline Tótal R Factor & & 22.78 & & 1.84 \\
\hline Uir Faetor $-1 / R=$ & & 0.04 & & 0.54 \\
\hline Glazinglalass boors & Properfies & Novilues & Metalsoórs & 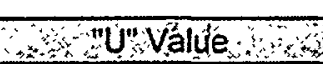 \\
\hline Winter and Summer & Dbl Pain / Tinted & 0.55 & 1-3/4" Metal Door & 0.39 \\
\hline GlazinglGiass Doors & Properties & Shading coefficient & & \\
\hline Glazing & Dbl Pain / Tinted & 0.55 & & \\
\hline Shyiftes & Double Pain & 0.50 & & \\
\hline
\end{tabular}




\begin{tabular}{|c|c|c|c|c|c|c|}
\hline \multirow{3}{*}{\begin{tabular}{|l} 
Project: \\
Calculated by: \\
Zone: \\
\end{tabular}} & \multicolumn{3}{|c|}{ CsIXTRU Grout Project Scenario 1} & \multirow{2}{*}{\multicolumn{3}{|c|}{ Checked By: }} \\
\hline & \multirow{2}{*}{ B. K. Grover } & \multicolumn{2}{|c|}{ Date: Oct. 19,98} & & & \\
\hline & & & & Design Time & 5 & 10 am to $5 \mathrm{pm}$ \\
\hline \multicolumn{7}{|c|}{ 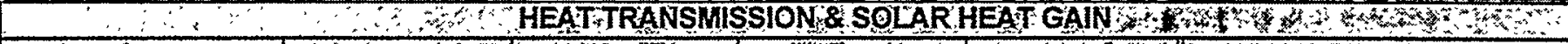 } \\
\hline CONPONENT & $\begin{array}{l}\text { GroSSAREA } \\
\text { (ETA2) }\end{array}$ & YISAREA & $\begin{array}{l}\text { TEMP DIFE } \\
\text { (DEG } \mathrm{A} \text { X }\end{array}$ & 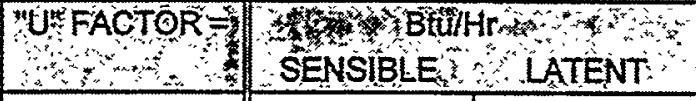 & \multicolumn{2}{|c|}{$\begin{array}{l}\text { STO } \\
\text { SENSIBLE W LATENT: }\end{array}$} \\
\hline & & & & 0.04 & 38,920 & \\
\hline ACTUAL AREA: & 41,500 & 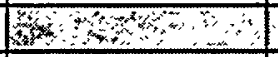 & $\because x+3$ & $6+4$ & 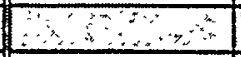 & $4:$ \\
\hline NORTH WALL & 2,816 & 2,675 & 25 & 0.06 & $.3,969$ & \\
\hline N:E WALL & & & 25 & 0.06 & & \\
\hline EASTWALL & 4,960 & 4,861 & 27 & 0.06 & 7,789 & \\
\hline SEEWALL ${ }^{\prime \prime} x$ & & & 27 & 0.06 & & \\
\hline SOLTHWALL & 2,816 & 2,816 & 31 & 0.06 & 5,181 & \\
\hline S:WiWALL & & & 61 & 0.06 & & \\
\hline WEST WALLL & 4,960 & 4,861 & 72 & 0.06 & 20,770 & \\
\hline NWWWALL : & & & 55 & 0.06 & & \\
\hline GLASS TRANS, & 339 & 339 & 20 & 0.55 & 3,731 & \\
\hline \multicolumn{7}{|l|}{ MISO, } \\
\hline \multicolumn{7}{|l|}{ MISG } \\
\hline COMPONENAS & AREA (FI 2 ) & X ${ }^{*} \mathrm{SHG}^{\prime}$ & $\times C L F$, & $\times \mathrm{SC}_{3}$ & 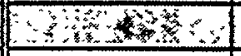 & \\
\hline NGLASSSOLARE & 141 & 37 & 0.75 & 0.55 & 2,149 & \\
\hline N:EKGIASS:SOLAR & & 159 & 0.23 & 0.55 & & \\
\hline EE GUASS SOLAR & 99 & 215 & 0.23 & 0.55 & 2,698 & \\
\hline S:EXGLASSSSOLAR & & 179 & 0.29 & 0.55 & & \\
\hline S.GLASSSOLAR & & 128 & 0.41 & 0.55 & & \\
\hline SWW GKASSSSOLAR & & 179 & 0.59 & 0.55 & & \\
\hline W GEASS SOLAR & 99 & 215 & 0.56 & 0.55 & 6,569 & \\
\hline NiWKGLASS SOELAR & & 159 & 0.51 & 0.55 & & \\
\hline SKMLTGHT SOLAR & & \begin{tabular}{|l|}
254 \\
\end{tabular} & 0.56 & 0.50 & & \\
\hline \multicolumn{7}{|c|}{ 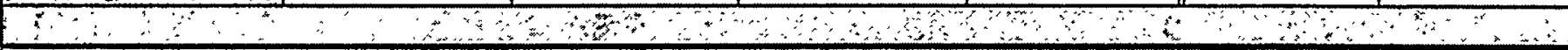 } \\
\hline \multicolumn{7}{|c|}{ 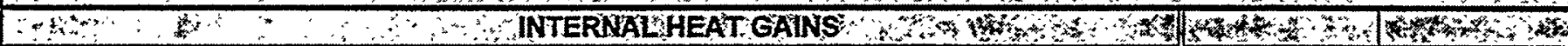 } \\
\hline GFM per Person: $=$ & - & \multicolumn{2}{|c|}{ Réoplécooling Lód Factor (CLF) } & 1.00 & \\
\hline Number of Occupants & - & \multicolumn{2}{|c|}{ Lighting Cooling Load Factor (CLF) } & 0.89 & \multicolumn{2}{|c|}{ 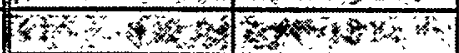 } \\
\hline Btuper Occupant: & - & ESensibleXCEF & - & * Hátent & & \\
\hline - Lightș & - & W WHrík 2 & & $\times 3413 \times O L=$ & & \\
\hline$\cdots$ Pó̂́é & - & 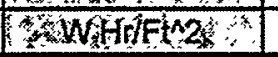 & & I $\times 3 \times 3413=$ & & \\
\hline \multirow{2}{*}{\multicolumn{5}{|c|}{ 准 }} & \multirow{2}{*}{ 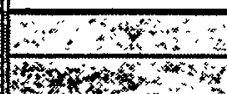 } & $\alpha+4, \cdots$ \\
\hline $4 \quad \frac{1}{4} 40^{28}$ & 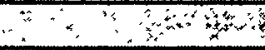 & COUTSIDEARTHE & AT GAIN & & & sols \\
\hline$\because ; \quad$ & CEA & H, & 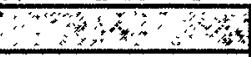 & Constant & \% & gen \\
\hline Sénsible & & 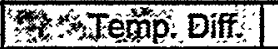 & 20 & 0.92 & & - \\
\hline Lafent : & & Ratiósifurence & -0.0022 & 4840 & - & Neg. Value \\
\hline$\therefore$ & $\because$ & 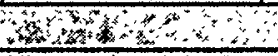 & 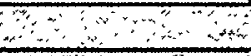 & SUBTOTAL & 91,776 & \\
\hline & & 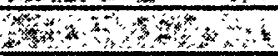 & Safey Factor=y & 1.10 & 100,954 & \\
\hline & & 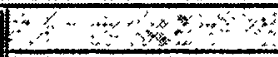 & 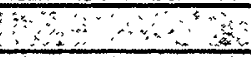 & 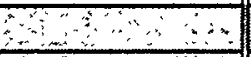 & $\cdots \cdots$ & \\
\hline & & $5 x+3 y, y$ & TO & tal Heat Gains: & 100,954 & \\
\hline & & Combined Total & 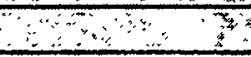 & $\mathrm{MBH}, \mathrm{s}$ & 101 & \\
\hline & & 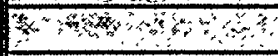 & $8 \div 28$ & TONS & 8.4 & \\
\hline & & Tofill Coolling Load & 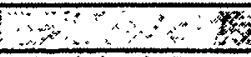 & B tufft 2 , w & 2.4 & \\
\hline & • & 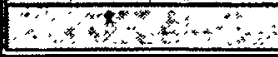 & 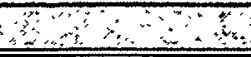 & Exitron & 4933 & \\
\hline
\end{tabular}




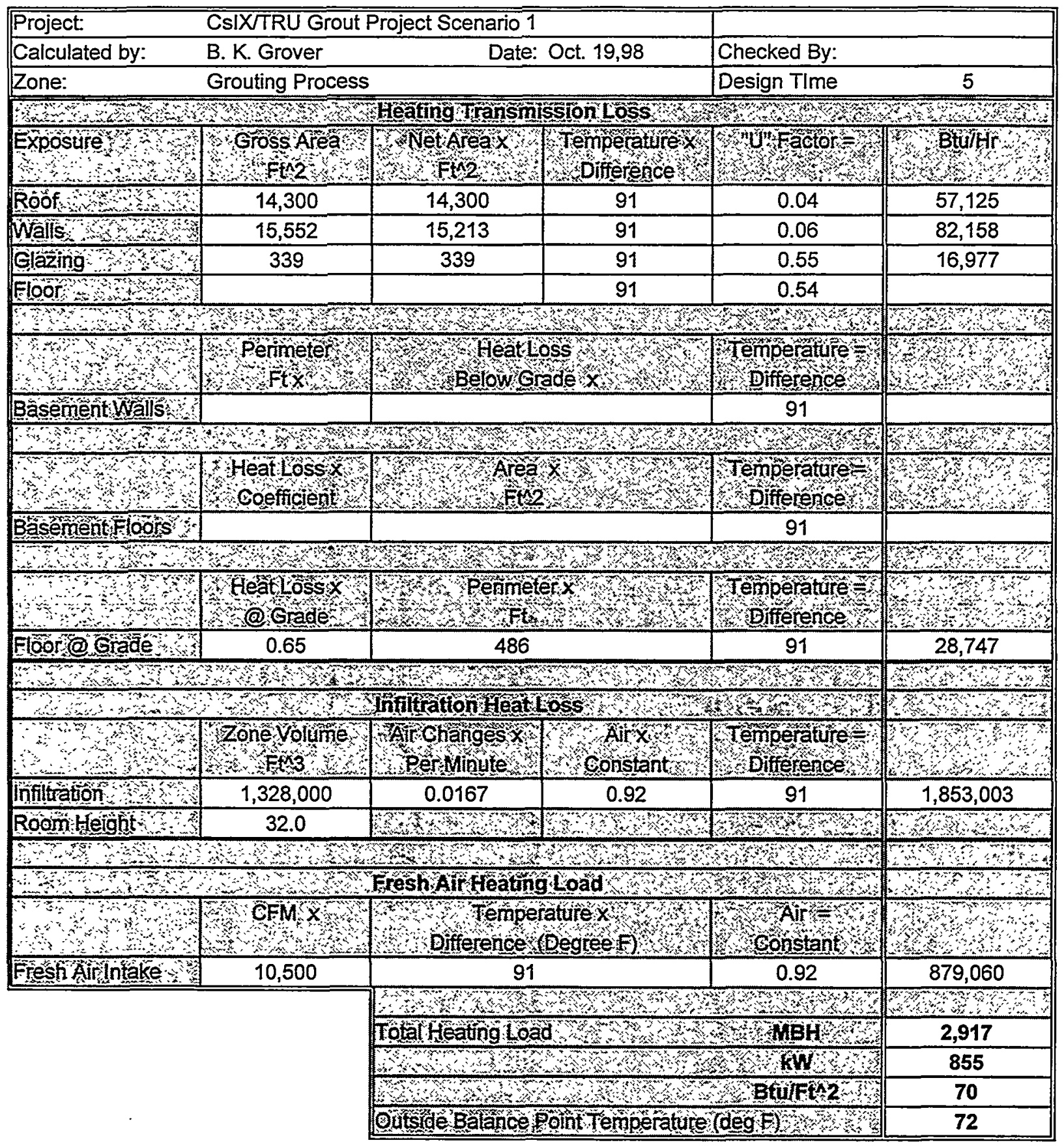




\begin{tabular}{|llrc|}
\hline Project: & CsIXTRU Grout Project Scenario 1 & Latitude - degrees: & \\
\hline Calculated by: & B. K. Grover & Longitude - degrees: & 112 \\
\hline Site Location & INEEL & Elevation - Ft: & 4938 \\
\hline
\end{tabular}

\begin{tabular}{|c|c|c|c|c|}
\hline 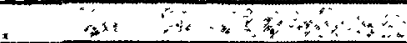 & 45 & Design Condit & & $6 \%$ \\
\hline 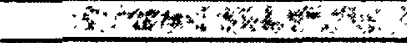 & Outsidestemperature & Inside Temperature & 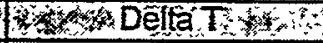 & Cooling Air Humitity \\
\hline Whoter Design: $975 \%$ & -19 & 72 & 91 & Rato Diference \\
\hline Sumíner Design:@2.5\% & 95 & 75 & 20 & -0.0022 \\
\hline
\end{tabular}

\begin{tabular}{|c|c|c|c|c|}
\hline 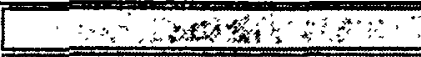 & & Zone U"Factors & 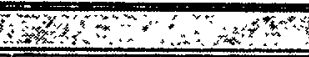 & 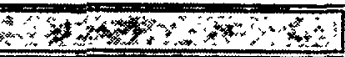 \\
\hline 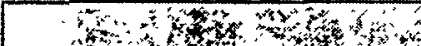 & Hallt W Wall & 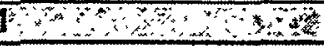 & $7628 \times 1$, Wal & 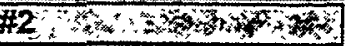 \\
\hline O Walls, & Construction & $R^{n}$ Value & KConstruction & Is \\
\hline Outsíde Air Film & 15 MPH Moving Air & 0.17 & 15 MPH Moving Air & 0.17 \\
\hline Outside'Surface & & & & \\
\hline Outer Sheathing, & & & & \\
\hline 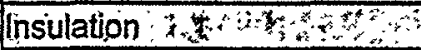 & $\mathrm{R}-16$ & 16.00 & & \\
\hline Inside:Surfácen & & & & \\
\hline Inside Air film $\mathrm{s}$, & Still Air & 0.68 & Still Air & 0.68 \\
\hline Other " ofen & & & & \\
\hline 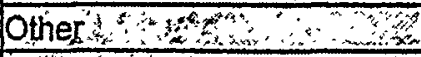 & & & & \\
\hline 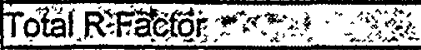 & & 16.85 & & 0.85 \\
\hline U." FactoritiR = & & 0.06 & & 1.18 \\
\hline 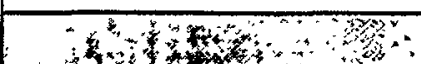 & Roof & 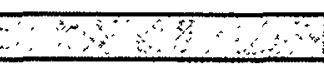 & 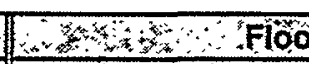 & Sand \\
\hline Holoos and Roofs & Construction & V V V V Iue & 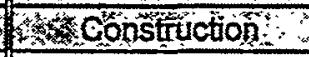 & 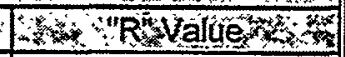 \\
\hline Qutșidé Alr Film & 15 MPH Moving Air & 0.17 & Still Air & 0.92 \\
\hline 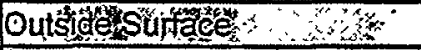 & & & & \\
\hline Outétsheathiog, & & & & \\
\hline 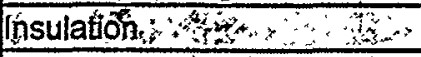 & R-22 & 22 & & \\
\hline Inside Surface! & & & & \\
\hline Inside Airfilm $\mathrm{g}$, & Still Air & 0.61 & Still Air & 0.92 \\
\hline Qther $x$ & & & & \\
\hline Other : & & & & \\
\hline Total R Factón & & 22.78 & & 1.84 \\
\hline 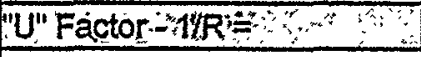 & & 0.04 & & 0.54 \\
\hline GlazinglGlassDoors & Próperties 7 r. & 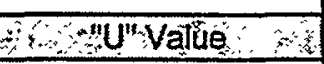 & MétalDoors & 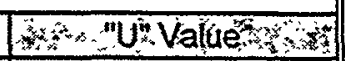 \\
\hline Winter and summer & Dbl Pain / Tinted & 0.55 & 1-3/4" Metal Door & 0.39 \\
\hline Glazinglglass'Doors & $\therefore$ Properties $\%$ in & Shading Coefficients & & \\
\hline rostazing & Dbl Pain / Tinted & 0.55 & & \\
\hline$\therefore$ SSkJifies & Double Pain & 0.50 & & \\
\hline
\end{tabular}




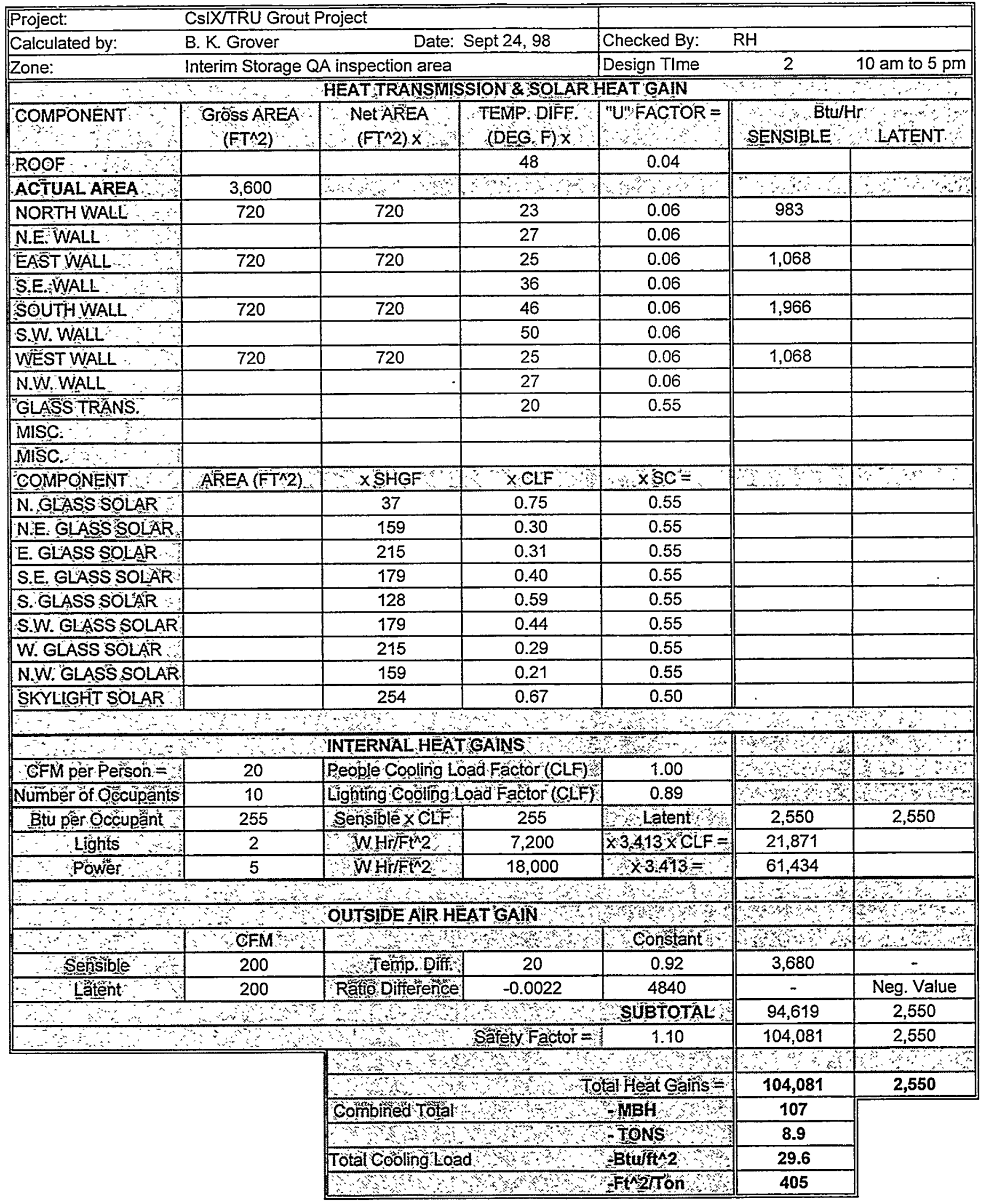




\begin{tabular}{|c|c|c|c|c|c|}
\hline Project: & CsIX/TRU Grout & Project & & & \\
\hline Calculated by: & B. K. Grover & Dat & Sept 24, 98 & Checked By: & $\overline{\mathrm{RH}}$ \\
\hline Zone: & Interim Storage & A inspection are & & Design TIme & 2 \\
\hline & & Hêating Trănsr & ssiôn Loss's & & \\
\hline Exposure & $\begin{array}{c}\text { Gross Area } \\
\mathrm{F}^{\wedge} 2\end{array}$ & $\begin{array}{c}\text { Net Arêa } \mathrm{X} \\
\mathrm{F}^{\wedge} 2\end{array}$ & $\begin{array}{c}\text { Temṕperaturé } x \\
\text { Difference }\end{array}$ & "U" Factór = & BtulHr \\
\hline Roof & 3,600 & & 91 & 0.04 & \\
\hline Walls & 2,880 & 2,880 & 91 & 0.06 & 15,554 \\
\hline Glazing & & & 91 & 0.55 & \\
\hline Floor & & & 91 & 0.54 & \\
\hline & & & & & \\
\hline & $\begin{array}{c}\text { Perimeter } \\
\text { Ftx }\end{array}$ & $\begin{array}{l}\text { Heat } \\
\text { Below } 0\end{array}$ & ade $\mathrm{x}$ & $\begin{array}{c}\text { Têmpêrâture }= \\
\text { Difference }\end{array}$ & \\
\hline Basement Walls & & & & 91 & \\
\hline & & & & & \\
\hline & $\begin{array}{l}\text { Heat Loss } x \\
\text { Coefficient }\end{array}$ & $\begin{array}{l}\text { Are } \\
\mathrm{Ft}^{\prime}\end{array}$ & $\bar{x}$ & $\begin{array}{c}\text { Temperature }= \\
\text { Difference }\end{array}$ & \\
\hline Basement Floors & & & & 91 & \\
\hline & & & & & \\
\hline - & $\begin{array}{l}\text { Heat Loss } x \\
\text { (c) Grade }\end{array}$ & Perím & $5 x$ & $\begin{array}{c}\text { Témpérature } \\
\text { Difference }\end{array}$ & \\
\hline Floor@Grade & 0.65 & 240 & & 91 & 14,196 \\
\hline & & Infiltration Hêa & oss & & \\
\hline & $\begin{array}{c}\text { Zone Volume } \\
\mathrm{F}_{3} \mathrm{t}^{\wedge} 3\end{array}$ & $\begin{array}{l}\text { Air Chángès } \mathrm{x} \\
\text { Per Minute }\end{array}$ & $\begin{array}{c}\text { Air } X \\
\text { Constant }\end{array}$ & $\begin{array}{c}\text { Tempêrature }= \\
\text { Difference }\end{array}$ & \\
\hline Infiltration : & 43,200 & 0.0167 & 0.92 & 91 & 60,278 \\
\hline Room Height & 12.0 & $\because ":$ & $\therefore \quad$. & $\because \quad \cdots$ & $:$ \\
\hline & & Fresh Air Heati & Load & & \\
\hline & CFM $x$ & $\begin{array}{r}\text { Temp } \\
\text { Difference }\end{array}$ & Degree $F$ & $\begin{array}{l}\text { Air }= \\
\text { Constant }\end{array}$ & \\
\hline Fresh Air Intáke, & 200 & 9 & & 0.92 & 16,744 \\
\hline & & & . & 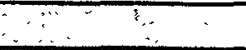 & $\because \cdots$ \\
\hline & & Tótal Heating Ló & & $\mathrm{MBH}$ & 107 \\
\hline & & $\therefore \quad \therefore$ & & $\mathbf{k W}$ & 31 \\
\hline & & & & Btu/Ft^2 & 30 \\
\hline & & Outside Balance & oint Temperature & $(\operatorname{deg} F)$ & (1) \\
\hline
\end{tabular}




\begin{tabular}{|c|c|c|c|}
\hline Project: & CsIXIRU Grout Project & Latitude - degrees: & 43 \\
\hline Calculated by: & B. K. Grover & Longitude - degrees: & 112 \\
\hline Site Location & INEEL & Elevation - Ft: & 4938 \\
\hline
\end{tabular}

\begin{tabular}{|c|c|c|c|c|}
\hline$\because \because \because \because \because$, & 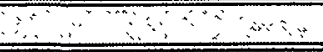 & Désign Cónóitions & 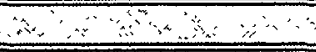 & $\therefore x^{n}$ \\
\hline$\therefore 4$ & Oútside Temperature & Inside Temperatứre & DeltaT & Cóoling Air Hứmitity. \\
\hline Winter Design@ @ 97.5\%: & -19 & 72 & 91 & Ratio Differencé \\
\hline SummerDesign@2.5\% & 95 & 75 & 20 & -0.0022 \\
\hline
\end{tabular}

\begin{tabular}{|c|c|c|c|c|}
\hline 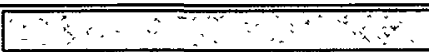 & $\therefore$ & Zone "u"Factórs & $\therefore+\cdots, \cdots$ & $8 \%$ \\
\hline \multirow{2}{*}{ Walls } & \multicolumn{2}{|c|}{ Wall \#1, } & \multicolumn{2}{|c|}{ Wall 2} \\
\hline & Constuction & "R" Value & Cônstruction & $\because " \mathrm{R}^{\prime \prime}$ Value \\
\hline Qutside Air Film & 15 MPH Moving Air & 0.17 & 15 MPH Moving Air & 0.17 \\
\hline \multicolumn{5}{|l|}{ Outside Surface : } \\
\hline \multicolumn{5}{|l|}{ Oû́ter Sheathing : : : } \\
\hline Insulation & $R-16$ & 16.00 & & \\
\hline \multicolumn{5}{|l|}{ Inside Surface : } \\
\hline Ínsid̆ Air Film & Still Air & 0.68 & Still Air & 0.68 \\
\hline \multicolumn{5}{|l|}{ Öthèr .... } \\
\hline \multicolumn{5}{|l|}{ Other } \\
\hline Total R Factor & & 16.85 & & 0.85 \\
\hline "Unagtor $-1 / R=\therefore$ & & 0.06 & & 1.18 \\
\hline \multirow{2}{*}{ Floors and Roofs } & \multicolumn{2}{|l|}{$\therefore$ Rôf } & \multicolumn{2}{|l|}{$\because \because " \because \quad \therefore$ Floor } \\
\hline & Construction & "R" Valúe $\because$ & $\therefore$ Cônstruction : & $\because$ "R" Value \\
\hline Oúfșide Air Film & 15 MPH Moving Air & 0.17 & Still Air & 0.92 \\
\hline \multicolumn{5}{|l|}{ Oútsidde Surface } \\
\hline \multicolumn{5}{|l|}{ Outer Sheathing } \\
\hline Insúlation & $\mathrm{R}-22$ & 22 & & \\
\hline \multicolumn{5}{|l|}{ Inside Surface } \\
\hline Inside Air Film & Still Air & 0.61 & Still Air & 0.92 \\
\hline \multicolumn{5}{|l|}{ Other $\therefore$} \\
\hline \multicolumn{5}{|l|}{ Other $\because \quad \cdots$} \\
\hline Total Ractor & & 22.78 & & 1.84 \\
\hline "U"Factor - $1 / R=$ & & 0.04 & & 0.54 \\
\hline GlazínglGiass Doors & Properties $:$ : & "Un" Value & $\because$ Métál Doôrs & "U" Value \\
\hline$\therefore$ Winter and Summer & Dbl Pain / Tinted & 0.55 & 1-3/4" Metal Door & 0.39 \\
\hline
\end{tabular}

GläzinglGlass Doors d Rropenties Glazing 


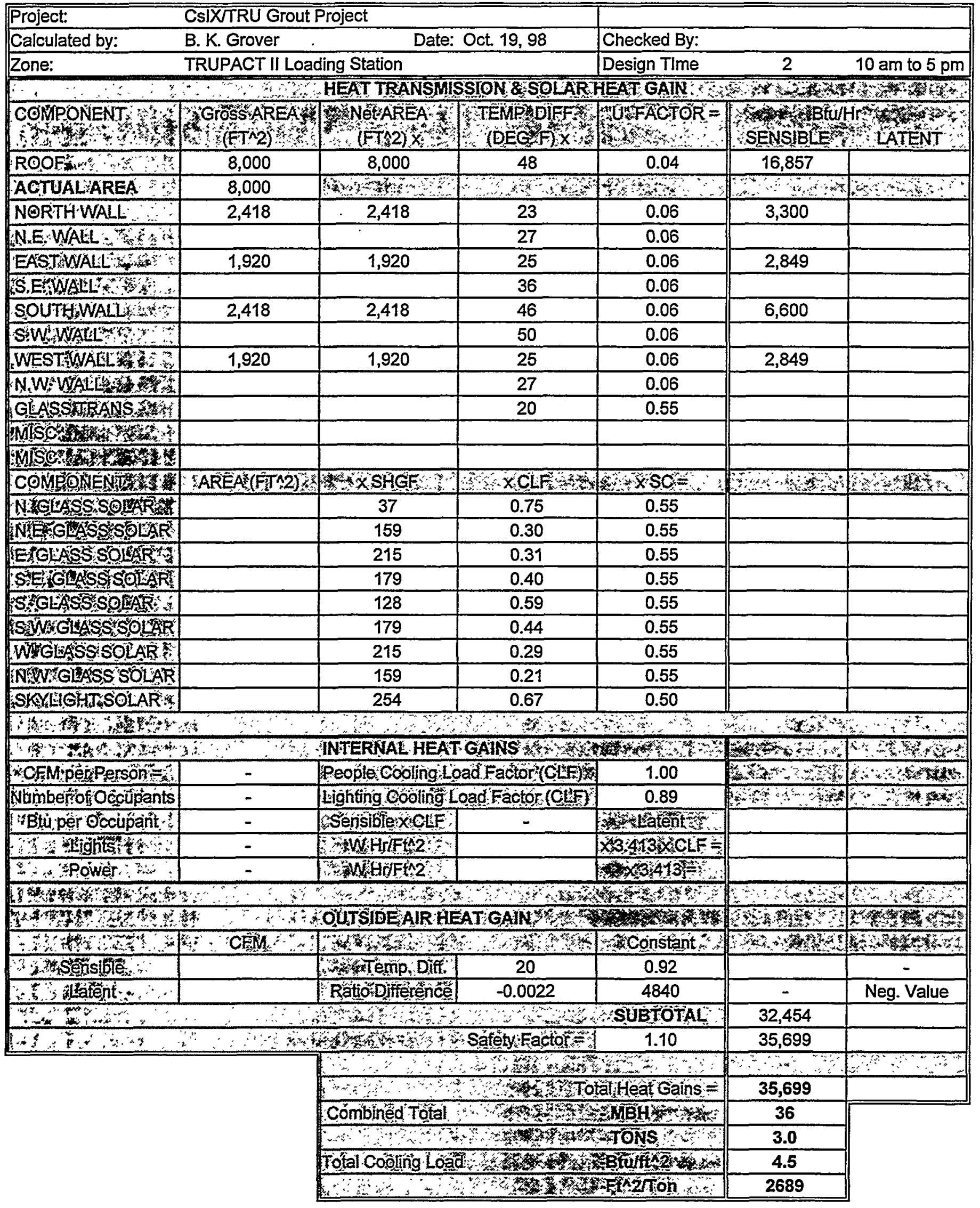




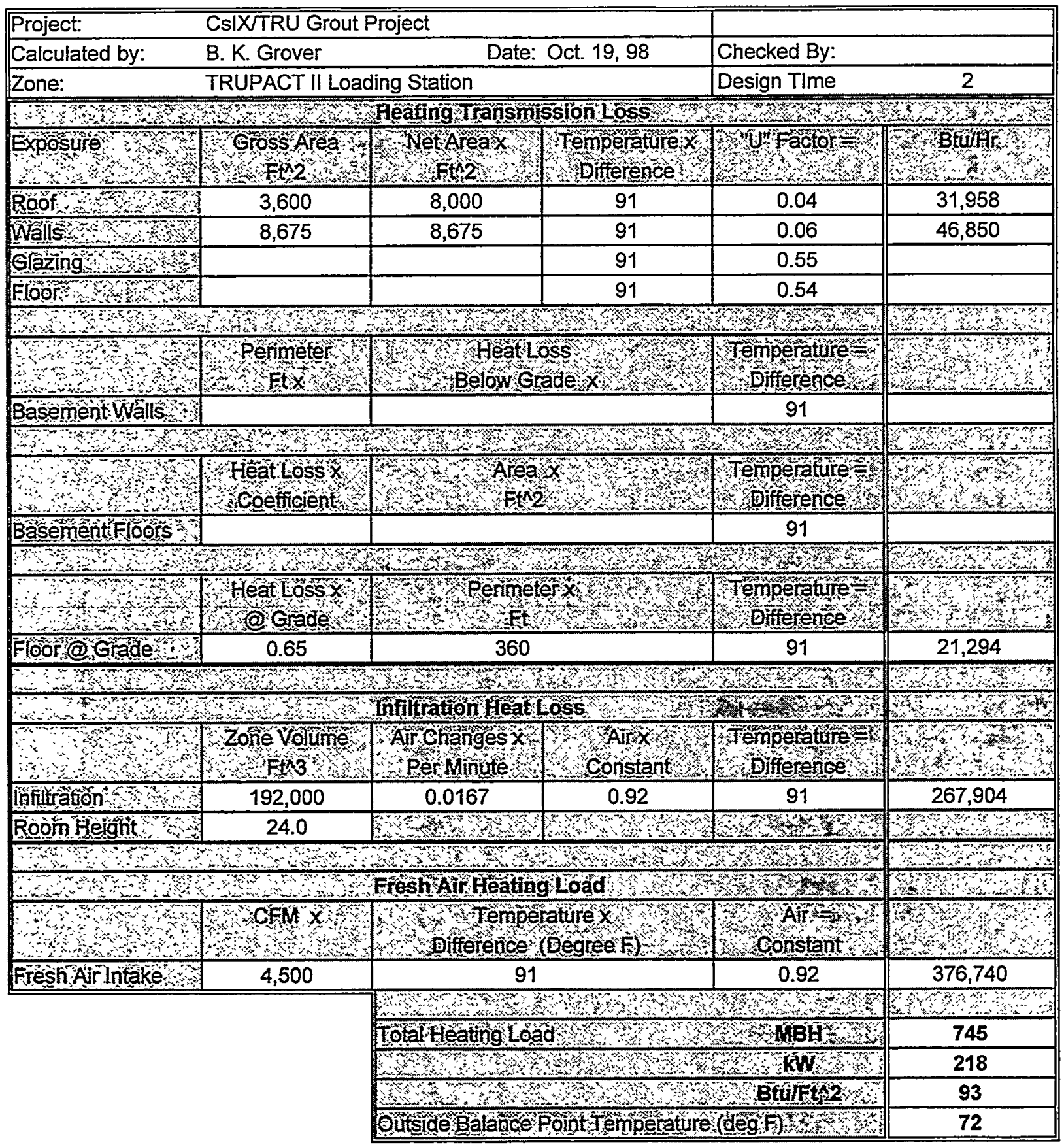




\begin{tabular}{|llrc|}
\hline Project: & CsIXTRU Grout Project & Latitude - degrees: & 43 \\
\hline Calculated by: & B. K. Grover & Longitude - degrees: & 112 \\
\hline Site Location & INEEL & Elevation - Ft & 4938 \\
\hline
\end{tabular}

\begin{tabular}{|c|c|c|c|c|}
\hline ... & $3 x+4, x$ & Design Conditions: & 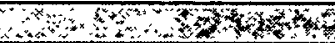 & $4 x$ \\
\hline$x_{0} x_{2}+\cdots$ & Oufside Temperature & Thside: Temperature & 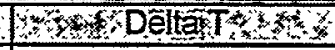 & Cooling Air Humitity \\
\hline WinterDesign@97.5\% & -19 & 72 & 91 & Ratio Difference $\mathrm{x}$ \\
\hline Sumimer Désign @2.5\% & 95 & 75 & 20 & -0.0022 \\
\hline
\end{tabular}

\begin{tabular}{|c|c|c|c|c|}
\hline \multirow{3}{*}{ Walls } & \multicolumn{4}{|c|}{ Zone "U" Factors e } \\
\hline & \multicolumn{2}{|c|}{ 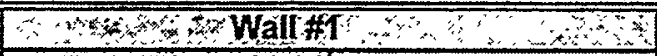 } & \multicolumn{2}{|c|}{ 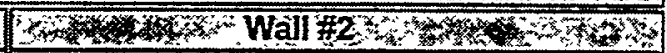 } \\
\hline & 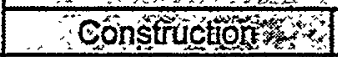 & $\therefore \quad R^{n}$ Valuécis & He Constructón & 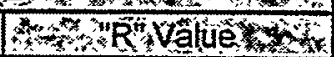 \\
\hline Qutsidè Air Film & 15 MPH Moving Air & 0.17 & 15 MPH Moving Air & 0.17 \\
\hline \multicolumn{5}{|l|}{ Outśide Surface : : : } \\
\hline \multicolumn{5}{|l|}{ Outer Sheathing } \\
\hline Insulation & R-16 & 16.00 & & \\
\hline \multicolumn{5}{|c|}{ Insider Sürace : $: \therefore 40$} \\
\hline Inside Air Film : & Still Air & 0.68 & Still Air & 0.68 \\
\hline \\
\hline Other & & & & \\
\hline Tofal:R Factor & & 16.85 & & 0.85 \\
\hline U" Factor - $1 / \mathrm{R}=$ & & 0.06 & & 1.18 \\
\hline \multicolumn{5}{|l|}{ 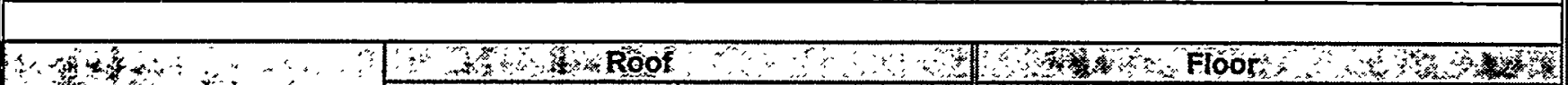 } \\
\hline WFloors,and Roofs & $\therefore$ Construetion & $\Rightarrow$ Rajue & Wo construction & 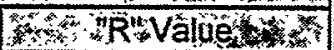 \\
\hline Qutside & 15 MPH Moving Air & 0.17 & Still Air & 0.92 \\
\hline \multicolumn{5}{|l|}{ 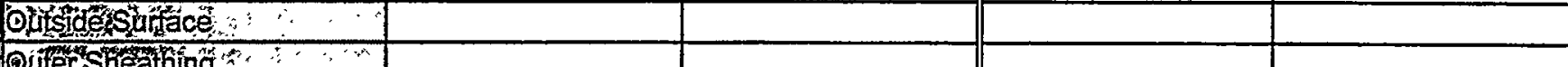 } \\
\hline \multicolumn{5}{|l|}{ Qufershienthing } \\
\hline Lisulatión & R-22 & 22 & & \\
\hline \multicolumn{5}{|l|}{ Iñide Surfạte : : } \\
\hline Inside:Aî́ Film & Still Air & 0.61 & Still Air & 0.92 \\
\hline \multicolumn{5}{|l|}{ Qther : } \\
\hline \multicolumn{5}{|l|}{ Othè } \\
\hline Totál.R.Factor: : & & 22.78 & & 1.84 \\
\hline U"Factor - 1/R= & & 0.04 & & 0.54 \\
\hline GlazinglGlass Doors & Propértes, $\%$ & 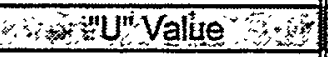 & 1. THetal Doors & 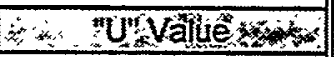 \\
\hline Winter and Summer & Dbl Pain / Tinted & 0.55 & 1-3/4" Metal Door & 0.39 \\
\hline GlạzinglGilaśs Dóors, & Propéties nxt & Shading Coefficient & & \\
\hline$\because$ Glázing & Dbl Pain./ Tinted & 0.55 & & \\
\hline Skylitês & Double Pain & 0.50 & & \\
\hline
\end{tabular}


Attachment D

CsIX/TRU Feasibility Study

Sketches and Drawings 


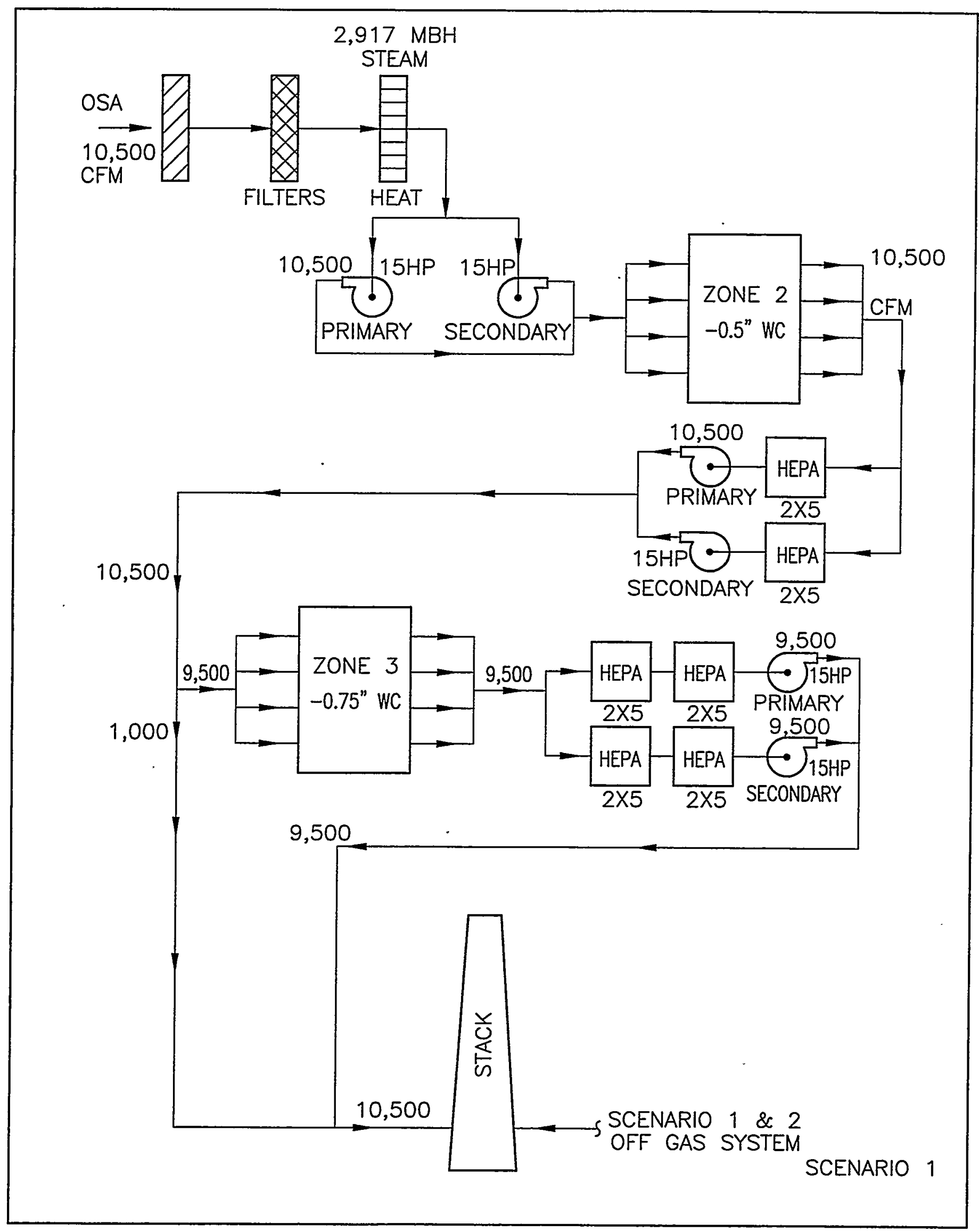




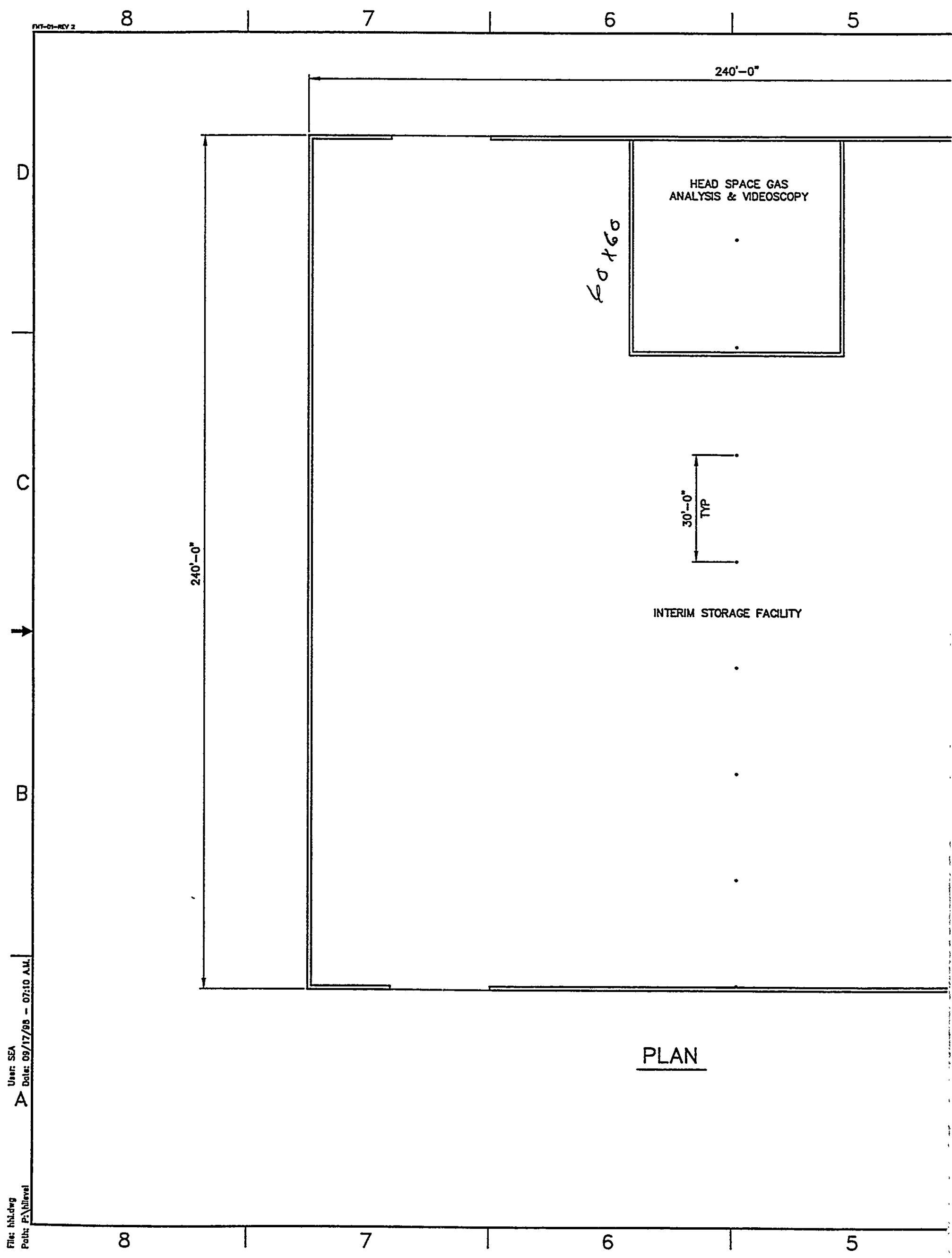




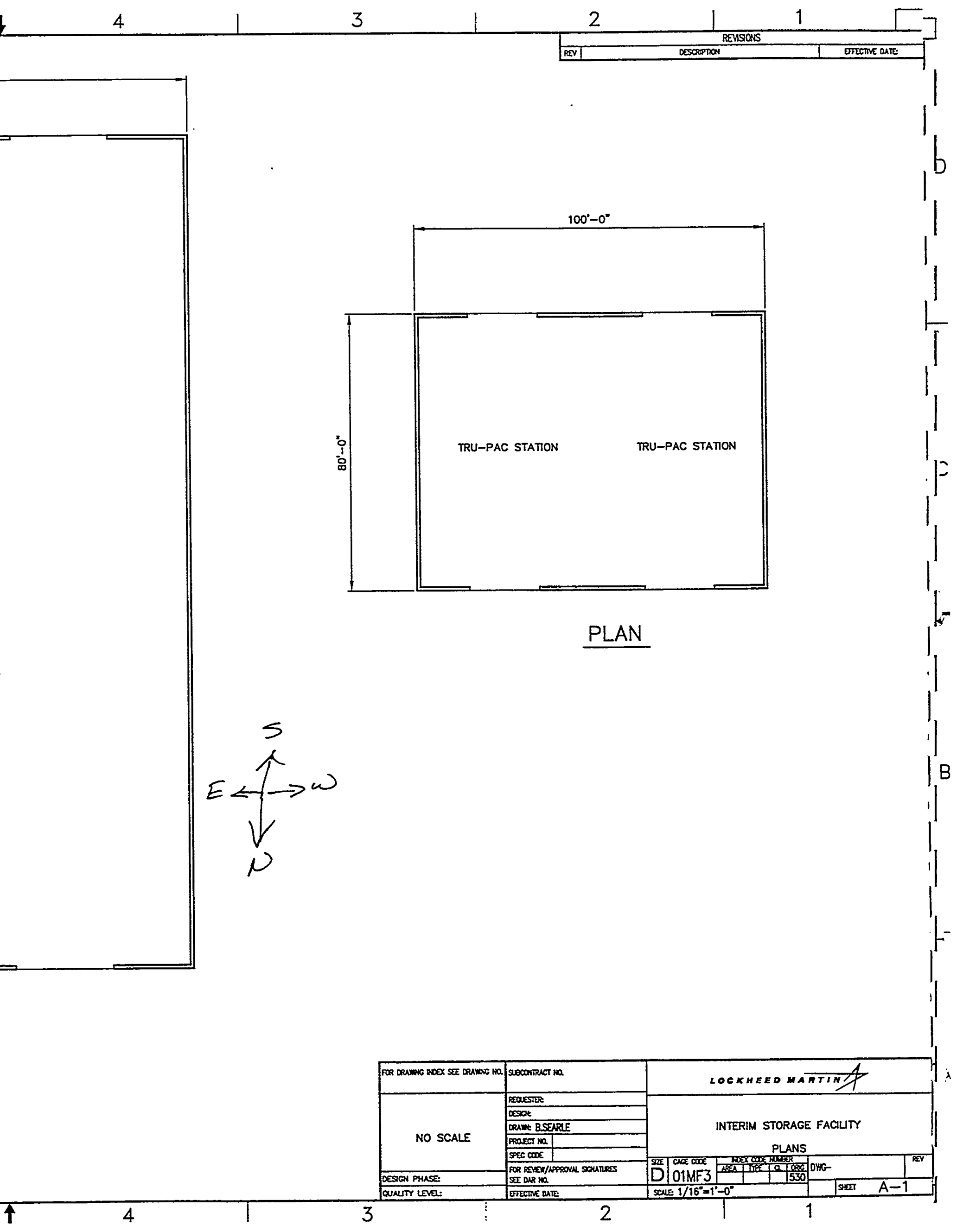




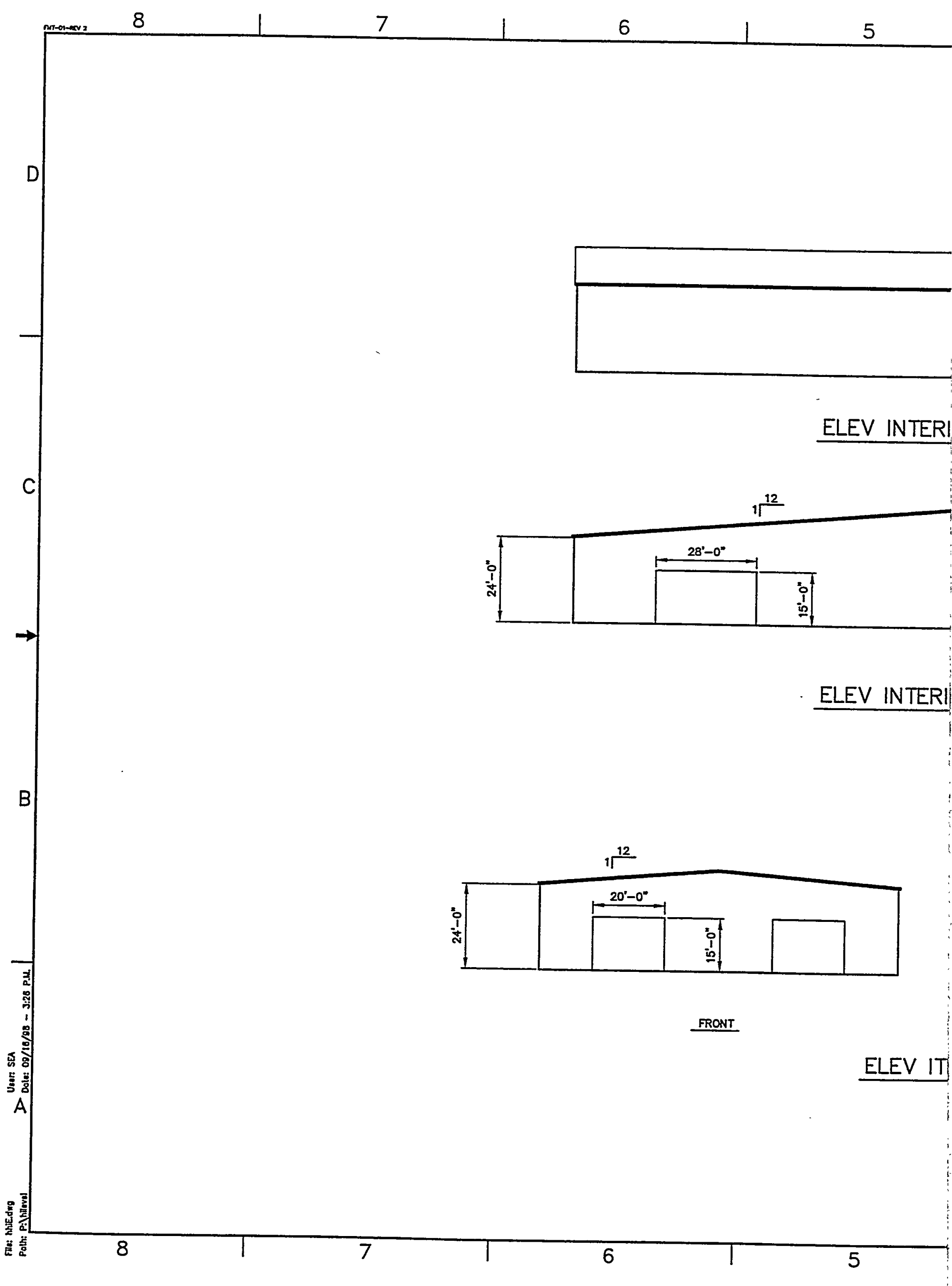




\begin{tabular}{|l|ll|l|l|l|}
\hline & 3 & 2 & 1 & 1 \\
\hline
\end{tabular}

SIDE

STORAGE FACILITY

C

FRONT

STORAGE FACILITY

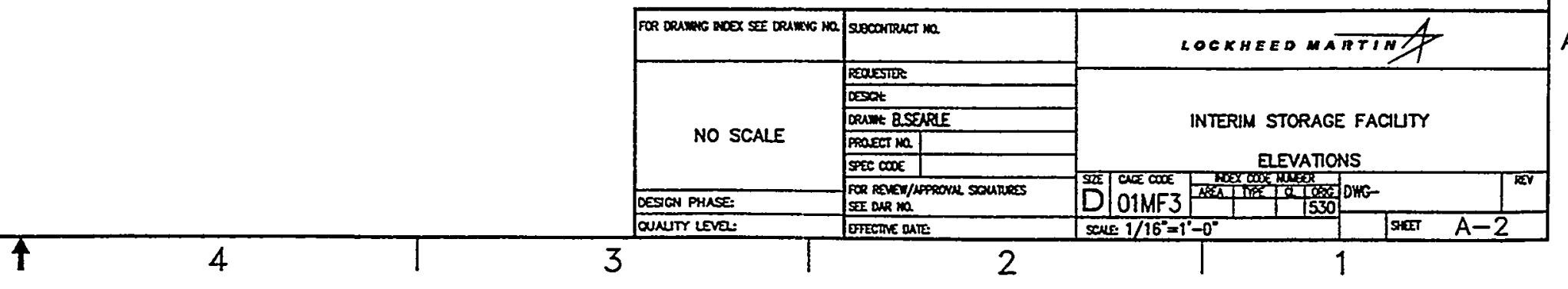




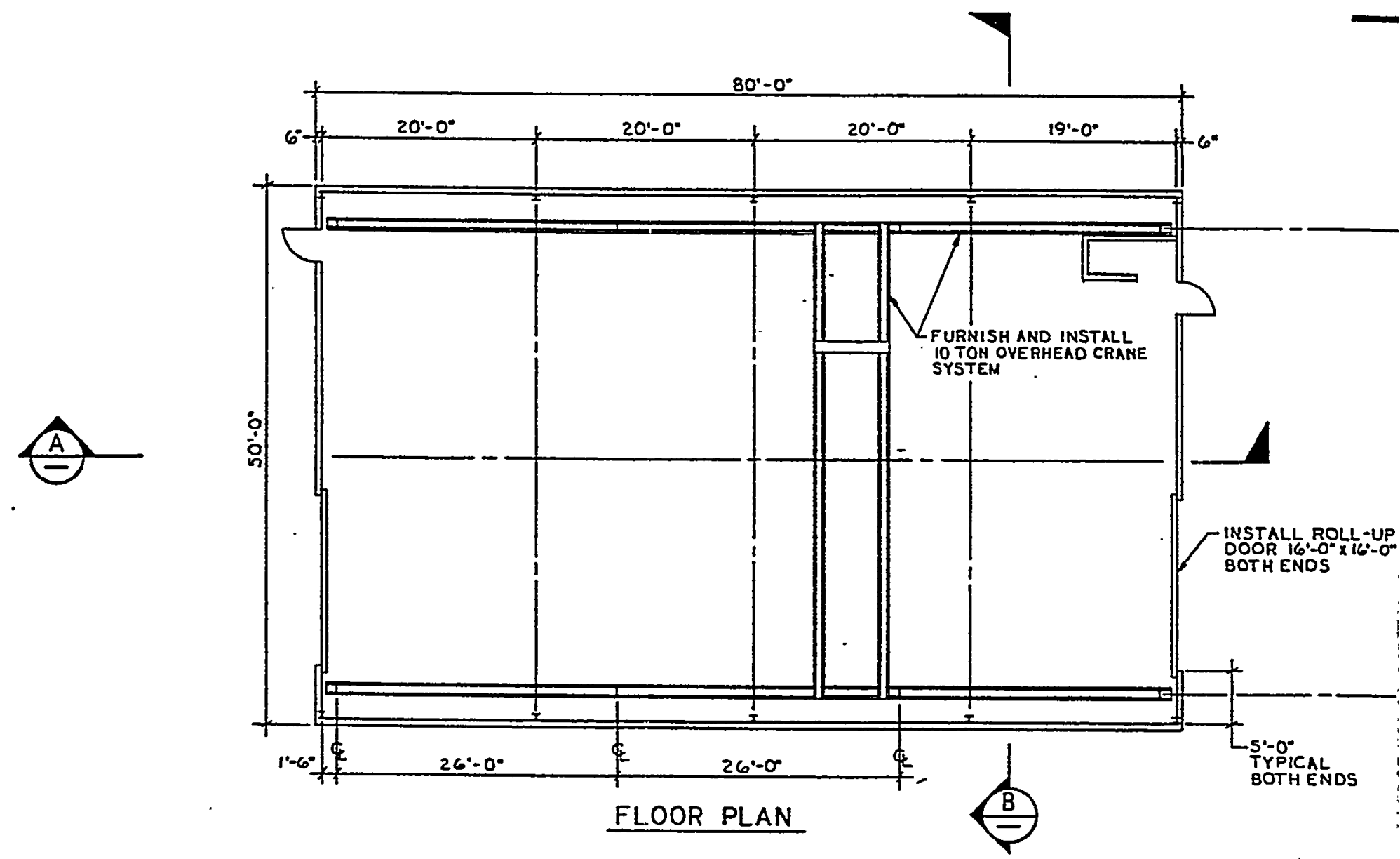

C

BULPING OIMENSIONS MRANE.

MMODATE

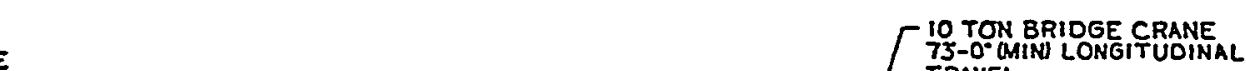

73-OON TRAVEL

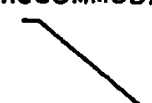

CRANE SUPPORT COLUMNS SUPPORT SYSTEM DESIGNED AND INSTALLED AS PER DIVISION I OF SPECIFICATION FOR SEISMIC ZONE 3 REQUIREMENTS.

PROVIOE BERI (1) 


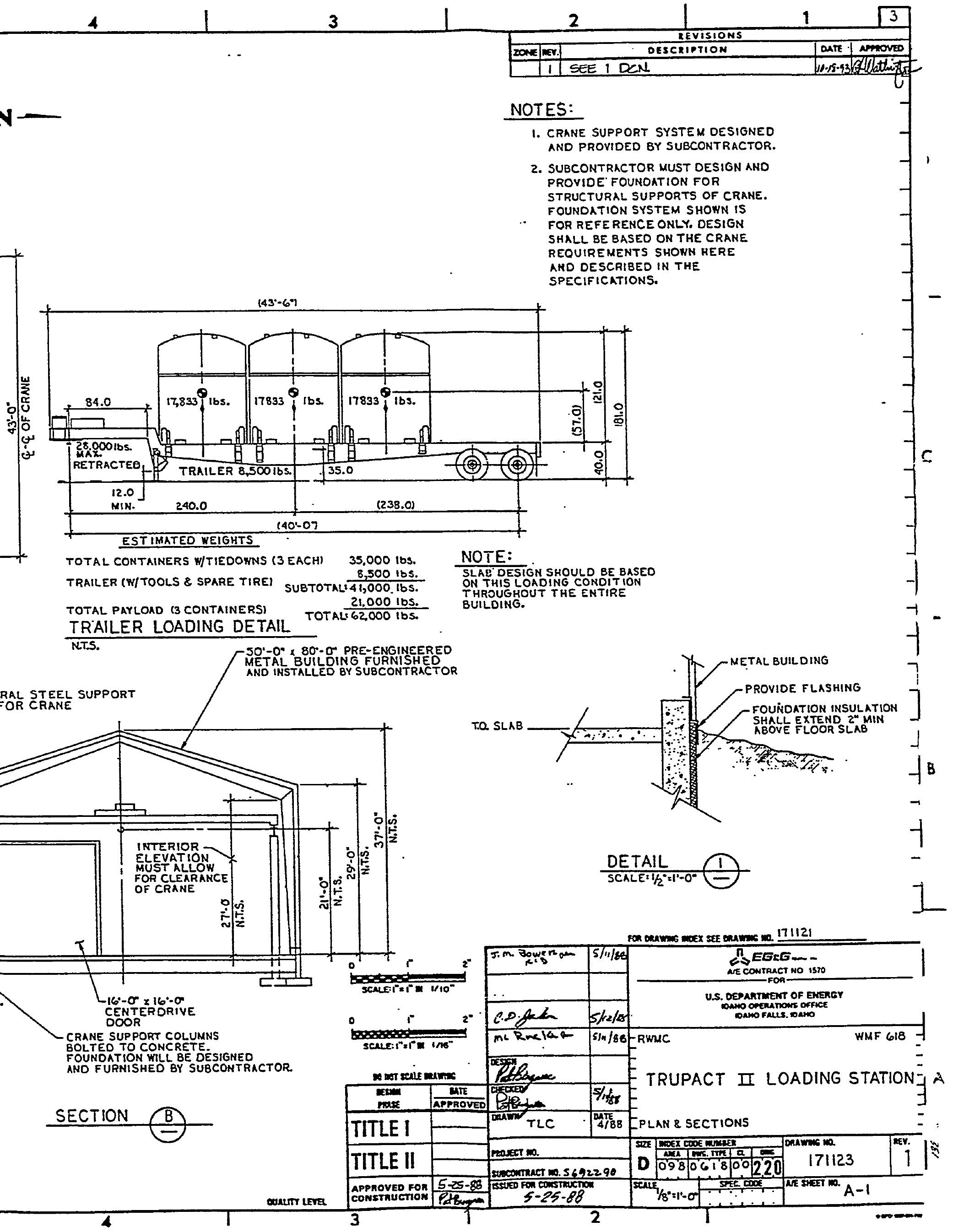


Project File Number $\quad$ 02BM1

Project/Task CsIX/TRU Grout Feasibility Study

Subtask D.5 Facility Design

Title: HVAC - Scenario 2

Summary:

This EDF describes documents requirements, assumptions, description, and calculations for the HVAC system required for Scenario 2.

EDF Contents:

- Attachment A - Detailed Process Description w/Assumptions \& Requirements.

- Attachment B - Equipment list for the Scenario 2 HVAC System.

- Attachment $\mathrm{C}$ - Supporting Calculations

- Attachment D - Sketches and Drawings

Distribution (complete package): C. W. Olsen MS 3211; K. L. Williams MS 3765; Project File; A copy of this EDF will be included in External Report No. INEEL/EXT $=98-00869$.

Distribution (summary package only):

\begin{tabular}{|c|c|c|c|c|c|}
\hline $\begin{array}{l}\text { Author } \\
\text { B. K. } \\
\text { Grover }\end{array}$ & $\begin{array}{l}\text { Dept. } \\
4130\end{array}$ & Reviewed & Date $10^{10 / 2 y / 98}$ & 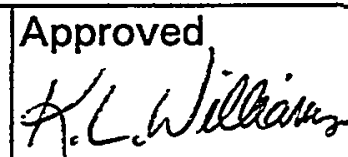 & $\begin{array}{l}\text { Date } \\
-10 / 27 / 98\end{array}$ \\
\hline
\end{tabular}




\author{
Attachment A \\ CsIX/TRU Feasibility Study
}

\title{
General
}

This EDF describes the HVAC system required for the Scenario 2 Grout Facility. The facility in scenario 2 has a smaller building and thus a smaller HVAC system. Other common facilities such as the Interim Storage Facility and TRUPACT II loading facility remain unchanged. See EDF-CsIX-011 for information on the interim Storage and TRUPACT II HVAC systems. Off-Gas is covered under EDFCsIX-013.

The HVAC system is comprised of three different zones for the Grouting facility. Each zone has its own air handling system. The primary and secondary zones have redundant systems with one system connected to backup power for continuous operation. The system is described below.

\section{Assumptions}

1. Eight air changes per hour for primary confinement.

2. Four air changes per hour for secondary confinement.

3. 20-CFM per person of outside air for administrative areas.

4. Only roughing filters are required on secondary zone outside air intake.

5. Only primary zone return air will require redundant HEPA filtration prior to discharge to the stack.

\section{Requirements}

1. The CsIX/TRU Grout building will be required to have two ventilation confinement zones per DOE Order 6430.1A.

2. Ventilation for primary and secondary zones are once through systems.

3. Steam heating will be used where applicable.

4. HEPA filtration will be required on both the primary and secondary zones. 


\section{Process Description}

The HVAC system for scenario 2 is very similar to scenario 1 . There are three primary zones. These zones are primary confinement, secondary confinement, and office space. Pressures of the different zones are +0.1 inch W.C. for office space, -0.5 inch W.C. for secondary confinement space, and -0.75 inch W.C. for primary confinement space.

The office space of the grouting facility will have a packaged type through the wall system. Since there is only two spaces that require cooling and heating a package type unit will be used. The unit will have two separate airhandling units and one heat pump. The $11 / 2$-ton heat pump will be mounted on a concrete pad just outside the facility by the office space and serve both airhandlers. The airhandling units will be located in the conditioned space and will supply a total of 400-CFM of air with $16 \mathrm{MBH}$ total heating capacity. 100-CFM of outside air will be introduced to the spaces by the wall mounted airhandling units. Estimated annual energy consumption for heating is $6621 \mathrm{kWh}$ per year and $1722 \mathrm{kWh}$ per year cooling. See calculations in Appendix $C$ for details on heating and cooling loads.

The heating and ventilation system for secondary confinement will be located on the east side of the second floor in the facility. Outside air will be taken into the system through a louvered inlet on the east wall. Total outside airflow is 6500 CFM for the secondary confinement system. CFM is based on a volumetric basis. 4 air changes per hour for the area served was used to develop the airflow requirements of the secondary air system. The air will be rough filtered, heated, and distributed to various areas throughout the building. The secondary confinement air system will be the main source of heating for the building. Heating will be from steam coils located on the outside air intake. Total capacity for the steam coil is $804 \mathrm{MBH}$ using the AE design criteria for the INEEL. Estimated annual energy consumption for the secondary system using a single measure check is $1.16 \times 10^{6} \mathrm{lbs}$. of steam per year. There will be two $71 / 2 \mathrm{Hp}$ airhandlers for supply to the secondary confinement areas. The primary airhandling unit will be connected to backup power. There are two airhandlers for maintenance and failure purposes. Once air is passed through the secondary zone, it will be HEPA filtered prior to being supplied to the primary zone or discharge to the stack. Two $2 \times 3$ HEPA filter housings will be installed, one HEPA housing will serve one airhandler. See airflow schematic in Appendix $D$ for more detail on airflow patterns and equipment layout. See calculations in Appendix $C$ for details on heating load.

Exhaust from the secondary confinement zone will be from another set of airhandlers. One of these two exhaust airhandlers will be connected to backup power for maintenance and failure purposes. Air exhausted from the secondary 
confinement system is either supplied to the primary confinement areas or exhausted to the stack. These $10 \mathrm{Hp}$ exhaust airhandlers are downstream of the HEPA filter housings. One airhandler is connected to one of the $2 \times 3 \mathrm{HEPA}$ housings. See air flow schematic in Appendix $D$ for more detail on equipment connection and airflows.

The Ventilation system for the primary confinement zone will be located on the eastern side of second floor in the facility, see drawing 2-A7. The primary containment ventilation system will receive air from the secondary containment system. The air discharged from the secondary zone is single HEPA filtered before entering into the primary zone. No heating or cooling capability is provided for the primary confinement zone system. Ductwork will be welded SST and be routed in areas not occupied by personnel. The primary zone system does not require the airflow that the secondary system does and for this reason, the secondary containment system bypasses $3000-$ CFM to the stack. See Scenario 2 airflow sketch in Appendix D for more detailed information on flow pattern. 3400-CFM of air is supplied to the primary confinement zone. CFM quantities are based on a volumetric basis. Primary confinement zone air requirements are 8 air changes per hour for the area served. The $5 \mathrm{Hp}$ airhandler and HEPA filter system is duplicated for maintenance and failure purposes. The primary airhandling unit will be connected to back up power. Once air has passed through the primary confinement zone, it will be double HEPA filtered and discharge to the stack.

The two common facilities, the Interim Storage and TRUPACT II Loading Facility, HVAC systems are described in EDF-CsIX-011. See that EDF for more information on those facilities. 


\author{
Attachment B \\ CsIX/TRU Feasibility Study \\ Equipment list for the Scenario 2 HVAC System
}

Grouting Facility

Office space airhandlers - 400-CFM, packaged split system

Condenser -1 ton cooling, pad mounted, DX, $1.2 \mathrm{kWatt}$

Secondary Confinement - Backward inclined Utility fan, Class II, 6400-CFM,

3.4 inch W.C., SST, $7.5 \mathrm{Hp}, 2$ each, one connected to backup power HEPA filter housing $-2 \times 3,6400-C F M$, SST, 2 each

Steam heat coils $-804 \mathrm{MBH}$ total load

Primary confinement supply fan - Backward inclined utility fan, Class II, 6400-CFM

6.75 inch W.C., SST, $10 \mathrm{Hp}, 2$ each, one connected to backup power

Exhaust fans - Backward inclined utility fan, Class II, 3400-CFM,

6.75 inch W.C., SST, $5 \mathrm{Hp}, 2$ each, one connected to backup power HEPA filter housing $-2 \times 2$, double filter, 3400-CFM, SST, 2 each 
Attachment C

CsIX/TRU Feasibility Study

Supporting calculations 
\begin{tabular}{l|l|l} 
10/20/g8 & Scenario & Energy Consume \\
& OFFICE SPACE: Single Measure Check
\end{tabular}

From Pales:

$$
\begin{aligned}
& B L C=U A=26.25=U A \\
& E_{\text {cool }}=\frac{24 * B L C * C D D *\left(1+\alpha_{0}\right)}{C O P} \\
&=\frac{24 * 26.25 * 343 *(1+0.1)}{2.8} . \\
&=84.892 \times 10^{3} \frac{\text { Btu }}{\text { Tear }}
\end{aligned}
$$

OTHER GAIUS:

$$
\begin{aligned}
& \text { GSA: } 1840 \mathrm{Btu} / \mathrm{Ar}_{-} \\
& E_{8 .}: \frac{5,785 \mathrm{Btul} / \mathrm{H}}{7625 \frac{\mathrm{Btu}}{1+r}} \\
& \text { Total }_{0} \\
& E_{I G}=?, 625 * 10 * 76=5.79 \times 10^{\circ} \frac{\text { Btu }}{\text { Sear }}
\end{aligned}
$$

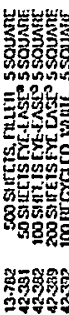

Assumed: $\quad \alpha_{0}=0.1$$$
\begin{aligned}
& \alpha_{D}=0.1 \\
& C O P=2.8 \text { A }
\end{aligned}
$$$$
C D D=343
$$

$$
\begin{aligned}
\text { operation }= & 10 \text { Hr/ Day } \\
& 76 \text { Day/ lea }
\end{aligned}
$$

Total Energy Consumption:

$$
\begin{aligned}
F_{\text {total }} & =5.79 \times 10^{6}+84.8 \times 10^{3}=5.88 \frac{8^{6} \mathrm{~B}_{0}}{i_{\text {ea }}} \\
& =1,722 \frac{\mathrm{kwt4}}{\varphi_{\text {ear }}}
\end{aligned}
$$




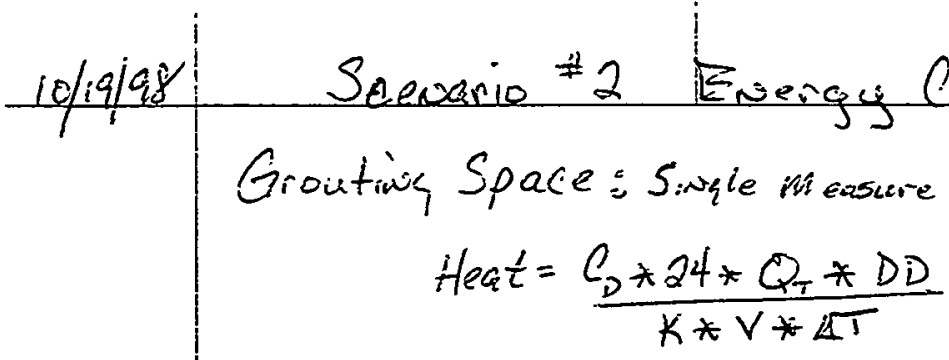

$$
\begin{aligned}
& =\frac{0.62 * 21 * 804,000 * 8640}{1,0 * 10 * 91}=1.135 \times 10^{9} \frac{\text { Bin }}{\text { Year }}
\end{aligned}
$$

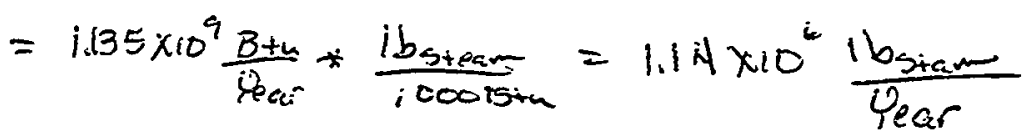

$$
\begin{aligned}
& C_{D}=.62 \\
& A=1.0 \\
& V=1.0 \\
& D D=8640 \\
& D T=91 . \mathrm{F} \\
& Q_{\tau}=804,100 \frac{\mathrm{B}+\mathrm{H}}{\mathrm{H} \lambda}
\end{aligned}
$$

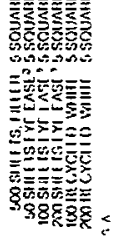

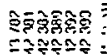

OFFice Space: Single Measure Method

Assumed

$$
\begin{aligned}
& \text { Heat }=\frac{C_{D} * 24 * Q_{T} * D D}{h * V * \Delta T} \\
& =\frac{0.62 * 24 * 16,000 * 8640}{1.0 * 1.0 * 91}=22.6 \times 10^{6} \frac{\text { Btu }}{Y_{\text {ear }}}
\end{aligned}
$$

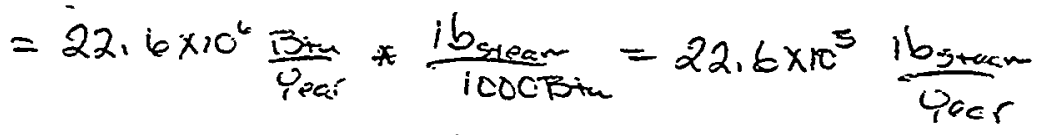

$$
\begin{aligned}
& =22.6 \times 10^{6} \frac{B+u}{4 e a} * \frac{K \omega H}{343 B+u}=6,621 \mathrm{Kwis}
\end{aligned}
$$

BXS

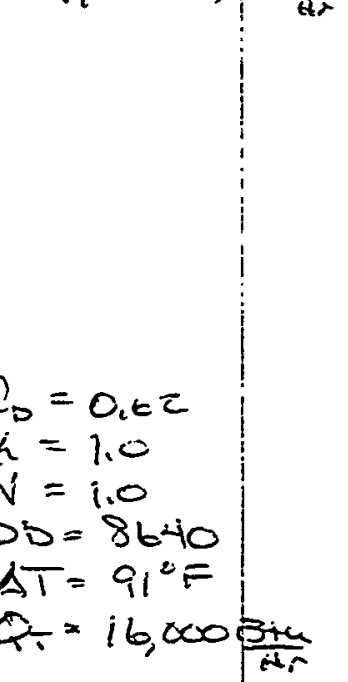

Total Strain Heat Consumption-Seevario 2 Grout Facility

$$
22.6 \times 10^{3}+1.14 \times 10^{6}=1.16 \times 10^{6} \frac{i b_{5 \times 20}}{Y_{\text {ear }}}
$$




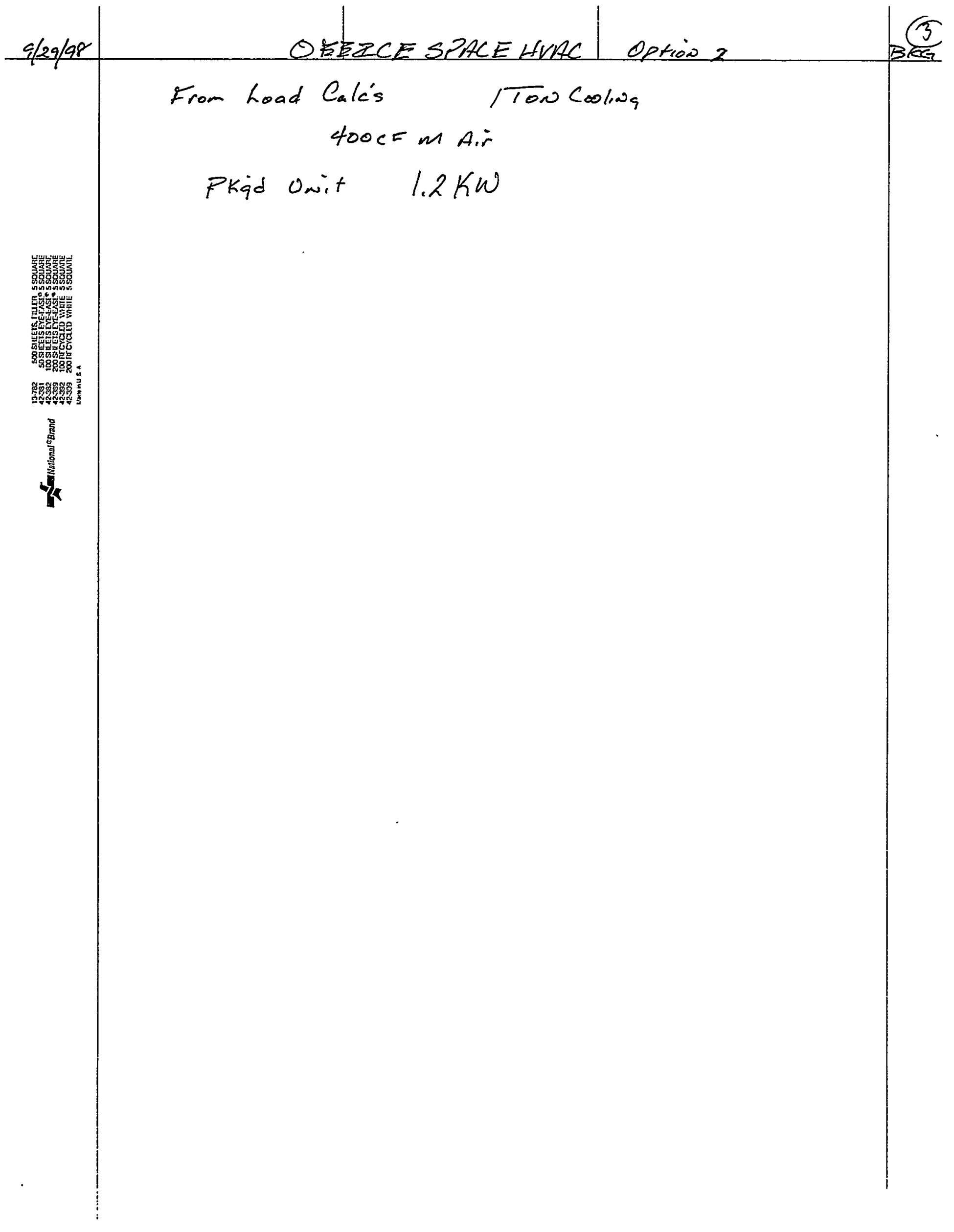




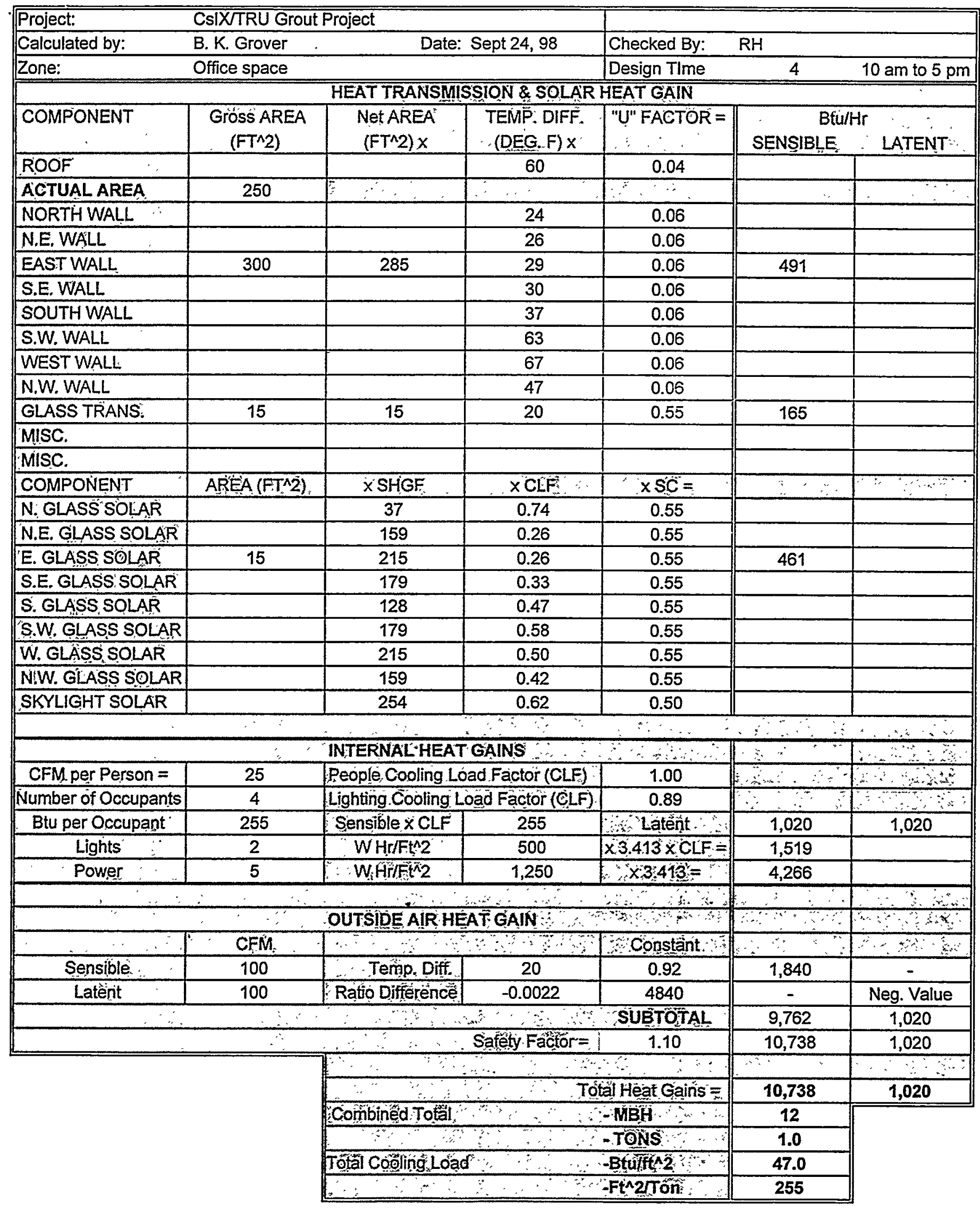




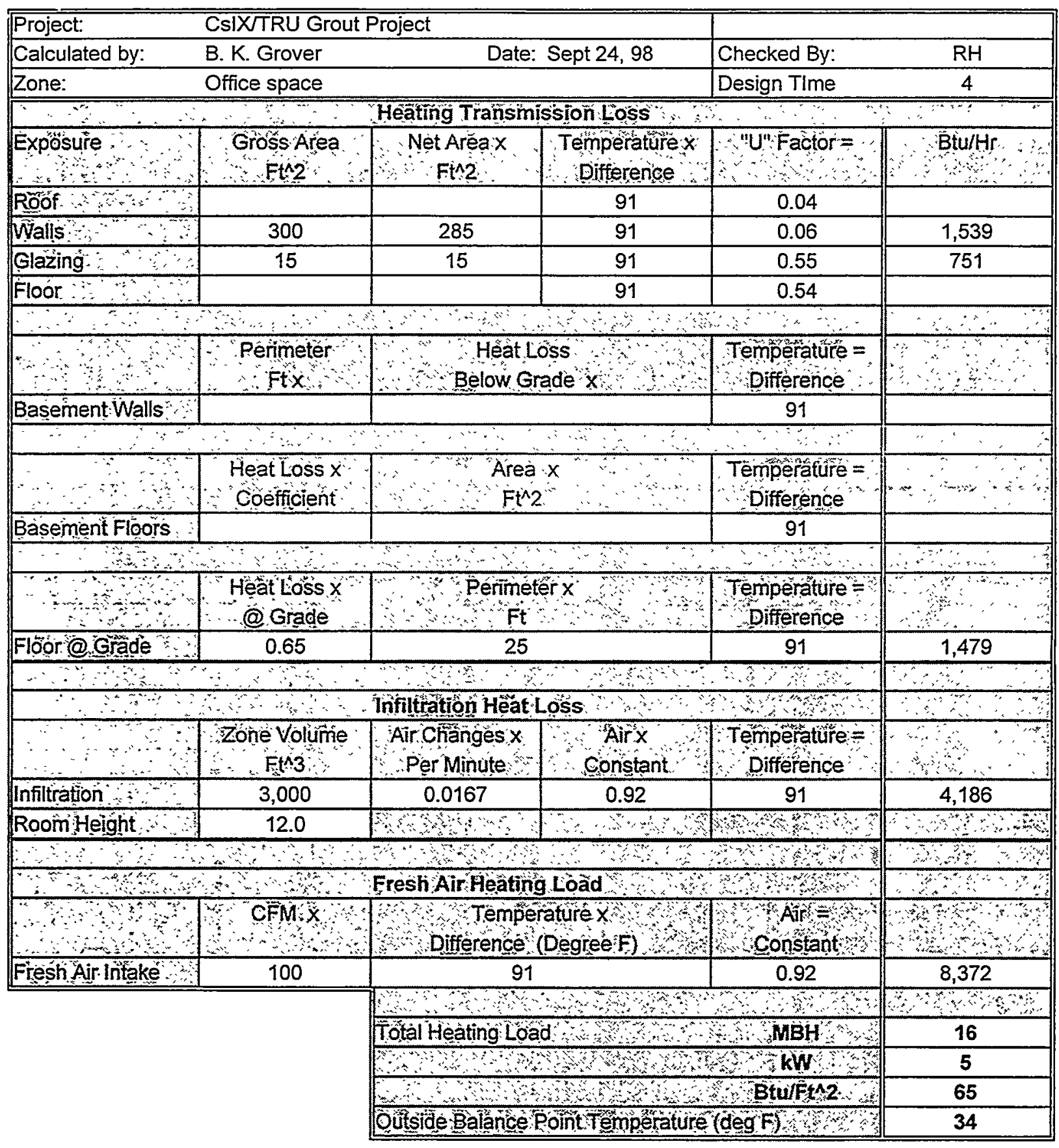




\begin{tabular}{|llcc|}
\hline Project: & CsIX/TRU Grout Project & Latitude - degrees: & 43 \\
\hline Calculated by: & B. K. Grover & Longitude - degrees: & 112 \\
\hline Site Location & INEEL & Elevation - Ft: & 4938 \\
\hline
\end{tabular}

\begin{tabular}{|c|c|c|c|c|}
\hline \multicolumn{5}{|c|}{ Deșign Conditionns } \\
\hline & Outside Témperăture & Inside Température & $\therefore$ Delta T & Coóling Air Humitity. \\
\hline Winter Désign@ @97.5\% & -19 & 72 & 91 & Ràtió Difference \\
\hline Sumimer Design @2.5\% & 95 & 75 & 20 & -0.0022 \\
\hline
\end{tabular}

\begin{tabular}{|c|c|c|c|c|}
\hline \multicolumn{5}{|c|}{ Zơne "Ua" Factôrs } \\
\hline \multirow{2}{*}{ Walls } & \multicolumn{2}{|c|}{ Wall \#1: } & \multicolumn{2}{|c|}{ Wall } \\
\hline & Construetion & "R"Value & Construction & "R" Value \\
\hline Outside Air Film & 15 MPH Moving Air & 0.17 & 15 MPH Moving Air & 0.17 \\
\hline \multicolumn{5}{|l|}{ Outside Surface } \\
\hline \multicolumn{5}{|l|}{ Outer Sheathing } \\
\hline Insulation & $\mathrm{R}-16$ & 16.00 & & \\
\hline \multicolumn{5}{|l|}{ inside Surface } \\
\hline Inside Air Film & Still Air & 0.68 & Still Air & 0.68 \\
\hline \multicolumn{5}{|l|}{ Other } \\
\hline \multicolumn{5}{|l|}{ Other } \\
\hline Tótal R' Factór & & 16.85 & & 0.85 \\
\hline "U" Factor - 1/R = & & 0.06 & & 1.18 \\
\hline \multirow[b]{2}{*}{ Floors, and Roofs } & \multicolumn{2}{|l|}{ Roof } & \multicolumn{2}{|l|}{ Flōor } \\
\hline & Cónstruction & "R" Values & Cónstruction : & "R" Value \\
\hline Oufsidể Air Film & 15 MPH Moving Air & 0.17 & Still Air & 0.92 \\
\hline \multicolumn{5}{|l|}{ Outsidè Surfácé } \\
\hline \multicolumn{5}{|l|}{ Outer Sheathing } \\
\hline Insulation & R-22 & 22 & & \\
\hline \multicolumn{5}{|l|}{ Inside Surfàcé } \\
\hline Inside Air Film & Still Air & 0.61 & Still Air & 0.92 \\
\hline \multicolumn{5}{|l|}{ Other ... } \\
\hline \multicolumn{5}{|l|}{ Other } \\
\hline Totál R Factor & & 22.78 & & 1.84 \\
\hline "U" Factor - 1/R = & & 0.04 & & 0.54 \\
\hline Glazinginglass Doors & $\therefore$ Properties & "U": Value & Metal Doors & "U" Value \\
\hline Winter and Summer & Dbl Pain / Tinted & 0.55 & 1-3/4" Metal Door & 0.39 \\
\hline GlazinglGlass Doors & $\therefore$ Properties & Shading Coefficjent & & \\
\hline Glazing. & Dbl Pain / Tinted & 0.55 & & \\
\hline Skýlités & Double Pain & 0.50 & & \\
\hline
\end{tabular}




\begin{tabular}{|c|c|c|c|c|c|c|}
\hline Project: & CsiX/TRU Grout & roject Scenario 2 & & & & \\
\hline Calculated by: & B. K. Grover & Date: & Oct. 19,98 & Checked By: & & \\
\hline Zone: & Grouting Process & & & Design Tlme & 4 & 10 am to $5 \mathrm{pm}$ \\
\hline Ph & Whow & MEATTTRANSMIS & SSION\&SOLAR & 1EATEAINK & 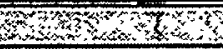 & 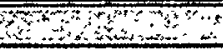 \\
\hline COMPONENT & $\begin{array}{l}\text { OGTSS RREA } \\
\text { (ET/2) }\end{array}$ & N NetSAREA & TEMP BIF & YYSARTR & SENSIBEE & $\begin{array}{l}\text { 4r, } \\
\text { LATENT }\end{array}$ \\
\hline$R O O F$ F & 6,350 & 6,350 & 60 & 0.04 & 16,725 & \\
\hline ACTUALAREA & 11,446 & S & Nons & Pon & Wras & 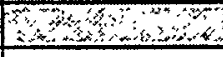 \\
\hline NORTHWALL & 1,640 & 1,640 & 24 & 0.06 & 2,336 & \\
\hline NE WALE & & & 26 & 0.06 & & \\
\hline EAST WAEL & 2,000 & 2,000 & 29 & 0.06 & 3,442 & \\
\hline SEXVALL & & & 30 & 0.06 & & \\
\hline SOUTHLNALL & 1,640 & 1,640 & 37 & 0.06 & 3,601 & \\
\hline SWW NALL & & & 63 & 0.06 & & \\
\hline WESTWNALL ${ }^{2}$ & 2,300 & 2,300 & 67 & 0.06 & 9,145 & \\
\hline NWW WALLS & & & 47 & 0.06 & & \\
\hline GUSS TRANS & & & 20 & 0.55 & & \\
\hline 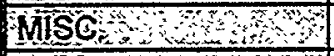 & & & & & & \\
\hline $\mathrm{MISC}$ & & & & & & \\
\hline GONPONENT & AREA $(\mathrm{GT} 2)$ & BXSHER & NWEEF & $\alpha \times S Q=1$ & Why & 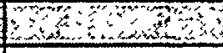 \\
\hline N Gl ${ }^{2} S S$ SOLAR & & 37 & 0.74 & 0.55 & & \\
\hline NESGISSS SOLSR & & 159 & 0.26 & 0.55 & & \\
\hline EE GLASS SOLAR & & 215 & 0.26 & 0.55 & & \\
\hline SE GLASSSSOLAR & & 179 & 0.33 & 0.55 & & \\
\hline S GLASSSOLAR & & 128 & 0.47 & 0.55 & & \\
\hline SWU SLASSSSOLAR & & 179 & 0.58 & 0.55 & & \\
\hline W GLASSSOLAR & & 215 & 0.50 & 0.55 & & \\
\hline NW G WASSSOLR & & 159 & 0.42 & 0.55 & & \\
\hline SKKLIGHT SOLAR & & 254 & 0.62 & 0.50 & & \\
\hline 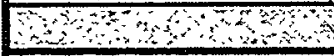 & W & on & ros & 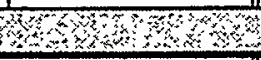 & 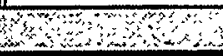 & $6<\alpha_{0}$ \\
\hline 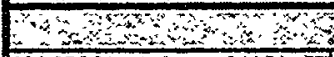 & $8 \mathrm{Now}$ & WNTERNALHEAT & EGAINS & XKon, & Whan & 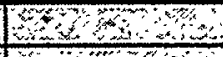 \\
\hline CEM Merpersonston & - & People Cooling E & oadFactor(CCF) & 1.00 & own & 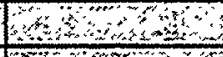 \\
\hline Numbersof Occupants & - & Gighing cooling L & oad Factor $(C G)$ & 0.89 & $3 x+4$ & Whom \\
\hline Btu spe Occupan & - & Sensible X CLE & - & Wringtent , & & \\
\hline An lights & - & 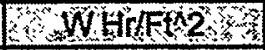 & & $\times 3413 \times C \mathrm{~L}$ & & \\
\hline$\because \angle$ power & - & SSWHAFt2 & & W $\times 3,413=2$ & & \\
\hline $6 \mathrm{ng}, \mathrm{s}$ & W & Wans? & Now & 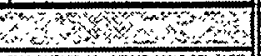 & Now & \\
\hline 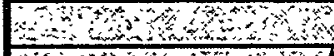 & 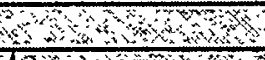 & OUISIDE AIRHE & SATGAIN & WN, & $6+2$ & $8,6, \%, 1,4$ \\
\hline ond & WCFMY & W & and & oonsant & 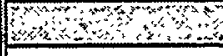 & 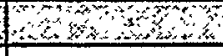 \\
\hline WSensible & & 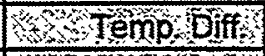 & 20 & 0.92 & & - \\
\hline Latent & & Ratio onfferenge & -0.0022 & 4840 & - & Neg. Value \\
\hline rowas & (4) & 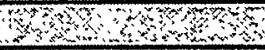 & 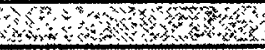 & SUBTOTAL & 35,250 & \\
\hline $6 a+4$ & Wan & 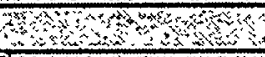 & Salfyy Factors & 1.10 & 38,775 & \\
\hline & & W w w & 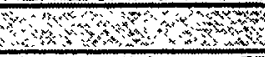 & 20 & 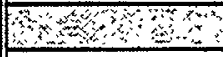 & 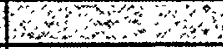 \\
\hline & & W. & 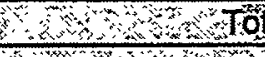 & fll Heat Gains & 38,775 & \\
\hline & & eonbine do Tols & 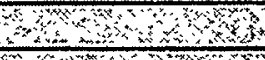 & 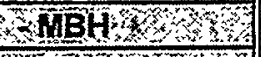 & 39 & \\
\hline & & $8+4$ & 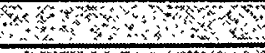 & NTONS $\mathrm{K}$ & 3.2 & \\
\hline & & Totar $G_{0}$ oling & 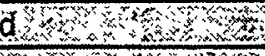 & Btulth 2 & 3.4 & \\
\hline & & $\mathrm{K}, \mathrm{n}$ & $\alpha$ & FENTO & 3542 & \\
\hline
\end{tabular}




\begin{tabular}{|c|c|c|c|c|c|}
\hline Project: & CsIXTRU Grout F & Project Scenario 2 & & & \\
\hline Calculated by: & B. K. Grover & Date: & Oct. 19,98 & Checked By: & \\
\hline Zone: & Grouting Process & & & Design TIme & 4 \\
\hline 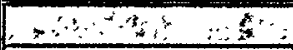 & $\therefore=1+36 x$ & Heáting Transm & SSION LOSS & 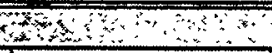 & 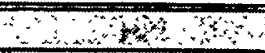 \\
\hline 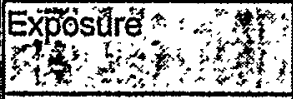 & $\begin{array}{l}\text { Gross Area } \\
\text { FH } 2\end{array}$ & NotArea $\times$ & $\begin{array}{l}\text { Temperattre } \\
\text { Difference }\end{array}$ & 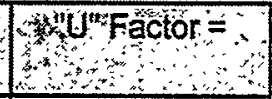 & Bturar \\
\hline Roof is a & 6,350 & 6,350 & 91 & 0.04 & 25,367 \\
\hline Walls & 7,580 & 7,580 & 91 & 0.06 & 40,936 \\
\hline Glażing $\quad:$ & & & 91 & 0.55 & \\
\hline Floor $; y=$ & & & 91 & 0.54 & \\
\hline 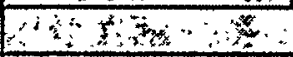 & 46 & 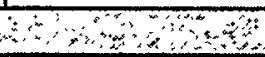 & कर & 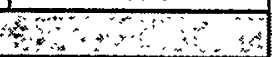 & and \\
\hline 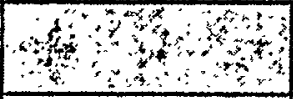 & $\begin{array}{c}\text { Perimeter } \\
\text { Ft }\end{array}$ & $\begin{array}{r}\text { HeatL } \\
\text { Below Gr }\end{array}$ & 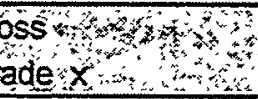 & $\begin{array}{l}\text { Temperafture }= \\
\text { Difference, }\end{array}$ & \\
\hline Basement Walls: & & & & 91 & \\
\hline 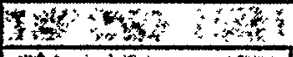 & 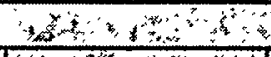 & 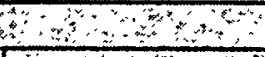 & 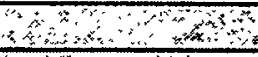 & 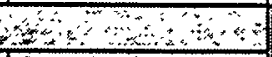 & H A \\
\hline Wy & \begin{tabular}{|} 
Heat Lóss \\
Coefficient \\
\end{tabular} & W & 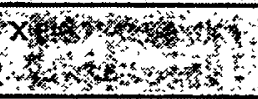 & Temperature & ( \\
\hline Basement'Floors & & & & 91 & \\
\hline 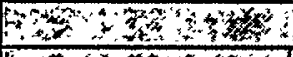 & 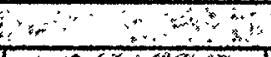 & 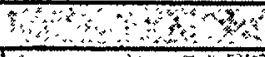 & $y$ & 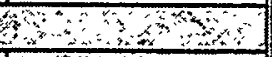 & 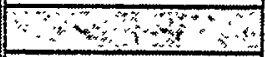 \\
\hline 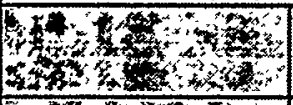 & 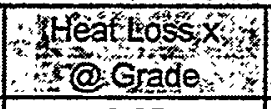 & 4nosermet & 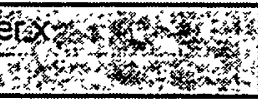 & $\begin{array}{l}\text { Tempepature } \\
\text { tu Differences }\end{array}$ & 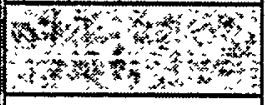 \\
\hline Elordotade & 0.65 & 340 & & 91 & 20,111 \\
\hline 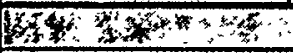 & $x^{4}+\cdots$ & 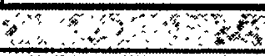 & 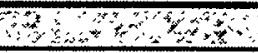 & 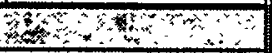 & $\therefore$ a \\
\hline 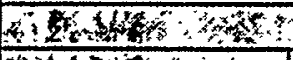 & 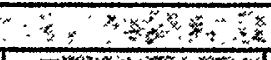 & Trififration $\mathrm{Heat}$ & oss & 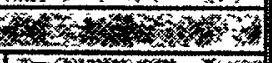 & (x) \\
\hline 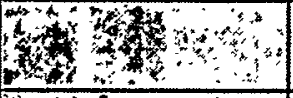 & \begin{tabular}{|c|} 
Zome volume \\
Ftr
\end{tabular} & $\begin{array}{l}\text { Air Changes } \\
\text { \& Perminute }\end{array}$ & 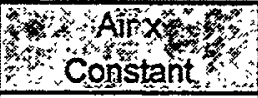 & $\begin{array}{l}\text { Temperature } \\
\text { soifference }\end{array}$ & 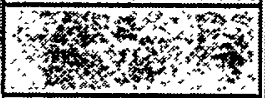 \\
\hline Philtration & 130,141 & 0.0167 & 0.92 & 91 & 181,590 \\
\hline Roomihejight is is & 11.4 & 40 & $x_{0}^{2}$ & b & 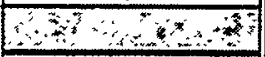 \\
\hline 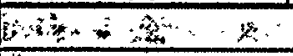 & $\cdots$ & 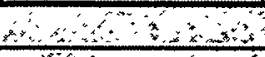 & 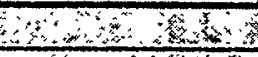 & 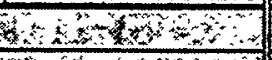 & 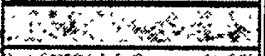 \\
\hline $8,844^{3}=$ & $\because$ & Fresh Air Heating & Load & $x+4 x+4$ & 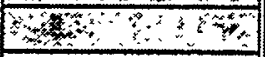 \\
\hline 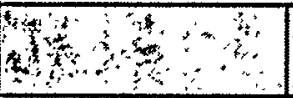 & $\therefore$ CFM $x$ & $\begin{array}{r}\text { RE Tempe } \\
\text { Difference } \\
\end{array}$ & $\begin{array}{l}\text { aturex } \\
\text { Degrea }\end{array}$ & 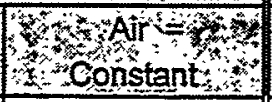 & \% \\
\hline Fresth Air Intake & 6,400 & 91 & & 0.92 & 535,808 \\
\hline & & 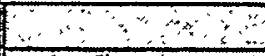 & 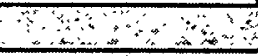 & $y^{2}+2$ & 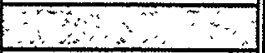 \\
\hline & & Total Heating Loa & 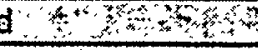 & 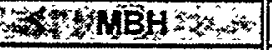 & 804 \\
\hline & & 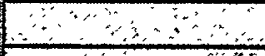 & 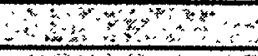 & $8, \mathbf{k W}$ & 236 \\
\hline & & 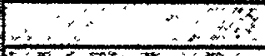 & 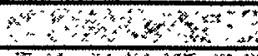 & BfulFt^ & 70 \\
\hline & & Oótside Balancé $\mathrm{F}$ & oinf Temperature & $(d \mathrm{eg}, \vec{f})$ & 72 \\
\hline
\end{tabular}




\begin{tabular}{|llcc|}
\hline Project: & CsIXTRU Grout Project Scenario 2 & Latitude - degrees: & 43 \\
\hline Calculated by: & B. K. Grover & Longitude - degrees: & 112 \\
\hline Site Location & INEEL & Elevation - Ft: & 4938 \\
\hline
\end{tabular}

\begin{tabular}{|c|c|c|c|c|}
\hline 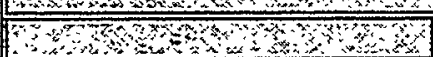 & Toüside Temperature & Inside Temperature. & WX Y Belta T & 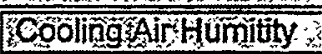 \\
\hline Wnte D esign @ $975 \%$ & -19 & 72 & 91 & RRationoiference \\
\hline Sunmer besign @ $25 \%$, & 95 & 75 & 20 & -0.0022 \\
\hline
\end{tabular}

\begin{tabular}{|c|c|c|c|c|}
\hline \multicolumn{5}{|c|}{ 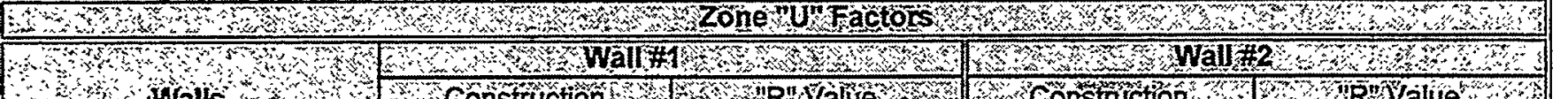 } \\
\hline OutsideAir Fim & 15 MPH Moving Air & 0.17 & $15 \mathrm{MPH}$ Moving Air & 0.17 \\
\hline \multicolumn{5}{|c|}{ Outside Sulface } \\
\hline \multicolumn{5}{|l|}{ Oúfer stheathing } \\
\hline isulation & $\mathrm{R}-16$ & 16.00 & & \\
\hline \multicolumn{5}{|l|}{ inside Sunfấ } \\
\hline 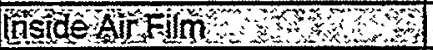 & Still Air & 0.68 & Still Air & 0.68 \\
\hline \multicolumn{5}{|c|}{ 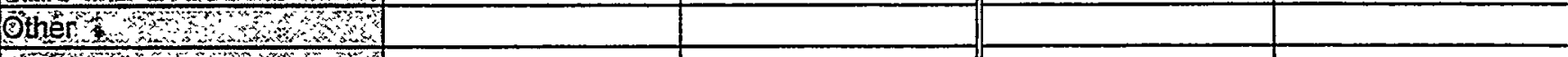 } \\
\hline \multicolumn{5}{|l|}{ 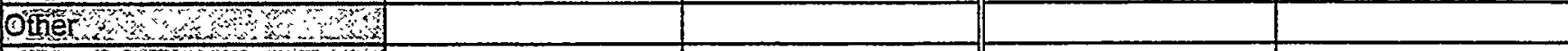 } \\
\hline 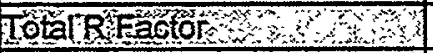 & & 16.85 & & 0.85 \\
\hline 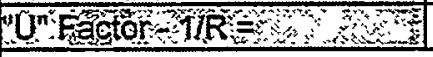 & & 0.06 & & 1.18 \\
\hline 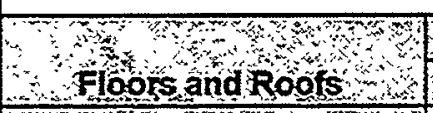 & 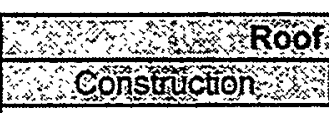 & 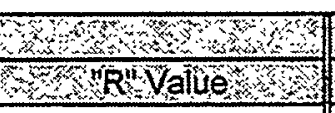 & 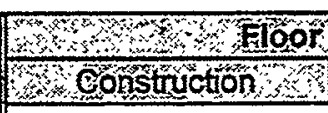 & 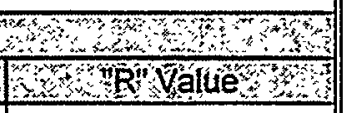 \\
\hline 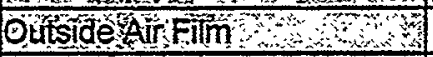 & $15 \mathrm{MPH}$ Moving Air & 0.17 & Still Air & 0.92 \\
\hline \multicolumn{5}{|c|}{ Outside sufface } \\
\hline \multicolumn{5}{|l|}{ Outersheathing } \\
\hline Insulation $\quad, \quad, \quad 3$ & $\mathrm{R}-22$ & 22 & & \\
\hline \multicolumn{5}{|l|}{ nsidersurface } \\
\hline Inside Air Eilm & Still Air & 0.61 & Still Air & 0.92 \\
\hline \multicolumn{5}{|c|}{ Other } \\
\hline \multicolumn{5}{|l|}{ Qtier } \\
\hline Totaldeactor & & 22.78 & & 1.84 \\
\hline 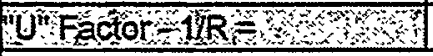 & & 0.04 & & 0.54 \\
\hline GlazinglGlass Doors & 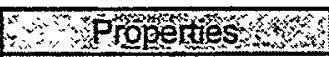 & 2) & 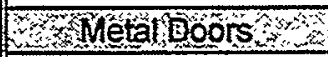 & wowalue \\
\hline Winter and summer $\alpha$ & Dbl Pain / Tinted & 0.55 & 1-3/4" Metal Door & \begin{tabular}{|l|}
0.39 \\
\end{tabular} \\
\hline Glazingolass Doors & ARropdes & Shading ooeffieng & & \\
\hline W S lazing $\alpha$ & Dbl Pain / Tinted & 0.55 & & \\
\hline Skylites & Double Pain & 0.50 & & \\
\hline
\end{tabular}




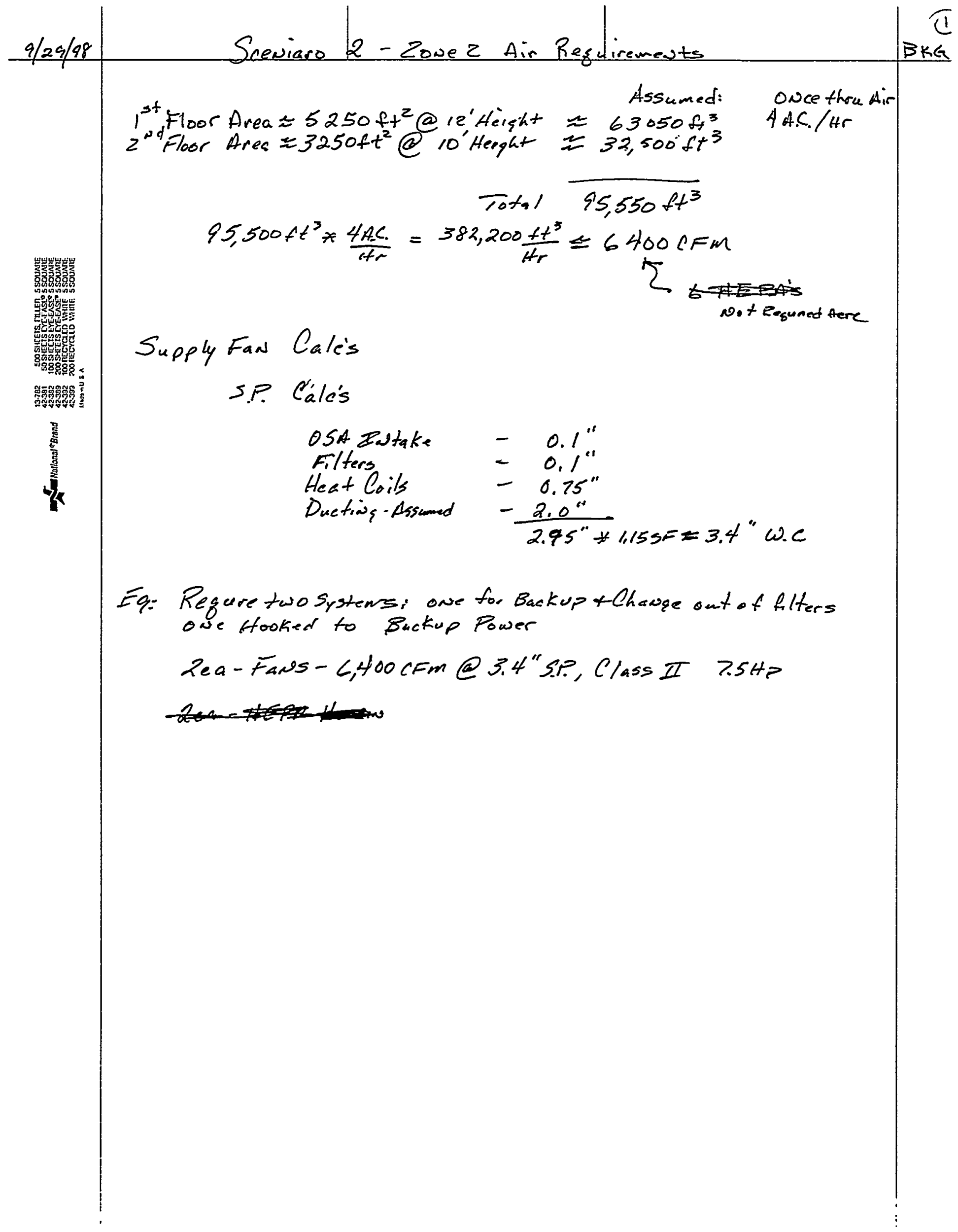




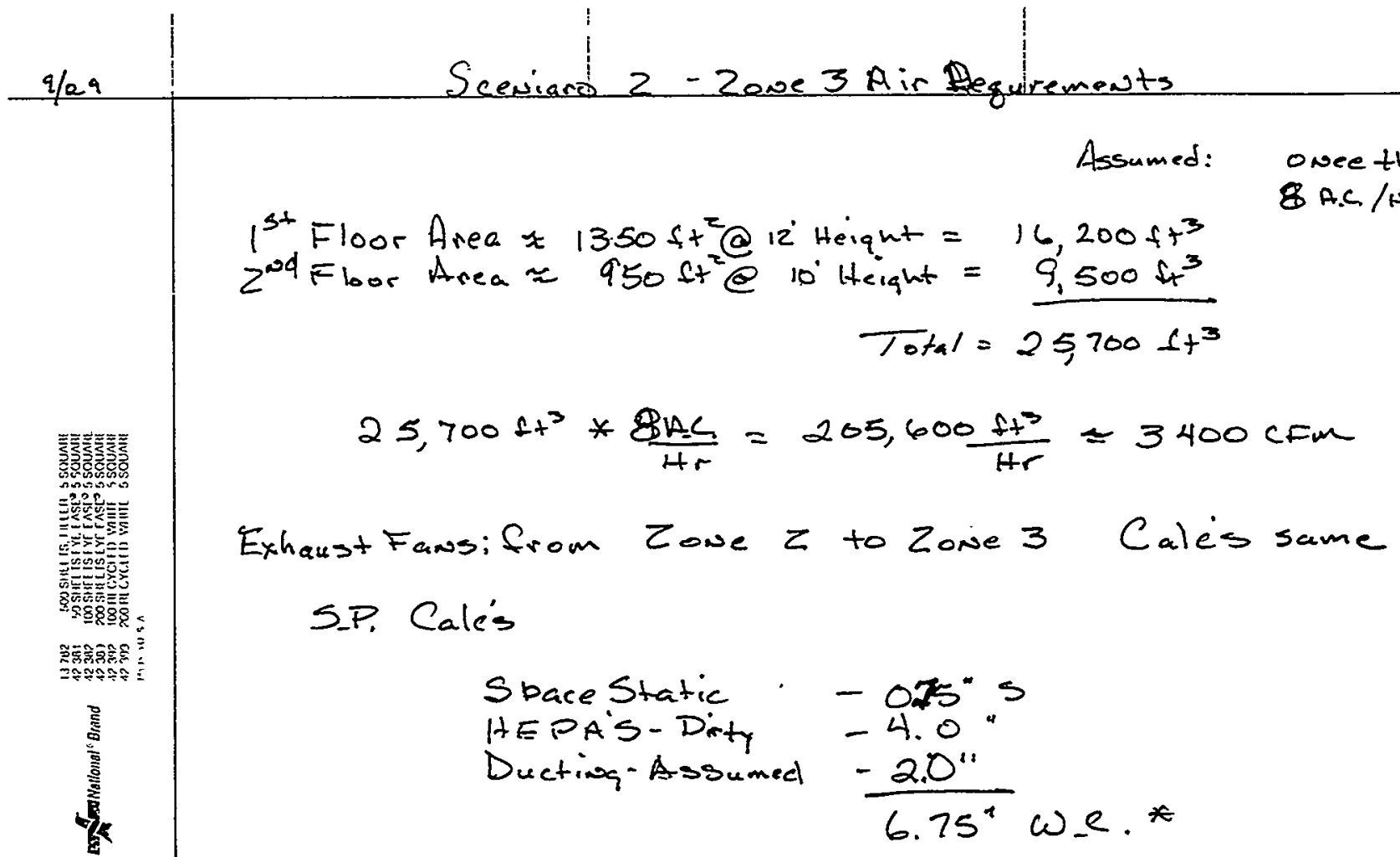

(2)

13 kg

* Supply from zane $z$ to Love 3 Assumed same as Exhaust to stack. HEPA's sized from Lowe 2 Air flow

Eq: Require two systems, one for Back op and Change Out. owe system weeds to be hooked up to Backup Power

Exhaust: Zone $Z$ to Zone 3

2e-Fans-6400cFm@6.75"S.R, ClassII 10 Hp

2 ea- HEPA Housings - $6400 \mathrm{cFm} \Rightarrow$ ZN B

Exhaust howe 3 to Stack

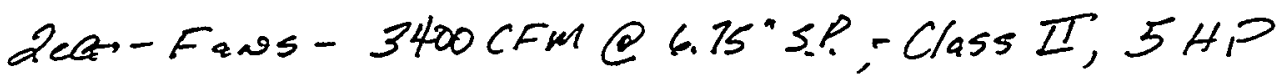

Lea - HEPA's Housing - $3400 \mathrm{cFm}-2 \times 2$ 
431.02

$06 / 17 / 97$

Rev. \#04
ENGINEERING DESIGN FILE

Attachment D

CsIX/TRU Feasibility Study

Sketches and Drawings
Functional File Number - SPR-CsIX-11

EDF Serial Number - EDF-CsIX-012

Page 1 of 1 


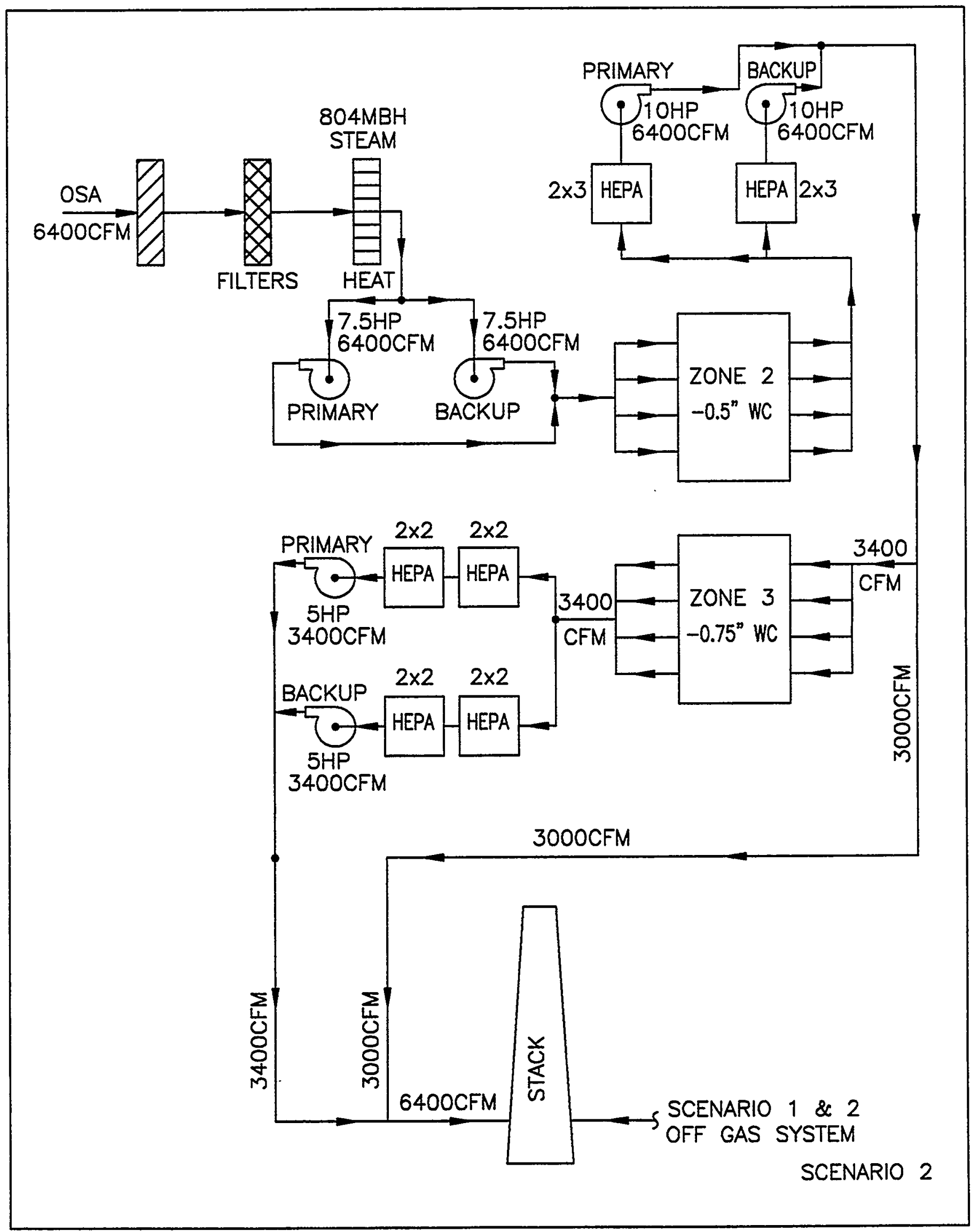


Project File Number $\quad 02 B M 1$

Project/Task CsIX/TRU Grout Feasibility Study

Subtask D.5 Facility Design

Title: Off-Gas

Summary:

This EDF describes documents requirements, assumptions, description, and calculations for the Off-Gas system required.

EDF Contents:

- Attachment A - Detailed Process Description w/Assumptions \& Requirements.

- Attachment B - Equipment list for the Off-Gas System.

- Attachment C - Supporting Calculations

- Attachment D - Sketches and Drawings

Distribution (complete package): C. W. Olsen MS 3211; K. L. Williams MS 3765; Project File; A copy of this EDF will be included in External Report No. INEEL/EXT $=98-00869$.

Distribution (summary package only):

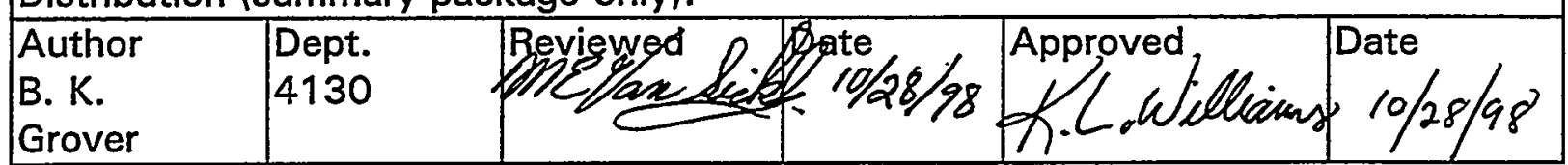




\author{
Attachment A \\ CsIX/TRU Feasibility Study
}

General

This EDF describes the Off-Gas system required for the Scenario 1 and 2 Grout Processing Facilities. HVAC is covered under EDF-CsIX-011 and EDF-CsIX-012.

\title{
Assumptions
}

1. Moisture will be removed from the off-gas system by the condensers.

2. No carbon filter absorbers are needed.

3. The off-gas systems for both scenarios have the same air flow and pressures.

\section{$\underline{\text { Requirements }}$}

1. Double HEPA filtration is required per AE standards.

2. 2 inch W.C. negative pressure required at vessels.

3. Two identical and independent systems are required.

4. One of the two off-gas systems will be connected to backup power.

\section{Process Description}

The off-gas system for both scenarios are very similar. Equipment connected to the system will remove moisture at the source. Condensers are located at various locations throughout the facilities. Condensers are located on the CsIX Resin Dryer, UDS Rotary Evaporator, Dewatering Stations, and at the inlet to the off-gas fan. The condensers are located at the point of generation. Connections to various vessels in the facilities are also connected to the off-gas system. Total offgas flow will be 500-CFM, see Appendix $C$ for more detailed calculations. Two independent systems will be installed. This will allow change out of the HEPA filters and failure of a system without process shut down. One of the two $2.5 \mathrm{Hp}$ fan systems will be connected to backup power. Each system will have a double HEPA filter housing with a $1 \times 1$ configuration. Just prior to the off-gas fan, there will be a mist eliminator and a superheater. Further analysis on installation of a carbon absorber should be investigated. 


\section{Attachment B \\ CsIX/TRU Feasibility Study \\ Equipment list for Off-gas System}

Off-gas fans -2 each, $4 \mathrm{ft}$ by $4 \mathrm{ft}$, SST, $2.5 \mathrm{Hp}$, one connected to backup power

HEPA filter housings $-1 \times 1$ housing, double filters, 2 each

Note:

Condensers, mist eliminators, and the superheater are listed in EDF-CsIX001. 
Attachment C

CsIX/TRU Feasibility Study

Supporting calculations 


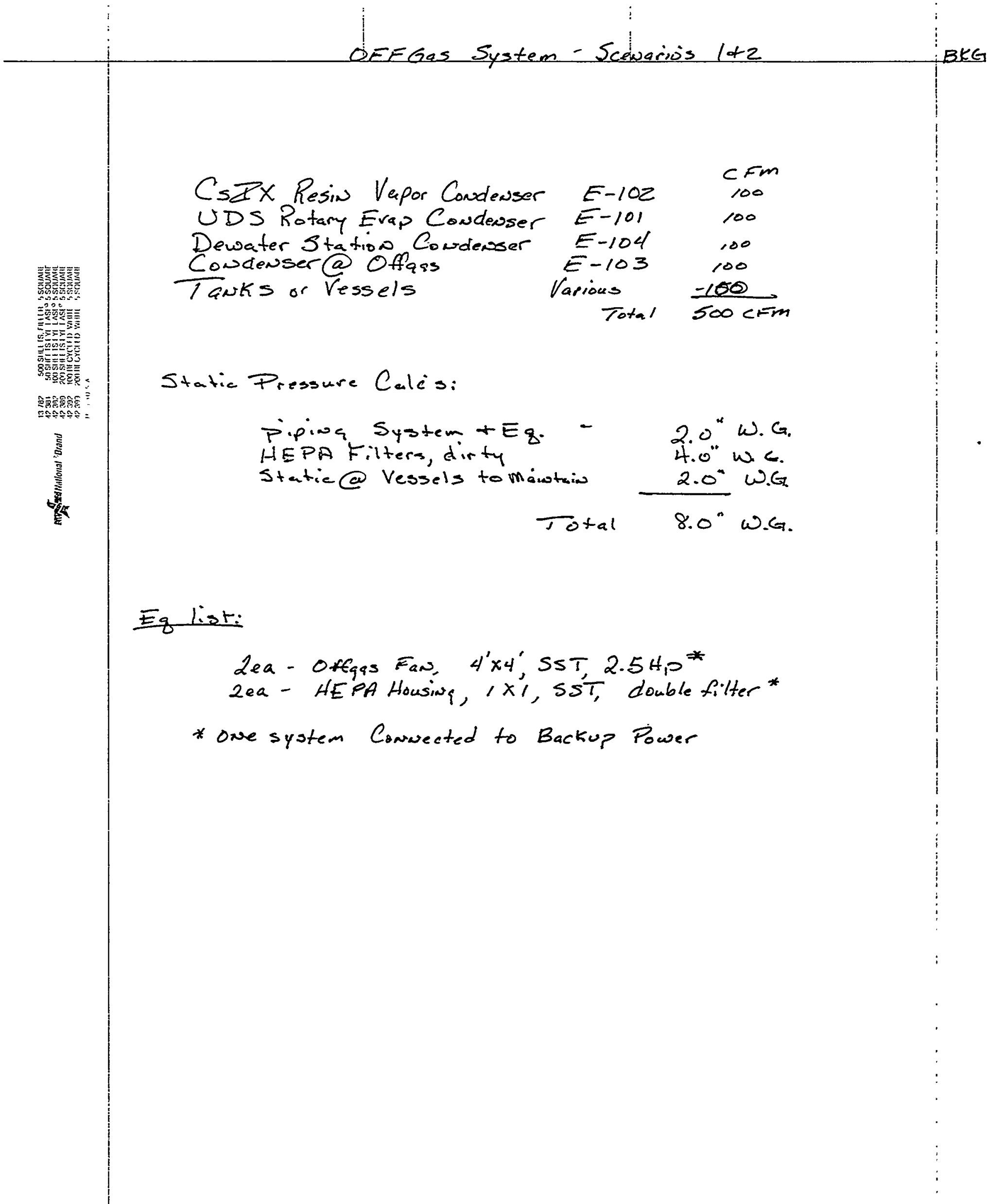


Attachment D

CsIX/TRU Feasibility Study

Sketches and Drawings 
ENGINEERING DATA

\begin{tabular}{|c|c|c|c|}
\hline $\begin{array}{c}\text { Approximate } \\
\text { Weight (Lb) }\end{array}$ & Class & $\begin{array}{c}\text { Max. Motor } \\
\text { Frame Size }\end{array}$ & $\begin{array}{c}W^{2} \\
(L b-F t 2)\end{array}$ \\
\hline (C/F) & II & $445 T$ & 1 \\
\hline
\end{tabular}

\begin{tabular}{|c|}
\hline $\begin{array}{c}\text { Motor } \\
\text { Location }\end{array}$ \\
\hline $\mathrm{W}$ \\
\hline
\end{tabular}
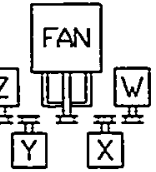

CONFIGURATION

\begin{tabular}{|c|c|c|}
\hline Arrangement & $\begin{array}{c}\text { Discharge } \\
\text { Position }\end{array}$ & Rotation \\
\hline 1 & Top Horizontal & $\mathrm{CW}$ \\
\hline
\end{tabular}

INSTALLATION

\begin{tabular}{|c|c|c|}
\hline $\begin{array}{c}\text { Inlet } \\
\text { Conditions }\end{array}$ & $\begin{array}{c}\text { Outlet } \\
\text { Conditions }\end{array}$ & Inlet Vanes \\
\hline Standard & Standard & None \\
\hline
\end{tabular}

\section{MOTOR SPECS}

\begin{tabular}{|c|c|c|c|}
\hline$H p$ & $V / C / P$ & Enclosure & RPM \\
\hline 3 & $460 / 60 / 3$ & Open Drip Proof & 3600 \\
\hline
\end{tabular}

\section{B 1 Neries 41 Backward Inclined Single Width Centrifugal Fan}

Standard Construction Features

- Series 41 Class I, II, and III fans feature continuously welded steel housings on all sizes 7-73 - All Class III fans are fully welded and supplied with outlet flanges - Heavy duty, self aligning ball or roller pillow bearings - Fully welded backward inclined wheel - Discharge collars for slip-fit duct connections on fan sizes 7-30 - Inlet collars on Arr. 1,9,10 - Unpunched flanged discharges are standard on Class I and II fan sizes 33-73 and all downblast fans - Polished solid steel shafts

- All structural parts are phosphatized and coated with Permatector.

\section{PERFORMANCE}

\begin{tabular}{|c|c|c|c|c|c|c|c|c|}
\hline Qty. & Model & $\begin{array}{c}\text { Volume } \\
\text { (CFM) }\end{array}$ & $\begin{array}{c}\text { SP } \\
(\ln \text { WC) }\end{array}$ & $\begin{array}{c}\text { CSP } \\
(\ln \text { WC) }\end{array}$ & $\begin{array}{c}\text { TS } \\
(\mathrm{Ft} / \mathrm{m})\end{array}$ & $\begin{array}{c}\text { OV } \\
(\mathrm{Ft} / \mathrm{m})\end{array}$ & FRPM & $\begin{array}{c}\text { Power } \\
(\mathrm{BHp})\end{array}$ \\
\hline 1 & 8-BISW-41 & 500 & 8.000 & 8.000 & 13667 & 775 & 4972 & 2.28 \\
\hline
\end{tabular}

SOUND

\begin{tabular}{|c|c|c|c|c|c|c|c|c|c|c|}
\hline \multicolumn{8}{|c|}{ Inlet / Outlet Sound Power by Octave Band } & \multirow{2}{*}{ LwA } & \multirow{2}{*}{ dBA } & \multirow{2}{*}{$\begin{array}{l}\text { Noise } \\
\text { Criteri }\end{array}$} \\
\hline 63 & 125 & 250 & 500 & 1000 & 2000 & 4000 & 8000 & & & \\
\hline $100 / 108$ & $100 / 107$ & & & & & & & & & \\
\hline
\end{tabular}

AIR DENSITY

\begin{tabular}{|c|c|c|}
\hline $\begin{array}{c}\text { Elevation } \\
(\mathrm{Ft})\end{array}$ & $\begin{array}{c}\text { Airstream } \\
\text { Temp. (F) }\end{array}$ & $\begin{array}{c}\text { Startup } \\
\text { Temp. }(\mathrm{F})\end{array}$ \\
\hline 4800 & 70 & 70 \\
\hline
\end{tabular}

LWA - A weighted sound power level, based on ANSI S1.4. $\mathrm{dBA}$ - A weighted sound pressure level, based on $11.5 \mathrm{~dB}$ attenuation per octave band at 5 feet. Noise Criteria (NC) level based on an average attenuation of $11.5 \mathrm{~dB}$ per octave band at 5 feet.

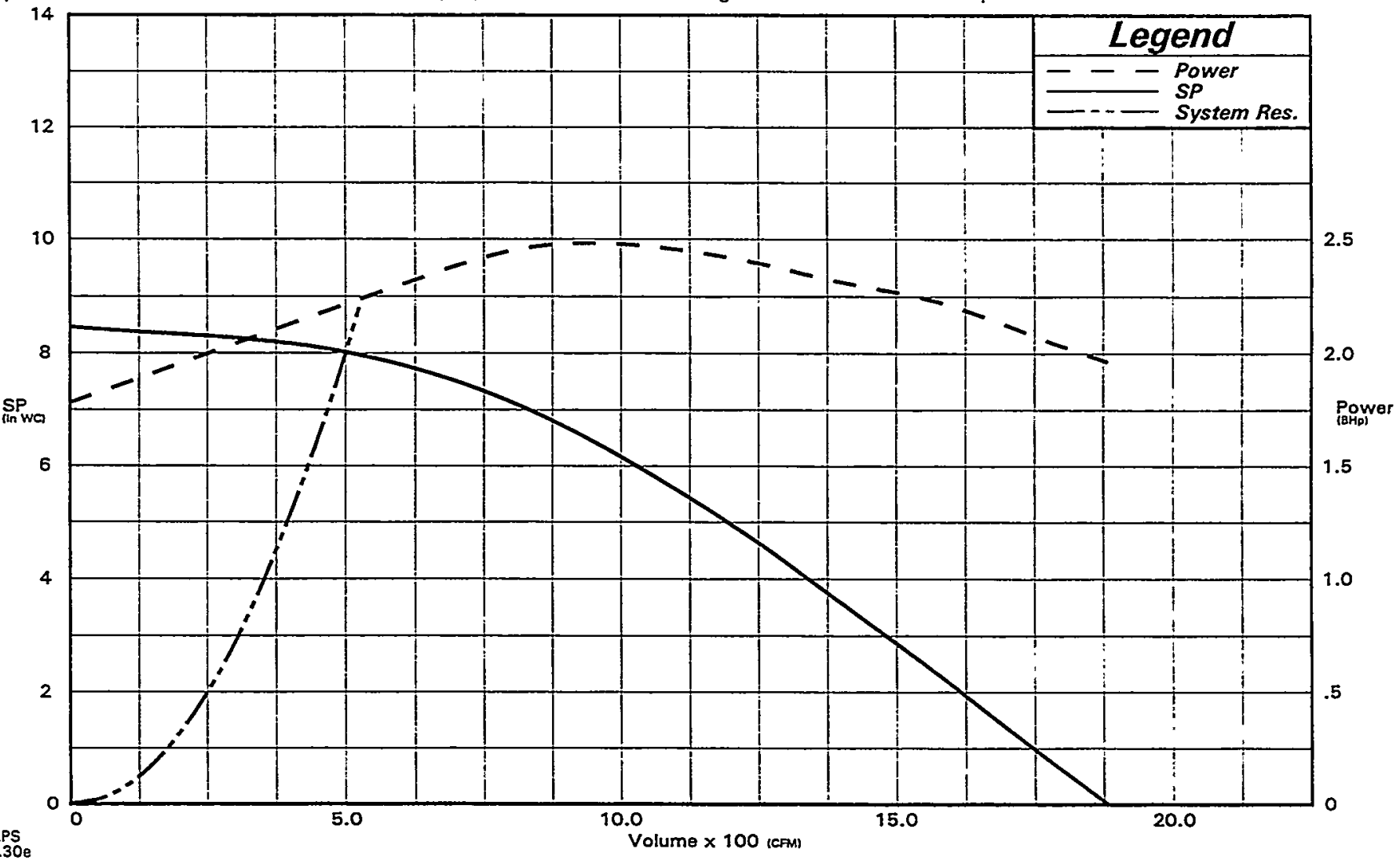



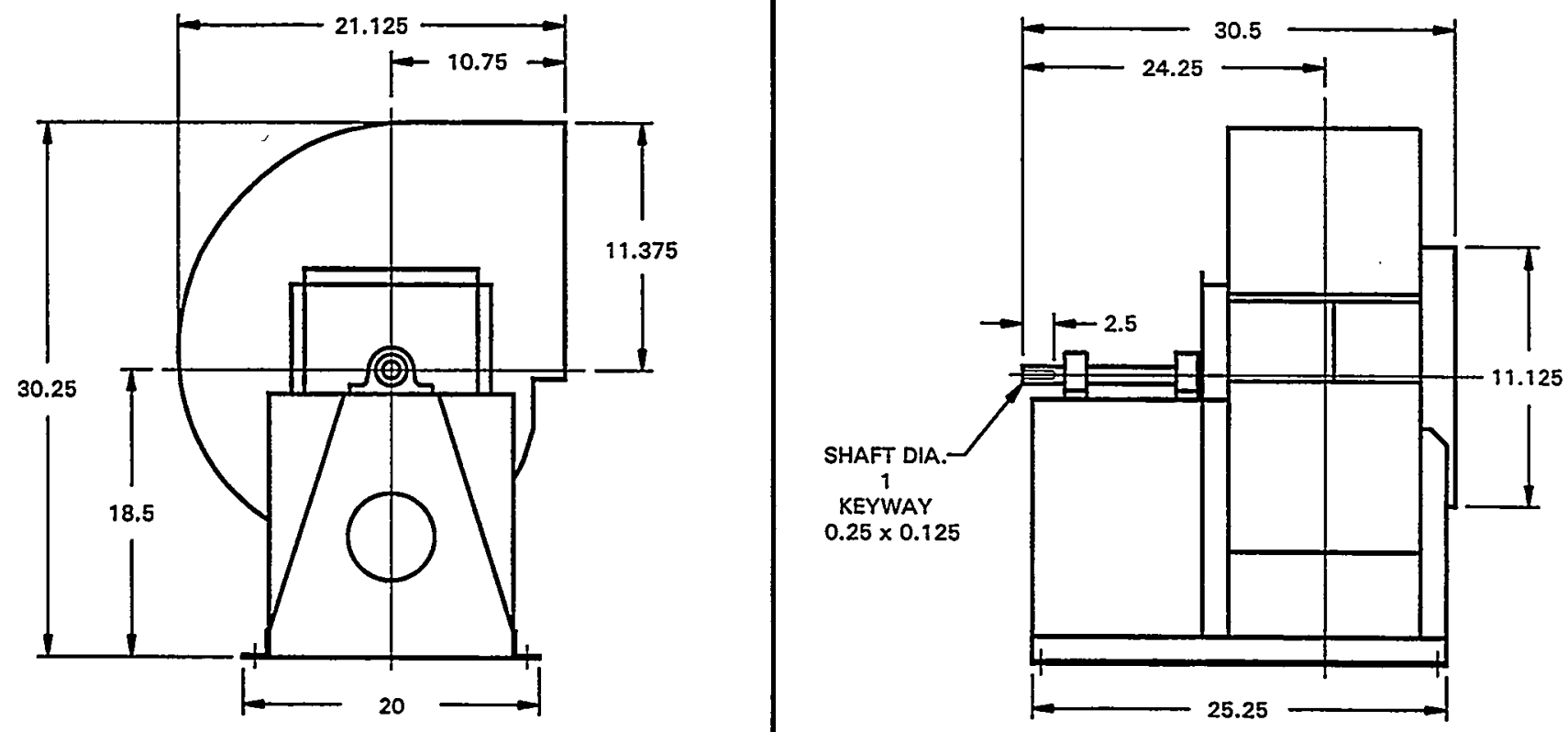

SIDE VIEW

END VIEW
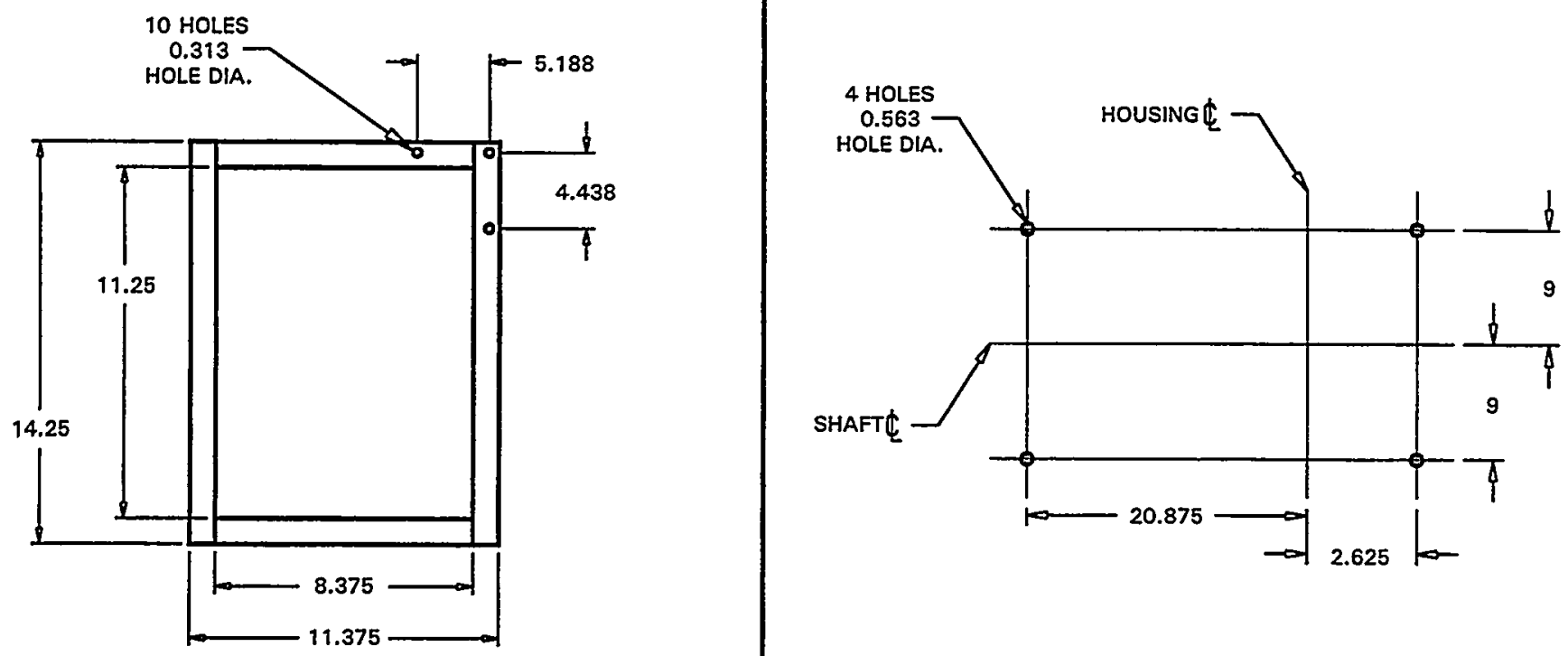


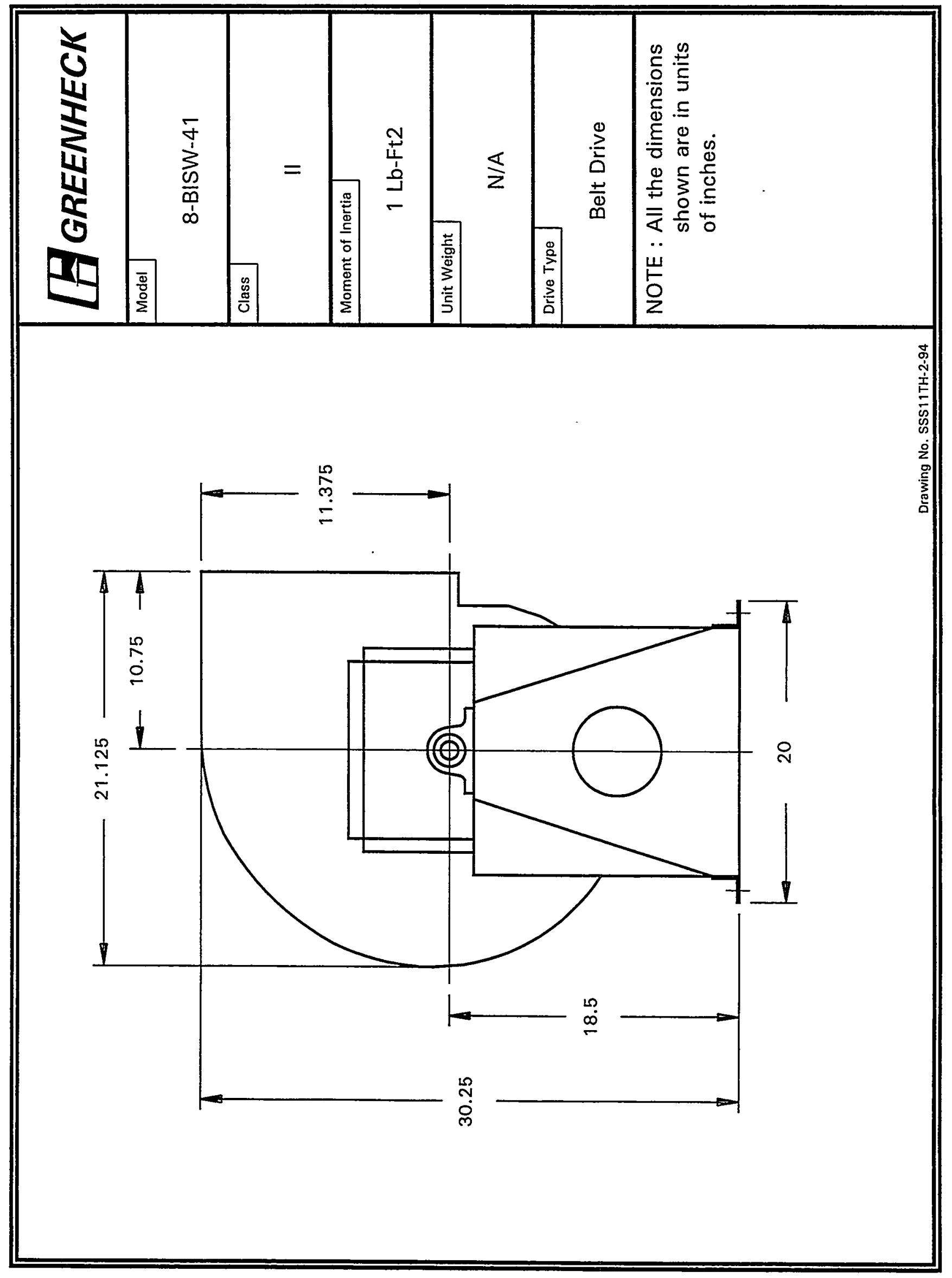


Project File Number

02BM1

Project/Task CsIX/TRU Grout Feasibility Study

Subtask

\section{Title: ELECTRICAL REOUIREMENTS}

Summary: Facility Engineering has been tasked with a Feasibility Study examining the feasibility and estimating cost for a process to treat the sodium bearing waste and newly generated liquid waste for removal from the tank farm by the year 2012. In this EDF, a study was performed to determine the amount of electrical power, the source of electrical power, and a summary of the loads. For Scenario 1, all-in-one facility, the connected load would be 698 kVA with demand of 471 kVA. The standby power would be 88 kVA. For Scenario 2, grouting only in new building, the connected loads would be $569 \mathrm{kVA}$, demand load would be $381 \mathrm{kVA}$, and standby would be $53 \mathrm{kVA}$. The major differences in loads are due to lighting, miscellaneous, and $H \& V$ loads. The grouting only building is substantially less in size than the all-in-one building. The actual process loads are approximately the same. Either Scenario would be supported from INTEC Loadcenter 14 using 480 VAC, 3 phase. A combination of new and existing duct banks would be used to route the power to the new buildings. In Scenario 2, approximately $60 \mathrm{kVA}$ of load would be added to NWCF. However, the net effect would likely be much less since existing loads in NWCF would likely be removed.

Distribution (complete package):

Distribution (summary package only):

\begin{tabular}{|c|c|c|c|c|c|}
\hline 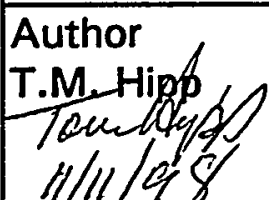 & \begin{tabular}{|l} 
Dept. \\
Facility \\
Engineering
\end{tabular} & Reviewed & $\begin{array}{l}\text { Date } \\
11 / 12 / 98\end{array}$ & Approved & $\begin{array}{l}\text { Date } \\
11 / 12 / 98\end{array}$ \\
\hline
\end{tabular}

See Management Control Procedure (MCP) 6 for instructions on use of this form. 


\section{CESIUM ION EXCHANGE GROUTING PROJECT}

\subsection{ELECTRICAL REQUIREMENTS}

\subsection{EXISTING AND PLANNED ELECTRICAL UTILTIIES AT INTEC}

The INTEC ties into the $138 \mathrm{kV}$ INEEL loop at Substation 2 which is located outside the area fence to the south. Substation 2 transforms the $138 \mathrm{kV}$ to $13.8 \mathrm{kV}$ and provides power to Substations 10 and 15 which are located within the INTEC complex. Power at $13.8 \mathrm{kV}$ is then distributed from these substation through the complex. The Electrical and Utility System Upgrade (EUSU) project is currently under construction. The EUSU Project will install a new $13.8 \mathrm{kV}$ electrical distribution system throughout the complex. This new system will provide greater safety, additional capacity and greater reliability.

Currently, standby power is provided by each facility. Standby generators are located at various facilities and operate as an island of power during a normal power outage. The EUSU project will construct a standby power plant, install new standby generators and tie existing generators into the standby power system. Standby power will then be distributed through the complex by the new $13.8 \mathrm{kV}$ distribution system, The Utility Control System (UCS) provides control of both the normal and standby power distribution systems.

\subsection{POWER REQUIREMENTS}

\subsubsection{NORMAL POWER}

The electrical requirements of the Cesium lon Exchange Grouting Project (CsIX) were analyzed and determined to be $698 \mathrm{kVA}$ connected, with a demand projected to be $471 \mathrm{kVA}$. The results of the analysis are shown in Tables 1 and 2. The major loads with the CsIX project are various lighting, heating, and motor loads. The motor loads consist of both process and ventilation motors.

Normal power will be supplied to the CsIX Project by $480 \mathrm{kV}$ feeders from Loadcenter 14. A combination of new and existing duct banks will be used to route the feeders. 


\subsubsection{STANDBY POWER}

The requirements for standby power for the CsIX facilities were analyzed and determined to be 88 kVA. The results of the analysis are shown in Table 3. The standby loads for the CsIX facilities will consist of the following:

- UPS Normal and Bypass feeders

- Selected lighting and miscellaneous loads

- Exhaust and supply fans

- Other process loads

Standby power to the CsIX facilities will be provided over the normal power distribution system from Substation 60 . Shedding of the normal power loads will be performed by the UCS. The UCS will control the operation of circuit breakers and equipment to ensure that only those loads requiring standby power remain in operation

\subsubsection{UNINTERRUBTABLE POWER SUPPLY (UPS)}

A solid state UPS with a static transfer switch will be provided. The UPS will be provided with a 15 minute battery backup and feed a 208Y/120 Volt panel. The UPS and the panel will be located in the electrical room and support the following loads: Voice paging/evacuation systems, environmental monitoring system, control system, and other critical loads.

\subsection{LIGHTING}

\subsubsection{INTERIOR LIGHTING}

Lighting in office areas and other low ceiling areas will be supplied by recessed pendant mounted fluorescent fixtures. These fixtures will be operated at 277 Volts and will be locally switched. Motion detectors will be utilized areas of low occupancy. Lighting in high bay areas will be will be supplied by metal halide fixtures operating at 277 volts. The metal halide fixtures will be switched at the lighting panel.

\subsubsection{CELL LIGHTING}

Lighting in the cells will be designed to allow for remote operation of the equipment via an in cell CCTV system or by operation through cell windows. 
Lighting of the cells will be indirect. Translucent panels will be provided on the top of the cells and on the side walls as required. Fixtures will be mounted so that maximum light is directed into the cells and the fixtures are easily accessible for maintenance. The light source will be metal halide with the design will taking into account light loss through the translucent panels as well as the light lost in viewing operations through the cell windows.

\subsubsection{EXTERIOR LIGHTING}

The exterior of the building will be illuminated with high pressure sodium wall pack fixtures mounted over each personnel door. Loading areas will be provided with high pressure sodium wall pack fixtures on each side of the doors. The need for area lighting in maneuvering areas will be evaluated during the conceptual and final designs.

\subsubsection{EMERGENCY AND EXIT LIGHTING}

Emergency egress lighting will be in accordance with NFPA 101, Life Safety Code. In areas where illumination is provided by fluorescent fixtures, selected fixtures will be provided with integral battery back up. In areas where illumination is provided by metal halide fixtures, selected fixtures will be provided with a quartz lamp which will be used for emergency lighting. The quartz lamp will be connected to the UPS system or will be provided with an integral battery.

\subsection{DESIGN DESCRIPTION}

\subsubsection{SITE CONDITIONS}

Electrical equipment will be rated for continuous operation at an elevation of 5,000 feet above sea level.

\subsubsection{HAZARDOUS LOCATIONS}

Several areas within the facility will be used for storing or handling of hazardous materials. An evaluation will be performed during the conceptual design and the final design to determine the effects of these materials on the installation of electrical equipment. Flammable and Combustible Liquids Codes NFPA 30: National Electrical Code, NFPA 70; and Recommended Practice for Classification of Class I Hazardous Locations for Electrical installations in Chemical Process Areas, NFPA 497A will be used in making the determination. 


\subsubsection{SERVICES}

\subsubsection{PRIMARY SERVICE}

A single feed from Loadcenter 14 will supply $480 \mathrm{~V}$ power to the CsIX facilities. The feeders will be routed through both new and existing duct banks.

\subsubsection{STANDBY POWER}

Standby power will be provided at $480 \mathrm{~V}$ over the existing distribution system. The existing UCS in conjunction with the CsIX control system will control selected circuit breakers to limit the standby power distribution only to those loads deemed necessary.

\subsubsection{UTILIZATION VOLTAGES}

Two utilization voltages will be provided for the CsiX facilities. Transformers Lloadcenters 14 will transform $13.8 \mathrm{kV}$ to $480 \mathrm{Y} / 277$ Volt; transformers down stream will transform the 480 Volts to $208 \mathrm{Y} / 120$ Volts.

Electrical loads will be assigned voltages as follows:

- Motors $1 / 2$ HP to less than $100 \mathrm{HP}-480 \mathrm{~V}, 3$ phase

- Resistive loads 1 kVA to less than 100 kVA - 480V, 3 phase

- Motors less than $1 / 2$ HP $120 \mathrm{~V}$, single phase

- Miscellaneous loads less than 1 kVA - 120V, single phase

- Lighting - 277V single phase.

\subsubsection{EQUIPMENT}

\subsubsection{TRANSFORMERS}

Transformers 5,000 kVA and below will be cast coil, dry type transformers.

\subsubsection{LIGHTING}

Lighting levels will conform the llluminating Engineer's Society (IES) handbook and standard practices at the INTEC. In general, the design will provide the following illumination levels:

- Work Stations

- Work Areas

- Non-Work Areas
70 foot candles 30 to 50 foot candles depending on activity 10 foot candles, 50 where data is obtained 


\subsubsection{GROUNDING}

Grounding at the INTEC is accomplished with bare copper conductors installed in all duct banks and ground rods installed in every manhole. This in turn is solidly connected to the casing of the deep wells. Facilities and structures throughout the INTEC are connected to this ground system. Therefore, the CsIX facilities will be connected to the ground system. Grounding within the facilities will be accomplished in accordance with the National Electrical Code and IEEE Standard 142-1991, "IEEE Recommended Practice for Grounding of Industrial and Commercial Power Systems."

\subsubsection{LIGHTNING PROTECTION}

Lightning protection will be provided in accordance with NFPA 78, "Lightning Protection Code."

\subsection{SCENARIOS}

Two scenarios have been proposed for the CsIX Project. In scenario 1, the ion exchange and grouting would be performed in the same new building. In scenario 2, the grouting would be performed in a new building while the ion exchange would be performed in the existing New Waste Calcining Facility (NWCF). Tables 4, 5, and 6 give the loads for the $2^{\text {nd }}$ scenario. As expected, the loads are less in scenario 2 primarily because of lighting, miscellaneous, and $H \& V$ loads since the grouting building would be smaller in floor space as compared to the combined process facility of scenario 1 . The actual process and transport load differences are relatively minor. In scenario 2, the connected load would be 569 kVA, the demand load would be $381 \mathrm{kVA}$, and the standby load would be $53 \mathrm{kVA}$. This compares to 698,471 , and 88 kVA respectively for the loads of scenario 1 . Scenario 2 will also add a maximum of $60 \mathrm{kVA}$ to the load at NWCF. This load will consist of approximately twelve motors of five horsepower or less. The net impact of this addition will likely be less than $60 \mathrm{KVA}$ since existing loads at NWCF will be removed to accommodate the ion exchange process. 
TABLE 1

CsIX GROUTING PROJECT

CONNECTED LOAD STUDY

Scenario 1

\begin{tabular}{|c|c|}
\hline Load & KVA \\
\hline $\begin{array}{l}\text { Lighting loads: } \\
\text { Processing building } \\
\text { Interim storage } \\
\text { TruPac }\end{array}$ & $\begin{array}{r}93 \\
58 \\
8\end{array}$ \\
\hline $\begin{array}{l}\text { Miscellaneous loads: } \\
\text { Process building } \\
\text { Interim storage } \\
\text { TruPac }\end{array}$ & $\begin{array}{r}41 \\
4 \\
4\end{array}$ \\
\hline $\begin{array}{l}\text { Process loads: } \\
\text { Process building } \\
\text { Dewatering room } \\
\text { Cure room } \\
\text { Interim Storage } \\
\text { TruPac }\end{array}$ & $\begin{array}{l}81 \\
14 \\
11 \\
22 \\
10\end{array}$ \\
\hline $\begin{array}{l}\text { Cranes: } \\
\quad \text { Processing }\end{array}$ & 70 \\
\hline $\begin{array}{l}\text { Tansporting loads: } \\
\text { Process building }\end{array}$ & 72 \\
\hline $\begin{array}{l}\text { H\&V: } \\
\text { Processing } \\
\text { Interim storage } \\
\text { TruPac }\end{array}$ & $\begin{array}{r}56 \\
34 \\
120\end{array}$ \\
\hline Total connected KVA & 698 \\
\hline
\end{tabular}


TABLE 2

CsIX GROUTING PROJECT

DEMAND LOAD STUDY

Scenario 1

\begin{tabular}{|c|c|c|}
\hline Load & Demand & KVA \\
\hline $\begin{array}{l}\text { Lighting loads: } \\
\text { Processing building } \\
\text { Interim storage } \\
\text { TruPac }\end{array}$ & $\begin{array}{l}90 \% \\
90 \% \\
90 \%\end{array}$ & $\begin{array}{r}83 \\
52 \\
7\end{array}$ \\
\hline $\begin{array}{l}\text { Miscellaneous loads: } \\
\text { Process building } \\
\text { Interim storage } \\
\text { TruPac }\end{array}$ & $\begin{array}{l}50 \% \\
50 \% \\
50 \%\end{array}$ & $\begin{array}{r}21 \\
2 \\
2\end{array}$ \\
\hline $\begin{array}{l}\text { Process loads: } \\
\text { Process building } \\
\text { Dewatering room } \\
\text { Cure room } \\
\text { Interim } \\
\text { TruPac }\end{array}$ & $\begin{array}{r}\text { specified } \\
25 \% \\
25 \% \\
25 \% \\
25 \%\end{array}$ & $\begin{array}{r}72 \\
4 \\
3 \\
5 \\
3\end{array}$ \\
\hline $\begin{array}{l}\text { Cranes: } \\
\text { Processing } \\
\text { Tansporting loads: } \\
\text { Process building }\end{array}$ & $10 \%$ & $\begin{array}{r}7 \\
18\end{array}$ \\
\hline $\begin{array}{l}\text { H\&V: } \\
\text { Processing } \\
\text { Interim storage } \\
\text { TruPac }\end{array}$ & $\begin{array}{r}100 \% \operatorname{Prcs} \\
\text { s } \\
100 \% \\
100 \%\end{array}$ & $\begin{array}{r}45 \\
34 \\
120\end{array}$ \\
\hline Total connected KVA & & 471 \\
\hline
\end{tabular}


TABLE 3

\section{CsIX GROUTING PROJECT STANDBY LOAD STUDY Scenario 1}

\begin{tabular}{|l|r|r|}
\hline \multicolumn{1}{|c|}{ Load } & Standby & KVA \\
\hline Lighting loads: & $10 \%$ of $90 \%$ & 8 \\
Processing building & $10 \%$ o f90\% & 6 \\
Interim storage & $10 \%$ of $90 \%$ & 1 \\
TruPac & & \\
Miscellaneous loads: & $10 \%$ of $50 \%$ & 2 \\
$\quad$ Process building & $10 \%$ of $50 \%$ & .2 \\
Interim storage & $10 \%$ of $50 \%$ & .2 \\
TruPac & & \\
& & 7 \\
Process loads: & $10 \%$ of 72 & .4 \\
Process building & $10 \%$ of $25 \%$ & .3 \\
$\quad$ Dewatering room & $10 \%$ of $25 \%$ & 1 \\
$\quad$ Cure room & $10 \%$ o f25\% & 2 \\
Interim Storage & $10 \%$ of $25 \%$ & \\
Tansporting loads: & & \\
Process building & & \\
H\&V: & $10 \%$ of $100 \%$ & \\
Processing & $10 \%$ of $100 \%$ & \\
Interim storage & $10 \%$ of $100 \%$ & \\
TruPac & & \\
\hline Total connected KVA & & \\
& & \\
\hline
\end{tabular}


TABLE 4

\section{CsIX GROUTING PROJECT CONNECTED LOAD STUDY Scenario 2}

\begin{tabular}{|l|r|}
\hline \multicolumn{1}{|c|}{ Load } & KVA \\
\hline Lighting loads: & 33 \\
Processing building & 58 \\
Interim storage & 8 \\
TruPac & \\
Miscellaneous loads: & 12 \\
Process building & 4 \\
Interim storage & 4 \\
TruPac & \\
Process loads: & 81 \\
Process building & 14 \\
Dewatering room & 11 \\
Cure room & 22 \\
Interim Storage & 10 \\
TruPac & \\
Cranes: & 70 \\
Processing & \\
Tansporting loads: & \\
Process building & 72 \\
H\&V: & \\
Processing & 120 \\
Interim storage & \\
TruPac & \\
\hline Total connected KVA & \\
& \\
\hline
\end{tabular}


TABLE 5

CsIX GROUTING PROJECT

DEMAND LOAD STUDY

Scenario 2

\begin{tabular}{|c|c|c|}
\hline Load & Demand & KVA \\
\hline $\begin{array}{l}\text { Lighting loads: } \\
\text { Processing building } \\
\text { Interim storage } \\
\text { TruPac }\end{array}$ & $\begin{array}{l}90 \% \\
90 \% \\
90 \%\end{array}$ & $\begin{array}{r}30 \\
52 \\
7\end{array}$ \\
\hline $\begin{array}{l}\text { Miscellaneous loads: } \\
\text { Process building } \\
\text { Interim storage } \\
\text { TruPac }\end{array}$ & $\begin{array}{l}50 \% \\
50 \% \\
50 \%\end{array}$ & $\begin{array}{l}6 \\
2 \\
2\end{array}$ \\
\hline $\begin{array}{l}\text { Process loads: } \\
\text { Process building } \\
\text { Dewatering room } \\
\text { Cure room } \\
\text { Interim } \\
\text { TruPac }\end{array}$ & $\begin{array}{r}\text { specified } \\
25 \% \\
25 \% \\
25 \% \\
25 \%\end{array}$ & $\begin{array}{r}72 \\
4 \\
3 \\
5 \\
3\end{array}$ \\
\hline $\begin{array}{l}\text { Cranes: } \\
\text { Processing } \\
\text { Tansporting loads: } \\
\text { Process building }\end{array}$ & $\begin{array}{l}10 \% \\
25 \%\end{array}$ & 7 \\
\hline $\begin{array}{l}\text { H\&V: } \\
\text { Processing } \\
\text { Interim storage } \\
\text { TruPac }\end{array}$ & $\begin{array}{r}100 \% \operatorname{Prcs} \\
\mathrm{s} \\
100 \% \\
100 \% \\
\end{array}$ & $\begin{array}{r}16 \\
34 \\
120\end{array}$ \\
\hline Total connected KVA & & 381 \\
\hline
\end{tabular}


TABLE 6

\section{CsIX GROUTING PROJECT STANDBY LOAD STUDY Scenario 2}

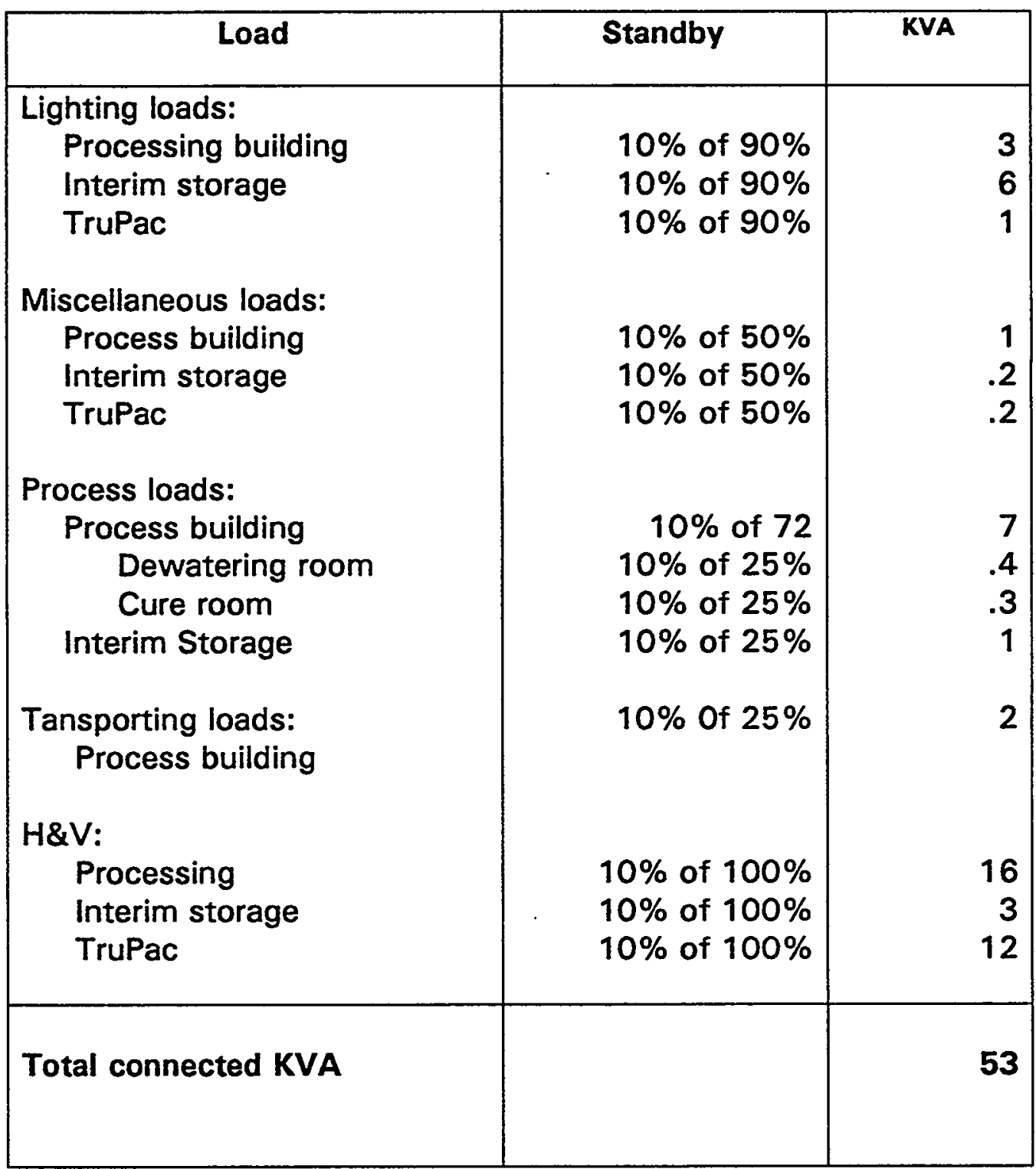


Project File Number $\quad$ O2BM1

Project/Task CsIX/TRU Grout Feasibility Study

Subtask C.4 Determine Waste Radiation Levels

\begin{tabular}{l} 
Title: CsIX/TRU Component Projected Radiation Fields \\
\hline Summary: This EDF contains: \\
1. A description of the cases considered and basis used to calculate proj \\
radiation fields for in-process materials and completed waste forms. \\
2. Compilation of radiation field and heat generation results. \\
3. Implications of results and recommendations for further refinement of \\
process assumptions.
\end{tabular}

Distribution (complete package): H. K. Peterson, S. J. Losinski, K. L. Williams, C. W. Olsen Distribution (summary package only): J. J. McCarthy

\begin{tabular}{|c|c|c|c|c|c|}
\hline $\begin{array}{l}\text { Author } \\
\text { H. K. Peterson } \\
\text { S. J. Losinski }\end{array}$ & 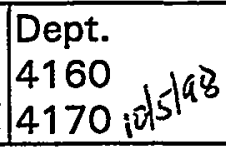 & $\begin{array}{l}\text { Reviewed } \\
\text { T.MM Domald }\end{array}$ & $\mid \begin{array}{l}\text { Date } \\
10-5-48\end{array}$ & $\begin{array}{l}\text { Approved } \\
\text { K.L.Williams } \\
\text { K.C.CWillease }\end{array}$ & $\begin{array}{l}\text { Date } \\
10 / 5 / 98\end{array}$ \\
\hline
\end{tabular}




\section{Evaluation Description}

\section{Objective}

This EDF contains an evaluation of projected radiation fields and heat generation rates for various intermediate products and completed waste forms of the CsIX/TRU Grout process. A description of the process is included in EDF-CsIX-001.

An objective of performing this evaluation was to determine if the processed waste generated from the CsIX/TRU Grout process would meet waste acceptance criteria for disposal at WIPP as a contact handled waste. A second objective was to determine approximate radiation fields for major pieces of process equipment in the CsIX/TRU Grout process so that hot cell shielding and equipment placement could be considered in a facility layout.

Two iterations of the analysis were performed. The first iteration used radionuclide concentration data from liquid in tank farm tank WM-181, which is expected to generate the highest dose rate stabilized grout based on Cs137 concentration. The second iteration used "Averaged" radionuclide concentrations for all tank farm waste processed in the CsIX/TRU Grout process.

Variants of these iterations were also performed to evaluate waste form sensitivity to process assumptions related to filtration of undissolved solids (UDS).

\section{Radionuclide Source Data}

The radiation source data used for this evaluation are contained in EDF-CsIX001. A detailed description of the assumptions used to generate the data are included in that document. It should be noted that many assumptions were required to generate source data and many of these assumptions may have a significant impact on the analysis. Areas of uncertainty include:

- Tank Farm Volumes and Composition

- HLLW Evaporation Schedule and Performance

- Newly Generated Liquid Waste Volumes and Composition

- Tank Farm Liquid Transfer

- UDS Quantities and Filtration Performance

- Cesium Removal Performance of the lon Exchange Resin

- Neutralization and Grouting Formulation and Grout Density 
In all cases the radiological source data has been decayed to Jan. 2008.

\section{Equipment/ Waste Package Size}

The following flow streams and equipment geometry were used in the first iteration of this study. This iteration represents processing of SBW from tank WM-181. Flow stream numbers refer to the material balance presented in Section 7 of EDF-CsIX-001.

a. Flow Stream 101 , in a cylindrical tank $10^{\prime}$ tall $X 8^{\prime}$ diameter (full), with $1 / 2^{\prime \prime}$ 304 SS wall thickness. This calculation represents SBW from WM-181 which has been steam jetted into Liquid Waste Feed Tanks.

b. Flow Stream 107, in a standard steel 55 gal drum that is full. This calculation represents dried UDS in a full 55 gal drum located at the rotary evaporator.

c. Flow Stream 107diluted by a factor of 10 with water, in a cylindrical tank $5^{\prime}$ tall $\times 4$ ' diameter, with $3 / 8^{\prime \prime} 304$ SS wall thickness. This represents the expected backwash slurry of UDS and DI water from the pressure leaf filtration system.

d. Flow Stream 108, in a cylindrical tank $8^{\prime}$ tall $X 1.33^{\prime}$ diameter, with $3 / 8^{\prime \prime} 304$ SS wall thickness, with .25 cubic meters dispersed uniformly in the cylinder. This represents an ion exchange column in which the IONSIV IE-911 resin has been full loaded with cesium.

e. Flow Stream 106, in a cylindrical tank $10^{\prime}$ tall $X 8^{\prime}$ diameter (full), with $1 / 2^{\prime \prime}$ 304 SS wall thickness. This calculation represents the liquid effluent from the ion exchange column.

f. Flow Stream 108, in a cylindrical tank $15^{\prime}$ tall $X 8^{\prime}$ diameter (full), with $1 / 2^{\prime \prime}$ 304 SS wall thickness. This calculation represents the spent and dried IONSIV IE-911 resin total inventory in the Spent Resin Storage Tank at the end of processing all SBW and NGLW.

g. Flow Stream 106, in a half cylindrical tank $3^{\prime}$ long $X 2^{\prime}$ diameter (full), with 3/8" 304 SS wall thickness. This calculation represents the grout mixer filled with a batch charge of liquid waste, before addition of other grout ingredients. 
h. Flow Stream 117, in a cylindrical tank $3^{\prime}$ long X 2' diameter (full), with 3/8" 304 SS wall thickness. This represents the grout mixer with a completed batch of grout that is ready to be cast into a drum.

i. Flow Stream 117, in a standard steel 55 gal drum that is full. This represents the final grout waste form.

The following flow streams and equipment geometry were used in the second iteration of this study. It should be noted that in this iteration SBW and NGLW were evaporated (in the HLLWE) to a slightly higher radionuclide concentration than the previously described iteration. This iteration represents the "average" processing of all SBW and NGLW. Also included are calculations that indicate sensitivity to the fate of UDS after filtration. Flow stream numbers refer to the material balance presented in Section 6 of EDF-CsIX-001.

1. Flow Stream 101, in a cylindrical tank $10^{\prime}$ tall $X 8^{\prime}$ diameter (full), with $1 / 2^{\prime \prime}$ 304 SS wall thickness. This calculation represents average liquid feed which has been steam jetted into Liquid Waste Feed Tanks.

2. Flow Stream 107, in a standard steel 55 gal drum that is full. This calculation represents dried UDS in a full 55 gal drum located at the rotary evaporator.

3. Flow Stream 107 diluted by a factor of 10 with water, in a cylindrical tank $5^{\prime}$ tall $X$ 4' diameter, with $3 / 8^{\prime \prime} 304$ SS wail thickness. This represents the expected backwash slurry of UDS and DI water from the pressure leaf filtration system.

4. Flow Stream 108, in a cylindrical tank $8^{\prime}$ tall $X 1.33^{\prime}$ diameter, with $3 / 8^{\prime \prime} 304$ SS wall thickness, with .25 cubic meters dispersed uniformly in the cylinder. This represents an ion exchange column in which the IONSIV IE-911 resin has been full loaded with cesium.

5. Flow Stream 106, in a cylindrical tank $10^{\prime}$ tall $X 8^{\prime}$ diameter (full), with $1 / 2^{\prime \prime}$ 304 SS wall thickness. This calculation represents the liquid effluent from the ion exchange column.

6. Flow Stream 108, in a cylindrical tank $15^{\prime}$ tall $X 8^{\prime}$ diameter (full), with $1 / 2^{\prime \prime}$ 304 SS wall thickness. This calculation represents the spent and dried IONSIV IE-911 resin total inventory in the Spent Resin Storage Tank at the end of processing all SBW and NGLW. This calculation assumes that the $20 \%$ UDS which is not collected in the filtration system will pass through the ion exchange column as well. 
7. Flow Stream $108 \mathrm{a}$, in a cylindrical tank $15^{\prime}$ tall $X 8^{\prime}$ diameter (full), with $1 / 2^{\prime \prime}$ 304 SS wall thickness. This calculation represents the spent and dried IONSIV IE-911 resin total inventory in the Spent Resin Storage Tank at the end of processing all SBW and NGLW. This calculation assumes that the $20 \%$ UDS which is not collected in the filtration system will be filtered out in the ion exchange column.

8. Flow Stream 113, in a half cylindrical tank $3^{\prime}$ long $X 2^{\prime}$ diameter (full), with $3 / 8$ " 304 SS wall thickness. This calculation represents the grout mixer filled with a batch charge of liquid waste, before addition of other grout ingredients.

9. Flow Stream 117, in a cylindrical tank $3^{\prime}$ long $X 2$ ' diameter (full), with $3 / 8^{\prime \prime}$ 304 SS wall thickness. This represents the grout mixer with a completed batch of grout that is ready to be cast into a drum. This iteration assumes that there are no UDS in the grout.

10. Flow Stream 117, in a standard steel 55 gal drum that is full. This represents the final grout waste form. This iteration assumes that there are no UDS in the grout.

11. Flow Stream 117- $\mathrm{A}$, in a standard steel 55 gal drum that is full. This represents the final grout waste form. This iteration assumes that all UDS passing the filtration system is incorporated in the grout.

\section{Evaluation Method}

The calculations were made with the MicroShield, Version 5.01 computer code. 


\section{Evaluation Results}

Table 2-1 presents the results of radiation field and heat generation calculations for the iteration of processing SBW from tank WM-181. All radiation fields are reported as dose at contact and heat generation is reported as total heat per container. Details of the analysis and the radionuclide tables are included as Attachment 1.

Table 2-1. Radiation Field and Heat Generation for Processing WM-181.

\begin{tabular}{|c|l|r|r|}
\hline Item & Description & Radiation Field & Heat Generation \\
\hline & & @ Contact & Per Container \\
\hline a & Liquid Waste Feed Tanks & $13.3 \mathrm{R} / \mathrm{hr}$. & \\
\hline b & Drum of Dried UDS & $49 \mathrm{R} / \mathrm{hr}$. & \\
\hline c & UDS Filtrate Slurry Tank & $7.8 \mathrm{R} / \mathrm{hr}$. & \\
\hline $\mathrm{d}$ & Loaded lon Exchange Column & $2675 \mathrm{R} / \mathrm{hr}$. & \\
\hline e & lon Exchange Liquid Effluent Tank & $170 \mathrm{mR} / \mathrm{hr}$. & \\
\hline $\mathrm{f}$ & Spent lon Exchange Resin Tank & $5360 \mathrm{R} / \mathrm{hr}$. & \\
\hline $\mathrm{g}$ & Mixer With Liquid Waste & $88 \mathrm{mR} / \mathrm{hr}$. & \\
\hline $\mathrm{h}$ & Mixer With Grout Batch & $58 \mathrm{mR} / \mathrm{hr}$. & \\
\hline i & Finished Grout Waste Drum & $65 \mathrm{mR} / \mathrm{hr}$. & \\
\hline
\end{tabular}


431.02\#

$06 / 17 / 97$

Rev. \#04
ENGINEERING DESIGN FILE
Functional File Number - SPR-CsIX-13 EDF Serial Number - EDF-CsIX-15

Page 7 of 10

Table 2-2 presents the results of radiation field and heat generation calculations for the iteration of processing average SBW and NGLW. All radiation fields are reported as dose at contact and heat generation is reported as total heat per container. Details of the analysis and the radionuclide tables are included as Attachment 2.

Table 2-2. Radiation Field and Heat Generation for Processing Average Tank Waste.

\begin{tabular}{|c|l|r|r|}
\hline Item & Description & Radiation Field & Heat Generation \\
\hline & & @ Contact & Per Container \\
\hline 1 & Liquid Waste Feed Tanks & $14.4 \mathrm{R} / \mathrm{hr}$. & $7.24 \mathrm{~W}$ \\
\hline 2 & Drum of Dried UDS & $48.6 \mathrm{R} / \mathrm{hr}$. & $0.94 \mathrm{~W}$ \\
\hline 3 & UDS Filtrate Slurry Tank & $7.8 \mathrm{R} / \mathrm{hr}$. & $0.8 \mathrm{~W}$ \\
\hline 4 & Loaded lon Exchange Column & $2630 \mathrm{R} / \mathrm{hr}$. & $24 \mathrm{~W}$ \\
\hline 5 & lon Exchange Liquid Effluent Tank & $185 \mathrm{mR} / \mathrm{hr}$. & $3.98 \mathrm{~W}$ \\
\hline 6 & Spent lon Exchange Resin Tank & $7626 \mathrm{R} / \mathrm{hr}$. & $2050 \mathrm{~W}$ \\
\hline 7 & $\begin{array}{l}\text { Spent lon Exchange Resin Tank } \\
\text { (+UDS) }\end{array}$ & $10650 \mathrm{R} / \mathrm{hr}$. & $2840 \mathrm{~W}$ \\
\hline 8 & Mixer With Liquid Waste & $90 \mathrm{mR} / \mathrm{hr}$. & $0.037 \mathrm{~W}$ \\
\hline 9 & Mixer With Grout Batch & $70 \mathrm{mR} / \mathrm{hr}$. & $0.047 \mathrm{~W}$ \\
\hline 10 & Finished Grout Waste Drum & $115 \mathrm{mR} / \mathrm{hr}$. & $0.037 \mathrm{~W}$ \\
\hline 11 & Finished Grout Waste Drum (+UDS) & $130 \mathrm{mR} / \mathrm{hr}$. & $0.037 \mathrm{~W}$ \\
\hline
\end{tabular}

\section{Implications of Results and Recommendations}

The results of this evaluation indicate that the quantity and fate of UDS plays a significant influence in the radiation dose rate of waste forms and intermediate products. Most notably the radiation field of the spent ion exchange resin increased by approximately $40 \%$ when it was assumed that all UDS that made it through the filtration system was captured in the ion exchange column.

It was also noted that UDS concentration had a moderate effect on the radiation dose rate of the finished grout product. The increase was calculated to be approximately $13 \%$, which may be significant for waste products that are approaching the contact handled threshold limit.

The results of the evaluation also indicate that the degree of evaporation of wastes in the tank farm can have a significant influence of the radiation dose rate of the grouted waste. Implications of this analysis indicate that the actual management of tank farm wastes and their associated evaporation/concentration schedules may have a significant impact on the grouted wasteform. 
It is recommended that additional characterization of the inventory of UDS in the tank farm be performed (radionuclide speciation, particle size, and quantities).

It is also recommended that technological development of filtration equipment with UDS be conducted. Also, data must be collected on the performance of the ion exchange resin with respect to filtration of UDS. 
431.02\#

$06 / 17 / 97$

Rev. \#04
ENGINEERING DESIGN FILE

\section{Attachment 1}

Functional File Number - SPR-CsIX-13

EDF Serial Number - EDF-CsIX-15

Page 9 of 10 


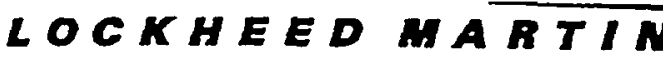

Lockheed Martin Idaho Technologies Company INTERDEPARTMENTAL COMMUNICATION

Date: $\quad$ August 6, 1998

To:

S. J. Losinski

MS 3625

$6-5962$

From:

H. K. Peterson

MS 2107

$6-8657$

Subject: $\quad$ CS IX/TRU COMPONENT PROJECTED RADIATION FIELDS -- HKP-12-98

References: a) MicroShield, Version 5.01, Grove Engineering, Rockville MD

Contact/near surface ( $1 \mathrm{~cm}$ from item surface) radiation field calculations have been made for the requested Cs DX/TRU Feasibility Study components and are summarized below. The calculations were made with the MicroShield, Version 5.01, computer code (Ref.a) and input/output listings for each are attached for reference. Also attached, for documentation purposes, are copies of the spreadsheet pages that list the stream numbers and the respective radiological concentrations.

a) T-101 A \& B Liquid Waste Feed Tank, assumed to be a right circular cylinder 10 feet tall, 8 $f t$ in diameter, with a wall of $1 / 2$ inches stainless steel (SS), and containing 14,234 liters of Stream 101. The calculated radiation field at $1 \mathrm{~cm}$ from the tank midline is $13.3 \mathrm{R} / \mathrm{hr}$.

b) EV-101 Rotary Evaporator, assumed to be the same as a standard 55-gal drum containing $291.2 \mathrm{~kg}$ of Stream 107. The calculated radiation field for this component is $49 \mathrm{R} / \mathrm{hr}$.

c) T-108 UDS Slurry Tank, assumed to be $5 \mathrm{ft}$ tall, $4 \mathrm{ft}$ in diameter, with a $1 / 4$ inch SS wall, and containing $249 \mathrm{~kg}$ of Stream 107 diluted by a factor of 10 with water. The calculated radiation field is $7.8 \mathrm{R} / \mathrm{hr}$.

d) V-104A Cesium Ion Exchange Column, assumed to be $8 \mathrm{ft}$ tall, $1.33 \mathrm{ft}$ in diameter, with a $3 / 8$ inch SS wall, and containing $0.25 \mathrm{~m}^{3}$ of Stream 108 distributed uniformly in the column. The radiation field for this component is calculated to be $2675 \mathrm{R} / \mathrm{hr}$.

e) T-103 A \& B Cs IX Effluent Tank, assumed to be $10 \mathrm{ft}$ tall, $8 \mathrm{ft}$ diameter, with a $1 / 2$ inch SS wall, and containing 14234 liters of Stream 106. The radiation field for this component is $170 \mathrm{mR} / \mathrm{hr}$.

f) T-112 Spent Resin Storage Tank, assumed to be $15 \mathrm{ft}$ high, $8 \mathrm{ft}$ in diameter, with a $1 / 2$ inch SS wall, and containing $21,350 \mathrm{~kg}$ of Stream 108. The radiation field is calculated to be 5360 $\mathrm{R} / \mathrm{hr}$.

g) M-101 Mixer, assumed to be a half cylinder $2 \mathrm{ft}$ in diameter, $3 \mathrm{ft}$ long, with a $3 / 8$-inch SS wall and containing 133.4 liters of Stream 106. The radiation field is calculated to be 88 $\mathrm{mR} / \mathrm{hr}$. 
Page 2

h) M-101 Mixer, assumed to be a full cylinder $3 \mathrm{ft}$ long, $2 \mathrm{ft}$ in diameter, with a 3/8-inch SS wall, and containing $453.7 \mathrm{~kg}$ of Stream 117. The radiation field for this component is calculated to be $58 \mathrm{mR} / \mathrm{hr}$.

i) Waste Drum, assumed to be a standard 55 - gal drum with $239 \mathrm{~kg}$ of grouted waste from Stream 117. The radiation field for this package is calculated to be $65 \mathrm{mR} / \mathrm{hr}$.

If there are any questions or comments concerning this analysis, please call me at the abovelisted number.
hkp
cc:
T. S. Bohn, LMITCO, MS 4138

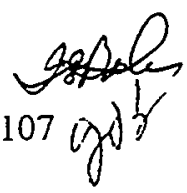
HKP Letter File 
Microshield $\nabla 5.01$ (5.01-00121)

Lockheed Martin Idaho Technologies Company

Page : 1

DOS File: WM181101.MS5

Run Date: July 31, 1998

Run Time: 8:51:44 AM

Duration: 00:01:49

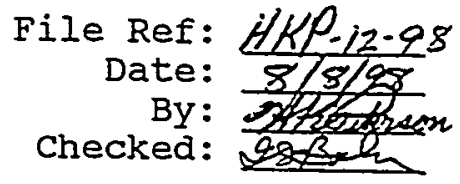

Case Title: FM-181

Description: T-101 A \& B Iiquid Faste Feed Tanks - Surface Rad. Geometry: 7 - cylinder volume - side Shields

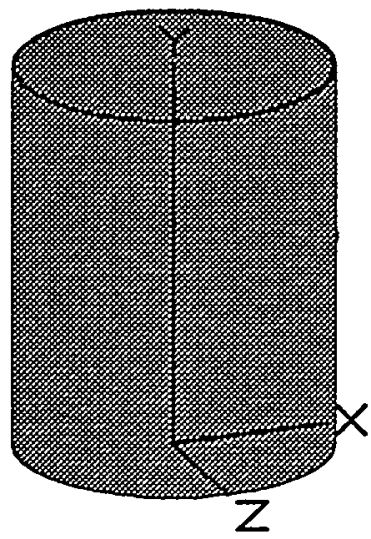

$\begin{array}{lrrr}\text { Height } & 304.8 \mathrm{~cm} & 10 \mathrm{ft} 0.0 \mathrm{in}- \\ \text { Radius } & 121.92 \mathrm{~cm} & 4 \mathrm{ft}-\end{array}$

Dose Points

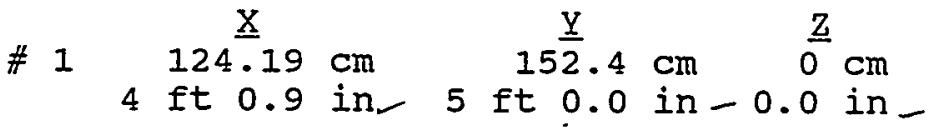

Shields

Shield Name Dimension Material Density

Source $\quad 1.42 e+07 \mathrm{~cm}^{3}$ Mixed $\rightarrow 1.3$

Concrete 0.3

Shield 1

Transition

Water 1

Air Gap

$1.27 \mathrm{~cm}$ Iron 7.86 -

$1.0 \mathrm{~cm}$ Air 0.0011

Air 0.00122

Source Input

Grouping Method : Standard Indices

Number of Groups : 25

Iower Energy Cutoff : 0.015

Photons < 0.015 : Excluded

Library : ICRP-38

\begin{tabular}{|c|c|c|c|}
\hline Tuclide & curies & becav & $\underline{\mu \mathrm{Ci}} / \mathrm{cm}^{3}$ \\
\hline$A m-241$ & $5.8640 e+000$ & $2.1697 e+011$ & $4.1198 e-001$ \\
\hline$m-243$ & $2.6600 e-003$ & $9.8420 e+007$ & \\
\hline$a-137 m$ & $6.3197 e+002$ & $.3383 e+013$ & $4.4400 e+001$ \\
\hline$e-144$ & $1900 e-006$ & $503 e+005$ & $e-002$ \\
\hline$m-242$ & -002 & & \\
\hline $\mathrm{Cm}-244$ & -001 & 009 & +002 \\
\hline $20-60$ & $0070 e+000$ & +010 & $1.4100 \mathrm{e}-001$ \\
\hline s- - & $2.3200 e-001$ & 009 & $.0308 e+002$ \\
\hline$s-1$ & .002 & 008 & $2+001$ \\
\hline$s-137$ & 6.68 & 2.47 & +006 \\
\hline$E u-152$ & $e-002$ & 009 & +002 \\
\hline$E u-154$ & $e+000$ & & $2+003$ \\
\hline $\mathrm{Eu}-1$ & -001 & 2.4 & +003 \\
\hline$[-3$ & $e-001$ & 1.27 & $e-002$ \\
\hline$[-129$ & $e-003$ & 3.49 & $6.6399 e-004$ \\
\hline$i-63$ & +000 & 010 & $8 e-001$ \\
\hline$p-237$ & -003 & & $3 e+001$ \\
\hline $\mathrm{Pm}-147$ & 1.65 & 6.10 & $1.1599 \mathrm{e}-001 / 4.291$ \\
\hline $\operatorname{Pr}-144$ & $e-006$ & 2.62 & $4.9792 \bar{e}-00 \overline{7} i$ \\
\hline $\mathrm{Pu}-238$ & $1.6226 e+001$ & $6.0036 e+011$ & 1.1400 e +000 \\
\hline$P u-239$ & ee-001 & $e+010$ & $2.6199 e-002$ \\
\hline $\mathrm{Pu}-2$ & $1.0450 e-001$ & $3.8665 e+009$ & $7.3418 e-003$ \\
\hline$P u-241$ & $4.8680 e+000$ & $1.8012 e+011$ & $3.4201 e-001$ \\
\hline
\end{tabular}


Page : 2

DOS File: WM181101.MS5

Run Date: July 31, 1998

Run Time: 8:51:44 AM

Duration: 00:01:49

\begin{tabular}{|c|c|c|c|}
\hline Nuclide & curies & becouerels & $\mu \mathrm{Ci} / \mathrm{cm}^{3}$ \\
\hline$P u-242$ & $2.4700 e-004$ & $9.1390 e+006$ & $1.7353 e-005 v$ \\
\hline $\mathrm{Rh}-106$ & $1.6800 e-004$ & $6.2160 e+006$ & $1.1803 e=005$ \\
\hline$R u-106$ & $1.6800 e-004$ & $6.2160 e+006$ & $1.1803 e-005$ \\
\hline $\mathrm{Sb}-125$ & $2.1070 e-001$ & $7.7959 e+009$ & $1.4803 e-002$ \\
\hline$S m-151$ & $1.1460 e+001$ & $4.2402 e+011$ & $8.0514 e-001$ \\
\hline Sr-90 & $6.3767 e+002$ & $2.3594 e+013$ & $4.4800 e+001$ \\
\hline$T C-99$ & $3.5580 e-001$ & $1.3165 e+010$ & $2.4997 e-002$ \\
\hline Th -230 & $1.4800 e-006$ & $5.4760 e+004$ & $1.0398 e-007$ \\
\hline Th-231 & $2.1200 e-004$ & $7.8440 e+006$ & $1.4894 e-005$ \\
\hline $\mathrm{U}-233$ & $2.2600 e-008$ & $8.3620 e+002$ & $1.5878 e-009 / 5.8748 e-005$ \\
\hline$U-234$ & $2.4480 e-002$ & $9.0576 e+008$ & $1.7199 e-003$ \\
\hline$U-235$ & $6.1300 e-004$ & $2.2681 e+007$ & $4.3067 e-0051.5935 e+000$ \\
\hline$U-236$ & $2.1640 e-003$ & $8.0068 e+007$ & $1.5203 e-004-5.6253 e+000$ \\
\hline$U-238$ & $6.0490 e-004$ & $2.2381 e+007$ & $4.2498 \mathrm{e}-0051.5724 \mathrm{e}+000$ \\
\hline$Y-90$ & $6.3767 e+002$ & $2.3594 e+013$ & $4.4800 e+001 / 1.6576 e+006$ \\
\hline
\end{tabular}

Integration Parameters

Radial

Circumferential

24

$y$ Direction (axial)

24

24

$\begin{array}{cc}\frac{\text { Energy }}{\text { MeV }} & \begin{array}{c}\text { Activity } \\ \text { photons/se }\end{array} \\ 0.015 & 5.637 e+10 \\ 0.02 & 7.244 e+10 \\ 0.03 & 1.426 e+12 \\ 0.04 & 3.746 e+11 \\ 0.05 & 9.532 e+09 \\ 0.06 & 7.786 e+10 \\ 0.08 & 7.873 e+09 \\ 0.1 & 6.336 e+10 \\ 0.15 & 2.212 e+08 \\ 0.2 & 1.052 e+10 \\ 0.3 & 1.048 e+09 \\ 0.4 & 3.758 e+09 \\ 0.5 & 1.586 e+09 \\ 0.6 & 2.101 e+13 \\ 0.8 & 6.188 e+10 \\ 1.0 & 1.196 e+11 \\ 1.5 & 1.306 e+11 \\ 2.0 & 3.469 e+06 \\ 3.0 & 3.717 e+03\end{array}$

Results

Fluence Rate Exposure Rate Exposure Rate

Fluence Rate

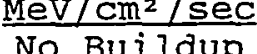

$8.067 e-264$

$1.523 e-117$

$3.167 e-35$

9. $318 e-15$

$1.602 e-08$

$5.190 e-04$

$5.814 e-02$

7.379 et00

3. $126 e-01$

$3.883 e+01$

1.028 et 01

$6.722 e+01$

4. 430 et 01

8.402 et 05

$4.343 e+03$

$1.298 e+04$

3. $112 e+04$

1. 420 et 00

$3.158 e-03$

$\mathrm{MeV} / \mathrm{cm}^{2} / \mathrm{sec}$

With Buildup

$1.743 e-21$

$5.738 \mathrm{e}-20$

1. $007 e-16$

$1.563 e-09$

$7.061 e-04$

6.038 e+00

$8.297 e+01$

$2.874 e+03$

$2.737 e+01$

1. $627 \mathrm{e}+03$

$2.035 e+02$

$8.895 e+02$

4.348 e+02

$6.632 e+06$

2. 545 e+0 4

6. $170 e+04$

1. 084 e+0 5

$4.168 e+00$

$7.549 e-03$

$\frac{\text { Exposure }}{\mathrm{mR} / \mathrm{hr}}$

No Buildup

$6.919 e-265$

$5.276 e-119$

$3.138 e-37$

4. $121 \mathrm{e}-17$

4. $268 \mathrm{e}-11$

$1.031 \mathrm{e}-06$

9.201e-05

1. $129 e-02$

$5.148 e-04$

$6.853 e-02$

1. 951 e-02

1.310e-01

8. $696 e-02$

1. 640 e+03

8. 260e+00

$2.392 e+01$

$5.236 e+01$

$2.196 e-03$

$4.285 e-06$

$6.834 e+06$

$1.725 e+03$
$\mathrm{mR} / \mathrm{hr}$

with Buildup

1. $495 e-22$

$1.988 e-21$

$9.977 e-19$

$6.913 e-12$

$1.881 e-06$

1. 199e-02

1. $313 e-01$

4. 397e+00

$4.508 e-02$

2.871 e+00

3. 861e-01

$1.733 e+00$

$8.536 e-01$

1. 294 e+04

4.841e+01

$1.137 e+02$

$1.825 e+02$

$6.445 e-03$

$1.024 e-05$

$1.330 e+04 v$ 
Microshield v5.01 (5.01-00121)

Lockheed Martin Idaho Technologies Company

Page : 1

DOS File: WM181107.MS5

Run Date: August 2, 1998

Run Time: $8: 31: 55 \mathrm{AM}$

Duration: $00: 01: 54$

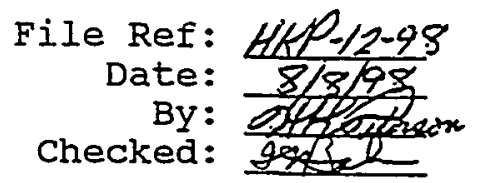

Case Title: FM181-107

Description: 55-gal drum loaded with $291.2 \mathrm{~kg}$ of stream 107 . Geometry: 7 - Cylinder Volume - Side Shields

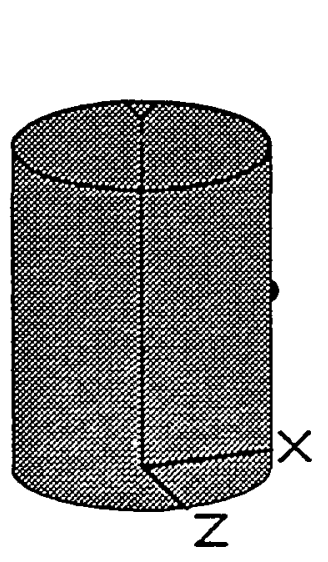

\begin{tabular}{lrr}
\multicolumn{5}{c}{ Source Dimensions } \\
Height & $77.598 \mathrm{~cm}$ & $2 \mathrm{ft} 6.6 \mathrm{in}$ \\
Radius & $29.21 \mathrm{~cm}$ & $11.5 \mathrm{in}$
\end{tabular}

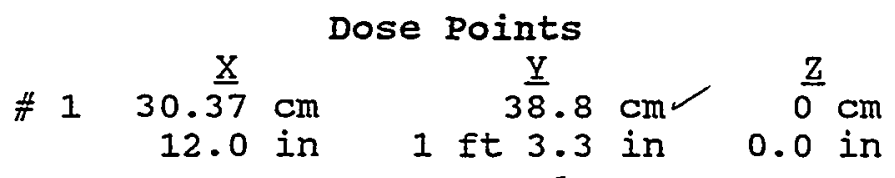

Shields

Shield Name Dimension MaterialDensity Source 2.08e+05 $\mathrm{cm}^{3}$ Concrete $1.4-$ Shield $1 \quad .16 \mathrm{~cm}_{2}$ Iron 7.86 Transition $\quad 1.0 \mathrm{~cm}$ Air 0.0011 Air Gap Air 0.00122

\section{Source Input \\ Grouping Method : Standard Indices \\ Number of Groups : 25 \\ Lower Energy Cutoff : 0.015 \\ Photons < 0.015 : Excluded}

Library : ICRP-38

Nuclide

Am-241

$\mathrm{Ba}-137 \mathrm{~m}$

$\mathrm{Ce}-144$

Co-60

Cs -134

Cs -137

$\mathrm{Np}-237$

Pr -144

$\mathrm{Pu}-238$

Pu-239

$\mathrm{Pu}-240$

$\mathrm{Pu}-241$

$\mathrm{Pu}-242$

$\mathrm{Rh}-106$

Ru-106

$\mathrm{Sb}-125$

Sr -90

$\mathrm{U}-234$

$\mathrm{U}-235$

$\mathrm{U}-236$

$\mathrm{U}-238$

$\mathrm{Y}-90$ becquerels

$3.4650 \mathrm{e}-001 \mathrm{1}$ 1.2821e+010

$3.6090 e+001 / 1 / 3353 e+012$

$7.7460 e-010 / 2.8660 e+001$

$8.0660 e-003-2.9844 e+008$

$1.4360 e-003 \sqrt{5} .3132 e+007$

$3.8150 e+001 / 1.4116 e+012$

$5.0700 e-003 / 1.8759 e+008$

$7.7460 e-010 \leq / 2.8660 e+001$

$2.0730 e+001 / 7.6701 e+011$

$1.1120 e+000 \checkmark 4.1144$ e+010

$3.4900 e-001 V 1.2913 e+010$

$7.1930 \mathrm{e}+000 \vee 2.6614 \mathrm{e}+011$

$5.1830 e-004 / 1.9177 e+007$

$7.1340 e-006 \vee 2.6396 e+005$

$7.1340 \mathrm{e}-006 \mathrm{~V} / 2.6396 \mathrm{e}+005$

$1.6250 e-001 / 6.0125 e+009$

5. $1250 e+000 / 1.8963 e+011$

$6.9300 e-003 / 2.5641 e+008$

$1.5260 e-004 \sqrt{5.6462 e+006}$

$7.2200 e-005 / 2.6714 \mathrm{e}+006$

$2.2600 e-005 \quad 8.3620 e+005$

$5.1250 e+000 / 1.8963 e+011$ $\mu \mathrm{Ci} / \mathrm{cm}^{3}$

$1.6659 e+000$

$1.7351 e+002$

$3.7240 e-009$

$3.8779 e-002$

$6.9038 e-003$

$1.8341 \mathrm{e}+002$

$2.4375 e-002$

$3.7240 e-009$

$9.9663 e+001$

$5.3462 e+000$

$1.6779 e+000$

$3.4582 e+001$

$2.4918 e-003$

$3.4298 e-005$

$3.4298 e-005$

$7.8125 e-001$

$2.4639 e+001$

$3.3317 e-002$

$7.3365 e-004$

$3.4712 \mathrm{e}-004$

$1.0865 e-004$

2. $4639 e+001$
$\mathrm{Ba} / \mathrm{cm}^{3}$

$6.1637 e+004$

$6.4199 e+006$

$1.3779 e-004$

$1.4348 e+003$

$2.5544 e+002$

$6.7863 e+006$

$9.0187 e+002$

$1.3779 e-004$

$3.6875 e+006$

$1.9781 e+005$

$6.2082 e+004$

$1.2795 e+006$

$9.2198 e+001$

$1.2690 e+000$

$1.2690 \mathrm{e}+000$

$2.8906 e+004$

$9.1166 e+005$

$1.2327 e+003$

$2.7145 e+001$

$1.2843 e+001$

$4.0202 e+000$

$9.1166 e+005$

Buildup

The material reference is : source 
Page : 2

DOS'File: WM181107.MS5

Run Date: August 2, 1998

Run Time: 8:31:55 AM

Duration: 00:01:54

Integration Parameters

Radial

Circumferential

Y Direction (axial) $\quad 26$

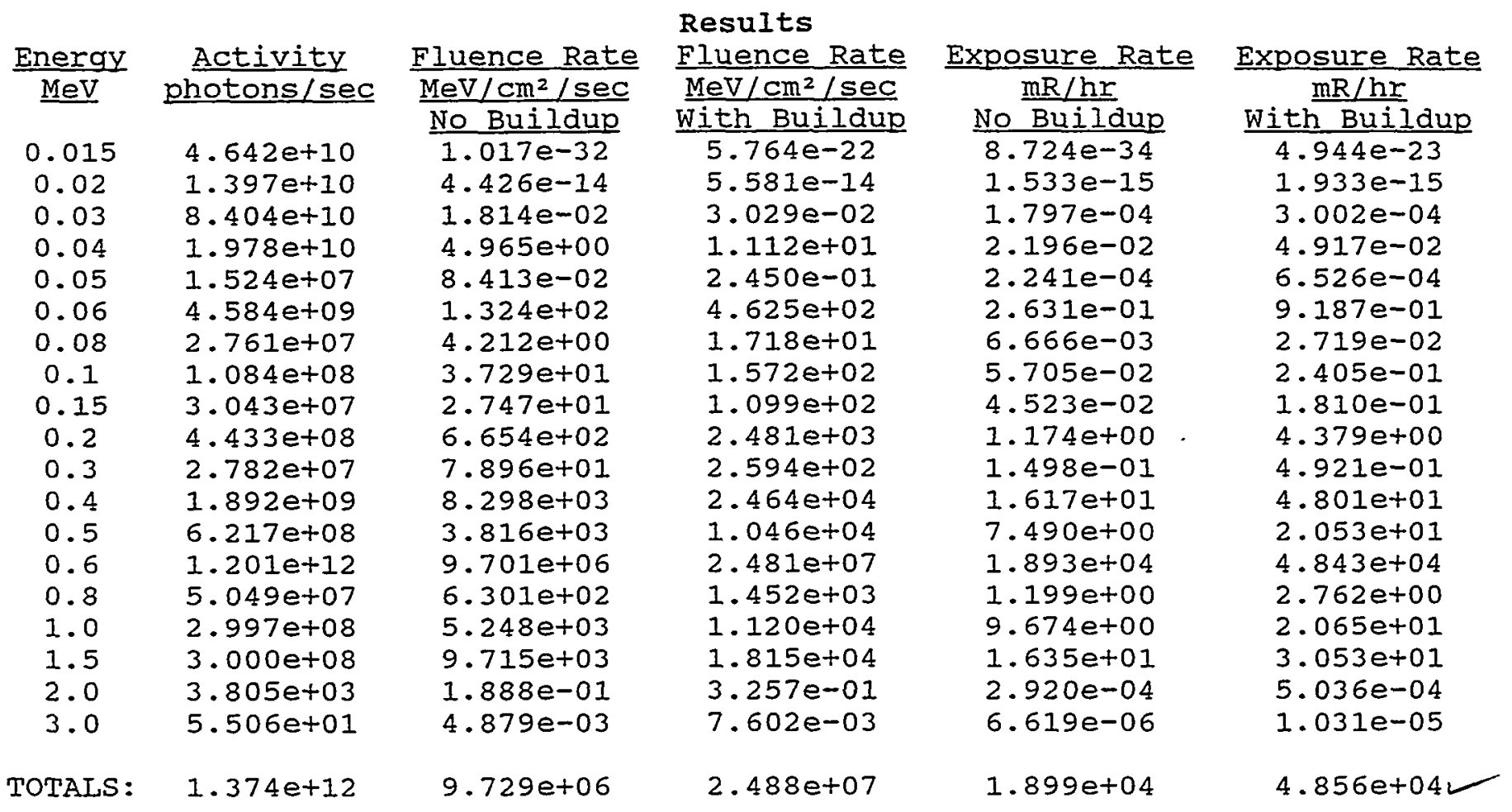


Microshield $\nabla 5.01$ (5.01-00121)

Page : 1

Iockheed Martin Idaho Technologies Company

DOS File: WM181171.MS5

Run Date: August 2, 1998

Run Time: 9:12:34 AM

File Ref:

Date:

By:

Checked:

Duration: 00:02:12

Case Title: WM181-107

Description: UDS Slurry tank (T-108) with $249 \mathrm{~kg}$ stream 107 Geometry: 7 - Cylinder volume - side shields

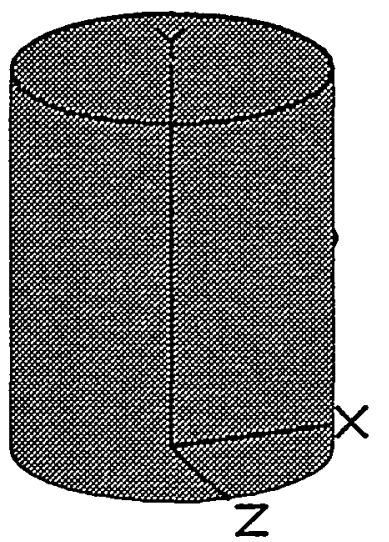

Nuclide

Am-241

Ba-137m

$\mathrm{Ce}-144$

Co-60

Cs -134

Cs -137

$\mathrm{Np}-237$

Pr -144

$\mathrm{Pu}-238$

$\mathrm{Pu}-239$

$\mathrm{Pu}-240$

$\mathrm{Pu}-241$

$\mathrm{Pu}-242$

Rh-106

$\mathrm{Ru}-106$

$\mathrm{Sb}-125$

Sr -90

$\mathrm{U}-234$

$\mathrm{U}-235$

$\mathrm{U}-236$

$\mathrm{U}-238$

$\mathrm{Y}-90$
Height Radius

Source Dimensions

$152.4 \mathrm{~cm}$

$61.0 \mathrm{~cm}$

5 ft 0.0 in

Dose Points

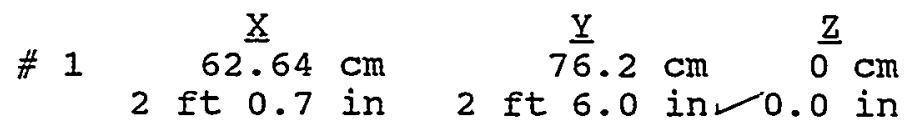

Shields

Shield Name Dimension MaterialDensity Source

$1.78 e+06 \mathrm{~cm}^{3}$ Mixed $\rightarrow 1.13$

Concrete 0.14

Shield 1

Transition

Air Gap

Water 0.99

m Tron 7.86

$1.0 \mathrm{~cm}$ - Air

0.0011

Air

0.00122

Source Input

Grouping Method : Standard Indices

Number of Groups : 25

Lower Energy Cutoff : 0.015

Photons < 0.015 : Excluded

\section{Library : ICRP-38}

curies $2.9680 \mathrm{e}-001 \frac{0}{1.0982 e+010}$ $3.0910 e+001 / 1.1437 e+012$ $6.6300 e-010 \sqrt{2} .4531 e+001$ $6.9090 e-003 / 2.5563 e+008$ $1.2300 e-003 / 4.5510 e+007$ $3.2670 e+001 \cup 1.2088 e+012$ $4.3400 e-003 \quad 1.6058 e+008$ $6.6300 e-010 \mathrm{2} .4531 e+001$ $1.7760 e+001 / 6.5712 e+011$ $9.5280 e-001 / 3.5254 e+010$ $2.9930 e-001 / .1074 e+010$ $6.1610 e+000 / 2.2796 e+011$ $4.4400 e-004 / 7.6428 e+007$ $6.1110 e-006 / 2.2611 e+005$ $6.1110 e-006 \sqrt{2} .2611 e+005$ $1.3900 e-001 V 5.1430 e+009$ $4.3900 e+000 \mathrm{~V} / .6243 e+011$ $5.9360 e-003 / 2.1963 e+008$ $1.3070 e-004 / 4.8359 e+006$ $6.1850 e-005 \mathrm{~V} / 2885 e+006$ $1.9350 e-005 \checkmark z .1595 e+005$ $4.3900 e+000 / 1.6243 e+011$ $\mu \mathrm{Ci} / \mathrm{cm}^{3}$

$1.6660 \mathrm{e}-001$

$1.7350 e+001$

$3.7215 e-010$

$3.8781 e-003$

$6.9042 e-004$

$1.8338 e+001$

$2.4361 e-003$

$3.7215 e-010$

$9.9689 e+000$

$5.3482 e-001$

$1.6800 e-001$

$3.4583 e+000$

$2.4922 e-004$

$3.4302 e-006$

$3.4302 e-006$

$7.8023 e-002$

$2.4642 e+000$

$3.3320 e-003$

$7.3364 e-005$

$3.4717 e-005$

$1.0861 e-005$

$2.4642 e+000$
$\mathrm{Ba} / \mathrm{cm}^{3}$

6.1641 e +003

$6.4196 \mathrm{e}+005$

$1.3770 e-005$

$1.4349 e+002$

$2.5545 e+001$

$6.7851 e+005$

$9.0136 e+001$

$1.3770 e-005$

$3.6885 e+005$

$1.9788 \mathrm{e}+004$

$6.2160 e+003$

$1.2796 e+005$

$9.2213 e+000$

$1.2692 e-001$

$1.2692 e-001$

$2.8868 e+003$

$9.1174 \mathrm{e}+004$

$1.2328 \mathrm{e}+002$

$2.7145 e+000$

$1.2845 \mathrm{e}+000$

$4.0187 e-001$

$9.1174 e+004$ 
Page : 2

DOS File: WM181171.MS5

Run Date: August 2, 1998

Run Time: $9: 12: 34$ AM

Duration: 00:02:12

Buildup

The material reference is : source

\section{Integration Parameters}

Radial

Circumferential

$Y$ Direction (axial)

Energy MeV
0.015
0.02
0.03
0.04
0.05
0.06
0.08
0.1
0.15
0.2
0.3
0.4
0.5
0.6
0.8
1.0
1.5
2.0
3.0
$3.977 e+10$
1. $197 \mathrm{e}+10$
$7.198 e+10$
$1.694 e+10$
$1.306 e+07$
$3.926 e+09$
2. 363 et 07
$9.282 e+07$
$2.604 e+07$
3. $792 e+08$
2. 380 e+07
1. $618 e+09$
5. 318 e+08
1. $029 e+12$
$4.325 e+07$
2. $567 e+08$
2. $570 e+08$
3. $259 e+03$
$4.716 e+01$

Activity photons/sec

TOTALS:
Fluence Rate $\mathrm{MeV} / \mathrm{Cm}^{2} / \mathrm{sec}$ No Buildup $7.905 e-128$ $4.889 e-57$

1. $498 e-16$ $9.645 e-07$

$6.636 e-06$

1. $425 e-01$

3. $751 e-02$

$7.418 e-01$

$1.073 e+00$

3. $210 e+01$

4. $498 \mathrm{e}+00$

5. 140 e+02

2. $502 e+02$

$6.653 e+05$

4. $623 e+01$

4. $056 e+02$

8.247 e+02

1. $711 e-02$

4.840 e-04

$6.674 e+05$
Results

Fluence Rate $\mathrm{MeV} / \mathrm{cm}^{2} / \mathrm{sec}$ With Buildup

$9.819 e-21$

$8.984 e-20$

$6.395 e-12$

$1.808 e-02$

$3.354 e-02$

2.421 e+ 02

1.427 et 01

1. 147 e+02

$5.032 e+01$

$8.459 e+02$

$6.096 e+01$

4. $949 e+03$

1.821 e+03

4.002 et06

2. 140 e+02

$1.554 \mathrm{e}+03$

2. 411 eto3

4. 318e-02

$1.012 \mathrm{e}-03$

$4.014 e+06$
Exposure Rate $\mathrm{mR} / \mathrm{hr}$

No Builaup

$6.780 e-129$

$1.694 \mathrm{e}-58$

$1.484 \mathrm{e}-18$

$4.266 e-09$

$1.768 \mathrm{e}-08$

$2.831 \mathrm{e}-04$

$5.936 e-05$

$1.135 e-03$

$1.767 e-03$

$5.666 e-02$

$8.533 e-03$

$1.001 e+00$

4.911e-01

$1.299 \mathrm{e}+03$

$8.793 e-02$

$7.476 e-01$

$1.388 e+00$

$2.646 e-05$

$6.566 e-07$

$1.302 e+03$
Exposure Rate $\mathrm{mR} / \mathrm{hr}$ With Buildup

$8.422 \mathrm{e}-22$

$3.112 e-21$

$6.338 e-14$

$7.994 \mathrm{e}-05$

$8.934 \mathrm{e}-05$

4.810 e-01

$2.258 \mathrm{e}-02$

$1.755 \mathrm{e}-01$

$8.287 e-02$

$1.493 e+00$

$1.156 \mathrm{e}-01$

$9.643 e+00$

$3.575 e+00$

$7.811 e+03$

$4.071 e-01$

$2.865 e+00$

$4.056 \mathrm{e}+00$

$6.677 e-05$

$1.373 e-06$

$7.833 e+03$ 
Microshield v5.01 (5.01-00121)

Lockheed Martin Idaho Technologies Company

Page : 1

DOS File: CSIEXI.MS5

Run Date: August 4, 1998

Run Time: 10:30:38 AM

Duration: 00:03:25

File Ref:

Date:

By:

Checked;

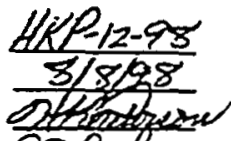

Case Title: Cs I Exch Col

Description: Cs Ion Exchange column with 250 I of stream 108 Geometry: 7 - Cylinder Volume - side shields

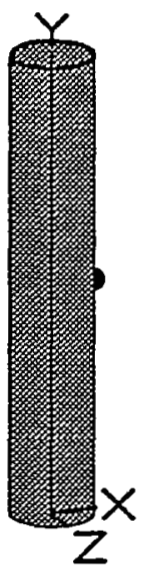

Nuclide

Am-241

$\mathrm{Ba}-137 \mathrm{~m}$

$\mathrm{Ce}-144$

Co-60

Cs -134

Cs -135

Cs -137

$\mathrm{Np}-237$

Pr -144

$\mathrm{Pu}-238$

$\mathrm{Pu}-239$

$\mathrm{Pu}-240$

$\mathrm{Pu}-241$

$\mathrm{Pu}-242$

$\mathrm{Rh}-106$

Ru-106

$\mathrm{Sb}-125$

Sr -90

$\mathrm{U}-234$

$\mathrm{U}-235$

$\mathrm{U}-236$

$\mathrm{U}-238$

$Y-90$
Height

Radius

Source Dimensions

$243.84 \mathrm{~cm}$

$20.32 \mathrm{~cm}$

$8 \mathrm{ft}$

8.0 in $\gamma$

Dose Points

Shields

Shield Name Dimension MaterialDensity Source $3.16 e+05 \mathrm{~cm}^{3}$ Mixed $\rightarrow 2$

Shield I

Transition

Air Gap

Carbon 1.8

Water 0.2

$.953 \mathrm{~cm}$ Iron 7.86

$1.0 \mathrm{~cm}-$ Air 0.0011

Air 0.00122

Source Input

Grouping Method : Standard Indices

Number of Groups : 25

Lower Energy Cutoff : 0.015

Photons < 0.015 : Excluded

Library : ICRP-38

becauerels

curies

9.3750e-003r $3.4688 e+008$

$5.1320 e+003 \quad 1.8988 e+014$

$2.1000 e-011 \sim 7.7700 e-001$

$2.1900 e-004 / 8.1030 e+006$

$1.8800 e+000 / 6.9560 e+010$

$1.0330 \mathrm{e}-001 \sqrt{3.8221 \mathrm{e}+009}$

$5.4250 e+003 \checkmark 2.0073 e+014$

$1.3780 e-004 \checkmark 5.0986 e+006$

2.1000e-011 7.7700e-001

$5.6250 e-001 v 2.0813 e+010$

$3.0250 e-002 \checkmark 1.1193 e+009$

$9.4750 e-003 \checkmark 3.5058 e+008$

$1.9550 \mathrm{e}-0017.2335 \mathrm{e}+009$

$1.4100 e-005 \checkmark / 5.2170 e+005$

$1.9380 e-007 \vee 7.1706 e+003$

$1.9380 \mathrm{e}-007 / 7.1706 \mathrm{e}+003$

$4.4000 e-003 / 1.6280 e+008$

$1.3900 e-001 / 5.1430 e+009$

$1.8800 e-0046.9560 e+006$

$4.1500 e-006 / 1.5355 e+005$

$1.9650 e-006 / 7.2705 e+004$

$6.1500 e-007 \checkmark 2.2755 e+004$

$1.3900 e-001 \checkmark 5.1430 e+009$ $\mu \mathrm{Ci} / \mathrm{cm}^{3} \ldots$

$2.9639 e-002$

$1.6225 e+004$

$6.6392 e-011$

$6.9238 e-004$

5.9437 e+000

$3.2659 e-001$

$1.7151 \mathrm{e}+004$

$4.3566 e-004$

$6.6392 e-011$

$1.7784 e+000$

$9.5636 e-002$

$2.9956 e-002$

$6.1808 e-001$

$4.4578 e-005$

$6.1271 e-007$

$6.1271 e-007$

$1.3911 \mathrm{e}-002$

$4.3945 e-001$

$5.9437 e-004$

$1.3120 e-005$

$6.2124 e-006$

$1.9443 e-006$

$4.3945 e-001$
$\mathrm{Bg} / \mathrm{cm}^{3}$

$1.0967 e+003$

$6.0032 e+008$

$2.4565 e-006$

$2.5618 e+001$

$2.1992 e+005$

$1.2084 \mathrm{e}+004$

$6.3460 e+008$

$1.6119 e+001$

$2.4565 e-006$

$6.5799 e+004$

$3.5385 e+003$

$1.1084 \mathrm{e}+003$

$2.2869 e+004$

$1.6494 e+000$

$2.2670 e-002$

$2.2670 e-002$

$5.1470 e+002$

$1.6260 e+004$

$2.1992 e+001$

$4.8545 e-001$

$2.2986 e-001$

$7.1941 e-002$

$1.6260 e+004$ 


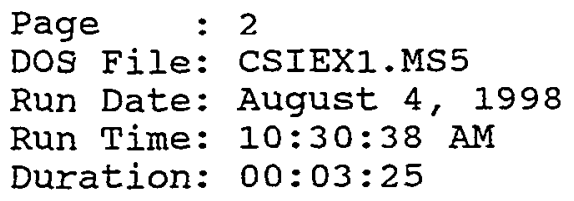

\section{Buildup \\ The material reference is : Source}

Radial

Integration Parameters
Circumferential

$Y$ Direction (axial)
29

29

31

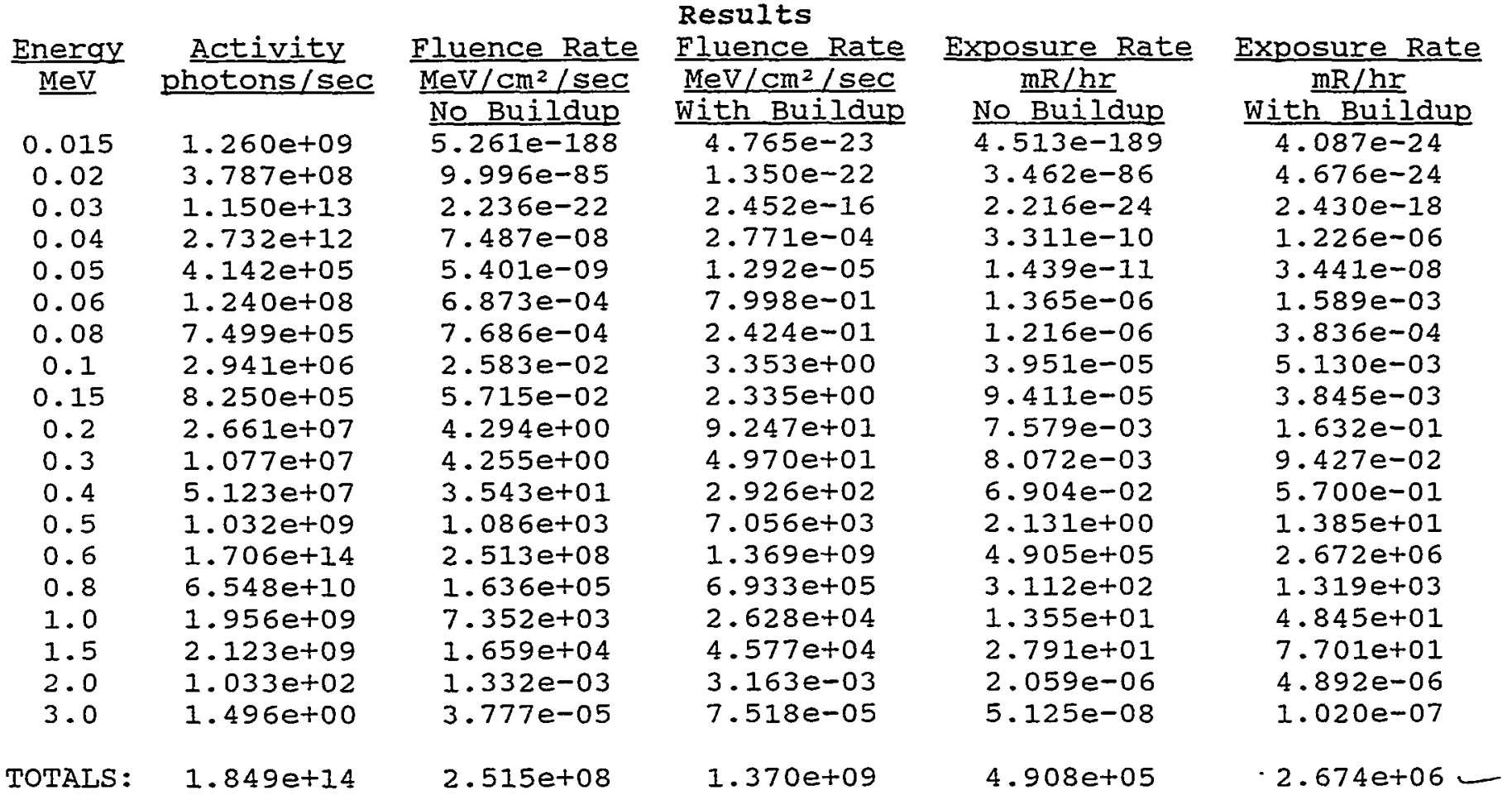


Microshield v5.01 (5.01-00121)

Lockheed Martin Idaho Technologies Company

Page : 1

DOS File: WM181106.MS5

Run Date: August 5, 1998

Run Time: 5:54:40 PM

Duration: 00:03:55

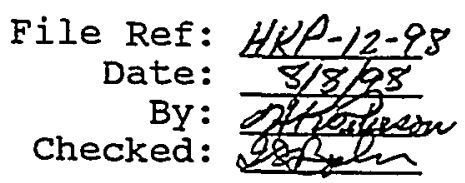

Case Title: Cs IX Effluent tnk

Description: Cs IX Effluent tank (T-103 A\&B) W/14234 I st \#106 Geometry: 7 - Cylinder volume - side shields

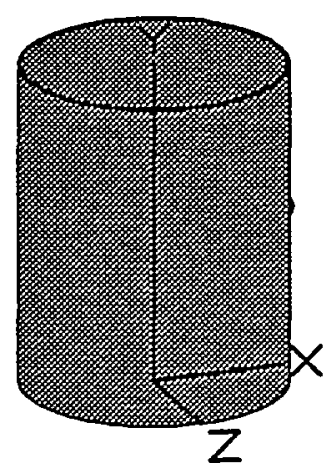

$\begin{array}{lrl} & \text { Source Dimensions } & \\ \text { Height } & 304.8 \mathrm{~cm} & 10 \mathrm{ft} 0.0 \mathrm{in} \text { - } \\ \text { Radius } & 121.92 \mathrm{~cm} & 4 \mathrm{ft} .\end{array}$

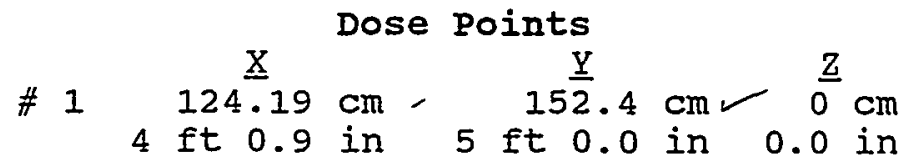

shields

Shield Name Dimension Material Density

$\begin{array}{lccl}\text { Source } & 1.42 e+07 \mathrm{~cm}^{3} & \text { Water } & 1 \\ \text { Shield 1 } & 1.27 \mathrm{~cm} \text { - Iron } & 7.86 \\ \text { Transition } & 1.0 \mathrm{~cm} \text { Air } & 0.0011^{\prime} \text { - } \\ \text { Air Gap } & & \text { Air } & 0.00122,\end{array}$

Source Input

Grouping Method : Standard Indices

Number of Groups : 25

Lower Energy Cutoff : 0.015

Photons < 0.015 : Excluded

Library : ICRP-38

\begin{tabular}{|c|c|c|c|}
\hline Juclide & curies & becquerels & $\mu \mathrm{Ci} / \mathrm{cm}^{3}$ \\
\hline$A m-241$ & $5.2660 e+000$ & $1.9484 e+011$ & $3.6997 e-001$ \\
\hline$A m-243$ & $.3900 e-003$ & $8.8430 e+007$ & $2128 e+000$ \\
\hline $\mathrm{Ba}-137 \mathrm{~m}$ & $5.6800 e-001$ & $2.1016 e+010$ & $3.9906 e-002$ \\
\hline $\mathrm{Ce}-144$ & $1.2350 e+000$ & $4.5695 e+010$ & \\
\hline$c m-242$ & $e-002$ & $4.6139 e+008$ & \\
\hline $\mathrm{Cm}-244$ & $1.6370 e-001$ & $6.0569 e+009$ & $1.1501 e-002$ \\
\hline Co- 60 & $1.8080 e-003$ & $16 e+007$ & $1.2702 e-004$ \\
\hline Cs -134 & $780 e-004$ & $7.6886 e+006$ & $1.4599 e-005$ \\
\hline Cs -135 & $1.1460 e-005$ & $4.2402 e+005$ & $8.0514 e-007$ \\
\hline Cs -137 & $6.0000 e-001$ & 2.22 & $4.2154 e-002$ \\
\hline & De-002 & & $1.6169 e+002$ \\
\hline$E u-154$ & Detooo & $1.2747 \mathrm{e}$ & $2.4203 e-001$ \\
\hline$E u-155$ & $6.0500 e-001$ & $2.2385 e+010$ & $4.2505 e-002$ \\
\hline $\mathrm{H}-3$ & $3.0890 e-001$ & $1.1429 \mathrm{e}$ & $2.1702 e-002$ \\
\hline & -003 & & 2.2088 \\
\hline $\mathrm{Ni}$ & De+00o & 5.5315 & $1.0503 e-001$ \\
\hline $\mathrm{Np}-237$ & $4.9680 e-003$ & $1.8382 e+008$ & $3.4903 e-004$ \\
\hline$P m-147$ & $1.4800 e+000$ & $5.4760 e+010$ & $1.0398 e-001$ \\
\hline Pr- & $e+000$ & 4.56 & $8.6767 e-002$ \\
\hline$P u-238$ & $1.4660 e+001$ & $5.4242 e+011$ & $1.0300 e+000$ \\
\hline $\mathrm{Pu}-239$ & $3.3450 e-001$ & $1.2377 e+010$ & $2.3501 e-002$ \\
\hline $\mathrm{Pu}-240$ & $9.3900 e-002$ & $3.4743 e+009$ & $6.5971 e-003$ \\
\hline$P u-241$ & $3840 e+000$ & $1 e+011$ & $3.0800 e-001$ \\
\hline & $200 e-004$ & $8.2140 e+006$ & $1.5597 e-005$ \\
\hline & & $3 e+006$ & $1.000<e-0 u=$ \\
\hline
\end{tabular}


Page : 2

DOS File: WM181106.MS5

Run Date: August 5, 1998

Run Time: 5:54:40 PM

Duration: 00:03:55

$\begin{array}{lc}\text { Nuclide } & \text { curies } \\ \text { Ru-106 } & 1.5090 e-004 \\ \mathrm{Sb}-125 & 1.8930 \mathrm{e}-001 \\ \mathrm{Sm}-151 & 1.0310 \mathrm{e}+001 \\ \mathrm{Sr}-90 & 5.7220 \mathrm{e}+002 \\ \mathrm{~T}-99 & 3.1880 \mathrm{e}-001 \\ \mathrm{Th}-230 & 1.3300 \mathrm{e}-006 \\ \mathrm{Th}-231 & 1.9070 \mathrm{e}-004 \\ \mathrm{U}-232 & 4.3270 \mathrm{e}-005 \\ \mathrm{U}-233 & 2.0350 \mathrm{e}-008 \\ \mathrm{U}-234 & 2.1900 \mathrm{e}-002 \\ \mathrm{U}-235 & 5.5080 \mathrm{e}-004 \\ \mathrm{U}-236 & 1.9500 \mathrm{e}-003 \\ \mathrm{U}-238 & 5.4370 \mathrm{e}-004 \\ \mathrm{Y}-90 & 5.7220 \mathrm{e}+002\end{array}$

becquerels

$5.5833 e+006$

$7.0041 e+009$

$3.8147 e+011$

$2.1171 e+013$

$1.1796 e+010$

$4.9210 e+004$

$7.0559 e+006$

$1.6010 e+006$

$7.5295 e+002$

$8.1030 e+008$

$2.0380 e+007$

$7.2150 e+007$

$2.0117 e+007$

$2.1171 e+013$ $\frac{\mu \mathrm{Ci} / \mathrm{cm}^{3}}{1.0602 e-005 / 3.9226 e-001}$
$1.3300 e-002 / 4.9208 \mathrm{Bg} / \mathrm{cm}^{3}$
$7.2434 \mathrm{e}-001 / 2.6801 \mathrm{e}+004$
$4.0201 \mathrm{e}+001 / 1.4874 \mathrm{e}+006$
$2.2398 \mathrm{e}-002 / 8.2872 \mathrm{e}+002$
$9.3441 \mathrm{e}-008 / 3.4573 \mathrm{e}-003$
$1.3398 \mathrm{e}-005 / 4.9572 \mathrm{e}-001$
$3.0400 \mathrm{e}-006 / 1.1248 \mathrm{e}-001$
$1.4297 \mathrm{e}-009 / 5.2899 \mathrm{e}-005$
$1.5386 \mathrm{e}-003 / 5.6929 \mathrm{e}+001$
$3.8697 \mathrm{e}-005 / 1.4318 \mathrm{e}+000$
$1.3700 \mathrm{e}-004 / 5.0690 \mathrm{~s}+000$
$3.8198 \mathrm{e}-005 / 1.4133 \mathrm{e}+000$
$4.0201 \mathrm{e}+001 / 1.4874 \mathrm{e}+006$

Buildup

The material reference is : source

Radial

\section{Integration parameters}

Circumferential

$y$ Direction (axial)
32

32

31

MeV

$\begin{array}{ll}0.015 & 5.081 e+10 \\ 0.02 & 6.510 e+10 \\ 0.03 & 9.947 e+09 \\ 0.04 & 3.960 e+10 \\ 0.05 & 8.631 e+09 \\ 0.06 & 6.994 e+10 \\ 0.08 & 7.997 e+09 \\ 0.1 & 5.719 e+10 \\ 0.15 & 5.134 e+09 \\ 0.2 & 9.463 e+09 \\ 0.3 & 9.360 e+08 \\ 0.4 & 3.378 e+09 \\ 0.5 & 1.313 e+09 \\ 0.6 & 3.036 e+10 \\ 0.8 & 4.841 e+10 \\ 1.0 & 4.069 e+10 \\ 1.5 & 5.066 e+10 \\ 2.0 & 3.561 e+08 \\ 3.0 & 9.562 e+04\end{array}$

TOTALS: $\quad 4.999 e+11$
Fluence Rate $\mathrm{MeV} / \mathrm{cm}^{2} / \mathrm{sec}$ With Buildup

$7.027 e-23$

$2.727 e-22$

$9.026 e-22$

$1.121 \mathrm{e}-12$

$1.205 e-05$

$2.327 e-01$

$1.041 e+01$

$5.690 e+02$

2. $375 e+02$

$7.311 e+02$

$1.153 e+02$

$5.706 e+02$

2. 841 e+02

$8.116 e+03$

$1.835 e+04$

$2.060 e+04$

4.461 et 04

$4.718 e+02$

$2.242 e-01$

$9.467 e+04$
Exposure Rate $\mathrm{mR} / \mathrm{hr}$

No Buildup

$5.594 e-253$

$7.218 e-114$

$8.308 e-38$

$2.163 e-17$

$9.441 \mathrm{e}-11$

1. $600 e-06$

$1.197 e-04$

1. $204 \mathrm{e}-02$

$1.400 e-02$

7.324 e-02

$2.103 e-02$

1. $434 \mathrm{e}-01$

$8.824 e-02$

2. $917 e+00$

8. $002 e+00$

$1.012 e+01$

2. $539 e+01$

2. $829 e-01$

1.390e-04

$4.707 e+01$
Exposure Rate $\mathrm{mR} / \mathrm{hr}$

With Buildup

$6.027 e-24$

$9.447 e-24$

$8.945 e-24$

$4.958 e-15$

3. $211 \mathrm{e}-08$

$4.623 e-04$

1. $647 \mathrm{e}-02$

8.705e-01

3. $912 e-01$

$1.290 \mathrm{e}+00$

2. 187 e-01

1. $112 \mathrm{e}+00$

$5.577 e-01$

1. $584 \mathrm{e}+01$

$3.491 e+01$

$3.797 e+01$

$7.506 e+01$

$7.296 e-01$

$3.041 e-04$

I. $690 \mathrm{e}+02 \checkmark$ 
Microshield v5.01 (5.01-00121)

Page : 1

DOS File: WM181SRT.MS5

Run Date: August 11, 1998

Run Time: 5:30:29 PM

Duration: $00: 04: 14$

File Ref:

Date:

By:

Checked:

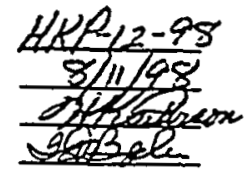

Case Title: T-112 spent Resin Tk

Description: T-112 Spent Resin Storage Tank with $21,350 \mathrm{~kg}$ of st \#108 Geometry: 7 - Cylinder volume - side shields

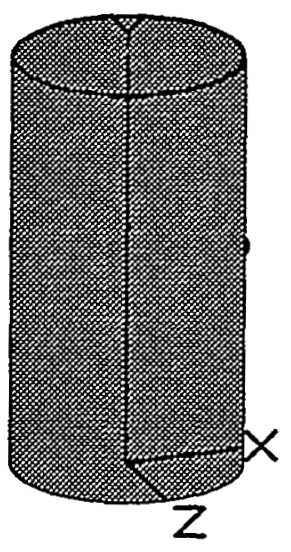

$$
\text { Source Input }
$$

Grouping Method : standard Indices Number of Groups : 25 Lower Energy Cutoff : 0.015 Photons < 0.015 : Excluded Library : ICRP-38

Nuclide Am-241 $\mathrm{Ba}-137 \mathrm{~m}$ $\mathrm{Ce}-144$ Co-60 Cs -134 Cs -135 Cs -137 $\mathrm{Np}-237$ Pr-144 Pu-238 Pu-239 $\mathrm{Pu}-240$ Pu-241 $\mathrm{Pu}-242$ Rh-106 Ru-106 $\mathrm{Sb}-125$ $\mathrm{Sr}-90$ $\mathrm{U}-234$ $\mathrm{U}-235$ $\mathrm{U}-236$ $\mathrm{U}-238$ $\mathrm{Y}-90$ becquerels

curies

8.0100e-001

$4.3830 e+005$

$1.7900 e-009$

$1.8700 e-002$

$1.6060 e+002$

$8.8200 e+000$

$4.6330 \mathrm{e}+005$

$1.1760 e-002$

$1.7900 e-009$

$4.8000 e+001$

$2.5800 e+000$

$8.0900 e-001$

$1.6700 \mathrm{e}+001$

$1.2040 e-003$

$1.6500 e-005$

$1.6500 e-005$

$3.7600 e-001$

$1.1870 \mathrm{e}+001$

$1.6060 e-002$

$3.5400 e-004$

$1.6780 e-004$

$5.2500 e-005$

1.1870e+001
$2.9637 e+010$

$1.6217 e+016$

$6.6230 e+001$

$6.9190 e+008$

$5.9422 e+012$

$3.2634 e+011$

$1.7142 e+016$

$4.3512 e+008$

$6.6230 e+001$

$1.7760 e+012$

$9.5460 e+010$

$2.9933 e+010$

$6.1790 e+011$

$4.4548 \mathrm{e}+007$

$6.1050 e+005$

$6.1050 e+005$

$1.3912 e+010$

4.3919e+011

$5.9422 e+008$

$1.3098 e+007$

$6.2086 e+006$

$1.9425 e+006$

$4.3919 e+011$ $\mu \mathrm{Ci} / \mathrm{cm}^{3}$

$3.7517 e-002$

$2.0529 e+004$

$8.3839 e-011$

$8.7586 e-004$

$7.5221 e+000$

$4.1311 e-001$

$2.1700 e+004$

$5.5081 e-004$

$8.3839 e-011$

$2.2482 e+000$

$1.2084 e-001$

$3.7892 e-002$

$7.8219 e-001$

$5.6392 e-005$

$7.7282 e-007$

$7.7282 e-007$

$1.7611 e-002$

$5.5596 e-001$

$7.5221 e-004$

$1.6580 e-005$

$7.8593 e-006$

$2.4590 e-006$

$5.5596 e-001$
$\mathrm{Bg} / \mathrm{cm}^{3}$

$1.3881 e+003$

7.5957 e+008

$3.1020 e-006$

$3.2407 e+001$

$2.783 .2 e+005$

$1.5285 \mathrm{e}+004$

$8.0289 e+008$

$2.0380 e+001$

$3.1020 e-006$

$8.3183 e+004$

$4.4711 e+003$

$1.4020 e+003$

$2.8941 e+004$

$2.0865 e+000$

$2.8594 \mathrm{e}-002$

$2.8594 e-002$

$6.5160 \mathrm{e}+002$

$2.0571 e+004$

$2.7832 \mathrm{e}+001$

$6.1348 e-001$

$2.9080 e-001$

$9.0982 e-002$

$2.0571 e+004$
$15 \mathrm{ft}$

$4 \mathrm{ft}$ 
Page : 2

DOS File: WM181SRT.MS5

Run Date: August 11, 1998

Run Time: 5:30:29 PM

Duration: $00: 04: 14$

Buildup

The material reference is : source

Integration Parameters

Radial 31

Circumferential

31

$Y$ Direction (axial)

33

Results

Fluence Rate

Fluence photons/sec

015

$\mathrm{MeV} / \mathrm{cm}^{2} / \mathrm{sec}$ No Buildup

0.015

$1.075 e+11$

0.02

0.03

3. $233 e+10$

$2.410 e-260$

1. $220 \mathrm{e}-116$

$4.379 e-32$

0.04

$9.821 e+14$

0.05

$2.333 e+14$

0.06

$3.534 e+07$

0.08

1. 060 e+10

0.1

$6.401 e+07$

0.15

0.2

2. 510 e+08

$7.045 e+07$

0.3

2.274 e+09

0.4

$9.200 e+08$

0.5

$4.378 e+09$

0.6

$8.819 e+10$

0.8

$1.457 e+16$

$5.593 e+12$

1. 0

1.5

$1.671 \mathrm{e}+11$

$1.813 e+11$

2. 0

8. 818 et 03

3.0

1.274 e+02

TOTALS: $\quad 1.579 e+16$
$6.559 e-12$

5. $317 e-11$

5. $417 e-05$

$3.012 e-04$

$1.740 e-02$

$5.825 e-02$

$4.955 e+00$

$5.398 e+00$

$4.712 e+01$

1. $490 e+03$

$3.534 e+08$

$2.393 e+05$

1. $109 e+04$

2. $656 e+04$

$2.224 e-03$

$6.682 e-05$

$3.537 e+08$

\section{$\mathrm{MeV} / \mathrm{cm}^{2} / \mathrm{sec}$}

With Buildup

1.655e-21

1. $153 e-20$

2. $991 e-14$

$6.443 e-07$

1. $520 \mathrm{e}-06$

4. $369 e-01$

3. $425 e-01$

6.084 et 00

5.097 et00

2. $026 e+02$

1. $052 \mathrm{e}+02$

$6.105 e+02$

1. $437 \mathrm{e}+04$

2. $742 e+09$

1. $383 e+06$

$5.216 e+04$

9. $187 e+04$

$6.480 e-03$

1. 590 e-04

$2.744 e+09$
Exposure Rate $\mathrm{mR} / \mathrm{hr}$

No Buildup

2.068e-26I

$4.225 e-118$

$4.340 e-34$

2. $901 e-14$

$1.417 e-13$

$1.076 e-07$

$4.766 e-07$

2. $662 e-05$

$9.592 e-05$

8.745e-03

$1.024 e-02$

9. 182 e-02

2. $925 e+00$

$6.898 e+05$

$4.552 e+02$

2. $044 e+01$

$4.468 e+01$

3. $439 e-06$

$9.065 e-08$

$6.903 e+05$
Exposure Rate $\mathrm{mR} / \mathrm{hr}$ with Buildup

1. $419 \mathrm{e}-22$

3. $993 e-22$

$2.964 \mathrm{e}-16$

2. 850e-09

4.050e-09

8. $679 e-04$

5. $420 e-04$

$9.308 e-03$

8. 394e-03

3. 576e-01

1. 995 e-01

1. $189 e+00$

2. $821 e+01$

$5.353 e+06$

$2.630 e+03$

9. $614 e+01$

1. $546 e+02$

$1.002 e-05$

$2.157 e-07$

$5.356 e+06$ 
Microshield v5.01 (5.01-00121)

Lockheed Martin Idaho Technologies Company

Page : 1

DOS File: WM18116A.MS5

Run Date: August 4, 1998

Run Time: 11:18:10 AM

Duration: 00:01:35

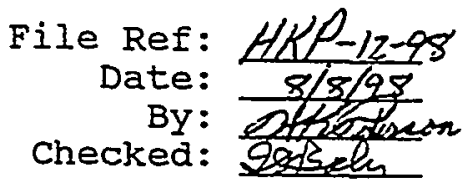

Case Title: M-101 Mixer

Description: M-101 Mixer (1/2 cyl) with 133.4 I of stream \#106 Geometry: 7 - cylinder volume - side shields

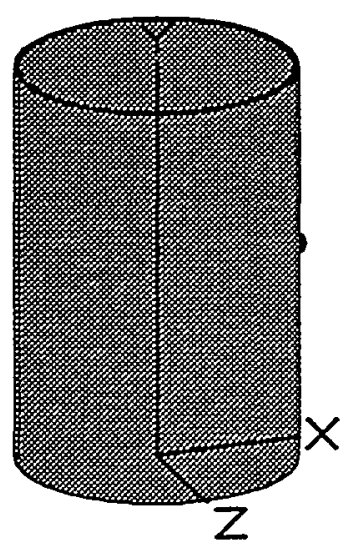

\begin{tabular}{lll}
\multicolumn{5}{c}{ Source Dimensions } \\
Height & $91.44 \mathrm{~cm}$ & $3 \mathrm{ft}-$ \\
Radius & $30.48 \mathrm{~cm}$ & $1 \mathrm{ft}-$
\end{tabular}

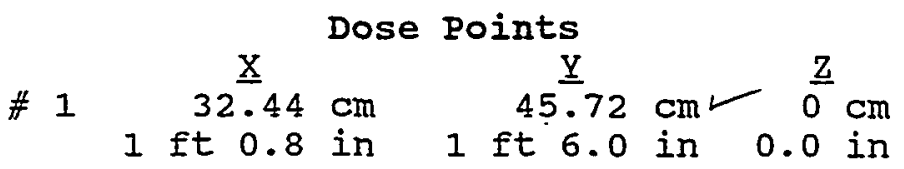

Shields

Shield Name Dimension Material Density

Source $\quad 2.67 e+05 \mathrm{~cm}^{3}$ Mixed $\rightarrow 1.27$

Concrete 0.27

Shield 1

Transition

Water 1

Air Gap

$.953 \mathrm{~cm}$

Iron 7.86

$1.0 \mathrm{~cm}$

Air

0.0011

Source Input

Grouping Method : standard Indices

Number of Groups : 25

Lower Energy Cutoff : 0.015

Photons < 0.015 : Excluded

\section{Library : ICRP-38}

\begin{tabular}{|c|c|c|c|}
\hline Nuclide & becquerels & $\mu \mathrm{Ci} / \mathrm{cm}^{3}$ & $\mathrm{Bg} / \mathrm{cm}^{3}$ \\
\hline$A m-241$ & L.8267e+009 & $1.8499 e-001$ & $6.8446 e+003$ \\
\hline$A m-243$ & $2.2400 e-005$ & $8.3933 e-005$ & $3.1055 e+000$ \\
\hline$a-137$ & $e+008$ & $1.9960 e-002$ & +002 \\
\hline $\mathrm{Ce}-144$ & $2846 e+008$ & $4.3390 e-002$ & $1.6054 \mathrm{e}+003$ \\
\hline $\mathrm{Cm}-242$ & $1.1690 e-004$ & $2 e-004$ & $1.6207 e+001$ \\
\hline $\mathrm{Cm}-244$ & $1.5340 e-003$ & $9 e-003$ & $2.1267 e+002$ \\
\hline Co-60 & 005 & -005 & $2.3430 e+000$ \\
\hline$C s-134$ & 004 & -006 & 2.70 \\
\hline$c s-135$ & 003 & 007 & $e-002$ \\
\hline $\mathrm{Cs}-1$ & 008 & 2.3 & $e+002$ \\
\hline Eu-: & $e-00$ & 2.1 & $2+001$ \\
\hline$E u-154$ & $e-002$ & -001 & $7 e+003$ \\
\hline $\mathrm{Eu}-155$ & $e-00$ & 2.1 & $e+002$ \\
\hline $\mathrm{H}-\mathrm{S}$ & & 1. & +002 \\
\hline & De-005 & 2.9 & $1.1045 \mathrm{e}+001$ \\
\hline $\mathrm{Ni}-63$ & $1.4000 e-002 U$ & $5.2458 e-002$ & $1.9409 e+003$ \\
\hline$N p-237$ & $.6570 e-005$ & 1.7 & $6.4564 e+000$ \\
\hline$P m-147$ & $5.1356 e+008$ & 5.2 & $1.9243 e+003$ \\
\hline $\operatorname{Pr}-144$ & $.1580 e-002$ & 4.3 & $4 e+003$ \\
\hline $\mathrm{Pu}-238$ & $1.3700 e-001$ & 5.1 & $1.8994 \mathrm{e}+004$ \\
\hline $\mathrm{Pu}-239$ & $3.1360 e-003$ & $1.1751 e-002$ & $4.3477 e+002$ \\
\hline & $.8100 e-004 \sqrt{3.2597 e+007}$ & $3.3011 e-003$ & $1.2214 e+002$ \\
\hline $\mathrm{Pu}-241$ & $4.1100 e-002 v$ & $1.5400 e-001$ & $5.6981 e+003$ \\
\hline
\end{tabular}


Page : 2

DóS File: WM18116A.MS5

Run Date: August 4, 1998

Run Time: 11:18:10 AM

Duration: 00:01:35

\begin{tabular}{|c|c|c|c|}
\hline Nuclide & curies & $\mu \mathrm{Ci} / \mathrm{cm}^{3}$ & $\mathrm{Bg} / \mathrm{cm}^{3}$ \\
\hline$P u-242$ & $2.0800 e-00$ & $7.7938 e-006$ & $2.8837 e-00$ \\
\hline-106 & $18 e+004$ & $5.2983 e-006$ & \\
\hline $\mathrm{Ru}-106$ & $140 e-006$ & $5.2983 e-006$ & $9604 e-001$ \\
\hline $\mathrm{Sb}-125$ & $1.7700 e-003$ & $6.6322 e-003$ & $4539 e+002$ \\
\hline 51 & $e-002$ & 3.6196 & +004 \\
\hline $\operatorname{Sr}-90$ & +011 & $2.0084 e+001$ & $7.4311 e+005$ \\
\hline TC. & De-003 & 1.1204 & +002 \\
\hline & 02 & 4.68 & $5 e-003$ \\
\hline Th- & $e-006 \mathrm{r}$ & -006 & $9 e-001$ \\
\hline$U-232$ & $4.0600 e-007$ & $1.5213 e-006$ & $5.6287 e-002$ \\
\hline$U-233$ & $9100 e-010$ & $7.1568 e-010$ & $2.6480 e-005$ \\
\hline & & 7.68 & $21 e+001$ \\
\hline & 5005 & 1.93356 & $7.1538 \mathrm{e}-001$ \\
\hline$U-2$ & $.8280 e-005 / 6.7636 e+005$ & $6.8495 e-005$ & $2.5343 e+000$ \\
\hline$U-238$ & $5.1000 e-006$ & $1.9110 e-005$ & $0706 e-001$ \\
\hline$Y-90$ & $5.3600 e+000$ & $2.0084 e+001$ & $7.4311 e+005$ \\
\hline
\end{tabular}

Buildup

The material reference is : source

Integration Parameters

Radial

Circumferential

23

$Y$ Direction (axial)

23

\begin{tabular}{|c|c|}
\hline Energy & Activity \\
\hline MeV & photons/sec \\
\hline 0.015 & $4.754 e+08$ \\
\hline 0.02 & $6.101 e+08$ \\
\hline 0.03 & $9.318 e+07$ \\
\hline 0.04 & $3.712 e+08$ \\
\hline 0.05 & $8.090 e+07$ \\
\hline 0.06 & $6.557 e+08$ \\
\hline 0.08 & $7.496 e+07$ \\
\hline 0.1 & $5.360 e+08$ \\
\hline 0.15 & $4.814 e+07$ \\
\hline 0.2 & $8.868 e+07$ \\
\hline 0.3 & $8.772 e+06$ \\
\hline 0.4 & $3.161 e+07$ \\
\hline 0.5 & $1.229 e+07$ \\
\hline 0.6 & $2.846 e+08$ \\
\hline 0.8 & $4.538 e+08$ \\
\hline 1.0 & $3.814 e+08$ \\
\hline 1.5 & $4.748 e+08$ \\
\hline 2.0 & $3.339 e+06$ \\
\hline 3.0 & $8.966 e+02$ \\
\hline
\end{tabular}

TOTALS: $\quad 4.685 e+09$
Fluence Rate $\mathrm{MeV} / \mathrm{cm}^{2} / \mathrm{sec}$ No Buildup $6.068 e-190$

$6.616 e-85$

$2.249 e-27$

$1.401 \mathrm{e}-11$

$1.494 \mathrm{e}-06$

$5.396 e-03$

$1.254 \mathrm{e}-01$

$8.050 e+00$

$5.899 e+00$

$2.552 e+01$

6.211 et 00

$3.930 e+01$

$2.326 e+01$

$7.553 e+02$

$2.044 e+03$

$2.585 e+03$

$6.683 e+03$

$7.731 \mathrm{e}+01$

$4.031 e-02$

$1.225 e+04$
Results

Fluence Rate $\mathrm{MeV} / \mathrm{cm}^{2} / \mathrm{sec}$ With Buildup

$2.118 e-22$

$7.182 e-21$

$9.912 \mathrm{e}-20$

$6.508 e-07$

2. $005 e-02$

$2.297 e+01$

$9.530 e+01$

$2.072 e+03$

$3.900 e+02$

$8.384 e+02$

$9.884 \mathrm{e}+01$

$4.245 e+02$

$1.872 \mathrm{e}+02$

$4.927 e+03$

$1.001 e+04$

$1.032 e+04$

$1.977 e+04$

$1.937 \mathrm{e}+02$

$8.248 e-02$

$4.936 e+04$
Exposure Rate $\mathrm{mR} / \mathrm{hr}$

No Buildup

$5.205 e-191$

$2.292 e-86$

$2.228 e-29$

$6.197 e-14$

$3.979 e-09$

$1.072 e-05$

$1.984 \mathrm{e}-04$

$1.232 e-02$

$9.714 e-03$

$4.504 e-02$

$1.178 e-02$

$7.657 e-02$

$4.565 e-02$

$1.474 \mathrm{e}+00$

$3.888 e+00$

$4.764 \mathrm{e}+00$

$1.124 e+01$

$1.196 e-01$

$5.468 e-05$

$2.169 e+01$
Exposure Rate $\mathrm{mR} / \mathrm{hr}$ With Buildup

$1.817 e-23$

$2.488 e-22$

$9.824 e-22$

$2.878 \mathrm{e}-09$

$5.341 \mathrm{e}-05$

$4.562 e-02$

$1.508 \mathrm{e}-01$

$3.170 e+00$

$6.422 e-01$

$1.480 \mathrm{e}+00$

$1.875 e-01$

8.271e-01

$3.674 \mathrm{e}-0 \mathrm{I}$

$9.617 \mathrm{e}+00$

$1.904 e+01$

$1.903 e+01$

$3.327 e+01$

2. $996 e-01$

$1.119 e-04$

$8.813 e+01$ 
Microshield v5.01 (5.01-00121)

Lockheed Martin Idaho Technologies Company

Page : 1

DOS File: WM181117.MS5

Run Date: August 4, 1998

Run Time: 11:28:52 AM

Duration: 00:02:17

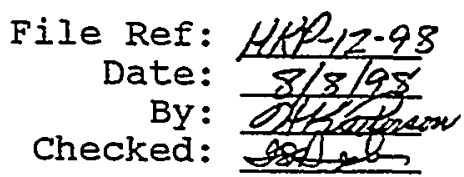

Case Title: M-101 Mixer

Description: M-101 Mixer (full cylinder) with $453.7 \mathrm{~kg}$ of stream \#117 Geometry: 7 - cylinder volume - Side shields

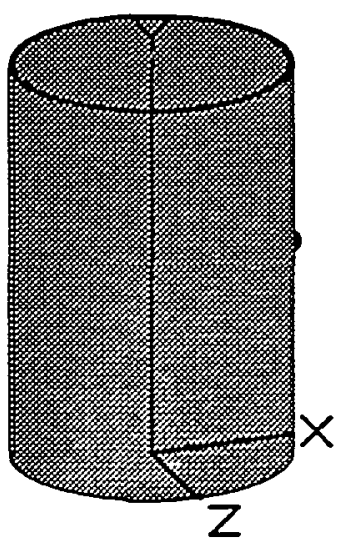

Source Input

Grouping Method : Standard Indices

Number of Groups : 25

Lower Energy Cutoff : 0.015

Photons < 0.015 : Excluded

\section{Library : ICRP-38}

\begin{tabular}{|c|c|}
\hline & \\
\hline & $1326 e+00$ \\
\hline & \\
\hline & \\
\hline & $1.3474 \mathrm{e}-002$ \\
\hline & $1.3613 e-004$ \\
\hline & \\
\hline & te \\
\hline & $9 e-006$ \\
\hline & \\
\hline & \\
\hline $\mathrm{su}$ & $3 e-004$ \\
\hline & $e-00$ \\
\hline & \\
\hline $\mathrm{H}-$ & 00 \\
\hline & \\
\hline & 1.63 \\
\hline & \\
\hline & 1.34 \\
\hline & $1 e-001 / 5.9$ \\
\hline & $3 e+008$ \\
\hline & $1.0<$ \\
\hline & 907 \\
\hline & \\
\hline
\end{tabular}

$2.1597 e-001$

$1.7845 e-001$
$\underline{\mathrm{Ba} / \mathrm{cm}^{3}}$ $9.7586 e-005$

$2.3306 e-002$

$5.0486 e-002$

$5.1006 e-004$

$6.6645 e-003$

$7.3783 e-005$

8. $5165 e-006$

$4.6749 e-007$

$2.4655 e-002$

$2.5334 e-003$

$1.4051 e-001$

$2.4646 e-002$

$1.2631 e-002$

$3.4679 e-004$

$6.1193 e-002$

$6.0519 e-002$

$5.0486 e-002$

$5.9844 e-001$

$1.3685 e-002$

$3.8416 e-003$

$9.0954 e-006$

$7.9908 \mathrm{e}+003$

$3.6107 e+000$

$8.6234 e+002$

$1.8680 e+003$

$1.8872 e+001$

$2.4659 e+002$

$2.7300 e+000$

$3.1511 e-001$

$1.7297 e-002$

$9.1225 e+002$

$9.3737 e+001$

$5.1990 e+003$

$9.1190 e+002$

$4.6734 \mathrm{e}+002$

$1.2831 \mathrm{e}+001$

$2.2641 e+003$

$2.2392 e+003$

$1.8680 e+003$

$2.2142 \mathrm{e}+004$

$5.0633 e+002$

$1.4214 \mathrm{e}+002$

$6.6027 e+003$
$3 \mathrm{ft}$

$1 \mathrm{ft}$

Dose Points

$45.72 \mathrm{~cm}$
ft $6.0 \mathrm{in}$
$\quad 0.0 \mathrm{~cm}$

shields

mension Material Density

$3.3653 e-001$ 
Page : 2

DOS File: WM181117.MS5

Run Date: August 4, 1998

Run Time: 11:28:52 AM

Duration: 00:02:17

Nuclide
Rh-106
Ru-106
$\mathrm{Sb}-125$
$\mathrm{Sm}-151$
$\mathrm{~S}-90$
$\mathrm{~T}-99$
$\mathrm{Th}-230$
$\mathrm{Th}-231$
$\mathrm{U}-232$
$\mathrm{U}-233$
$\mathrm{U}-234$
$\mathrm{U}-235$
$\mathrm{U}-236$
$\mathrm{U}-238$
$\mathrm{Y}-90$

$\frac{\text { curies }}{1.6515 e-006} \frac{\text { becquerels }}{6.1106 e+004}$
$1.6515 e-006$
$2.0644 e-003 / 7.6382 e+007$
$1.1250 e-001 / 4.1625 e+009$
$6.2625 e+000 / 2.3171 e+011$
$3.4845 e-003 / 1.2893 e+008$
$1.4520 e-008 / 5.3724 e+002$
$2.0824 e-006 / 7.7048 e+004$
$4.7175 e-007 / 7.7455 e+004$
$2.2185 e-010 / 8.2084 e+000$
$2.3955 e-004 / 8.8634 e+006$
$6.0338 e-006 / 2.2325 e+005$
$2.1233 e-005 / 7.8560 e+005$
$5.9438 e-006 / 2.1992 e+005$
$6.2625 e+000 / 2.3171 e+011$

$\mu \mathrm{Ci} / \mathrm{cm}^{3}$ $6.1882 e-006$

$6.1882 e-006$

$7.7352 e-003$

$4.2154 e-001$

$2.3466 e+001$

$1.3056 e-002$

$5.4406 e-008$

$7.8027 e-006$

$1.7676 e-006$

$8.3127 e-010$

$8.9759 e-004$

$2.2608 e-005$

$7.9558 e-005$

$2.2271 e-005$

$2.3466 e+001$
$\mathrm{Bg} / \mathrm{cm}^{3}$
$2.2896 e-001$
$2.2896 e-001$
$2.8620 e+002$
$1.5597 e+004$
$8.6823 e+005$
$4.8309 e+002$
$2.0130 e-003$
$2.8870 e-001$
$6.5403 e-002$
$3.0757 e-005$
$3.3211 e+001$
$8.3651 e-001$
$2.9437 e+000$
$8.2404 e-001$
$8.6823 e+005$

The material reference is : Source

\section{Integration Parameters}

Radial

Circumferential

$Y$ Direction (axial)
26

26

26

\begin{tabular}{|c|c|c|c|c|c|}
\hline \multirow{3}{*}{$\frac{\text { Energy }}{\underline{\text { MeV }}}$} & Activity & Fluence Rate & $\begin{array}{l}\text { Results } \\
\text { Fluence Rate }\end{array}$ & Exposure Rate & Exposure Rate \\
\hline & photons/sec & $\mathrm{MeV} / \mathrm{cm}^{2} / \mathrm{sec}$ & $\mathrm{MeV} / \mathrm{cm}^{2} / \mathrm{sec}$ & $\mathrm{mR} / \mathrm{hr}$ & $\mathrm{mR} / \mathrm{hr}$ \\
\hline & & No Buildup & With Buildup & No Buildup & With Buildup \\
\hline 0.015 & $5.540 e+08$ & $2.821 e-191$ & $8.103 e-24$ & $2.420 e-192$ & $6.950 e-25$ \\
\hline 0.02 & $7.120 e+08$ & $1.313 e-85$ & $2.579 e-23$ & $4.547 e-87$ & $8.935 e-25$ \\
\hline 0.03 & $1.084 e+08$ & $9.738 e-28$ & $3.654 e-23$ & $9.651 e-30$ & $3.621 e-25$ \\
\hline 0.04 & $4.312 e+08$ & $6.857 e-12$ & $4.270 e-10$ & $3.033 e-14$ & $1.888 e-12$ \\
\hline 0.05 & $9.393 e+07$ & $7.935 e-07$ & $7.654 e-05$ & $2.114 e-09$ & $2.039 e-07$ \\
\hline 0.06 & $7.655 e+08$ & $3.269 e-03$ & $3.186 e-01$ & $6.494 e-06$ & $6.328 e-04$ \\
\hline 0.08 & $8.673 e+07$ & $8.937 e-02$ & $5.510 e+00$ & $1.414 \mathrm{e}-04$ & $8.719 e-03$ \\
\hline 0.1 & $6.223 e+08$ & $6.184 e+00$ & $2.346 e+02$ & $9.460 e-03$ & $3.589 e-01$ \\
\hline 0.15 & $5.599 e+07$ & $4.800 e+00$ & $8.537 e+01$ & $7.905 e-03$ & $1.406 e-01$ \\
\hline 0.2 & $1.030 e+08$ & $2.105 e+01$ & $2.529 e+02$ & $3.715 e-02$ & $4.464 e-01$ \\
\hline 0.3 & $1.018 e+07$ & $5.168 e+00$ & $4.066 e+01$ & $9.803 e-03$ & $7.712 e-02$ \\
\hline 0.4 & $3.682 e+07$ & $3.291 e+01$ & $2.022 e+02$ & $6.413 e-02$ & $3.941 e-01$ \\
\hline 0.5 & $1.431 e+07$ & $1.952 e+01$ & $1.006 e+02$ & $3.831 e-02$ & $1.974 e-01$ \\
\hline 0.6 & $3.318 e+08$ & $6.360 e+02$ & $2.867 e+03$ & $1.241 e+00$ & $5.596 e+00$ \\
\hline 0.8 & $5.270 e+08$ & $1.722 \mathrm{e}+03$ & $6.398 e+03$ & $3.275 e+00$ & $1.217 e+01$ \\
\hline 1.0 & $4.429 e+08$ & $2.187 e+03$ & $7.109 e+03$ & $4.031 e+00$ & $1.310 e+01$ \\
\hline 1.5 & $5.514 e+08$ & $5.717 e+03$ & $1.493 e+04$ & $9.618 e+00$ & $2.512 e+01$ \\
\hline 2.0 & $3.885 e+06$ & $6.683 e+01$ & $1.542 e+02$ & $1.034 e-01$ & $2.384 e-01$ \\
\hline 3.0 & $1.043 e+03$ & $3.526 e-02$ & $6.966 e-02$ & $4.783 e-05$ & $9.451 e-05$ \\
\hline TOTALS: & $5.451 e+09$ & $1.042 e+04$ & $3.238 e+04$ & $1.844 e+01$ & $5.785 e+01-$ \\
\hline
\end{tabular}


Page : 1

DOS File: WD117.MS5

Run Date: August 4, 1998

Run Time: 11:36:46 AM

Duration: $00: 02: 14$
Microshield v5.01 (5.01-00121)

Lockheed Martin Idaho Technologies Company

\section{Case Title: Waste Drum \\ Description: Waste Drum with $239 \mathrm{~kg}$ of grouted waste from st \#117 Geometry: 7 - Cylinder volume - side shields}

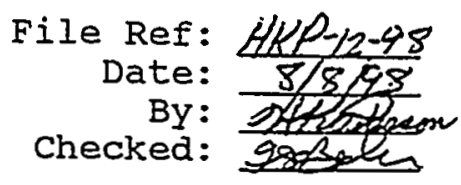

\begin{tabular}{|c|c|c|c|}
\hline \multirow[b]{2}{*}{ Nuclide } & \multicolumn{2}{|c|}{$\begin{array}{c}\text { Source Input } \\
\text { Grouping Method: Standard Indices } \\
\text { Number of Groups : } 25 \\
\text { Lower Energy Cutoff : } 0.015 \\
\text { Photons < } 0.015: \text { Excluded } \\
\text { Iibrary : ICRP-38 }\end{array}$} & \multirow[b]{2}{*}{$\mathrm{Bg} / \mathrm{cm}^{3}$} \\
\hline & curies becquerels & $\mu \mathrm{Ci} / \mathrm{cm}^{3}$ & \\
\hline$\overline{A m-24 I}$ & $3 . \overline{0300 e-002}$ & $1 . \overline{6185 e-001}$ & $5 . \overline{9886 e+0} 03$ \\
\hline$A m-243$ & $1.3700 e-005$ & $7.3182 e-005$ & $2.7077 e+000$ \\
\hline $7 \mathrm{~m}$ & $3.2780 e-003$ & $1.7510 e-002$ & $6.4788 e+002$ \\
\hline$e-1$ & $6270 e+008$ & $3.7926 e-002$ & $1.4033 \mathrm{e}+003$ \\
\hline$m-242$ & $.6529 e+006$ & $3.8300 e-004$ & $171 e+001$ \\
\hline$c m-2$ & $4658 e+007$ & $5.0036 e-003$ & ee+002 \\
\hline $50-6$ & $.8369 e+005$ & 5.539 & $e+000$ \\
\hline$s-$ & $4289 e+004$ & $6.3941 e-006$ & -001 \\
\hline$: s-13$ & $4309 e+003$ & $3.5095 e-007$ & $985 e-002$ \\
\hline Cs -137 & $2821 e+008$ & $1.8509 e-002$ & $8484 e+002$ \\
\hline $\mathrm{Eu}-152$ & $172 e+007$ & $1.9017 e-003$ & $e+001$ \\
\hline Eu- & $3112 e+008$ & 1.055 & +003 \\
\hline$E u-1$ & $2821 e+008$ & $1.8509 e-002$ & $e+002$ \\
\hline $\mathrm{H}-3$ & $5675 e+007$ & $9.4816 e-003$ & $3.5082 e+002$ \\
\hline$I-129$ & $4.8750 e-005$ & $2.6041 e-004$ & $9.6352 e+000$ \\
\hline & $1831 e+008$ & $4.5955 e-0$ & $3 e+003$ \\
\hline & $.0612 e+006$ & 1.532 & -000 \\
\hline & $.1476 e+008$ & $4.5442 e-002$ & $1.6814 \mathrm{e}+003$ \\
\hline Pr-144 & $7.0970 e-003$ & $3.7910 e-002$ & $1.4027 e+003$ \\
\hline & $3.1117 e+009$ & $4.4924 e-001$ & $1.6622 e+004$ \\
\hline & $7.1188 e+007$ & & $3.8027 e+002$ \\
\hline & $1.9980 e+007$ & $2.8845 e-003$ & $1.0673 e+002$ \\
\hline & $0 e-002 \sqrt{9.2833 e+008}$ & $1.3402 e-001$ & $e+003$ \\
\hline
\end{tabular}

Source Dimensions

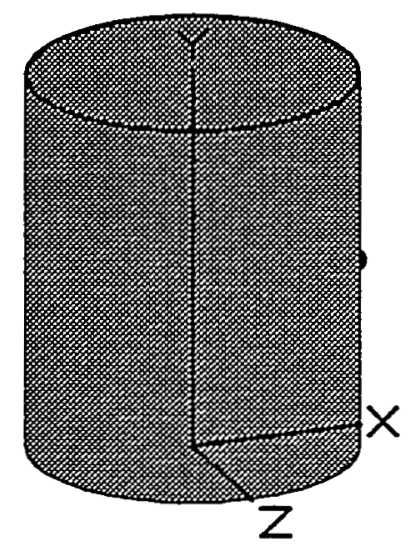

Shield 1 Transition Air Gap

\section{Dose Points} $\underline{Y}$ $31.04 \mathrm{~cm}$ 1 ft 0.2 in

Shields

Shield Name Dimension MaterialDensity Source $1.87 \mathrm{e}+05 \mathrm{~cm}^{3}$ Mixed $\rightarrow 1.9$ concrete 1.8 $.16 \mathrm{~cm}$ Iron 7.86 $1.0 \mathrm{~cm}$ Air 0.0011 Air 0.00122 
Page $:$ : 2

DOS Filé: WD117.MS5

Run Date: August 4, 1998

Run Time: 11:36:46 AM

Duration: 00:02:14

\begin{tabular}{|c|c|c|c|}
\hline Nuclide & becouerels & $\mu \mathrm{Ci} / \mathrm{cm}^{3}$ & $\mathrm{Bg} / \mathrm{cm}^{3}$ \\
\hline$\overline{P u-242}$ & $1.2 \overline{2780 e-006} \sqrt{4.7286 e+004}$ & $6 . \overline{8267 e-006}$ & $2 . \overline{5259 e-001}$ \\
\hline $\mathrm{Rh}-106$ & $8.6980 e-007 / 3.2183 e+004$ & $4.6462 e-006$ & 1.719 le-00I \\
\hline Ru-106 & $8.6980 e-007 \checkmark 3.2183 e+004$ & $4.6462 e-006$ & $1.7191 \mathrm{e}-001$ \\
\hline$S b-125$ & $1.0870 e-003 / 4.0219 e+007$ & $5.8065 e-003$ & $2.1484 \mathrm{e}+002$ \\
\hline$S m-151$ & $5.9260 e-002,-2.1926 e+009$ & $3.1655 e-001$ & $1.1712 e+004$ \\
\hline $\mathrm{Sr}-90$ & $3.2980 e+0001.2203 e+011$ & $1.7617 e+001$ & $6.5183 e+005$ \\
\hline TC-99 & $1.8350 e-003 / 6.7895 e+007$ & $9.8021 e-003$ & $3.6268 e+002$ \\
\hline Th -230 & $7.6470 e-009 / 2.8294 e+002$ & $4.0848 e-008$ & $1.5114 \mathrm{e}-003$ \\
\hline $\mathrm{Th}-231$ & $1.0970 e-006 / 4.0589 e+004$ & $5.8599 e-006$ & $2.1682 e-001$ \\
\hline $\mathrm{U}-232$ & $2.4850 e-007 / g .1945 e+003$ & $1.3274 e-006$ & $4.9115 e-002$ \\
\hline $\mathrm{U}-233$ & $1.1690 e-010 / 4.3253 e+000$ & $6.2445 e-010$ & $2.3105 e-005$ \\
\hline $\mathrm{U}-234$ & $1.2620 e-004 / 4.6694 e+006$ & $6.7413 e-004$ & $2.4943 e+001$ \\
\hline $\mathrm{U}-235$ & $3.1780 e-006$ & $1.6976 e-005$ & $6.2811 e-001$ \\
\hline $\mathrm{U}-236$ & $1.1180 e-005 \checkmark 4.1366 e+005$ & $5.9721 e-005$ & $2.2097 e+000$ \\
\hline$U-238$ & $3.1300 e-006 / 1.1581 e+005$ & $1.6720 e^{-005}$ & $6.1863 e-001$ \\
\hline$Y-90$ & $3.2980 e+0001.2203 e+011$ & $1.7617 \mathrm{e}+001$ & $6.5183 e+005$ \\
\hline
\end{tabular}

\section{Integration Parameters}

Radial

Circumferential

$Y$ Direction (axial)
26
26
26

Results

Energy

Activity

Eluence Rate

Fluence Rate

$\underline{\mathrm{MeV}}$

0.015 photons/sec

\begin{tabular}{l}
\hline $\mathrm{MeV} / \mathrm{cm}^{2} / \mathrm{sec}$ \\
\hline No Buildup \\
\hline $1.648 \mathrm{e}-34$ \\
$1.386 e-15$ \\
$1.041 e-05$ \\
$4.695 e-02$ \\
$2.267 e-01$ \\
$9.636 e+00$ \\
$5.754 e+00$ \\
$9.248 e+01$ \\
$2.178 e+01$ \\
$6.661 e+01$ \\
$1.246 e+01$ \\
$6.979 e+01$ \\
$3.804 e+01$ \\
$1.164 e+03$ \\
$2.869 e+03$ \\
$3.400 e+03$ \\
$7.914 e+03$ \\
$8.630 e+01$ \\
$4.210 e-02$
\end{tabular}

$\mathrm{MeV} / \mathrm{cm}^{2} / \mathrm{sec}$

With Buildup

2. $918 e+08$

0.02

$3.744 \mathrm{e}+08$

0.03

0.04

0.05

0.06

0.08

0.1

0.15

0.2

0.3

0.4

0.5

0.6

0.8

1.0

1. 5

2.0

3.0

$5.721 e+07$

2. 272 et08

$4.949 e+07$

$4.024 e+08$

$4.583 e+07$

3. 280 e+08

2. $951 \mathrm{e}+07$

$5.428 e+07$

5. $361 e+06$

1. $939 e+07$

$7.535 e+06$

1. $748 \mathrm{e}+08$

2. 777 e+08

2. 334 e+08

2. $906 e+08$

2. $046 e+06$

$5.495 e+02$

TOTALS: $2.871 e+09$
$3.412 \mathrm{e}-15$

$5.337 e-05$

$4.095 e-01$

2. 571 et 00

1. $149 e+02$

$6.014 \mathrm{e}+01$

8.122e+02

1. $399 e+02$

3. $474 \mathrm{e}+02$

5. $094 \mathrm{e}+01$

2. $451 e+02$

1. $194 \mathrm{e}+02$

$3.353 e+03$

7.272 et03

7.900 et03

1. $586 \mathrm{e}+04$

1. $586 \mathrm{e}+02$

$6.929 e-02$

$1.575 e+04$

$3.643 e+04$
$5.269 e-24$

Exposure Rate $\mathrm{mR} / \mathrm{hr}$

No Buildup

$1.414 \mathrm{e}-35$

$4.802 e-17$

$1.031 e-07$

2. $076 e-04$

$6.038 e-04$

$1.914 e-02$

9.105e-03

1. $415 \mathrm{e}-01$

3. 587 e-02

$1.176 \mathrm{e}-01$

2. $364 e-02$

1. $360 \mathrm{e}-01$

$7.466 e-02$

$2.272 e+00$

5. 457 e+0o

6. $267 e+00$

1. $332 \mathrm{e}+01$

$1.335 e-01$

$5.712 e-05$

$2.800 e+01$
Exposure Rate $\mathrm{mR} / \mathrm{hr}$ With Buildup

$4.520 e-25$

1. $182 e-16$

$5.290 e-07$

$1.811 \mathrm{e}-03$

$6.848 e-03$

$2.283 e-01$

$9.517 e-02$

1. $243 \mathrm{e}+00$

2. $303 e-01$

$6.131 e-01$

$9.663 e-02$

$4.776 \mathrm{e}-01$

$2.344 e-01$

6. $545 \mathrm{e}+00$

1. 383 e+01

1. $456 e+01$

2. $668 e+01$

2. $453 e-01$

9.401e-05

$6.509 e+01=$ 


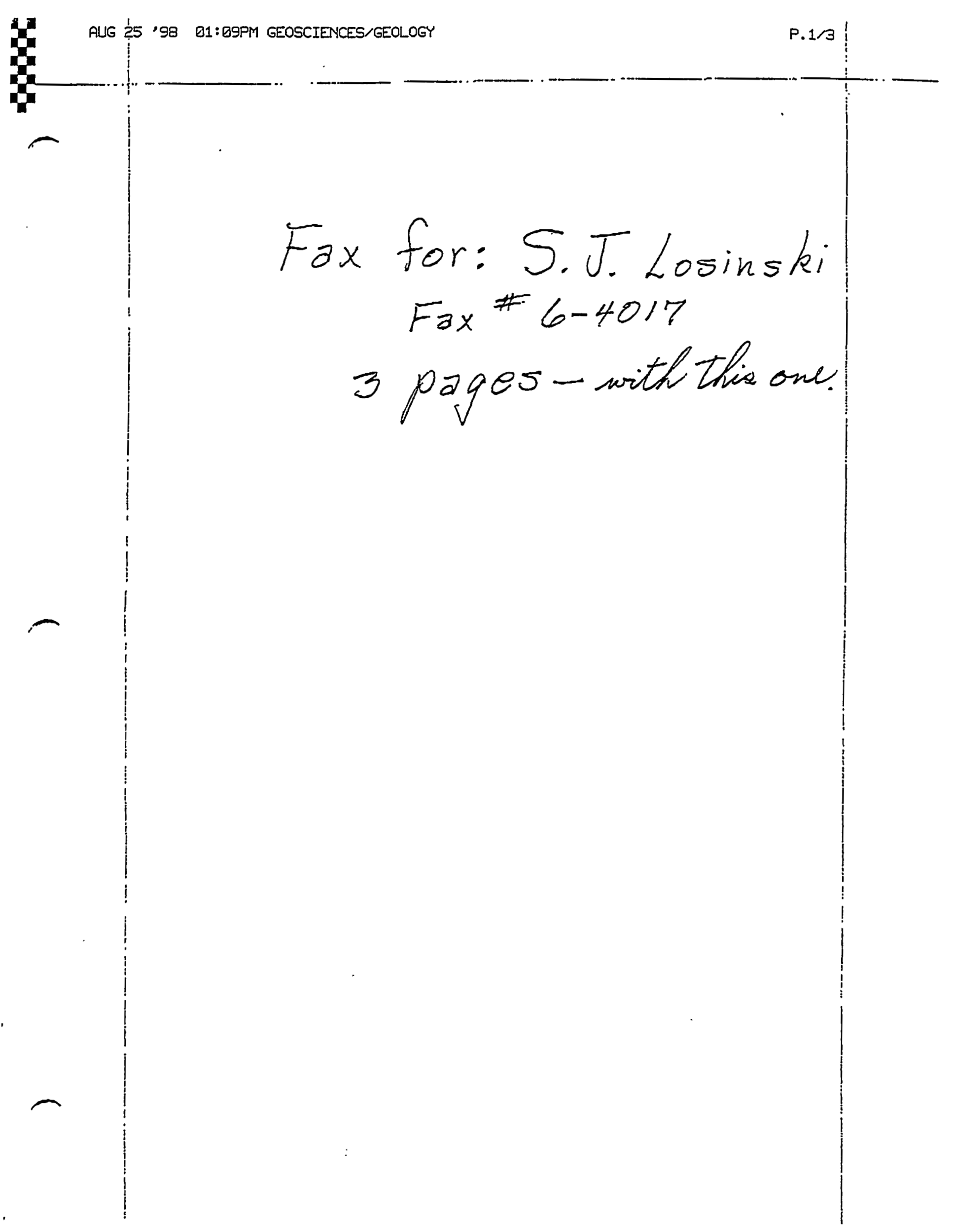


Microshield v5.01 (5.01-00121)

Lockheed Martin Idaho Technologies company Case File Heat Generation Case File: W181107.MS5 Case Title: Th181-107

$\begin{array}{lc}\text { Isotope } & \frac{\text { Activity }}{\text { Curies }} \\ \text { Am-241 } & 3.4650 e-001 \\ \text { Ba-137m } & 3.6090 e+001 \\ \text { Ce-144 } & 7.7460 e-010 \\ \text { Co-60 } & 8.0660 e-003 \\ \text { Cs-134 } & 1.4360 e-003 \\ \text { Cs-137 } & 3.8150 e+001 \\ \text { Np-237 } & 5.0700 e-003 \\ \text { Pr-144 } & 7.7460 e-010 \\ \text { Pu-238 } & 2.0730 e+001 \\ \text { Pu-239 } & 1.1120 e+000 \\ \text { Pu-240 } & 3.4900 e-001 \\ \text { Pu-241 } & 7.1930 e+000 \\ \text { Pu-242 } & 5.1830 e-004 \\ \text { Rh-106 } & 7.1340 e-006 \\ \text { Ru-106 } & 7.1340 e-006 \\ \text { Sb-125 } & 1.6250 e-001 \\ S I-90 & 5.1250 e+000 \\ U-234 & 6.9300 e-003 \\ U-235 & 1.5260 e-004 \\ U-236 & 7.2200 e-005 \\ U-238 & 2.2600 e-005 \\ \text { Y-90 } & 5.1250 e+000\end{array}$

Activity Bequerels

$1.2821 e+010$

$1.3353 e+012$

$2.8660 e+001$

$2.9844 e+008$

$5.3132 e+007$

$1.4116 \mathrm{e}+012$

$1.8759 e+008$

$2.8660 e+001$

$7.6701 e+011$

$4.1144 \mathrm{e}+010$

$1.2913 \mathrm{e}+010$

2. $6614 e+011$

$1.9177 e+007$

$2.6396 e+005$

$2.6396 e+005$

$6.0125 e+009$

$1.8963 e+011$

$2.5641 e+008$

$5.6462 e+006$

$2.6714 e+006$

$8.3620 e+005$

$1.8963 e+011$

Total

\section{Heat Generation Watts/Curie}

$$
\begin{aligned}
& 3.2755 e-002 \\
& 3.5403 e-003 \\
& 6.0991 e-004 \\
& 1.5417 e-002 \\
& 1.0149 e-002 \\
& 1.1086 e-003 \\
& 2.8548 e-002 \\
& 7.3470 e-003 \\
& 3.2552 e-002 \\
& 3.0531 e-002 \\
& 3.0591 e-002 \\
& 3.1773 e-005 \\
& 2.9012 e-002 \\
& 9.5865 e-003 \\
& 5.9466 e-005 \\
& 3.0911 e-003 \\
& 1.1603 e-003 \\
& 2.8232 e-002 \\
& 2.7030 e-002 \\
& 2.6729 e-002 \\
& 2.4838 e-002 \\
& 5.5407 e-003
\end{aligned}
$$

Heat Generation:

\section{Eeat Generation Watts}

$1.1350 e-002$

$1.2777 e-001$

$4.7244 \mathrm{e}-013$

$1.2435 \mathrm{e}-004$

$1.4574 \mathrm{e}-005$

$4.2291 e-002$

$1.4474 \mathrm{e}-004$

$5.6910 e-012$

$6.7481 e-001$

$3.3951 e-002$

$1.0676 e-002$

$2.2854 e-004$

$1.5037 e-005$

$6.8390 \mathrm{e}-008$

$4.2423 e-010$

$5.0231 e-004$

$5.9465 e-003$

$1.9565 e-004$

$4.1248 e-006$

$1.9299 e-006$

$5.6134 e-007$

$2.8396 \mathrm{e}-002$

$9.3642 \mathrm{e}-001$

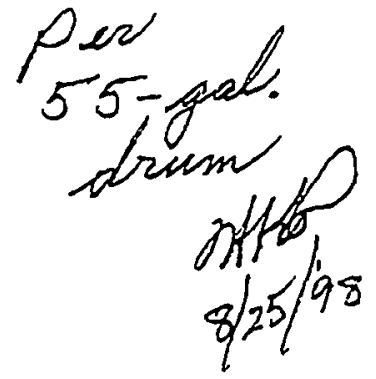


Hicroshiela v5.01 (5.01-00121)

Iockheed Hartin Idaho Technologies Company Case File geat Generation

Case File: WM181SRT.U55

Case Title: T-112 spent Resin Tk

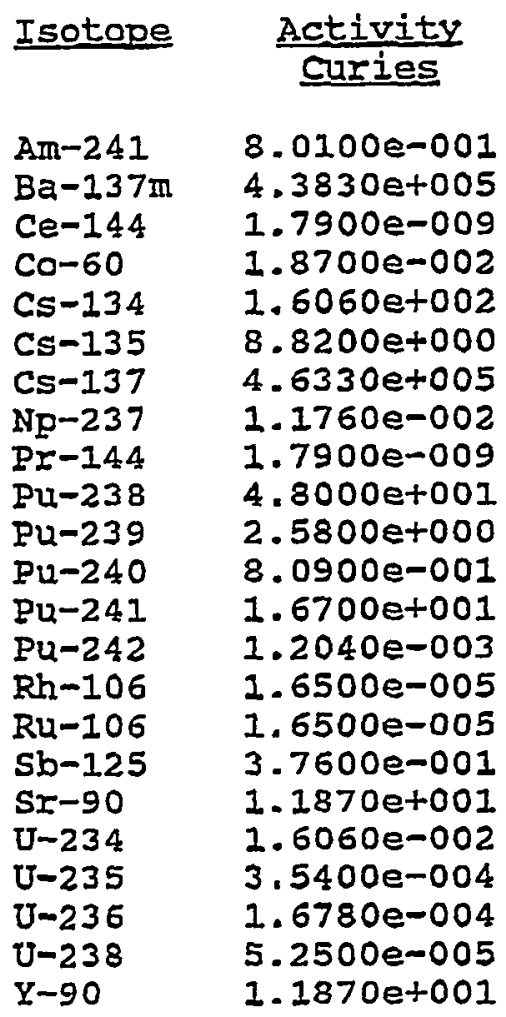

\section{Activity \\ Bequerels}

$2.9637 e+010$

$1.6217 \mathrm{e}+016$

$6.6230 e+001$

$6.9190 e+008$

$5.9422 \mathrm{e}+012$

$3.2634 \mathrm{e}+011$

$1.7142 \mathrm{e}+016$

$4.3512 \mathrm{e}+008$

$6.6230 e+001$

$1.7760 e+012$

$9.5460 e+010$

$2.9933 \mathrm{e}+010$

$6.1790 e+011$

$4,4548 \mathrm{e}+007$

$6.1050 e+005$

$6.1050 e+005$

$1.3912 \mathrm{e}+010$

$4.3919 e+011$

$5.9422 e+008$

$1.3098 \mathrm{e}+007$

$6.2086 \mathrm{e}+006$

$1.9425 e+006$

$4.3919 e+011$

Total
Heat Generation Watts/Curie

$3.2755 e-002$

$3.5403 e-003$

$6.0991 \mathrm{e}-004$

$1.5417 e-002$

$1.0149 e-002$

$3.9913 e-004$

$1.1086 \mathrm{e}-003$

$2.8548 e-002$

$7.3470 e-003$

$3.2552 e-002$

$3.0531 e-002$

$3.0591 e-002$

$3.1773 \mathrm{e}-005$

$2.9012 e-002$

$9.5865 e-003$

$5.9466 \mathrm{e}-005$

$3.0911 \mathrm{e}-003$

$1.1603 e-003$

$2.8232 e-002$

$2.7030 e-002$

$2.6729 e-002$

2. $4838 e-002$

$5.5407 e-003$

Heat Generation:
Heat Generation Watts

$2.6237 e-002$

$1.5517 \mathrm{e}+003$

$1.0917 e-012$

$2.8829 e-004$

$1.6299 \mathrm{e}+000$

$3.5203 e-003$

$5.1359 e+002$

$3.3573 e-004$

$1.3251 e-011$

$1.5625 e+000$

$7.8771 e-002$

2. $4748 e-002$

5.3061e-004

3. $4930 e-005$

$1.5818 e-007$

$9.8119 e-010$

$1.1623 e-003$

$1.3773 e-002$

$4.5341 e-004$

$9.5688 e-006$

$4.4852 e-006$

$1.3040 e-006$

$6.5768 \mathrm{e}-002$

$2.0687 e+003$

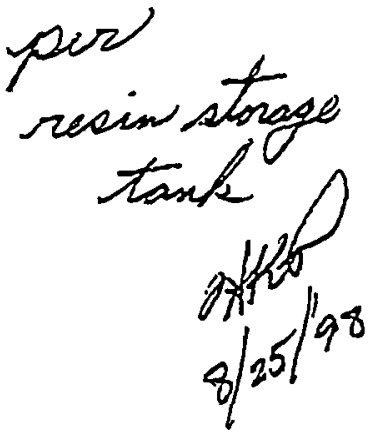


431.02\#

$06 / 17 / 97$

Rev. \#04

\section{ENGINEERING DESIGN FILE}

Functional File Number - SPR-CsIX-13

EDF Serial Number - EDF-CsIX-15

Page 10 of 10

Attachment 2 
Date: $\quad$ September 30,1998
To:
From:
S. J. Losinski
MS 3625
$6-5962$
From:
H. K. Peterson OAftereterson MS 2107
6-8657
Subject: “AVERAGE" CS IX/TRU COMPONENT PROJECTED RADIATION FIELDS -- HKP-18-98

References: a) MicroShield, Version 5.01, Grove Engineering, Rockville MD

Contact/near surface ( $1 \mathrm{~cm}$ from item surface) radiation field calculations for "average" conditions have been made for the requested Cs IX/TRU Feasibility Study components and are summarized below. Also projected are the heat generation rates for these components for the corresponding source term concentrations. The calculations were made with the MicroShield, Version 5.01, computer code (Ref.a) and input/output listings for each are attached for reference.

a) (Item \#1) Assumed to be T-101 A \& B Liquid Waste Feed Tank, which is a right circular cylinder 10 feet tall, $8 \mathrm{ft}$ in diameter, with a wall of $1 / 2$ inches stainless steel (SS), and containing 14,234 liters of Stream 103. The calculated radiation field at $1 \mathrm{~cm}$ from the tank midline is $14.4 \mathrm{R} / \mathrm{hr}$ and the heat generation rate (HGR) is 7.24 watts.

b) (Item \#2) Assumed to be EV-101 Rotary Evaporator, which is assumed to be the same as a standard 55-gal drum containing $291.2 \mathrm{~kg}$ of Stream 107. The calculated radiation field for this component is $48.6 \mathrm{R} / \mathrm{hr}$ and the HGR is 0.94 watts.

c) (Item \#3) Assumed to be the T-108 UDS Slurry Tank, which is $5 \mathrm{ft}$ tall, $4 \mathrm{ft}$ in diameter, with a 3/8-inch SS wall, and containing $249 \mathrm{~kg}$ of Stream 107 diluted by a factor of 10 with water. The calculated radiation field is $7.8 \mathrm{R} / \mathrm{hr}$ and the HGR is 0.8 watts.

d) (Item \#4) Assumed to be the V-104A Cesium Ion Exchange Column, modeled to be $8 \mathrm{ft}$ tall, $1.33 \mathrm{ft}$ in diameter, with a $3 / 8$ inch SS wall, and containing $0.25 \mathrm{~m}^{3}$ of Stream 108 distributed uniformly in the column. The radiation field for this component is calculated to be $2630 \mathrm{R} / \mathrm{hr}$ and the HGR is 24 watts.

e) (Item \#5) Assumed to be the T-103 A \& B Cs IX Effluent Tank and modeled to be $10 \mathrm{ft} \mathrm{tall}$, $8 \mathrm{ft}$ diameter, with a $1 / 2$ inch SS wall, and containing 14234 liters of Stream 106. The calculated radiation field for this component is $185 \mathrm{mR} / \mathrm{hr}$ and the HGR is 4 watts.

f) (Item \#6) Assumed to be the T-112 Spent Resin Storage Tank and modeled to be $15 \mathrm{fth}$ high, $8 \mathrm{ft}$ in diameter, with a $1 / 2$ inch SS wall, and containing $23112 \mathrm{~kg}$ of Stream 108 . The radiation field is calculated to be $7630 \mathrm{R} / \mathrm{hr}$ and the HGR is 2050 watts. 


\section{S. J. Losinski}

September 30, 1998

HKP-18-98

Page 2

g) (Item \#7) A recalculation of the T-112 Spent Resin Storage Tank containing 23,112 kg of Stream 108a modeled as in item \#6. The radiation field is calculated to be $10,650 \mathrm{R} / \mathrm{hr}$ and the HGR is 2840 watts.

h) (Item \#8) Modeled the same as the M-101 Mixer, assumed to be a half cylinder $2 \mathrm{ft}$ in diameter, $3 \mathrm{ft}$ long, with a 3/8-inch SS wall and containing 133.4 liters of Stream 113 . The radiation field is calculated to be $90 \mathrm{mR} / \mathrm{hr}$ and the HGR is 0.04 watts.

i) (Item \#9) Modeled the same as the M-101 Mixer, assumed to be a full cylinder $3 \mathrm{ft}$ long, $2 \mathrm{ft}$ in diameter, with a 3/8-inch SS wall, and containing $507.3 \mathrm{~kg}$ of Stream 117. The radiation field for this component is calculated to be $70 \mathrm{mR} / \mathrm{hr}$ and the HGR is 0.05 watts.

j) (Item \#10) Modeled the same as a Waste Drum, assumed to be a standard 55-gal drum with $395.2 \mathrm{~kg}$ of grouted waste from Stream 117. The radiation field for this package is calculated to be $115 \mathrm{mR} / \mathrm{hr}$ and has an HGR of 0.04 watts.

k) (Item \#11) Modeled the same as the component of item \#10 but containing $395.2 \mathrm{~kg}$ of Stream $117 \mathrm{a}$. The radiation field is calculated to be $130 \mathrm{mR} / \mathrm{hr}$ and the HGR is 0.04 watts.

If there are any questions or comments concerning this analysis, please call me at the abovelisted number.

$h \mathrm{kp}$

Attachments

cc:

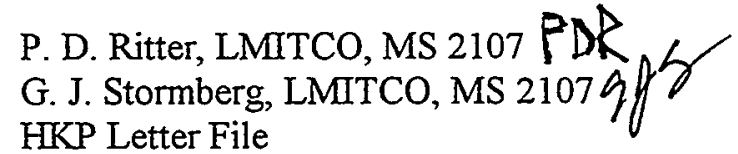


Microshield V5.01 (5.01-00121)

Page : 1

Lockheed Martin Idaho Technologies Company

DOS File: 181103SL.MS5

Run Date: September 24, 1998

Run Time: $8: 40: 42$ AM

Duration: $00: 01: 47$

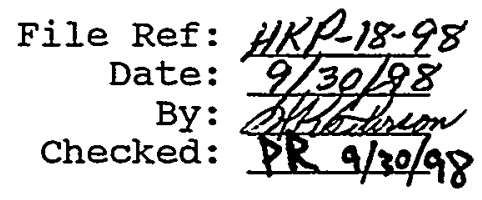

Case Title: WM-181103

Description: T-101 A \& B Liquid Waste Feed Tanks - Surface Rad./WS103 Geometry: 7 - cylinder volume - side Shields

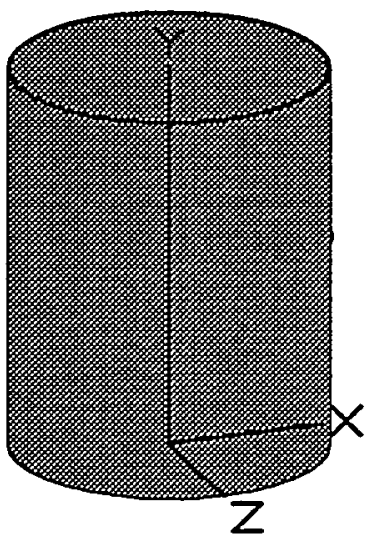

\begin{tabular}{lrrr}
\multicolumn{7}{c}{ Source Dimensions } \\
Height & $304.8 \mathrm{~cm}$ & $10 \mathrm{ft}$ & $0.0 \mathrm{in}$ \\
Radius & $121.92 \mathrm{~cm}$ & $4 \mathrm{ft}$
\end{tabular}

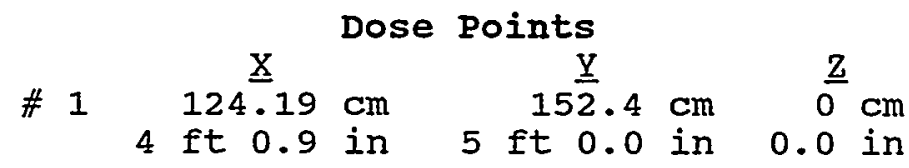

Shields

Shield Name Dimension Material Density Source $\quad 1.42 e+07 \mathrm{~cm}^{3}$ Mixed $\rightarrow 1.3$

Concrete 0.3

Shield 1

Transition

Water 1

Air Gap

$1.27 \mathrm{~cm}$

Iron 7.86

$1.0 \mathrm{~cm}$

Air

0.0011

Air 0.00122

Source Input

Grouping Method : Standard Indices

Number of Groups : 25

Lower Energy Cutoff : 0.015

Photons < 0.015 : Excluded

Library : ICRP-38

Nuclide

Am-241

$\mathrm{Am}-243$

$\mathrm{Ba}-137 \mathrm{~m}$

$\mathrm{Ce}-144$

$\mathrm{Cm}-242$

$\mathrm{Cm}-244$

Co-60

Cs -134

Cs -135

Cs -137

$\mathrm{Eu}-152$

Eu-154

$\mathrm{Eu}-155$

$\mathrm{H}-3$

$I-129$

$\mathrm{Ni}-63$

$\mathrm{Np}-237$

Pm-147

Pr-144

$\mathrm{Pu}-238$

$\mathrm{Pu}-239$

$\mathrm{Pu}-240$

$\mathrm{Pu}-241$ becquerels

2.4431e+011

$1.1033 e+008$

$2.5640 e+013$

$3.1365 e+010$

$5.7794 e+008$

$7.7700 e+009$

2.7047 e+010

$2.2592 \mathrm{e}+010$

$5.0172 e+008$

$2.7163 e+013$

$1.4763 e+009$

$8.5655 e+010$

$3.7248 e+010$

$1.5866 e+010$

$3.6253 e+009$

$2.5956 e+010$

$1.3398 e+009$

$7.1965 e+010$

$3.1365 e+010$

$4.5251 e+011$

$3.4517 e+010$

$9.9826 e+009$

$1.9965 e+011$ $\mu \mathrm{Ci} / \mathrm{cm}^{3}$

4.6390e-001

2.0950e-004

$4.8685 e+001$

$5.9556 e-002$

$1.0974 \mathrm{e}-003$

$1.4754 \mathrm{e}-002$

$5.1357 e-002$

$4.2898 \mathrm{e}-002$

$9.5268 \mathrm{e}-004$

$5.1578 e+001$

$2.8032 e-003$

$1.6264 \mathrm{e}-001$

$7.0727 e-002$

$3.0126 e-002$

$6.8837 e-003$

4. $9285 e-002$

$2.5440 e-003$

$1.3665 e-001$

$5.9556 e-002$

$8.5923 e-001$

$6.5542 \mathrm{e}-002$

$1.8955 \mathrm{e}-002$

$3.7910 e-001$
$\mathrm{Bg} / \mathrm{cm}^{3}$

$1.7164 \mathrm{e}+004$

$7.7517 e+000$

$1.8013 e+006$

$2.2036 e+003$

4.0604 e+001

$5.4589 e+002$

$1.9002 \mathrm{e}+003$

$1.5872 \mathrm{e}+003$

$3.5249 e+001$

$1.9084 \mathrm{e}+006$

$1.0372 \mathrm{e}+002$

$6.0178 \mathrm{e}+003$

$2.6169 e+003$

$1.1147 \mathrm{e}+003$

2. $5470 \mathrm{e}+002$

$1.8235 \mathrm{e}+003$

$9.4127 e+001$

$5.0560 e+003$

$2.2036 e+003$

$3.1792 e+004$

$2.4251 e+003$

$7.0134 \mathrm{e}+002$

$1.4027 e+004$ 
Page : 2

DOS File: 181103SL.MS5

Run Date: September 24, 1998

Run Time: 8:40:42 AM

Duration: 00:01:47

\begin{tabular}{|c|c|c|c|c|}
\hline Nuclide & curies & becquerels & $\mu \mathrm{Ci} / \mathrm{cm}^{3}$ & $\underline{\mathrm{Bg} / \mathrm{cm}^{3}}$ \\
\hline $\mathrm{Pu}-242$ & $2.0870 e-004$ & $7.7219 e+006$ & $1.4662 e-005$ & $5.4251 e-001$ \\
\hline$R h-106$ & $4.6400 e-003$ & $1.7168 e+008$ & $3.2599 \mathrm{e}-004$ & $1.2062 e+001$ \\
\hline$R u-106$ & $4.6400 e-003$ & $1.7168 e+008$ & $3.2599 e-004$ & $1.2062 e+001$ \\
\hline $\mathrm{Sb}-125$ & $1.5480 e-001$ & $5.7276 e+009$ & $1.0876 e-002$ & $4.0240 e+002$ \\
\hline$S m-151$ & $6.8590 e+000$ & $2.5378 e+011$ & $4.8189 e-001$ & $1.7830 e+004$ \\
\hline Sr-90 & $4.8706 e+002$ & $1.8021 e+013$ & $3.4219 e+001$ & $1.2661 e+006$ \\
\hline TC-99 & $2.1160 e-001$ & $7.8292 e+009$ & $1.4866 e-002$ & $5.5005 e+002$ \\
\hline $\operatorname{Th}-230$ & $9.7130 e-006$ & $3.5938 e+005$ & $6.8240 e-007$ & $2.5249 e-002$ \\
\hline$T h-231$ & $1.3920 e-003$ & $5.1504 e+007$ & $9.7797 e-005$ & $3.6185 e+000$ \\
\hline $\mathrm{U}-232$ & $2.7400 e-005$ & $1.0138 e+006$ & $1.9250 e-006$ & $7.1226 e-002$ \\
\hline$U-233$ & $1.4770 e-007$ & $5.4649 e+003$ & $1.0377 e-008$ & $3.8394 e-004$ \\
\hline$U-234$ & $1.3900 \mathrm{e}-002$ & $5.1430 e+008$ & $9.7656 e-004$ & $3.6133 e+001$ \\
\hline$U-235$ & $3.4600 e-004$ & $1.2802 \mathrm{e}+007$ & $2.4309 e-005$ & $8.9942 e-001$ \\
\hline$U-236$ & $6.7590 e-004$ & $2.5008 e$ & -005 & $1.7570 e+000$ \\
\hline$U-238$ & $3.7300 e-004$ & $1.3801 e+007$ & $2.6206 e-005$ & $9.6961 e-001$ \\
\hline$Y-90$ & $4.8706 e+002$ & $1.8021 e+013$ & $3.4219 e+001$ & $1.2661 e+006$ \\
\hline
\end{tabular}

Integration Parameters

Radial

Circumferential

$Y$ Direction (axial)

24

24

24

$\begin{array}{cc}\frac{\text { Energy }}{\text { MeV }} & \begin{array}{c}\text { Activity } \\ \text { photons/sec }\end{array} \\ 0.015 & 5.103 e+10 \\ 0.02 & 7.875 e+10 \\ 0.03 & 1.565 e+12 \\ 0.04 & 3.999 e+11 \\ 0.05 & 7.095 e+09 \\ 0.06 & 8.780 e+10 \\ 0.08 & 1.250 e+10 \\ 0.1 & 4.313 e+10 \\ 0.15 & 3.557 e+09 \\ 0.2 & 6.442 e+09 \\ 0.3 & 6.213 e+08 \\ 0.4 & 2.589 e+09 \\ 0.5 & 1.352 e+09 \\ 0.6 & 2.305 e+13 \\ 0.8 & 5.378 e+10 \\ 1.0 & 5.493 e+10 \\ 1.5 & 6.171 e+10 \\ 2.0 & 2.450 e+08 \\ 3.0 & 9.479 e+04 \\ & \end{array}$

TOTALS: $\quad 2.548 e+13$
Fluence Rate $\mathrm{MeV} / \mathrm{Cm}^{2} / \mathrm{sec}$ No Buildup

$7.302 e-264$ $1.656 e-117$

3. $475 e-35$

$9.947 e-15$

$1.192 e-08$

$5.853 e-04$

$9.234 e-02$

5.024 e+00

$5.026 e+00$

2. $378 e+01$

$6.098 e+00$

4.630 et 01

$3.776 e+01$

9.218 e+05

$3.774 e+03$

5. 960 e+03

1. 471 e+04

$1.003 e+02$

$8.053 e-02$

$9.464 e+05$
Results

Fluence Rate $\mathrm{MeV} / \mathrm{cm}^{2} / \mathrm{sec}$ With Buildup

1. $578 \mathrm{e}-21$

$6.237 e-20$

1. $105 e-16$

1. 669 e-09

$5.255 e-04$

$6.809 e+00$

1. $318 e+02$

1.956 et03

4. 401 et 02

9.961e+02

1. 207 et 02

6.127e+02

3. $707 e+02$

$7.275 e+06$

2. 212 e+04

2. 834 e+04

5. $125 e+04$

2. $944 \mathrm{e}+02$

1. $925 e-01$

$7.382 e+06$
Exposure Rate $\mathrm{mR} / \mathrm{hr}$

No Buildup

$6.263 e-265$

$5.735 e-119$

$3.444 \mathrm{e}-37$

$4.399 e-17$

$3.176 e-11$

$1.162 e-06$

$1.461 \mathrm{e}-04$

$7.686 \mathrm{e}-03$

$8.277 e-03$

$4.197 e-02$

$1.157 \mathrm{e}-02$

$9.021 e-02$

$7.413 e-02$

$1.799 e+03$

$7.179 e+00$

$1.099 e+01$

$2.474 \mathrm{e}+01$

$1.551 e-01$

$1.093 e-04$

$1.842 e+03$
Exposure Rate $\mathrm{mR} / \mathrm{hr}$ With Buildup

$1.354 \mathrm{e}-22$

2. $161 e-21$

$1.095 \mathrm{e}-18$

7. $381 e-12$

$1.400 e-06$

$1.352 e-02$

$2.085 e-01$

$2.993 e+00$

$7.248 e-01$

$1.758 e+00$

2. $289 e-01$

$1.194 \mathrm{e}+00$

$7.276 e-01$

$1.420 e+04$

$4.208 e+01$

5.224 e+01

8. $622 e+01$

$4.552 e-01$

2. $612 e-04$

$1.439 e+04$ 
Microshield v5.01 (5.01-00121)

Lockheed Martin Idaho Technologies Company Case File Heat Generation Case File: 181103SL.MS5

Case Title: WM-181103

\begin{tabular}{|c|c|c|}
\hline Isotope & $\frac{\text { Activity }}{\text { Curies }}$ & $\frac{\text { Activity }}{\text { Bequerels }}$ \\
\hline $\mathrm{Am}-241$ & $6.6030 e+000$ & $2.4431 e+011$ \\
\hline$A m-243$ & $2.9820 e-003$ & $1.1033 e+008$ \\
\hline $\mathrm{Ba}-137 \mathrm{~m}$ & $6.9296 e+002$ & $2.5640 e+013$ \\
\hline $\mathrm{Ce}-144$ & $8.4770 e-001$ & $3.1365 e+010$ \\
\hline $\mathrm{Cm}-242$ & $1.5620 e-002$ & $5.7794 e+008$ \\
\hline $\mathrm{Cm}-244$ & $2.1000 e-001$ & $7.7700 e+009$ \\
\hline Co-60 & $7.3100 e-001$ & $2.7047 e+010$ \\
\hline Cs -134 & $6.1060 e-001$ & $2.2592 e+010$ \\
\hline Cs-135 & $1.3560 e-002$ & $5.0172 e+008$ \\
\hline Cs -137 & $7.3414 e+002$ & $2.7163 e+013$ \\
\hline $\mathrm{Eu}-152$ & $3.9900 e-002$ & $1.4763 e+009$ \\
\hline$E u-154$ & $2.3150 e+000$ & $8.5655 e+010$ \\
\hline Eu-155 & $1.0067 e+000$ & $3.7248 e+010$ \\
\hline $\mathrm{H}-3$ & $4.2880 e-001$ & $1.5866 \mathrm{e}+010$ \\
\hline$I-129$ & $9.7980 e-002$ & $3.6253 e+009$ \\
\hline $\mathrm{Ni}-63$ & $7.0150 e-001$ & $2.5956 e+010$ \\
\hline$N p-237$ & $3.6210 e-002$ & $1.3398 e+009$ \\
\hline$P m-147$ & $1.9450 e+000$ & $7.1965 e+010$ \\
\hline $\operatorname{Pr}-144$ & $8.4770 e-001$ & $3.1365 e+010$ \\
\hline$P u-238$ & $1.2230 e+001$ & $4.5251 e+011$ \\
\hline$P u-239$ & $9.3290 e-001$ & $3.4517 e+010$ \\
\hline $\mathrm{Pu}-240$ & $2.6980 \mathrm{e}-001$ & $9.9826 e+009$ \\
\hline$P u-241$ & $5.3960 e+000$ & $1.9965 e+011$ \\
\hline$P u-242$ & $2.0870 e-004$ & $7.7219 e+006$ \\
\hline$R h-106$ & $4.6400 e-003$ & $1.7168 e+008$ \\
\hline Ru-106 & $4.6400 e-003$ & $1.7168 e+008$ \\
\hline$S b-125$ & $1.5480 e-001$ & $5.7276 e+009$ \\
\hline$S m-151$ & $6.8590 e+000$ & $2.5378 e+011$ \\
\hline$S r-90$ & $4.8706 e+002$ & $1.8021 e+013$ \\
\hline TC-99 & $2.1160 e-001$ & $7.8292 e+009$ \\
\hline Th-230 & $9.7130 e-006$ & $3.5938 e+005$ \\
\hline Th-231 & $1.3920 e-003$ & $5.1504 e+007$ \\
\hline $\mathrm{U}-232$ & $2.7400 e-005$ & $1.0138 e+006$ \\
\hline$U-233$ & $1.4770 \mathrm{e}-007$ & $5.4649 e+003$ \\
\hline$U-234$ & $1.3900 e-002$ & $5.1430 e+008$ \\
\hline$U-235$ & $3.4600 e-004$ & $1.2802 e+007$ \\
\hline$U-236$ & $6.7590 e-004$ & $2.5008 e+007$ \\
\hline$U-238$ & $3.7300 e-004$ & $1.3801 e+007$ \\
\hline$Y-90$ & $4.8706 e+002$ & $1.8021 \mathrm{e}+013$ \\
\hline
\end{tabular}

Total

\section{Heat Generation Watts/Curie}

$3.2755 e-002$

$3.1599 e-002$

$3.5403 e-003$

$6.0991 e-004$

3. $6195 \mathrm{e}-002$

$3.4375 e-002$

$1.5417 \mathrm{e}-002$

$1.0149 e-002$

$3.9913 e-004$

$1.1086 \mathrm{e}-003$

$7.3864 \mathrm{e}-003$

$8.7221 e-003$

$6.4902 e-004$

$3.3688 e-005$

$4.7222 e-004$

$1.0154 \mathrm{e}-004$

$2.8548 \mathrm{e}-002$

3. $6732 \mathrm{e}-004$

$7.3470 e-003$

$3.2552 \mathrm{e}-002$

$3.0531 e-002$

3. $0591 \mathrm{e}-002$

$3.1773 e-005$

$2.9012 e-002$

$9.5865 e-003$

$5.9466 \mathrm{e}-005$

$3.0911 e-003$

$1.1669 \mathrm{e}-004$

$1.1603 e-003$

$6.0024 e-004$

2.7709e-002

$7.1148 \mathrm{e}-004$

$3.1460 e-002$

$2.8571 e-002$

$2.8232 e-002$

$2.7030 e-002$

$2.6729 e-002$

$2.4838 e-002$

$5.5407 e-003$

Heat Generation: $\frac{\text { Heat Generation }}{\text { Watts }}$

$2.1628 e-001$

$9.4230 e-005$

$2.4533 \mathrm{e}+000$

$5.1702 e-004$

$5.6537 e-004$

$7.2188 \mathrm{e}-003$

$1.1270 \mathrm{e}-002$

$6.1969 e-003$

$5.4122 e-006$

$8.1383 e-001$

$2.9472 \mathrm{e}-004$

$2.0192 e-002$

$6.5337 e-004$

$1.4445 \mathrm{e}-005$

$4.6268 e-005$

$7.1233 e-005$

$1.0337 e-003$

$7.1444 \mathrm{e}-004$

$6.2280 e-003$

$3.9811 e-001$

$2.8483 e-002$

$8.2535 e-003$

$1.7145 e-004$

$6.0548 e-006$

$4.4482 e-005$

$2.7592 e-007$

$4.7851 e-004$

$8.0035 e-004$

$5.6514 \mathrm{e}-001$

$1.2701 e-004$

$2.6914 e-007$

$9.9037 e-007$

$8.6201 e-007$

4.2200e-009

$3.9243 e-004$

$9.3525 e-006$

$1.8066 \mathrm{e}-005$

$9.2646 e-006$

2.6987 e +000

$7.2392 e+000$ 
Microshield v5.01 (5.01-00121)

Iockheed Martin Idaho Technologies company

Page : 1

DOS File: WM181107.MS5

Run Date: September 24, 1998

Run Time: 9:10:56 AM

Duration: 00:01:46

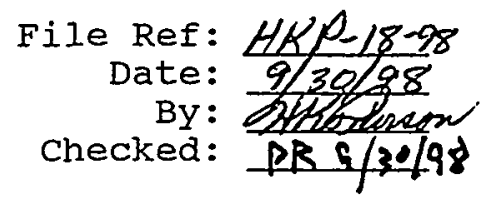

Case Title: FM181-107

Description: 55-gal drum loaded with $291.2 \mathrm{~kg}$ of stream 107 Geometry: 7 - cylinder volume - side Shields

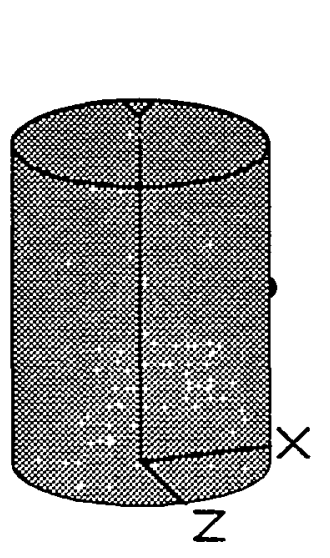

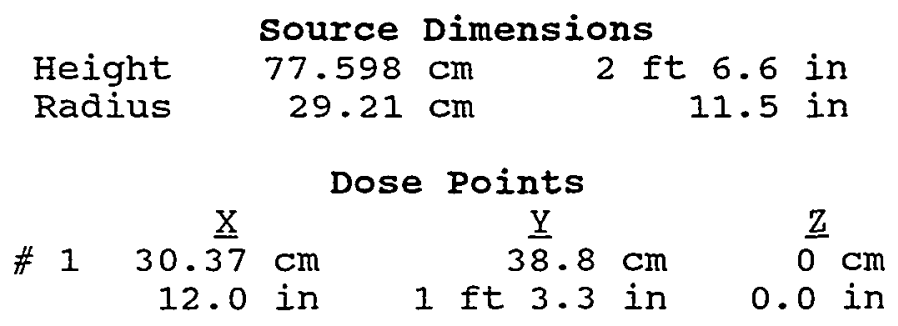

$\begin{array}{lccl}\text { Shield Name } & \begin{array}{c}\text { Shields } \\ \text { Dimension }\end{array} & \text { Material Density } \\ \text { Source } & 2.08 \mathrm{0}+05 \mathrm{~cm}^{3} & \text { Concrete } & 1.4 \\ \text { Shield } 1 & .16 \mathrm{~cm} & \text { Iron } & 7.86 \\ \text { Transition } & 1.0 \mathrm{~cm} & \text { Air } & 0.0011 \\ \text { Air Gap } & & \text { Air } & 0.00122\end{array}$

Source Input

Grouping Method : Standard Indices

Number of Groups : 25

Lower Energy Cutoff : 0.015

Photons < 0.015 : Excluded

Library : ICRP-38

$\begin{array}{lc}\text { Nuclide } & \text { curies } \\ \text { Am-241 } & 3.4650 e-001 \\ \mathrm{Ba}-137 \mathrm{~m} & 3.6090 \mathrm{e}+001 \\ \mathrm{Ce}-144 & 7.7460 \mathrm{e}-010 \\ \mathrm{Co}-60 & 8.0660 \mathrm{e}-003 \\ \mathrm{Cs}-134 & 1.4360 \mathrm{e}-003 \\ \mathrm{Cs}-137 & 3.8150 \mathrm{e}+001 \\ \mathrm{~Np}-237 & 5.0700 \mathrm{e}-003 \\ \mathrm{Pr}-144 & 7.7460 \mathrm{e}-010 \\ \mathrm{Pu}-238 & 2.0730 \mathrm{e}+001 \\ \mathrm{Pu}-239 & 1.1120 \mathrm{e}+000 \\ \mathrm{Pu}-240 & 3.4900 \mathrm{e}-001 \\ \mathrm{Pu}-241 & 7.1930 \mathrm{e}+000 \\ \mathrm{Pu}-242 & 5.1830 \mathrm{e}-004 \\ \mathrm{Rh}-106 & 7.1340 \mathrm{e}-006 \\ \mathrm{Ru}-106 & 7.1340 \mathrm{e}-006 \\ \mathrm{Sb}-125 & 1.6250 \mathrm{e}-001 \\ \mathrm{Sr}-90 & 5.1250 \mathrm{e}+000 \\ \mathrm{U}-234 & 6.9300 \mathrm{e}-003 \\ \mathrm{U}-235 & 1.5260 \mathrm{e}-004 \\ \mathrm{U}-236 & 7.2200 \mathrm{e}-005 \\ \mathrm{U}-238 & 2.2600 \mathrm{e}-005 \\ \mathrm{Y}-90 & 5.1250 \mathrm{e}+000\end{array}$

becquerels

$1.2821 \mathrm{e}+010$

$1.3353 e+012$

$2.8660 e+001$

$2.9844 \mathrm{e}+008$

$5.3132 e+007$

$1.4116 e+012$

$1.8759 e+008$

$2.8660 e+001$

$7.6701 e+011$

$4.1144 e+010$

$1.2913 e+010$

$2.6614 \mathrm{e}+011$

$1.9177 e+007$

$2.6396 e+005$

$2.6396 e+005$

$6.0125 e+009$

$1.8963 e+011$

$2.5641 e+008$

$5.6462 e+006$

$2.6714 e+006$

$8.3620 e+005$

$1.8963 e+011$ $\mu \mathrm{Ci} / \mathrm{cm}^{3}$

$1.6659 e+000$

$1.7351 e+002$

$3.7240 e-009$

$3.8779 e-002$

$6.9038 e-003$

$1.8341 \mathrm{e}+002$

$2.4375 e-002$

$3.7240 e-009$

$9.9663 e+001$

$5.3462 e+000$

$1.6779 e+000$

$3.4582 e+001$

$2.4918 \mathrm{e}-003$

$3.4298 e-005$

$3.4298 e-005$

$7.8125 e-001$

$2.4639 e+001$

$3.3317 e-002$

$7.3365 e-004$

$3.4712 e-004$

$1.0865 e-004$

$2.4639 e+001$
$\mathrm{Ba} / \mathrm{cm}^{3}$

$6.1637 e+004$

$6.4199 e+006$

$1.3779 e-004$

$1.4348 \mathrm{e}+003$

$2.5544 e+002$

$6.7863 e+006$

$9.0187 e+002$

$1.3779 e-004$

$3.6875 e+006$

$1.9781 e+005$

$6.2082 e+004$

$1.2795 e+006$

$9.2198 e+001$

$1.2690 e+000$

$1.2690 e+000$

$2.8906 e+004$

$9.1166 e+005$

$1.2327 e+003$

$2.7145 e+001$

$1.2843 e+001$

$4.0202 e+000$

$9.1166 e+005$ 
Page : 2

DOS File: WM181107.MS5

Run Date: September 24, 1998

Run Time: 9:10:56 AM

Duration: $00: 01: 46$

Radial

Integration Parameters

Circumferential

24

$\mathrm{Y}$ Direction (axial) 26

\begin{tabular}{|c|c|c|c|c|c|}
\hline Energy & Activity & Fluence Rate & Fluence Rate & Exposure Rate & Exposure Rate \\
\hline $\mathrm{MeV}$ & photons/sec & $\frac{\mathrm{MeV} / \mathrm{cm}^{2} / \mathrm{sec}}{\text { No Buildup }}$ & $\frac{\mathrm{MeV} / \mathrm{cm}^{2} / \mathrm{sec}}{\text { With Buildup }}$ & No $\frac{\mathrm{mR} / \mathrm{hr}}{\text { Buildup }}$ & $\frac{\mathrm{mR} / \mathrm{hr}}{\text { with } \mathrm{Buil} \text { duo }}$ \\
\hline 0.015 & $4.642 e+10$ & $1.017 e-32$ & $5.764 e-22$ & $8.724 e-34$ & $4.944 e-23$ \\
\hline 0.02 & $1.397 e+10$ & $4.426 e-14$ & $5.581 e-14$ & $1.533 \mathrm{e}-15$ & $1.933 e-15$ \\
\hline 0.03 & $8.404 e+10$ & $1.814 e-02$ & $3.029 e-02$ & $1.797 e-04$ & $3.002 e-04$ \\
\hline 0.04 & $1.978 e+10$ & $4.965 e+00$ & $1.112 \mathrm{e}+01$ & $2.196 e-02$ & $4.917 e-02$ \\
\hline 0.05 & 1.524 e +07 & $8.413 e-02$ & $2.450 e-01$ & $2.241 e-04$ & $6.526 e-04$ \\
\hline 0.06 & $4.584 e+09$ & $1.324 e+02$ & $4.625 e+02$ & $2.631 e-01$ & $9.187 e-01$ \\
\hline 0.08 & $2.761 e+07$ & $4.212 e+00$ & $1.718 e+01$ & $6.666 e-03$ & $2.719 e-02$ \\
\hline 0.1 & $1.084 e+08$ & $3.729 e+01$ & $1.572 \mathrm{e}+02$ & $5.705 e-02$ & $2.405 e-01$ \\
\hline 0.15 & $3.043 e+07$ & $2.747 e+01$ & $1.099 e+02$ & $4.523 e-02$ & $1.810 e-01$ \\
\hline 0.2 & $4.433 e+08$ & $6.654 e+02$ & $2.481 e+03$ & 1.174 etoo & $4.379 e+00$ \\
\hline 0.3 & $2.782 e+07$ & $7.896 e+01$ & $2.594 e+02$ & $1.498 e-01$ & $4.921 e-01$ \\
\hline 0.4 & $1.892 e+09$ & $8.298 e+03$ & $2.464 e+04$ & $1.617 e+01$ & $4.801 e+01$ \\
\hline 0.5 & $6.217 e+08$ & $3.816 e+03$ & $1.046 e+04$ & $7.490 \mathrm{e}+00$ & $2.053 e+01$ \\
\hline 0.6 & $1.201 e+12$ & $9.701 e+06$ & $2.481 e+07$ & $1.893 e+04$ & $4.843 e+04$ \\
\hline 0.8 & $5.049 e+07$ & $6.301 e+02$ & $1.452 e+03$ & $1.199 e+00$ & $2.762 e+00$ \\
\hline 1.0 & $2.997 e+08$ & $5.248 e+03$ & $1.120 e+04$ & $9.674 e+00$ & $2.065 e+01$ \\
\hline 1.5 & $3.000 e+08$ & $9.715 e+03$ & $1.815 e+04$ & $1.635 e+01$ & $3.053 e+01$ \\
\hline 2.0 & $3.805 e+03$ & $1.888 e-01$ & $3.257 e-01$ & $2.920 e-04$ & $5.036 e-04$ \\
\hline 3.0 & $5.506 e+01$ & $4.879 e-03$ & $7.602 e-03$ & $6.619 e-06$ & $1.031 e-05$ \\
\hline COTALS & $1.374 e+12$ & $9.729 e+06$ & $2.488 e+07$ & $1.899 e+04$ & $4.856 e+04$ \\
\hline
\end{tabular}


Microshield v5.01 (5.01-00121)

Lockheed Martin Idaho Technologies Company

Case File Heat Generation

Case File: WM181107.MS5

Case Title: FM181-107

\begin{tabular}{|c|c|}
\hline Isotope & $\frac{\text { Activity }}{\text { Curies }}$ \\
\hline$A m-241$ & $3.4650 e-001$ \\
\hline $\mathrm{Ba}-137 \mathrm{~m}$ & $3.6090 e+001$ \\
\hline $\mathrm{Ce}-144$ & $7.7460 \mathrm{e}-010$ \\
\hline co-60 & $8.0660 e-003$ \\
\hline Cs -134 & $1.4360 e-003$ \\
\hline Cs -137 & $3.8150 \mathrm{e}+001$ \\
\hline$N p-237$ & $5.0700 e-003$ \\
\hline Pr-144 & $7.7460 \mathrm{e}-010$ \\
\hline $\mathrm{Pu}-238$ & $2.0730 e+001$ \\
\hline $\mathrm{Pu}-239$ & $1.1120 \mathrm{e}+000$ \\
\hline $\mathrm{Pu}-240$ & $3.4900 e-001$ \\
\hline $\mathrm{Pu}-241$ & $7.1930 e+000$ \\
\hline $\mathrm{Pu}-242$ & $5.1830 e-004$ \\
\hline $\mathrm{Rh}-106$ & $7.1340 e-006$ \\
\hline $\mathrm{Ru}-106$ & $7.1340 e-006$ \\
\hline$S b-125$ & $1.6250 e-001$ \\
\hline$S r-90$ & $5.1250 e+000$ \\
\hline$U-234$ & $6.9300 e-003$ \\
\hline$U-235$ & $1.5260 \mathrm{e}-004$ \\
\hline$U-236$ & $7.2200 e-005$ \\
\hline$U-238$ & $2.2600 e-005$ \\
\hline$Y-90$ & $5.1250 e+000$ \\
\hline
\end{tabular}

\section{Activity \\ Bequerels}

$1.2821 e+010$

$1.3353 e+012$

$2.8660 \mathrm{e}+001$

$2.9844 \mathrm{e}+008$

$5.3132 e+007$

$1.4116 e+012$

$1.8759 e+008$

$2.8660 e+001$

$7.6701 e+011$

$4.1144 \mathrm{e}+010$

$1.2913 e+010$

2. $6614 \mathrm{e}+011$

$1.9177 e+007$

2. $6396 e+005$

$2.6396 \mathrm{e}+005$

$6.0125 e+009$

$1.8963 e+011$

$2.5641 e+008$

$5.6462 e+006$

2. $6714 \mathrm{e}+006$

8. 3620 et 005

1. $8963 e+011$

Total
Heat Generation Watts/Curie

$3.2755 e-002$

$3.5403 e-003$

$6.0991 e-004$

1. $5417 \mathrm{e}-002$

$1.0149 e-002$

$1.1086 e-003$

$2.8548 e-002$

$7.3470 e-003$

3. $2552 \mathrm{e}-002$

$3.0531 e-002$

3. $0591 e-002$

$3.1773 e-005$

$2.9012 e-002$

$9.5865 e-003$

$5.9466 e-005$

$3.0911 e-003$

$1.1603 e-003$

$2.8232 e-002$

$2.7030 e-002$

$2.6729 e-002$

$2.4838 e-002$

$5.5407 e-003$

Heat Generation: $\frac{\text { Heat Generation }}{\text { Watts }}$

$1.1350 \mathrm{e}-002$

$1.2777 e-001$

$4.7244 e-013$

$1.2435 \mathrm{e}-004$

$1.4574 \mathrm{e}-005$

$4.2291 e-002$

$1.4474 \mathrm{e}-004$

$5.6910 \mathrm{e}-012$

$6.7481 e-001$

$3.3951 e-002$

$1.0676 e-002$

$2.2854 \mathrm{e}-004$

$1.5037 e-005$

$6.8390 e-008$

$4.2423 e-010$

$5.0231 e-004$

$5.9465 e-003$

$1.9565 e-004$

$4.1248 e-006$

$1.9299 e-006$

$5.6134 e-007$

$2.8396 e-002$

$9.3642 e-001$ 
Microshield v5.01 (5.01-00121)

Lockheed Martin Idaho Technologies Company

Page : 1

DoS File: WM181171.MS5

Run Date: September 24, 1998

Run Time: 9:25:54 AM

File Ref: HkP-18-98

Date:

By:

Duration: 00:02:06

Case Title: FM181-107

Description: UDS slurry tank (T-108) with $249 \mathrm{~kg}$ stream 107 (Dil) Geometry: 7 - Cylinder Volume - side Shields

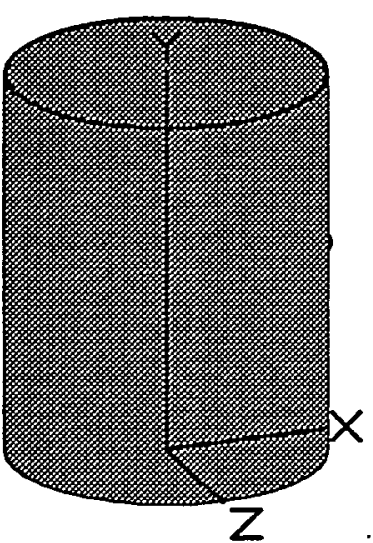

Source Input

Grouping Method : standard Indices

Number of Groups : 25

Lower Energy Cutoff : 0.015

Photons $<0.015$ : Excluded

Iibrary : ICRP-38

Nuclide

$\mathrm{Am}-241$

curies

$2.9680 e-001$

$\mathrm{Ba}-137 \mathrm{~m}$

$3.0910 e+001$

$\mathrm{Ce}-144$

$6.6300 e-010$

Co- 60

$6.9090 e-003$

Cs-134

$1.2300 e-003$

Cs -137

$\mathrm{Np}-237$

$3.2670 e+001$

$4.3400 e-003$

Pr-144

$\mathrm{Pu}-238$

$\mathrm{Pu}-239$

Pu-240

$\mathrm{Pu}-241$

$\mathrm{Pu}-242$

Rh-106

Ru-106

$\mathrm{Sb}-125$

Sr -90

$\mathrm{U}-234$

$\mathrm{U}-235$

$\mathrm{U}-236$

$\mathrm{U}-238$

$\mathrm{Y}-90$ becquerels

$1.0982 \mathrm{e}+010$

$1.1437 e+012$

$2.4531 e+001$

$2.5563 \mathrm{e}+008$

$4.5510 e+007$

$1.2088 \mathrm{e}+012$

$1.6058 \mathrm{e}+008$

$2.4531 \mathrm{e}+001$

$6.5712 e+011$

$3.5254 \mathrm{e}+010$

$1.1074 \mathrm{e}+010$

$2.2796 e+011$

$1.6428 \mathrm{e}+007$

2.2611 e+005

$2.2611 e+005$

$5.1430 e+009$

$1.6243 e+011$

$2.1963 e+008$

$4.8359 e+006$

$2.2885 e+006$

$7.1595 \mathrm{e}+005$

$1.6243 e+011$ $\mu \mathrm{Ci} / \mathrm{cm}^{3}$

$1.6660 \mathrm{e}-001$

$1.7350 e+001$

$3.7215 e-010$

$3.8781 e-003$

$6.9042 e-004$

$1.8338 e+001$

$2.4361 e-003$

$3.7215 e-010$

$9.9689 e+000$

$5.3482 e-001$

$1.6800 e-001$

$3.4583 e+000$

$2.4922 \mathrm{e}-004$

$3.4302 e-006$

$3.4302 e-006$

$7.8023 e-002$

$2.4642 \mathrm{e}+000$

$3.3320 e-003$

$7.3364 e-005$

$1.0861 e-005$

$2.4642 e+000$
$3.4717 e-005$
Source Dimensions

$152.4 \mathrm{~cm}$

5 ft 0.0 in

$61.0 \mathrm{~cm}$

2 ft 0.0 in

Dose Points

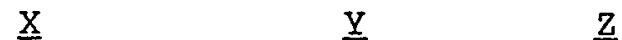

2 ft 6.0 in 0.0 in

$\stackrel{\mathrm{Z}}{0} \mathrm{~cm}$

shields

ield Name Dimension MaterialDensity

$1.78 \mathrm{e}+06 \mathrm{~cm}^{3}$ Mixed $\rightarrow 1.13$

Concrete 0.14

Water 0.99

$.635 \mathrm{~cm}$ Iron 7.86

$1.0 \mathrm{~cm}$ Air 0.0011

Air 0.00122
$\mathrm{Bg} / \mathrm{cm}^{3}$

$6.1641 e+003$

$6.4196 e+005$

$1.3770 e-005$

$1.4349 e+002$

$2.5545 e+001$

$6.7851 e+005$

$9.0136 e+001$

$1.3770 e-005$

$3.6885 e+005$

$1.9788 \mathrm{e}+004$

$6.2160 e+003$

$1.2796 e+005$

$9.2213 e+000$

$1.2692 \mathrm{e}-001$

$1.2692 e-001$

$2.8868 e+003$

$9.1174 \mathrm{e}+004$

$1.2328 e+002$

$2.7145 e+000$

$1.2845 e+000$

$4.0187 e-001$

$9.1174 e+004$ 
Page : 2

DOS File: WM181171.MS5

Run Date: September 24, 1998

Run Time: 9:25:54 AM

Duration: 00:02:06

Buildup

The material reference is : Source

Radial

Integration Parameters

Circumferential

26

$Y$ Direction (axial) 26

\begin{tabular}{|c|c|c|c|c|c|}
\hline Energy & Activity & Fluence Rate & Fluence Rate & Exposure Rate & Exposure Rate \\
\hline $\mathrm{MeV}$ & photons/sec & $\mathrm{MeV} / \mathrm{cm}^{2} / \mathrm{sec}$ & $\mathrm{MeV} / \mathrm{cm}^{2} / \mathrm{sec}$ & $\mathrm{mR} / \mathrm{hr}$ & $\mathrm{mR} / \mathrm{hr}$ \\
\hline & & No Buildup & with Buildup & No Buildup & With Buildup \\
\hline 0.015 & $3.977 e+10$ & $7.905 e-128$ & $9.819 e-21$ & $6.780 e-129$ & $8.422 e-22$ \\
\hline 0.02 & $1.197 e+10$ & $4.889 e-57$ & $8.984 e-20$ & $1.694 e-58$ & $3.112 e-21$ \\
\hline 0.03 & $7.198 e+10$ & $1.498 e-16$ & $6.395 e-12$ & $1.484 e-18$ & $6.338 e-14$ \\
\hline 0.04 & $1.694 e+10$ & $9.645 e-07$ & $1.808 e-02$ & $4.266 e-09$ & $7.994 e-05$ \\
\hline 0.05 & $1.306 e+07$ & $6.636 e-06$ & $3.354 e-02$ & $1.768 e-08$ & $8.934 e-05$ \\
\hline 0.06 & $3.926 e+09$ & $1.425 e-01$ & $2.421 e+02$ & $2.831 e-04$ & $4.810 e-01$ \\
\hline 0.08 & $2.363 e+07$ & $3.751 e-02$ & $1.427 e+01$ & $5.936 e-05$ & $2.258 e-02$ \\
\hline 0.1 & $9.282 e+07$ & $7.418 e-01$ & $1.147 e+02$ & $1.135 e-03$ & $1.755 e-01$ \\
\hline 0.15 & $2.604 e+07$ & $1.073 e+00$ & $5.032 e+01$ & $1.767 e-03$ & $8.287 e-02$ \\
\hline 0.2 & $3.792 e+08$ & $3.210 e+01$ & $8.459 e+02$ & $5.666 e-02$ & $1.493 e+00$ \\
\hline 0.3 & $2.380 e+07$ & $4.498 e+00$ & $6.096 e+01$ & $8.533 e-03$ & $1.156 \mathrm{e}-01$ \\
\hline 0.4 & $1.618 e+09$ & $5.140 e+02$ & $4.949 e+03$ & $1.001 e+00$ & $9.643 e+00$ \\
\hline 0.5 & $5.318 e+08$ & $2.502 e+02$ & $1.821 e+03$ & $4.911 e-01$ & $3.575 e+00$ \\
\hline 0.6 & $1.029 e+12$ & $6.653 e+05$ & $4.002 e+06$ & $1.299 e+03$ & $7.811 e+03$ \\
\hline 0.8 & $4.325 e+07$ & $4.623 e+01$ & $2.140 e+02$ & $8.793 e-02$ & $4.071 e-01$ \\
\hline 1.0 & $2.567 e+08$ & $4.056 e+02$ & $1.554 e+03$ & $7.476 e-01$ & $2.865 e+00$ \\
\hline 1.5 & $2.570 e+08$ & $8.247 e+02$ & $2.411 e+03$ & $1.388 e+00$ & $4.056 e+00$ \\
\hline 2.0 & $3.259 e+03$ & $1.711 \mathrm{e}-02$ & $4.318 e-02$ & $2.646 e-05$ & $6.677 e-05$ \\
\hline 3.0 & $4.716 e+01$ & $4.840 e-04$ & $1.012 e-03$ & $6.566 e-07$ & $1.373 e-06$ \\
\hline TOTALS & $1.176 e+12$ & $6.674 e+05$ & $4.014 e+06$ & $1.302 e+03$ & $7.833 e+03$ \\
\hline
\end{tabular}


Microshield v5.01 (5.01-00121)

Lockheed Martin Idaho Technologies Company

Case File Heat Generation

Case File: WM181171.MS5

Case Title: WM181-107

$\begin{array}{lc}\text { Isotope } & \frac{\text { Activity }}{\text { Curies }} \\ \mathrm{Am}-241 & 2.9680 \mathrm{e}-001 \\ \mathrm{Ba}-137 \mathrm{~m} & 3.0910 \mathrm{e}+001 \\ \mathrm{Ce}-144 & 6.6300 \mathrm{e}-010 \\ \mathrm{Co}-60 & 6.9090 \mathrm{e}-003 \\ \mathrm{Cs}-134 & 1.2300 \mathrm{e}-003 \\ \mathrm{Cs}-137 & 3.2670 \mathrm{e}+001 \\ \mathrm{~Np}-237 & 4.3400 \mathrm{e}-003 \\ \mathrm{Pr}-144 & 6.6300 \mathrm{e}-010 \\ \mathrm{Pu}-238 & 1.7760 \mathrm{e}+001 \\ \mathrm{Pu}-239 & 9.5280 \mathrm{e}-001 \\ \mathrm{Pu}-240 & 2.9930 \mathrm{e}-001 \\ \mathrm{Pu}-241 & 6.1610 \mathrm{e}+000 \\ \mathrm{Pu}-242 & 4.4400 \mathrm{e}-004 \\ \mathrm{Rh}-106 & 6.1110 \mathrm{e}-006 \\ \mathrm{Ru}-106 & 6.1110 \mathrm{e}-006 \\ \mathrm{Sb}-125 & 1.3900 \mathrm{e}-001 \\ \mathrm{Sr}-90 & 4.3900 \mathrm{e}+000 \\ \mathrm{U}-234 & 5.9360 \mathrm{e}-003 \\ \mathrm{U}-235 & 1.3070 \mathrm{e}-004 \\ \mathrm{U}-236 & 6.1850 \mathrm{e}-005 \\ \mathrm{U}-238 & 1.9350 \mathrm{e}-005 \\ \mathrm{Y}-90 & 4.3900 \mathrm{e}+000\end{array}$

\section{Activity \\ Bequerels}

$1.0982 e+010$

1.1437 e+012

2.4531 e+001

$2.5563 e+008$

$4.5510 e+007$

$1.2088 e+012$

$1.6058 \mathrm{e}+008$

2.4531 e+001

$6.5712 e+011$

3.5254 e +010

1.1074 et010

$2.2796 e+011$

$1.6428 e+007$

2.2611e+005

2.2611 e+005

$5.1430 e+009$

1. $6243 \mathrm{e}+011$

$2.1963 e+008$

$4.8359 e+006$

2.2885 et006

$7.1595 e+005$

$1.6243 e+011$

Total
Heat Generation Watts/Curie

$3.2755 \mathrm{e}-002$

$3.5403 e-003$

$6.0991 e-004$

$1.5417 e-002$

$1.0149 e-002$

$1.1086 \mathrm{e}-003$

$2.8548 e-002$

$7.3470 e-003$

$3.2552 e-002$

$3.0531 e-002$

$3.0591 e-002$

$3.1773 e-005$

$2.9012 e-002$

$9.5865 e-003$

$5.9466 e-005$

$3.0911 \mathrm{e}-003$

$1.1603 e-003$

$2.8232 e-002$

$2.7030 e-002$

$2.6729 e-002$

$2.4838 e-002$

$5.5407 e-003$

Heat Generation:
Heat Generation Watts

$9.7216 \mathrm{e}-003$

$1.0943 e-001$

$4.0437 e-013$

$1.0651 \mathrm{e}-004$

$1.2483 e-005$

$3.6216 e-002$

$1.2390 e-004$

4.8710e-012

$5.7813 e-001$

$2.9090 e-002$

$9.1560 e-003$

$1.9575 e-004$

$1.2881 \mathrm{e}-005$

$5.8583 e-008$

$3.6340 e-010$

$4.2967 e-004$

$5.0937 e-003$

$1.6759 \mathrm{e}-004$

$3.5329 e-006$

1. $6532 \mathrm{e}-006$

$4.8061 e-007$

$2.4324 e-002$

$8.0221 e-001$ 
Microshield v5.01 (5.01-00121)

Lockheed Martin Idaho Technologies Company

Page : 1

DOS File: 181108SL.MS5

Run Date: September 24, 1998

Run Time: 10:01:15 AM

File Ref: $4 K \mathrm{~N}_{-18-98}$

Date:

By:

Duration: 00:03:10

Checked:

Case Title: Cs I Exch Col

Description: Cs Ion Exchange column with $270 \mathrm{~kg}$ of stream 108 Geometry: 7 - Cylinder volume - Side shields

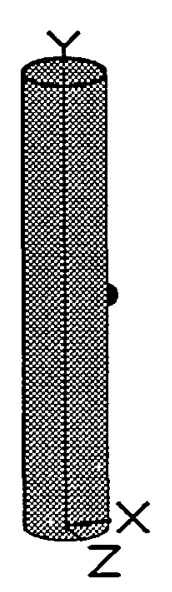

Source Input
Grouping Method : Standard Indic
Number of Groups : 25
Lower Energy Cutoff : 0.015
photons $0.015:$ Excluded

Iibrary : ICRP-38

Nuclide

Am-241

$\mathrm{Ba}-137 \mathrm{~m}$

$\mathrm{Ce}-144$

Co-60

Cs -134

Cs -135

Cs -137

$\mathrm{Np}-237$

$\mathrm{Pr}-144$

$\mathrm{Pu}-238$

$\mathrm{Pu}-239$

$\mathrm{Pu}-240$

$\mathrm{Pu}-241$

$\mathrm{Pu}-242$

$\mathrm{Rh}-106$

$\mathrm{Ru}-106$

$\mathrm{Sb}-125$

$\mathrm{S} x-90$

$\mathrm{U}-234$

$\mathrm{U}-235$

$\mathrm{U}-236$

$\mathrm{U}-238$

$\mathrm{Y}-90$ becquerels

$3.3781 e+009$

$1.8618 e+014$

$7.5480 e+000$

$7.8810 e+007$

$1.6184 \mathrm{e}+011$

$3.6149 e+009$

$1.9680 e+014$

$4.9580 e+007$

$7.5480 e+000$

$2.0280 e+011$

$1.0889 e+010$

$3.4166 e+009$

$7.0522 e+010$

$5.0838 e+006$

$6.9819 e+004$

$6.9819 e+004$

$1.5884 e+009$

$5.0135 e+010$

$6.7821 e+007$

$1.4874 \mathrm{e}+006$

$7.0670 e+005$

$2.2078 e+005$

$5.0135 e+010$ $\underline{\mu \mathrm{Ci} / \mathrm{cm}^{3}}$

$2.8865 e-001$

$1.5909 e+004$

$6.4495 e-010$

$6.7341 \mathrm{e}-003$

$1.3829 e+001$

$3.0888 e-001$

$1.6816 \mathrm{e}+004$

$4.2365 e-003$

$6.4495 e-010$

1. $7328 \mathrm{e}+001$

$9.3044 e-001$

$2.9194 e-001$

$6.0259 e+000$

$4.3439 e-004$

$5.9658 e-006$

$5.9658 \mathrm{e}-006$

$1.3572 \mathrm{e}-001$

$4.2839 e+000$

$5.7951 e-003$

$1.2709 e-004$

$6.0385 e-005$

$1.8865 \mathrm{e}-005$

$4.2839 \mathrm{e}+000$
$\mathrm{Ba} / \mathrm{cm}^{3}$

$1.0680 e+004$

$5.8863 e+008$

$2.3863 e-005$

$2.4916 e+002$

$5.1166 e+005$

$1.1429 e+004$

$6.2220 e+008$

1. $5675 e+002$

$2.3863 e-005$

$6.4115 e+005$

$3.4426 e+004$

$1.0802 e+004$

$2.2296 e+005$

$1.6073 e+001$

$2.2074 e-001$

$2.2074 \mathrm{e}-001$

$5.0218 e+003$

$1.5850 e+005$

$2.1442 e+002$

$4.7025 e+000$

$2.2343 e+000$

$6.9800 e-001$

$1.5850 e+005$
$8 \mathrm{ft}$

8.0 in
$\underline{\mathrm{Z}}$
$0 \mathrm{~cm}$
$0.0 \mathrm{in}$ 
Page: 2

DOS File: 181108SL.MS5

Run Date: September 24, 1998

Run Time: 10:01:15 AM

Duration: 00:03:10

Buildup

The material reference is : Source

Radial

Integration Parameters

Circumferential

29

$Y$ Direction (axial) 31

\begin{tabular}{|c|c|c|c|c|c|}
\hline Energy & Activity & Fluence Rate & $\begin{array}{l}\text { Results } \\
\text { Fluence Rate }\end{array}$ & Exposure Rate & Exposure Rate \\
\hline $\mathrm{MeV}$ & photons/sec & $\mathrm{MeV} / \mathrm{cm}^{2} / \mathrm{sec}$ & $\mathrm{MeV} / \mathrm{cm}^{2} / \mathrm{sec}$ & $\mathrm{mR} / \mathrm{hr}$ & $\mathrm{mR} / \mathrm{hr}$ \\
\hline & & No Buildup & With Buildup & No Buildup & With Buildup \\
\hline 0.015 & $1.227 e+10$ & $5.126 e-187$ & $4.643 e-22$ & $4.397 e-188$ & $3.982 e-23$ \\
\hline 0.02 & $3.690 e+09$ & $9.738 \dot{e}-84$ & $1.315 e-21$ & $3.373 e-85$ & $4.556 e-23$ \\
\hline 0.03 & $1.128 e+13$ & $2.193 e-22$ & $2.404 e-16$ & $2.173 e-24$ & $2.383 e-18$ \\
\hline 0.04 & $2.679 e+12$ & $7.342 e-08$ & $2.718 e-04$ & $3.247 e-10$ & $1.202 e-06$ \\
\hline 0.05 & $4.033 e+06$ & $5.259 e-08$ & $1.258 e-04$ & $1.401 \mathrm{e}-10$ & $3.350 e-07$ \\
\hline 0.06 & $1.208 e+09$ & $6.693 e-03$ & $7.789 e+00$ & $1.329 e-05$ & $1.547 e-02$ \\
\hline 0.08 & 7.294 e+06 & $7.475 e-03$ & $2.357 e+00$ & $1.183 e-05$ & $3.730 e-03$ \\
\hline 0.1 & $2.865 e+07$ & $2.516 e-01$ & $3.267 e+01$ & $3.850 e-04$ & $4.998 e-02$ \\
\hline 0.15 & $8.041 e+06$ & $5.570 e-01$ & $2.276 e+01$ & $9.172 e-04$ & $3.748 e-02$ \\
\hline 0.2 & $1.511 e+08$ & $2.438 e+01$ & $5.250 e+02$ & $4.303 e-02$ & $9.266 e-01$ \\
\hline 0.3 & $3.065 e+07$ & $1.211 e+01$ & $1.414 \mathrm{e}+02$ & $2.297 e-02$ & $2.683 e-01$ \\
\hline 0.4 & $4.998 e+08$ & $3.457 e+02$ & $2.854 e+03$ & $6.736 e-01$ & $5.562 e+00$ \\
\hline 0.5 & $2.527 e+09$ & $2.657 e+03$ & $1.727 e+04$ & $5.215 e+00$ & $3.390 e+01$ \\
\hline 0.6 & $1.673 e+14$ & $2.466 e+08$ & $1.343 e+09$ & $4.813 e+05$ & $2.622 e+06$ \\
\hline 0.8 & $1.523 e+11$ & $3.806 e+05$ & $1.613 e+06$ & $7.240 e+02$ & $3.068 e+03$ \\
\hline 1.0 & $4.610 e+09$ & $1.733 e+04$ & $6.195 e+04$ & $3.194 e+01$ & $1.142 e+02$ \\
\hline 1.5 & $4.999 e+09$ & $3.907 e+04$ & $1.078 e+05$ & $6.573 e+01$ & $1.813 e+02$ \\
\hline 2.0 & $1.005 e+03$ & $1.295 e-02$ & $3.077 e-02$ & $2.003 e-05$ & $4.758 e-05$ \\
\hline 3.0 & $1.456 e+01$ & $3.677 e-04$ & $7.319 e-04$ & $4.989 e-07$ & $9.929 e-07$ \\
\hline TOTALS & $1.815 e+14$ & $2.470 e+08$ & $1.345 e+09$ & $4.821 e+05$ & $2.626 e+06$ \\
\hline
\end{tabular}


Microshield v5.01 (5.01-00121)

Lockheed Martin Idaho Technologies Company Case File Heat Generation

Case File: 181108SL.MS5

Case Title: Cs I Exch Col

\begin{tabular}{|c|c|}
\hline Isotope & $\frac{\text { Activity }}{\text { curies }}$ \\
\hline $\mathrm{Am}-241$ & $9.1300 e-002$ \\
\hline $\mathrm{Ba}-137 \mathrm{~m}$ & $5.0320 e+003$ \\
\hline $\mathrm{Ce}-144$ & $2.0400 e-010$ \\
\hline Co-6o & $2.1300 e-003$ \\
\hline Cs -134 & $4.3740 e+000$ \\
\hline Cs -135 & $9.7700 \mathrm{e}-002$ \\
\hline Cs -137 & $5.3190 e+003$ \\
\hline$N p-237$ & $1.3400 \mathrm{e}-003$ \\
\hline Pr -144 & $2.0400 e-010$ \\
\hline $\mathrm{Pu}-238$ & $5.4810 e+000$ \\
\hline $\mathrm{Pu}-239$ & $2.9430 e-001$ \\
\hline$P u-240$ & $9.2340 \mathrm{e}-002$ \\
\hline $\mathrm{Pu}-241$ & $1.9060 e+000$ \\
\hline$P u-242$ & $1.3740 e-004$ \\
\hline$R h-106$ & $1.8870 e-006$ \\
\hline$R u-106$ & $1.8870 e-006$ \\
\hline$S b-125$ & $4.2930 e-002$ \\
\hline Sr-90 & $1.3550 e+000$ \\
\hline $\mathrm{U}-234$ & $1.8330 e-003$ \\
\hline$U-235$ & $4.0200 e-005$ \\
\hline$U-236$ & $1.9100 e-005$ \\
\hline$U-238$ & $5.9670 e-006$ \\
\hline$Y-90$ & $1.3550 e+000$ \\
\hline
\end{tabular}

\section{Activity \\ Bequerels}

$3.3781 e+009$

$1.8618 e+014$

$7.5480 e+000$

$7.8810 e+007$

$1.6184 \mathrm{e}+011$

$3.6149 e+009$

$1.9680 e+014$

$4.9580 e+007$

$7.5480 \mathrm{e}+000$

$2.0280 e+011$

$1.0889 e+010$

$3.4166 \mathrm{e}+009$

$7.0522 \mathrm{e}+010$

$5.0838 e+006$

$6.9819 e+004$

$6.9819 e+004$

$1.5884 \mathrm{e}+009$

$5.0135 e+010$

$6.7821 e+007$

$1.4874 \mathrm{e}+006$

$7.0670 e+005$

$2.2078 e+005$

$5.0135 e+010$

Total

\section{Heat Generation Watts/Curie}

$3.2755 e-002$

$3.5403 e-003$

$6.0991 e-004$

$1.5417 \mathrm{e}-002$

$1.0149 e-002$

3. $9913 e-004$

$1.1086 e-003$

$2.8548 e-002$

$7.3470 e-003$

$3.2552 e-002$

$3.0531 e-002$

3.0591e-002

$3.1773 e-005$

$2.9012 e-002$

$9.5865 e-003$

$5.9466 e-005$

$3.0911 e-003$

$1.1603 e-003$

$2.8232 e-002$

2. $7030 e-002$

2. $6729 e-002$

$2.4838 e-002$

$5.5407 e-003$

Heat Generation:

\section{Heat Generation Watts}

$2.9905 e-003$

$1.7815 \mathrm{e}+001$

$1.2442 \mathrm{e}-013$

$3.2837 e-005$

$4.4391 e-002$

$3.8995 e-005$

$5.8964 \mathrm{e}+000$

$3.8255 e-005$

$1.4988 \mathrm{e}-012$

$1.7842 e-001$

$8.9854 e-003$

$2.8248 e-003$

$6.0559 e-005$

$3.9862 e-006$

$1.8090 e-008$

$1.1221 \mathrm{e}-010$

$1.3270 e-004$

$1.5722 \mathrm{e}-003$

$5.1750 e-005$

$1.0866 e-006$

$5.1053 e-007$

$1.4821 \mathrm{e}-007$

$7.5076 e-003$

$2.3958 e+001$ 
Microshield v5.01 (5.01-00121)

Lockheed Martin Idaho Technologies Company

Page : 1

DOS File: 181106SI.MS5

Run Date: September 24, 1998

Run Time: 1:56:10 PM

File Ref:

Date:

By:

Checked:

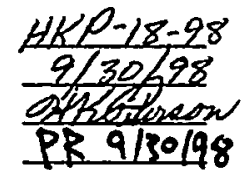

Duration: $00: 03: 57$

Case Title: Cs IX Effluent tnk

Description: Cs IX Effluent tank (T-103 A\&B) w/14234 I st \#106 Geometry: 7 - Cylinder volume - side shields

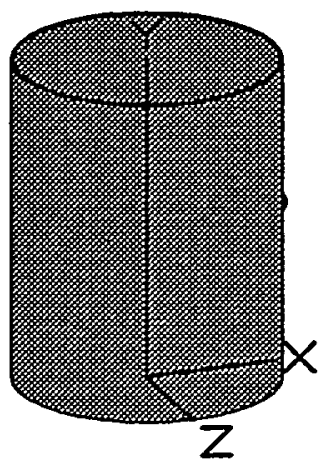

Source Input

Grouping Method : Standard Indices

Number of Groups : 25

Lower Energy Cutoff : 0.015

Photons < 0.015 : Excluded

Iibrary : ICRP-38

Nuclide

Am-241

$\mathrm{Am}-243$

$\mathrm{Ba}-137 \mathrm{~m}$

$\mathrm{Ce}-144$

Cm-242

Cm-244

Co-60

Cs -134

Cs -135

Cs -137

Eu-152

Eu-154

Eu-155

$\mathrm{H}-3$

$I-129$

$\mathrm{Ni}-63$

$\mathrm{Np}-237$

Pm-147

Pr-144

$\mathrm{Pu}-238$

$\mathrm{Pu}-239$

$\mathrm{Pu}-240$

$\mathrm{Pu}-241$

$\mathrm{Pu}-242$

$\mathrm{Rh}-106$ becquerels

$2.4490 e+011$

$1.1059 \mathrm{e}+008$

$2.5759 e+010$

$3.1443 e+010$

$5.7942 \mathrm{e}+008$

$7.7959 e+009$

$2.7121 e+010$

$2.2644 \mathrm{e}+007$

$5.0283 e+005$

$2.7228 \mathrm{e}+010$

$1.4800 e+009$

$8.5840 e+010$

$3.7333 e+010$

$1.5906 \mathrm{e}+010$

$3.6334 \mathrm{e}+009$

$2.6018 e+010$

$1.3431 e+009$

$7.2150 e+010$

$3.1443 e+010$

$4.5362 e+011$

$3.4595 e+010$

$1.0005 e+010$

$2.0013 e+011$

$7.7330 \mathrm{e}+006$

$1.7205 e+008$ $\mu \mathrm{Ci} / \mathrm{cm}^{3}$

$4.6503 e-001$

$2.1000 e-004$

$4.8912 e-002$

$5.9704 \mathrm{e}-002$

$1.1002 e-003$

$1.4803 e-002$

$5.1498 e-002$

$4.2997 e-005$

$9.5478 \mathrm{e}-007$

$5.1702 e-002$

$2.8103 e-003$

$1.6299 \mathrm{e}-001$

$7.0889 e-002$

$3.0203 e-002$

$6.8992 e-003$

$4.9404 e^{-002}$

$2.5503 e-003$

$1.3700 e-001$

$5.9704 e-002$

8. $6134 \mathrm{e}-001$

$6.5690 e-002$

$1.8997 e-002$

$3.8002 e-001$

$1.4684 e-005$

$3.2669 e-004$
$\mathrm{Bg} / \mathrm{cm}^{3}$

$1.7206 e+004$

$7.7699 e+000$

$1.8098 \mathrm{e}+003$

$2.2090 e+003$

$4.0708 e+001$

$5.4771 e+002$

$1.9054 \mathrm{e}+003$

$1.5909 e+000$

$3.5327 e-002$

$1.9130 e+003$

$1.0398 \mathrm{e}+002$

$6.0308 e+003$

$2.6229 e+003$

$1.1175 \mathrm{e}+003$

$2.5527 e+002$

$1.8280 e+003$

9.4361 e+001

$5.0690 e+003$

2. $2090 e+003$

3. $1870 e+004$

$2.4305 e+003$

$7.0290 \mathrm{e}+002$

$1.4061 \mathrm{e}+004$

$5.4329 e-001$

$1.2088 e+001$ 
Page : 2

DOS File: 181106SI.MS5

Run Date: September 24, 1998

Run Time: 1:56:10 PM

Duration: 00:03:57

\begin{tabular}{|c|c|c|c|c|}
\hline Nuclide & curies & becquerels & $\mu \mathrm{Ci} / \mathrm{cm}^{3}$ & $\mathrm{Bg} / \mathrm{cm}^{3}$ \\
\hline $\mathrm{Ru}-106$ & $4 . \overline{6500 e-003}$ & $1.7205 e+008$ & $3.2669 e-004$ & $1 . \overline{2088 e+001}$ \\
\hline$S b-125$ & $1.5500 \mathrm{e}-001$ & 5.7350 e+009 & $1.0890 e-002$ & $4.0292 e+002$ \\
\hline$S m-151$ & $6.8750 \mathrm{e}+000$ & $2.5438 e+011$ & $4.8301 e-001$ & 1.7871 e+004 \\
\hline $\mathrm{Sr}-90$ & $4.8820 e+002$ & $1.8063 e+013$ & $3.4299 e+001$ & $1.2691 e+006$ \\
\hline TC-99 & $2.1200 e-001$ & $7.8440 e+009$ & $1.4894 e-002$ & $5.5109 e+002$ \\
\hline$T h-230$ & $9.7360 e-006$ & $3.6023 e+005$ & $6.8402 e-007$ & $2.5309 e-002$ \\
\hline$T h-231$ & $1.3950 \mathrm{e}-003$ & $5.1615 e+007$ & $9.8008 e-005$ & $3.6263 e+000$ \\
\hline $\mathrm{U}-232$ & $2.7470 e-005$ & $1.0164 e+006$ & $1.9299 e-006$ & $7.1408 e-002$ \\
\hline $\mathrm{U}-233$ & $1.4800 e-007$ & $5.4760 e+003$ & $1.0398 e-008$ & $3.8472 e-004$ \\
\hline $\mathrm{U}-234$ & $1.3940 e-002$ & $5.1578 e+008$ & $9.7937 e-004$ & $3.6237 e+001$ \\
\hline$U-235$ & $3.4700 e-004$ & $1.2839 e+007$ & $2.4379 e-005$ & $9.0202 e-001$ \\
\hline $\mathrm{U}-236$ & $6.7750 e-004$ & $2.5068 e+007$ & $4.7599 e-005$ & $1.7611 e+000$ \\
\hline$U-238$ & $3.7440 e-004$ & $1.3853 e+007$ & $2.6304 e-005$ & $9.7325 e-001$ \\
\hline$Y-90$ & 4.8820 e+002 & $1.8063 e+013$ & $3.4299 e+001$ & $1.2691 \mathrm{e}+006$ \\
\hline
\end{tabular}

\section{Integration parameters}

Radial

Circumferential

$Y$ Direction (axial)
32

32

31

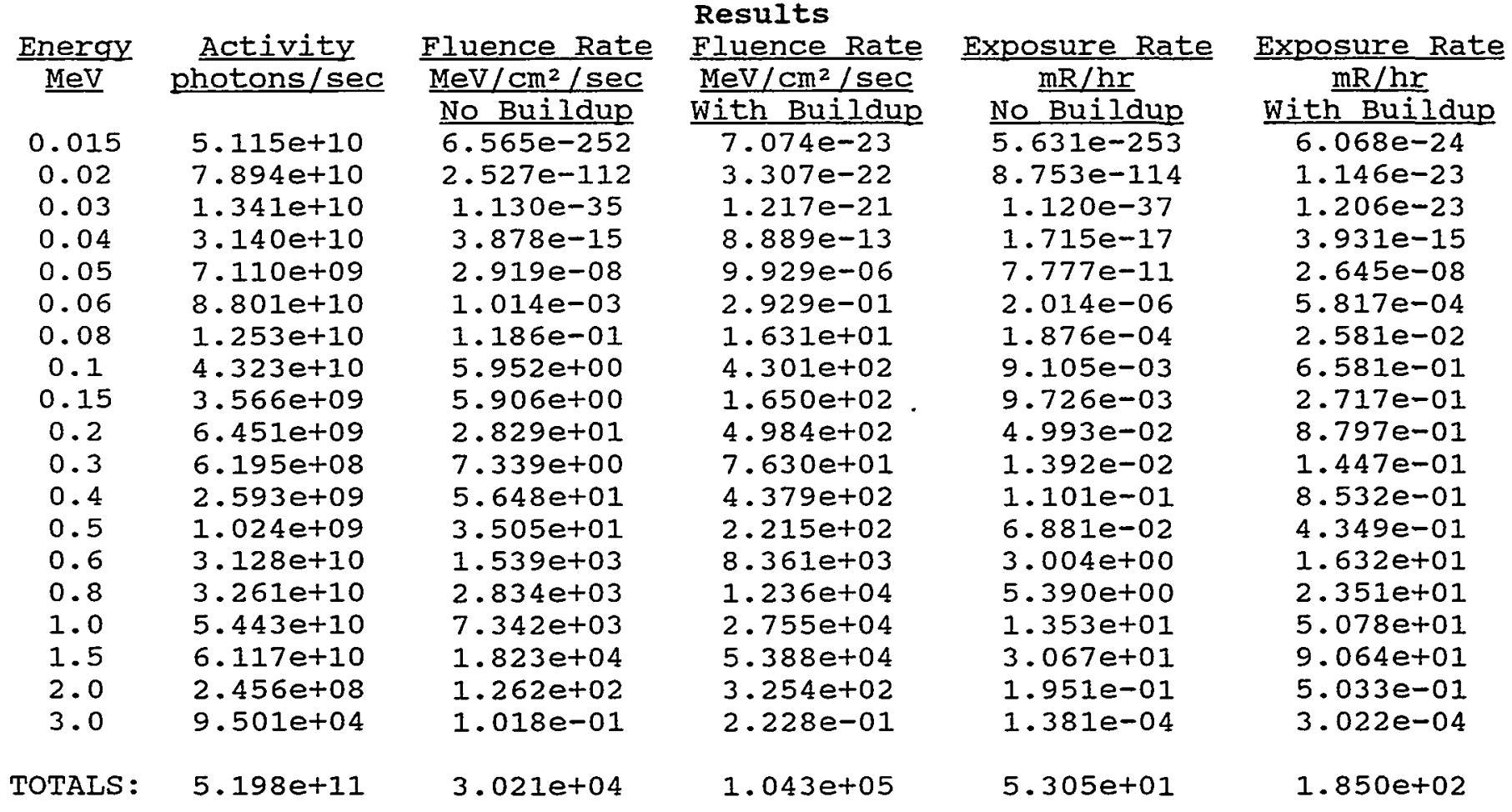


Microshield v5.01 (5.01-00121)

Lockheed Martin Idaho Technologies Company Case File Heat Generation

Case File: 181106SL.MS5

Case Title: Cs IX Effluent tnk

\begin{tabular}{|c|c|}
\hline Isotope & $\frac{\text { Activity }}{\text { Curies }}$ \\
\hline$A m-241$ & $6.6190 \mathrm{e}+000$ \\
\hline$A m-243$ & $2.9890 \mathrm{e}-003$ \\
\hline $\mathrm{Ba}-137 \mathrm{~m}$ & $6.9620 e-001$ \\
\hline $\mathrm{Ce}-144$ & $8.4980 e-001$ \\
\hline $\mathrm{Cm}-242$ & $1.5660 e-002$ \\
\hline $\mathrm{Cm}-244$ & $2.1070 e-001$ \\
\hline Co-60 & $7.3300 \mathrm{e}-001$ \\
\hline Cs -134 & $6.1200 e-004$ \\
\hline$C s-135$ & $1.3590 e-005$ \\
\hline Cs -137 & $7.3590 e-001$ \\
\hline$E u-152$ & $4.0000 e-002$ \\
\hline$E u-154$ & $2.3200 e+000$ \\
\hline $\mathrm{Eu}-155$ & $1.0090 e+000$ \\
\hline $\mathrm{H}-3$ & $4.2990 e-001$ \\
\hline$I-129$ & $9.8200 e-002$ \\
\hline $\mathrm{Ni}-63$ & $7.0320 e-001$ \\
\hline $\mathrm{Np}-237$ & $3.6300 e-002$ \\
\hline$P m-147$ & $1.9500 \mathrm{e}+000$ \\
\hline $\operatorname{Pr}-144$ & $8.4980 e-001$ \\
\hline $\mathrm{Pu}-238$ & $1.2260 \mathrm{e}+001$ \\
\hline$P u-239$ & $9.3500 e-001$ \\
\hline $\mathrm{Pu}-240$ & $2.7040 e-001$ \\
\hline$P u-241$ & $5.4090 e+000$ \\
\hline$P u-242$ & $2.0900 e-004$ \\
\hline$R h-106$ & $4.6500 e-003$ \\
\hline $\mathrm{Ru}-106$ & $4.6500 e-003$ \\
\hline$S b-125$ & $1.5500 \mathrm{e}-001$ \\
\hline$S m-151$ & $6.8750 e+000$ \\
\hline$s r-90$ & $4.8820 e+002$ \\
\hline TC-99 & $2.1200 e-001$ \\
\hline$T h-230$ & $9.7360 e-006$ \\
\hline Th-231 & $1.3950 e-003$ \\
\hline$U-232$ & $2.7470 e-005$ \\
\hline $\mathrm{U}-233$ & $1.4800 e-007$ \\
\hline$U-234$ & $1.3940 e-002$ \\
\hline $\mathrm{U}-235$ & $3.4700 e-004$ \\
\hline $\mathrm{U}-236$ & $6.7750 e-004$ \\
\hline$U-238$ & $3.7440 e-004$ \\
\hline$Y-90$ & $4.8820 e+002$ \\
\hline
\end{tabular}

$\frac{\text { Activity }}{\text { Bequerels }}$

$2.4490 e+011$

$1.1059 e+008$

$2.5759 e+010$

$3.1443 e+010$

$5.7942 e+008$

$7.7959 e+009$

$2.7121 e+010$

$2.2644 e+007$

$5.0283 e+005$

2.7228 e+010

$1.4800 e+009$

8.5840 et010

$3.7333 e+010$

$1.5906 \mathrm{e}+010$

$3.6334 \mathrm{e}+009$

2. $6018 e+010$

1.3431 e+009

7.2150 e+010

$3.1443 e+010$

4.5362 e+011

$3.4595 e+010$

$1.0005 e+010$

$2.0013 e+011$

7.7330 e+006

$1.7205 e+008$

1.7205 e+008

$5.7350 e+009$

$2.5438 e+011$

$1.8063 e+013$

7.8440 e+009

$3.6023 e+005$

$5.1615 e+007$

1.0164 e+006

$5.4760 e+003$

5.1578 e+008

$1.2839 e+007$

$2.5068 \mathrm{e}+007$

$1.3853 \mathrm{e}+007$

$1.8063 e+013$

Total

\section{Heat Generation Watts/Curie}

$3.2755 e-002$

$3.1599 e-002$

$3.5403 e-003$

$6.0991 e-004$

$3.6195 e-002$

$3.4375 \mathrm{e}-002$

$1.5417 \mathrm{e}-002$

$1.0149 e-002$

$3.9913 e-004$

$1.1086 \mathrm{e}-003$

$7.3864 \mathrm{e}-003$

$8.7221 e-003$

$6.4902 e-004$

$3.3688 e-005$

$4.7222 \mathrm{e}-004$

$1.0154 \mathrm{e}-004$

$2.8548 \mathrm{e}-002$

$3.6732 e-004$

$7.3470 e-003$

$3.2552 e-002$

$3.0531 e-002$

$3.0591 e-002$

$3.1773 e-005$

2. $9012 e-002$

$9.5865 e-003$

$5.9466 \mathrm{e}-005$

$3.0911 \mathrm{e}-003$

$1.1669 e-004$

$1.1603 e-003$

$6.0024 \mathrm{e}-004$

$2.7709 e-002$

$7.1148 \mathrm{e}-004$

$3.1460 e-002$

$2.8571 e-002$

$2.8232 \mathrm{e}-002$

$2.7030 e-002$

2. $6729 e-002$

$2.4838 \mathrm{e}-002$

$5.5407 e-003$

Heat Generation:
Heat Generation Watts

$2.1680 e-001$

$9.4451 e-005$

$2.4647 e-003$

$5.1830 e-004$

$5.6682 e-004$

$7.2429 e-003$

$1.1300 e-002$

$6.2111 \mathrm{e}-006$

$5.4241 e-009$

8.1579e-004

$2.9546 e-004$

2. $0235 e-002$

$6.5486 e-004$

$1.4482 \mathrm{e}-005$

$4.6372 e-005$

$7.1406 \mathrm{e}-005$

$1.0363 e-003$

$7.1628 \mathrm{e}-004$

$6.2435 e-003$

$3.9909 e-001$

$2.8547 e-002$

$8.2719 e-003$

$1.7186 e-004$

$6.0635 e-006$

$4.4577 e-005$

$2.7652 e-007$

$4.7913 e-004$

$8.0221 e-004$

$5.6646 \mathrm{e}-001$

$1.2725 e-004$

2. $6978 \mathrm{e}-007$

$9.9251 e-007$

8. $6422 e-007$

$4.2285 e-009$

$3.9356 e-004$

$9.3796 e-006$

$1.8109 e-005$

$9.2993 e-006$

2.7050 e+000

$3.9785 e+000$ 
Microshiela v5.01 (5.01-00121)

Iockheed Martin Idaho Technologies Company

Page : 1

DOS File: T112108.MS5

Run Date: September 24, 1998

Run Time: 2:16:24 PM

Date:

By:

Duration: 00:04:05

Case Title: T-112 spent Resin TK

Description: T-112 spent Resin storage Tank with $23,112 \mathrm{~kg}$ of st \# 108 Geometry: 7 - cylinder Volume - side shields

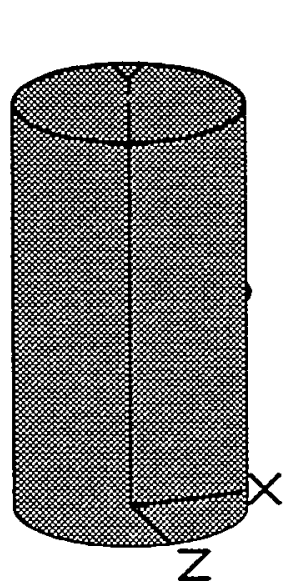

Source Dimensions

Source Input

Grouping Method : Standard Indices

Number of Groups : 25

Lower Energy Cutoff : 0.015

Photons < 0.015 : Excluded

Library : ICRP-38

\begin{tabular}{|c|c|c|c|c|}
\hline Nuclide & curies & becquerels & $\mu \mathrm{Ci} / \mathrm{cm}^{3}$ & $\mathrm{Bg} / \mathrm{cm}^{3}$ \\
\hline$A m-241$ & $7.8119 e+000$ & $2.8904 e+011$ & $3.6589 e-001$ & $1.3538 e+004$ \\
\hline$B a-137 m$ & $4.3070 e+005$ & $1.5936 e+016$ & $2.0173 e+004$ & 1008 \\
\hline $\mathrm{Ce}-144$ & $00-008$ & 6.47 & $8.1966 e-$ & \\
\hline $\mathrm{Co}-60$ & .8260 e-001 & 6.7 & $8.5525 e-003$ & 002 \\
\hline$s-134$ & $0 e+002$ & 13 & 1.7644 & \\
\hline$C s-135$ & $0 e+000$ & 3.0 & 3.9189 & L. $4500 e$ \\
\hline$C s-137$ & $0 e+005$ & 1.6 & 2.13 & 1.090 \\
\hline$N p-237$ & pe-001 & 4.25 & $5.3816 e$ & 1.9912 \\
\hline Pr-144 & $e-008$ & 6.4 & 8.19 & .0327 \\
\hline $\mathrm{Pu}-238$ & bet002 & 1.7 & 2.1976 & .1312 \\
\hline$P u-239$ & $e+001$ & 9.3 & 1.17 & \\
\hline $\mathrm{Pu}-240$ & be+000 & 2.9 & 3.7020 & 1.3098 \\
\hline $\mathrm{Pu}-241$ & $e+002$ & 6.0 & 7.6439 & \\
\hline$P u-242$ & je-002 & $4 \cdot 3$ & $5.5081 \epsilon$ & 1 \\
\hline $\mathrm{Rh}-106$ & $1.6160 e-004$ & 5.9 & 7.5689 & .8005 \\
\hline $\mathrm{Ru}-106$ & $0 e-004$ & 5.9 & $7.5689 e-$ & 1 \\
\hline $\mathrm{Sb}-125$ & 3.6750 eto00 & +011 & $1.7213 e-$ & $6.3687 e+c$ \\
\hline $\mathrm{Sr}-90$ & $1.1600 e+002$ & $e+012$ & $5.4332 \mathrm{e}$ & \\
\hline$U-234$ & $1.5690 e-001$ & +009 & $7.3488 e-003$ & $2.7191 \mathrm{e}+\mathrm{c}$ \\
\hline $\mathrm{U}-235$ & $3.4440 e-003$ & 1.2 & & \\
\hline $\mathrm{U}-236$ & $5.7300 e-003$ & $e+008$ & $2.6838 e-004$ & $9.9300 e+000$ \\
\hline$U-238$ & $5.1000 e-004$ & & $2.3887 e-005$ & \\
\hline$Y-90$ & 1.1600 et 002 & 4.2920 eto12 & $5.4332 e+000$ & $2.0103 e+005$ \\
\hline
\end{tabular}




$\begin{array}{ll}\text { Page }: & 2 \\ \text { DOS File: } & \text { T112108.MS5 } \\ \text { Run Date: September 24, } 1998 \\ \text { Run Time: } 2: 16: 24 \text { PM } \\ \text { Duration: } 00: 04: 05\end{array}$

Buildup

The material reference is : source

\begin{tabular}{ll}
\multicolumn{2}{c}{ Integration Parameters } \\
Radial 31 \\
Circumferential & 31 \\
$Y$ Direction (axial) & 33
\end{tabular}

\begin{tabular}{|c|c|c|c|c|c|}
\hline$\frac{\text { Energy }}{\underline{\mathrm{MeV}}}$ & $\frac{\text { Activity }}{\text { photons/sec }}$ & $\frac{\text { Fluence Rate }}{\frac{\text { MeV/cm } / \text { / sec }}{\text { No Buildup }}}$ & $\begin{array}{l}\text { Results } \\
\text { Fluence Rate } \\
\frac{\text { MeV/cm } / \text { sec }}{\text { With Buildup }}\end{array}$ & $\frac{\text { Exposure Rate }}{\text { No } \frac{\mathrm{mR} / \mathrm{hr}}{\mathrm{Buildup}}}$ & $\frac{\text { Exposure Rate }}{\frac{\mathrm{mR} / \mathrm{hr}}{\text { With Buildup }}}$ \\
\hline 0.015 & $1.051 e+12$ & $\overline{1.237 e-253}$ & $6.779 e-20$ & $1.061 \mathrm{e}-254$ & $5.815 e-21$ \\
\hline 0.02 & $3.158 e+11$ & $4.053 e-113$ & $6.653 e-19$ & $1.404 e-114$ & $2.304 e-20$ \\
\hline 0.03 & $9.652 e+14$ & $2.423 e-31$ & $2.012 e-13$ & $2.401 e-33$ & $1.994 e-15$ \\
\hline 0.04 & $2.293 e+14$ & $1.345 e-11$ & $5.503 e-06$ & $5.949 e-14$ & $2.434 e-08$ \\
\hline 0.05 & $3.453 e+08$ & $7.938 e-10$ & $6.229 e-05$ & $2.115 e-12$ & $1.659 e-07$ \\
\hline 0.06 & $1.033 e+11$ & $7.224 e-04$ & $1.230 e+01$ & $1.435 e-06$ & $2.442 e-02$ \\
\hline 0.08 & $6.252 e+08$ & $3.786 e-03$ & $7.098 e+00$ & $5.991 e-06$ & $1.123 e-02$ \\
\hline 0.1 & $2.453 e+09$ & $2.165 e-01$ & $1.118 e+02$ & $3.312 e-04$ & $1.710 e-01$ \\
\hline 0.15 & $6.884 e+08$ & $7.215 e-01$ & $7.643 e+01$ & $1.188 e-03$ & $1.259 e-01$ \\
\hline 0.2 & $1.295 e+10$ & $3.575 e+01$ & $1.884 e+03$ & $6.310 e-02$ & $3.325 e+00$ \\
\hline 0.3 & $2.636 e+09$ & $1.958 e+01$ & $4.604 e+02$ & $3.714 e-02$ & $8.733 e-01$ \\
\hline 0.4 & $4.279 e+10$ & $5.834 e+02$ & $9.033 e+03$ & $1.137 \mathrm{e}+00$ & $1.760 e+01$ \\
\hline 0.5 & $2.175 e+11$ & $4.656 e+03$ & $5.205 e+04$ & 9.140 e +00 & $1.022 e+02$ \\
\hline 0.6 & $1.432 e+16$ & $4.404 e+08$ & $3.902 e+09$ & $8.595 e+05$ & $7.617 e+06$ \\
\hline 0.8 & $1.312 e+13$ & $7.115 e+05$ & $4.567 e+06$ & $1.353 e+03$ & $8.687 e+03$ \\
\hline 1.0 & $3.970 e+11$ & $3.341 e+04$ & $1.706 e+05$ & $6.158 e+01$ & $3.144 e+02$ \\
\hline 1.5 & $4.305 e+11$ & $7.993 e+04$ & $2.921 e+05$ & $1.345 e+02$ & $4.915 e+02$ \\
\hline 2.0 & $8.614 e+04$ & $2.756 e-02$ & $8.409 e-02$ & $4.262 e-05$ & $1.300 e-04$ \\
\hline 3.0 & $1.247 e+03$ & $8.314 e-04$ & $2.037 e-03$ & $1.128 e-06$ & $2.763 e-06$ \\
\hline TOTALS: & $1.553 e+16$ & $4.412 e+08$ & $3.907 e+09$ & $8.611 e+05$ & $7.626 e+06$ \\
\hline
\end{tabular}


Microshield v5.01 (5.01-00121)

Lockheed Martin Idaho Technologies Company

Case File Heat Generation

Case File: T112108.MS5

Case Title: $T-112$ spent Resin Tk

Isotope

$\mathrm{Am}-241$
$\mathrm{Ba}-137 \mathrm{~m}$
$\mathrm{Ce}-144$
$\mathrm{C}-60$
$\mathrm{Cs}-134$
$\mathrm{Cs}-135$
$\mathrm{Cs}-137$
$\mathrm{~Np}-237$
$\mathrm{Pr}-144$
$\mathrm{Pu}-238$
$\mathrm{Pu}-239$
$\mathrm{Pu}-240$
$\mathrm{Pu}-241$
$\mathrm{Pu}-242$
$\mathrm{Rh}-106$
$\mathrm{Ru}-106$
$\mathrm{Sb}-125$
$\mathrm{Sr}-90$
$\mathrm{U}-234$
$\mathrm{U}-235$
$\mathrm{U}-236$
$\mathrm{U}-238$
$\mathrm{Y}-90$

\section{Activity} Curies

$7.8119 e+000$

$4.3070 e+005$

$1.7500 \mathrm{e}-008$

$1.8260 e-001$

$3.7670 \mathrm{e}+002$

$8.3670 \mathrm{e}+000$

$4.5530 \mathrm{e}+005$

$1.1490 \mathrm{e}-001$

$1.7500 e-008$

$4.6920 e+002$

2. $5190 e+001$

$7.9040 e+000$

1. $6320 e+002$

$1.1760 \mathrm{e}-002$

$1.6160 e-004$

1. $6160 e-004$

$3.6750 e+000$

$1.1600 e+002$

$1.5690 e-001$

$3.4440 e-003$

$5.7300 e-003$

$5.1000 e-004$

$1.1600 e+002$
Activity

Bequerels

$2.8904 e+011$

$1.5936 e+016$

$6.4750 e+002$

$6.7562 e+009$

$1.3938 e+013$

$3.0958 e+011$

$1.6846 \mathrm{e}+016$

$4.2513 e+009$

$6.4750 e+002$

$1.7360 e+013$

$9.3203 e+011$

$2.9245 e+011$

$6.0384 e+012$

$4.3512 e+008$

$5.9792 e+006$

$5.9792 e+006$

$1.3598 \mathrm{e}+011$

$4.2920 e+012$

$5.8053 e+009$

$1.2743 e+008$

$2.1201 e+008$

$1.8870 e+007$

$4.2920 e+012$

Total $\frac{\text { Heat Generation }}{\text { Watts/Curie }}$

$3.2755 e-002$

$3.5403 e-003$

$6.0991 e-004$

1. $5417 \mathrm{e}-002$

1. $0149 e-002$

$3.9913 e-004$

$1.1086 \mathrm{e}-003$

$2.8548 e-002$

$7.3470 \mathrm{e}-003$

$3.2552 e-002$

3. $0531 e-002$

3. $0591 e-002$

$3.1773 e-005$

2. $9012 \mathrm{e}-002$

$9.5865 e-003$

$5.9466 \mathrm{e}-005$

$3.0911 e-003$

$1.1603 e-003$

$2.8232 e-002$

2. $7030 e-002$

2. $6729 e-002$

2. $4838 e-002$

$5.5407 e-003$

Heat Generation:

\section{Heat Generation Watts}

$2.5588 e-001$

$1.5248 e+003$

$1.0673 e-011$

$2.8151 e-003$

$3.8231 e+000$

$3.3395 e-003$

$5.0473 e+002$

$3.2802 e-003$

$1.2857 e-010$

$1.5273 e+001$

$7.6908 e-001$

$2.4179 e-001$

$5.1854 e-003$

$3.4118 e-004$

$1.5492 e-006$

$9.6097 e-009$

$1.1360 e-002$

$1.3459 e-001$

$4.4297 e-003$

$9.3093 e-005$

$1.5316 \mathrm{e}-004$

$1.2667 e-005$

$6.4272 e-001$

$2.0507 e+003$ 
Microshield v5.01 (5.01-00121)

Lockheed Martin Idaho Technologies Company

Page : 1

DOS File: T112108A.MS5

Run Date: September 24, 1998

Run Time: $2: 28: 02 \mathrm{PM}$

File Ref:

Date:

By:

Duration: $00: 02: 02$

checked:

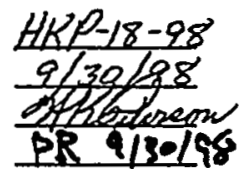

Case Title: T-112 spent Resin Tk

Description: T-112 spent Resin Storage Tank with $23,112 \mathrm{~kg}$ of $\mathrm{st} \# 108 \mathrm{a}$ Geometry: 7 - cylinder Volume - Side shields

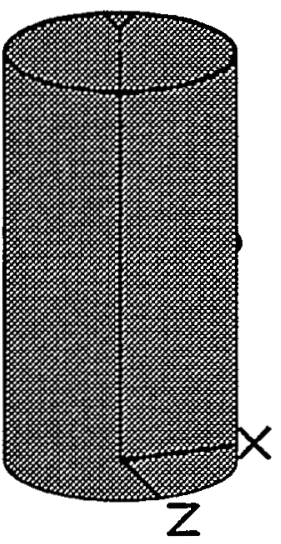

$$
\text { Source Dimensions }
$$

Height $\quad 457.2 \mathrm{~cm} \quad 15 \mathrm{ft}$

Dose Points

$\underline{\mathrm{X}} .19 \mathrm{~cm} \quad 22 \frac{\underline{\mathrm{Y}}}{8.6 \mathrm{~cm}} \quad \underline{\mathrm{Z}} \mathrm{cm}$

$\begin{array}{llrl}\# 1 & 124.19 \mathrm{~cm} & 228.6 \mathrm{~cm} & 0 \mathrm{~cm} \\ & 4 \mathrm{ft} 0.9 \mathrm{in} & 7 \mathrm{ft} 6.0 \mathrm{in} & 0.0 \mathrm{in}\end{array}$

Shields

Shield Name Dimension MaterialDensity Source 2.14e+07 $\mathrm{cm}^{3}$ Mixed $\rightarrow 1.08$

Concrete 0.08

Shield 1 Water 1

Transition

$1.27 \mathrm{~cm}$ Iron 7.86

Air Gap

$1.0 \mathrm{~cm}$

Air

0.0011

Source Input

Grouping Method : Standard Indices

Number of Groups : 25

Lower Energy Cutoff : 0.015

Photons < 0.015 : Excluded

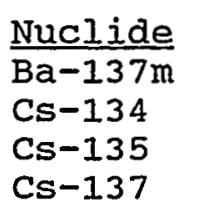

Nuclide Cs -134

Cs -137

Library : ICRP-38

$\begin{array}{cccc}\text { curies } & \frac{\text { becquerels }}{2.2248 e+016} & 2.816 \mathrm{ci} / \mathrm{cm}^{3} & \frac{\mathrm{Bg} / \mathrm{cm}^{3}}{6.0130 e+005} \\ 5.2695 e+002 & 1.9497 e+013 & 2.4681 e+001 & 1.0420 \mathrm{e}+009 \\ 1.1720 \mathrm{e}+001 & 4.3364 \mathrm{e}+011 & 5.4894 \mathrm{e}-001 & 2.0311 \mathrm{e}+0004 \\ 6.3560 e+005 & 2.3517 \mathrm{e}+016 & 2.9770 \mathrm{e}+004 & 1.1015 \mathrm{e}+009\end{array}$

Buildup

The material reference is : source

Integration Parameters

Radial Circumferential $Y$ Direction (axial)
31

31

33
Eneray MeV

0.03

0.04

0.2

0.3
Activity photons/sec

$$
\begin{aligned}
& 1.347 e+15 \\
& 3.201 e+14 \\
& 4.094 e+09 \\
& 2.808 e+09
\end{aligned}
$$

Fluence Rate $\mathrm{MeV} / \mathrm{Cm}^{2} / \mathrm{sec}$ No Buildup

$3.382 e-31$ $1.878 \mathrm{e}-11$ $1.130 \mathrm{e}+01$ $2.085 e+01$
Results

Fluence Rate $\mathrm{MeV} / \mathrm{cm}^{2} / \mathrm{sec}$ With Buildup

$2.808 e-13$

$7.682 e-06$

$5.955 e+02$

$4.904 \mathrm{e}+02$
Exposure Rate Exposure Rate $\mathrm{mR} / \mathrm{hr}$

$$
\text { No } \frac{\mathrm{mR} / \mathrm{hr}}{\mathrm{Build}}
$$

$3.352 e-33$

$8.305 e-14$

$1.995 e-02$

$3.956 e-02$
With Buildup

$2.783 e-15$

$3.397 e-08$

$1.051 e+00$

$9.301 e-01$ 
Page : 2

DOS File: T112108A.MS5

Run Date: September 24, 1998

Run Time: 2:28:02 PM

Duration: 00:02:02

\begin{tabular}{|c|c|c|c|c|c|}
\hline Energy & Activity & Fluence Rate & Fluence Rate & Exposure Rate & Exposure Rate \\
\hline $\mathrm{MeV}$ & photons/sec & $\frac{\mathrm{MeV} / \mathrm{cm}^{2} / \mathrm{sec}}{\text { No Buildup }}$ & $\frac{\mathrm{MeV} / \mathrm{cm}^{2} / \mathrm{sec}}{\text { With Buildup }}$ & No $\frac{\mathrm{mR} / \mathrm{hr}}{\mathrm{Bujldup}}$ & With $\frac{\mathrm{mR} / \mathrm{hr}}{\mathrm{Buil}}$ \\
\hline 0.5 & $2.847 e+11$ & $6.093 e+03$ & $6.811 e+04$ & $1.196 \mathrm{e}+01$ & $1.337 e+02$ \\
\hline 0.6 & $2.000 e+16$ & $6.148 e+08$ & $5.448 e+09$ & $1.200 e+06$ & $1.063 e+07$ \\
\hline 0.8 & $1.835 e+13$ & $9.953 e+05$ & $6.389 e+06$ & $1.893 e+03$ & $1.215 e+04$ \\
\hline 1.0 & $5.459 e+11$ & $4.594 e+04$ & $2.345 e+05$ & $8.467 e+01$ & $4.323 e+02$ \\
\hline 1.5 & $5.927 e+11$ & $1.101 e+05$ & $4.022 e+05$ & $1.852 e+02$ & $6.767 e+02$ \\
\hline TOTALS: & $2.168 e+16$ & $6.159 e+08$ & $5.455 e+09$ & $1.202 e+06$ & $1.065 e+07$ \\
\hline
\end{tabular}


Microshield v5.01 (5.01-00121)

Lockheed Martin Idaho Technologies Company

Case File Heat Generation

Case File: T112108A.MS5

Case Title: T-112 Spent Resin Tk

\section{Isotope Activity Curies}

$\mathrm{Ba}-137 \mathrm{~m}$
$\mathrm{Cs}-134$
$\mathrm{Cs}-135$
$\mathrm{Cs}-137$
Activity Bequerels
$6.0130 e+005$
$5.2695 e+002$
1.1720 e+001
$6.3560 e+005$

Heat Generation Watts/Curie

$2.2248 e+016$

$1.9497 e+013$

$4.3364 e+011$

$2.3517 e+016$

$$
\begin{aligned}
& 3.5403 e-003 \\
& 1.0149 e-002 \\
& 3.9913 e-004 \\
& 1.1086 e-003
\end{aligned}
$$

Total

Heat Generation:
Heat Generation Watts

$2.1288 e+003$

$5.3480 e+000$

$4.6778 e-003$

$7.0460 e+002$

$2.8387 e+003$ 
Microshield v5.01 (5.01-00121)

Lockheed Martin Idaho Technologies Company

Page : 1

DOS File: 181113SL.MS5

Run Date: September 24, 1998

Run Time: 2:53:01 PM

File Ref: HKP $18-98$

Date:

By:

Duration: 00:01:31

Checked:

Case Title: M-101 Mixer

Description: M-101 Mixer (1/2 cyl) with 133.41 of stream \# 113 Geometry: 7 - cylinder volume - side shields

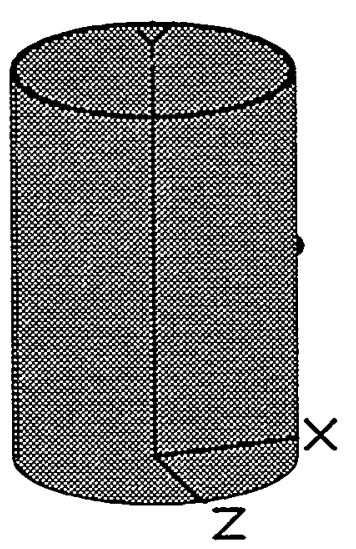

\begin{tabular}{lll}
\multicolumn{5}{c}{ Source Dimensions } \\
Height & $91.44 \mathrm{~cm}$ & $3 \mathrm{ft}$ \\
Radius & $30.48 \mathrm{~cm}$ & $1 \mathrm{ft}$
\end{tabular}

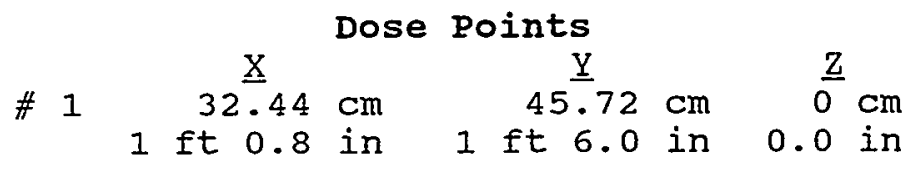

\begin{tabular}{|c|c|c|c|}
\hline \multicolumn{4}{|c|}{ Shields } \\
\hline Source & $2.67 e+05 \mathrm{~cm}^{3}$ & $\begin{array}{l}\text { Mixed } \\
\text { Concret }\end{array}$ & \\
\hline & & Water & \\
\hline Shi & $.953 \mathrm{~cm}$ & Iron & 7.8 \\
\hline ition & $1.0 \mathrm{~cm}$ & Air & 0.0011 \\
\hline Air Gap & & & \\
\hline
\end{tabular}

Source Input

Grouping Method : Standard Indices

Number of Groups : 25

Lower Energy Cutoff : 0.015

Photons < 0.015 : Excluded

Library : ICRP-38

\begin{tabular}{|c|c|c|c|c|}
\hline Nuclide & curies & becquerels & $\mu \mathrm{Ci} / \mathrm{cm}^{3}$ & \\
\hline$\overline{A m-241}$ & $6.1100 e-002$ & $2.2607 e+009$ & $2 . \overline{2894 e-001}$ & $8.4708 e+003$ \\
\hline$m-243$ & $2.7600 e-005$ & $.0212 e+006$ & $1.0342 e-004$ & -00 \\
\hline$a-137 m$ & $0 e-003$ & 008 & & \\
\hline$e-144$ & $0 e-003$ & 008 & & 003 \\
\hline & De-004 & & 04 & +001 \\
\hline & & & 03 & +002 \\
\hline $0-60$ & $0 e-003$ & & & 002 \\
\hline$s-1$ & 06 & & & 7.82 \\
\hline$c s-1$ & -007 & 03 & .007 & 002 \\
\hline$C s-1$ & & & & 02 \\
\hline & & & & 001 \\
\hline$E u-154$ & & 08 & 02 & 003 \\
\hline$E u-155$ & 003 & 008 & -002 & +003 \\
\hline $1-3$ & 003 & & 02 & 5.492 \\
\hline-129 & & & & \\
\hline$N i-63$ & & & & +002 \\
\hline$p-237$ & & & -003 & $4.6416 e+001$ \\
\hline$m-147$ & 02 & be+008 & -002 & $2.4955 e+003$ \\
\hline & & $3 e+008$ & $91 e-002$ & $1.0875 e+003$ \\
\hline & & & & $e+004$ \\
\hline & & & & $1.1965 e+003$ \\
\hline & & & & \\
\hline & $u \in-u v z$ & $1.8463 e+009$ & $1.8698 e-001$ & LOLet \\
\hline
\end{tabular}


Page : 2

DOS File: 181113SL.MS5

Run Date: September 24, 1998

Run Time: 2:53:01 PM

Duration: 00:01:31

$\begin{array}{lc}\text { Nuclide } & \text { curies } \\ \text { Pu-242 } & 1.9340 e-006 \\ \text { Rh-106 } & 4.2950 e-005 \\ \text { Ru-106 } & 4.2950 e-005 \\ \text { Sb-125 } & 1.4400 e-003 \\ \text { Sm-151 } & 6.3500 e-002 \\ \text { Sr-90 } & 4.5090 e+000 \\ \text { Tc-99 } & 1.9600 e-003 \\ \text { Th-230 } & 8.9900 e-008 \\ \text { Th-231 } & 1.2870 e-005 \\ \mathrm{U}-232 & 2.5350 e-007 \\ \mathrm{U}-233 & 1.3740 e-009 \\ \mathrm{U}-234 & 1.2860 \mathrm{e}-004 \\ \mathrm{U}-235 & 3.2000 \mathrm{e}-006 \\ \mathrm{U}-236 & 6.2560 \mathrm{e}-006 \\ \mathrm{U}-238 & 3.4550 \mathrm{e}-006 \\ \mathrm{Y}-90 & 4.5090 \mathrm{e}+000\end{array}$

becquerels

$7.1558 e+004$

$1.5892 \mathrm{e}+006$

$1.5892 \mathrm{e}+006$

$5.3280 e+007$

$2.3495 e+009$

$1.6683 e+011$

$7.2520 e+007$

$3.3263 e+003$

$4.7619 e+005$

$9.3795 e+003$

$5.0838 e+001$

$4.7582 e+006$

$1.1840 e+005$

$2.3147 e+005$

$1.2784 e+005$

$1.6683 e+011$

$\frac{\mu \mathrm{Ci} / \mathrm{cm}^{3}}{2467 e-006}$
$1.6093 e-004$
$1.6093 e-004$
$5.3957 e-003$
$2.3793 e-001$
$1.6895 e+001$
$7.3441 e-003$
$3.3686 e-007$
$4.8224 e-005$
$9.4987 e-007$
$5.1484 e-009$
$4.8186 e-004$
$1.1990 e-005$
$2.3441 e-005$
$1.2946 e-005$
$1.6895 e+001$

$\frac{\mathrm{Bq} / \mathrm{cm}^{3}}{2.6813 e-001}$
$5.9545 e+000$
$5.9545 e+000$
$1.9964 e+002$
$8.8036 e+003$
$6.2512 e+005$
$2.7173 e+002$
$1.2464 e-002$
$1.7843 e+000$
$3.5145 e-002$
$1.9049 e-004$
$1.7829 e+001$
$4.4365 e-001$
$8.6733 e-001$
$4.7900 e-001$
$6.2512 e+005$

\section{The material reference is : source}

\begin{tabular}{ll}
\multicolumn{2}{c}{ Integration Parameters } \\
Radial & 23 \\
Circumferential & 23 \\
Y Direction (axial) & 23
\end{tabular}

\begin{tabular}{c} 
Energy \\
\hline MeV \\
0.015 \\
0.02 \\
0.03 \\
0.04 \\
0.05 \\
0.06 \\
0.08 \\
0.1 \\
0.15 \\
0.2 \\
0.3 \\
0.4 \\
0.5 \\
0.6 \\
0.8 \\
1.0 \\
1.5 \\
2.0 \\
3.0
\end{tabular}

Activity photons/sec

$4.718 e+08$

$7.286 e+08$

$1.239 e+08$

$2.892 e+08$

$6.548 e+07$

8.124 e+08

$1.156 e+08$

$3.979 e+08$

$5.938 e+07$

$5.716 e+06$

$2.402 e+07$

$9.474 \mathrm{e}+06$

2.884 e+08

$3.000 e+08$

$5.013 e+08$

$5.634 \mathrm{e}+08$

2.267 et06

$8.772 e+02$
3. $291 \mathrm{e}+07$

TOTALS:
Fluence Rate $\mathrm{MeV} / \mathrm{cm}^{2} / \mathrm{sec}$ No Buildup

$3.416 e-190$

$6.178 e-85$

2.771e-27

$1.041 e-11$

$1.159 e-06$

$6.432 e-03$

$1.869 e-01$

$5.790 e+00$

$3.916 e+00$

1.660 e+01

$3.934 \mathrm{e}+00$

$2.903 e+01$

$1.743 e+01$

$7.444 \mathrm{e}+02$

$1.315 e+03$

$3.308 e+03$

$7.730 e+03$

$5.123 e+01$

3. $856 e-02$
Results

Fluence Rate

$\mathrm{MeV} / \mathrm{cm}^{2} / \mathrm{sec}$ With Buildup

$1.767 e-22$

$6.917 e-21$

$1.044 \mathrm{e}-19$

$4.114 e-07$

1. 391e-02

2. $523 e+01$

$1.348 e+02$

$1.433 \mathrm{e}+03$

$2.546 e+02$

5. $317 e+02$

$6.154 \mathrm{e}+01$

$3.086 e+02$

$1.387 e+02$

$4.810 e+03$

$6.399 e+03$

1.317 e+04

$2.286 e+04$

$1.284 \mathrm{e}+02$

$7.902 e-02$
Exposure Rate $\mathrm{mR} / \mathrm{hr}$

No Buildup

$2.930 e-191$

$2.140 e-86$

$2.746 e-29$

$4.603 e-14$

$3.089 e-09$

$1.278 e-05$

$2.958 e-04$

$8.858 \mathrm{e}-03$

$6.449 e-03$

$2.930 e-02$

$7.462 \mathrm{e}-03$

$5.655 e-02$

3. $422 e-02$

$1.453 e+00$

2. 501 e+00

$6.097 e+00$

1.300e+01

$7.923 e-02$

$5.231 e-05$

$2.328 e+01$
Exposure Rate $\mathrm{mR} / \mathrm{hr}$

With Buildup

$1.515 e-23$

$2.396 e-22$

$1.035 e-21$

$1.820 e-09$

$3.705 e-05$

5. $012 e-02$

$2.134 e-01$

$2.192 e+00$

$4.192 e-01$

9.384e-01

$1.167 e-01$

$6.014 e-01$

$2.723 e-01$

$9.389 e+00$

1.217e+01

$2.427 e+01$

$3.846 e+01$

$1.985 e-01$

$1.072 e-04$

$5.025 e+04$

$8.930 e+01$ 
Microshield v5.01 (5.01-00121)

Iockheed Martin Idaho Technologies Company

Case File Heat Generation

Case File: 181113SL.MS5

Case Title: M-101 Mixer

$\begin{array}{lcc}\text { Isotope } & \text { Activity } & \text { Activity } \\ & \text { curies } & \text { Bequerels } \\ \text { Am-241 } & 6.1100 e-002 & 2.2607 e+009 \\ \text { Am-243 } & 2.7600 e-005 & 1.0212 e+006 \\ \text { Ba-137m } & 6.4200 e-003 & 2.3754 e+008 \\ \text { Ce-144 } & 7.8440 e-003 & 2.9023 e+008 \\ \text { Cm-242 } & 1.4400 e-004 & 5.3280 e+006 \\ \text { Cm-244 } & 1.9480 e-003 & 7.2076 e+007 \\ \text { Co-60 } & 6.7600 e-003 & 2.5012 e+008 \\ \text { Cs-134 } & 5.6430 e-006 & 2.0879 e+005 \\ \text { Cs-135 } & 1.2500 e-007 & 4.6250 e+003 \\ \text { Cs-137 } & 6.7900 e-003 & 2.5123 e+008 \\ \text { Eu-152 } & 3.6950 e-004 & 1.3672 e+007 \\ \text { Eu-154 } & 2.1340 e-002 & 7.8958 e+008 \\ \text { Eu-155 } & 9.3100 e-003 & 3.4447 e+008 \\ \text { H-3 } & 3.9620 e-003 & 1.4659 e+008 \\ \text { I-129 } & 9.0580 e-004 & 3.3515 e+007 \\ \text { Ni-63 } & 6.4800 e-003 & 2.3976 e+008 \\ \text { Np-237 } & 3.3480 e-004 & 1.2388 e+007 \\ \text { Pm-147 } & 1.8000 e-002 & 6.6600 e+008 \\ \text { Pr-144 } & 7.8440 e-003 & 2.9023 e+008 \\ \text { Pu-238 } & 1.1300 e-001 & 4.1810 e+009 \\ \text { Pu-239 } & 8.6300 e-003 & 3.1931 e+008 \\ \text { Pu-240 } & 2.4900 e-003 & 9.2130 e+007 \\ \text { Pu-241 } & 4.9900 e-002 & 1.8463 e+009 \\ \text { Pu-242 } & 1.9340 e-006 & 7.1558 e+004 \\ \text { Rh-106 } & 4.2950 e-005 & 1.5892 e+006 \\ \text { Ru-106 } & 4.2950 e-005 & 1.5892 e+006 \\ \text { Sb-125 } & 1.4400 e-003 & 5.3280 e+007 \\ \text { Sm-151 } & 6.3500 e-002 & 2.3495 e+009 \\ \text { Sr-90 } & 4.5090 e+000 & 1.6683 e+011 \\ \text { Tc-99 } & 1.9600 e-003 & 7.2520 e+007 \\ \text { Th-230 } & 8.9900 e-008 & 3.3263 e+003 \\ \text { Th-231 } & 1.2870 e-005 & 4.7619 e+005 \\ \mathrm{U}-232 & 2.5350 e-007 & 9.3795 e+003 \\ \mathrm{U}-233 & 1.3740 e-009 & 5.0838 e+001 \\ \mathrm{U}-234 & 1.2860 e-004 & 4.7582 e+006 \\ \mathrm{U}-235 & 3.2000 e-006 & 1.1840 e+005 \\ \mathrm{U}-236 & 6.2560 e-006 & 2.3147 e+005 \\ \mathrm{U}-238 & 3.4550 e-006 & 1.2784 e+005 \\ \mathrm{Y}-90 & 4.5090 e+000 & 1.6683 e+011 \\ & & \\ & & \end{array}$

Total

\section{Heat Generation Watts/Curie}

$3.2755 e-002$

$3.1599 e-002$

$3.5403 e-003$

$6.0991 e-004$

$3.6195 e-002$

$3.4375 e-002$

$1.5417 e-002$

$1.0149 e-002$

$3.9913 e-004$

$1.1086 e-003$

$7.3864 e-003$

$8.7221 e-003$

$6.4902 e-004$

$3.3688 e-005$

$4.7222 e-004$

$1.0154 e-004$

$2.8548 \mathrm{e}-002$

3. $6732 e-004$

$7.3470 e-003$

$3.2552 e-002$

$3.0531 e-002$

3. $0591 e-002$

$3.1773 e-005$

$2.9012 e-002$

$9.5865 e-003$

$5.9466 \mathrm{e}-005$

$3.0911 e-003$

$1.1669 e-004$

1. $1603 e-003$

$6.0024 e-004$

$2.7709 e-002$

$7.1148 \mathrm{e}-004$

$3.1460 e-002$

2.8571e-002

$2.8232 e-002$

$2.7030 e-002$

$2.6729 e-002$

$2.4838 \mathrm{e}-002$

$5.5407 e-003$

Heat Generation: $\frac{\text { Heat Generation }}{\text { Watts }}$

$2.0013 e-003$

$8.7215 e-007$

$2.2728 e-005$

$4.7841 e-006$

$5.2121 e-006$

$6.6963 e-005$

$1.0422 \mathrm{e}-004$

$5.7270 e-008$

$4.9891 e-011$

$7.5271 e-006$

$2.7293 e-006$

$1.8613 e-004$

$6.0424 \mathrm{e}-006$

1.3347e-007

$4.2774 e-007$

$6.5800 e-007$

$9.5580 e-006$

$6.6118 e-006$

$5.7630 e-005$

$3.6784 \mathrm{e}-003$

$2.6349 \mathrm{e}-004$

$7.6172 e-005$

$1.5855 e-006$

$5.6109 e-008$

$4.1174 e-007$

$2.5541 \mathrm{e}-009$

$4.4512 e-006$

$7.4095 e-006$

$5.2318 \mathrm{e}-003$

$1.1765 e-006$

$2.4911 e-009$

$9.1567 e-009$

$7.9752 e-009$

$3.9257 e-011$

$3.6307 e-006$

8. $6497 e-008$

1. $6722 \mathrm{e}-007$

$8.5815 \mathrm{e}-008$

$2.4983 e-002$

$3.6736 e-002$ 
Microshield v5.01 (5.01-00121)

Lockheed Martin Idaho Technologies Company

Page : 1

DOS File: 181117SL.MS5

Run Date: September 24, 1998

Run Time: 4:11:09 PM

File Ref:

Date:

By:

Duration: 00:02:13

Checked:

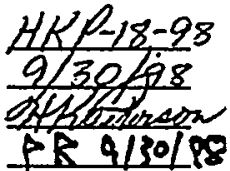

Case Title: M-101 Mixer

Description: M-101 Mixer (full cylinder) with $507.3 \mathrm{~kg}$ of stream \#117 Geometry: 7 - cylinder volume - side shields

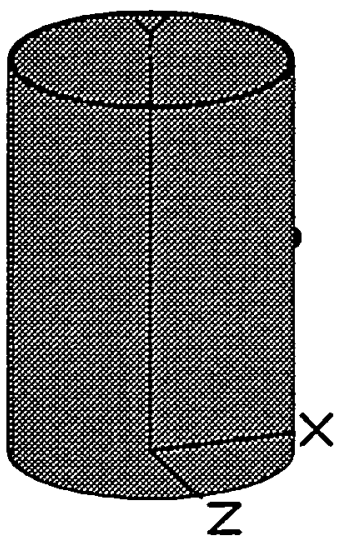

$\begin{array}{lc}\text { Nuclide } & \begin{array}{c}\text { curies } \\ \text { Am-241 }\end{array} \\ \text { Am-243 } & 3.5600 \mathrm{e}-002 \\ \mathrm{Ba}-137 \mathrm{~m} & 8.2500 \mathrm{e}-005 \\ \mathrm{Ce}-144 & 1.0100 \mathrm{e}-002 \\ \mathrm{Cm}-242 & 1.8600 \mathrm{e}-004 \\ \mathrm{Cm}-244 & 2.5100 \mathrm{e}-003 \\ \mathrm{Co}-60 & 8.7300 \mathrm{e}-003 \\ \mathrm{Cs}-134 & 7.2500 \mathrm{e}-006 \\ \mathrm{Cs}-135 & 1.6100 \mathrm{e}-007 \\ \mathrm{Cs}-137 & 8.7300 \mathrm{e}-003 \\ \mathrm{Eu}-152 & 4.7600 \mathrm{e}-004 \\ \mathrm{Eu}-154 & 2.7500 \mathrm{e}-002 \\ \mathrm{Eu}-155 & 1.2020 \mathrm{e}-002 \\ \mathrm{H}-3 & 5.1200 \mathrm{e}-003 \\ \mathrm{I}-129 & 1.1700 \mathrm{e}-003 \\ \mathrm{Ni}-63 & 8.3700 \mathrm{e}-003 \\ \mathrm{Pm}-147 & 2.3200 \mathrm{e}-002 \\ \mathrm{Pr}-144 & 1.0100 \mathrm{e}-002 \\ \mathrm{Pu}-238 & 1.4600 \mathrm{e}-001 \\ \mathrm{Pu}-239 & 1.1100 \mathrm{e}-002 \\ \mathrm{Pu}-240 & 3.2200 \mathrm{e}-003 \\ \mathrm{Pu}-241 & 6.4400 \mathrm{e}-002 \\ \mathrm{Pu}-242 & 2.5000 \mathrm{e}-006\end{array}$
Height Radius Source Dimensions $91.44 \mathrm{~cm}$ $30.48 \mathrm{~cm}$ $3 \mathrm{ft}$ $1 \mathrm{ft}$

Dose Points

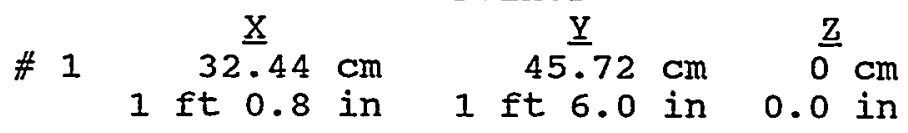

Shields

Shield Name Dimension Material Density source $2.67 e+05 \mathrm{~cm}^{3}$ Mixed $\rightarrow 1.9$

Concrete 1.7

Shield 1 Transition Air Gap Water 0.2

$\begin{array}{llll}.953 \mathrm{~cm} & \text { Iron } & 7.86 \\ 1.0 \mathrm{~cm} & \text { Air } & 0.0011\end{array}$

Source Input

Grouping Method : Standard Indices

Number of Groups : 25 Lower Energy Cutoff : 0.015

Photons < 0.015 : Excluded

Library : ICRP-38

becquerels

$2.9082 e+009$

$1.3135 e+006$

$3.0525 e+008$

$3.7370 e+008$

$6.8820 e+006$

9.2870 e+007

$3.2301 e+008$

$2.6825 e+005$

$5.9570 e+003$

3.2301 e+008

$1.7612 \mathrm{e}+007$

$1.0175 e+009$

$4.4474 e+008$

1.8944 e+008

$4.3290 e+007$

$3.0969 e+008$

$8.5840 e+008$

$3.7370 e+008$

$5.4020 e+009$

4. $1070 \mathrm{e}+008$

1. $1914 \mathrm{e}+008$

$2.3828 e+009$

$9.2500 e+004$ $\mu \mathrm{Ci} / \mathrm{cm}^{3}$

$2.9451 e-001$

$1.3302 e-004$

$3.0913 e-002$

$3.7845 e-002$

$6.9694 e-004$

$9.4050 e-003$

$3.2711 e-002$

$2.7166 e-005$

$6.0327 e-007$

$3.2711 e-002$

$1.7836 e-003$

$1.0304 e-001$

$4.5039 e-002$

$1.9185 e-002$

$4.3840 e-003$

$3.1362 e-002$

$8.6930 e-002$

3. $7845 e-002$

$5.4706 e-001$

$4.1592 e-002$

$1.2065 e-002$

$2.4131 e-001$

$9.3675 e-006$
$\mathrm{Bg} / \mathrm{cm}^{3}$

$1.0897 e+004$

$4.9217 e+000$

$1.1438 e+003$

$1.4003 e+003$

$2.5787 e+001$

$3.4798 e+002$

$1.2103 e+003$

$1.0051 e+000$

$2.2321 e-002$

$1.2103 e+003$

$6.5992 e+001$

$3.8126 e+003$

$1.6664 e+003$

$7.0983 e+002$

$1.6221 \mathrm{e}+002$

$1.1604 \mathrm{e}+003$

$3.2164 e+003$

$1.4003 e+003$

$2.0241 e+004$

$1.5389 e+003$

$4.4642 e+002$

$8.9284 \mathrm{e}+003$

$3.4660 e-001$ 
Page : 2

DOS File: 181117SI.MS5

Run Date: September 24, 1998

Run Time: 4:11:09 PM

Duration: $00: 02: 13$

\begin{tabular}{|c|c|c|c|c|}
\hline Nuclide & curies & becquerels & $\mu \mathrm{Ci} / \mathrm{cm}^{3}$ & $\mathrm{Bg} / \mathrm{cm}^{3}$ \\
\hline $\mathrm{Rh}-106$ & $5.5300 e-005$ & $2.0461 e+006$ & $2.0721 e-004$ & $7 . \overline{6667 e+000}$ \\
\hline Ru-106 & $5.5300 e-005$ & $2.0461 e+006$ & $2.0721 e-004$ & $7.6667 e+000$ \\
\hline$S b-125$ & $1.8500 e-003$ & $6.8450 e+007$ & $6.9320 e-003$ & $2.5648 e+002$ \\
\hline $\mathrm{Sm}-151$ & $8.1700 e-002$ & $3.0229 e+009$ & $3.0613 e-001$ & $1.1327 \mathrm{e}+004$ \\
\hline Sr-90 & $5.7800 e+000$ & $2.1386 e+011$ & $2.1658 \mathrm{e}+001$ & $8.0133 e+005$ \\
\hline Tc-99 & $2.5200 e-003$ & $9.3240 e+007$ & $9.4424 e-003$ & $3.4937 e+002$ \\
\hline$T h-230$ & $1.1600 e-007$ & $4.2920 e+003$ & $4.3465 e-007$ & $1.6082 e-002$ \\
\hline$T h-231$ & $1.6600 e-005$ & $6.1420 e+005$ & $6.2200 e-005$ & $2.3014 e+000$ \\
\hline $\mathrm{U}-232$ & $3.2700 e-007$ & $1.2099 e+004$ & $1.2253 e-006$ & $4.5335 e-002$ \\
\hline $\mathrm{U}-233$ & $1.7700 e-009$ & $6.5490 e+001$ & $6.6322 e-009$ & $2.4539 e-004$ \\
\hline$U-234$ & $1.6600 e-004$ & 6.1420 e+006 & $6.2200 e-004$ & 2.3014 e+001 \\
\hline$U-235$ & $4.1300 e-006$ & $1.5281 e+005$ & $1.5475 e-005$ & $5.7258 e-001$ \\
\hline $\mathrm{U}-236$ & $8.0700 e-006$ & $2.9859 e+005$ & $3.0238 e-005$ & $1.1188 e+000$ \\
\hline$U-238$ & $4.4600 e-006$ & $1.6502 \mathrm{e}+005$ & $1.6712 e-005$ & $6.1833 e-001$ \\
\hline$Y-90$ & $5.7800 e+000$ & $2.1386 e+011$ & $2.1658 \mathrm{e}+001$ & $8.0133 e+005$ \\
\hline
\end{tabular}

Integration Parameters

Radial 26

Circumferential

26

$Y$ Direction (axial)

26

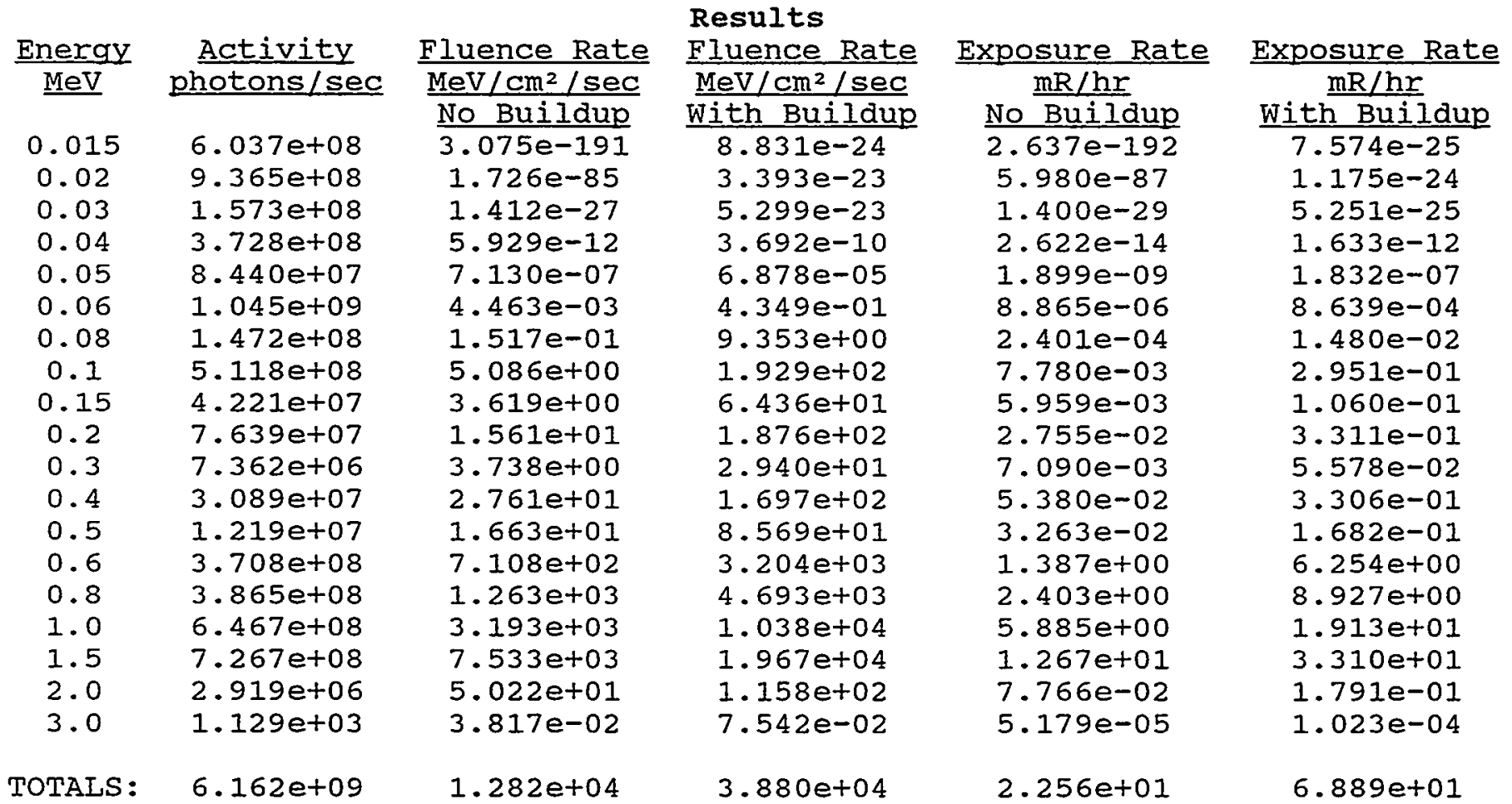


Microshield v5.01 (5.01-00121)

Lockheed Martin Idaho Technologies Company Case File Heat Generation Case File: 181117SL.MS5 Case Title: M-101 Mixer

\begin{tabular}{|c|c|}
\hline Isotope & $\frac{\text { Activity }}{\text { Curies }}$ \\
\hline$A m-241$ & $7.8600 e-002$ \\
\hline$A m-243$ & $3.5500 \mathrm{e}-005$ \\
\hline $\mathrm{Ba}-137 \mathrm{~m}$ & $8.2500 e-003$ \\
\hline $\mathrm{Ce}-144$ & $1.0100 e-002$ \\
\hline $\mathrm{Cm}-242$ & $1.8600 e-004$ \\
\hline $\mathrm{Cm}-244$ & $2.5100 e-003$ \\
\hline Co-60 & $8.7300 e-003$ \\
\hline Cs -134 & $7.2500 \mathrm{e}-006$ \\
\hline$C s-135$ & $1.6100 e-007$ \\
\hline Cs -137 & $8.7300 e-003$ \\
\hline $\mathrm{Eu}-152$ & $4.7600 e-004$ \\
\hline$E u-154$ & $2.7500 e-002$ \\
\hline$E u-155$ & $1.2020 e-002$ \\
\hline $\mathrm{H}-3$ & $5.1200 e-003$ \\
\hline$I-129$ & $1.1700 e-003$ \\
\hline $\mathrm{Ni}-63$ & $8.3700 e-003$ \\
\hline$P m-147$ & $2.3200 e-002$ \\
\hline Pr-144 & $1.0100 e-002$ \\
\hline $\mathrm{Pu}-238$ & $1.4600 e-001$ \\
\hline $\mathrm{Pu}-239$ & $1.1100 e-002$ \\
\hline $\mathrm{Pu}-240$ & $3.2200 e-003$ \\
\hline$P u-241$ & $6.4400 e-002$ \\
\hline $\mathrm{Pu}-242$ & $2.5000 e-006$ \\
\hline $\mathrm{Rh}-106$ & $5.5300 e-005$ \\
\hline$R u-106$ & $5.5300 e-005$ \\
\hline$S b-125$ & $1.8500 e-003$ \\
\hline$S m-151$ & $8.1700 e-002$ \\
\hline Sr-90 & $5.7800 e+000$ \\
\hline TC-99 & $2.5200 e-003$ \\
\hline Th-230 & $1.1600 \mathrm{e}-007$ \\
\hline Th-231 & $1.6600 e-005$ \\
\hline $\mathrm{U}-232$ & $3.2700 e-007$ \\
\hline$U-233$ & $1.7700 e-009$ \\
\hline$U-234$ & $1.6600 e-004$ \\
\hline$U-235$ & $4.1300 e-006$ \\
\hline$U-236$ & $8.0700 e-006$ \\
\hline$U-238$ & $4.4600 e-006$ \\
\hline$Y-90$ & $5.7800 e+000$ \\
\hline
\end{tabular}

\section{Activity Bequerels}

$2.9082 e+009$

$1.3135 e+006$

$3.0525 \mathrm{e}+008$

$3.7370 e+008$

$6.8820 e+006$

$9.2870 e+007$

$3.2301 e+008$

2. $6825 \mathrm{e}+005$

$5.9570 e+003$

$3.2301 e+008$

$1.7612 \mathrm{e}+007$

$1.0175 e+009$

$4.4474 e+008$

$1.8944 \mathrm{e}+008$

$4.3290 e+007$

$3.0969 e+008$

$8.5840 \mathrm{e}+008$

3.7370 e+008

$5.4020 e+009$

$4.1070 e+008$

$1.1914 \mathrm{e}+008$

$2.3828 e+009$

$9.2500 e+004$

$2.0461 e+006$

$2.0461 e+006$

$6.8450 e+007$

3. $0229 e+009$

$2.1386 e+011$

$9.3240 e+007$

$4.2920 e+003$

$6.1420 e+005$

1.2099e+004

$6.5490 e+001$

$6.1420 e+006$

$1.5281 e+005$

$2.9859 e+005$

$1.6502 e+005$

$2.1386 e+011$

\section{Heat Generation Watts/Curie}

\begin{abstract}
$3.2755 e-002$
3.1599e-002

$3.5403 e-003$

6.0991e-004

3. $6195 e-002$

3. $4375 e-002$

1. $5417 e-002$

$1.0149 e-002$

$3.9913 e-004$

$1.1086 e-003$

$7.3864 e-003$

$8.7221 e-003$

$6.4902 e-004$

$3.3688 e-005$

$4.7222 e-004$

$1.0154 e-004$

$3.6732 e-004$

$7.3470 e-003$

$3.2552 e-002$

$3.0531 e-002$

$3.0591 e-002$

$3.1773 e-005$

$2.9012 e-002$

$9.5865 e-003$

$5.9466 e-005$

$3.0911 e-003$

$1.1669 e-004$

$1.1603 e-003$

$6.0024 e-004$

$2.7709 e-002$

$7.1148 e-004$

$3.1460 e-002$

2.8571e-002

$2.8232 e-002$

$2.7030 e-002$

$2.6729 e-002$

$2.4838 \mathrm{e}-002$

$5.5407 e-003$
\end{abstract}

Total
Heat Generation:

\section{Heat Generation Watts}

$2.5745 e-003$

$1.1218 e-006$

$2.9207 e-005$

$6.1601 e-006$

$6.7323 e-006$

$8.6282 e-005$

$1.3459 e-004$

$7.3579 e-008$

$6.4260 e-011$

$9.6777 e-006$

$3.5159 e-006$

$2.3986 e-004$

$7.8013 e-006$

$1.7248 e-007$

$5.5250 e-007$

$8.4992 e-007$

$8.5219 e-006$

$7.4205 e-005$

$4.7526 \mathrm{e}-003$

$3.3890 e-004$

$9.8504 \mathrm{e}-005$

$2.0462 e-006$

$7.2530 e-008$

$5.3014 \mathrm{e}-007$

$3.2885 e-009$

$5.7186 e-006$

$9.5332 \mathrm{e}-006$

$6.7065 e-003$

$1.5126 e-006$

3. $2143 e-009$

$1.1810 e-008$

$1.0288 e-008$

$5.0571 e-011$

$4.6866 e-006$

$1.1164 e-007$

2. $1571 e-007$

$1.1078 e-007$

$3.2025 e-002$

4. 7130 e-002 
Microshield v5.01 (5.01-00121)

Lockheed Martin Idaho Technologies Company

Page : 1

DOS File: WD117SL.MS5

Run Date: September 24, 1998

Run Time: $4: 33: 44$ PM

Duration: $00: 02: 10$

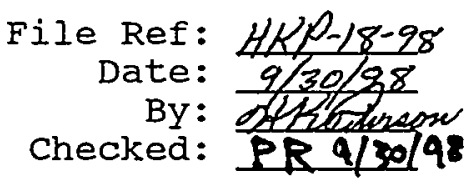

Case Title: Waste Drum

Description: Waste Drum with $395.2 \mathrm{~kg}$ of grouted waste from st \#117 Geometry: 7 - cylinder volume - side shields

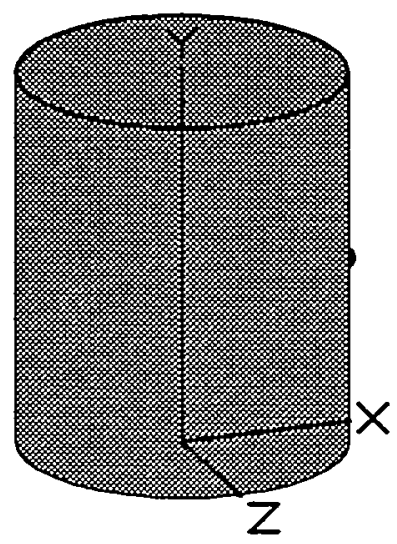

$\begin{array}{lcr} & \text { Source Dimensions } \\ \text { Height } & 69.84 \mathrm{~cm} & 2 \mathrm{ft} \\ \text { Radius } & 29.21 \mathrm{~cm} & 11.5 \text { in }\end{array}$

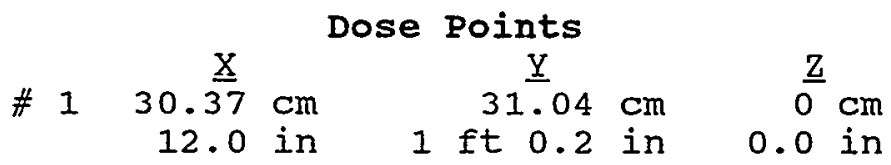

Shields

Shield Name Dimension Material Density Source $\quad 1.87 \mathrm{e}+05 \mathrm{~cm}^{3}$ Mixed $\rightarrow 1.9$

Concrete 1.8

Shield 1

Transition

Water 0.1

Air Gap

$.16 \mathrm{~cm}$ Iron 7.86

$1.0 \mathrm{~cm}$ Air 0.0011

Air 0.00122

Source Input

Grouping Method : standard Indices

Number of Groups : 25

Lower Energy Cutoff : 0.015

Photons < 0.015 : Excluded

Library : ICRP-38

\begin{tabular}{|c|c|c|c|c|}
\hline Nuclide & curies & becquerels & $\mu \mathrm{Ci} / \mathrm{cm}^{3}$ & $\mathrm{Bg} / \mathrm{cm}^{3}$ \\
\hline$A m-241$ & $6.1300 e-002$ & $2.2681 e+009$ & $3 . \overline{2745 e-001}$ & $1 . \overline{2116 e+004}$ \\
\hline & & $0249 e+006$ & $4797 e-004$ & \\
\hline & & & & \\
\hline & 003 & & $6 e-002$ & +003 \\
\hline & & & & $2.8658 e+001$ \\
\hline & & & & +002 \\
\hline & & & & 003 \\
\hline & 006 & & & \\
\hline & & & & 002 \\
\hline & & & & -003 \\
\hline & & & & 001 \\
\hline & & & & 003 \\
\hline & & & 02 & -003 \\
\hline & & & & +002 \\
\hline & & & & +002 \\
\hline & & & & .003 \\
\hline & & & & 1001 \\
\hline & & & 02 & +003 \\
\hline & & & & $1.5535 \mathrm{e}+003$ \\
\hline & & & & +004 \\
\hline & & & & \\
\hline & & & & $e+002$ \\
\hline $50-241$ & $5.0200 e-002$ & $1.8574 e+009$ & $2.6816 e-001$ & $9.9217 e+00$ \\
\hline
\end{tabular}


Page : 2

DoS File: WD117SL.MS5

Run Date: September 24, 1998

Run Time: $4: 33: 44$ PM

Duration: 00:02:10

$\begin{array}{lc}\text { Nuclide } & \text { curies } \\ \text { Pu-242 } & 1.9400 \mathrm{e}-006 \\ \mathrm{Rh}-106 & 4.3100 \mathrm{e}-005 \\ \mathrm{Ru}-106 & 4.3100 \mathrm{e}-005 \\ \mathrm{Sb}-125 & 1.4400 \mathrm{e}-003 \\ \mathrm{Sm}-151 & 6.3600 \mathrm{e}-002 \\ \mathrm{Sr}-90 & 4.5050 \mathrm{e}+000 \\ \mathrm{~T}-99 & 1.9600 \mathrm{e}-003 \\ \mathrm{Th}-230 & 9.0100 \mathrm{e}-008 \\ \mathrm{Th}-231 & 1.2900 \mathrm{e}-005 \\ \mathrm{U}-232 & 2.5500 \mathrm{e}-007 \\ \mathrm{U}-233 & 1.3750 \mathrm{e}-009 \\ \mathrm{U}-234 & 1.2900 \mathrm{e}-004 \\ \mathrm{U}-235 & 3.2200 \mathrm{e}-006 \\ \mathrm{U}-236 & 6.2800 \mathrm{e}-006 \\ \mathrm{U}-238 & 3.4700 \mathrm{e}-006 \\ \mathrm{Y}-90 & 4.5050 \mathrm{e}+000\end{array}$

becquerels

$7.1780 e+004$

$1.5947 e+006$

$1.5947 e+006$

$5.3280 e+007$

$2.3532 e+009$

$1.6669 e+011$

$7.2520 e+007$

$3.3337 e+003$

$4.7730 e+005$

$9.4350 e+003$

$5.0875 e+001$

$4.7730 e+006$

$1.1914 \mathrm{e}+005$

$2.3236 e+005$

$1.2839 \mathrm{e}+005$

$1.6669 e+011$ $\frac{\mu \mathrm{Ci} / \mathrm{cm}^{3}}{1.0363 e-005}$
$2.3023 e-004$
$2.3023 e-004$
$7.6921 e-003$
$3.3973 e-001$
$2.4065 e+001$
$1.0470 e-002$
$4.8129 e-007$
$6.8908 e-005$
$1.3621 e-006$
$7.3449 e-009$
$6.8908 e-004$
$1.7200 e-005$
$3.3546 e-005$
$1.8536 e-005$
$2.4065 e+001$

$\mathrm{Bg} / \mathrm{cm}^{3}$

$3.8343 e-001$

$8.5185 e+000$

$8.5185 e+000$

$2.8461 e+002$

$1.2570 e+004$

$8.9039 e+005$

$3.8738 e+002$

$1.7808 e-002$

$2.5496 \mathrm{e}+000$

$5.0399 e-002$

$2.7176 e-004$

$2.5496 e+001$

$6.3641 e-001$

$1.2412 \mathrm{e}+000$

$6.8583 e-001$

$8.9039 e+005$

Buildup

The material reference is : source

Integration Parameters

Radial Circumferential $Y$ Direction (axial)

26

26

26

$\begin{array}{ccc}\frac{\text { Energy }}{\text { MeV }} & \begin{array}{c}\text { Activity } \\ \text { photons/sec }\end{array} & \frac{\text { Fluence Rate }}{\text { MeV/cm } / \text { sec }} \\ 0.015 & 4.726 e+08 & \frac{\text { No Buildup }}{2.669 e-34} \\ 0.02 & 7.308 e+08 & 2.706 e-15 \\ 0.03 & 1.243 e+08 & 2.260 e-05 \\ 0.04 & 2.910 e+08 & 6.015 e-02 \\ 0.05 & 6.594 e+07 & 3.020 e-01 \\ 0.06 & 8.151 e+08 & 1.952 e+01 \\ 0.08 & 1.163 e+08 & 1.461 e+01 \\ 0.1 & 4.008 e+08 & 1.130 e+02 \\ 0.15 & 3.299 e+07 & 2.434 e+01 \\ 0.2 & 5.979 e+07 & 7.338 e+01 \\ 0.3 & 5.744 e+06 & 1.335 e+01 \\ 0.4 & 2.407 e+07 & 8.664 e+01 \\ 0.5 & 9.502 e+06 & 4.797 e+01 \\ 0.6 & 2.892 e+08 & 1.925 e+03 \\ 0.8 & 3.022 e+08 & 3.122 e+03 \\ 1.0 & 5.046 e+08 & 7.351 e+03 \\ 1.5 & 5.672 e+08 & 1.545 e+04 \\ 2.0 & 2.272 e+06 & 9.581 e+01 \\ 3.0 & 8.794 e+02 & 6.737 e-02 \\ & & \\ \text { TOTALS: } & 4.814 e+09 & 2.834 e+04\end{array}$

Results

Fluence Rate

$\mathrm{MeV} / \mathrm{cm}^{2} / \mathrm{sec}$

with Buildup

$8.532 e-24$

$6.659 e-15$

$1.159 e-04$

$5.246 e-01$

$3.425 e+00$

$2.328 e+02$

1.527 et02

$9.925 e+02$

$1.563 e+02$

$3.826 \mathrm{e}+02$

$5.459 e+01$

$3.043 e+02$

$1.506 e+02$

$5.546 e+03$

7.914 e +03

$1.708 e+04$

$3.095 e+04$

$1.761 \mathrm{e}+02$

1.109e-01
Exposure Rate $\mathrm{mR} / \mathrm{hr}$

No Buildup

$2.289 e-35$

$9.373 e-17$

$2.240 \mathrm{e}-07$

2. $660 \mathrm{e}-04$

8. $045 e-04$

$3.877 e-02$

$2.312 \mathrm{e}-02$

1. $729 e-01$

$4.009 e-02$

$1.295 e-01$

$2.533 e-02$

$1.688 \mathrm{e}-01$

$9.415 e-02$

$3.757 e+00$

$5.939 e+00$

$1.355 e+01$

$2.599 e+01$

$1.482 \mathrm{e}-01$

$9.140 e-05$

$5.008 e+01$
Exposure Rate $\mathrm{mR} / \mathrm{hr}$ With Buildup

$7.318 e-25$

$2.307 e-16$

$1.149 e-06$

$2.320 e-03$

$9.123 e-03$

$4.624 \mathrm{e}-01$

$2.416 e-01$

$1.518 \mathrm{e}+00$

$2.574 e-01$

$6.754 \mathrm{e}-01$

$1.035 e-01$

5. $929 e-01$

$2.956 e-01$

$1.082 \mathrm{e}+01$

$1.505 e+01$

$3.149 e+01$

$5.208 e+01$

$2.724 e-01$

$1.504 e-04$

$6.410 e+04$

$1.139 e+02$ 
Microshield v5.01 (5.01-00121)

Lockheed Martin Idaho Technologies Company Case File Heat Generation Case File: WD117SL.MS5 Case Title: Waste Drum

$\begin{array}{lc}\text { Isotope } & \text { Activity } \\ & \text { Curies } \\ \text { Am-241 } & 6.1300 e-002 \\ \text { Am-243 } & 2.7700 e-005 \\ \text { Ba-137m } & 6.4300 e-003 \\ \text { Ce-144 } & 7.8600 e-003 \\ \text { Cm-242 } & 1.4500 e-004 \\ \text { Cm-244 } & 1.9600 e-003 \\ \text { Co-60 } & 6.8000 e-003 \\ \text { Cs-134 } & 5.6500 e-006 \\ \text { Cs-135 } & 1.2600 e-007 \\ \text { Cs-137 } & 6.8000 e-003 \\ \text { Eu-152 } & 3.7100 e-004 \\ \text { Eu-154 } & 2.1500 e-002 \\ \text { Eu-155 } & 9.3700 e-003 \\ \text { H-3 } & 3.9900 e-003 \\ \text { I-129 } & 9.0900 e-004 \\ \text { Ni-63 } & 6.5200 e-003 \\ \text { Np-237 } & 3.3670 e-004 \\ \text { Pm-147 } & 1.8100 e-002 \\ \text { Pr-144 } & 7.8600 e-003 \\ \text { Pu-238 } & 1.1300 e-001 \\ \text { Pu-239 } & 8.6500 e-003 \\ \text { Pu-240 } & 2.5100 e-003 \\ \text { Pu-241 } & 5.0200 e-002 \\ \text { Pu-242 } & 1.9400 e-006 \\ \text { Rh-106 } & 4.3100 e-005 \\ \text { Ru-106 } & 4.3100 e-005 \\ \text { Sb-125 } & 1.4400 e-003 \\ \text { Sm-151 } & 6.3600 e-002 \\ \text { Sr-90 } & 4.5050 e+000 \\ \text { Tc-99 } & 1.9600 e-003 \\ \text { Th-230 } & 9.0100 e-008 \\ \text { Th-231 } & 1.2900 e-005 \\ \text { U-232 } & 2.5500 e-007 \\ \mathrm{U}-233 & 1.3750 e-009 \\ \text { U-234 } & 1.2900 e-004 \\ \text { U-235 } & 3.2200 e-006 \\ \mathrm{U}-236 & 6.2800 e-006 \\ \mathrm{U}-238 & 3.4700 e-006 \\ \mathrm{Y}-90 & 4.5050 e+000 \\ & \\ & \end{array}$

$\frac{\text { Activity }}{\text { Bequerels }}$

$2.2681 e+009$

$1.0249 e+006$

$2.3791 e+008$

$2.9082 e+008$

$5.3650 e+006$

$7.2520 e+007$

$2.5160 e+008$

$2.0905 e+005$

$4.6620 e+003$

$2.5160 e+008$

1.3727 e+007

$7.9550 e+008$

$3.4669 e+008$

$1.4763 e+008$

$3.3633 e+007$

$2.4124 \mathrm{e}+008$

$1.2458 e+007$

$6.6970 e+008$

2. $9082 e+008$

$4.1810 e+009$

3. $2005 e+008$

$9.2870 \mathrm{e}+007$

$1.8574 \mathrm{e}+009$

$7.1780 e+004$

$1.5947 e+006$

$1.5947 e+006$

$5.3280 e+007$

$2.3532 e+009$

$1.6669 e+011$

7.2520 e+007

$3.3337 e+003$

$4.7730 e+005$

$9.4350 \mathrm{e}+003$

$5.0875 e+001$

4.7730e+006

$1.1914 \mathrm{e}+005$

$2.3236 e+005$

$1.2839 e+005$

$1.6669 e+011$ $\frac{\text { Heat Generation }}{\text { Watts/Curie }}$

$3.2755 e-002$

$3.1599 e-002$

$3.5403 e-003$

6.0991e-004

3. $6195 e-002$

$3.4375 e-002$

$1.5417 e-002$

$1.0149 e-002$

$3.9913 e-004$

$1.1086 \mathrm{e}-003$

$7.3864 e-003$

$8.7221 e-003$

$6.4902 e-004$

$3.3688 e-005$

$4.7222 e-004$

$1.0154 e-004$

$2.8548 e-002$

$3.6732 e-004$

$7.3470 \mathrm{e}-003$

$3.2552 \mathrm{e}-002$

$3.0531 e-002$

3. $0591 e-002$

$3.1773 e-005$

$2.9012 e-002$

$9.5865 e-003$

$5.9466 e-005$

$3.0911 e-003$

$1.1669 e-004$

$1.1603 e-003$

$6.0024 e-004$

$2.7709 e-002$

$7.1148 \mathrm{e}-004$

$3.1460 e-002$

$2.8571 e-002$

$2.8232 e-002$

$2.7030 e-002$

$2.6729 e-002$

$2.4838 e-002$

$5.5407 e-003$

Total

Heat Generation:
Heat Generation Watts

$2.0079 e-003$

$8.7531 e-007$

$2.2764 \mathrm{e}-005$

$4.7939 e-006$

$5.2483 e-006$

$6.7376 e-005$

$1.0483 e-004$

$5.7341 e-008$

$5.0290 e-011$

$7.5382 e-006$

$2.7404 \mathrm{e}-006$

$1.8753 e-004$

$6.0813 e-006$

1. $3442 \mathrm{e}-007$

$4.2925 e-007$

$6.6206 e-007$

$9.6123 e-006$

$6.6485 \mathrm{e}-006$

$5.7747 e-005$

$3.6784 \mathrm{e}-003$

$2.6410 \mathrm{e}-004$

$7.6784 \mathrm{e}-005$

$1.5950 e-006$

$5.6283 e-008$

$4.1318 e-007$

$2.5630 e-009$

$4.4512 \mathrm{e}-006$

$7.4212 e-006$

$5.2272 \mathrm{e}-003$

$1.1765 e-006$

2. $4966 e-009$

9.1780e-009

$8.0224 \mathrm{e}-009$

$3.9285 e-011$

3. $6420 e-006$

$8.7038 e-008$

$1.6786 \mathrm{e}-007$

$8.6188 \mathrm{e}-008$

$2.4961 e-002$

$3.6719 e-002$ 
Microshield v5.01 (5.01-00121)

Page : 1

DoS File: WD117ASL.MS5

Run Date: September 25, 1998

Run Time: 10:00:19 AM

Duration: 00:02:09

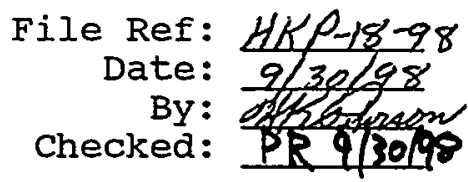

Case Title: Waste Drum

Description: Faste Drum with $395.2 \mathrm{~kg}$ of grouted waste from st \#117a Geometry: 7 - cylinder volume - side shields

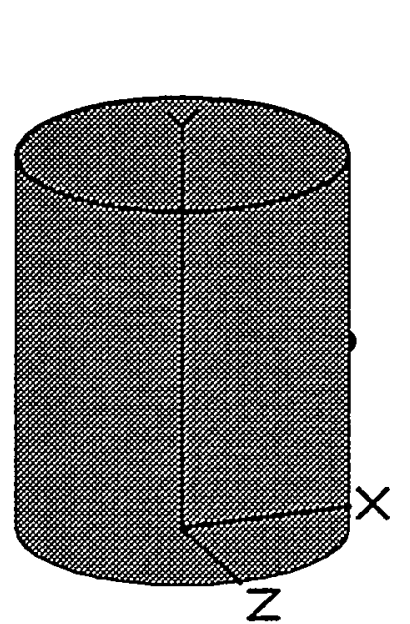

Nuclide

Am-241

$\mathrm{Am}-243$

$\mathrm{Ba}-137 \mathrm{~m}$

$\mathrm{Ce}-144$

$\mathrm{Cm}-242$

$\mathrm{Cm}-244$

Co-60

Cs -134

Cs -135

Cs -137

$\mathrm{Eu}-152$

Eu-154

$\mathrm{Eu}-155$

$\mathrm{H}-3$

$I-129$

$\mathrm{Ni}-63$

$\mathrm{Np}-237$

Pm-147

Pr-144

$\mathrm{Pu}-238$

$\mathrm{Pu}-239$

$\mathrm{Pu}-240$

$\mathrm{Pu}-241$

\section{Lockheed Martin Idaho Technologies Company}

\begin{tabular}{llr}
\multicolumn{5}{c}{ Source Dimensions } \\
Height & $69.84 \mathrm{~cm}$ & $2 \mathrm{ft} 3.5$ in \\
Radius & $29.21 \mathrm{~cm}$ & $11.5 \mathrm{in}$
\end{tabular}

\begin{tabular}{crrr}
\multicolumn{9}{c}{ Dose Points } \\
$\# 1 \quad 30.37 \mathrm{~cm}$ & $\underline{\mathrm{y}}$ & $\underline{\mathrm{Z}}$ \\
\# & $31.04 \mathrm{~cm}$ & $0 \mathrm{~cm}$ \\
& $12.0 \mathrm{in}$ & $1 \mathrm{ft} 0.2 \mathrm{in}$ & $0.0 \mathrm{in}$
\end{tabular}

shields

Shield Name Dimension Material Density source

$1.87 \mathrm{e}+05 \mathrm{~cm}^{3}$ Mixed $\rightarrow 1.9$

Shield 1

Transition

Air Gap

$\begin{array}{lll} & \text { Water } & 0.1 \\ .16 \mathrm{~cm} & \text { Iron } & 7.86 \\ 1.0 \mathrm{~cm} & \text { Air } & 0.0011 \\ & \text { Air } & 0.00122\end{array}$

Source Input

Grouping Method : Standard Indices

Number of Groups : 25

Lower Energy Cutoff : 0.015

Photons < 0.015 : Excluded

Iibrary : ICRP-38

becquerels $\quad \mu \mathrm{Ci} / \mathrm{cm}^{3}$

2.2681e+009

$1.0249 e+006$

$6.9190 e+008$

$2.9082 e+008$

$5.3650 e+006$

$7.2520 \mathrm{e}+007$

2. $5160 e+008$

$2.2829 e+005$

$4.6620 e+003$

$7.3260 e+008$

$1.3727 e+007$

$7.9550 e+008$

$3.4669 e+008$

$1.4763 e+008$

$3.3633 e+007$

$2.4124 \mathrm{e}+008$

$1.2458 e+007$

$6.6970 e+008$

$2.9082 e+008$

$4.4585 e+009$

$3.3485 e+008$

$9.6940 e+007$

$1.9447 e+009$
$3.2745 e-001$

$1.4797 e-004$

$9.9891 e-002$

$4.1986 e-002$

$7.7455 e-004$

$1.0470 e-002$

$3.6324 e-002$

$3.2959 e-005$

$6.7306 e-007$

$1.0577 e-001$

$1.9818 e-003$

$1.1485 e-001$

$5.0052 e-002$

$2.1314 e-002$

$4.8556 e-003$

$3.4828 e-002$

$1.7986 e-003$

$9.6686 e-002$

$4.1986 e-002$

$6.4368 e-001$

$4.8343 e-002$

$1.3995 e-002$

$2.8076 e-001$
$\mathrm{Bg} / \mathrm{cm}^{3}$

$1.2116 e+004$

$5.4747 e+000$

$3.6960 e+003$

$1.5535 \mathrm{e}+003$

$2.8658 e+001$

$3.8738 e+002$

$1.3440 e+003$

$1.2195 e+000$

$2.4903 e-002$

$3.9134 e+003$

$7.3326 e+001$

$4.2494 e+003$

$1.8519 e+003$

$7.8860 e+002$

$1.7966 e+002$

$1.2886 e+003$

$6.6547 e+001$

$3.5774 \mathrm{e}+003$

$1.5535 e+003$

$2.3816 e+004$

$1.7887 e+003$

$5.1783 e+002$

$1.0388 e+004$ 
Page : 2

DOS File: WD117ASL.MS5

Run Date: September 25, 1998

Run Time: 10:00:19 AM

Duration: 00:02:09

\begin{tabular}{|c|c|c|c|c|}
\hline Nuclide & curies & becquerels & $\mu \mathrm{Ci} / \mathrm{cm}^{3}$ & $\mathrm{Ba} / \mathrm{cm}^{3}$ \\
\hline $\mathrm{Pu}-242$ & $2 . \overline{1180 e-006}$ & $7.8366 e+004$ & $1.1314 e-005$ & $4.1861 e-001$ \\
\hline $\mathrm{Rh}-106$ & $4.3100 e-005$ & $1.5947 e+006$ & $2.3023 e-004$ & $8.5185 e+000$ \\
\hline $\mathrm{Ru}-106$ & $4.3100 e-005$ & $1.5947 e+006$ & $2.3023 e-004$ & $8.5185 e+000$ \\
\hline$S b-125$ & $1.4980 e-003$ & $5.5426 e+007$ & $8.0019 e-003$ & $2.9607 e+002$ \\
\hline$S m-151$ & $6.3600 e-002$ & $2.3532 e+009$ & $3.3973 e-001$ & $1.2570 \mathrm{e}+004$ \\
\hline$S r-90$ & $4.5450 e+000$ & $1.6817 e+011$ & $2.4278 e+001$ & $8.9829 e+005$ \\
\hline TC-99 & $1.9600 e-003$ & $7.2520 \mathrm{e}+007$ & $1.0470 e-002$ & $3.8738 e+002$ \\
\hline$T h-230$ & $9.0100 e-008$ & $3.3337 e+003$ & $4.8129 e-007$ & $1.7808 e-002$ \\
\hline Th-231 & $1.2900 e-005$ & $4.7730 e+005$ & $6.8908 e-005$ & $2.5496 \mathrm{e}+000$ \\
\hline $\mathrm{U}-232$ & $2.5500 e-007$ & $9.4350 e+003$ & $1.3621 e-006$ & $5.0399 e-002$ \\
\hline$U-233$ & $1.3750 e-009$ & $5.0875 e+001$ & $7.3449 e-009$ & $2.7176 e-004$ \\
\hline$U-234$ & $1.3100 e-004$ & $4.8470 e+006$ & $6.9977 e-004$ & $2.5891 \mathrm{e}+001$ \\
\hline $\mathrm{U}-235$ & $3.2700 e-006$ & $1.2099 e+005$ & $1.7467 e-005$ & 6.4630 e-001 \\
\hline$U-236$ & $6.2800 e-006$ & $2.3236 e+005$ & $3.3546 e-005$ & $1.2412 \mathrm{e}+000$ \\
\hline$U-238$ & $3.4800 e-006$ & $1.2876 e+005$ & $1.8589 e-005$ & $6.8780 e-001$ \\
\hline$Y-90$ & $4.5450 e+000$ & $1.6817 e+011$ & $2.4278 e+001$ & $8.9829 e+005$ \\
\hline
\end{tabular}

Integration Parameters

Radial

Circumferential

26

$Y$ Direction (axial)

26

26

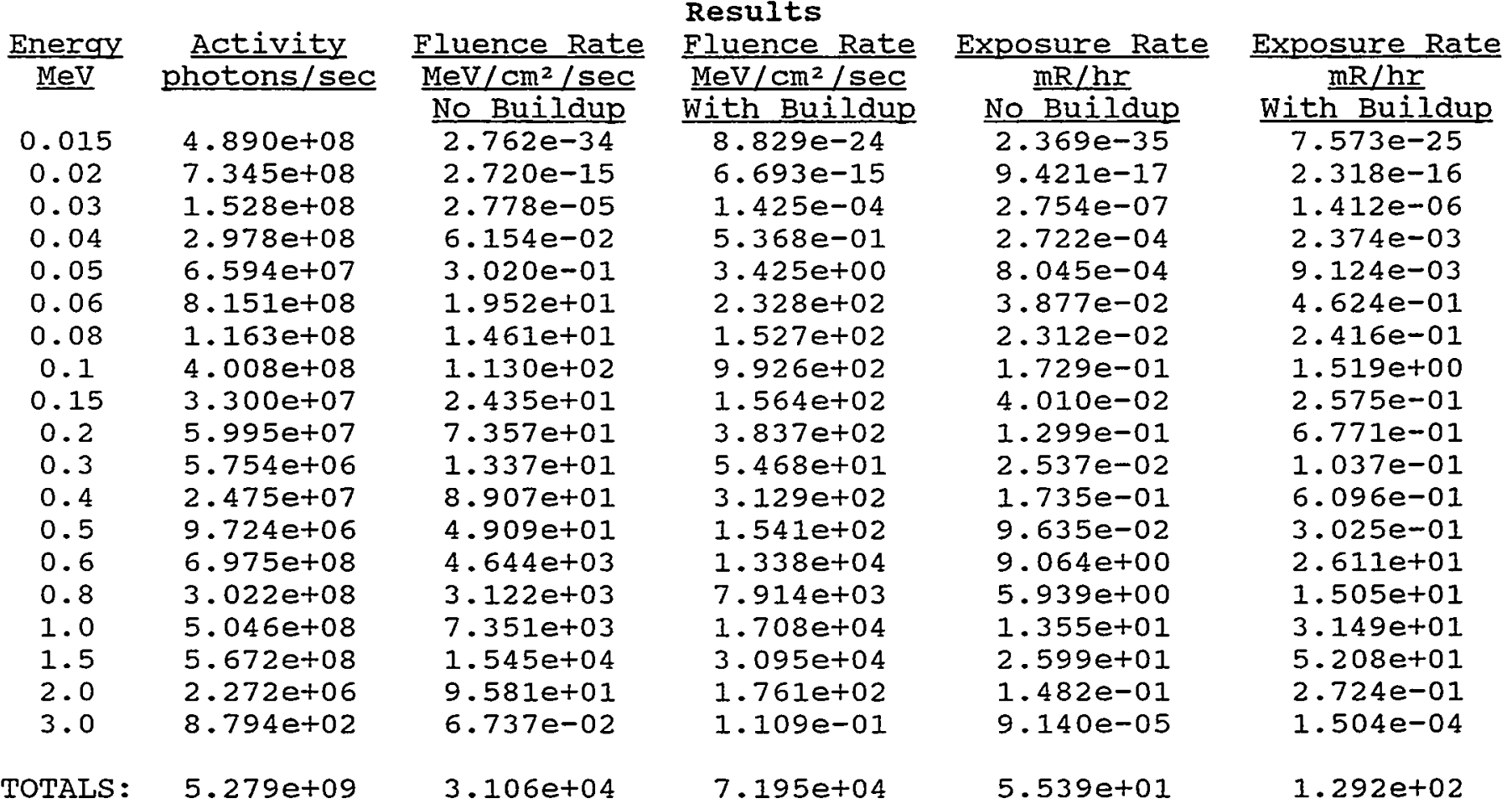


Microshield v5.01 (5.01-00121)

Lockheed Martin Idaho Technologies Company Case file Heat Generation Case File: WD117ASL.MS5 Case Title: Waste Drum

\begin{tabular}{lc} 
Isotope & Activity \\
& Curies \\
Am-241 & $6.1300 e-002$ \\
Am-243 & $2.7700 e-005$ \\
Ba-137m & $6.4300 e-003$ \\
Ce-144 & $7.8600 e-003$ \\
Cm-242 & $1.4500 e-004$ \\
Cm-244 & $1.9600 e-003$ \\
Co-60 & $6.8000 e-003$ \\
Cs-134 & $5.6500 e-006$ \\
Cs-135 & $1.2600 e-007$ \\
Cs-137 & $6.8000 e-003$ \\
Eu-152 & $3.7100 e-004$ \\
Eu-154 & $2.1500 e-002$ \\
Eu-155 & $9.3700 e-003$ \\
H-3 & $3.9900 e-003$ \\
I-129 & $9.0900 e-004$ \\
Ni-63 & $6.5200 e-003$ \\
Np-237 & $3.3670 e-004$ \\
Pm-147 & $1.8100 e-002$ \\
Pr-144 & $7.8600 e-003$ \\
Pu-238 & $1.1300 e-001$ \\
Pu-239 & $8.6500 e-003$ \\
Pu-240 & $2.5100 e-003$ \\
Pu-241 & $5.0200 e-002$ \\
Pu-242 & $1.9400 e-006$ \\
Rh-106 & $4.3100 e-005$ \\
Ru-106 & $4.3100 e-005$ \\
Sb-125 & $1.4400 e-003$ \\
Sm-151 & $6.3600 e-002$ \\
Sr-90 & $4.5050 e+000$ \\
Tc-99 & $1.9600 e-003$ \\
Th-230 & $9.0100 e-008$ \\
Th-231 & $1.2900 e-005$ \\
U-232 & $2.5500 e-007$ \\
U-233 & $1.3750 e-009$ \\
U-234 & $1.2900 e-004$ \\
U-235 & $3.2200 e-006$ \\
U-236 & $6.2800 e-006$ \\
U-238 & $3.4700 e-006$ \\
Y-90 & $4.5050 e+000$ \\
& \\
\hline
\end{tabular}

$\frac{\text { Activity }}{\text { Bequerels }}$

2.2681 e+009

$1.0249 e+006$

$2.3791 e+008$

$2.9082 e+008$

$5.3650 e+006$

$7.2520 e+007$

$2.5160 e+008$

$2.0905 e+005$

4.6620 et 003

$2.5160 e+008$

1.3727 e+007

$7.9550 \mathrm{e}+008$

$3.4669 e+008$

$1.4763 \mathrm{e}+008$

$3.3633 e+007$

$2.4124 \mathrm{e}+008$

$1.2458 \mathrm{e}+007$

$6.6970 e+008$

$2.9082 e+008$

4.1810 et009

$3.2005 e+008$

$9.2870 e+007$

$1.8574 \mathrm{e}+009$

$7.1780 e+004$

$1.5947 \mathrm{e}+006$

$1.5947 \mathrm{e}+006$

$5.3280 e+007$

$2.3532 \mathrm{e}+009$

$1.6669 e+011$

$7.2520 e+007$

3.3337 e+003

$4.7730 \mathrm{e}+005$

$9.4350 e+003$

$5.0875 e+001$

$4.7730 e+006$

$1.1914 \mathrm{e}+005$

$2.3236 e+005$

$1.2839 e+005$

$1.6669 e+011$

$\frac{\text { Heat Generation }}{\text { Watts/Curie }}$

$3.1599 e-002$

$3.5403 e-003$

$6.0991 e-004$

3. 6195e-002

$3.4375 \mathrm{e}-002$

1. $5417 \mathrm{e}-002$

1. $0149 e-002$

$3.9913 e-004$

$1.1086 \mathrm{e}-003$

$7.3864 e-003$

$8.7221 e-003$

$6.4902 e-004$

$3.3688 e-005$

$4.7222 e-004$

$1.0154 e-004$

$2.8548 e-002$

$3.6732 e-004$

$7.3470 e-003$

$3.2552 e-002$

$3.0531 e-002$

$3.0591 e-002$

$3.1773 e-005$

$2.9012 e-002$

$9.5865 e-003$

$5.9466 e-005$

$3.0911 e-003$

$1.1669 e-004$

$1.1603 e-003$

$6.0024 e-004$

$2.7709 e-002$

$7.1148 e-004$

$3.1460 e-002$

2. $8571 e-002$

$2.8232 e-002$

$2.7030 e-002$

2. $6729 e-002$

$2.4838 e-002$

$5.5407 e-003$

Heat Generation:
Heat Generation Watts

$2.0079 e-003$

$8.7531 e-007$

$2.2764 \mathrm{e}-005$

$4.7939 e-006$

$5.2483 e-006$

$6.7376 \mathrm{e}-005$

$1.0483 e-004$

$5.7341 e-008$

$5.0290 e-011$

$7.5382 \mathrm{e}-006$

$2.7404 \mathrm{e}-006$

$1.8753 e-004$

$6.0813 e-006$

1. $3442 \mathrm{e}-007$

$4.2925 e-007$

$6.6206 e-007$

$9.6123 e-006$

$6.6485 e-006$

$5.7747 e-005$

$3.6784 \mathrm{e}-003$

2. $6410 e-004$

$7.6784 \mathrm{e}-005$

$1.5950 \mathrm{e}-006$

$5.6283 e-008$

$4.1318 e-007$

$2.5630 e-009$

$4.4512 \mathrm{e}-006$

$7.4212 e-006$

$5.2272 e-003$

$1.1765 e-006$

$2.4966 e-009$

$9.1780 e-009$

$8.0224 e-009$

$3.9285 e-011$

$3.6420 e-006$

$8.7038 e-008$

$1.6786 e-007$

$8.6188 \mathrm{e}-008$

2. $4961 e-002$

$3.6719 e-002$

Total 
431.02

$08 / 12 / 98$

Rev. 06
ENGINEERING DESIGN FILE

1. Project File No. 02BM1

3. Subtask Determine Facility Shielding Requirements

4. Title: Shielding Requirements for High Level Waste Processing Components

5. Summary: This EDF contains preliminary information on shielding requirements for the subject study. It includes a letter (HKP-15-98) from H. K. Peterson summarizing the concrete wall thickness required based on preliminary design information.

MicroShield, Version 5.01, Grove Engineering, Rockville MD was used for calculations. The MicroShield calculation input/output sheets related to this work are attached to the letter.

Since the results are based on preliminary design assumptions, this EDF is not final. Future design efforts will need to finalize the shielding requirements. Pertinent names are shown in the signature block but the EDF is not signed.

This EDF should be used only for the purposes of this study and as a basis for shielding calculations for future design efforts.
Functional File No. SPR-CsIX-16

EDF No. EDF-CsIX-16

Page 1 of 1
2. Project/Task CsIX/TRU Grout Feasibility Study 


\section{LOCKHEED MARTINA7}

Lockheed Martin Idaho Technologies Company

INTERDEPARTMENTAL COMMUNICATION

Date:

August 31, 1998

To:

From:

J. L. Clapp

MS 3650

6-0307

MS 2107

$6-8657$

Subject: $\quad$ SHIELDING REQUIREMENTS FOR HIGH LEVEL WASTE PROCESSING COMPONENTS -- HKP-15-98

References: a) H. K. Peterson ltr to S. J. Losinski, CS IX/TRU COMPONENT PROJECTED

RADIATION FIELDS, HKP-12-98, August 6, 1998

b) MicroShield, Version 5.01, Grove Engineering, Rockville MD

The requested review of the shielding requirements for the high level waste processing components as spaced and projected on the preliminary floor plans has been completed. It should be remembered that since the radiation field is dependent on the distance from a source, the shielding thicknesses reported here are preliminary, since the plans are preliminary; if the distances to occupied areas change for the final drawings, the shield thicknesses could also change. The source term for the various components are as defined in reference a and calculations were performed with MicroShield (Ref.b). The requirements are summarized below.

Spent Resin Storage Tank - Since the Spent Resin Storage Tank is contained in a cell that has occupied areas (Control room, RadCon, and the stairwell) adjacent to it, the shielding should attenuate the radiation field from this component to $0.1 \mathrm{mR} / \mathrm{hr}$, in accordance with the requirements of MCP 91. The thickness of concrete that will adequately shield this component to $0.1 \mathrm{mR} / \mathrm{hr}$ for an adjacent full-time occupancy area is 38 inches.

The Cs IX Exchange columns are proposed for a cell that is above the Spent Resin Storage Tank cell. With the ion exchange columns placed as projected along the wall between this cell and the one containing the rotary dryer, 38 inches of concrete will be adequate to attenuate the radiation field to $0.1 \mathrm{mR} / \mathrm{hr}$ on the outside of this cell. Although this cell is projected to be 20 feet above ground level, there is a stairwell to the left of the cell and a resin prep location adjacent to the cell which could be full-time occupancy areas.

For the UDS (Undissolved Solids storage cell) it was assumed that the UDS would be stored in 55-gallon drums and stacked in the cell as opposed to storage in a single large tank. To provide the source term for these calculations, it was assumed that four 55-gallon drums were stacked on top of four other drums and placed against the shielding wall in question. To shield this cell on the outside wall will require 38 inches of concrete. This thickness can be relaxed somewhat on the other three walls because of adjacent, unoccupied cells and the P \& V corridor. Since the P \& V corridor will be an unoccupied area that will also contain substantial radiation sources, the wall between the operating corridor and the P \& V corridor should be a shielding wall. That 
wall, however, should only shield against the radiation sources within the P \& V corridor and not be responsible for shielding against the components contained within the cells. The outside wall for UDS storage should be 30 inches of concrete to attenuate the field from undissolved solids storage to $0.1 \mathrm{mR} / \mathrm{hr}$. The wall between that cell and the P \& V corridor can be reduced to 26 inches of concrete.

The cell housing the Rotary dryer and the slurry tank requires an outside wall of 26 inches of concrete to attenuate the radiation field from these components to $0.1 \mathrm{mR} / \mathrm{hr}$ on the outside of the building. This thickness can be relaxed for the wall between the P \& V corridor and the cell and will probably be sized for structural considerations.

The Waste Feed tanks (T-101A \& -B) will require 26 inches of concrete on the outside wall to reduce the field from these components to $0.1 \mathrm{mR} / \mathrm{hr}$ at the outside surface of the wall. The wall between this cell and the P \& V corridor could be reduced below the 26 inches and may be sized according to structural considerations.

The cell housing the IX effluent tanks will require 20 inches of concrete on the outside wall to attenuate the radiation field to $0.1 \mathrm{mR} / \mathrm{hr}$. The other walls of this cell can be constructed with concrete whose thickness can be based on structural considerations.

MicroShield calculational input/output listings are on file with the Letter File copy of this analysis should they be wished for reference.

If there are any questions or comments concerning this analysis, please call me at the abovelisted number.

$$
h k p
$$

cc: $\quad$ P. D. Ritter, LMITCO, MS 2107 PD

P. D. Ritter, LMITCO, MS 2107 PDR
G. J. Stormberg, LMITCO, MS 2107989 
Microshield v5.01 (5.01-00121)

Lockheed Martin Idaho Technologies company

Page : 1

DOS File: CSIEX1.MS5

Run Date: August 26, 1998

Run Time: 2:10:00 PM

File Ref:

Date:

By:

Duration: 00:03:31

Checked:

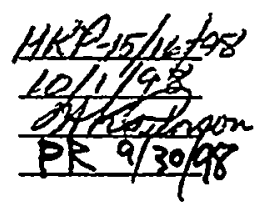

Case Title: Cs I Exch Col

Description: Shielding forcs Ion Exch col Cubicle with 2501 of stream 10 Geometry: 7 - Cylinder Volume - side Shields

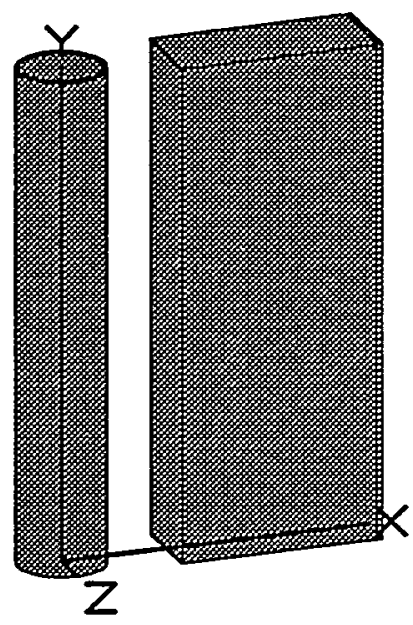

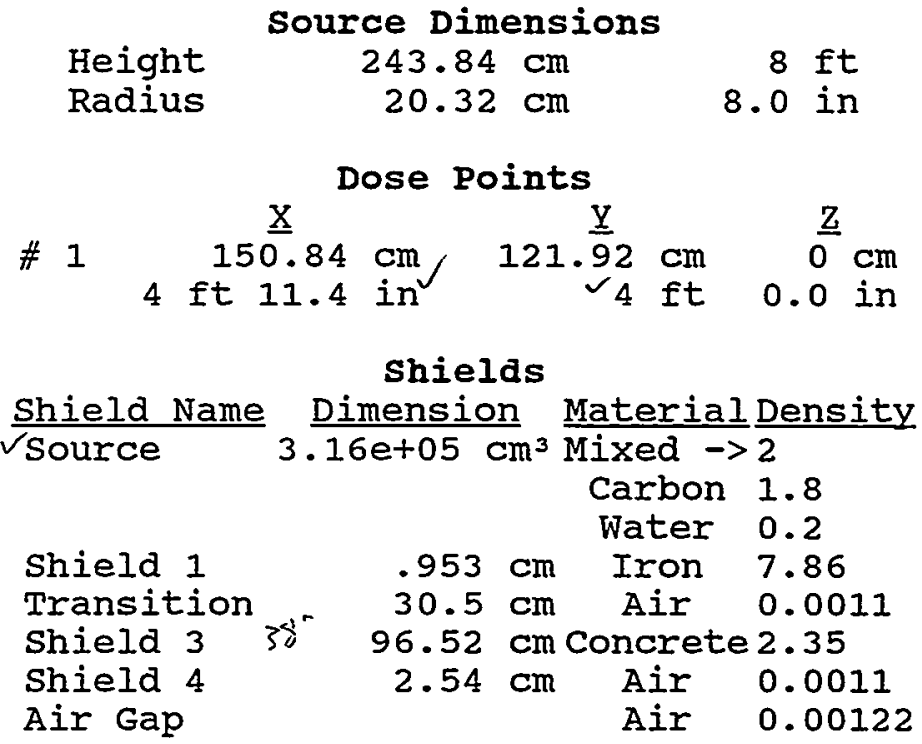

Source Input

Grouping Method : Standard Indices

Number of Groups : 25

Lower Energy Cutoff : 0.015

Photons < 0.015 : Excluded

Library : ICRP-38

\begin{tabular}{|c|c|c|c|c|}
\hline Nuclide & curies & becquerels & $\mu \mathrm{Ci} / \mathrm{cm}^{3}$ & $\mathrm{Bg} / \mathrm{cm}^{3}$ \\
\hline & $9 . \overline{3750 \mathrm{e}-0} 03$ & $3.4688 e+008$ & $2 . \overline{9639 e-002}$ & $1 . \overline{0967 e+00}$ \\
\hline & & & & \\
\hline & $e-011$ & 001 & & \\
\hline & De-004 & 06 & -004 & $18 e+00$ \\
\hline & $0 e+000$ & & & $1992 e+00$ \\
\hline & & & & 00 \\
\hline & 003 & & 004 & 008 \\
\hline & & & 004 & 501 \\
\hline & & & & -006 \\
\hline & 5 . & & & -004 \\
\hline & 3 . & & .002 & 003 \\
\hline & .003 & 3. & -002 & +003 \\
\hline & & & & +004 \\
\hline & & & & 00 \\
\hline & & & -007 & -00 \\
\hline & -007 & 7. & $6.1271 e-007$ & $e-002$ \\
\hline & $2-003$ & & $1.3911 e-002$ & $0 e+002$ \\
\hline & & & & $0 e+004$ \\
\hline & & & & \\
\hline & & & & \\
\hline$U-2$ & & $7.2705 e+$ & $6.2124 e-006$ & $2.2986 e-001$ \\
\hline
\end{tabular}


Page : 2

DOS File: CSIEX1.MS5

Run Date: August 26, 1998

Run Time: 2:10:00 PM

Duration: 00:03:31

\begin{tabular}{|c|c|c|c|c|}
\hline Nuclide & curies & becquerels & $\mu \mathrm{Ci} / \mathrm{cm}^{3}$ & $\mathrm{Bg} / \mathrm{cm}^{3}$ \\
\hline $\mathrm{U}-238$ & $6 . \overline{1500 \mathrm{e}-007}$ & $2.2755 e+004$ & $1 . \overline{9443 e-006}$ & $7 . \overline{1941 \mathrm{e}-002}$ \\
\hline$Y-90$ & $1.3900 e-001$ & $5.1430 e+009$ & $4.3945 e-001$ & $1.6260 \mathrm{e}+004$ \\
\hline
\end{tabular}

Radial

Integration Parameters

Circumferential

29

$Y$ Direction (axial)

29

31

$\begin{array}{cc}\frac{\text { Energy }}{\text { MeV }} & \begin{array}{c}\text { Activity } \\ \text { photons/sec }\end{array} \\ 0.015 & 1.260 e+09 \\ 0.02 & 3.787 e+08 \\ 0.03 & 1.150 e+13 \\ 0.04 & 2.732 e+12 \\ 0.05 & 4.142 e+05 \\ 0.06 & 1.240 e+08 \\ 0.08 & 7.499 e+05 \\ 0.1 & 2.941 e+06 \\ 0.15 & 8.250 e+05 \\ 0.2 & 2.661 e+07 \\ 0.3 & 1.077 e+07 \\ 0.4 & 5.123 e+07 \\ 0.5 & 1.032 e+09 \\ 0.6 & 1.706 e+14 \\ 0.8 & 6.548 e+10 \\ 1.0 & 1.956 e+09 \\ 1.5 & 2.123 e+09 \\ 2.0 & 1.033 e+02 \\ 3.0 & 1.496 e+00\end{array}$

Results

Fluence Rate

Fluence Rate

$\mathrm{MeV} / \mathrm{cm}^{2} / \mathrm{sec}$

$\mathrm{MeV} / \mathrm{cm}^{2} / \mathrm{sec}$

$\frac{\text { No Buildup }}{0.000 e+00}$

With Buildup

$0.000 e+00$

$5.434 e-25$

$5.400 e-136$

$2.571 e-25$

$1.726 \mathrm{e}-20$

$4.332 e-65$

$1.088 e-20$

$3.127 e-46$

$5.390 e-27$

$2.998 e-32$

$1.158 \mathrm{e}-23$

$6.769 e-25$

$1.869 e-20$

$6.725 e-23$

$4.522 \mathrm{e}-18$

$3.829 e-17$

$1.870 e-14$

$8.118 e-14$

$4.487 e-11$

$4.522 e-12$

$1.731 e-09$

$1.246 \mathrm{e}-07$

$5.215 \mathrm{e}-10$

$1.760 e-05$

$1.167 e-01$

$7.357 e-04$

$1.305 e+01$

$4.747 e-02$

$7.368 e-03$

$1.733 e-04$

$6.034 e-03$

$1.286 e-01$

$\frac{\text { Exposure }}{\mathrm{mR} / \mathrm{hr}}$

No Buildup

$0.000 e+00$

$0.000 e+00$

$5.352 e-138$

$1.916 e-67$

8. $331 e-49$

$5.954 e-35$

$1.071 e-27$

2. $859 e-23$

$6.305 e-20$

1. $433 e-16$

8.577e-15

$1.016 e-12$

2. $178 e-10$

$2.278 e-04$

1.399e-06

3. $194 e-07$

$1.015 e-05$

$3.953 e-12$

$4.988 e-10$

$6.767 e-13$

Exposure Rate

$3.612 e-08$

$4.252 e-09$

$2.396 e-04$ $\mathrm{mR} / \mathrm{hr}$

With Buildup

$4.661 e-26$

$8.905 e-27$

1. $711 \mathrm{e}-22$

$4.810 e-23$

$1.436 \mathrm{e}-29$

$2.301 e-26$

$1.064 e-25$

$6.918 e-21$

$3.080 e-17$

$7.920 \mathrm{e}-14$

$3.283 e-12$

$2.428 e-10$

$3.455 \mathrm{e}-08$

$2.547 \mathrm{e}-02$

$9.029 e-05$

$1.358 e-05$

$2.163 e-04$

$5.586 \mathrm{e}-11$

$5.769 e-12$

TOTALS: $\quad 1.849 \mathrm{e}+14$

$1.236 e-01$

$1.323 e+01$

$2.579 e-02$ 
Microshield v5.01 (5.01-00121)

Page : 1

Lockheed Martin Idaho Technologies Company

DOS File: UDSSTOR.MS5

Run Date: August 26, 1998

Run Time: 5:08:29 PM

File Ref:

Date:

By:

Duration: 00:02:15

Checked:

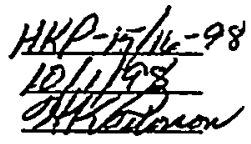
Case Title: UDS
Description: Shielding for uDs storage
Geometry: 13 - Rectangular volume

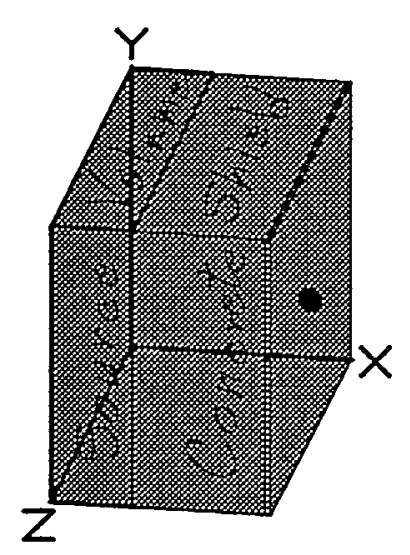

Source Dimensions

$\begin{array}{lrc}\text { Length } & 44.0 \mathrm{~cm} & 1 \mathrm{ft} 5.3 \mathrm{in} \\ \text { Width } & 243.8 \mathrm{~cm} & 7 \mathrm{ft} 12.0 \mathrm{in} \\ \text { Height } & 155.0 \mathrm{~cm} & 5 \mathrm{ft} 1.0 \mathrm{in}\end{array}$

Dose Points

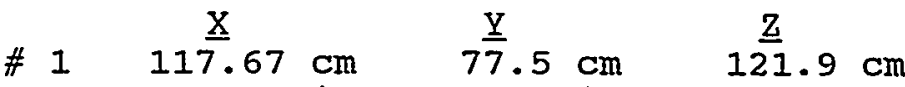

shields

Shield Name Dimension Material Density Source $\quad 1.66 \mathrm{e}+06 \mathrm{~cm}^{3}$ Concrete 1.4 Shield $128^{n} 71.12 \mathrm{~cm}$ Concrete 2.35 Shield $2 \quad 2.54 \mathrm{~cm}$ Air 0.0011 Air Gap

Air

0.00122

Source Input

Grouping Method : Standard Indices

Number of Groups : 25

Lower Energy Cutoff : 0.015

Photons < 0.015 : Excluded

Library : Grove

Nuclide
Ba-137m
$\mathrm{Ce}-144$
$\mathrm{Co}-60$
$\mathrm{Cs}-134$
$\mathrm{Cs}-137$
$\mathrm{Pr}-144$
$\mathrm{Rh}-106$
$\mathrm{Ru}-106$
$\mathrm{Sb}-125$
$\mathrm{Sr}-90$
$\mathrm{Y}-90$

curies

$2.8870 e+002$

$6.1970 e-009$

$6.4500 e-002$

$1.1520 e-002$

$3.0520 e+002$

6.1970e-009

$5.7100 e-005$

$5.7100 e-005$

$1.3000 e+000$

$4.1000 e+001$

$4.1000 e+001$ becquerels

$1.0682 \mathrm{e}+013$

$2.2929 e+002$

$2.3865 e+009$

$4.2624 \mathrm{e}+008$

$1.1292 \mathrm{e}+013$

$2.2929 e+002$

$2.1127 e+006$

$2.1127 e+006$

4. $8100 e+010$

$1.5170 e+012$

$1.5170 \mathrm{e}+012$ $\mu \mathrm{Ci} / \mathrm{cm}^{3}$

$1.7363 \mathrm{e}+002$

$3.7270 \mathrm{e}-009$

$3.8792 e-002$

$6.9284 e-003$

$1.8356 e+002$

$3.7270 e-009$

3. $4341 \mathrm{e}-005$

$3.4341 e-005$

$7.8185 e-001$

$2.4658 e+001$

$2.4658 e+001$
$\mathrm{Bg} / \mathrm{cm}^{3}$

$6.4244 \mathrm{e}+006$

1. $3790 \mathrm{e}-004$

$1.4353 \mathrm{e}+003$

$2.5635 e+002$

$6.7915 e+006$

$1.3790 e-004$

$1.2706 \mathrm{e}+000$

$1.2706 e+000$

$2.8929 e+004$

$9.1236 e+005$

$9.1236 e+005$

Buildup

The material reference is : Shield 1

\section{Integration Parameters}

$\mathrm{X}$ Direction

$Y$ Direction

$\mathrm{z}$ Direction

22

32

32
Energy

MeV

0.03
Activity photons/sec

$6.508 e+11$
Fluence Rate $\mathrm{MeV} / \mathrm{cm}^{2} / \mathrm{sec}$ No Buildup
Results

Fluence Rate $\mathrm{MeV} / \mathrm{cm}^{2} / \mathrm{sec}$ With Buildup $1.816 e-21$
Exposure Rate $\mathrm{mR} / \mathrm{hr}$ No Buildup

$2.421 e-84$
Exposure Rate $\mathrm{mR} / \mathrm{hr}$ With Buildup $1.800 e-23$ 
Page : 2

DOS File: UDSSTOR.MS5

Run Date: August 26, 1998

Run Time: 5:08:29 PM

Duration: 00:02:15

\begin{tabular}{|c|c|}
\hline Energy & Activity \\
\hline MeV & photons/sec \\
\hline 0.04 & $1.505 e+11$ \\
\hline 0.06 & $3.039 e-01$ \\
\hline 0.08 & $3.665 e+00$ \\
\hline 0.1 & $1.256 e+08$ \\
\hline 0.15 & $1.207 e+08$ \\
\hline 0.2 & 3.651 e+09 \\
\hline 0.3 & $2.008 e+08$ \\
\hline 0.4 & $1.506 \mathrm{e}+10$ \\
\hline 0.5 & $4.986 e+09$ \\
\hline 0.6 & $9.629 e+12$ \\
\hline 0.8 & $4.012 e+08$ \\
\hline 1.0 & $2.398 e+09$ \\
\hline 1.5 & $2.399 e+09$ \\
\hline 2.0 & $1.775 e+00$ \\
\hline
\end{tabular}

\begin{tabular}{l} 
Fluence Rate \\
\hline MeV/cm $2 /$ sec \\
\hline No Buildup \\
\hline $1.319 e-40$ \\
$3.486 e-30$ \\
$2.659 e-23$ \\
$2.308 e-13$ \\
$7.975 e-11$ \\
$4.731 e-08$ \\
$1.006 e-07$ \\
$8.496 e-05$ \\
$1.714 e-04$ \\
$1.380 e+00$ \\
$5.050 e-04$ \\
$1.516 e-02$ \\
$2.341 e-01$ \\
$9.760 e-10$
\end{tabular}

\begin{tabular}{l} 
Fluence Rate \\
\hline MeV/cm /sec \\
With Buildup \\
\hline 1.114 e-21 \\
$1.263 e-28$ \\
$1.799 e-21$ \\
$3.093 e-11$ \\
$1.966 e-08$ \\
$1.277 e-05$ \\
$1.966 e-05$ \\
$1.102 e-02$ \\
$1.552 e-02$ \\
$9.186 e+01$ \\
$2.068 e-02$ \\
$4.299 e-01$ \\
$3.581 e+00$ \\
$1.031 e-08$
\end{tabular}

Exposure Rate Exposure Rate $\mathrm{mR} / \mathrm{hr}$

No Buildup

$9.592 e+01$
$5.835 e-43$

$6.923 e-33$

$4.208 e-26$

3. $531 e-16$

1. $313 e-13$

8. 350 e-11

1. $909 e-10$

$1.655 \mathrm{e}-07$

3. $364 e-07$

$2.693 e-03$

9.606e-07

2. $794 e-05$

$3.939 e-04$

1. $509 \mathrm{e}-12$

$\mathrm{mR} / \mathrm{hr}$

With Buildup

4.926e-24

2. $509 e-31$

2. $847 e-24$

4. $732 e-14$

3. $237 e-11$

2. $255 e-08$

3. $729 e-08$

2. $147 e-05$

3. $046 e-05$

1. $793 e-01$

3. $934 e^{-05}$

$7.924 e-04$

$6.025 e-03$

1. $594 \mathrm{e}-11$

3. $117 e-03$

$1.862 e-01$
$1.630 e+00$ 
Microshield v5.01 (5.01-00121)

Lockheed Martin Idaho Technologies Company

Page : 1

DOS File: UDSSTOR.MS5

Run Date: August 26, 1998

Run Time: 5:12:41 PM

Duration: 00:02:15

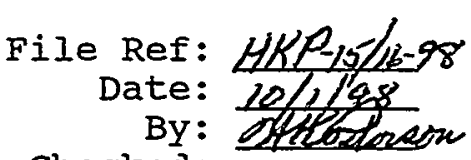

Checked:

By:

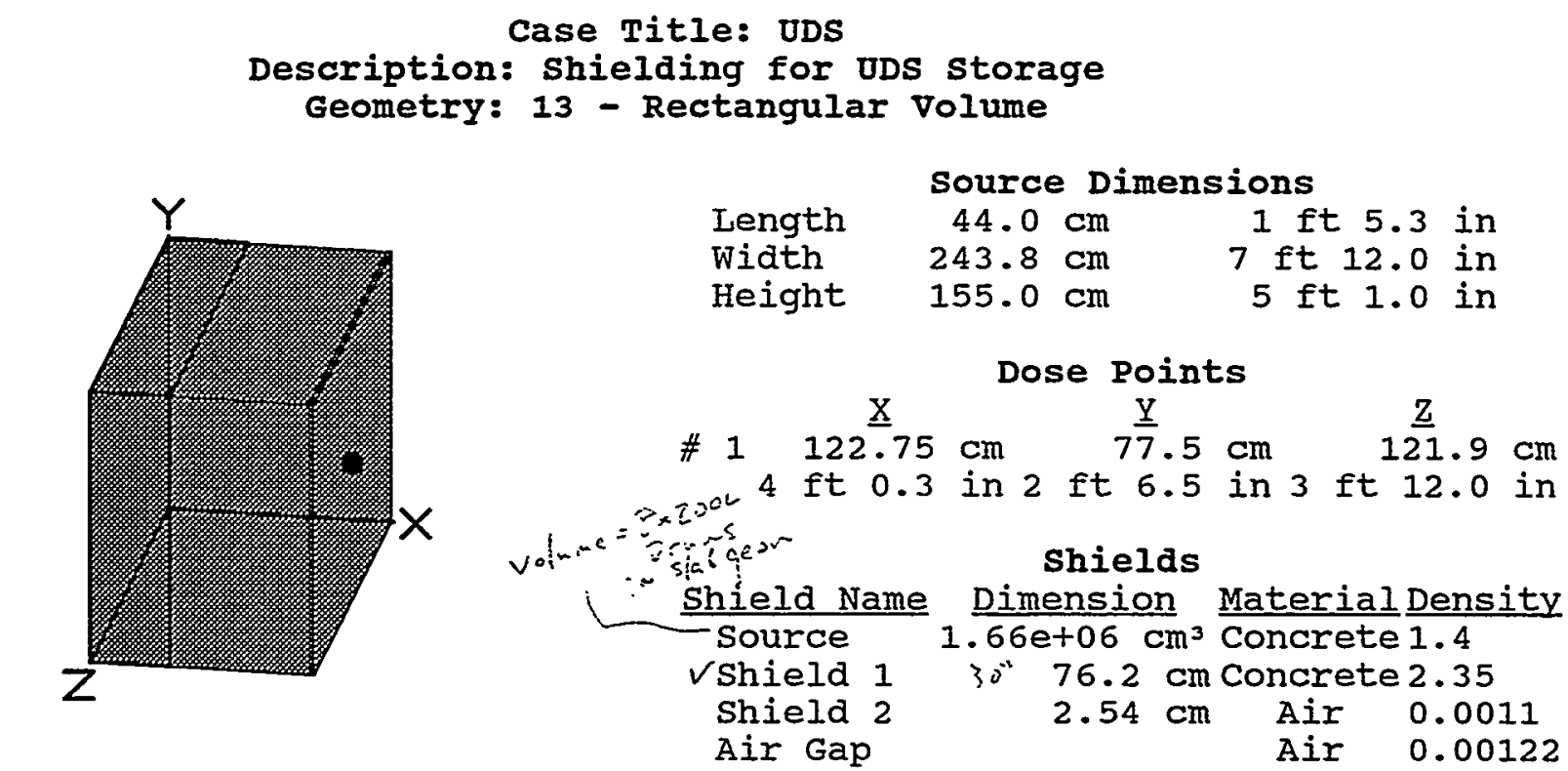

\begin{tabular}{|c|c|c|c|c|c|}
\hline & & & Results & & \\
\hline$\frac{\text { nergy }}{\text { MeV }}$ & $\frac{\text { Activity }}{\text { photons/sec }}$ & $\begin{array}{l}\text { Fluence Rate } \\
\mathrm{MeV} / \mathrm{cm}^{2} / \mathrm{sec}\end{array}$ & $\frac{\text { Fluence Rate }}{\mathrm{MeV} / \mathrm{cm}^{2} / \mathrm{sec}}$ & $\frac{\text { Exposure Raze }}{\mathrm{mR} / \mathrm{hr}}$ & $m R / h r$ \\
\hline & $6.508 e+11$ & $\frac{\text { No Buildup }}{2.737 e-88}$ & $\frac{\text { with Buildup }}{1.691 e^{-21}}$ & $\frac{\text { No Buildup }}{2.713 e-90}$ & $\frac{\text { With Buildup }}{1.676 e-23}$ \\
\hline
\end{tabular}

Integration Parameters

$\mathrm{X}$ Direction

$Y$ Direction

z Direction
Source Input

Number of Groups : 25

Lower Energy Cutoff : 0.015

Library : Grove

becquerels

$1.0682 e+013$

$2.2929 e+002$

$2.3865 e+009$

4.2624 e +008

$1.1292 e+013$

$2.2929 e+002$

$2.1127 e+006$

$2.1127 e+006$

$4.8100 e+010$

$1.5170 e+012$

$1.5170 e+012$ $\mu \mathrm{Ci} / \mathrm{cm}^{3}$
$1.7363 e+002$
$3.7270 e-009$
$3.8792 e-002$
$6.9284 e-003$
$1.8356 e+002$
$3.7270 e-009$
$3.4341 e-005$
$3.4341 e-005$
$7.8185 e-001$
$2.4658 e+001$
$2.4658 e+001$

$\mathrm{Ba} / \mathrm{cm}^{3}$

$6.4244 \mathrm{e}+006$

$1.3790 e-004$

$1.4353 e+003$

$2.5635 e+002$

$6.7915 e+006$

$1.3790 e-004$

$1.2706 \mathrm{e}+000$

$1.2706 e+000$

$2.8929 e+004$

$9.1236 e+005$

$9.1236 e+005$

Buildup

The material reference is : Shield 1

22

32

32

\section{Results}

Fluence Rate With Buildup

$\frac{\text { No Builaup }}{2.713 e-90}$

$1.676 \mathrm{e}-23$ 
Page : 2

DOS File: UDSSTOR.MS5

Run Date: August 26, 1998

Run Time: 5:12:41 PM

Duration: 00:02:15

\begin{tabular}{|c|c|c|c|c|c|}
\hline$\frac{\text { Energy }}{\text { MeV }}$ & $\frac{\text { Activity }}{\text { photons/sec }}$ & $\frac{\text { Fluence Rate }}{\frac{\text { MeV/cm } \text { cm }^{2} \text { sec }}{\text { No Buildup }}}$ & $\frac{\text { Fluence Rate }}{\frac{\text { MeV/cm } / \text { sec }}{\text { With Buildup }}}$ & $\frac{\text { Exposure Rate }}{\text { No } \frac{\mathrm{mR} / \mathrm{hr}}{\mathrm{Buildup}}}$ & $\begin{array}{l}\frac{\text { Exposure Rate }}{\text { mR/hr }} \\
\text { with Buildup }\end{array}$ \\
\hline 0.04 & $1.505 e+11$ & $1.395 e-43$ & $1.037 e-21$ & $6.169 e-46$ & $4.587 e-24$ \\
\hline 0.06 & $3.039 e-01$ & $1.230 e-31$ & $5.133 e-30$ & $2.443 e-34$ & $1.020 e-32$ \\
\hline 0.08 & $3.665 e+00$ & $2.285 e-24$ & $1.669 e-22$ & $3.616 e-27$ & $2.641 e-25$ \\
\hline 0.1 & $1.256 e+08$ & $2.835 e-14$ & $4.235 e-12$ & $4.338 e-17$ & $6.480 e-15$ \\
\hline 0.15 & $1.207 e+08$ & $1.411 e-11$ & $3.975 e-09$ & $2.324 e-14$ & $6.546 e-12$ \\
\hline 0.2 & $3.651 e+09$ & $9.995 e-09$ & $3.114 e-06$ & $1.764 e-11$ & $5.496 e-09$ \\
\hline 0.3 & $2.008 e+08$ & $2.628 e-08$ & $5.893 e-06$ & $4.985 e-11$ & $1.118 e-08$ \\
\hline 0.4 & $1.506 e+10$ & $2.546 e-05$ & $3.747 e-03$ & $4.961 e-08$ & $7.300 e-06$ \\
\hline 0.5 & $4.986 e+09$ & $5.683 e-05$ & $5.785 e-03$ & $1.115 e-07$ & $1.136 e-05$ \\
\hline 0.6 & $9.629 e+12$ & $4.952 e-01$ & $3.675 e+01$ & $9.665 e-04$ & $7.174 e-02$ \\
\hline 0.8 & $4.012 e+08$ & $2.040 e-04$ & $9.199 e-03$ & $3.880 e-07$ & $1.750 e-05$ \\
\hline 1.0 & $2.398 e+09$ & $6.672 e-03$ & $2.063 e-01$ & $1.230 e-05$ & $3.802 e-04$ \\
\hline 1.5 & $2.399 e+09$ & $1.187 e-01$ & $1.949 e+00$ & $1.998 e-04$ & $3.280 e-03$ \\
\hline 2.0 & $1.775 \mathrm{e}+00$ & $5.392 e-10$ & $6.064 e-09$ & $8.338 e-13$ & $9.377 e-12$ \\
\hline דגחר & $1.046 e+13$ & $6.209 e-01$ & $3.893 e+01$ & $1.179 e-03$ & $7.543 e-02$ \\
\hline
\end{tabular}


Microshield v5.01 (5.01-00121)

Page : 1

Lockheed Martin Idaho Technologies Company

DOS File: WM181107.MS5

Run Date: September 1, 1998

Run Time: 4:54:40 PM

Duration: 00:01:57

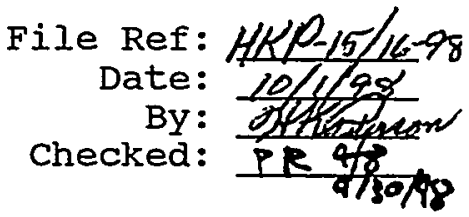

Case Title: WM181-107

Description: 55-gal drum loaded with $291.2 \mathrm{~kg}$ of stream 107 (Pb shield) Geometry: 7 - cylinder volume - side shields

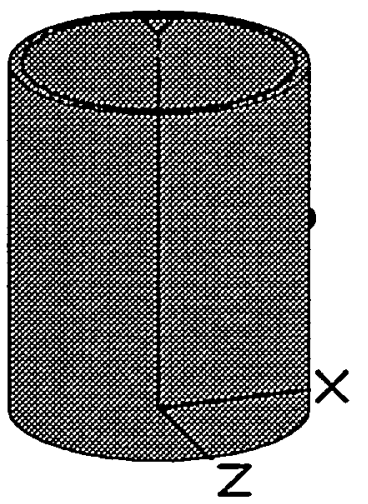

$\begin{array}{lrrr} & \text { Source } & \text { Dimensions } \\ \text { Height } & 77.598 \mathrm{~cm} & 2 \text { ft } 6.6 \text { in } \\ \text { Radius } & 29.21 \mathrm{~cm} & 11.5 \text { in }\end{array}$

\begin{tabular}{|c|c|c|c|c|}
\hline & & Dos & Points & \\
\hline 1 & $32^{x}$ & & $\frac{\underline{y}}{38}$ & $\underline{z}$ \\
\hline & ft 1.0 & in & 1 ft 3.3 in & 0.0 in \\
\hline
\end{tabular}

Shields

Shield Name Dimension Material Density Source $2.08 \mathrm{e}+05 \mathrm{~cm}^{3}$ Concrete 1.4

Shield $1 \quad .16 \mathrm{~cm}$ Iron 7.86

Shield $2 \quad 2.54 \mathrm{~cm}$ Lead 11.34

Transition $\quad 1.0 \mathrm{~cm}$ Air 0.0011

Air Gap

Air

0.00122

Source Input

Grouping Method : Standard Indices

Number of Groups : 25

Lower Energy Cutoff : 0.015

Photons < 0.015 : Excluded

Library : ICRP-38

\begin{tabular}{|c|c|c|c|c|}
\hline Nuclide & curies & becquerels & $\mu \mathrm{Ci} / \mathrm{cm}^{3}$ & $\mathrm{Bg} / \mathrm{cm}^{3}$ \\
\hline$A m-241$ & $3.4650 e-001$ & $1.2821 e+010$ & $1.6659 e+000$ & $6.1637 e+00$ \\
\hline $3 a-137 m$ & $.6090 e+001$ & $3353 e+012$ & $1.7351 \mathrm{e}+002$ & $199 e+0 c$ \\
\hline $\mathrm{Ce}-144$ & $460 e-010$ & $2.8660 e+001$ & $240 e-009$ & 1 \\
\hline Co-60 & $8.0660 e-003$ & $2.9844 e+008$ & $3.8779 e-002$ & $1.4348 e+003$ \\
\hline$C s-134$ & $1.4360 e-003$ & $5.3132 e+007$ & $6.9038 e-003$ & $2.5544 e+002$ \\
\hline Cs -137 & $3.8150 e+001$ & $1.4116 e+012$ & $1.8341 e+002$ & $6.7863 e+006$ \\
\hline $\mathrm{Np}-237$ & $5.0700 e-003$ & $1.8759 e+008$ & $2.4375 e-002$ & $e+00$ \\
\hline $\mathrm{Pr}-144$ & $7460 e-010$ & $2.8660 e+001$ & $3.7240 e-009$ & $1.3779 e-004$ \\
\hline 38 & $0 e+001$ & +011 & $9.9663 e+001$ & $3.6875 e+006$ \\
\hline $\mathrm{Pu}-239$ & $e+000$ & $4 e+010$ & $52 e+000$ & $1.9781 e+005$ \\
\hline $\mathrm{Pu}-240$ & $3.4900 e-001$ & $1.2913 e+010$ & $1.6779 e+000$ & $6.2082 e+004$ \\
\hline $\mathrm{Pu}-241$ & 7.1930 et000 & $2.6614 e+011$ & $3.4582 e+001$ & $1.2795 e+006$ \\
\hline & -004 & & $18 e-003$ & $9.2198 e+001$ \\
\hline $\mathrm{kh}-1$ & 7.13 & 2.6 & -005 & $90 e+00$ \\
\hline$R u-106$ & $7.1340 e-006$ & $2.6396 e+005$ & $3.4298 e-005$ & $1.2690 \mathrm{e}+000$ \\
\hline$S b-125$ & $1.6250 \mathrm{e}-001$ & $6.0125 e+009$ & $7.8125 e-001$ & $2.8906 e+004$ \\
\hline$S r-90$ & $5.1250 e+000$ & $1.8963 e+011$ & $2.4639 e+001$ & $9.1166 e+005$ \\
\hline$U-234$ & -003 & & $3.3317 €$ & $2327 e+003$ \\
\hline$U-235$ & $1.5260 e-004$ & $5.6462 e+0$ & $7.3365 e-004$ & $2.7145 \mathrm{e}+001$ \\
\hline$U-236$ & $7.2200 \mathrm{e}-005$ & $2.6714 e+006$ & $3.4712 e-004$ & $1.2843 e+001$ \\
\hline$U-238$ & $.2600 e-005$ & $8.3620 e+005$ & $1.0865 e-004$ & $4.0202 e+000$ \\
\hline$Y-90$ & $.1250 \mathrm{e}+000$ & $1.8963 e+011$ & $2.4639 e+001$ & $9.1166 e+005$ \\
\hline
\end{tabular}

Buildup 


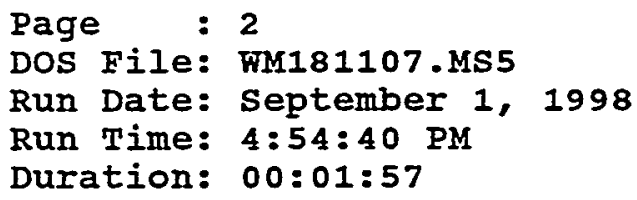

The material reference is : Shield 2

Radial Circumferential $Y$ Direction (axial)
24

24

26

\begin{tabular}{|c|c|c|c|c|c|}
\hline Energy & Activity & Fluence Rate & $\begin{array}{l}\text { Resulcs } \\
\text { Fluence Rate }\end{array}$ & Exposure Rate & Exposure Rate \\
\hline $\mathrm{MeV}$ & photons/sec & $\frac{\mathrm{MeV} / \mathrm{cm}^{2} / \mathrm{sec}}{\text { No Buildup }}$ & $\frac{\mathrm{MeV} / \mathrm{cm}^{2} / \mathrm{sec}}{\text { With Buildup }}$ & No $\frac{m R / h r}{\text { Buildup }}$ & With Buildup \\
\hline 0.015 & $4.642 e+10$ & $0.000 e+00$ & $4.288 e-22$ & $0.000 e+00$ & $3.678 e-23$ \\
\hline 0.02 & $1.397 e+10$ & $0.000 e+00$ & $1.720 e-22$ & $0.000 e+00$ & $5.959 e-24$ \\
\hline 0.03 & $8.404 e+10$ & $0.000 e+00$ & $1.596 e-21$ & $0.000 e+00$ & $1.582 e-23$ \\
\hline 0.04 & $1.978 e+10$ & $9.006 e-171$ & $5.151 e-22$ & $3.983 e-173$ & $2.278 e-24$ \\
\hline 0.05 & $1.524 \mathrm{e}+07$ & $2.275 e-96$ & $5.114 e-25$ & $6.060 e-99$ & $1.362 e-27$ \\
\hline 0.06 & $4.584 e+09$ & $2.936 e-57$ & $1.934 e-22$ & $5.832 e-60$ & $3.842 e-25$ \\
\hline 0.08 & $2.761 e+07$ & $2.886 e-28$ & $1.741 e-24$ & $4.567 e-31$ & $2.755 e-27$ \\
\hline 0.1 & $1.084 e+08$ & $5.130 e-68$ & $2.711 e-07$ & $7.848 e-71$ & $4.148 e-10$ \\
\hline 0.15 & $3.043 e+07$ & $6.969 e-25$ & $1.010 e-22$ & $1.148 e-27$ & $1.663 e-25$ \\
\hline 0.2 & $4.433 e+08$ & $5.135 e-11$ & $7.516 e-11$ & $9.063 e-14$ & $1.327 e-13$ \\
\hline 0.3 & $2.782 e+07$ & $1.601 e-04$ & $2.388 e-04$ & $3.036 e-07$ & $4.530 e-07$ \\
\hline 0.4 & $1.892 e+09$ & $2.611 e+00$ & $4.367 e+00$ & $5.087 e-03$ & $8.509 e-03$ \\
\hline 0.5 & $6.217 e+08$ & $1.037 e+01$ & $1.876 e+01$ & $2.036 e-02$ & $3.682 e-02$ \\
\hline 0.6 & $1.201 e+12$ & $8.213 e+04$ & $1.554 \mathrm{e}+05$ & $1.603 e+02$ & $3.033 e+02$ \\
\hline 0.8 & $5.049 e+07$ & $1.696 e+01$ & $3.434 e+01$ & $3.227 e-02$ & $6.532 e-02$ \\
\hline 1.0 & $2.997 e+08$ & $2.550 e+02$ & $5.317 e+02$ & $4.700 e-01$ & $9.802 e-01$ \\
\hline 1.5 & $3.000 e+08$ & $9.084 e+02$ & $1.861 e+03$ & $1.528 e+00$ & $3.131 e+00$ \\
\hline 2.0 & $3.805 e+03$ & $2.202 e-02$ & $4.459 e-02$ & $3.405 e-05$ & $6.895 e-05$ \\
\hline 3.0 & $5.506 e+01$ & $6.525 e-04$ & $1.255 e-03$ & $8.852 e-07$ & $1.703 e-06$ \\
\hline TOTALS: & $1.374 \mathrm{e}+12$ & $8.333 e+04$ & $1.578 e+05$ & $1.624 e+02$ & $3.075 e+02$ \\
\hline
\end{tabular}


Microshield v5.01 (5.01-00121)

Lockheed Martin Idaho Technologies Company

Page : 1

DOS File: WM181107.MS5

Run Date: September 1, 1998

Run Time: 4:51:24 PM

Duration: 00:01:58

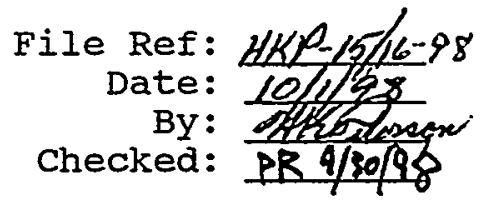

Case Title: WM181-107

Description: 55-gal drum loaded with $291.2 \mathrm{~kg}$ of stream 107 (Pb shield) Geometry: 7 - Cylinder Volume - side shields

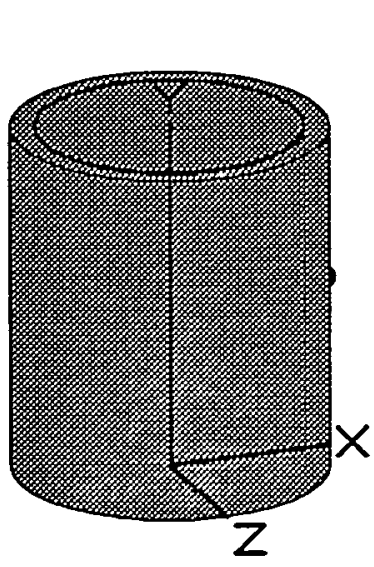

\begin{tabular}{lrrr}
\multicolumn{5}{c}{ Source } & Dimensions \\
Height & $77.598 \mathrm{~cm}$ & $2 \mathrm{ft}$ & $6.6 \mathrm{in}$ \\
Radius & $29.21 \mathrm{~cm}$ & $11.5 \mathrm{in}$
\end{tabular}

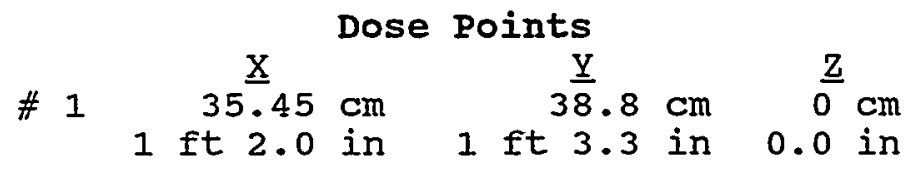

shields

Shield Name Dimension MaterialDensity

Source $2.08 \mathrm{e}+05 \mathrm{~cm}^{3}$ Concrete 1.4

Shield 1

$.16 \mathrm{~cm}$ Iron 7.86

Shield 2

Transition

$5.08 \mathrm{~cm}$

Lead

11.34

Air Gap

$1.0 \mathrm{~cm}$

Air

0.0011

Air

0.0011

Source Input

Grouping Method : standard Indices

Number of Groups : 25

Lower Energy Cutoff : 0.015

Photons < 0.015 : Excluded

\begin{tabular}{l} 
Nuclide \\
\hline $\mathrm{Am}-24 \mathrm{I}$ \\
$\mathrm{Ba}-137 \mathrm{~m}$ \\
$\mathrm{Ce}-144$ \\
$\mathrm{Co}-60$ \\
$\mathrm{Cs}-134$ \\
$\mathrm{Cs}-137$ \\
$\mathrm{~Np}-237$ \\
$\mathrm{Pr}-144$ \\
$\mathrm{Pu}-238$ \\
$\mathrm{Pu}-239$ \\
$\mathrm{Pu}-240$ \\
$\mathrm{Pu}-241$ \\
$\mathrm{Pu}-242$ \\
$\mathrm{Rh}-106$ \\
$\mathrm{Ru}-106$ \\
$\mathrm{Sb}-125$ \\
$\mathrm{Sr}-90$ \\
$\mathrm{U}-234$ \\
$\mathrm{U}-235$ \\
$\mathrm{U}-236$ \\
$\mathrm{U}-238$ \\
$\mathrm{Y}-90$
\end{tabular}

Library : ICRP-38

\section{curies}

$3.4650 e-001$

$3.6090 e+001$

$7.7460 e-010$

$8.0660 e-003$

$1.4360 e-003$

$3.8150 e+001$

$5.0700 e-003$

$7.7460 \mathrm{e}-010$

$2.0730 e+001$

$1.1120 \mathrm{e}+000$

$3.4900 e-001$

$7.1930 e+000$

$5.1830 e-004$

$7.1340 \mathrm{e}-006$

$7.1340 \mathrm{e}-006$

$1.6250 e-001$

$5.1250 e+000$

$6.9300 e-003$

$1.5260 \mathrm{e}-004$

$7.2200 e-005$

$2.2600 e-005$

$5.1250 e+000$ $\frac{\text { becquerels }}{1.2821 \mathrm{e}+010}$

$1.3353 e+012$

$2.8660 e+001$

$2.9844 e+008$

$5.3132 e+007$

$1.4116 \mathrm{e}+012$

$1.8759 e+008$

$2.8660 e+001$

$7.6701 e+011$

$4.1144 \mathrm{e}+010$

$1.2913 e+010$

2. 6614e+011

$1.9177 \mathrm{e}+007$

2. $6396 e+005$

2. $6396 \mathrm{e}+005$

$6.0125 e+009$

$1.8963 \mathrm{e}+011$

$2.5641 \mathrm{e}+008$

$5.6462 e+006$

$2.6714 \mathrm{e}+006$

$8.3620 e+005$

$1.8963 e+011$ $\mu \mathrm{Ci} / \mathrm{cm}^{3}$

$1.6659 \mathrm{e}+000$

$1.7351 e+002$

3. $7240 e-009$

$3.8779 e-002$

$6.9038 e-003$

1.8341 e+002

$2.4375 e-002$

$3.7240 e-009$

$9.9663 e+001$

$5.3462 e+000$

$1.6779 e+000$

$3.4582 e+001$

$2.4918 e-003$

$3.4298 \mathrm{e}-005$

$3.4298 e-005$

$7.8125 e-001$

2.4639 et001

$3.3317 e-002$

$7.3365 e-004$

$3.4712 e-004$

$1.0865 e-004$

$2.4639 e+001$
$\underline{\mathrm{Ba} / \mathrm{cm}^{3}}$

$6.1637 e+004$

$6.4199 e+006$

$1.3779 e-004$

$1.4348 e+003$

$2.5544 e+002$

$6.7863 e+006$

9.0187 e+002

$1.3779 e-004$

$3.6875 e+006$

$1.9781 e+005$

$6.2082 e+004$

$1.2795 e+006$

$9.2198 e+001$

$1.2690 e+000$

$1.2690 e+000$

$2.8906 e+004$

$9.1166 e+005$

$1.2327 e+003$

$2.7145 e+001$

$1.2843 e+001$

$4.0202 e+000$

$9.1166 \mathrm{e}+005$

Builaup 


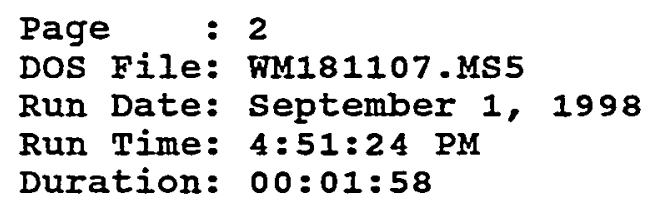

The material reference is : shield 2

Radial

Integration Parameters

Circumferential

24

Y Direction (axial) 26

\begin{tabular}{|c|c|c|c|c|c|}
\hline Energy & Activity & Fluence Rate & $\begin{array}{l}\text { Results } \\
\text { Fluence Rate }\end{array}$ & Exposure Rate & osure Rate \\
\hline $\mathrm{MeV}$ & photons/sec & $\mathrm{MeV} / \mathrm{cm}^{2} / \mathrm{sec}$ & $\mathrm{MeV} / \mathrm{cm}^{2} / \mathrm{sec}$ & $\mathrm{mR} / \mathrm{hr}$ & $\mathrm{mR} / \mathrm{hr}$ \\
\hline & & No Builaup & With Buildup & No Buildup & with Buildup \\
\hline 0.015 & $4.642 e+10$ & $0.000 e+00$ & $3.663 e-22$ & $0.000 e+00$ & $3.142 e-23$ \\
\hline 0.02 & $1.397 e+10$ & $0.000 e+00$ & $1.470 e-22$ & $0.000 e+00$ & $5.091 e-24$ \\
\hline 0.03 & $8.404 e+10$ & $0.000 e+00$ & $1.364 e-21$ & $0.000 e+00$ & $1.351 e-23$ \\
\hline 0.04 & $1.978 e+10$ & $0.000 e+00$ & $4.400 e-22$ & $0.000 e+00$ & $1.946 e-24$ \\
\hline 0.05 & $1.524 e+07$ & $1.517 e-189$ & $4.369 e-25$ & $4.042 e-192$ & $1.164 e-27$ \\
\hline 0.06 & $4.584 e+09$ & $1.203 e-114$ & $1.652 e-22$ & $2.389 e-117$ & $3.282 e-25$ \\
\hline 0.08 & $2.761 e+07$ & $2.721 e-55$ & $1.487 e-24$ & $4.306 e-58$ & $2.353 e-27$ \\
\hline 0.1 & $1.084 e+08$ & $2.620 e-135$ & $2.316 e-07$ & $4.009 e-138$ & $3.543 e-10$ \\
\hline 0.15 & $3.043 e+07$ & $3.140 e-49$ & $7.484 e-23$ & $5.170 e-52$ & $1.232 e-25$ \\
\hline 0.2 & $4.433 e+08$ & $4.061 e-23$ & $1.289 e-22$ & $7.167 e-26$ & $2.275 e-25$ \\
\hline 0.3 & $2.782 e+07$ & $1.601 e-09$ & $2.600 e-09$ & $3.037 e-12$ & $4.931 e-12$ \\
\hline 0.4 & $1.892 e+09$ & $2.729 e-03$ & $5.229 e-03$ & $5.318 e-06$ & $1.019 e-05$ \\
\hline 0.5 & $6.217 e+08$ & $7.460 e-02$ & $1.593 e-01$ & $1.464 e-04$ & $3.127 e-04$ \\
\hline 0.6 & $1.201 e+12$ & $1.597 e+03$ & $3.614 e+03$ & $3.117 e+00$ & $7.054 e+00$ \\
\hline 0.8 & $5.049 e+07$ & $8.892 e-01$ & $2.201 e+00$ & $1.691 e-03$ & $4.187 e-03$ \\
\hline 1.0 & $2.997 e+08$ & $2.195 e+01$ & $5.669 e+01$ & $4.046 e-02$ & $1.045 e-01$ \\
\hline 1.5 & $3.000 e+08$ & $1.344 \mathrm{e}+02$ & $3.480 e+02$ & $2.261 e-01$ & $5.855 e-01$ \\
\hline 2.0 & $3.805 e+03$ & $3.898 e-03$ & $1.006 e-02$ & $6.028 e-06$ & $1.555 e-05$ \\
\hline 3.0 & $5.506 e+01$ & $1.288 e-04$ & $3.159 e-04$ & $1.748 e-07$ & $4.285 e-07$ \\
\hline TOTALS & $1.374 e+12$ & $1.754 e+03$ & $4.021 e+03$ & $3.386 e+00$ & $7.749 e+00$ \\
\hline
\end{tabular}


Microshield v5.01 (5.01-00121)

Page : 1

DOS File: WM181107.MS5

Run Date: September 1, 1998

Run Time: 4:58:50 PM

Duration: $00: 01: 55$

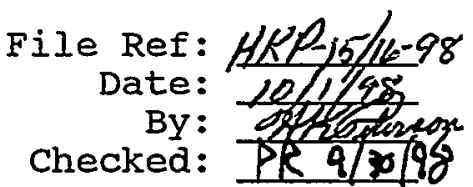

Case Title: WM181-107

Description: 55-gal drum loaded with $291.2 \mathrm{~kg}$ of stream 107 (Fe shield) Geometry: 7 - Cylinder volume - side Shields

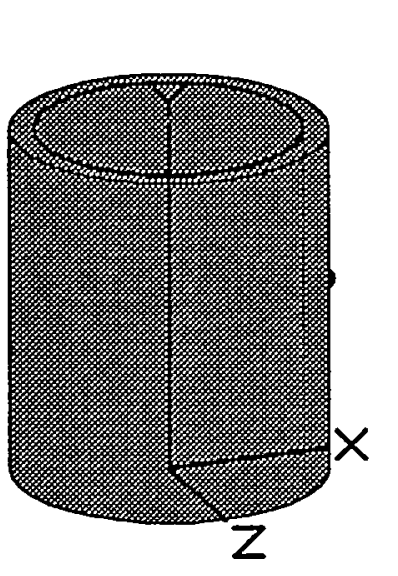

\begin{tabular}{lrrr}
\multicolumn{5}{c}{ Source Dimensions } \\
Height & $77.598 \mathrm{~cm}$ & $2 \mathrm{ft} 6.6$ in \\
Radius & $29.21 \mathrm{~cm}$ & $11.5 \mathrm{in}$
\end{tabular}

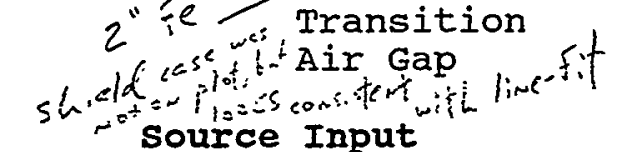

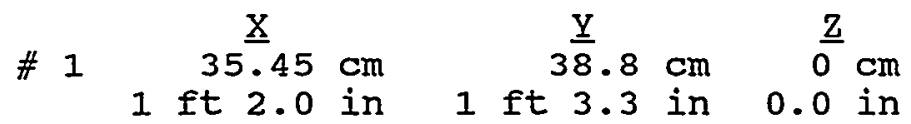

shields

Shield Name Dimension Material Density Source $2.08 \mathrm{e}+05 \mathrm{~cm}^{3}$ Concrete 1.4 Shield $1 . .16 \mathrm{~cm}$ Iron 7.86 Shield $2 \quad 5.08 \mathrm{~cm}$ Iron 7.86

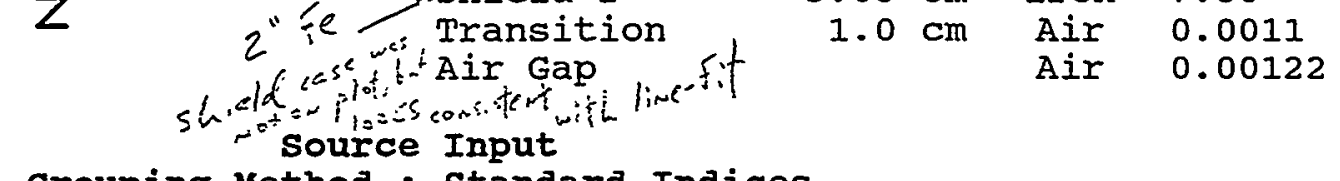
Grouping Method : Standard Indices Number of Groups : 25 Lower Energy Cutoff : 0.015 Photons < 0.015 : Excluded Library : ICRP-38

\begin{tabular}{|c|c|c|c|c|}
\hline Nuclide & curies & becquerels & $\mu \mathrm{Ci} / \mathrm{cm}^{3}$ & $\mathrm{Bg} / \mathrm{cm}^{3}$ \\
\hline $\mathrm{Am}-241$ & $3 . \overline{4650 e-001}$ & $1.2821 \mathrm{e}+010$ & $1 . \overline{6659 e+0} 00$ &.$\overline{1637 e+004}$ \\
\hline $\mathrm{Ba}-137 \mathrm{~m}$ & $3.6090 e+001$ & $1.3353 e+012$ & $1.7351 \mathrm{e}+002$ & +006 \\
\hline$e-144$ & 010 & $2.8660 \mathrm{e}+001$ & $7240 e-009$ & 1 \\
\hline Co-60 & $560 e-003$ & $2.9844 e+008$ & $8779 e-002$ & +003 \\
\hline $\mathrm{Cs}-134$ & $1.4360 e-003$ & $5.3132 e+007$ & $6.9038 e-003$ & $2.5544 \mathrm{e}+002$ \\
\hline Cs -137 & $3.8150 e+001$ & $1.4116 e+012$ & $1.8341 \mathrm{e}+002$ & $7863 e+006$ \\
\hline $\mathrm{Np}-237$ & $e-003$ & 1.87 & 2.4 & $7 e+002$ \\
\hline Pr-144 & $e-010$ & 2.86 & $0 e-009$ & $.3779 e-004$ \\
\hline $\mathrm{Pu}-238$ & $2.0730 e+001$ & 7.6701 & $9.9663 e$ & +006 \\
\hline $\mathrm{Pu}-2$ & +000 & & 000 & +005 \\
\hline $\mathrm{Pu}-$ & 3.49 & 1.2 & 1.67 & $2 e+004$ \\
\hline $\mathrm{Pu}-$ & $e+000$ & 2.66 & 3.458 & $5 e+006$ \\
\hline $\mathrm{Pu}-$ & $5.1830 e-004$ & $1.9177 e+007$ & $2.4918 e-003$ & $9.2198 e+001$ \\
\hline $\mathrm{Rh}-$ & $e-006$ & 2.63 & & +000 \\
\hline 06 & $e-006$ & 2.6 & 3.4 & $e+000$ \\
\hline & Je-001 & 6.0 & -001 & $6 e+004$ \\
\hline$S r-90$ & .1250 e +000 & $1.8963 e+011$ & $e+001$ & $9.1166 e+005$ \\
\hline$U-234$ & $.9300 e-003$ & $2.5641 e+008$ & $3.3317 e-002$ & $1.2327 e+003$ \\
\hline$U-235$ & $5260 e-004$ & & $5 e-004$ & $7145 e+001$ \\
\hline & $2200 e-005$ & $2.6714 \mathrm{e}+00$ & & $1.2843 e+001$ \\
\hline & $2600 e-005$ & $8.3620 e+0$ & $1.0865 e-004$ & $4.0202 e+000$ \\
\hline$Y-90$ & $1250 e+000$ & $1.8963 e+011$ & $2.4639 e+001$ & $9.1166 e+005$ \\
\hline
\end{tabular}

Buildup 


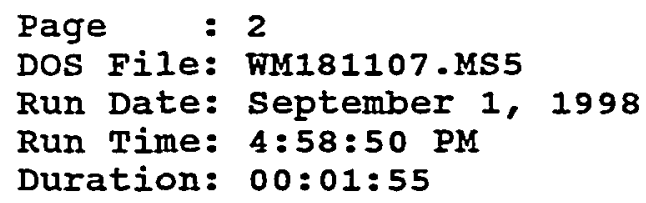

The material reference is : shield 2

Radial

\section{Integration Parameters}

Circumferential $Y$ Direction (axial)

$$
\begin{aligned}
& 24 \\
& 24 \\
& 26
\end{aligned}
$$

\section{Results}

$\begin{array}{cc}\frac{\text { Energy }}{\text { MeV }} & \begin{array}{c}\text { Activity } \\ \text { photons/se }\end{array} \\ 0.015 & 4.642 e+10 \\ 0.02 & 1.397 \mathrm{e}+10 \\ 0.03 & 8.404 \mathrm{e}+10 \\ 0.04 & 1.978 \mathrm{e}+10 \\ 0.05 & 1.524 \mathrm{e}+07 \\ 0.06 & 4.584 \mathrm{e}+09 \\ 0.08 & 2.761 \mathrm{e}+07 \\ 0.1 & 1.084 \mathrm{e}+08 \\ 0.15 & 3.043 e+07 \\ 0.2 & 4.433 \mathrm{e}+08 \\ 0.3 & 2.782 \mathrm{e}+07 \\ 0.4 & 1.892 \mathrm{e}+09 \\ 0.5 & 6.217 \mathrm{e}+08 \\ 0.6 & 1.201 \mathrm{e}+12 \\ 0.8 & 5.049 \mathrm{e}+07 \\ 1.0 & 2.997 \mathrm{e}+08 \\ 1.5 & 3.000 \mathrm{e}+08 \\ 2.0 & 3.805 \mathrm{e}+03 \\ 3.0 & 5.506 \mathrm{e}+01\end{array}$

Fluence Rate $\mathrm{MeV} / \mathrm{cm}^{2} / \mathrm{sec}$

No Buildup

$0.000 e+00$

$0.000 e+00$

$1.993 e-141$

$1.049 e-61$

$2.825 e-35$

$1.828 e-19$

$9.517 e-11$

$4.543 e-06$

$2.835 e-03$

$4.817 e-01$

2. $388 e-01$

$4.862 e+01$

$3.432 e+01$

$1.198 e+05$

$1.239 e+01$

$1.445 e+02$

$4.633 e+02$

$1.234 e-02$

$4.467 e-04$

$1.205 e+05$
Fluence Rate $\mathrm{MeV} / \mathrm{cm}^{2} / \mathrm{sec}$ With Buildup

$3.700 e-22$

$1.750 \mathrm{e}-22$

$1.446 e-21$

$5.204 \mathrm{e}-22$

$5.732 e-25$

$3.099 e-19$

2. $219 e-10$

$1.397 e-05$

1. $402 e-02$

$3.285 e+00$

$1.982 \mathrm{e}+00$

$4.098 e+02$

$2.726 e+02$

$8.788 e+05$

$7.719 e+01$

$7.779 e+02$

1.900 et03

$4.285 e-02$

1. $248 \mathrm{e}-03$

$8.822 e+05$
Exposure Rate $\mathrm{mR} / \mathrm{hr}$

No Buildup

$0.000 e+00$

$0.000 e+00$

$1.975 e-143$

$4.638 e-64$

$7.525 e-38$

$3.631 e-22$

$1.506 e-13$

$6.950 e-09$

$4.669 e-06$

$8.502 e-04$

$4.530 e-04$

$9.473 e-02$

$6.736 e-02$

$2.339 e+02$

$2.357 e-02$

$2.664 \mathrm{e}-01$

$7.794 e-01$

$1.908 e-05$

$6.061 e-07$

$2.352 e+02$
Exposure Rate $\mathrm{mR} / \mathrm{hr}$

With Buildup

$3.174 e-23$

$6.061 \mathrm{e}-24$

$1.433 e-23$

$2.301 e-24$

$1.527 \mathrm{e}-27$

$6.156 \mathrm{e}-22$

3. $511 e-13$

$2.137 e-08$

$2.308 e-05$

$5.798 e-03$

$3.759 e-03$

$7.984 \mathrm{e}-01$

$5.351 e-01$

1. $715 e+03$

$1.468 \mathrm{e}-01$

$1.434 \mathrm{e}+00$

$3.196 \mathrm{e}+00$

$6.626 e-05$

1. $693 e-06$

$1.721 e+03$ 
Microshield v5.01 (5.01-00121)

Page : 1

DOS File: WM181107.MS5

Run Date: September 1, 1998

Run Time: 5:03:43 PM

Duration: 00:01:54

Lockheed Martin Idaho Technologies Company

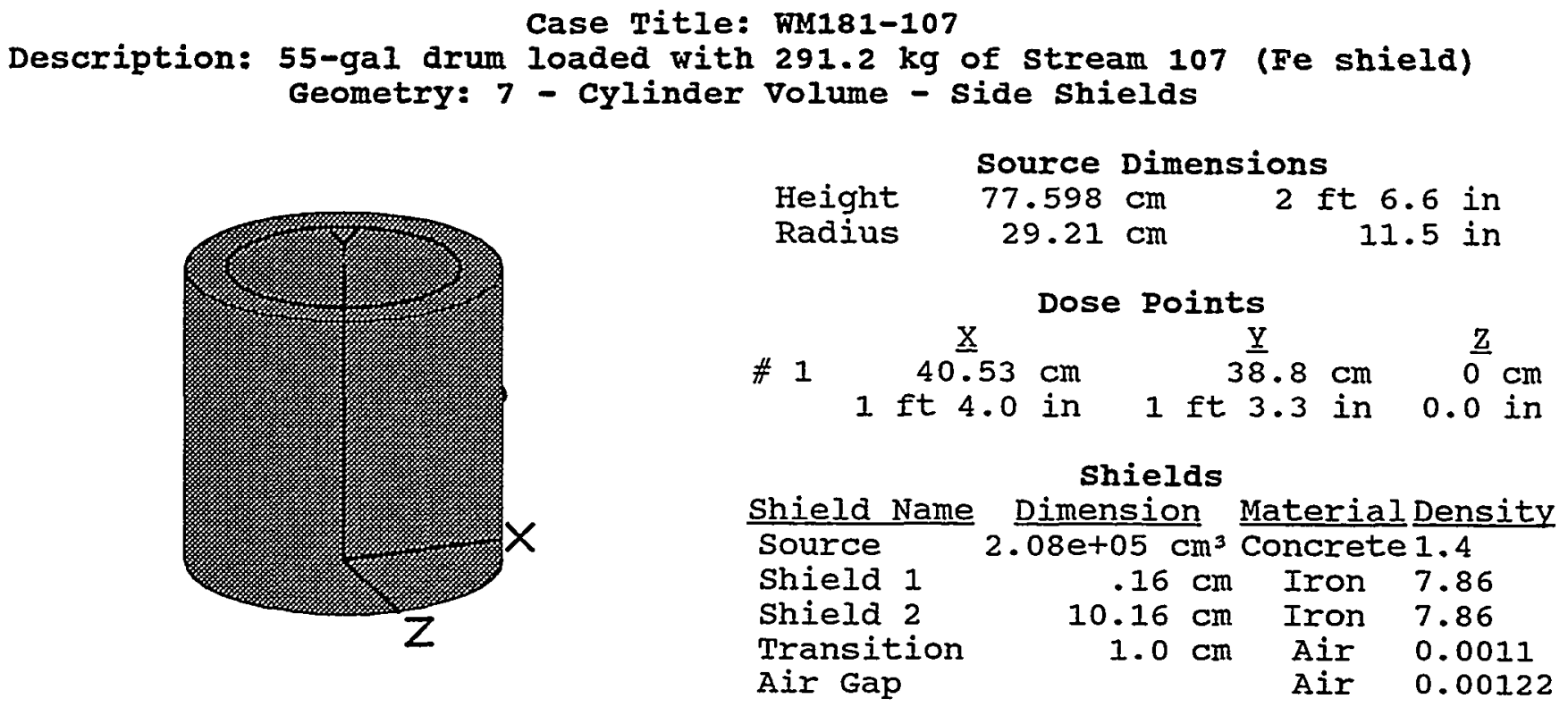

Source Input

Grouping Method : Standard Indices

Number of Groups : 25

Lower Energy Cutoff : 0.015

Photons < 0.015 : Excluded

Library : ICRP-38

$\begin{array}{lc}\text { Nuclide } & \begin{array}{c}\text { curies } \\ \text { Am-241 }\end{array} \\ \text { Ba-137m } & 3.6050 \mathrm{e}-001 \\ \mathrm{Ce}-144 & 7.7460 \mathrm{e}+001 \\ \mathrm{Co}-60 & 8.0660 \mathrm{e}-003 \\ \mathrm{Cs}-134 & 1.4360 \mathrm{e}-003 \\ \mathrm{Cs}-137 & 3.8150 \mathrm{e}+001 \\ \mathrm{~Np}-237 & 5.0700 \mathrm{e}-003 \\ \mathrm{Pr}-144 & 7.7460 \mathrm{e}-010 \\ \mathrm{Pu}-238 & 2.0730 \mathrm{e}+001 \\ \mathrm{Pu}-239 & 1.1120 \mathrm{e}+000 \\ \mathrm{Pu}-240 & 3.4900 \mathrm{e}-001 \\ \mathrm{Pu}-241 & 7.1930 \mathrm{e}+000 \\ \mathrm{Pu}-242 & 5.1830 \mathrm{e}-004 \\ \mathrm{Rh}-106 & 7.1340 \mathrm{e}-006 \\ \mathrm{Ru}-106 & 7.1340 \mathrm{e}-006 \\ \mathrm{Sb}-125 & 1.6250 \mathrm{e}-001 \\ \mathrm{Sr}-90 & 5.1250 \mathrm{e}+000 \\ \mathrm{U}-234 & 6.9300 \mathrm{e}-003 \\ \mathrm{U}-235 & 1.5260 \mathrm{e}-004 \\ \mathrm{U}-236 & 7.2200 \mathrm{e}-005 \\ \mathrm{U}-238 & 2.2600 \mathrm{e}-005 \\ \mathrm{Y}-90 & 5.1250 \mathrm{e}+000\end{array}$

becquerels

$1.2821 e+010$

$1.3353 e+012$

$2.8660 e+001$

$2.9844 \mathrm{e}+008$

$5.3132 e+007$

$1.4116 e+012$

$1.8759 e+008$

$2.8660 e+001$

$7.6701 e+011$

$4.1144 e+010$

$1.2913 e+010$

$2.6614 e+011$

$1.9177 e+007$

$2.6396 e+005$

$2.6396 e+005$

$6.0125 e+009$

$1.8963 e+011$

$2.5641 e+008$

$5.6462 e+006$

2. $6714 e+006$

$8.3620 e+005$

$1.8963 e+011$ $\mu \mathrm{Ci} / \mathrm{cm}^{3}$

$1.6659 e+000$

1.7351 e+002

$3.7240 e-009$

$3.8779 e-002$

$6.9038 e-003$

$1.8341 e+002$

$2.4375 e-002$

$3.7240 e-009$

$9.9663 e+001$

$5.3462 e+000$

$1.6779 e+000$

$3.4582 e+001$

$2.4918 e-003$

$3.4298 e-005$

$3.4298 e-005$

$7.8125 e-001$

$2.4639 e+001$

$3.3317 e-002$

$7.3365 e-004$

$3.4712 e-004$

$1.0865 e-004$

$2.4639 e+001$
File Ref:

Date:

Checked:

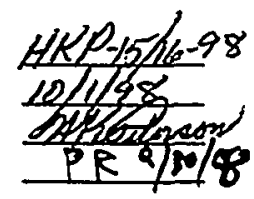




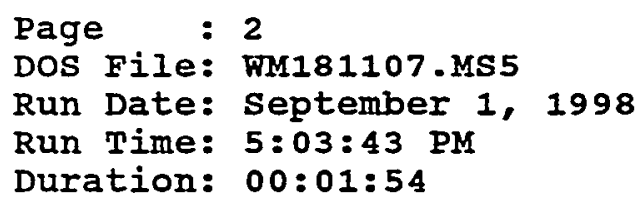

The material reference is : shield 2

Radial

Integration Parameters

Circumferential

$Y$ Direction (axial)

$$
\begin{aligned}
& 24 \\
& 24 \\
& 26
\end{aligned}
$$

\begin{tabular}{|c|c|c|c|c|c|}
\hline \multirow{3}{*}{$\frac{\text { Energy }}{\text { MeV }}$} & Activity & Fluence Rate & $\begin{array}{l}\text { Results } \\
\text { Fluence Rate }\end{array}$ & Exposure Rate & Exposure Rate \\
\hline & photons/sec & $\mathrm{MeV} / \mathrm{cm}^{2} / \mathrm{sec}$ & $\mathrm{MeV} / \mathrm{cm}^{2} / \mathrm{sec}$ & $\mathrm{mR} / \mathrm{hr}$ & $\mathrm{mR} / \mathrm{hr}$ \\
\hline & & No Buildup & with Buildup & No Buildup & With Buildup \\
\hline 0.015 & $4.642 e+10$ & $0.000 e+00$ & $2.832 e-22$ & $0.000 e+00$ & $2.429 e-23$ \\
\hline 0.02 & $1.397 e+10$ & $0.000 e+00$ & $1.339 e-22$ & $0.000 e+00$ & $4.640 e-24$ \\
\hline 0.03 & $8.404 e+10$ & $2.875 e-279$ & $1.107 e-21$ & $2.849 e-281$ & $1.097 e-23$ \\
\hline 0.04 & $1.978 e+10$ & $2.276 e-122$ & $3.983 e-22$ & $1.006 e-124$ & $1.762 e-24$ \\
\hline 0.05 & $1.524 e+07$ & $9.434 e-68$ & $4.388 e-25$ & $2.513 e-70$ & $1.169 e-27$ \\
\hline 0.06 & $4.584 e+09$ & $2.166 e-39$ & $1.927 e-22$ & $4.302 e-42$ & $3.827 e-25$ \\
\hline 0.08 & $2.761 e+07$ & $1.319 e-20$ & $3.476 e-20$ & $2.087 e-23$ & $5.501 e-23$ \\
\hline 0.1 & $1.084 e+08$ & $2.639 e-12$ & $1.014 \mathrm{e}-11$ & $4.037 e-15$ & $1.552 e-14$ \\
\hline 0.15 & $3.043 e+07$ & $1.001 \mathrm{e}-06$ & $7.269 \mathrm{e}-06$ & $1.649 e-09$ & $1.197 \mathrm{e}-08$ \\
\hline 0.2 & $4.433 e+08$ & $1.033 e-03$ & $1.190 e-02$ & $1.823 e-06$ & $2.101 e-05$ \\
\hline 0.3 & $2.782 e+07$ & $1.874 e-03$ & $2.921 e-02$ & $3.555 e-06$ & $5.540 e-05$ \\
\hline 0.4 & $1.892 e+09$ & $6.879 e-01$ & $1.125 e+01$ & $1.340 e-03$ & $2.192 e-02$ \\
\hline 0.5 & $6.217 e+08$ & $7.084 e-01$ & $1.085 e+01$ & $1.391 e-03$ & $2.130 e-02$ \\
\hline 0.6 & $1.201 e+12$ & $3.264 e+03$ & $4.520 \mathrm{e}+04$ & $6.371 e+00$ & $8.822 e+01$ \\
\hline 0.8 & $5.049 e+07$ & $5.044 e-01$ & $5.650 e+00$ & $9.594 e-04$ & $1.075 e-02$ \\
\hline 1.0 & $2.997 e+08$ & $7.841 e+00$ & $7.233 e+01$ & $1.445 e-02$ & $1.333 e-01$ \\
\hline 1.5 & $3.000 e+08$ & $3.993 e+01$ & $2.579 e+02$ & $6.717 e-02$ & $4.340 e-01$ \\
\hline 2.0 & $3.805 e+03$ & $1.382 e-03$ & $7.182 e-03$ & $2.137 e-06$ & $1.111 e-05$ \\
\hline 3.0 & $5.506 e+01$ & $6.597 e-05$ & $2.594 e-04$ & $8.950 e-08$ & $3.519 e-07$ \\
\hline TOTALS: & $1.374 e+12$ & $3.314 e+03$ & $4.556 e+04$ & $6.457 e+00$ & $8.884 e+01$ \\
\hline
\end{tabular}


Microshield v5.01 (5.01-00121)

Lockheed Martin Idaho Technologies Company

Page : 1

DOS File: WM181106.MS5

Run Date: September 9, 1998

Run Time: 8:41:31 AM

Duration: 00:04:11

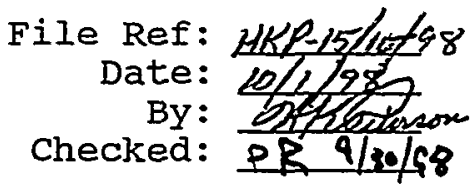

Case Title: Cs IX Effluent tnk

Description: Shielding 4 Cs IX Effluent tank (T-103 A\&B) w/14234 I St \#10 Geometry: 7 - Cylinder volume - side shields

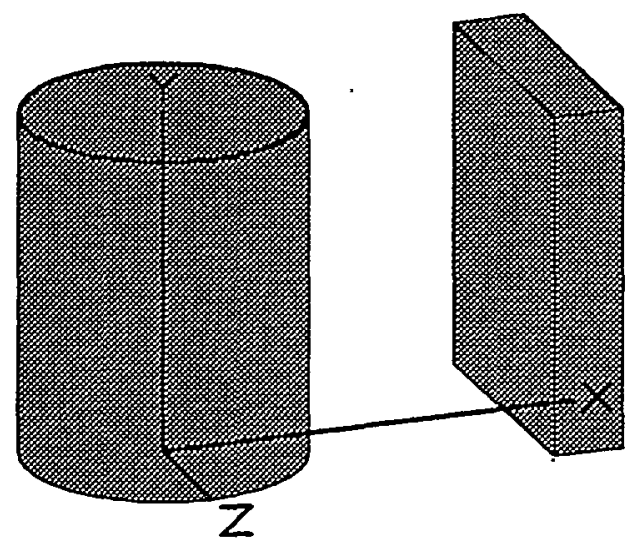

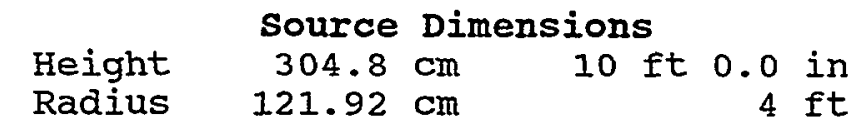

Dose Points

$\underline{x}$

Shields

Shield Name Dimension Material Density Source $1.42 \mathrm{e}+07 \mathrm{~cm}^{3}$ Water 1

Shield $1 \quad 1.27 \mathrm{~cm}$ Iron 7.86

Transition $182.88 \mathrm{~cm}$ Air 0.0011

Shield 3

Shield 4

Air Gap
$61.0 \mathrm{~cm}$ Concrete 2.35

$1.0 \mathrm{~cm}$ Air 0.0011

Air 0.00122

Source Input

Grouping Method : standard Indices

Number of Groups : 25

Lower Energy Cutoff : 0.015

Photons < 0.015 : Excluded

Iibrary : ICRP-38

Nuclide

Am-241

$\mathrm{Am}-243$

$\mathrm{Ba}-137 \mathrm{~m}$

$\mathrm{Ce}-144$

$\mathrm{Cm}-242$

$\mathrm{Cm}-244$

Co-60

Cs-134

Cs -135

Cs -137

Eu-152

Eu-154

Eu-155

$\mathrm{H}-3$

I-129

$\mathrm{Ni}-63$

$\mathrm{Np}-237$

Pm-147

Pr-144

Pu-238

Pu-239

$\mathrm{Pu}-240$

Pu-241 curies

5.2660 e+000

$2.3900 e-003$

$5.6800 e-001$

$1.2350 \mathrm{e}+000$

$1.2470 \mathrm{e}-002$

$1.6370 \mathrm{e}-001$

$1.8080 e-003$

$2.0780 e-004$

$1.1460 \mathrm{e}-005$

$6.0000 e-001$

$6.2200 e-002$

$3.4450 e+000$

$6.0500 e-001$

$3.0890 e-001$

$8.4970 e-003$

$1.4950 \mathrm{e}+000$

$4.9680 e-003$

$1.4800 \mathrm{e}+000$

$1.2350 e+000$

1.4660 e+001

$3.3450 e-001$

9.3900e-002

4.3840 et000 becquerels

$1.9484 e+011$

$8.8430 e+007$

$2.1016 e+010$

$4.5695 e+010$

$4.6139 e+008$

$6.0569 e+009$

$6.6896 e+007$

$7.6886 e+006$

$4.2402 e+005$

$2.2200 e+010$

$2.3014 e+009$

$1.2747 \mathrm{e}+011$

$2.2385 e+010$

$1.1429 e+010$

$3.1439 e+008$

$5.5315 e+010$

$1.8382 e+008$

$5.4760 e+010$

$4.5695 e+010$

$5.4242 \mathrm{e}+011$

$1.2377 e+010$

$3.4743 e+009$

$1.6221 e+011$ $\mu \mathrm{Ci} / \mathrm{cm}^{3}$

$3.6997 e-001$

$1.6791 \mathrm{e}-004$

$3.9906 e-002$

$8.6767 e-002$

8.7610e-004

$1.1501 e-002$

$1.2702 \mathrm{e}-004$

$1.4599 e-005$

$8.0514 e-007$

$4.2154 e-002$

$4.3699 e-003$

$2.4203 e-001$

$4.2505 e-002$

$2.1702 e-002$

$5.9697 e-004$

$1.0503 e-001$

$3.4903 e-004$

$1.0398 e-001$

8. $6767 e-002$

$1.0300 e+000$

$2.3501 e-002$

$6.5971 e-003$

$3.0800 e-001$
$\mathrm{Ba} / \mathrm{cm}^{3}$

$1.3689 e+004$

$6.2128 \mathrm{e}+000$

$1.4765 \mathrm{e}+003$

$3.2104 e+003$

$3.2416 e+001$

4.2554 et 002

$4.6999 e+000$

$5.4017 e-001$

$2.9790 e-002$

$1.5597 \mathrm{e}+003$

$1.6169 e+002$

$8.9552 e+003$

$1.5727 e+003$

$8.0298 e+002$

$2.2088 e+001$

$3.8862 e+003$

$1.2914 e+001$

$3.8472 e+003$

$3.2104 e+003$

$3.8108 e+004$

$8.6953 e+002$

$2.4409 e+002$

$1.1396 e+004$ 
Page : 2

DOS File: WM181106.MS5

Run Date: September 9, 1998

Run Time: 8:41:31 AM

Duration: 00:04:11

Nuclide
Pu-242
Rh-106
Ru-106
Sb-125
Sm-151
Sr-90
TC-99
Th-230
$\mathrm{Th}-231$
$\mathrm{U}-232$
$\mathrm{U}-233$
$\mathrm{U}-234$
$\mathrm{U}-235$
$\mathrm{U}-236$
$\mathrm{U}-238$
$\mathrm{Y}-90$

$\begin{array}{cc}\text { curies } & \text { becquerels } \\ 2.2200 e-004 & 8.2140 e+006 \\ 1.5090 e-004 & 5.5833 e+006 \\ 1.5090 e-004 & 5.5833 e+006 \\ 1.8930 e-001 & 7.0041 e+009 \\ 1.0310 e+001 & 3.8147 e+011 \\ 5.7220 e+002 & 2.1171 e+013 \\ 3.1880 e-001 & 1.1796 e+010 \\ 1.3300 e-006 & 4.9210 e+004 \\ 1.9070 e-004 & 7.0559 e+006 \\ 4.3270 e-005 & 1.6010 e+006 \\ 2.0350 e-008 & 7.5295 e+002 \\ 2.1900 e-002 & 8.1030 e+008 \\ 5.5080 e-004 & 2.0380 e+007 \\ 1.9500 e-003 & 7.2150 e+007 \\ 5.4370 e-004 & 2.0117 e+007 \\ 5.7220 e+002 & 2.1171 e+013\end{array}$

$\frac{\mu \mathrm{Ci} / \mathrm{cm}^{3}}{1.5597 e-005}$
$1.0602 e-005$
$1.0602 e-005$
$1.3300 e-002$
$7.2434 e-001$
$4.0201 e+001$
$2.2398 e-002$
$9.3441 e-008$
$1.3398 e-005$
$3.0400 e-006$
$1.4297 e-009$
$1.5386 e-003$
$3.8697 e-005$
$1.3700 e-004$
$3.8198 e-005$
$4.0201 e+001$

$\frac{\mu \mathrm{Ci} / \mathrm{cm}^{3}}{1.5597 \mathrm{e}-005}$

$\mathrm{Bg} / \mathrm{cm}^{3}$

$5.7709 e-001$

$3.9226 e-001$

3. $9226 \mathrm{e}-001$

$4.9208 e+002$

$2.6801 e+004$

1.4874 e+006

$8.2872 e+002$

$3.4573 e-003$

$4.9572 e-001$

$1.1248 \mathrm{e}-001$

$5.2899 e-005$

$5.6929 e+001$

$1.4318 e+000$

$5.0690 e+000$

1. $4133 e+000$

$1.4874 \mathrm{e}+006$

Buildup

The material reference is : shield 3

\section{Integration Parameters}

Radial Circumferential

$Y$ Direction (axial)

$$
\begin{aligned}
& 32 \\
& 32 \\
& 31
\end{aligned}
$$

$\begin{array}{cc}\frac{\text { Energy }}{\text { MeV }} & \begin{array}{c}\text { Activity } \\ \text { photons/se }\end{array} \\ 0.015 & 5.081 e+10 \\ 0.02 & 6.510 e+10 \\ 0.03 & 9.947 e+09 \\ 0.04 & 3.960 e+10 \\ 0.05 & 8.631 e+09 \\ 0.06 & 6.994 e+10 \\ 0.08 & 7.997 e+09 \\ 0.1 & 5.719 e+10 \\ 0.15 & 5.134 e+09 \\ 0.2 & 9.463 e+09 \\ 0.3 & 9.360 e+08 \\ 0.4 & 3.378 e+09 \\ 0.5 & 1.313 e+09 \\ 0.6 & 3.036 e+10 \\ 0.8 & 4.841 e+10 \\ 1.0 & 4.069 e+10 \\ 1.5 & 5.066 e+10 \\ 2.0 & 3.561 e+08 \\ 3.0 & 9.562 e+04\end{array}$

TOTALS: $\quad 4.999 e+11$
Fluence Rate $\mathrm{MeV} / \mathrm{cm}^{2} / \mathrm{sec}$ No Buildup

$0.000 e+00$

$0.000 e+00$

$2.032 e-107$

$3.346 e-51$

$9.080 e-32$

$7.925 e-22$

2. $981 e-15$

2. $119 e-11$

$1.654 e-09$

$6.571 e-08$

$2.235 e-07$

$7.840 e-06$

$1.635 e-05$

1. $418 e-03$

1. $680 \mathrm{e}-02$

$6.243 e-02$

9. $626 \mathrm{e}-01$

3. $316 e-02$

$6.213 e-05$

$1.076 e+00$
Results

Fluence Rate $\mathrm{MeV} / \mathrm{cm}^{2} / \mathrm{sec}$ with Buildup

$4.332 \mathrm{e}-24$

$8.733 e-24$

$2.951 e-24$

$3.115 e-23$

2. $220 e-23$

3. $599 e-20$

$2.065 e-13$

$2.735 e-09$

$3.562 e-07$

$1.488 e-05$

$3.608 e-05$

$8.467 e-04$

$1.243 e-03$

$7.988 e-02$

$5.899 e-01$

$1.536 e+00$

$1.306 e+01$

3. $148 e-01$

3. $817 e-04$

$1.558 \mathrm{e}+01$
Exposure Rate $\mathrm{mR} / \mathrm{hr}$

No Buildup

$0.000 e+00$

$0.000 e+00$

$2.014 e-109$

$1.480 e-53$

$2.419 e-34$

$1.574 e-24$

$4.717 e-18$

$3.242 e-14$

$2.723 e-12$

$1.160 e-10$

$4.240 e-10$

$1.528 \mathrm{e}-08$

$3.210 e-08$

$2.768 e-06$

$3.196 e-05$

$1.151 \mathrm{e}-04$

$1.620 \mathrm{e}-03$

$5.128 \mathrm{e}-05$

$8.429 e-08$

$1.821 \mathrm{e}-03$
Exposure Rate $\mathrm{mR} / \mathrm{hr}$

With Buildup

$3.716 e-25$

$3.025 e-25$

$2.925 e-26$

$1.378 e-25$

$5.913 e-26$

$7.149 e-23$

$3.268 e-16$

$4.184 e-12$

$5.866 \mathrm{e}-10$

$2.627 e-08$

$6.844 e-08$

$1.650 \mathrm{e}-06$

$2.440 e-06$

$1.559 e-04$

$1.122 \mathrm{e}-03$

$2.832 e-03$

2. $197 \mathrm{e}-02$

$4.869 e-04$

$5.179 e-07$

$2.657 e-02$ 
Microshield v5.01 (5.01-00121)

Lockheed Martin Idaho Technologies Company

Page : 1

DOS File: WM181106.MS5

Run Date: September 9, 1998

Run Time: $8: 46: 51$ AM

Duration: $00: 04: 21$

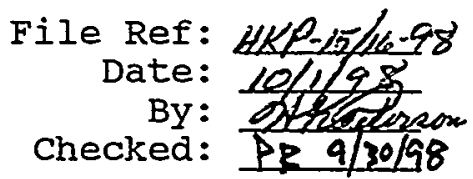

Case Title: Cs IX Effluent tnk

Description: Shielding 4 Cs IX Effluent tank (T-103 A\&B) w/14234 I St \#10 Geometry: 7 - cylinder Volume - side shields

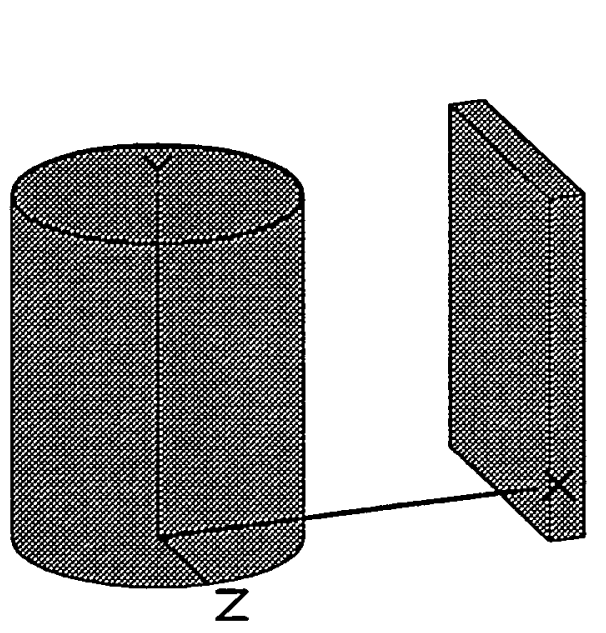

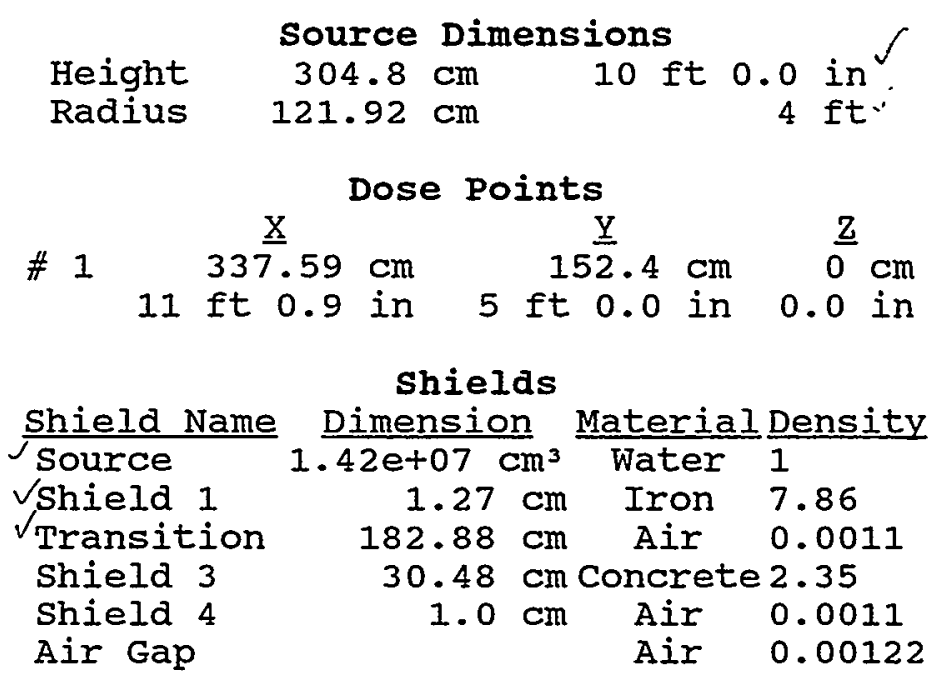

Source Input

Grouping Method : Standard Indices

Number of Groups : 25

Lower Energy Cutoff : 0.015

Photons < 0.015 : Excluded

Library : ICRP-38

\begin{tabular}{|c|c|c|c|c|}
\hline Nuclide & curies & becquerels & $\mu \mathrm{Ci} / \mathrm{cm}^{3}$ & $\mathrm{Bg} / \mathrm{cm}^{3}$ \\
\hline Am-241 & $.2660 e+000$ & $1.9484 \mathrm{e}+011$ & $3.6997 e-001$ & $1.3689 e+004$ \\
\hline & & $8.8430 e+007$ & & \\
\hline$-137 m$ & 001 & & & \\
\hline & 1000 & 010 & -002 & +003 \\
\hline$n-$ & $e-002$ & & -004 & +001 \\
\hline$m-$ & -001 & & -002 & +002 \\
\hline $0-$ & -003 & & 04 & 5000 \\
\hline 134 & -004 & & 005 & $e-001$ \\
\hline s- & -005 & & 007 & -002 \\
\hline & -001 & & & \\
\hline Eu- & -002 & & & +002 \\
\hline Eu- & 1000 & & 2.4 & +003 \\
\hline$E u-155$ & -001 & & 02 & +003 \\
\hline $\mathrm{H}-3$ & & & & +002 \\
\hline & 8. & & & \\
\hline i- & +000 & 5. & 1.0 & +003 \\
\hline$p-$ & -003 & & 004 & $1.2914 \mathrm{e}+001$ \\
\hline$P m-147$ & be+000 & 5.4 & $e-001$ & $e+003$ \\
\hline & & & & \\
\hline & +001 & & 000 & $e+00$ \\
\hline & $0 e-001$ & & -002 & $3 e+002$ \\
\hline & & & $71 e-003$ & $2.4409 e+002$ \\
\hline & & $6221 e+011$ & & \\
\hline
\end{tabular}


Page : 2

DOS File: WM181106.MS5

Run Date: September 9, 1998

Run Time: 8:46:51 AM

Duration: 00:04:21

Nuclide
$\mathrm{Pu}-242$
$\mathrm{Rh}-106$
$\mathrm{Ru}-106$
$\mathrm{Sb}-125$
$\mathrm{Sm}-151$
$\mathrm{~S}-90$
$\mathrm{~T}-99$
$\mathrm{Th}-230$
$\mathrm{Th}-231$
$\mathrm{U}-232$
$\mathrm{U}-233$
$\mathrm{U}-234$
$\mathrm{U}-235$
$\mathrm{U}-236$
$\mathrm{U}-238$
$\mathrm{Y}-90$

curies
$2.2200 \mathrm{e}-004$
$1.5090 \mathrm{e}-004$
$1.5090 \mathrm{e}-004$
$1.8930 \mathrm{e}-001$
$1.0310 \mathrm{e}+001$
$5.7220 \mathrm{e}+002$
$3.1880 \mathrm{e}-001$
$1.3300 \mathrm{e}-006$
$1.9070 \mathrm{e}-004$
$4.3270 \mathrm{e}-005$
$2.0350 \mathrm{e}-008$
$2.1900 \mathrm{e}-002$
$5.5080 \mathrm{e}-004$
$1.9500 \mathrm{e}-003$
$5.4370 \mathrm{e}-004$
$5.7220 \mathrm{e}+002$

becquerels

$8.2140 e+006$

$5.5833 e+006$

$\mu \mathrm{Ci} / \mathrm{cm}^{3}$

$\mathrm{Ba} / \mathrm{cm}^{3}$

$1.5597 e-005$

$5.7709 e-001$

$5.5833 e+006$

$7.0041 e+009$

$1.0602 e-005$

$3.9226 e-001$

$1.0602 e-005$

$3.9226 e-001$

$3.8147 e+011$

$1.3300 e-002$

$4.9208 e+002$

$2.1171 e+013$

$7.2434 \mathrm{e}-001$

$2.6801 e+004$

$1.1796 e+010$

$4.0201 e+001$

$1.4874 e+006$

$4.9210 e+004$

$7.0559 e+006$

$1.6010 e+006$

$2.2398 e-002$

$8.2872 e+002$

$9.3441 \mathrm{e}-008$

$3.4573 e-003$

$1.3398 e-005$

$4.9572 e-001$

$7.5295 e+002$

$3.0400 e-006$

$1.1248 e-001$

$1.4297 e-009$

$5.2899 e-005$

$8.1030 e+008$

$2.0380 e+007$

$1.5386 e-003$

$5.6929 e+001$

$3.8697 e-005$

$1.4318 e+000$

$7.2150 e+007$

$1.3700 e-004$

$5.0690 e+000$

$2.0117 e+007$

$3.8198 e-005$

$1.4133 e+000$

$2.1171 e+013$

$4.0201 e+001$

1. $4874 \mathrm{e}+006$

\section{Buildup}

The material reference is : shield 3

\begin{tabular}{ll}
\multicolumn{2}{c}{ Integration Parameters } \\
Radial & 32 \\
Circumferential & 32 \\
Y Direction (axial) & 31
\end{tabular}

\begin{tabular}{c} 
Energy \\
\hline MeV \\
0.015 \\
0.02 \\
0.03 \\
0.04 \\
0.05 \\
0.06 \\
0.08 \\
0.1 \\
0.15 \\
0.2 \\
0.3 \\
0.4 \\
0.5 \\
0.6 \\
0.8 \\
1.0 \\
1.5 \\
2.0 \\
3.0
\end{tabular}

\section{Activity photons/sec}

$5.081 e+10$

$6.510 e+10$

$9.947 e+09$

$3.960 e+10$

$8.631 e+09$

$6.994 e+10$

$7.997 e+09$

$5.719 e+10$

$5.134 e+09$

$9.463 e+09$

$9.360 e+08$

$3.378 e+09$

$1.313 e+09$

$3.036 e+10$

$4.841 e+10$

$4.069 e+10$

$5.066 e+10$

$3.561 e+08$

$9.562 e+04$

TOTALS:

$4.999 e+11$
Fluence Rate $\mathrm{MeV} / \mathrm{cm}^{2} / \mathrm{sec}$ No Buildup

$0.000 e+00$

$1.515 e-220$

$1.051 e-71$

2. $358 e-33$

$2.997 e-20$

$4.088 e-13$

$7.535 e-09$

$6.361 e-06$

$5.618 e-05$

$7.692 e-04$

$7.259 e-04$

$1.104 e-02$

$1.244 \mathrm{e}-02$

$6.668 e-01$

$3.838 e+00$

$8.423 e+00$

5.441 e+01

$1.105 e+00$

1. $122 e-03$

$6.847 e+01$
Results

Fluence Rate

$\mathrm{MeV} / \mathrm{cm}^{2} / \mathrm{sec}$

With Buildup

$5.143 e-24$

$1.037 \mathrm{e}-23$

$3.504 e-24$

$3.699 e-23$

$2.984 e-19$

8. $291 e-12$

$2.812 e-07$

$3.574 e-04$

4. $119 e-03$

$5.442 e-02$

$3.786 e-02$

$4.175 e-01$

$3.560 e-01$

$1.511 \mathrm{e}+01$

$6.011 e+01$

$9.956 e+01$

$4.047 e+02$

$6.221 e+00$

$4.499 e-03$

$5.865 e+02$
Exposure Rate $\mathrm{mR} / \mathrm{hr}$

No Buildup

$0.000 e+00$

$5.247 e-222$

$1.042 e-73$

$1.043 e-35$

$7.985 e-23$

$8.121 e-16$

$1.192 e-11$

$9.732 e-09$

$9.251 \mathrm{e}-08$

$1.358 e-06$

$1.377 e-06$

2. $151 e-05$

$2.442 e-05$

$1.301 e-03$

$7.300 e-03$

$1.553 e-02$

9. $154 \mathrm{e}-02$

$1.709 \mathrm{e}-03$

1. $522 e-06$

$1.174 e-01$
Exposure Rate $\mathrm{mR} / \mathrm{hr}$ With Buildup

$4.412 e-25$

$3.592 e-25$

$3.472 e-26$

1. $636 \mathrm{e}-25$

$7.948 e-22$

$1.647 e-14$

4. $450 e-10$

$5.468 e-07$

$6.783 e-06$

$9.605 e-05$

$7.181 e-05$

8. $135 e-04$

$6.987 e-04$

2. $949 e-02$

$1.143 e-01$

$1.835 e-01$

$6.808 \mathrm{e}-01$

$9.620 e-03$

$6.104 e-06$

$1.019 e+00$ 
Microshield v5.01 (5.01-00121)

Lockheed Martin Idaho Technologies Company

Page : 1

DOS File: SRSTSH.MS5

Run Date: August 25, 1998

Run Time: 1:44:40 PM

File Ref:

Date:

By:

Duration: 00:04:33

Checked:

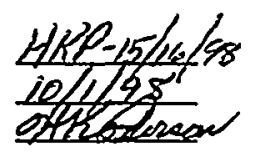

Case Title: T-112 Spent Resin Tk

Description: Shielding for $T-112$ Spent Resin Storage Tank Geometry: 7 - cylinder volume - Side Shields

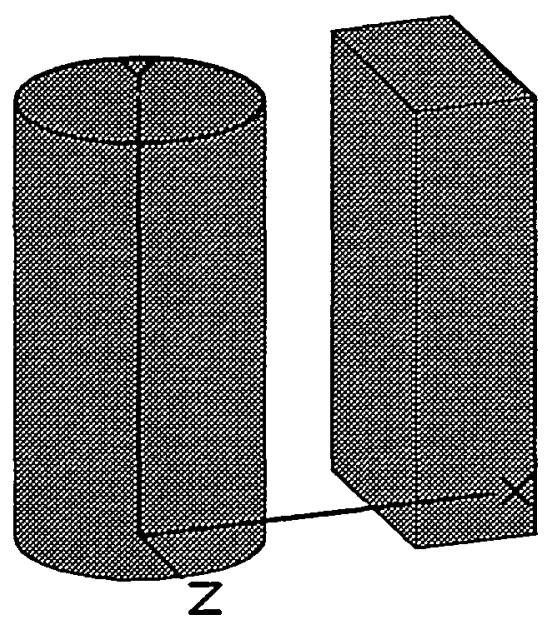

Height

Radius

\section{Source Dimensions}

$457.2 \mathrm{~cm}$

$121.92 \mathrm{~cm}$
$15 \mathrm{ft}$

$4 \mathrm{ft}$

Dose Points

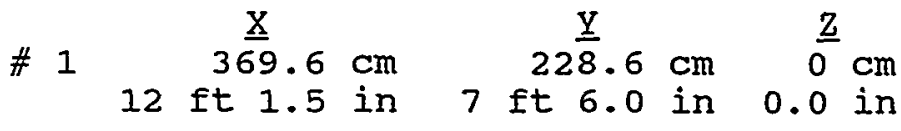

Shields

Shield Name Dimension Material Density

Source $\quad 2.14 \mathrm{e}+07 \mathrm{~cm}^{3}$ Mixed $\rightarrow 1.4$

Concrete 0.4

Water 1

Shield $1 \quad 1.27 \mathrm{~cm}$ Iron 7.86

Transition $121.92 \mathrm{~cm}$ Air 0.0011

Shield 3 ( $\left.\psi^{\prime}\right) 121.92 \mathrm{~cm}$ concrete 2.35

Shield 4

$2.54 \mathrm{~cm}$

Air

0.0011

Air Gap

0.00122

Source Input

Grouping Method : Standard Indices

Number of Groups : 25

Lower Energy Cutoff : 0.015

Photons < 0.015 : Excluded

\begin{tabular}{lc} 
Nuclide & curies \\
\hline $\mathrm{Am}-241$ & $8.0100 \mathrm{e}-001$ \\
$\mathrm{Ba}-137 \mathrm{~m}$ & $4.3830 \mathrm{e}+005$ \\
$\mathrm{Ce}-144$ & $1.7900 \mathrm{e}-009$ \\
$\mathrm{Co}-60$ & $1.8700 \mathrm{e}-002$ \\
$\mathrm{Cs}-134$ & $1.6060 \mathrm{e}+002$ \\
$\mathrm{Cs}-135$ & $8.8200 \mathrm{e}+000$ \\
$\mathrm{Cs}-137$ & $4.6330 \mathrm{e}+005$ \\
$\mathrm{~Np}-237$ & $1.1760 \mathrm{e}-002$ \\
$\mathrm{Pr}-144$ & $1.7900 \mathrm{e}-009$ \\
$\mathrm{Pu}-238$ & $4.8000 \mathrm{e}+001$ \\
$\mathrm{Pu}-239$ & $2.5800 \mathrm{e}+000$ \\
$\mathrm{Pu}-240$ & $8.0900 \mathrm{e}-001$ \\
$\mathrm{Pu}-241$ & $1.6700 \mathrm{e}+001$ \\
$\mathrm{Pu}-242$ & $1.2040 \mathrm{e}-003$ \\
$\mathrm{Rh}-106$ & $1.6500 \mathrm{e}-005$ \\
$\mathrm{Ru}-106$ & $1.6500 \mathrm{e}-005$ \\
$\mathrm{Sb}-125$ & $3.7600 \mathrm{e}-001$ \\
$\mathrm{Sr}-90$ & $1.1870 \mathrm{e}+001$ \\
$\mathrm{U}-234$ & $1.6060 \mathrm{e}-002$ \\
$\mathrm{U}-235$ & $3.5400 \mathrm{e}-004$ \\
$\mathrm{U}-236$ & $1.6780 \mathrm{e}-004$
\end{tabular}

Library : ICRP-38

becquerels $\quad \mu \mathrm{Ci} / \mathrm{cm}^{3}$

$2.9637 e+010 \quad 3.7517 e-002$

$1.6217 e+016 \quad 2.0529 e+004$

$6.6230 e+001 \quad 8.3839 e-011$

$6.9190 \mathrm{e}+008$

$5.9422 e+012$

$3.2634 \mathrm{e}+011$

$1.7142 e+016$

$4.3512 e+008$

$6.6230 e+001$

$1.7760 e+012$

$9.5460 \mathrm{e}+010$

$2.9933 e+010$

$6.1790 e+011$

$4.4548 e+007$

$6.1050 e+005$

6.1050 e+005

$1.3912 \mathrm{e}+010$

$4.3919 e+011$

$5.9422 e+008$

$1.3098 e+007$

$6.2086 e+006$
$8.7586 e-004$

7.5221 e+000

$4.1311 e-001$

$2.1700 e+004$

$5.5081 e-004$

$8.3839 e-011$

$2.2482 e+000$

$1.2084 e-001$

$3.7892 e-002$

$7.8219 e-001$

$5.6392 e-005$

$7.7282 e-007$

$7.7282 e-007$

$1.7611 \mathrm{e}-002$

$5.5596 e-001$

$7.5221 e-004$

$1.6580 e-005$

$7.8593 e-006$
$\mathrm{Bg} / \mathrm{cm}^{3}$

$1.3881 e+003$

7.5957 e+008

3.1020e-006

3.2407 e+001

$2.7832 e+005$

$1.5285 e+004$

$8.0289 e+008$

$2.0380 e+001$

3. $1020 e-006$

$8.3183 e+004$

$4.4711 \mathrm{e}+003$

$1.4020 e+003$

$2.8941 \mathrm{e}+004$

$2.0865 e+000$

$2.8594 e-002$

$2.8594 e-002$

$6.5160 e+002$

2.0571e+004

2.7832e+001

$6.1348 e-001$

2.9080e-001 
Page : 2

DOS File: SRSTSH.MS5

Run Date: August 25, 1998

Run Time: 1:44:40 PM

Duration: 00:04:33

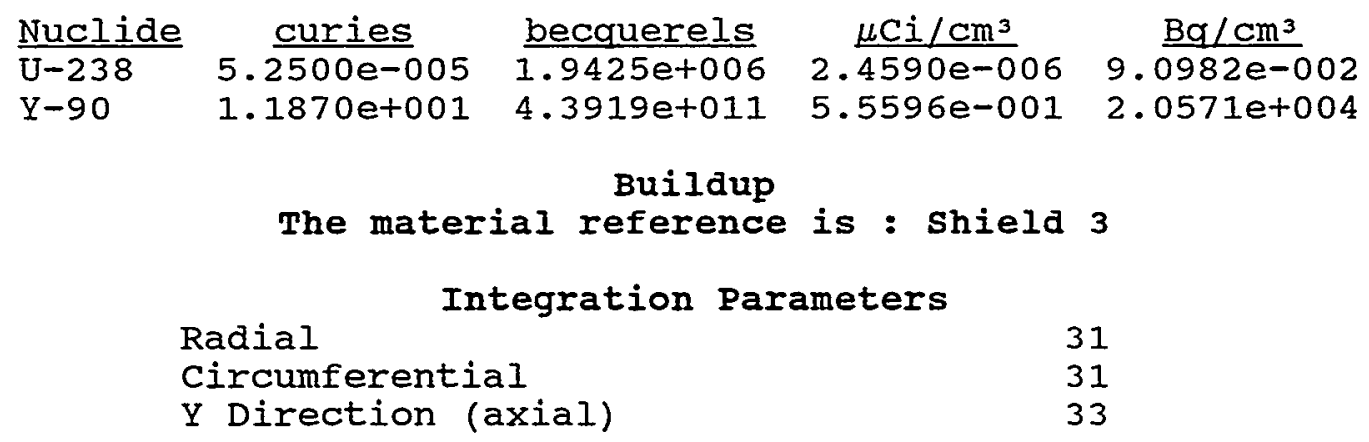

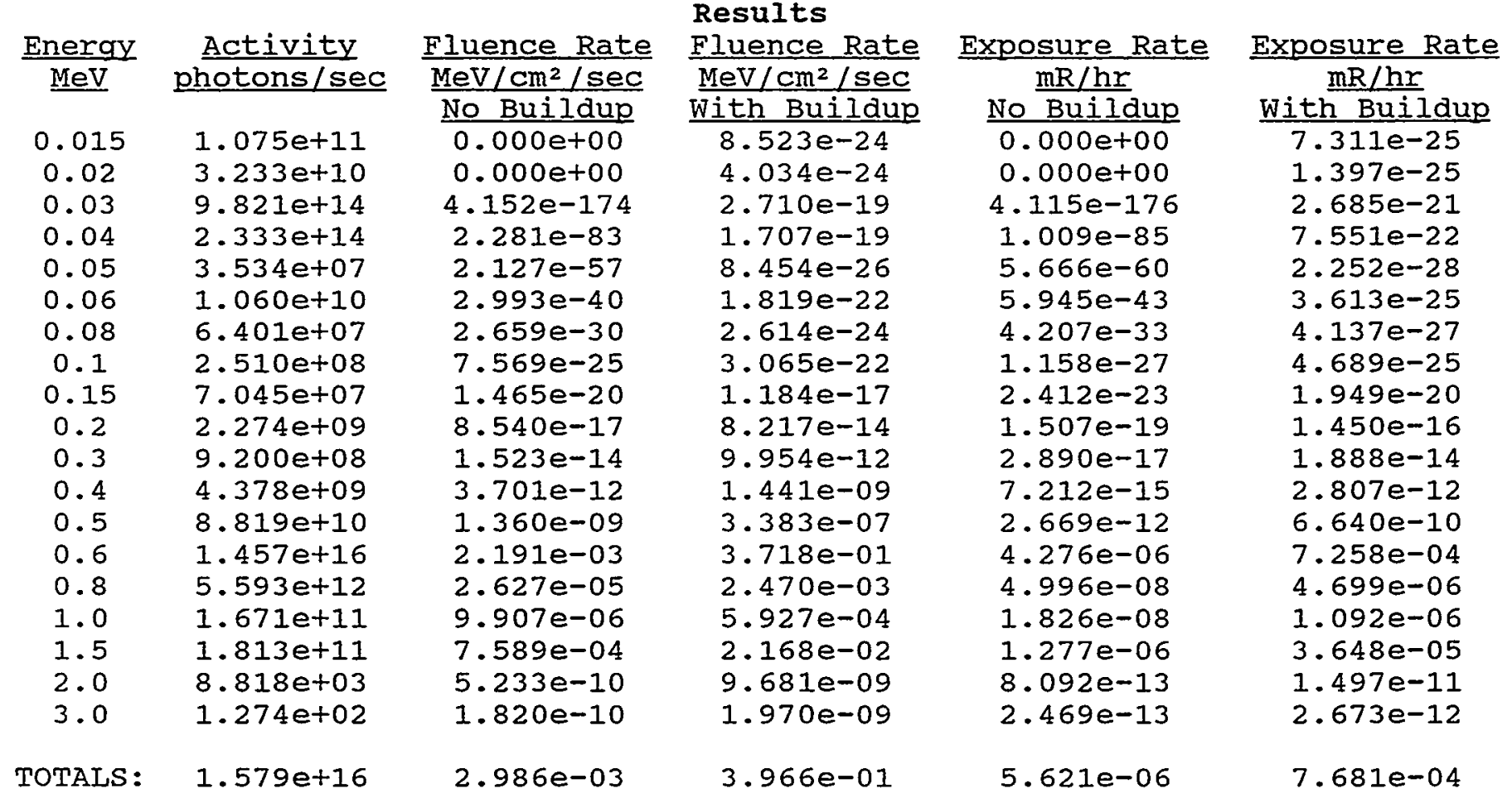


Microshield v5.01 (5.01-00121)

Lockheed Martin Idaho Technologies Company

Page : 1

DOS File: SRSTSH.MS5

Run Date: August 25, 1998

Run Time: 1:52:44 PM

File Ref:

Date:

By:

Duration: $00: 04: 31$

Checked:

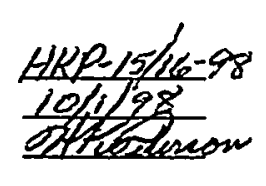

\begin{abstract}
Case Title: T-112 Spent Resin Tk
Description: Shielding for T-112 Spent Resin Storage Tank Geometry: 7 - cylinder volume - side Shields
\end{abstract}

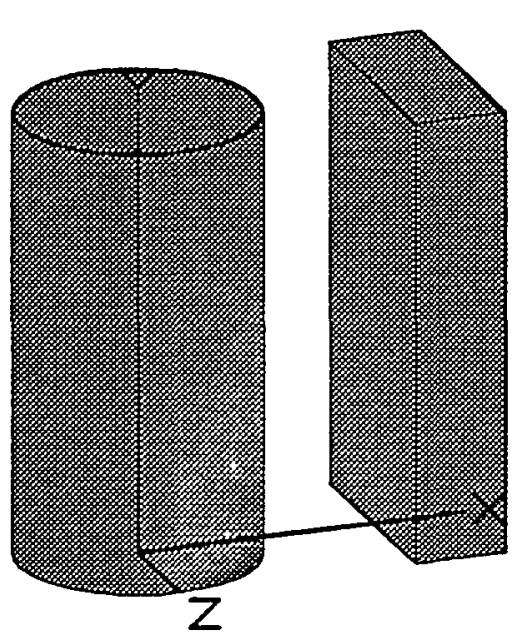

\author{
Source Dimensions \\ Height \\ Radius \\ $457.2 \mathrm{~cm}$
$121.92 \mathrm{~cm}$
}

$15 \mathrm{ft}$

$4 \mathrm{ft}$

Dose Points

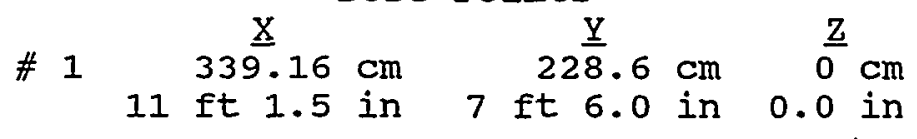

Shields

Shield Name Dimension MaterialDensity Source $2.14 \mathrm{e}+07 \mathrm{~cm}^{3}$ Mixed $\rightarrow 1.4$ Concrete 0.4

\begin{tabular}{lrlll} 
& \multicolumn{3}{r}{ Water } & 1 \\
Shield 1 & $1.27 \mathrm{~cm}$ & Iron & 7.86 \\
Transition & $121.92 \mathrm{~cm}$ Air & 0.0011 \\
Shield 3 (3') & $91.5 \mathrm{~cm}$ Concrete 2.35 \\
Shield 4 & $2.54 \mathrm{~cm}$ Air & 0.0011 \\
Air Gap & & & Air & 0.00122
\end{tabular}

Source Input

Grouping Method : standard Indices

Number of Groups : 25

Lower Energy Cutoff : 0.015

Photons < 0.015 : Excluded

Library : ICRP-38

Nuclide

Am-241

$\mathrm{Ba}-137 \mathrm{~m}$

$\mathrm{Ce}-144$

Co-60

Cs -134

Cs -135

Cs -137

$\mathrm{Np}-237$

Pr -144

$\mathrm{Pu}-238$

$\mathrm{Pu}-239$

$\mathrm{Pu}-240$

$\mathrm{Pu}-241$

$\mathrm{Pu}-242$

$\mathrm{Rh}-106$

Ru-106

$\mathrm{Sb}-125$

Sr -90

$\mathrm{U}-234$

$\mathrm{U}-235$

$\mathrm{U}-236$

\section{becquerels}

2.9637 e+010

$1.6217 \mathrm{e}+016$

$6.6230 e+001$

$6.9190 e+008$

$5.9422 \mathrm{e}+012$

$3.2634 \mathrm{e}+011$

$1.7142 e+016$

$4.3512 \mathrm{e}+008$

$6.6230 \mathrm{e}+001$

$1.7760 e+012$

$9.5460 e+010$

$2.9933 e+010$

$6.1790 \mathrm{e}+011$

$4.4548 e+007$

$6.1050 e+005$

$6.1050 e+005$

$1.3912 \mathrm{e}+010$

$4.3919 e+011$

$5.9422 \mathrm{e}+008$

$1.3098 e+007$

$6.2086 e+006$ $\mu \mathrm{Ci} / \mathrm{cm}^{3}$

3. $7517 e-002$

$2.0529 e+004$

$8.3839 e-011$

$8.7586 e-004$

$7.5221 e+000$

$4.1311 e-001$

$2.1700 e+004$

$5.5081 e-004$

$8.3839 e-011$

$2.2482 e+000$

$1.2084 e-001$

$3.7892 e-002$

$7.8219 e-001$

$5.6392 e-005$

$7.7282 e-007$

$7.7282 e-007$

$1.7611 e-002$

$5.5596 e-001$

$7.5221 e-004$

$1.6580 e-005$

$7.8593 e-006$
$\mathrm{Bg} / \mathrm{cm}^{3}$

$1.3881 \mathrm{e}+003$

$7.5957 e+008$

$3.1020 e-006$

$3.2407 e+001$

$2.7832 e+005$

$1.5285 e+004$

$8.0289 e+008$

$2.0380 e+001$

$3.1020 e-006$

$8.3183 e+004$

$4.4711 e+003$

$1.4020 e+003$

$2.8941 e+004$

$2.0865 e+000$

$2.8594 e-002$

$2.8594 e-002$

$6.5160 e+002$

$2.0571 e+004$

$2.7832 e+001$

$6.1348 e-001$

$2.9080 e-001$ 
Page : 2

DOS File: SRSTSH.MS5

Run Date: August 25, 1998

Run Time: 1:52:44 PM

Duration: 00:04:31

\begin{tabular}{|c|c|c|c|c|}
\hline $\begin{array}{l}\text { Nuclide } \\
\text { U-238 } \\
Y-90\end{array}$ & $\begin{array}{l}\text { curies } \\
5.2500 e-005 \\
1.1870 e+001\end{array}$ & $\begin{array}{l}\frac{\text { becquerels }}{1.9425 e+006} \\
4.3919 e+011\end{array}$ & $\begin{array}{c}\frac{\mu \mathrm{Ci} / \mathrm{cm}^{3}}{2.4590 \mathrm{e}-006} \\
5.5596 \mathrm{e}-001\end{array}$ & $\begin{array}{c}\frac{\mathrm{Bg} / \mathrm{cm}^{3}}{9.0982 e-002} \\
2.0571 e+004\end{array}$ \\
\hline & The mater & $\begin{array}{l}\text { Buildup } \\
\text { al reference }\end{array}$ & is : shield & 3 \\
\hline \multicolumn{5}{|c|}{ Integration Parameters } \\
\hline & $\begin{array}{l}\text { Radial } \\
\text { circumferenti }\end{array}$ & & & $\begin{array}{l}1 \\
1 \\
2\end{array}$ \\
\hline & $Y$ Direction ( & (xial) & & 3 \\
\hline
\end{tabular}

\begin{tabular}{|c|c|c|c|c|c|}
\hline Energy & Activity & Fluence Rate & Fluence Rate & Exposure Rate & Exposure Rate \\
\hline $\mathrm{MeV}$ & photons/sec & $\frac{\mathrm{MeV} / \mathrm{cm}^{2} / \mathrm{sec}}{\text { No Buildup }}$ & $\frac{\mathrm{MeV} / \mathrm{cm}^{2} / \mathrm{sec}}{\text { With Buildup }}$ & No $\frac{\mathrm{mR} / \mathrm{hr}}{\mathrm{Buildup}}$ & with $\frac{\mathrm{mR} / \mathrm{hr}}{\mathrm{Buil}}$ \\
\hline 0.015 & $1.075 e+11$ & $0.000 e+00$ & $1.000 e-23$ & $0.000 e+00$ & $8.581 e-25$ \\
\hline 0.02 & $3.233 e+10$ & $0.000 e+00$ & $4.735 e-24$ & 0.000 e +00 & $1.640 e-25$ \\
\hline 0.03 & $9.821 \mathrm{e}+14$ & $1.466 e-138$ & $3.181 e-19$ & $1.453 e-140$ & $3.152 e-21$ \\
\hline 0.04 & $2.333 e+14$ & $1.248 e-65$ & $2.004 e-19$ & $5.519 e-68$ & $8.863 e-22$ \\
\hline 0.05 & $3.534 e+07$ & $5.661 e-46$ & $9.923 e-26$ & $1.508 e-48$ & $2.643 e-28$ \\
\hline 0.06 & $1.060 e+10$ & $1.258 e-31$ & $2.135 e-22$ & $2.500 e-34$ & $4.242 e-25$ \\
\hline 0.08 & $6.401 e+07$ & $5.479 e-24$ & $5.393 e-22$ & $8.671 e-27$ & $8.535 e-25$ \\
\hline 0.1 & $2.510 e+08$ & $1.849 e-19$ & $4.292 e-17$ & $2.828 e-22$ & $6.566 e-20$ \\
\hline 0.15 & $7.045 e+07$ & $4.060 e-16$ & $1.857 e-13$ & $6.686 e-19$ & $3.058 e-16$ \\
\hline 0.2 & $2.274 e+09$ & $8.221 e-13$ & $4.219 e-10$ & $1.451 e-15$ & $7.446 e-13$ \\
\hline 0.3 & $9.200 e+08$ & $4.131 e-11$ & $1.473 e-08$ & $7.835 e-14$ & $2.795 e-11$ \\
\hline 0.4 & $4.378 e+09$ & $4.409 e-09$ & $9.893 e-07$ & $8.590 e-12$ & $1.928 e-09$ \\
\hline 0.5 & $8.819 e+10$ & $8.842 e-07$ & $1.327 e-04$ & $1.736 e-09$ & $2.604 e-07$ \\
\hline 0.6 & $1.457 e+16$ & $8.885 e-01$ & $9.451 \mathrm{e}+01$ & $1.734 e-03$ & $1.845 e-01$ \\
\hline 0.8 & $5.593 e+12$ & $5.252 e-03$ & $3.253 e-01$ & $9.989 e-06$ & $6.187 e-04$ \\
\hline 1.0 & $1.671 e+11$ & $1.183 e-03$ & $4.867 e-02$ & $2.181 e-06$ & $8.971 e-05$ \\
\hline 1.5 & $1.813 e+11$ & $3.875 e-02$ & $8.091 e-01$ & $6.520 e-05$ & $1.361 e-03$ \\
\hline 2.0 & $8.818 e+03$ & $1.596 e-08$ & $2.231 e-07$ & $2.468 e-11$ & $3.450 e-10$ \\
\hline 3.0 & $1.274 e+02$ & $3.056 e-09$ & $2.613 e-08$ & $4.147 e-12$ & $3.545 e-11$ \\
\hline TOTALS: & $1.579 e+16$ & $9.336 e-01$ & $9.570 e+01$ & $1.812 e-03$ & $1.866 e-01$ \\
\hline
\end{tabular}


Microshield v5.01 (5.01-00121)

Page : 1

DOS File: SRSTSH.MS5

Run Date: August 25, 1998

Run Time: 2:11:00 PM

Duration: 00:04:30

Lockheed Martin Idaho Technologies Company

\begin{abstract}
Case Title: T-112 Spent Resin Tk Description: Shielding for $T-112$ Spent Resin storage Tank Geometry: 7 - cylinder Volume - Side Shields
\end{abstract}

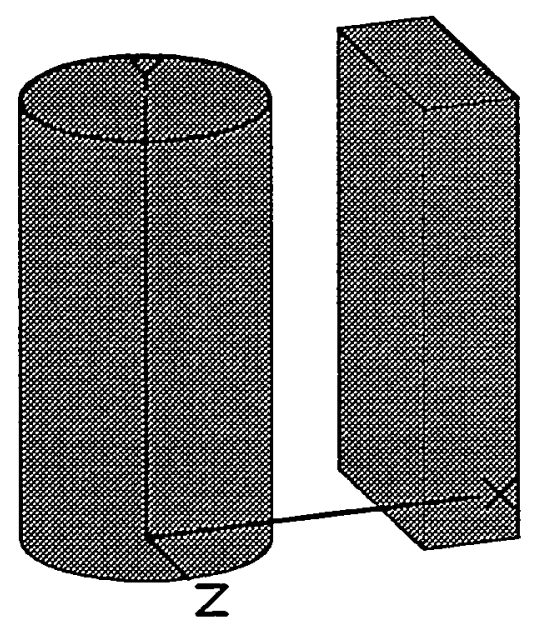

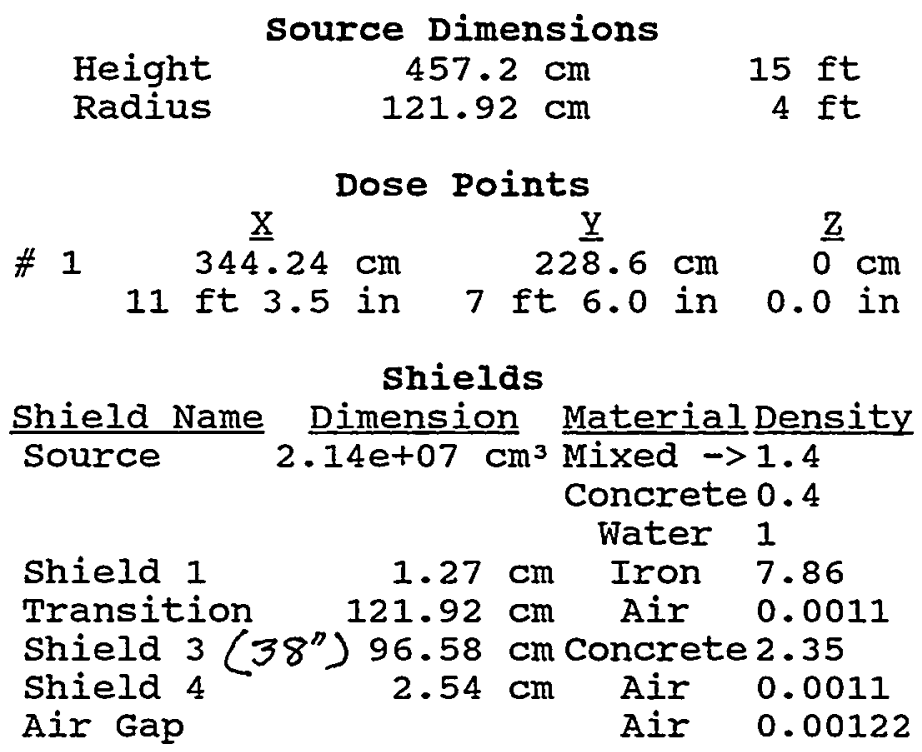

Source Input

Grouping Method : Standard Indices

Number of Groups : 25

Lower Energy Cutoff : 0.015

Photons < 0.015 : Excluded

\begin{tabular}{l} 
Nuclide \\
\hline $\mathrm{Am}-241$ \\
$\mathrm{Ba}-137 \mathrm{~m}$ \\
$\mathrm{Ce}-144$ \\
$\mathrm{Co}-60$ \\
$\mathrm{Cs}-134$ \\
$\mathrm{Cs}-135$ \\
$\mathrm{Cs}-137$ \\
$\mathrm{~Np}-237$ \\
$\mathrm{Pr}-144$ \\
$\mathrm{Pu}-238$ \\
$\mathrm{Pu}-239$ \\
$\mathrm{Pu}-240$ \\
$\mathrm{Pu}-241$ \\
$\mathrm{Pu}-242$ \\
$\mathrm{Rh}-106$ \\
$\mathrm{Ru}-106$ \\
$\mathrm{Sb}-125$ \\
$\mathrm{Sr}-90$ \\
$\mathrm{U}-234$ \\
$\mathrm{U}-235$ \\
$\mathrm{U}-236$
\end{tabular}

Nuclide

curies

8.0100e-001

$4.3830 e+005$

$1.7900 e-009$

$1.8700 e-002$

$1.6060 e+002$

$8.8200 e+000$

$4.6330 \mathrm{e}+005$

$1.1760 e-002$

$1.7900 e-009$

$4.8000 e+001$

$2.5800 e+000$

8.0900e-001

$1.6700 e+001$

$1.2040 e-003$

$1.6500 e-005$

$1.6500 e-005$

$3.7600 e-001$

$1.1870 e+001$

$1.6060 e-002$

$3.5400 e-004$

$1.6780 e-004$
Library : ICRP-38

becquerels $\quad \mu \mathrm{Ci} / \mathrm{cm}^{3}$

$2.9637 e+010 \quad 3.7517 e-002$

$1.6217 e+016 \quad 2.0529 e+004$

$6.6230 e+001$

$6.9190 e+008$

$5.9422 e+012$

$3.2634 e+011$

$1.7142 e+016$

$4.3512 e+008$

$6.6230 e+001$

$1.7760 e+012$

$9.5460 e+010$

$2.9933 e+010$

$6.1790 e+011$

$4.4548 e+007$

$6.1050 e+005$

$6.1050 e+005$

1.3912 e+010

$4.3919 e+011$

$5.9422 e+008$

$1.3098 e+007$

$6.2086 e+006$
$8.3839 e-011$

$8.7586 e-004$

$7.5221 \mathrm{e}+000$

$4.1311 e-001$

$2.1700 e+004$

$5.5081 e-004$

$8.3839 e-011$

$2.2482 e+000$

$1.2084 e-001$

$3.7892 e-002$

$7.8219 e-001$

$5.6392 e-005$

$7.7282 \mathrm{e}-007$

$7.7282 e-007$

$1.7611 \mathrm{e}-002$

$5.5596 e-001$

$7.5221 e-004$

$1.6580 \mathrm{e}-005$

$7.8593 e-006$

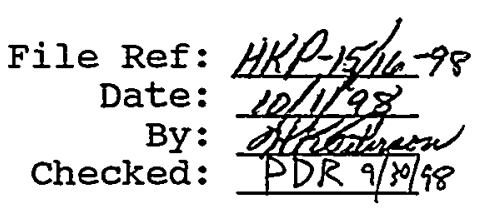


Page : 2

DOS File: SRSTSH.MS5

Run Date: August 25, 1998

Run Time: 2:11:00 PM

Duration: 00:04:30

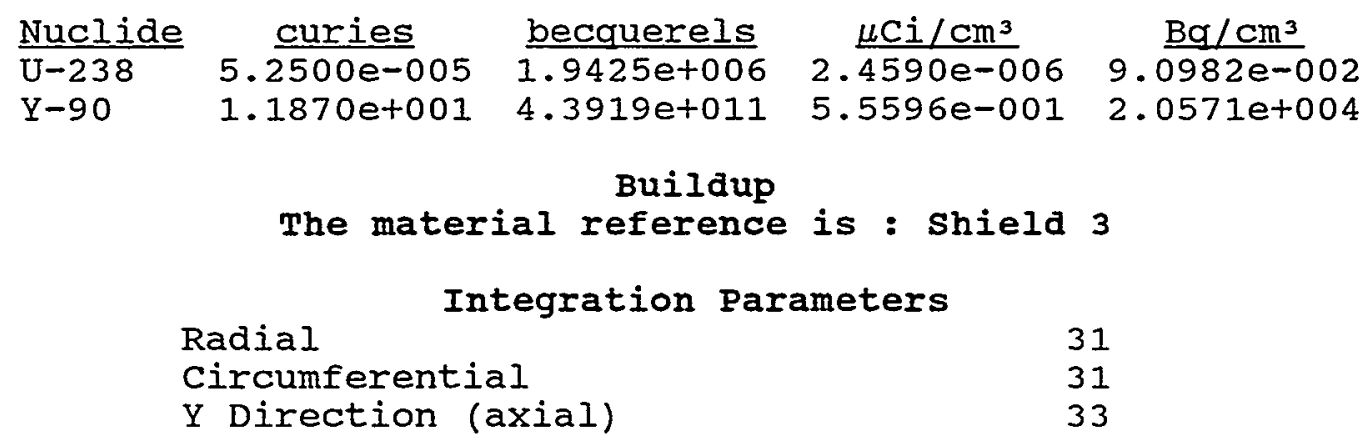

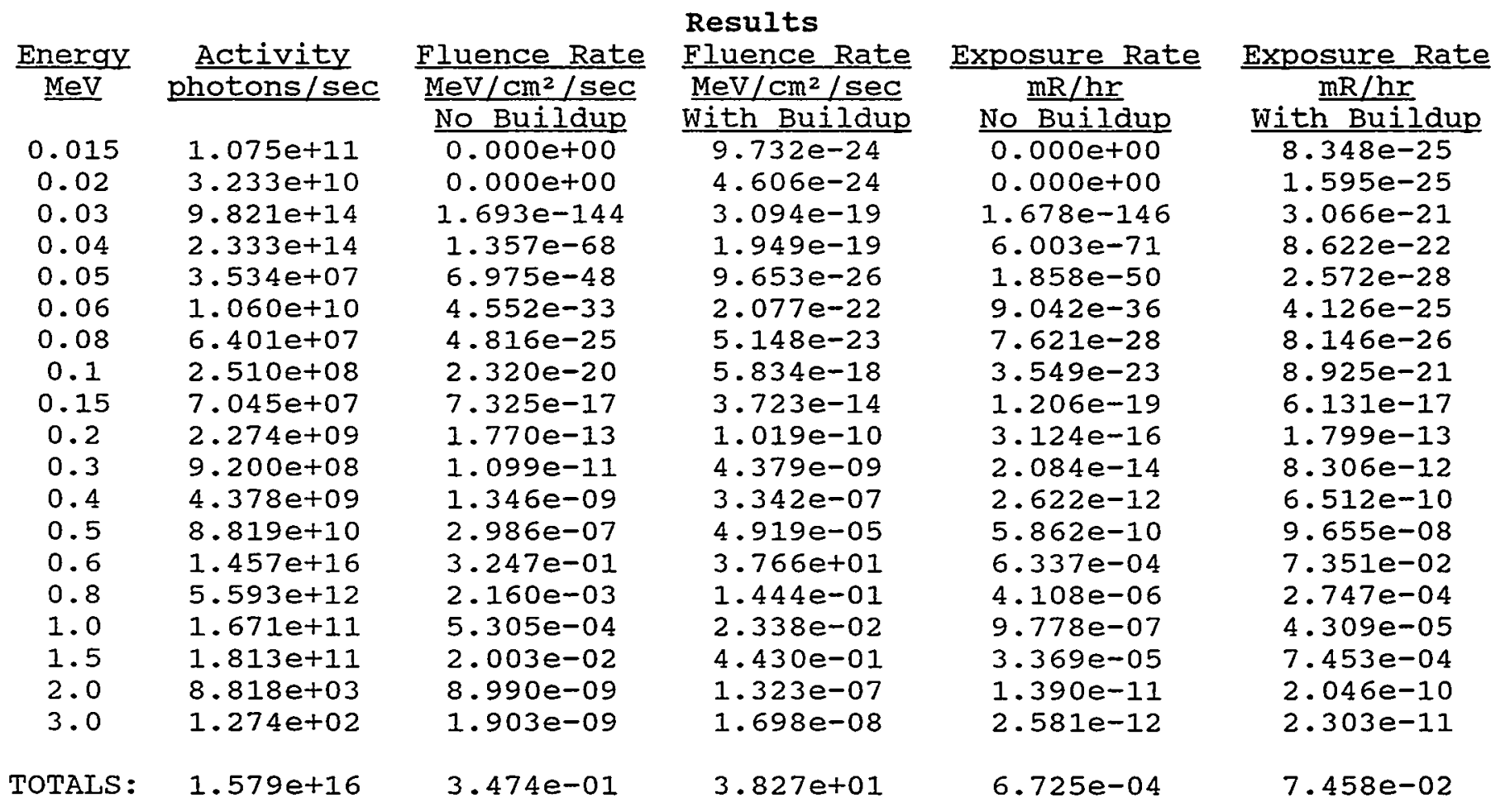


Microshield v5.01 (5.01-00121)

Lockheed Martin Idaho Technologies Company

Page : 1

DOS File: WM181101.MS5

Run Date: August 25, 1998

Run Time: 2:57:22 PM

Duration: $00: 01: 57$

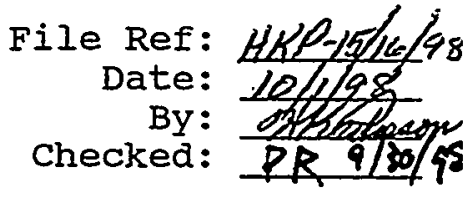
Case Title: WM-181
Description: Shielding for T-101 A \& B Liquid Waste Feed Tanks
Geometry: 7 - Cylinder Volume - Side Shields

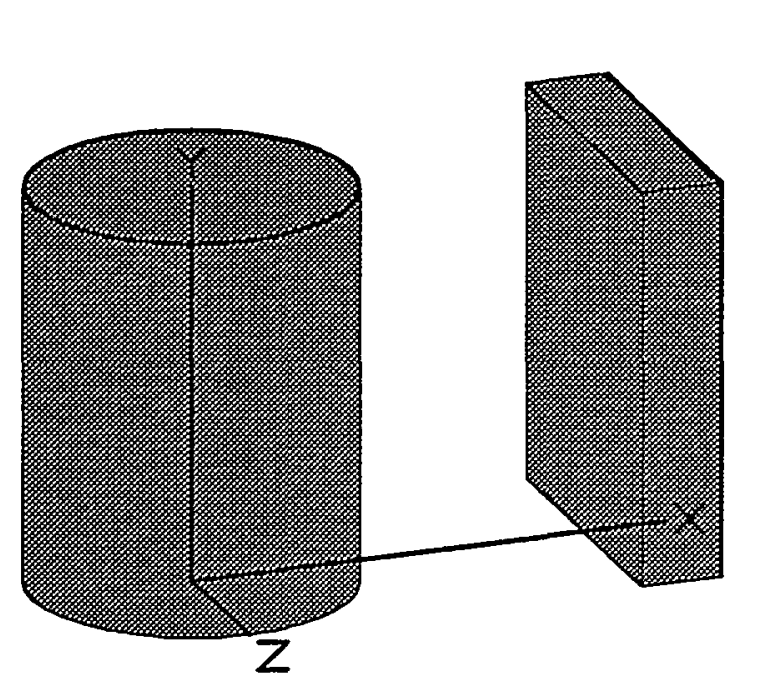

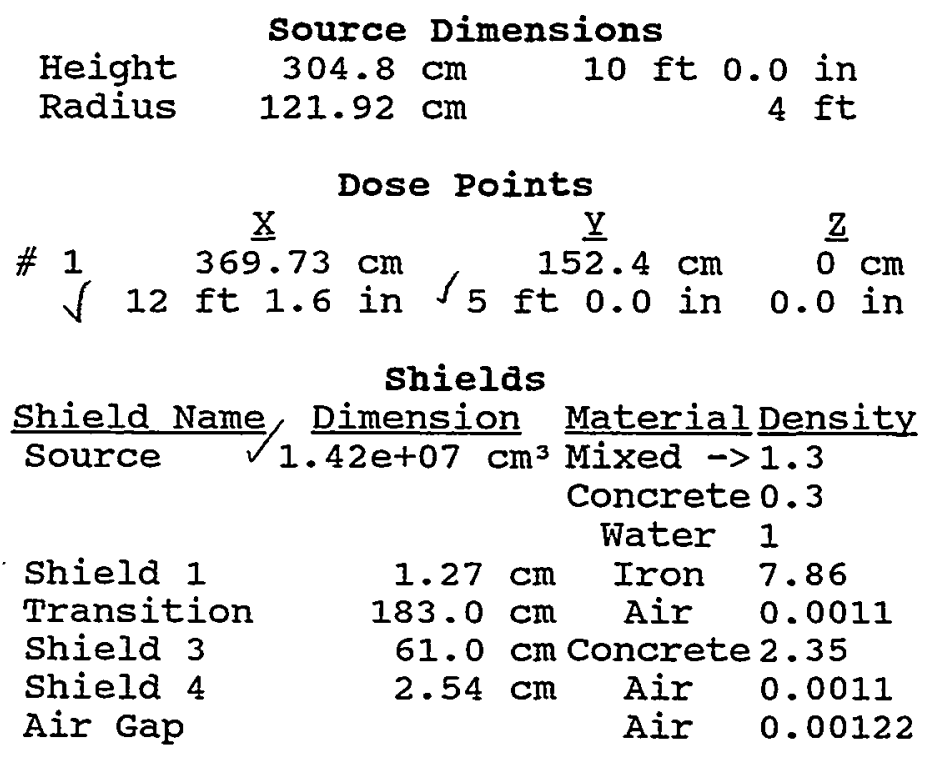

\section{Source Input \\ Grouping Method : Standard Indices \\ Number of Groups : 25 \\ Lower Energy Cutoff : 0.015 \\ Photons < 0.015 : Excluded}

Library : ICRP-38

Nuclide

Am-241

Am-243

$\mathrm{Ba}-137 \mathrm{~m}$

Ce-144

Cm-242

$\mathrm{Cm}-244$

Co-60

Cs-134

Cs-135

Cs -137

Eu-152

$\mathrm{Eu}-154$

Eu-155

$\mathrm{H}-3$

I-129

$\mathrm{Ni}-63$

Np-237

Pm-147

Pr-144

Pu-238

Pu-239 curies

$5.8640 \mathrm{e}+000$

$2.6600 e-003$

$6.3197 e+002$

$7.1900 e-006$

$1.3880 e-002$

$1.8080 e-001$

$2.0070 e+000$

$2.3200 e-001$

$1.2740 e-002$

$6.6898 e+002$

$6.9180 e-002$

$3.8290 e+000$

$6.7180 e-001$

3. $4450 e-001$

$9.4510 e-003$

$1.6650 \mathrm{e}+000$

$5.5370 e-003$

$1.6510 \mathrm{e}+000$

$7.0872 e-006$

$1.6226 \mathrm{e}+001$

3.7290e-001 becquerels

$2.1697 e+011$

$9.8420 e+007$

$2.3383 e+013$

2. $6603 e+005$

$5.1356 e+008$

$6.6896 \mathrm{e}+009$

$7.4259 e+010$

$8.5840 \mathrm{e}+009$

$4.7138 e+008$

$2.4752 e+013$

$2.5597 e+009$

$1.4167 \mathrm{e}+011$

$2.4857 e+010$

$1.2747 e+010$

$3.4969 e+008$

$6.1605 e+010$

$2.0487 e+008$

$6.1087 e+010$

$2.6223 e+005$

$6.0036 e+011$

$1.3797 e+010$ $\mu \mathrm{Ci} / \mathrm{cm}^{3}$

$4.1198 e-001$

$1.8688 e-004$

$4.4400 e+001$

$5.0514 e-007$

$9.7516 e-004$

$1.2702 e-002$

$1.4100 \mathrm{e}-001$

$1.6299 e-002$

$8.9507 e-004$

$4.7000 e+001$

$4.8603 e-003$

$2.6901 e-001$

$4.7198 e-002$

$2.4203 e-002$

$6.6399 e-004$

$1.1698 e-001$

$3.8901 e-004$

$1.1599 e-001$

$4.9792 e-007$

$1.1400 e+000$

$2.6199 e-002$
$\mathrm{Ba} / \mathrm{cm}^{3}$

$1.5243 e+004$

$6.9146 e+000$

$1.6428 \mathrm{e}+006$

$1.8690 e-002$

$3.6081 e+001$

$4.6999 e+002$

$5.2172 e+003$

$6.0308 e+002$

3.3117 et 001

$1.7390 e+006$

$1.7983 e+002$

$9.9534 e+003$

$1.7463 e+003$

$8.9552 e+002$

$2.4568 e+001$

$4.3281 e+003$

$1.4393 e+001$

$4.2917 e+003$

$1.8423 e-002$

$4.2179 e+004$

$9.6935 e+002$ 
Page : 2

DOS File: WM181101.MS5

Run Date: August 25, 1998

Run Time: 2:57:22 PM

Duration: 00:01:57

\begin{tabular}{|c|c|c|c|c|}
\hline Nuclide & curies & becquerels & $\underline{\mu \mathrm{Ci} / \mathrm{cm}^{3}}$ & $\mathrm{Bg} / \mathrm{cm}^{3}$ \\
\hline $\mathrm{Pu}-240$ & $1.0450 e-001$ & $3.8665 e+009$ & $7.3418 e-003$ & $2.7165 e+002$ \\
\hline$P u-241$ & $4.8680 e+000$ & $1.8012 e+011$ & $3.4201 e-001$ & $1.2654 e+004$ \\
\hline $\mathrm{Pu}-242$ & $2.4700 e-004$ & $9.1390 e+006$ & $1.7353 e-005$ & $6.4207 e-001$ \\
\hline $\mathrm{Rh}-106$ & $1.6800 e-004$ & $6.2160 e+006$ & $1.1803 e-005$ & $4.3671 e-001$ \\
\hline$R u-106$ & $1.6800 e-004$ & $6.2160 e+006$ & $1.1803 e-005$ & $4.3671 e-001$ \\
\hline $\mathrm{Sb}-125$ & $2.1070 e-001$ & $7.7959 e+009$ & $1.4803 e-002$ & $5.4771 e+002$ \\
\hline$S m-151$ & $1.1460 e+001$ & $4.2402 e+011$ & $8.0514 \mathrm{e}-001$ & $2.9790 e+004$ \\
\hline Sr-90 & $6.3767 e+002$ & $2.3594 e+013$ & $4.4800 e+001$ & $1.6576 e+006$ \\
\hline TC-99 & $3.5580 e-001$ & $1.3165 e+010$ & $2.4997 e-002$ & $9.2490 \mathrm{e}+002$ \\
\hline Th-230 & $1.4800 e-006$ & 5.47 & $1.0398 e-007$ & $3.8472 e-003$ \\
\hline$T h-231$ & $2.1200 e-004$ & $7.8440 e+006$ & $1.4894 e-005$ & $5.5109 e-001$ \\
\hline$U-233$ & $2.2600 e-008$ & $8.3620 e+002$ & $1.5878 e-009$ & $5.8748 e-005$ \\
\hline$U-234$ & $2.4480 e-002$ & $9.0576 e+008$ & $1.7199 e-003$ & $6.3635 e+001$ \\
\hline$U-235$ & $6.1300 e-004$ & $11 e+007$ & $4.3067 e-005$ & $1.5935 e+000$ \\
\hline$U-236$ & $2.1640 e-003$ & $8.0068 \mathrm{et}$ & $1.5203 e-004$ & $5.6253 e+000$ \\
\hline$U-238$ & $6.0490 e-004$ & $2.2381 e+007$ & $4.2498 e-005$ & $1.5724 \mathrm{e}+000$ \\
\hline$Y-90$ & $6.3767 e+002$ & $2.3594 e+013$ & $4.4800 e+001$ & $1.6576 e+006$ \\
\hline
\end{tabular}

Integration Parameters

Radial Circumferential $Y$ Direction (axial)

24

24

24

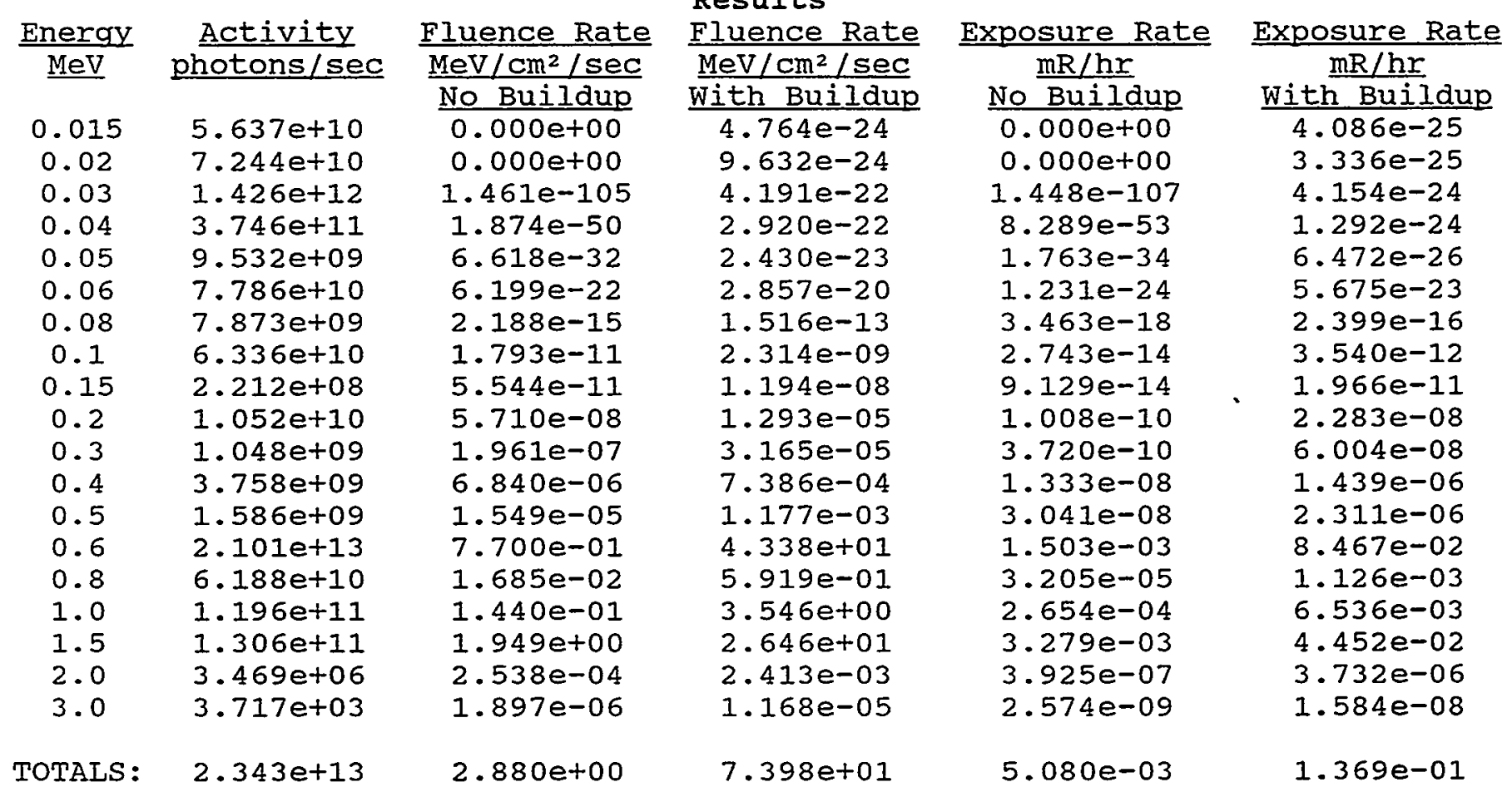


Microshield v5.01 (5.01-00121)

Iockheed Martin Idaho Technologies Company

Page : 1

DOS File: ROTDRY.MS5

Run Date: August 26, 1998

Run Time: 1:56:01 PM

Duration: 00:01:13

File Ref:

Date:

By:

Checked:

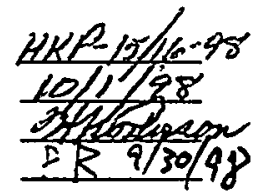

Case Title: WM181-107

Description: Shielding for Rotary dryer with $291.2 \mathrm{~kg}$ of stream 107 Geometry: 7 - cylinder volume - side shields

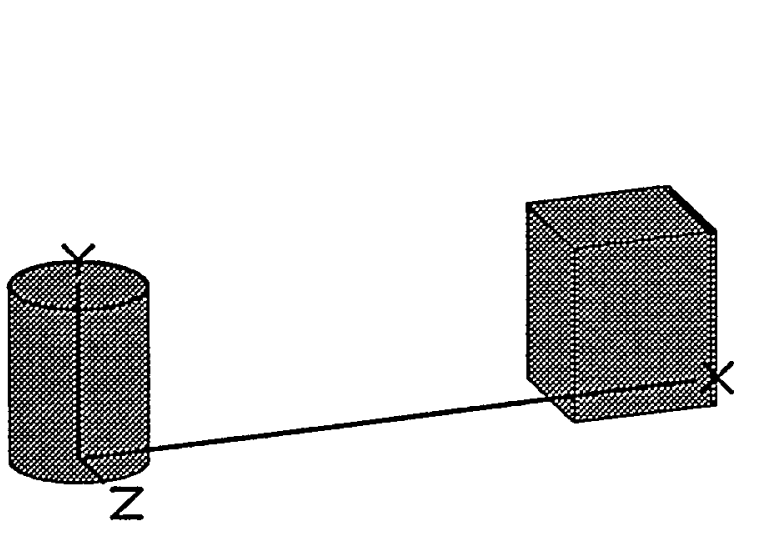

\begin{tabular}{lrrr}
\multicolumn{5}{c}{ Source } & Dimensions \\
Height & $77.598 \mathrm{~cm}$ & $2 \mathrm{ft} 6.6 \mathrm{in}$ \\
Radius & $29.21 \mathrm{~cm}$ & $11.5 \mathrm{in}$
\end{tabular}

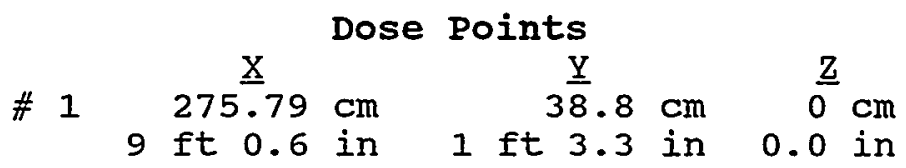

shields

Shield Name Dimension Material Density Source $2.08 \mathrm{e}+05 \mathrm{~cm}^{3}$ Concrete 1.4 Shield $1 \quad .16 \mathrm{~cm}$ Iron 7.86 Transition $182.88 \mathrm{~cm}$ Air 0.0011 Shield 3 ( $\Xi^{\prime}$ ) $61.0 \mathrm{~cm}$ Concrete 2.35 Shield 4 ( $2.54 \mathrm{~cm}$ Air 0.0011 Air Gap Air

0.00122

Source Input

Grouping Method : Standard Indices

Number of Groups : 25

Lower Energy Cutoff : 0.015

Photons < 0.015 : Excluded

\begin{tabular}{l} 
Nuclide \\
\hline $\mathrm{Am}-241$ \\
$\mathrm{Ba}-137 \mathrm{~m}$ \\
$\mathrm{Ce}-144$ \\
$\mathrm{Co}-60$ \\
$\mathrm{Cs}-134$ \\
$\mathrm{Cs}-137$ \\
$\mathrm{~Np}-237$ \\
$\mathrm{Pr}-144$ \\
$\mathrm{Pu}-238$ \\
$\mathrm{Pu}-239$ \\
$\mathrm{Pu}-240$ \\
$\mathrm{Pu}-241$ \\
$\mathrm{Pu}-242$ \\
$\mathrm{Rh}-106$ \\
$\mathrm{Ru}-106$ \\
$\mathrm{Sb}-125$ \\
$\mathrm{Sr}-90$ \\
$\mathrm{U}-234$ \\
$\mathrm{U}-235$ \\
$\mathrm{U}-236$ \\
$\mathrm{U}-238$ \\
$\mathrm{Y}-90$
\end{tabular}

curies $3.4650 e-001$

$3.6090 e+001$

$7.7460 e-010$

$8.0660 e-003$

$1.4360 \mathrm{e}-003$

$3.8150 e+001$

$5.0700 e-003$

$7.7460 e-010$

$2.0730 e+001$

1.1120 e +000

$3.4900 e-001$

$7.1930 e+000$

$5.1830 e-004$

$7.1340 e-006$

$7.1340 \mathrm{e}-006$

$1.6250 \mathrm{e}-001$

$5.1250 e+000$

$6.9300 e-003$

$1.5260 \mathrm{e}-004$

$7.2200 e-005$

$2.2600 e-005$

$5.1250 e+000$

Library : ICRP-38

becquerels

1.2821e+010

$1.3353 e+012$

$2.8660 e+001$

2.9844 e+008

$5.3132 e+007$

1. 4116 e+ 012

$1.8759 e+008$

2.8660 e+001

7.6701et011

4. 1144 et 010

$1.2913 e+010$

$2.6614 \mathrm{e}+011$

$1.9177 e+007$

2. $6396 e+005$

$2.6396 e+005$

$6.0125 e+009$

$1.8963 e+011$

2.5641 e+008

5. $6462 e+006$

2.6714 e+006

8.3620 et 005

$1.8963 e+011$ $\mu \mathrm{Ci} / \mathrm{cm}^{3}$

1. $6659 \mathrm{e}+000$

1.7351e+002

3. 7240 e-009

3. $8779 e-002$

$6.9038 e-003$

1. $8341 \mathrm{e}+002$

$2.4375 e-002$

$3.7240 e-009$

9. 9663 e+001

5. 3462 e+000

1. 6779 e+000

3. $4582 e+001$

2. $4918 \mathrm{e}-003$

3. $4298 \mathrm{e}-005$

3. $4298 \mathrm{e}-005$

7. $8125 e-001$

2. $4639 e+001$

3. 3317 e-002

$7.3365 e-004$

3. $4712 \mathrm{e}-004$

$1.0865 e-004$

2. $4639 e+001$
$\mathrm{Bg} / \mathrm{cm}^{3}$

$6.1637 e+004$

$6.4199 e+006$

$1.3779 e-004$

$1.4348 e+003$

$2.5544 e+002$

$6.7863 e+006$

$9.0187 e+002$

$1.3779 e-004$

$3.6875 e+006$

$1.9781 e+005$

$6.2082 e+004$

1. 2795 et 006

$9.2198 e+001$

$1.2690 \mathrm{e}+000$

$1.2690 \mathrm{e}+000$

$2.8906 e+004$

$9.1166 e+005$

1.2327 e+003

$2.7145 e+001$

$1.2843 e+001$

$4.0202 e+000$

$9.1166 e+005$ 
Page : 2

DOS File: ROTDRY.MS5

Run Date: August 26, 1998

Run Time: 1:56:01 PM

Duration: 00:01:13

\section{Buildup \\ The material reference is : Shield 3}

\begin{tabular}{ll}
\multicolumn{2}{c}{ Integration Parameters } \\
Radial 21 \\
Circumferential & 21 \\
$Y$ Direction (axial) & 21
\end{tabular}

\begin{tabular}{|c|c|c|c|c|c|}
\hline Energy & Activity & Fluence Rate & $\begin{array}{l}\text { Results } \\
\text { Fluence Rate }\end{array}$ & Exposure Rate & Exposure Rate \\
\hline $\mathrm{MeV}$ & photons/sec & $\mathrm{MeV} / \mathrm{cm}^{2} / \mathrm{sec}$ & $\mathrm{MeV} / \mathrm{cm}^{2} / \mathrm{sec}$ & $\mathrm{mR} / \mathrm{hr}$ & $\underline{\mathrm{mR} / \mathrm{hr}}$ \\
\hline & & No Buildup & with Buildup & No Buildup & With Buildup \\
\hline 0.015 & $4.642 e+10$ & $0.000 e+00$ & $7.074 e-24$ & $0.000 e+00$ & $6.067 e-25$ \\
\hline 0.02 & $1.397 e+10$ & $4.385 e-234$ & $3.349 e-24$ & $1.519 e-235$ & $1.160 e-25$ \\
\hline 0.03 & $8.404 e+10$ & $2.752 e-75$ & $4.456 e-23$ & $2.728 e-77$ & $4.416 e-25$ \\
\hline 0.04 & $1.978 e+10$ & $4.329 e-37$ & $2.781 e-23$ & $1.915 e-39$ & $1.230 e-25$ \\
\hline 0.05 & $1.524 e+07$ & $3.139 e-26$ & $3.726 e-25$ & $8.362 e-29$ & $9.925 e-28$ \\
\hline 0.06 & $4.584 e+09$ & $1.875 e-17$ & $5.423 e-16$ & $3.724 e-20$ & $1.077 e-18$ \\
\hline 0.08 & $2.761 e+07$ & $2.242 e-14$ & $1.278 e-12$ & $3.549 e-17$ & $2.022 e-15$ \\
\hline 0.1 & $1.084 e+08$ & $1.330 e-11$ & $1.393 e-09$ & $2.035 e-14$ & $2.132 e-12$ \\
\hline 0.15 & $3.043 e+07$ & $7.161 e-10$ & $1.262 e-07$ & $1.179 e-12$ & $2.077 e-10$ \\
\hline 0.2 & $4.433 e+08$ & $1.414 e-07$ & $2.618 e-05$ & $2.496 e-10$ & $4.620 e-08$ \\
\hline 0.3 & $2.782 e+07$ & $2.094 e-07$ & $2.788 e-05$ & $3.972 e-10$ & $5.288 e-08$ \\
\hline 0.4 & $1.892 e+09$ & $1.136 e-04$ & $1.024 e-02$ & $2.214 e-07$ & $1.996 e-05$ \\
\hline 0.5 & $6.217 e+08$ & $1.749 e-04$ & $1.119 e-02$ & $3.433 e-07$ & $2.197 e-05$ \\
\hline 0.6 & $1.201 e+12$ & $1.141 e+00$ & $5.449 e+01$ & $2.226 e-03$ & $1.064 e-01$ \\
\hline 0.8 & $5.049 e+07$ & $3.035 e-04$ & $9.139 e-03$ & $5.774 e-07$ & $1.738 e-05$ \\
\hline 1.0 & $2.997 e+08$ & $7.058 e-03$ & $1.502 e-01$ & $1.301 e-05$ & $2.769 e-04$ \\
\hline 1.5 & $3.000 e+08$ & $7.088 e-02$ & $8.427 e-01$ & $1.193 e-04$ & $1.418 e-03$ \\
\hline 2.0 & $3.805 e+03$ & $3.837 e-06$ & $3.209 e-05$ & $5.933 e-09$ & $4.962 e-08$ \\
\hline 3.0 & $5.506 e+01$ & $3.240 e-07$ & $1.763 e-06$ & $4.396 e-10$ & $2.392 e-09$ \\
\hline TOTALS: & $1.374 e+12$ & $1.219 e+00$ & $5.552 e+01$ & $2.360 e-03$ & $1.081 e-01$ \\
\hline
\end{tabular}


Microshield v5.01 (5.01-00121)

Lockheed Martin Idaho Technologies Company

Page : 1

DOS File: ROTDRY.MS5

Run Date: October 1, 1998

Run Time: 3:41:37 PM

Duration: 00:01:11

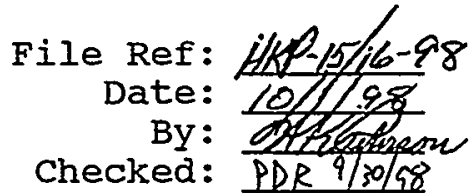

Case Title: WM181-107

Description: Shielding for Rotary dryer with $291.2 \mathrm{~kg}$ of stream 107 Geometry: 7 - Cylinder volume - side shields

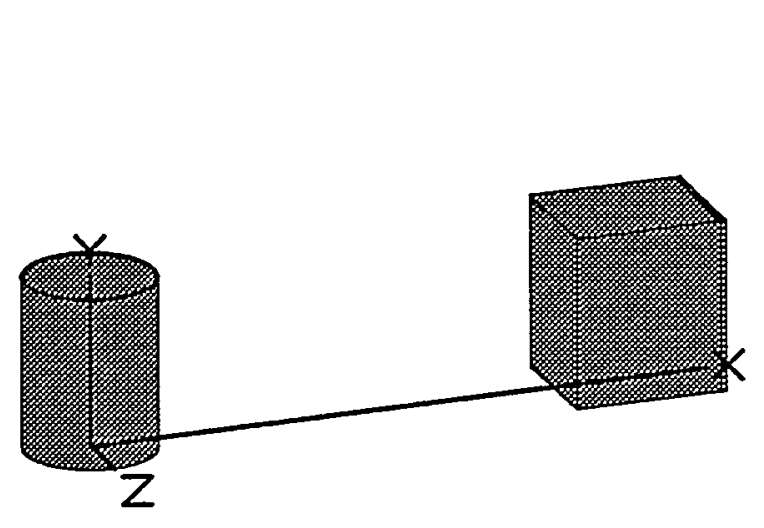

\begin{tabular}{lrrr}
\multicolumn{5}{c}{ Source Dimensions } \\
Height & $77.598 \mathrm{~cm}$ & $2 \mathrm{ft} 6.6 \mathrm{in}$ \\
Radius & $29.21 \mathrm{~cm}$ & $11.5 \mathrm{in}$
\end{tabular}

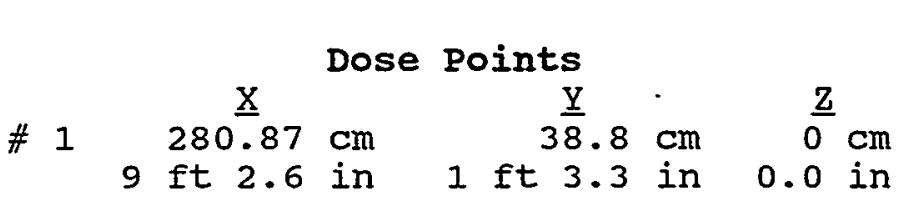

Shields

Shield Name Dimension Material Density Source $2.08 \mathrm{e}+05 \mathrm{~cm}^{3}$ Concrete 1.4 Shield $1 \quad .16 \mathrm{~cm}$ Iron 7.86 Transition $182.88 \mathrm{~cm}$ Air 0.0011 Shield $3 \quad 66.08 \mathrm{~cm}$ Concrete 2.35 Shield $4 \quad 2.54 \mathrm{~cm}$ Air 0.0011 Air Gap Air 0.00122

\section{Source Input \\ Grouping Method : Standard Indices} Number of Groups : 25

Lower Energy Cutoff : 0.015 Photons < 0.015 : Excluded Library : ICRP-38

\begin{tabular}{|c|c|c|c|}
\hline Nuclide & curies & becquerels & $\mu \mathrm{Ci} / \mathrm{cm}^{3}$ \\
\hline Am-241 & $3 . \overline{4650 e-001}$ & $\overline{1.2821 e+010}$ & $1 . \overline{6659 e+000}$ \\
\hline $\mathrm{Ba}-137 \mathrm{~m}$ & $3.6090 e+001$ & $1.3353 e+012$ & $1.7351 e+002$ \\
\hline $\mathrm{Ce}-144$ & $7460 e-010$ & $2.8660 e+001$ & -009 \\
\hline Co-60 & $0660 e-003$ & $2.9844 e+008$ & $3.8779 e-002$ \\
\hline$C s-134$ & $1.4360 e-003$ & $5.3132 e+007$ & $6.9038 e-003$ \\
\hline Cs -137 & $3.8150 e+001$ & $1.4116 \mathrm{e}+012$ & $1.8341 e+002$ \\
\hline $\mathrm{Np}-237$ & $5.0700 e-003$ & $1.8759 e+008$ & $2.4375 e-002$ \\
\hline $\operatorname{Pr}-144$ & $7.7460 e-010$ & $2.8660 e+001$ & $3.7240 e-009$ \\
\hline $\mathrm{Pu}-238$ & $2.0730 e+001$ & $7.6701 \mathrm{e}+011$ & $9.9663 e+001$ \\
\hline $\mathrm{Pu}-239$ & $1.1120 e+000$ & $4.1144 \mathrm{e}+010$ & $5.3462 e+000$ \\
\hline$P u-240$ & $3.4900 e-001$ & $1.2913 e+010$ & $1.6779 e+000$ \\
\hline$P u-241$ & $7.1930 e+000$ & $2.6614 \mathrm{e}+011$ & $3.4582 e+001$ \\
\hline$P u-242$ & $5.1830 e-004$ & $1.9177 e+007$ & $2.4918 e-003$ \\
\hline $\mathrm{Rh}-106$ & $7.1340 e-006$ & $96 e+005$ & $98 e-00$ \\
\hline & $7.1340 e-006$ & $2.6396 e+005$ & $3.4298 e-005$ \\
\hline$S b-125$ & $1.6250 e-001$ & $6.0125 e+009$ & $7.8125 e-001$ \\
\hline $\operatorname{Sr}-90$ & $5.1250 \mathrm{e}+000$ & $1.8963 e+011$ & $2.4639 e+001$ \\
\hline$U-234$ & $6.9300 e-003$ & $2.5641 e+008$ & $317 e-002$ \\
\hline & $5260 e-004$ & $5.6462 e+006$ & $7.3365 e-004$ \\
\hline$U-236$ & $7.2200 e-005$ & $2.6714 e+006$ & $3.4712 e-004$ \\
\hline$U-238$ & $2.2600 e-005$ & $8.3620 e+005$ & $1.0865 e-004$ \\
\hline$Y-90$ & $5.1250 e+000$ & $1.8963 e+011$ & $639 e+001$ \\
\hline
\end{tabular}

$\mathrm{Bg} / \mathrm{cm}^{3}$

6.1637 e+0 04

$6.4199 e+006$

$1.3779 e-004$

$1.4348 \mathrm{e}+003$

$2.5544 e+002$

$6.7863 e+006$

$9.0187 e+002$

$1.3779 e-004$

$3.6875 e+006$

1.9781 e+005

$6.2082 e+004$

$1.2795 \mathrm{e}+006$

$9.2198 \mathrm{e}+001$

$1.2690 e+000$

$1.2690 \mathrm{e}+000$

$2.8906 e+004$

$9.1166 \mathrm{e}+005$

$1.2327 \mathrm{e}+003$

2.7145e+001

$1.2843 e+001$

$4.0202 e+000$

$9.1166 \mathrm{e}+005$ 
Page : 2

DOS File: ROTDRY.MS5

Run Date: October 1, 1998

Run Time: 3:41:37 PM

Duration: 00:01:11

\section{Buildup \\ The material reference is : Shield 3}

Radial

Integration Parameters

Circumferential

$Y$ Direction (axial)

21

21

21

\begin{tabular}{|c|c|c|c|c|c|}
\hline$\frac{\text { Energy }}{\mathrm{MeV}}$ & $\frac{\text { Activity }}{\text { photons/sec }}$ & $\frac{\text { Fluence Rate }}{\mathrm{MeV} / \mathrm{cm}^{2} / \mathrm{sec}}$ & $\begin{array}{l}\text { Results } \\
\text { Fluence Rate } \\
\text { MeV/cm } 2 / \mathrm{sec}\end{array}$ & $\frac{\text { Exposure Rate }}{\mathrm{mR} / \mathrm{hr}}$ & $\frac{\text { Exposure Rate }}{\mathrm{mR} / \mathrm{hr}}$ \\
\hline & & No Buildup & With Buildup & No Buildup & With Buildup \\
\hline 0.015 & $4.642 e+10$ & $0.000 e+00$ & $6.820 e-24$ & $0.000 e+00$ & $5.850 e-25$ \\
\hline 0.02 & $1.397 e+10$ & $2.621 e-252$ & $3.229 e-24$ & $9.078 e-254$ & $1.119 e-25$ \\
\hline 0.03 & $8.404 e+10$ & $3.127 e-81$ & $4.297 e-23$ & $3.099 e-83$ & $4.258 e-25$ \\
\hline 0.04 & $1.978 e+10$ & $4.660 e-40$ & $2.682 e-23$ & $2.061 e-42$ & $1.186 e-25$ \\
\hline 0.05 & $1.524 e+07$ & $3.843 e-28$ & $6.800 e-26$ & $1.024 e-30$ & $1.811 e-28$ \\
\hline 0.06 & $4.584 e+09$ & $6.757 e-19$ & $2.225 e-17$ & $1.342 e-21$ & $4.420 e-20$ \\
\hline 0.08 & $2.761 e+07$ & $1.970 e-15$ & $1.230 e-13$ & $3.117 e-18$ & $1.946 e-16$ \\
\hline 0.1 & $1.084 e+08$ & $1.671 \mathrm{e}-12$ & $1.972 \mathrm{e}-10$ & $2.556 e-15$ & $3.018 e-13$ \\
\hline 0.15 & $3.043 e+07$ & $1.296 \mathrm{e}-10$ & $2.655 e-08$ & $2.134 e-13$ & $4.372 e-11$ \\
\hline 0.2 & $4.433 e+08$ & $3.054 e-08$ & $6.659 e-06$ & $5.391 e-11$ & $1.175 e-08$ \\
\hline 0.3 & $2.782 e+07$ & $5.591 e-08$ & $8.723 e-06$ & $1.060 e-10$ & $1.655 e-08$ \\
\hline 0.4 & $1.892 e+09$ & $3.481 e-05$ & $3.631 e-03$ & $6.783 e-08$ & $7.076 e-06$ \\
\hline 0.5 & $6.217 e+08$ & $5.927 e-05$ & $4.344 e-03$ & $1.163 e-07$ & $8.526 e-06$ \\
\hline 0.6 & $1.201 e+12$ & $4.182 e-01$ & $2.268 e+01$ & $8.163 e-04$ & $4.426 e-02$ \\
\hline 0.8 & $5.049 e+07$ & $1.252 e-04$ & $4.221 e-03$ & $2.382 e-07$ & $8.028 e-06$ \\
\hline 1.0 & $2.997 e+08$ & $3.173 e-03$ & $7.481 e-02$ & $5.849 e-06$ & $1.379 e-04$ \\
\hline 1.5 & $3.000 e+08$ & $3.671 e-02$ & $4.749 e-01$ & $6.176 e-05$ & $7.991 e-04$ \\
\hline 2.0 & $3.805 e+03$ & $2.165 e-06$ & $1.951 e-05$ & $3.347 e-09$ & $3.017 e-08$ \\
\hline 3.0 & $5.506 e+01$ & $2.018 e-07$ & $1.169 e-06$ & $2.738 e-10$ & $1.586 e-09$ \\
\hline TOTALS & $1.374 e+12$ & $4.583 e-01$ & $2.324 e+01$ & $8.843 e-04$ & $4.522 e-02$ \\
\hline
\end{tabular}


- roject File Number

02BD7

Project/Task HIW EIS Supporting Studies

Subtask

Title: Regulatory Requirements for the Design, Construction, and Operations of the ICPP Proposed Waste Processing Facilities

Summary: This EDF identifies and provides the existing environmental regulations and codes pertaining to the design, construction, operations, and performance of the proposed waste treatment and storage facilities at the Idaho Chemical Processing Plant (ICPP). This study also presents an assessment of the current NRC regulations and their potential applicability to the proposed facilities if the facilities were to be licensed by the NRC in the future. The NRC requirements for regulating DOE facilities or activities have not been defined yet. The NRC requirements to be applied will need to be determined by the appropriate NRC and DOE Task Forces.

The principal sources of requirements for the design, construction, and operations presented here are the Department of Energy (DOE), the DOE Idaho Operations Office (nOE-ID), the Environmental Protection Agency (EPA), the Idaho laws andregulations, the National Environmental Policy Act (NEPA), and other local codes and standards. The proposed facilities under consideration in this study will provide waste retrievial, treatment, and interim storage capabilities. They will process various wastes that are considered mixed wastes. These are wastes that contain both radioactive and RCRA hazardous constituents. The RCRA constituents include characteristic heavy metals and "listed" hazardous constituents, as defined in 40 CFR 261, subparts $C$ and $D$. The management of the wastes, as well as the facilities, is subject to the : requirements of both the EPA and the Atomic Energy Act.(AEA). The specific requirements for radioactive waste maniagement developed under the $\mathrm{AEA}$ are administered through the $\mathrm{DOE}$. The proposed treament facilities are expected to process several types of waste and to convert them to distinct waste formis that are suitable for disposal. The regulatory requirements for the disposal of the various waste forms resulting from the proposed treatment options and the criteria of the potential target repositories are described in detail in INEEL/EXI-97-01147. It is assumed that the wastes resulting from the treatment options will be delisted and will no longer be considered RCRA hazardous waste prior to being sent to interim storage facilities.

Existing NRC requirements apply to commercial, nOn-DOE, facilities. The degree of applicability of these requirements to the proposed facilities should be determined by the NRC and the DOE, with input from the DOE contractor. Of the existing NRC regulations, it has been determined in this study that 10 CFR 61. will apply to the proposed near-surface disposal facility for the grouted Low-Activity Waste (LAW) or grouted LIW, and 10 CFR 72 will be applicable to the proposed interim storage facilities for the vitrified, Hot Isostatic Pressed (HIIPed), or grouted High-Level Waste (HIW), and for the liquid High-Activity Waste (HAW) and vitrified HAW storage facilities. Independently, it was determined by Leroy and Morgan in 'Nuclear Regulatory Commission (NRC) Licensing Assessment for the Idaho National Engineering and Environmental - Laboratory (INEEL) High-Level Waste program," April 23, 1997, that 10 CFR 30 and 10 CFR 70 
will apply to the following facilities:

- 10 CFR 30 for the LAW collection and grouting facilities and for the collection and treatment of the IIW from the INEEL ongoing operations.

- 10 CFR 70. for the separations facility, for the interim storage of liquid HAW resulting from the separation processes; and for the HAW vitrification facility.

- 10 CFR 70 for the HIL vitrification, HIPing, or grouring facilities.

- 10 CFR 70 for the calcine retrieval and dissolution facilities.

Additional NRC regulations applicable to the proposed facilities are in 10 CFR 2, 10 CFR 19, 10 CFR 20, 10 CFR 21, 10 CFR 50, $10^{\prime}$ CFR 51, 10 CFR 52, and 10 CFR 73. 10 CFR 71 and 49 CER 173 (Department of Transportation) contain requirements for the packaging and transportation of waste. These requirements could have impact on the design and operations of the storage facilities. The existing facilities that will be modified to be used for storage of treated HIW or HAW are anticipated to be exempted by DOE from any further jurisdiction of NRC. Such a jurisdiction would be excessively difficult costly, and complex to apply. All the work requirements for the modification of the existing facilities are expected to he performed in accordance with the DOERW/0333P, "Quality Assurance Reckirements and Description.".

IfDOE facilities become regulated by NRC, the jurisdiction of other currently government applicable authorities will not automatically or necessarily cease. In particular, it is expected that. local, State, Federal EPA, and some DOE regulatory requirements woùuld still apply.

In addition to all the regulatory requirements established by the various government authorities discussed above, the schedule of the construction and operations of the proposed facilities must. meet the terms and the dates of the commitments as stated in the Settlement Agreement between the State of Idaho and the DOE.

Distribution (complete package): HIW EIS Library, also contained in INEEI-EXT-01389 (VWO) INEELEXT-97-01392 (HWO), INEEL-EXT-97-01400 (CWO), INEEL-EXT-97-01399 (DCWO), and INEEL-EXI97-01428 (TRU Separations), S. I. Austad MS 3650, J. B. Bosley MS 3428, R. E. Dafoe MS 37.65, W. H. Landman MS 3625, A. E. Lee MS 3765, D. A. Lopez MS 3765, B. R. Helm MS 3765, J. J. McCartihy MS 3625, T. A. Solle MS 3428, N. E. Russell MS 3765, D. S. Vandel MS 3625.

Distribution (summary package only): D. J. Harrell MS 3211, K. L. Williams MS 3765 .

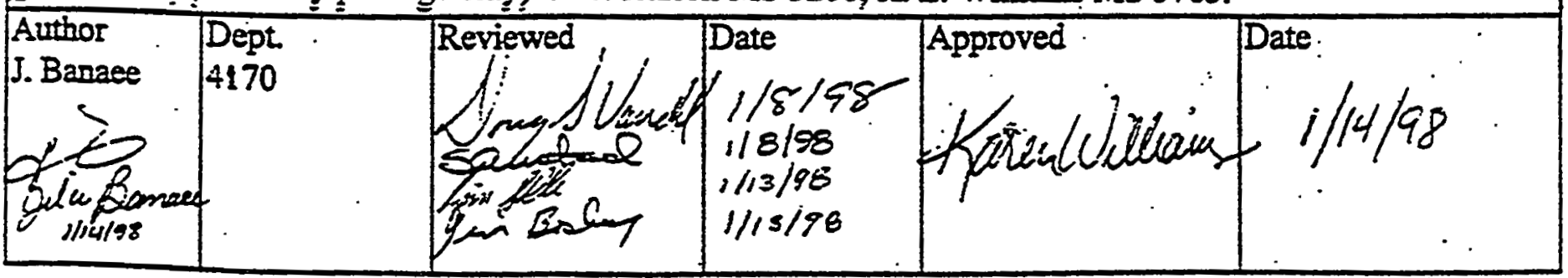




\section{INTRODUCTION}

This study identifies and presents the existing environmental regulations and standard codes for the design, construction, operations, and performance of the proposed waste treatment and storage facilities at the Idaho Chemical Processing Plant (ICPP). Also, it provides the applicable existing Nuclear Regulatory Commission (NRC) regulations and guidance documents to the proposed facilities if they were to be licensed by the XNRC in the future. The existing licensing process and related licensing issues applicable to the proposed facilities are also discussed.

The environmental regulations are primarily based on safety and health considerations. The standards define the requirements that protect human health and the environment. The principal sources for the standard regulations and codes presented here are:

- Environmental Protection Agency

- Idaho Laws and regulations

- National Environmental Policy Act (NEPA)

- Department of Energy

- DOE Idaho Operations Office (DOE-ID)

- Other applicable ständards and codes

In addition to the current regulations, the governing standards for the facility design and performance considerations of the future (i.e., DOE Orders 435.1) drafted at the time of. preparation of this report are also presented.

The facilities under consideration in this study will provide waste retrieval, treatment, and interim storage capabilities. They will process.various wastes that are considered mixed wastes. These are wastes that contain both radioactive and Resource Conservation and Recovery Act (RCRA) hazardous constituents. The RCRA constituents include characteristic heavy metals and "listed" hazardouls constiturents, as defined in 40 CFR 261; subparts C and D. The management of the wastes, as well as the facilities, is subject to the requirements of both the EPA and the Atomic Energy Act (AEA). . The specific standards for radioactive waste management developed under AEA are administered through the DOE.

The proposed treatment facilities are expected to process several types of waste to convert them to distinct waste forms that are suitable for disposal. The wastes resulting frorri the proposed treatment options are assumed to be delisted prior to being transported to the proposed interim storage facilities. The waste forms are produced starting from a variety of mixed wastes including high-level liquid waste (HIIW), Sodium-bearing liquid waste (SBW), and other radioactive wastes form ongoing operations (1996-2012), known as the newly generated wastes (NGW). These wastes have been stored in the Tank Farm at the ICPP. Most of the HILW have been calcined and stored at the Calcined Solids Storage Facility (CSSF).

The EPA has established treatment standards under the RCRA Land Disposal Restrictions (LDRs), in 40 CFR 268, for hazardous waste constituents prior to land disposal. The regulatory 
requirements for the disposal of the various waste forms resulting from the proposed options and the criteria of the potential target repositories are described in detail in INEEL/EXT-97-01147.

\section{PROPOSED WASTE TREATMENT OPTIONS}

Several treatment options are being proposed including foü non-separations and two separation processes. In addition, a no-action alternative will likely be considered, defined as the continuation of the current practice of calcination and storage in stainless steel bins at ICPP. The non-separation options include: (a) vitrified waste option (VWO), (b) Hot Isostatic Pressed (HIP) waste option (HWO), (c) direct cementitious waste option (DCWO), and (d) cementitious waste option (CWO). The separation processes are expected to generate up to three different waste streams; designated as high activity waste (HAW), low activity waste (LAW), and transuranic (TRU) waste. These options are summarized below:

\section{Vitrified Waste Option}

This option involves the following steps: 1) calcination of HLLW, vitrification of all the calcine wastes (existing and future) and placing in canisters ( $2^{\prime} \times 10^{\prime}$ or other canisters approved by the repository/NRC), and 3) interimstorage prior to shipment to a HILW repository.

Process duration: 20 year Schedule

\section{HIIP Waste Option (HWO)}

As in the VWO, the wastes will be calcined but instead of being vitrified, they will be directly HIIP processed and placed in canisters (2' $\times 10^{\prime}$ or other canisters approved by the repository/NRC), and 3 ) will be sent to an interim storage facility prior to shipment to and disposal at a. HIW repository.

Process duration: 20 year schedule

\section{Cementitious Waste Option (CWO)}

This option includes calcining the HLIW, retrieving the calcine wastes, and recalcining with the SBW in the modified New Waste Calcining Facility (NWCF), grouting in canisters (2' $\times 10^{\prime}$ canisters), and sending to an interim storage facility for transport to and ultimate disposal at an off-site HIW disposal facility. It is proposed that the cementitious waste would be suitable for disposal at the Nevada Test Site (NTS) using Greater Confinement Disposal (GCD) facility. 
Currently, the GCD facility has not been approved for disposal of HIW and waste acceptance requirements for the GCD facility have not been defined. Pursuant to the 1987 Nuclear Waste Policy Amendments Act (NWPAA), the Yucca Mountain in Nevada is designated for characterization as the only candidate site for a HLW geologic repository. However, projections of future wastes suggests a need for a second repository at some time in the future; or expansion of the first potential repository. Criteria for acceptance and disposal of waste at the potential HIW repository at the Yucca Mountain have not been finalized. The current waste acceptance criteria are preliminary at the present time. These criteria are covered in detail in INEEL/EXT. 97-01147.

Process duration: 5 year schedule

\section{Direct Cementitious Waste Option}

The DCWO consists of step 1 of the VWO, then direct grouting of all the calcined wastes and packaging in canisters ( $2^{\prime} \times 10^{\prime}$ or other canisters approved by the repository/NRC), and interim storage prior to shipment to an off-site HIW repository such as the NTS-GCD, if approved for the HLW disposal, or possibly to a potential HLW geologic repository at the Yit Mountain. This option is planned to have the same processing time as the CWO with the difference in starting date.

\section{.. Separations Options}

Two. waste separation options have been proposed: HAW/AW, known as full separations, and TRU separations. The full separations option involves calcining the HLW and SBW, retrieving and dissolving the calcine; and feeding the dissolved calcine and.fie remaining liquid SBW and the NGW to a waste separations facility to separate them into the HAW and LAW streams. The NGW, if classified as LIW, would bypass the separations facility and would ultimately be combined with the LAW.

In the TRU separations, as in the HAW/LAW separation option, the dissolved calcine and the :remaining liquid SBW, and the NGW will be fed to a waste separations system. Two separation alternatives are being considered under this option, designated as (1) TRU/LA Class C. and (2) TRU/LAW Class A/HAW. In the first alternative, the wastes would be separated into TRU waste and LAW. The TRU waste fraction is expected to contain alpha-emitting TRU radionuclides with half-lives greater than twenty years. The remaining waste stream, designated as LAW Class $C$, wouild contain Cesium (Cs) and strontium (SE) isotopes, and low activity waste portion. It is anticipated that the IAW in this alternative would meet the definition of NRC LLW Class $\mathrm{C}$. In the second altemative, $\mathrm{Cs}$ and $\mathrm{Sr}$ will be separated as HAW, and the remaining waste from separations will be designated as TRU and LAW. The LAW is expected to meet the definition of NRC ILW Class A. 
The HAW is planned to be vitrified using the same process described in the VWO and be shipped to a HLW geological repository for permanent disposal. The HAW containing isolated Cs and Sr would be stored at the INEEL awaiting disposal in a HLW geologic repository or an alternate approved disposal facility. The TRU waste stream would be converted to a solid form to be send to the Waste Isolation Pilot Plant (WIPP) for disposal. The LAW would be grouted and shipped to a LLW disposal facility. The disposal options being considered for the grouted LAW are the CSSF, the tanks at ICPP, or a LIW near-surface disposal facility. The requirements for the design and operations of a near-surface LLW disposal facility are covered in detail in EDF-FDO-008. This reference is contained in INEEL/EXT-98-00051.

Based on the NRC source term definition of HLW (10 CFR 60.2), the HAW, LAW and the TRU waste streams are actually considered $H L W$. These wastes do not conform to the existing classification for radioactive waste. Although, the separations alternatives and the resultant waste streams may be technically and economically feasible and attractive, they will need to . receive evaluation, redefinition of types of waste, and the concurrence of applicable government authorities. It is assumed that a determination will be made by the appropriate authorities (e.g., the DOE and the NRC) that the TRU waste and the IAW streams meet the TRU waste and the NRC LIW-Class A or Class C definition, respectively. The LAW also meets the definition of . incidental waste in the NRC evaluation of HLW separation procespes at Hanford Site (58 FR, "State of Washington and Oregon, Denial for Petition for Rulemakng," U.S. Nuclear Regulatory Commission, "March 4, 1993, p. 12342.): The HAW would be considered HLW and can be classified as HLW.

\section{ASSUMPTIONS}

1) All of the wastes produced from the treatment and storage facilities will meet the requirements enforced by the EPA, the DOE, the DOT, the NRC, and other potential target repositories for the disposal.

2) Under EPA 40 CFR 268.42(b), an equivalency petition for using alternative waste treatment - technologies including CWO and DCWO to borosilicate glass ${ }^{2}$ will be granted by the EPA.

3) All of the wastes resulting from the various treatment options will be delisted prior to being transported to the interim storage facilities. The EPA Upfront Exclusions for the petitioned wastes will be granted to delist the RCRA listed hazardous waste codes. The EPA delisting

\footnotetext{
-Vitrification using borosilicate glass technology is considered by the EPA a best demonstrated available technology (BDAT) for treatment of mixed HIW (55 Federal Register (FR), June 1, 1990, p.22627). DOE's stidies of glass-ceramic process and comparison of glass-ceramic process and waste form with borosilicate glass waste have shown that glass-ceramic waste form meets the definition of EPA vitrification and borosilicate glass. EPA has concurred with the DOE conclusion and has determined that the glass-ceramic process is an acceptable technology to meet BDAT (see 57 FR, May 26, 1992, p. 22024).
} 
criteria and processes for preparing a delisting petition are contained in "Petitions to Delist Hazardous Wastes: A Guidance Manual," Second Edition, PB93-169365, March 1993. The Upfront Exclusions may be granted for wastes and/or waste residues that have not been generated, but will be generated in the future. The EPA will evaluate the petitioned wastes based on available information such as the characteristics of the untreated wastes, process description, and bench-scale or pilot scale treatment data.

4) An off-site facility would need to be approved for the disposal of HLW resulting from the CWO, DCWO, and HWO.

\section{ENVIRONMENTAL REGULATIONS, DOE CRITERIA, AND OTHER STANDARD CODES}

\subsection{FEDERAL AND STATE LAWS AND REGULATIONS}

\section{Resource Conservation and Recovery Act}
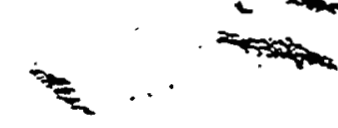

The RCRA has established minimum national standard requirements which apply to owners or operators of all facilities that treat, store, or dispose of hazardous waste. The State of Idaho has the authority to implement the RCRA requirements through the Idaho Department of Health and Welfare (IDHW). The State of Idaho adopted the Federal RCRA regulations, pursuant to the Idaho Hazardous Waste Management Act of 1983. The regulations are incorporated by reference as provided in the Federal 'requirements under 40 CFR into the "Tdaho Rules and Standards for Hazardous Waste", under administrative code known as Idaho A d̈ministrative Procedures Act (IDAPA) 16.01.05.

The RCRA requirements applicable to the hazardous waste facilities are defined in 40 CFR 264 (IDAPA 16.01.05.008), "Standards for Owners and Operators of Hazardous Waste Treatment, Storage, and Disposal Facilities" and in 40 CFR 270 (IDAPA 16.01.05.012), "EPA Administerè Permit Programs: the Hazardous Waste Permit Program."

40 CFR 264 sets regulatory requirements for the design, construction, and operation of the facility, quality assurance program, teșting and maintenance of the equipment, air emission standards, groundwater protection standards, security, inspectiop personnel training, preparedness and prevention, contingency plan and emergency procedures, manifest system and record keeping, closure and post-closure, financial requirements, and use and management of containers.

The existing hazardous waste facilities used for any future hazardous waste management activities can continue operations while meeting the requirements in 40 CFR 265 (16.01.05.009), 
"Interim Status Standards for Owners and Operators of Hazardous Waste Treatment, Storage, and Disposal Facilities". This will allow the existing facilities to continue operations while meeting minimum operational requirements defined in 40 CFR 265. To be qualified for interim status, the existing facility must have been in operation.or under construction on November 19, 1980 or have been in operation when the facility became subject to the RCRA requirements.

40 CFR 262.34 (subpart of 40 CFR 262 - IDAPA 16.01.05.006), "Accumulation Time" and 40 CFR 268.50 (subpart of 40 CFR 268 - IDAPA 16.01.05.011), "Prohibitions on Storage of Restricted Wastes" contain requiremènts, conditions, and time limits for storage of hazardous wastes. Based on 40 CFR 262.34, a generator may accumulate hazardous waste on-site for 90 days or less without a permit or an interim status, provided that the generator meet the conditions speciffed in 40 CFR 262.34. A generator who accumulates hazardous waste for more than 90 days is an operator of a storage facility and is subject to the requirements of 40 CFR 264 and 40 CFR 265, and the permit requirements of 40 CFR 270 unless the generator has been granted an extension to the 90-day period by the EPA. A 30-day extension may be granted at the discretion of the EPA on a case-by-case basis. A generator who accumulates hazardous waste greater than 100 kilograms but less than 1000 kilograms in a calendar month may accumulate hazardous waste on-site for 180 days or less without a permit or without an interim status provided the generator meets the requirements stated in 40 CFR 262.34.

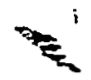

Under 40 CFR 268.50, the storage of hazardous wastes such as those present in the Tank Farm is prohibited, unless the following conditions are met:

(1) A generator stores such wastes in tanks, containers, or containment buildings on-site solely to facilitate proper recovery, treatment, or disposal and the generator complies with the requirements in 40 CFR 262.34, 40 CFR 264, and 40 CFR 265.

(2) An owner/operator, of ä hazardous waste treatment, storage, or disposal facility stores such wastes in tanks, containers, or containment buildings to facilitate proper recovery, treatment, or disposal, and an owner/operator must comply with the operating record requirements specfifed in 40 CFR 264.73 and 40 CFR 265.73.

An owner/operator of a treatment, storage, or disposal facility may store hazardous waste :restricted from land disposal beyond one year provided that the owner/operator proves to the EPA that such storage was solely for the purpose of facilitating proper recovery, treatment, or disposal.

The prohibition in storage does not apply to hazardous wastes that meet the IDR treatment standards and treatment equivalency as defined in $268.42(\mathrm{~b})$. Etowever, if the waste is still listed, the generator must comply with the RCRA requirements for hazardous waste storage. 


\section{Permit Requirements}

Various permits are required prior to the construction and operations, and during the operations of the proposed facilities. These include RCRA permit, air emissions permit, wastewater discharges permit, etc. In addition, separate permits may be needed once the processes or activities are better known, as individual pilot plant operations or modifications to the existing facilities/systems may require separate permits. The applicable permit requirements are described below. A summary of the permit requirements and regulatory drivers is presented Section 5 .

\section{RCRA Permit}

All facilities that treat, store, or dispose of hazardous wastes are required to obtain a RCRA permit during the active life (including the closure period).. 40 CFR 270 establishes the requirements for obtaining a Permit. A RCRA Permit application consists of two parts, Part A and Part B. Part A of the permit application is a short standard form that collects-aeneral information about the treatment, storage, or disposal facility. Part B of the persitapplication includes a much more detailed technical description of the facility. the permit application covers all aspects of the design, construction, operation, monitoring, and maintenance of the facility. The requirements for Part A permit application are in $4 \theta$ CFR 270.13, and for Part B Permit application are in 40 CFR 270.14 through 29.

Once the owner or operator of a facility has submitted a permit application, the regulator conducts an in-depth evaluation to determine if the application satisfies the RCRA requirements. For the new hazardous waste facilities, Parts A and Part B of the permit application must be submitted a least 180 days prior to physical construction is expected to commence. For the existing hazardous waste facilities, the requirements to submit an application is satisfied by submitting only Part A application to operate under interim status until the permitting agepey sets a date for submitting Part B of the application.

\section{Air Permit}

Air permits will be required from the State of Idaho and/or EPA Region $X$ prior to construction and operations of each of the treatment, storage, and disposal facilities with radioactive and nonradioactive emissions sources. The existing facilities that will be used for the proposed waste processing activities may require permit modification if the existing permit does not satisfy the permit requirements for the proposed use.

The Clean Air Act (CAA) sets permit requirements and emission standard limits. The CAA requirements are implemented by the IDHW under Idaho codes (see Idaho Codes and regulations below) or by EPA Region X. The CAA implementing regulations are in 40 CFR $50,52,60,61$, $62,63,70,77$, and 124 . The air permit requirements are briefly described below. 
For nonrad emissions a Permit to Construct (PTC) will be required from the IDHW for each of the new emission points prior to the construction or modifications of a facility. Individual pilot plant systems may require separate PTCs once the processes are known. Hazardous Air Pollutants (HAPs) and the Toxic Air Pollutants (TAPs) will need to be quantified prior to permitting.

The National Emission Standards for Hazardous Air Pollutants (NESHAPs), Subpart H sets the standards for the radioactive air emissions. The proposed facilities will qualify as radiological sources. if they emit any radiological emissions. They are regulated by the EPA under the NESHAPS and the State of Idaho for radioactive air emissions. The state of Idaho treats the INEEL as one large facility. Currently, the maximum off-site limits for the total INEEL radiological emissions is $10 \mathrm{mrem} / \mathrm{yr}$.

The National Ambient Air Quality Pollutants Standards (NAAQS) has established requirements for particulate matter, sulfur dioxide, ozone, nitrogen dioxide, carbon monoxide, fluoride, and lead. If their emissions are significant as defined in IDAPA 16.01.01.88; they must comply with the requirements of the Prevention to Significant Deterioration (PSD). The Best Available Control Technology (BACT) must be used to control pollutants if compliance wituthe PSD is required.

The air emissions must be calculated for each of the new facilitiềs to determine the permit -requirements and compliance with the regulatory standards, and to identify how they impact the sitewide total emissions at the INEEL because the State of Idaho treats the INEEL as one large facility. The determination of the expected air emissions is usually done during the Title II design.

Appendix D to 40 CFR 61 should be used for estimating the radioniuclide emissions from the new sources to determine if a NESHAPs approval to construct is needed. If the estimated dose is greater than $0.1 \mathrm{mrem} / \mathrm{year}$, the NESHAPs application will be required. In such case, a PSD evaluation will required by the State of Idaho.

Some of the process vents associated with the hazardous waste treatment units must meet the RCRA air emission standards in 40 CFR 264/265 Subparts AA. A process vent is any openended stack or pipe that is vented to the atmosphere. 40 CFR 264/265 Subparts BB and CC contain air emissions standards which are applicable only to certain types of processes.such as equipment leaks, tanks, and containers.

The EPA proposed MACT Rule which enforces limits on air polliutants applies to hazardous waste incinerators or other comparable facilities which burn hazardous waste and/or are qualified as an incinerator by the EPA. This study assumes that the MACT Rule will be applicable to the vitrification and HIPing facilities. The MACT Rule sets emission limits for dioxin/furan, hydrocarbons, chlorine, carbon monoxide, lead, cadmium, mercury, antimony, arsenic, beryllium, chromium, and particulate matter. 


\section{Wastewater Effluent Discharges and Drinking Water Pemit}

The EPA has established requirements for stormwater and nonstormwater discharges into the environment under the National Pollution Discharge Elimination System (NPDES). The NPDES contains the requirements that control the discharge of pollutants to waters of the U.S. (e.g., Big Lost River) as defined in the Clean Water Act (CWA) in 40 CFR 122. These sources can include sanitary, industrial processes, and storm water runoff from industrial and construction areas.

A permit under the NPDES is required for storm and nonstorm waters (e.g., service water, sewer ${ }^{-}$ discharges). The INEEL has a general NPDES permit. Therefore, the existing INEEL NPDES permit should be evaluated to determine if there is a need for modifications of the INEEL permit or addendum to the permit to satisfy the permit requirements for the proposed facilities.

Wastewater Land Application Permits are required for construction, modifications, and operation of facilities that dispose of municipal and industrial wastewater to the land surface. The requirements are defined in 40 CFR 122 (CWA).

Pursuant to the CWA, the facilities that engage in storing, transferring, and consuming oil and oil products which could reasonably be expected to discharge oil in the Big Lost River or other waters of the U.S. must have Spill Control Prevention and Countermeasures Plans. The-Plans are required if the oil discharges are in harmful quantities that violate the applicable water quality standards and cause harm to the hurwan health and environment.

\section{Idaho Laws and Regulations}

This section lists the Idaho codes and standards for air and water pollution control and for releases into the environment: The standards are based on the Federal requirements established by the CAA, the CWA, and the RCRA.

Idaho Code 39-44, "Hazardous Waste Management Act"

IDAPA 16.01.05, "Rules and Standards for Hazardous Waste"

IDAPA 16.01.01, "Rules for the Control of Air Pollution in Idaho"

IDAPA.16.01.01.161, "Toxic Substances"

IDAPA 16.01.01.210, "Demonstration of Reconstruction Compliance with Toxic Standards"

IDAPA 16.01.01.575, "Air Quality Standards and Area Classification"

IDAPA 16.01.01.650, "Rules for Control of Fugitive Dust"

IDAPA 16.01.09, "Idaho Radiation Control Rules"

Idaho Code 39-36, "Water Quality Act"

IDAPA 16.01.02, "Water Quality Standards and Wastewater Treatment"

IDAPA 16.01.08.500, "Design Standards for Public Drinking water Systems".

IDAPA 16.01.08.551, "Construction Requirement for Public Water Systems" 
IDAPA 16.01.08, "Idaho Rules for Public Drinking Water Systems"

IDAPA 16.01.17, "Wastewater Land Application Permit Regulations"

\section{CFR 191, "Environmental Radiation Protection Standards for Management and} Disposal of Spent Nuclear Fuel, High-Level Waste and Transuranic Waste".

The EPA has set radiation protection requirements for management of radioactive waste in 40 CFR 19i. The radiation protection standards for management and storage of radioactive wastes apply to:

(a) Radiation doses received by members of the public as a result of the management (except for transportation) and storage of spent nuclear fuel or HLW or TRU waste at any facility regulated by the NRC or by Agreement States, to the extent that such management and storage operations are not subject to the provisions of 40 CFR 190, "Environmental Radiation Protection Standards for Nuclear Power Operations"; and

(b) Radiation doses received by members of the public as a result of the managenent and storage of spent nuclear fuel or HIW or TRU waste at any disposal facilithat is operated by the DOE and that is not regulated by the NRC or by Agreement States.

\section{CFR 257, "Criteria for Classification of Solid Waste Disposal Facilities and Practices".}

The proposed ILW land disposal facility at the INEEL will be comparable to the LLW disposal facility of the Radioactive Waste Management Complex (RWMC). The Idaho-Department of Environmental Quality (DEQ) evaluated the applicability of federal and state regulations to the $R W M C L L W$ disposal facility. The $D E Q^{b}$ has concluded that, in addition to other requirements, disposal of waste at RWMC is subject to Subtitle D landfill standards, 40 CFR 257. The environmental standards required by 40 CFR 257 are based on safety and health considerations which protect humar health, wildlife, and the environment. The DOE or the NRC requirements for design and performance of a LLW disposal facility are much more stringent than those in 40 .CFR 257 and supersede the subtitle $D$ landfill standards.

40 CFR 257 requires that disposal facilities or practices in floodplains not restrict the flow of the base flood, reduce the temporary water storage capacity of the floodplain, or result in washout of solid wastes which pose a hazard to human life, wildlife, land or water resources. The disposal facilities shall not cause a discharge of pollutants into waters of the United States. Such a discharge would be a violation of the NPDES. Also, the facilities must not contaminate any underground drinking water source beyond solid waste facility boundary or beyond an altemative specified boundary.

DEQ letter to Jay Mitchell, Manager of LMITCO NEPA/Permitting, July 23, 1996. 


\subsection{DEPARTMENT OF ENERGY}

The AEA of 1954 authorizes the DOE to establish standard criteria to ensure safe operations of its facilities, and to protect human health and to minimize dangers to life and property. The DOE has developed a series of Orders and Directives. They contain standards that require the planning, design, and construction of.DOE facilities be performed in a manner that will satisfy all applicable Federal, State, and local environmental, safety and health laws and regulations, and the DOE criteria. The DOE and DOE-ID standards applicable to this study include the following:

\section{DOE Order 6430.1A, "General Design Criteria"}

Compliance with DOE Order 6430.1A is mandatory under the current LMITCO contract DOEID Notice 430.1A, "Life Cycle Asset Management-ID expectations," requires that for facilities under the purview of the Defense Nuclear Facilities Safety Board (DNFSB), 6430.1A remains effective until 10 CFR 830.340, "Maintenance Mirnagement", and DOE Order 420.1, "Facility Safety", are finalized and incorporated into the IMITCO contract.

DOE-ID Notice $430.1 \mathrm{~A}$ establishes the DOE-ID expectations of the contractor in areas covered by DOE Order 430.1, "Life Cycle Asset Management", in managing the INEEL. This order incorporates priviate industry standards safety design criteria, and requires additional nuclear safety criteria for nuclear facilities.

DOE Order 6430.1A provides general and specific design standards, guidance, and practices for use in the DOE facilities. The stardards are to provide levels of design for occupant life safety, reduction in loss of government property, functioning essential operations and confinement of radioactive and hazardous material. Division 13, Section 1300, General Requirements, and Section 1324, Radioactive Solid Waste Facilities, address general and specific design criteria. Also, Section 0900-99.0, Nonreactor Nuclear Facilities, contains additional criteria relevant to :-

\footnotetext{
"The term "defense" nuclear facility has not been defined anywhere in the regulations or by the DOE yet. I have spoken with a number of people in the IMITCO Mecharical, Civil, and Industrial Engineering Department and the DOE-ID (David Crandall, Scott. Jensen, Lee Williams, and others) to learn what might constitute a "defense" nuclear facility and to find out the difference between a defense nuclear facility and a nuciear facility. They were not sure about the definition of a "defense" nuclear facility. However, they all believe that the facilities under consideration in this stady could be considered "defense" nuclear facilities because they will be used for management of the DOE defense related wastes. According to Scot Jensen, the RWMC LLW disposal facility is considered a "defense" nuclear facility.
}

The definition of a nuclear facility is in MCP-2446. Based on this document, a "nuclear facility" is a facility with operations that involve radioactive and/or fissionable material in such form and quantity that a nuelear hazard potentially exits to the employees or the general public. A nuclear facility includes noureactor and reactor facilities. 
facility design. All of these criteria provide minimally acceptable requirements for the facility design. It should be noted that the applicable local building codes and models always take precedence on the issues covered in the DOE order and provide additional design requirements not covered in the DOE order.

\section{DOE-ID, “Architectural Engineering Standards” (AE).}

The AE contains general design requirements such as those defined in DOE Order 6430.1A, and additional specific construction codes. The following is a list of some of the applicable standards and codes included in the $\mathrm{AE}$ documents:

ICBO UBC, "Uniform Building Code, latest edition"

ICBO UFC, "Uniform Fire Code, latest edition".

29 CFR 1910, "Occupational Safety and Health Standards"

29 CFR 1926, "Safety and Health Regulations for Construction"

ADAAG, "Americans with Disabilities Act (ADA) - Accessibility Guidelines" -

ASCE-4-86, "Seismic Ainalysis of Safety-Related Nuclear Structures"

ASCE-7-93, "Minimum Design Loads for Buildings and Structures?.

DOE-STD-93, "Natural Phenomena Hazard Performance categorization"

DOE-STD-1020-94, "Natural Phenomena Hazard Design and Evaluation Criteria for

Deparment of Energy Facilities"

DOE-STD-1021-93, "Natural Phenomena Hazards Performance Categorization Guidelines for

Structures, Systems, and Components"

\section{DOE Order 5820.2A, "Radioactive Waste Management"}

DOE Order $5820.2 \mathrm{~A}$ established policies and criteria for management of HLW, TRU; andXIW. The Order requires that radioactive and mixed waste be managed in a manner that is in compliance with all applicable Federal, State, and local environmental, safety, and health regulations and laws and DOE criteria.

Design objectives for facilities shall assure protection of the public and operating personnel from hazards associated with normal HLW operations; accident conditions, and the effects of natural phenomena. Other objectives are compliance with the DOE policies regarding nuclear safety, quality assurance, contingency plans, training, fire protection, pollution control, and safeguards and security protection for waste and protection of essential operations from the effects of potential accidents.

The development of large scale waste treatment facilities shall be supported by the appropriate documentation such as NEPA documentation, construction design report including projected 
waste throughputs, and treatment methods, construction and operating cost estimates, and Safety Analysis Report (SAR).

All new HIL handling, transfer, and storage facilities shall be doubly contained. Where required, ventilation and filtration systems shall be provided to maintain radionuclide releases" within the guidelines specified in DOE Order 5481.1B, "Safety Analysis and Review System", DOE Order 5480.23, "Nuclear Safety Analysis Report,"and other applicable orders discussed in this EDF. Ventilation systems shall be provided where the possibility exists for generating flammable and explosive mixtures of gases (e.g., hydrogen or organic).

Nuclear criticality safety considerations and controls shall be evaluated for normal operations and, before any significant operational changes are made, to protect against an uncontrolled nuclear criticality incident. Each facility shall utilize remote maintenance features and other appropriate techniques to minimize personnel radiation exposure in accordance with DOE . 5481.1B, "Environment, Safety, and Health Program for Department of Energy Operations," DOE Order 5480.23, and DOE Order 5480.24, "Nuclear Criticality Safety."

Monitoring, surveillance, and leak detection capability shall be incorporated the engineering systems (e.g., liquid level sensing devices and alarms for high-leve waste liquid systems) to provide rapid identification of failed containment, and measurement of abnormal temperatures. The following, at a minimum, shall be monitored: temperaturejpressure; radioactivity in . ventilation exhaust, and liquid effluent streams associated with HLW facilities. Where the possibility exists for the generation of flammable and explosive mixtures of gases, monitoring shall be conducted.

Training and qualification standards shall be developed and an up-to-date record of training status shall be maintained." Worker safety training must comply with the requirements of DOE 5480.iB and applicable Orders. Quality Assurance consistent with DOE Order 5700.6C, "Quality Assurance", shall be conducted in accordance with applicable requirements of the American National Standards Institute and other applicable codes.

- As in HIW facilities, the TRU and LIW treatment and storage facilities must be equipped with monitoring, surveillance, and leak detection capabilities. The DOE Order 5820.2A requires that" the TRU temporary storage area at the generator site, prior to shipment to the WIPP, be designed, constructed, operated, and monitored to minimize the possibility of fire, explosion, or accidental release of waste to the environment. The activities to assure the self storage of IRU waste shall also be consistent with the RCRA requirements and 40 CFR 191. In this study, no interim storage area is planned for the TRU waste because it expected that the TRU waste will be roadready for shipment to the WIPP. A temporary staging or package transfer area may be required for the waste container handling prior to the TRU waste transfer to the WIPP.

LLW disposal performance must be in a manner that assures external exposure to the waste and concentrations of radioactive material which may be released into surface water, ground, water, soil, plants and animals results in and effective dose equivalent that does not exceed $25 \mathrm{mrem} / \mathrm{yr}$ 
to any member of the public. Releases to the atmosphiere shall meet the requirements of $40 \mathrm{CFR}$ 61. Reasonable effort should be made to maintain releases of radioactivity in effluents to the general environment as low as is reasonably achievable.

\section{DOE Order 435.1 (Draft), "Radioactive Waste Management"}

Currently, a draf DOE Order 435.1 has been issued by the-DOE for review. This order will replace the current DOE Order 5820.2A, Radioactive Waste Management. The cancellation of this order does not, by itself modify or otherwise affect contractual obligation with the order. Therefore, the provisions of the 5820.2A will remain in effect until the LMITCO contract is modified to delete the reference to the requirements in the canceled order.

DOE Order 435.1 requires that facility siting and design be in compliance with all federal, state, and local laws and regulations, and be performed in accordance with the requirements in DOE Manual 435.1, Radioactive Waste Management Manual, and with other applicable DOE Orders.

DOE Manual 435.1 further describes and establishes the requirements of DOE Order 435.1 for management of DOE HIW, TRU, and LEW. Based on the DOE Manual 435i waste storage, pre-treatment, and treatment facilities design and operation are requitired to comply with the following applicable Orders and regulations.

DOE 0 151.1, "Comprehensive Emergency Management System"

DOE O 420.1", "Facility Safety"

DOE 0 430.1, "Life-Cycle Asset Management"

DOE 0440.1 , "Worker Protection Management for DOE Federal and Contractor Employees"

DOE 0 460.1A, "Packaging and Transportation Safety"

DOE 0 4330.4B, "Maintenance Management Program"

DOE O 5400.1, "General Environmental Protection Requirements."

DOE 0 5400.5, "Radiation' Protection of the Public and the Environment

DOE $O 5480.19$, "Conduct of Operations Requirements for DOE Facilities"

DOE O 5480.20A, "Personnel Selection, Qualification, and Training Requirements for DंOE Nuclear Facilities"

DOE 0 5480.21, "Unreviewed Safety Questions"

DOE O 5480.22, "Technical Safety Requirements"

DOE O 5480.23, "Nuclear Safety Analysis Reports"

DOE 0 6430.1A, General Design Criteria"

10 CFR 820, "Procedural Rules for DOE Nuclear Activities".

10 CFR 830.120, "Quality Assurance Requirements 10 CFR 83ุ. Protection"

10 CFR 1021, "National Environmental Policy Act Implementing Procedures"

29 CFR 1910, "Occupational Safety and Health Standards"

"DOE Order 420.1 will replace DOE Order 548024, "Nuclear Criticality Safety." 
40. CFR 61, National Emission Standards for Hazardous Air Pollutants"

40 CFR 264, "Standards for Owners and Operators of Hazardous Waste Storage, Treatment, and - Disposal Facilities"

40 CFR 265, "Interim Status Standards for Owners and Operators of Hazardous Waste Storage, Treatment, and Disposal Facilities"

$49^{\circ}$ CFR $106-110$ Subchapter A, "Hazardous Materials Transportation"

49 CFR 171-180 Subchapter C, "Hazardous Materials Regulations"

\section{DOE Order 5400.5, "Radiation Protection of the Public and the Environment"}

DOE Order 5400.5 establishes standards and requirements for operations of the DOE and contractors to protect the public and environment against undue risk from radiation

Chapter II, Requirements for Radiation Protection of the Public and the Environment, specifies that exposure of members of the public to radiation sources as a consequence of all routine DOE activities shall not cause, in a year, an effective dose equivalent greater than 100 aren. The 100 mrem limit is the sum of the effective dose equivalent from exposure to radiatio external to the body during the year plus the committed effective dosse equivalent from radionuclides taken into the body (radioactive decay inside the body) during the year. Exposure of members of the public to radioactive materials released to the entmosphere as a consequence of routine DOE activities shall not cause members of the public to receive an effective dose equivalent greater than 10 mrem arinually.

Chapter IV, Residual Radioactive Material, presents radiological protection requirements and guidelines for cleanup of ressidual radioactive riaterial and management of the resulting residues and release of property. Basic dose limits, guidelines and authorized limits for allowable levels of residual radioactive material, and control of the radioactive wastes and residues are provided.

\section{DOE Order 5480.23, "Nuclear Safety Analysis Reports"}

This Order establishes requirements for DOE-owned nuclear facilities and operations, and for contractors responsible for the design, construction, operation, decontamination, or. decommissioning of nuclear facilities to develop safety analyses that establish and evaluate the adequacy of the safety bases of the facilities. The SAR required by this Order documents the results of the safety analysis. 


\section{DOE Order 5480.28, “Natural Phenomena Hazard Mitigation"}

The requirements provided in this order shall be used in conjunction with the general design criteria in DOE 6430.1A and other departmental design criteria as applicable. DOE Order 5480.28 requires that facilities structures, systems, and components (SSCs) be designed and constructed to. withstand the effects of natural phenomena hazards. An objective. for all SSCs is to prevent loss of structural integrity endangering life safety. An additional objective for selected SSCs or site activities is to prevent loss of capability to perform functions consistent with: (1) importance to safety for workers and the public; (2) impact on the environment; (3) repair/replacement costs; or (4) programmatic mission.

\section{DOE Order 5700.6C, “Quality Assurance”}

The provisions of this Order apply to the work performed by all Departmental Elements and management and operating (M\&O) contractors as provided by law and/or contract and as implemented by the Department's Contracting Officer. If conflicts between this and other Deparmental Orders exist, the quality assurance requirements of DOE 5700 . ST take precedent. Work licensed by the NRC or an NRC Agreement State and subjecto the quality assurance requirements of that agency are excluded from this Order.

\subsection{NATIONAL ENVIRONMENTAL POLICY ACT}

Under 10 CFR 1021, the NEPA establishes national policy procedures promoting awareness of the environmental impacts of major federal activities during the planning and decisionmaking stages of a project. The NEPA requires all agencies of the federal government prepare a detailed EIS describing potential effects of the proposed major federal actions that may be significantly. affect the quality of the human environment.

All federal facilities under the NEPA are encouraged, to the extent practicable, to incorporate Pollution Prevention/Waste Minimization (P2/WMin) criteria and recycling in the/planning stages and in the design of the new facilities or modifications to the existing facilities. The P2/WMin and recycling activities will make facilities more efficient and compatible with future environmental regulations and increase energy efficiency and conservation.

\subsection{OTHER STANDARDS AND CODES}

In adiition to the requirements discussed above, this section provides a list of other applicable standard codes pertinent to health and safety. 
1) General design of structures, systems, and components

Uniform Building Code, International Conference of Building Officials American Institute of Steel Construction.

American Welding.Society Standards

American Concrete Institute

DOE-ID Welding Procedure Specification Manual

DOE-STD-1027-92, "Hazard Categorization and Accident Analysis Techniques" for Compliance with DOE Order 5480.23; "Nuclear Safety Analysis Reports"

The hazard categorization is based on a simple approach which is intended to meet DOE Order 5480.23 requirements for a preliminary assessment and hazard categorization. DOE Order 5480.23, states that a hazard categorization of the DOE facilities is to be performed on processes, operations, or activities and not necessarily whole facilities.

DOE-STD-3007-93, “Guidelines for Preparing Criticality Safety Evaluations at Department of Energy Non-Reactor Nuclear Facilities".

DOE-STD-3009-94, “Preparation Guide for U.S. DOE Nonreactox-ezuclear Facility Safety Analysis Reports"

\section{2) Radiological control design features}

INEL Radiological. Control Manual

10 CFR 835", "Occupational Radiation Protection"

3) Fire design features :

Uniforn Fire Códe, Western Fire Chiefs Association and International conference of

Building Officials

National Fire Protection Association (NFPA)

Uniform Building Code, Section 505 (e)

DOE Order 5480.7A, "Fire Protection"

4) Seismic design feature, Flood design features, and Wind design features

DOE-STD-1020-94(CH-1) Natural Phenomena Hazards Designand Evaluation Critéria for -

Deparment of Energy. Facilities

40 CFR 264.18

40 CFR 270.14

DOE Order 5480.11 has been canceled and replaced by 10 CFR 835. 


\section{SUMMARY OF REGULATORY DRIVERS AND PERMIT REQUIREMENTS}

The following table presents a summary of the Federal and State regulatory requirements applicable to the construction and operations of the proposed facilities.

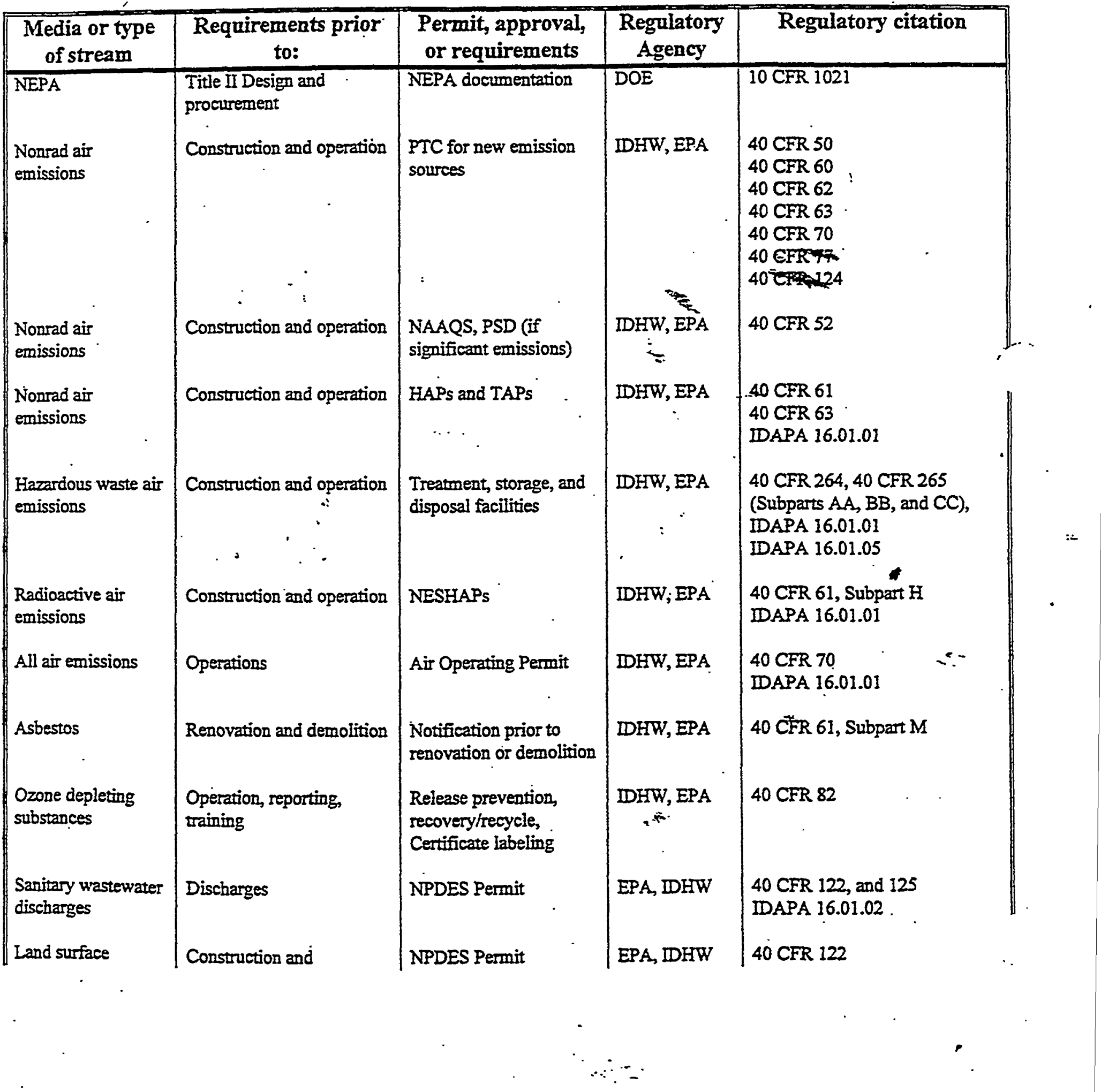




\begin{tabular}{|c|c|c|c|c|}
\hline $\begin{array}{l}\text { wastewater } \\
\text { discharges }\end{array}$ & Operations & & $\vdots$ & IDAPA 16.01 .02 \\
\hline $\begin{array}{l}\text { Storm wastewater } \\
\text { and nonstorm } \\
\text { wastewater } \\
\text { discharges }\end{array}$ & $\begin{array}{l}\text { Construction and } \\
\text { Operations } \\
. \\
.\end{array}$ & $\begin{array}{l}\text { NPDES Permit or } \\
\text { compliance writh Idaho } \\
\text { Water Quality Standards }\end{array}$ & EPA & 40 CFR 122 \\
\hline $\begin{array}{l}\text { Drinking water } \\
\text { supply }\end{array}$ & $\begin{array}{l}\text { Construction and } \\
\text { operations } \\
\text {. }\end{array}$ & $\begin{array}{l}\text { Approval of Engineering } \\
\text { Plans, Cross Connection } \\
\text { Control Plans, Report, } \\
\text { and Spec. }\end{array}$ & $\begin{array}{c}\text { IDHW, EPA } \\
. \quad .\end{array}$ & $\begin{array}{l}40 \text { CFR 141, } \\
40 \text { CFR 143, } \\
\text { IDAPA 16.01.08. }\end{array}$ \\
\hline $\begin{array}{l}\text { Hazardous waste } \\
\text { treatment, storage, } \\
\text { Disposal }\end{array}$ & $\begin{array}{l}\text { Construction, operation, } \\
\text { and maintenance of new } \\
\text { facilities or modifications } \\
\text { of existing facilities }\end{array}$ & $\begin{array}{l}\text { Hazardous Waste Permit } \\
\text { (Part A and B) }\end{array}$ & IDHW, EPA & $\begin{array}{l}40 \text { CFR } 270 \\
40 \text { CFR } 264 \\
40 \text { CFR } 265 \\
\vdots\end{array}$ \\
\hline $\begin{array}{l}\text { Underground } \\
\text { storage Tanks } \\
\text { (UTSs) } \\
\text { Land disposal of . } \\
\text { suaste }\end{array}$ & $\begin{array}{l}\text { Construction and } \\
\text { operations } \\
\text { Construetion, operations, } \\
\text { disposal }\end{array}$ & Technical standards & $\begin{array}{c}\text { IDHW, EPA } \\
\cdot \\
\text { IDHW, EPA }\end{array}$ & $\begin{array}{l}40 \text { CFR } 280 \\
40 \text { CFR 268, } 40 \text { CFR 257, } \\
\text { IDAPA } 16.01 .05,10 \text { CFR } 61\end{array}$ \\
\hline
\end{tabular}

\section{SETTLEMENT AGREEMENT}

The State of Idaho and the DOE signed an agreement on October 16, 1995. The Agreement contains several commitments for the treatment of the HIW and SBW and their transfer out of Idaho. Based on the Agreement, all remaining liquid HIW must be calcined by June 30 , 1998, and calcination of all SBW must be completed by December 31, 2012. The Agreement requires that all HWW be treated and be road-ready to be moved out of Idaho for disposal by the year 2035. The calcination and the proposed treatment shall provide for completion of treatment of all calcine wastes by December 31, 2035 .

It is stated in the Agreement that the DOE, as soon as practicable, commence the procurement of:a treatment facility at INEEL for the treatment of mixed waste. The DOE shall execute a procurement contract for the Facility by June 1, 1997, complete construction of the Facility by . December 31, 2002, and commence operation of the Facility by March 31, 2003.

Commencement of construction is contingent upon Idaho approving necessary permits.

Based on the Agreement, the DOE shall accelerate efforts to evaluate altematives for the treatment of calcined waste so as to put it into a form suitable for transport to a permanent repository or an interim storage facility outside Idaho. To support this effort, the DOE shall solicit proposais for feasibility studies by July 1, 1997, and shall commence negotiating a plan and schedule with the State of Idaho for calcine treatment by December 31, 1999. The plan and schedule shall provide for completion of the treatment of all calcined waste located at the INEEL 
by a date established by the Record of Decision (ROD) for the EIS that analyzes the alternatives for treatment of such waste. The State of Idaho expressly reserves its right to seek appropriate relief from the Court in the event that the date established in the ROD for the EIS that analyzes the alternatives for treatment of such waste is significantly later than the DOE's target date.

\section{NUCLEAR REGULATORYCOMOMISSION}

The purpose of this study is to provide an assessment of the current NRC regulations and their potential applicability to the proposed facilities if the facilities were to be licensed by the NRC in the future. The NRC requirement for regulating the DOE facilities or activities have not been defined yet. The requirements will need to be determined by the NRC and DOE Task Forces. Existing NRC regulations apply to commercial, non-DOE, facilities. The degree of applicability of the NRC requirements to the proposed facilities should be determined by the NRC and DOE, with input from the DOE contractor. If DOE facilities become regulated by $\mathrm{NRC}$, the jurisdiction of other currently government applicable authorities will not automatically or necessarily cease. In particular, it is expected that local, State, Federal EPA, and SOthe DOE regulatory requirements :would still apply.

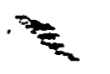

- Currently, the NRC is not authorized by law to license DOE facilities for:

- HIW processing such as those for vitrification, solidification, Cs and Srextraction,

- short term storage of $H L W$, for IRU waste storage and disposal from DOE activities, and

- . DOE LLW processing; storage, and disposal.

However, based on the recent DOE proposal, the NRC could take responsibilities for regulating the DOE nuclear facilities. Existing NRC regulations are compiled in $10 \mathrm{CFR}$, titled "Energy". Thése regulations follow a similar philosophy as the DOE, the EPA, and other codes and standards previously discussed above. The Commission has also issued a number of regulatory guides (e.g., NUREG) and other guidance documents which provide acceptable methods for complying with the NRC regulations. They contain criteria for facility design, operations, and for safety and health.

Of the existing NRC regulations, it has been determined in this study that 10 CFR 61 will apply to the proposed LAW or the LIW disposal facility, and 10 CFR 72 will be applicable to the proposed interim storage facilities for the vitrified, HIPed, or grouted $H W W$, and for the liquid HAW and vitrified HAW storage facilities. Independently, it was determined by Leroy and Morgan in "Nuclear Regulatory Commission (NRC) Licensing Assessment for the Idaho National Engineering and Environmental Laboratory (INEEL) High-Level Waste program," April 23, 1997, that 10 CFR 30 and 10 CFR 70 will apply to the following facilities: 
- 10 CFR 30 for the LAW collection and grouting facilities and for the collection and treatment of the LLW from the INEEL ongoing operations.

- 10.CFR 70 for the separations facility, for the interim storage of liquid HAW from the separation processes, and for the HAW vitrification facility.

- 10 CFR 70 for the HLW vitrification. HIPing, or grouting facilities.

- 10 CFR 70 for the calcine retrieval and dissolution facilities.

10 CFR 30, "Rules of General Applicability to Domestic Licensing of Byproduct Materialf"; and 10 CFR 70, "Domestic Licensing of Special Nuclear Material", are not specifically or directly applicable to the facilities listed above. According to Steve LeRoy (personal communication, 12/03/97), they are the only ones which came close to being applicable to the proposed treatment, separations, and retrieval facilities. It is believed that certain elements of 10 CFR 30 and 10 CFR 70 could potentially be applicable to licensing of the proposed facilities. The fact remains that NRC will most likely have to promulgate new regulations specifically for the DOE HLW, LLW, and calcine retrieval and treatment facilities or to revise the requirements of 10 CFR 30 and 10 .CFR 70 if they were to apply them to the proposed facilities.

Additional NRC regulations that are applicable to all of the proposed facilitiesare in 10 CFR 2, 10 CFR 19, 10 CFR 20, 10 CFR 21, 10 CFR 50, 10 CFR 51, 10 CF 52 , and 10 CFR 73. 10 CFR 71 and 49 CFR 173 (Department of Transportation) contain requirements for the packaging and transportation of radioactive wastes. These requirements weuld have impact on the design and operations of the storage facilities.

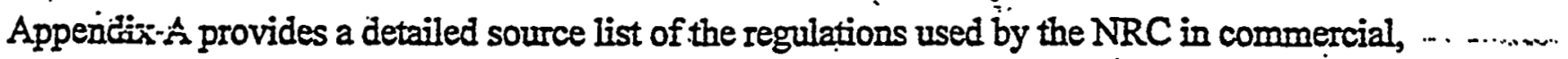
non-reactor, nuclear facilities. The regulations are primarily based on the health and safety considerations. The list includes applicable parts and subparts of 10 CFR $20,21,30,50,51,61$, 70 , and 72 as well as related guidance documents. The requirements and guidance documents are listed under the following categories: 1) radioactive waste mänagement , 2) design of structures, components, equipment, and systems, 3) electric power, utility services, and fire protection, 4) radiation protection, 5) conduct of operation, 6 ) safety analysis report criteria, 7 ) quality assurance, and 8) decommissioning.

The existing facilities that will be modified to be used for storage of HLW are expected to be exempted by DOE from any further jurisdiction of NRC. Such a jurisdiction would be excessively difficult, costly, and complex to apply. All the work requirements for the modification of the existing facilities are expected to be performed in accordance with the DOE/RW/0333P, "Quality Assurance Requirements and Description" The requirements in DOE/RW/0333P are endorsed by the Office of Civilian Radioactive Waste Management

\footnotetext{
'Byproduct material means any radioactive material (except special nuclear material) yielded in or made radioactive by exposure to the radiation incident to the process of producing or utilizing special nuciear material. Special nuclear material means (1) piutonium, uranium 233 , uranium enriched in the isotope 233 or in the isotope 235 , and any other material which the Commission determines to be special nuclear material, but does not include source material; or (2) any material artifieially enriched by any of the foregoing but does not include-source material.
} 
(OCRWM) which carries out the DOE mission for safe design and operation of a HLW geologic repository and a HLW storage facility.

\section{Current Licensing Process}

Most of the discussion in this section is based on the jnformation contained in the DOE-STD. 101-92, "Compilation of Nuclear Safety Criteria Potential Application to the DOE Nonreactor Facilities" and in the report by Morgan and LeRoy, "Nuclear Regulatory Commission (NRC) Licensing Assessment for the Idaho National Engineering and Environmental Laboratory (INEEL) High-Level Waste program," April 23, 1997.

The applicable NRC regulations that define licensing processes are in 10 CFR'2, 10 CFR 30, 10 CFR 51, and 10 CFR 61 for LLW facilities and in 10 CFR 2, 10 CFR 50, 10 CFR 52, 10 CFR 70 ; and 10 CFR 72 for HLW or HAW facilities. The licensing of a nuclear facility requires preparation and submittal of an application and a number of supporting documents to the NRC such as SAR, environmental report (ER), quality assurance document, training plan, monitoring plan, and safeguards and security plan. The following is a generic description of the various documents that will be applicable to the proposed facilities.

The ER must meet the NRC requirements in 10 CFR 51. Appendix A, Section 7 provides regulatory sources containing quality assurance procedures for the facility design, construction, and operations. The quality assurance requirements in DOE/RW-0333P are expected to be used for the existing facilities that will be modified to be used for storage of HLW or HAW. The SAR documents the adequacy of safety analysis for a nuclear facility to ensure that the facility can be designed, constructed, operated, maintained, shut down, and decommissioned safely and in compliance with applicable laws and regulations. The SAR criteria must meet the regulations listed in Appendix A, Section 6. The training, monitoring, and safeguards and security plans used by the license applicant to protect health and minimize danger to life or property must be developed in accordance with the applicable regulations. The training program should inclade an analysis of the job, learning objectives and performance criteria, procedures for personnel monitoring, procedures to avoid accidents, etc. It is assumed that the DOE will retain the responsibility for safeguard and security for its facilities.

The NRC licensing process is divided into four stages: pre-application stage, application review stage, construction and operating license stage; and decontamination and site closure stage. The licensing duration from submittal of the application to receipt of the license is expected to take three to five years or longer.

Pre-application stage is prior to filing a license application with the NRC. It entails the development of the license application and the pre-submittal communications with the NRC. This includes the NRC and DOE interactions to clearly define the NRC acceptance criteria against which the ICPP proposed facilities license application will be reviewed. 
The application review stage describes the activities after submittal of the license application to the NRC. A notification will be published in the Federal Register for public hearing when the NRC receives the application. This application review stage begins with a review process referred to as a "Docketing Review" which is usually performed within 1 to 3 months. This review is to ensure that the application is complete and contains the necessary information: The Docketing Review. process is followed by a detailed safety review of the application by the NRC staff. The NRC will ensure that the regulatory requirements are met as established in the regulations. The NRC usually requests additional information during this review which can be extensive and delay the review: Submittal of high quality, complete, and detailed SAR will reduce the request for additional information, hence the review time.

The construction and operating license stage follows the receipt of the license. The NRC will have the regulatory oversight during construction and operations.

NRC issues a license for certain time period. Before a facility license expires, a decommissioning plan will be developed by the DOE for review by the NRC. It is expected that the EPA will regulate the decontamination and decommissioning activities. Before the final closure, the DOE must submit a closire plan to the NRC for review. The closure plan must describe how the ownet/operator will conduct clean-up, what cleaneup levels will be attained, and how clean-up will be verified. The plan also includes a post-closure, and long term monitoring and maintenance. Upon review and acceptance, theNRR will airthorize closure. Monitoring will be performed during the post-closure plan in accordance with the applicable requirements. When all the monitoring and control requirements are met-the license will be terminated.

\section{LLW Near-Surface Disposal}

10 CFR 61, Licensing Requirements for Land Disposal of Radioäctive Waste

10 CFR 61 contains specific technical requirements and performance objectives applicable to near-surface disposal of radioactive wastes. It contains requirements for design, operation, closure and post-closure, and monitoring. Near-surface disposal involves disposal of waste in the uppermost portion of the earth, approximately 30 meters or 100 feet of natural grade. The NRC maintains that the use of shallow land disposal is adequate for protection of individuals and the public, when properly sited, designed, and operated, as required by: 10 CFR" 61 .

Design, operation, and closure of the land disposal facility must ensure protection of any individual inadvertently intruding into the disposal site and occupying the site or contacting the waste at any time after active institutional controls over the disposal site are removed. Operations at the land disposal facility must be conducted in compliance with the standards for radiation protection set in 10 CFR 20, except for releases of radioactivity in effluents from the land disposal facility, which shall be governed by 10 CFR 61.41. At the time a license application is submitted, the applicant shall have conducted a preoperational monitoring program to provide basic environmental data on the disposal site characteristics. The applicant shall 
obtain information about the ecology, meteorology, climate, hydrology, geology, geochemistry, and seismology of the disposal site. For those characteristics that are subject to seasonal variation, data must cover at least a twelve month period.

The regulations for near surface disposal of radioactive wastes include a waste classification system which divides the wastes into three classes: Class A, B, and C. The classification system is based on the overall disposal hazards of the wastes. Certain minimum requirements must be met for all waste Classes as provided in 10 CFR 61.56 (a). In addition; Class $B$ and $C$ wastes are required to have structural stability as discussed in 10 CFR 61.56(b): The detailed information regarding the NRC requirements for a LLW disposal facility can be found in EDF-FDO-008.

\section{HIW Storage}

10 CFR 72, "Licensing Requirements for the Independent Storage of Spent Nuclear Fuel and High-Level Radioactive Waste"

10 CFR 72 contains reguiations and procedures that are applicable to HIW of interim storage facilities. The regulations in this part establish requirements for the issuance of licenses to the DOE to receive, transfer, package, and possess IIIW, spent fuel, and other radioactive materials associated with spent fuel and HLW storage, in a monitored retrievable storage facility (MRS)g. This part also defines requirements for the safety design features of the facility structure and equipment. It requires that structures, systems, and components be designed, fabricated, erected, and tested to provide protection against environmental conditions and natural phenomena such as earthquakes, tornadoes, lighting, hurricanes, and floods. The facilities should also be designed to prevent massive collapse of building structures or the dropping of heavy objects as a result of building structural failure on the spent fuel or high-level radioactive waste or on to structures, systems, and components important to safety. If the facilities are - located over an aquifer which is a major water resource, measures must be taken to preclucte the transport of radioactive materials to the environment through this potential pathway.

Structures, systems, and components against fires and expiosions must be designed and located:-: so that they can continue to perform their safety functions effectively under credible fire and explosion exposure conditions. Noncombustible and heat-resistant materials must be used wherever practical, particularly in locations vital to the control of radioactive materials and to the maintenance of safety control functions. Explosion and fire detection, alarm, and suppression systems shall be designed and provided with sufficient capacity and capability to minimize the adverse effects of fires and explosions on structures, systems, and components important to safety.

\footnotetext{
${ }^{8}$ Pursuant to the Nuciear Waste Policy Act, a MRS is an option for providing safe and reliable long-term storage of HIW or spent nuclear fuel. However, disposal of HLW and spent fuel in a repository should proceed regardiess of any construction of a MRS pursuant to the Act.
} 
Other features that are important to safety must be designed to permit inspection, maintenance, and testing. Emergency capability must be designed to provide for accessibility to the equipment of onsite and available offsite emergency facilities and services such as hospitals, fire and police departments, ambulance service, and other emergency agencies.

Ventilation systems and off-gas systems must be provided where necessary to ensure the confinement of airborne radioactive particulate materials during normal or off-normal conditions. Storage confinement systems must have the capability for continuous monitoring in a manner such that the licensee will be able to determine when corrective action needs to be taken to maintain safe storage conditions. Instrumentation and control systems must be provided to monitor systems that are important to safety over anticipated ranges for normal operation and off-normal operation. Those instruments and control systems that must remain operational under accident conditions must be identified in the SAR.

Control room or control area must be designed to permit occipancy and actions to be taken to monitor the facilities under normal conditions, and to provide safe control .

of the facilities under off-normal or accident conditions. Utility or other serviceswyst be designed to meet emergency conditions.
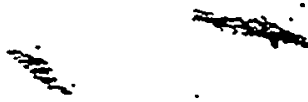

It is required that FI W be packaged in a manner that allows handling and retrievability without the release of radioactive materials to the environment or radiation exposures in excess of 10 CFR 20, "Standards for Protection Against Radiation", limits. The package must be designed to confine the high-level radioactive waste for the duration of the license. During normal operations and anticipated occurrerices, the annual dose equivivalent to any real individual who is located beyond the controlled area must not exceed 25 mrem to the whole body, 75 mrem to the thyroid and 25 mrem to any other organ as a result of exposure to planned discharges of radioactive materials and decay products excepted, to the general environment, and direct radiation from operations. Operational restrictions must be established to meet as low as is reasonably achievable. (ALARA) objectives for radioactive materials in effluents and dire radiation levels associated with storage operations. Operational limits must be established for radioactive materials in effluents and direct radiation levels associated operations to meet the limits given above.

\section{CFR 72.124, "Criteria for nuclear criticality safety"}

The design of handling, packaging, transfer, and storage systems must include margins of safety for the nuclear criticality parameters that are commensurate withethe uncertainties in the data and methods used in calculations. It must demonstrate safety for the handling, packaging, transfer and storage conditions and in the nature of the immediate environment under accident conditions.

When practicable the design of an MRS must be based on favorable geometry, permanently fixed neutron absorbing materials (poisons), or both. Where solid neutron absorbing materials are used, the design shall provide for positive means to verify their continued efficacy. 
A criticality monitoring system shall be maintained in each area where special nuclear material is handled, used, or stored which will energize clearly audible alarm signals if accidental criticality occurs. Monitoring of dry storage areas where special nuclear material is packaged in its stored configuration under a license issued under this subpart is not required.

10 CFR 72.128, "Criteria for spent fuel, high-level radioactive waste, and other radioactive waste storage and handling"

The regulations of this subpart require that $H I W$ storage and other systems that might contain or handle radioactive materials be designed to ensure adequate safety under normal and accident conditions. These systems must be designed with: (1) a capability to test and monitor components important to safety, and suitable shielding for radioactive protection under normal and accident conditions, (2) confinement systems, (3) a heat-removal capability having testability and reliability consistent with its importance to safety, and (4) means to minimize the quantity of radioactive wastes generated. Provisions must be made for the packing of site-generated lowlevel wastes in a form suitable for storage onsite awaiting transfer to disposal sitesm 


\section{APPENDIX A}

The following lists the current NRC requirements and guides applied to the areas of safety addressed in the SAR. The requirements are listed under the following categories: 1) radioactive waste management , 2) design of structures, components, equipment, and systems, 3) electric power, utility services, and fire protection, 4) radiation protection, 5) conduct of operation, 6) safety analysis report criteria, 7) quality assurance, and 8) decommissioning.

\section{1) Radioactive waste management}

This section identifies criteria for the control, collection, handling, processing, storage; and disposal of liquid, gaseous, and solid wastes that may contain radioactive materials, and the instrumentation used to monitor the release of radioactive materials. Also, as previously discussed, all RCRA hazardous and radioactive waste (mixed waste) management facilities are also subject to EPA RCRA regulations.

10 CFR 30, "Rules of General Applicability to Domestic Licensing of Byproduct Material" 10 CFR 61, "Licensing Requirements for Land Disposal of Radioactive Waste"

10 CFR 70.59, "Effluent Monitoring Reporting Requirements".

10 CFR 72.104, "Criteria in Effluents and Direct Radiation in Effluents and Direct Radiation from an ISFSI or MRS" :

D. 10 CFR 72.128, "Criteria for Spent Fuel, High-Level Radioactive Waste, and Other Radioactive Waste Storage and Handling"

Regulatory' Guide 1.21, "Measuring, Evaluating, and Reporting Radioactivity in Solid Wastes and Releases of Radioactive Materials in Liquid and Gaseous Eflluents from Light-WaterCooled Nuclear Power Plants."

Regulatory Guide 1.143, "Design Guidance for Radioactive Waste Management Systems, Structures, and Components Installed in Light-Water-Cooled Nuclear Power Plants."

Regulatory Guide 3.10, "Liquid. Waste Treatment Design Guide for Plutonium Processing and Fuel Fabrication Plants."

Regulatory Guide 3.13, "Guide for Acceptable Waste Storage Methods at UF6 Production Plants." 
Regulatory Guide 3.20, "Process Off-gas Systems for Fuel Reprocessing Plants."

Regulatory Guide 3.49, "Design of an Independent Spent Fuel Storage Installation (Water-Basin Type)."

Regulatory Guide 3.60, "Design of an Independent Spent Fuel Storage Installation (Dry Storage)."

Regulatory Guide 4.18, "Standard Format and Contents of Environmental Reports for Nearsurface Disposal of Radioactive Waste."

NUREG-1199, "Standard Format and Content of a License Application for a Low-level Radioactive Waste Disposal Facility.".

NUREG-1200, "Standard Review Plan for the Review of a License Application for a Radioactive Low-level Waste Disposal Facility." .

NUREG-1300, "Standard Review Plan for the Review of a License Applicatienar a Radioactive Low-level Waste Disposal Facility."

NUREG-0800, Section 11.2, "Liquid Waste Management Systems."

NUREG-0800, Section 11.3, "Gaseous Waste Management Systems."

NUREG-0800, Section 11.4, "Solid Waste Management Systems."

NUREG-0800, Section 11.5, "Process and Effluents radiological Monitoring."

NUREG-1567, "Offgas Treatment and Ventilation."

2) Design of structures; components, equipment and systems

10 CFR 21, "Reporting of Defects and Noncompliance"

10 CFR 50.34, "Contents of Applications: Technical Information"

10 CFR 50, Appendix F, "Policy. Relating to the Siting of Fuel Reprocessing Plants and Related Waste Management Facilities"

10 CFR 61.51, "Disposal Site Design for Land Disposal"

10 CFR 6.1.52, "Land Disposal Facility Operation and Disposal Site Closure" 
10 CFR 61.54, "Alternative Requirements for Design and Operations"

10 CFR 70, "Domestic Licensing of Special Nuclear material"

- 10 CFR 72, "Licensing Requirements for the Independent Storage of Spent Nuclear Fuel and High-Level Radioactive Waste"

10 CFR 72.120, "General Considerations"

10 CFR 72.122, "Overall Requirements"

10 CFR 72.124, “Criteria for Nuclear Criticality Safety"

10.CFR 72.126, "Criteria for Radiological Protection"

10 CFR 72.128, "Criteria for Spent Fuel, High-Level Radioactive-Waste, and Other Radioactive Waste Storage and Hand-ling"

. 10 CFR 72.130, "Criteria for Dẹcommissioning"

Regulatory Guide, 3.10, "Liquid Waste Treament System Desiğn Guide for Plutonium Processing and Fuel Fabrication Plants."

Regulatory Guidë 3.12, "General Design Guide for Ventilation Systems of Plutonium Processing and Fuel Fabrication Plants."

Regulatory Guide 3.20, "Process Off-gas Systems for Fuel Reprocéssing Plants"

Regulatory Guide 3.32, "General Design Guide for Ventilation Systems for Fuel Reprocesting Plants."

Regulatory Guide 3.38, "General Fire Protection Guide for Fuel Reprocessing Plants.”

Regulatory Guide 3.56, "General Guidance for Designing, Testing, Operating, and Maintaining Emission Control Devices at Uranium Mills."

Regulatory Guide 5.25, "Design Considerations for Minimizing Residual Holdups of Special .Nuclear Material in Equipment for Wet process Operations:" .*-

\section{Seismic systems criteria}

10 CFR 61.12, "Specific Technical Information" 
10 CFR 72.120, "General Considerations"

Regulatory Guide 1.29, "Seismic Design Classification."

Regulatory. Guide 3.14, "Seismic Design Classification for Plutonium Processing and Fuel Fabrication Plants.

\section{Wind and Tornado Loading criteria}

10 CFR 61.12, "Specific Technical Information"

10 CFR 72.40, "Issuance of License"

10 CFR 72.90, "General Considerations"”

10 CFR 72.92, "Design Basis External Natural Events"

10 CFR 72.98, "Tdentifying Regions Around an ISFSI or MRS Site"

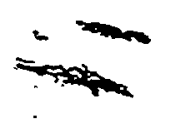

10 CFR 72.122, "Overall Requirements"

Regulatory Guide 1.76, "Design Basis Tornado for Nuclear Power Plants."

Regulatory Guide 3.10, "Liquid Waste Treatment Design Guide for Plutonium Processing and Fuel Fabrication Plants."

Regulatory Guide 3.12, "General Design Guide for Ventilation Systems of Plutonium Processing and Fuel Fabrication Plants."

Regulatory Guide 3.16, "General Fire Protection Guide for Plutonium Processing and Fuel Fabrication Plants."

Regulatory Guide 3.18, "Confinement Barriers and Systems for Fuel Reprocessing Plants.

Regulatory Guide 3.20, "Process Offgas Systems for Fuel-Reprocessing Plants."

Regulatory Guide 3.31, "Emergency Water Supply Systems for Fuel Reprocessing Plants."

Regulatory Guide 3.32, "General Design Guide for Ventilation Systems for Fuel Reprocessing Plants."

Regulatory Guide 3.38, "General Fire Protection Guide for Fuel Reprocessing Plants." 
Regulatory Guide 3.49, “Design of an Independent Spent Fuel Storage Installation, (Water Basin Type)."

Regulatory Guide 3.53, "Applicability of Existing Regulatory Guides to the Design and Operation of an Independent Spent Fuel Storage Installation."

Regulatory Guide 3.60, "Design of an Independent Spent Fuel Storage Installation (Dry Storage)."

NUREG/CR-3874, "Near-Ground Tomado Wind Fields," McDonald, J.R, Texas Tech. University, July 1984.

NUREG/CR-3848, "Experimental Investigation of Unsteady. Tomadic Wind Loads on Structures," Jischke, M.C., Oklahoma Teaching Hospitals, June 1984.

NUREG/CR-3058, "A Methodology for Tornado Hazard Probability Assessment." McDonald, J.R., Texas Tech. University, October 1983.

NUREG/CR-2944, "Tornado Damage Risk Assessment" Reinholy T.A. and Ellingwood, B., National Bureau of Standards, February 1983.

NUREG/CR-2565, "Structural Performance of HEPA Filters Under Simulated Tornado Conditions," Horak, H.L. and Smith, P.R., Los Alamos National Laboratory, May 1982.

NUREG/CR-2014, "Kinematics of Translating Tomado Wind Fields," Peterson, R.E., Texas Tech. University, April 1981.

NUREG/CR-158̨5, "Modeling Tornado Dynamics," Aeronauticä Research Association, September 1980.

\section{Water level (flood) design}

10 CFR 61.12, "Specific Technical Information".

10 CFR 61.50, "Disposal Site Suitability Requirements for Iand Disposal"

10 CFR 72.40, "Issuance of Iicense"

10 CFR 72.90, “General Considerations"

10 CFR 72.92, "Design Basis External Natural Events"

10 CFR 72.94, "Design Basis Extemal Man-Induced Events" 
10 CFR 72.98, "Identifying regions Around and ISFSI or MRS Site"

10 CFR 72.122, "Overall Requirements"

Regulatory Guide 3.10, "Liquid Waste Treatment Design Guide for Plutonium Processing and Fuel Fabrication Plants."

Regulatory Guide 3.11, "Design, Construction, and Inspection of Embankment Retention Systems for Uranium Mills."

Regulatory Guide 3.18, "Confinement Barriers and Systems for Fuel Reprocessing Plants.

Regulatory Guide 3.31, "Emergency Water Supply Systems for Fuel Reprocessing Plants."

Regulatory Guide 3.40, "Design Basis Floods for Fuel Reprocessing Plants and for Plutonium Processing and Fuel Fabrication Plants."

Regulatory. Guide 3.49, "Design of an Independent Spent Fuel Stordge Installation, (Water Basin. Type)."

Regulatory Guide 3.53, "Applicability of Existing Regulatory Guides to the-Design and Operation of an Independent Spent Fuel Storage Installation."

Regulatory Guide 3.60, "Design of an Independent Spent Fuel Storage Installation (Dry Storage)."

NUREG/CR-2678, "Flood-Risk Analysis Methodology Development Project - Final Report" Wagner, D.P. et al., Oak Ridge National Laboratory, July 1982.

\section{Missile protection}

10 CFR 61.12, "Specific Technical Information"

10 CFR 72.40, "Issuance of License".

10 CFR 72.90, "General Considerations"

10 CFR 72.92, "Design Basis External Natural Events"

10 CFR 72.94, "Design Basis External Man-Induced Events"

10 CFR 72.98; "Tdentifying Regions Around an ISFSI or MRS Site" 
10 CFR 72.122, "Overall Requirements"

Regulatory Guide 3.10, "Liquid Waste Treatment Design Guide for Plutonium Processing and Fuel Fabrication Plants."

Regulatory Guide 3.12, "General Design Guide for Ventilation Systems of Plutonium Processing and Fuel Fabrication Plants."

Regulatory Guide 3.16, "General Fire Protection Guide for Plutonium Processing and Fuel Fabrication Plants."

Regulatory Guide 3.18, "Confinement Barriers and Systems for Fuel Reprocessing Plants."

'Regulatory Guide 3.20, "Process Off-gas Systems for Fuel Reprocessing Plants."

Regulatory Guide 3.31, "Emergency Water Supply Systems for Fuel Reprocessize Plants."

Regulatory Guide 3.32, "General Design Guide for Ventilation Stems for Fuel Reprocessing Plants."

Regulatory Guide 3.38, "General Fire Protection Guide for.Fuel Reprocessing Plants."

Regulatory Guide 3.49, "Design of an Indeppendent Spent Fuel Stoziso-Installation, (Water Basin Type).”

Regulatory Guide 3.53, “Äpplicability of Existing Regulatory Guides to the Design and Operation of an Independent Spent Fuel Storage Installation."

Regulatory Guide 3.60, "Design of an Independent Spent Fuel Storage Installation (Dry Storage)."

NUREG-0533, “Aircraft Impact risk Assessment, Data Base for Assessment of Air Carrier Impact Risk in the Vicinity of Airports," USNRC, July 1979.

NUREG/CR-2462, "Capacity of Nuclear Power Plant Structures to Resist Blast Loading," Kennedy, R.P. et al., Sandia National Laboratories, September 1983.

NUREG/CR-2859, "Evaluation of Aircraft Crash Hazards for Nuclear Power Plants," Kot, C.A. et al., Argonne National Laboratory, September 1982. 


\section{Seismic design}

10 CFR 61.12, "Specific Technical Information"

10 CFR 70.22, "Contents of Applications"

10 CFR 70.23, "Requirements for the Approval of Applications"

10 CFR 72.40, "Issuance of License"

10 CFR 72.90, "General Considerations" .

10 CFR 72.92, "Design Basis External Natural Events"

10 CFR 72.98, "Tdentifying Regions Around an ISFSI or MRS Site"

10 CFR 72.102 "Geological and Seismological Characteristics".

10 CFR 72.122, "Overall Requirements"
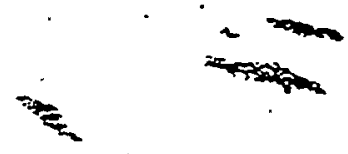

Regulatory Guide 3.10, "Liquid Waste Treatment Design Guide for Plutonium Processing and Fuel Fabrication Plants."

Regulatory Guide 3.12, "General Design Guide for Ventîation Systems of Plutonium Processing and Fuel Fabrication Plants."

Regulatory Guide 3.14, "Seismic Design Classification for Plutonium Processing and Fuel Fabrication Planț."

Regulatory Guide 3.16, "General Fire Protection Guide for Plutonium Processing and Fuel Fabrication Plants."

Regulatory Guide 3.17, "Earthquake Instrumentation for Fuel Reprocessing Plants."

Regulatory Guide 3.18, “Confinement Barriers and Systems for Fuel Reprocessing Plants. Regulatory Guide 3.20, "Process Off-gas Systems for Fuel Reprocessing Plants."

Regulatory Guide 3.31, "Emergency Water Supply Systems fờ Fuel Reprocessing Plants."

Regulatory Guide 3.32, "General Design Guide for Ventilation Systems for Fuel Reprocessing Plants."

Regulatory Guide 3.38, "General Fire Protection Guide for Fuel Reprocessing Plants." 
Regulatory Guide 3.49, "Design of an Independent Spent Fuel Storage Installation, (Water Basin Type)."

Regulatory Guide 3.53, "Applicability of Existing Regulatory Guides to the Design and Operation of an Independent Spent Fuel Storage Installation."

Regulatory Guide 3.60, "Design of an Independent Spent Fuel Storage Installation (Dry Storage)."

NUREG/CR-1069, "Effects of Earthquakes on Underground Facilities: Literature Review and Discussion," Carpenter, D.W. and Chung, D.C., Lawrence Livermore National Laboratory, June 1986.

\section{Ventilation and process off-gas systems}

10 CFR 72.132, "Overall Requirements"

Regulatory Guide 1.140, "Design, Testing, and Maintenance for Normal Ventilation Systems."

Regulatory Guide 1.52, "Design, Testing, and Maintenance aCriteria for Post-accident engineered Safety Feature Ventilation Systems.”

Regulatory Guide 3.12, "General Design Guide for Ventilation Systems of Plutonium Processing and Fuel Fabrication Plants."

Regulatory Guide 3.20, "Process Off-gas Systems for Fuel Reprocessing Plants."

Regulatory Guide 3.32, "General Design Guide for Ventilation Systems for Fuel Reprocessing Plants:"

Regulatory Guide 3.49, "Design of an Independent Spent Fuel Storage Installation (Water Basin

Type)."

Regulatory Guide 3.60, "Design of an Independent Spent Fuel Storage Installation (Dry Storage)."

NUREG-1567, Section 11.4.1.3 (DRAFT), "Ventilation offgas System Design Feature." 
3) Electrical power, utility services, and fire protection

10 CFR 50.55(a), "Codes and Standards"

10 CFR 72.122; "Overall Requirements".

Regulatory Guide 1.108, "Periodic Testing of Diesei Generator Units Used as Onsite Electric Power Systems at Nuclear Power Plants."

Regulatory Guide 3.14, "Seismic Design Classification for Plutonium Processing and Fuel Fabrication Plants."

Regulatory Guide 3.16, "General Fire Protection Guide for Plutonium Processing and Fuel Fabrication Plants."

Regulatory Guide 3.38, "General Fire Protection Guide for Fuel Reprocessing Plants."

\section{4) Radiation protection}
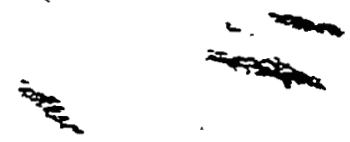

The criteria identified in this chapter are for the radiation protection of operating, construction, and maintenance personnel during normal and anticipated operational occurrences. The compilation includes criteria for facility equipment design and programs to minimize and monitor radiation exposure to meet tirestandards for protection against radiation of 10 CFR 20 .

10 CFR 19.12, "Instructions to Workers"

10 CFR 20, "Standards for Protection Against Radiation"

10 CFR 61.41, "Protection of the General Population From Releases of Radioactivity"

10 CFR 61.43, "Protection of Individuals During Operations"

10 CFR 72.44, "License Conditions"

10 CFR 72.104," "Criteria for Radioactive Materials in Effluents and Direct Radiation from an ISFSI.or MRS"

10 CFR 72.126, "Criteria for Radiological Protection"

Regulatory Guide 3.6, "Content of Technical Specifications for Fuel Reprocessing Plants."

Regulatory Guide 8.1, "Radiation Symbol." 
Regulatory Guide 8.2, "Administrative Practices in Radiation Monitoring."

Regulatory Guide 8.10, "Operating Philosophy for Maintaining Occupational Radiation Exposuress as Low as is Reasonably Achievable."

Regulatory Guide 8.24, "Health Physics Surveys During Enriched Uranium Processing and Fuel Fabrication."

\section{5) Conduct of operations}

The criteria identified in this chapter address training, emergency planning, plant procedures, and the maintenance of records and reporting.

10 CFR 61, "Licensing Requirements for Land Disposal of Radioactive Waste"

10 CFRR 51, "Environmental Protection Regulations for Domestic Licensing and Related Regulatory Functions".

- 10 CFR 70, "Domestic Iicensing of Special Nuclear Material":
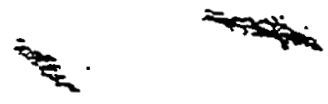

\section{CFR 72.190, "Operator Requirements"}

10 CFR 72:192, "Operator Training and Certification Program".

10 CFR 72.194, "Physical Requirements."

Regulatory Gựde 3.28, "Welder Qualification for Welding in Areas of Limited Accessibility in Fuel Reprocessing Plants and in Plutonium Processing and Fuel Fabrication Plants:" .

Regulatory Guide 3.42, "Emergency Planning for Fuel Cycle Facilities and Plants Iicensed Under 10 CFR Parts 50 and 70.

\section{6) Safety analysis report criteria}

10 CFR 20, "Standards for Protection Against Radiation"

10 CFR 30, "Rules of General Applicability to Domestic Licensing of Byproduct Material"

10 CFR 50.33, "Contents of Application, General Information"

10 CFR 50.36(b), "Environmental Conditions" 
10 CFR 50.55, "Conditions of Construction Permits"

10 CFR 50.71, "Maintenance of Records, making Reports"

10 CFR 61, "Licensing Requirements for Land Disposal of Radioactive Waste"

10 CFR 61.10, "Contents of Application"

10 CFR 70, "Domestic Licensing of Special Nuclear Material"

10 CFR 70.22, "Contents of Application"

10 CFR 70.23, "Requirements for the Approval of Applications"

10 CFR 72, "Licensing Requirements for the Independent Storage of Spent Nuclear Fuel and High-Level Radioactive Waste”

10 CFR 72.22, "Contents of Application: General and Financial Information"

10 CFR 72.24, "Contents of Application: Technical Information"

10 CFR 72.30, "Decommissioning Planning, Including Financing and Record Keeping"

10 CFR 72.48,-"Cluanges, Tests, and Experiments"

Regulatory Guide 3.15, "Standard Format and Content of License Application for Storage Only of Unirradiated Power Reactor Fuel and Associated Radioactive Material."

Regulatory Guide 3.25, "Standard Format and Content of Safety Analysis Reports for Uranium Enrichment Facilities."

Regulatory Guide 3.26, "Standard Format and Content of Safety Analysis Reports for Fuel Reprocessing Plants."

Regulatory Guide 3.39, "Standard. Format and Content of License Applications" for Plutonium Processing and Fuel Fabrication Plants." .

Regulatory Guide 3.44, "Standard Format and Content for the Safety Analysis Report for an Independent Spent Fuel Storage Installation (Water-Basin Type)."

Regulatory Guide 3.48, "Standard Format and Content for the Safety Analysis Report for an Independent Spent Fuel Storage Installation or Monitored Retrievable Storage Installation (Dry Storage)." 
Regulatory Guide 3.50, "Standard Format and Content for a License Application to Store Spent Fuel and High-Level Radioactive Waste."

Regulatory Guide 3.52, "Standard Format and Content for the Health and Safety Sections of License Renewal Applications for Uranium Processing and Fuel Fabrication."

Regulatory Guide 3.55, "Standard Format and Content for the Health and Safety Sections of License Renewal Applications for Uranium Hexafluoride Production"

Regulatory Guide 3.61, "Standard Format and Content for a Topical Safety.Analysis Report for a Spent Fuel Dry Storage Cask"

Regulatory Guide 3.62, "Standard Format and Content for the Safety Aralysis Report for Onsite Storage of Spent Fuel Storage Casks."

\section{Accidentanalysis}

The criteria in this chapter are for initiating events that resuit in a criticality accident. 10 CFR 50.34, "Contents of Applications: Techrical Information"

10 CFR 61.13, "Technical Analyses"

10 CFR 70.22, "Content of Applications"

10 CFR 70.23, "Requirements for the Approval of Applications":

$$
\text { . } 2
$$

10 CFR 72.24, "Contents of Application: Technical Information"

10 CFR 100.11, "Determination of Exclusion Area, Low Population Zone, and Population Center Distance".

Regulatory Guide 1.25, “Assumptions Used for Evaluating the Potential Radialogical Consequences of a Fuel-Handiing Accident in the Fuel-Handling and Storage Facility for Boiling and Pressurized Water Reactors.”

Regulatory Guide 1.91, "Evaluations of Explosions Postulated.ti Occur on Transportation Routes Near Nuclear Power Plants.".

Regulatory Guide 1.113, "Estimating Aquatic Dispersion of Effluents from Accidental and Routine Releases." 
Regulatory Guide 1.145, "Atmospheric Dispersion Models for Potential Accident Consequence Assessments at Nuclear Power Plants."

NUREG-1320, "Nuclear Fuel Cycle Facility Accident Analysis Handbook," USNRC, May 1988.

NUREG-1179, "Rupture of Model 48Y UF6 Cylinder and Release of Uranium Hexafluoride," USNRC, February 1986.

NUREG-0772, "The Effects of Natural Phenomena on the Exxon Nuclear Company Mixed- : Oxide Fabrication Plant at Richland Washington," USNRC, September 1980.

NUREG/CR-4303, "High-Level Waste Preclosure Systems Safety Analysis," GA Technologies, Inc., September 1985.

NUREG/CR-3682, "Nuclear Fuel Cycle Risk Assessment-Review and Evaluation of Existing Methods," Pelto, P.J. et al., Battelle Pacific Northwest Laboratories, May 1984.

NUREG/CR-3210, “Lowi-Level Waste Risk Methodology Development," Cō et al., EG\&G Inc., May 1983.

NUREG/CR-3139, "Scenarios and Analytical Methods for UF6-Releases at NRC-Licensed Fuel Cycle Facilities," Simantov, M. et al., Oak Ridge National Laboratory, July 1984.

7) Quality assurance during design, construction, and operation

10 CFR 50.4; "Written Communications"

10 CFR 50.55, "Conditions of Construction Permits"

10 CFR 50, Appendix B, "Quality Assurance Criteria for Nuclear Power Plants and Fuel Reprocessing Plants"

10 CFR 61.12, "Specific Technical Information"

10 CFR 72.40, "Issuance of License"

10 CFR 72 Subpart G, "Quality Assurance"

Regulatory Guide 1.30, "Quality Assurance Requirements for the Installation, Inspection, and Testing of Instrumentation and Electric Equipment."

Regulatory Guide 2.3, "Quality Verification for Plate-Type Uranium-Aluminum Fuel Elements for Use in Research Reactors." 
Regulatory Guide 3.3, "Quality Assurance Program Requirements for Fuel Reprocessing Plants and for Plutonium Processing and Fuel Fabrication Plants."

Regulatory Guide 3.10, 'Tiquid Waste Treatment System Design Guide for Plutonium Processing and Fuel Fabrication Plants.

Regulatory Guide 3.12, "General Design Guide for Ventilation Systems or Plutonium Processing and Fuel Fabrication Plants." :

\section{8) Decommissioning}

10 CFR 50, Appendix F, 'Policy Relating to the Siting of Fuel Reprocessing Plants and Related Waste Management Facilities."

10 CFR 50.75, "Reporting and Record Keeping for Decommissioning Planning"

10 CFR 50.82, "Application for Termination of Licenses"

10 CFR 61.12, "Specific Technical Information"
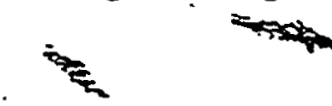

10 CFR 61.14, "Institutional Information"

10 CFR 61.23, "Standards for Issuance of a License"

10 CFR 61.24, "Conditions of Licenses"

10 CFR 61.28, "Contents of Application for Closure"

10 CFR 61.29, "Post Closure Observation and Maintenance"

10 CFR 61.30, "Transfer of License"

10 CFR 61.31, "Termination of License"

10 CFR 61.40, "General Requirement".

10 CFR 61.42, "Protection of Individuals From Inadvertent Intrusion"

10 CFR 61.44, "Stability of the Disposal Site After Closure"

10 CFR 61.52, "Land Disposal Facility Operation and Disposal Site Closure" 
10 CFR 61.53, "Environmental Monitoring"

10 CFR 61.62, "Funding for Disposal Site Closure and Stabilization"

10 CFR 70.25, "Financial Assurance and Record Keeping for Decommissioning" .

10 CFRं 70.38, "Expiration and Termination of Licenses" "

10 CFR 72.30, "Decommissioning Planning Including Financing and Record Keeping"

10 CFR 72.40, "Issuance of License"

10 CFR 72.54, "Application for Termination of License"

10 CFR 72.130, "Criteria for Decommissioning"

Regulatory Guide 3.65, "Standard Format and Content of Decommissioning Plawer for Iicensees Under 10 CFR Parts 30, 40, and 70."

NUREG-0436, Rev. 1 and Supplements 1 and 2, "Plan for Reevaluation of NRC Policy on Decommissioning of Nuclear Facilities," USNRC, December 197.8.

NUREG-0278, Vol. 1 \& Vol. 2, "Technology, Safety, and Costs of Decommissioning a Reference Nuclear Fuel Reprocessing Plant" Schneider, KJ. et ai.; Battelle Pacific Northwest Laboratory, October 1977.

NUREG/CR-1754, Addendum 1, "Technology, Safety, and Costs of Decommissioning Reference Non-Euel-Cyclẹ Nuclear Facilities," Short, S.M., Pacific Northwest Laboratory, October 1989. 
Project File Number $\quad$ 02BD7

Project/Task Waste Treatment Project Feasibility Studies

Subtask WTF Design Requirements

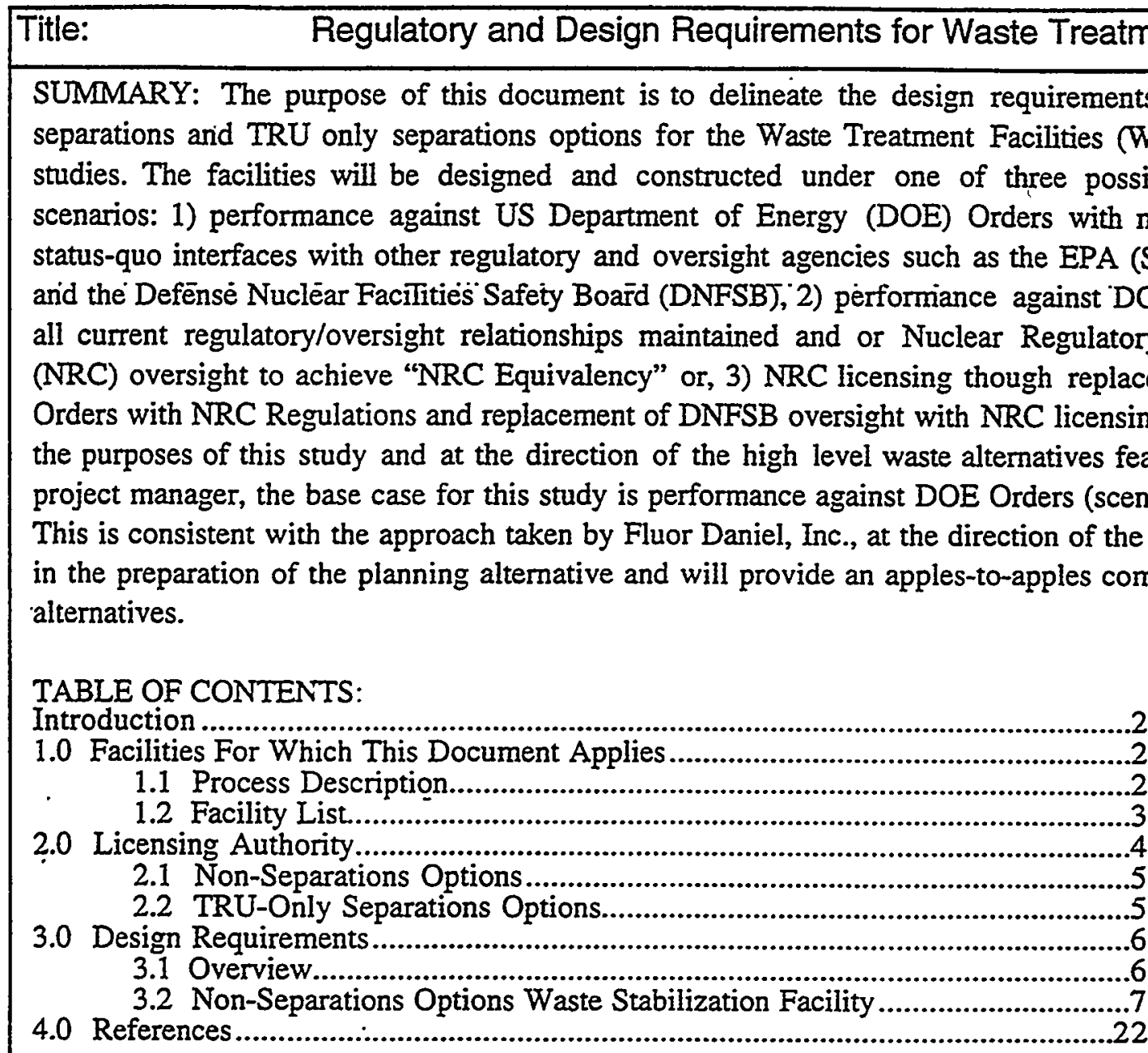

Distribution (complete package):

Distribution (summary package only):

\begin{tabular}{|c|c|c|c|c|c|}
\hline $\begin{array}{l}\text { Author } \\
\text { P.M. Rice } \\
\text { S.L. Austad, P.E. } \\
\text { L.E, Guillen, P.E. }\end{array}$ & Dept. & 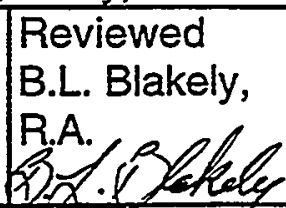 & $\begin{array}{l}\text { Date } \\
\text { 1/27/98 }\end{array}$ & Approved & $2 / 2 / 98$ \\
\hline $\begin{array}{l}\text { bointing } \\
\text { sauptes }\end{array}$ & & $\begin{array}{l}\text { LMITCO } \\
\text { Review }\end{array}$ & Date & $\begin{array}{l}\text { LMITCO } \\
\text { Approval }\end{array}$ & Date \\
\hline
\end{tabular}




\section{Introduction}

The purpose of this document is to delineate the design requirements for the non-separations and TRU only separations options for the Waste Treatment Facilities (WTF) feasibility studies. The facilities will be designed and constructed under one of three possible regulatory scenarios: 1) performance against US Department of Energy (DOE) Orders with maintenance of status-quo interfaces with other regulatory and oversight agencies such as the EPA (State of Idaho) and the Defense Nuclear Facilities Safety Board (DNFSB), 2) performance against DOE Orders with all current regulatory/oversight relationships maintained and or Nuclear Regulatory Commission (NRC) oversight to achieve "NRC Equivalency" or, 3) NRC licensing though replacement of DOE Orders with NRC Regulations and replacement of DNFSB oversight with NRC licensing process. For the purposes of this study and at the direction of the high level waste altematives feasibility studies project manager, the base case for this study is performance against DOE Orders (scenario 1, above). This is consistent with the approach taken by Fluor Daniel, Inc., at the direction of the HLW Program in the preparation of the planning alternative and will provide an apples-to-apples comparison of the alternatives.

With the ground rules clearly established above, the following is a discussion of how they will be selectively applied/ignored.

This EDF will identify the applicable DOE orders, regulations and guidance documents that would be used in the design of the facilities. There are currently no NRC regulations in place for the licensing of waste processing facilities such as those discussed herein. The only WTF activities for which the NRC has been routinely involved is the licensing of waste storage and disposal facilities. Never-the-less, NRC requirements are looming on the horizon. In addition, the waste products to be produced are in many cases destined for NRC licensed storage facilities. Therefore, there are some NRC requirements that just cannot be ignored. Thus, where appropriate, NRC regulations are explicitly specified in the design requirements. Where specific design criteria is provided under NRC regulations and guidance documents whether directly applicable or for similar facilities, it will be referenced. This will be useful in helping to determine the cost differential between DOE regulatory/oversite and NRC licensing requirements.

\subsection{Facilities For Which This Document Applies}

\subsection{Process Description}

The following is a very brief description of each of the processes that this document will consider:

Direct Vitrification Direct vitrification is a process for converting calcine into a glass waste. In the direct vitrification process, calcine is mixed with "frit" materials and fed to a melter, which would operate at a temperature of around $1100^{\circ} \mathrm{C}$. Numerous small-scale tests have been performed to determine frit formulations and glass properties, primarily leachability. No pilot data or design data is available for direct vitrification of calcine. 
Direct Grout Grouting is an ambient temperature process for solidifying or stabilizing a waste material. Grouting utilizes hydraulic cement that hardens by chemical interactions with water, and various additives which may aid dispersion, control hardening, control pumping characteristics or enhance retention of certain contaminants in the waste. -

Cementitious Waste Process Darryl Siemer has proposed a direct grouting process ${ }^{33}$ with differences from that described above. Siemer suggests mixing existing calcine with existing SBW, and recalcining the resulting slurry in the existing calciner, using sugar as additive to permit calcination of high-sodium waste. This recalcined waste would then be mixed with a combination of cementitious.agents and water, and transferred to a stainless steel can. After setting at ambient temperature, the can is transferred to an autoclave and cured with steam. If further processed by HIPing, the can would be vented and placed in a furnace to remove volatiles. Then the can would be transferred to the HIP chamber, an inert gas added to pressurize to $30-125 \mathrm{MPa}$ (4350-18,100 $\mathrm{psi}$ ), and the can heated at $850-1050^{\circ} \mathrm{C}$ for the required "soak" time.

Hot Isostatic Press (HIP) The HIP process uses high pressure and high temperature to convert calcine or other solid wastes to a glass-ceramic waste form. In a conceptual flowsheet proposed for processing calcine, calcine from storage is mixed with frit or other additives, fed to a HIP can, the can sealed and decontaminated, and then isostatically pressed in a furnace. Processing temperatures for the HIP process are similar to vitrification, typically $1050-1100^{\circ} \mathrm{C}$. The typical HIP operating pressure is 20,000 psi.

TRU Waste Alternative In this alternative, calcine is dissolved and actinides removed from the resulting solution by the TRUEX process. TRUEX wash effluents and raffinate along with other ICPP low level wastes, are evaporated, denitrated and grouted. The TRUEX strip effluent is evaporated, denitrated and then packaged for shipment to WIPP. An alternative to denitration would be to neutralize and evaporate the effluents from separation.

\subsection{Facility List}

Below is a list of the primary, main ancillary, and common support facilities that will be required for each of the options discussed herein:

1. Non-Separations Direct Vitrification Option:

- Vitrification Facility

2. Non-Separations Direct Grout Option:

- Grouting Facility

3. Non-Separations Calcine Hot Isostatic Press (HIP) Option:

- Calcine HIP Facility 
4. Non-Separations Cementitious Waste Option:

- Calcine Slurry and Grouting Facility

5. TRU-Only Separations:

- TRU Separations Facility

- TRU Product Handing, Packaging and Lag Storage Facility (TRU-only Separations Option):

- Class C Grouted Waste Interim Storage Facility:

In addition, a number of common support facilities/systems will be required to support the above facilities which include:

- Calcine Retrieval System (may vary depending on the process design for each option)

- Temporary Calcine (surge or staging) Storage

- Interim HLW Storage Facility

Note: For all of these alternatives, the study ends with interim storage of the waste product prior to shipment. Thus none of the studies includes facilities for receiving and internment of the final waste product at a repository.

\subsection{Licensing Authority}

As previously stated, for the purposes of this effort, the base case for this study is performance against DOE Orders. The following discussion is presented to defined under what NRC regulations each of the above referenced facilities would be licensed if NRC licensing were the preferred approach. This information is provided for reference only.

In early 1997 the INEEL Spent Nuclear Fuel, High-Level Waste and Related Programs prepared a licensing assessment report for proposed INEEL ICPP High Activity Waste Treatment Facilities (Idaho Chemical Processing Plant High Activity Waste Treatment Project Regulatory Assessment Report, prepared by R. G. Morgan and S. E. Leroy, Duke Engineering Services, Inc. S. E. Leroy letter to V. L. Jacobson, dated April 25, 1997) (1). The report provided an assessment of how the proposed ICPP Waste Treatment Facilities could be licensed under existing NRC regulations and processes. The report identifies the applicable NRC regulations and guidance documents that would be used in the licensing process. It also identifies those areas where additional NRC guidance documents, regulations, or rulemaking may be necessary.

The above referenced report specifically addresses the facilities defined in the "preferred alternative', whereas this EDF is examining other methods of processing and disposing of the 
calcined wastes at ICPP. For the purpose of defining the licensing criteria for the options discussed in this EDF, the above referenced report will be used. Licensing criteria will be based on similarities between the preferred alternative facilities and the facilities described here.

\subsection{Non-Separations Options (Primary and Ancillary Facilities)}

The non-separations options include: 1) direct vitrification, 2) direct grouting, 3) calcine HIPing, and 4) the Cementitious Waste options. Each option will include a facility to perform the appropriate operations to produce the end product (e.g., vitrification facility, direct grouting facility, HIPing facility, and a calcine slurry and grouting facility for the Cementitious Waste option).

The non-separations options are similar in scope to the High Activity Waste Treatment (HAWT) Facilities described in reference 1. The HAWT facilities include a calcine retrieval, transport, and receiving system; a calcine dissolution process; a high activity waste vitrification process; and vitrified product storage. The non-separations options include a calcine retrieval, transport, and receiving system; a waste stabilization process (vitrification, grouting, HIPing); and product storage.

The facilities which will be licensed are the Waste Stabilization (WS) Facilities (e.g., the vitrification, grouting, HIPing facilities), the temporary calcine (surge or staging) storage tanks associated with the receipt of the feed stock, the calcine retrieval system, and the interim HLW storage facility.

Based on the existing NRC regulations and rulemaking activities, it is expected that the following licenses would be required to support the non-separations option plan:

- Waste Stabilization (WS) Facilities (e.g., the vitrification, grouting, HIPing facilities) would require a 10 CFR Part 70 license

- Temporary calcine (surge or staging) storage tanks associated with the receipt of the feed stock would require a $10 \mathrm{CFR}$ Part 70 license.

- Calcine retrieval system would require a 10 CFR Part 70 license.

- Interim (temporary) HLW storage facility would require a 10 CFR Part 72 license.

Other NRC regulations that are applicable to the design, licensing, and operations of the facilities will be addressed later in this EDF.

\subsection{TRU-Only Separations Options (Primary and Ancillary Facilities)}

As with the non-separations options, the TRU-only separations options licensing requirements were derived by similarity to the preferred option HAWT facilities.

The facilities which will be licensed are the TRU Separations (TS) facility, TRU product handing, packaging and lag storage facility, class $\mathrm{C}$ grouted waste interim storage facility, the 
temporary calcine (surge or staging) storage tanks associated with the receipt of the feed stock, and the calcine retrieval system.

Based on the existing NRC regulations and rulemaking activities, it is expected that the following licenses would be required to support the non-separations option plan:

- TRU Separations (TS) facilities, including product handling, packaging, and lag storage would require a $10 \mathrm{CFR}$ Part 70 license

- Temporary calcine (surge or staging) storage tanks associated with the receipt of the feed stock would require a $10 \mathrm{CFR}$ Part 70 license.

- Calcine retrieval system would require a $10 \mathrm{CFR}$ Part 70 license.

Other NRC regulations that are applicable to the design, licensing, and operations of the facilities will be addressed later in this EDF.

\subsection{Design Requirements}

\subsection{Overview}

The criteria contained in this document are based only on the rudimentary descriptions of the processes presented in section 1.1. As the design is developed further, some of the criteria may become nonapplicable, and others will be identified. The purpose here is to provide a set of highlevel requirements to guide the development of the conceptual designs of the facilities and provide a reasonable basis for cost estimating purposes. In general this document will not attempt to cover criteria outside of the design and construction of the facilities. Process criteria such as the waste form acceptance criteria, treatment standards and so forth will be addressed by others.

\subsubsection{A Note on NRC Regulations}

NRC regulations are contained in Title 10, Energy of the Code of Federal Regulations. CFR's have the authority of legal mandates, and require compliance, under penalty of law, by all affected parties. NRC generates guidance documents as needed to provide clarification and elaboration of the regulations, describe information to be included in the reports, and give acceptance criteria. These publications truly are guidance documents which are not required to be followed but provide suggested methods for achieving successful licensing. Guidance documents include:

\section{NRC Regulatory Guides}

Regulatory Guides delineate acceptable methods of meeting NRC requirements. Different methods for meeting these requirements may be used if justified but the licensees usually attempt to use the Regulatory Guide methods because alternate approaches require extensive justification and additional NRC review. The use of the guides simplifies and shortens the licensing process. 
There are over 480 Regulatory Guides that have been issued to support the licensing of commercial nuclear facilities. While many of the guides apply to nuclear reactors, others, such as those describing waste storage may be viewed as applicable to the WTF. However, no definitive guidance is available for the type of facilities discussed below.

\section{NRC Reports}

NRC Reports (NUREGs) and other NRC reports developed by contractors are published on a variety of technical and regulatory issues. They may pertain to specific proceedings such as Safety Evaluation reports or Environmental Impact statements.

\section{NRC Technical Positions}

Technical Position and Staff Position Papers are also prepared by the NRC as a means of providing guidance on requirements for specific facilities regulated by the agency.

\section{Generic Communications}

NRC Generic communications include NRC Information Notes, Generic Letters and NRC Bulletins. These documents provide the licensees with specific information on problems or matters of interest to the licensee.

\section{National Standards}

NRC regulations and documents often incorporate or refer to national codes such as the ASME boiler and pressure vessel codes. These codes then become a requirement and are used in developing design criteria. If the licensee wishes an exception, the exception must be identified and basis for the exception agreed to during the licensing process.

\subsection{Non-Separations Options Waste Stabilization Facility}

The non-separations options include: 1) direct vitrification, 2) direct grouting, 3) calcine HIPing, and 4) the Cementitious Waste options. Each option will include a facility to perform the appropriate operations to produce the end product (e.g., vitrification facility, direct grouting facility, HIPing facility, and a calcine slurry and grouting facility for the Cementitious Waste option). The requirements for supporting (ancillary) facilities will be discussed under separate headings.

\subsubsection{Civil Requirements}

\subsubsection{Site Development}

A suitable site shall be located for the Waste Stabilization Facility at the INEEL in the vicinity of the Idaho Chemical Processing Plant (ICPP) with the proximity to waste sources, utilities, other facilities, vehicular access, shipping and storage capability, and future growth. A study of the impact of this facility on site utilities and infrastructure shall be performed. Information regarding 
topography, soil conditions, subsurface rock formations, road and structure locations shall be included in the- final site-decision process.

\subsubsection{Flood Design}

Flood design shall be in accordance with DOE-STD-1020. Additionally, if the facility is a RCRA facility, design shall be in accordance with 40 CFR 270.14. This standard requires the facility to be located above the 100-yr flood elevation or for engineered barriers against flooding of the site to be constructed. If the facility is a TSCA facility, 40 CFR 761.65 requires the facility to be located above the 100-yr flood elevation with no allowance for engineered barriers against flooding.

\subsubsection{Surface Drainage}

Design for surface drainage from local precipitation shall be in accordance with DOE-STD1020 and should be consistent with the ICPP site drainage plan. The INEEL site specific local precipitation standard for a 25 -year, 6 -hour storm is 1.4 inches total.

\subsubsection{Subsurface Investigation and Surveying}

Surveying and subsurface investigation for design shall be conducted to determine depth of rock, confirm soil characteristics and evaluate existing soil for chemical and radiological contamination. Locations of ground surface interferences and site characteristics shall be determined with a survey of the site.

\subsubsection{Soil Excavation and Shoring}

Specifications for excavation work shall require that excavations comply with OSHA Standards, 29 CFR 1926, Subpart P (and Subpart U if blasting is necessary), Subsection 1926.641. Where major complex temporary support systems such as shoring, cribbing, sheet piling, etc. are required, they shall be fully design by the $A E$ as part of the design package.

\subsubsection{Paving and Surfacing}

Paving shall be provided around the building for parking areas and access roads. All paved areas adjacent to buildings and structures shall have a $1 \%$ minimum slope away from the buildings or structures... Unpaved areas_shall.be sloped $2 \%$. minimum.

Design for paved roads shall conform to Idaho State Highway Standard Specifications and AASHTO HS-20 loading. Geometric design of all roads, streets, access drives and parking areas shall comply with AASHTO. Other loadings such as those imposed by transfer cask operations shall be incorporated into pavement design where applicable.

\subsubsection{Slabs, Sidewalks, and Stoops}


Sidewalks, door stoops, and approaches shall be provided at all building personnel exits or vehicle openings. Sidewalks shall be installed to provide a safe and efficient means for personnel to access doorways and walk to other nearby facilities. Concrete slabs, door stoops, truck ramps, etc., shall be sloped at least $2 \%$.

\subsubsection{Physical Protection}

The facility shall be located within the ICPP security system and fence. Construction of the new facility may take place outside of the existing main security fence if an equivalent level of security protection is established.

\subsubsection{Underground Utilities}

Existing underground ICPP utilities (sewer, potable and fire water systems) shall be extended as necessary to provide necessary services. Design of potable systems shall be in accordance with the State of Idaho Department of Health and Welfare, Idaho Regulations for Public Drinking Water Systems. Sanitary waste water shall be routed to the ICPP sewage treatment system. Water used for cleaning of the hot cells, if applicable, shall be removed by floor drains or sumps, filtered, contained in double containment tanks, monitored for hazardous materials, and if allowable, routed to the sanitary sewer system:...

\subsubsection{Site Demolition}

Site demolition, as required, will be dependent of the final site location.

\section{2:3 Architectural Requirements}

\subsubsection{General .....}

Architectural designs shall be in accordance with the DOE-ID Architectural Engineering Standards, DOE 6430.1A, and the following design criteria. The facility shall have a minimum design life of 40 years. Interim Storage-Facility design life shall be 50 years minimum. The facility shall be planned and laid out on the basis of repetitive or discrete processing steps. The need for safe normal and emergency access, egress and internal traffic flow shall be considered.

Energy conservation shall be given attention during planning and design in accordance with 10 CFR 435, Energy Conservation Voluntary Performance Standards for New Buildings, Mandatory for Federal Buildings.

\subsection{Hot Cell.}

Hot Cell design shall be based upon a Uniform Building Code (UBC) occupancy classification of Group $\mathrm{H}$, Division 7. Occupancy separations and construction types shall be designed in accordance with the UBC. 
Layout of the Hot Cell area shall include a buffer area for personnel entering and exiting the cell and shielded viewing windows for remote operations.

The Hot Cell shall include shielded, impervious and decontaminable walls, floors and ceilings as appropriate. The hot cell walls shall provide sufficient shielding to protect personnel from gamma and neutron radiation. The dose rate at the exterior of the Hot Cell wall in the operating gallery shall be below $0.1 \mathrm{mrem} / \mathrm{hr}$.

\subsection{Buffer Area.}

A Buffer Area shall be provided between the Hot Cell and other areas. Design shall be based upon a UBC occupancy classification of Group H, Division 7. Facilities for changing anti-c clothing and personnel monitoring (frisking) devices shall be provided adjacent to the Hot Cell. A shielding labyrinth leading from the Hot Cell to the Buffer Area and then to an Anti-C Change Room shall be provided. These areas. shall be separated from each other and the pressure barriers-maintained.

The Buffer Area shall provide space for discarded protective clothing used in the Hot Cell and a step off pad for frisking of contamination by PCM machine. All surfaces in the Buffer Area shall be impervious and decontaminable as well as the floors and walls of the Anti-C room.

\subsection{Operating Galleries.}

Operating galleries shall be provided as required by view angles and retraction/repair of remote equipment. Space and utilities shall be provided to accommodate remote equipment operations. Operating galleries shall be separated from the Hot Cell by a concrete shielded wall.

\subsubsection{T.4 Utility Support Arèas.}

Utility Support Areas design shall be based upon a UBC occupancy classification of $\mathrm{H}-7$ and shall be designed to accommodate remote and contact maintenance of equipment.

\subsection{Equipment Maintenance Areas.}

Crane maintenance areas shall be provided to support maintenance of in-cell equipment.

\subsection{Administrative Areas.}

The Administrative Area design, which includes office and support areas, shall be based upon a UBC occupancy classification of Group B.

The Administrative Area shall include a minimum of three offices for a shift supervisor and HP support personnel. A Ready Room shall be provided for conduct of meetings and work breaks. Men's and women's lavatories, showers, lockers, and change facilities shall be provided. Storage space and a janitor's room shall also be provided. 


\subsubsection{Building Features}

Materials selected for the walls shall address durability, low maintenance, shielding, insulation and decontamination. The walls shall meet the recommended $R$ value of the DOE-ID A/E Standards. The UBC Construction Type of II-N shall be used for the Facility.

The entire surface area of the contaminated work areas shall be decontaminable. Where wash down or decontamination activities are to be located, the floors shall be sloped to drains that lead to appropriate holding tanks.

Devices (such as door types or air lock arrangements) shall maintain pressure barriers for the hot cell and operating gallery areas.

\subsubsection{Structural-Requirements -}

\subsubsection{General}

Structural design shall be in accordance with the DOE-ID Architectural Engineering Standards, DOE O 420.1, and DOE-STD-1020.

\subsubsection{Classification and Design Loads}

The performance categories for SSCs shall be established using DOE-STD-1021, Natural Phenomena Hazards Design and Evaluation Criteria for Department of Energy Facilities." Sitespecific studies and hazard assessments of the site, as.needed, shall be developed in accordance with . DOE-STD-1022, "Natural Phenomena Hazards Site Characterization Criteria" and DOE-STD1023, "Natural Phenomena Hazards Assessment Criteria."

All permanent and transient loads that could exist or be developed during normal operations of the facility shall be considered in the design of the facility. Loads to be considered shall include: dead, live, thermal, lateral soil, snow, natural phenomena, seismic, wind, flood, off-normal operating and accident loads, and load combinations.

Dead and live loads shall be determined in accordance with ANSVASCE 7, "Minimum Design Loads for Buildings and Other Structures." Loadings due to natural phenomena hazards (wind, seismic, flood, etc.) shall be determined in accordance with DOE-STD-1020, Natural Phenomena Hazards Design and Evaluation Criteria for Department of Energy Facilities."

In accordance with DOE-STD-1020, the Uniform Building Code shall be used as the basis for seismic design for Performance Category 1 and 2 SSCs. The seismic input control motion for the INEEL for Performance Category 3 SSCs is specified by appropriately scaling the USNRC R.G. 1.60 horizontal spectra $(0.18 \mathrm{~g})$. The input motion is assumed to occur in the free-field at the top of a real or hypothetical rock outcrop near the facility location. The vertical input spectra shall be taken as $2 / 3$ of the horizontal spectra. A detailed soil amplification analysis or the soil surface spectra shall be taken to equal the rock outcrop spectra multiplied by: 
(a) 1.2 for soil overburden up to $20 \mathrm{ft}$.

(b) 1.5 for soil overburden between $20 \mathrm{ft}$ and $50 \mathrm{ft}$.

Snow loads shall be determined in accordance with ANSI/ASCE 7, with a ground snow load of 35 psf and a minimum roof snow load of 30 psf. Tornado loads are not anticipated and need not be included. Load combinations shall be determined in accordance with ANSI/ASCE 7.

3.2.4.2.I NRC Specific - To meet NRC requirements, seismic loads shall be determined in accordance with 10 CFR 72, Subparts D and E, 10 CFR 100, and USNRC Reg. Guide 1.6. Tornado loads shall be determined in accordance with ANSI/ANS-2.3. Load combinations shall be designed using applicable load combinations and stress limits stipulated in ANSI/AISC N690 and ANSUACI349.

\subsubsection{Footings and Foundations}

Footings shall be designed to support the structure and keep differential settlement within allowable limits. Design frost depth shall be 5 - $\mathrm{ft}$ below grade. The Hot Cell and shielded storage areas shall be provided with continuous reinforced grade beams or wall footings as required for shielding.

\subsubsection{Structural Features}

The Hot Cell walls and roof design shall be consistent with shielding and loading requirements. Other shielded area walls and roofs shall be designed consistent with shielding and loading requiremients. The structural design must support crane systems.

\subsubsection{Handling Requirements}

\subsubsection{Cranes (Critical Lift Devices Only)}

All crane designs shall meet the ASME NOG-1 and where applicable, CMAA 70, Crane Manufacturers Association of America, Inc., Specification for Electric Overhead Traveling Cranes and CMAA 74-1987, Specifications for Top Running and Under Running Single Girder Electric Overhead Traveling Cranes Utilizing Under Running Trolley Hoist.

In addition, all cranes shall meet the requirements of NUREG-0612, Control of Heavy Loads at Nuclear Power Plants.

Cranes shall have true vertical lift on the hoist and all motions shall be the slowest that are commercially available to allow for more precise control when placing or picking objects.

\subsubsection{Work Platforms}


The facility shall be equipped with decontaminable work platforms that shall provide a safe and convenient elevated work area for personnel as required. Design of the platforms and their means of access shall be in accordance with OSHA 1910. Removable guard rails may be utilized as necessary to meet process and handling requirements.

\subsubsection{HVAC Requirements}

All HVAC systems shall be in accordance with Regulatory Guides RG 1.140 Design, testing, and maintenance for normal ventilation systems, RG 1 Design, testing, and maintenance criteria for post-accident engineered safety feature ventilation systems, NUREG-0800 Section 9.4.3 Auxiliary and radioactive waste area ventilation, NUREG-0800 Section 11.3 Gaseous waste management systems, NUREG 0800 Section 11.5 Process and effluent radiological monitoring, NUREG-1567 Offgas treatment and ventilation, NUREG-1567 (Draft) Section 11.4.1.3, Ventilation offgas system design features.

HVAC systems shall be in accordance with 29 CFR 1910, Occupational Safety and Health Standards for General Industry, Subpart $G$ (Occupational Health and Environmental Control) and Subpart $Z$ (Toxic and Hazardous Substances).

The HVAC systems shall meet the air quality requirements addressed in 40 CFR 50-53, and 58 .

The HVAC systems shall be in accordance with ANSI/ASME N509-1989, Nuclear Power Plant Air Cleaning Units and Components and ANSIASME N510-1989, Testing of Nuclear AirCleaning Systems.

The HVAC systems shall meet the requirements of Idaho Administrative Procedures Act (IDAPA) 16.01.01000-01999.

The HVAC systems shall be in accordance with MIL-F-51068C (Filter: Particulate High Efficiency, Fire Resistant) and MIL-F-51079A (Filter Medium: Fire Resistant, High Efficiency).

The Hot Cell atmospheric pressure shall be controlled during hot operations to $-0.6 \mathrm{~W}$. G. (or lower) below atmospheric pressure.

Heating loads shall be based on a minimum winter outdoor design temperature of $-14^{\circ} \mathrm{F}$. Cooling loads shall be based on temperatures of $93^{\circ} \mathrm{F}$ dry bulb and $61^{\circ} \mathrm{F}$ wet bulb. The HVAC system should maintain a minimum temperature of $65^{\circ} \mathrm{F}$ in the winter and approximately $76^{\circ} \mathrm{F}$ in the summer in the operations area (not including the vitrification cell). The HVAC system must maintain a minimum temperature of $65^{\circ} \mathrm{F}$ in the winter and approximately $72^{\circ} \mathrm{F}$ in the summer in the Administrative areas. HVAC design for indoor temperature conditioning shall be based on ASHRAE 90. 
Air shall flow from areas of least contamination potential to areas of highest contamination potential. The HVAC system shall collect exhaust air from contamination control areas and pass it through HEPA filters prior to discharge to the atmosphere.

\subsubsection{Mechanical Utilities Requirements}

Mechanical utilities systems shall meet the requirements of the ASME Code for Pressure Piping B31.

\subsubsection{Compressed Air}

Compressed air for plant and instrument air shall be provided for pneumatically operated HVAC system equipment and other pneumatic operations in the facility. The system design for compressed air shall be in accordance with 29 CFR 1910, Occupational Safety and Health Standards for General Industry, Subpart $M$ (Compressed Gas and Compressed Air Equipment). Instrument air shall be ISO-141 Grade or better.

\subsubsection{Compressed Gas}

Argon compressed gas shall be supplied for welding processes

Helium compressed gas shall be supplied for pressure testing and inerting operations.

The system design for compressed gas shall be in accordance with 29 CFR 1910, Occupational Safety and Health Standards for General Industry, Subpart H (Hazardous Materials) and Subpart M (Compressed Gas and Compressed Air Equipment).

\subsubsection{Potable Water}

Potäble water, including hot water where applicable, shall bë provided to the facility to service water closets, urinal(s), sinks, showers, shower/eyewash facilities, evaporative coolant units, drinking fountains, and miscellaneous ports.

Cross-connection control shall be in accordance with the Idaho Code (IDAPA 16.01.08), "The Cross Connection Control Manual, Accepted Procedure and Practice" (Pacific Northwest Section of American Water Works Association), and the Foundation for Cross Connection Control and Hydraulic Research (University of Southern California.)

\subsubsection{Waste Systems}

3.2.9.4.1 Liquid Waste-Liquid waste system(s) shall be provided for in the Hot Cells and other process areas. The liquid waste systems shall be designed in accordance with NUREG-0800 Section 11.2 Liquid waste management systems. Condensate from HVAC equipment shall be disposed of using the liquid waste system. Liquid waste shall be collected and tested prior to being pumped into the waste line 
3.2.9.4.2 Sanitary Systems-The sanitation system design shall be in accordance with 29 CFR 1910, Occupational Safety and Health Standards for General Industry, Subpart J (General Environmental Controls). Sanitary sewer drains, cleanouts, and vents shall be provided as needed

\subsubsection{Fire Protection}

Fire water shall be provided in accordance with DOE 6430.1A, DOE Order 420.1, and the DOE-ID Architectural Engineering Standards. Fire protection systems shall ensure nuclear criticality and suppressant-HLW chemical reactions cannot occur. All underground fire water lines shall be cathodically protected and meet State of Idaho requirements for minimum distances from potable water piping.

\subsubsection{Steam}

Steam shall be provided and routed to the HVAC system as required. The steam lines shall be insulated.

\subsubsection{Electrical Requirements}

The criteria for the electrical design of the WTF is based on requirements from NFPA, ANSI, Factory Mutual (FM), DOE O 420.1 and 29 CFR 1910, Occupational Safety and Health Standards for General Industry, Subpart $S$ (Electrical).

Electrical design and installation shall incorporate the most efficient methods of penetration, shielding integrity retention, efficiency, and operational convenience.

The facility shall require an electrical room a communications room, and an Uninterruptable Power Supply (UPS) room.

\subsubsection{Power}

The electric power system shall be designed to provide standard power to the facility and emergency electrical supply to essential instrumentation, emergency lighting, emergency communications, and physical security systems. Standby power shall be supplied for the Hot Cells, process areas, and HVAC system exhaust fan.

An Uninterruptable Power Supply (UPS) shall provide emergency power. The UPS shall support the Fire Alarm, Voice Paging, HVAC, Radiation Monitoring and Alarm, and security systems. There shall sufficient battery capacity to carry the rated load for a minimum of 30 minutes.

\subsubsection{Grounding}

Grounding shall be provided in accordance with the DOE-ID Architectural Engineering standards. 


\subsubsection{Cathodic Protection}

Utility piping shall be protected through connection to the existing ICPP cathodic protection system. A testing/bonding station shall be included to periodically monitor the cathodic protection system.

\subsubsection{Lighting}

Interior and exterior lighting shall be designed and inciuded in accordance with current Illuminating Engineering Society (IES) recommendations. Emergency and exit lighting shall be provided at each means of egress. Hot Cell lighting shall be provided by high-pressure sodium fixtures.

\subsubsection{Lightning Protection}

A lightning protection system shall be included and shall be designed in accordance with NFPA 780.

\subsubsection{HVAC Controls}

A HVAC control system shall be provided. It shall be a smart system that can automatically generate control signals to change HVAC equipment operating parameters based on signal received from various monitors. A computer monitor shall be provided in the Shift Supervisor's office for reviewing the operating status of the system and making adjustments to control setpoints.

Instrumentation shall be provided to detect and alarm both high and low differential pressure across filters in the HVAC system. Instrumentation shall be provided to initiate isolation of the HVAC system filters in the event of fire detection.

\subsubsection{Equipment Controls}

Facility control, process control, and data acquisition systems shall be provided.

Remotely controlled CCTV cameras shall be provided in the Hot Cells and process areas for general visual observation, operations, inspection, and documentation. Each Hot Cell window shall be equipped with a visual inspection station which shall include two high resolution cameras; a monitor; camera controls for pan, tilt, and zoom functions; and recording capability for archival purposes.

Instrumentation shall be provided to measure and record the facility structural response to an earthquake.

A system shall be provided for the collection of alarms from the HVAC system and other alarms. This shall be located in the Shift Supervisor's office. 


\subsubsection{Radiation Monitoring and Alarms}

Radiation detection instrumentation shall be provided to warn operating personnel of radiation and airborne radioactivity levels above set limits. The RAMs shall alarm locally and remotely in the RadCon office.

Stack monitoring shall be provided for the detection of radioactive particulates in the air exhaust stream. These instruments shall comply with ANSI-N42.17B-1989.

Provisions shall be made in the design for monitoring groundwater in the vicinity of the storage area for radioactive contamination.

Activity monitors shall be provided in the wash water collected from the Hot Cells and process areas.

\subsubsection{Communications and Alarms}

Voice and data telecommunications lines shall be provided throughout the occupied areas of the facility.. The existing Broadband Local Area.Network (LAN) shall be made available..in the facility. Access ports shall be provided in all normally occupied offices.

Fire alarm, emergency voice paging, and evacuation alarm systems shall be compatible with existing systems at ICPP.

\subsubsection{Data Acquisition and Recording}

A data entry station shall be provided to record and monitor all fuel movements. The stations shall be linked for data communications.

\subsubsection{Security Systems}

Physical protection of the facility shall be in accordance with 10 CFR 73 and 10 CFR 72 , Subpart $\mathrm{H}$.

\subsubsection{Design Life Requirements}

Design life of the facility and equipment shall be 30 years and have maintainable or replaceable life of 60 years

\subsubsection{Safety Requirements}

\subsubsection{Safety Classification}

The facility is assumed to be a Hazard Category 2. 


\subsubsection{Construction}

The design of utility services and distribution systems that are important to safety shall include redundant systems to the extent necessary to maintain, with adequate capacity, the ability to perform safety functions assuming a single failure.'

The facility and its systems important to safety ${ }^{2}$ shall be designed to be evaluated by appropriate tests or by other means acceptable to the NRC to demonstrate that they will reasonably maintain confinement of radioactive material under normal, off-normal, and credible accident conditions.

Structures, systems, and components important to safety shall be designed and located so that they can continue to perform their safety functions effectively under credible fire and explosion exposure conditions.

The design of the facility shall include provisions to protect against nuclear criticality that might otherwise result from the operation or the failure of fire suppression or decontamination systems.

Material handling, packaging, transfer, and storage systems shall be designed to be maintained subcritical under the worst case moderated and reflected conditions, and to ensure that, before a nuclear criticality accident is possible, at least two unlikely, independent, and concurrent or sequential changes must occur in the conditions essential to nuclear criticality safety.

Each entrance or access point into a high radiation area shall have either a control device that energizes a conspicuous visible or audible alarm signal so that the individual entering the high radiation area and the supervisor of the activity are made aware of the entry; or entryways that are locked, except during periods when access to the areas is required, with positive control over each individual entry.

Process materials that are reactive with water or other chemicals shall be protected from exposure to those materials.

The facility shall be designed to prevent the dropping of critical loads under normal and off normal conditions including the design basis accidents (DBAs) that they shall withstand.

1. A single failure is an occurrence that results in the loss of capability of a component to perform its intended safety function(s). A multiple failure, i.e., loss of capability of several components, resulting from a single occurrence, is considered to be a single failure. Systems are considered to be designed against an assumed single failure if neither (1) a single failure of any active component (assuming passive components function properly) nor, (2) a single failure of any passive component. (assuming active components function properly) results in loss-of -the system's capability to perform its safety function(s).

2. Structures, systems, and components important to safety mean those features of the Storage Facility whose function is: (1) To maintain the conditions required to store spent fuel safely, (2) To prevent damage to the spent fuel waste container during handling and storage, or (3) To provide reasonable assurance that spent fuel can be received, handled, packaged, stored, and retrieved without undue risk to the health and safety of the public. 
The facility shall be designed to be able to recover from accidents involving dropping of critical loads.

In-cell equipment shall be designed for recovery from all possible conditions to the extent that manned entry into the cell, for maintenance, can be accomplished.

Fire doors shall be provided as required by UBC, UFC, NFPA-80, and NFPA-101. In addition, all fire doors and frames shall meet all requirements of the Underwriters Laboratories and shall bear the UL or FM label. Fire doors and frames shall be constructed from metal. Structural members, such as steel channels embedded in wall openings, shall not substitute as door frames. All fire doors shall be provided with fitted frames which are anchored to, but separate from, the building structural members. The fire doors shall contain windows fitted with UL approved safety glass which is not removable from the outside of the door. Their installation shall meet all of the requirements of NFPA-80 and NFPA-101.

The facility design shall mitigate natural phenomena hazards. The design shall address common cause effects and interactions for: earthquakes, volcanic events, tornadoes, hurricanes, high winds, floods, excessive rains, excessive snow, ice cover, lightning, and fires. The secondary natural phenomena include drought, fog, frost, high temperatures, low temperatures, landslides, subsidence, surface collapse, uplift, storm surges, and waterspouts. Damage and failure will be considered for systems, structures, and components. In addition, the facility shall have instrumentation or other means to detect and record the occurrence and severity of seismic events.

\subsubsection{Operation}

Radiation protection for occupational workers shall be per 10 CFR 835 (Occupational Radiation Protection) and the INEEL Radiological Control Manual

Facility design features and physical controls shall ensure occupational exposure is maintained ALARA during normal and off-normal operations

Personnel radiation exposure levels throughout facility shall not exceed $0.1 \mathrm{mr} / \mathrm{hr}$ for continuously-occupied areas.

The following radiation zones (as described in the DOE-ID AE Standards) shall apply during operations: TBD

Safe access will be provided to all packages, vehicles, and installed components for purposes of testing, inspection, and maintenance.

\subsubsection{Environmental Requirements}

Facility emission limits shall be per requirements listed in EDF-WTS-003, Section 5. 
Administrative controls and Best Available Control Technology shall be used to minimize the impacts of air emissions

The facility processes and equipment shall be designed to limit solid waste generation of LLW and industrial (cold) waste

Solid radioactive waste produced by operations shall be packaged in standard RWMC $4 \times 4$ x8-ft plywood boxes for contact-handled (CH) LLW or INEL Mark III concrete containers for remote-handled (RH) LLW, and shipped to RWMC for disposal

Means for measuring the amount and concentrations of radionuclides in effluents during normal operations, and under accident conditions, shall be provided for effluent control systems

Warm liquid waste shall be controlled and verified to meet the criteria for existing ICPP handling systems, and shall be transferred to those systems

If all or part of the facility is located outside of existing ICPP fences, the use of new land shall not exceed 551 acres when combined with other storage systems included in DOE/EIS-0203-F.

\subsubsection{Safeguards and Security}

The materials are not attractive as defined in DOE Order 5633.3B.

A data management system shall be provided to keep records. The data management system shall meet the requirements of 10 CFR 72, Licensing Requirements for the Independent Storage of Spent Nuclear Fuel and High-Level Radioactive Waste, Subpart D (Records, Reports, Inspections, and Enforcement).

Dual records shall be maintained to ensure an off-normal event cannot result in the loss of the sole records. These records shall be retained for as long as the material is stored, and for a period of five years after the material is disposed of or transferred.

The following are NRC-requirements that may or may not apply

Equipment shall be provided to conduct a physical inventory of all material in storage at intervals not to exceed 12 months unless otherwise directed by the Commission. A copy of the current inventory shall be retained as a record until the Commission terminates the license.

Physical protection of the facility and materials shall be in accordance with 10 CFR 72 , Licensing Requirements for the Independent Storage of Spent Nuclear Fuel and High-Level Radioactive Waste, Subpart H (Physical Protection); 10 CFR 73, Physical Protection of Plants and Materials; and 10 CFR 1046, Physical Protection of Security Interests.

\subsubsection{Quality Assurance Requirements}


The applicable portions of DOE/RW-0333P, Quality Assurance Requirements and Description, shall be invoked as the baseline requirements document for developing and implementing quality assurance programs. These requirements apply to activities related to interim storage or disposal, including characterization for data collection, conditioning, or placing into a form for disposal. In addition, the EM-WAPS Rev. 01, Waste Acceptance Product Specifications for Vitrified High-Level Waste Forms, May 1995, also imposes a QA Program consistent with the QA requirements under the DOE/RW-0333P.

All purchased items will be restricted to those not suspect/counterfeit, misrepresented, used, or other than represented/advertised in accordance with INEL-95/227, "Guidelines for Identifying Suspect/Counterfeit Material."

Recörds, reports, and irispections shall be done in accordance with 10 CFR"72, Licensing Requirements for the Independent Storage of Spent Nuclear Fuel, Subpart D (Records, Reports, Inspections, and Enforcement).

Training of personnel shall be performed in accordance with 10 CFR 72, Licensing Requirements for the Independent Storage of Spent Nuclear Fuel, Subpart I (Training and Certification of Personnel). 


\subsection{References}

Nuclear Regulatory Commission (NRC) Licensing Assessment for the Idaho National Engineering and Environmental Laboratory (INEEL) High-Level Waste Program, prepared by R. G. Morgan and S. E. Leroy, Duke Engineering Services, Inc., April 23, 1997. Referenced in Idaho Chemical Processing Plant High Activity Waste Treatment Project Regulatory Assessment Report - SEL-11-97, prepared by S. E. Leroy, dated April 25, 1997. 


\section{Appendix F}

\section{Equipment List}


N-New CsiX/Grout Bldg, G - New Grout BIdg, NWCF - Existing Waste Calcining Facility, S - New Storage Bldg, n/a - not a part o

\begin{tabular}{|c|c|c|c|c|c|c|c|}
\hline \multirow{3}{*}{$\begin{array}{c}\text { Equip- } \\
\text { ment } \\
\text { Number }\end{array}$} & \multirow{3}{*}{$\begin{array}{c}\text { Equipment } \\
\text { Name }\end{array}$} & \multirow{2}{*}{\multicolumn{2}{|c|}{ Scenario }} & \multirow{3}{*}{\begin{tabular}{|c|}
$\begin{array}{c}\text { Equipment } \\
\text { Type }\end{array}$ \\
\end{tabular}} & \multirow{3}{*}{$\begin{array}{c}\text { Additional } \\
\text { Description }\end{array}$} & \multirow{3}{*}{$\begin{array}{c}\text { Main } \\
\text { System }\end{array}$} & \multirow{3}{*}{ Subs } \\
\hline & & & & & & & \\
\hline & & $\begin{array}{c}1 \\
\text { Bldg }\end{array}$ & $\begin{array}{c}2 \\
\text { Bldg }\end{array}$ & & & & \\
\hline \multicolumn{8}{|c|}{ Truck Unloading Station for New CsIX/Grout Bldg or New Grout Bldg } \\
\hline & Dust Control Mechanism & $\mathbf{N}$ & G & Filter & & Material storage & \\
\hline & Dust Control Mechanism & $\mathrm{N}$ & G & Filter & & Material storag & \\
\hline & Dust Control Mechanism & $\mathbf{N}$ & G & Filter & & Material storage & \\
\hline & Dust Control Mechanism & $\mathrm{N}$ & $G$ & Filter & & Material storage & \\
\hline & Air Enductor & N & G & Eductor & Venturi & Material storage & \\
\hline & Air Enductor & $\mathrm{N}$ & G & Eductor & Venturi & Material storag & \\
\hline & Air Enductor & N & G & Eductor & Venturi & Material storag & \\
\hline & Air Enductor & N & $\mathbf{G}$ & Eductor & Venturi & Material storag & \\
\hline \multicolumn{8}{|c|}{ Liquid Waste Feed Transfer Station } \\
\hline & Air Jet Pump & $\mathbf{N}$ & NWCF & Pump & & Waste feed & \\
\hline \multicolumn{8}{|c|}{ CsIX Removal Equipment in the New CxIS/Grout BIdg or NWCF } \\
\hline$T-101 A$ & Liquid Waste Feed Tank & $N$ & $n / a$ & Tank & Flat bottom & Waste feed & \\
\hline$T-101 B$ & Liquid Waste Feed Tank & $N$ & $\mathrm{n} / \mathrm{a}$ & Tank & Flat bottom & Waste feed & \\
\hline$T-101 A^{*}$ & Liquid Waste Feed Tank & $\mathrm{n} / \mathrm{a}$ & NWCF & Tank & Existing VES-NCC-102 & Waste feed & \\
\hline$T-101 B^{*}$ & Liquid Waste Feed Tank & $\mathrm{n} / \mathrm{a}$ & NWCF & Tank & Existing VES-NCC-102 & Waste feed & \\
\hline P-101A & Liquid Waste Feed Pump & $\mathbf{N}$ & NWCF & Pump & Metering & Waste feed & \\
\hline$P-101 B$ & Liquid Waste Feed Pump & $N$ & NWCF & Pump & Metering & Waste feed & \\
\hline$F-101 A$ & UDS Filter & $\mathbf{N}$ & NWCF & Filter & Pressure leaf & UDS & UDS filtr \\
\hline$F-101 B$ & UDS Filter & $N$ & NWCF & Filter & Pressure leaf & UDS & UDS filtr \\
\hline$T-108$ & UDS Slurry Tank & $N$ & NWCF & Tank & Flat botttom-air sparge & UDS & UDS dry \\
\hline$P-107$ & UDS Slurry Pump & $N$ & NWCF & Pump & Metering & UDS & UDS dry \\
\hline$E V-101$ & Rotary Evaporator & $N$ & NWCF & Evaporator & Wiped Film & UDS & UDS dry \\
\hline E-101 & UDS Drier Vapor Condenser & $\mathbf{N}$ & NWCF & Condenser & Shell \& tube & UDS & UDS dry \\
\hline P.110 & Condensate Pump & $N$ & na & Pump & Centrif, gravity for 2 & UDS & UDS dry \\
\hline B-101 & Cs Sorbant Bin & $N$ & NWCF & Bin & Cone bottom & lon exchange & Material \\
\hline ED-105 & Air Eductor & $\mathbf{N}$ & NWCF & Eductor & Venturi & lon exchange & Material \\
\hline$V-105$ & Resin Prep Tank & $N$ & $\mathrm{n} / \mathrm{a}$ & Tank & & lon exchange & Resin prd \\
\hline$V-105^{*}$ & Resin Prep Tank & $\mathrm{n} / \mathrm{a}$ & NWCF & Tank & Existing VES-NCM-120 & lon exchange & Resin pr \\
\hline P-114 & Resin Sluice Pump & $N$ & $n / a$ & Pump & High clearance diaphram & lon exchange & Resin pri \\
\hline$P-114^{*}$ & Resin Sluice Pump & $\mathrm{n} / \mathrm{a}$ & NWCF & Pump & Existing P-NCM-220 & Ion exchange & Resin prę \\
\hline$V-104 \mathrm{~A}$ & Cesium Ion Exchange Columns & $N$ & NWCF & Column & & lon exchange & CslX strit \\
\hline$V-104 B$ & Cesium Ion Exchange Columns & $N$ & NWCF & Column & & lon exchange & CslX strif \\
\hline$V-104 C$ & Cesium Ion Exchange Columns & $N$ & NWCF & Column & & lon exchange & CslX strit \\
\hline $\mathrm{F}-106 \mathrm{~A}$ & Resin Fines Filter & $N$ & NWCF & Filter & Cartridge & Ion exchange & CsIX strin \\
\hline$F-106 B$ & Resin Fines Filter & $N$ & NWCF & Filter & Cartridge & lon exchange & CslX strif \\
\hline$T-103 A$ & IX Effluent Hold Tank & $N$ & NWCF & Tank & Flat bottom & Ion exchange & CsiX strip \\
\hline$T-103 B$ & IX Effluent Hold Tank & $N$ & NWCF & Tank & Flat bottom & lon exchange & CsIX strip \\
\hline$P-103 A$ & CsIX Effluent Pump & $\mathrm{N}$ & NWCF & Pump & Centrifugal & Ion exchange & CslX strip \\
\hline$P-103 B$ & CsIX Effluent Pump & $\mathrm{N}$ & NWCF & Pump & Centrifugal & Ion exchange & CsiX strip \\
\hline P-112 & Spent CXIX Sorbant Pump & N & NWCF & Pump & High clearance diaphram & lon exchange & CsIX strip \\
\hline$V-106$ & Waste Resin Prep Column & $\mathbf{N}$ & NWCF & Column & & lon exchange & Spent res \\
\hline P-104A & Fines Recycle Pump & $N$ & NWCF & Pump & Centrifugal & lon exchange & Spent res \\
\hline P-104B & Fines Recycie Pump & $\mathbf{N}$ & NWCF & Pump & Centrifugal & Ion exchange & Spent res \\
\hline$F-102$ & Cs Sorbant Fines Screen & N & NWCF & Filter & Cartridge & Ion exchange & Spent res \\
\hline$D-102$ & Cs Sorbant Dryer & $N$ & NWCF & Dryer & Hollow flight & Ion exchange & Spent res \\
\hline$E-102$ & Vapor Condenser & $\mathbf{N}$ & NWCF & Condenser & Shell \& tube & lon exchange & Spent res \\
\hline
\end{tabular}

The Scenario \# Bldg columns indicate the building location of equipment for each scenario. 


\begin{tabular}{|c|c|c|c|c|c|c|c|c|c|}
\hline cated scen & & & & Size & & & & & \\
\hline $\begin{array}{l}\text { Flow Diag } \\
\text { Drawing }\end{array}$ & Ref & $\begin{array}{l}\text { Capacity } \\
\text { (cu. meter }\end{array}$ & Length & $\begin{array}{c}\text { Width or } \\
\text { Dia. }\end{array}$ & Height & Weight & $\begin{array}{c}\text { Electrical } \\
\text { Requirements }\end{array}$ & & Radiation \\
\hline Number & Dwg & or noted) & $(\mathrm{ft})$ & $(\mathrm{ft})$ & $(\mathrm{ft})$ & (lbs) & \begin{tabular}{l|l}
$(W)$ & $(\mathrm{HP})$ \\
\end{tabular} & Material & Field \\
\hline
\end{tabular}

2
2
2
2
2
2
2
2

\section{3}

3

3

3

3

3

4

4

4

4

4

4

4

2

2

6

6

6

6

5

5
5

5

5

5

5

5

5

5

5

5

6

6

6

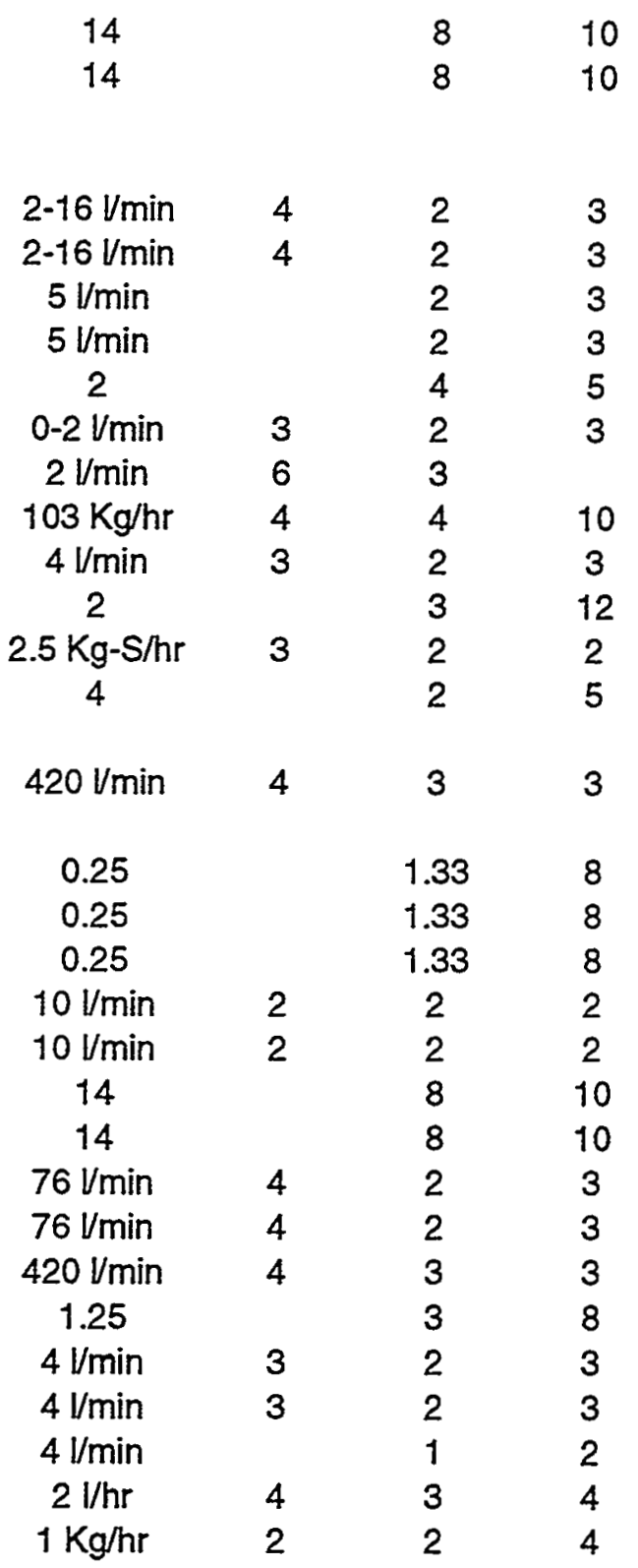

SST

SST

SST

SST

SST

SST

SST

SST

SST

SST

SST

SST

Carbon ste€ None

SST

SST

SST

SST

SST

SST

SST

SST

SST

SST

SST

SST

SST

SST

SST

SST

SST

SST 
INTEC Liquid Waste CsIX

N - New CsIXJGrout Bldg, G - New Grout Bldg, NWCF - Existing Waste Calcining Facility, S - New Storage Bldg, n/a - not a part of

\begin{tabular}{|c|c|c|c|c|c|c|c|}
\hline \multirow{2}{*}{$\begin{array}{c}\text { Equip- } \\
\text { ment } \\
\text { Number }\end{array}$} & \multirow[b]{2}{*}{$\begin{array}{c}\text { Equipment } \\
\text { Name }\end{array}$} & \multicolumn{2}{|c|}{ Scenario } & \multirow[b]{2}{*}{$\begin{array}{c}\text { Equipment } \\
\text { Type }\end{array}$} & \multirow[b]{2}{*}{$\begin{array}{c}\text { Additional } \\
\text { Description }\end{array}$} & \multirow[b]{2}{*}{$\begin{array}{c}\text { Main } \\
\text { System } \\
\end{array}$} & \multirow[b]{2}{*}{ Subsy } \\
\hline & & $\begin{array}{c}1 \\
\text { Bldg }\end{array}$ & $\begin{array}{c}2 \\
\text { Bldg }\end{array}$ & & & & \\
\hline$P-113$ & Sorbant Drier Condensate Pump & $N$ & n/a & Pump & Centrif, gravity for 2 & lon exchange & Spent re \\
\hline$T-112$ & Spent Resin Storage Tank & $N$ & NWCF & Tank & Cone bottom & lon exchange & Spent $r$ \\
\hline \multicolumn{8}{|c|}{ UDS Loading Cell in New CxIS/Grout Bldg or NWCF } \\
\hline & Staging Conveyor & $\mathrm{N}$ & NWCF & Conveyor & Chain driven & UDS & UDS I \\
\hline & Decon Booth & $N$ & NWCF & Booth & Shielded, w/window & UDS & UDS \\
\hline & Decon Booth Door & $N$ & NWCF & Door & Shielded & UDS & UDS L \\
\hline & Decon Booth Door & $\mathbf{N}$ & NWCF & Door & Shielded & UDS & UDS ! \\
\hline & Rotation Table & $N$ & NWCF & Mechanism & & UDS & UDS \\
\hline & Decon Conveyor & $N$ & NWCF & Conveyor & & UDS & UDS L \\
\hline & Decon Equipment & N & NWCF & Mechanism & & UDS & UDS L \\
\hline & Inspection Equipment & $N$ & NWCF & Mechanism & Area swipe remote mech & UDS & UDS L \\
\hline & Cell Door & N & NWCF & Door & Electric motor driven & UDS & UDS L \\
\hline & Load Out Conveyor & $N$ & NWCF & Conveyor & Self powered rollers & UDS & UDS L \\
\hline & Lift Table & $N$ & NWCF & Lift & Air operated & UDS & UDS L \\
\hline & Lift Table Conveyor & $N$ & NWCF & Conveyor & & UDS & UDS L \\
\hline & Drum Dust Tight Discharge Cone & $N$ & NWCF & Mechanism & Air operated & UDS & UDS L \\
\hline & Shielded Drum & $N$ & NWCF & Drum & w/dust tight inlet cone & UDS & UDS 1 \\
\hline \multicolumn{8}{|c|}{ Grouting Equipment in New CxIS/Grout Bldg or New Grout Bldg } \\
\hline B-102 & CaO Bin & $N$ & G & Bin & Cone bottom & Grouting & Material \\
\hline$B-103$ & Portland Cement Bin & $\mathbf{N}$ & $\therefore \mathbf{G}$ & Bin & Cone bottom & Grouting & Material \\
\hline$B-104$ & Flyash Bin & $\mathbf{N}$ & $\mathbf{G}$ & Bin & Cone bottom & Grouting & Material \\
\hline$B-105$ & Blast Furnace Slag Bin & $\mathbf{N}$ & $\mathbf{G}$ & Bin & Cone bottom & Grouting & Material \\
\hline ED-101 & Air Eductor & $\mathbf{N}$ & $\mathbf{G}$ & Eductor & Venturi & Grouting & Material \\
\hline ED-102 & Air Eductor & $N$ & $\mathbf{G}$ & Eductor & Venturi & Grouting & Materie \\
\hline ED-103 & Air Eductor & $\mathbf{N}$ & $\mathbf{G}$ & Eductor & Venturi & Grouting & Material \\
\hline ED-104 & Air Eductor & $\mathbf{N}$ & $\mathbf{G}$ & Eductor & Venturi & Grouting & Material \\
\hline B-107 & CaO Weight Bin & $N$ & $\mathbf{G}$ & Bin & Cone bottom & Grouting & Grout \\
\hline$T-104 A$ & Grout Feed Tank & N & $\mathbf{G}$ & Tank & Flat bottom & Grouting & Grout \\
\hline$T-104 B$ & Grout Feed Tank & $\mathbf{N}$ & $\mathbf{G}$ & Tank & Flat bottom & Grouting & Grout \\
\hline$N-101 A$ & ph Sampler/Neutralizer & $N$ & $\mathbf{G}$ & Neutralizer & Auto batch & Grouting & Grout \\
\hline$N-101 B$ & ph Sampler/Neutralizer & $N$ & $\mathbf{G}$ & Neutralizer & Auto batch & Grouting & Grout \\
\hline$P-105 A$ & Grout Mixer Feed Pump & $\mathbf{N}$ & $\mathbf{G}$ & Pump & Metering & Grouting & Grout \\
\hline$P-105 B$ & Grout Mixer Feed Pump & $N$ & $\mathbf{G}$ & Pump & Metering & Grouting & Grout \\
\hline$F-106$ & Knockout Filter & N & $\mathbf{G}$ & Filter & & Grouting & Grout \\
\hline$B-108 A$ & Dry Ingredient Weigh Bin & N & $\mathbf{G}$ & Bin & Cone bottom & Grouting & Grout \\
\hline$B-108 B$ & Dry Ingredient Weigh Bin & $N$ & $\mathbf{G}$ & Bin & Cone bottom & Grouting & Grout \\
\hline$B-108 C$ & Dry Ingredient Weigh Bin & $N$ & $\mathbf{G}$ & Bin & Cone bottom & Grouting & Grout \\
\hline C-101A & Solids Feed Conveyor & $N$ & $\mathbf{G}$ & Conveyor & Vibratory & Grouting & Grout ! \\
\hline C-101B & Solids Feed Conveyor & $N$ & $\mathbf{G}$ & Conveyor & Vibratory & Grouting & Grout \\
\hline$C-101 C$ & Solids Feed Conveyor & $\mathbf{N}$ & $\mathbf{G}$ & Conveyor & Vibratory & Grouting & Grout : \\
\hline$M-101 A$ & Grout Mixer & $\mathbf{N}$ & $\mathbf{G}$ & Mixer & Horizontal helical & Grouting & Grout r \\
\hline$M-101 B$ & Grout Mixer & $\mathbf{N}$ & $\mathbf{G}$ & Mixer & Horizontal helical & Grouting & Grout $r$ \\
\hline$M-101 C$ & Grout Mixer & $\mathbf{N}$ & $\mathbf{G}$ & Mixer & Horizontal helical & Grouting & Grout mi \\
\hline$B-106 A$ & Dry Grout Admixtures Bin & $\mathbf{N}$ & $\mathbf{G}$ & Bin & Cone bottom & Grouting & Material \\
\hline$B-106 B$ & Liquid Grout Admixtures Tank & $\mathbf{N}$ & $\mathbf{G}$ & Tank & & Grouting & Materia \\
\hline$B-106 C$ & Liquid Grout Admixtures Tank & $\mathbf{N}$ & $\mathbf{G}$ & Tank & & Grouting & Materie \\
\hline$P-106$ & Decon Agent Pump & $\mathbf{N}$ & $\mathbf{G}$ & Pump & Centrifugal-HP & Grouting & Decon \\
\hline
\end{tabular}

The Scenario \# Bldg columns indicate the building location of equipment for each scenario. 
icated scenario. Flow Diag

Drawing Number

Ref
Dwg

\begin{tabular}{|c|c|c|}
\hline $\begin{array}{c}\text { Capacity } \\
\text { (cu. meter } \\
\text { or noted) }\end{array}$ & $\begin{array}{c}\text { Length } \\
(\mathrm{ft})\end{array}$ & Widt \\
\hline $\begin{array}{c}4 \mathrm{~V} / \mathrm{min} \\
15\end{array}$ & 3 & \\
\end{tabular}

Size

6

6

4

3

1.00

4

idith or

Dia. Height

Weight

(ft) (ft)
(lbs)

$\begin{array}{cc}2 & 3 \\ 8 & 15\end{array}$

Electrical
Requirements

Material

Radiation

SST Field

(HP)

Field

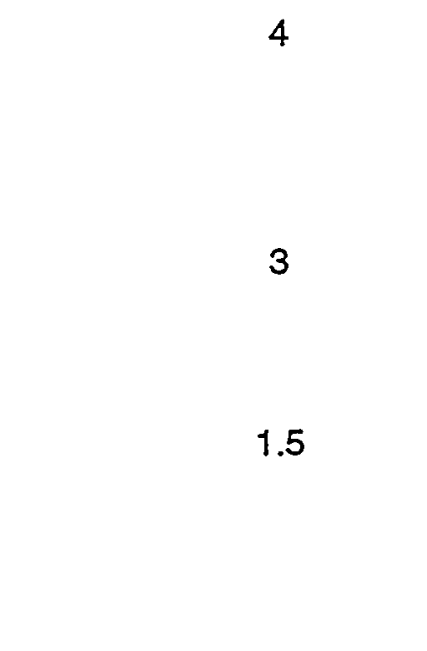

3

3

1,200

1,200

300

3

$\begin{array}{ccc}300 & & \\ 600 & 0.50 & \text { SST } \\ 1,200 & S S T \\ & S S T \\ & S S T \\ & S S T \\ & \text { SST }\end{array}$

0.50 SST

0.50 SST

0.50 SST

1.00 SST

SST

7 ga. SST

SST

ST

$\begin{array}{lllll}38 & 10 & 25 & \text { Carbon ste } & \text { None } \\ 34 & 10 & 23 & \text { Carbon ste€ } & \text { None } \\ 34 & 10 & 23 & \text { Carbon ste€ } & \text { None } \\ 58 & 12 & 26 & \text { Carbon ste€ } & \text { None }\end{array}$

$2-16 V / \min \quad 4 \quad 2 \quad 3$

SST

SST

SST

SST

SST

SST

SST

Carbon steel

Carbon steel

Carbon steel

SST

SST

SST

SST

SST

SST

Carbon stee None

FW Plastic

FW Plastic

SST 
N-NeW CsIX/Grout Bldg, G - New Grout Bldg, NWCF - Existing Waste Calcining Facility, S - New Storage Bldg, n/a - not a part

\begin{tabular}{|c|c|c|c|c|c|c|c|}
\hline \multirow{2}{*}{$\begin{array}{c}\text { Equip- } \\
\text { ment } \\
\text { Number }\end{array}$} & \multirow[b]{2}{*}{$\begin{array}{l}\text { Equipment } \\
\text { Name }\end{array}$} & \multicolumn{2}{|c|}{ Scenario } & \multirow[b]{2}{*}{$\begin{array}{c}\text { Equipment } \\
\text { Type }\end{array}$} & \multirow[b]{2}{*}{$\begin{array}{l}\text { Additional } \\
\text { Description }\end{array}$} & \multirow[b]{2}{*}{$\begin{array}{c}\text { Main } \\
\text { System }\end{array}$} & \multirow[b]{2}{*}{ Sub: } \\
\hline & & $\begin{array}{c}1 \\
\text { Bidg }\end{array}$ & $\begin{array}{c}2 \\
\text { Bldg }\end{array}$ & & & & \\
\hline$P-115$ & Metering Pump/Admixtures & $\mathrm{N}$ & $\mathbf{G}$ & Pump & Metering & Grouting & Grout \\
\hline$T-106$ & Mixer Wash Tank & $N$ & G & Tank & Flat bottom & Grouting & Decon \\
\hline P-116 & Decon Return Pump & $N$ & $\mathbf{G}$ & Pump & Centrifugal & Grouting & Decon \\
\hline$F-105$ & Spent Decon Solution Filter & N & $\mathbf{G}$ & Filter & Cartridge & Grouting & Decon \\
\hline \multicolumn{8}{|c|}{ Grouted Waste Drum Fill/Drum Seal Area } \\
\hline & Storage Area Conveyor & N & $\mathbf{G}$ & Conveyor & & Grouting & Drum i \\
\hline & SST Airlock Enclosure & N & $\mathbf{G}$ & Booth & & Grouting & Drum $f$ \\
\hline & Airlock Door & $\mathrm{N}$ & $\mathbf{G}$ & Door & Electric motor driven & Grouting & Drum $f$ \\
\hline & Airlock Door & N & $\mathbf{G}$ & Door & Electric motor driven & Grouting & Drum $f$ \\
\hline & Airlock Conveyor & N & $G$ & Conveyor & & Grouting & Drum $f$ \\
\hline & Main Inlet Conveyor & N & $G$ & Conveyor & Self powered rollers & Grouting & Drum $\mathrm{f}$ \\
\hline & Transverse Section Lifts & N & $\mathbf{G}$ & Liff & \multicolumn{2}{|c|}{ Belt driven w/ifting mech. Grouting } & Drum $f$ \\
\hline & Transverse Section Lifts & N & $G$ & Lift & \multicolumn{2}{|c|}{ Belt driven w/ifting mech. Grouting } & Drum $\mathrm{f}$ \\
\hline & Mixer Booth Inlet Conveyor & N & 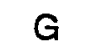 & Conveyor & Self powered rollers & Grouting & Drum $\mathrm{f}$ \\
\hline & Mixer Booth & $N$ & $G$ & Booth & & Grouting & Drum fi: \\
\hline & Mixer Booth Conveyor & N & $\mathbf{G}$ & Conveyor & Self powered rollers & Grouting & Drum fi \\
\hline & Fill Assembly & N & $G$ & Mechanism & Telescoping fill tube & Grouting & Drum fi \\
\hline & Lid Placement Booth & N & $G$ & Booth & & Grouting & Drum fi \\
\hline & Lid Placement Exit Door & $N$ & $\mathbf{G}$ & Door & Electric motor driven & Grouting & Drum fi \\
\hline & Lid Placement Inlet Conveyor & $N$ & $G$ & Conveyor & Self powered rollers & Grouting & Drum fi \\
\hline & Lid Placement Exit Conveyor & $N$ & $\mathbf{G}$ & Conveyor & Self powered rollers & Grouting & Drum fi \\
\hline & Lid Placement Conveyor & $\mathbf{N}$ & $\mathbf{G}$ & Conveyor & Belt driven w/lifting mech. & Grouting & Drum fi \\
\hline & Rotatiing Table & $N$ & $\mathbf{G}$ & Mechanism & Electric motor driven & Grouting & Drum fi \\
\hline & Drum Rim Cleaning Mechanicsm & $N$ & $\mathbf{G}$ & Mechanism & Remote arm w/wiper & Grouting & Drum fi \\
\hline & Lid Placement Assembly & $\mathbf{N}$ & $\mathbf{G}$ & Mechanism & & Grouting & Drum fi \\
\hline & Transfer Section Tunnel & $N$ & $\mathbf{G}$ & Booth & Glovebox & Grouting & Drum fi \\
\hline & Transfer Section Inlet Conveyor & $N$ & $G$ & Conveyor & Self powered rollers & Grouting & Drum fi \\
\hline & Transfer Section Inlet Conveyor & $\mathbf{N}$ & $\mathbf{G}$ & Conveyor & Self powered rollers & Grouting & Drum fi \\
\hline & Transfer Section Inlet Conveyor & $N$ & $\mathbf{G}$ & Conveyor & Self powered rollers & Grouting & Drum fi \\
\hline & Transfer Section Exit Converyor & $\mathbf{N}$ & $\mathbf{G}$ & Conveyor & Self powered rollers & Grouting & Drum fi \\
\hline & Transfer Section Exit Converyor & $\mathbf{N}$ & $\mathbf{G}$ & Conveyor & Self powered rollers & Grouting & Drum fi \\
\hline & Transfer Section Exit Converyor & $N$ & $\mathbf{G}$ & Conveyor & Self powered rollers & Grouting & Drum fi \\
\hline & Transfer Table & N & $\mathbf{G}$ & Lift & Belt driven w/litting mech. & Grouting & Drum fi \\
\hline & Transfer Table & $N$ & $\mathbf{G}$ & Lift & Beft driven w/lifting mech. & Grouting & Drum fi \\
\hline & Transfer Table & $\mathbf{N}$ & $\mathbf{G}$ & Lift & Belt driven w/lifting mech. & Grouting & Drum fi \\
\hline & Transverse Conveyor & N & $\mathbf{G}$ & Conveyor & Self powered rollers & Grouting & Drum fi \\
\hline & Transverse Conveyor & N & $\mathbf{G}$ & Conveyor & Self powered rollers & Grouting & Drum fi \\
\hline & Inspection/Decon Booth & N & $\mathbf{G}$ & Booth & & Grouting & Drum fi \\
\hline & Booth Door & N & $\mathbf{G}$ & Door & & Grouting & Drum fi \\
\hline & Booth Door & $N$ & $\mathbf{G}$ & Door & & Grouting & Drum fi \\
\hline & Inspect/Decon Inlet Conveyor & N & $\mathbf{G}$ & Conveyor & Self powered rollers & Grouting & Drum fil \\
\hline & Inspect/Decon Exit Conveyor & N & $\mathbf{G}$ & Conveyor & Self powered rollers & Grouting & Drum fil \\
\hline & Inspect/Decon Conveyor & N & $\mathbf{G}$ & Conveyor & Belt driven w/lifting mech. & Grouting & Drum fil \\
\hline & Rotatiing Table & N & $\mathbf{G}$ & Mechanism & Electric motor driven & Grouting & Drum fil \\
\hline & Decon Equipment & N & $\mathbf{G}$ & Mechanism & w/solution collection & Grouting & Drum fi \\
\hline & Inspection Equipment & $N$ & $\mathbf{G}$ & Mechanism & Automatic swipe & Grouting & Drum fil \\
\hline & Discharge Section Tunnel & $N$ & $\mathbf{G}$ & Booth & Glovebox & Grouting & Drum fil \\
\hline
\end{tabular}

The Scenario \# Bldg columns indicate the building location of equipment for each scenario. 
\begin{tabular}{r|r|} 
ndicated scenario. \\
Flow Diag
\end{tabular}

Drawing

tem

Number

$n$
Ref
Dwg

\begin{tabular}{c|c|c|}
$\begin{array}{c}\text { Capacity } \\
\text { (cu. meter } \\
\text { or noted) }\end{array}$ & $\begin{array}{c}\text { Length } \\
\text { (ft) }\end{array}$ & Widt \\
\hline
\end{tabular}

Size

\begin{tabular}{|l|l|l|l|}
$\begin{array}{l}\text { Width or } \\
\text { Dia. }\end{array}$ & $\begin{array}{c}\text { Height } \\
\text { (ft) }\end{array}$ & Weight & $\begin{array}{c}\text { Electrical } \\
\text { Requirements }\end{array}$ \\
\cline { 3 - 4 } & &
\end{tabular}

$1 \mathrm{l} / \mathrm{min}$

1

$76 \mathrm{l} / \mathrm{min}$

$76 \mathrm{l} / \mathrm{min}$

2

3

5

42

3

(HP)

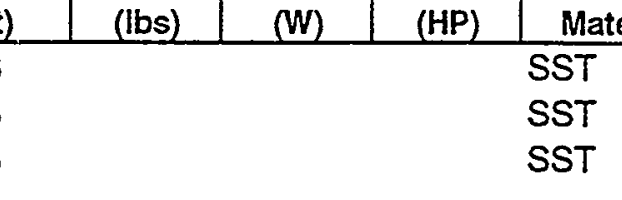

Radiation

Field

$3 \quad 1,200$

63

8

8

8

8

8

8

8

8

8

8

8

8

8

8

8

8

8

8

8

8

8

8

8

8

8

8

8

8

8

8

9

9

9

9

9

9

9

9

$1.5 \quad 3$

1.53

33

600

600

2.00

1.00

0.25

2.00

SST

7 ga. SST

1,200

1,200

1,200

1,200

1,200

1,200

0.50 SST

0.50 SST

1.50

1.50

0.50

0.50 SST

7 ga. SST

7 ga. SST

$3 \quad 3$

33

33

33

33

3

3

3

$5.5 \quad 3$

5.53

1,000

31,000

\subsection{SST}

3.50 SST

3.50 SST

1.53

1.53

33

3

600

600

$0.50 \quad S S T$

0.50 SST

1,200

3,000

7 ga. SST 
INTEC Liquid Waste CsI)

N-New CsIX/Grout BIdg, G - New Grout Bldg, NWCF - Existing Waste Calcining Facility, S - New Storage Bldg, na - not a part of

\begin{tabular}{|c|c|c|c|c|c|c|c|}
\hline \multirow{2}{*}{$\begin{array}{c}\text { Equip- } \\
\text { ment } \\
\text { Number }\end{array}$} & \multirow[b]{2}{*}{$\begin{array}{l}\text { Equipment } \\
\text { Name }\end{array}$} & \multicolumn{2}{|c|}{ Scenario } & \multirow[b]{2}{*}{$\begin{array}{c}\text { Equipment } \\
\text { Type }\end{array}$} & \multirow[b]{2}{*}{$\begin{array}{l}\text { Additional } \\
\text { Description }\end{array}$} & \multirow[b]{2}{*}{$\begin{array}{c}\text { Main } \\
\text { System }\end{array}$} & \multirow[b]{2}{*}{ Subs } \\
\hline & & $\begin{array}{c}1 \\
\text { Bldg }\end{array}$ & $\begin{array}{c}2 \\
\text { Bldg }\end{array}$ & & & & \\
\hline & Discharge Section Inlet Conveyor & $\mathrm{N}$ & $\vec{G}$ & Conveyor & Self powered rollers & Grouting & Drum fil \\
\hline & Discharge Section Inlet Conveyor & N & G & Conveyor & Self powered rollers & Grouting & Drum fil \\
\hline & Discharge Section Inlet Conveyor & $N$ & $G$ & Conveyor & Self powered rollers & Grouting & Drum fil \\
\hline & Main Discharge Conveyor & $N$ & $G$ & Conveyor & Self powered rollers & Grouting & Drum fil \\
\hline & Transfer Section Lifts & $N$ & $\mathbf{G}$ & Lift & Belt driven w/lifting mech. & Grouting & Drum fil \\
\hline & Transfer Section Lifts & N & $\mathbf{G}$ & Lift & Belt driven w/lifting mech. & Grouting & Drum fil \\
\hline & Transfer Section Lifts & N & G & Lift & Belt driven w/ifting mech. & Grouting & Drum \\
\hline & SST Airlock Enclosure & $N$ & $\mathbf{G}$ & Booth & & Grouting & Drum fil \\
\hline & Airlock Door & $N$ & $\mathbf{G}$ & Door & Electric motor driven & Grouting & Drum fil \\
\hline & Airlock Door & $N$ & $\mathbf{G}$ & Door & Electric motor driven & Grouting & Drum \\
\hline & Airlock Conveyor & $N$ & G & Conveyor & Self powered rollers & Grouting & Drum \\
\hline & Tilt \& Pan Cameras (14 required) & $\mathbf{N}$ & G & Camera & w/zoom capability & Grouting & Drum \\
\hline \multicolumn{8}{|c|}{ Grout Curing Room } \\
\hline & Inlet Staging Enclosure & $N$ & G & Booth & w/windows \& access & Grouting & Curing \\
\hline & Inlet Staging Conveyor & $N$ & $\mathbf{G}$ & Conveyor & Selfpowered rollers & Grouting & Curing \\
\hline & Drum Lift Enclosure & $N$ & $\mathbf{G}$ & Booth & w/windows \& access & Grouting & Curing \\
\hline & Drum Lift & $\mathbf{N}$ & $\mathbf{G}$ & Lift & $4 \mathrm{ft} \mathrm{lift}$ & Grouting & Curing \\
\hline & Drum Lift Conveyor & $N$ & $\mathbf{G}$ & Conveyor & Selfpowered rollers & Grouting & Curing \\
\hline & Cure Line Enclosure & $\mathbf{N}$ & $\mathbf{G}$ & Booth & whwindows \& access & Grouting & Curing \\
\hline & Cure Line Conveyor & $N$ & $\mathbf{G}$ & Conveyor & Selfpowered rollers & Grouting & Curing \\
\hline & Cure Line Conveyor & $\mathbf{N}$ & $\mathbf{G}$ & Conveyor & Selfpowered rollers & Grouting & Curing \\
\hline & 180 Degree Conveyor & $\mathbf{N}$ & $\mathbf{G}$ & Conveyor & Chain driven & Grouting & Curing \\
\hline & 180 Degree Conveyor & $N$ & $\mathbf{G}$ & Conveyor & Chain driven & Grouting & Curing \\
\hline & 180 Degree Conveyor & $N$ & $\mathbf{G}$ & Conveyor & Chain driven & Grouting & Curing \\
\hline & 180 Degree Conveyor & $\mathbf{N}$ & $\mathbf{G}$ & Conveyor & Chain driven & Grouting & Curing \\
\hline & 180 Degree Conveyor & $N$ & $\mathbf{G}$ & Conveyor & Chain driven & Grouting & Curing \\
\hline & 180 Degree Conveyor & $\mathbf{N}$ & G & Conveyor & Chain driven & Grouting & Curing \\
\hline & 180 Degree Conveyor & $N$ & $\mathbf{G}$ & Conveyor & Chain driven & Grouting & Curing \\
\hline & 180 Degree Conveyor & $N$ & G & Conveyor & Chain driven & Grouting & Curing \\
\hline & Cure Line Conveyor & $N$ & $\mathbf{G}$ & Conveyor & Chain driven & Grouting & Curing \\
\hline & Cure Line Conveyor & $N$ & $\mathrm{G}$ & Conveyor & Chain driven & Grouting & Curing \\
\hline & Cure Line Conveyor & $\mathbf{N}$ & $\mathbf{G}$ & Conveyor & Chain driven & Grouting & Curing \\
\hline & Cure Line Conveyor & $\mathbf{N}$ & $\mathbf{G}$ & Conveyor & Chain driven & Grouting & Curing \\
\hline & Cure Line Conveyor & $N$ & G & Conveyor & Chain driven & Grouting & Curing \\
\hline & Cure Line Conveyor & $N$ & $G$ & Conveyor & Chain driven & Grouting & Curing \\
\hline & Cure Line Conveyor & $\mathbf{N}$ & $\mathbf{G}$ & Conveyor & Chain driven & Grouting & Curing \\
\hline & Cure Line Conveyor & $N$ & $\mathbf{G}$ & Conveyor & Chain driven & Grouting & Curing \\
\hline & Drum Lift Enclosure & $N$ & $\mathbf{G}$ & Booth & w/windows \& access & Grouting & Curing \\
\hline & Drum Lift & $N$ & $\mathbf{G}$ & Lift & $4 \mathrm{ft} \mathrm{lift}$ & Grouting & Curing \\
\hline & Drum Lift Conveyor & $N$ & G & Conveyor & Selfpowered rollers & Grouting & Curing \\
\hline & Staging Conveyor & $\mathbf{N}$ & $\mathbf{G}$ & Conveyor & Selfpowered rollers & Grouting & Curing \\
\hline & Drum Elevator Enclosure & $\mathbf{N}$ & $\mathbf{G}$ & Booth & w/windows \& access & Grouting & Curing \\
\hline & Drum Elevator Door & $\mathbf{N}$ & $\mathbf{G}$ & Door & Electric motor driven & Grouting & Curing \\
\hline & Drum Elevator to 2nd Floor & $\mathbf{N}$ & $\mathbf{G}$ & Lift & $12 \mathrm{ft} \mathrm{lift}$ & Grouting & Curing \\
\hline \multicolumn{8}{|c|}{ Grout Dewatering Room } \\
\hline & Inlet Indexing Lift Conveyor & $\mathbf{N}$ & G & Conveyor & Self powered rollers & Grouting & Dewe \\
\hline & Indexing Lift Table & $\mathbf{N}$ & G & Lift & & Grouting & Dewa \\
\hline
\end{tabular}

The Scenario \# Bldg columns indicate the building location of equipment for each scenario. 
dicated scenario.

Flow Diag

m

Drawing Ref

Number Dwg

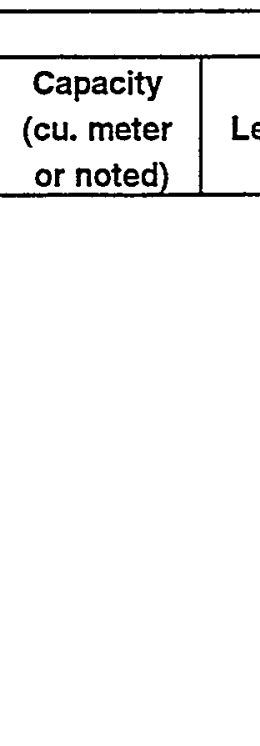

5

\section{3}

10

10

10

10

10

10

10

10

10

10

10

10

10

10

10

10

10

10

10

10

10

10

10

10

10

10

10

10

10

10

10

3

33

33

33

(

(1)

(2)

6

6

33

63

9

9

9

9

9

9

133

133

133

133

133

$13 \quad 3$

$13 \quad 3$

133

33

33

1.53

33

3

3

\begin{tabular}{|c|c|c|c|}
\hline \multicolumn{4}{|c|}{ Size } \\
\hline & Width or & & \\
\hline $\begin{array}{l}\text { ength } \\
\text { (ft) }\end{array}$ & $\begin{array}{l}\text { Dia. } \\
\text { (ft) }\end{array}$ & $\begin{array}{l}\text { Height } \\
\text { (ft) }\end{array}$ & $\begin{array}{l}\text { Weight } \\
\text { (lbs) }\end{array}$ \\
\hline
\end{tabular}

Electrical

Requirements

(W) $1 \mathrm{HP})$

1,200

1,200

1,200

9,000

3

1,200

1,800

5,600

33

63
2.50

2.50

2.50

7 ga SST

0.50 SST

0.50 SST

$1,200 \quad$ SST

7 ga. SST

1,200 SST

1,200

SST

2.00 SST

2.00 SST

2.00 SST

2.00 SST

2.00 SST

2.00 SST

2.00 SST

2.00 SST

1.00 SST

1.00 SST

1.00 SST

1.00 SST

1.00 SST

1.00 SST

1.00 SST

1.00 SST

7 ga. SST

1.50 SST

$1,200 \quad$ SST

400

SST

7 ga. SST

0.50 SST

2.00 SST

1,200

SST

2.00 SST 
N - New CsIX/Grout Bldg, G - New Grout Bldg, NWCF - Existing Waste Calcining Facility, S - New Storage Bldg, n/a - not a part

\begin{tabular}{|c|c|c|c|c|c|c|c|}
\hline \multirow{3}{*}{$\begin{array}{c}\text { Equip- } \\
\text { ment } \\
\text { Number }\end{array}$} & \multirow{3}{*}{$\begin{array}{c}\text { Equipment } \\
\text { Name } \\
\end{array}$} & \multicolumn{2}{|c|}{ Scenario } & \multirow{3}{*}{$\begin{array}{l}\text { Equipment } \\
\text { Type }\end{array}$} & \multirow{3}{*}{$\begin{array}{l}\text { Additional } \\
\text { Description }\end{array}$} & \multirow{3}{*}{$\begin{array}{l}\text { Main } \\
\text { System }\end{array}$} & \multirow[b]{3}{*}{ Sub } \\
\hline & & 1 & 2 & & & & \\
\hline & & Bldg & Bldg & & & & \\
\hline & Indexing Arm & $N$ & $G$ & Mechanism & Roller assembly arem & Grouting & Dewa \\
\hline & Dewatering Station Conveyor & $N$ & $\mathbf{G}$ & Conveyor & Self powered rollers & Grouting & Dewa \\
\hline & 90 deg Transfer and Lift & $\mathrm{N}$ & $G$ & Lift & Chain transfer & Grouting & Dewa \\
\hline & Dewatering Station Conveyor & $N$ & $\mathbf{G}$ & Conveyor & Self powered rollers & Grouting & Dewa \\
\hline & Air Heaters ( 9 ea.) & $N$ & $\mathbf{G}$ & Heater & & Grouting & Dewal \\
\hline & Dewatering Station Line Lift & $N$ & $G$ & Lift & & Grouting & Dewal \\
\hline & Dewatering Station Line Lift & $\mathrm{N}$ & G & Lift & & Grouting & Dewal \\
\hline & Dewatering Station Line Lift & $N$ & $G$ & Lift & & Grouting & Dewat \\
\hline & Dewatering Station Line Lift & $N$ & $\mathbf{G}$ & Lift & & Grouting & Dewat \\
\hline & Dewatering Station Line Lift & $N$ & $\mathbf{G}$ & Liff & & Grouting & Dewat \\
\hline & Dewatering Station Line Lift & N & $\mathbf{G}$ & Lift & & Grouting & Dewat \\
\hline & Dewatering Station Line Lift & $N$ & G & Lift & & Grouting & Dewat \\
\hline & Dewatering Station Line Lift & $\mathbf{N}$ & $\mathbf{G}$ & Lift & & Grouting & Dewat \\
\hline & Dewatering Station Line Lift & $N$ & $\mathbf{G}$ & Lift & & Grouting & Dewat \\
\hline & Drum Off Load Conveyor & $N$ & G & Conveyor & Self powered rollers & Grouting & Dewat \\
\hline & 90 deg Transfer and Lift & $\mathrm{N}$ & $\mathbf{G}$ & Lift & Chain transfer & Grouting & Dewat \\
\hline & Hydraulic Drum Lift & N & $\mathbf{G}$ & Lift & & Grouting & Dewat \\
\hline$E-104$ & Vapor Condenser & N & $\mathbf{G}$ & Condenser & Shell \& tube & Grouting & Dewat. \\
\hline$P-118$ & Condensate Pump & $N$ & $\mathbf{G}$ & Pump & Centrifugal & Grouting & Dewat \\
\hline \multicolumn{8}{|c|}{ Grout Recycle Station } \\
\hline & Oven & $\mathbf{N}$ & $\mathbf{G}$ & Oven & Moderate Temperature & Grouting & Grout I \\
\hline$E-105$ & Vapor Condenser & $N$ & $\mathbf{G}$ & Condenser & Shell \& tube & Grouting & Grout \\
\hline \multicolumn{8}{|c|}{ Decontamination/Waste Water Equipment } \\
\hline$T-102$ & DI Water Feed Tank & N & $\mathbf{G}$ & Tank & Flat bottom & Decon/Wash & \\
\hline$T-109$ & Decontamination Agent Acid & $\mathbf{N}$ & n/a & Tank & & Decon/Wash & \\
\hline$T-109^{*}$ & Decontamination Agent Acid & $\mathrm{n} / \mathrm{a}$ & NWCF & Tank & Existing VES-NCM-117 & Decon/Wash & \\
\hline$T-110$ & Decontamination Agent Base & $\mathbf{N}$ & $\mathrm{n} / \mathrm{a}$ & Tank & & Decon/Wash & \\
\hline$T-110^{*}$ & Decontamination Agent Acid & $\mathrm{n} / \mathrm{a}$ & NWCF & Tank & Existing VES-NCM-118 & Decon/Wash & \\
\hline$P-102 A$ & DI Water Pump & $\mathbf{N}$ & $\mathbf{G}$ & Pump & Centrifugal & Decon/Wash & \\
\hline$P-102 B$ & DI Water Pump & N & $\mathbf{G}$ & Pump & Centrifugal & Decon/Wash & \\
\hline$P-108$ & Acid Decon Pump & $\mathbf{N}$ & $\mathbf{G}$ & Pump & Centrifugal & Decon/Wash & \\
\hline$P-108^{*}$ & Acid Decon Pump & $\mathrm{n} / \mathrm{a}$ & NWCF & Pump & Existing P-NCM-217 & Decon/Wash & \\
\hline P-109 & Base Decon Pump & $\mathbf{N}$ & $\mathbf{G}$ & Pump & Centrifugal & Decon/Wash & \\
\hline P-109* & Base Decon Pump & na & NWCF & Pump & Existing P-NCM-218 & Decon/Wash & \\
\hline$T-111$ & Waste Water Tank & $\mathrm{N}$ & G & Tank & Horizontal cylindrical & Waste water & Waste \\
\hline$T-111^{*}$ & Waste Water Tank & $\mathrm{n} / \mathrm{a}$ & NWCF & Tank & Existing VES-NCC-119 & Waste water & Waste \\
\hline$T-111^{*}$ & Waste Water Tank & $\mathrm{n} / \mathrm{a}$ & NWCF & Tank & Existing VES-NCC-122 & Waste water & Waste \\
\hline$P-111 A$ & Waste Water Pump & $\mathbf{N}$ & $\mathbf{G}$ & Pump & Centrifugal & Waste water & \\
\hline$P-111 B$ & Waste Water Pump & $N$ & $\mathbf{G}$ & Pump & Centrifugal & Waste water & \\
\hline$P-111^{*}$ & Waste Water Pump & $n / a$ & NWCF & Pump & Existing JET-NCC-519 & Waste water & \\
\hline$P-117$ & Decon Return Pump & $N$ & G & Pump & Centrifugal & DeconWash & Drum fi \\
\hline \multicolumn{8}{|c|}{ HVAC Equipment for the New CsIX/Grout Bldg or New Grout Bldg } \\
\hline E-103 & VOG Condenser & $N$ & $\mathbf{G}$ & Condenser & & HVAC & Offgas \\
\hline$V-101$ & VOG Vapor Demister & $\mathrm{N}$ & $\mathbf{G}$ & Column & & HVAC & Offgas \\
\hline $\mathrm{EH}-101$ & VOG Superheater & N & $\mathbf{G}$ & Superheater & & HVAC & Offgas \\
\hline$F-103$ & HEPA Prefilter & $\mathrm{N}$ & $\mathbf{G}$ & Filter & & HVAC & Offgas \\
\hline$F-104 A$ & HEPA Filter (Stage 1) & $N$ & $\mathbf{G}$ & Filter & & HVAC & Offgas \\
\hline
\end{tabular}

The Scenario \# Bldg columns indicate the building location of equipment for each scenario. 


\begin{tabular}{|c|c|c|c|c|c|c|c|c|c|c|}
\hline \multicolumn{2}{|l|}{ dicated scenario. } & \multicolumn{5}{|c|}{ Size } & & & \multirow[b]{3}{*}{ Material } & \multirow{3}{*}{$\begin{array}{c}\text { Radiation } \\
\text { Field }\end{array}$} \\
\hline \multirow{2}{*}{$\begin{array}{c}\text { Flow Diag } \\
\text { Drawing } \\
\text { Number }\end{array}$} & \multirow{2}{*}{$\begin{array}{l}\text { Ref } \\
\text { Dwg }\end{array}$} & \multirow{2}{*}{$\begin{array}{l}\text { Capacity } \\
\text { (cu. meter } \\
\text { or noted) }\end{array}$} & \multirow{2}{*}{$\begin{array}{l}\text { Length } \\
\text { (ft) }\end{array}$} & \multirow{2}{*}{$\begin{array}{c}\text { Width or } \\
\text { Dia. } \\
(\mathrm{ft})\end{array}$} & \multirow{2}{*}{$\begin{array}{l}\text { Height } \\
\text { (ft) }\end{array}$} & \multirow{2}{*}{$\begin{array}{l}\text { Weight } \\
\text { (lbs) }\end{array}$} & \multicolumn{2}{|c|}{$\begin{array}{c}\text { Electrical } \\
\text { Requirements }\end{array}$} & & \\
\hline & & & & & & & (W) & $(\mathrm{HP})$ & & \\
\hline $9 \quad 10$ & & & & & & & & & & \\
\hline 10 & & & 30 & 3 & & & 5,600 & & SST & \\
\hline 10 & & & 3 & 3 & & & & 1.50 & SST & \\
\hline 10 & & & 3 & 3 & & & 1,200 & & SST & \\
\hline 10 & & & & & & & 900 & & & \\
\hline 10 & & & & & & & & 0.50 & SST & \\
\hline 10 & & & & & & & & & & \\
\hline 10 & & & & & & & & & & \\
\hline 10 & & & & & & & & & & \\
\hline 10 & & & & & & & & & & \\
\hline 10 & & & & & & & & & & \\
\hline 10 & & & & & & & & & & \\
\hline 10 & & & & & & & & & & \\
\hline 10 & & & & & & & & & & \\
\hline 10 & & & 10 & 3 & & & 2,200 & & SST & \\
\hline 10 & & & 3 & 3 & & & & 1.50 & SST & \\
\hline 10 & & & & & & & & & & \\
\hline 10 & & $2 \mathrm{Kg} / \mathrm{hr}$ & 2 & 2 & 4 & & & & SST & \\
\hline 10 & & $4 \mathrm{l} / \mathrm{min}$ & 3 & 2 & 3 & & & & SST & \\
\hline 11 & & & & & & & & & & \\
\hline 11 & & $2 \mathrm{Kg} / \mathrm{hr}$ & 2 & 2 & 4 & & & & SST & \\
\hline 2 & & 4 & & 5 & 8 & & & & FW Plastic & \\
\hline 2 & & 4 & & 5 & 8 & & & & SST & \\
\hline 2 & & 4 & & 5 & 8 & & & & & \\
\hline 2 & & 4 & & 5 & 8 & & & & SST & \\
\hline 2 & & 4 & & 5 & 8 & & & & & \\
\hline 2 & & $420 \mathrm{~V} / \mathrm{min}$ & 4 & 3 & 3 & & & & SST & \\
\hline 2 & & $420 \mathrm{~V} / \mathrm{min}$ & 4 & 3 & 3 & & & & SST & \\
\hline 2 & & $20 \mathrm{~V} / \mathrm{min}$ & 3 & 2 & 3 & & & & SST & \\
\hline 2 & & & & & & & & & & \\
\hline 2 & & $20 \mathrm{Vmin}$ & 3 & 2 & 3 & & & & SST & \\
\hline 2 & & & & & & & & & & \\
\hline 3 & & 19 & 14 & 8 & & & & & SST & \\
\hline 3 & & & & & & & & & & \\
\hline 3 & & & & & & & & & & \\
\hline 3 & & $76 \mathrm{~V} / \mathrm{min}$ & 4 & 2 & 3 & & & & SST & \\
\hline 3 & & $76 \mathrm{~V} \min$ & 4 & 2 & 3 & & & & SST & \\
\hline 9 & & $20 \mathrm{~V} / \mathrm{min}$ & 3 & 2 & 3 & & & & SST & \\
\hline 12 & & & & & & & & & & \\
\hline 12 & & & & & & & & & & \\
\hline 12 & & & & & & & & & SST & \\
\hline 12 & & & & 2 & 2 & & & & SST & \\
\hline
\end{tabular}


N - New CsIX/Grout Bldg, G - New Grout Bldg, NWCF - Existing Waste Calcining Facility, S - New Storage Bldg, na - not a part of

\begin{tabular}{|c|c|c|c|c|c|c|c|}
\hline \multirow{2}{*}{$\begin{array}{c}\text { Equip- } \\
\text { ment } \\
\text { Number }\end{array}$} & \multirow[b]{2}{*}{$\begin{array}{c}\begin{array}{c}\text { Equipment } \\
\text { Name }\end{array} \\
\end{array}$} & \multicolumn{2}{|c|}{ Scenario } & \multirow[b]{2}{*}{$\begin{array}{l}\text { Equipment } \\
\text { Type }\end{array}$} & \multirow[b]{2}{*}{$\begin{array}{l}\text { Additional } \\
\text { Description }\end{array}$} & \multirow[b]{2}{*}{$\begin{array}{c}\text { Main } \\
\text { System }\end{array}$} & \multirow[b]{2}{*}{ Subsy } \\
\hline & & $\begin{array}{c}1 \\
\text { Bldg }\end{array}$ & $\begin{array}{c}2 \\
\text { Bidg }\end{array}$ & & & & \\
\hline$F-104 B$ & HEPA Filter (Stage 2) & $\mathrm{N}$ & $G$ & Filter & & HVAC & Offgas \\
\hline$B L-101 A$ & Off Gas Blower & $N$ & $G$ & Blower & & HVAC & Offgas \\
\hline \multirow[t]{27}{*}{$B L-101 B$} & Off Gas Blower & N & $\mathbf{G}$ & Blower & & HVAC & Offgas \\
\hline & Heating Coils & $N$ & $\mathbf{G}$ & Heating Coil & Steam & HVAC & Zone 2 \\
\hline & Rough Prefilter & $\mathrm{N}$ & $\mathbf{G}$ & Filter & & HVAC & Zone 2 \\
\hline & Zone 2 HEPA Filter Housing & N & $\mathbf{G}$ & Filter & $2 \times 5$ redundant & HVAC & Zone 2 \\
\hline & Zone 2 HEPA Filter Housing & $\mathrm{N}$ & $G$ & Filter & $2 \times 5$ redundant & HVAC & Zone 2 \\
\hline & Zone 2 Supply Fan & $N$ & $\mathrm{n} / \mathrm{a}$ & Blower & $10500 \mathrm{~cm} @ 3.4^{\mathrm{u}}$ W.C & HVAC & Zone 2 \\
\hline & Zone 2 Supply Fan & $\mathrm{N}$ & $\mathrm{n} / \mathrm{a}$ & Blower & $10500 \mathrm{cfm} @ 3.4^{\mathrm{n}}$ W.C & HVAC & Zone 2 \\
\hline & Zone 3 Exhaust Fan & N & $\mathrm{n} / \mathrm{a}$ & Blower & $9500 \mathrm{cfm} @ 6.75^{\mathfrak{n}}$ W.C. & HVAC & Zone3 \\
\hline & Zone 3 Exhaust Fan & N & $\mathrm{n} / \mathrm{a}$ & Blower & $9500 \mathrm{cfm} \otimes 6.75^{\mathrm{u}}$ W.C. & HVAC & Zone3 \\
\hline & Zone 3 Exhaust HEPA Filter & $N$ & $n / a$ & Filter & $2 \times 5$ redundant & HVAC & Zone3 \\
\hline & Zone 3 Exhaust HEPA Filter & N & na & Filter & $2 \times 5$ redundant & HVAC & Zone3 \\
\hline & Zone 3 Inlet Fan & N & n/a & Blower & $10500 \mathrm{~cm} @ 6.75^{\prime \prime}$ W.C. & HVAC & Zone3 \\
\hline & Zone 3 Inlet Fan & N & $n / a$ & Blower & $10500 \mathrm{cfm} @ 6.75^{\mathrm{n}}$ W.C. & HVAC & Zone3 \\
\hline & Zone 3 Supply HEPA Filter & $N$ & $\mathrm{n} / \mathrm{a}$ & Filter & $2 \times 5$ redundant & HVAC & Zone3 \\
\hline & Zone 3 Supply HEPA Filter & $N$ & $\mathrm{n} / \mathrm{a}$ & Filter & $2 \times 5$ redundant & HVAC & Zone3 \\
\hline & Office Space HVAC Unit & $\mathrm{n} / \mathrm{a}$ & $G$ & HVAC Unit & Pkged unit, $400 \mathrm{cfm}, 1 \mathrm{~T}$ & HVAC & Office \\
\hline & Zone 2 Supply Fan & $\mathrm{n} / \mathrm{a}$ & $\mathbf{G}$ & Blower & $6,400 \mathrm{cfm}$ @ $3.4^{\mathrm{N}}$ W.C. & HVAC & Zone 2 \\
\hline & Zone 2 Supply Fan & $\mathrm{n} / \mathrm{a}$ & $\mathbf{G}$ & Blower & $6,400 \mathrm{~cm} @ 3.4^{\mathrm{n}}$ W.C. & HVAC & Zone 2 \\
\hline & Zone 3 Exhaust Fan & $\mathrm{n} / \mathrm{a}$ & $\mathbf{G}$ & Blower & $3400 \mathrm{cfm} @ 6.75^{\mathrm{n}}$ W.C. & HVAC & Zone3 \\
\hline & Zone 3 Exhaust Fan & $\mathrm{n} / \mathrm{a}$ & $\mathbf{G}$ & Blower & $3400 \mathrm{cfm} @ 6.75^{\mathrm{u}}$ W.C. & HVAC & Zone3 \\
\hline & Zone 3 Exhaust HEPA Filter & $\mathrm{n} / \mathrm{a}$ & $\mathbf{G}$ & Filter & $2 \times 2$ redundant & HVAC & Zone3 \\
\hline & Zone 3 Exhaust HEPA Filter & $\mathrm{n} / \mathrm{a}$ & $\mathbf{G}$ & Filter & $2 \times 2$ redundant & HVAC & Zone3 \\
\hline & Zone 3 Inlet Fan & $\mathrm{n} / \mathrm{a}$ & $\mathbf{G}$ & Blower & $6400 \mathrm{cfm} @ 6.75^{\mathrm{n}}$ W.C. & HVAC & Zone3 \\
\hline & Zone 3 Inlet Fan & $n / a$ & $\mathbf{G}$ & Blower & $6400 \mathrm{cfm} @ 6.75^{\mathrm{u}}$ W.C. & HVAC & Zone3 \\
\hline & Zone 3 Supply HEPA Filter & $n / a$ & $\mathbf{G}$ & Filter & $2 \times 3$ redundant & HVAC & Zone3 \\
\hline & Zone 3 Supply HEPA Filter & $n / a$ & $G$ & Filter & $2 \times 3$ redundant & HVAC & Zone3 \\
\hline & \multicolumn{7}{|c|}{ Existing HVAC Equipment in NWCF } \\
\hline$E-103^{*}$ & VOG Condenser & $\mathrm{n} / \mathrm{a}$ & NWCF & Condenser & Existing HE-NCC-336 & HVAC & Offgas \\
\hline$V-101^{*}$ & VOG Vapor Demister & $\mathrm{n} / \mathrm{a}$ & NWCF & Column & Existing VES-NCC-1360 & HVAC & Offgas \\
\hline $\mathrm{EH}-101^{*}$ & VOG Superheater & $\mathrm{n} / \mathrm{a}$ & NWCF & Superheater & Existing HE-NCC-335 & HVAC & Offgas \\
\hline$F-103 / 10$ & 4HEPA Prefilter \& Filter & $\mathrm{n} / \mathrm{a}$ & NWCF & Filter & Existing F-NCC-130 & HVAC & Offgas \\
\hline BL-101* & Off Gas Blower & $\mathrm{n} / \mathrm{a}$ & NWCF & Blower & Existing BLO-NCC-242 & HVAC & Offgas \\
\hline \multicolumn{8}{|c|}{ Electrical Equipment } \\
\hline & Motor Control Center & N & $\mathbf{G}$ & Electrical & & & \\
\hline & UPS & $N$ & $\mathbf{G}$ & Electrical & $30-50 \mathrm{kVA}$ & & \\
\hline \multicolumn{8}{|c|}{ Cranes, PAR's \& Manipulators } \\
\hline & UDS Unloading Area Crane & $\mathbf{N}$ & NWCF & Crane & & UDS & Operatic \\
\hline & P\&V Corridor Crane & $N$ & G & Crane & & Operations & \\
\hline & Cell Hatch Crane & N & $\mathbf{G}$ & Crane & & Operations & \\
\hline & Maintenance Monorail & N & $\mathbf{G}$ & Crane & & Operations & \\
\hline & P\&V Corridor PAR & $\mathbf{N}$ & $\mathrm{n} / \mathrm{a}$ & Crane & & Operations & \\
\hline & Maintenance PAR & $N$ & $n / a$ & Crane & & Operations & \\
\hline & UDS Drying Cell Manipulator & $N$ & $\mathrm{n} / \mathrm{a}$ & Manipulator & & UDS & Mainten \\
\hline & Waste Resin Cell Manipulator & $N$ & $\mathrm{n} / \mathrm{a}$ & Manipulator & & lon exchange & Mainten \\
\hline & TRUPACT Building Crane & $S$ & $S$ & Crane & 10 ton bridge crane & Trupact & Interim : \\
\hline
\end{tabular}

The Scenario \# Bldg columns indicate the building location of equipment for each scenario. 


\begin{tabular}{|c|c|c|c|c|c|c|c|c|c|c|c|}
\hline \multicolumn{3}{|c|}{ icated scenario. } & \multicolumn{5}{|c|}{ Size } & & & & \\
\hline \multirow[b]{2}{*}{$\underline{\mathrm{m}}$} & \multirow{2}{*}{$\begin{array}{l}\text { Flow Diag } \\
\text { Drawing } \\
\text { Number }\end{array}$} & \multirow{2}{*}{$\begin{array}{l}\text { Ref } \\
\text { Dwg }\end{array}$} & \multirow{2}{*}{$\begin{array}{l}\text { Capacity } \\
\text { (cu. meter } \\
\text { or noted) }\end{array}$} & \multirow{2}{*}{$\begin{array}{c}\text { Length } \\
\text { (ft) }\end{array}$} & \multirow{2}{*}{$\begin{array}{c}\text { Width or } \\
\text { Dia. } \\
(\mathrm{ft})\end{array}$} & \multirow{2}{*}{$\begin{array}{l}\text { Height } \\
\text { (ft) }\end{array}$} & \multirow{2}{*}{$\begin{array}{c}\text { Weight } \\
\text { (lbs) }\end{array}$} & \multicolumn{2}{|c|}{$\begin{array}{c}\text { Electrical } \\
\text { Requirements }\end{array}$} & \multirow[b]{2}{*}{ Material } & \multirow{2}{*}{$\begin{array}{c}\text { Radiation } \\
\text { Field }\end{array}$} \\
\hline & & & & & & & & $(W)$ & (HP) & & \\
\hline \multicolumn{12}{|c|}{12} \\
\hline & 12 & & & 4 & 4 & & & & 2.50 & SST & \\
\hline & 12 & & & 4 & 4 & & & & 2.50 & SST & \\
\hline
\end{tabular}

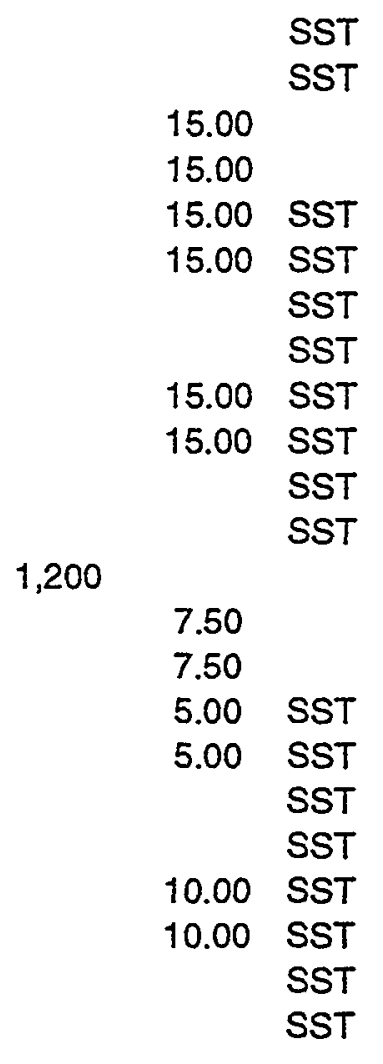


N - New CsIX/Grout Bldg, G - New Grout Bldg, NWCF - Existing Waste Calcining Facility, S - New Storage Bldg, n/a - not a part

\begin{tabular}{|c|c|}
\hline $\begin{array}{c}\text { Equip- } \\
\text { ment } \\
\text { Number }\end{array}$ & $\begin{array}{c}\text { Equipment } \\
\text { Name }\end{array}$ \\
\cline { 2 - 3 }
\end{tabular}

\begin{tabular}{|c|c|}
\hline \multicolumn{2}{|c|}{ Scenario } \\
\hline $\begin{array}{c}1 \\
\text { Bldg }\end{array}$ & B \\
\hline $\mathrm{S}$
\end{tabular}

\begin{tabular}{|c|c|}
\hline 2 & \\
Bldg & \\
\hline$S$ &
\end{tabular}

Equipment
Type

10 ton

Additional

Miscellaneous Equipment

Freight Elevator

Drum Movement Forklift

Drum Storage Forklift

Drum Movement Lifting Fixtures

$N \quad G \quad$ Lift

$N \quad G$ Forklift

Drum Movement Lifting Fixtures $S \quad S$ Mechanism

Head Space Sampling \& Video Inspection (Storage Building)

Inlet/Load Conveyor
Index Table Conveyor
Index Lift Table
Index Arm
Airlock
Airlock Door
Airlock Door
Airlock Conveyor
Glovebox
Glovebox Inlet Door
Glovebox Coneyor
Video Camera
Headspace Sample Tube
Mechanical Swip Arm
Hood Enclosure
Filter Removal Arm
Filter Install Arm
Glovebox Outlet Door
Airlock
Airlock Door
Airlock Door
Airlock Conveyor
Unload Conveyor
Glovebox Exhaust Fan
Glovebox Exhaust Fan
Airlock Exhaust Fan
Airlock Exhaust Fan
Video Control Panel
.

HVAC Equpment for the New Storage Bldg

Interim Storage Air Handler

Interim Storage Condenser (10T)

Interim Storage Exhaust Fan

Interim Storage Exhaust Fan

S
S
S
S
S
S
S
S
S
S
S
S
S
S
S
S
S
S
S
S
S
S
S
S
S
S
S

$S$

S

$\mathrm{s}$

S

S

$S$

Conveyor

Conveyor Self powered rollers

Mechanism

Mechanism

Booth

Door

Door

Conveyor

Booth

Door

Conveyor

Electric motor driven

Electric motor driven

Self powered rollers

w/window \& HEPA

Electric motor driven

Camera

Self powered rollers

Mechanism

Hardened, rotatable

Mechanism

Auto swipe, remote

Booth

Clear

Mechanism Automated

Mechanism Automated

Door

Electric motor driven

Booth

Door

Door

Electric motor driven

Electric motor driven

Conveyor

Self powered rollers

Conveyor

Chain drive

Blower

One on backup power

Blower

Blower

One on backup power

Blower

Mechanism

mon

$s$

S

S

TRUPACT II Building
Stretch Wrap Machines
Stretch Wrap Machines
Pallet Weight Machine
Pallet Weight Machine
Vacuum Pump

$S$

$\begin{array}{lll}\text { S } & \text { S } & \text { Mechanism } \\ \text { S } & \text { S } & \text { Mechanism } \\ \text { S } & \text { S } & \text { Mechanism } \\ \text { S } & \text { S } & \text { Mechanism } \\ \text { S } & \text { S } & \text { Mechanism } 34 \mathrm{cfm}\end{array}$

S HVAC Unit $3200 \mathrm{cfm} @ 2.75^{\mathrm{x}}$ S.P.

$S$ Condenser Air cooled, pad mount

S Blower $1400 \mathrm{cfm}, 2$ speed

S Blower $1400 \mathrm{cfm}, 2$ speed

The Scenario \# Bldg columns indicate the building location of equipment for each scenario. 


\begin{tabular}{|c|c|c|c|c|c|c|c|c|c|c|}
\hline \multicolumn{2}{|l|}{ dicated scenario. } & \multicolumn{5}{|c|}{ Size } & \multirow{2}{*}{\multicolumn{2}{|c|}{$\begin{array}{c}\text { Electrical } \\
\text { Requirements }\end{array}$}} & \multirow[b]{3}{*}{ Material } & \\
\hline \begin{tabular}{|c|c}
$\begin{array}{c}\text { Flow Diag } \\
\text { Drawing }\end{array}$ \\
\end{tabular} & Ref & $\begin{array}{l}\text { Capacity } \\
\text { (cu. meter }\end{array}$ & Length & $\begin{array}{l}\text { Width or } \\
\text { Dia. }\end{array}$ & Height & Weight & & & & \multirow{2}{*}{$\begin{array}{l}\text { Radiation } \\
\text { Field }\end{array}$} \\
\hline Number & Dwg & or noted) & (ft) & $(\mathrm{ft})$ & $(\mathrm{ft})$ & (lbs) & $(W)$ & (HP) & & \\
\hline
\end{tabular}

orage 10T

orage

orage

orage

rage

rage

Jrage

Jrage

rage

rage

rage

Jrage

Jrage

rage

Jrage

rage

Jrage

rage

rrage

Jrage

Jrage

rage

rage

rage
43

103
3

3

1.5

43

53

$\begin{array}{cc}10 & 3 \\ 3 & 3 \\ & 1.5\end{array}$

3

$\begin{array}{lll} & 2.00 & \text { SST } \\ 1,200 & & \text { SST } \\ & 2.00 & \text { SST } \\ & & 7 \text { ga. SST } \\ & & \\ & 0.50 & \text { SST } \\ & 0.50 & \text { SST } \\ 1,600 & & \text { SST } \\ & & 7 \text { ga. SST } \\ & 0.50 & \text { SST } \\ 2,000 & & \text { SST } \\ 1,500 & & \\ & & \\ 3,000 & & \\ & & \text { Plastic } \\ 400 & & \text { SST } \\ 400 & & \text { SST } \\ & & \\ & & 7 \text { ga. SST } \\ & 0.50 & \text { SST } \\ & 0.50 & \text { SST } \\ & & \text { SST } \\ 1,600 & 2.00 & \text { SST } \\ & 2.00 & \\ & 2.00 & \\ & 1.00 & \\ & 1.00 & \\ & & \end{array}$

10,200

7.50

7.50

$\begin{array}{ll}6 & 6 \\ 6 & 6 \\ 4 & 4 \\ 4 & 4\end{array}$


N - New CsIX/Grout Bldg, G - New Grout BIdg, NWCF - Existing Waste Calcining Facility, S - New Storage Bldg, na - not a part of

\begin{tabular}{|c|c|c|c|c|c|c|c|}
\hline \multirow{2}{*}{$\begin{array}{c}\text { Equip- } \\
\text { ment } \\
\text { Number }\end{array}$} & \multirow[b]{2}{*}{$\begin{array}{c}\text { Equipment } \\
\text { Name }\end{array}$} & \multicolumn{2}{|c|}{ Scenario } & \multirow[b]{2}{*}{$\begin{array}{c}\text { Equipment } \\
\text { Type }\end{array}$} & \multirow[b]{2}{*}{$\begin{array}{l}\text { Additional } \\
\text { Description }\end{array}$} & \multirow[b]{2}{*}{$\begin{array}{c}\text { Main } \\
\text { System }\end{array}$} & \multirow[b]{2}{*}{ Subsy } \\
\hline & & $\begin{array}{c}1 \\
\text { Bldg }\end{array}$ & $\begin{array}{c}2 \\
\text { Bldg }\end{array}$ & & & & \\
\hline & Vacuum Pump & $S$ & $S$ & Mechanism & $34 \mathrm{cfm}$ & Trupact & Interim : \\
\hline & 12 Electric Unit Heaters & $S$ & S & HVAC Unit & $20 \mathrm{Kw}$ & Trupact & Interin \\
\hline & Exhaust Fan & $S$ & S & Blower & Sidewall, 2 speed & Trupact & Interin \\
\hline & Exhaust Fan & $S$ & $S$ & Blower & Sidewall, 2 speed & Trupact & Interin \\
\hline
\end{tabular}

\section{TOTALS}




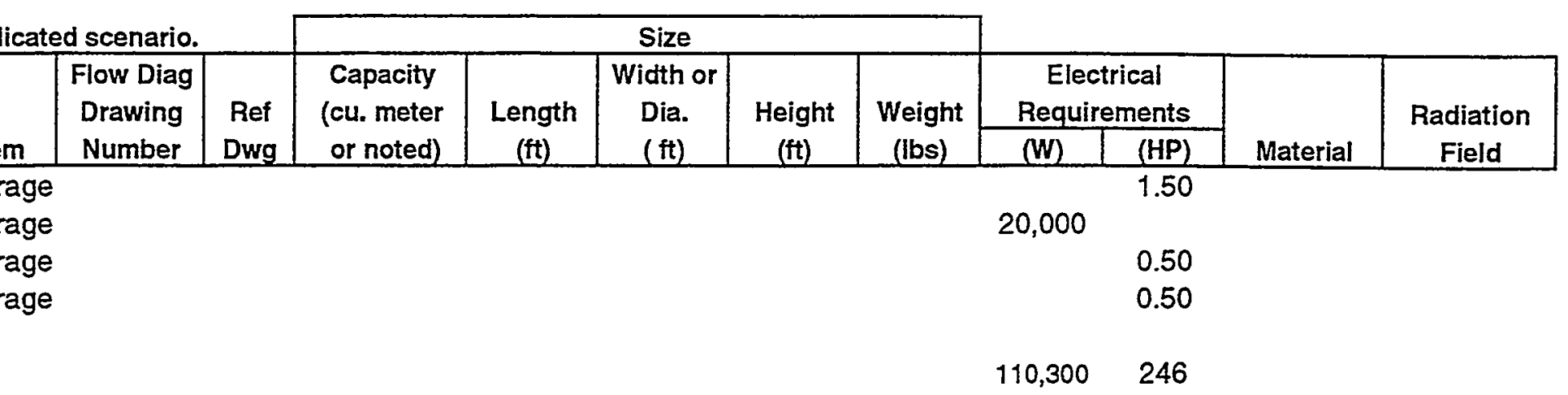


Appendix G

List of Applicable Regulations, Codes, and Standards 
Reference

Title

Applies to

Code of Federal Regulations

10 CFR 435 Energy Conservation Voluntary

Performance Standards for New

Buildings, Mandatory for Federal

Buildings

10 CFR 820

$10 \mathrm{CFR}$

830.120

10 CFR 835

10 CFR 1021

10 CFR 1046

20 CFR 1910

29 CFR 1926

40 CFR 61

40 CFR 191

40 CFR 264

40 CFR 265

Activities

CFR 835

1508) for General Industry

Occupational Safety and Health

Regulations for Construction

National Emission Standards for

Hazardous Air Pollutants

Transuranic Radioactive Wastes
Procedural Rules for DOE Nuclear

Quality Assurance Requirements for 10

Occupational Radiation Protection

National Environmental Policy Act

(NEPA) Implementing Procedures (Also

in DOE O 5440.1D and 40 CFR 1500-

Physical Protection of Security Interests

Occupational Safety and Health Standards

Environmental Radiation Protection

Standards for Management and Disposal

of Spent Nuclear Fuel, High-Level and

Standards for Owners and Operators of

Hazardous Waste Treatment, Storage and

Disposal Facilities (DAPA 16.01.05.008)

Interim Status Standards for Owners and

Operators of Hazardous Waste Storage, Treatment and Disposal Facilities

40 CFR 270.14 Contents of Part B: General Requirements

State of Idaho, Statutes, Rules and Regulations

Idaho Code 39- Hazardous Waste Management Act

44

IDAPA

16.01 .01

IDAPA

16.01.01.161

IDAPA

16.01.01.210

IDAPA

16.01.01.575
Rules for the Control of Air Pollution in

Idaho

Toxic Substances

Demonstration of Reconstruction

Compliance with Toxic Standards

Air Quality Standards and Area

Classification
$\mathrm{H} \& \mathrm{~V}$ system, lighting systems, building insulation.

Procedures

Quality assurance activities

Radiation protection for occupational workers

Project pollution prevention systems

Security systems

Total facility and supporting systems

Construction of facility and supporting systems

Air pollution control and monitoring systems

Radiation confinement structures and systems

Regulatory requirements for design, construction and operation of the facility Operational procedures, and design requirements.

Interim storage facility design

Control of hazardous waste

Air pollution control equipment Control of toxic substances

Control of hazardous waste

Air pollution control equipment 
Appendix G Regulations, Codes and Standards

Reference Title

Applies to

\begin{tabular}{|c|c|c|}
\hline $\begin{array}{l}\text { DAPA } \\
16.01 .01 .650\end{array}$ & Rules for Control of Fugitive Dust & $\begin{array}{l}\text { Construction or demolition } \\
\text { activities }\end{array}$ \\
\hline DAPA & Water Quality Standards and Wastewater & Waste water system \\
\hline 16.01 .02 & Treatment & \\
\hline $\begin{array}{l}\text { DAPA } \\
16.01 .05\end{array}$ & Rules and Standards for Hazardous Waste & $\begin{array}{l}\text { RCRA regulations as adopted } \\
\text { by the State of Idaho }\end{array}$ \\
\hline $\begin{array}{l}\text { WAPA } \\
16.01 .08\end{array}$ & $\begin{array}{l}\text { Idaho Rules for Public Drinking Water } \\
\text { Systems }\end{array}$ & Potable water utility system \\
\hline $\begin{array}{l}\text { DDAPA } \\
16.01 .09\end{array}$ & Idaho Radiation Control Rules & $\begin{array}{l}\text { Worker protection, radiation } \\
\text { shielding }\end{array}$ \\
\hline
\end{tabular}

DOE Orders

DOE O 151.1 Comprehensive Emergency Management System

DOE O $420.1 \quad$ Facility Safety

DOE O 430.1

Life Cycle Cost Asset Management

DOE $O 430.2$

In House Energy Management

DOE O 435.1

Radioactive Waste Management

Facility emergency plans

Criticality control, fire

protection, design for natural

phenomena

Facility design criteria

H\&V System

Replaces DOE 5820.2A when

(Draft)

approved and incorporated into the site contract.

DOE O 440.1 Wiorker Protection Management for DOE Federal and Contractor Employees

DOE 460.1A Packaging and Transportation Safety

DOE 5400.1 General Environmental Protection

Requirements

DOE 5400.5 Radiation Protection of the Public and the Environment

DOE 5480.4 Environmental Protection, Safety, and Health Protection Standards

DOE 5480.7A Fire Protection

DOE 5480.11 Radiation Protection for Occupational Workers

DOE 5480.19 Conduct of Operations Requirements for DOE Facilities

DOE 5480.20A Personnel Selection, Qualification and

Training Requirements for DOE Nuclear Facilities

DOE 5480.21 Unreviewed Safety Questions

DOE 5480.22 Technical Safety Requirements

DOE 5480.23 Nuclear Safety Analysis Reports

Systems to protect employees

Packaging system

Environmental protection features

Features to limit radiation dose to the public

Requires compliance to the OSHA standards

Replaced by 10 CFR 835 .

Operational procedures

Requirements for personnel

DOE 5480.28 Natural Phenomena Hazard Mitigation

Safety issues

Safety features

Safety analysis

Design of structures, systems

and components to resist

natural phenomena 
Appendix G Regulations, Codes and Standards

\begin{tabular}{cll} 
Reference & \multicolumn{1}{c}{ Title } & \multicolumn{1}{c}{ Applies to } \\
\hline DOE 5481.1B & Environmental, Safety, and Health & Safety analysis \\
& Program for DOE Operations & Quality assurance activities \\
DOE 5700.6C & Quality Assurance & $\begin{array}{l}\text { Appropriate documentation of } \\
\text { features for radioactive waste }\end{array}$ \\
& Radioactive Waste Management & management
\end{tabular}

See Note 3.

DOE-ID References

DOE-ID AES DOE-ID Architectural Engineering

Standards (AES)

INEEL Radiological Control Manual

INEEL Welding Manual

Total Facility and supporting

systems

Worker protection, radiation

shielding

On-site welding

DOE Standards and Guides for Use in Complying with DOE Orders

DOE STD 1027 Hazard Categorization and Accident

Analysis Techniques

Compliance with DOE

5480.23

DOE STD 3007 Guidelines for Preparing Criticality Safety

Evaluations at DOE Non-Reactor Nuclear

Facilities

DOE STD 3009 Preparation Guide. for U.S. DOE

Nonreactor Nuclear Facility Safety

Safety analysis/evaluations

Analysis Reports

DOE Standards and Guides Imposed by the DOE-ID AES

DOE STD 1020 Natural Phenomena Hazards Design and Evaluation Criteria for Department of Energy Facilities

DOE STD 1021 Natural Phenomena Hazards Performance Categorization Guidelines for Structures, Systems, and Components (SSC).

DOE STD 1061 Fire Protection Design Criteria

DOE HDBK DOE Fire Protection Handbook

1062

Facility and system design for natural phenomena

Categorization of SSC's for design per DOE STD 1020

Fire protection systems and facility materials

Fire protection systems

National Codes and Standards Imposed by the DOE-ID AES Site and Civil

AASHTO Geometric Design of Highways and Streets

FM

FM 1-28

\section{Architectural}

Factory Mutual Engineering Association Approval Guide

Wind Loads to Roof Systems and Roof Deck Securement
Geometric design of roads, streets, access drives and parking lots

Facility materials and construction Loading on roof systems 
Appendix G Regulations, Codes and Standards

\begin{tabular}{|c|c|c|}
\hline Reference & $\begin{array}{r}\text { Title } \\
\end{array}$ & Applies to \\
\hline $\mathrm{ICBO} \mathrm{UBC}$ & Uniform Building Code & $\begin{array}{l}\text { Systems, structures and } \\
\text { components not covered by } \\
\text { other codes or standards }\end{array}$ \\
\hline NFPA 80 & Fire Doors and Windows & Fire doors and windows \\
\hline NFPA 101 & Life Safety Code & $\begin{array}{l}\text { Life safety systems, building } \\
\text { layout }\end{array}$ \\
\hline \multirow[t]{2}{*}{ UL } & $\begin{array}{l}\text { Underwriters Laboratories Fire Resistance } \\
\text { Directory }\end{array}$ & $\begin{array}{l}\text { Facility construction and } \\
\text { materials and applicable } \\
\text { equipment. }\end{array}$ \\
\hline & Structural & \\
\hline AISC (ASD) & $\begin{array}{l}\text { Specification for Structural Steel } \\
\text { Buildings (or) }\end{array}$ & Structural steel \\
\hline AISC (LRFD) & $\begin{array}{l}\text { LRFD Specification for Structural Steel } \\
\text { Buildings }\end{array}$ & Structural steel \\
\hline ACI 318 & $\begin{array}{l}\text { Building Code Requirements for } \\
\text { Reinforced Concrete }\end{array}$ & Non-safety related concrete \\
\hline ACI 349 & $\begin{array}{l}\text { Code Requirements for Nuclear Safety } \\
\text { Related Concrete Structures }\end{array}$ & $\begin{array}{l}\text { Safety related concrete } \\
\text { structures }\end{array}$ \\
\hline $\begin{array}{l}\text { ANSI/AISC } \\
\text { N690 }\end{array}$ & $\begin{array}{l}\text { Specification for the Design, Fabrication, } \\
\text { and Erection of Steel Safety Related } \\
\text { Structures of Nuclear Facilities }\end{array}$ & Safety related structural steel \\
\hline ANSI/ASCE 4 & $\begin{array}{l}\text { Seismic Analysis of Safety-Related } \\
\text { Nuclear Structures (optional) }\end{array}$ & $\begin{array}{l}\text { Seismic analysis of safety } \\
\text { related structures }\end{array}$ \\
\hline ANSI/ASCE 7 & $\begin{array}{l}\text { Minimum Design Loads for Buildings and } \\
\text { Other Structures }\end{array}$ & Building design loads \\
\hline AWS D1.1 & $\begin{array}{l}\text { Structural Welding Code - Steel } \\
\text { Mechanical (General) }\end{array}$ & Welding of structural steel \\
\hline \multirow[t]{2}{*}{ IAPMCO/ICBO } & Uniform Mechanical Code & $\begin{array}{l}\text { Mechanical systems not } \\
\text { covered by other standards }\end{array}$ \\
\hline & $\begin{array}{l}\text { Mechanical (Piping Systems and } \\
\text { Vessels) }\end{array}$ & \\
\hline ANSI B31.1 & Power Piping & $\begin{array}{l}\text { Steam and condensate piping } \\
\text { systems not covered by B31.9. }\end{array}$ \\
\hline ANSI B31.3 & $\begin{array}{l}\text { Chemical Plant and Petroeum Refinery } \\
\text { Piping }\end{array}$ & Process piping systems \\
\hline ANSI B31.9 & Building Service Piping & $\begin{array}{l}\text { Building service piping } \\
\text { systems (nonpotable water, air, } \\
\text { etc.) }\end{array}$ \\
\hline ASME & $\begin{array}{l}\text { Boiler and Pressure Vessel Codes } \\
\text { Mechanical (HVAC Systems) }\end{array}$ & Pressure vessels \\
\hline ASHRAE 90 & $\begin{array}{l}\text { Efficient Design of New Buildings Except } \\
\text { Low-Rise Residential Buildings }\end{array}$ & HVAC systems \\
\hline IAPMCO UPC & Uniform Plumbing Code & $\begin{array}{l}\text { Plumbing systems (potable } \\
\text { water, sewer) within the } \\
\text { facility }\end{array}$ \\
\hline
\end{tabular}




\section{Appendix G Regulations, Codes and Standards}

Reference

Title

Applies to

\begin{tabular}{|c|c|c|}
\hline NFPA 90 & $\begin{array}{l}\text { Standard for the Installation of Air } \\
\text { Conditioning and Ventilating System } \\
\text { Mechanical (Cranes and Hoists) }\end{array}$ & HVAC systems \\
\hline ANSI B30.16 & Overhead Hoists (Underhung) & Overhead hoists \\
\hline ASME B30.2 & Overhead and Gantry Cranes & Cranes \\
\hline ASME NOG-1 & $\begin{array}{l}\text { Rules for Construction of Overhead and } \\
\text { Gantry Cranes }\end{array}$ & $\begin{array}{l}\text { Overhead cranes carrying } \\
\text { nuclear material }\end{array}$ \\
\hline CMAA 70 & $\begin{array}{l}\text { Specification for Electrical Overhead } \\
\text { Traveling Cranes }\end{array}$ & $\begin{array}{l}\text { Overhead electrical cranes not } \\
\text { covered by CMAA } 74 \text {. }\end{array}$ \\
\hline CMAA 74 & $\begin{array}{l}\text { Specification for Top Running and Under } \\
\text { Running Single Girder Electric Overhead } \\
\text { Traveling Cranes } \\
\text { Fire Protection }\end{array}$ & Single girder overhead cranes \\
\hline ICBO UFC & Uniform Fire Code & Fire protection systems \\
\hline NFPA 13 & Installation of Sprinkler Systems & Fire sprinkler system \\
\hline NFPA 24 & $\begin{array}{l}\text { Installation of Private Fire Service Mains } \\
\text { and Their Appurtenances } \\
\text { Electrical }\end{array}$ & Firewater supply system \\
\hline ANSI C2 & National Electrical Safety Code (NESC) & Electrical systems \\
\hline IES & Lighting Handbook & Lighting system \\
\hline NFPA 70 & National Electrical Code & Electrical systems \\
\hline NFPA 72 & National Fire Alarm Code & $\begin{array}{l}\text { Fire alarms, emergency } \\
\text { notification system }\end{array}$ \\
\hline NFPA 780 & $\begin{array}{l}\text { Lightning Protection Code } \\
\text { Radiation Monitoring }\end{array}$ & Lightning protection \\
\hline $\begin{array}{l}\text { ANSI N42.17 } \\
\text { Series }\end{array}$ & $\begin{array}{l}\text { Radiation Instrumentation Performance } \\
\text { Specifications for Health Physics } \\
\text { Instrumentation }\end{array}$ & $\begin{array}{l}\text { Radiation monitoring } \\
\text { equipment }\end{array}$ \\
\hline
\end{tabular}

Other National Codes and Standards Applicable to this Project Mechanical (HVAC systems)

ANSI/ASME Nuclear Power Plant Air Cleaning Units N509 and Components

ANSI/ASME Testing of Nuclear Air Cleaning Systems

N510

NFPA 801

Fire Protection

Facilites Handling Radioactive Materials

Electrical

NFPA 110 Emergency and Standby Power Systems

HVAC systems for primary and secondary containment HVAC systems for primary and secondary containment

Fire protection systems, and materials

Emergency and standby power systems 


\section{Appendix G Regulations, Codes and Standards}

Note 1. Additional requirements that will affect this project are found in EDF-WTS-003, Regulatory Requirements for the Design, Construction, and Operations of the ICPP Proposed Waste Processing Facilities

Note 2. Additional information is also found in EDF-WTS-004, Regulatory and Design Requirements for Waste Treatment Facilities.

Note 3. DOE 6430.1A is currently imposed by the LMITCO/DOE contract "for specific facilities under the purview of the Defense Nuclear Facilities Safety Board". However, it will not apply after 10 CFR 830.340 and DOE O 420.1 are finalized and incorporated into the contract. It may be used as a guide for design but it will not be used as a requirement for this project. 